\title{
HOMER Economic Models - US Navy
}

\author{
Kurt S. Myers \\ Jason W. Bush
}

February 2016

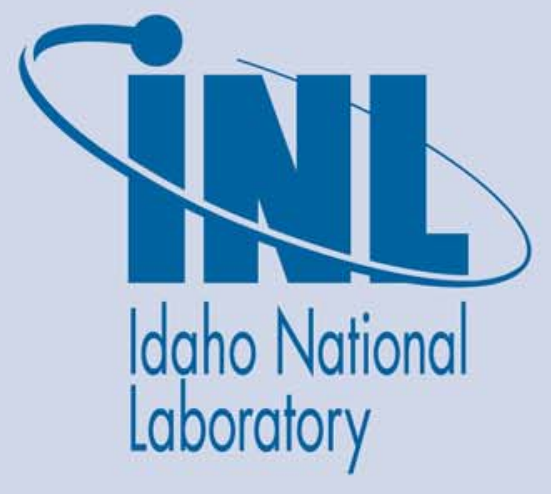

The INL is a U.S. Department of Energy National Laboratory operated by Battelle Energy Alliance 


\section{DISCLAIMER}

This information was prepared as an account of work sponsored by an agency of the U.S. Government. Neither the U.S. Government nor any agency thereof, nor any of their employees, makes any warranty, expressed or implied, or assumes any legal liability or responsibility for the accuracy, completeness, or usefulness, of any information, apparatus, product, or process disclosed, or represents that its use would not infringe privately owned rights. References herein to any specific commercial product, process, or service by trade name, trade mark, manufacturer, or otherwise, does not necessarily constitute or imply its endorsement, recommendation, or favoring by the U.S. Government or any agency thereof. The views and opinions of authors expressed herein do not necessarily state or reflect those of the U.S. Government or any agency thereof. 
INL/EXT-16-38069

Revision 0

\section{HOMER Economic Models - US Navy}

Kurt S. Myers

Jason W. Bush

February 2016

Idaho National Laboratory

Originating Organization [optional]

Idaho Falls, Idaho 83415

http://www.inl.gov

Prepared for the

U.S. Department of Energy

Office of Energy Efficiency \& Renewable Energy

Under DOE Idaho Operations Office

Contract DE-AC07-05ID14517 


\section{CONTENTS}

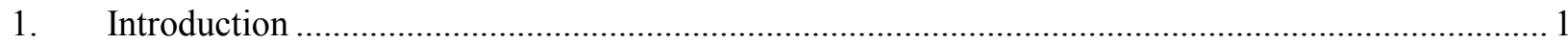

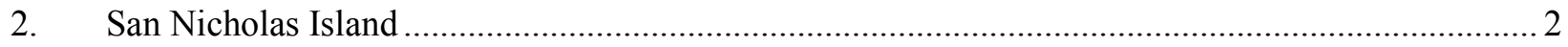

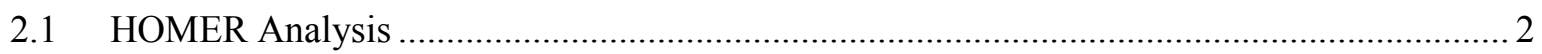

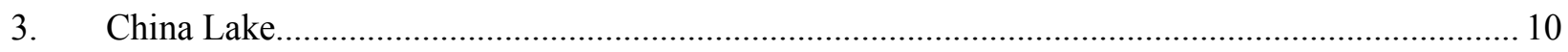

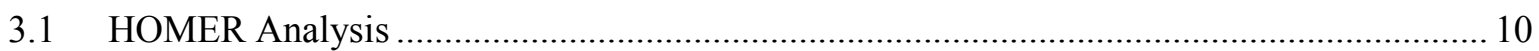

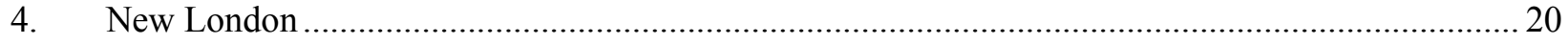

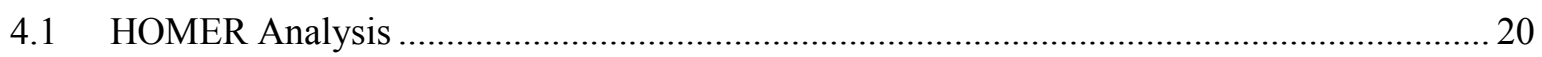

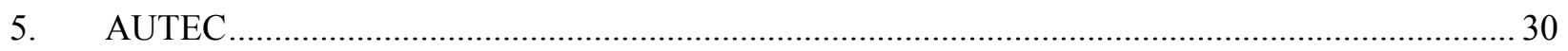

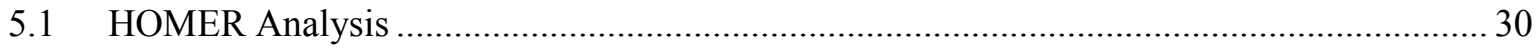

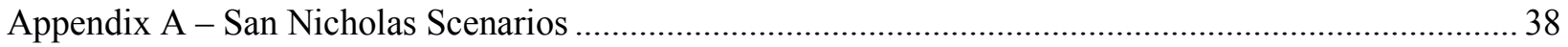

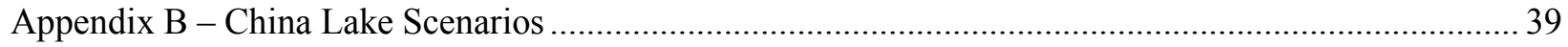

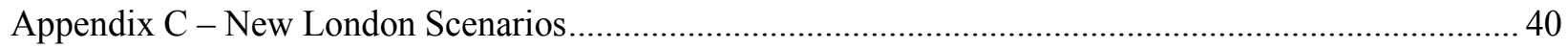

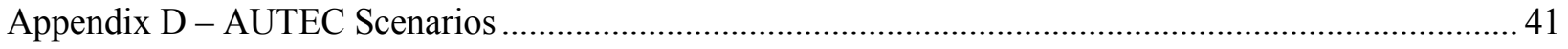

\section{FIGURES}

Figure 1: Navy locations modeled in HOMER .................................................................................. 1

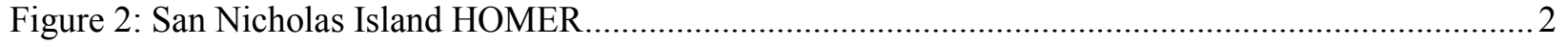

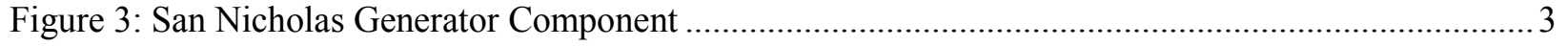

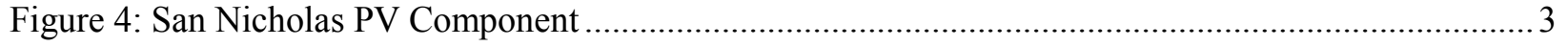

Figure 5: San Nicholas Wind Turbine Component...........................................................................

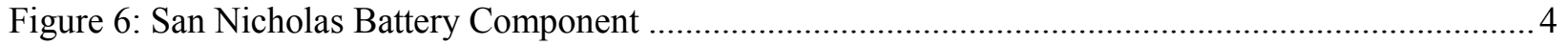

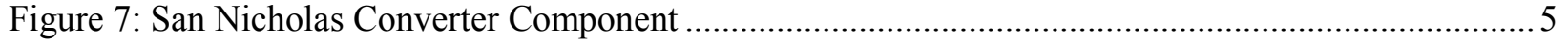

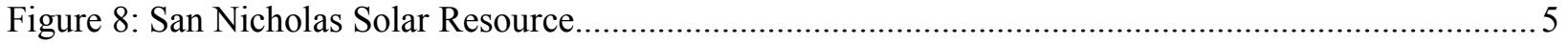

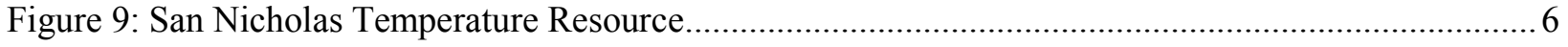

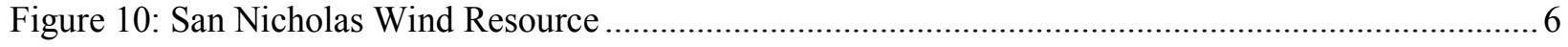

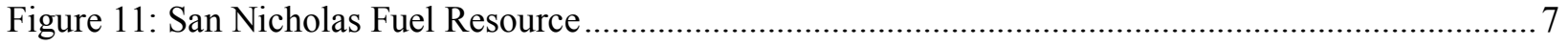

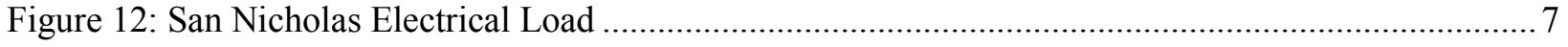

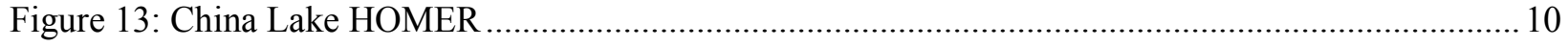

Figure 14: China Lake Generator Component ................................................................................... 11

Figure 15: China Lake Sun Power PV Component …........................................................................ 11 
Figure 16: China Lake PV Distributed Component .......................................................................... 12

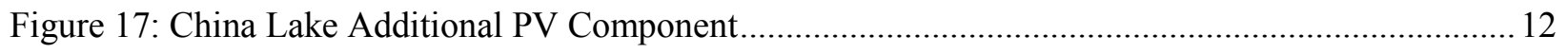

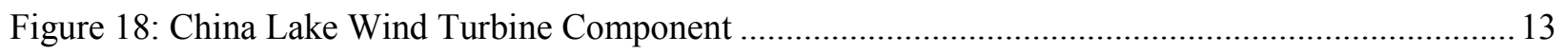

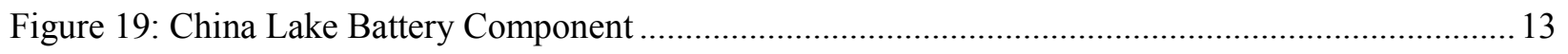

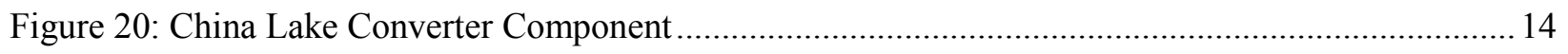

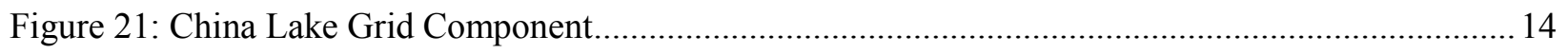

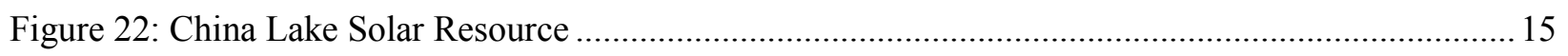

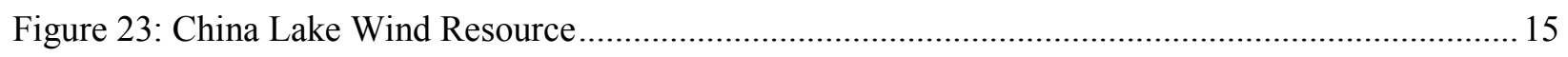

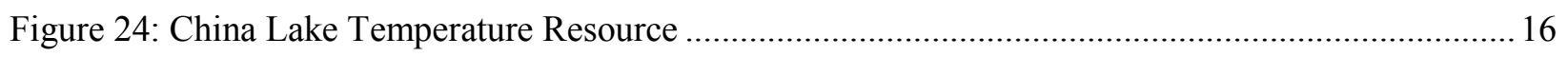

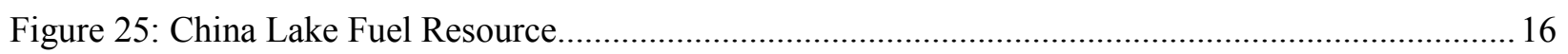

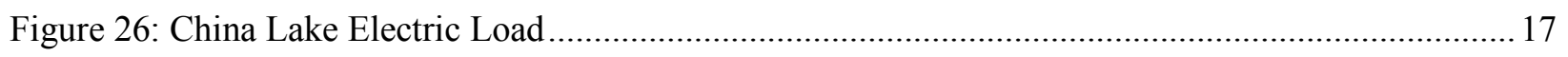

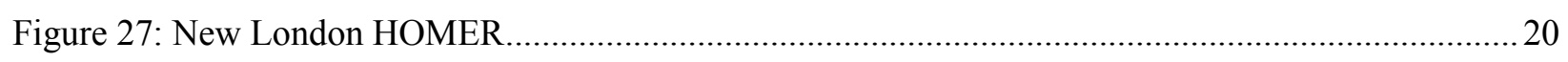

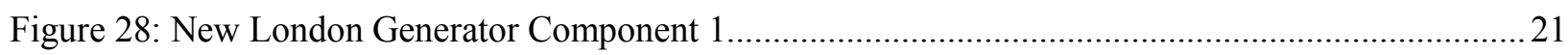

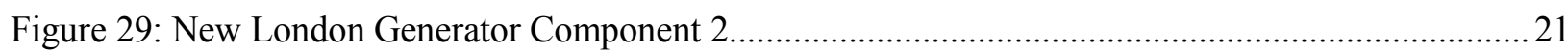

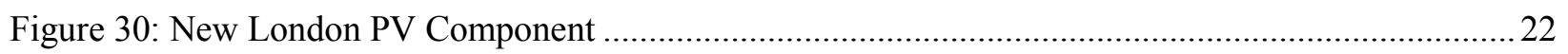

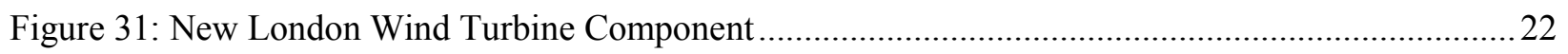

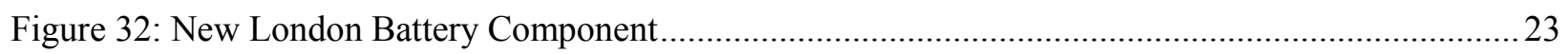

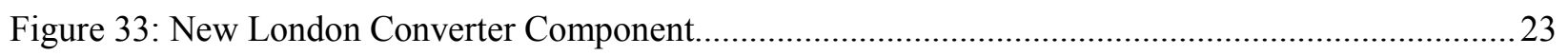

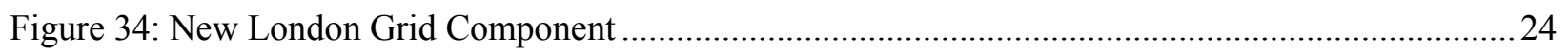

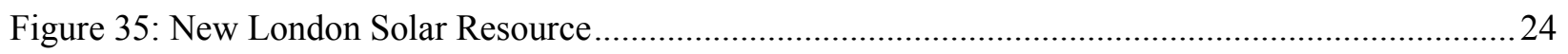

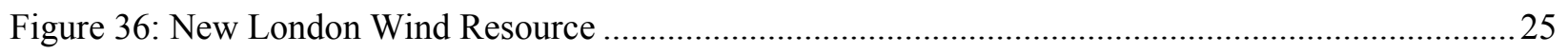

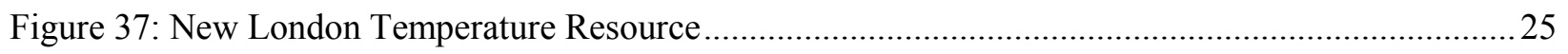

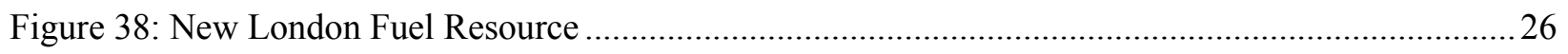

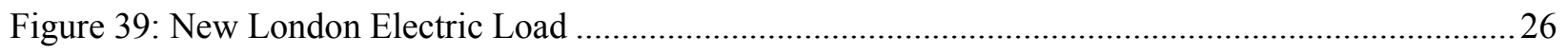

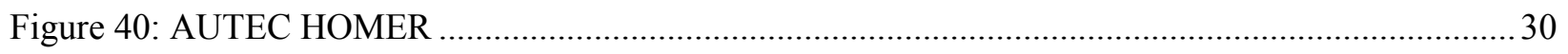

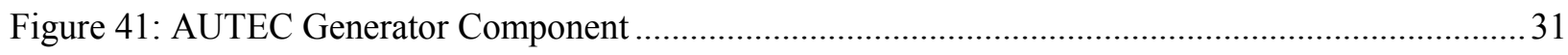

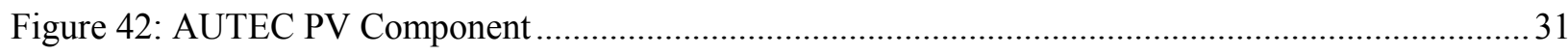

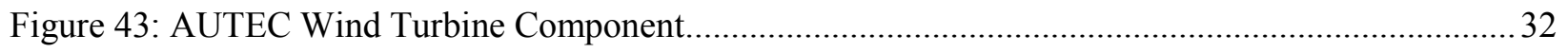

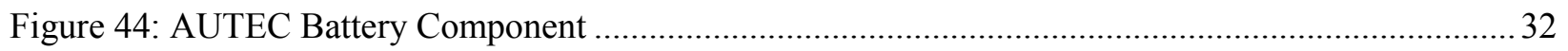

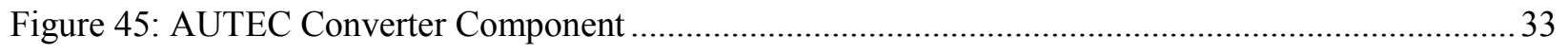

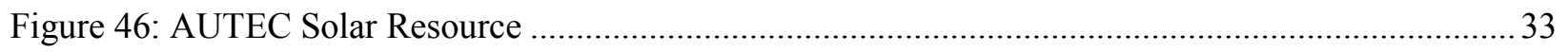

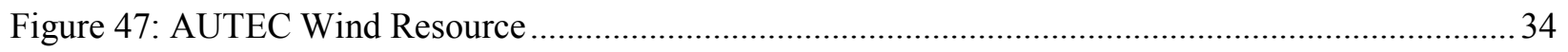

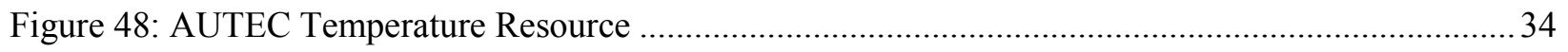

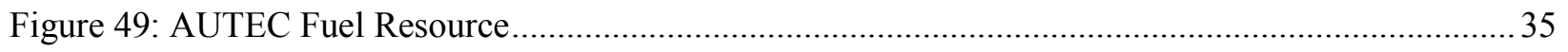

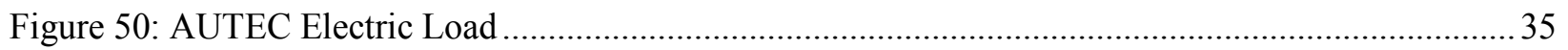




\section{TABLES}

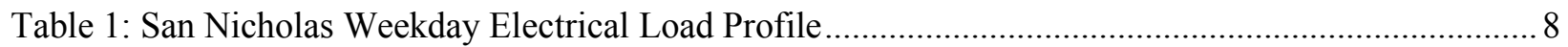

Table 2: San Nicholas Weekend Electrical Load Profile ......................................................................... 8

Table 3: San Nicholas Sensitivity \& Optimization Results ...................................................................... 9

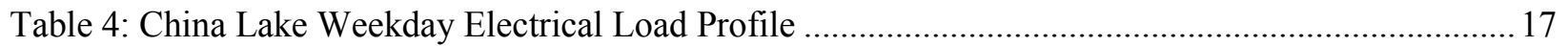

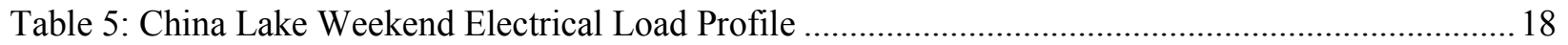

Table 6: China Lake Grid-tied Sensitivity \& Optimization Results ....................................................... 19

Table 7: China Lake Island Sensitivity \& Optimization Results ............................................................. 19

Table 8: New London Weekday Electrical Load Profile .......................................................................... 27

Table 9: New London Weekend Electrical Load Profile ......................................................................... 27

Table 10: New London Grid-tied Sensitivity \& Optimization Results .....................................................28

Table 11: New London Island Sensitivity \& Optimization Results...........................................................29

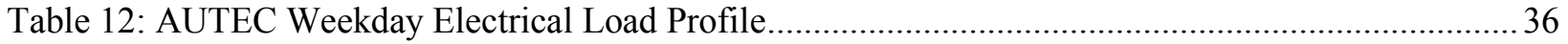

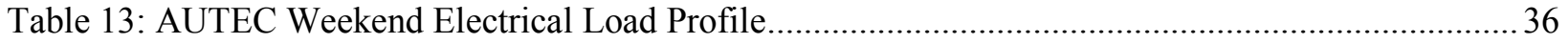

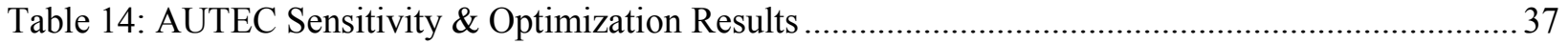




\section{HOMER Economic Models - US Navy}

\section{Introduction}

Figure 1: Navy locations modeled in HOMER
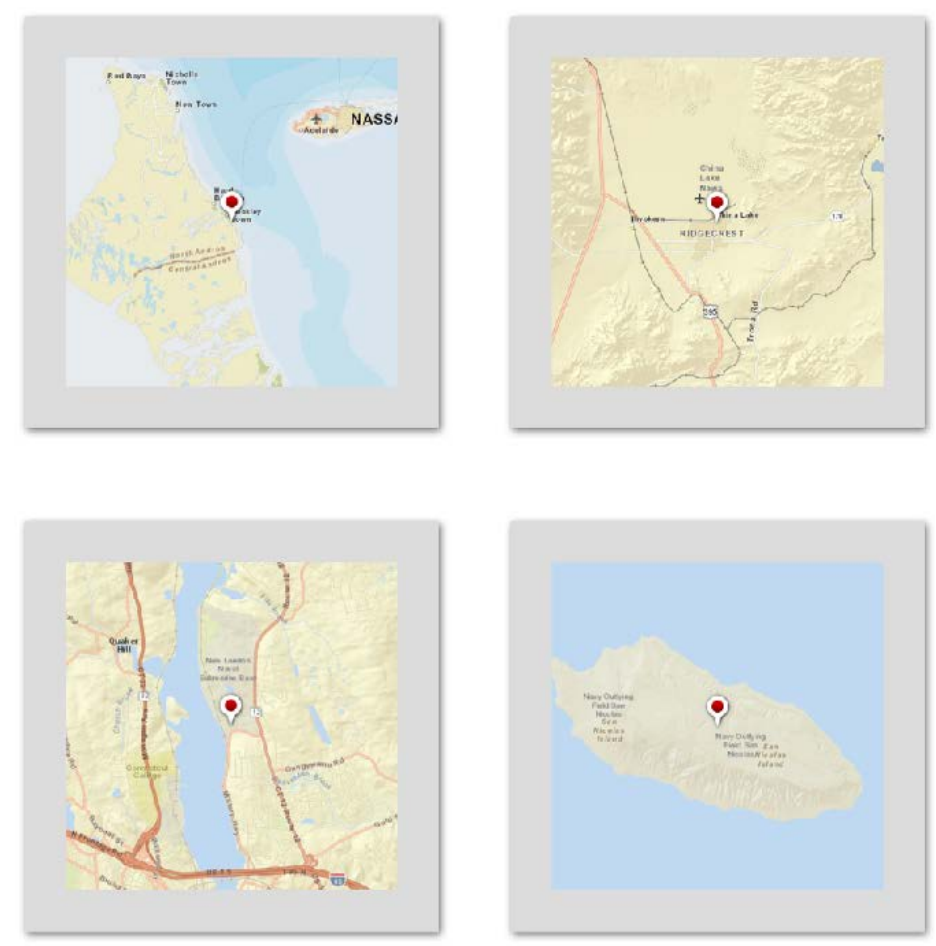

This LETTER REPORT has been prepared by Idaho National Laboratory for US Navy NAVFAC EXWC to support in testing pre-commercial SIREN (Simulated Integration of Renewable Energy Networks) computer software models. In the logistics mode SIREN software simulates the combination of renewable power sources (solar arrays, wind turbines, and energy storage systems) in supplying an electrical demand. NAVFAC EXWC will create SIREN software logistics models of existing or planned renewable energy projects at five Navy locations (San Nicolas Island, AUTEC, New London, \& China Lake), and INL will deliver additional HOMER computer models for comparative analysis. In the transient mode SIREN simulates the short time-scale variation of electrical parameters when a power outage or other destabilizing event occurs. In the HOMER model, a variety of inputs are entered such as location coordinates, Generators, PV arrays, Wind Turbines, Batteries, Converters, Grid costs/usage, Solar resources, Wind resources, Temperatures, Fuels, and Electric Loads. HOMER's optimization and sensitivity analysis algorithms then evaluate the economic and technical feasibility of these technology options and account for variations in technology costs, electric load, and energy resource availability. The Navy can then use HOMER's optimization and sensitivity results to compare to those of the SIREN model.

The U.S. Department of Energy (DOE) Idaho National Laboratory (INL) possesses unique expertise and experience in the software, hardware, and systems design for the integration of renewable energy into the electrical grid. NAVFAC EXWC will draw upon this expertise to complete mission requirements. 


\section{San Nicholas Island}

\subsection{HOMER Analysis}

Figure 2: San Nicholas Island HOMER

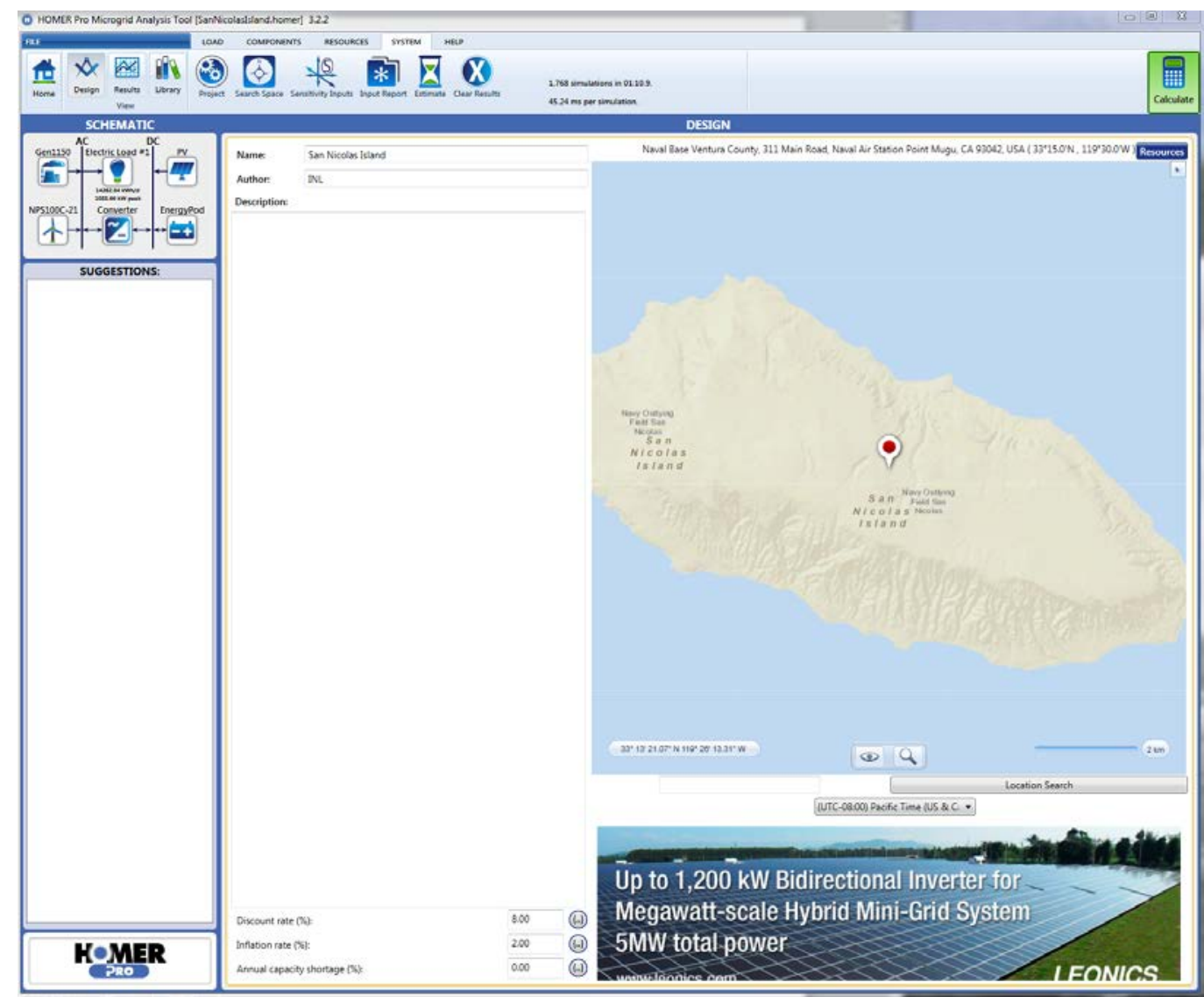

The HOMER (Hybrid Optimization of Multiple Energy Resources) model greatly simplifies the task of designing hybrid renewable microgrids, whether remote or attached to a larger grid. HOMER's optimization and sensitivity analysis algorithms allow you to evaluate the economic and technical feasibility of a large number of technology options and to account for variations in technology costs, electric load, and energy resource availability.

For San Nicholas Island inputs we have included:

- $\quad 1-1150 \mathrm{~kW}$ Generator

- 7 - 100kW Northern Power Wind Turbines

- Flat panel fixed PV up from $250 \mathrm{~kW}$ up to $2000 \mathrm{~kW}$

- $\quad 280 \mathrm{~kW} / 1 \mathrm{MWh}$ Primus Power EnergyPod Battery (allowed up to 4)

- System Converter up to $2000 \mathrm{~kW}$

This model used the location coordinates of $33^{\circ} 15.05^{\prime} \mathrm{N}, 119^{\circ} 29.97^{\prime} \mathrm{W}$ and wind resource data from the $50 \mathrm{~m}$ NREL Met tower. The solar resource was downloaded from the NREL Solar database and the electrical load data was analysis of spreadsheet data provided for Dec13, Jan14, Feb14, Jun14, Jul14, \& Aug14. Homer requires 1 year of hourly/monthly data so a linear interpolation algorithm was used to fill 
in the missing months. A temperature resource was also included and was downloaded from NASA's surface and solar database. Refer to following figures:

Figure 3: San Nicholas Generator Component

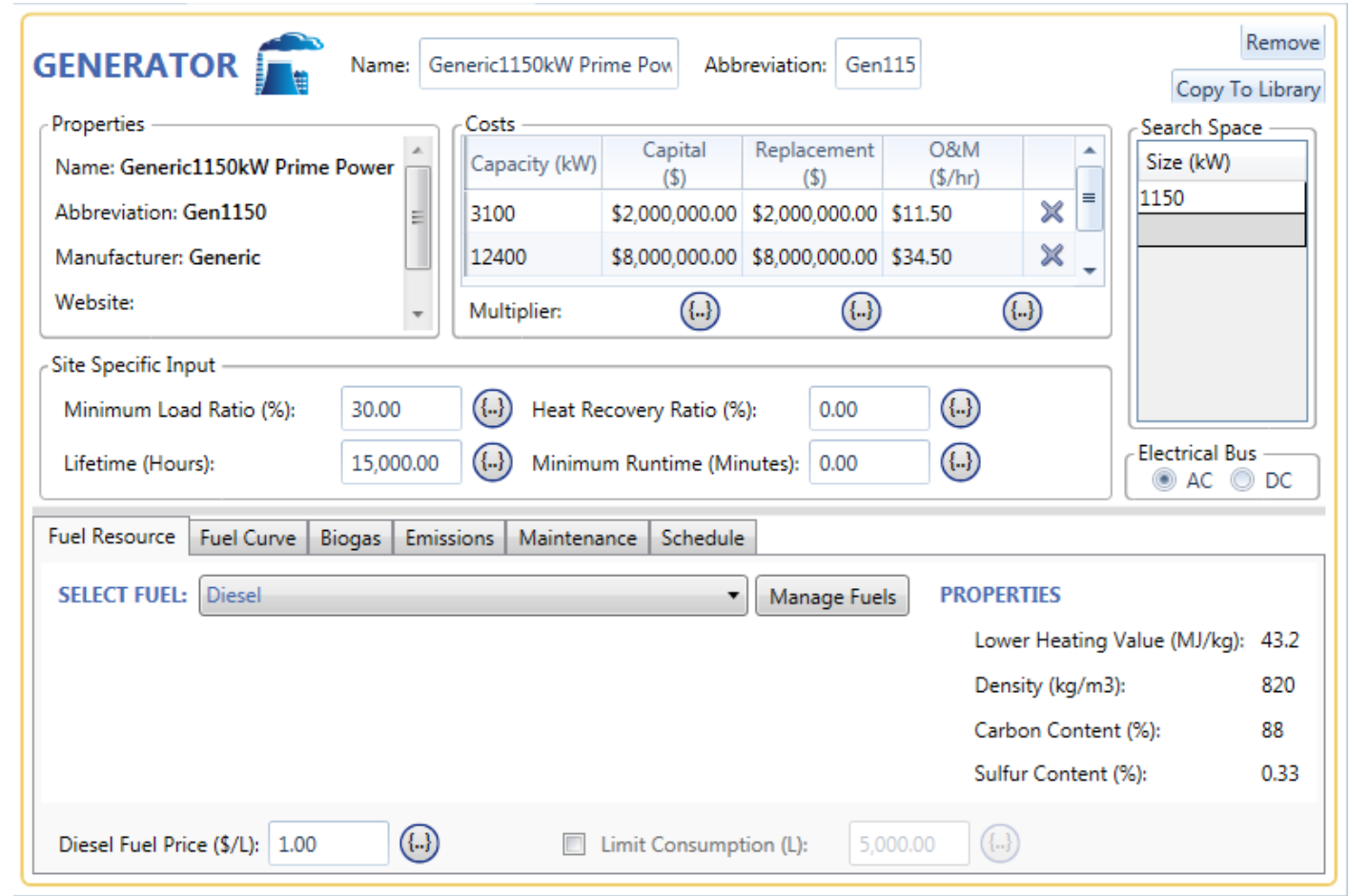

Figure 4: San Nicholas PV Component

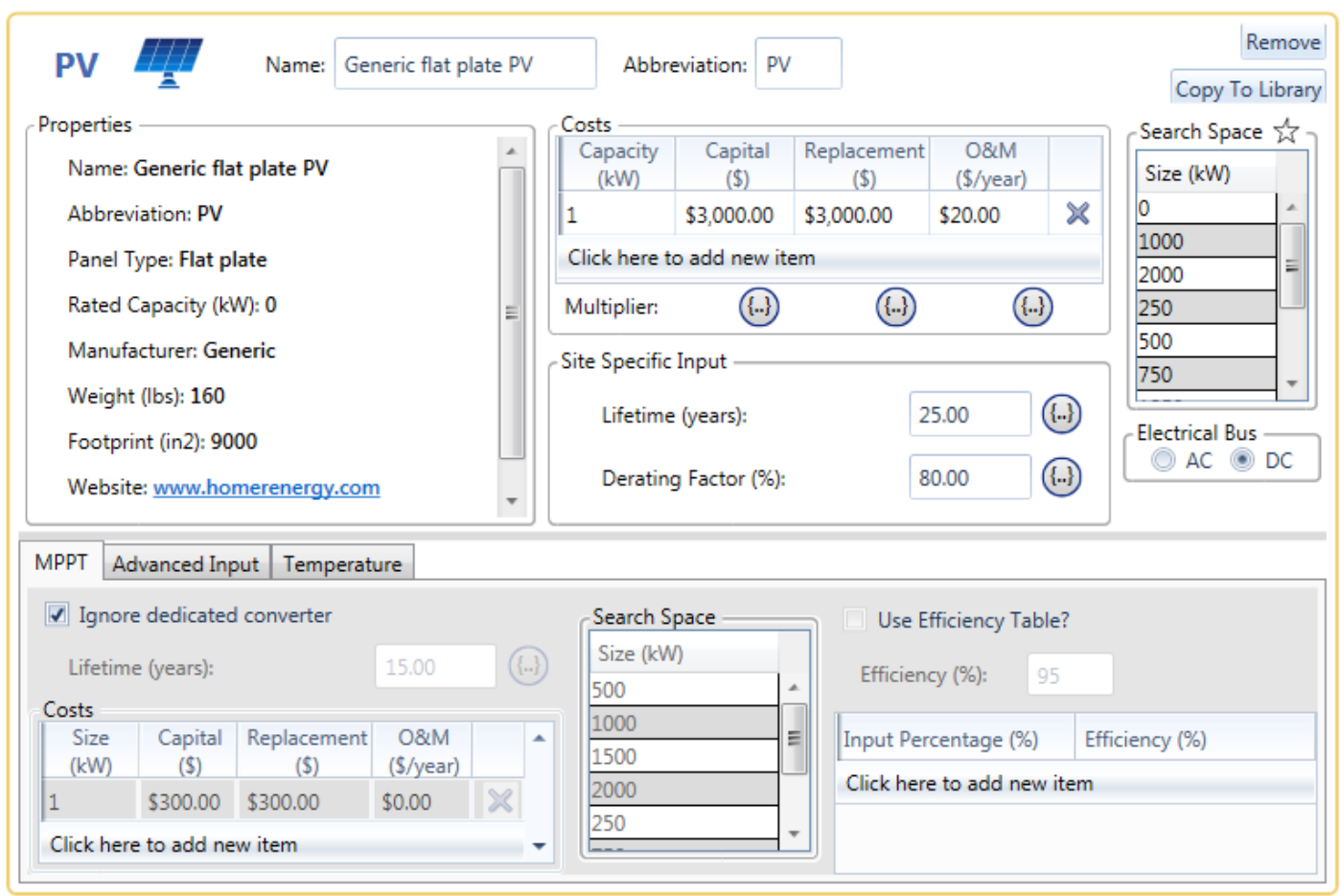


Figure 5: San Nicholas Wind Turbine Component

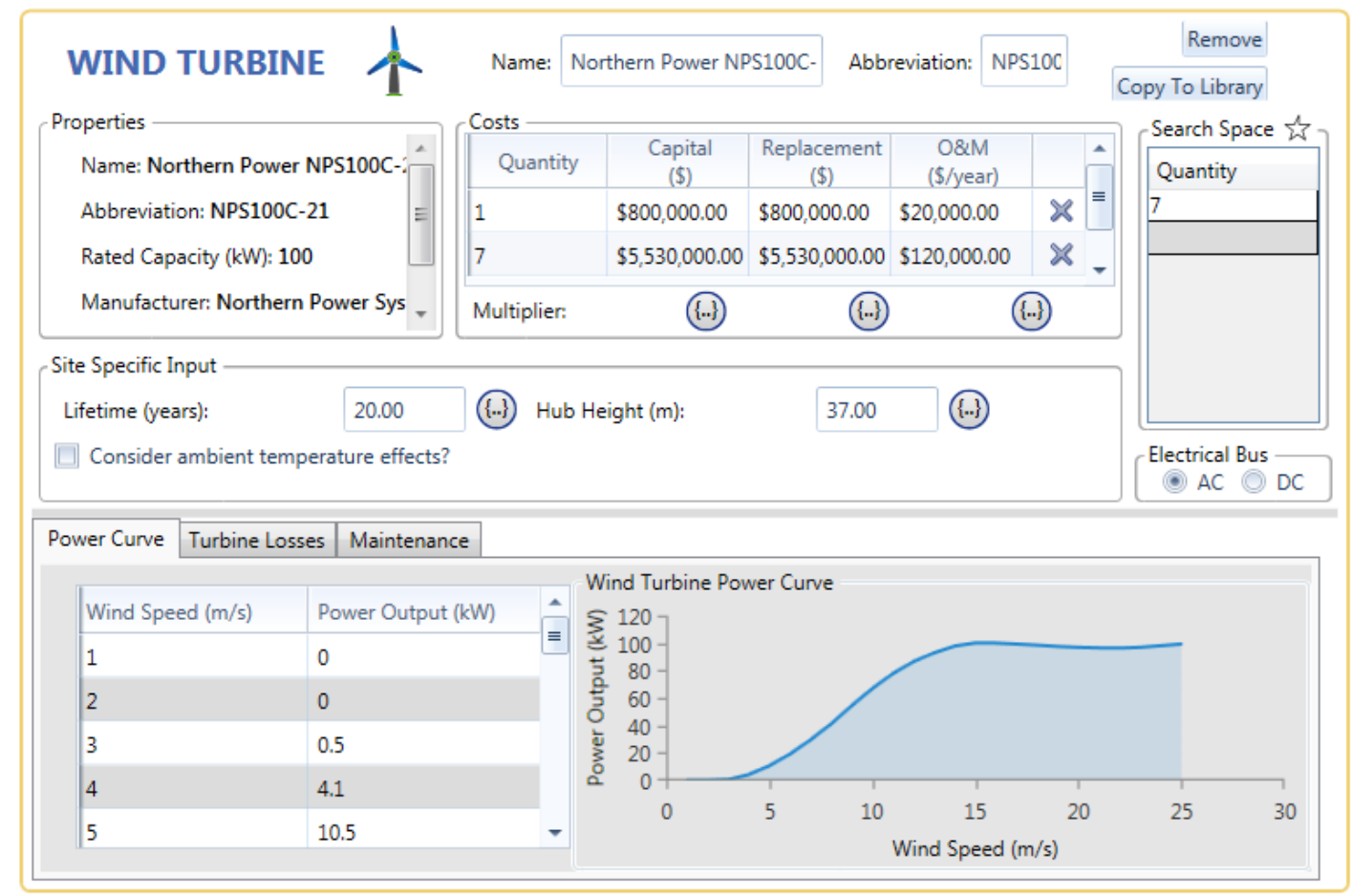

Figure 6: San Nicholas Battery Component

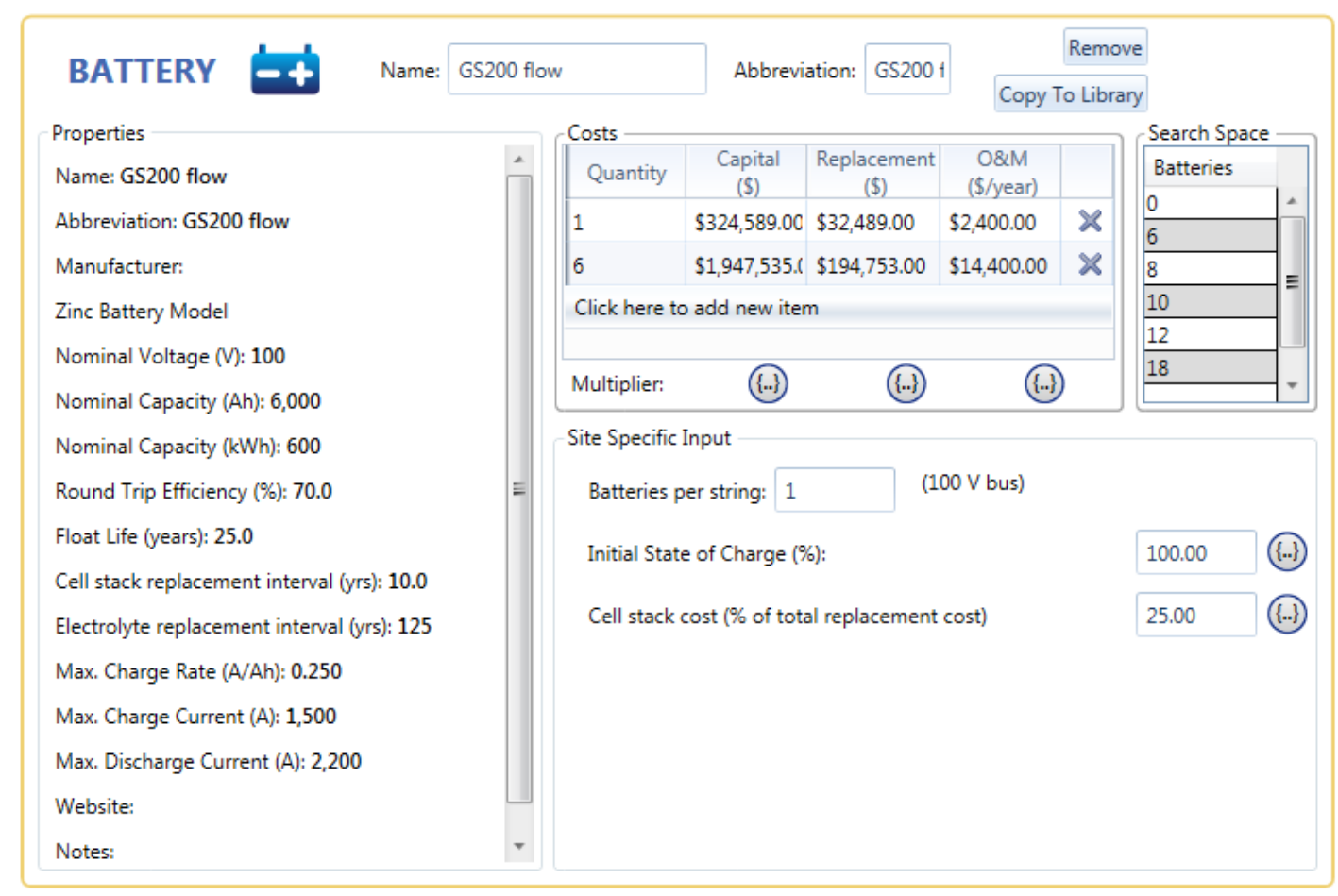


Figure 7: San Nicholas Converter Component

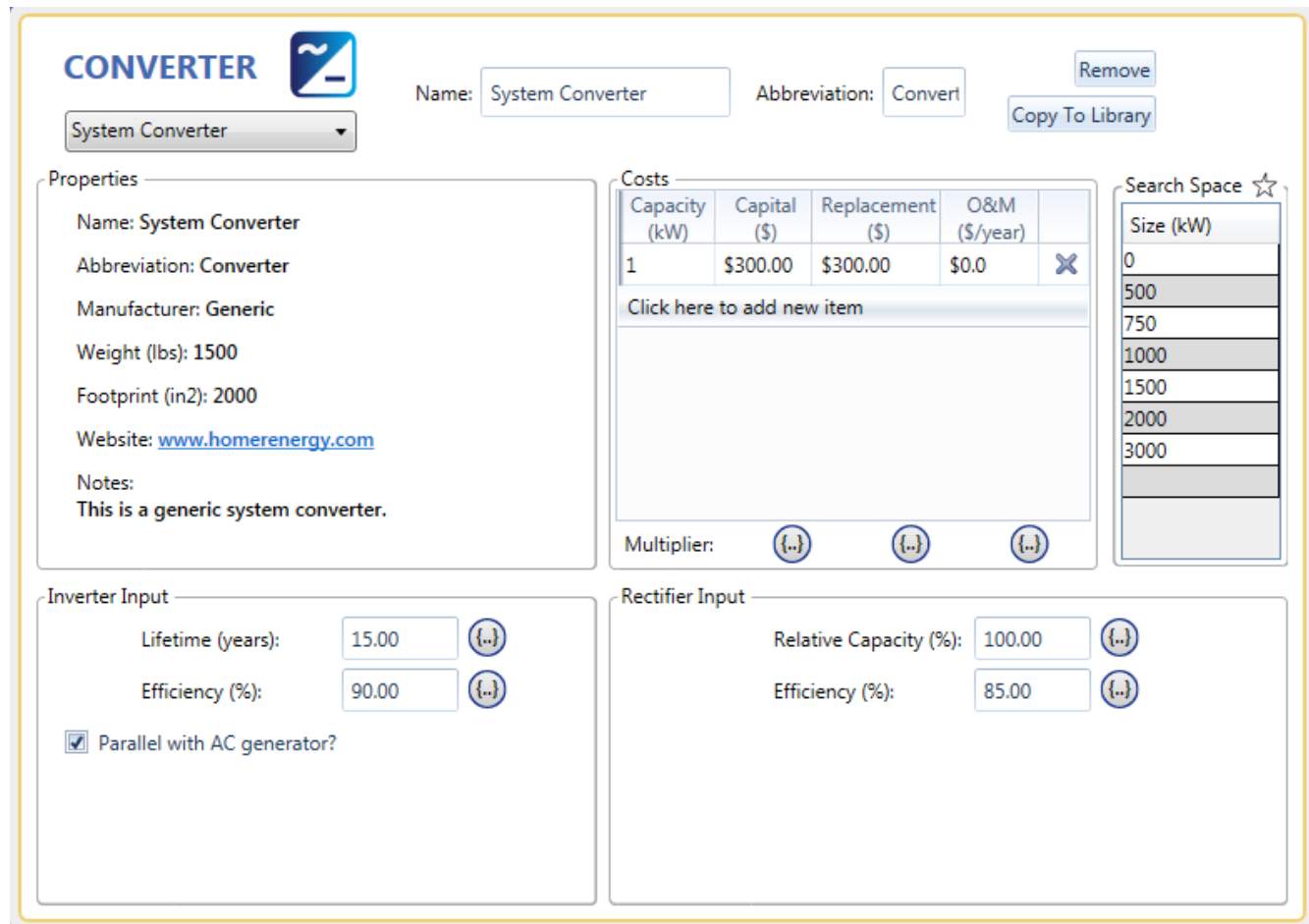

Figure 8: San Nicholas Solar Resource

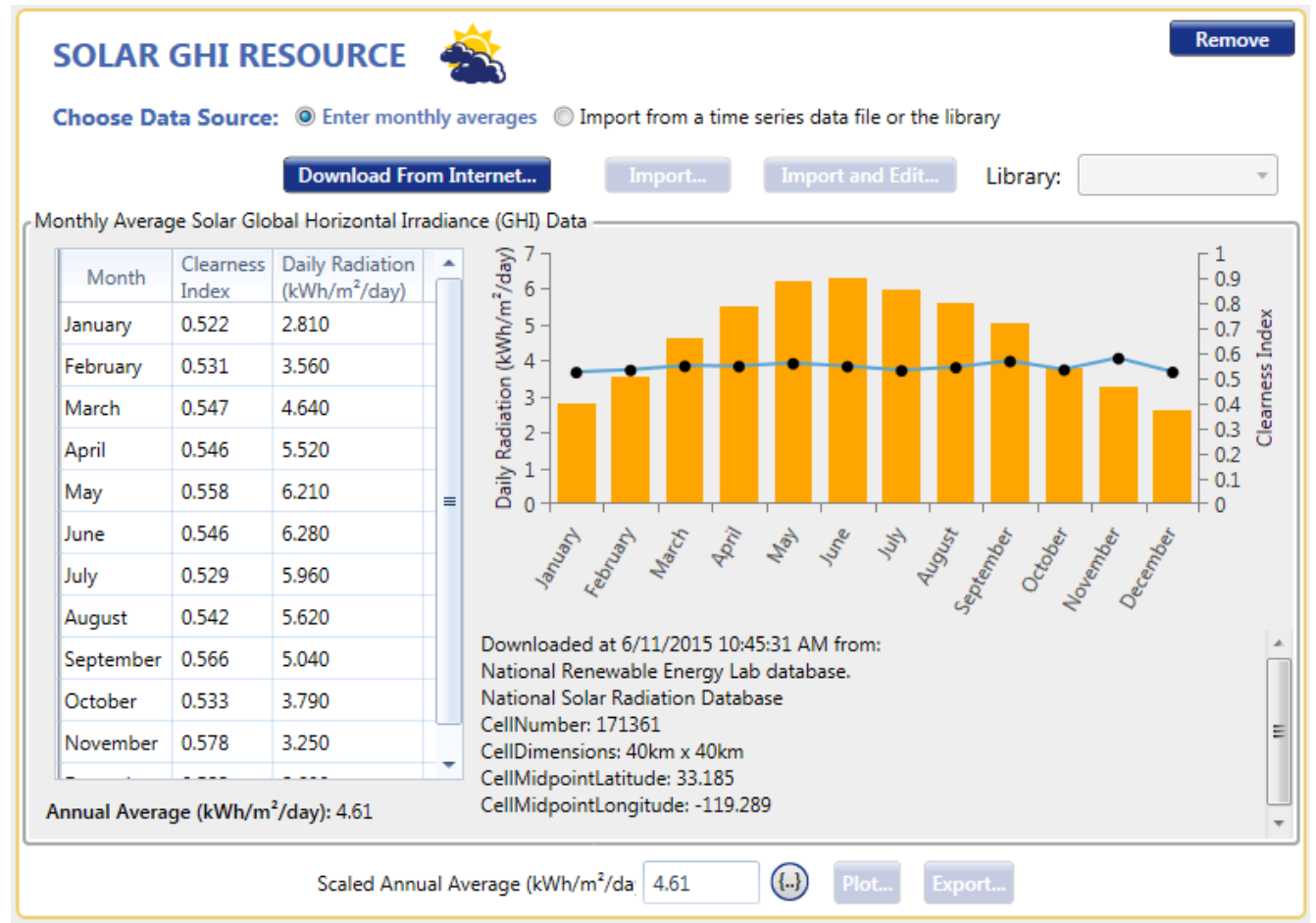


Figure 9: San Nicholas Temperature Resource

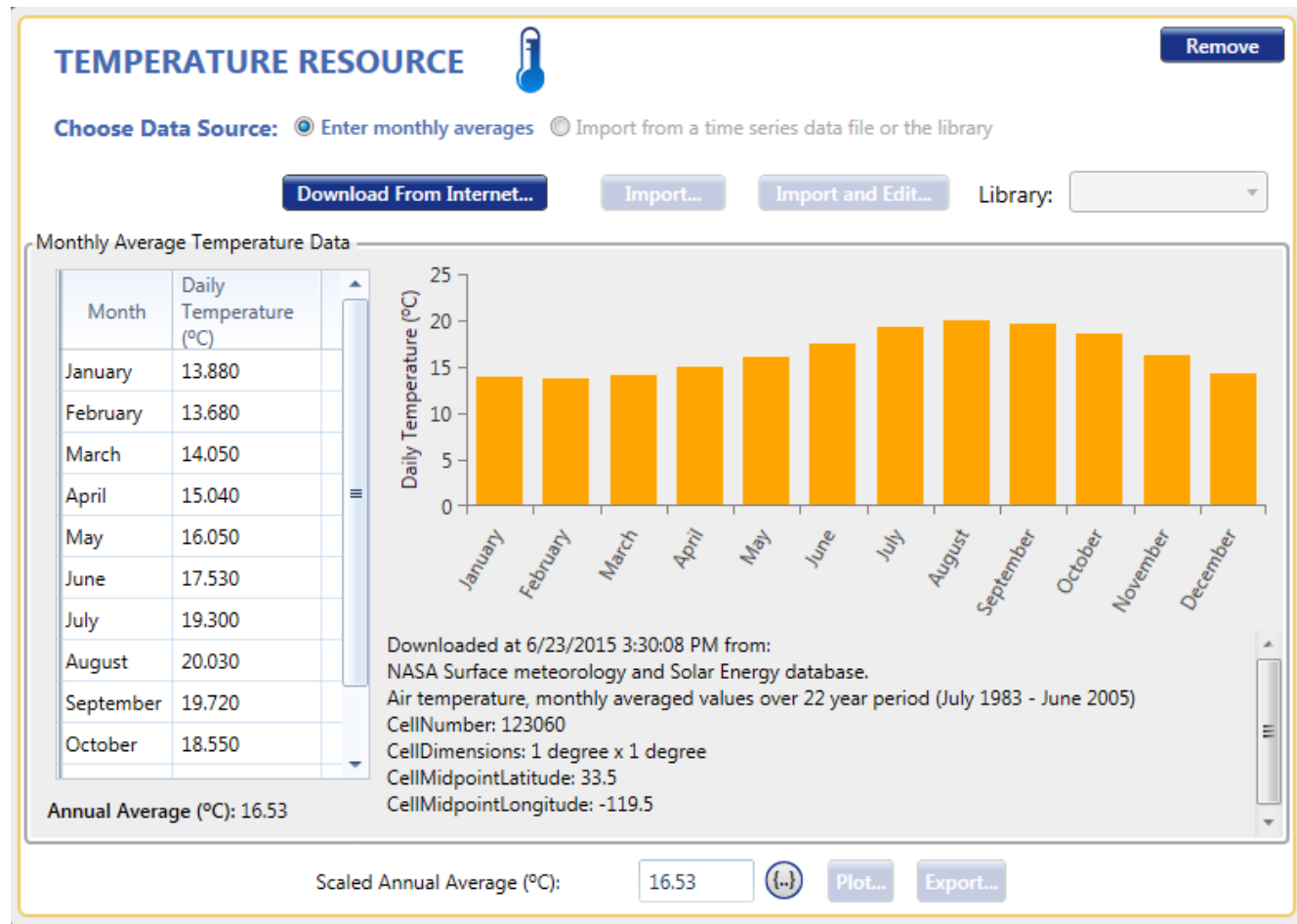

Figure 10: San Nicholas Wind Resource

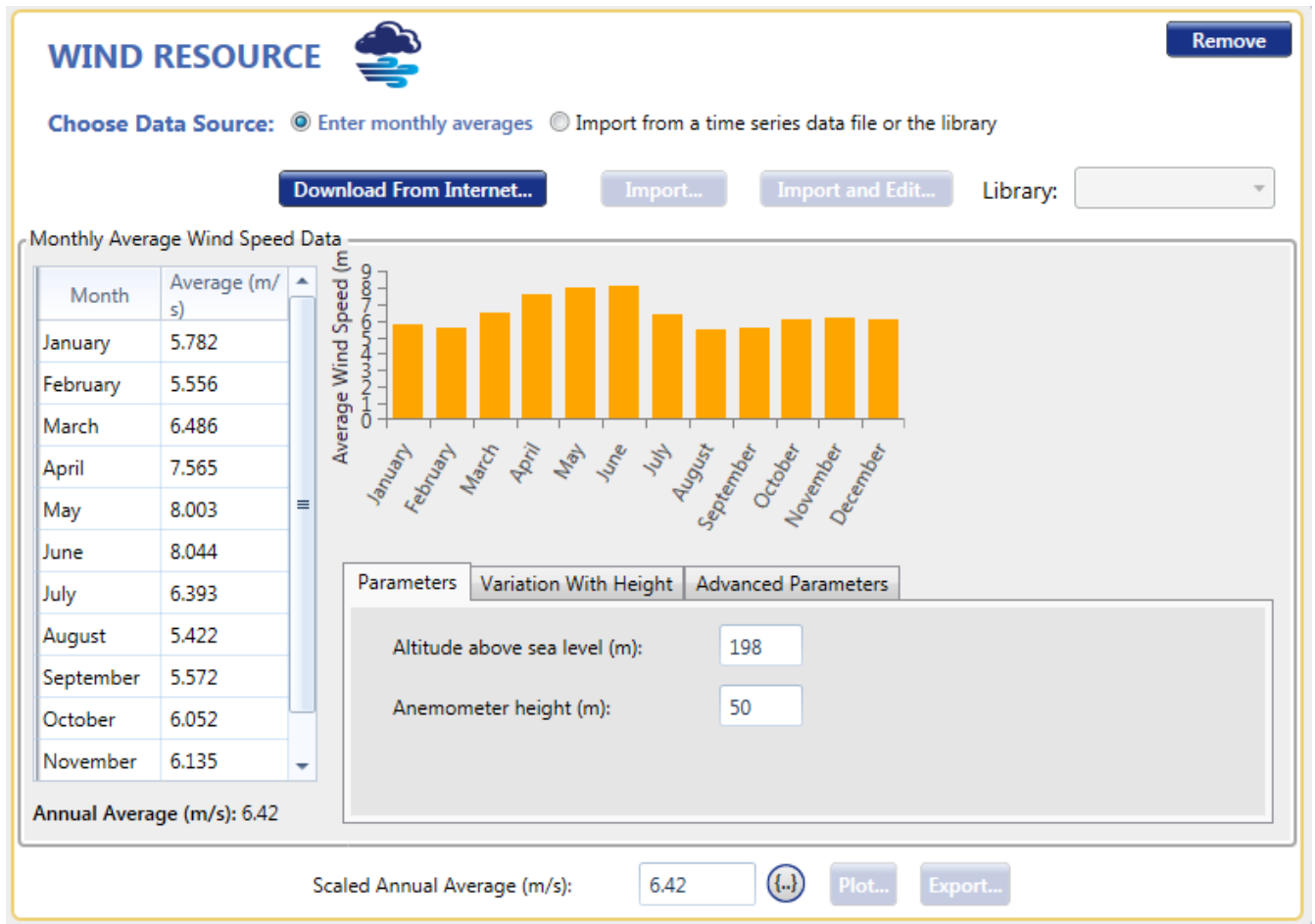


Figure 11: San Nicholas Fuel Resource

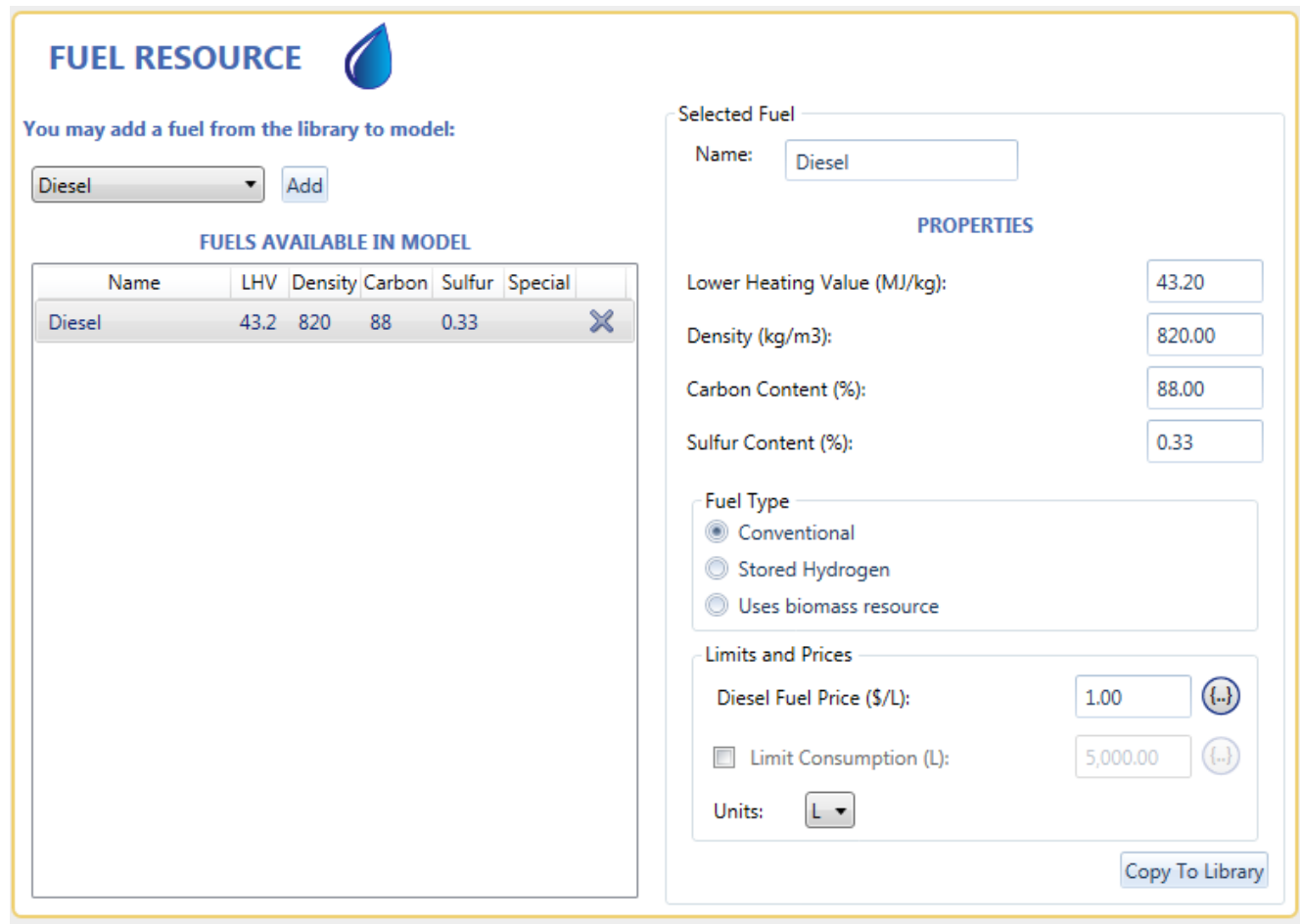

Figure 12: San Nicholas Electrical Load

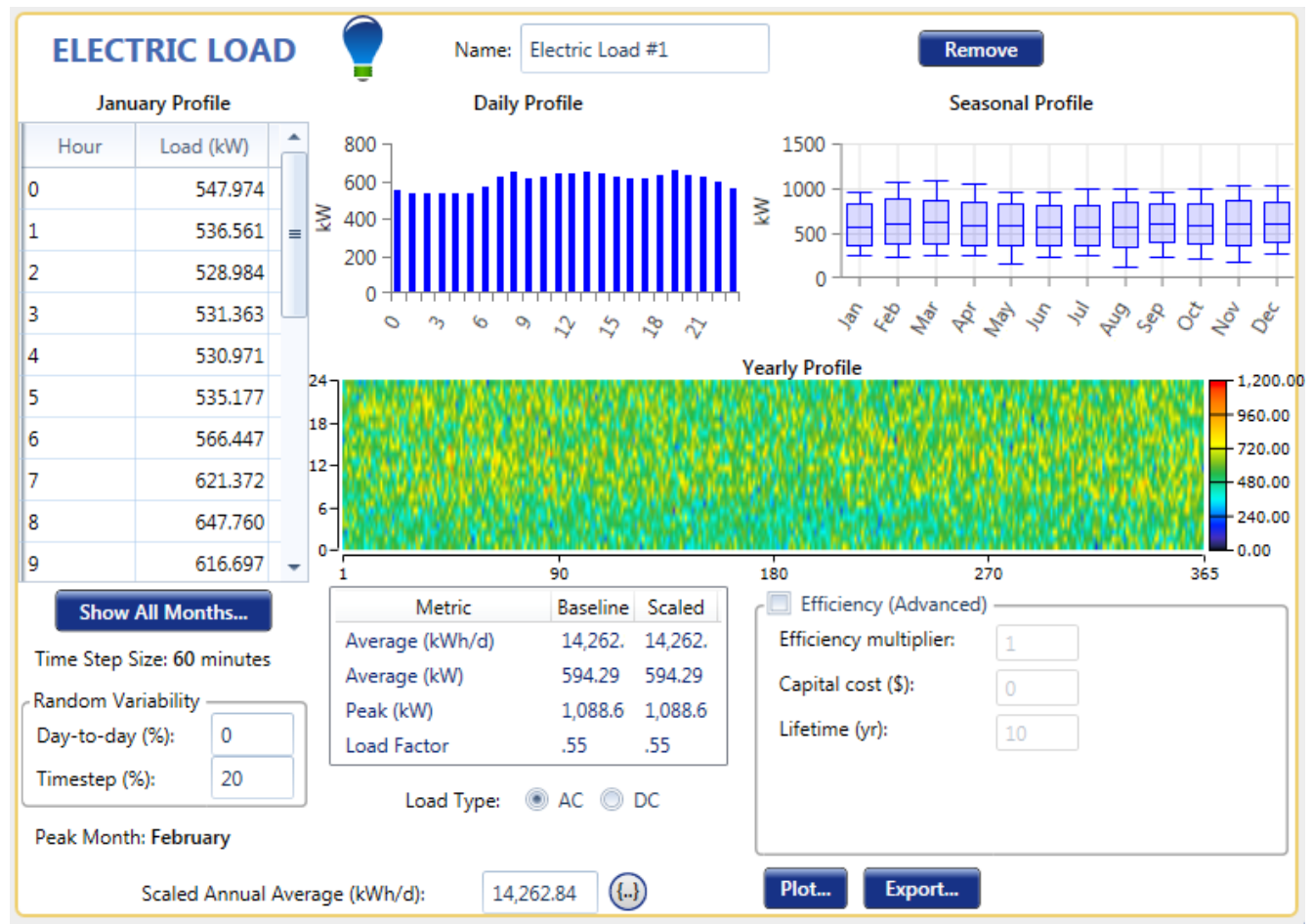


Table 1: San Nicholas Weekday Electrical Load Profile

\begin{tabular}{|c|c|c|c|c|c|c|c|c|c|c|c|c|c|c|}
\hline \multicolumn{3}{|c|}{ Yearly Load Data } & . & & & & & & & & & & & 8 \\
\hline \multicolumn{15}{|c|}{ Weekdays Weekends } \\
\hline Hour & January & February & March & April & May & June & July & August & September & October & November & December & & \\
\hline 0 & 547.974 & 593.185 & 579.855 & 566.525 & 553.195 & 539.865 & 558.336 & 549.486 & 554.771 & 560.056 & 565.341 & 570.625 & & \\
\hline 1 & 536.561 & 574.664 & 561.702 & 548.740 & 535.777 & 522.815 & 532.095 & 535.680 & 543.881 & 552.082 & 560.283 & 568.484 & & \\
\hline 2 & 528.984 & 564.099 & 553.209 & 542.320 & 531.430 & 520.541 & 521.476 & 528.633 & 540.315 & 551.996 & 563.678 & 575.359 & & \\
\hline 3 & 531.363 & 558.677 & 548.350 & 538.023 & 527.696 & 517.369 & 513.492 & 530.260 & 542.844 & 555.429 & 568.014 & 580.599 & & \\
\hline 4 & 530.971 & 558.768 & 548.596 & 538.424 & 528.252 & 518.080 & 509.861 & 524.630 & 539.118 & 553.607 & 568.095 & 582.584 & & \\
\hline 5 & 535.177 & 559.591 & 550.432 & 541.273 & 532.113 & 522.954 & 508.847 & 523.279 & 538.722 & 554.166 & 569.609 & 585.053 & & \\
\hline 6 & 566.447 & 592.180 & 579.653 & 567.127 & 554.600 & 542.073 & 528.058 & 540.901 & 558.313 & 575.726 & 593.139 & 610.552 & & \\
\hline 7 & 621.372 & 652.066 & 633.398 & 614.731 & 596.064 & 577.397 & 562.712 & 571.472 & 593.012 & 614.552 & 636.092 & 657.631 & & \\
\hline 8 & 647.760 & 679.646 & 663.181 & 646.717 & 630.252 & 613.788 & 603.834 & 610.988 & 626.580 & 642.171 & 657.763 & 673.354 & & \\
\hline 9 & 616.697 & 653.596 & 643.884 & 634.172 & 624.461 & 614.749 & 588.493 & 597.899 & 609.116 & 620.332 & 631.549 & 642.765 & & \\
\hline 10 & 623.293 & 650.785 & 641.238 & 631.691 & 622.144 & 612.598 & 585.485 & 603.180 & 613.907 & 624.633 & 635.359 & 646.086 & & \\
\hline 11 & 642.215 & 665.117 & 653.712 & 642.307 & 630.901 & 619.496 & 605.651 & 629.669 & 636.235 & 642.801 & 649.367 & 655.932 & & \\
\hline 12 & 642.929 & 676.620 & 667.304 & 657.989 & 648.673 & 639.357 & 635.024 & 665.564 & 663.608 & 661.652 & 659.696 & 657.740 & & \\
\hline 13 & 648.937 & 673.879 & 665.867 & 657.855 & 649.843 & 641.831 & 637.781 & 666.824 & 662.937 & 659.050 & 655.163 & 651.277 & & \\
\hline 14 & 644.339 & 668.228 & 659.934 & 651.640 & 643.346 & 635.053 & 635.613 & 666.025 & 660.212 & 654.399 & 648.586 & 642.773 & & \\
\hline 15 & 618.877 & 642.911 & 633.265 & 623.619 & 613.973 & 604.328 & 620.501 & 649.403 & 642.084 & 634.764 & 627.445 & 620.125 & & \\
\hline 16 & 614.892 & 633.473 & 621.834 & 610.196 & 598.557 & 586.919 & 608.213 & 629.635 & 624.982 & 620.328 & 615.674 & 611.020 & & \\
\hline 17 & 614.732 & 637.849 & 623.467 & 609.085 & 594.703 & 580.320 & 609.035 & 624.901 & 623.809 & 622.717 & 621.624 & 620.532 & & \\
\hline 18 & 633.123 & 655.364 & 640.364 & 625.364 & 610.364 & 595.364 & 626.838 & 623.064 & 628.366 & 633.669 & 638.971 & 644.273 & & \\
\hline 19 & 655.368 & 674.115 & 655.186 & 636.257 & 617.329 & 598.400 & 616.953 & 619.647 & 631.032 & 642.417 & 653.802 & 665.187 & & \\
\hline 20 & 634.487 & 681.723 & 659.610 & 637.496 & 615.383 & 593.269 & 604.133 & 617.679 & 622.950 & 628.222 & 633.493 & 638.764 & & \\
\hline 21 & 619.023 & 668.705 & 648.587 & 628.468 & 608.349 & 588.230 & 605.152 & 611.252 & 616.093 & 620.934 & 625.775 & 630.616 & & \\
\hline 22 & 599.282 & 643.063 & 625.564 & 608.065 & 590.566 & 573.066 & 589.937 & 602.057 & 605.869 & 609.681 & 613.493 & 617.305 & & \\
\hline 23 & 561.628 & 613.245 & 601.882 & 590.520 & 579.158 & 567.795 & 579.781 & 574.229 & 577.893 & 581.556 & 585.219 & 588.882 & & \\
\hline
\end{tabular}

Table 2: San Nicholas Weekend Electrical Load Profile

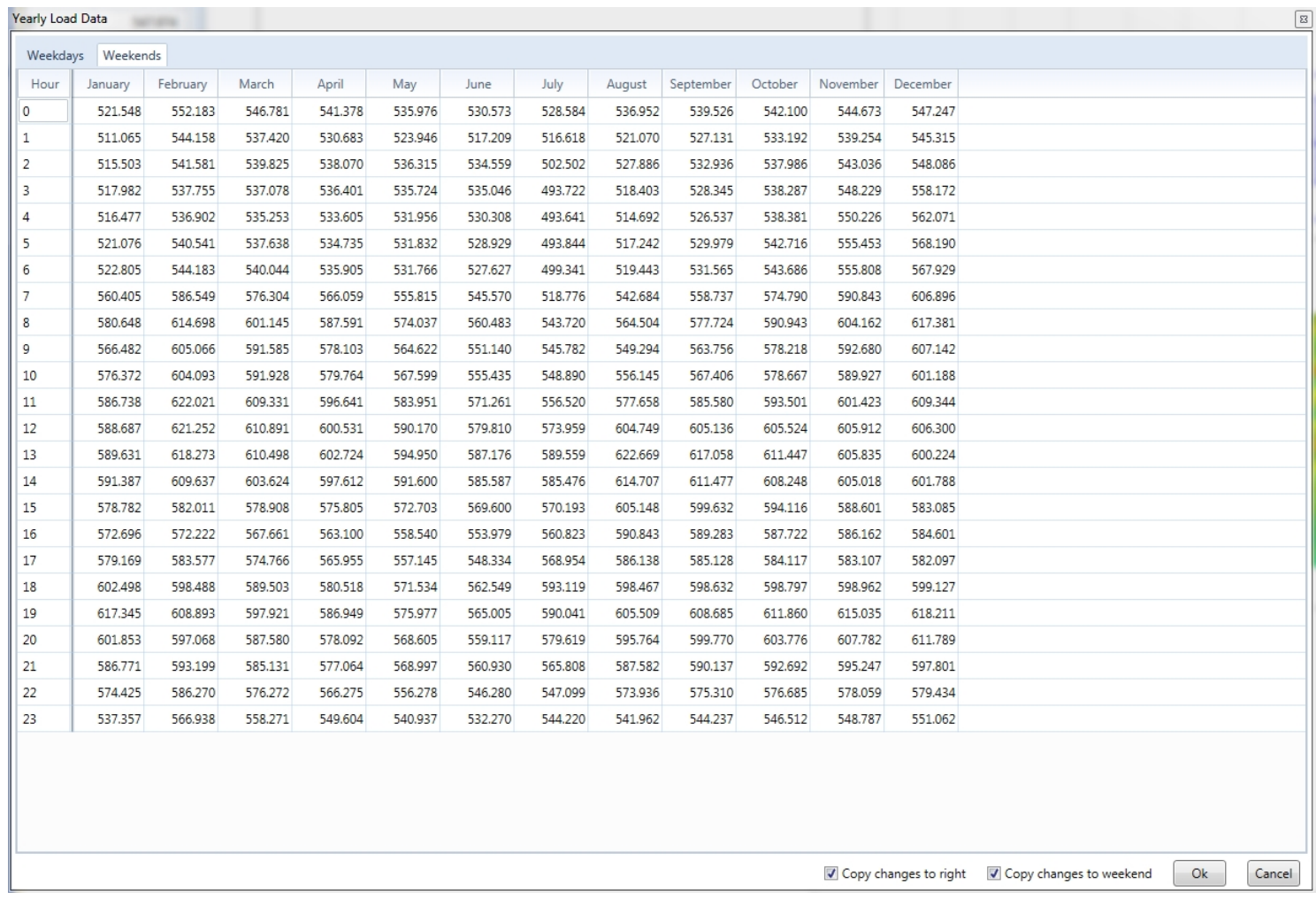


Two of the components are already at SNI (Wind Turbines \& Generator). Inputs for these two components were set so HOMER always looks at using the 7 wind turbines and the 1 genset. HOMER then has the option to consider different amounts of PV \& batteries, and different size system converter. These are the simulation results produced for the optimization cases:

Table 3: San Nicholas Sensitivity \& Optimization Results

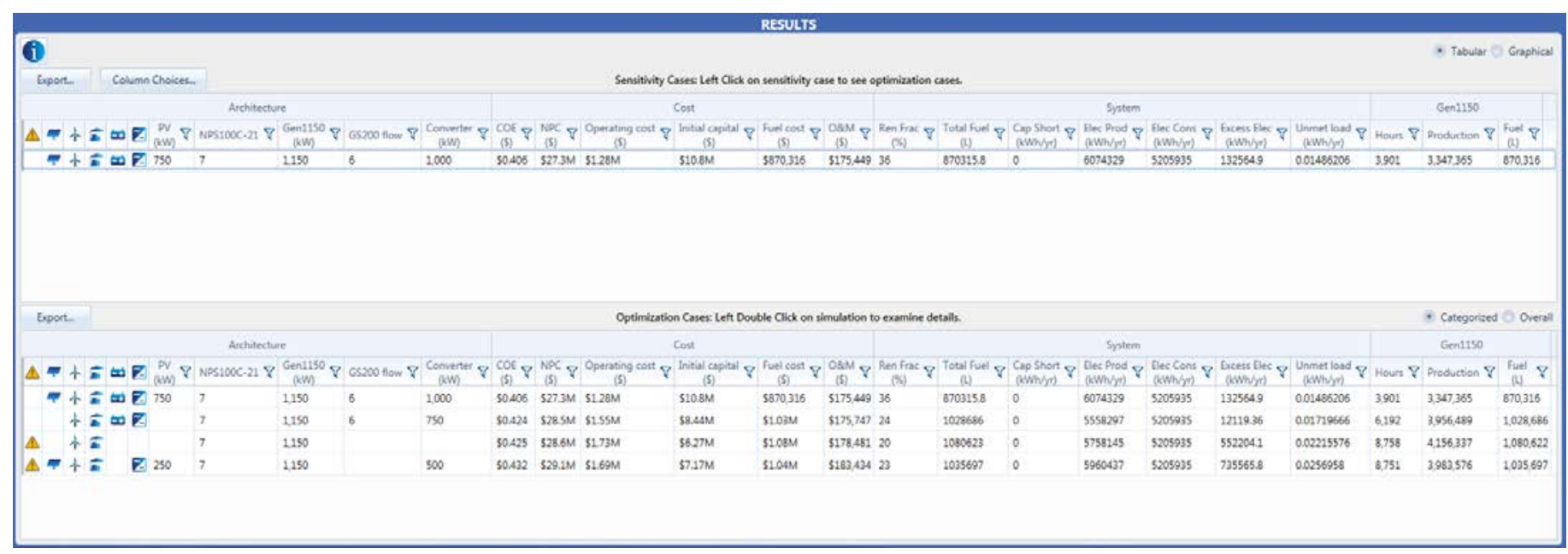

The top case (HOMER optimized selection) includes $750 \mathrm{~kW} \mathrm{PV}, 7-100 \mathrm{~kW}$ wind turbines, $1150 \mathrm{~kW}$ Genset, 6 Vizn GS200 Flow batteries (1MW) and a 1MW system converter. For this case the cost of energy is calculated at $\$ 0.406$ and the renewable fraction is $36 \%$. With the input parameters HOMER was given the above cases were the only ones found feasible. See case pdf's for further details on each simulation. 


\section{China Lake}

\subsection{HOMER Analysis}

Figure 13: China Lake HOMER

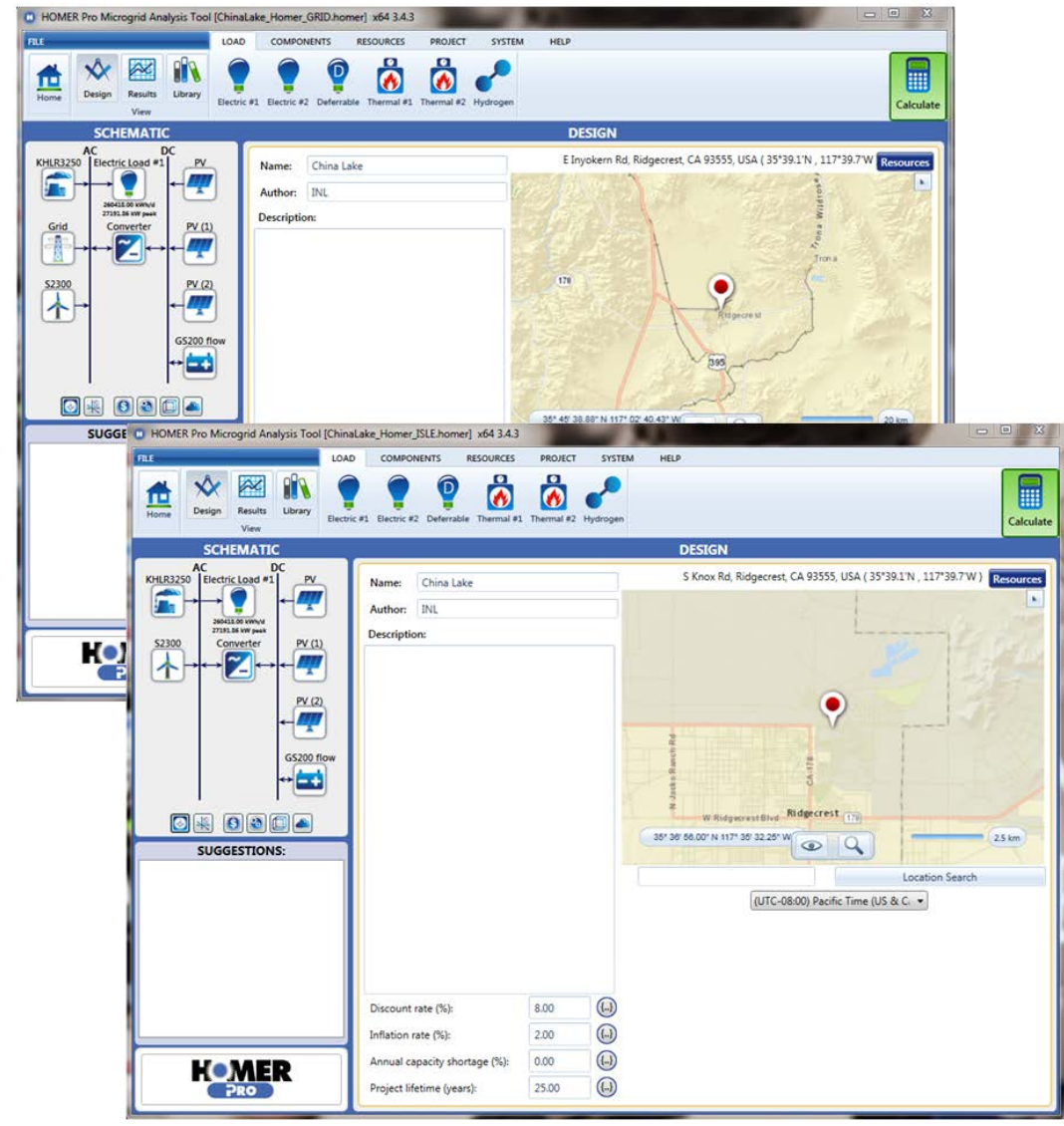

The HOMER (Hybrid Optimization of Multiple Energy Resources) model greatly simplifies the task of designing hybrid renewable microgrids, whether remote or attached to a larger grid. HOMER's optimization and sensitivity analysis algorithms allow you to evaluate the economic and technical feasibility of a large number of technology options and to account for variations in technology costs, electric load, and energy resource availability.

Multiple inputs are entered into the HOMER model such as location coordinates of $35^{\circ} 39.1^{\prime} \mathrm{N}$, $117^{\circ} 39.7^{\prime}$ W which is near Naval Air Weapons Station China Lake's main gate. Resources and components are the main inputs that are needed to make a HOMER model. For China Lake the components used consist of Generator, PV, Wind Turbine, Battery, Converter and the Grid. INL has created cost inputs for these components based on vendor/market data and past experience. The resource inputs created for China Lake consist of Solar, Wind, Temperature, Fuels, and Electric Load. Refer to figures $(1-11)$. The solar, wind, \& temperature resources were downloaded from the NASA Surface meteorology and Solar Energy database and contained monthly averaged values for a 10 to 22 year period $(1983-2005)$. The fuel resource consists of a Diesel profile that was generated from current market information. The electrical load data was an analysis of naval power load spreadsheet data provided for the year 2010. Based on the power data HOMER estimates annual peak load as 27,191.86 kW. Grid purchase inputs are then set as $12000,15000,20000,25000, \& 27200 \mathrm{kWs}$ with grid sales set as $0 \mathrm{kWs}$. Note in the China Lake model; both the grid-tied and off-grid (island) systems are modeled. 
Figure 14: China Lake Generator Component

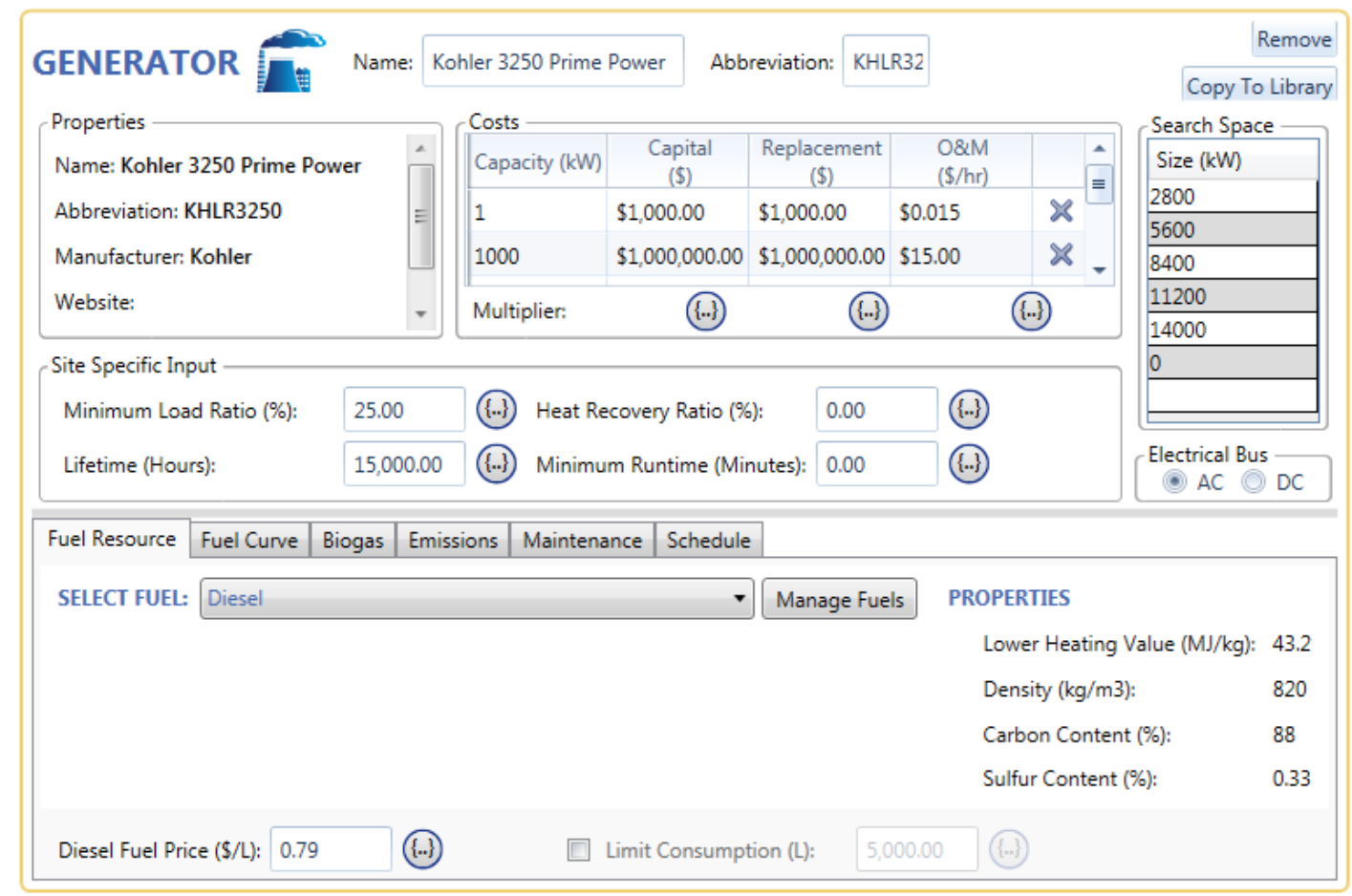

Figure 15: China Lake Sun Power PV Component

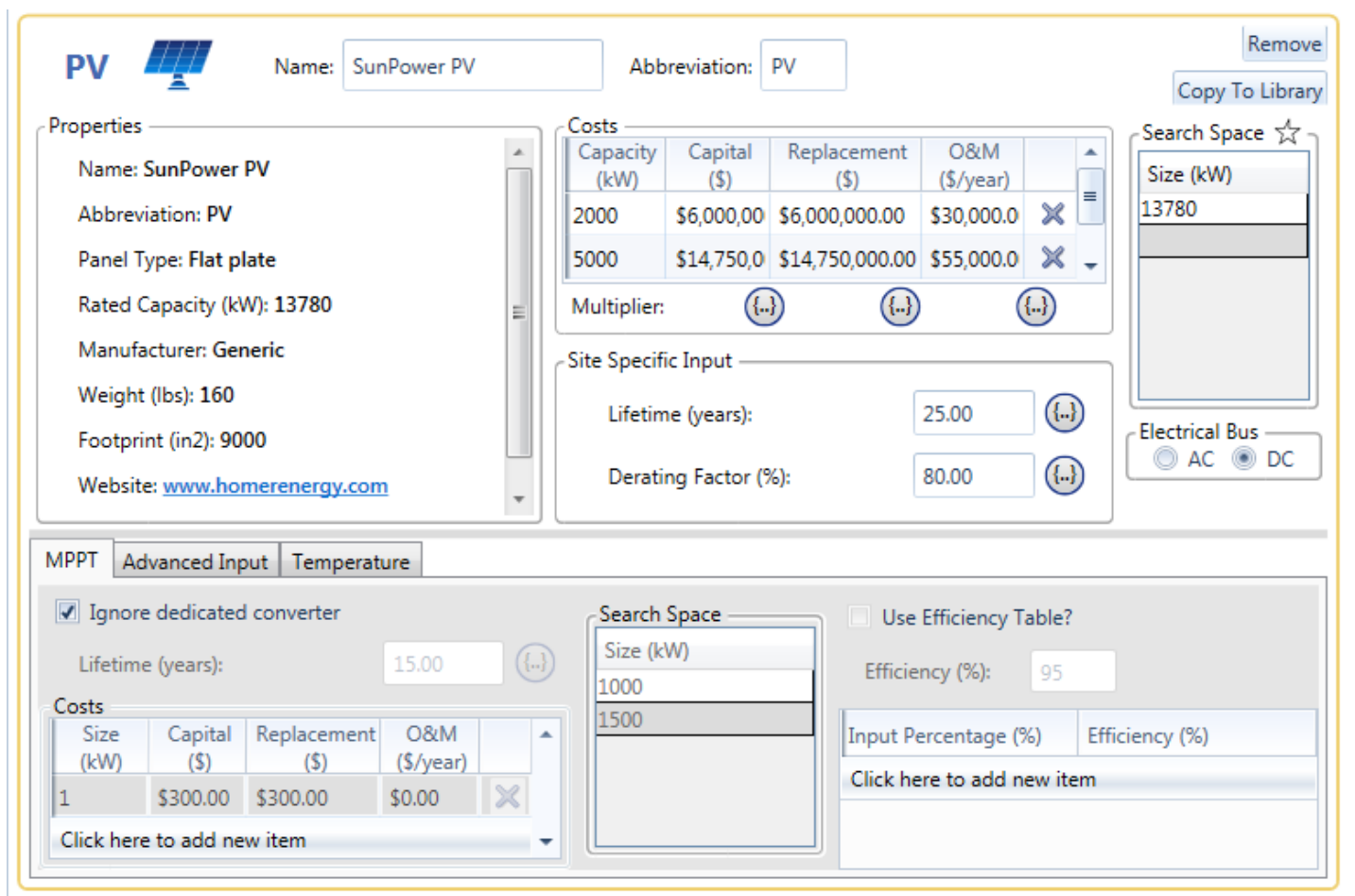


Figure 16: China Lake PV Distributed Component

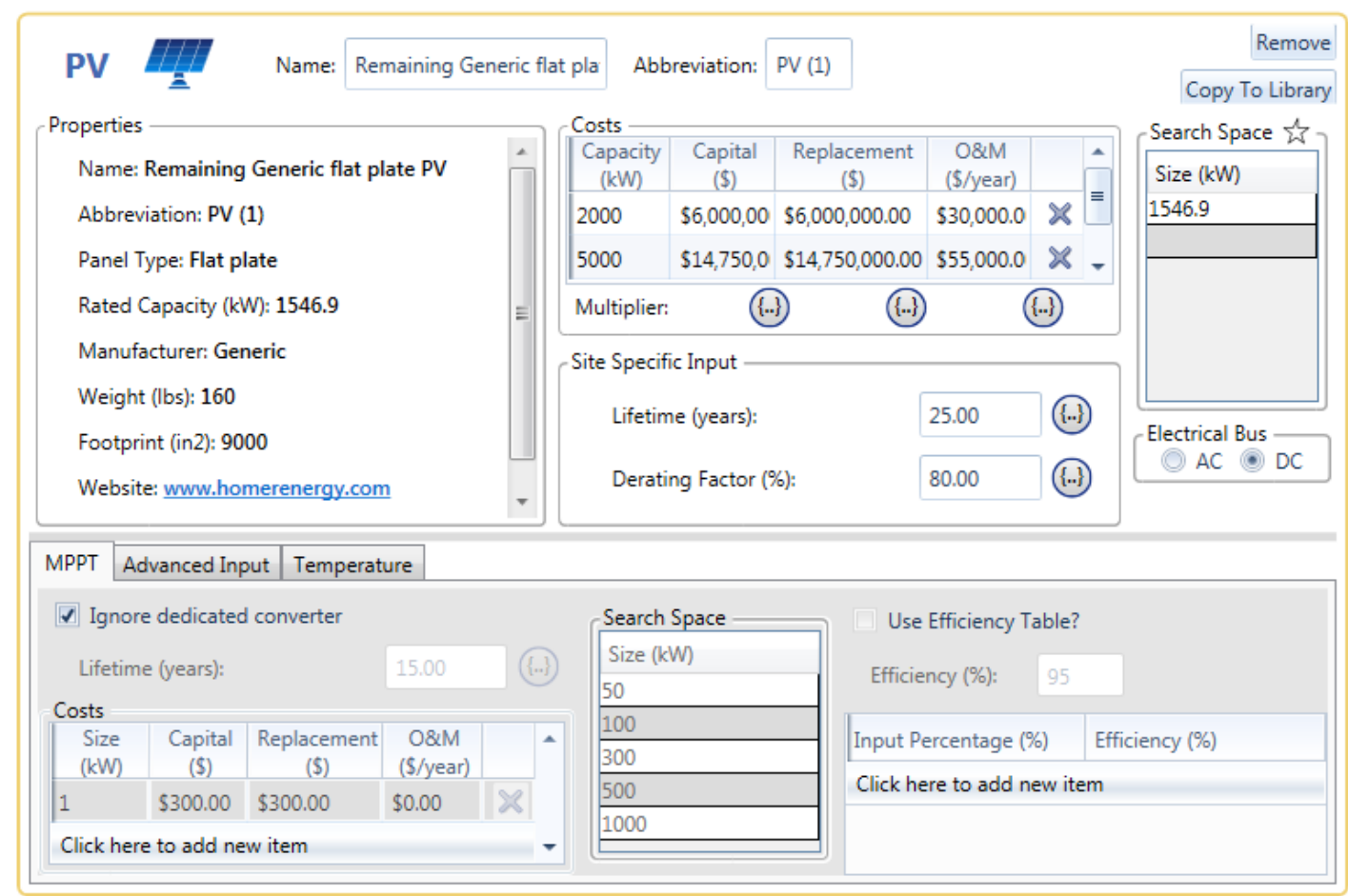

Figure 17: China Lake Additional PV Component

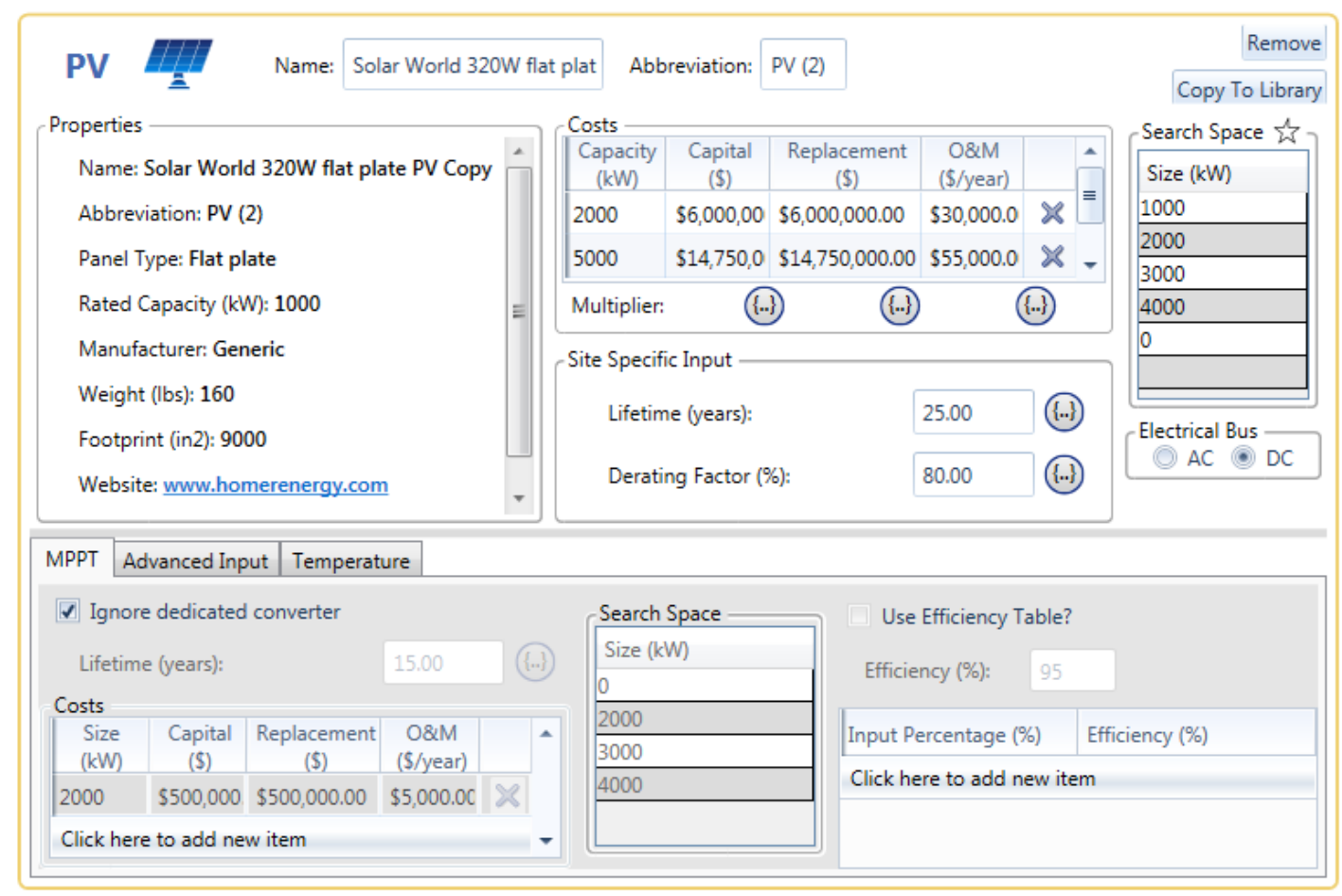


Figure 18: China Lake Wind Turbine Component

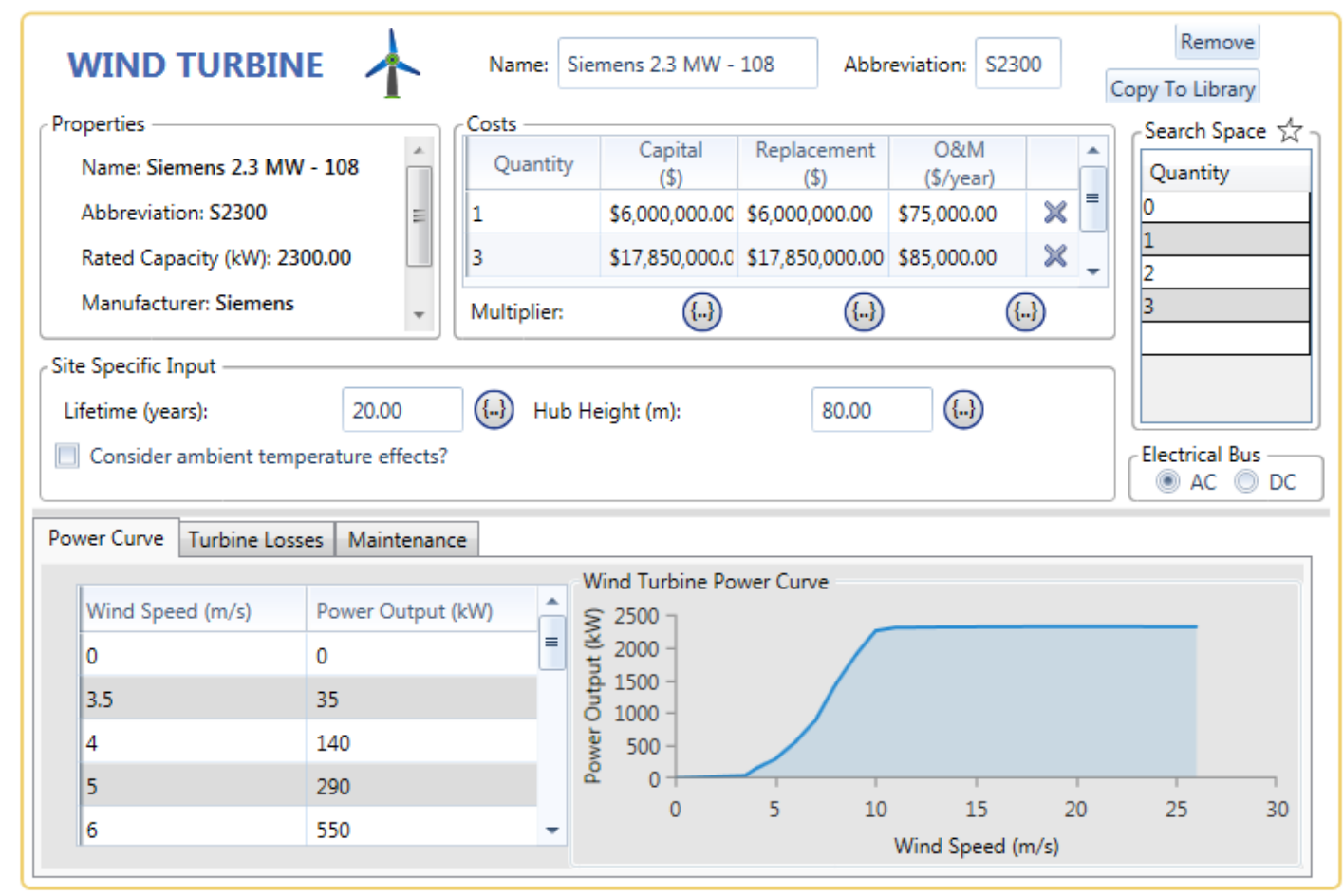

Figure 19: China Lake Battery Component

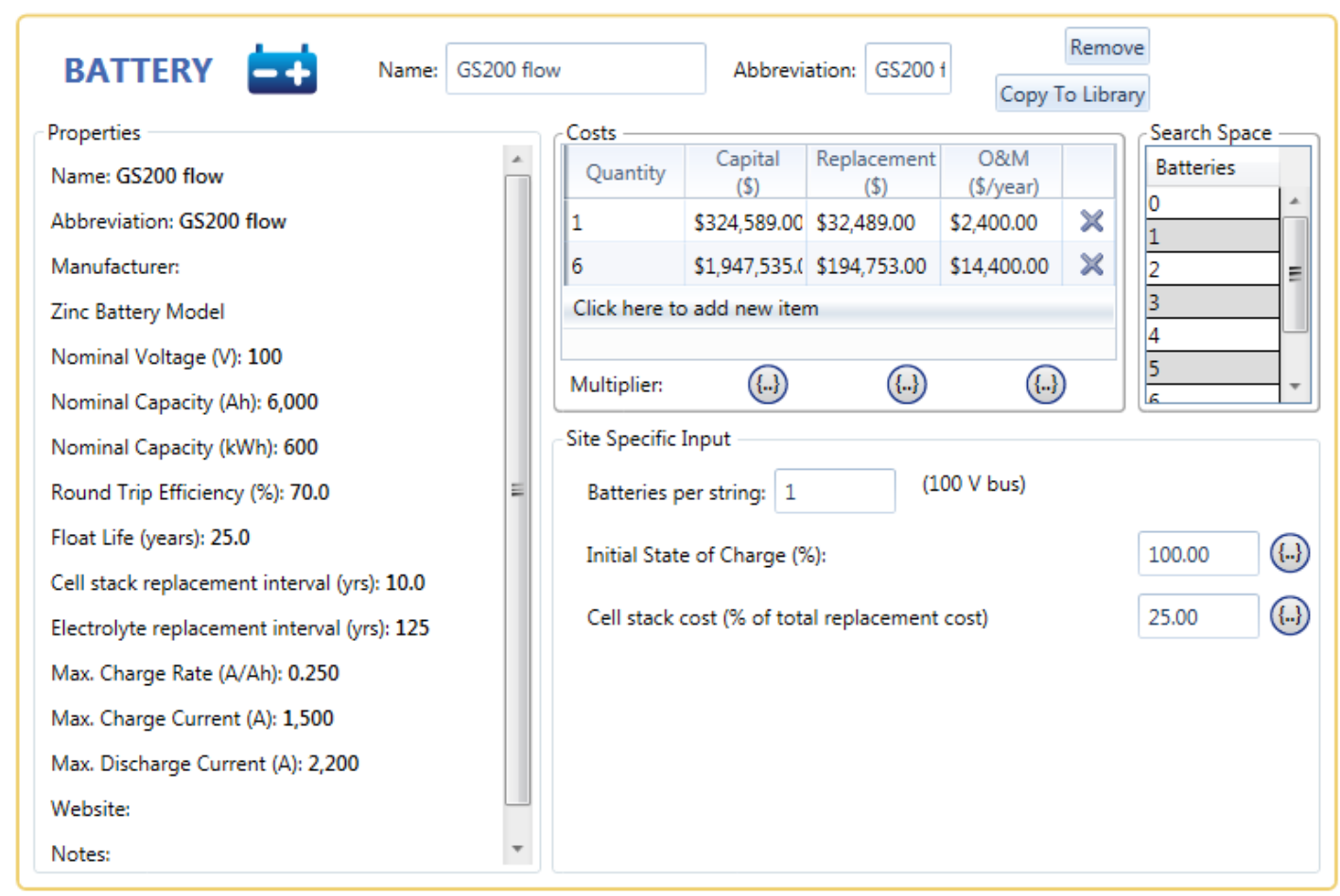


Figure 20: China Lake Converter Component

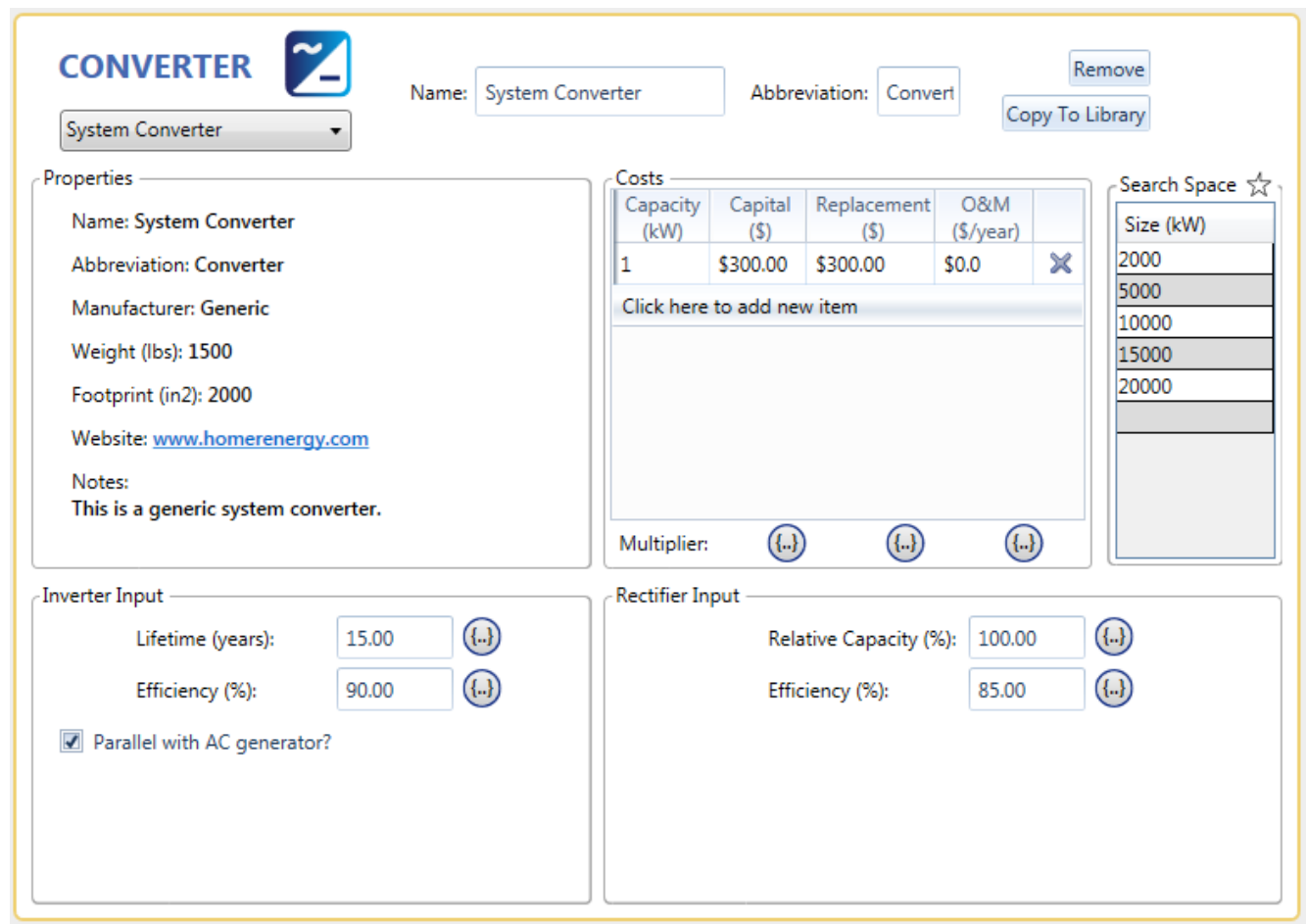

Figure 21: China Lake Grid Component

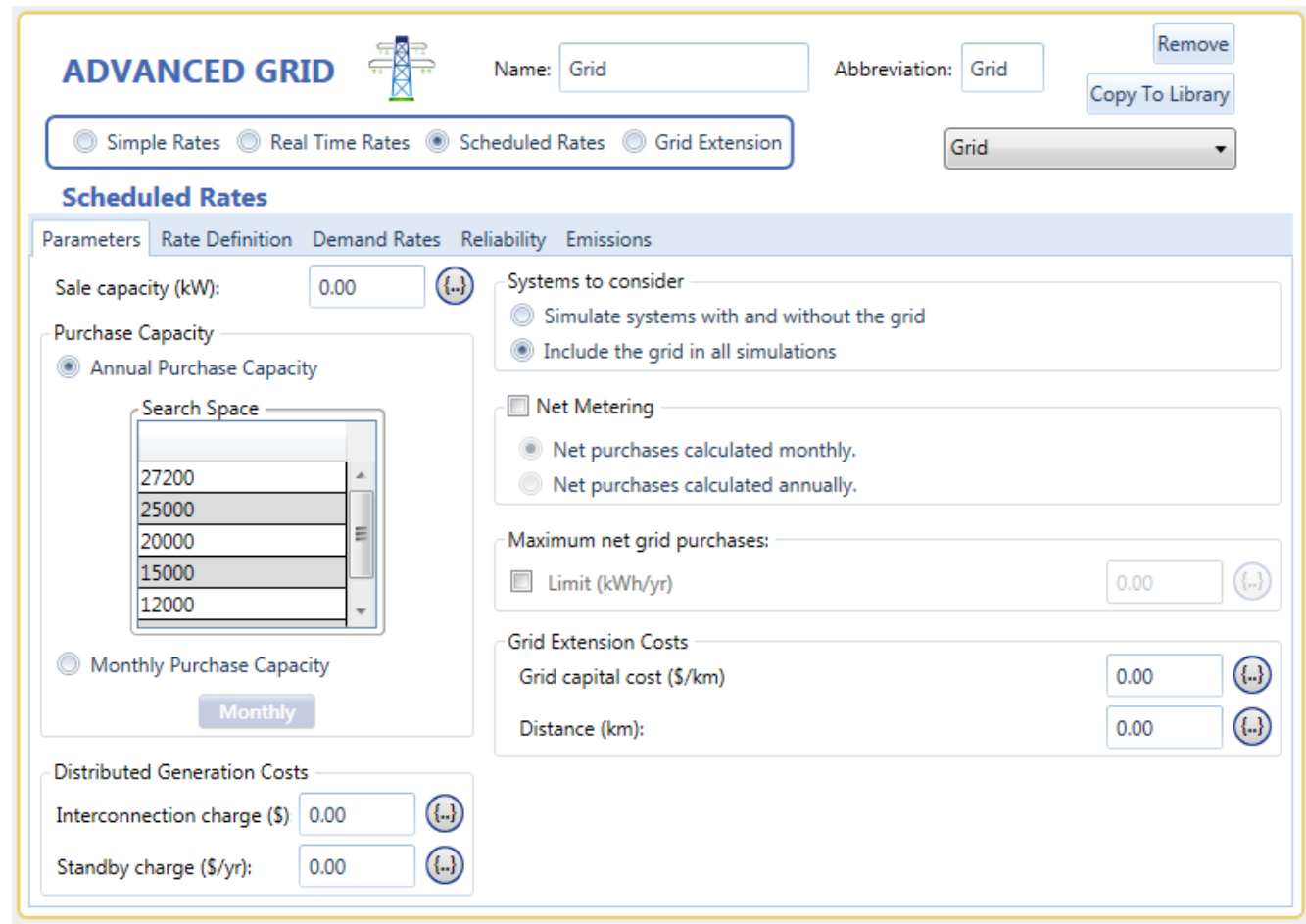


Figure 22: China Lake Solar Resource

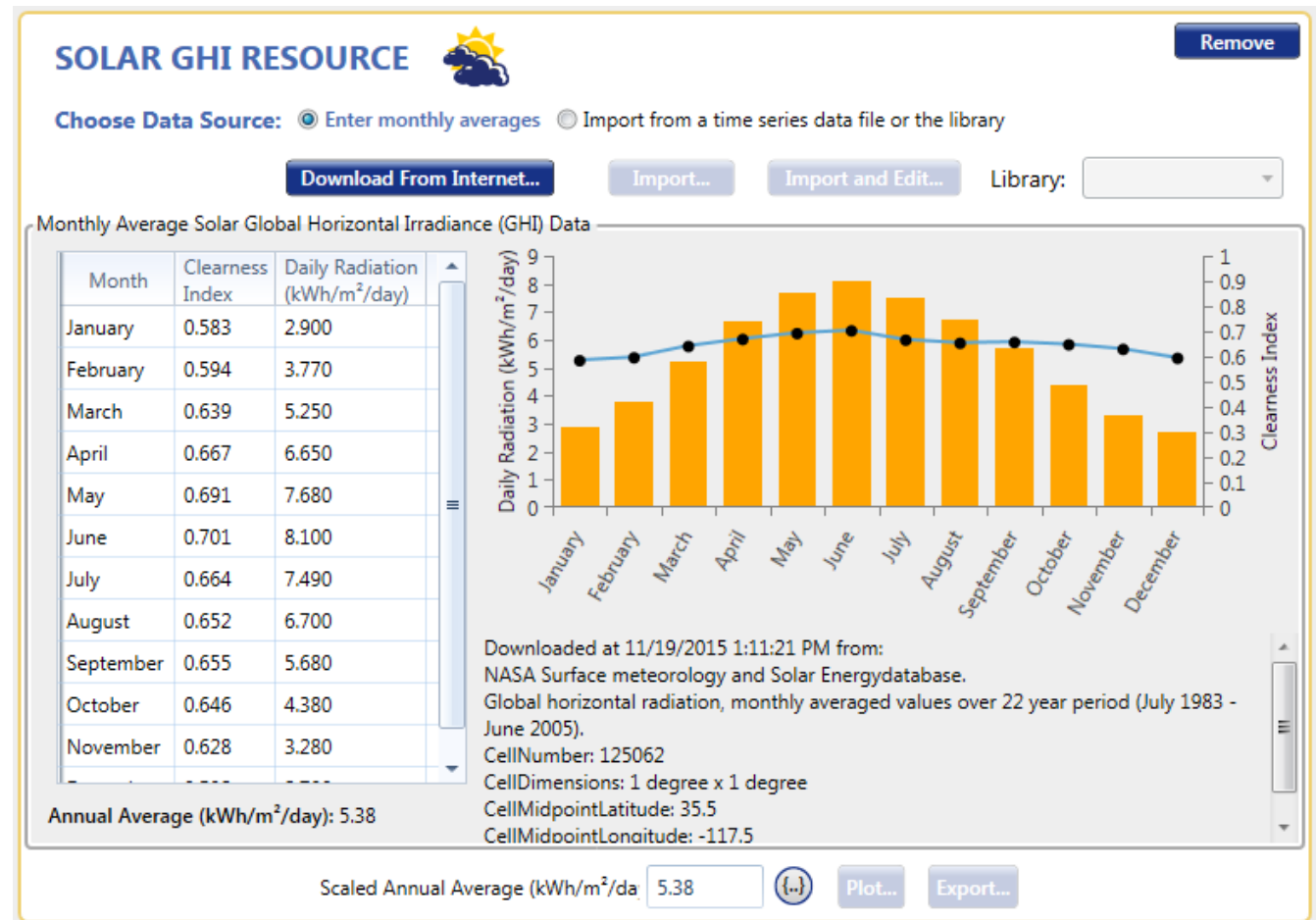

Figure 23: China Lake Wind Resource

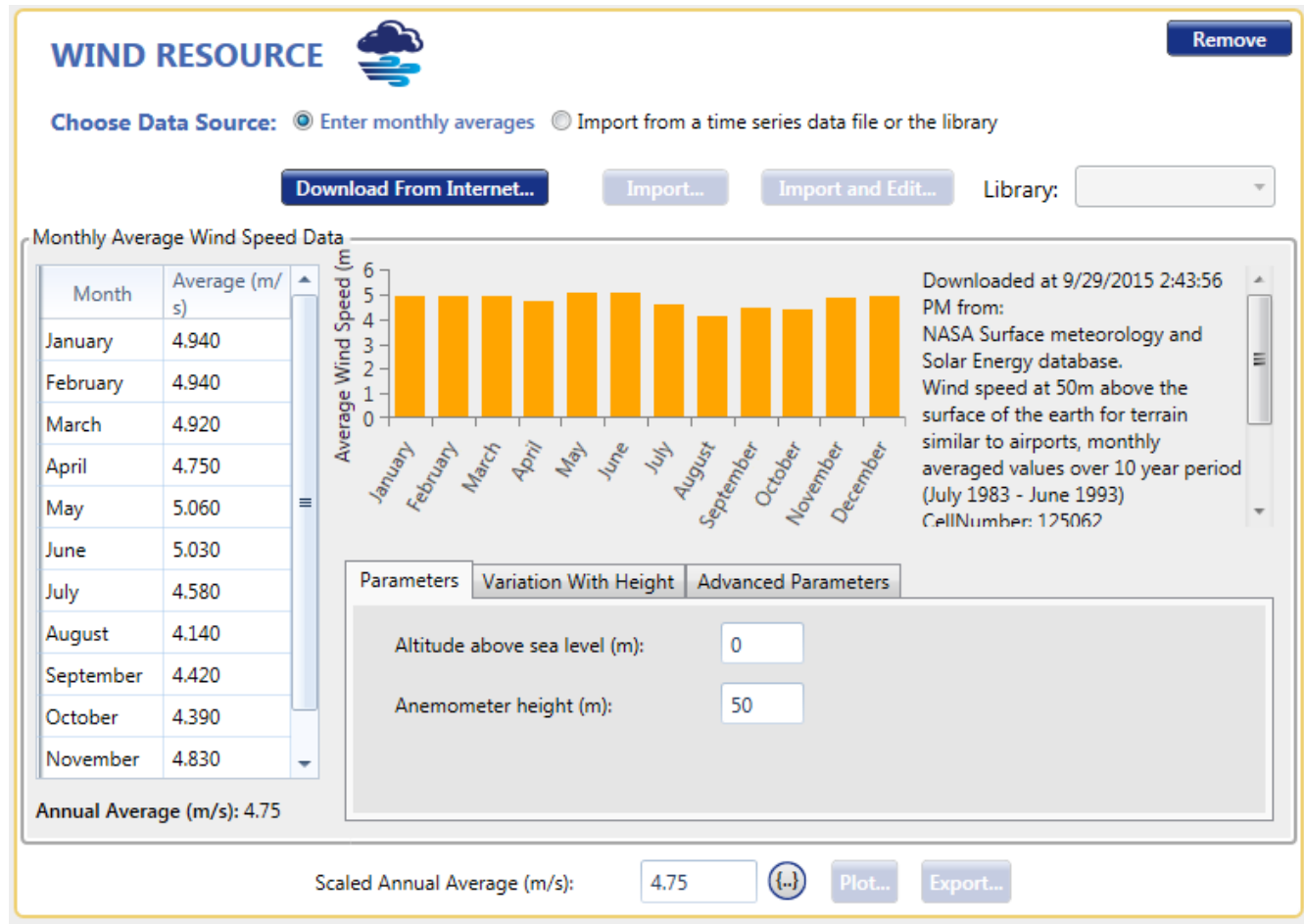


Figure 24: China Lake Temperature Resource

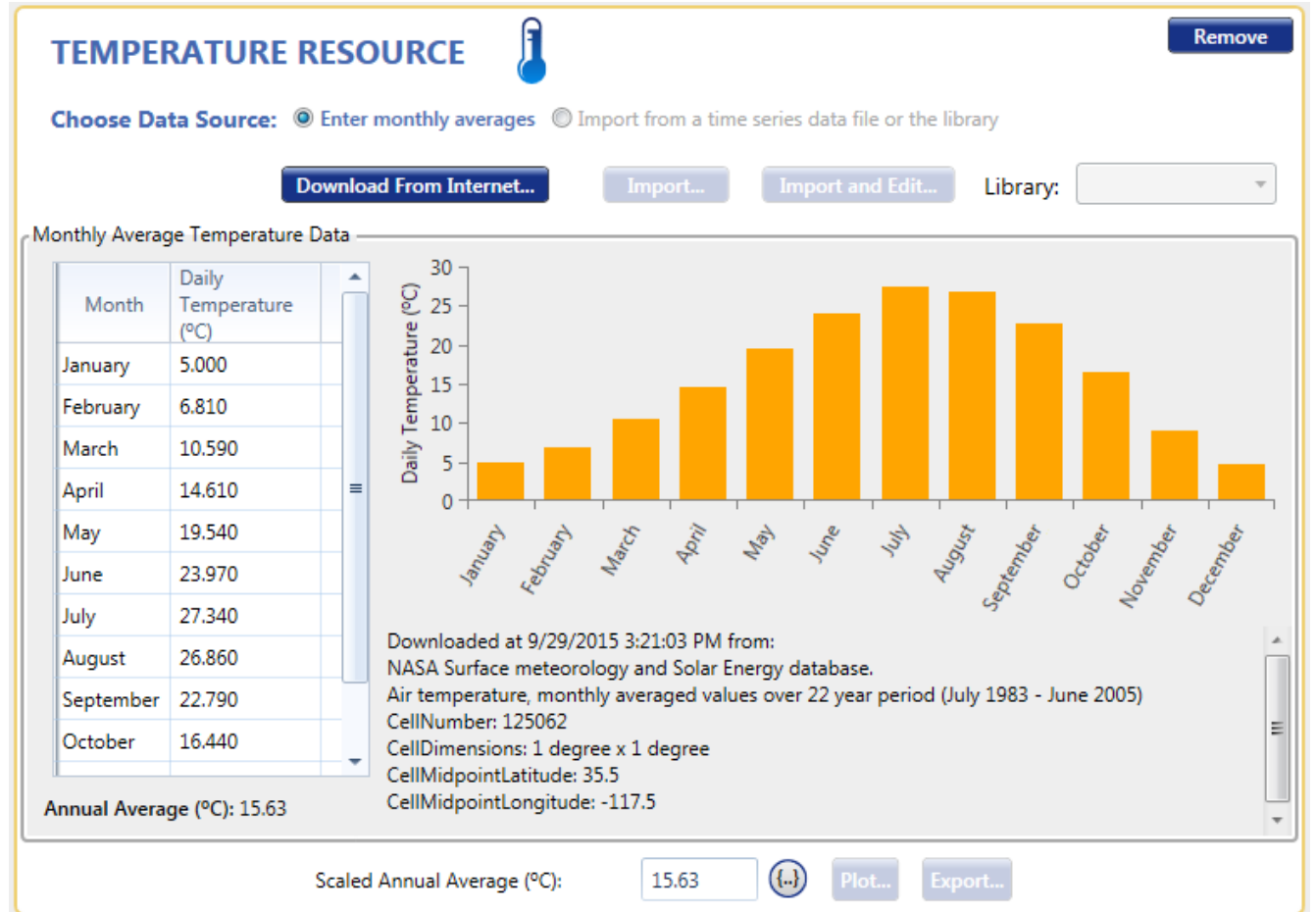

Figure 25: China Lake Fuel Resource

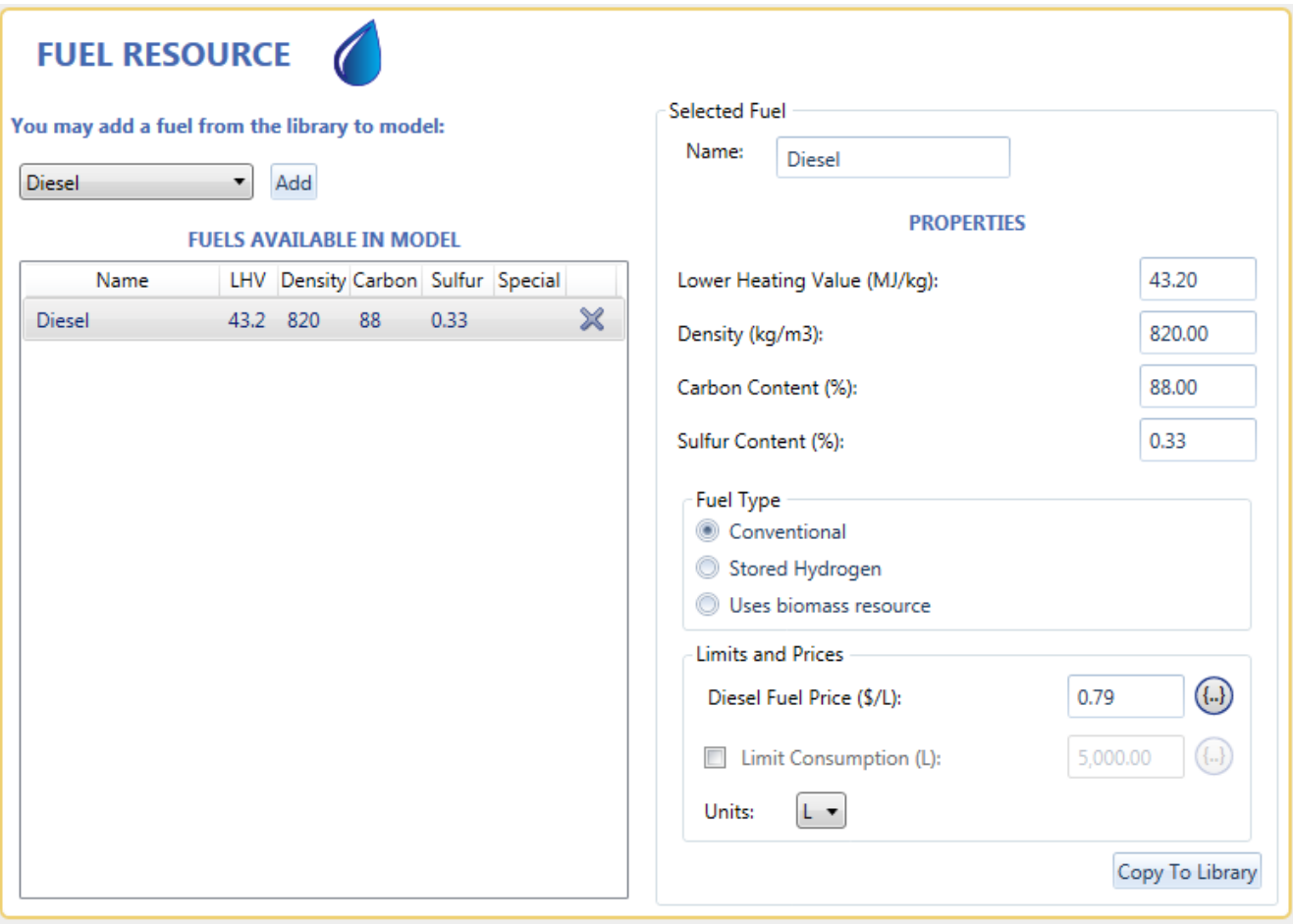


Figure 26: China Lake Electric Load

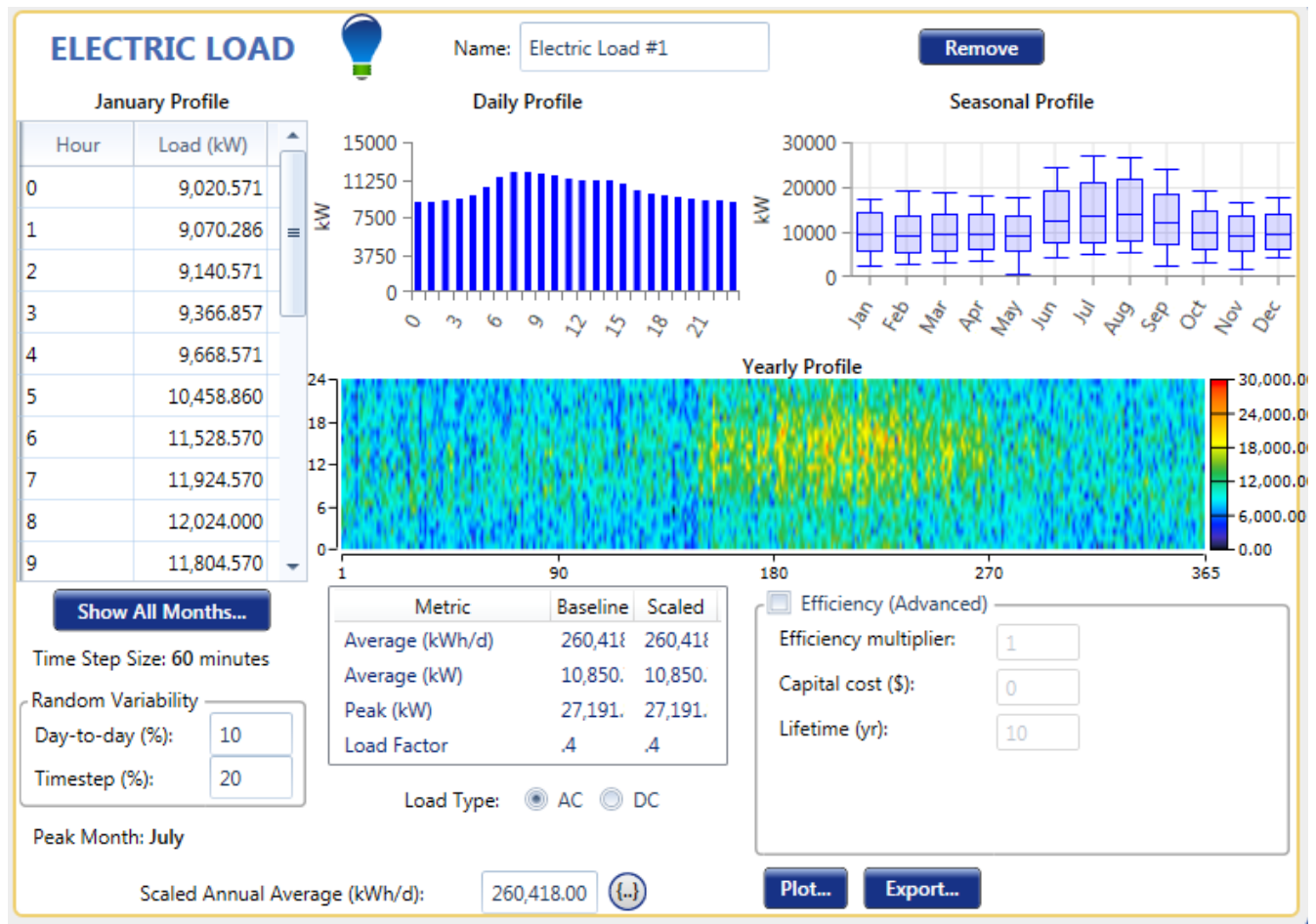

Table 4: China Lake Weekday Electrical Load Profile

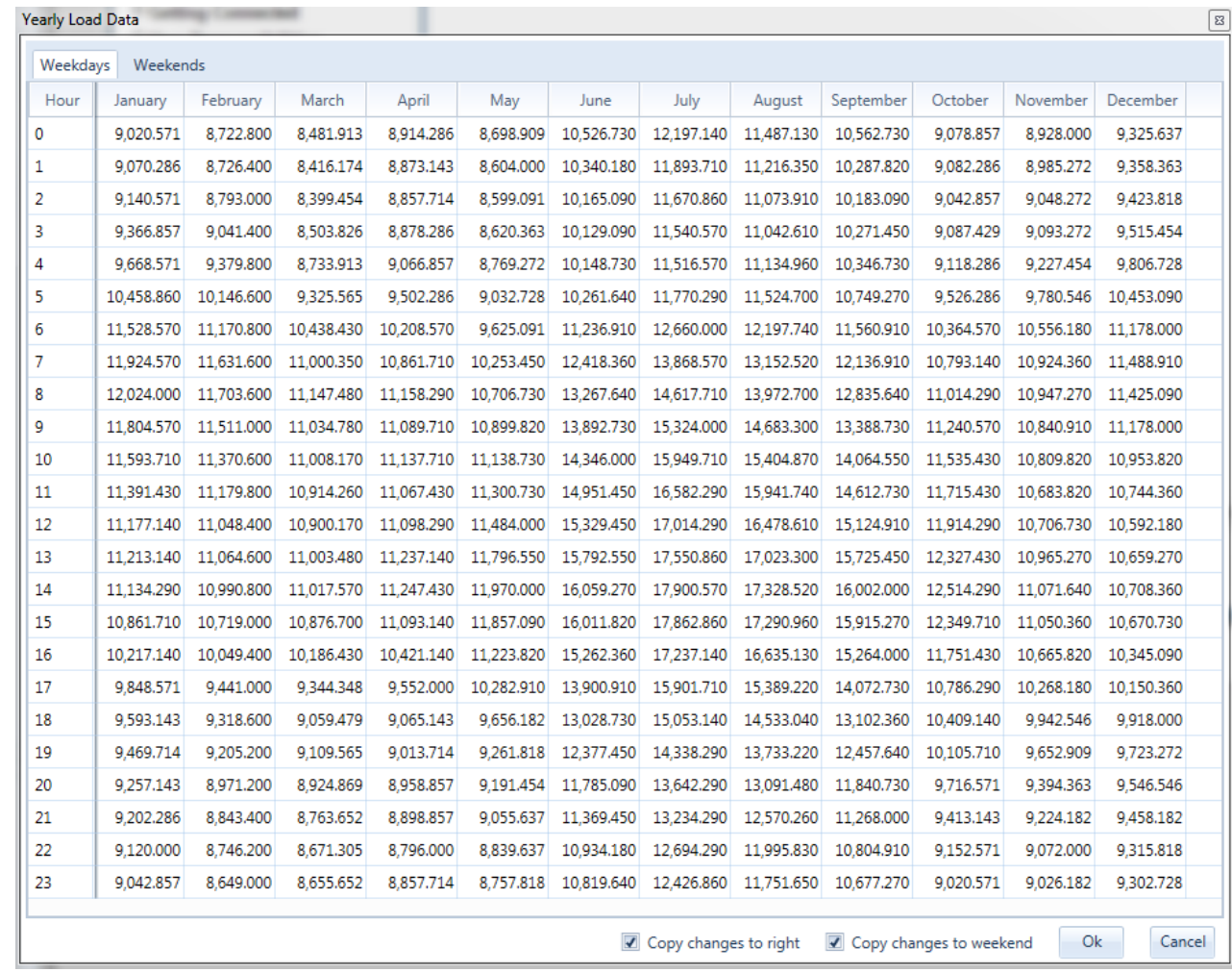


Table 5: China Lake Weekend Electrical Load Profile

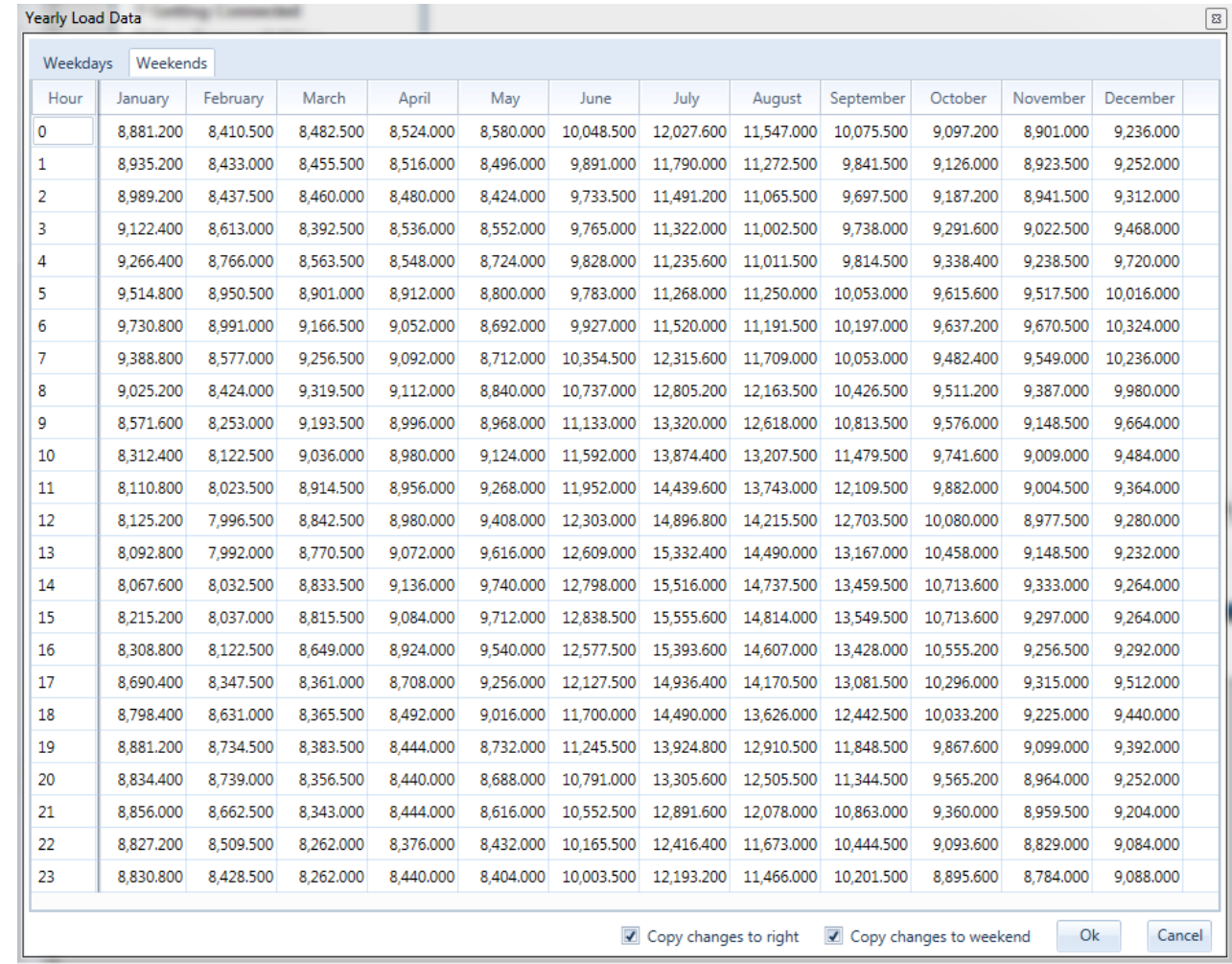

In both the grid-tied and island mode scenarios the two PV components (PV \& PV (1)) are already installed at China Lake (13.78 MW project and various other solar projects that total 1,546.9 kW). Inputs for these two components were set so HOMER always looks at using the currently installed PV generation. HOMER then has the option to consider other amounts of generation such as additional PV, wind, gensets, \& batteries, as well as different size system converter. These are the simulation results produced for the optimization cases:

1) Grid-tied:

a) $0-3250 \mathrm{~kW}$ Generators

b) $0-2.3 \mathrm{MW}$ Siemens 108 Wind Turbines

c) $13,780 \mathrm{~kW}$ Existing Flat Panel PV

d) 1,547 kW Existing Flat Panel PV

e) $0 \mathrm{MW}$ Additional Flat panel fixed PV

f) 0 - GS200 Flow Battery $(0 \mathrm{~kW})$

g) $10,000 \mathrm{~kW}$ System Converter

h) $27,200 \mathrm{~kW}$ Grid Purchases

i) $\mathrm{COE}=\$ 0.169$

2) Island:

a) $6-3250 \mathrm{~kW}$ Generator

b) $6-2.3 \mathrm{MW}$ Siemens 108 Wind Turbines

c) $13,780 \mathrm{~kW}$ Existing Flat Panel PV

d) $1,547 \mathrm{~kW}$ Existing Flat Panel PV 

e) $6 \mathrm{MW}$ Additional Flat panel fixed PV
f) 70 - ViZn GS200 Flow Battery ()
g) $20,000 \mathrm{~kW}$ System Converter
h) $\mathrm{COE}=\$ 0.272$

Figures $12 \& 13$ show the complete simulation results produced for the optimization cases:

\section{Grid-tied:}

Table 6: China Lake Grid-tied Sensitivity \& Optimization Results

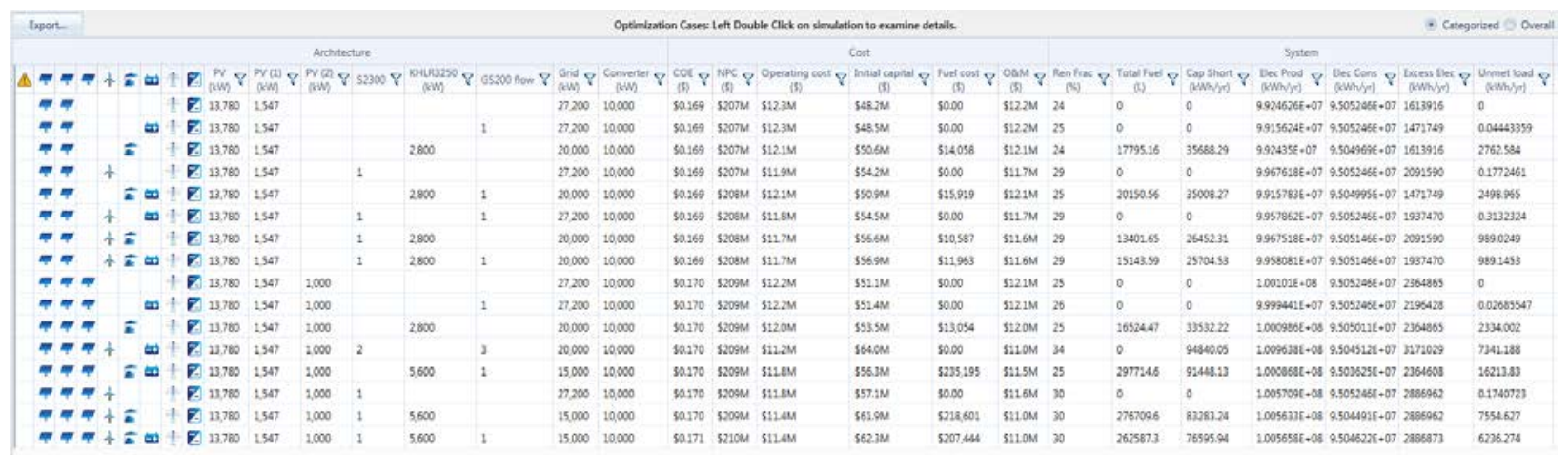

Island:

Table 7: China Lake Island Sensitivity \& Optimization Results

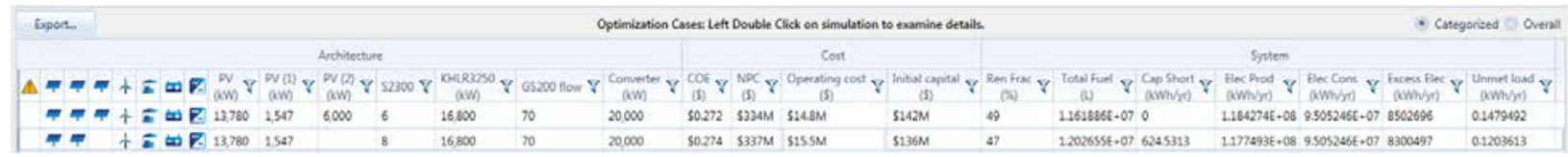

With the input parameters HOMER was given these were the only cases it found feasible. See case pdf's in appendix for further details on each simulation. 


\section{New London}

\subsection{HOMER Analysis}

Figure 27: New London HOMER

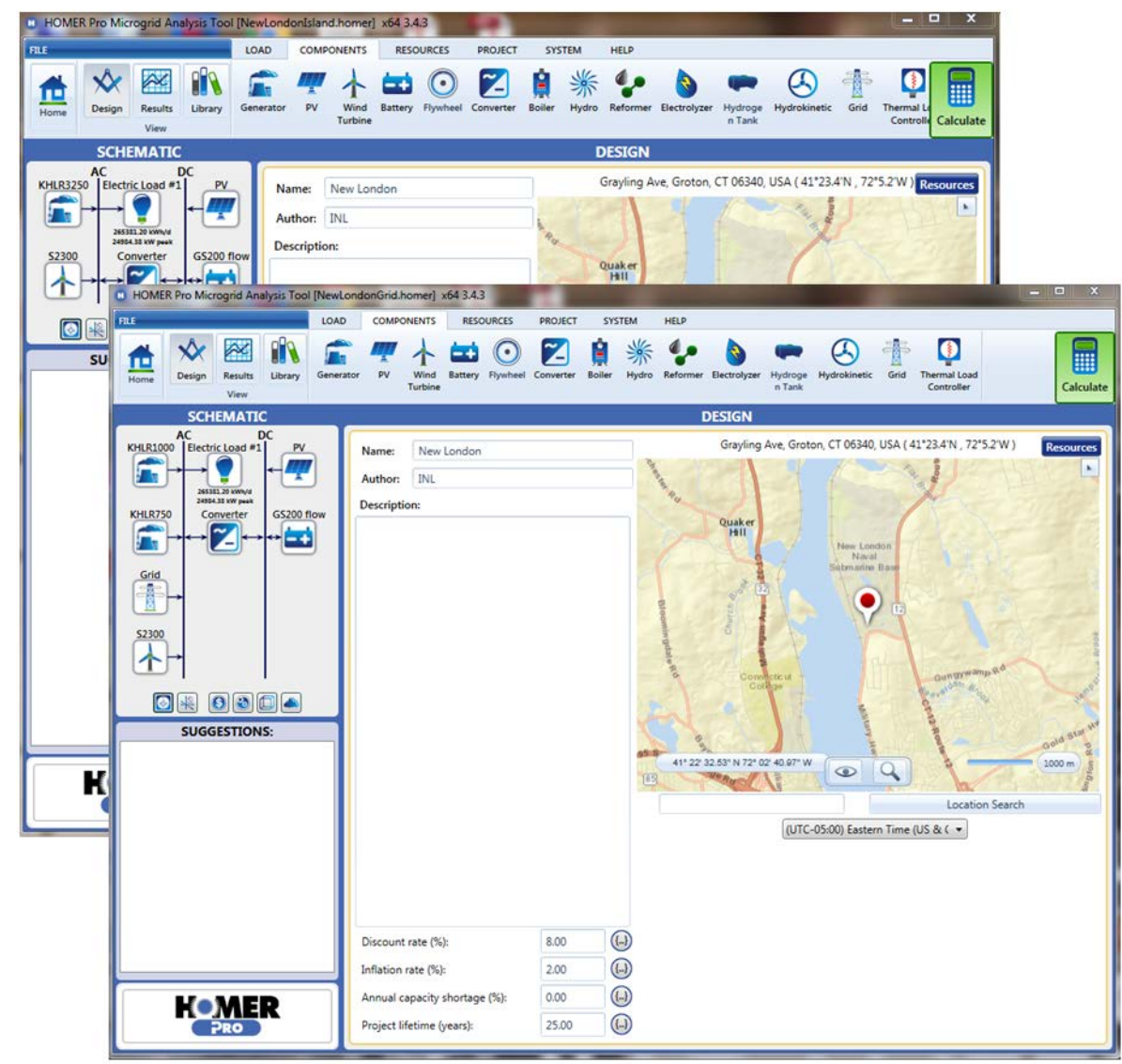

The HOMER (Hybrid Optimization of Multiple Energy Resources) model greatly simplifies the task of designing hybrid renewable microgrids, whether remote or attached to a larger grid. HOMER's optimization and sensitivity analysis algorithms allow you to evaluate the economic and technical feasibility of a large number of technology options and to account for variations in technology costs, electric load, and energy resource availability.

Multiple inputs are entered into the HOMER model such as location coordinates of $41^{\circ} 23.4^{\prime} \mathrm{N}$, $72^{\circ} 5.2^{\prime} \mathrm{W}$ which is near Grayling Ave on Naval Submarine Base New London. Resources and components are the main inputs that are needed to make a HOMER model. For New London the components used consist of Generator, PV, Wind Turbine, Battery, Converter and the Grid. INL has created cost inputs for these components based on vendor/market data and past experience. The resource inputs created consist of Solar, Wind, Temperature, Fuels, and Electric Load. Refer to figures (1 - 9). The solar, wind, \& temperature resources were downloaded from the NASA Surface meteorology and Solar Energy database and contained monthly averaged values for a 10 to 22 year period $(1983-2005)$. The fuel resource consists of a Diesel profile that was generated from current market information. The electrical load data was an analysis of naval power load spreadsheet data provided for the year 2013. Based on the power data HOMER estimates annual peak load as $24,984.38 \mathrm{~kW}$. Grid purchase inputs are then set as $12000,15000,20000,25000, \& 27200 \mathrm{kWs}$ with grid sales set as $0 \mathrm{kWs}$. Note in the New London model; both the grid-tied and off-grid (island) systems are modeled. 
Figure 28: New London Generator Component 1

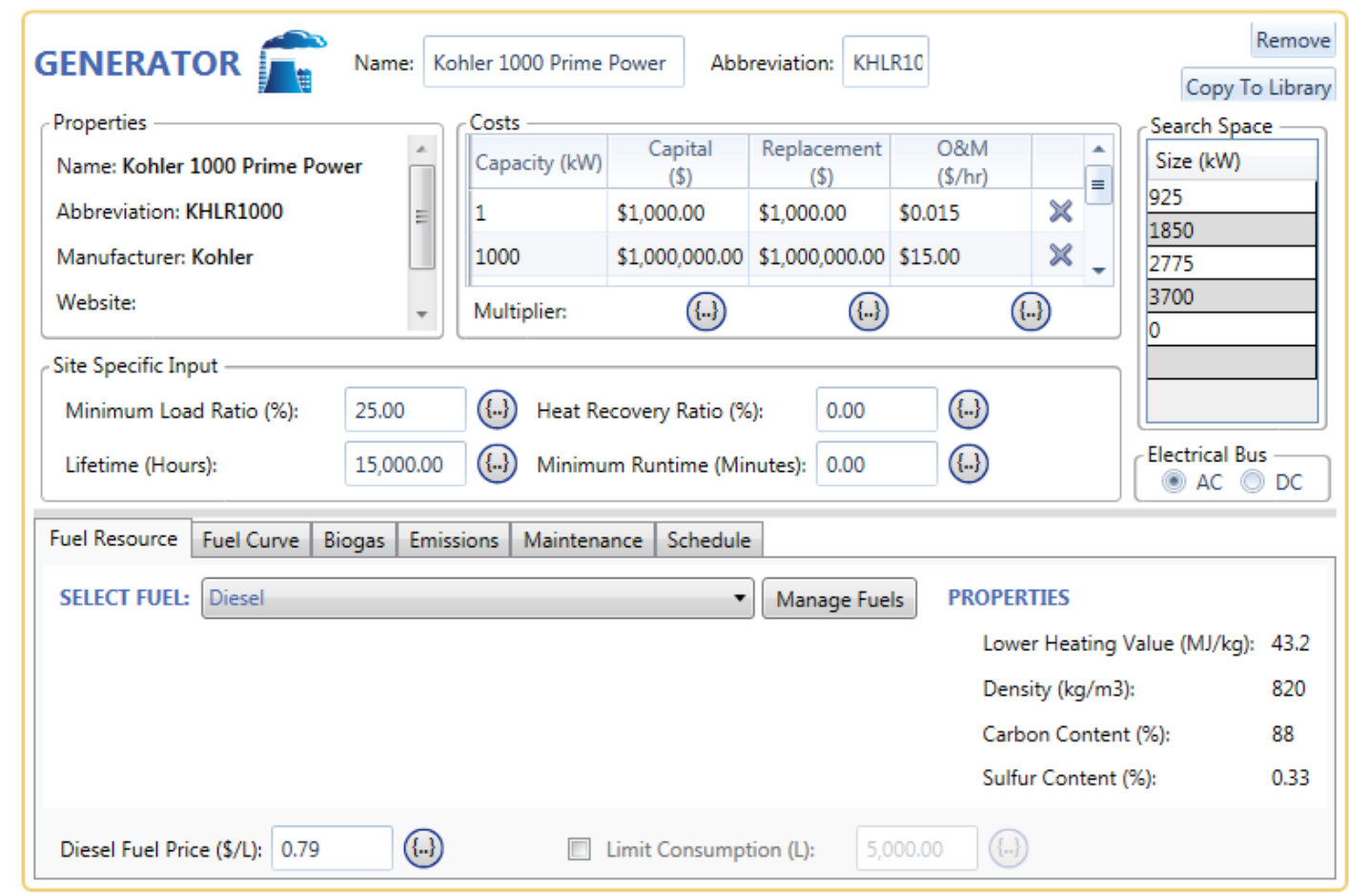

Figure 29: New London Generator Component 2

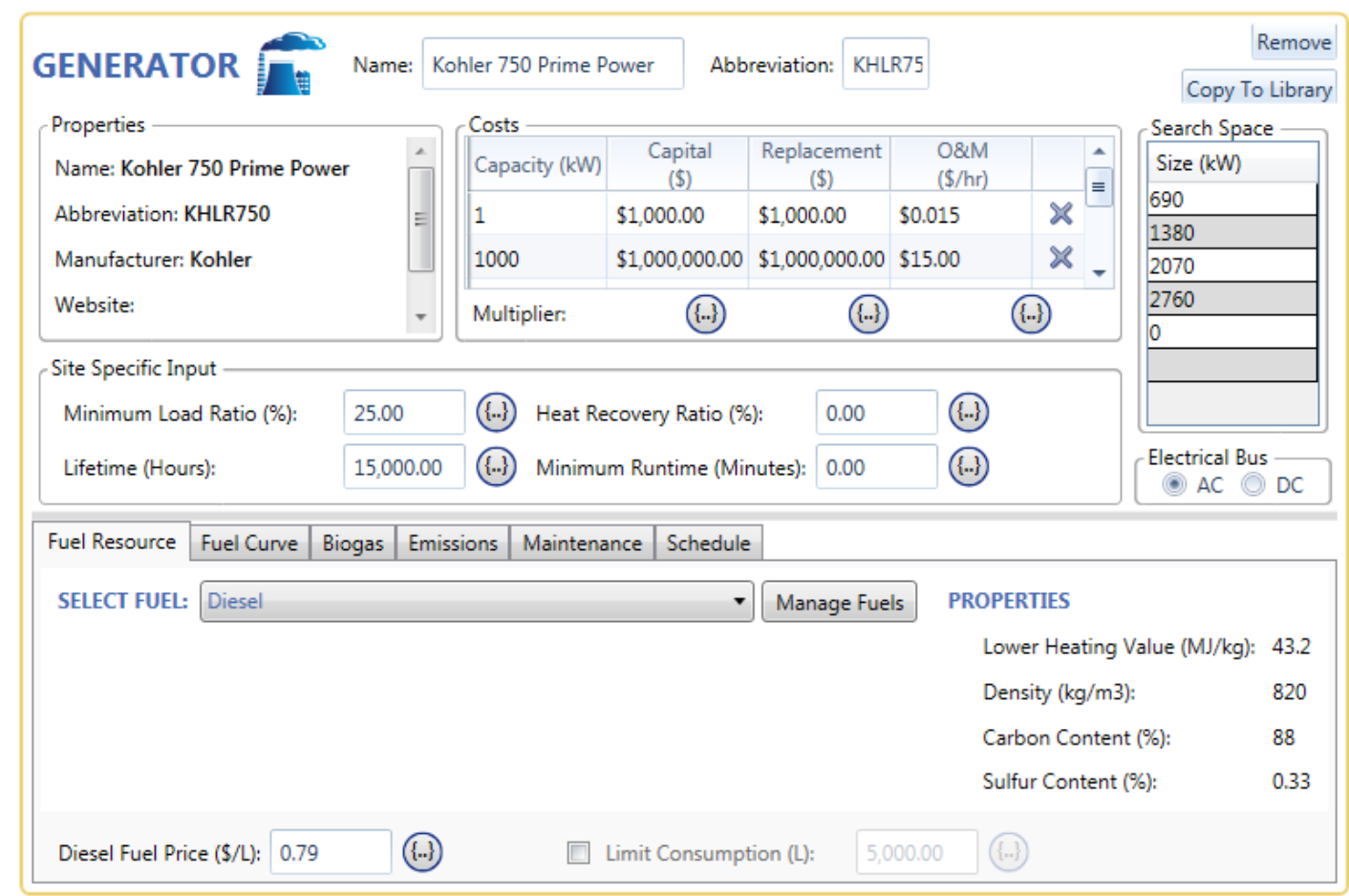


Figure 30: New London PV Component

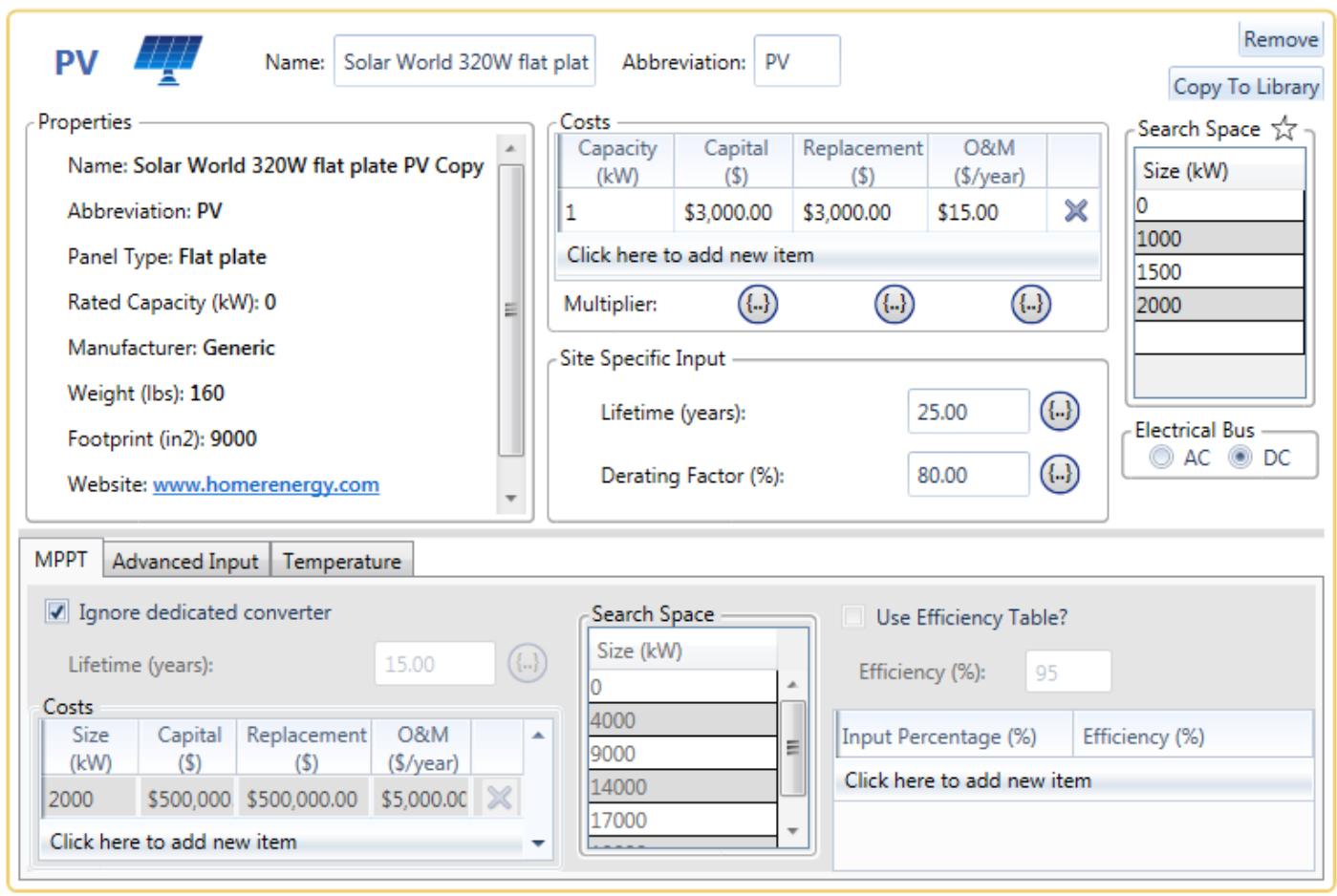

Figure 31: New London Wind Turbine Component

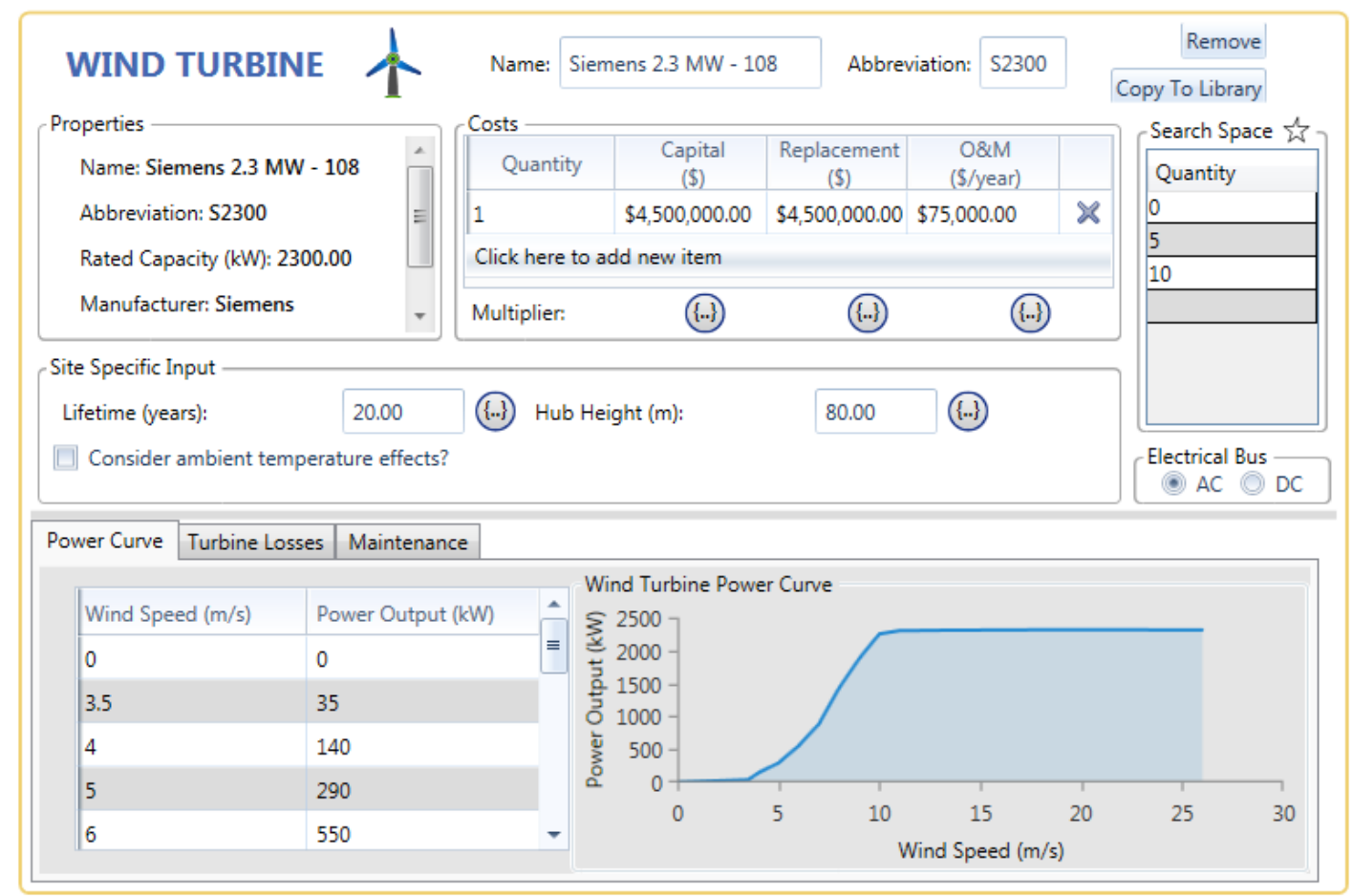


Figure 32: New London Battery Component

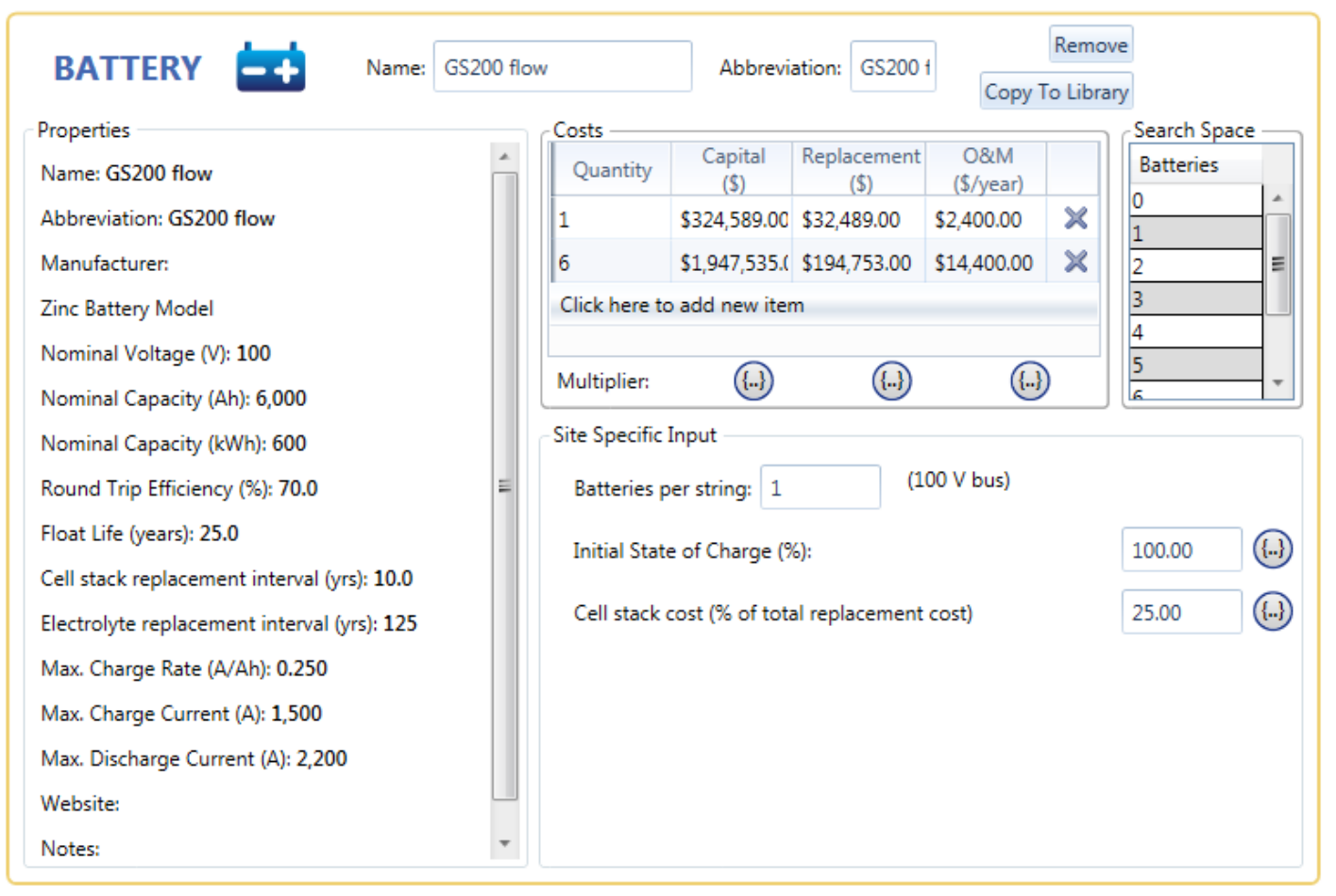

Figure 33: New London Converter Component

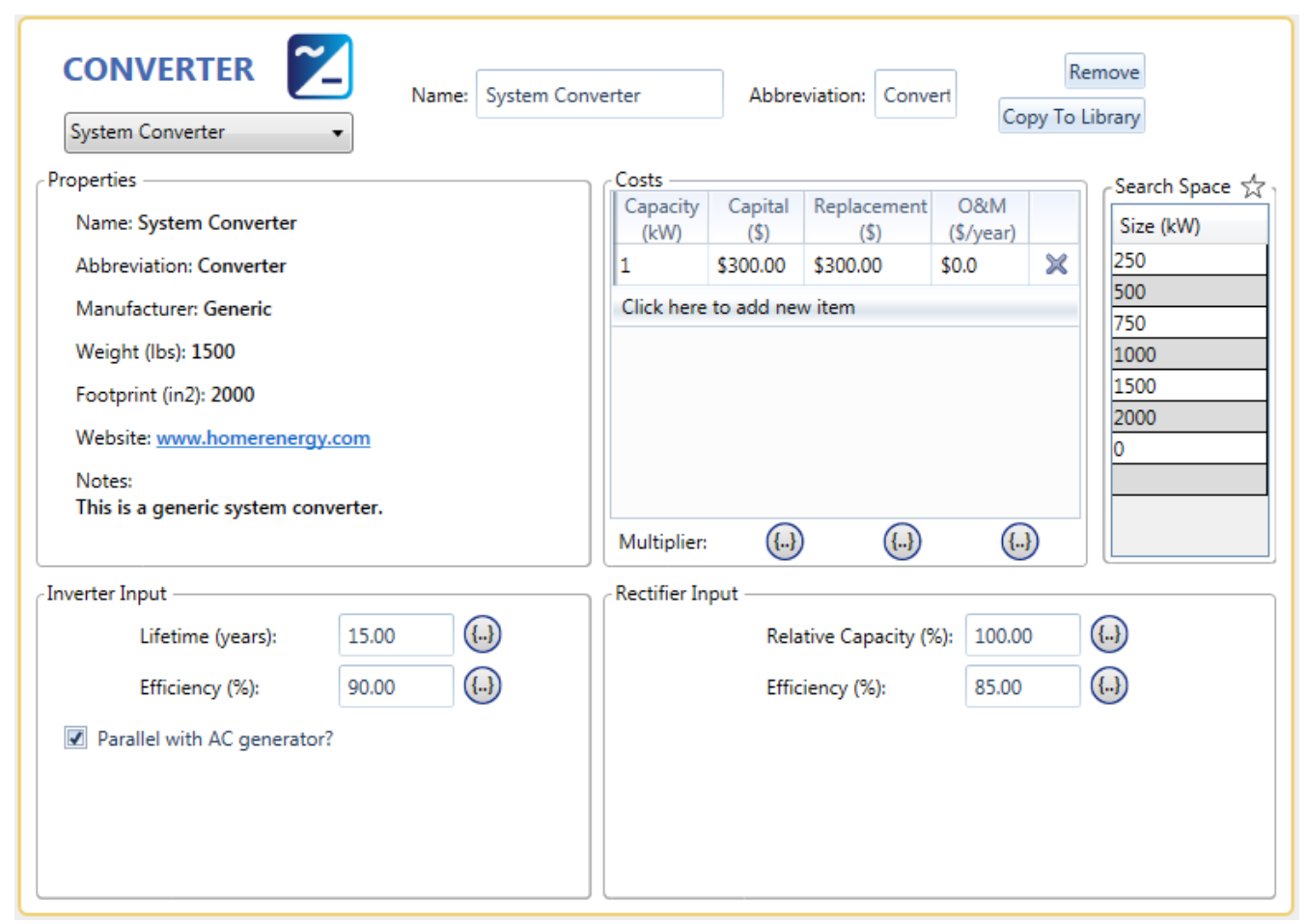


Figure 34: New London Grid Component

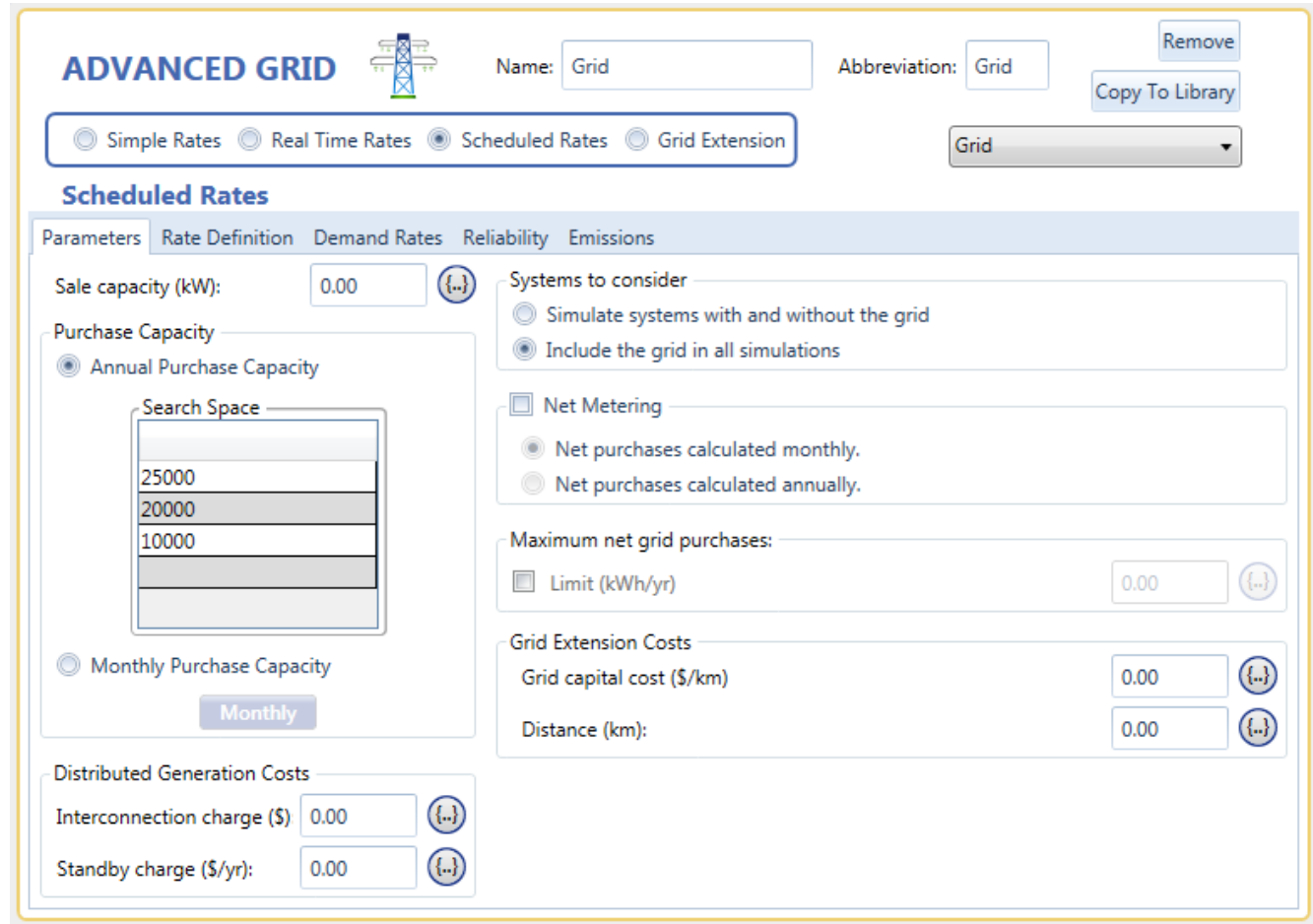

Figure 35: New London Solar Resource

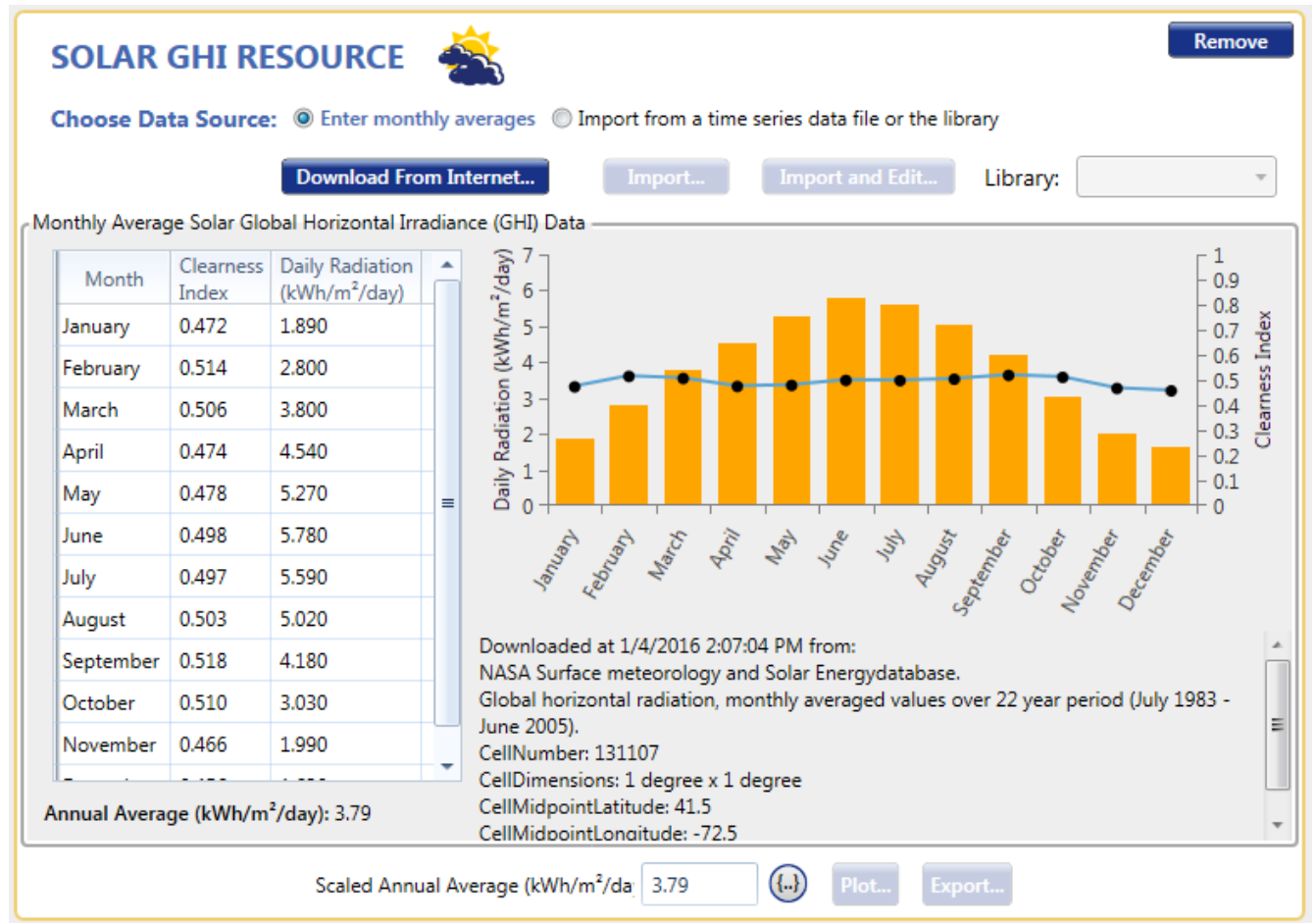


Figure 36: New London Wind Resource

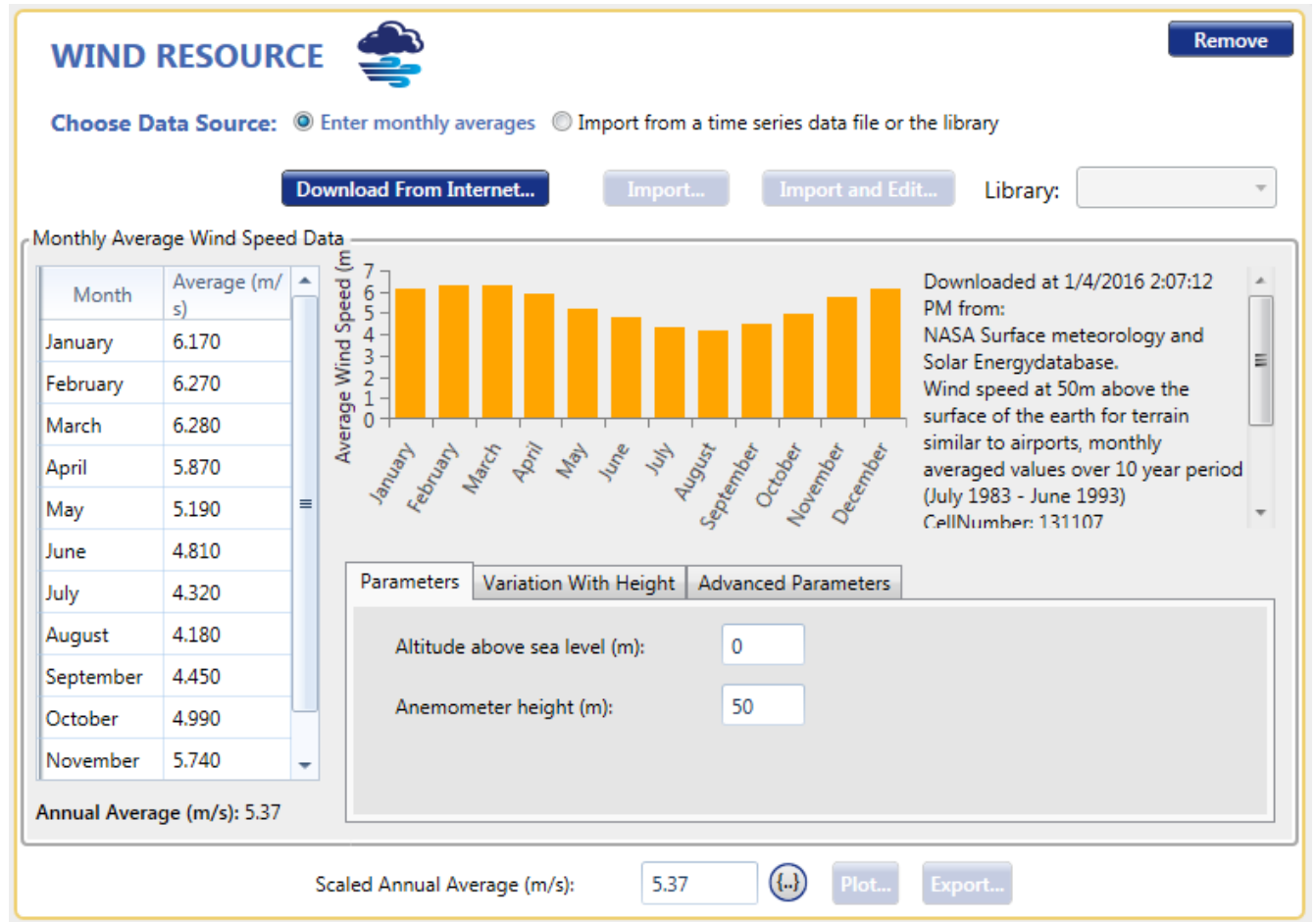

Figure 37: New London Temperature Resource

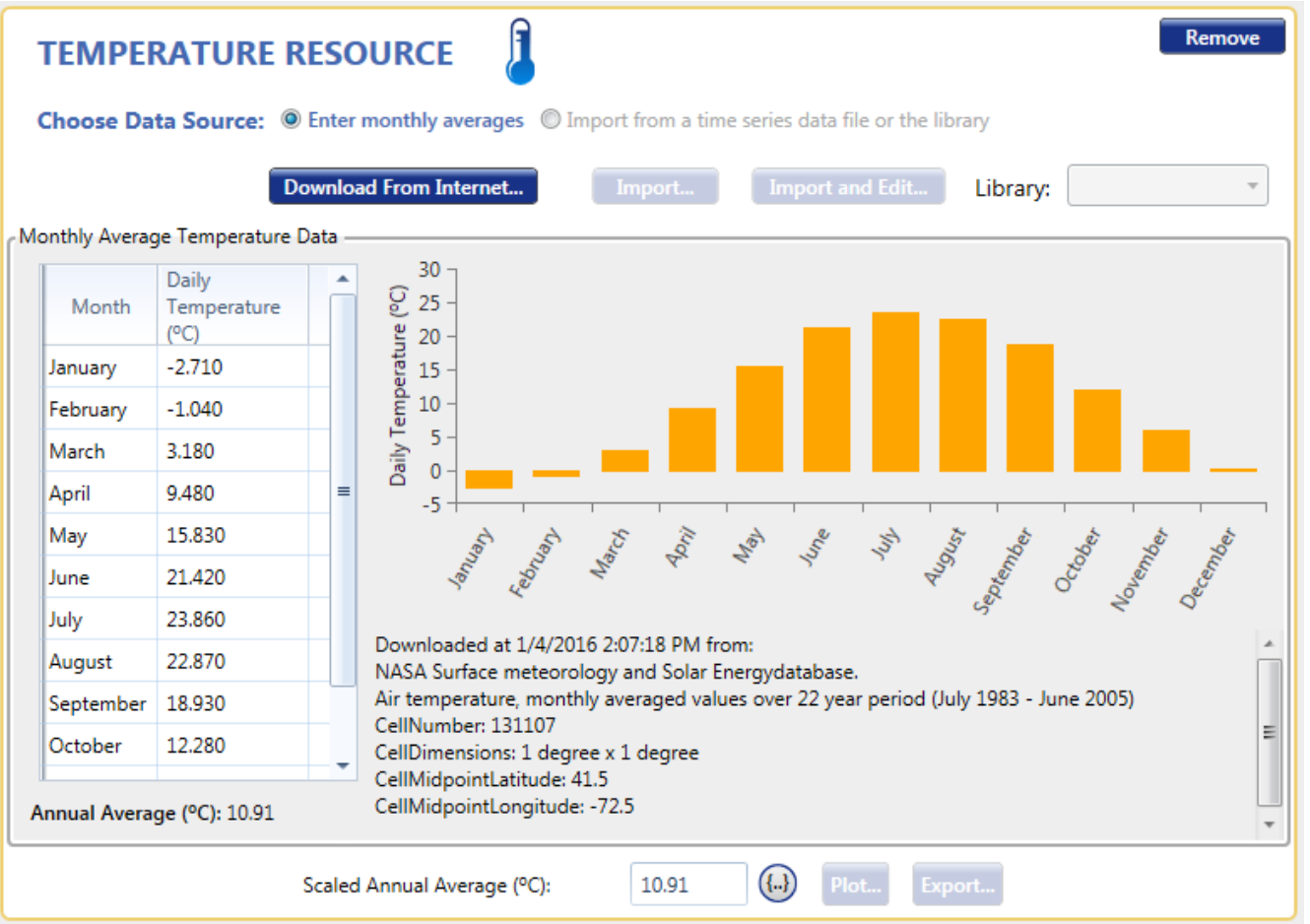


Figure 38: New London Fuel Resource

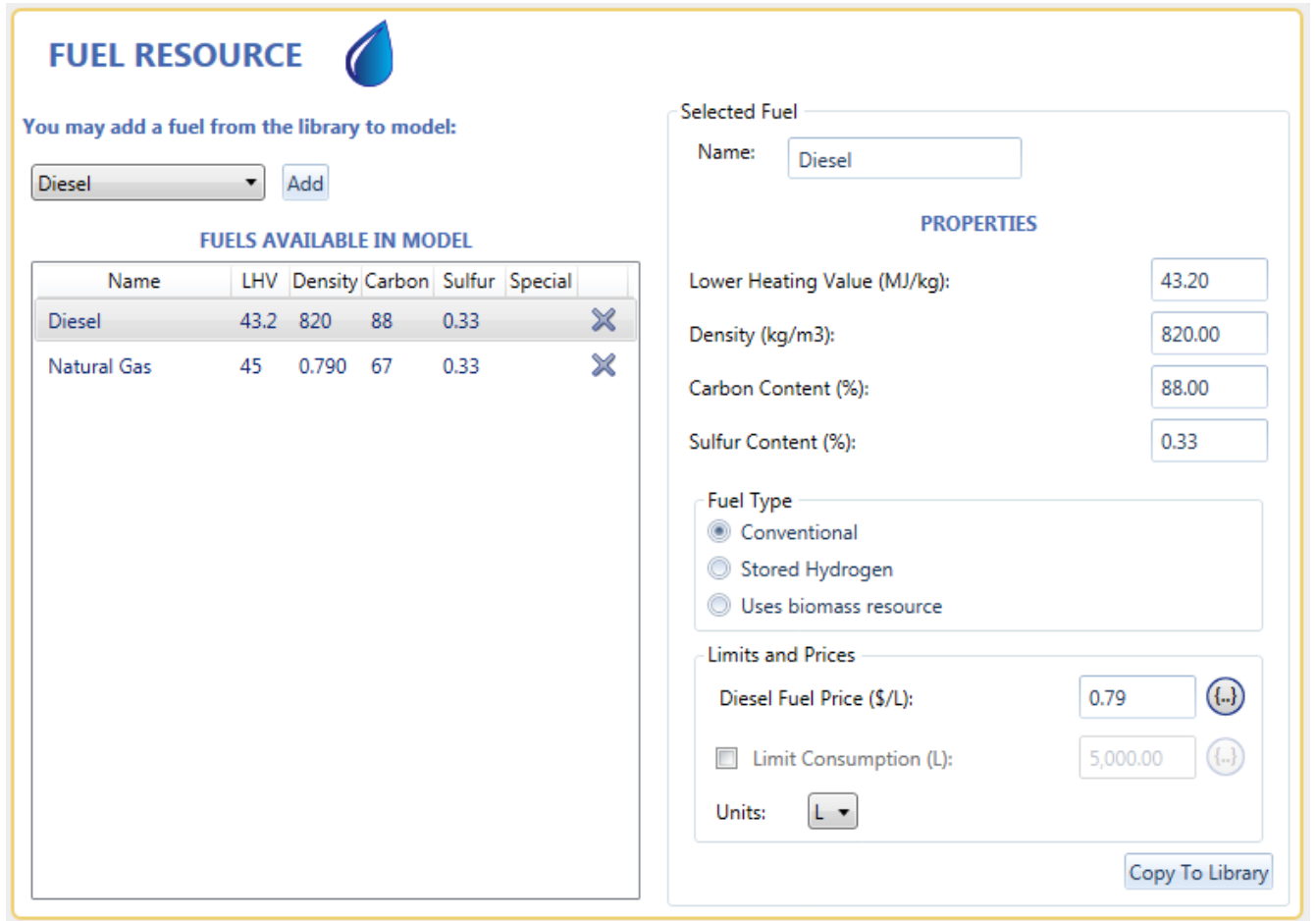

Figure 39: New London Electric Load

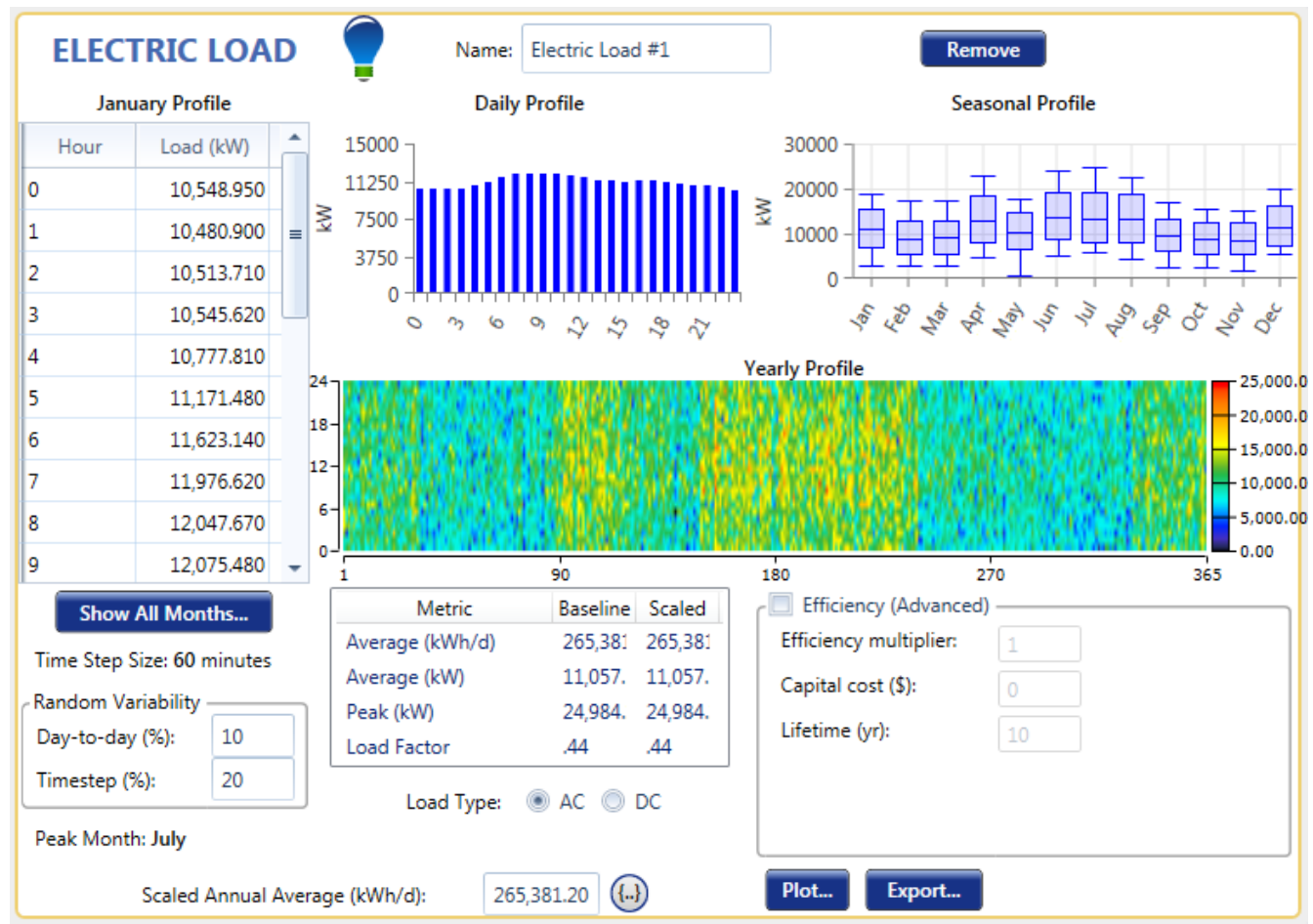


Table 8: New London Weekday Electrical Load Profile

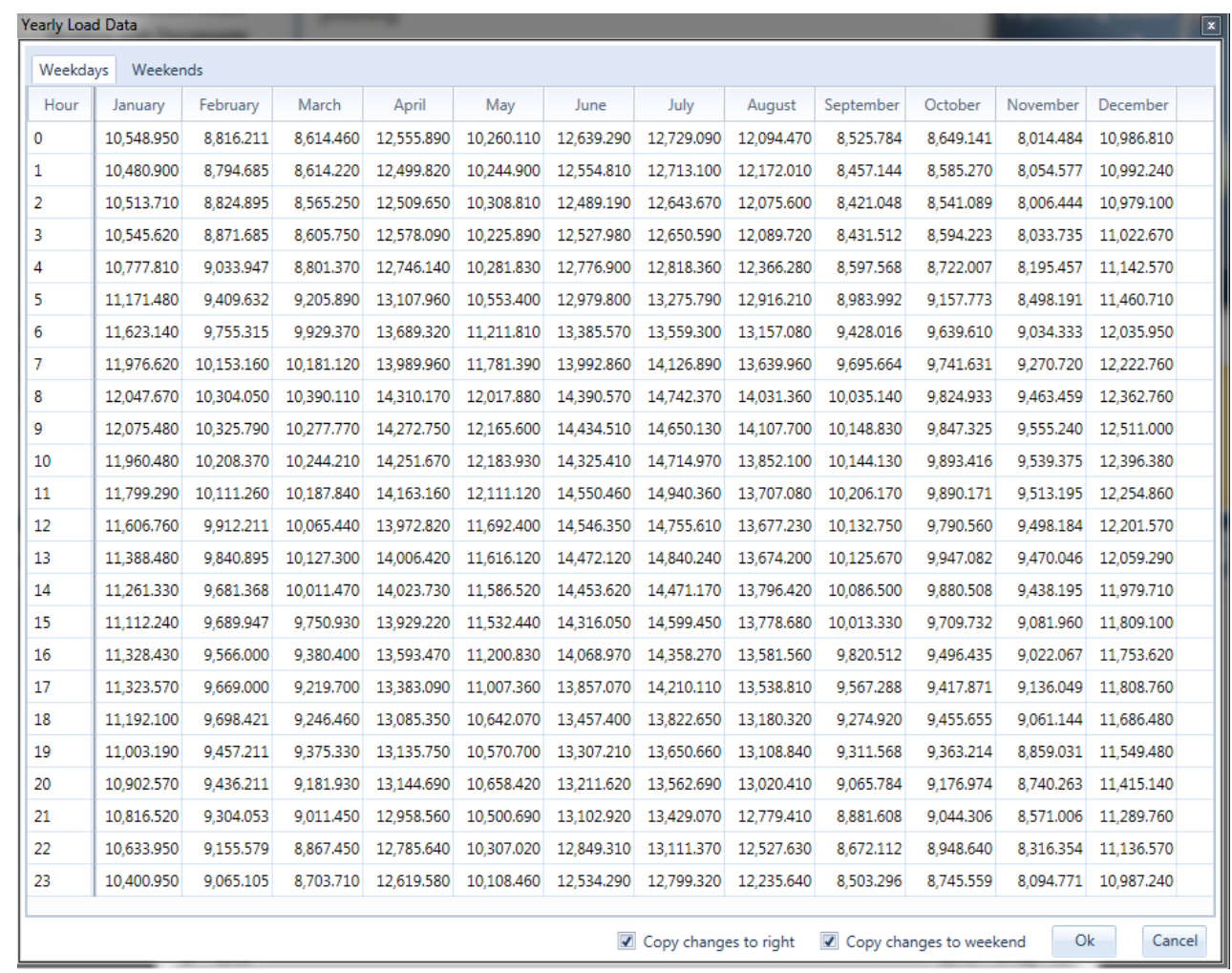

Table 9: New London Weekend Electrical Load Profile

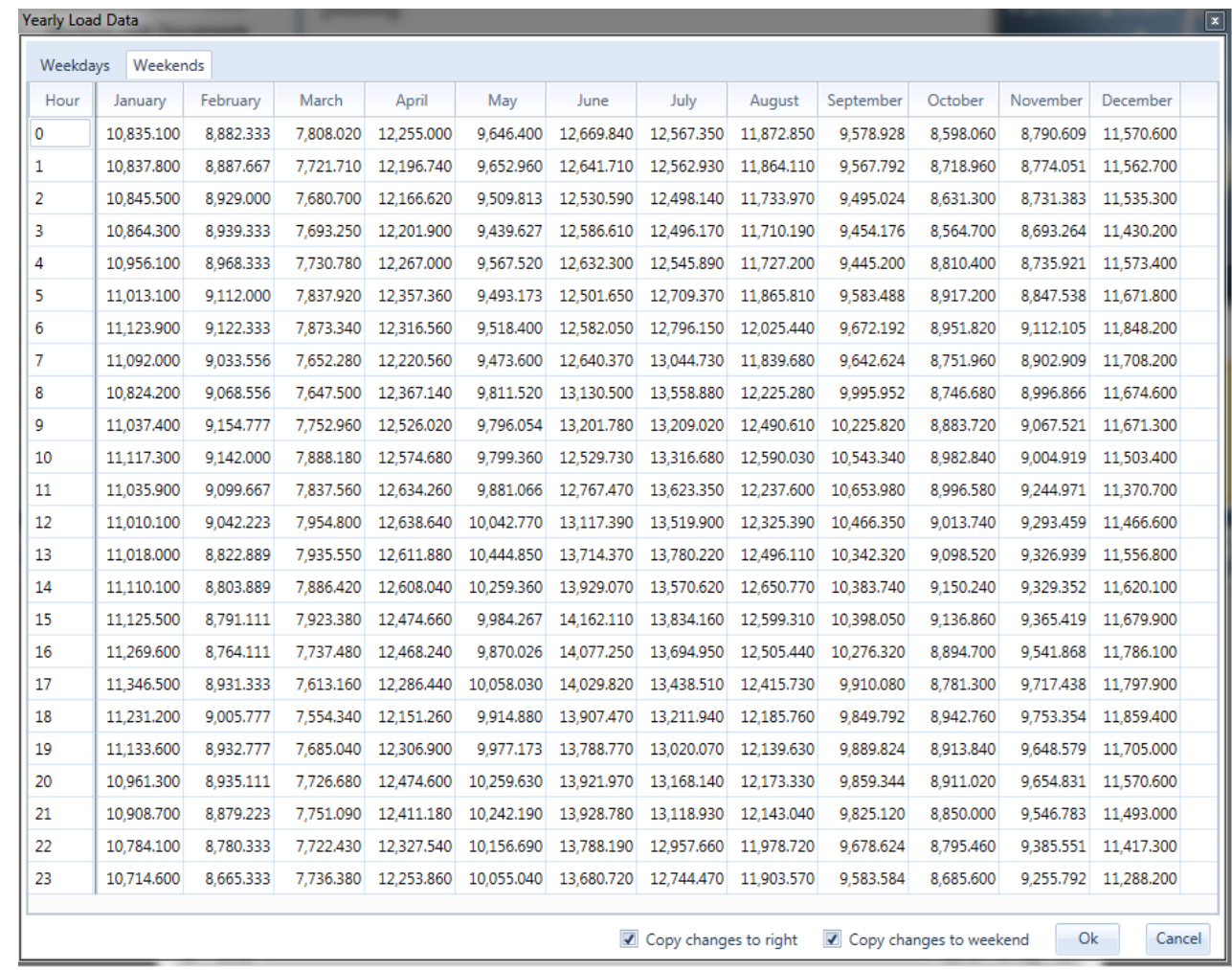


These are the simulation results produced for the optimization cases:

3) Grid-tied:
a) $1-1000 \mathrm{~kW}$ Generators
b) $5-2.3 \mathrm{MW}$ Siemens 108 Wind Turbines
c) 0 MW Flat panel fixed PV
d) 0 - GS200 Flow Battery $(0 \mathrm{~kW})$
e) 20,000 kW Grid Purchases
f) $\mathrm{COE}=\$ 0.145$

4) Island:
a) $6-3250 \mathrm{~kW}$ Generator
b) $15-2.3 \mathrm{MW}$ Siemens 108 Wind Turbines
c) $0 \mathrm{MW}$ Flat panel fixed PV
d) 60 - ViZn GS200 Flow Battery ()
e) $24,000 \mathrm{~kW}$ System Converter
f) $\mathrm{COE}=\$ 0.263$

Figures $10 \& 11$ show the complete simulation results produced for the optimization cases: Grid-tied:

Table 10: New London Grid-tied Sensitivity \& Optimization Results

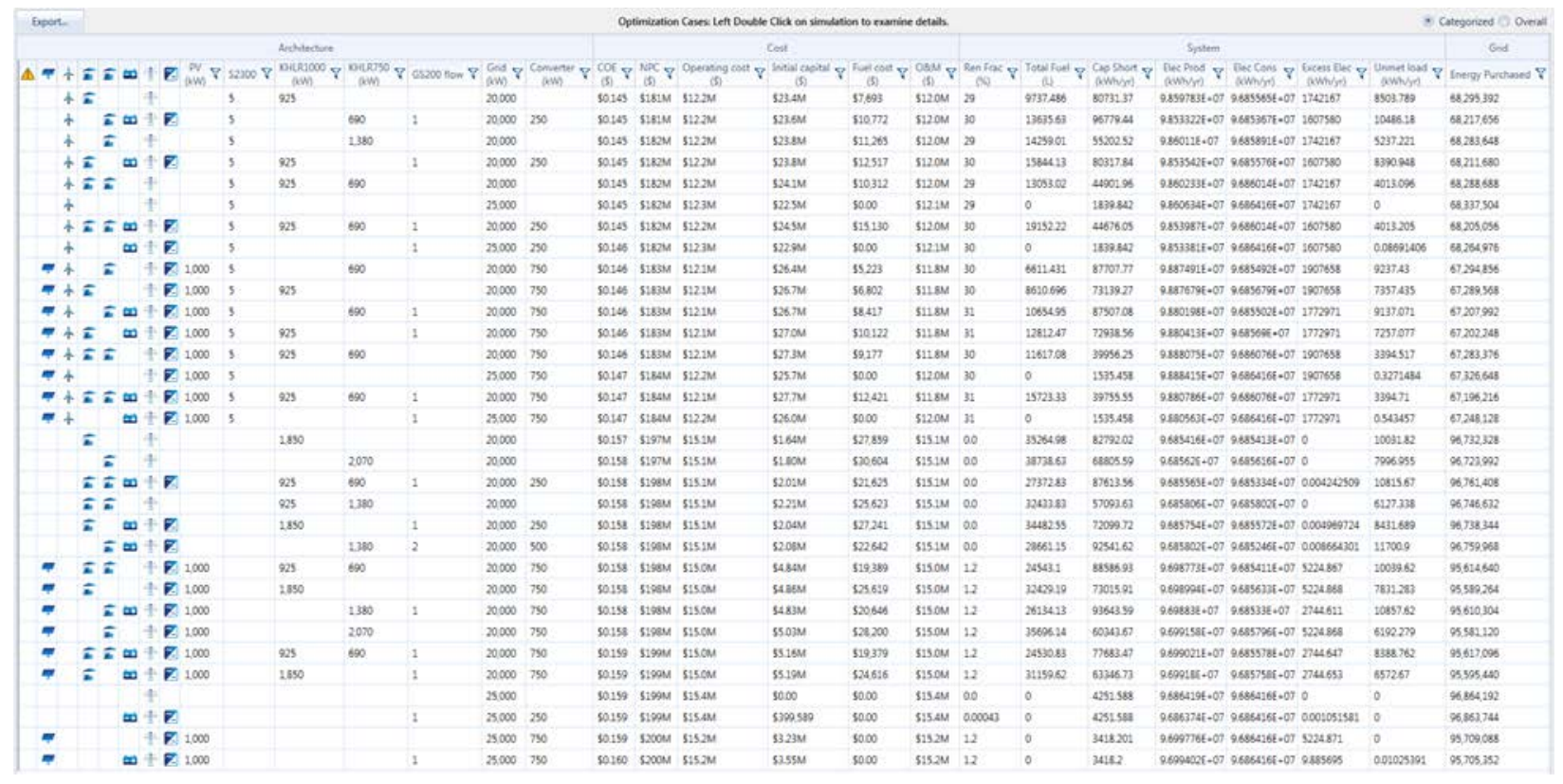


Island:

Table 11: New London Island Sensitivity \& Optimization Results

\begin{tabular}{|c|c|c|c|c|c|c|c|c|c|c|c|c|c|c|c|c|c|}
\hline \multicolumn{2}{|c|}{ Export. } & \multicolumn{14}{|c|}{ Optimization Cases: Left Double Click on simulation to examine detals. } & \multicolumn{2}{|c|}{ - Conegorized O Overall } \\
\hline \multicolumn{6}{|c|}{ Acchitiecture } & \multicolumn{6}{|c|}{ Cost } & \multicolumn{6}{|c|}{ Sprtem } \\
\hline$\Delta=+$ & 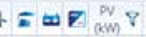 & 523008 & 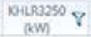 & Cs200 flow 8 & Comenter 8 & ${ }_{(3)}^{\cot 8}$ & ${ }_{(5)}^{N A C} Y$ & $\begin{array}{l}\text { Operating cost } \nabla \\
(5)\end{array}$ & $\begin{array}{l}\text { - Intial capital } 8 \\
\text { (3) }\end{array}$ & - Fuel cost 8 & ${ }_{(5)}^{0.92} \nabla$ & - Ren froc $\nabla$ & - Total fuel \& Cap Short 8 & $\begin{array}{l}\text { Des Prod } \nabla \\
\text { (kWhyr) }\end{array}$ & $\begin{array}{l}\text { Dles Cons } \\
(\text { WWh } / y)\end{array}$ & bicess tiec 8 & 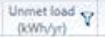 \\
\hline & 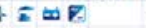 & is & 16800 & 60 & 24000 & 50.263 & $5330 \mathrm{M}$ & \$17.2M & s109M & $59.31 \mathrm{M}$ & S3:83M & 48 & $21784286-072328.557$ & $24091986+08$ & $9.6864166+07$ & $73.5579736-07$ & 0.1142578 \\
\hline$m+$ & $\boldsymbol{\Sigma} \boldsymbol{\Sigma} \pm \boldsymbol{\nabla} 5,000$ & is & 16,800 & 60 & 24000 & 50.265 & $5331 \mathrm{M}$ & 516.0M & \$124M & s8.62M & $53.74 \mathrm{M}$ & 52 & $20902676=07 \quad 2328.557$ & $24360926 \cdot 08$ & $96064266=07$ & $73.8246396 \cdot 07$ & 0.15625 \\
\hline m & $\bar{z}=0$ [ 30000 & & 16800 & 80 & 24,000 & s0.342 & S428M & S22.AM & S136m & \$243M & $53.90 \mathrm{M}$ & 20 & $2810356=07 \quad 27528.73$ & $2158162 \mathrm{E}+08$ & $9.6859176-07$ & 7 1550152 & 4992:927 \\
\hline$\Delta=+$ & $=\nabla 5000$ & is & 19,600 & & 24000 & s0.354 & 5443M & $526.0 \mathrm{M}$ & S107M & $510.2 \mathrm{M}$ & $57.18 \mathrm{M}$ & 44 & $12973521=07 \quad 95672.95$ & $1516524 E+08$ & $9.68563 E=07$ & S.A552116-07 & 7857.158 \\
\hline$\Delta+$ & +5 & 30 & 19,600 & & & 50.363 & SASAM & $523.3 \mathrm{M}$ & $5152 \mathrm{M}$ & s8.50M & $5720 \mathrm{M}$ & 33 & $10756346=0794180.11$ & $2.2671956-06$ & $96655996-07$ & $7.299635 \mathrm{E} * 06$ & 8263.7 \\
\hline & $\bar{z} \pm \boldsymbol{Z}$ & & 16,800 & 80 & 24000 & $\$ 0.370$ & $5463 \mathrm{M}$ & $532.1 \mathrm{M}$ & $5480 \mathrm{M}$ & $520.8 \mathrm{M}$ & S493M & $\infty$ & $2.6348006-0795012.64$ & $1.228196-06$ & $9.66214996-07$ & 0.2796204 & 42667.16 \\
\hline & $\bar{E}$ & & 22,400 & & & 50.005 & $5507 \mathrm{M}$ & $537.7 \mathrm{M}$ & 519.8M & S10.2M & SQS6M & $\infty 0$ & $23011236-07 \quad 52801.31$ & $9.7033266-07$ & $9.6858655-07$ & 7.174600 .8 & 5506.633 \\
\hline$\Delta=$ & E. 5,000 & & 22,400 & & 24000 & S0.415 & $5520 \mathrm{M}$ & $537.0 \mathrm{M}$ & $542.0 \mathrm{M}$ & $5172 \mathrm{M}$ & $58.63 \mathrm{M}$ & 5.5 & $2.1743926-07 \quad 26735.8$ & $9.0017376-07$ & $9.6662815-07$ & 7. $\$ 473565$ & 1350.797 \\
\hline
\end{tabular}

With the input parameters HOMER was given these were the only cases it found feasible. See case pdf's in appendix for further details on each simulation. 


\section{AUTEC}

\subsection{HOMER Analysis}

Figure 40: AUTEC HOMER

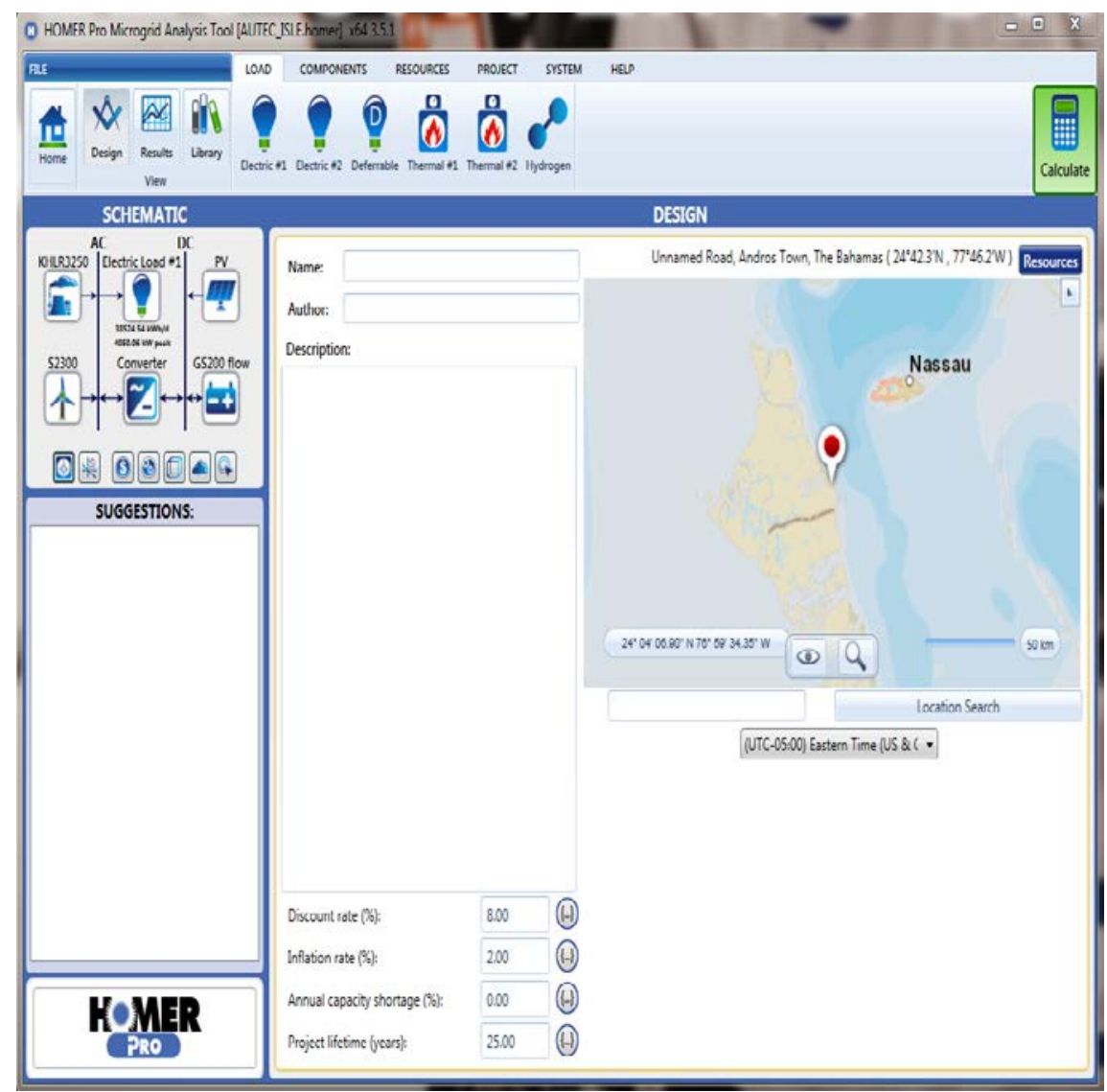

The HOMER (Hybrid Optimization of Multiple Energy Resources) model greatly simplifies the task of designing hybrid renewable microgrids, whether remote or attached to a larger grid. HOMER's optimization and sensitivity analysis algorithms allow you to evaluate the economic and technical feasibility of a large number of technology options and to account for variations in technology costs, electric load, and energy resource availability.

For Autec inputs we have included:

- Up to $5-3250 \mathrm{~kW}$ Generator

- Up to 3-2300kW Siemens 108 Wind Turbines

- Flat panel fixed PV up to $4000 \mathrm{~kW}$

- $1 \mathrm{MW} / 3 \mathrm{MWh}$ Primus Power EnergyPod Battery (allowed up to 20)

- System Converter up to $5000 \mathrm{~kW}$

This model used the location coordinates of $24^{\circ} 42.3^{\prime} \mathrm{N}, 77^{\circ} 46.2^{\prime} \mathrm{W}$ and wind \& solar resource data from NASA's surface and solar database. The electrical load data was not provided for Autec so an interpolated load was created from other similar military base average load data. Needless to say, the more accurate the data a model has, the more accurate the results are; any actual Autec load data could 
improve model accuracy. A temperature resource was included and was also downloaded from NASA's surface and solar database. Refer to following figures:

Figure 41: AUTEC Generator Component

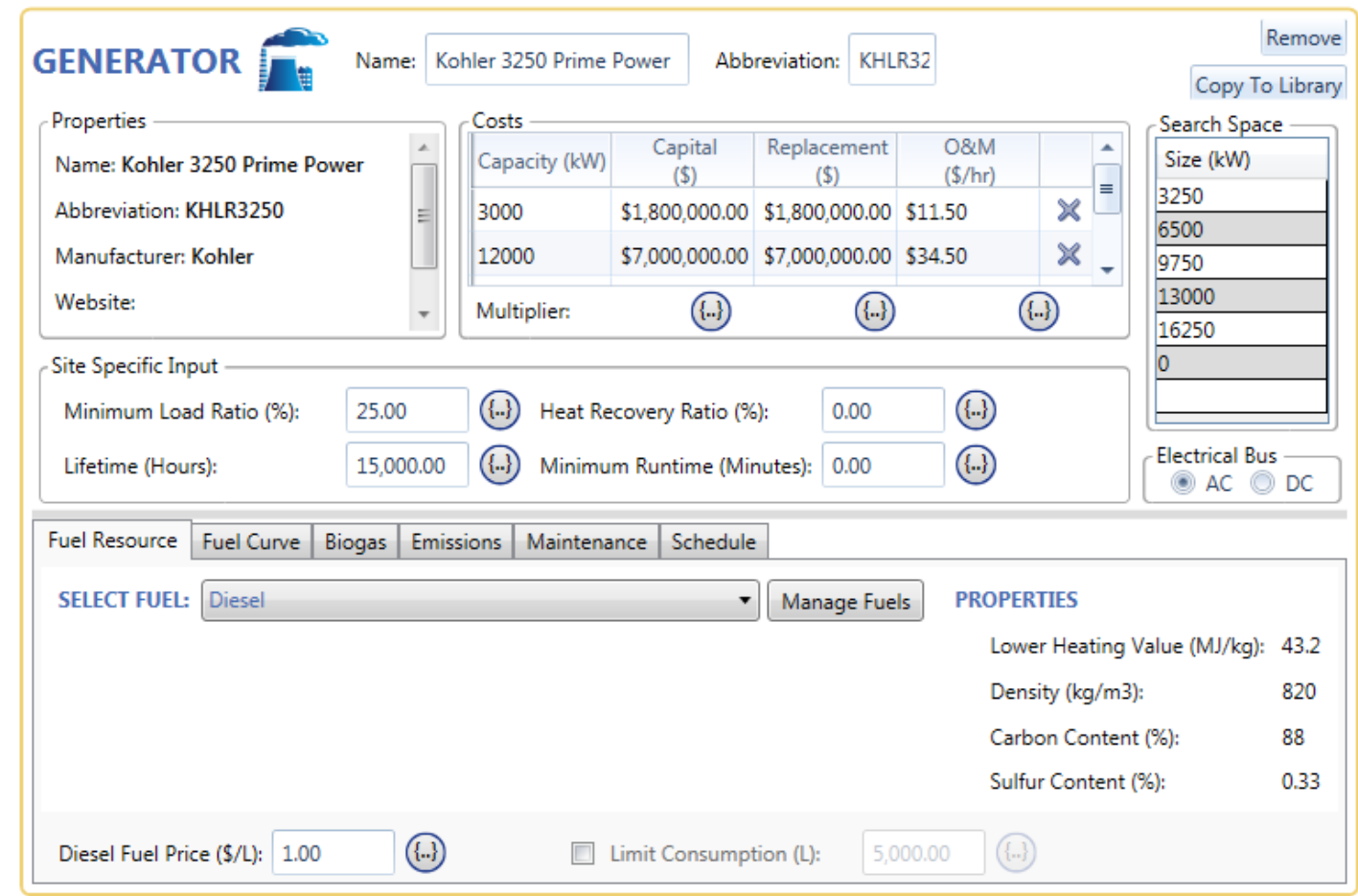

Figure 42: AUTEC PV Component

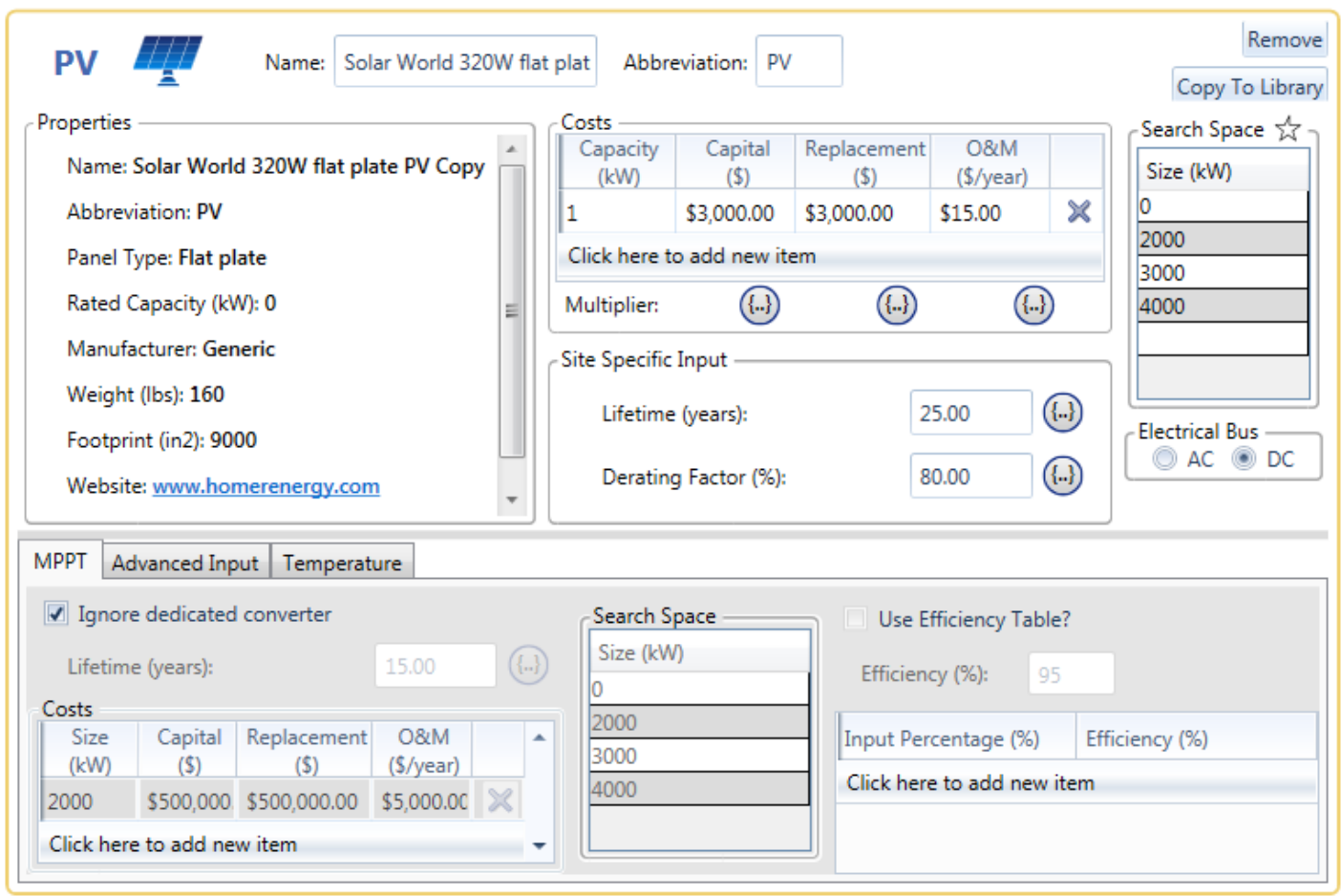


Figure 43: AUTEC Wind Turbine Component

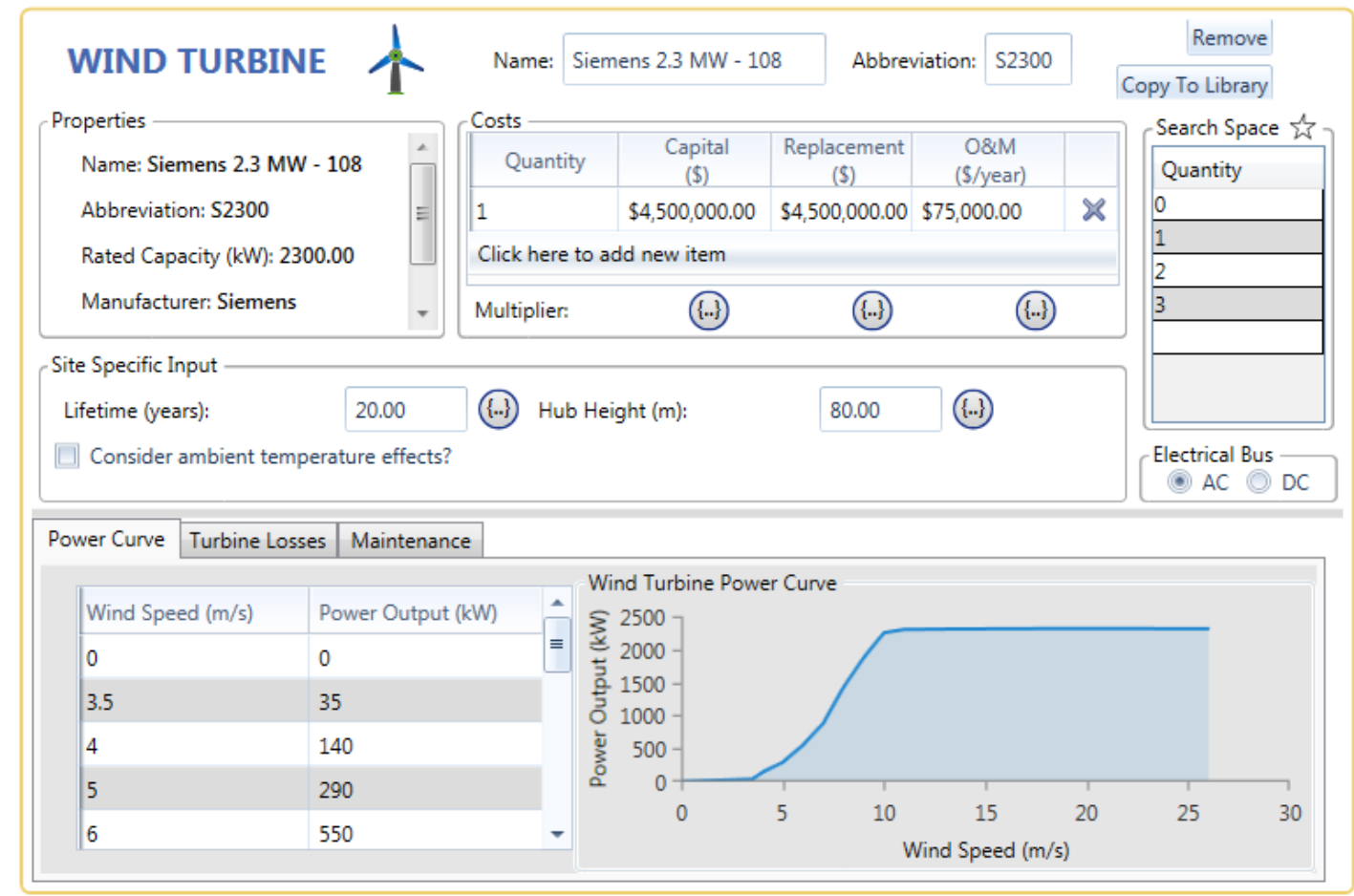

Figure 44: AUTEC Battery Component

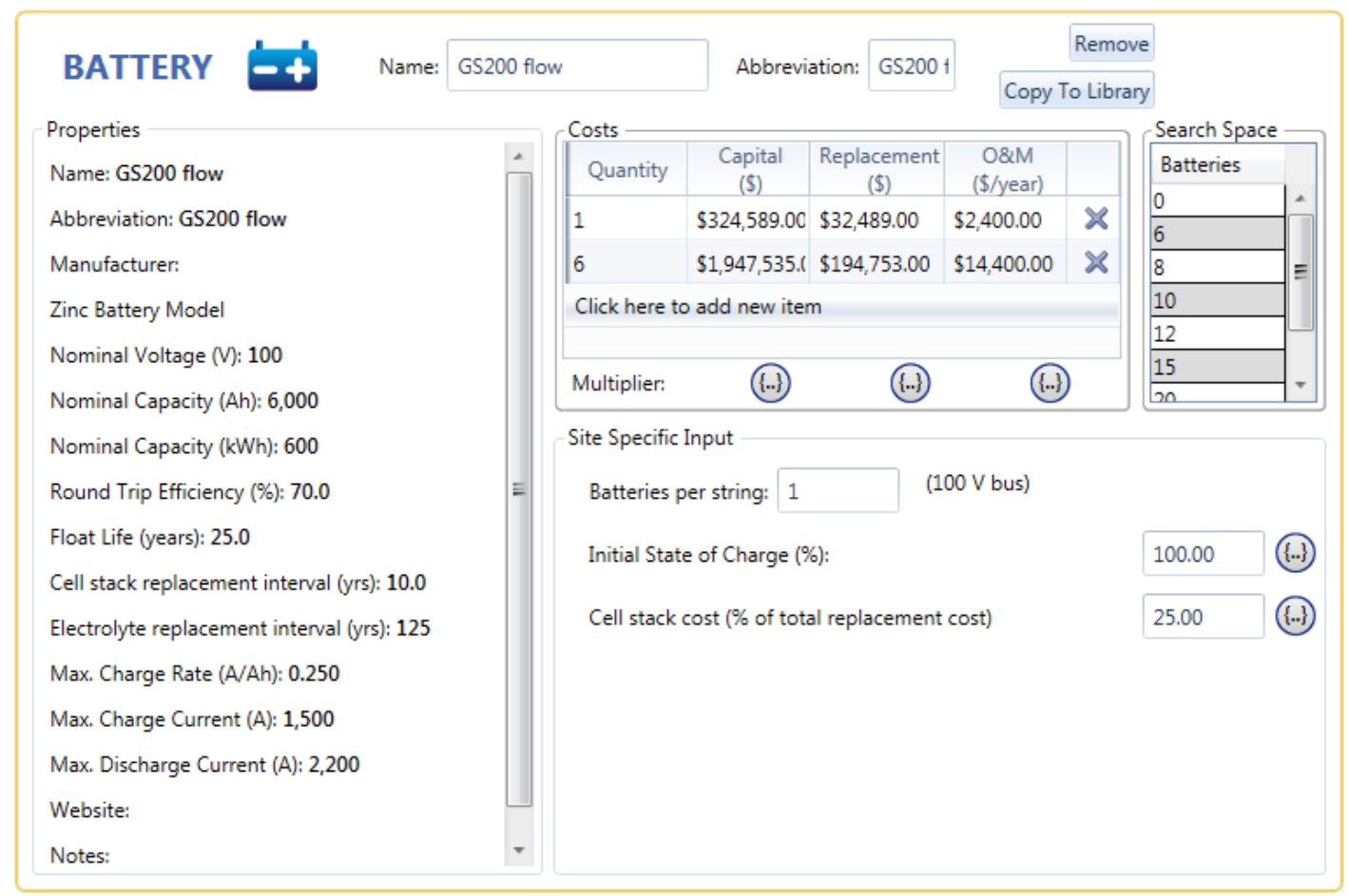


Figure 45: AUTEC Converter Component

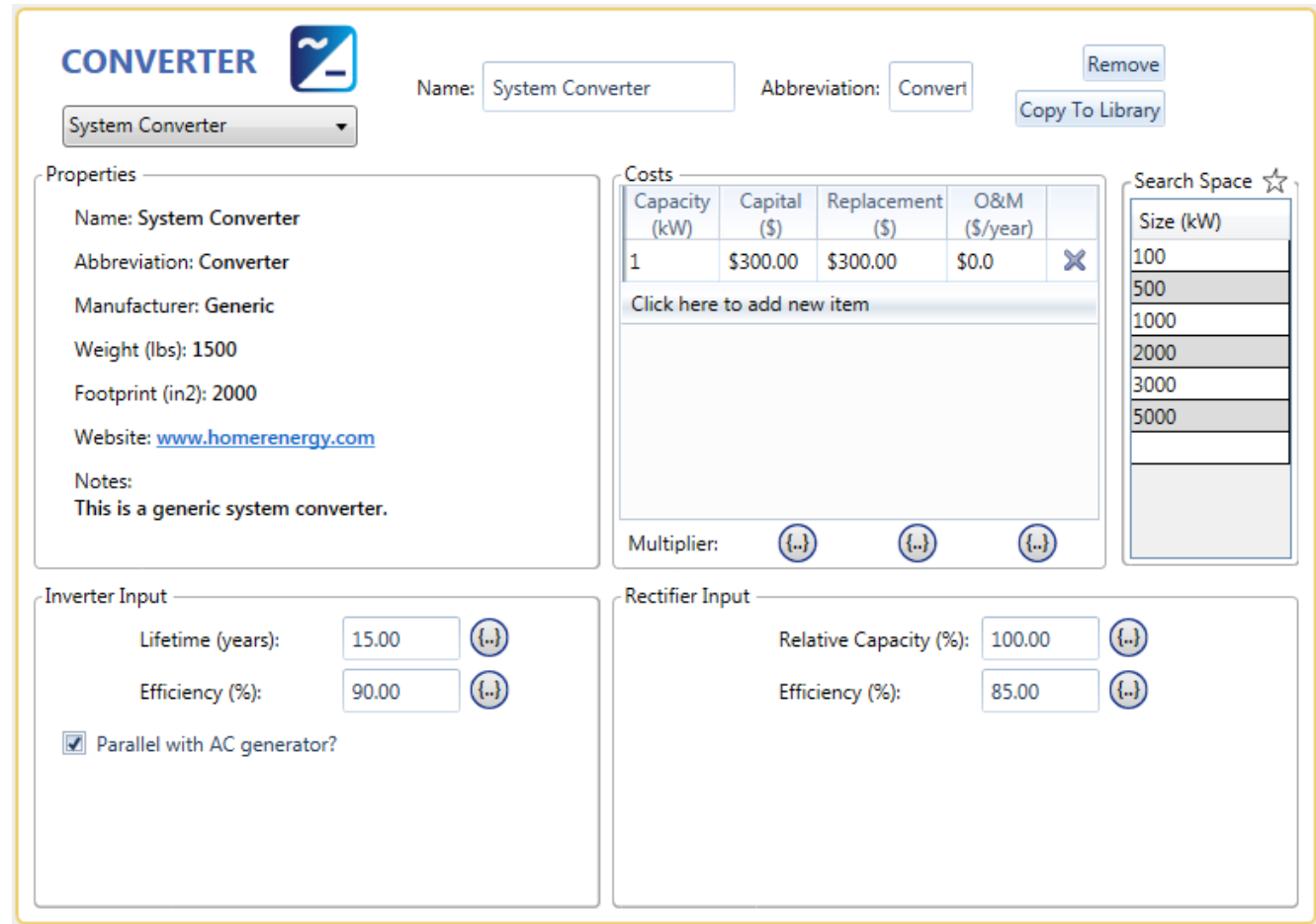

Figure 46: AUTEC Solar Resource

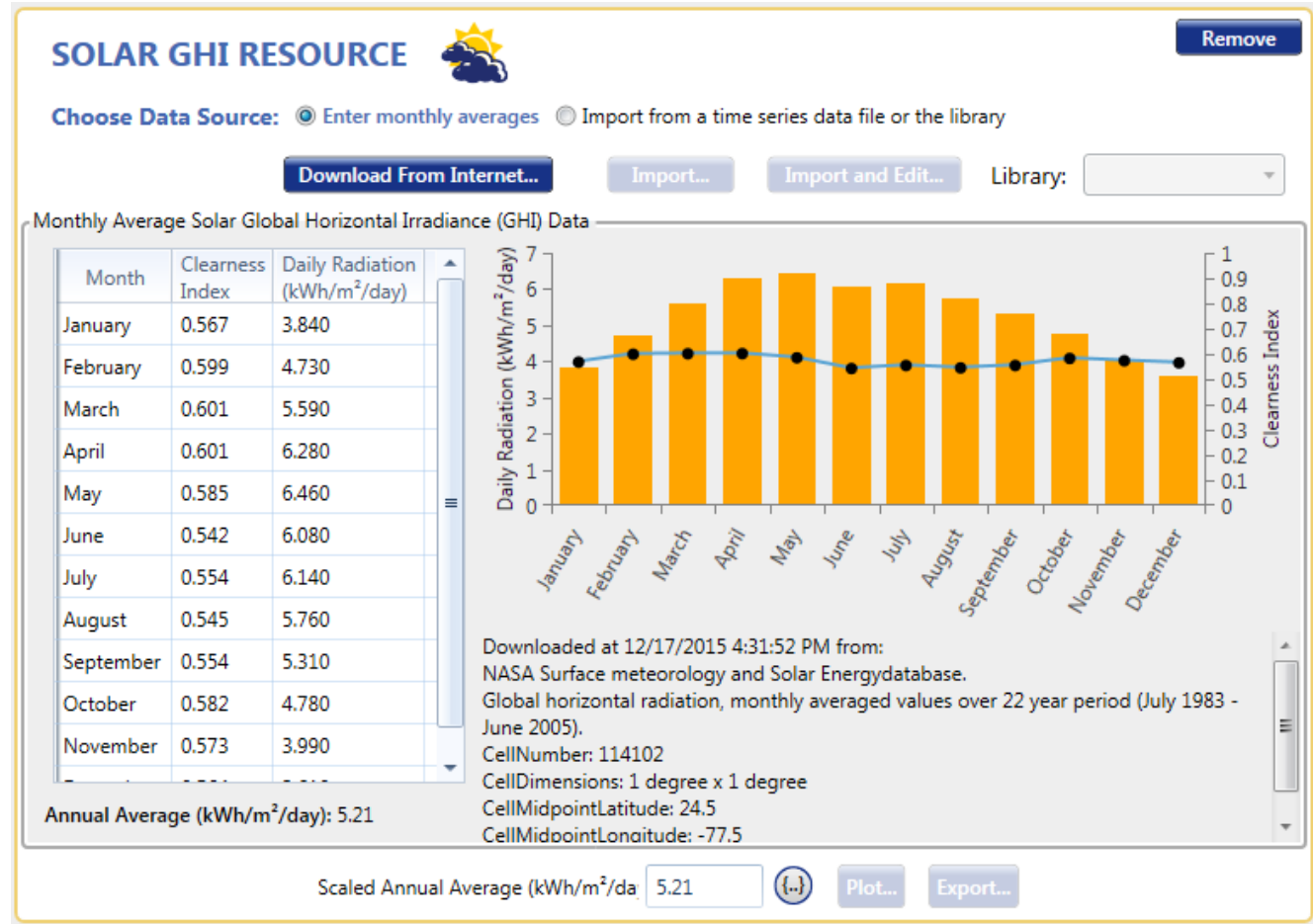


Figure 47: AUTEC Wind Resource

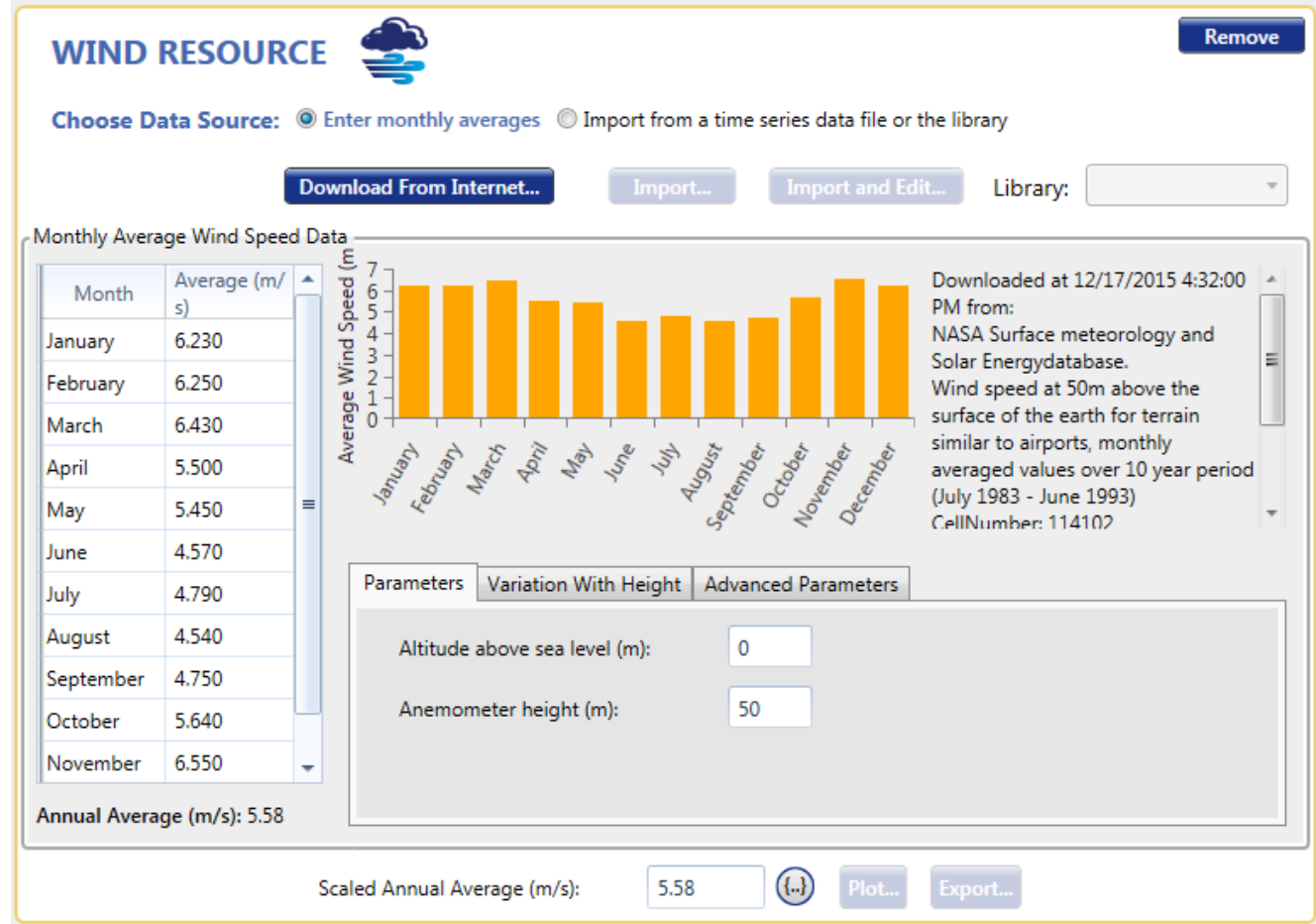

Figure 48: AUTEC Temperature Resource

TEMPERATURE RESOURCE f

Choose Data Source: (1) Enter monthly averages Import from a time series data file or the library

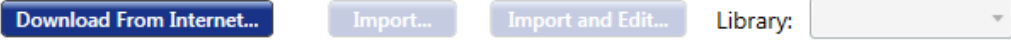

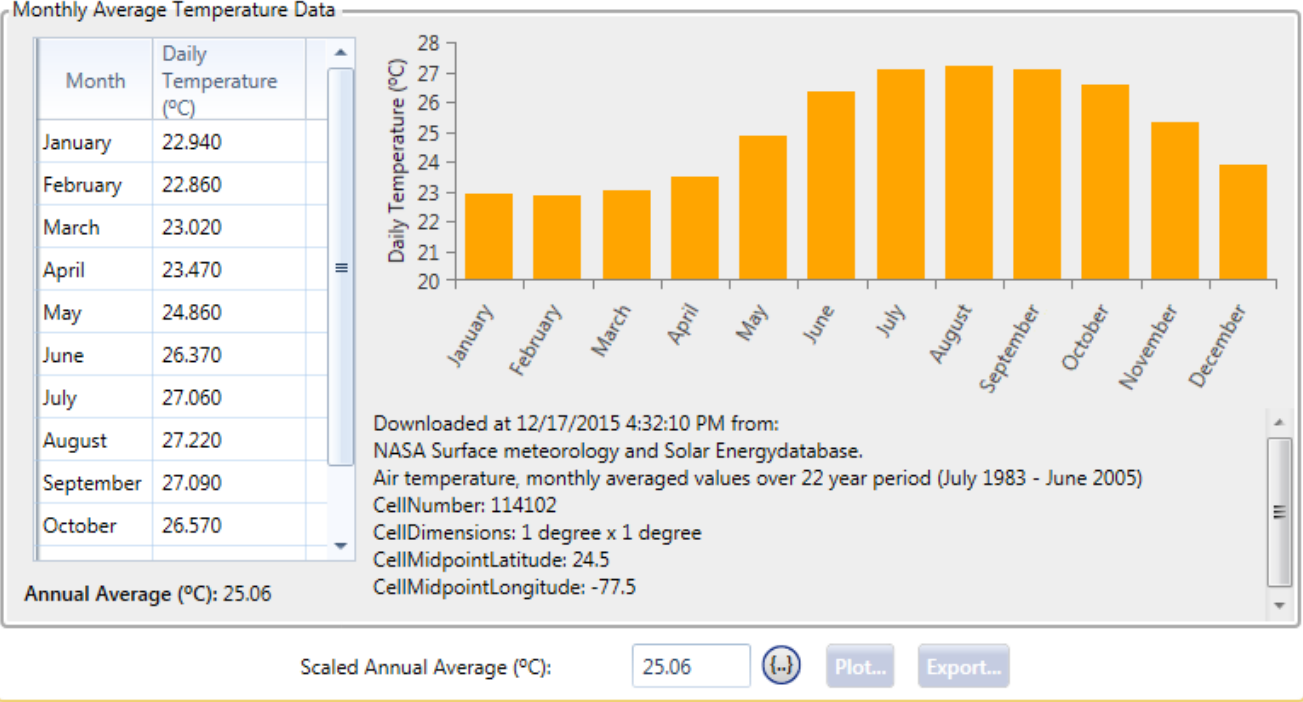


Figure 49: AUTEC Fuel Resource

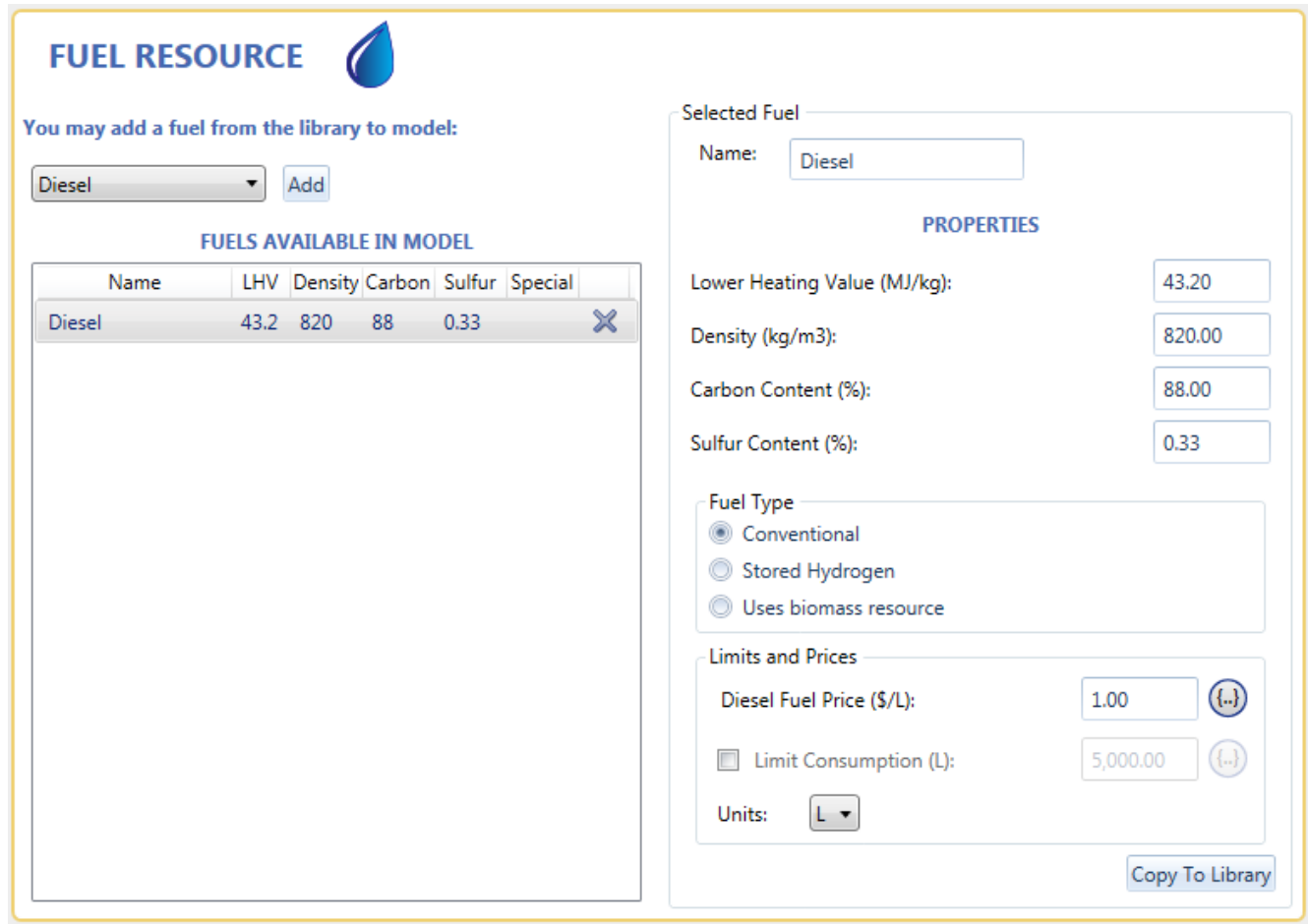

Figure 50: AUTEC Electric Load

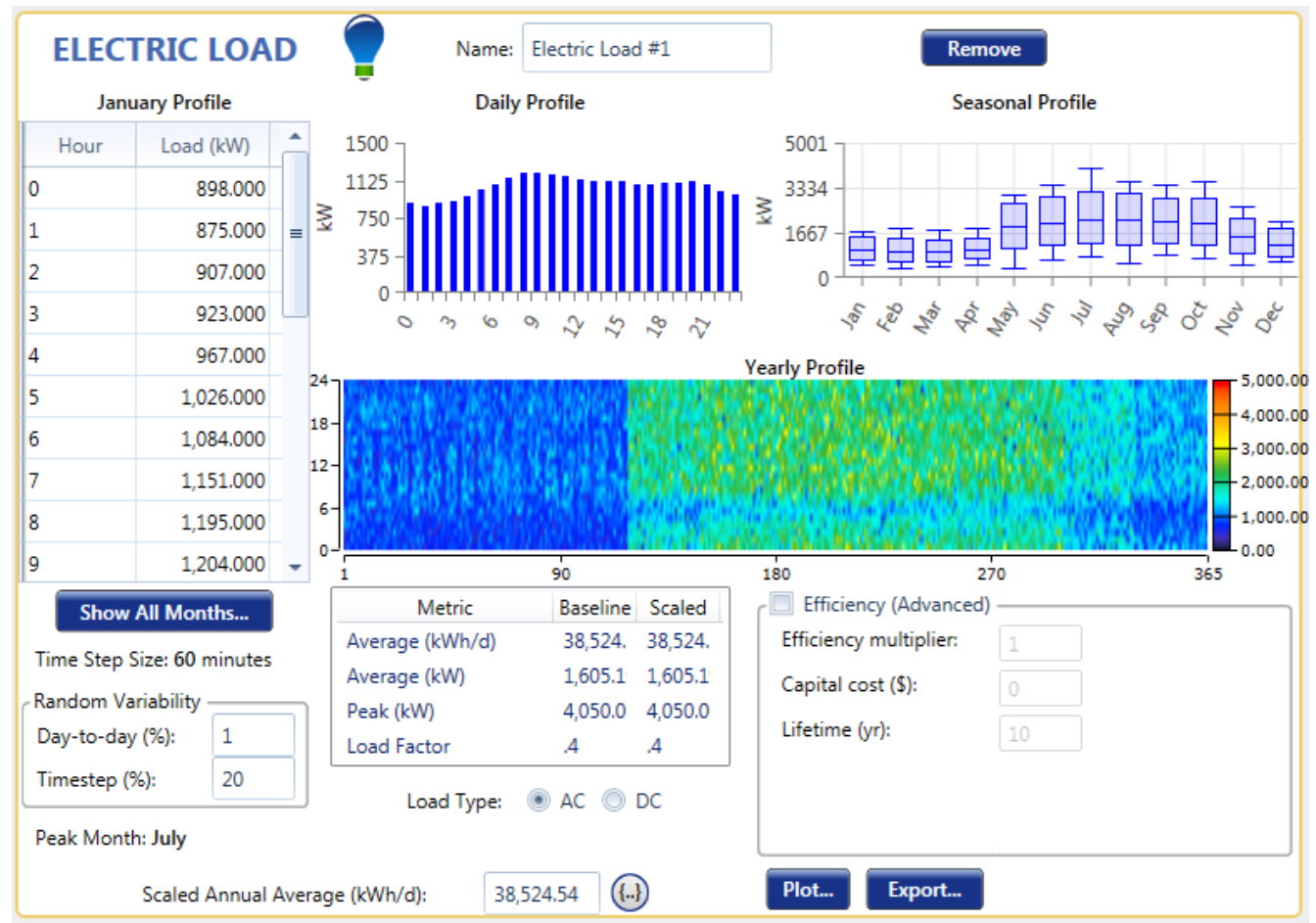


Table 12: AUTEC Weekday Electrical Load Profile

\begin{tabular}{|c|c|c|c|c|c|c|c|c|c|c|c|c|}
\hline \multicolumn{4}{|c|}{ Yearly Load Data } & \multicolumn{2}{|c|}{$=$} & \multirow[t]{2}{*}{ 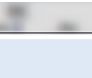 } & \multicolumn{4}{|c|}{ 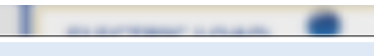 } & \multicolumn{2}{|r|}{8} \\
\hline \multicolumn{12}{|c|}{ Weekdays } & \\
\hline Hour & January & February & March & April & May & June & July & August & September & October & November & December \\
\hline 0 & 898.000 & 878.000 & 865.000 & 884.000 & $1,911.000$ & $2,111.000$ & $2,211.000$ & $2,161.000$ & $2,098.000$ & $2,086.000$ & $1,492.000$ & $1,192.000$ \\
\hline 1 & 875.000 & 865.000 & 852.000 & 871.000 & $1,817.000$ & $2,017.000$ & $2,117.000$ & $2,067.000$ & $2,004.000$ & $1,992.000$ & $1,434.000$ & $1,134.000$ \\
\hline 2 & 907.000 & 896.000 & 883.000 & 902.000 & $1,681.000$ & $1,881.000$ & $1,981.000$ & $1,931.000$ & $1,868.000$ & $1,856.000$ & $1,381.000$ & $1,081.000$ \\
\hline 3 & 923.000 & 913.000 & 900.000 & 919.000 & $1,605.000$ & $1,805.000$ & $1,905.000$ & $1,855.000$ & $1,792.000$ & $1,780.000$ & $1,352.000$ & $1,052.000$ \\
\hline 4 & 967.000 & 956.000 & 943.000 & 962.000 & $1,401.000$ & $1,601.000$ & $1,701.000$ & $1,651.000$ & $1,588.000$ & $1,576.000$ & $1,271.000$ & 971.000 \\
\hline 5 & $1,026.000$ & $1,015.000$ & $1,002.000$ & $1,021.000$ & $1,256.000$ & $1,456.000$ & $1,556.000$ & $1,506.000$ & $1,443.000$ & $1,431.000$ & $1,228.000$ & 928.000 \\
\hline 6 & $1,084.000$ & $1,074,000$ & $1,061.000$ & $1,080.000$ & $1,414,000$ & $1,614,000$ & $1,714,000$ & $1,664,000$ & $1,601.000$ & $1,589.000$ & $1,337.000$ & $1,037.000$ \\
\hline 7 & $1,151.000$ & $1,141.000$ & $1,128.000$ & $1,147.000$ & $1,695.000$ & $1,895.000$ & $1,995.000$ & $1,945.000$ & $1,882.000$ & $1,870.000$ & $1,511.000$ & $1,211.000$ \\
\hline 8 & $1,195.000$ & $1,184.000$ & $1,171.000$ & $1,190.000$ & $1,976.000$ & $2,176.000$ & $2,276.000$ & $2,226.000$ & $2,163.000$ & $2,151.000$ & $1,673.000$ & $1,373.000$ \\
\hline 9 & $1,204,000$ & $1,194.000$ & $1,181.000$ & $1,200.000$ & $2,089.000$ & $2,289.000$ & $2,389.000$ & $2,339.000$ & $2,276.000$ & $2,264.000$ & $1,734,000$ & $1,434.000$ \\
\hline 10 & $1,182.000$ & $1,172.000$ & $1,159.000$ & $1,178.000$ & $2,099.000$ & $2,299.000$ & $2,399.000$ & $2,349.000$ & $2,286.000$ & $2,274.000$ & $1,728.000$ & $1,428.000$ \\
\hline 11 & $1,162.000$ & $1,151.000$ & $1,138.000$ & $1,157.000$ & $2,100.000$ & $2,300.000$ & $2,400.000$ & $2,350.000$ & $2,287.000$ & $2,275.000$ & $1,718.000$ & $1,418.000$ \\
\hline 12 & $1,140.000$ & $1,130.000$ & $1,117.000$ & $1,136.000$ & $2,100.000$ & $2,300.000$ & $2,400.000$ & $2,350.000$ & $2,287.000$ & $2,275.000$ & $1,708.000$ & $1,408.000$ \\
\hline 13 & $1,118.000$ & $1,107.000$ & $1,094.000$ & $1,113.000$ & $2,114,000$ & $2,314.000$ & $2,414.000$ & $2,364.000$ & $2,301.000$ & $2,289.000$ & $1,703.000$ & $1,403.000$ \\
\hline 14 & $1,125.000$ & $1,105.000$ & $1,092.000$ & $1,111.000$ & $2,115.000$ & $2,315.000$ & $2,415.000$ & $2,365.000$ & $2,302.000$ & $2,290.000$ & $1,708.000$ & $1,408.000$ \\
\hline 15 & $1,115.000$ & $1,105.000$ & $1,092.000$ & $1,111.000$ & $2,112.000$ & $2,312.000$ & $2,412.000$ & $2,362.000$ & $2,299.000$ & $2,287.000$ & $1,701.000$ & $1,401.000$ \\
\hline 16 & $1,080.000$ & $1,070.000$ & $1,057.000$ & $1,076.000$ & $2,100.000$ & $2,300.000$ & $2,400.000$ & $2,350.000$ & $2,287.000$ & $2,275.000$ & $1,678.000$ & $1,378.000$ \\
\hline 17 & $1,085.000$ & $1,075.000$ & $1,062.000$ & $1,081.000$ & $2,056.000$ & $2,256.000$ & $2,356.000$ & $2,306.000$ & $2,243.000$ & $2,231.000$ & $1,658.000$ & $1,358.000$ \\
\hline 18 & $1,099.000$ & $1,079.000$ & $1,066.000$ & $1,085.000$ & $2,000.000$ & $2,200.000$ & $2,300.000$ & $2,250.000$ & $2,187.000$ & $2,175.000$ & $1,637.000$ & $1,337.000$ \\
\hline 19 & $1,107.000$ & $1,100.000$ & $1,087.000$ & $1,106.000$ & $1,997.000$ & $2,197.000$ & $2,297.000$ & $2,247.000$ & $2,184,000$ & $2,172.000$ & $1,639.000$ & $1,339.000$ \\
\hline 20 & $1,110.000$ & $1,099.000$ & $1,086.000$ & $1,105.000$ & $2,083.000$ & $2,283.000$ & $2,383.000$ & $2,333.000$ & $2,270.000$ & $2,258.000$ & $1,684.000$ & $1,384.000$ \\
\hline 21 & $1,090.000$ & $1,080.000$ & $1,067.000$ & $1,086.000$ & $2,045.000$ & $2,245.000$ & $2,345.000$ & $2,295.000$ & $2,232.000$ & $2,220.000$ & $1,655.000$ & $1,355.000$ \\
\hline 22 & $1,025.000$ & $1,015.000$ & $1,002.000$ & $1,021.000$ & $2,005.000$ & $2,205.000$ & $2,305.000$ & $2,255.000$ & $2,192.000$ & $2,180.000$ & $1,603.000$ & $1,303.000$ \\
\hline 23 & 991.000 & 981.000 & 968.000 & 987.000 & $1,911.000$ & $2,111.000$ & $2,211.000$ & $2,161.000$ & $2,098.000$ & $2,086.000$ & $1,539.000$ & $1,239.000$ \\
\hline & & & & & & & & & & to weekend & Ok & Cancel \\
\hline
\end{tabular}

Table 13: AUTEC Weekend Electrical Load Profile

\begin{tabular}{|c|c|c|c|c|c|c|c|c|c|c|c|c|}
\hline \multicolumn{4}{|c|}{ Yearly Load Data } & \multicolumn{2}{|c|}{$\mathrm{s}$} & \multirow[t]{2}{*}{ 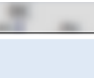 } & \multicolumn{4}{|c|}{ 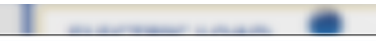 } & \multicolumn{2}{|r|}{$\varepsilon$} \\
\hline \multicolumn{12}{|c|}{ Weekdays Weekends } & \\
\hline Hour & January & February & March & April & May & June & July & August & September & October & November & December \\
\hline 0 & 884.000 & 847.000 & 865.000 & 845.000 & $1,899.000$ & $2,063.000$ & $2,194,000$ & $2,167.000$ & $2,049.000$ & $2,088.000$ & $1,489.000$ & $1,183.000$ \\
\hline 1 & 862.000 & 836.000 & 856.000 & 835.000 & $1,806.000$ & $1,972.000$ & $2,107.000$ & $2,073.000$ & $1,960.000$ & $1,997.000$ & $1,428.000$ & $1,123.000$ \\
\hline 2 & 892.000 & 860.000 & 889.000 & 864.000 & $1,663.000$ & $1,838.000$ & $1,963.000$ & $1,930.000$ & $1,819.000$ & $1,870.000$ & $1,370.000$ & $1,070.000$ \\
\hline 3 & 899.000 & 870.000 & 889.000 & 885.000 & $1,598.000$ & $1,769.000$ & $1,883.000$ & $1,851.000$ & $1,739.000$ & $1,801.000$ & $1,345.000$ & $1,047.000$ \\
\hline 4 & 927.000 & 895.000 & 926.000 & 910.000 & $1,396.000$ & $1,569.000$ & $1,673.000$ & $1,639.000$ & $1,535.000$ & $1,598.000$ & $1,272.000$ & 963.000 \\
\hline 5 & 931.000 & 895.000 & 960.000 & 962.000 & $1,232.000$ & $1,408.000$ & $1,505.000$ & $1,478.000$ & $1,373.000$ & $1,440.000$ & $1,202.000$ & 884.000 \\
\hline 6 & 905.000 & 856.000 & 934.000 & 964.000 & $1,321,000$ & $1,483.000$ & $1,600.000$ & $1,564.000$ & $1,465.000$ & $1,517.000$ & $1,248.000$ & 951.000 \\
\hline 7 & 898.000 & 836.000 & 954.000 & 970.000 & $1,541.000$ & $1,689.000$ & $1,840.000$ & $1,801.000$ & $1,674.000$ & $1,739.000$ & $1,373.000$ & $1,085.000$ \\
\hline 8 & 895.000 & 856.000 & 988.000 & 985.000 & $1,789.000$ & $1,923.000$ & $2,094.000$ & $2,045.000$ & $1,922.000$ & $2,000.000$ & $1,517.000$ & $1,228.000$ \\
\hline 9 & 881.000 & 868.000 & 997.000 & 991.000 & $1,896.000$ & $2,013.000$ & $2,189.000$ & $2,133.000$ & $2,019.000$ & $2,098.000$ & $1,565.000$ & $1,283.000$ \\
\hline 10 & 854.000 & 847.000 & 962.000 & 962.000 & $1,898.000$ & $2,024.000$ & $2,192.000$ & $2,130.000$ & $2,028.000$ & $2,095.000$ & $1,548.000$ & $1,281.000$ \\
\hline 11 & 834.000 & 835.000 & 938.000 & 946.000 & $1,896.000$ & $2,000.000$ & $2,185.000$ & $2,130.000$ & $2,036.000$ & $2,091.000$ & $1,550.000$ & $1,280.000$ \\
\hline 12 & 835.000 & 825.000 & 911.000 & 924.000 & $1,892.000$ & $1,997.000$ & $2,188.000$ & $2,124,000$ & $2,045.000$ & $2,092.000$ & $1,535.000$ & $1,276.000$ \\
\hline 13 & 806.000 & 800.000 & 871.000 & 896.000 & $1,896.000$ & $1,995.000$ & $2,192,000$ & $2,110.000$ & $2,045.000$ & $2,102.000$ & $1,521.000$ & $1,260.000$ \\
\hline 14 & 819.000 & 809.000 & 874.000 & 900.000 & $1,892.000$ & $1,989.000$ & $2,177.000$ & $2,106.000$ & $2,048.000$ & $2,110.000$ & $1,534.000$ & $1,263.000$ \\
\hline 15 & 850.000 & 837.000 & 886.000 & 910.000 & $1,898.000$ & $1,995.000$ & $2,181.000$ & $2,114.000$ & $2,062.000$ & $2,123.000$ & $1,526.000$ & $1,260.000$ \\
\hline 16 & 889.000 & 877.000 & 903.000 & 926.000 & $1,932.000$ & $2,032.000$ & $2,216.000$ & $2,147.000$ & $2,104.000$ & $2,156.000$ & $1,537.000$ & $1,272.000$ \\
\hline 17 & 969.000 & 966.000 & 964.000 & 997.000 & $1,954.000$ & $2,079.000$ & $2,260.000$ & $2,184.000$ & $2,144.000$ & $2,182.000$ & $1,563.000$ & $1,294.000$ \\
\hline 18 & $1,020.000$ & $1,010.000$ & 997.000 & $1,028.000$ & $1,936.000$ & $2,067.000$ & $2,244.000$ & $2,160.000$ & $2,121.000$ & $2,138.000$ & $1,565.000$ & $1,289.000$ \\
\hline 19 & $1,048.000$ & $1,053.000$ & $1,014.000$ & $1,049.000$ & $1,944.000$ & $2,084.000$ & $2,256.000$ & $2,165.000$ & $2,123.000$ & $2,148.000$ & $1,584.000$ & $1,306.000$ \\
\hline 20 & $1,067.000$ & $1,076.000$ & $1,029.000$ & $1,053.000$ & $2,032.000$ & $2,183.000$ & $2,349.000$ & $2,274.000$ & $2,220.000$ & $2,242.000$ & $1,641.000$ & $1,354.000$ \\
\hline 21 & $1,056.000$ & $1,062.000$ & $1,025.000$ & $1,041.000$ & $2,001.000$ & $2,164.000$ & $2,311.000$ & $2,246.000$ & $2,192.000$ & $2,215.000$ & $1,629.000$ & $1,330.000$ \\
\hline 22 & 996.000 & 991.000 & 961.000 & 979.000 & $1,964.000$ & $2,128.000$ & $2,277.000$ & $2,223.000$ & $2,156.000$ & $2,174.000$ & $1,578.000$ & $1,280.000$ \\
\hline \multirow[t]{2}{*}{23} & 970.000 & 959.000 & 929.000 & 945.000 & $1,876.000$ & $2,030.000$ & $2,188.000$ & $2,133.000$ & $2,051.000$ & $2,074.000$ & $1,515.000$ & $1,217.000$ \\
\hline & & & & & & \multicolumn{3}{|c|}{ 1. Copy changes to right } & spy crange & $s$ to weekend & Ok & Cancel \\
\hline
\end{tabular}


HOMER has the option to consider different amounts of generator, wind, PV, batteries, and different size system converter. These are the simulation results produced for the optimization cases:

Table 14: AUTEC Sensitivity \& Optimization Results

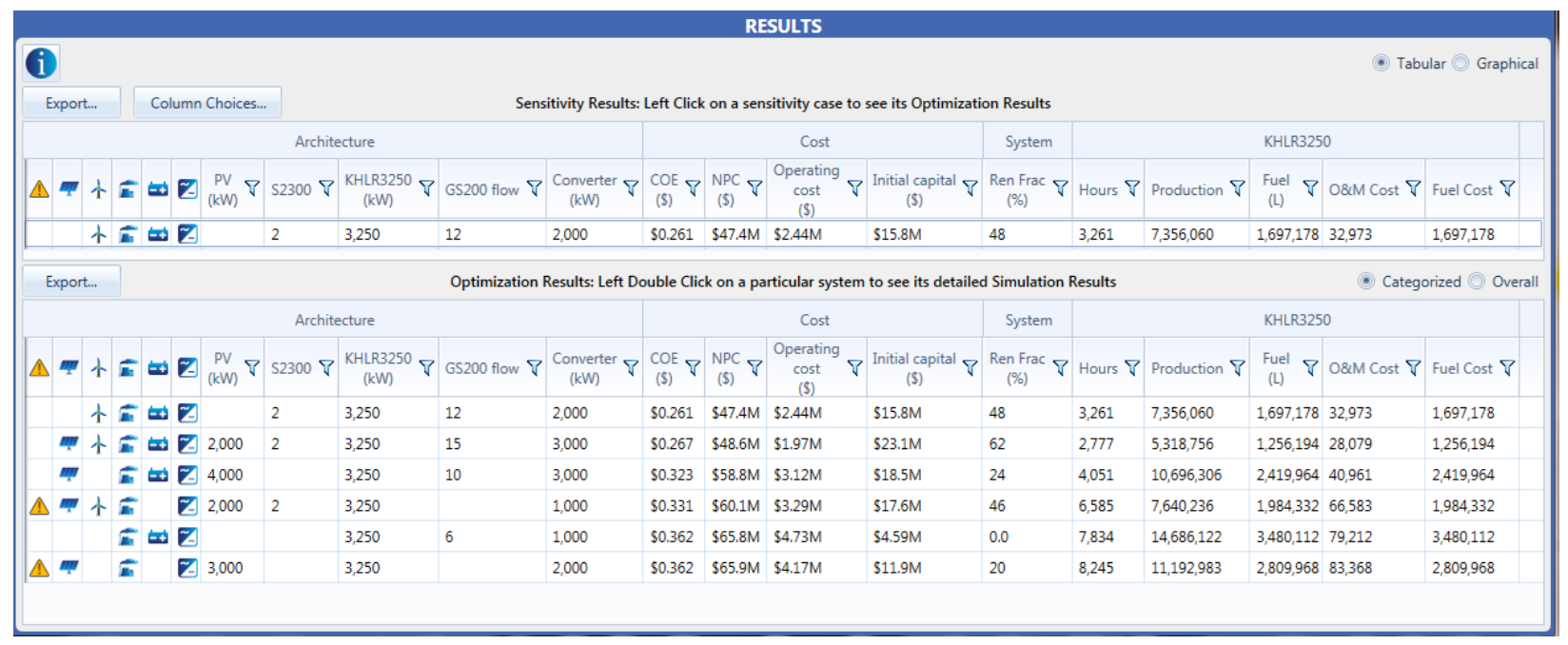

The top case (HOMER optimized selection) includes 2 - 2300kW wind turbines, 1 - 3250kW Genset, 12 Vizn GS200 Flow batteries and a 2MW system converter. For this case the cost of energy is calculated at $\$ 0.261$ and the renewable fraction is $48 \%$. With the input parameters HOMER was given the several other cases were found feasible. See each cases pdf's for further details on each simulation. 


\section{Appendix A - San Nicholas Scenarios}

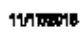

System Report

System archltecture

\begin{tabular}{|c|c|c|c|}
\hline PV & Eenerlc fiat ple PV & 750 & WW \\
\hline Wind Turbing & Nothem Poner NPS1006-21 & 7 & \\
\hline Generator & Ceneric1150kW Prime Ponwer & 1,150 & $\mathbf{W W}$ \\
\hline Buttery & Gs200 flaw & 6 & atring: \\
\hline Corwinter & Syatem Conwerter & 1,000 & WW \\
\hline Didpateh Strateosy & Cycle Charping & & \\
\hline
\end{tabular}

\section{Cost summary}

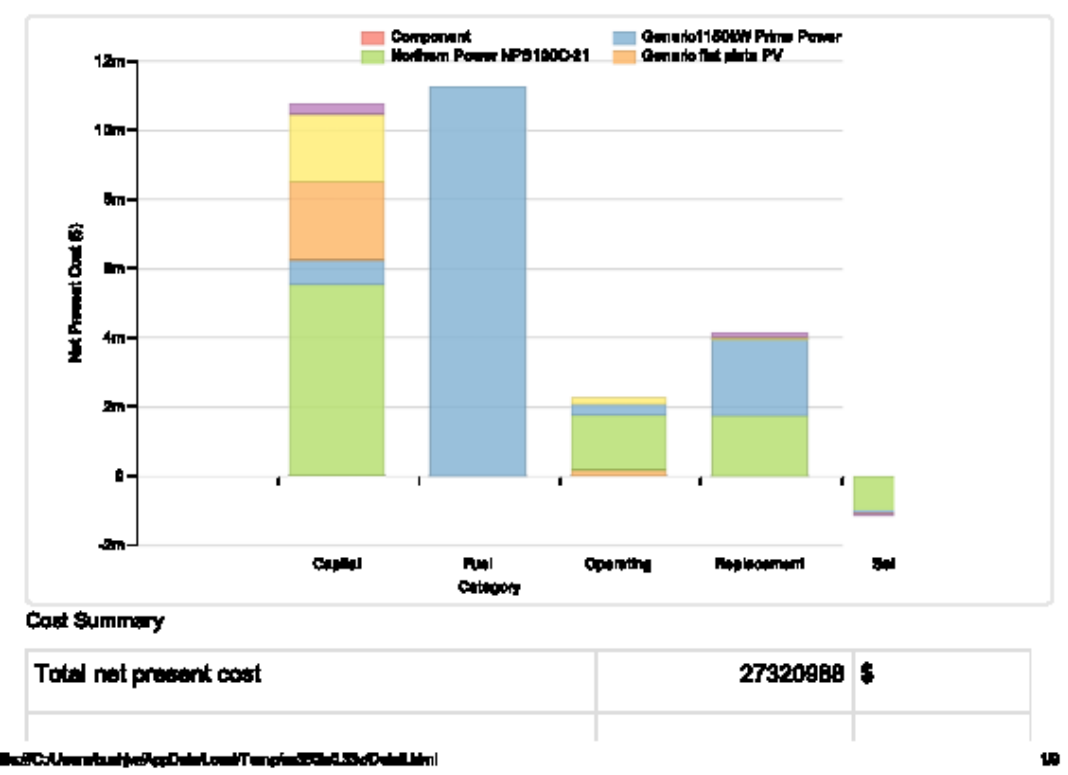




\section{System Report}

\section{System architecture}

\begin{tabular}{|c|c|c|c|}
\hline PV & Generic flat plate PV & 750 & $\mathrm{~kW}$ \\
\hline Wind Turbine & Northern Power NPS100C-21 & 7 & \\
\hline Generator & Generic1150kW Prime Power & 1,150 & kW \\
\hline Battery & GS200 flow & 6 & strings \\
\hline Converter & System Converter & 1,000 & kW \\
\hline Dispatch Strategy & Cycle Charging & & \\
\hline
\end{tabular}

\section{Cost summary}

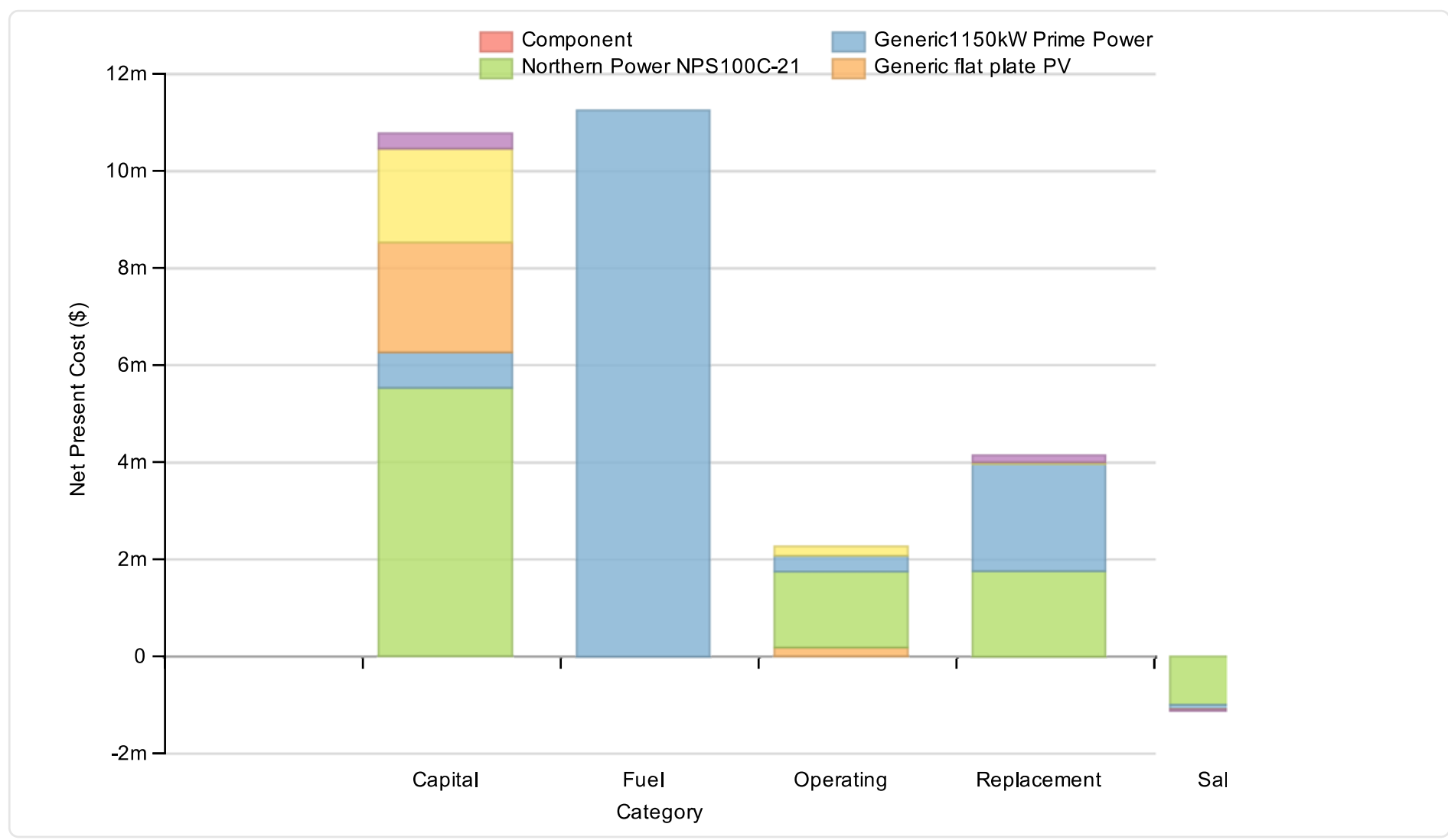

Cost Summary

Total net present cost 


\section{Net Present Costs}

\begin{tabular}{|l|r|r|r|r|r|r|}
\hline Component & Capital & Replacement & O\&M & Fuel & Salvage & Total \\
\hline $\begin{array}{l}\text { Generic flat } \\
\text { plate PV }\end{array}$ & $2,250,000$ & 0 & 193,913 & 0 & 0 & $2,443,913$ \\
\hline $\begin{array}{l}\text { Northern Power } \\
\text { NPS100C-21 }\end{array}$ & $5,530,000$ & $1,763,003$ & $1,551,301$ & 0 & $-993,565$ & $7,850,739$ \\
\hline $\begin{array}{l}\text { Generic1150kW } \\
\text { Prime Power }\end{array}$ & 741,936 & $2,211,004$ & 336,744 & $11,251,017$ & $-88,572$ & $14,452,129$ \\
\hline GS200 flow & $1,947,535$ & 43,013 & 186,156 & 0 & $-5,832$ & $2,170,872$ \\
\hline Converter & 300,000 & 127,282 & 0 & 0 & $-23,956$ & 403,326 \\
\hline System & $10,769,471$ & $4,144,301$ & $2,268,114$ & $11,251,017$ & $-1,111,924$ & $27,320,979$ \\
\hline
\end{tabular}

\section{Annualized Costs}

\begin{tabular}{|l|r|r|r|r|r|r|}
\hline Component & Capital & Replacement & O\&M & Fuel & Salvage & Total \\
\hline Generic flat plate PV & 174,047 & 0 & 15,000 & 0 & 0 & 189,047 \\
\hline $\begin{array}{l}\text { Northern Power NPS100C- } \\
21\end{array}$ & 427,770 & 136,376 & 120,000 & 0 & $-76,857$ & 607,289 \\
\hline $\begin{array}{l}\text { Generic1150kW Prime } \\
\text { Power }\end{array}$ & 57,392 & 171,031 & 26,049 & 870,315 & $-6,851$ & $1,117,936$ \\
\hline GS200 flow & 150,650 & 3,327 & 14,400 & 0 & -451 & 167,926 \\
\hline Converter & 23,206 & 9,846 & 0 & 0 & $-1,853$ & 31,199 \\
\hline System & 833,066 & 320,580 & 175,449 & 870,315 & $-86,012$ & $2,113,398$ \\
\hline
\end{tabular}




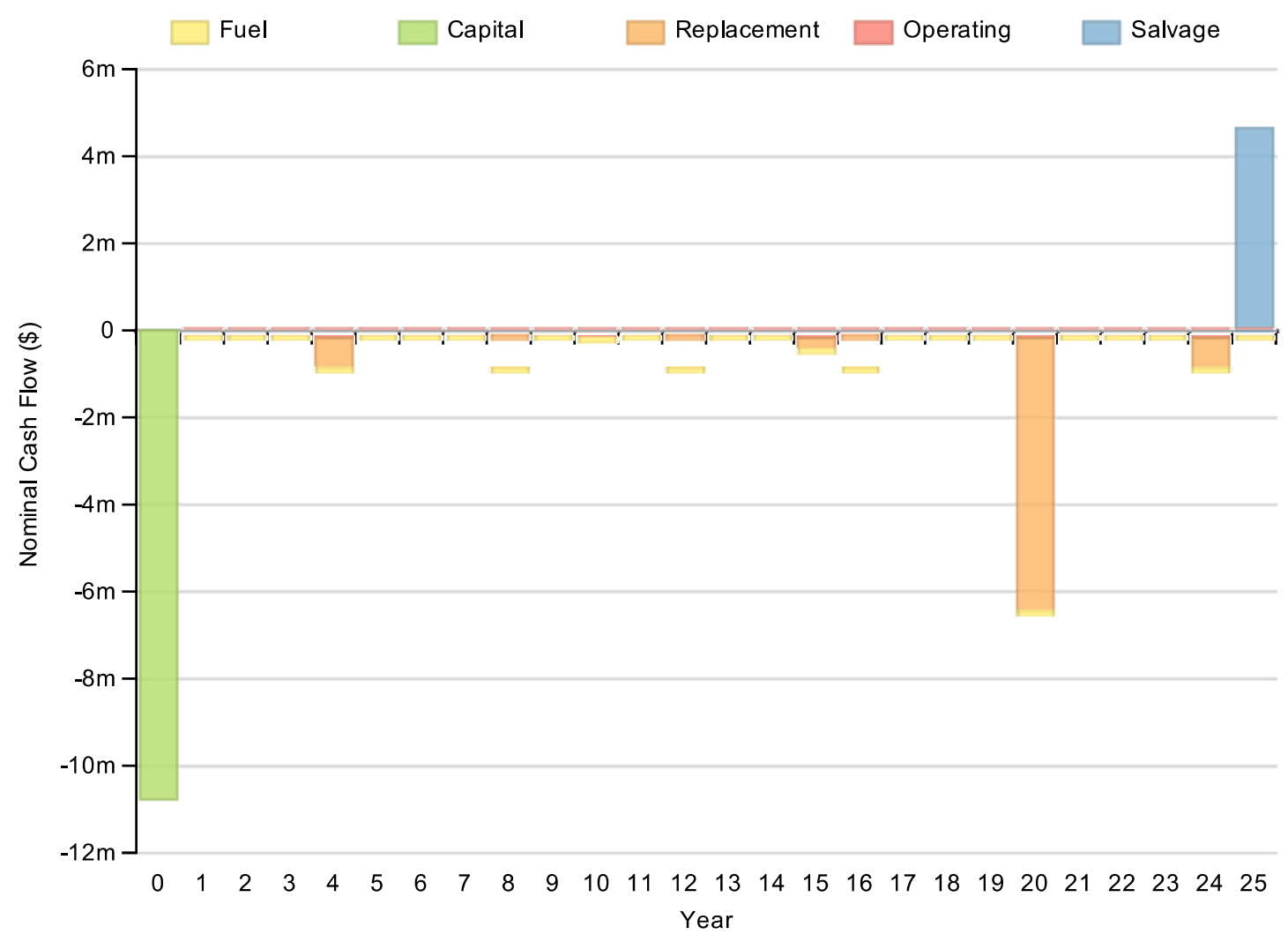

\section{Electrical}

\begin{tabular}{|l|r|l|}
\hline Quantity & Value & Units \\
\hline Excess electricity & 132565 & $\mathrm{kWh} / \mathrm{yr}$ \\
\hline Unmet load & $0 \mathrm{kWh} / \mathrm{yr}$ \\
\hline Capacity shortage & $0 \mathrm{kWh} / \mathrm{yr}$ \\
\hline Renewable fraction & 0 & \\
\hline
\end{tabular}

\section{Component}

PV

Generator

Wind Turbine

Total

\section{Fraction (\%)}

$1,125,156$

$3,347,365$

$1,601,808$

$6,074,329$

100 
AC primary load

DC primary load

0

Total

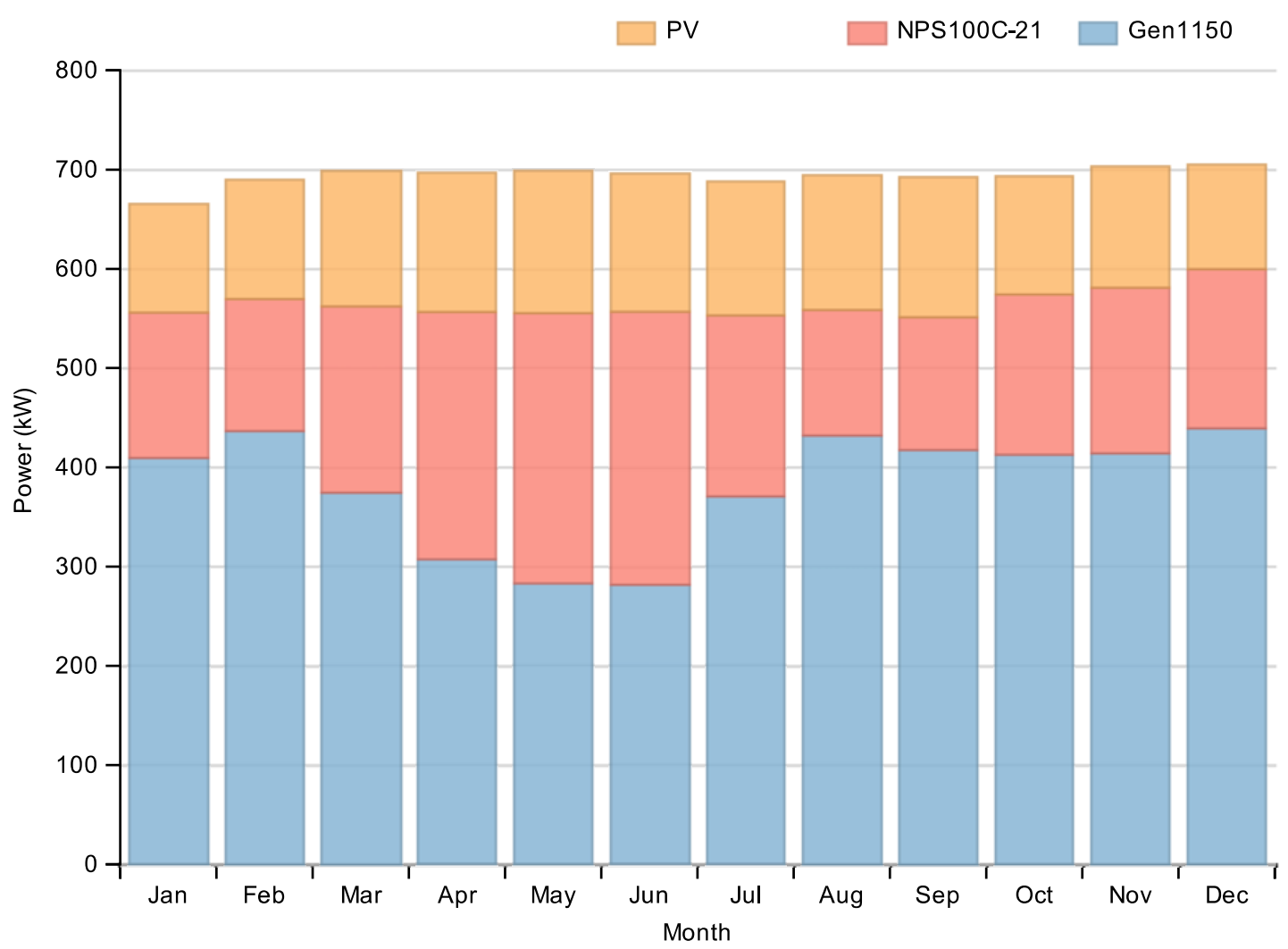

PV:Generic flat plate PV

\begin{tabular}{|l|r|l|}
\hline Quantity & Value & Units \\
\hline Rated capacity & 750 & $\mathrm{~kW}$ \\
\hline Mean output & 128 & $\mathrm{~kW}$ \\
\hline Mean output & 3082.60 & $\mathrm{kWh} / \mathrm{d}$ \\
\hline Capacity factor & 17.13 & $\%$ \\
\hline Total production & 1125156 & $\mathrm{kWh} / \mathrm{yr}$ \\
\hline Minimum output & 0.00 & $\mathrm{~kW}$ \\
\hline Maximum output & 758.97 & $\mathrm{~kW}$ \\
\hline
\end{tabular}




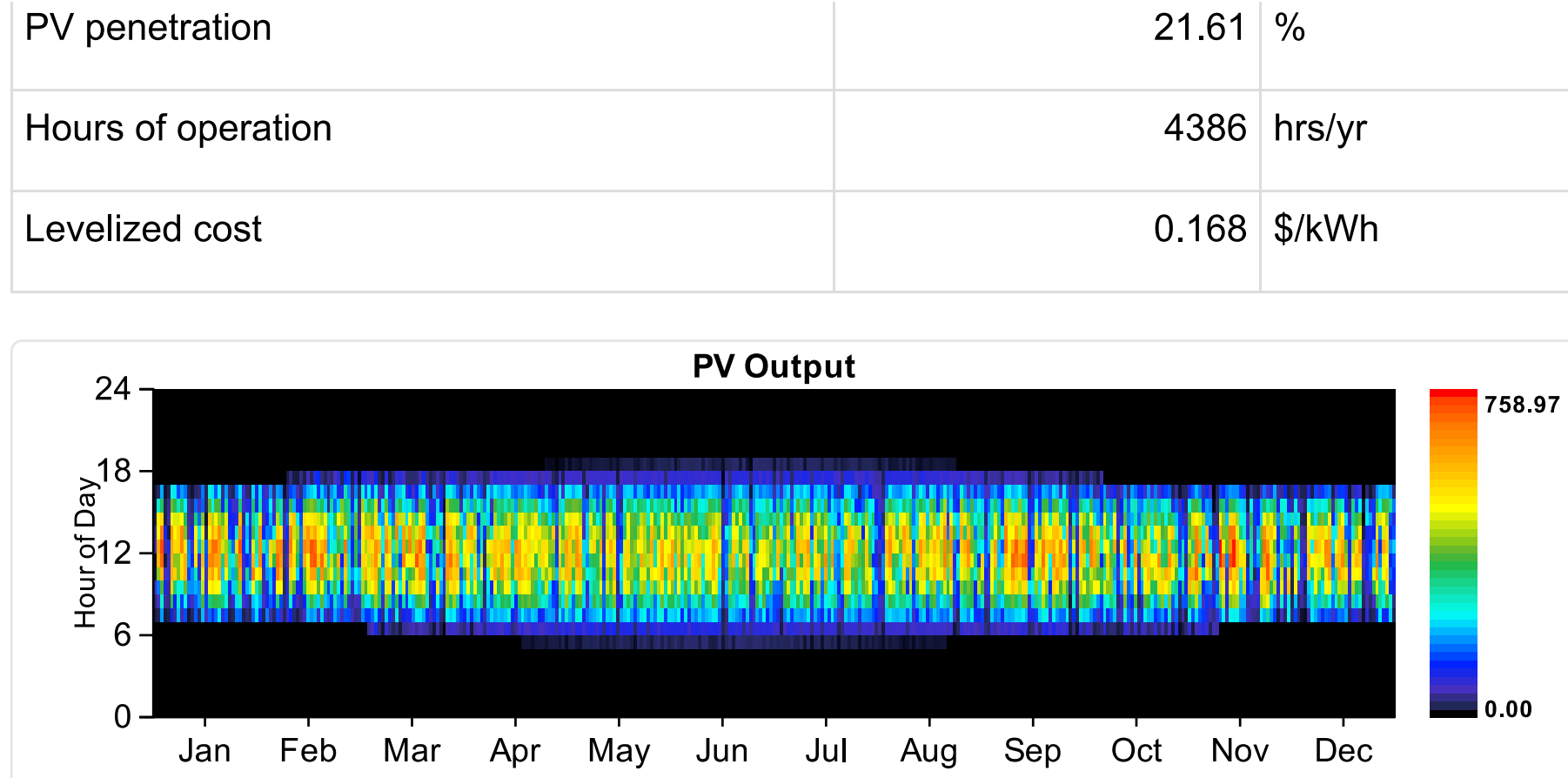

\section{Wind Turbine:Northern Power NPS100C-21}

\begin{tabular}{|l|r|l|}
\hline Quantity & Value & Units \\
\hline Total rated capacity & 700 & $\mathrm{~kW}$ \\
\hline Mean output & 183 & $\mathrm{~kW}$ \\
\hline Capacity factor & 26.12 & $\%$ \\
\hline Total production & 1601808 & $\mathrm{kWh} / \mathrm{yr}$ \\
\hline Minimum output & 0.00 & $\mathrm{~kW}$ \\
\hline Maximum output & 686.75 & $\mathrm{~kW}$ \\
\hline Wind penetration & $30.77 \%$ & $\%$ \\
\hline Hours of operation & 8108 & $\mathrm{hrs} / \mathrm{yr}$ \\
\hline Levelized cost & 0.379 & $\$ / \mathrm{kWh}$ \\
\hline
\end{tabular}




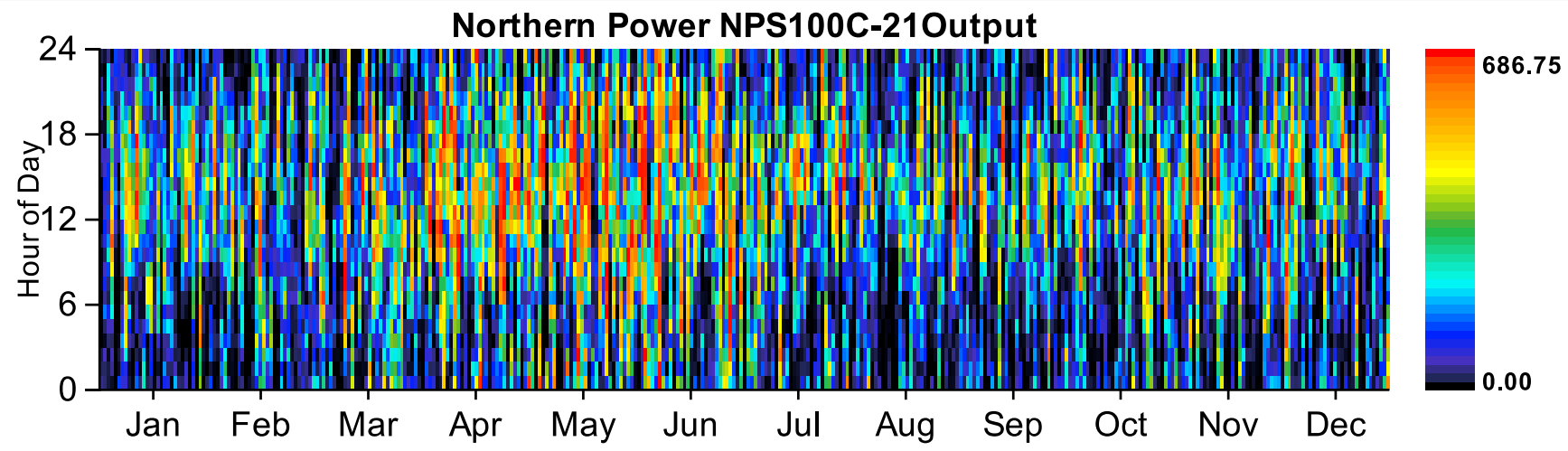

Generator:Generic1150kW Prime Power

\begin{tabular}{|c|c|c|}
\hline Quantity & Value & Units \\
\hline Hours of operation & 3901 & $\mathrm{hrs} / \mathrm{yr}$ \\
\hline Number of starts & 950 & starts/yr \\
\hline Operational life & 4 & $\mathrm{yr}$ \\
\hline Fixed generation cost & 56.14 & $\$ / \mathrm{hr}$ \\
\hline Marginal generation cost & 0.26 & $\$ / k W h$ \\
\hline Electrical production & 3347365 & $\mathrm{kWh} / \mathrm{yr}$ \\
\hline Mean electrical output & 858 & $\mathrm{~kW}$ \\
\hline Min. electrical output & 345 & kW \\
\hline Max. electrical output & 1150 & $\mathrm{~kW}$ \\
\hline Fuel consumption & 870316 & L/yr \\
\hline Specific fuel consumption & 0.26 & $\mathrm{~L} / \mathrm{kWh}$ \\
\hline Fuel energy input & 8563909 & $\mathrm{kWh} / \mathrm{yr}$ \\
\hline Mean electrical efficiency & 39 & $\%$ \\
\hline
\end{tabular}




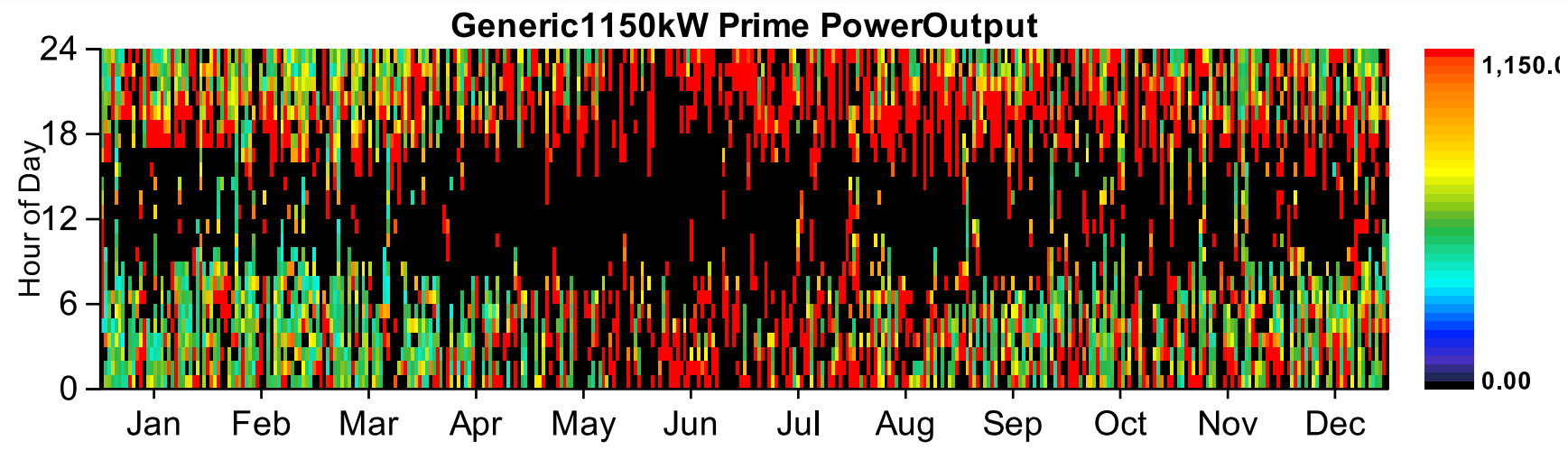

\section{Battery:GS200 flow}

\section{Quantity}

String size

Strings in parallel

Batteries

Bus voltage

Value

\section{Quantity}

Nominal capacity

Usable nominal capacity

Autonomy

Lifetime throughput

Battery wear cost

Average energy cost

Energy in

Energy out

Storage depletion
Value

\begin{tabular}{r|l|l} 
& Units \\
3600 & $\mathrm{kWh}$ \\
\hline 3600 & $\mathrm{kWh}$ \\
\hline 6 & $\mathrm{hr}$ \\
\hline 0 & \\
\hline 0.000 & $\$ / \mathrm{kWh}$ \\
\hline 0.356 & $\$ / \mathrm{kWh}$ \\
\hline 1282096 & $\mathrm{kWh} / \mathrm{yr}$ \\
\hline 897551 & $\mathrm{kWh} / \mathrm{yr}$ \\
\hline 101 & $\mathrm{kWh} / \mathrm{yr}$ \\
\hline
\end{tabular}


Losses

Annual throughput

$1072780 \mathrm{kWh} / \mathrm{yr}$

Expected life

$25 \mathrm{yr}$

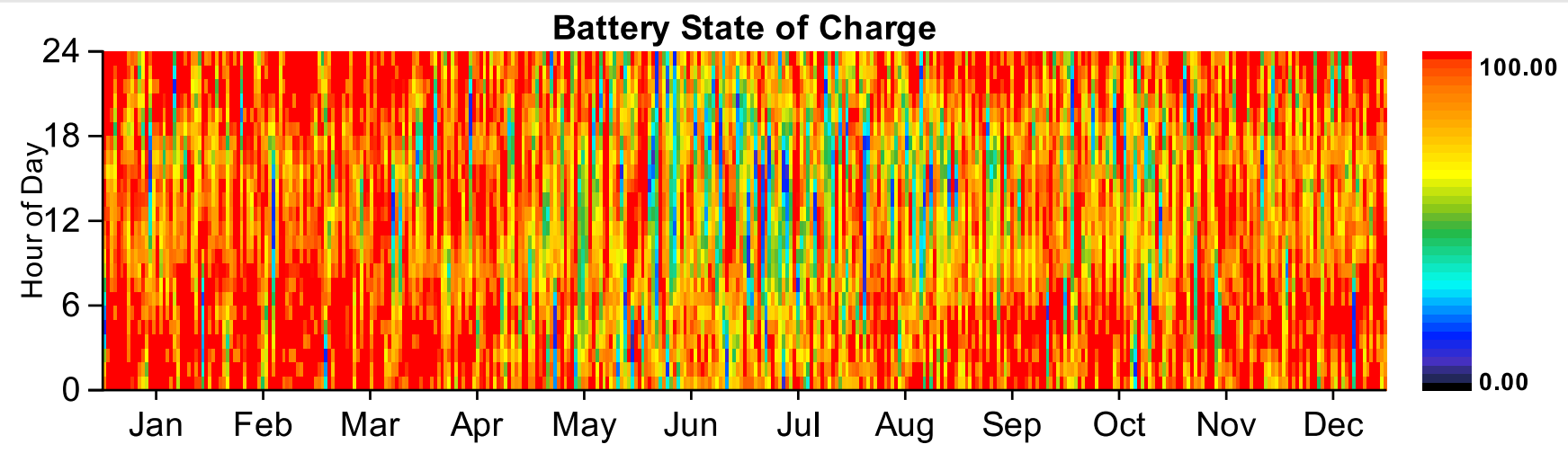

Converter

\begin{tabular}{|l|r|r|l|}
\hline Quantity & Inverter & Rectifier & Units \\
\hline Capacity & 1,000 & 900 & $\mathrm{~kW}$ \\
\hline Mean output & 172 & 119 & $\mathrm{~kW}$ \\
\hline Minimum output & 0 & 0 & $\mathrm{~kW}$ \\
\hline Maximum output & 860 & 1,000 & $\mathrm{~kW}$ \\
\hline Capacity factor & 17 & 12 & $\%$ \\
\hline Hours of operation & 4,730 & 2,572 & $\mathrm{hrs} / \mathrm{yr}$ \\
\hline Energy in & $1,673,117$ & $1,226,471$ & $\mathrm{kWh} / \mathrm{yr}$ \\
\hline Energy out & $1,505,805$ & $1,042,500$ & $\mathrm{kWh} / \mathrm{yr}$ \\
\hline Losses & 167,312 & 183,971 & $\mathrm{kWh} / \mathrm{yr}$ \\
\hline
\end{tabular}



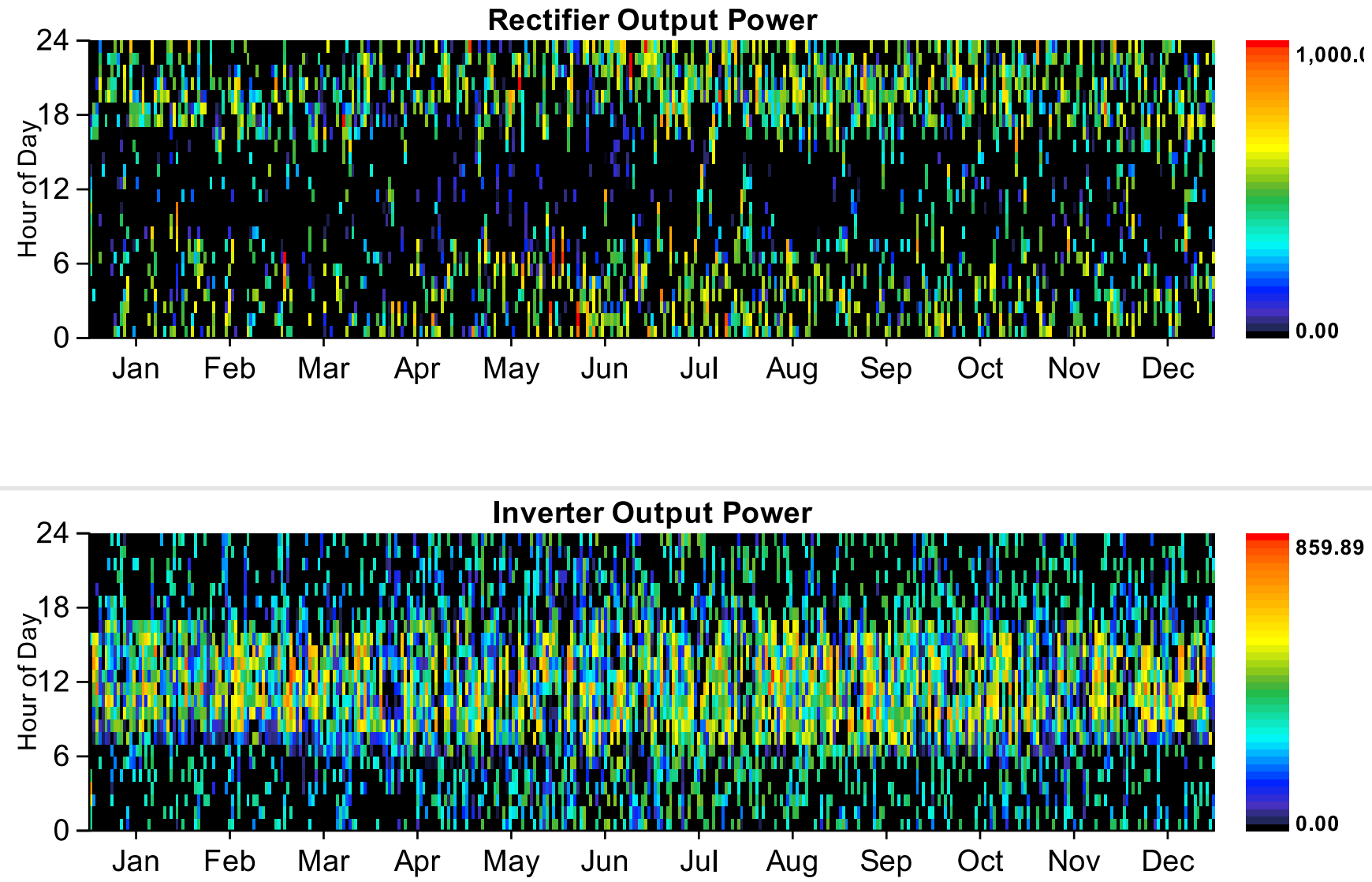

\section{Emissions}

\section{Pollutant}

Carbon dioxide

Carbon monoxide

Unburned hydrocarbons

Particulate matter

Sulfur dioxide

Nitrogen oxides

\section{Emissions}

$2284157 \mathrm{~kg} / \mathrm{yr}$

$9574 \mathrm{~kg} / \mathrm{yr}$

$1097 \mathrm{~kg} / \mathrm{yr}$

$274 \mathrm{~kg} / \mathrm{yr}$

$4703 \mathrm{~kg} / \mathrm{yr}$

$9574 \mathrm{~kg} / \mathrm{yr}$

HOMER Energy, LLC @ 2015 


\section{System Report}

\section{System architecture}

\begin{tabular}{|c|c|c|c|}
\hline Wind Turbine & Northern Power NPS100C-21 & 7 & \\
\hline Generator & Generic1150kW Prime Power & 1,150 & $\mathrm{~kW}$ \\
\hline Battery & GS200 flow & 6 & strings \\
\hline Converter & System Converter & 750 & $\mathrm{~kW}$ \\
\hline Dispatch Strategy & Cycle Charging & & \\
\hline
\end{tabular}

\section{Cost summary}

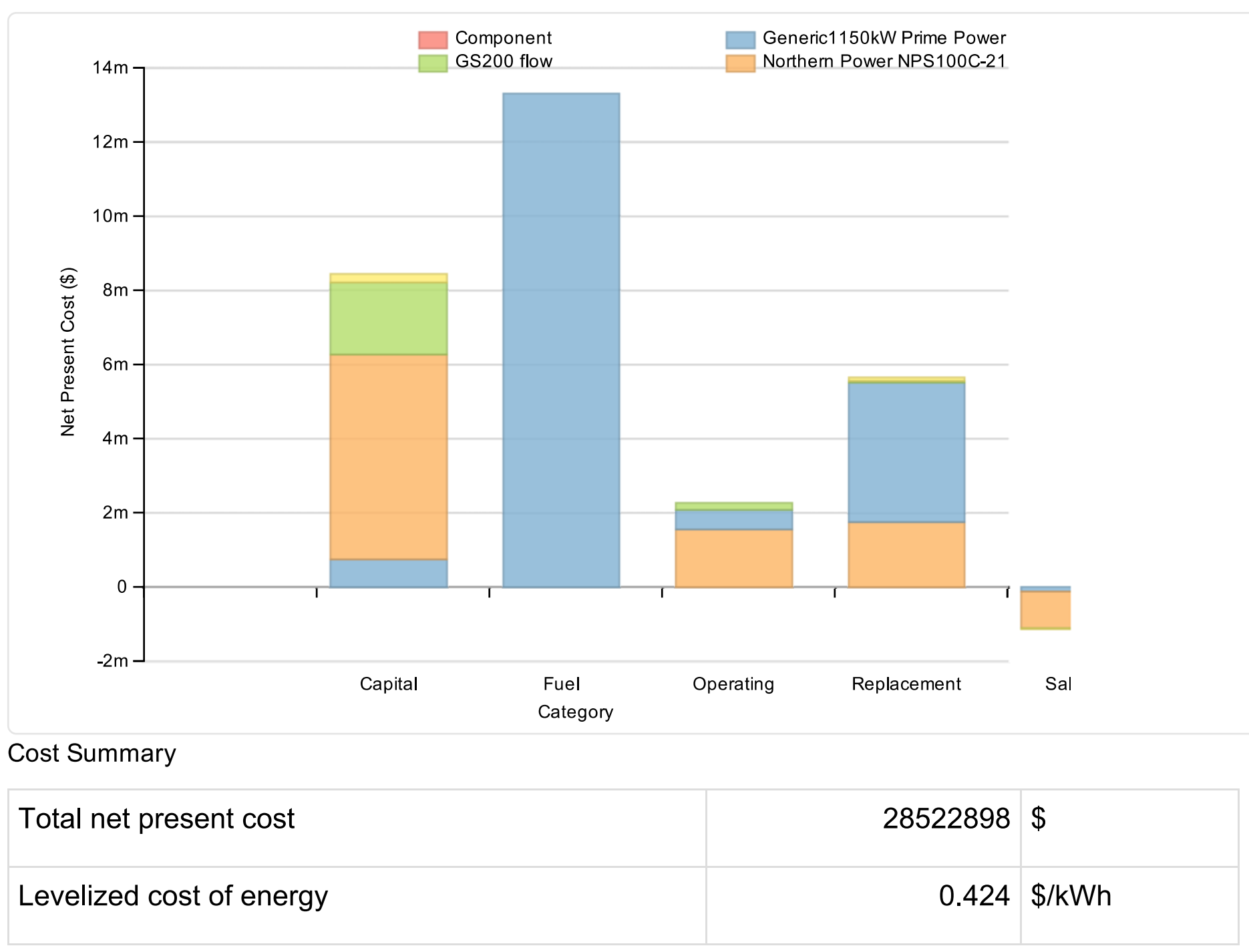




\section{Net Present Costs}

\begin{tabular}{|l|r|r|r|r|r|r|}
\hline Component & Capital & Replacement & O\&M & Fuel & Salvage & \multicolumn{1}{l|}{ Total } \\
\hline $\begin{array}{l}\text { Northern Power } \\
\text { NPS100C-21 }\end{array}$ & $5,530,000$ & $1,763,003$ & $1,551,301$ & 0 & $-993,565$ & $7,850,739$ \\
\hline $\begin{array}{l}\text { Generic1150kW } \\
\text { Prime Power }\end{array}$ & 741,936 & $3,744,850$ & 534,508 & $13,298,348$ & $-120,861$ & $18,198,781$ \\
\hline GS200 flow & $1,947,535$ & 43,013 & 186,156 & 0 & $-5,832$ & $2,170,872$ \\
\hline Converter & 225,000 & 95,462 & 0 & 0 & $-17,967$ & 302,495 \\
\hline System & $8,444,470$ & $5,646,327$ & $2,271,966$ & $13,298,348$ & $-1,138,224$ & $28,522,887$ \\
\hline
\end{tabular}

\section{Annualized Costs}

\begin{tabular}{|l|r|r|r|r|r|r|}
\hline Component & Capital & Replacement & O\&M & Fuel & Salvage & Total \\
\hline $\begin{array}{l}\text { Northern Power } \\
\text { NPS100C-21 }\end{array}$ & 427,770 & 136,376 & 120,000 & 0 & $-76,857$ & 607,289 \\
\hline $\begin{array}{l}\text { Generic1150kW Prime } \\
\text { Power }\end{array}$ & 57,392 & 289,681 & 41,347 & $1,028,685$ & $-9,349$ & $1,407,756$ \\
\hline GS200 flow & 150,650 & 3,327 & 14,400 & 0 & -451 & 167,926 \\
\hline Converter & 17,405 & 7,384 & 0 & 0 & $-1,390$ & 23,400 \\
\hline System & 653,217 & 436,768 & 175,746 & $1,028,685$ & $-88,047$ & $2,206,369$ \\
\hline
\end{tabular}




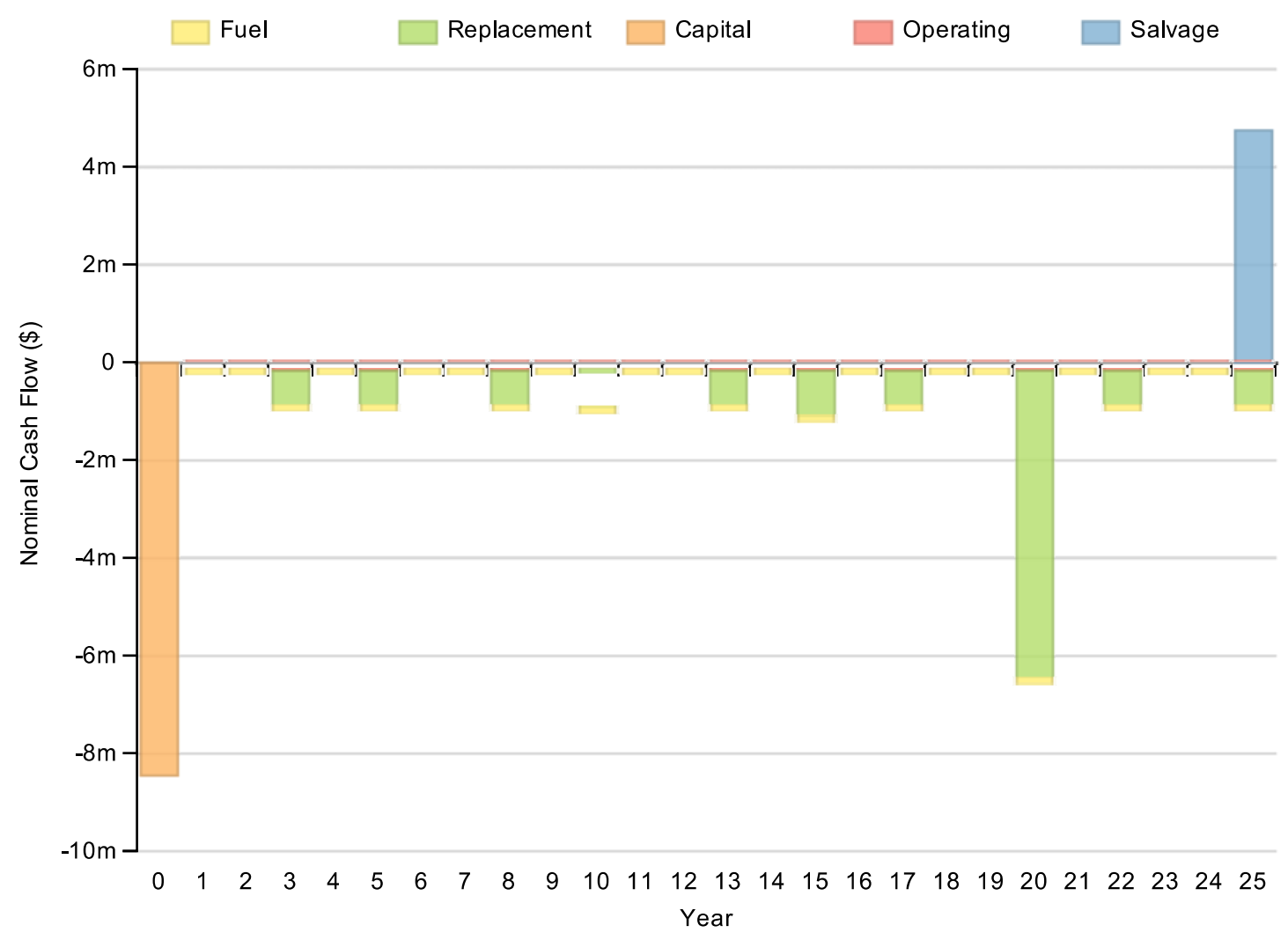

\section{Electrical}

\section{Quantity}

Excess electricity

Unmet load

Capacity shortage

Renewable fraction

\section{Component}

Generator

Wind Turbine

Total
Value

Production(kWh/yr)

$3,956,489$

$1,601,808$

$5,558,297$
Units

$12119 \mathrm{kWh} / \mathrm{yr}$

$0 \mathrm{kWh} / \mathrm{yr}$

$0 \mathrm{kWh} / \mathrm{yr}$

0
Fraction (\%)

Load

Consumption(kWh/yr)

Fraction (\%)

AC primary load 
DC primary load

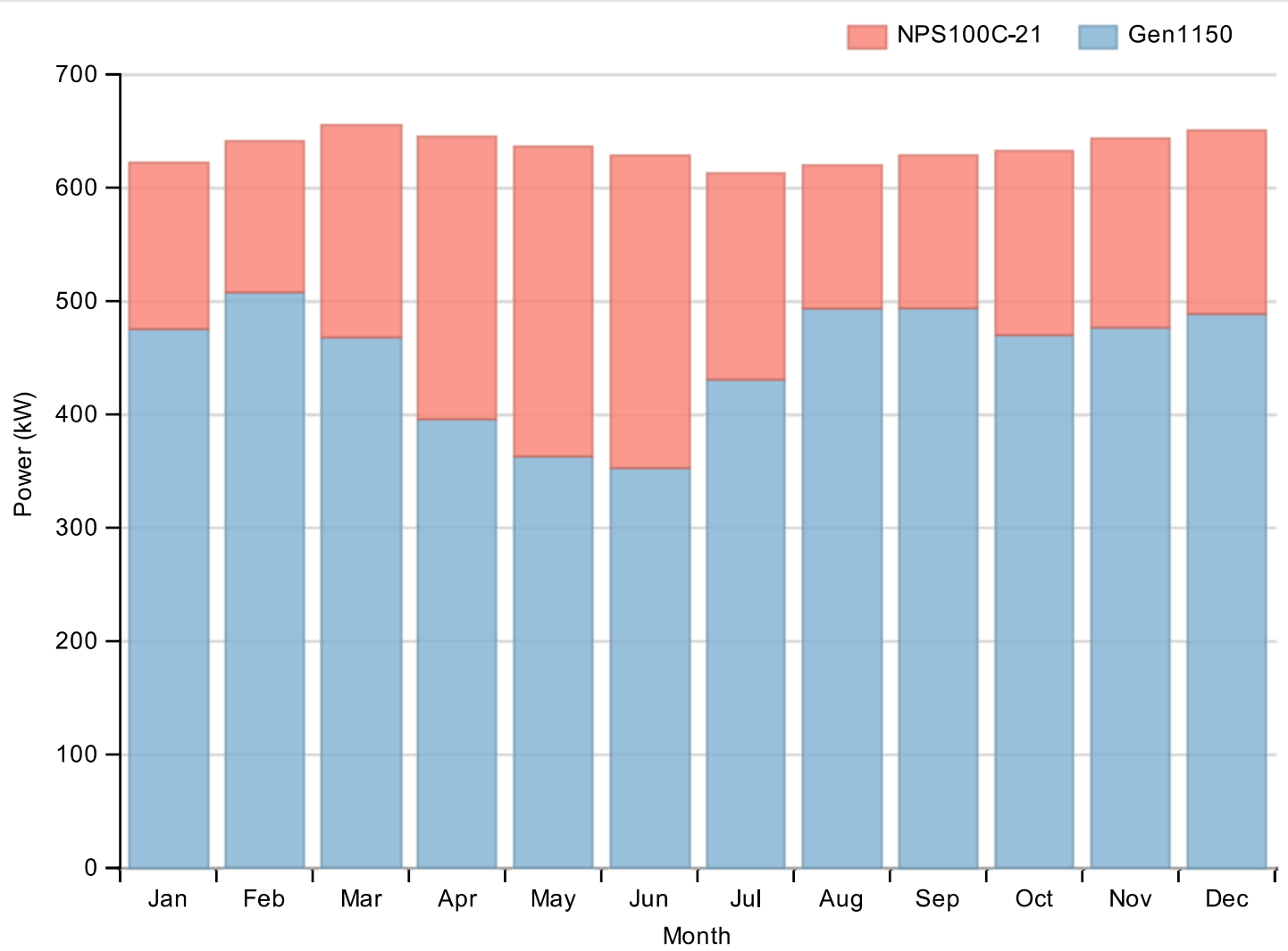

\section{Wind Turbine:Northern Power NPS100C-21}

\begin{tabular}{|l|r|l|}
\hline Quantity & Value & Units \\
\hline Total rated capacity & 700 & $\mathrm{~kW}$ \\
\hline Mean output & 183 & $\mathrm{~kW}$ \\
\hline Capacity factor & 26.12 & $\%$ \\
\hline Total production & 1601808 & $\mathrm{kWh} / \mathrm{yr}$ \\
\hline Minimum output & 0.00 & $\mathrm{~kW}$ \\
\hline Maximum output & 686.75 & $\mathrm{~kW}$ \\
\hline Wind penetration & 30.77 & $\%$ \\
\hline Hours of operation & 8108 & $\mathrm{hrs} / \mathrm{yr}$ \\
\hline
\end{tabular}




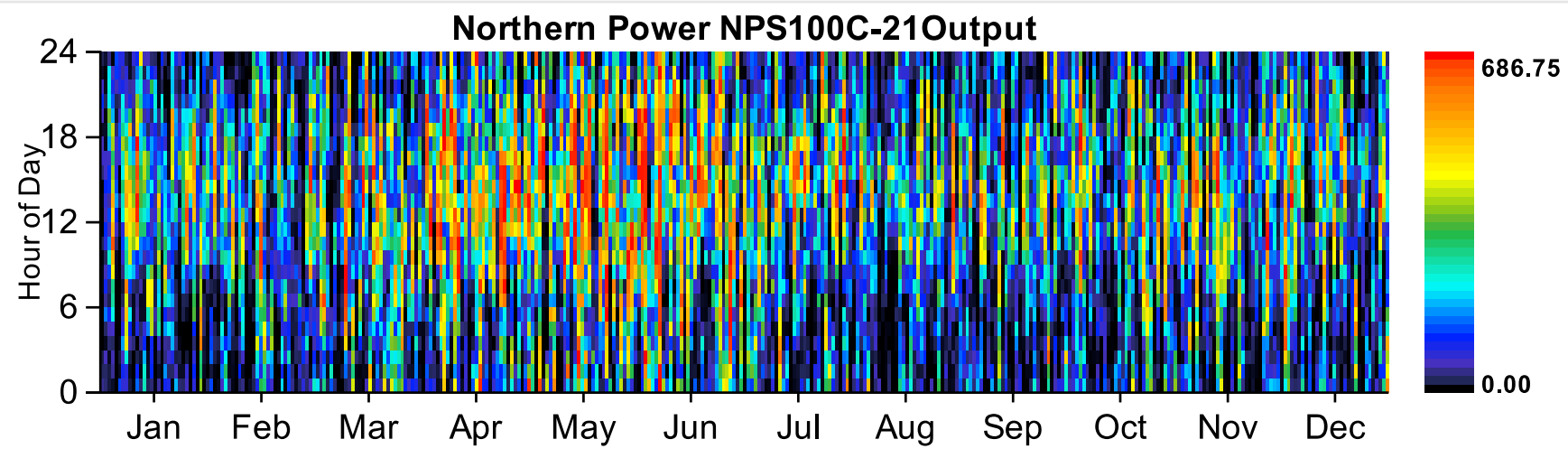

\section{Generator:Generic1150kW Prime Power}

\begin{tabular}{|c|c|c|}
\hline Quantity & Value & Units \\
\hline Hours of operation & 6192 & hrs/yr \\
\hline Number of starts & 883 & starts/yr \\
\hline Operational life & 2 & $\mathrm{yr}$ \\
\hline Fixed generation cost & 56.14 & $\$ / \mathrm{hr}$ \\
\hline Marginal generation cost & 0.26 & $\$ / k W h$ \\
\hline Electrical production & 3956489 & $\mathrm{kWh} / \mathrm{yr}$ \\
\hline Mean electrical output & 639 & $\mathrm{~kW}$ \\
\hline Min. electrical output & 345 & $\mathrm{~kW}$ \\
\hline Max. electrical output & 1150 & $\mathrm{~kW}$ \\
\hline Fuel consumption & 1028686 & L/yr \\
\hline Specific fuel consumption & 0.26 & $\mathrm{~L} / \mathrm{kWh}$ \\
\hline Fuel energy input & 10122272 & $\mathrm{kWh} / \mathrm{yr}$ \\
\hline Mean electrical efficiency & 39 & $\%$ \\
\hline
\end{tabular}




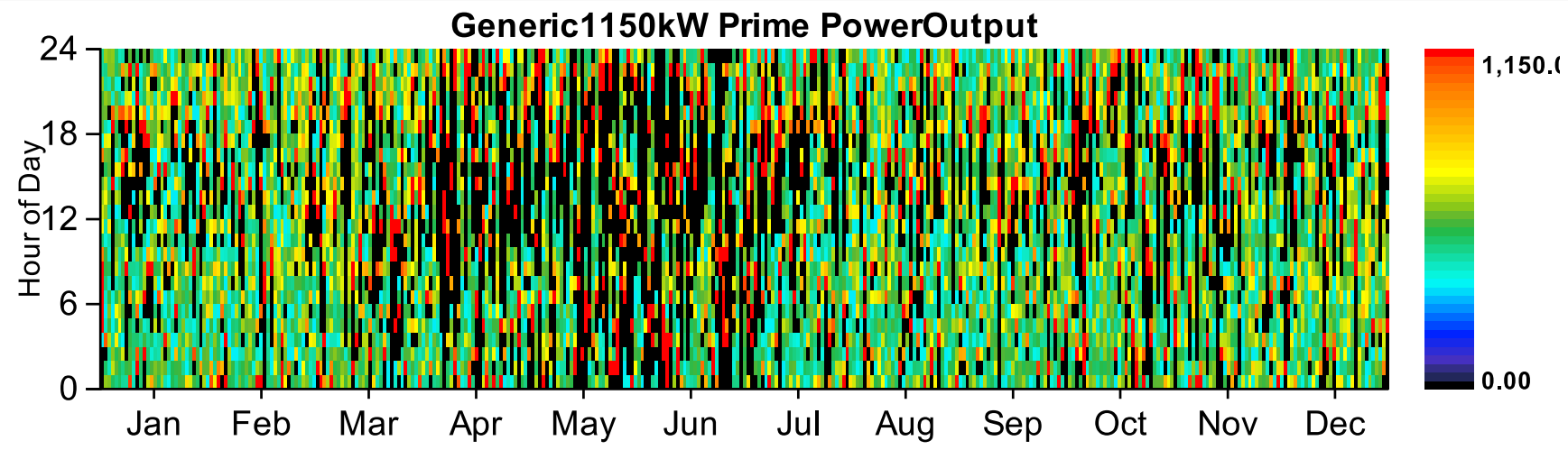

\section{Battery:GS200 flow}

\section{Quantity}

String size

Strings in parallel

Batteries

Bus voltage

\section{Value}

\section{Quantity}

Nominal capacity

Usable nominal capacity

Autonomy

Lifetime throughput

Battery wear cost

Average energy cost

Energy in

Energy out

Storage depletion
Value

\begin{tabular}{|l|l|}
\hline 3600 & Units \\
\hline 3600 & kWh \\
\hline
\end{tabular}

$6 \mathrm{hr}$

0

$0.000 \$ / \mathrm{kWh}$

$0.410 \$ / \mathrm{kWh}$

$622609 \mathrm{kWh} / \mathrm{yr}$

$435826 \mathrm{kWh} / \mathrm{yr}$

$0 \mathrm{kWh} / \mathrm{yr}$ 
Losses

Annual throughput

$520912 \mathrm{kWh} / \mathrm{yr}$

Expected life

$25 \mathrm{yr}$

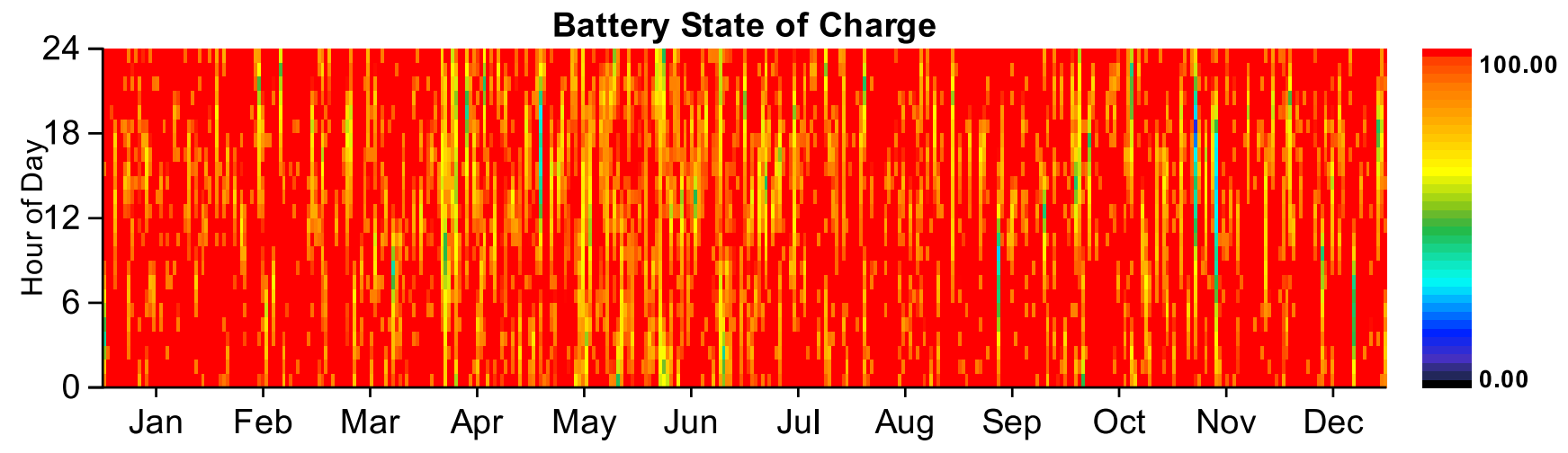

Converter

\begin{tabular}{|l|r|r|l|}
\hline Quantity & Inverter & Rectifier & Units \\
\hline Capacity & 750 & 675 & $\mathrm{~kW}$ \\
\hline Mean output & 45 & 71 & $\mathrm{~kW}$ \\
\hline Minimum output & 0 & $0 \mathrm{~kW}$ \\
\hline Maximum output & 558 & $750 \mathrm{~kW}$ \\
\hline Capacity factor & 6 & $9 \%$ & $\%$ \\
\hline Hours of operation & 2,074 & 1,771 & $\mathrm{hrs} / \mathrm{yr}$ \\
\hline Energy in & 435,826 & 732,481 & $\mathrm{kWh} / \mathrm{yr}$ \\
\hline Energy out & 392,244 & 622,609 & $\mathrm{kWh} / \mathrm{yr}$ \\
\hline Losses & 43,583 & 109,872 & $\mathrm{kWh} / \mathrm{yr}$ \\
\hline
\end{tabular}



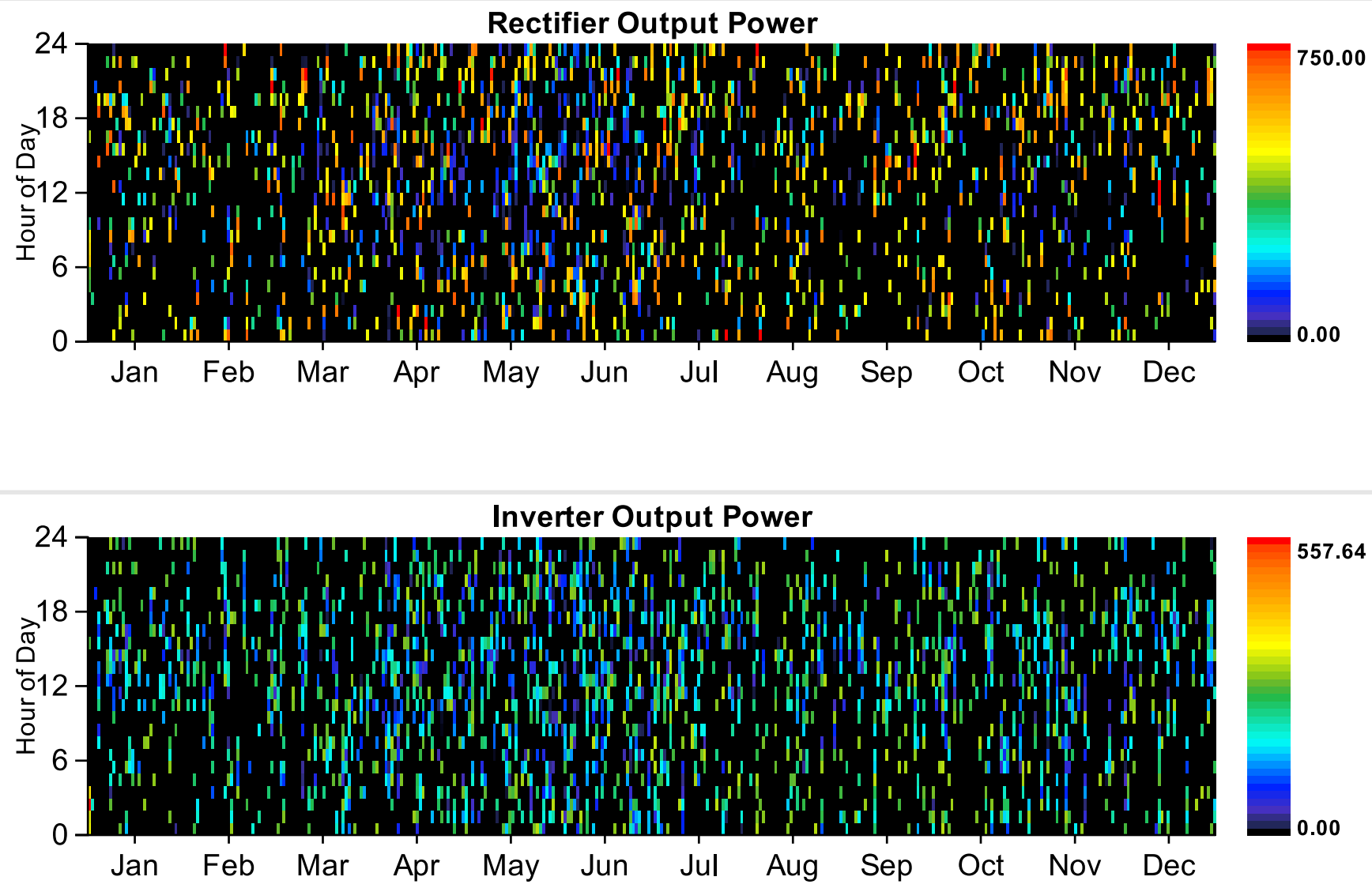

\section{Emissions}

\begin{tabular}{|l|r|l|}
\hline Pollutant & Emissions & Units \\
\hline Carbon dioxide & 2699802 & $\mathrm{~kg} / \mathrm{yr}$ \\
\hline Carbon monoxide & 11316 & $\mathrm{~kg} / \mathrm{yr}$ \\
\hline Unburned hydrocarbons & 1296 & $\mathrm{~kg} / \mathrm{yr}$ \\
\hline Particulate matter & 324 & $\mathrm{~kg} / \mathrm{yr}$ \\
\hline Sulfur dioxide & 5559 & $\mathrm{~kg} / \mathrm{yr}$ \\
\hline Nitrogen oxides & 11316 & $\mathrm{~kg} / \mathrm{yr}$ \\
\hline
\end{tabular}

HOMER Energy, LLC @ 2015 


\section{System Report}

\section{System architecture}

\begin{tabular}{|l|l|r|}
\hline Wind Turbine & Northern Power NPS100C-21 & 7 \\
\hline Generator & Generic1150kW Prime Power & $1,150 \mathrm{~kW}$ \\
\hline Dispatch Strategy & Cycle Charging & \\
\hline
\end{tabular}

\section{Cost summary}

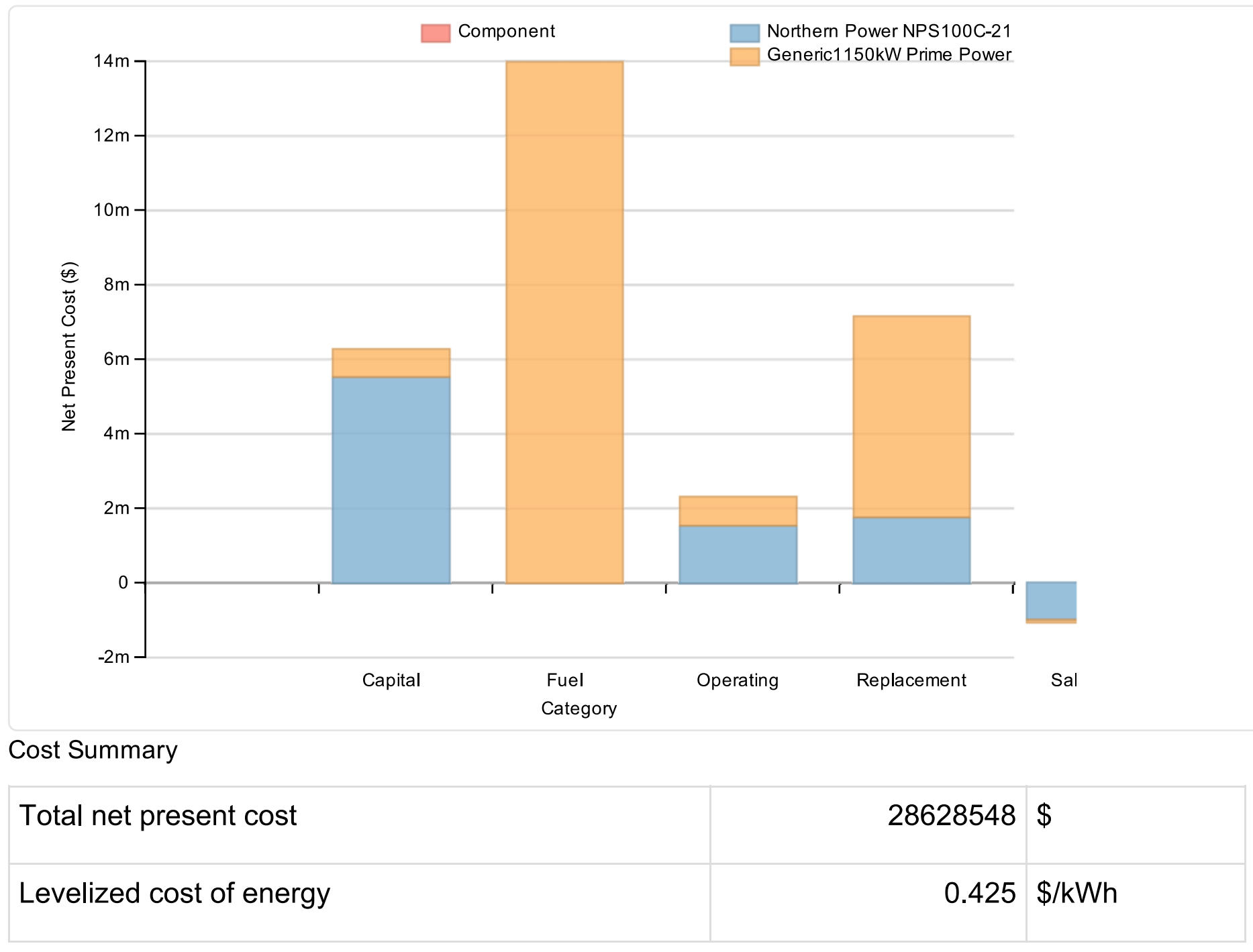

\section{Net Present Costs}

\begin{tabular}{|c|c|c|c|c|c|c|}
\hline Component & Capital & Replacement & O\&M & Fuel & Salvage & Total \\
\hline Niauthaun na..... & E con กnก & 1 דת & 1 r51 Dnd & $n$ & Dก) Eef & \\
\hline
\end{tabular}




\begin{tabular}{|c|c|c|c|c|c|c|}
\hline $\begin{array}{l}\text { Ivortiern rower } \\
\text { NPS100C-21 }\end{array}$ & ৩,১১U,UUU & I, ו ০J,UUs & I,כ ו, ו I & $U$ & -yys,000 & I, , \\
\hline $\begin{array}{l}\text { Generic1150kW } \\
\text { Prime Power }\end{array}$ & 741,936 & $5,381,779$ & 756,012 & $13,969,756$ & $-71,687$ & $20,777,796$ \\
\hline System & $6,271,936$ & $7,144,782$ & $2,307,313$ & $13,969,756$ & $-1,065,251$ & $28,628,536$ \\
\hline
\end{tabular}

\section{Annualized Costs}

\begin{tabular}{|l|l|r|r|r|r|r|}
\hline Component & Capital & Replacement & O\&M & Fuel & Salvage & Total \\
\hline $\begin{array}{l}\text { Northern Power } \\
\text { NPS100C-21 }\end{array}$ & 427,770 & 136,376 & 120,000 & 0 & $-76,857$ & 607,289 \\
\hline $\begin{array}{l}\text { Generic1150kW Prime } \\
\text { Power }\end{array}$ & 57,392 & 416,304 & 58,481 & $1,080,622$ & $-5,545$ & $1,607,254$ \\
\hline System & 485,162 & 552,680 & 178,481 & $1,080,622$ & $-82,402$ & $2,214,543$ \\
\hline
\end{tabular}

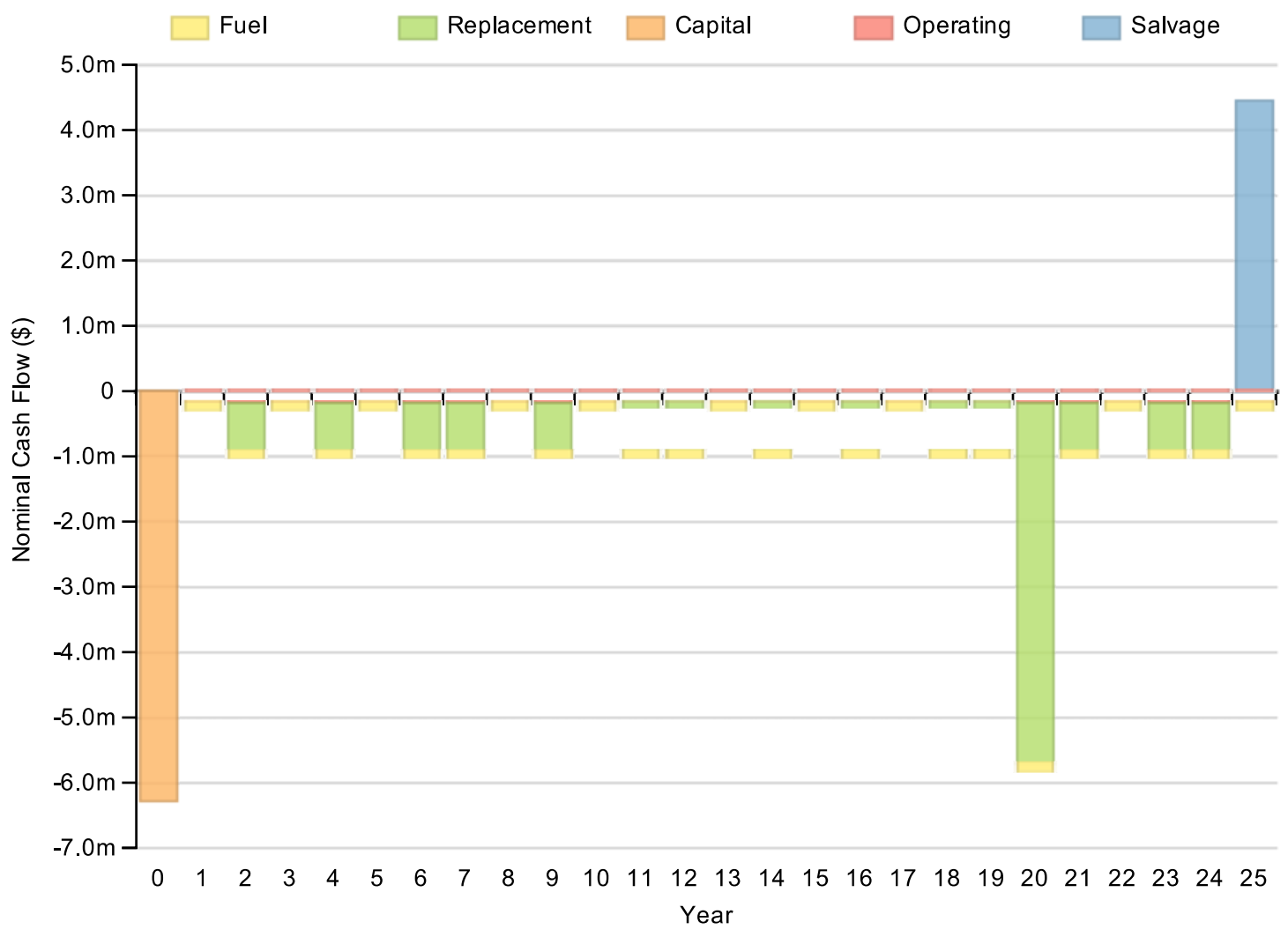

\section{Electrical}

\section{Quantity}




\begin{tabular}{|l|r|l|}
\hline Excess electricity & 552204 & $\mathrm{kWh} / \mathrm{yr}$ \\
\hline Unmet load & $0 \mathrm{kWh} / \mathrm{yr}$ \\
\hline Capacity shortage & $0 \mathrm{kWh} / \mathrm{yr}$ \\
\hline Renewable fraction & 0 & \\
\hline
\end{tabular}

\section{Component}

Generator

Wind Turbine

Total
Production(kWh/yr)

Fraction (\%)

$4,156,337$

$1,601,808$

$5,758,145$
Load

AC primary load

DC primary load

Total
Consumption(kWh/yr)
Fraction (\%)

$5,205,935$

100

0

0

\section{8}

.




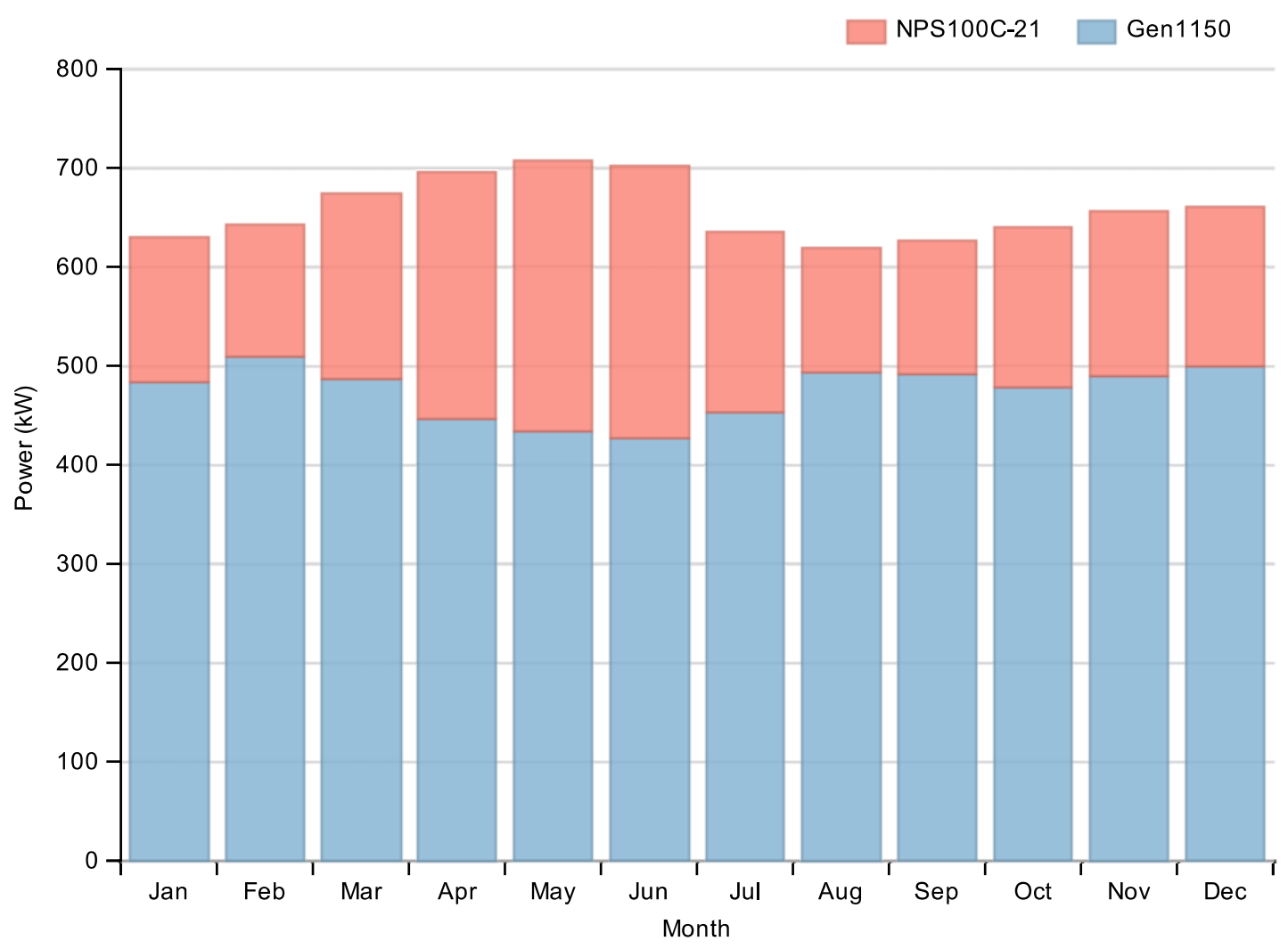

\section{Wind Turbine:Northern Power NPS100C-21}

\begin{tabular}{|l|r|l|}
\hline Quantity & Value & Units \\
\hline Total rated capacity & 183 & $\mathrm{~kW}$ \\
\hline Mean output & 26.12 & $\%$ \\
\hline Capacity factor & 1601808 & $\mathrm{kWh} / \mathrm{yr}$ \\
\hline Total production & 0.00 & $\mathrm{~kW}$ \\
\hline Minimum output & 686.75 & $\mathrm{~kW}$ \\
\hline Maximum output & 30.77 & $\%$ \\
\hline Wind penetration & 8108 & $\mathrm{hrs} / \mathrm{yr}$ \\
\hline Hours of operation & 0.379 & $\$ / \mathrm{kWh}$ \\
\hline Levelized cost & & \\
\hline
\end{tabular}




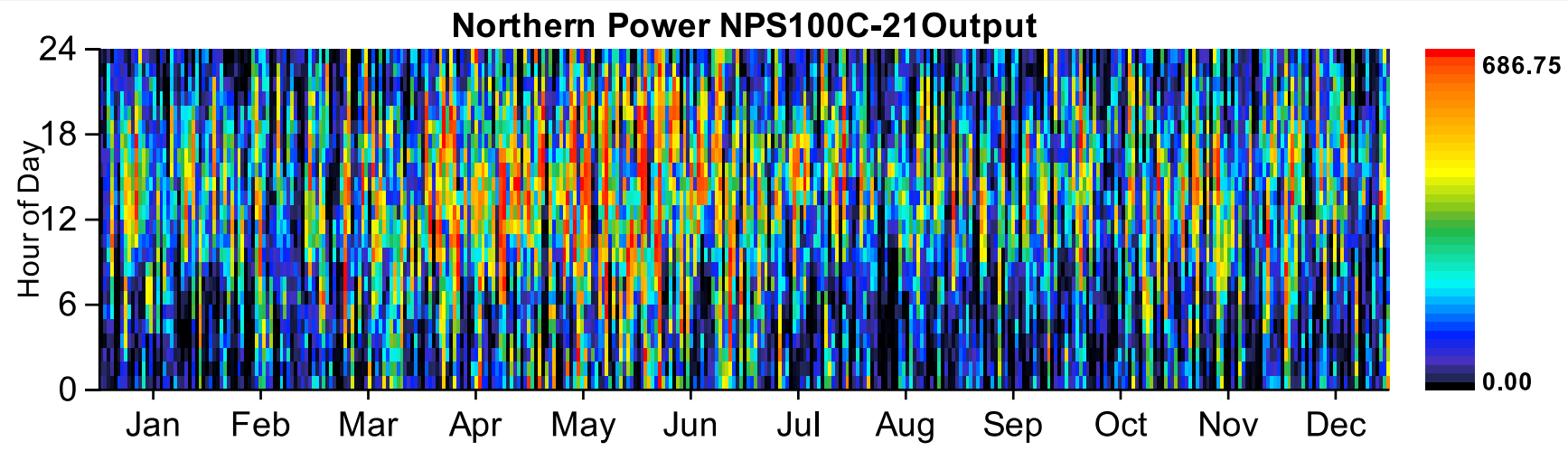

Generator:Generic1150kW Prime Power

\begin{tabular}{|l|r|l|l|}
\hline Quantity & Value & Units \\
\hline Hours of operation & 8758 & hrs/yr \\
\hline Number of starts & 3 & starts/yr \\
\hline Operational life & 2 & $\mathrm{yr}$ \\
\hline Fixed generation cost & 56.14 & $\$ / \mathrm{hr}$ \\
\hline Marginal generation cost & 0.26 & $\$ / \mathrm{kWh}$ \\
\hline Electrical production & 4156337 & $\mathrm{kWh} / \mathrm{yr}$ \\
\hline Mean electrical output & 475 & $\mathrm{~kW}$ \\
\hline Min. electrical output & 345 & $\mathrm{~kW}$ \\
\hline Max. electrical output & 1018 & $\mathrm{~kW}$ \\
\hline Fuel consumption & 1080623 & $\mathrm{~L} / \mathrm{yr}$ \\
\hline Specific fuel consumption & 0.26 & $\mathrm{~L} / \mathrm{kWh}$ \\
\hline Fuel energy input & 10633327 & $\mathrm{kWh} / \mathrm{yr}$ \\
\hline Mean electrical efficiency & $39 \%$ & \\
\hline
\end{tabular}




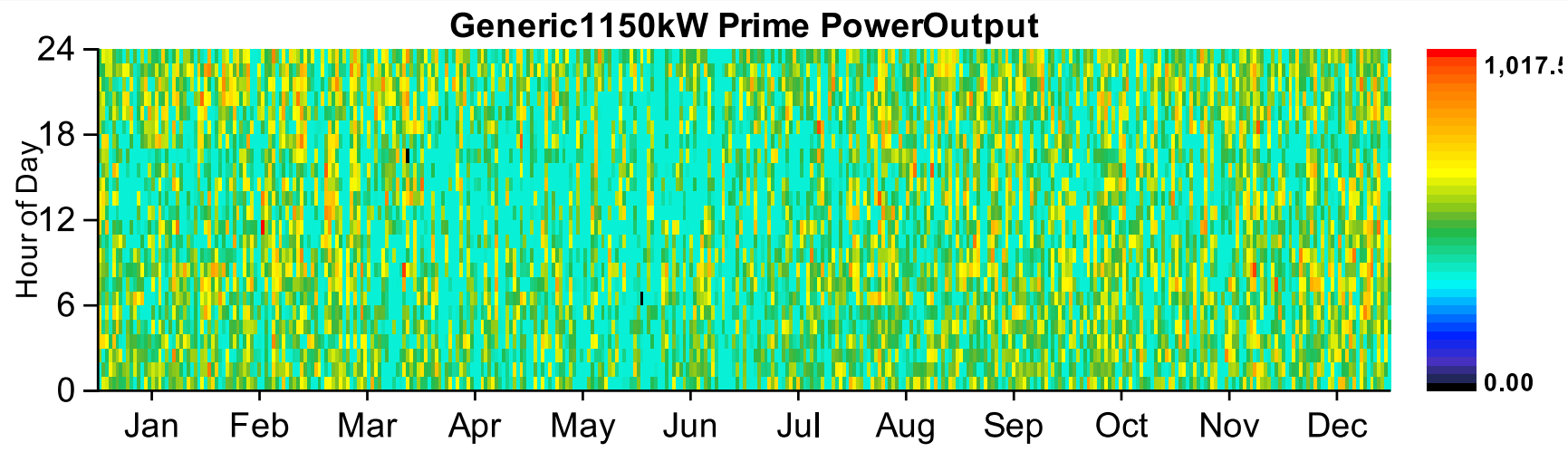

\section{Emissions}

\begin{tabular}{|l|r|l|}
\hline Pollutant & Emissions & Units \\
\hline Carbon dioxide & 2836110 & $\mathrm{~kg} / \mathrm{yr}$ \\
\hline Carbon monoxide & 11887 & $\mathrm{~kg} / \mathrm{yr}$ \\
\hline Unburned hydrocarbons & 1362 & $\mathrm{~kg} / \mathrm{yr}$ \\
\hline Particulate matter & 340 & $\mathrm{~kg} / \mathrm{yr}$ \\
\hline Sulfur dioxide & 5839 & $\mathrm{~kg} / \mathrm{yr}$ \\
\hline Nitrogen oxides & 11887 & $\mathrm{~kg} / \mathrm{yr}$ \\
\hline
\end{tabular}

HOMER Energy, LLC @ 2015 


\section{System Report}

\section{System architecture}

\begin{tabular}{|l|l|r|}
\hline PV & Generic flat plate PV & $250 \mathrm{~kW}$ \\
\hline Wind Turbine & Northern Power NPS100C-21 & 7 \\
\hline Generator & Generic1150kW Prime Power & $1,150 \mathrm{~kW}$ \\
\hline Converter & System Converter & $500 \mathrm{~kW}$ \\
\hline Dispatch Strategy & Cycle Charging & \\
\hline
\end{tabular}

\section{Cost summary}

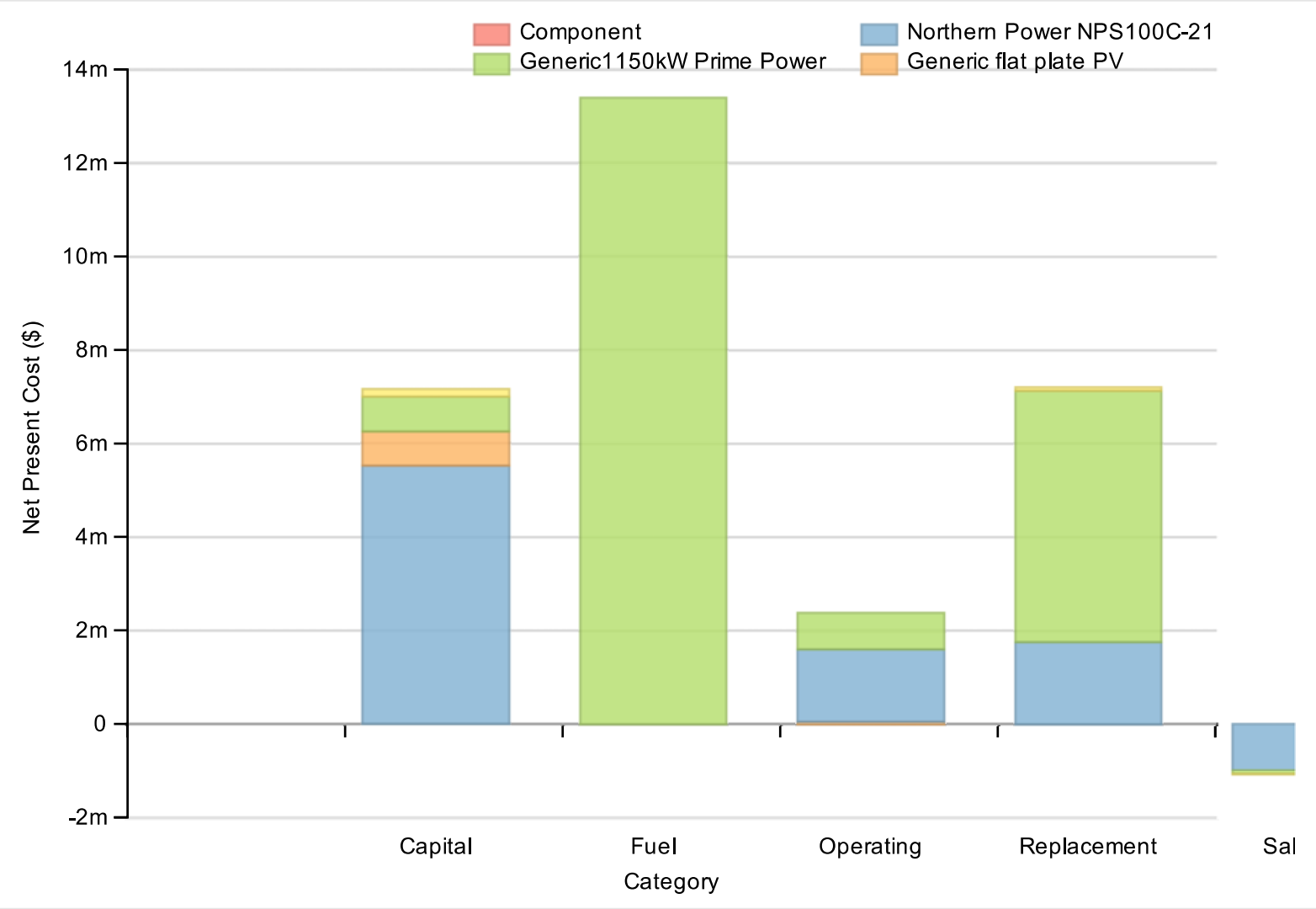

Cost Summary

Total net present cost

Levelized cost of energy
$29058882 \$$

$0.432 \$ / k W h$ 


\section{Net Present Costs}

\begin{tabular}{|l|r|r|r|r|r|r|}
\hline Component & Capital & Replacement & O\&M & Fuel & Salvage & Total \\
\hline $\begin{array}{l}\text { Generic flat plate } \\
\text { PV }\end{array}$ & 750,000 & 0 & 64,638 & 0 & 0 & 814,638 \\
\hline $\begin{array}{l}\text { Northern Power } \\
\text { NPS100C-21 }\end{array}$ & $5,530,000$ & $1,763,003$ & $1,551,301$ & 0 & $-993,565$ & $7,850,739$ \\
\hline $\begin{array}{l}\text { Generic1150kW } \\
\text { Prime Power }\end{array}$ & 741,936 & $5,379,269$ & 755,407 & $13,388,983$ & $-73,761$ & $20,191,834$ \\
\hline Converter & 150,000 & 63,641 & 0 & 0 & $-11,978$ & 201,663 \\
\hline System & $7,171,936$ & $7,205,913$ & $2,371,346$ & $13,388,983$ & $-1,079,304$ & $29,058,874$ \\
\hline
\end{tabular}

\section{Annualized Costs}

\begin{tabular}{|l|r|r|r|r|r|r|}
\hline Component & Capital & Replacement & O\&M & Fuel & Salvage & Total \\
\hline Generic flat plate PV & 58,016 & 0 & 5,000 & 0 & 0 & 63,016 \\
\hline $\begin{array}{l}\text { Northern Power } \\
\text { NPS100C-21 }\end{array}$ & 427,770 & 136,376 & 120,000 & 0 & $-76,857$ & 607,289 \\
\hline $\begin{array}{l}\text { Generic1150kW Prime } \\
\text { Power }\end{array}$ & 57,392 & 416,110 & 58,434 & $1,035,696$ & $-5,706$ & $1,561,926$ \\
\hline Converter & 11,603 & 4,923 & 0 & 0 & -927 & 15,599 \\
\hline System & 554,781 & 557,409 & 183,434 & $1,035,696$ & $-83,489$ & $2,247,831$ \\
\hline
\end{tabular}




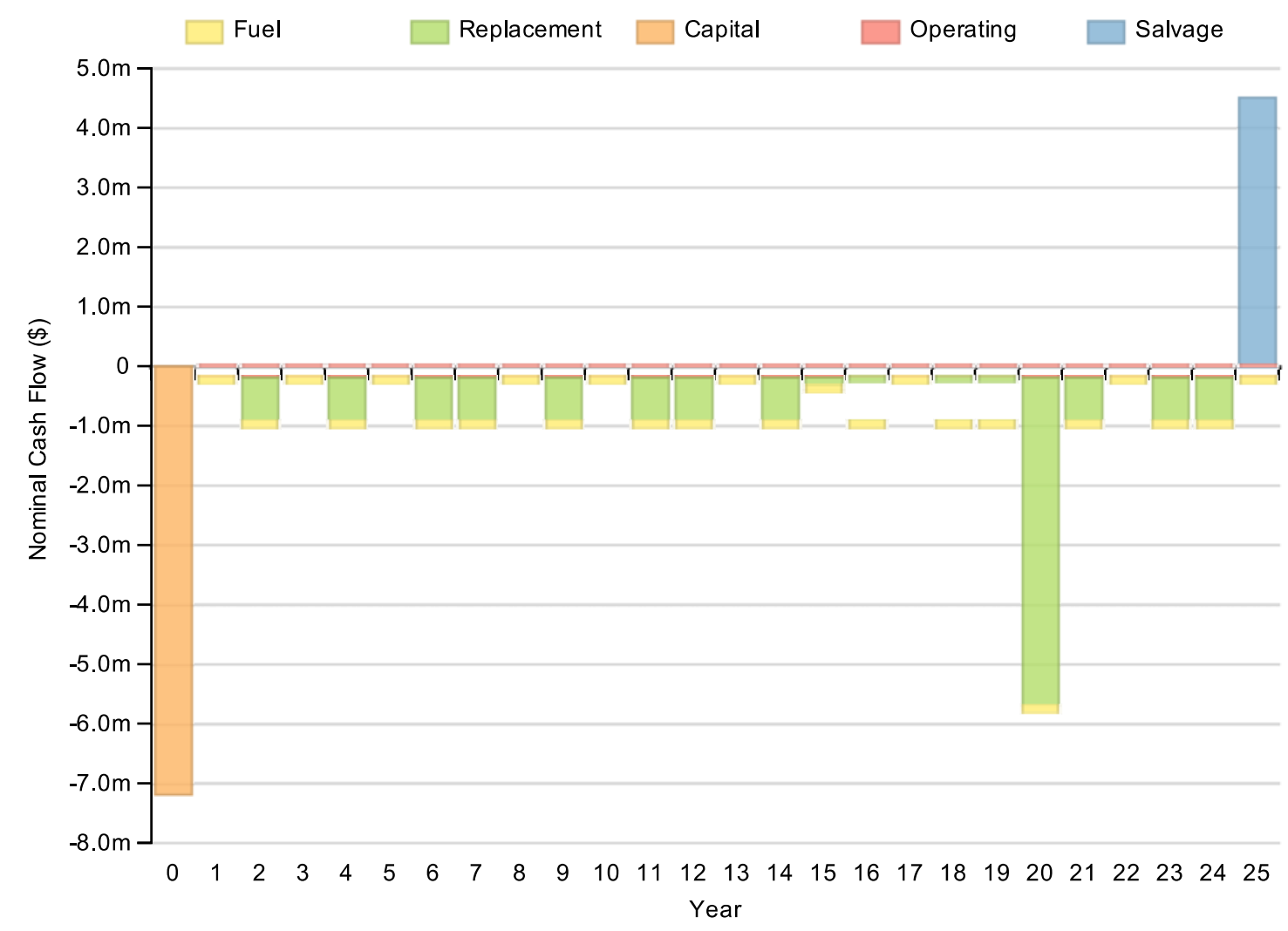

\section{Electrical}

\section{Quantity}

Excess electricity

Unmet load

Capacity shortage

Renewable fraction

\section{Component}

PV

Generator

Wind Turbine

Total

\section{Production(kWh/yr)}

Value

(n)

\begin{tabular}{r|l|l|}
735566 & $\mathrm{kWh} / \mathrm{yr}$ \\
\hline 0 & $\mathrm{kWh} / \mathrm{yr}$ \\
\hline 0 & $\mathrm{kWh} / \mathrm{yr}$ \\
\hline 0 & \\
\hline
\end{tabular}


AC primary load

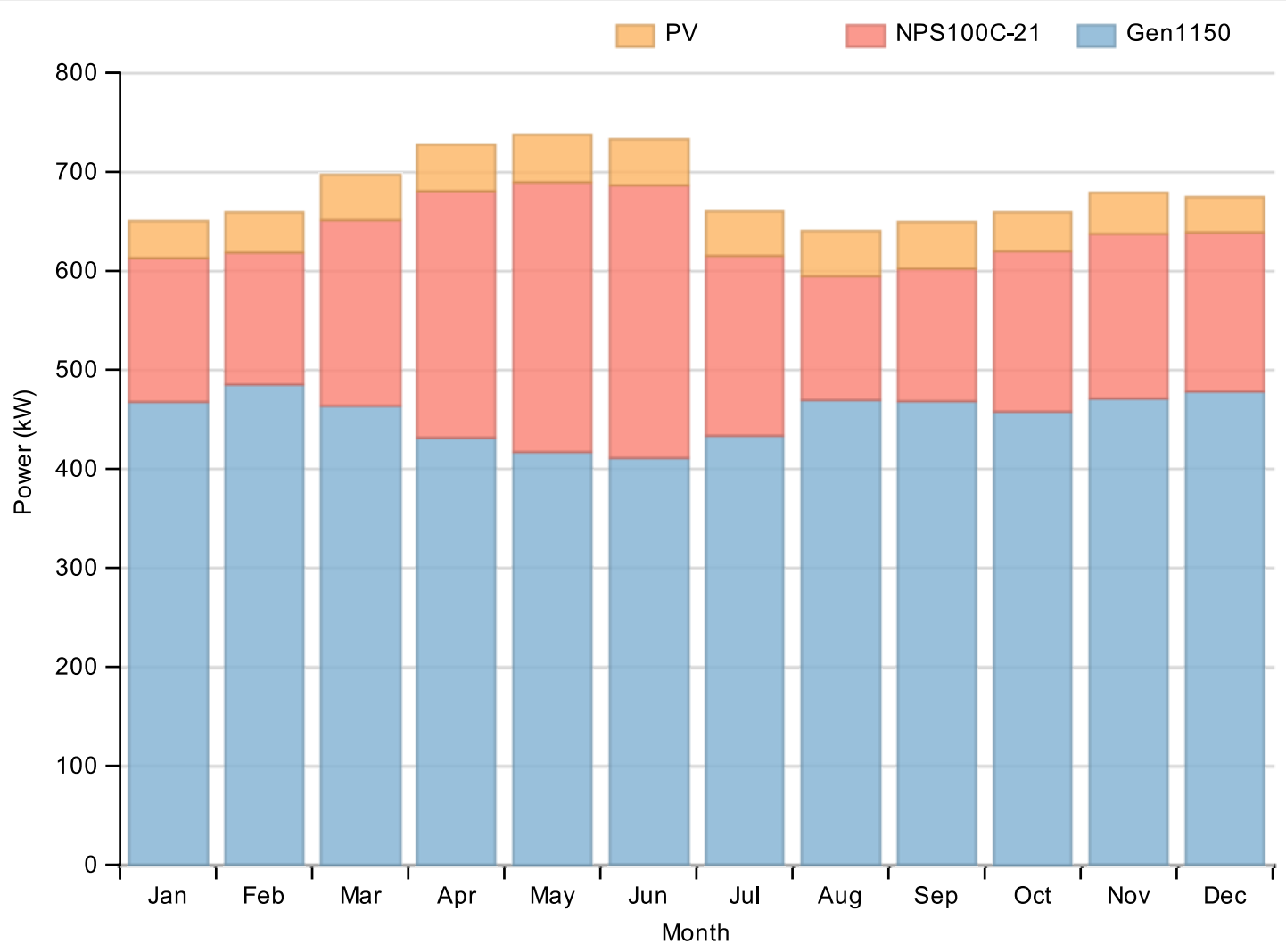

\section{PV:Generic flat plate PV}

\begin{tabular}{|l|r|l|}
\hline Quantity & Value & Units \\
\hline Rated capacity & 250 & $\mathrm{~kW}$ \\
\hline Mean output & 43 & $\mathrm{~kW}$ \\
\hline Mean output & 1027.50 & $\mathrm{kWh} / \mathrm{d}$ \\
\hline Capacity factor & 17.13 & $\%$ \\
\hline Total production & 375052 & $\mathrm{kWh} / \mathrm{yr}$ \\
\hline Minimum output & 0.00 & $\mathrm{~kW}$ \\
\hline Maximum output & 252.99 & $\mathrm{~kW}$ \\
\hline
\end{tabular}




\section{PV penetration}

Hours of operation

Levelized cost

\begin{tabular}{|l|l|}
\hline 7.20 & $\%$ \\
\hline 4386 & $\mathrm{hrs} / \mathrm{yr}$ \\
\hline 0.168 & $\$ / \mathrm{kWh}$ \\
\hline
\end{tabular}

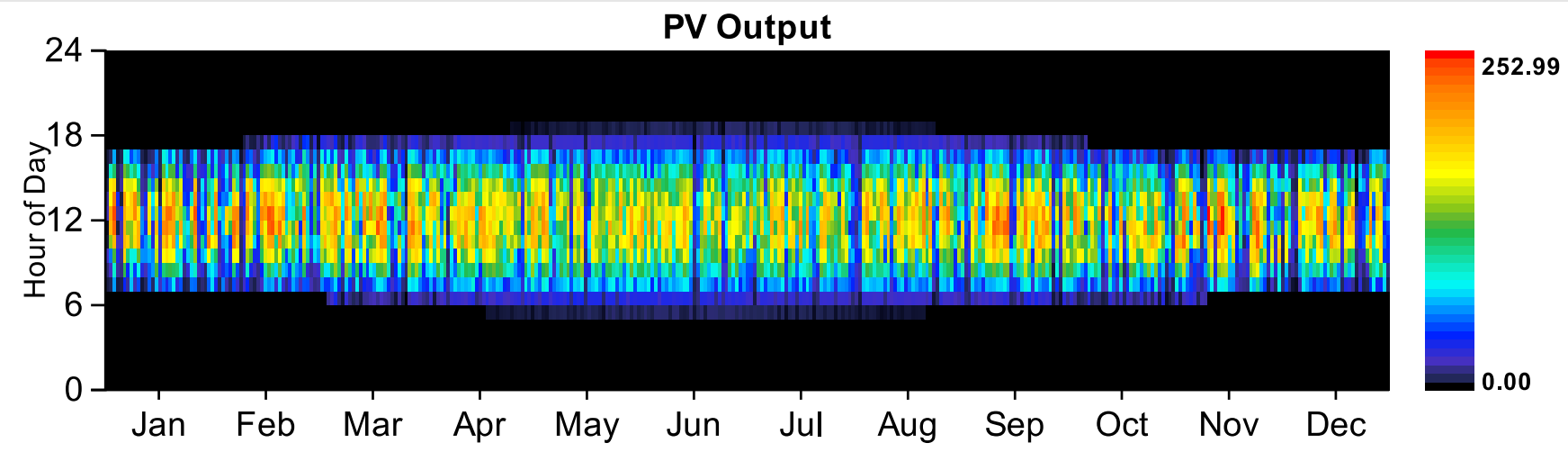

Wind Turbine:Northern Power NPS100C-21

\begin{tabular}{|l|r|l|}
\hline Quantity & Value & Units \\
\hline Total rated capacity & 700 & $\mathrm{~kW}$ \\
\hline Mean output & 183 & $\mathrm{~kW}$ \\
\hline Capacity factor & 26.12 & $\%$ \\
\hline Total production & 1601808 & $\mathrm{kWh} / \mathrm{yr}$ \\
\hline Minimum output & 0.00 & $\mathrm{~kW}$ \\
\hline Maximum output & 686.75 & $\mathrm{~kW}$ \\
\hline Wind penetration & $30.77 \%$ & $\%$ \\
\hline Hours of operation & 8108 & $\mathrm{hrs} / \mathrm{yr}$ \\
\hline Levelized cost & 0.379 & $\$ / \mathrm{kWh}$ \\
\hline
\end{tabular}




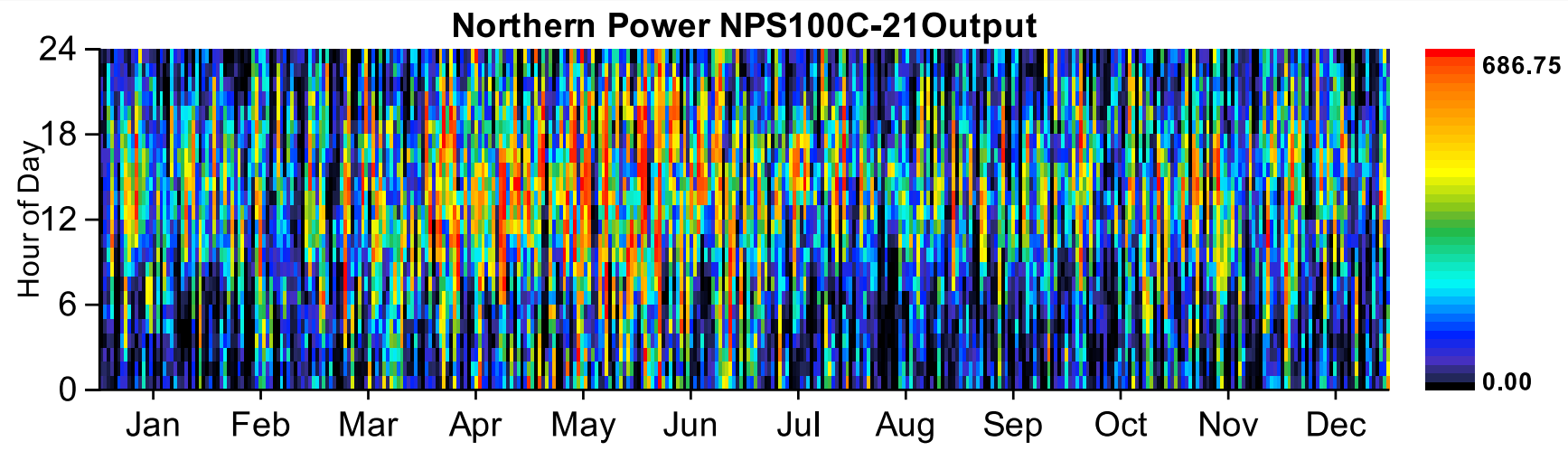

Generator:Generic1150kW Prime Power

\begin{tabular}{|l|r|l|}
\hline Quantity & Value & Units \\
\hline Hours of operation & 8751 & $\mathrm{hrs} / \mathrm{yr}$ \\
\hline Number of starts & 10 & starts/yr \\
\hline Operational life & 2 & $\mathrm{yr}$ \\
\hline Fixed generation cost & 56.14 & $\$ / \mathrm{hr}$ \\
\hline Marginal generation cost & 0.26 & $\$ / \mathrm{kWh}$ \\
\hline Electrical production & 3983576 & $\mathrm{kWh} / \mathrm{yr}$ \\
\hline Mean electrical output & 455 & $\mathrm{~kW}$ \\
\hline Min. electrical output & 345 & $\mathrm{~kW}$ \\
\hline Max. electrical output & 979 & $\mathrm{~kW}$ \\
\hline Fuel consumption & 1035697 & $\mathrm{~L} / \mathrm{yr}$ \\
\hline Specific fuel consumption & 0.26 & $\mathrm{~L} / \mathrm{kWh}$ \\
\hline Fuel energy input & 10191258 & $\mathrm{kWh} / \mathrm{yr}$ \\
\hline Mean electrical efficiency & $39 \%$ & $\%$ \\
\hline
\end{tabular}




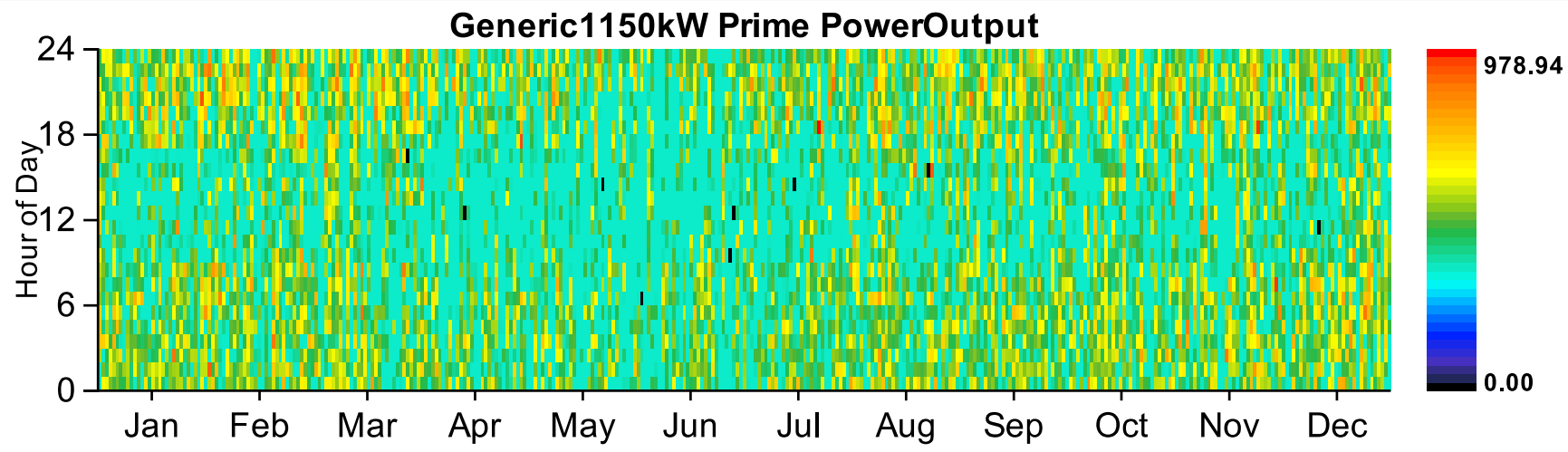

Converter

\begin{tabular}{|l|r|r|l|}
\hline Quantity & Inverter & Rectifier & Units \\
\hline Capacity & 500 & 450 & $\mathrm{~kW}$ \\
\hline Mean output & 19 & $0 \mathrm{~kW}$ \\
\hline Minimum output & 0 & $0 \mathrm{~kW}$ \\
\hline Maximum output & 217 & $0 \mathrm{~kW}$ \\
\hline Capacity factor & 4 & 0 & $\%$ \\
\hline Hours of operation & 2,661 & 0 & $\mathrm{hrs} / \mathrm{yr}$ \\
\hline Energy in & 189,274 & 0 & $\mathrm{kWh} / \mathrm{yr}$ \\
\hline Energy out & 170,347 & 0 & $\mathrm{kWh} / \mathrm{yr}$ \\
\hline Losses & 18,927 & 0 & $\mathrm{kWh} / \mathrm{yr}$ \\
\hline
\end{tabular}



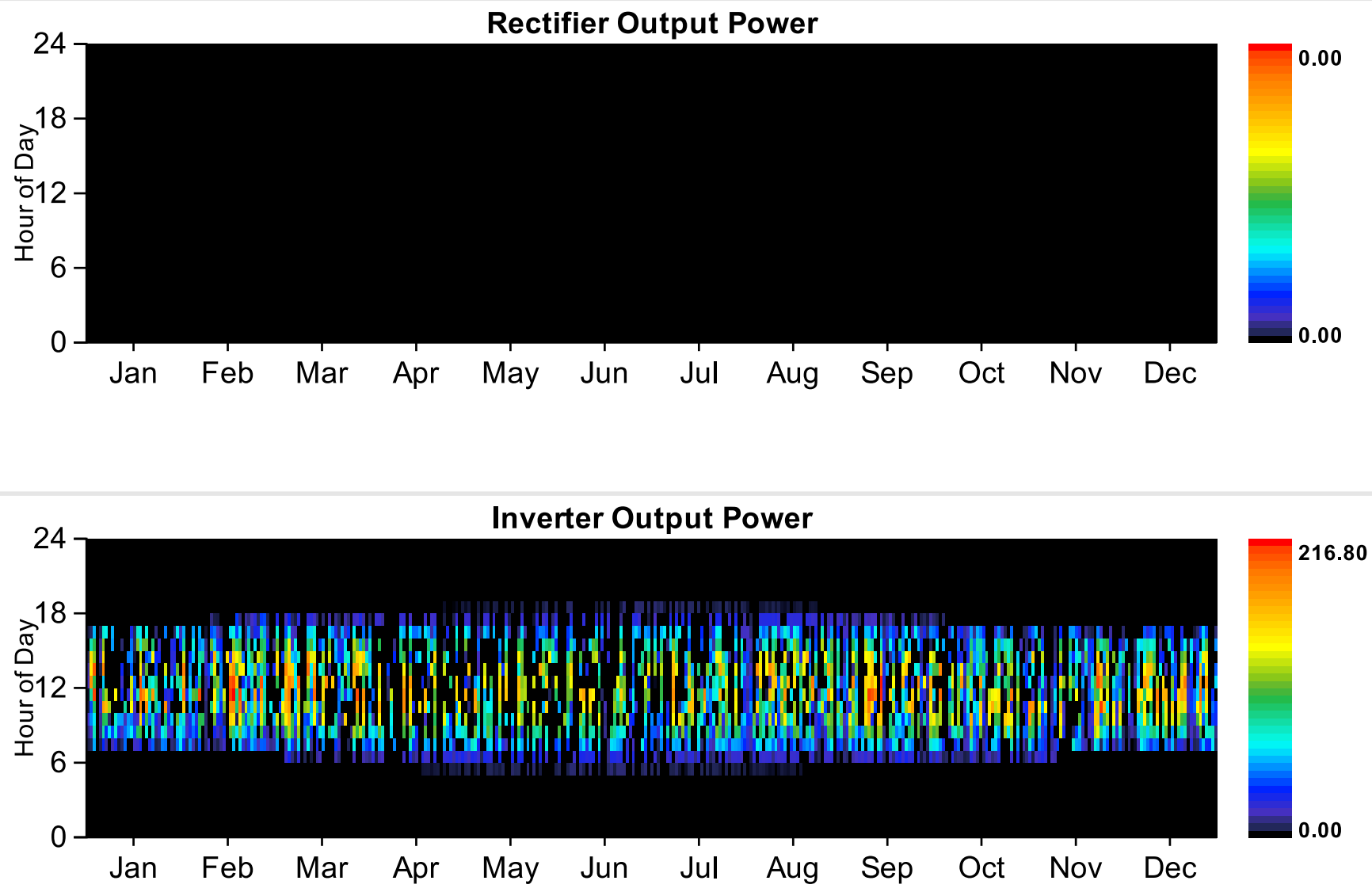

\section{Emissions}

\begin{tabular}{|l|r|l|}
\hline Pollutant & Emissions & Units \\
\hline Carbon dioxide & 2718202 & $\mathrm{~kg} / \mathrm{yr}$ \\
\hline Carbon monoxide & 11393 & $\mathrm{~kg} / \mathrm{yr}$ \\
\hline Unburned hydrocarbons & 1305 & $\mathrm{~kg} / \mathrm{yr}$ \\
\hline Particulate matter & 326 & $\mathrm{~kg} / \mathrm{yr}$ \\
\hline Sulfur dioxide & 5597 & $\mathrm{~kg} / \mathrm{yr}$ \\
\hline Nitrogen oxides & $11393 \mathrm{~kg} / \mathrm{yr}$ \\
\hline
\end{tabular}

HOMER Energy, LLC @ 2015 


\section{Appendix B - China Lake Scenarios}

System Report

System architecture

\begin{tabular}{|l|l|r|}
\hline PV & SunPower PV & $13,780 \mathrm{~kW}$ \\
\hline PV \#2 & Remaining Generic flat plate PV & $1,547 \mathrm{~kW}$ \\
\hline Comverter & System Corverter & $10,000 \mathrm{~kW}$ \\
\hline Grid & Grid & $27,200 \mathrm{~kW}$ \\
\hline Dispatch Stralegy & Cyde Charging & \\
\hline
\end{tabular}

Cost summary

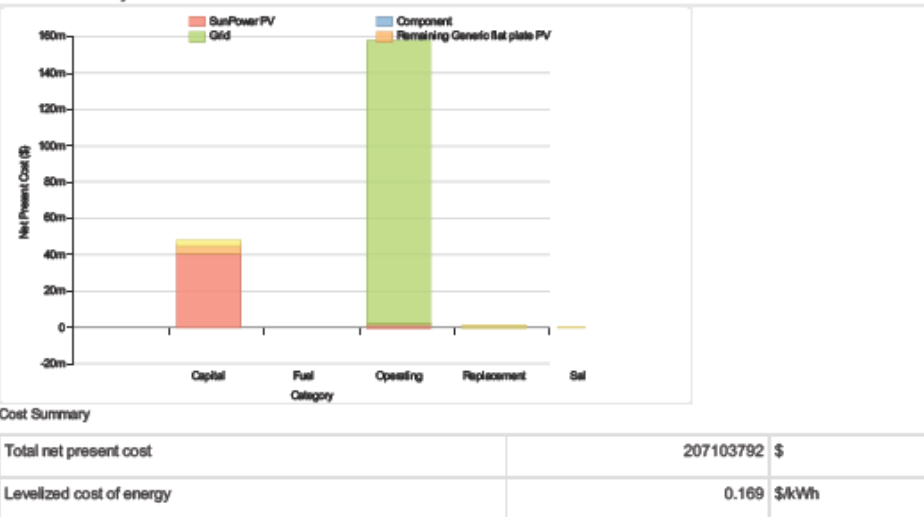

Net Present Costs

\begin{tabular}{|l|r|r|r|r|r|r|}
\hline Component & Capital & Replacement & \multicolumn{1}{l|}{ O\&M } & Fuel & Salvage & \multicolumn{1}{l}{ Total } \\
\hline SunPower PV & $40,358,332$ & 0 & $1,656,876$ & 0 & 0 & $42,015,206$ \\
\hline Remaining Generic flat plate PV & $4,678,459$ & 0 & 339,013 & 0 & 0 & $5,017,472$ \\
\hline Grid & 0 & 0 & $155,698,736$ & 0 & 0 & $155,698,736$ \\
\hline Comverter & $3,000,000$ & $1,272,821$ & 0 & 0 & $-239,558$ & $4,033,263$ \\
\hline System & $48,036,792$ & $1,272,821$ & $157,694,624$ & 0 & $-239,558$ & $206,764,679$ \\
\hline
\end{tabular}

Annualized Costs

\begin{tabular}{|l|r|r|r|r|r|r|}
\hline Component & Capital & Replacement & \multicolumn{1}{l|}{ O\&M } & Fuel Salvage & \multicolumn{2}{l|}{ Total } \\
\hline SunPower PV & $3,121,894$ & 0 & 128,167 & 0 & 0 & $3,250,061$ \\
\hline Remaining Generic flat plate PV & 361,899 & 0 & 26,224 & 0 & 0 & 388,123 \\
\hline Grid & 0 & 0 & $12,043,979$ & 0 & 0 & $12,043,979$ \\
\hline
\end{tabular}


System Report

System architecture

\begin{tabular}{|l|l|r|}
\hline PV & SunPower PV & 13,780 \\
\hline PV \#2 & Remaining Generic flat plate PV & 1,547 \\
\hline Converter & System Converter & 10,000 \\
\hline Grid & Grid & $\mathrm{kW}$ \\
\hline Dispatch Strategy & Cycle Charging & $27,200 \mathrm{~kW}$ \\
\hline
\end{tabular}

\section{Cost summary}

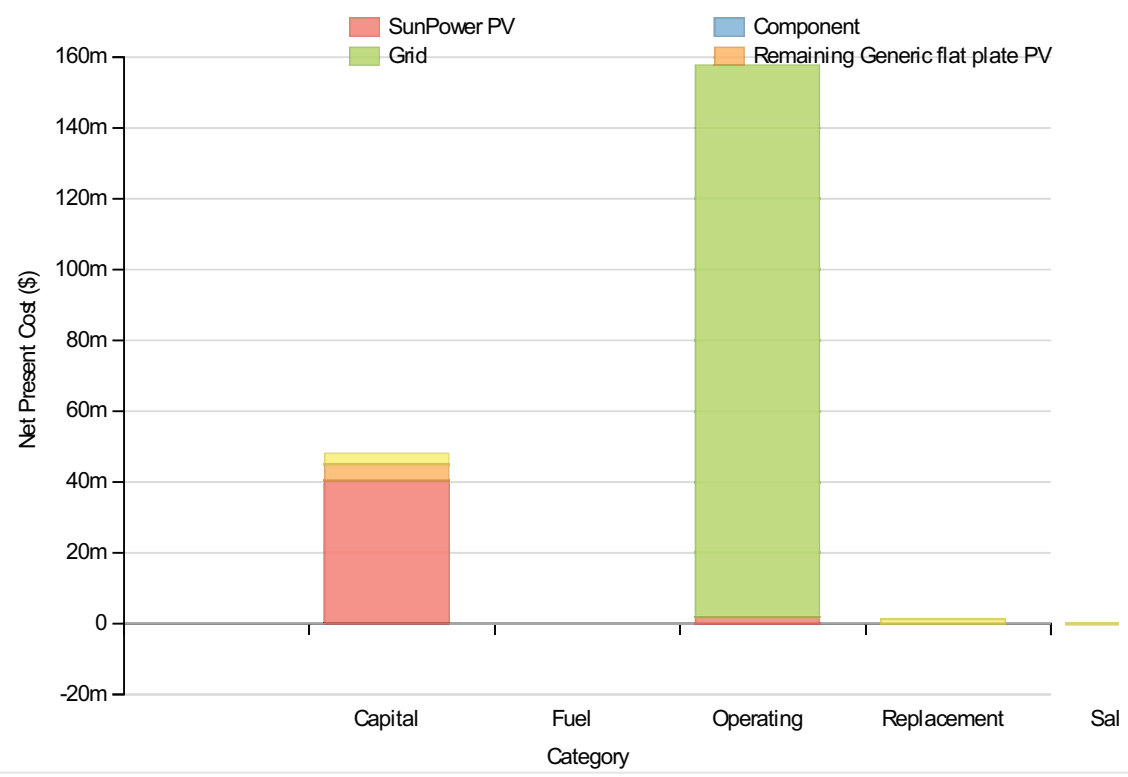

Cost Summary

Total net present cost

$207103792 \$$

Levelized cost of energy

$0.169 \$ / k W h$

Net Present Costs

\begin{tabular}{|c|c|c|c|c|c|c|}
\hline Component & Capital & Replacement & O\&M & Fuel & Salvage & Total \\
\hline SunPower PV & $40,358,332$ & 0 & $1,656,876$ & 0 & 0 & $42,015,208$ \\
\hline Remaining Generic flat plate PV & $4,678,459$ & 0 & 339,013 & 0 & 0 & $5,017,472$ \\
\hline Grid & 0 & 0 & $155,698,736$ & 0 & 0 & $155,698,736$ \\
\hline Converter & $3,000,000$ & $1,272,821$ & 0 & 0 & $-239,558$ & $4,033,263$ \\
\hline System & $48,036,792$ & $1,272,821$ & $157,694,624$ & 0 & $-239,558$ & $206,764,679$ \\
\hline
\end{tabular}

\section{Annualized Costs}

\begin{tabular}{|l|r|r|r|r|r|r|}
\hline Component & Capital & Replacement & O\&M & Fuel & Salvage & Total \\
\hline SunPower PV & $3,121,894$ & 0 & 128,167 & 0 & 0 \\
\hline Remaining Generic flat plate PV & 361,899 & 0 & 26,224 & 0 & 0 \\
\hline Grid & 0 & 0 & $12,043,979$ & 0 & 0 \\
\hline
\end{tabular}




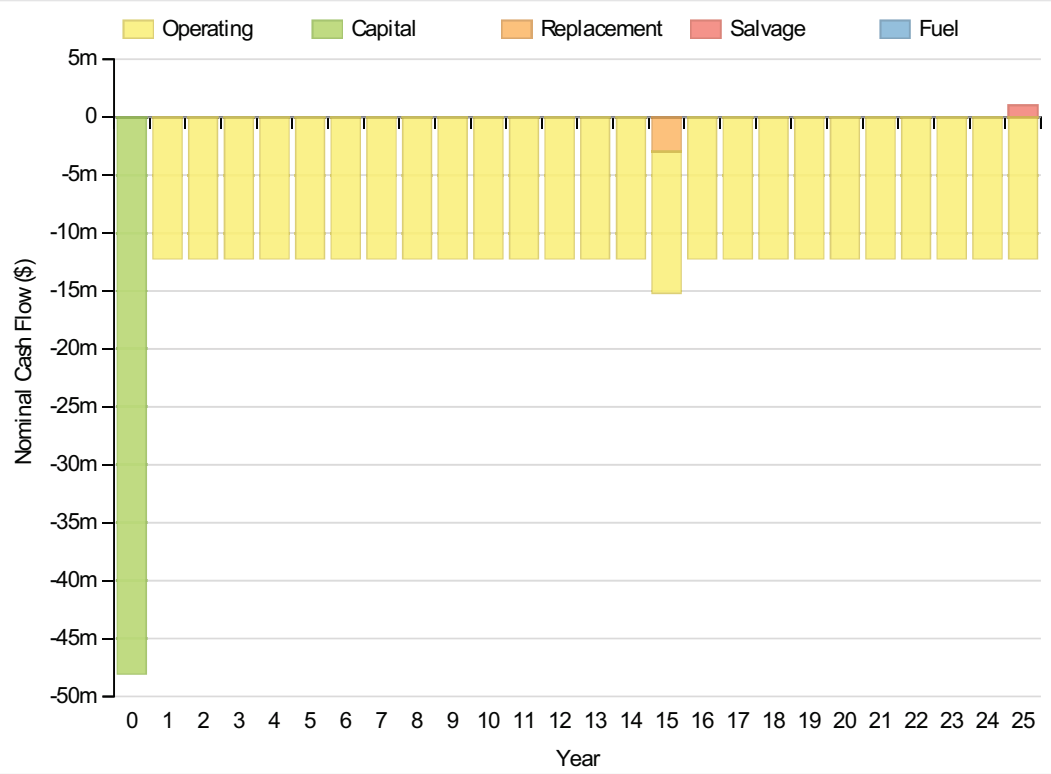

\section{Electrical}

\begin{tabular}{|c|c|c|c|c|}
\hline Quantity & & Value & & Units \\
\hline Excess electricity & & & 613916 & $\mathrm{kWh} / \mathrm{yr}$ \\
\hline Unmet load & & & 0 & $\mathrm{kWh} / \mathrm{yr}$ \\
\hline Capacity shortage & & & 0 & $\mathrm{kWh} / \mathrm{yr}$ \\
\hline Renewable fraction & & & 0 & \\
\hline Component & Production(kWh/yr) & & Fractio & n (\%) \\
\hline PV & & $24,644,568$ & & 25 \\
\hline PV & & $2,766,525$ & & 3 \\
\hline Grid Purchases & & $71,835,160$ & & 72 \\
\hline Total & & $99,246,256$ & & 100 \\
\hline Load & Consumption(kWh/yr) & & Fract & tion (\%) \\
\hline AC primary load & & $95,052,456$ & & 100 \\
\hline DC primary load & & & 0 & c \\
\hline Total & & $95,052,456$ & & 100 \\
\hline
\end{tabular}




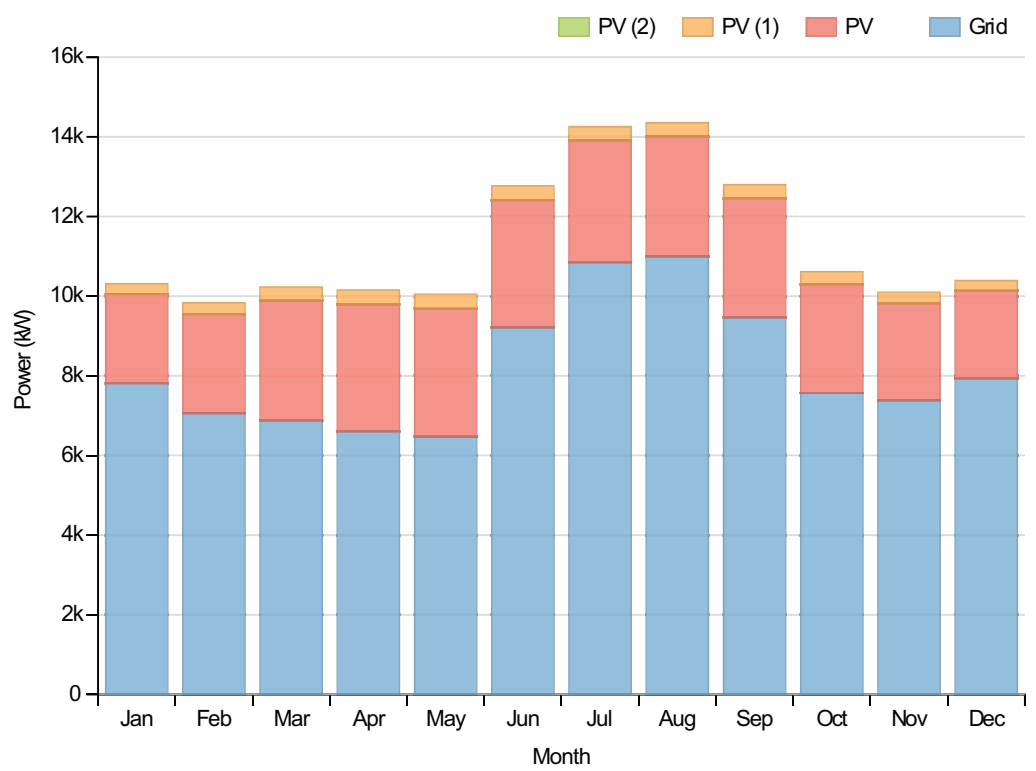

\section{PV:SunPower PV}

\begin{tabular}{|l|c|l|}
\hline Quantity & Value & Units \\
\hline Rated capacity & $13780 \mathrm{~kW}$ \\
\hline Mean output & $2813 \mathrm{~kW}$ \\
\hline Mean output & $67519.00 \mathrm{kWh} / \mathrm{d}$ \\
\hline Capacity factor & $20.42 \%$ \\
\hline Total production & $24644568 \mathrm{kWh} / \mathrm{yr}$ \\
\hline Minimum output & $0.00 \mathrm{~kW}$ \\
\hline Maximum output & $13941.00 \mathrm{~kW}$ \\
\hline PV penetration & $25.93 \%$ \\
\hline Hours of operation & $\%$ & 4386 \\
\hline Levelized cost & $\mathrm{hrs} / \mathrm{yr}$ \\
\hline
\end{tabular}

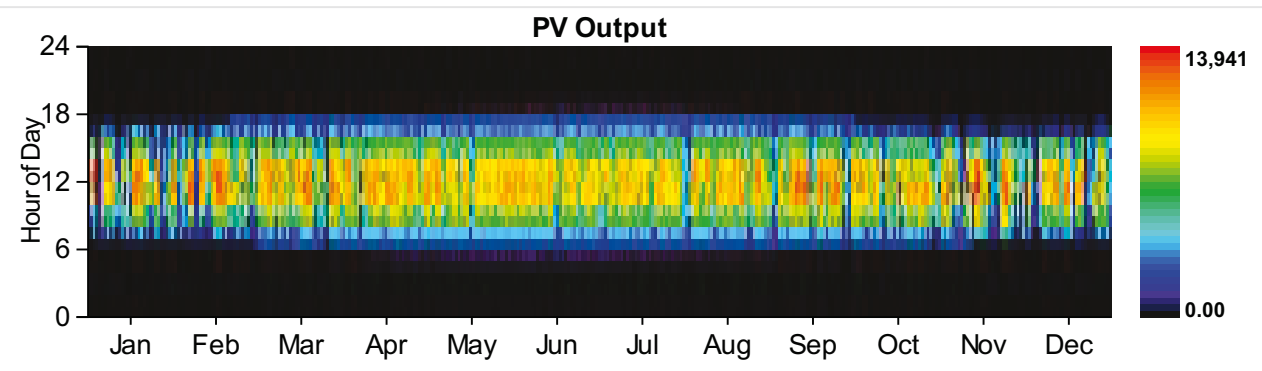

\section{PV:Remaining Generic flat plate PV}

\begin{tabular}{|l|c|c|}
\hline Quantity & Value & Units \\
\hline Rated capacity & $1547 \mathrm{~kW}$ \\
\hline Mean output & $316 \mathrm{~kW}$ \\
\hline Mean output & $7579.50 \mathrm{kWh} / \mathrm{d}$ \\
\hline Capacity factor & $20.42 \%$ \\
\hline
\end{tabular}


§etahtripduction

Minimum output

Maximum output

PV penetration

Hours of operation

Levelized cost

\section{5 kifites}

$0.00 \mathrm{~kW}$

1565.00 kW

$2.91 \%$

4386 hrs/yr

$0.014 \$ / k W h$

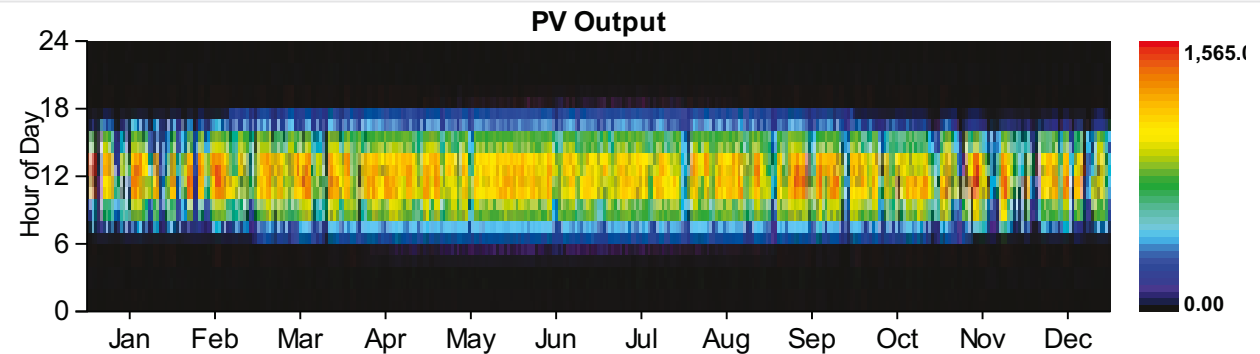

Converter

\begin{tabular}{|c|c|c|c|}
\hline Quantity & Inverter & Rectifier & Units \\
\hline Capacity & 10,000 & 9,000 & $\mathrm{~kW}$ \\
\hline Mean output & 2,650 & 0 & $\mathrm{~kW}$ \\
\hline Minimum output & 0 & 0 & $\mathrm{~kW}$ \\
\hline Maximum output & 10,000 & 0 & $\mathrm{~kW}$ \\
\hline Capacity factor & 27 & 0 & $\%$ \\
\hline Hours of operation & 4,386 & 0 & hrs/yr \\
\hline Energy in & $25,797,274$ & 0 & $\mathrm{kWh} / \mathrm{yr}$ \\
\hline Energy out & $23,217,452$ & 0 & $\mathrm{kWh} / \mathrm{yr}$ \\
\hline Losses & $2,579,822$ & 0 & $\mathrm{kWh} / \mathrm{yr}$ \\
\hline
\end{tabular}

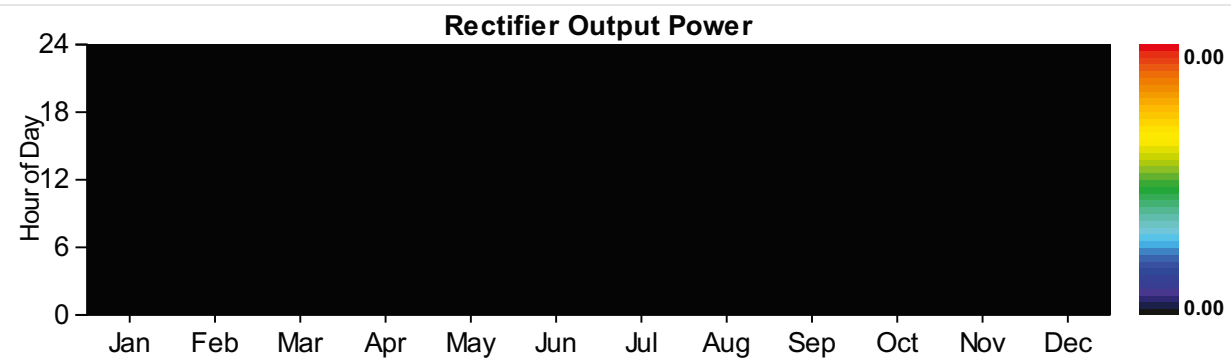




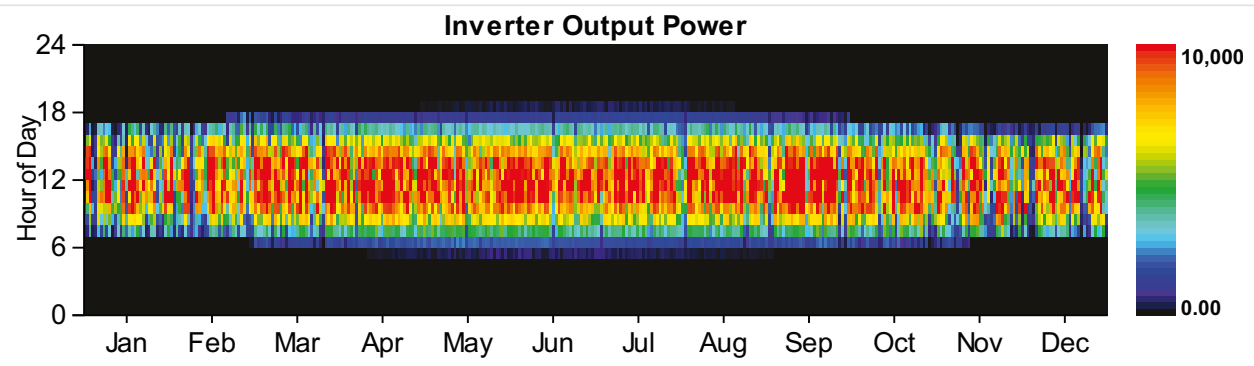

Grid

Rate: Rate 1 Demand

\begin{tabular}{|c|c|c|c|c|c|c|}
\hline Resources.ReportingService_GeneratelnputsReport_Month & $\begin{array}{l}\text { Energy } \\
\text { Purchased } \\
\text { (kWh) }\end{array}$ & $\begin{array}{l}\text { Energy } \\
\text { Sold } \\
\text { (kWh) }\end{array}$ & $\begin{array}{l}\text { Net } \\
\text { Purchases } \\
\text { (kWh) }\end{array}$ & $\begin{array}{l}\text { Peak } \\
\text { Demand } \\
(\mathbf{k W})\end{array}$ & $\begin{array}{l}\text { Energy } \\
\text { Charge } \\
\text { (\$) }\end{array}$ & $\begin{array}{l}\text { Demand } \\
\text { Charge } \\
(\$)\end{array}$ \\
\hline January & 0 & 0 & 0 & 17,079 & 0 & 273,262 \\
\hline February & 0 & 0 & 0 & 15,668 & 0 & 250,685 \\
\hline March & 0 & 0 & 0 & 17,075 & 0 & 273,199 \\
\hline April & 0 & 0 & 0 & 14,394 & 0 & 230,299 \\
\hline May & 0 & 0 & 0 & 14,850 & 0 & 237,602 \\
\hline June & 0 & 0 & 0 & 20,019 & 0 & 320,305 \\
\hline July & 0 & 0 & 0 & 24,169 & 0 & 386,703 \\
\hline August & 0 & 0 & 0 & 22,951 & 0 & 367,211 \\
\hline September & 0 & 0 & 0 & 18,833 & 0 & 301,321 \\
\hline October & 0 & 0 & 0 & 16,050 & 0 & 256,794 \\
\hline November & 0 & 0 & 0 & 15,751 & 0 & 252,018 \\
\hline December & 0 & 0 & 0 & 17,148 & 0 & 274,366 \\
\hline Annual & 0 & 0 & 0 & 24,169 & 0 & $3,423,765$ \\
\hline
\end{tabular}

Rate: Rate 1

\begin{tabular}{|c|c|c|c|c|c|c|}
\hline Resources.ReportingService_GeneratelnputsReport_Month & $\begin{array}{l}\text { Energy } \\
\text { Purchased } \\
\text { (kWh) }\end{array}$ & $\begin{array}{l}\text { Energy } \\
\text { Sold } \\
\text { (kWh) }\end{array}$ & $\begin{array}{l}\text { Net } \\
\text { Purchases } \\
\text { (kWh) }\end{array}$ & $\begin{array}{l}\text { Peak } \\
\text { Demand } \\
\text { (kW) }\end{array}$ & $\begin{array}{l}\text { Energy } \\
\text { Charge } \\
(\$)\end{array}$ & $\begin{array}{l}\text { Demand } \\
\text { Charge } \\
(\$)\end{array}$ \\
\hline January & $5,808,431$ & 0 & $5,808,431$ & 0 & 697,012 & 0 \\
\hline February & $4,748,350$ & 0 & $4,748,350$ & 0 & 569,802 & 0 \\
\hline March & $5,124,164$ & 0 & $5,124,164$ & 0 & 614,900 & 0 \\
\hline April & $4,760,968$ & 0 & $4,760,968$ & 0 & 571,316 & 0 \\
\hline May & $4,811,302$ & 0 & $4,811,302$ & 0 & 577,356 & 0 \\
\hline June & $6,639,290$ & 0 & $6,639,290$ & 0 & 796,715 & 0 \\
\hline July & $8,073,620$ & 0 & $8,073,620$ & 0 & 968,834 & 0 \\
\hline August & $8,188,394$ & 0 & $8,188,394$ & 0 & 982,607 & 0 \\
\hline
\end{tabular}




\begin{tabular}{|c|c|c|c|c|c|c|}
\hline Rectoberrces.ReportingService_GeneratelnputsReport_Month & $\begin{array}{l}\text { EÂergy } 598 \\
\text { Purchased } \\
\text { (kWh31,799 }\end{array}$ & $\begin{array}{l}\text { Energy } \\
\text { Sold } \\
\text { (kWh) } 0\end{array}$ & $\begin{array}{l}\text { Nêt819,598 } \\
\text { Purchases } \\
\text { (kWhi) }\end{array}$ & $\begin{array}{l}\text { Peak } 0 \\
\text { Demand } \\
(\mathbf{k W}) \quad 0\end{array}$ & $\begin{array}{l}\text { ERefg } 352 \\
\text { Charge } \\
(\$)^{75,816}\end{array}$ & $\begin{array}{l}\text { Demand } \\
\text { Charge } \\
(\$)\end{array}$ \\
\hline November & $5,318,139$ & 0 & $5,318,139$ & 0 & 638,177 & 0 \\
\hline December & $5,911,110$ & 0 & $5,911,110$ & 0 & 709,333 & 0 \\
\hline Annual & $71,835,160$ & 0 & $71,835,160$ & 0 & $8,620,220$ & 0 \\
\hline
\end{tabular}

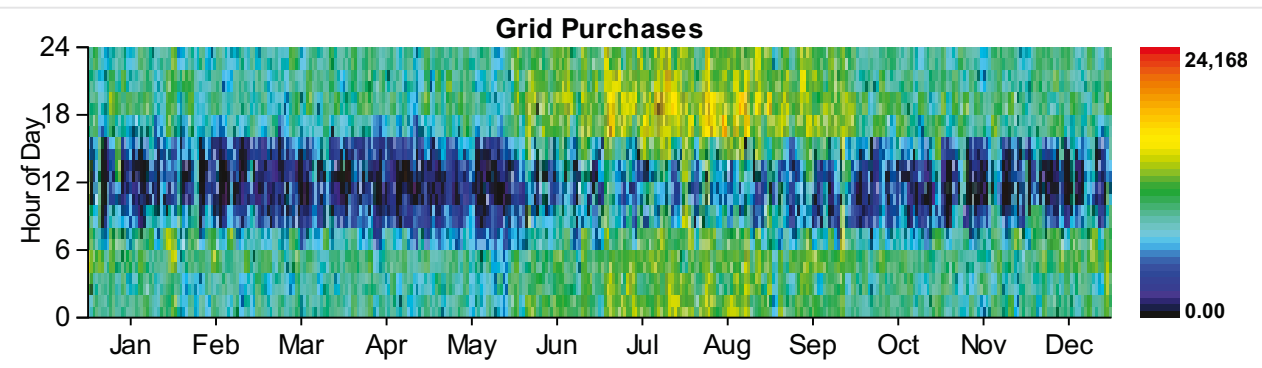

\section{Emissions}

\begin{tabular}{|l|r|l|}
\hline Pollutant & Emissions & Units \\
\hline Carbon dioxide & 45399820 & $\mathrm{~kg} / \mathrm{yr}$ \\
\hline Carbon monoxide & 0 & $\mathrm{~kg} / \mathrm{yr}$ \\
\hline Unburned hydrocarbons & 0 & $\mathrm{~kg} / \mathrm{yr}$ \\
\hline Particulate matter & 0 & $\mathrm{~kg} / \mathrm{yr}$ \\
\hline Sulfur dioxide & $196828 \mathrm{~kg} / \mathrm{yr}$ \\
\hline Nitrogen oxides & $96259 \mathrm{~kg} / \mathrm{yr}$ \\
\hline
\end{tabular}

HOMER Energy, LLC @ 2016 
System Report

System architecture

\begin{tabular}{|l|l|r|}
\hline PV & SunPower PV & 13,780 \\
\hline PV \#2 & Remaining Generic flat plate PV & 1,547 \\
\hline Battery & GS200 flow & 1 \\
\hline Converter & System Converter & $10,000 \mathrm{~kW}$ \\
\hline Grid & Grid & 27,200 \\
\hline Dispatch Strategy & Cycle Charging & $\mathrm{kW}$ \\
\hline
\end{tabular}

Cost summary

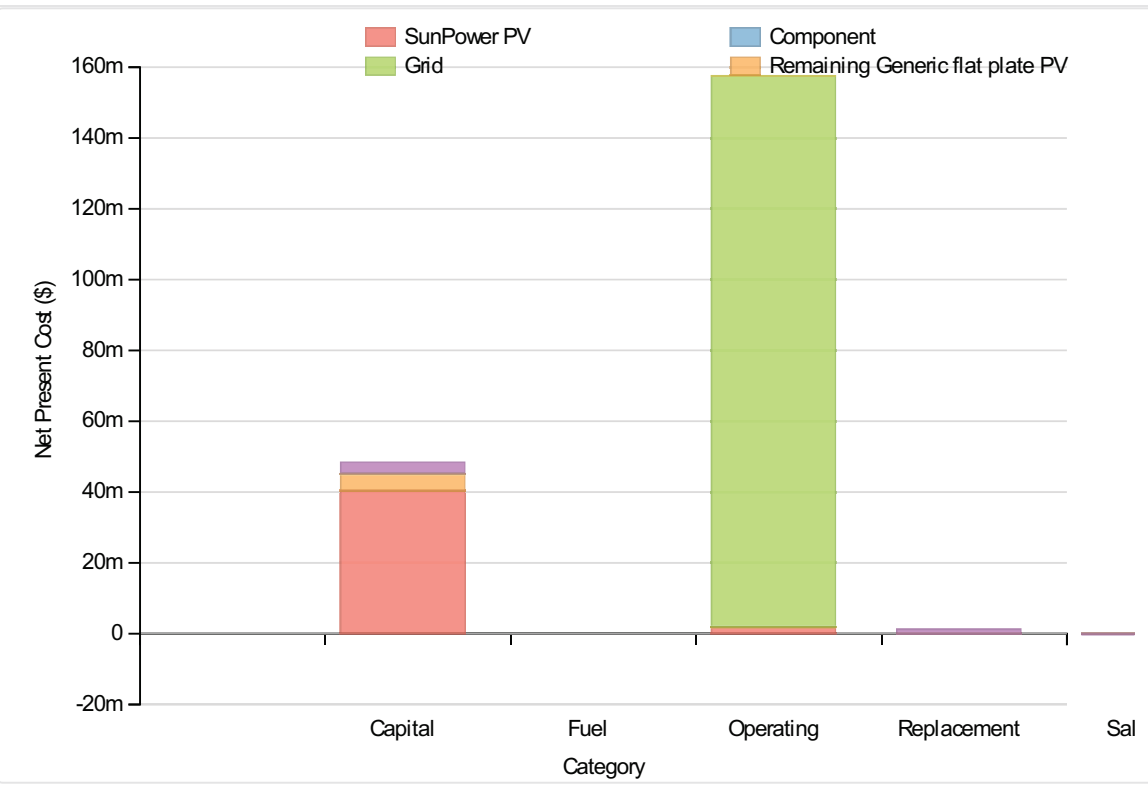

Cost Summary

Total net present cost

$207291696 \$$

Levelized cost of energy

$0.169 \$ / \mathrm{kWh}$

\section{Net Present Costs}

\begin{tabular}{|c|c|c|c|c|c|c|}
\hline Component & Capital & Replacement & O\&M & Fuel & Salvage & Total \\
\hline SunPower PV & $40,358,332$ & 0 & $1,656,876$ & 0 & 0 & $42,015,208$ \\
\hline Remaining Generic flat plate PV & $4,678,459$ & 0 & 339,013 & 0 & 0 & $5,017,472$ \\
\hline Grid & 0 & 0 & $155,524,832$ & 0 & 0 & $155,524,832$ \\
\hline GS200 flow & 324,589 & 7,176 & 31,026 & 0 & -973 & 361,818 \\
\hline Converter & $3,000,000$ & $1,272,821$ & 0 & 0 & $-239,558$ & $4,033,263$ \\
\hline System & $48,361,380$ & $1,279,996$ & $157,551,744$ & 0 & $-240,530$ & $206,952,590$ \\
\hline
\end{tabular}

Annualized Costs

\begin{tabular}{|l|r|r|r|r|r|r|}
\hline Component & Capital & Replacement & O\&M & Fuel & Salvage & Total \\
\hline SunPower PV & $3,121,894$ & 0 & 128,167 & 0 & 0 & $3,250,061$ \\
\hline
\end{tabular}


Bompipinenfeneric flat plate PV

Grid

GS200 flow

Converter

System
Capitž̨p 1,899 Replacement

0 O\&M 26,224

Fuel 0 Salvage 0

Total 388,123

\begin{tabular}{|l|l|l|l|l|l|}
\hline 0 & 0 & $12,030,527$ & 0 & 0 & $12,030,527$ \\
\hline
\end{tabular}

25,108

555

2,400

0

$-75$

27,988

232,063

98,458

0

0

$-18,531$

311,990

$3,740,965$

99,013

$12,187,317$

$\begin{array}{ll}0 & -18,606\end{array}$

$16,008,689$

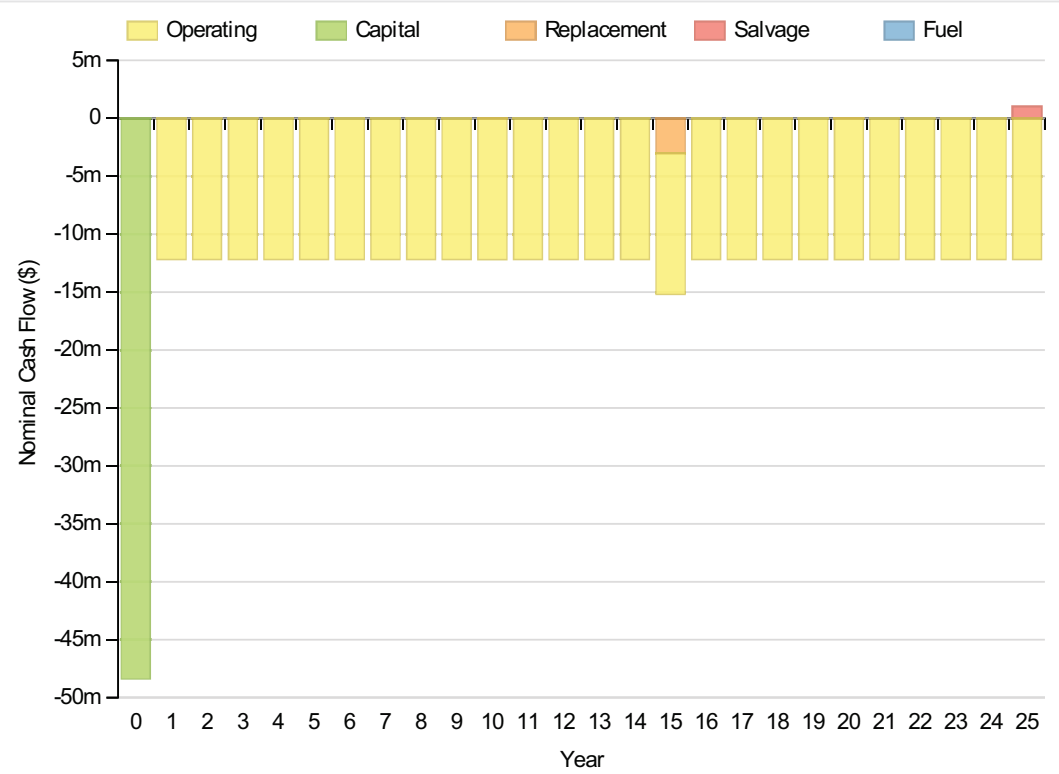

\section{Electrical}

\section{Quantity}

Excess electricity

Unmet load

Capacity shortage

Renewable fraction
Value

ralue




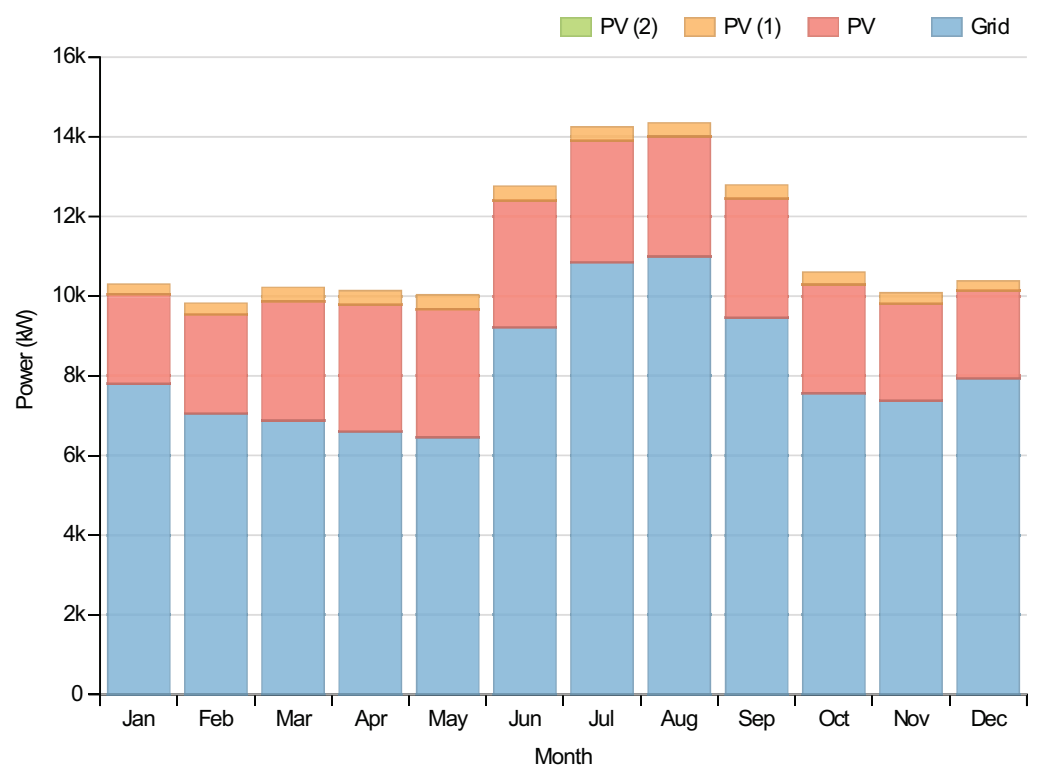

\section{PV:SunPower PV}

\begin{tabular}{|l|c|l|}
\hline Quantity & Value & Units \\
\hline Rated capacity & $13780 \mathrm{~kW}$ \\
\hline Mean output & $2813 \mathrm{~kW}$ \\
\hline Mean output & $67519.00 \mathrm{kWh} / \mathrm{d}$ \\
\hline Capacity factor & $20.42 \%$ \\
\hline Total production & $24644568 \mathrm{kWh} / \mathrm{yr}$ \\
\hline Minimum output & $0.00 \mathrm{~kW}$ \\
\hline Maximum output & $13941.00 \mathrm{~kW}$ \\
\hline PV penetration & $25.93 \%$ \\
\hline Hours of operation & $\%$ & 4386 \\
\hline Levelized cost & $\mathrm{hrs} / \mathrm{yr}$ \\
\hline
\end{tabular}

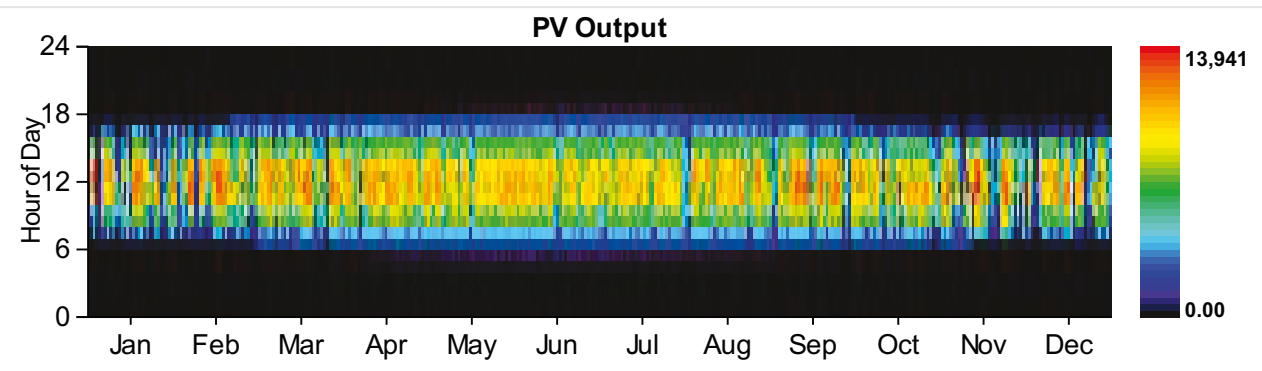

\section{PV:Remaining Generic flat plate PV}

\begin{tabular}{|l|c|l|}
\hline Quantity & Value & Units \\
\hline Rated capacity & $1547 \mathrm{~kW}$ \\
\hline Mean output & $316 \mathrm{~kW}$ \\
\hline Mean output & $7579.50 \mathrm{kWh} / \mathrm{d}$ \\
\hline Capacity factor & $20.42 \%$ \\
\hline
\end{tabular}


Qetahirefduction

Minimum output

Maximum output

PV penetration

Hours of operation

Levelized cost
2766525 UNAtlyr

$0.00 \mathrm{~kW}$

1565.00 kW

$2.91 \%$

$4386 \mathrm{hrs} / \mathrm{yr}$

$0.014 \$ / k W h$

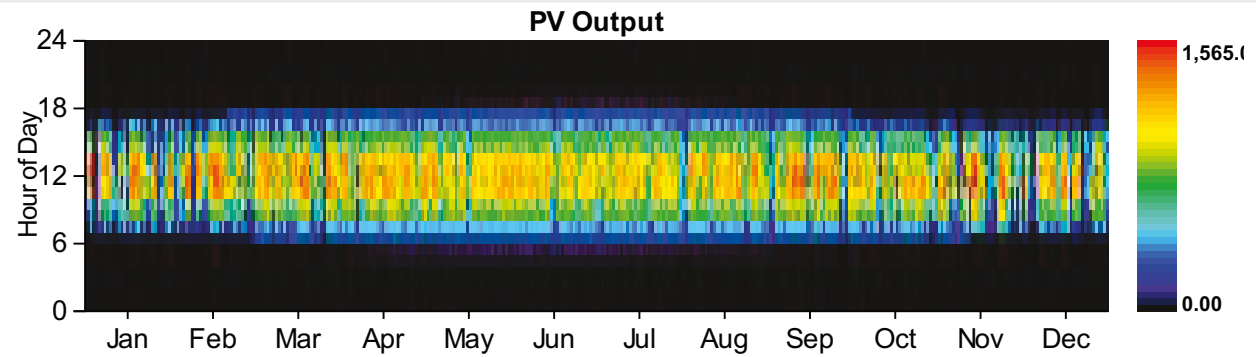

\section{Battery:GS200 flow}

Quantity $\quad$ Value

String size

Strings in parallel

Batteries

Bus voltage

\begin{tabular}{|c|c|c|}
\hline Quantity & Value & Units \\
\hline Nominal capacity & 600 & kWh \\
\hline Usable nominal capacity & 600 & kWh \\
\hline Autonomy & 0 & $\mathrm{hr}$ \\
\hline Lifetime throughput & 0 & \\
\hline Battery wear cost & 0.000 & $\$ / k W h$ \\
\hline Average energy cost & 0.000 & $\$ / k W h$ \\
\hline Energy in & 142166 & $\mathrm{kWh} / \mathrm{yr}$ \\
\hline Energy out & 100019 & $\mathrm{kWh} / \mathrm{yr}$ \\
\hline Storage depletion & 600 & $\mathrm{kWh} / \mathrm{yr}$ \\
\hline Losses & 41547 & $\mathrm{kWh} / \mathrm{yr}$ \\
\hline Annual throughput & 119545 & $\mathrm{kWh} / \mathrm{yr}$ \\
\hline Expected life & 25 & $\mathrm{yr}$ \\
\hline
\end{tabular}




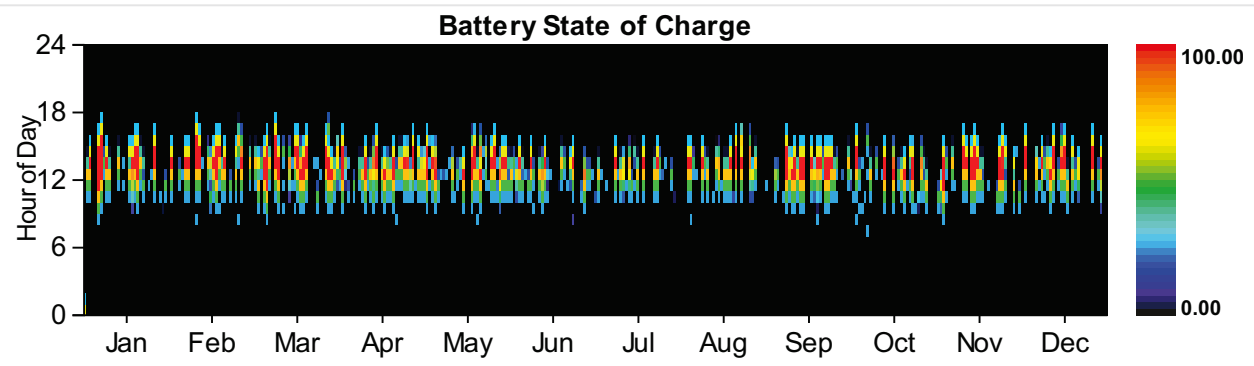

Converter

\begin{tabular}{|c|c|c|c|}
\hline Quantity & Inverter & Rectifier & Units \\
\hline Capacity & 10,000 & 9,000 & $\mathrm{~kW}$ \\
\hline Mean output & 2,661 & 0 & $\mathrm{~kW}$ \\
\hline Minimum output & 0 & 0 & $\mathrm{~kW}$ \\
\hline Maximum output & 10,000 & 0 & $\mathrm{~kW}$ \\
\hline Capacity factor & 27 & 0 & $\%$ \\
\hline Hours of operation & 4,471 & 0 & $\mathrm{hrs} / \mathrm{yr}$ \\
\hline Energy in & $25,897,278$ & 0 & $\mathrm{kWh} / \mathrm{yr}$ \\
\hline Energy out & $23,307,446$ & 0 & $\mathrm{kWh} / \mathrm{yr}$ \\
\hline Losses & $2,589,832$ & 0 & $\mathrm{kWh} / \mathrm{yr}$ \\
\hline
\end{tabular}
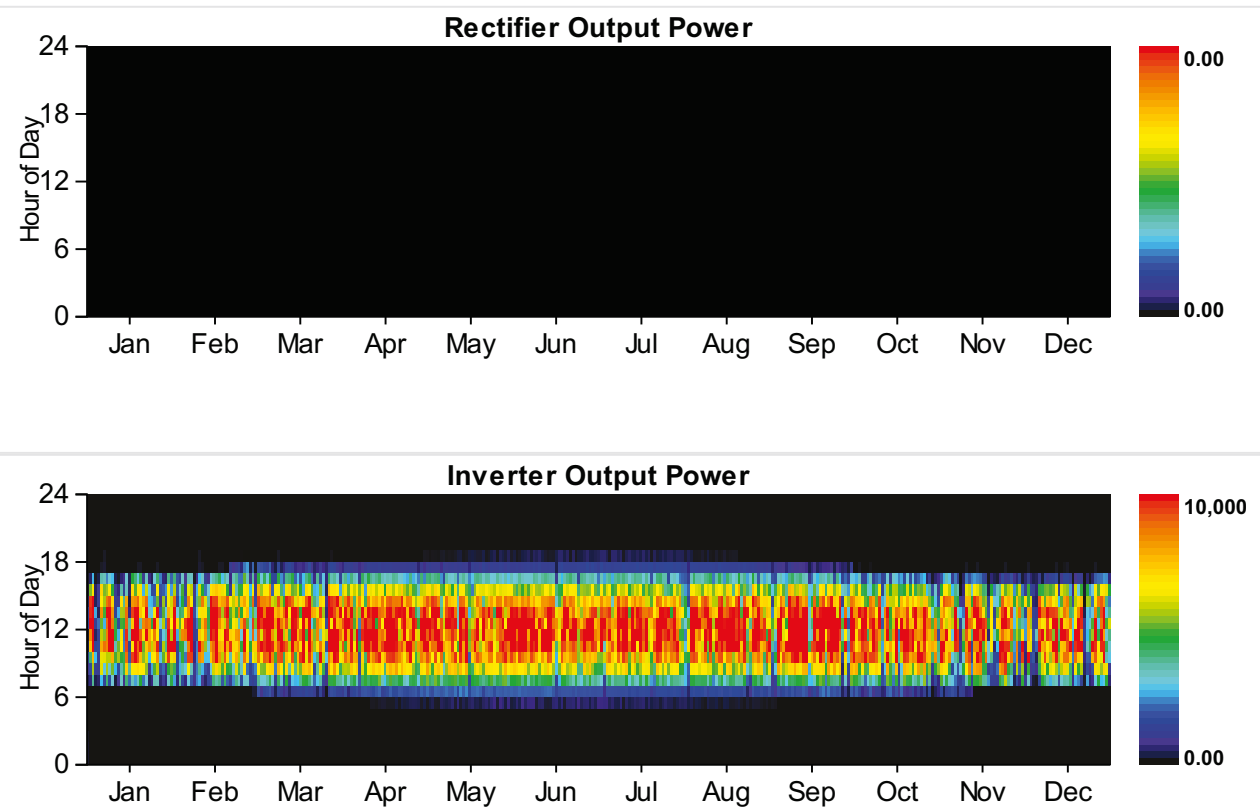

Grid

Rate: Rate 1 Demand

\begin{tabular}{|c|c|c|c|c|c|c|}
\hline Resources.ReportingService_GeneratelnputsReport_Month & $\begin{array}{l}\text { Energy } \\
\text { Purchased } \\
\text { (kWh) }\end{array}$ & $\begin{array}{l}\text { Energy } \\
\text { Sold } \\
\text { (kWh) }\end{array}$ & $\begin{array}{l}\text { Net } \\
\text { Purchases } \\
\text { (kWh) }\end{array}$ & $\begin{array}{l}\text { Peak } \\
\text { Demand } \\
(\text { kW) }\end{array}$ & $\begin{array}{l}\text { Energy } \\
\text { Charge } \\
\text { (\$) }\end{array}$ & $\begin{array}{l}\text { Demand } \\
\text { Charge } \\
(\$)\end{array}$ \\
\hline January & 0 & 0 & 0 & 17,079 & 0 & 273,262 \\
\hline February & 0 & 0 & 0 & 15,668 & 0 & 250,685 \\
\hline
\end{tabular}




\begin{tabular}{|c|c|c|c|c|c|c|}
\hline March & Energy 0 & Energy $\varphi^{0}$ & Net $\quad 0$ & Peakn075 & Energy & Dênảieg \\
\hline $\begin{array}{l}\text { April } \\
\text { Resources.ReportingService_GeneratelnputsReport_Month }\end{array}$ & $\begin{array}{l}\text { Purchased } \\
\text { (kWh) }\end{array}$ & $\begin{array}{l}\text { Sold } 0 \\
\text { (kWh) }\end{array}$ & $\begin{array}{l}\text { Purchase } \\
\text { (kWh) }\end{array}$ & $\begin{array}{l}\text { Depu,gigd } \\
\text { (kW) }\end{array}$ & $\begin{array}{l}\text { Charge } \\
\text { (\$) }\end{array}$ & $\begin{array}{l}\text { Chargeg9 } \\
\text { (\$) }\end{array}$ \\
\hline May & 0 & 0 & 0 & 14,850 & 0 & 237,602 \\
\hline June & 0 & 0 & 0 & 20,019 & 0 & 320,305 \\
\hline July & 0 & 0 & 0 & 24,169 & 0 & 386,703 \\
\hline August & 0 & 0 & 0 & 22,785 & 0 & 364,561 \\
\hline September & 0 & 0 & 0 & 18,833 & 0 & 301,321 \\
\hline October & 0 & 0 & 0 & 16,050 & 0 & 256,794 \\
\hline November & 0 & 0 & 0 & 15,751 & 0 & 252,018 \\
\hline December & 0 & 0 & 0 & 17,148 & 0 & 274,366 \\
\hline Annual & 0 & 0 & 0 & 24,169 & 0 & $3,421,115$ \\
\hline
\end{tabular}

Rate: Rate 1

\begin{tabular}{|c|c|c|c|c|c|c|}
\hline Resources.ReportingService_GeneratelnputsReport_Month & $\begin{array}{l}\text { Energy } \\
\text { Purchased } \\
\text { (kWh) }\end{array}$ & $\begin{array}{l}\text { Energy } \\
\text { Sold } \\
\text { (kWh) }\end{array}$ & $\begin{array}{l}\text { Net } \\
\text { Purchases } \\
\text { (kWh) }\end{array}$ & $\begin{array}{l}\text { Peak } \\
\text { Demand } \\
\text { (kW) }\end{array}$ & $\begin{array}{l}\text { Energy } \\
\text { Charge } \\
\text { (\$) }\end{array}$ & $\begin{array}{l}\text { Demand } \\
\text { Charge } \\
\text { (\$) }\end{array}$ \\
\hline January & $5,801,195$ & 0 & $5,801,195$ & 0 & 696,143 & 0 \\
\hline February & $4,740,909$ & 0 & $4,740,909$ & 0 & 568,909 & 0 \\
\hline March & $5,114,871$ & 0 & $5,114,871$ & 0 & 613,785 & 0 \\
\hline April & $4,751,667$ & 0 & $4,751,667$ & 0 & 570,200 & 0 \\
\hline May & $4,801,942$ & 0 & $4,801,942$ & 0 & 576,233 & 0 \\
\hline June & $6,632,296$ & 0 & $6,632,296$ & 0 & 795,875 & 0 \\
\hline July & $8,068,444$ & 0 & $8,068,444$ & 0 & 968,213 & 0 \\
\hline August & $8,182,007$ & 0 & $8,182,007$ & 0 & 981,841 & 0 \\
\hline September & $6,811,501$ & 0 & $6,811,501$ & 0 & 817,380 & 0 \\
\hline October & $5,624,914$ & 0 & $5,624,914$ & 0 & 674,990 & 0 \\
\hline November & $5,310,932$ & 0 & $5,310,932$ & 0 & 637,312 & 0 \\
\hline December & $5,904,472$ & 0 & $5,904,472$ & 0 & 708,537 & 0 \\
\hline Annual & $71,745,144$ & 0 & $71,745,144$ & 0 & $8,609,417$ & 0 \\
\hline
\end{tabular}

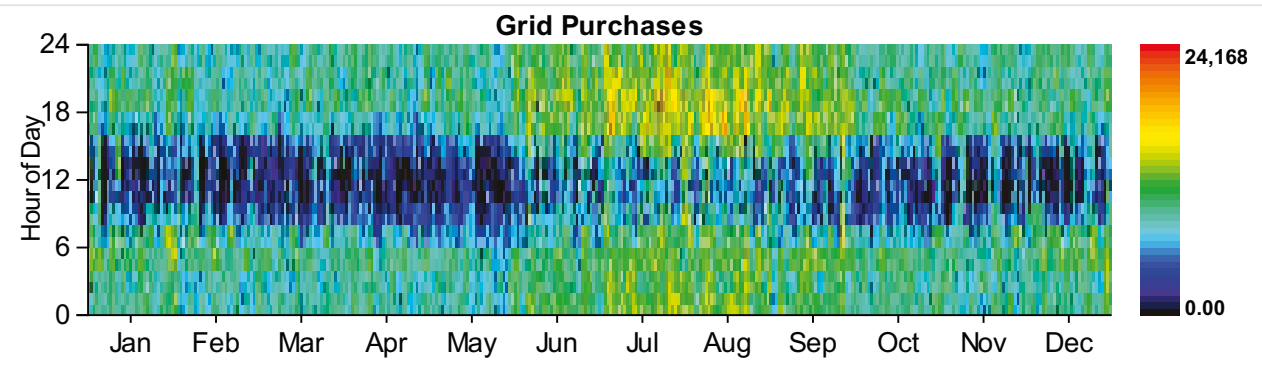


טו וטוסטוויו וי

\begin{tabular}{|l|r|l|}
\hline Pollutant & Emissions & Units \\
\hline Carbon dioxide & $45342932 \mathrm{~kg} / \mathrm{yr}$ \\
\hline Carbon monoxide & 0 & $\mathrm{~kg} / \mathrm{yr}$ \\
\hline Unburned hydrocarbons & 0 & $\mathrm{~kg} / \mathrm{yr}$ \\
\hline Particulate matter & $0 \mathrm{~kg} / \mathrm{yr}$ \\
\hline Sulfur dioxide & $196582 \mathrm{~kg} / \mathrm{yr}$ \\
\hline Nitrogen oxides & $96138 \mathrm{~kg} / \mathrm{yr}$
\end{tabular}

HOMER Energy, LLC @ 2016 
System Report

System architecture

\begin{tabular}{|l|l|r|}
\hline PV & SunPower PV & 13,780 \\
\hline PV \#2 & Remaining Generic flat plate PV & 1,547 \\
\hline Generator & Kohler 3250 Prime Power & 2,800 \\
\hline Converter & System Converter & 10,000 \\
\hline Grid & Grid & $\mathrm{kW}$ \\
\hline Dispatch Strategy & Cycle Charging & 20,000 \\
\hline
\end{tabular}

Cost summary

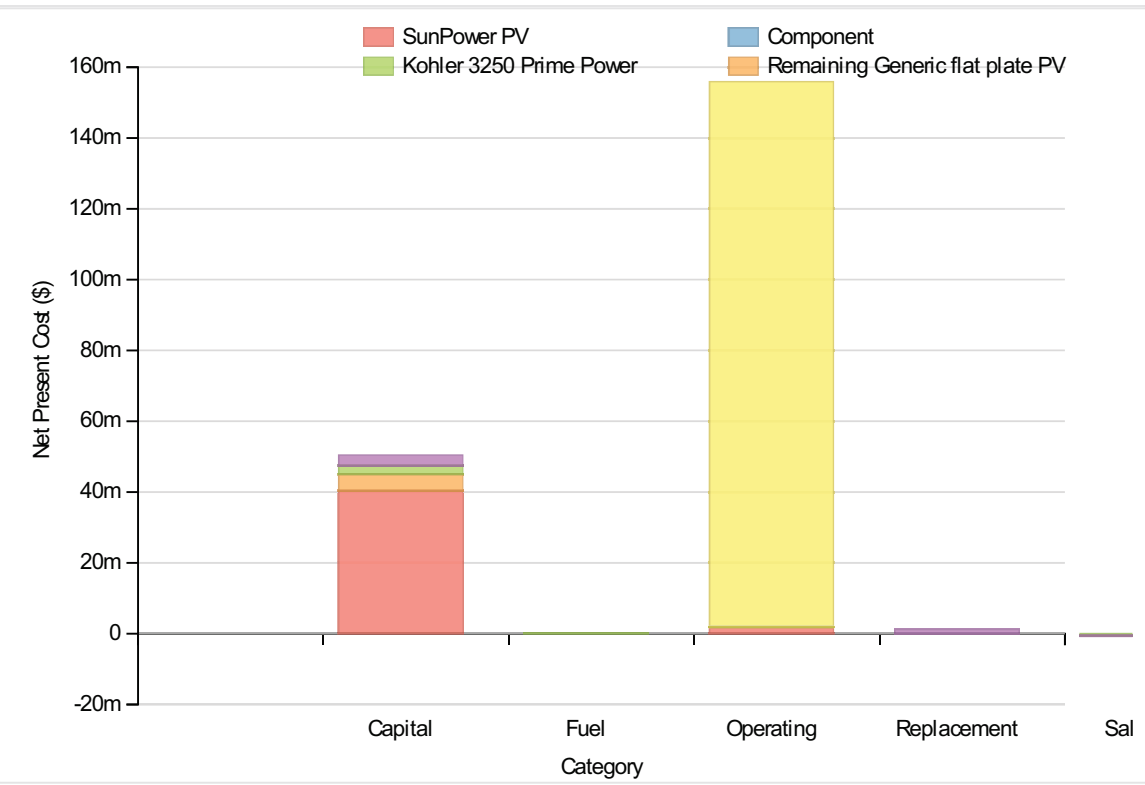

Cost Summary

Total net present cost

$207309664 \$$

Levelized cost of energy

$0.169 \$ / \mathrm{kWh}$

\section{Net Present Costs}

\begin{tabular}{|c|c|c|c|c|c|c|}
\hline Component & Capital & Replacement & O\&M & Fuel & Salvage & Total \\
\hline SunPower PV & $40,358,332$ & 0 & $1,656,876$ & 0 & 0 & $42,015,208$ \\
\hline Remaining Generic flat plate PV & $4,678,459$ & 0 & 339,013 & 0 & 0 & $5,017,472$ \\
\hline Kohler 3250 Prime Power & $2,350,000$ & 0 & 41,055 & 181,737 & $-488,837$ & $2,083,955$ \\
\hline Grid & 0 & 0 & $153,820,640$ & 0 & 0 & $153,820,640$ \\
\hline Converter & $3,000,000$ & $1,272,821$ & 0 & 0 & $-239,558$ & $4,033,263$ \\
\hline System & $50,386,792$ & $1,272,821$ & $155,857,632$ & 181,737 & $-728,395$ & $206,970,587$ \\
\hline
\end{tabular}

Annualized Costs

\begin{tabular}{|l|r|r|r|r|r|r|}
\hline Component & Capital & Replacement & O\&M & Fuel & Salvage & Total \\
\hline SunPower PV & $3,121,894$ & 0 & 128,167 & 0 & 0 \\
\hline
\end{tabular}


Zompipinenfeneric flat plate PV

Kohler 3250 Prime Power

Grid

Converter

System

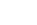

\section{Capiż̧1,899}

181,783

0

232,063

$3,897,639$
Replacement

0 O\&M

26,224 Fuel

0 Salvage 0 Total 388,123

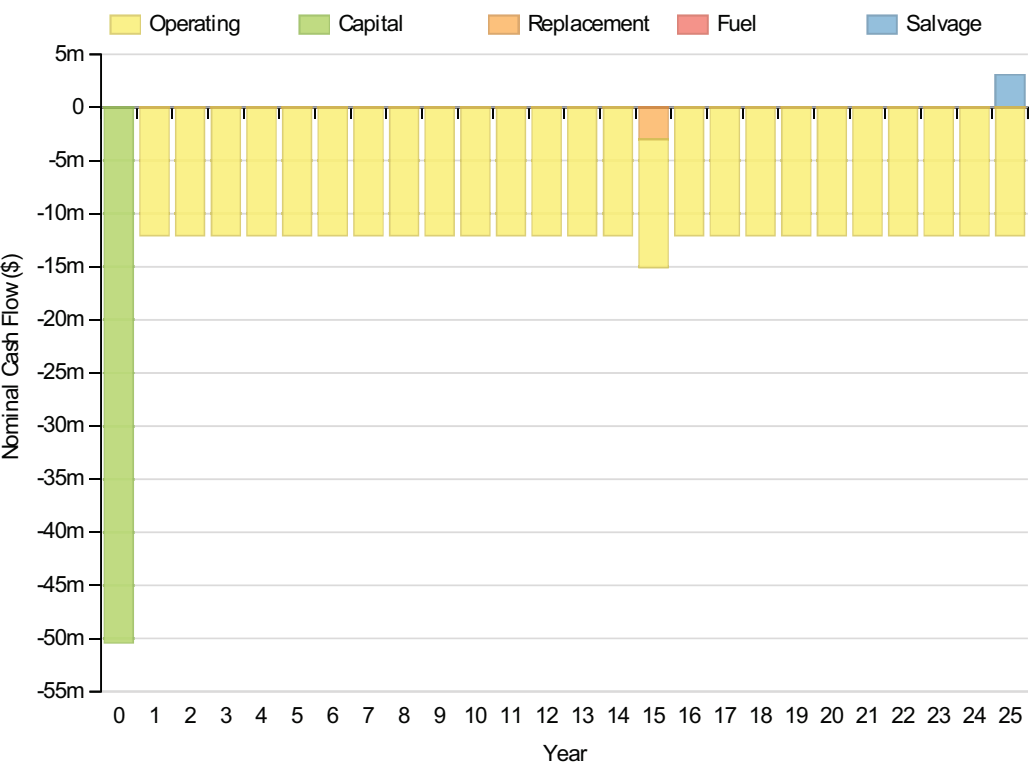

\section{Electrical}

\section{Quantity}

Excess electricity

Unmet load

Capacity shortage

Renewable fraction
Value

\section{Component}

PV

PV

Generator

Grid Purchases

Total

Load

AC primary load

DC primary load

Total
Production(kWh/yr)

Fraction (\%)

$24,644,568$

$2,766,525$

71,939

Consumption(kWh/yr)

Fraction (\%) 


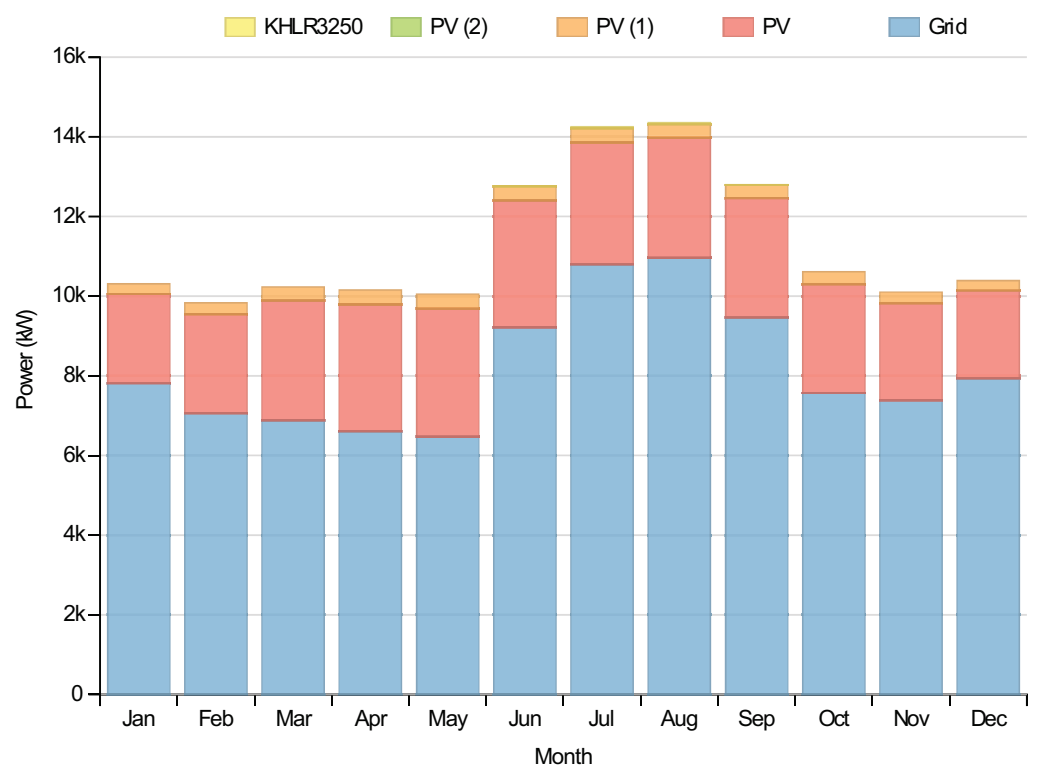

\section{PV:SunPower PV}

\begin{tabular}{|l|l|l|}
\hline Quantity & Value & Units \\
\hline Rated capacity & $13780 \mathrm{~kW}$ \\
\hline Mean output & $2813 \mathrm{~kW}$ \\
\hline Mean output & $67519.00 \mathrm{kWh} / \mathrm{d}$ \\
\hline Capacity factor & $20.42 \%$ \\
\hline Total production & $24644568 \mathrm{kWh} / \mathrm{yr}$ \\
\hline Minimum output & $0.00 \mathrm{~kW}$ \\
\hline Maximum output & 13941.00 & $\mathrm{~kW}$ \\
\hline PV penetration & $25.93 \%$ & $\%$ \\
\hline Hours of operation & 4386 & $\mathrm{hrs} / \mathrm{yr}$ \\
\hline Levelized cost & & $0.119 / \mathrm{kWh}$ \\
\hline
\end{tabular}

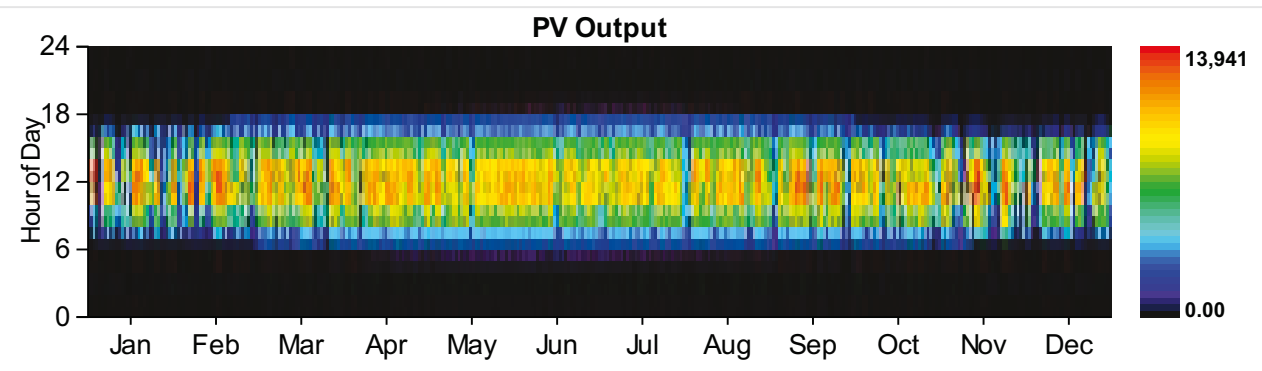

\section{PV:Remaining Generic flat plate PV}

\begin{tabular}{|l|c|l|}
\hline Quantity & Value & Units \\
\hline Rated capacity & $1547 \mathrm{~kW}$ \\
\hline Mean output & $316 \mathrm{~kW}$ \\
\hline Mean output & $7579.50 \mathrm{kWh} / \mathrm{d}$ \\
\hline Capacity factor & $20.42 \%$ \\
\hline
\end{tabular}




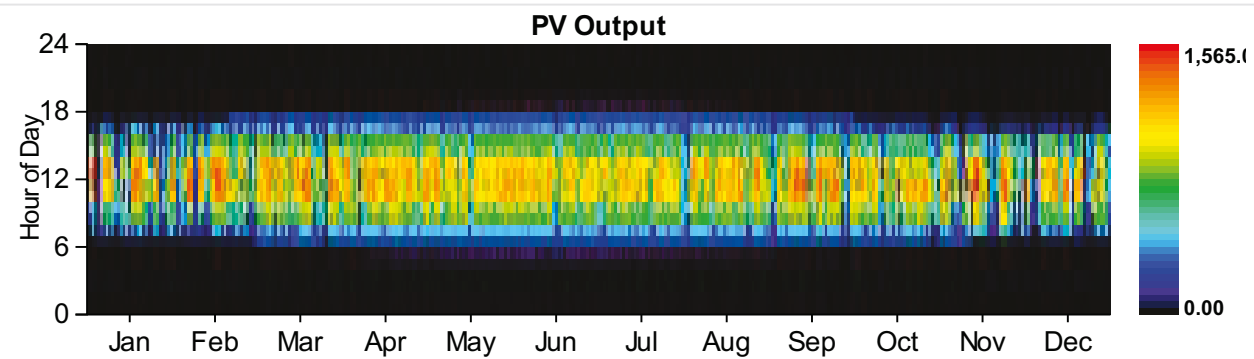

\section{Generator:Kohler 3250 Prime Power}

\section{Quantity}

Hours of operation

Number of starts

Operational life

Fixed generation cost

Marginal generation cost

Electrical production

Mean electrical output

Min. electrical output

Max. electrical output

Fuel consumption

Specific fuel consumption

Fuel energy input

Mean electrical efficiency

\begin{tabular}{|r|l|l|}
\hline Value & Units \\
\hline 79 & hrs/yr \\
\hline 190 & starts/yr \\
\hline 207.82 & $\$ / h r$ \\
\hline 0.18 & $\$ / \mathrm{kWh}$ \\
\hline 71939 & $\mathrm{kWh} / \mathrm{yr}$ \\
\hline 911 & $\mathrm{~kW}$ \\
\hline 700 & $\mathrm{~kW}$ \\
\hline 2800 & $\mathrm{~kW}$ \\
\hline 17795 & $\mathrm{~L} / \mathrm{yr}$ \\
\hline 0.25 & $\mathrm{~L} / \mathrm{kWh}$ \\
\hline 175104 & $\mathrm{kWh} / \mathrm{yr}$ \\
\hline 41 & $\%$ \\
\hline
\end{tabular}

Kohler 3250 Prime PowerOutput

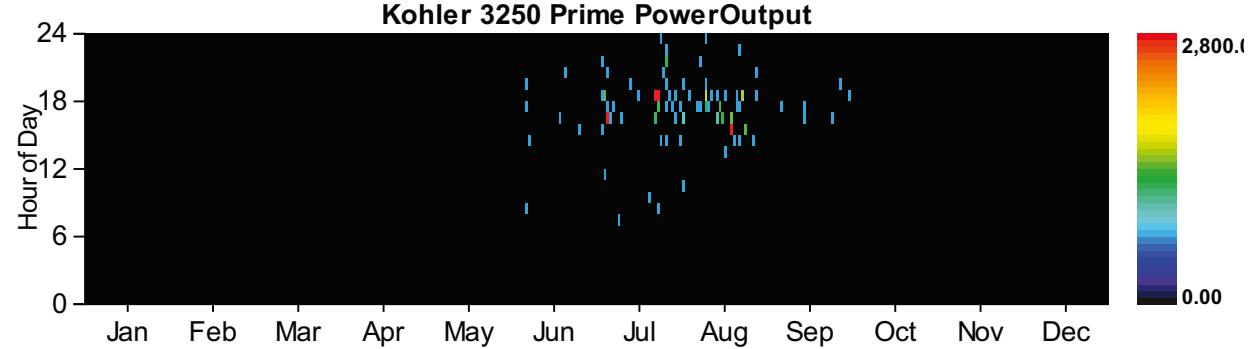




\begin{tabular}{|c|c|c|c|}
\hline Quantity & Inverter & Rectifier & Units \\
\hline Capacity & 10,000 & 9,000 & $\mathrm{~kW}$ \\
\hline Mean output & 2,650 & 0 & $\mathrm{~kW}$ \\
\hline Minimum output & 0 & 0 & $\mathrm{~kW}$ \\
\hline Maximum output & 10,000 & 0 & $\mathrm{~kW}$ \\
\hline Capacity factor & 27 & 0 & $\%$ \\
\hline Hours of operation & 4,386 & 0 & $\mathrm{hrs} / \mathrm{yr}$ \\
\hline Energy in & $25,797,274$ & 0 & $\mathrm{kWh} / \mathrm{yr}$ \\
\hline Energy out & $23,217,452$ & 0 & $\mathrm{kWh} / \mathrm{yr}$ \\
\hline Losses & $2,579,822$ & 0 & $\mathrm{kWh} / \mathrm{yr}$ \\
\hline
\end{tabular}
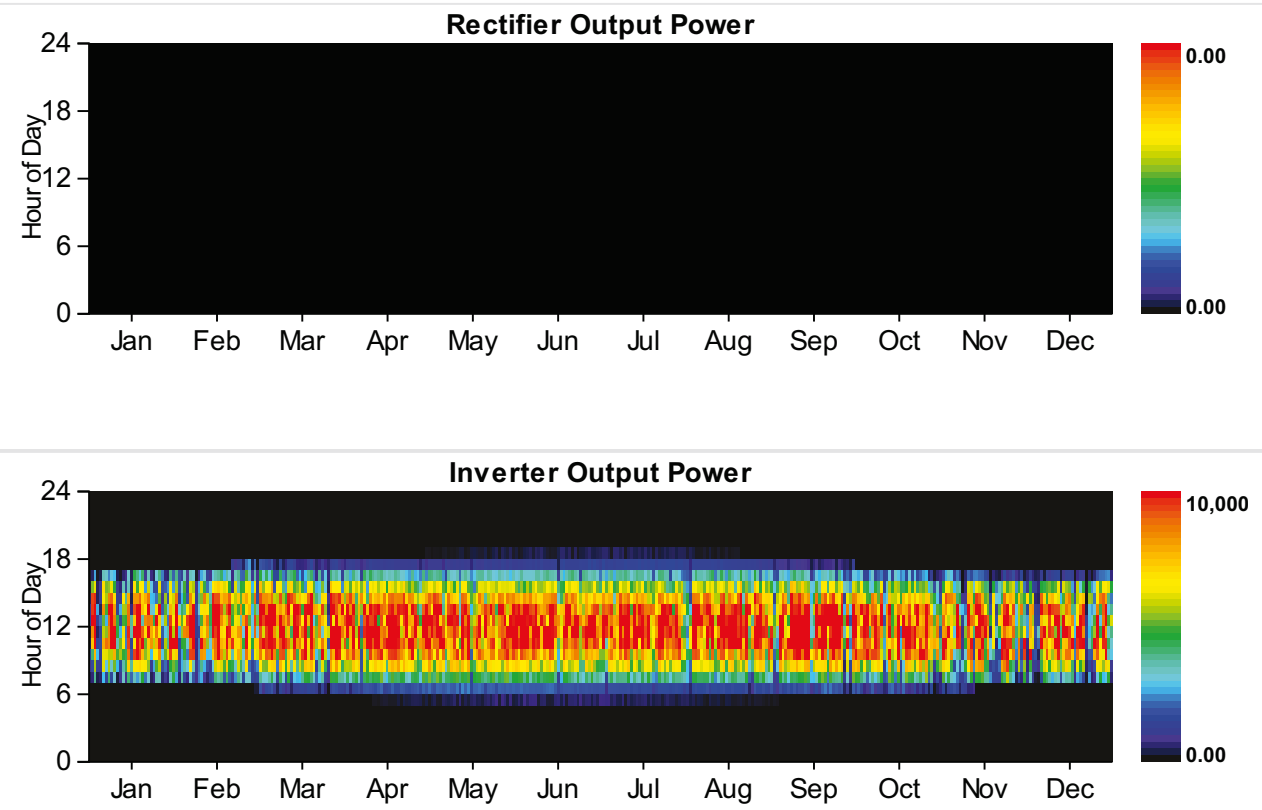

\section{Grid}

Rate: Rate 1 Demand

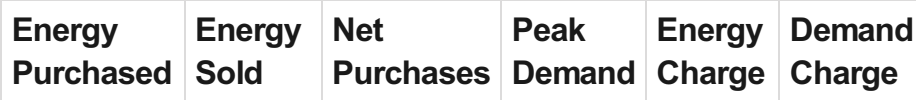

Resources.ReportingService_GeneratelnputsReport_Month

(kWh)

(kWh)

(kWh)

(kW)

(\$)

(\$)

\begin{tabular}{|c|c|c|c|c|c|c|}
\hline January & 0 & 0 & 0 & 17,079 & 0 & 273,262 \\
\hline February & 0 & 0 & 0 & 15,668 & 0 & 250,685 \\
\hline March & 0 & 0 & 0 & 17,075 & 0 & 273,199 \\
\hline April & 0 & 0 & 0 & 14,394 & 0 & 230,299 \\
\hline May & 0 & 0 & 0 & 14,850 & 0 & 237,602 \\
\hline June & 0 & 0 & 0 & 19,319 & 0 & 309,105 \\
\hline July & 0 & 0 & 0 & 20,000 & 0 & 320,000 \\
\hline August & 0 & 0 & 0 & 20,000 & 0 & 320,000 \\
\hline
\end{tabular}




\begin{tabular}{|c|c|c|c|c|c|c|}
\hline September & Energy 0 & Energy & Net $\quad 0$ & Peàk133 & Energy & 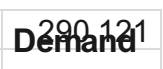 \\
\hline $\begin{array}{l}\text { October } \\
\text { Resources.ReportingService_GeneratelnputsReport_Month }\end{array}$ & $\begin{array}{l}\text { Purchased } \\
\text { (kWh) }\end{array}$ & $\begin{array}{l}\text { Sold } 0 \\
\text { (kWh) }\end{array}$ & $\begin{array}{l}\text { Purchase } \\
\text { (kWh) }\end{array}$ & $\begin{array}{l}\text { Démिaईa } \\
\text { (kW) }\end{array}$ & $\begin{array}{l}\text { Charge } \\
\text { (\$) }\end{array}$ & $\begin{array}{l}\text { CB5iggieg4 } \\
\text { (\$) }\end{array}$ \\
\hline November & 0 & 0 & 0 & 15,751 & 0 & 252,018 \\
\hline December & 0 & 0 & 0 & 17,148 & 0 & 274,366 \\
\hline Annual & 0 & 0 & 0 & 20,000 & 0 & $3,287,451$ \\
\hline
\end{tabular}

Rate: Rate 1

\begin{tabular}{|c|c|c|c|c|c|c|}
\hline Resources.ReportingService_GeneratelnputsReport_Month & $\begin{array}{l}\text { Energy } \\
\text { Purchased } \\
\text { (kWh) }\end{array}$ & $\begin{array}{l}\text { Energy } \\
\text { Sold } \\
\text { (kWh) }\end{array}$ & $\begin{array}{l}\text { Net } \\
\text { Purchases } \\
\text { (kWh) }\end{array}$ & $\begin{array}{l}\text { Peak } \\
\text { Demand } \\
(\mathbf{k W})\end{array}$ & $\begin{array}{l}\text { Energy } \\
\text { Charge } \\
\text { (\$) }\end{array}$ & $\begin{array}{l}\text { Demand } \\
\text { Charge } \\
(\$)\end{array}$ \\
\hline January & $5,808,431$ & 0 & $5,808,431$ & 0 & 697,012 & 0 \\
\hline February & $4,748,350$ & 0 & $4,748,350$ & 0 & 569,802 & 0 \\
\hline March & $5,124,164$ & 0 & $5,124,164$ & 0 & 614,900 & 0 \\
\hline April & $4,760,968$ & 0 & $4,760,968$ & 0 & 571,316 & 0 \\
\hline May & $4,811,302$ & 0 & $4,811,302$ & 0 & 577,356 & 0 \\
\hline June & $6,634,390$ & 0 & $6,634,390$ & 0 & 796,127 & 0 \\
\hline July & $8,039,810$ & 0 & $8,039,810$ & 0 & 964,777 & 0 \\
\hline August & $8,156,603$ & 0 & $8,156,603$ & 0 & 978,792 & 0 \\
\hline September & $6,815,398$ & 0 & $6,815,398$ & 0 & 817,848 & 0 \\
\hline October & $5,631,799$ & 0 & $5,631,799$ & 0 & 675,816 & 0 \\
\hline November & $5,318,139$ & 0 & $5,318,139$ & 0 & 638,177 & 0 \\
\hline December & $5,911,110$ & 0 & $5,911,110$ & 0 & 709,333 & 0 \\
\hline Annual & $71,760,464$ & 0 & $71,760,464$ & 0 & $8,611,256$ & 0 \\
\hline
\end{tabular}

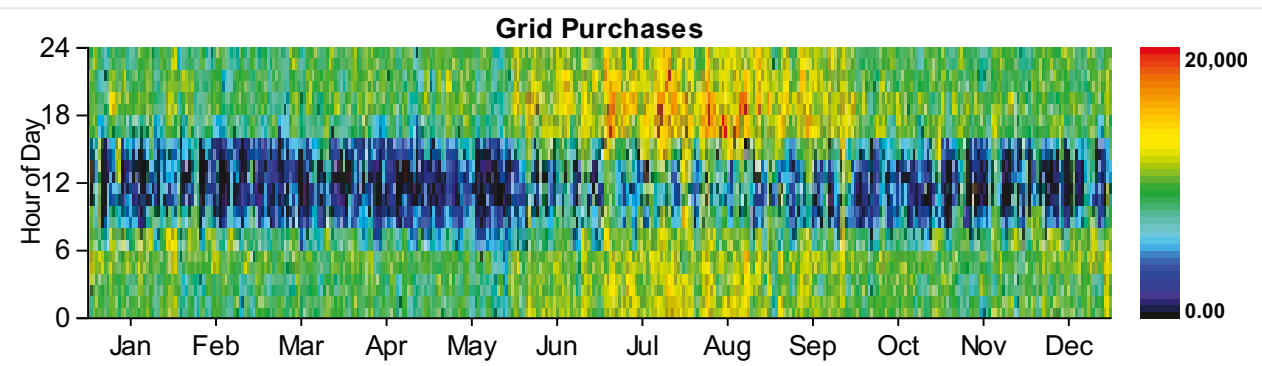

\section{Emissions}

\section{Pollutant}

Carbon dioxide

Carbon monoxide

Unburned hydrocarbons

Particulate matter

\section{Emissions}

Units

45399316 kg/yr

$196 \mathrm{~kg} / \mathrm{yr}$

$22 \mathrm{~kg} / \mathrm{yr}$

$6 \mathrm{~kg} / \mathrm{yr}$ 
HOMER Energy, LLC @ 2016 
System Report

System architecture

\begin{tabular}{|l|l|r|}
\hline PV & SunPower PV & 13,780 \\
\hline PV \#2 & Remaining Generic flat plate PV & 1,547 \\
\hline Wind Turbine & Siemens $2.3 \mathrm{MW}-108$ & 1 \\
\hline Converter & System Converter & 10,000 \\
\hline Grid & Grid & $\mathrm{kW}$ \\
\hline Dispatch Strategy & Cycle Charging & 27,200 \\
\hline
\end{tabular}

Cost summary

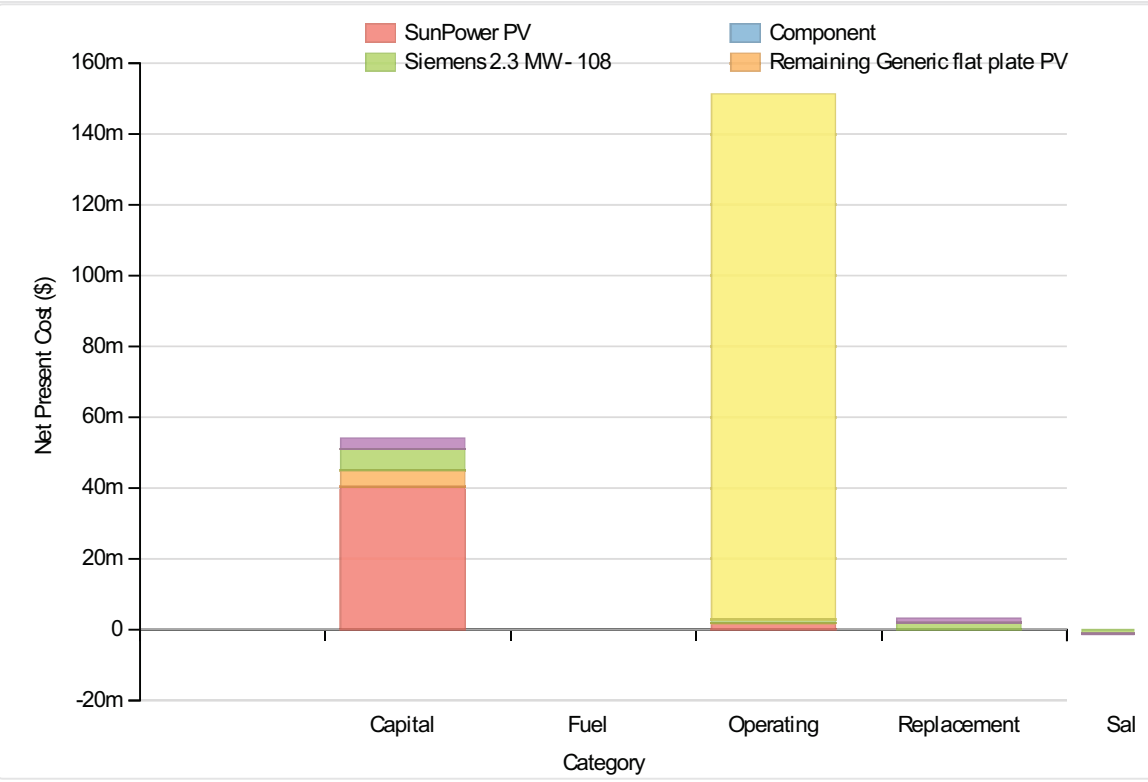

Cost Summary

Total net present cost

$207496480 \$$

Levelized cost of energy

$0.169 \$ / \mathrm{kWh}$

\section{Net Present Costs}

\begin{tabular}{|c|c|c|c|c|c|c|}
\hline Component & Capital & Replacement & O\&M & Fuel & Salvage & Total \\
\hline SunPower PV & $40,358,332$ & 0 & $1,656,876$ & 0 & 0 & $42,015,208$ \\
\hline Remaining Generic flat plate PV & $4,678,459$ & 0 & 339,013 & 0 & 0 & $5,017,472$ \\
\hline Siemens 2.3 MW - 108 & $6,000,000$ & $1,912,842$ & 969,563 & 0 & $-1,078,009$ & $7,804,396$ \\
\hline Grid & 0 & 0 & $148,287,040$ & 0 & 0 & $148,287,040$ \\
\hline Converter & $3,000,000$ & $1,272,821$ & 0 & 0 & $-239,558$ & $4,033,263$ \\
\hline System & $54,036,792$ & $3,185,663$ & $151,252,480$ & 0 & $-1,317,567$ & $207,157,368$ \\
\hline
\end{tabular}

Annualized Costs

\begin{tabular}{|l|r|r|r|r|r|r|}
\hline Component & Capital & Replacement & O\&M & Fuel & Salvage & Total \\
\hline SunPower PV & $3,121,894$ & 0 & 128,167 & 0 & 0 \\
\hline
\end{tabular}


Zompipinenfeneric flat plate PV

Siemens 2.3 MW - 108

Grid

Converter

System
Capit绝1,899

464,126

0

232,063

$4,179,983$

$\square$ Fuel

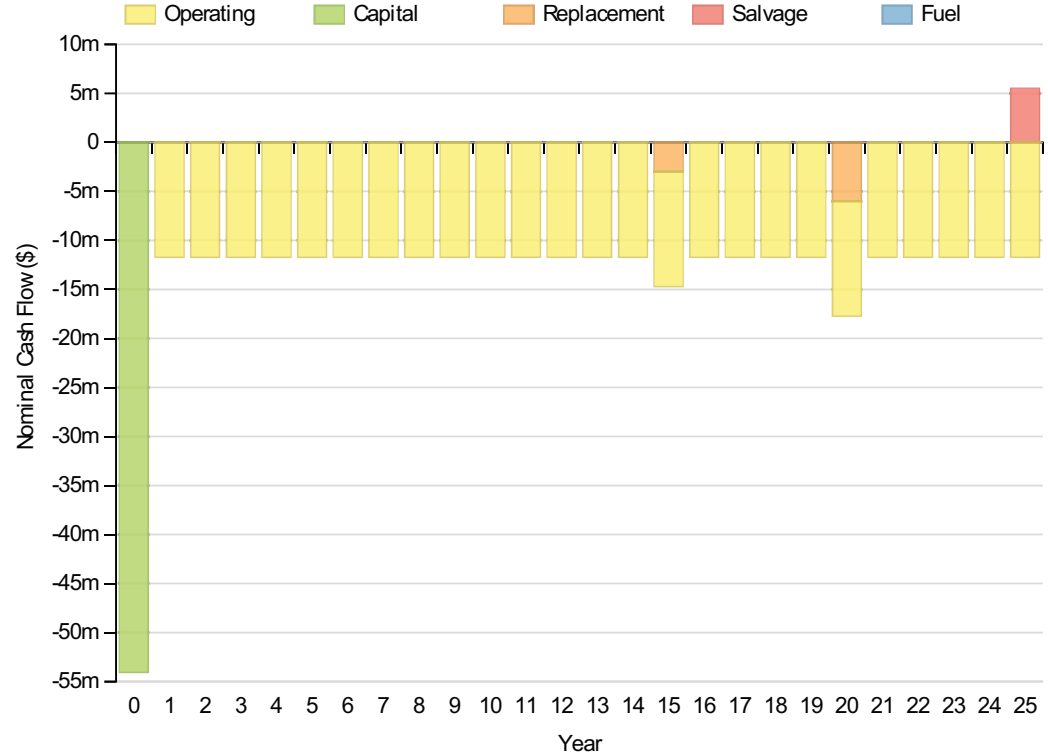

\section{Electrical}

\section{Quantity}

Excess electricity

Unmet load

Capacity shortage

Renewable fraction

\section{Component}

PV

PV

Wind Turbine

Grid Purchases

Total

Load

AC primary load

DC primary load

Total
Value

$0 \mathrm{kWh} / \mathrm{yr}$

$0 \mathrm{kWh} / \mathrm{yr}$

0
Production(kWh/yr)

Fraction (\%)

$24,644,568$

$2,766,525$

$4,674,352$

$67,590,728$

$99,676,176$

Consumption(kWh/yr) 


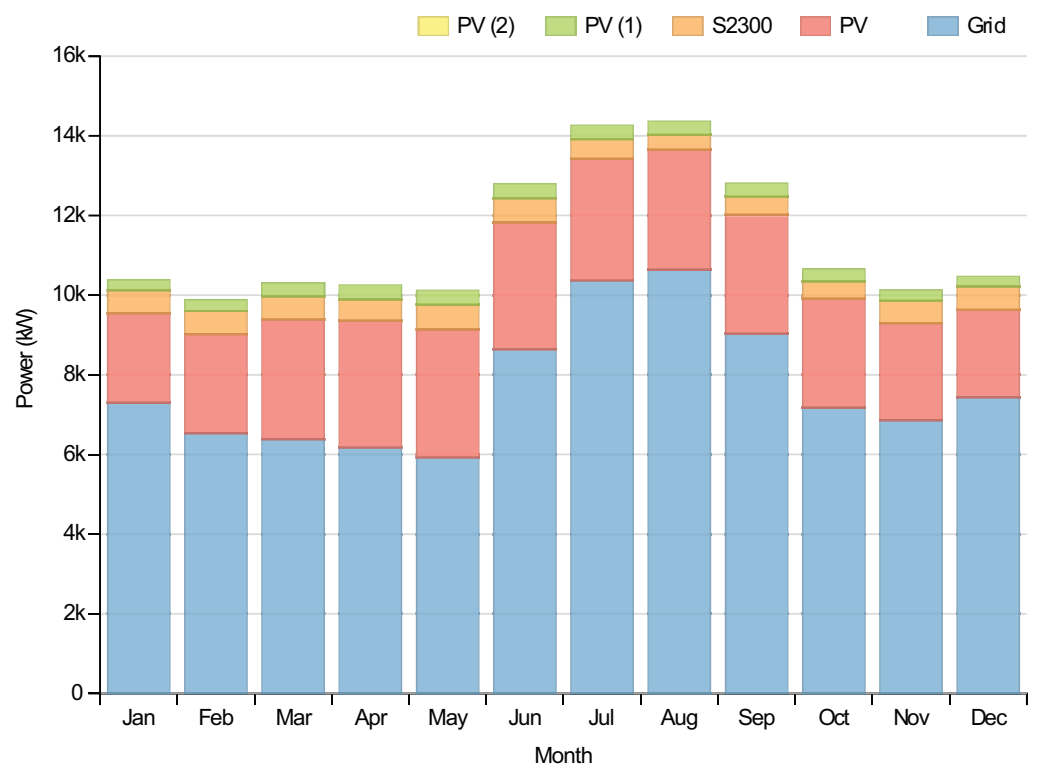

\section{PV:SunPower PV}

\begin{tabular}{|l|l|l|}
\hline Quantity & Value & Units \\
\hline Rated capacity & $13780 \mathrm{~kW}$ \\
\hline Mean output & $2813 \mathrm{~kW}$ \\
\hline Mean output & $67519.00 \mathrm{kWh} / \mathrm{d}$ \\
\hline Capacity factor & $20.42 \%$ \\
\hline Total production & $24644568 \mathrm{kWh} / \mathrm{yr}$ \\
\hline Minimum output & $0.00 \mathrm{~kW}$ \\
\hline Maximum output & 13941.00 & $\mathrm{~kW}$ \\
\hline PV penetration & $25.93 \%$ & $\%$ \\
\hline Hours of operation & 4386 & $\mathrm{hrs} / \mathrm{yr}$ \\
\hline Levelized cost & & $0.119 / \mathrm{kWh}$ \\
\hline
\end{tabular}

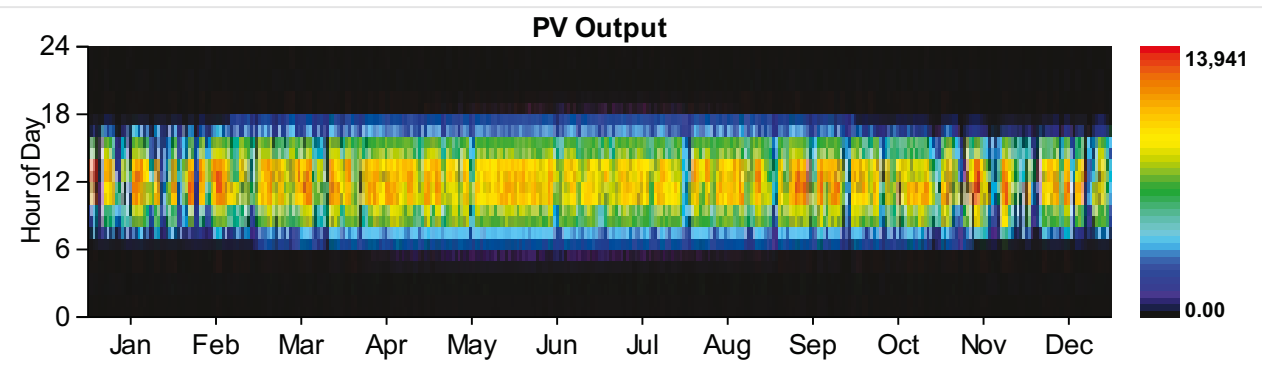

\section{PV:Remaining Generic flat plate PV}

\begin{tabular}{|l|c|l|}
\hline Quantity & Value & Units \\
\hline Rated capacity & $1547 \mathrm{~kW}$ \\
\hline Mean output & $316 \mathrm{~kW}$ \\
\hline Mean output & $7579.50 \mathrm{kWh} / \mathrm{d}$ \\
\hline Capacity factor & $20.42 \%$ \\
\hline
\end{tabular}


Eetahtripduction

Minimum output

Maximum output

PV penetration

Hours of operation

Levelized cost

\section{5 uNtes}

$0.00 \mathrm{~kW}$

$1565.00 \mathrm{~kW}$

$2.91 \%$

$4386 \mathrm{hrs} / \mathrm{yr}$

$0.014 \$ / k W h$

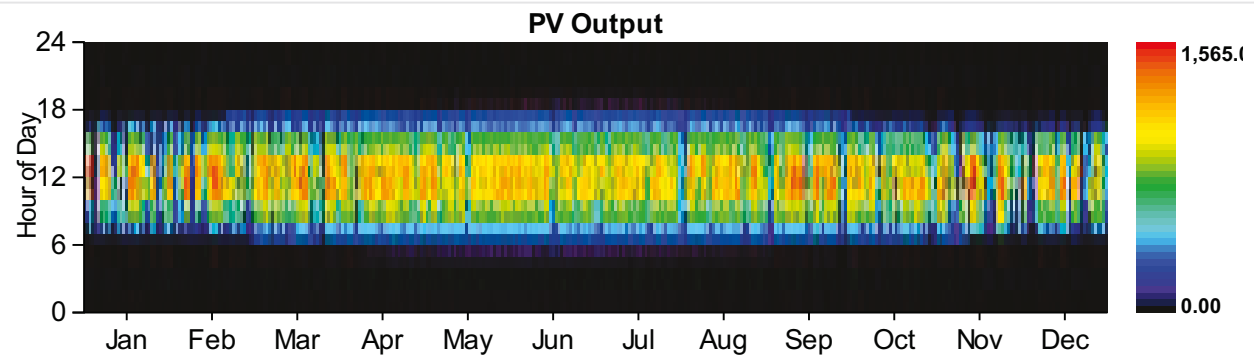

Wind Turbine:Siemens 2.3 MW - 108

\section{Quantity}

Total rated capacity

Mean output

Capacity factor

Total production

Minimum output

Maximum output

Wind penetration

Hours of operation

Levelized cost

\begin{tabular}{|r|l|l|}
\hline Value & Units \\
\hline 2300 & $\mathrm{~kW}$ \\
\hline 534 & $\mathrm{~kW}$ \\
\hline 23.20 & $\%$ \\
\hline 4674352 & $\mathrm{kWh} / \mathrm{yr}$ \\
\hline 0.49 & $\mathrm{~kW}$ \\
\hline 2312.70 & $\mathrm{~kW}$ \\
\hline 4.92 & $\%$ \\
\hline 8760 & $\mathrm{hrs} / \mathrm{yr}$ \\
\hline 0.129 & $\$ / \mathrm{kWh}$ \\
\hline
\end{tabular}

\section{Converter}

\begin{tabular}{|l|r|r|r|}
\hline Quantity & Inverter & Rectifier & Units \\
\hline Capacity & 10,000 & 9,000 & $\mathrm{~kW}$ \\
\hline Mean output & 2,601 & $\mathrm{~kW}$ \\
\hline Minimum output & 0 & $\mathrm{~kW}$ \\
\hline Maximum output & 10,000 & 0 \\
\hline Capacity factor & 26 & 0 & $\mathrm{~kW}$ \\
\hline Hours of operation & 4,386 & 0 & $\mathrm{hrs} / \mathrm{yr}$ \\
\hline Energy in & $25,319,570$ & 0 & $\mathrm{kWh} / \mathrm{yr}$ \\
\hline Energy out & $22,787,530$ & 0 & $\mathrm{kWh} / \mathrm{yr}$ \\
\hline
\end{tabular}



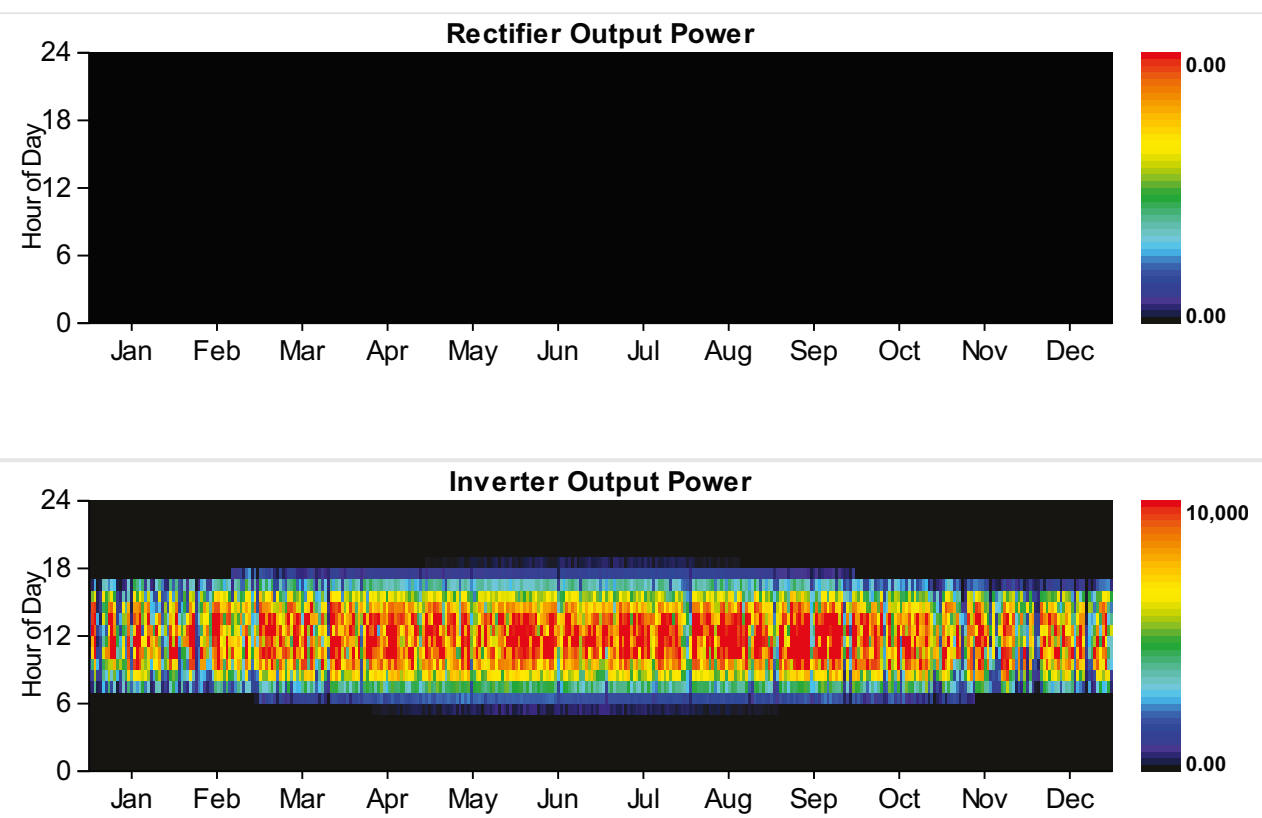

Grid

Rate: Rate 1 Demand

\begin{tabular}{|c|c|c|c|c|c|c|}
\hline Resources.ReportingService_GeneratelnputsReport_Month & $\begin{array}{l}\text { Energy } \\
\text { Purchased } \\
\text { (kWh) }\end{array}$ & $\begin{array}{l}\text { Energy } \\
\text { Sold } \\
(\mathbf{k W h})\end{array}$ & $\begin{array}{l}\text { Net } \\
\text { Purchases } \\
\text { (kWh) }\end{array}$ & $\begin{array}{l}\text { Peak } \\
\text { Demand } \\
(\mathbf{k W})\end{array}$ & $\begin{array}{l}\text { Energy } \\
\text { Charge } \\
(\$)\end{array}$ & $\begin{array}{l}\text { Demand } \\
\text { Charge } \\
\text { (\$) }\end{array}$ \\
\hline January & 0 & 0 & 0 & 16,288 & 0 & 260,605 \\
\hline February & 0 & 0 & 0 & 15,654 & 0 & 250,459 \\
\hline March & 0 & 0 & 0 & 17,043 & 0 & 272,688 \\
\hline April & 0 & 0 & 0 & 14,366 & 0 & 229,856 \\
\hline May & 0 & 0 & 0 & 13,700 & 0 & 219,202 \\
\hline June & 0 & 0 & 0 & 20,004 & 0 & 320,060 \\
\hline July & 0 & 0 & 0 & 23,789 & 0 & 380,622 \\
\hline August & 0 & 0 & 0 & 22,424 & 0 & 358,782 \\
\hline September & 0 & 0 & 0 & 18,548 & 0 & 296,765 \\
\hline October & 0 & 0 & 0 & 16,023 & 0 & 256,374 \\
\hline November & 0 & 0 & 0 & 15,278 & 0 & 244,445 \\
\hline December & 0 & 0 & 0 & 16,870 & 0 & 269,913 \\
\hline Annual & 0 & 0 & 0 & 23,789 & 0 & $3,359,769$ \\
\hline
\end{tabular}

Rate: Rate 1

\begin{tabular}{|c|c|c|c|c|c|c|}
\hline Resources.ReportingService_GeneratelnputsReport_Month & $\begin{array}{l}\text { Energy } \\
\text { Purchased } \\
\text { (kWh) }\end{array}$ & $\begin{array}{l}\text { Energy } \\
\text { Sold } \\
\text { (kWh) }\end{array}$ & $\begin{array}{l}\text { Net } \\
\text { Purchases } \\
\text { (kWh) }\end{array}$ & $\begin{array}{l}\text { Peak } \\
\text { Demand } \\
(\mathbf{k W})\end{array}$ & $\begin{array}{l}\text { Energy } \\
\text { Charge } \\
\text { (\$) }\end{array}$ & $\begin{array}{l}\text { Demand } \\
\text { Charge } \\
(\$)\end{array}$ \\
\hline January & $5,427,674$ & 0 & $5,427,674$ & 0 & 651,321 & 0 \\
\hline February & $4,390,087$ & 0 & $4,390,087$ & 0 & 526,810 & 0 \\
\hline
\end{tabular}




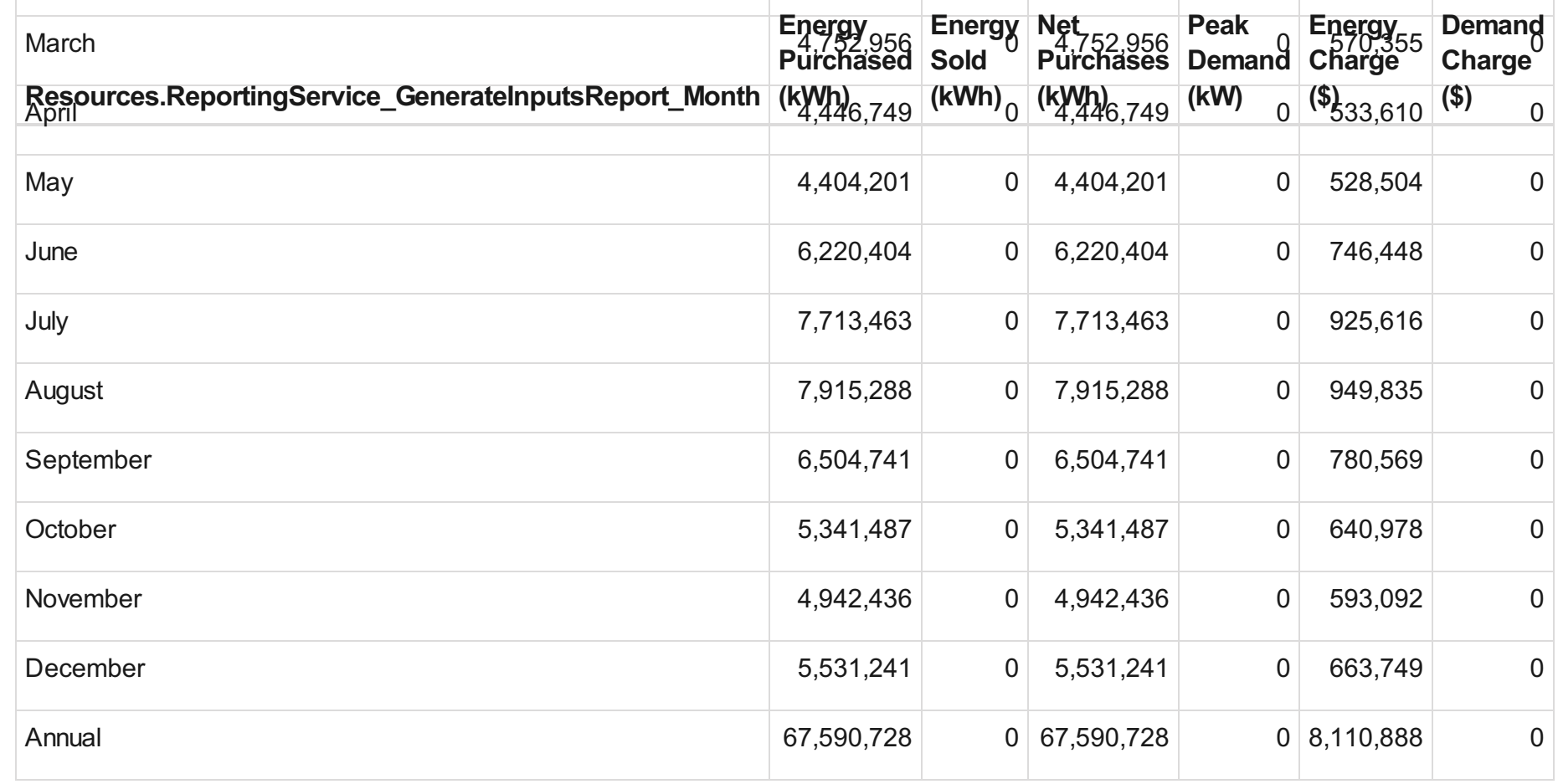

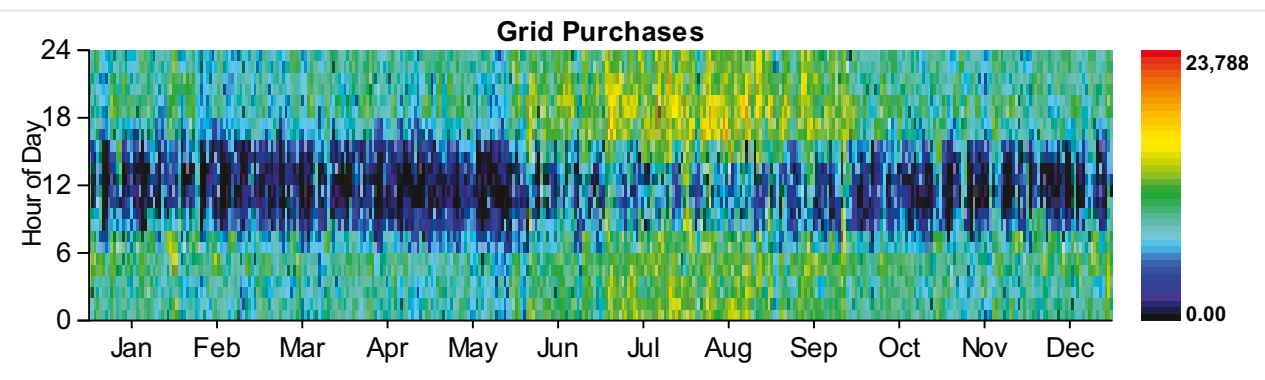

\section{Emissions}

\begin{tabular}{|l|r|l|}
\hline Pollutant & Emissions & Units \\
\hline Carbon dioxide & 42717340 & $\mathrm{~kg} / \mathrm{yr}$ \\
\hline Carbon monoxide & 0 & $\mathrm{~kg} / \mathrm{yr}$ \\
\hline Unburned hydrocarbons & $0 \mathrm{~kg} / \mathrm{yr}$ \\
\hline Particulate matter & 0 & $\mathrm{~kg} / \mathrm{yr}$ \\
\hline Sulfur dioxide & $185199 \mathrm{~kg} / \mathrm{yr}$ \\
\hline Nitrogen oxides & $90572 \mathrm{~kg} / \mathrm{yr}$ \\
\hline
\end{tabular}


System Report

System architecture

\begin{tabular}{|c|c|c|c|}
\hline PV & SunPower PV & 13,780 & $\mathrm{~kW}$ \\
\hline PV \#2 & Remaining Generic flat plate PV & 1,547 & $\mathrm{~kW}$ \\
\hline Generator & Kohler 3250 Prime Power & 2,800 & $\mathrm{~kW}$ \\
\hline Battery & GS200 flow & 1 & strings \\
\hline Converter & System Converter & 10,000 & $\mathrm{~kW}$ \\
\hline Grid & Grid & 20,000 & $\mathrm{~kW}$ \\
\hline Dispatch Strategy & Cycle Charging & & \\
\hline
\end{tabular}

\section{Cost summary}

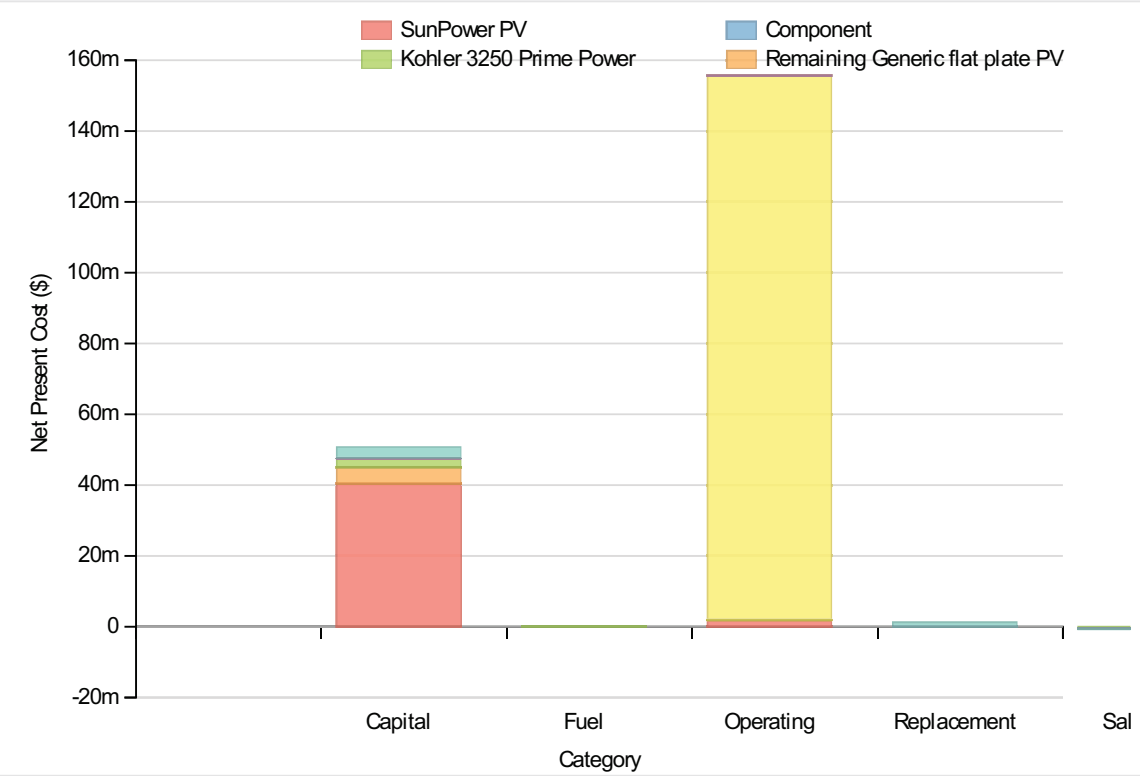

Cost Summary

Total net present cost

$207545360 \$$

Levelized cost of energy

$0.169 \$ / k W h$

Net Present Costs

\begin{tabular}{|c|c|c|c|c|c|c|}
\hline Component & Capital & Replacement & O\&M & Fuel & Salvage & Total \\
\hline SunPower PV & $40,358,332$ & 0 & $1,656,876$ & 0 & 0 & $42,015,208$ \\
\hline Remaining Generic flat plate PV & $4,678,459$ & 0 & 339,013 & 0 & 0 & $5,017,472$ \\
\hline Kohler 3250 Prime Power & $2,350,000$ & 0 & 40,535 & 205,792 & $-489,775$ & $2,106,552$ \\
\hline Grid & 0 & 0 & $153,671,936$ & 0 & 0 & $153,671,936$ \\
\hline GS200 flow & 324,589 & 7,176 & 31,026 & 0 & -973 & 361,818 \\
\hline Converter & $3,000,000$ & $1,272,821$ & 0 & 0 & $-239,558$ & $4,033,263$ \\
\hline System & $50,711,380$ & $1,279,996$ & $155,739,392$ & 205,792 & $-730,306$ & $207,206,254$ \\
\hline
\end{tabular}




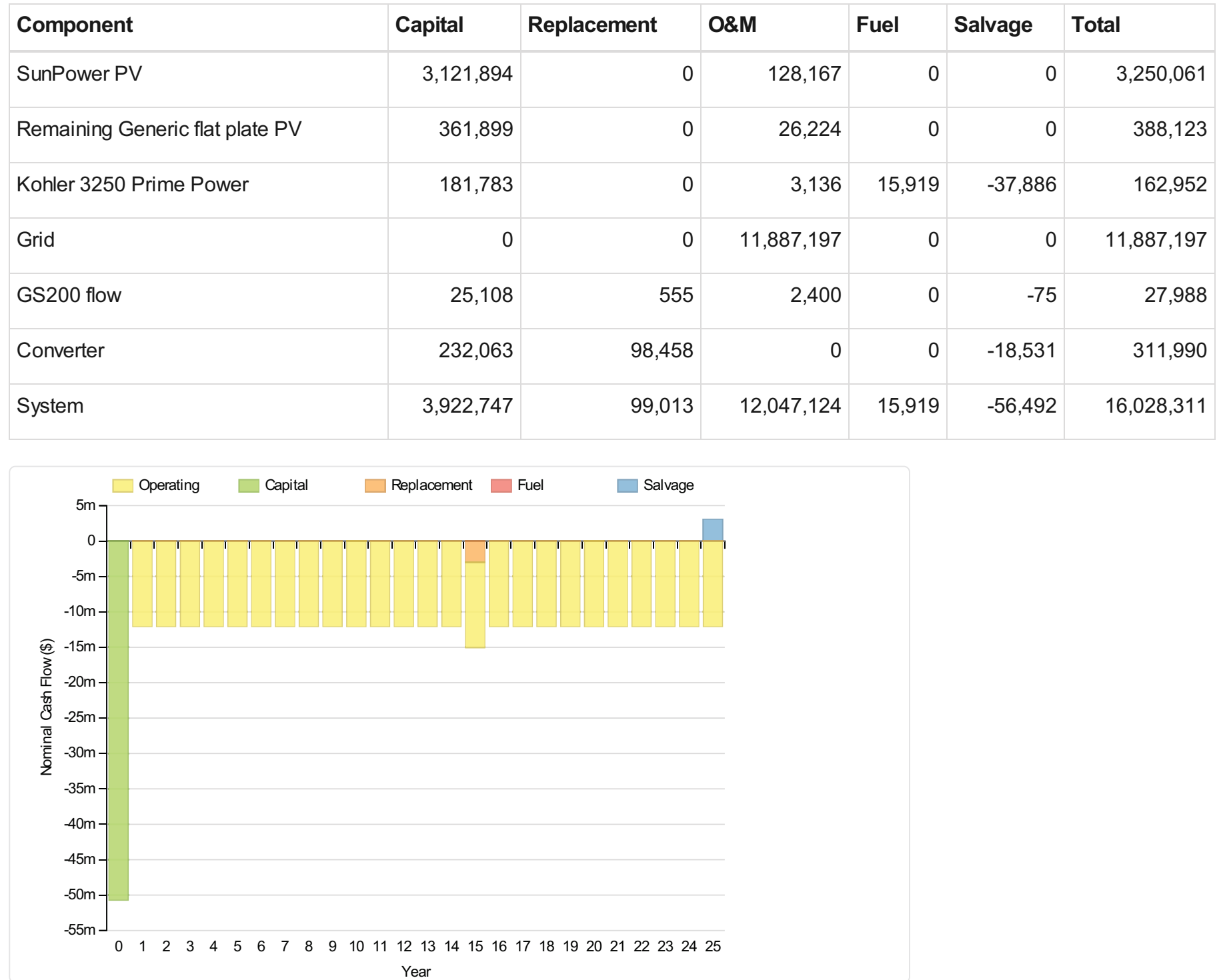

\section{Electrical}

\section{Quantity}

Excess electricity

Unmet load

Capacity shortage

Renewable fraction

\section{Component}

PV

PV

Generator

Grid Purchases

Total

Load

Consumption(kWh/yr)

Production(kWh/yr)
Value

Fraction (\%)

$24,644,568$

$2,766,525$

3

82,145

0

$71,664,592$

72

$99,157,832$ 


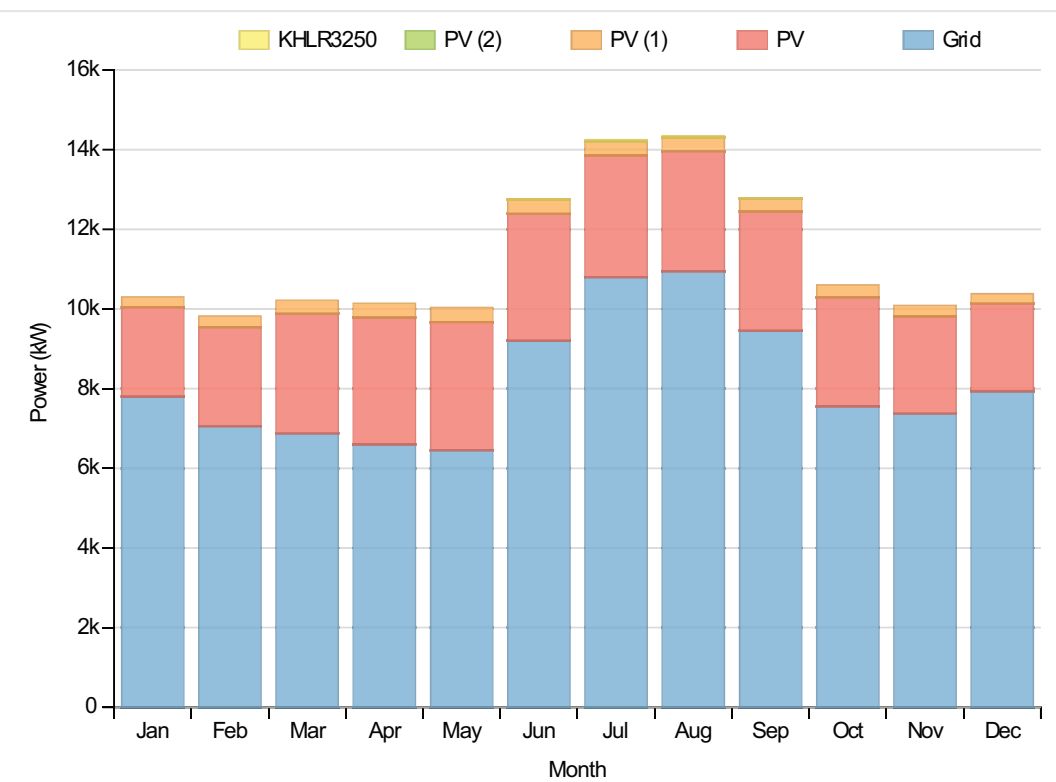

PV:SunPower PV

\begin{tabular}{|c|c|c|}
\hline Quantity & Value & Units \\
\hline Rated capacity & 13780 & $\mathrm{~kW}$ \\
\hline Mean output & 2813 & $\mathrm{~kW}$ \\
\hline Mean output & 67519.00 & $\mathrm{kWh} / \mathrm{d}$ \\
\hline Capacity factor & 20.42 & $\%$ \\
\hline Total production & 24644568 & $\mathrm{kWh} / \mathrm{yr}$ \\
\hline Minimum output & 0.00 & $\mathrm{~kW}$ \\
\hline Maximum output & 13941.00 & $\mathrm{~kW}$ \\
\hline PV penetration & 25.93 & $\%$ \\
\hline Hours of operation & 4386 & hrs/yr \\
\hline Levelized cost & 0.119 & $\$ / \mathrm{kWh}$ \\
\hline
\end{tabular}

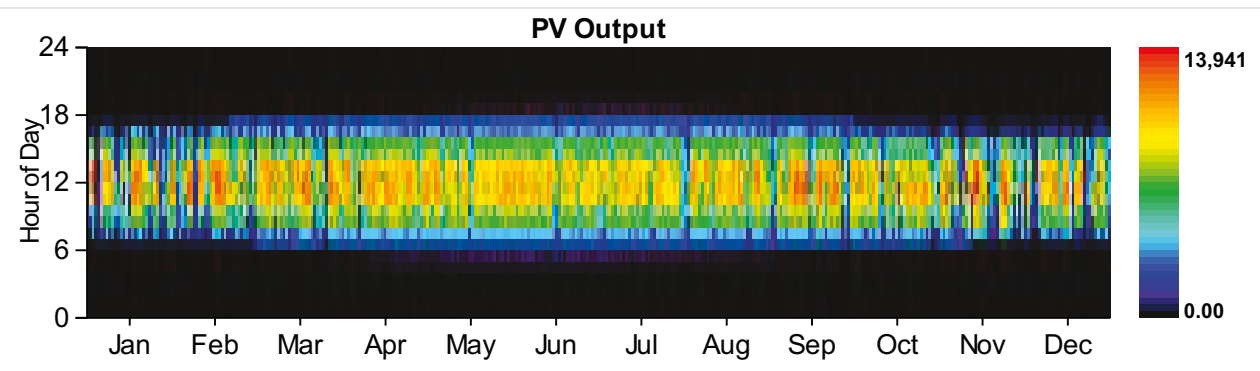

\section{PV:Remaining Generic flat plate PV}

\section{Quantity}

Rated capacity

Mean output

Mean output

\section{Value}

Units

1547 kW

316 kW 


\begin{tabular}{|c|c|c|c|}
\hline Quarafity factor & Value & 20.42 & Units \\
\hline Total production & & 2766525 & $\mathrm{kWh} / \mathrm{yr}$ \\
\hline Minimum output & & 0.00 & $\mathrm{~kW}$ \\
\hline Maximum output & & 1565.00 & $\mathrm{~kW}$ \\
\hline PV penetration & & 2.91 & $\%$ \\
\hline Hours of operation & & 4386 & $\mathrm{hrs} / \mathrm{yr}$ \\
\hline Levelized cost & & 0.014 & $\$ / k W h$ \\
\hline
\end{tabular}

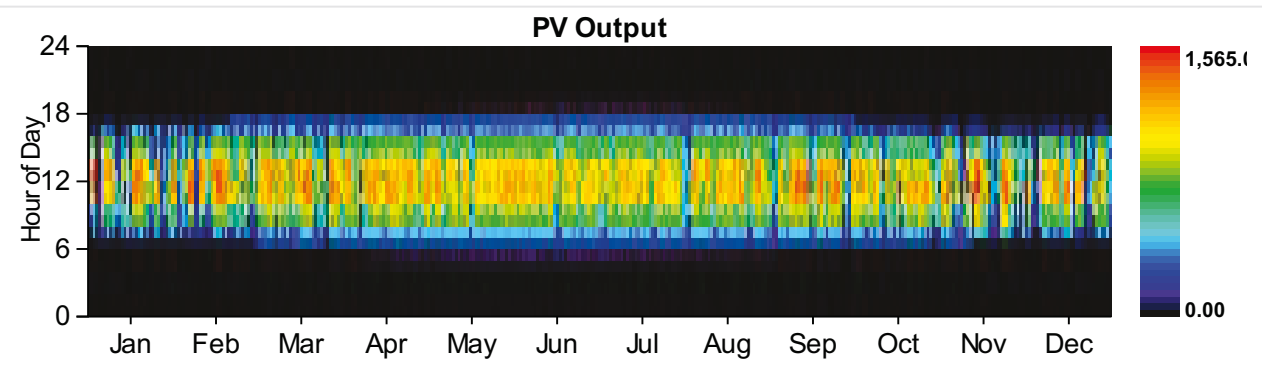

Generator:Kohler 3250 Prime Power

\section{Quantity}

Hours of operation

Number of starts

Operational life

Fixed generation cost

Marginal generation cost

Electrical production

Mean electrical output

Min. electrical output

Max. electrical output

Fuel consumption

Specific fuel consumption

Fuel energy input

Mean electrical efficiency

\section{Value}

Units

$78 \mathrm{hrs} / \mathrm{yr}$

70 starts/yr

$192 \mathrm{yr}$

$207.82 \$ / h r$

$0.18 \$ / k W h$

$82145 \mathrm{kWh} / \mathrm{yr}$

1053 kW

700 kW

$2800 \mathrm{~kW}$

20151 L/yr

$0.25 \mathrm{~L} / \mathrm{kWh}$

$198282 \mathrm{kWh} / \mathrm{yr}$

$41 \%$ 


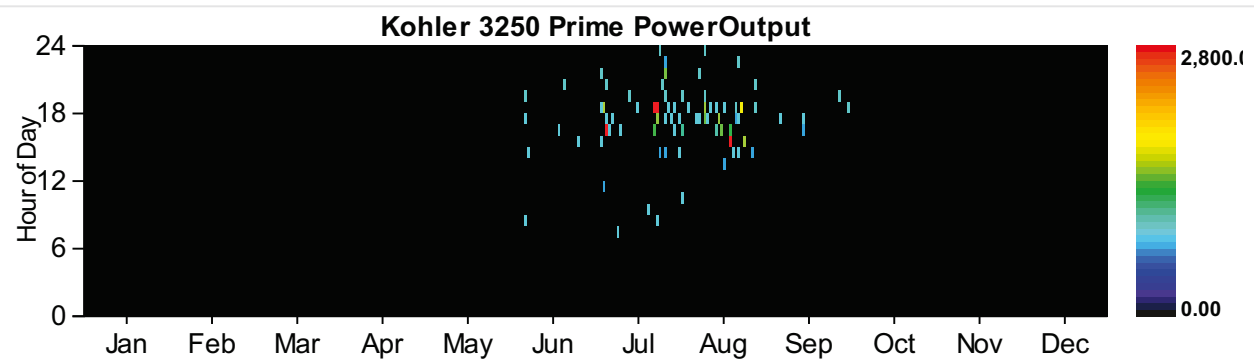

\section{Battery:GS200 flow}

\section{Quantity}

Value

String size

Strings in parallel

Batteries

Bus voltage

\section{Quantity}

Value

Units

Nominal capacity

600 kWh

Usable nominal capacity

Autonomy

600 kWh

Lifetime throughput

Battery wear cost

$0 \mathrm{hr}$

Average energy cost

$0.000 \$ / k W h$

Energy in

Energy out

Storage depletion

0

\section{Losses}

Annual throughput

Expected life

\begin{tabular}{|r|r|}
\hline 0.000 & $\$ / \mathrm{kWh}$ \\
\hline 0.010 & $\$ / \mathrm{kWh}$ \\
\hline 153371 & $\mathrm{kWh} / \mathrm{yr}$ \\
\hline 107862 & $\mathrm{kWh} / \mathrm{yr}$ \\
\hline 600 & $\mathrm{kWh} / \mathrm{yr}$ \\
\hline 44909 & $\mathrm{kWh} / \mathrm{yr}$ \\
\hline 128920 & $\mathrm{kWh} / \mathrm{yr}$ \\
\hline 25 & $\mathrm{yr}$ \\
\hline
\end{tabular}

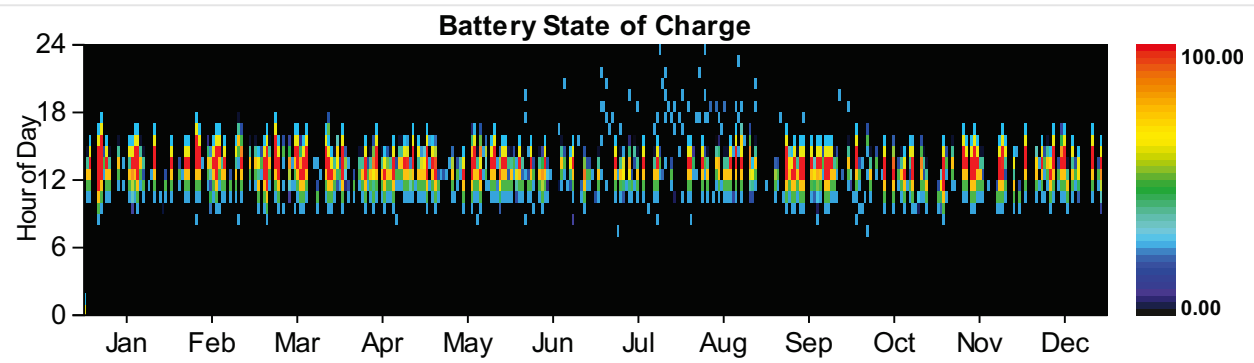

\section{Converter}

\section{Quantity}

Capacity

\section{Inverter}

Rectifier

Units

10,000




\begin{tabular}{|c|c|c|c|}
\hline Q日aneftyput & Inverter & Rectifier & kWits \\
\hline Minimum output & 0 & 0 & $\mathrm{~kW}$ \\
\hline Maximum output & 10,000 & 179 & $\mathrm{~kW}$ \\
\hline Capacity factor & 27 & 0 & $\%$ \\
\hline Hours of operation & 4,491 & 23 & $\mathrm{hrs} / \mathrm{yr}$ \\
\hline Energy in & $25,897,782$ & 4,561 & $\mathrm{kWh} / \mathrm{yr}$ \\
\hline Energy out & $23,307,908$ & 3,877 & $\mathrm{kWh} / \mathrm{yr}$ \\
\hline Losses & $2,589,874$ & 684 & $\mathrm{kWh} / \mathrm{yr}$ \\
\hline
\end{tabular}
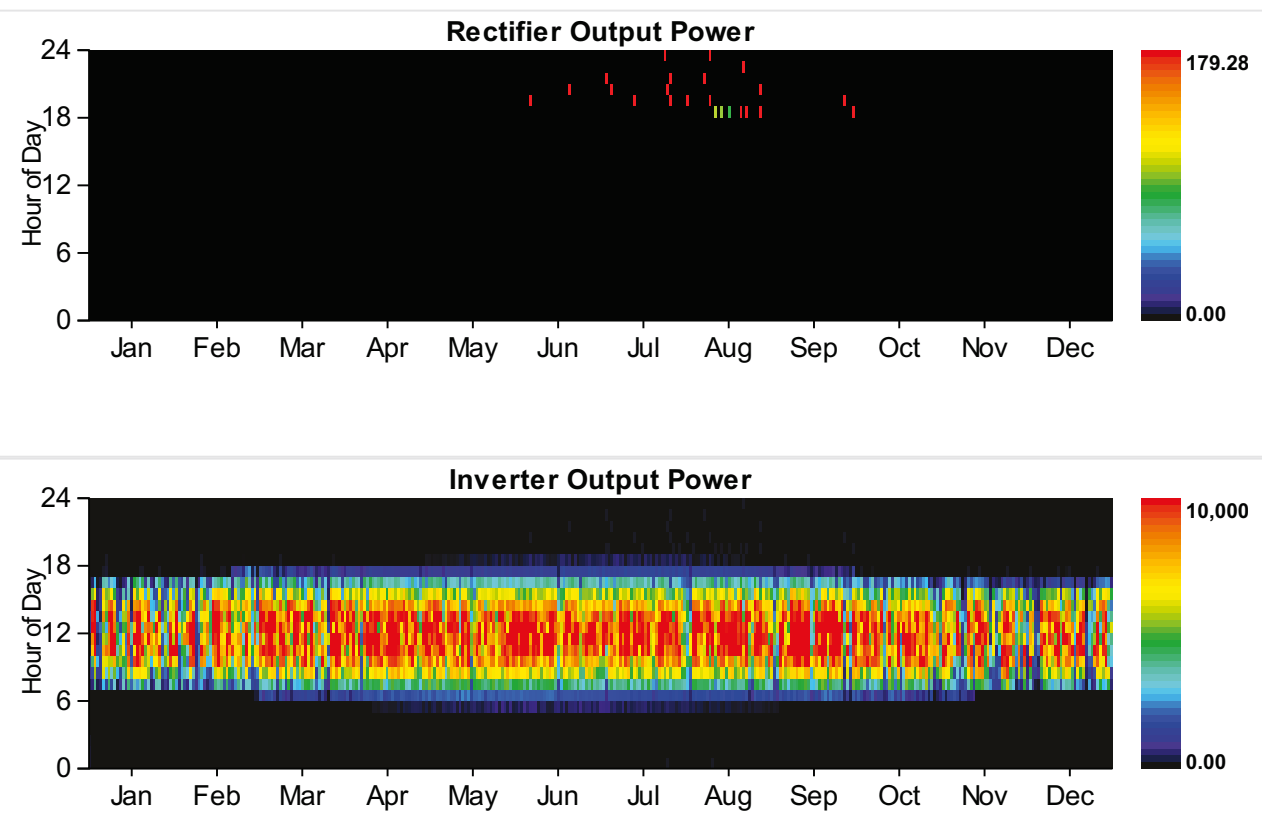

\section{Grid}

Rate: Rate 1 Demand

\begin{tabular}{|c|c|c|c|c|c|c|}
\hline Resources.ReportingService_GeneratelnputsReport_Month & $\begin{array}{l}\text { Energy } \\
\text { Purchased } \\
\text { (kWh) }\end{array}$ & $\begin{array}{l}\text { Energy } \\
\text { Sold } \\
\text { (kWh) }\end{array}$ & $\begin{array}{l}\text { Net } \\
\text { Purchases } \\
\text { (kWh) }\end{array}$ & $\begin{array}{l}\text { Peak } \\
\text { Demand } \\
(\mathbf{k W})\end{array}$ & $\begin{array}{l}\text { Energy } \\
\text { Charge } \\
\text { (\$) }\end{array}$ & $\begin{array}{l}\text { Demand } \\
\text { Charge } \\
(\$)\end{array}$ \\
\hline January & 0 & 0 & 0 & 17,079 & 0 & 273,262 \\
\hline February & 0 & 0 & 0 & 15,668 & 0 & 250,685 \\
\hline March & 0 & 0 & 0 & 17,075 & 0 & 273,199 \\
\hline April & 0 & 0 & 0 & 14,394 & 0 & 230,299 \\
\hline May & 0 & 0 & 0 & 14,850 & 0 & 237,602 \\
\hline June & 0 & 0 & 0 & 19,319 & 0 & 309,105 \\
\hline July & 0 & 0 & 0 & 20,000 & 0 & 320,000 \\
\hline August & 0 & 0 & 0 & 20,000 & 0 & 320,000 \\
\hline September & 0 & 0 & 0 & 18,133 & 0 & 290,121 \\
\hline October & 0 & 0 & 0 & 16,050 & 0 & 256,794 \\
\hline
\end{tabular}




\begin{tabular}{|c|c|c|c|c|c|c|}
\hline November & Energy 0 & Energy & Net 0 & Peak751 & Energy & DéรFâAd8 \\
\hline $\begin{array}{l}\text { December } \\
\text { Resources.ReportingService_GeneratelnputsReport_Month }\end{array}$ & $\begin{array}{l}\text { Purchased } \\
\text { (kWh) }\end{array}$ & $\begin{array}{l}\text { Sold } 0 \\
\text { (kWh) }\end{array}$ & $\begin{array}{l}\text { Purchases } \\
\text { (kWh) }\end{array}$ & $\begin{array}{l}\text { Demanad } \\
\text { (kW) }\end{array}$ & $\begin{array}{l}\text { Charge } \\
\text { (\$) }\end{array}$ & $\begin{array}{l}\text { Changs } 66 \\
\text { (\$) }\end{array}$ \\
\hline Annual & 0 & 0 & 0 & 20,000 & 0 & $3,287,451$ \\
\hline
\end{tabular}

Rate: Rate 1

\begin{tabular}{|c|c|c|c|c|c|c|}
\hline Resources.ReportingService_GeneratelnputsReport_Month & $\begin{array}{l}\text { Energy } \\
\text { Purchased } \\
\text { (kWh) }\end{array}$ & $\begin{array}{l}\text { Energy } \\
\text { Sold } \\
\text { (kWh) }\end{array}$ & $\begin{array}{l}\text { Net } \\
\text { Purchases } \\
\text { (kWh) }\end{array}$ & $\begin{array}{l}\text { Peak } \\
\text { Demand } \\
(\mathbf{k W})\end{array}$ & $\begin{array}{l}\text { Energy } \\
\text { Charge } \\
(\$)\end{array}$ & $\begin{array}{l}\text { Demand } \\
\text { Charge } \\
\text { (\$) }\end{array}$ \\
\hline January & $5,801,195$ & 0 & $5,801,195$ & 0 & 696,143 & 0 \\
\hline February & $4,740,909$ & 0 & $4,740,909$ & 0 & 568,909 & 0 \\
\hline March & $5,114,871$ & 0 & $5,114,871$ & 0 & 613,785 & 0 \\
\hline April & $4,751,667$ & 0 & $4,751,667$ & 0 & 570,200 & 0 \\
\hline May & $4,801,942$ & 0 & $4,801,942$ & 0 & 576,233 & 0 \\
\hline June & $6,626,605$ & 0 & $6,626,605$ & 0 & 795,193 & 0 \\
\hline July & $8,031,922$ & 0 & $8,031,922$ & 0 & 963,831 & 0 \\
\hline August & $8,147,624$ & 0 & $8,147,624$ & 0 & 977,715 & 0 \\
\hline September & $6,807,550$ & 0 & $6,807,550$ & 0 & 816,906 & 0 \\
\hline October & $5,624,914$ & 0 & $5,624,914$ & 0 & 674,990 & 0 \\
\hline November & $5,310,932$ & 0 & $5,310,932$ & 0 & 637,312 & 0 \\
\hline December & $5,904,472$ & 0 & $5,904,472$ & 0 & 708,537 & 0 \\
\hline Annual & $71,664,592$ & 0 & $71,664,592$ & 0 & $8,599,752$ & 0 \\
\hline
\end{tabular}

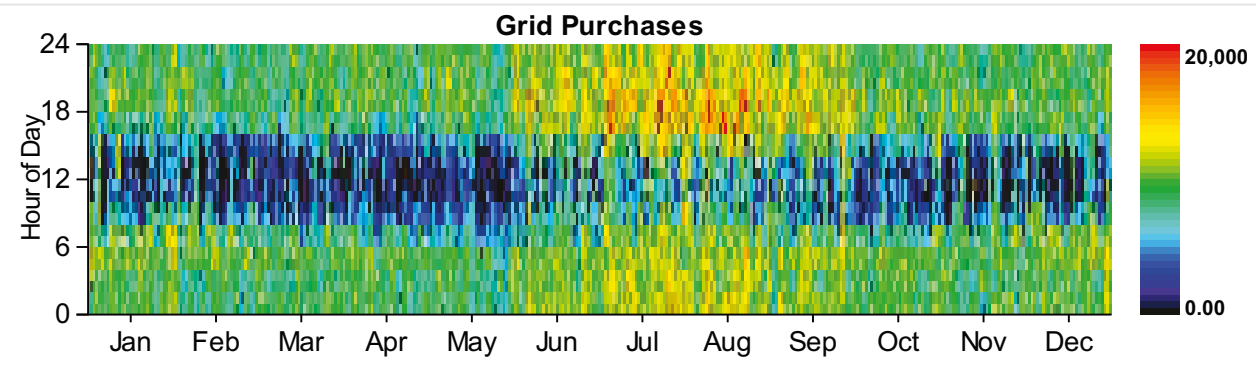

\section{Emissions}

\section{Pollutant}

Carbon dioxide

Carbon monoxide

Unburned hydrocarbons

Particulate matter

Sulfur dioxide

Nitrogen oxides
Emissions

Units

$45344908 \mathrm{~kg} / \mathrm{yr}$

$222 \mathrm{~kg} / \mathrm{yr}$

$25 \mathrm{~kg} / \mathrm{yr}$

$6 \mathrm{~kg} / \mathrm{yr}$

$196470 \mathrm{~kg} / \mathrm{yr}$

$96252 \mathrm{~kg} / \mathrm{yr}$ 
System Report

System architecture

\begin{tabular}{|l|l|r|}
\hline PV & SunPower PV & 13,780 \\
\hline PV \#2 & Remaining Generic flat plate PV & 1,547 \\
\hline Wind Turbine & Siemens $2.3 \mathrm{~kW}-108$ & 1 \\
\hline Battery & GS200 flow & 1 strings \\
\hline Converter & System Converter & $10,000 \mathrm{~kW}$ \\
\hline Grid & Grid & $27,200 \mathrm{~kW}$ \\
\hline Dispatch Strategy & Cycle Charging & $\mathrm{k}$ \\
\hline
\end{tabular}

\section{Cost summary}

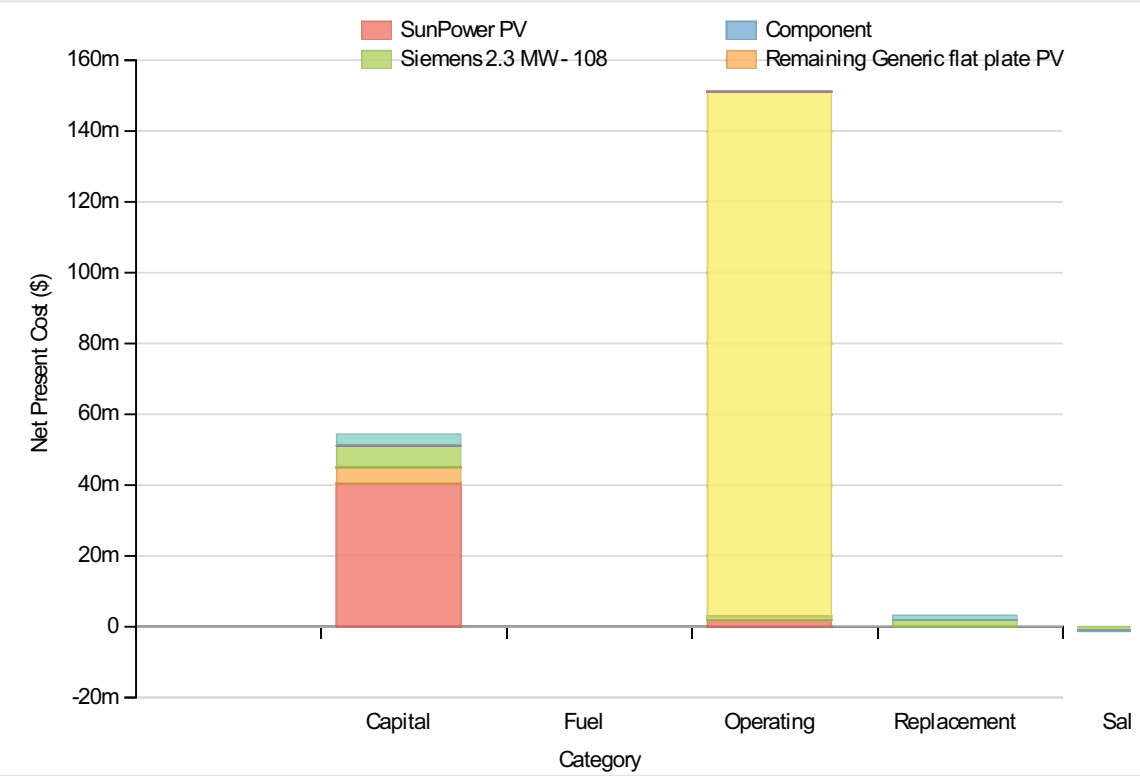

Cost Summary

Total net present cost

207672672 \$

Levelized cost of energy

$0.169 \$ / k W h$

Net Present Costs

\begin{tabular}{|c|c|c|c|c|c|c|}
\hline Component & Capital & Replacement & O\&M & Fuel & Salvage & Total \\
\hline SunPower PV & $40,358,332$ & 0 & $1,656,876$ & 0 & 0 & $42,015,208$ \\
\hline Remaining Generic flat plate PV & $4,678,459$ & 0 & 339,013 & 0 & 0 & $5,017,472$ \\
\hline Siemens 2.3 MW - 108 & $6,000,000$ & $1,912,842$ & 969,563 & 0 & $-1,078,009$ & $7,804,396$ \\
\hline Grid & 0 & 0 & $148,101,408$ & 0 & 0 & $148,101,408$ \\
\hline GS200 flow & 324,589 & 7,176 & 31,026 & 0 & -973 & 361,818 \\
\hline Converter & $3,000,000$ & $1,272,821$ & 0 & 0 & $-239,558$ & $4,033,263$ \\
\hline System & $54,361,380$ & $3,192,838$ & $151,097,872$ & 0 & $-1,318,539$ & $207,333,551$ \\
\hline
\end{tabular}




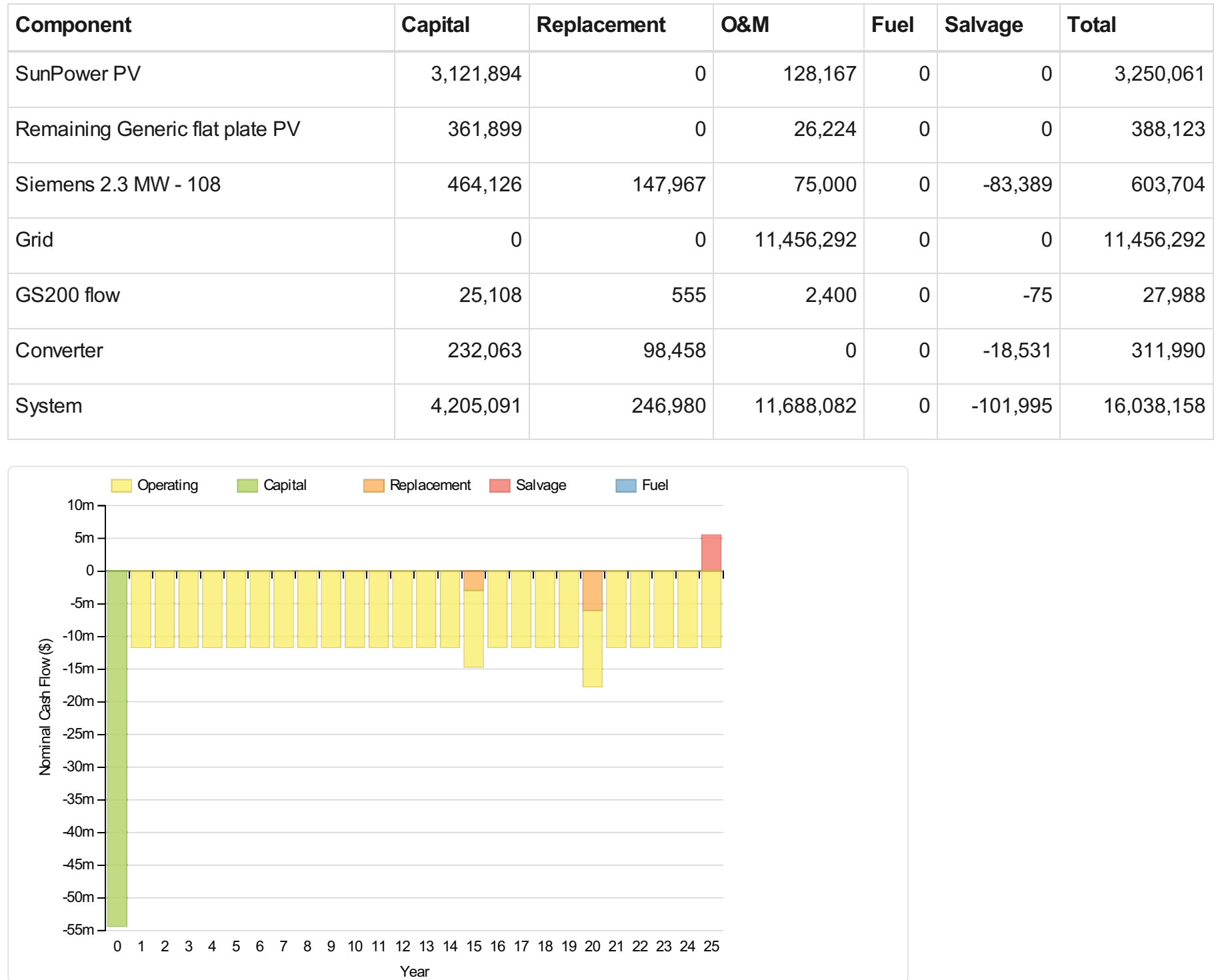

\section{Electrical}

\section{Quantity}

Excess electricity

Unmet load

Capacity shortage

Renewable fraction

Component

PV

PV

Wind Turbine

Grid Purchases

Total

Load

Consumption(kWh/yr)

\section{Production(kWh/yr)}

\section{Value}$$
1937470 \mathrm{kWh} / \mathrm{yr}
$$$$
0 \mathrm{kWh} / \mathrm{yr}
$$$$
0 \mathrm{kWh} / \mathrm{yr}
$$

0

Fraction (\%)

$2,766,525$ 


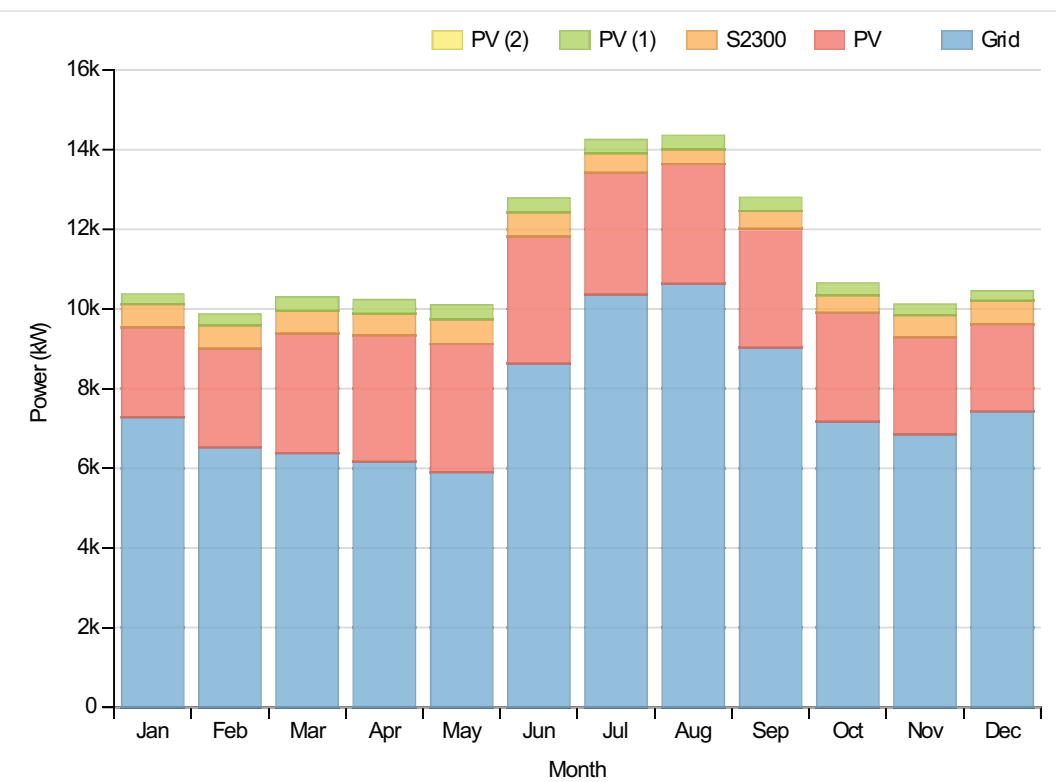

PV:SunPower PV

\begin{tabular}{|c|c|c|}
\hline Quantity & Value & Units \\
\hline Rated capacity & 13780 & $\mathrm{~kW}$ \\
\hline Mean output & 2813 & $\mathrm{~kW}$ \\
\hline Mean output & 67519.00 & $\mathrm{kWh} / \mathrm{d}$ \\
\hline Capacity factor & 20.42 & $\%$ \\
\hline Total production & 24644568 & $\mathrm{kWh} / \mathrm{yr}$ \\
\hline Minimum output & 0.00 & $\mathrm{~kW}$ \\
\hline Maximum output & 13941.00 & $\mathrm{~kW}$ \\
\hline PV penetration & 25.93 & $\%$ \\
\hline Hours of operation & 4386 & hrs/yr \\
\hline Levelized cost & 0.119 & $\$ / \mathrm{kWh}$ \\
\hline
\end{tabular}

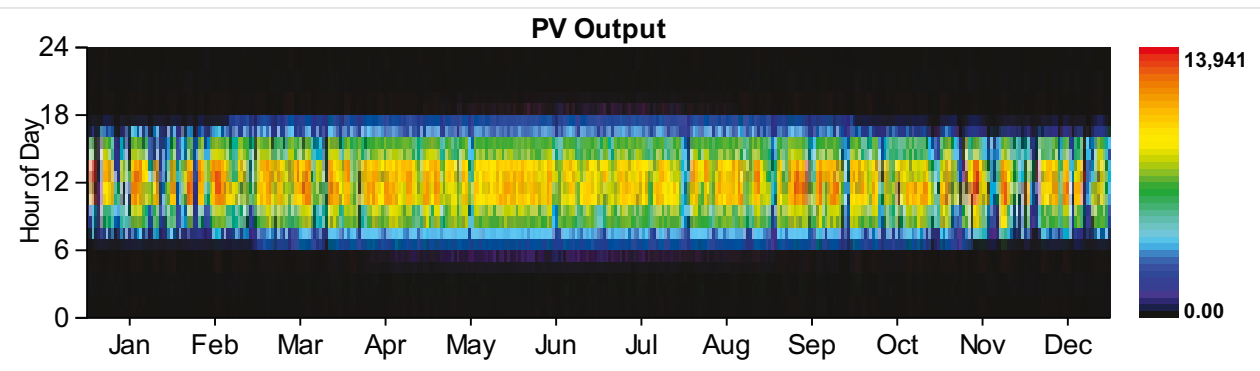

\section{PV:Remaining Generic flat plate PV}

\section{Quantity}

Rated capacity

Mean output

Mean output

\section{Value}

Units

1547 kW

$316 \mathrm{~kW}$ 


\begin{tabular}{|c|c|c|c|}
\hline 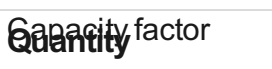 & Value & 20.42 & Units \\
\hline Total production & & 2766525 & $\mathrm{kWh} / \mathrm{yr}$ \\
\hline Minimum output & & 0.00 & $\mathrm{~kW}$ \\
\hline Maximum output & & 1565.00 & $\mathrm{~kW}$ \\
\hline PV penetration & & 2.91 & $\%$ \\
\hline Hours of operation & & 4386 & $\mathrm{hrs} / \mathrm{yr}$ \\
\hline Levelized cost & & 0.014 & $\$ / k W h$ \\
\hline
\end{tabular}

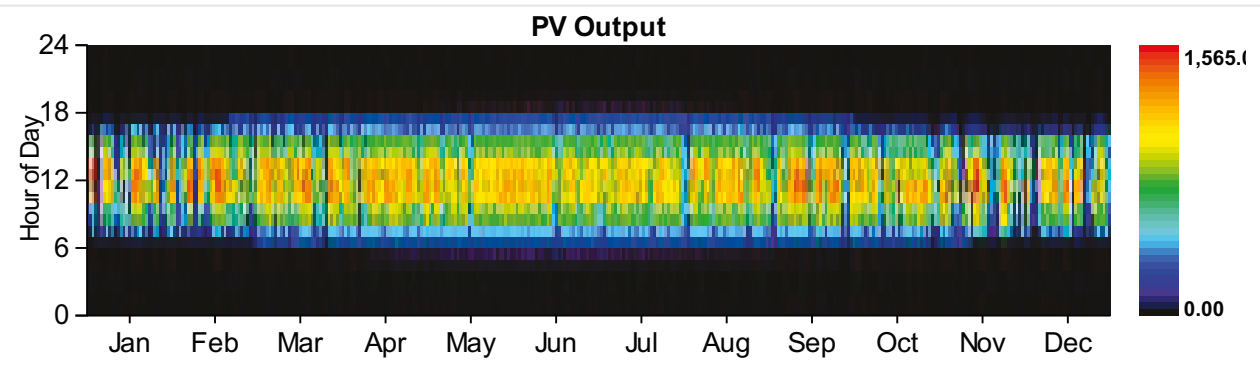

Wind Turbine:Siemens 2.3 MW - 108

\section{Quantity}

Total rated capacity

Mean output

Capacity factor

Total production

Minimum output

Maximum output

Wind penetration

Hours of operation

Levelized cost

\begin{tabular}{|r|r|}
\hline Value & Units \\
\hline 2300 & $\mathrm{~kW}$ \\
\hline 534 & $\mathrm{~kW}$ \\
\hline 23.20 & $\%$ \\
\hline 4674352 & $\mathrm{kWh} / \mathrm{yr}$ \\
\hline 0.49 & $\mathrm{~kW}$ \\
\hline 2312.70 & $\mathrm{~kW}$ \\
\hline 4.92 & $\%$ \\
\hline 8760 & $\mathrm{hrs} / \mathrm{yr}$ \\
\hline 0.129 & $\$ / \mathrm{kWh}$ \\
\hline
\end{tabular}

\section{Battery:GS200 flow}

\section{Quantity}

String size

Strings in parallel

Batteries

Bus voltage

\section{Value}

Quantity

Value

Units

Nominal capacity

Usable nominal capacity
$600 \mathrm{kWh}$

$600 \mathrm{kWh}$ 


\section{Qutentivy}

Lifetime throughput

Battery wear cost

Average energy cost

Energy in

Energy out

Storage depletion

Losses

Annual throughput

Expected life

\section{Value}

0 Units

0

\begin{tabular}{|r|l|}
\hline 0.000 & $\$ / \mathrm{kWh}$ \\
\hline 0.000 & $\$ / \mathrm{kWh}$ \\
\hline 154117 & $\mathrm{kWh} / \mathrm{yr}$ \\
\hline 108384 & $\mathrm{kWh} / \mathrm{yr}$ \\
\hline 600 & $\mathrm{kWh} / \mathrm{yr}$ \\
\hline 45132 & $\mathrm{kWh} / \mathrm{yr}$ \\
\hline 129544 & $\mathrm{kWh} / \mathrm{yr}$ \\
\hline 25 & $\mathrm{yr}$ \\
\hline
\end{tabular}

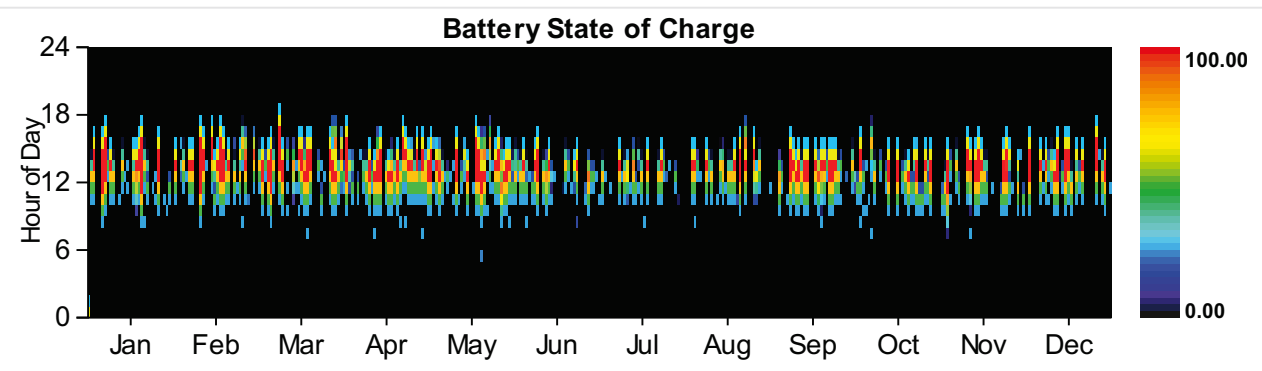

\section{Converter}

\begin{tabular}{|l|r|r|r|}
\hline Quantity & Inverter & Rectifier & Units \\
\hline Capacity & 10,000 & 9,000 & $\mathrm{~kW}$ \\
\hline Mean output & 2,612 & 0 & $\mathrm{~kW}$ \\
\hline Minimum output & 0 & $0 \mathrm{~kW}$ \\
\hline Maximum output & 10,000 & 0 & $\mathrm{~kW}$ \\
\hline Capacity factor & 26 & 0 & $\%$ \\
\hline Hours of operation & 4,575 & 48 & $\mathrm{hrs} / \mathrm{yr}$ \\
\hline Energy in & $25,427,954$ & 0 & $\mathrm{kWh} / \mathrm{yr}$ \\
\hline Energy out & $22,885,056$ & 0 & $\mathrm{kWh} / \mathrm{yr}$ \\
\hline Losses & $2,542,898$ & $\mathrm{kWh} / \mathrm{yr}$ \\
\hline
\end{tabular}

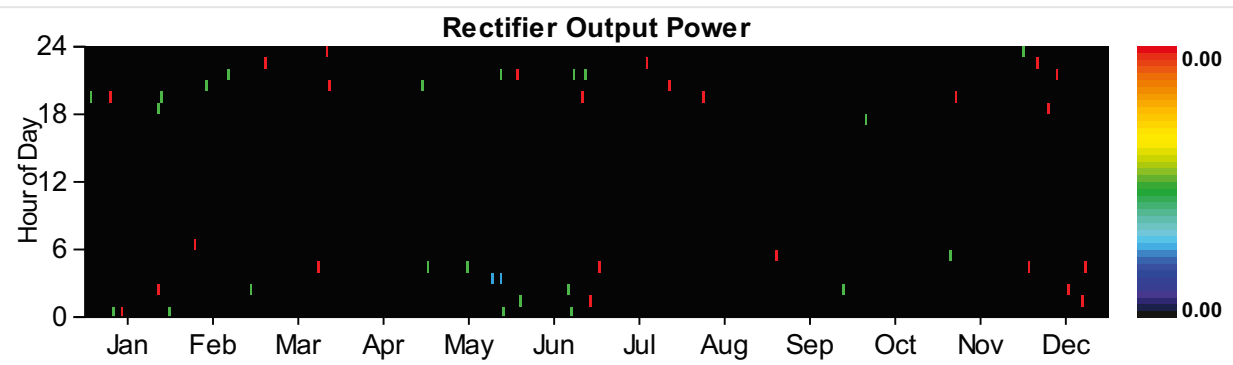




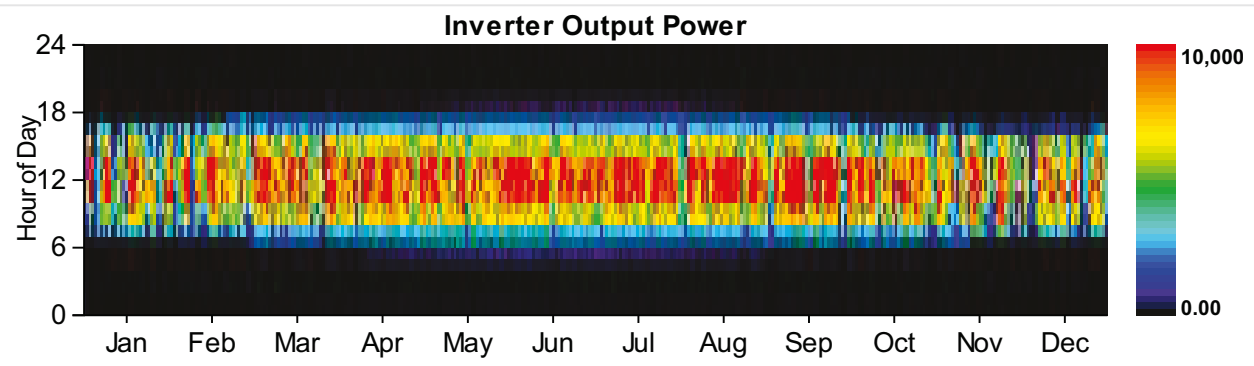

Grid

Rate: Rate 1 Demand

\begin{tabular}{|c|c|c|c|c|c|c|}
\hline Resources.ReportingService_GeneratelnputsReport_Month & $\begin{array}{l}\text { Energy } \\
\text { Purchased } \\
\text { (kWh) }\end{array}$ & $\begin{array}{l}\text { Energy } \\
\text { Sold } \\
\text { (kWh) }\end{array}$ & $\begin{array}{l}\text { Net } \\
\text { Purchases } \\
\text { (kWh) }\end{array}$ & $\begin{array}{l}\text { Peak } \\
\text { Demand } \\
(\mathbf{k W})\end{array}$ & $\begin{array}{l}\text { Energy } \\
\text { Charge } \\
\text { (\$) }\end{array}$ & $\begin{array}{l}\text { Demand } \\
\text { Charge } \\
(\$)\end{array}$ \\
\hline January & 0 & 0 & 0 & 16,288 & 0 & 260,605 \\
\hline February & 0 & 0 & 0 & 15,654 & 0 & 250,459 \\
\hline March & 0 & 0 & 0 & 17,043 & 0 & 272,688 \\
\hline April & 0 & 0 & 0 & 14,366 & 0 & 229,856 \\
\hline May & 0 & 0 & 0 & 13,700 & 0 & 219,202 \\
\hline June & 0 & 0 & 0 & 20,004 & 0 & 320,060 \\
\hline July & 0 & 0 & 0 & 23,789 & 0 & 380,622 \\
\hline August & 0 & 0 & 0 & 22,258 & 0 & 356,132 \\
\hline September & 0 & 0 & 0 & 18,548 & 0 & 296,765 \\
\hline October & 0 & 0 & 0 & 16,023 & 0 & 256,374 \\
\hline November & 0 & 0 & 0 & 15,278 & 0 & 244,445 \\
\hline December & 0 & 0 & 0 & 16,870 & 0 & 269,913 \\
\hline Annual & 0 & 0 & 0 & 23,789 & 0 & $3,357,119$ \\
\hline
\end{tabular}

Rate: Rate 1

\begin{tabular}{|c|c|c|c|c|c|c|}
\hline Resources.ReportingService_GeneratelnputsReport_Month & $\begin{array}{l}\text { Energy } \\
\text { Purchased } \\
\text { (kWh) }\end{array}$ & $\begin{array}{l}\text { Energy } \\
\text { Sold } \\
\text { (kWh) }\end{array}$ & $\begin{array}{l}\text { Net } \\
\text { Purchases } \\
\text { (kWh) }\end{array}$ & $\begin{array}{l}\text { Peak } \\
\text { Demand } \\
(\mathbf{k W})\end{array}$ & $\begin{array}{l}\text { Energy } \\
\text { Charge } \\
\text { (\$) }\end{array}$ & $\begin{array}{l}\text { Demand } \\
\text { Charge } \\
(\$)\end{array}$ \\
\hline January & $5,419,880$ & 0 & $5,419,880$ & 0 & 650,386 & 0 \\
\hline February & $4,381,999$ & 0 & $4,381,999$ & 0 & 525,840 & 0 \\
\hline March & $4,743,226$ & 0 & $4,743,226$ & 0 & 569,187 & 0 \\
\hline April & $4,435,979$ & 0 & $4,435,979$ & 0 & 532,317 & 0 \\
\hline May & $4,393,103$ & 0 & $4,393,103$ & 0 & 527,172 & 0 \\
\hline June & $6,213,113$ & 0 & $6,213,113$ & 0 & 745,574 & 0 \\
\hline July & $7,708,083$ & 0 & $7,708,083$ & 0 & 924,970 & 0 \\
\hline August & $7,908,765$ & 0 & $7,908,765$ & 0 & 949,052 & 0 \\
\hline
\end{tabular}




\begin{tabular}{|c|c|c|c|c|c|c|}
\hline $\begin{array}{l}\text { September } \\
\text { Rectoberrces.ReportingService_GeneratelnputsReport_Month }\end{array}$ & $\begin{array}{l}\text { EÁerge, } 302 \\
\text { Purchased } \\
\text { (kwh) } \\
\text { (kw3,870 }\end{array}$ & $\begin{array}{l}\text { Energ9 } \\
\text { Sold } \\
(\mathbf{k W h})^{0}\end{array}$ & $\begin{array}{l}\text { Nêt496,302 } \\
\text { Purchases } \\
\text { (kWh) }\end{array}$ & $\begin{array}{l}\text { Peak } 0 \\
\text { Demand } \\
(\mathbf{k W})\end{array}$ & $\begin{array}{l}\text { Eherg } 556 \\
\text { Charge } \\
(\$)^{40,064}\end{array}$ & $\begin{array}{l}\text { Demand } \\
\text { Charge } \\
(\$)\end{array}$ \\
\hline November & $4,934,859$ & 0 & $4,934,859$ & 0 & 592,183 & 0 \\
\hline December & $5,523,998$ & 0 & $5,523,998$ & 0 & 662,880 & 0 \\
\hline Annual & $67,493,176$ & 0 & $67,493,176$ & 0 & $8,099,180$ & 0 \\
\hline
\end{tabular}

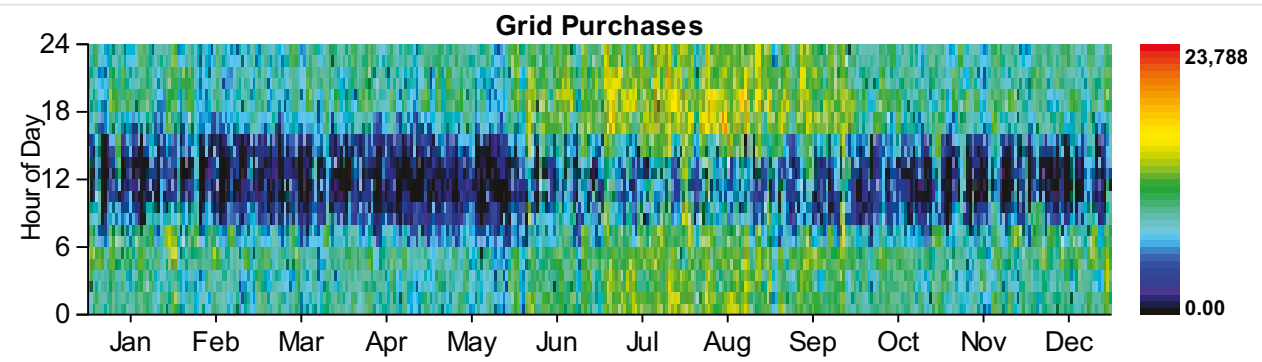

\section{Emissions}

\begin{tabular}{|l|r|l|}
\hline Pollutant & Emissions & Units \\
\hline Carbon dioxide & 42655688 & $\mathrm{~kg} / \mathrm{yr}$ \\
\hline Carbon monoxide & 0 & $\mathrm{~kg} / \mathrm{yr}$ \\
\hline Unburned hydrocarbons & 0 & $\mathrm{~kg} / \mathrm{yr}$ \\
\hline Particulate matter & 0 & $\mathrm{~kg} / \mathrm{yr}$ \\
\hline Sulfur dioxide & $184931 \mathrm{~kg} / \mathrm{yr}$ \\
\hline Nitrogen oxides & $90441 \mathrm{~kg} / \mathrm{yr}$ \\
\hline
\end{tabular}


System Report

System architecture

\begin{tabular}{|c|c|c|c|}
\hline PV & SunPower PV & 13,780 & $\mathrm{~kW}$ \\
\hline PV \#2 & Remaining Generic flat plate PV & 1,547 & $\mathrm{~kW}$ \\
\hline Wind Turbine & Siemens $2.3 \mathrm{MW}-108$ & 1 & \\
\hline Generator & Kohler 3250 Prime Power & 2,800 & $\mathrm{~kW}$ \\
\hline Converter & System Converter & 10,000 & $\mathrm{~kW}$ \\
\hline Grid & Grid & 20,000 & $\mathrm{~kW}$ \\
\hline Dispatch Strategy & Cycle Charging & & \\
\hline
\end{tabular}

\section{Cost summary}

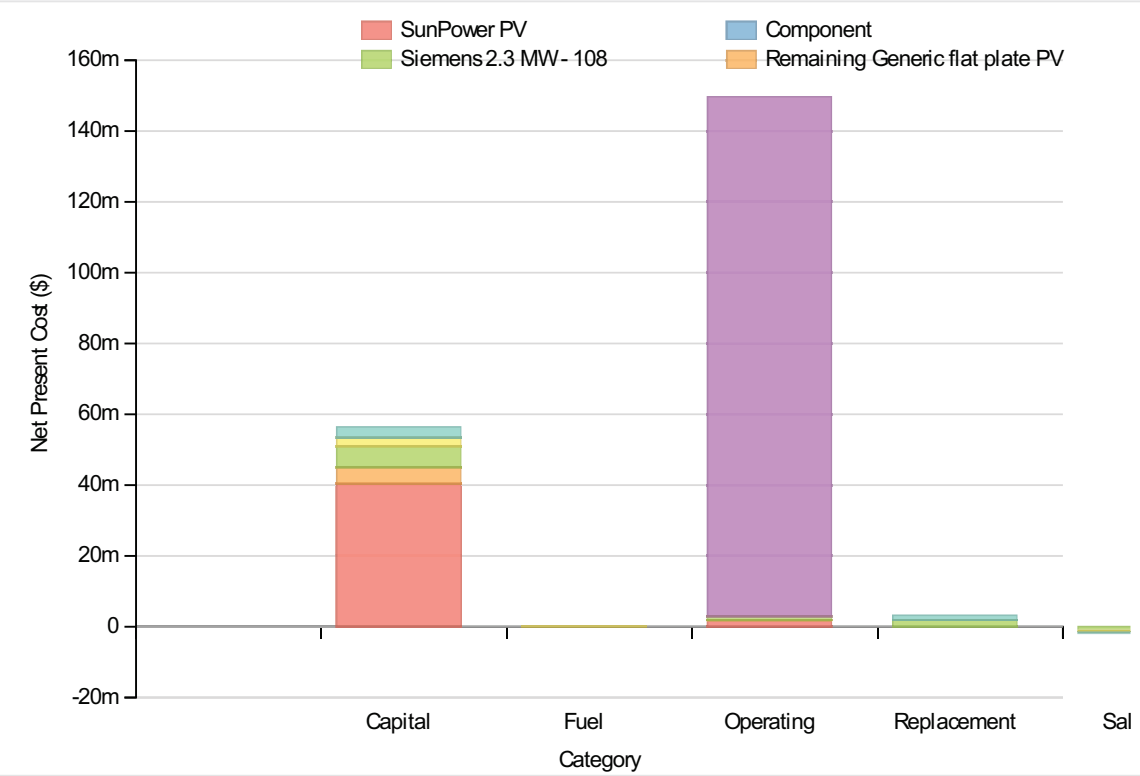

Cost Summary

Total net present cost

$207858560 \$$

Levelized cost of energy

$0.169 \$ / k W h$

Net Present Costs

\begin{tabular}{|c|c|c|c|c|c|c|}
\hline Component & Capital & Replacement & O\&M & Fuel & Salvage & Total \\
\hline SunPower PV & $40,358,332$ & 0 & $1,656,876$ & 0 & 0 & $42,015,208$ \\
\hline Remaining Generic flat plate PV & $4,678,459$ & 0 & 339,013 & 0 & 0 & $5,017,472$ \\
\hline Siemens 2.3 MW - 108 & $6,000,000$ & $1,912,842$ & 969,563 & 0 & $-1,078,009$ & $7,804,396$ \\
\hline Kohler 3250 Prime Power & $2,350,000$ & 0 & 34,819 & 136,867 & $-500,096$ & $2,021,590$ \\
\hline Grid & 0 & 0 & $146,627,520$ & 0 & 0 & $146,627,520$ \\
\hline Converter & $3,000,000$ & $1,272,821$ & 0 & 0 & $-239,558$ & $4,033,263$ \\
\hline System & $56,386,792$ & $3,185,663$ & $149,627,760$ & 136,867 & $-1,817,663$ & $207,519,419$ \\
\hline
\end{tabular}




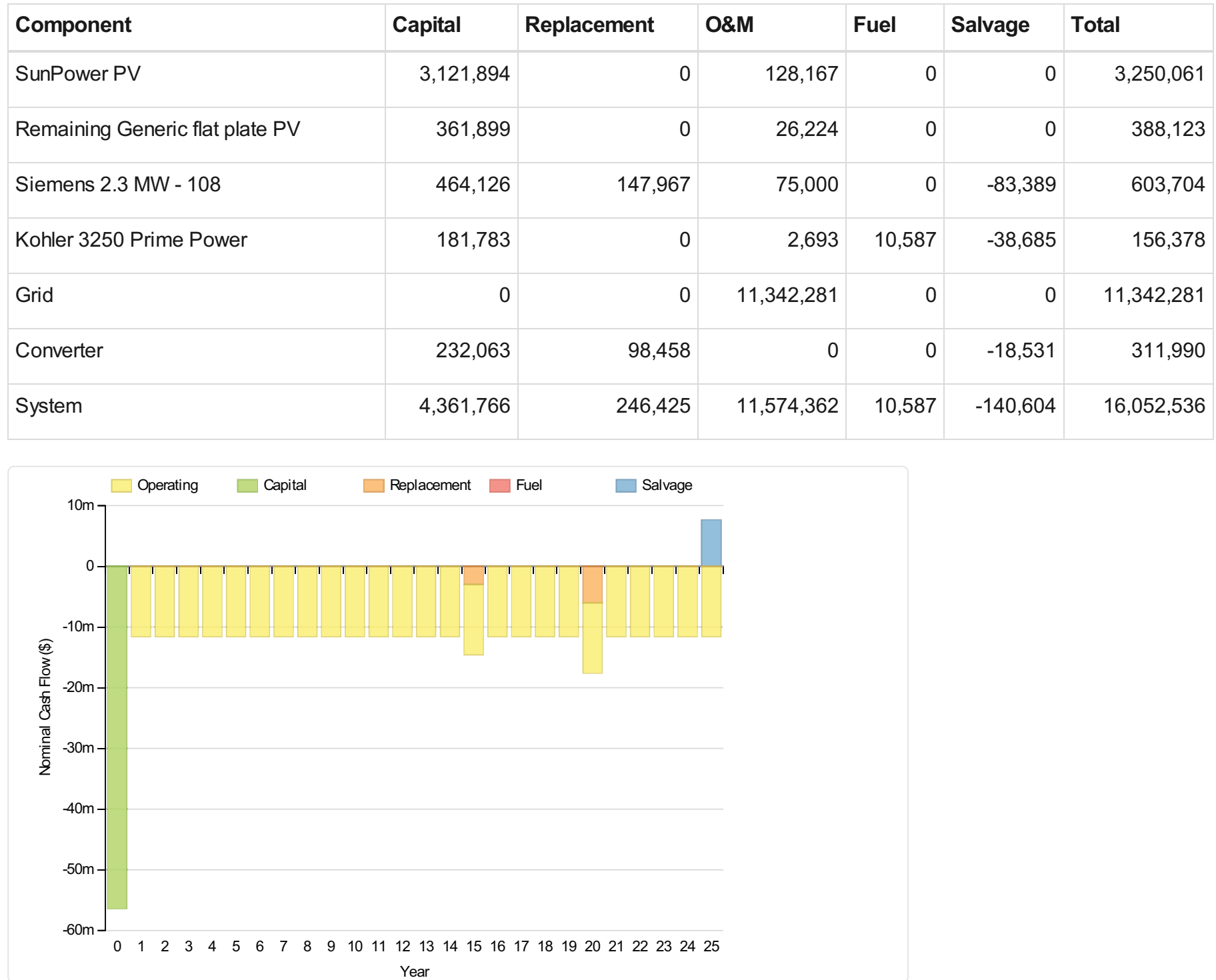

\section{Electrical}

\section{Quantity}

Excess electricity

Unmet load

Capacity shortage

Renewable fraction

\section{Component}

PV

PV

Generator

Wind Turbine

Grid Purchases

Total

\section{Value}

$2091590 \mathrm{kWh} / \mathrm{yr}$

$989 \mathrm{kWh} / \mathrm{yr}$
$26452 \mathrm{kWh} / \mathrm{yr}$

0

\section{Production(kWh/yr)}

$24,644,568$

Fraction (\%)

$2,766,525$

53,730 


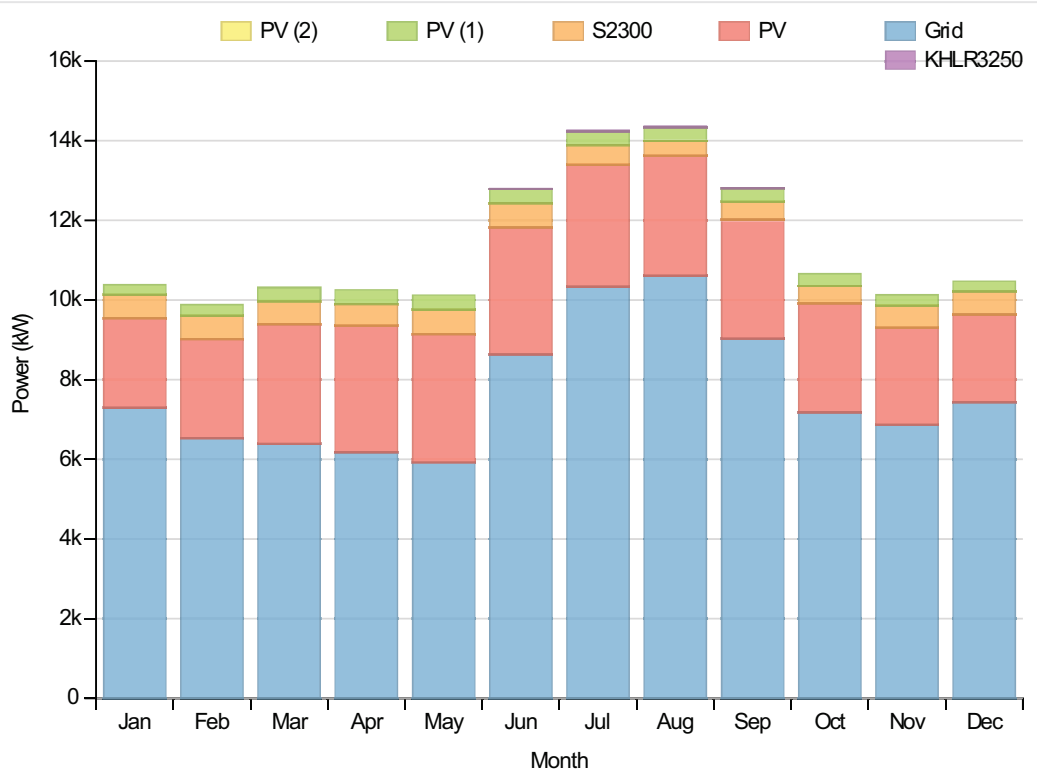

\section{PV:SunPower PV}

\begin{tabular}{|c|c|c|}
\hline Quantity & Value & Units \\
\hline Rated capacity & 13780 & $\mathrm{~kW}$ \\
\hline Mean output & 2813 & $\mathrm{~kW}$ \\
\hline Mean output & 67519.00 & $\mathrm{kWh} / \mathrm{d}$ \\
\hline Capacity factor & 20.42 & $\%$ \\
\hline Total production & 24644568 & $\mathrm{kWh} / \mathrm{yr}$ \\
\hline Minimum output & 0.00 & $\mathrm{~kW}$ \\
\hline Maximum output & 13941.00 & $\mathrm{~kW}$ \\
\hline PV penetration & 25.93 & $\%$ \\
\hline Hours of operation & 4386 & $\mathrm{hrs} / \mathrm{yr}$ \\
\hline Levelized cost & 0.119 & $\$ / k W h$ \\
\hline
\end{tabular}

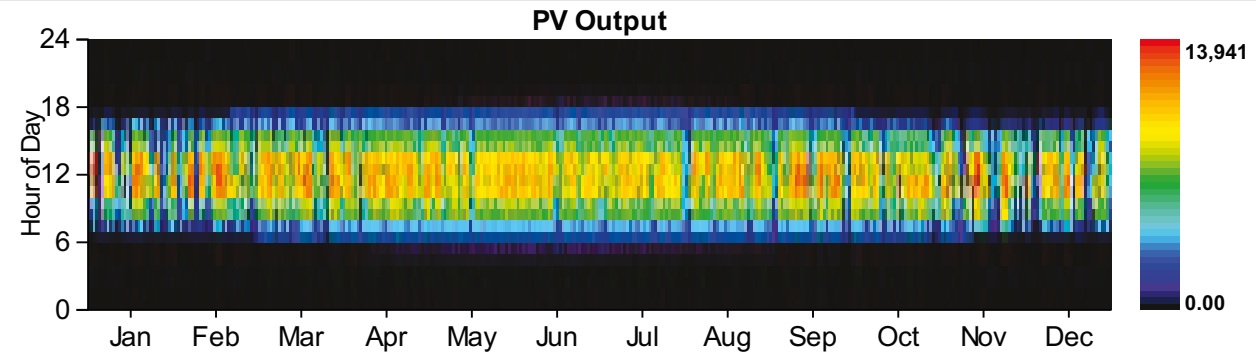

PV:Remaining Generic flat plate PV

\begin{tabular}{|l|c|c|}
\hline Quantity & Value & Units \\
\hline Rated capacity & $1547 \mathrm{~kW}$ \\
\hline Mean output & $316 \mathrm{~kW}$ \\
\hline
\end{tabular}


Qearnfityput

Capacity factor

Total production

Minimum output

Maximum output

PV penetration

Hours of operation

Levelized cost
7579.50 kflifts

$20.42 \%$

$2766525 \mathrm{kWh} / \mathrm{yr}$

$0.00 \mathrm{~kW}$

$1565.00 \mathrm{~kW}$

$2.91 \%$

$4386 \mathrm{hrs} / \mathrm{yr}$

$0.014 \$ / \mathrm{kWh}$

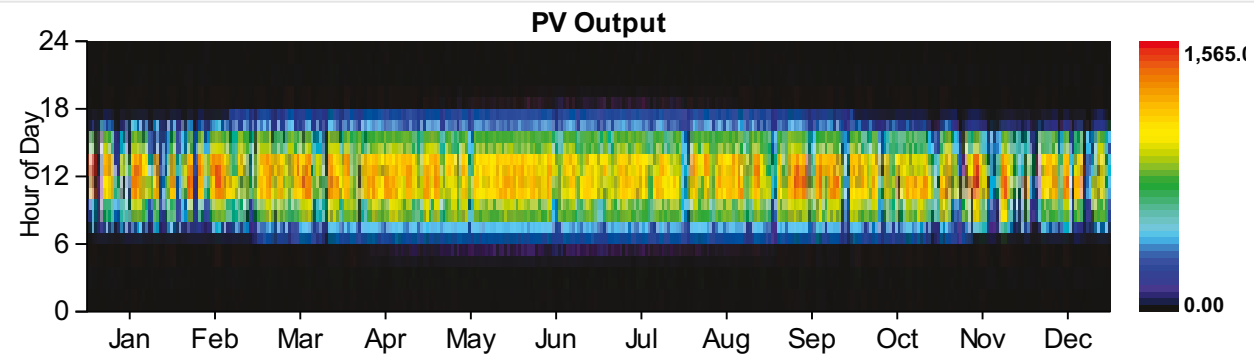

Wind Turbine:Siemens 2.3 MW - 108

\begin{tabular}{|c|c|c|}
\hline Quantity & Value & Units \\
\hline Total rated capacity & 2300 & $\mathrm{~kW}$ \\
\hline Mean output & 534 & $\mathrm{~kW}$ \\
\hline Capacity factor & 23.20 & $\%$ \\
\hline Total production & 4674352 & $\mathrm{kWh} / \mathrm{yr}$ \\
\hline Minimum output & 0.49 & $\mathrm{~kW}$ \\
\hline Maximum output & 2312.70 & $\mathrm{~kW}$ \\
\hline Wind penetration & 4.92 & $\%$ \\
\hline Hours of operation & 8760 & $\mathrm{hrs} / \mathrm{yr}$ \\
\hline Levelized cost & 0.129 & $\$ / \mathrm{kWh}$ \\
\hline
\end{tabular}

\section{Generator:Kohler 3250 Prime Power}

\section{Quantity}

Hours of operation

Number of starts

Operational life

Fixed generation cost

Marginal generation cost

Electrical production
Value

\section{Units}

$67 \mathrm{hrs} / \mathrm{yr}$

61 starts/yr

$224 \mathrm{yr}$

$207.82 \$ / h r$

$0.18 \$ / k W h$

$53730 \mathrm{kWh} / \mathrm{yr}$ 
Qeanetectrical output

Min. electrical output

Max. electrical output

Fuel consumption

Specific fuel consumption

Fuel energy input

Mean electrical efficiency
802 JNits

700 kW

$2800 \mathrm{~kW}$

13402 L/yr

$0.25 \mathrm{~L} / \mathrm{kWh}$

$131872 \mathrm{kWh} / \mathrm{yr}$

$41 \%$

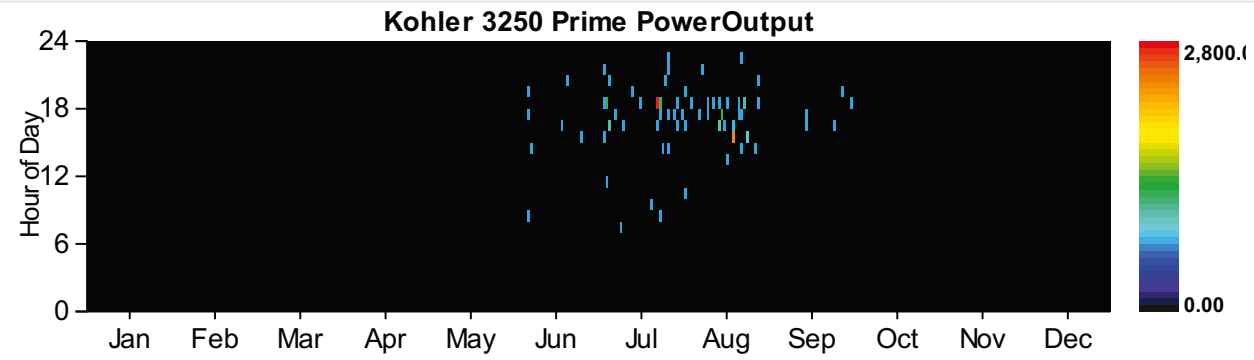

Converter

\begin{tabular}{|c|c|c|c|}
\hline Quantity & Inverter & Rectifier & Units \\
\hline Capacity & 10,000 & 9,000 & $\mathrm{~kW}$ \\
\hline Mean output & 2,601 & 0 & $\mathrm{~kW}$ \\
\hline Minimum output & 0 & 0 & $\mathrm{~kW}$ \\
\hline Maximum output & 10,000 & 0 & $\mathrm{~kW}$ \\
\hline Capacity factor & 26 & 0 & $\%$ \\
\hline Hours of operation & 4,386 & 0 & $\mathrm{hrs} / \mathrm{yr}$ \\
\hline Energy in & $25,319,570$ & 0 & $\mathrm{kWh} / \mathrm{yr}$ \\
\hline Energy out & $22,787,530$ & 0 & $\mathrm{kWh} / \mathrm{yr}$ \\
\hline Losses & $2,532,040$ & 0 & $\mathrm{kWh} / \mathrm{yr}$ \\
\hline
\end{tabular}

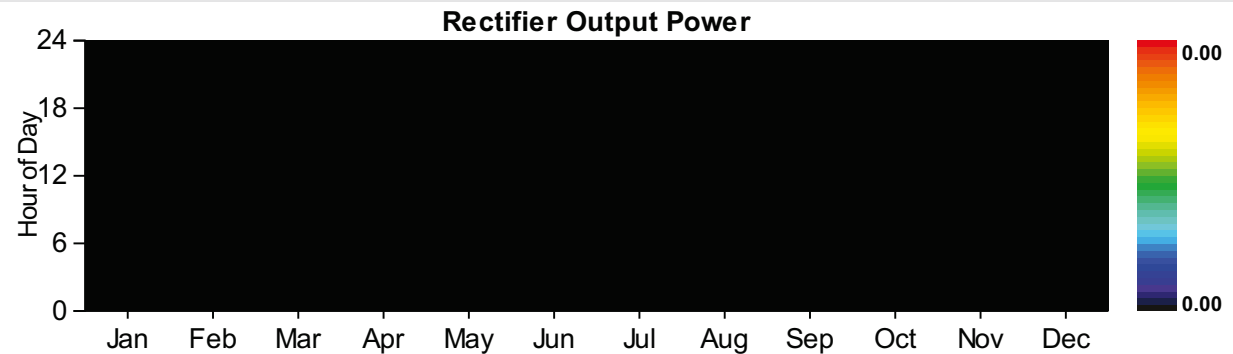




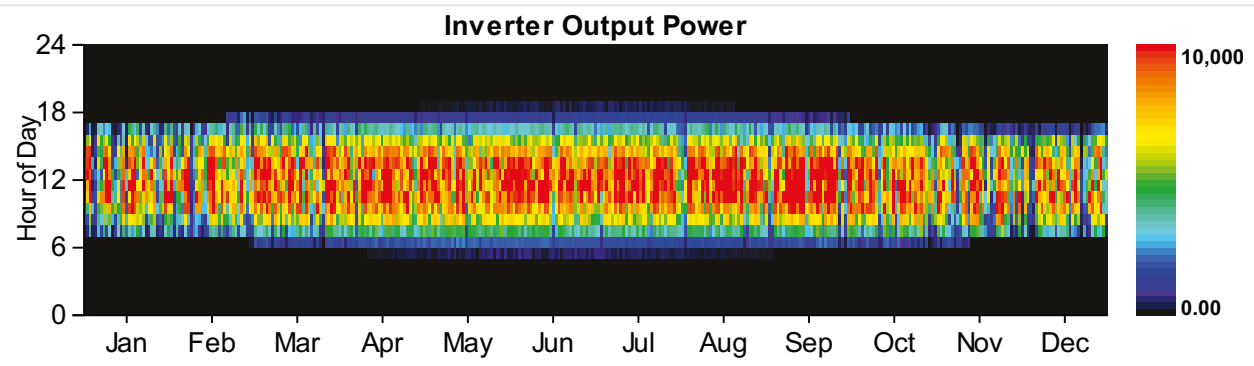

Grid

Rate: Rate 1 Demand

\begin{tabular}{|c|c|c|c|c|c|c|}
\hline Resources.ReportingService_GeneratelnputsReport_Month & $\begin{array}{l}\text { Energy } \\
\text { Purchased } \\
\text { (kWh) }\end{array}$ & $\begin{array}{l}\text { Energy } \\
\text { Sold } \\
\text { (kWh) }\end{array}$ & $\begin{array}{l}\text { Net } \\
\text { Purchases } \\
\text { (kWh) }\end{array}$ & $\begin{array}{l}\text { Peak } \\
\text { Demand } \\
(\mathbf{k W})\end{array}$ & $\begin{array}{l}\text { Energy } \\
\text { Charge } \\
\text { (\$) }\end{array}$ & $\begin{array}{l}\text { Demand } \\
\text { Charge } \\
(\$)\end{array}$ \\
\hline January & 0 & 0 & 0 & 16,288 & 0 & 260,605 \\
\hline February & 0 & 0 & 0 & 15,654 & 0 & 250,459 \\
\hline March & 0 & 0 & 0 & 17,043 & 0 & 272,688 \\
\hline April & 0 & 0 & 0 & 14,366 & 0 & 229,856 \\
\hline May & 0 & 0 & 0 & 13,700 & 0 & 219,202 \\
\hline June & 0 & 0 & 0 & 19,304 & 0 & 308,860 \\
\hline July & 0 & 0 & 0 & 20,000 & 0 & 320,000 \\
\hline August & 0 & 0 & 0 & 20,000 & 0 & 320,000 \\
\hline September & 0 & 0 & 0 & 17,848 & 0 & 285,565 \\
\hline October & 0 & 0 & 0 & 16,023 & 0 & 256,374 \\
\hline November & 0 & 0 & 0 & 15,278 & 0 & 244,445 \\
\hline December & 0 & 0 & 0 & 16,870 & 0 & 269,913 \\
\hline Annual & 0 & 0 & 0 & 20,000 & 0 & $3,237,966$ \\
\hline
\end{tabular}

Rate: Rate 1

\begin{tabular}{|c|c|c|c|c|c|c|}
\hline Resources.ReportingService_GeneratelnputsReport_Month & $\begin{array}{l}\text { Energy } \\
\text { Purchased } \\
\text { (kWh) }\end{array}$ & $\begin{array}{l}\text { Energy } \\
\text { Sold } \\
\text { (kWh) }\end{array}$ & $\begin{array}{l}\text { Net } \\
\text { Purchases } \\
\text { (kWh) }\end{array}$ & $\begin{array}{l}\text { Peak } \\
\text { Demand } \\
(\mathbf{k W})\end{array}$ & $\begin{array}{l}\text { Energy } \\
\text { Charge } \\
(\$)\end{array}$ & $\begin{array}{l}\text { Demand } \\
\text { Charge } \\
\text { (\$) }\end{array}$ \\
\hline January & $5,427,674$ & 0 & $5,427,674$ & 0 & 651,321 & 0 \\
\hline February & $4,390,087$ & 0 & $4,390,087$ & 0 & 526,810 & 0 \\
\hline March & $4,752,956$ & 0 & $4,752,956$ & 0 & 570,355 & 0 \\
\hline April & $4,446,749$ & 0 & $4,446,749$ & 0 & 533,610 & 0 \\
\hline May & $4,404,201$ & 0 & $4,404,201$ & 0 & 528,504 & 0 \\
\hline June & $6,215,504$ & 0 & $6,215,504$ & 0 & 745,860 & 0 \\
\hline July & $7,689,997$ & 0 & $7,689,997$ & 0 & 922,800 & 0 \\
\hline August & $7,892,434$ & 0 & $7,892,434$ & 0 & 947,092 & 0 \\
\hline
\end{tabular}




\begin{tabular}{|c|c|c|c|c|c|c|}
\hline Rctobeurces.ReportingService_GeneratelnputsReport_Month & 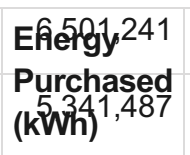 & $\begin{array}{l}\text { Energ9 } \\
\text { Sold } \\
(\mathbf{k W h})^{0}\end{array}$ & $\begin{array}{l}\text { Net501,241 } \\
\text { Purchases } \\
\text { (kWh') }\end{array}$ & $\begin{array}{l}\text { Peak } 0 \\
\text { Demand } \\
(\mathbf{k W}) \quad 0\end{array}$ & $\begin{array}{l}\text { Erhergyt49 } \\
\text { Charge } \\
(\$)^{40,978}\end{array}$ & $\begin{array}{l}\text { Demand } \\
\text { Charge } \\
(\$)\end{array}$ \\
\hline November & $4,942,436$ & 0 & $4,942,436$ & 0 & 593,092 & 0 \\
\hline December & $5,531,241$ & 0 & $5,531,241$ & 0 & 663,749 & 0 \\
\hline Annual & $67,536,008$ & 0 & $67,536,008$ & 0 & $8,104,321$ & 0 \\
\hline
\end{tabular}

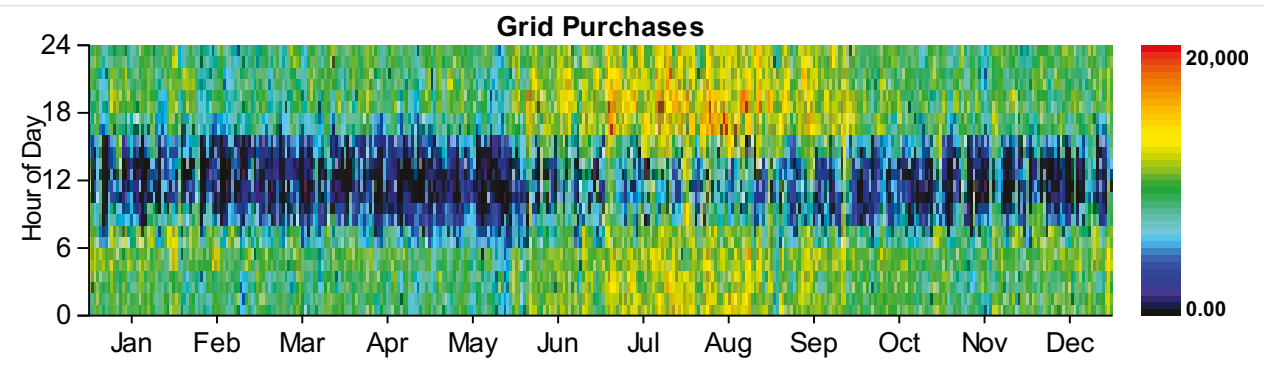

\section{Emissions}

\begin{tabular}{|l|r|l|}
\hline Pollutant & Emissions & Units \\
\hline Carbon dioxide & 42717928 & $\mathrm{~kg} / \mathrm{yr}$ \\
\hline Carbon monoxide & 147 & $\mathrm{~kg} / \mathrm{yr}$ \\
\hline Unburned hydrocarbons & $17 \mathrm{~kg} / \mathrm{yr}$ \\
\hline Particulate matter & $4 \mathrm{~kg} / \mathrm{yr}$ \\
\hline Sulfur dioxide & $185121 \mathrm{~kg} / \mathrm{yr}$ \\
\hline Nitrogen oxides & $90646 \mathrm{~kg} / \mathrm{yr}$ \\
\hline
\end{tabular}


System Report

System architecture

\begin{tabular}{|l|l|r|}
\hline PV & SunPower PV & 13,780 \\
\hline PV \#2 & Remaining Generic flat plate PV & 1,547 \\
\hline Wind Turbine & Siemens $2.3 \mathrm{MW}-108$ & 1 \\
\hline Generator & Kohler 3250 Prime Power & 2,800 \\
\hline Battery & GS200 flow & 1 \\
\hline Converter & System Converter & strings \\
\hline Grid & Grid & 10,000 \\
\hline Dispatch Strategy & Cycle Charging & 20,000 \\
\hline
\end{tabular}

\section{Cost summary}

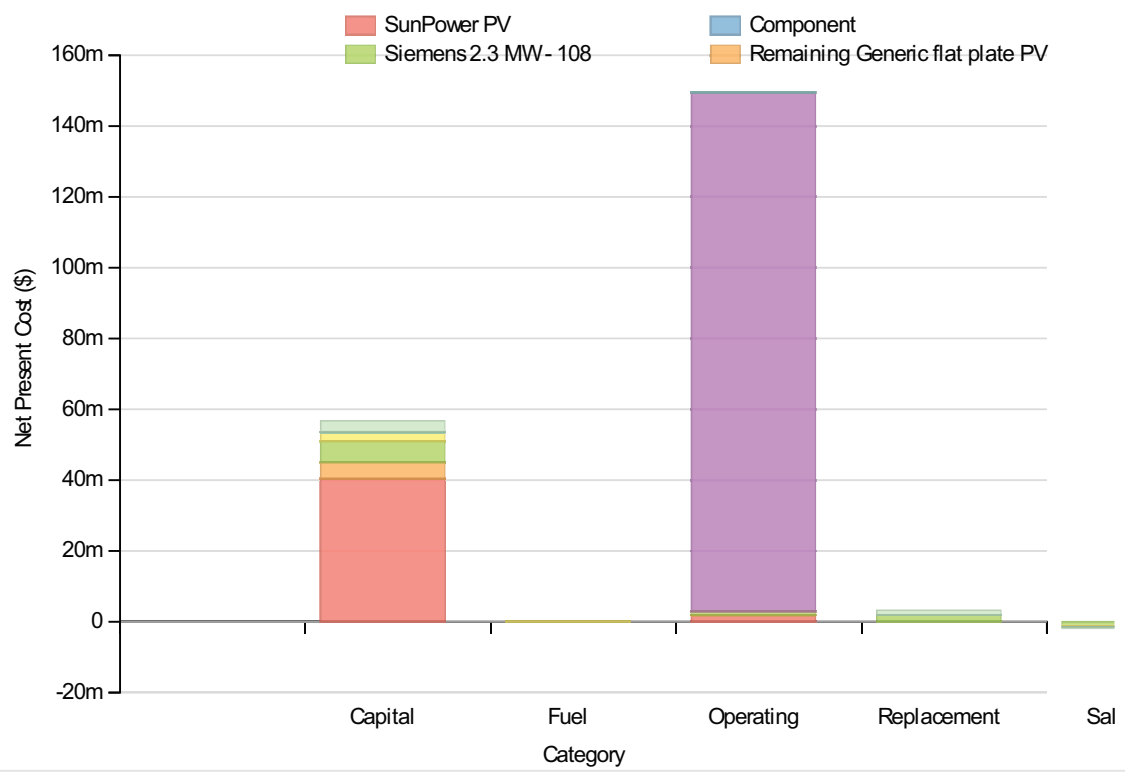

Cost Summary

Total net present cost

$208078544 \$$

Levelized cost of energy

$0.169 \$ / \mathrm{kWh}$

\section{Net Present Costs}

\begin{tabular}{|c|c|c|c|c|c|c|}
\hline Component & Capital & Replacement & O\&M & Fuel & Salvage & Total \\
\hline SunPower PV & $40,358,332$ & 0 & $1,656,876$ & 0 & 0 & $42,015,208$ \\
\hline Remaining Generic flat plate PV & $4,678,459$ & 0 & 339,013 & 0 & 0 & $5,017,472$ \\
\hline Siemens 2.3 MW - 108 & $6,000,000$ & $1,912,842$ & 969,563 & 0 & $-1,078,009$ & $7,804,396$ \\
\hline Kohler 3250 Prime Power & $2,350,000$ & 0 & 34,299 & 154,657 & $-501,035$ & $2,037,921$ \\
\hline Grid & 0 & 0 & $146,469,344$ & 0 & 0 & $146,469,344$ \\
\hline GS200 flow & 324,589 & 7,176 & 31,026 & 0 & -973 & 361,818 \\
\hline Converter & $3,000,000$ & $1,272,821$ & 0 & 0 & $-239,558$ & $4,033,263$ \\
\hline
\end{tabular}




\section{Annualized Costs}

\begin{tabular}{|c|c|c|c|c|c|c|}
\hline Component & Capital & Replacement & O\&M & Fuel & Salvage & Total \\
\hline SunPower PV & $3,121,894$ & 0 & 128,167 & 0 & 0 & $3,250,061$ \\
\hline Remaining Generic flat plate PV & 361,899 & 0 & 26,224 & 0 & 0 & 388,123 \\
\hline Siemens 2.3 MW - 108 & 464,126 & 147,967 & 75,000 & 0 & $-83,389$ & 603,704 \\
\hline Kohler 3250 Prime Power & 181,783 & 0 & 2,653 & 11,963 & $-38,757$ & 157,642 \\
\hline Grid & 0 & 0 & $11,330,045$ & 0 & 0 & $11,330,045$ \\
\hline GS200 flow & 25,108 & 555 & 2,400 & 0 & -75 & 27,988 \\
\hline Converter & 232,063 & 98,458 & 0 & 0 & $-18,531$ & 311,990 \\
\hline System & $4,386,874$ & 246,980 & $11,564,491$ & 11,963 & $-140,752$ & $16,069,556$ \\
\hline
\end{tabular}

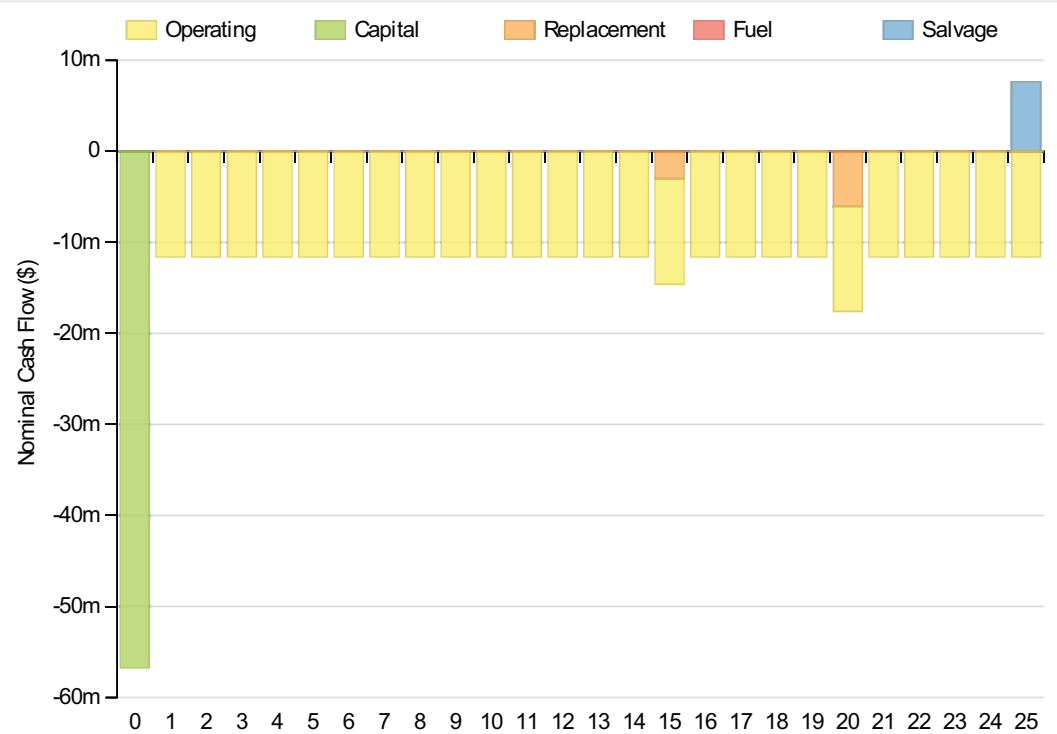

Year

Electrical

\begin{tabular}{|c|c|c|c|c|}
\hline \multirow{2}{*}{\multicolumn{2}{|c|}{$\begin{array}{l}\text { Quantity } \\
\text { Excess electricity }\end{array}$}} & \multicolumn{2}{|l|}{ Value } & Units \\
\hline & & & 1937470 & $\mathrm{kWh} / \mathrm{yr}$ \\
\hline Unmet load & & & 989 & $\mathrm{kWh} / \mathrm{yr}$ \\
\hline Capacity shortage & & & 25705 & $\mathrm{kWh} / \mathrm{yr}$ \\
\hline Renewable fraction & & & 0 & \\
\hline Component & Production(kWh/yr) & & Fractio & n (\%) \\
\hline PV & & $24,644,568$ & & 25 \\
\hline PV & & $2,766,525$ & & 3 \\
\hline Generator & & 61,293 & & 0 \\
\hline Wind Turbine & & $4,674,352$ & & 5 \\
\hline Grid Purchases & & $67,434,064$ & & 68 \\
\hline
\end{tabular}




\section{Load}

AC primary load

DC primary load

Total

\section{Consumption(kWh/yr)}

Fraction (\%)

$95,051,464$

0

$95,051,464$
100

0

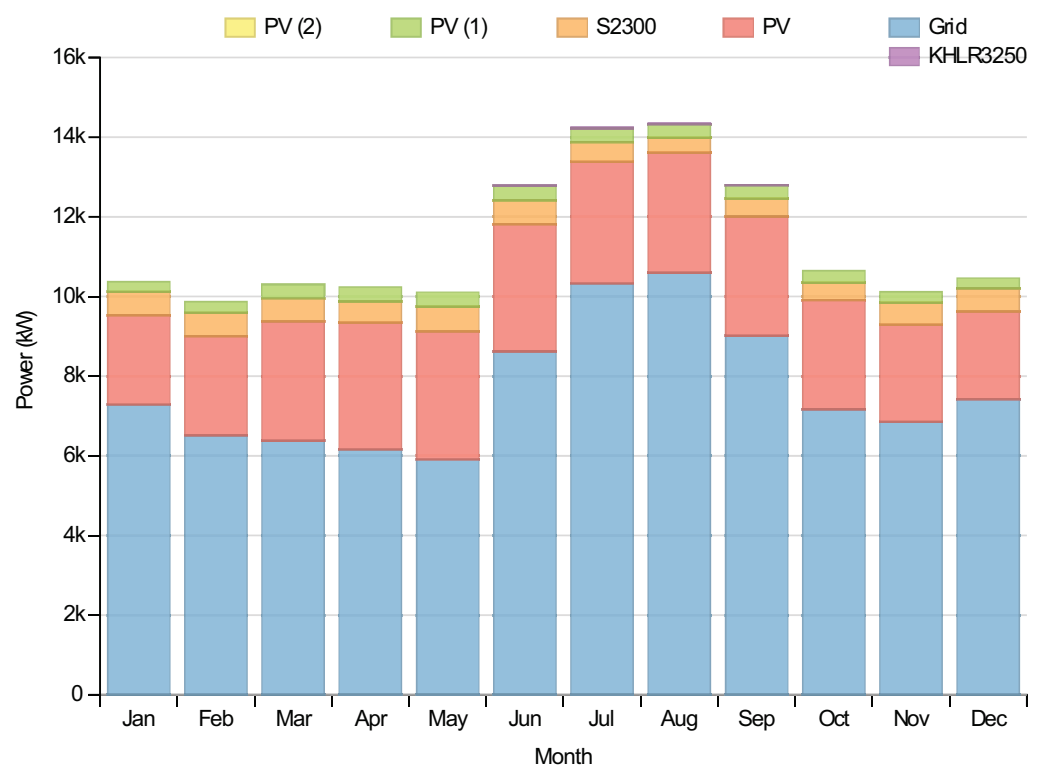

\section{PV:SunPower PV}

\section{Quantity}

Rated capacity

Mean output

Mean output

Capacity factor

Total production

Minimum output

Maximum output

PV penetration

Hours of operation

Levelized cost

\section{Value}

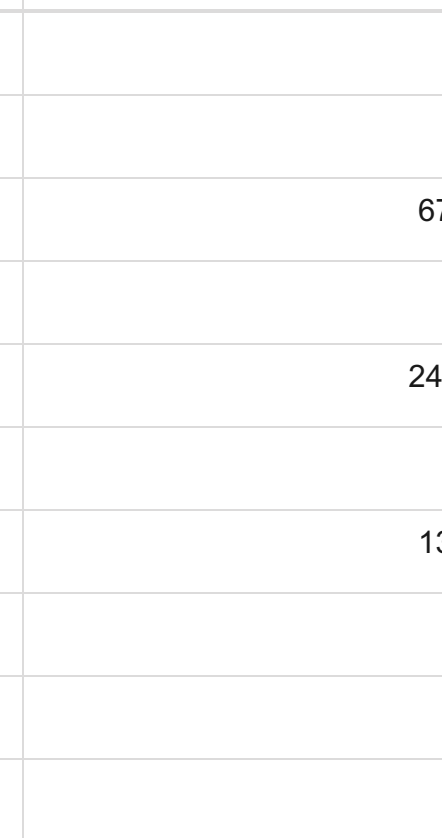

\begin{tabular}{|r|l|}
\hline 13780 & $\mathrm{~kW}$ \\
\hline 2813 & $\mathrm{~kW}$ \\
\hline 67519.00 & $\mathrm{kWh} / \mathrm{d}$ \\
\hline 20.42 & $\%$ \\
\hline 24644568 & $\mathrm{kWh} / \mathrm{yr}$ \\
\hline 0.00 & $\mathrm{~kW}$ \\
\hline 13941.00 & $\mathrm{~kW}$ \\
\hline 25.93 & $\%$ \\
\hline 4386 & $\mathrm{hrs} / \mathrm{yr}$ \\
\hline 0.119 & $\$ / \mathrm{kWh}$ \\
\hline
\end{tabular}

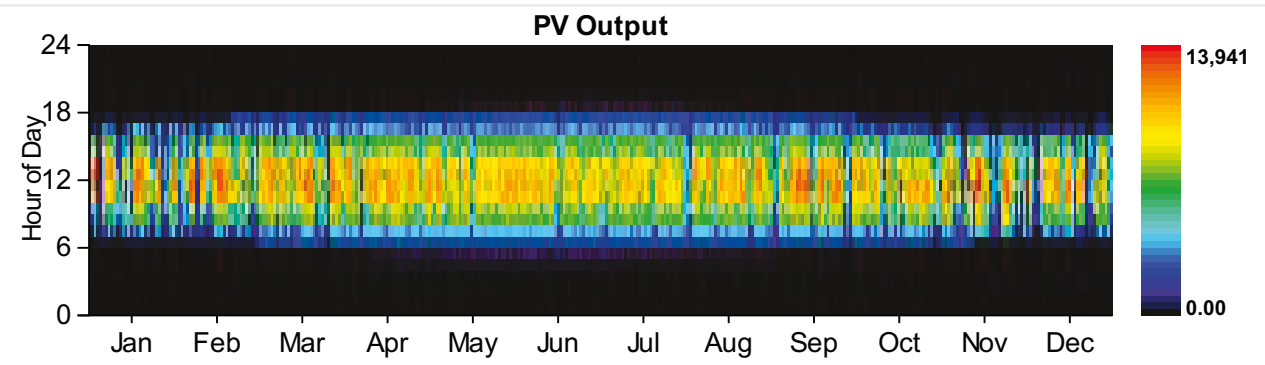




\section{Quantity}

Rated capacity

Mean output

Mean output

Capacity factor

Total production

Minimum output

Maximum output

PV penetration

Hours of operation

Levelized cost
Value

Units

1547 kW

316 kW

$7579.50 \mathrm{kWh} / \mathrm{d}$

$20.42 \%$

$2766525 \mathrm{kWh} / \mathrm{yr}$

$0.00 \mathrm{~kW}$

$1565.00 \mathrm{~kW}$

$2.91 \%$

$4386 \mathrm{hrs} / \mathrm{yr}$

$0.014 \$ / k W h$

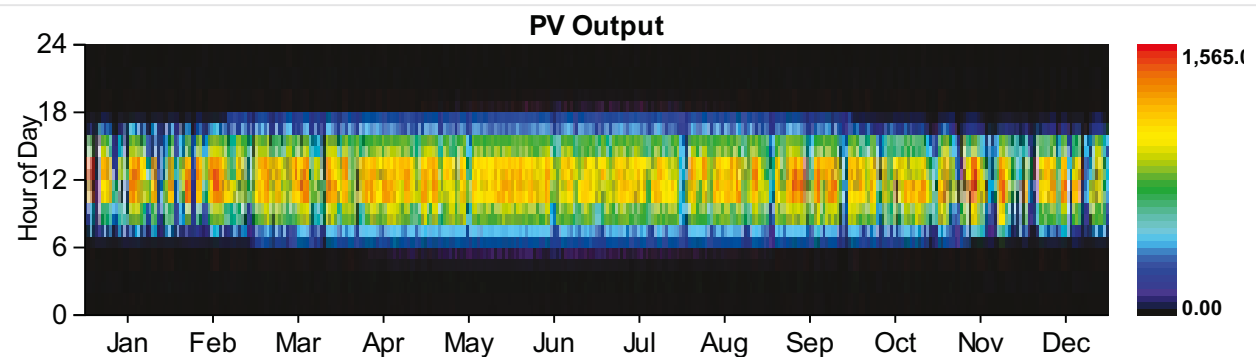

Wind Turbine:Siemens 2.3 MW - 108

\section{Quantity}

Total rated capacity

Mean output

Capacity factor

Total production

Minimum output

Maximum output

Wind penetration

Hours of operation

Levelized cost

\begin{tabular}{|r|l|l|}
\hline Value & Units \\
\hline 2300 & $\mathrm{~kW}$ \\
\hline 534 & $\mathrm{~kW}$ \\
\hline 23.20 & $\%$ \\
\hline 4674352 & $\mathrm{kWh} / \mathrm{yr}$ \\
\hline 0.49 & $\mathrm{~kW}$ \\
\hline 2312.70 & $\mathrm{~kW}$ \\
\hline 4.92 & $\%$ \\
\hline 8760 & $\mathrm{hrs} / \mathrm{yr}$ \\
\hline 0.129 & $\$ / \mathrm{kWh}$ \\
\hline
\end{tabular}

Generator:Kohler 3250 Prime Power

\section{Quantity}

Value

Units

Hours of operation

Number of starts

Operational life 
Qixâิ

Marginal generation cost

Electrical production

Mean electrical output

Min. electrical output

Max. electrical output

Fuel consumption

Specific fuel consumption

Fuel energy input

Mean electrical efficiency

\subsection{Bhlits}

$0.18 \$ / k W h$

$61293 \mathrm{kWh} / \mathrm{yr}$

$929 \mathrm{~kW}$

$700 \mathrm{~kW}$

2800 kW

15144 L/yr

$0.25 \mathrm{~L} / \mathrm{kWh}$

149013 kWh/yr

$41 \%$

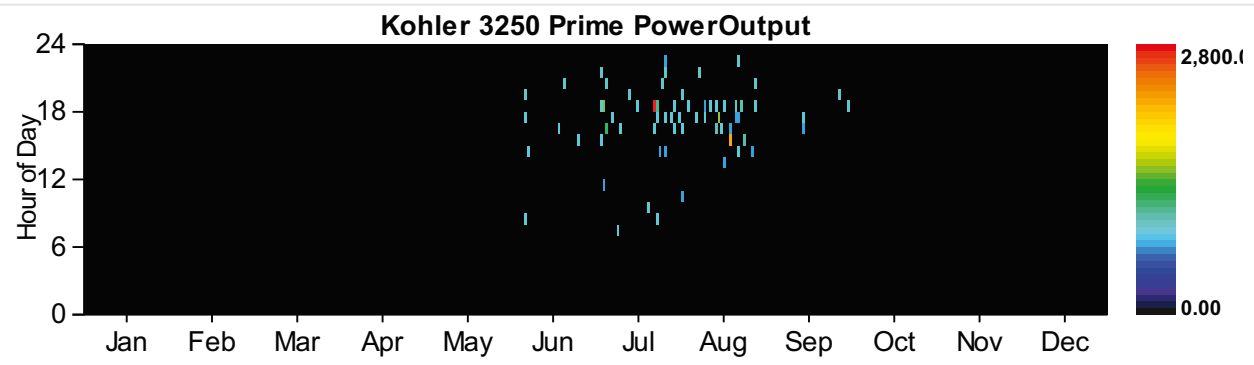

\section{Battery:GS200 flow}

\section{Quantity}

Value

String size

Strings in parallel

Batteries

Bus voltage

\section{Quantity}

Nominal capacity

Usable nominal capacity

Autonomy

Lifetime throughput

Battery wear cost

Average energy cost

Energy in

Energy out

Storage depletion

Losses
Value

Units

$600 \mathrm{kWh}$

$600 \mathrm{kWh}$

$0 \mathrm{hr}$

0

$0.000 \$ / k W h$

$0.008 \$ / k W h$

$162812 \mathrm{kWh} / \mathrm{yr}$

$114471 \mathrm{kWh} / \mathrm{yr}$

$600 \mathrm{kWh} / \mathrm{yr}$

$47741 \mathrm{kWh} / \mathrm{yr}$ 


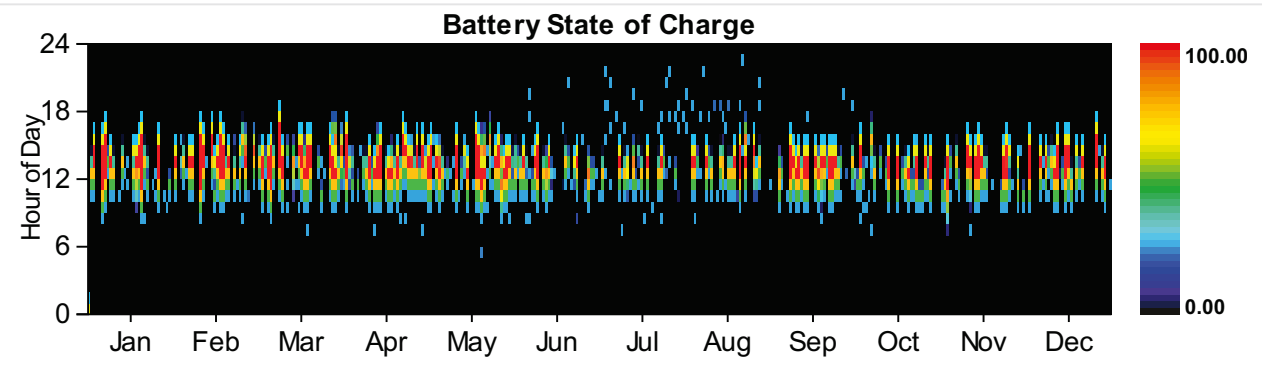

\section{Converter}

\begin{tabular}{|c|c|c|c|}
\hline Quantity & Inverter & Rectifier & Units \\
\hline Capacity & 10,000 & 9,000 & $\mathrm{~kW}$ \\
\hline Mean output & 2,613 & 0 & $\mathrm{~kW}$ \\
\hline Minimum output & 0 & 0 & $\mathrm{~kW}$ \\
\hline Maximum output & 10,000 & 179 & $\mathrm{~kW}$ \\
\hline Capacity factor & 26 & 0 & $\%$ \\
\hline Hours of operation & 4,588 & 66 & hrs/yr \\
\hline Energy in & $25,428,318$ & 3,506 & $\mathrm{kWh} / \mathrm{yr}$ \\
\hline Energy out & $22,885,384$ & 2,980 & $\mathrm{kWh} / \mathrm{yr}$ \\
\hline Losses & $2,542,934$ & 526 & $\mathrm{kWh} / \mathrm{yr}$ \\
\hline
\end{tabular}
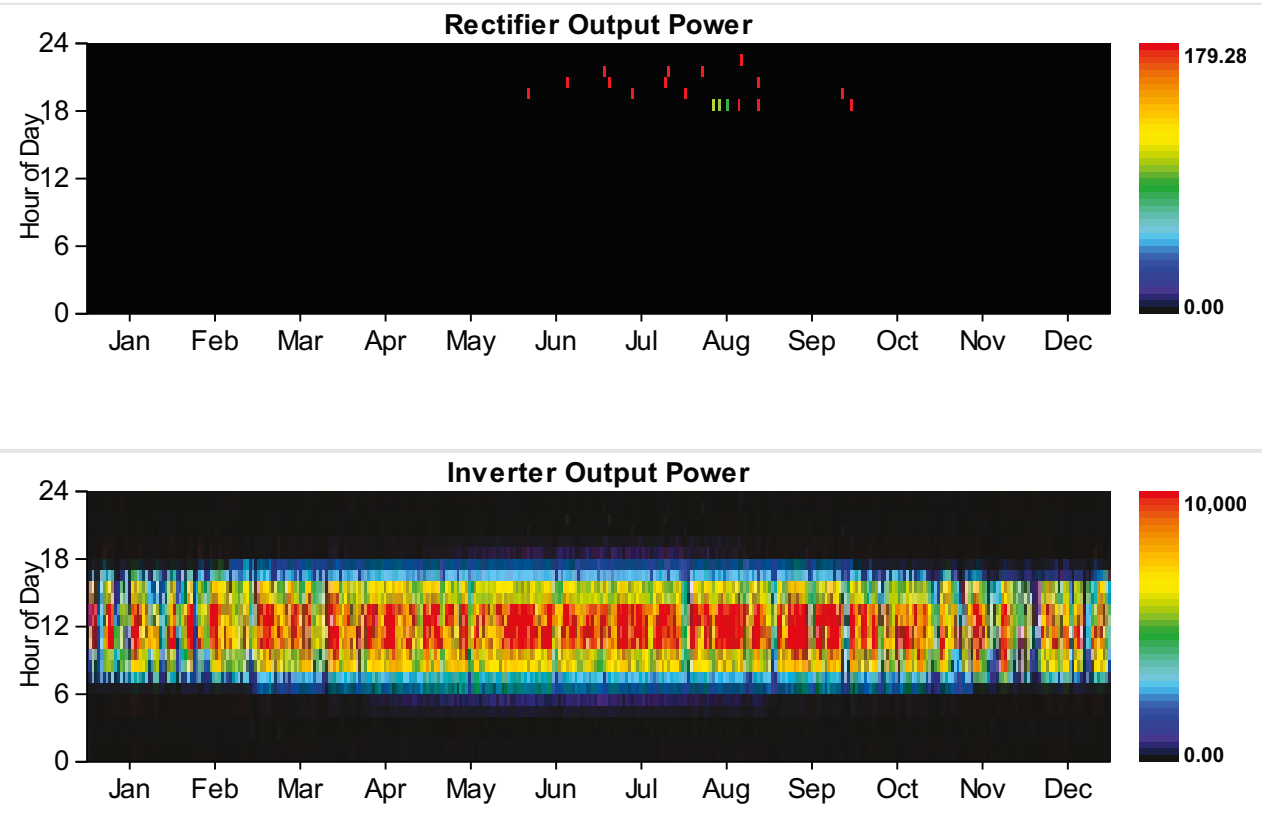

Grid

Rate: Rate 1 Demand

\begin{tabular}{|l|l|l|l|l|l|l|l} 
& Energy & Energy & Net & Peak & Energy & Demand \\
Resources.ReportingService_GeneratelnputsReport_Month & $\begin{array}{l}\text { Purchased } \\
\text { Sold }\end{array}$ & $\begin{array}{l}\text { Purchases } \\
\text { Sold }\end{array}$ & $\mathbf{k W h}$ & $\mathbf{k W h})$ & $\mathbf{( k W )}$ & $\mathbf{( \$ )}$ & $\mathbf{( \$ )}$
\end{tabular}




\begin{tabular}{|c|c|c|c|c|c|c|}
\hline Regrartes.ReportingService_GeneratelnputsReport_Month & $\begin{array}{lr}\text { Energy } & 0 \\
\text { Purchased } \\
\text { (kWh) }\end{array}$ & $\begin{array}{l}\text { Energyo } \\
\text { Sold } \\
\text { (kWh) } 0\end{array}$ & $\begin{array}{lr}\text { Net } & 0 \\
\text { Purchases } \\
(\mathbf{k W h}) \quad 0\end{array}$ & $\begin{array}{l}\text { Péak288 } \\
\text { Demand } \\
\text { (kW5),654 }\end{array}$ & $\begin{array}{l}\text { Energy } \\
\text { Charge } \\
(\$) \quad 0\end{array}$ & $\begin{array}{l}\text { Dêfpafex } \\
\text { Charge } \\
\text { (\$千50,459 }\end{array}$ \\
\hline March & 0 & 0 & 0 & 17,043 & 0 & 272,688 \\
\hline April & 0 & 0 & 0 & 14,366 & 0 & 229,856 \\
\hline May & 0 & 0 & 0 & 13,700 & 0 & 219,202 \\
\hline June & 0 & 0 & 0 & 19,304 & 0 & 308,860 \\
\hline July & 0 & 0 & 0 & 20,000 & 0 & 320,000 \\
\hline August & 0 & 0 & 0 & 20,000 & 0 & 320,000 \\
\hline September & 0 & 0 & 0 & 17,848 & 0 & 285,565 \\
\hline October & 0 & 0 & 0 & 16,023 & 0 & 256,374 \\
\hline November & 0 & 0 & 0 & 15,278 & 0 & 244,445 \\
\hline December & 0 & 0 & 0 & 16,870 & 0 & 269,913 \\
\hline Annual & 0 & 0 & 0 & 20,000 & 0 & $3,237,966$ \\
\hline
\end{tabular}

Rate: Rate 1

\begin{tabular}{|c|c|c|c|c|c|c|}
\hline Resources.ReportingService_GeneratelnputsReport_Month & $\begin{array}{l}\text { Energy } \\
\text { Purchased } \\
\text { (kWh) }\end{array}$ & $\begin{array}{l}\text { Energy } \\
\text { Sold } \\
\text { (kWh) }\end{array}$ & $\begin{array}{l}\text { Net } \\
\text { Purchases } \\
\text { (kWh) }\end{array}$ & $\begin{array}{l}\text { Peak } \\
\text { Demand } \\
(\mathbf{k W})\end{array}$ & $\begin{array}{l}\text { Energy } \\
\text { Charge } \\
\text { (\$) }\end{array}$ & $\begin{array}{l}\text { Demand } \\
\text { Charge } \\
(\$)\end{array}$ \\
\hline January & $5,419,880$ & 0 & $5,419,880$ & 0 & 650,386 & 0 \\
\hline February & $4,381,999$ & 0 & $4,381,999$ & 0 & 525,840 & 0 \\
\hline March & $4,743,226$ & 0 & $4,743,226$ & 0 & 569,187 & 0 \\
\hline April & $4,435,979$ & 0 & $4,435,979$ & 0 & 532,317 & 0 \\
\hline May & $4,393,103$ & 0 & $4,393,103$ & 0 & 527,172 & 0 \\
\hline June & $6,207,422$ & 0 & $6,207,422$ & 0 & 744,891 & 0 \\
\hline July & $7,682,359$ & 0 & $7,682,359$ & 0 & 921,883 & 0 \\
\hline August & $7,884,209$ & 0 & $7,884,209$ & 0 & 946,105 & 0 \\
\hline September & $6,493,163$ & 0 & $6,493,163$ & 0 & 779,180 & 0 \\
\hline October & $5,333,870$ & 0 & $5,333,870$ & 0 & 640,064 & 0 \\
\hline November & $4,934,859$ & 0 & $4,934,859$ & 0 & 592,183 & 0 \\
\hline December & $5,523,998$ & 0 & $5,523,998$ & 0 & 662,880 & 0 \\
\hline Annual & $67,434,064$ & 0 & $67,434,064$ & 0 & $8,092,087$ & 0 \\
\hline
\end{tabular}




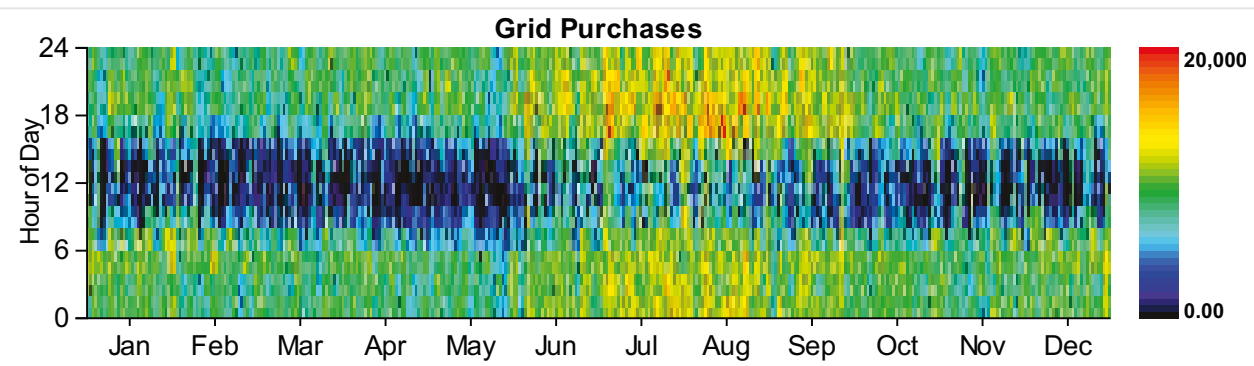

\section{Emissions}

\begin{tabular}{|l|r|l|}
\hline Pollutant & Emissions & Units \\
\hline Carbon dioxide & 42658072 & $\mathrm{~kg} / \mathrm{yr}$ \\
\hline Carbon monoxide & $167 \mathrm{~kg} / \mathrm{yr}$ \\
\hline Unburned hydrocarbons & $19 \mathrm{~kg} / \mathrm{yr}$ \\
\hline Particulate matter & $5 \mathrm{~kg} / \mathrm{yr}$ \\
\hline Sulfur dioxide & $184851 \mathrm{~kg} / \mathrm{yr}$ \\
\hline Nitrogen oxides & $90528 \mathrm{~kg} / \mathrm{yr}$ \\
\hline
\end{tabular}

HOMER Energy, LLC @ 2016 
System Report

System architecture

\begin{tabular}{|l|l|r|}
\hline PV & SunPower PV & 13,780 \\
\hline PV \#2 & Remaining Generic flat plate PV & 1,547 \\
\hline PV \#3 & Solar World 320W flat plate PV Copy & 1,000 \\
\hline Converter & System Converter & 10,000 \\
\hline Grid & Grid & $\mathrm{kW}$ \\
\hline Dispatch Strategy & Cycle Charging & 27,200 \\
\hline
\end{tabular}

Cost summary

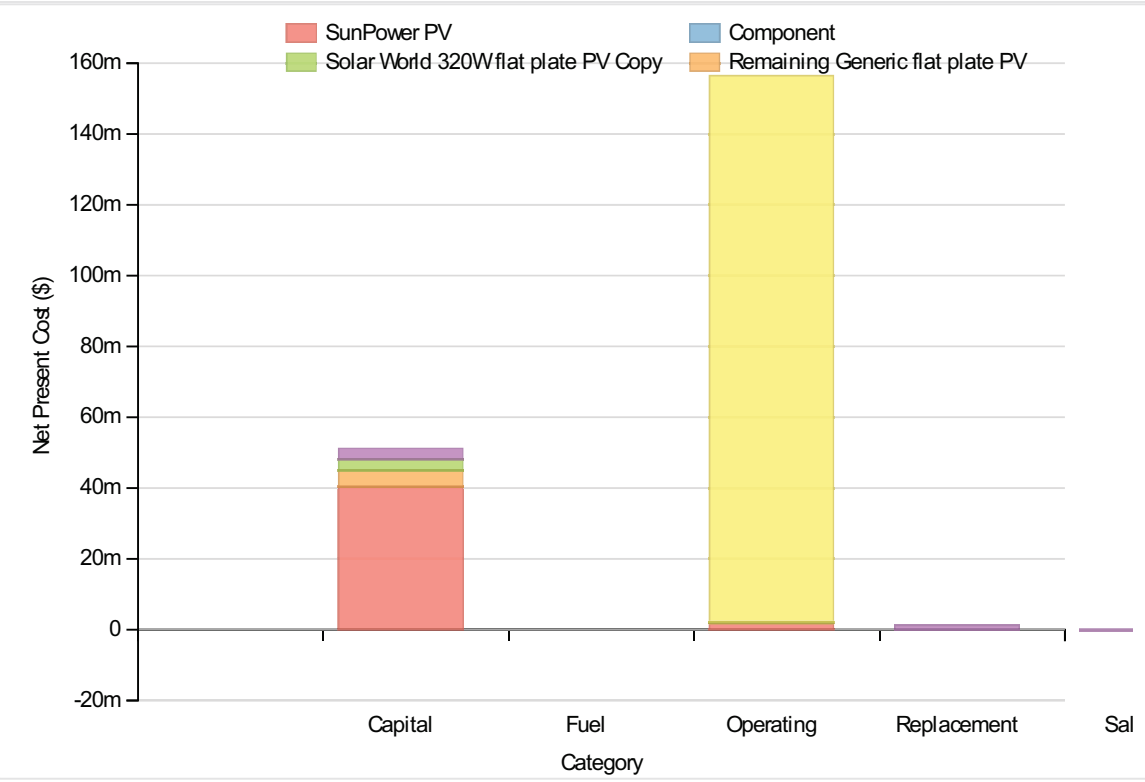

Cost Summary

Total net present cost

$208627504 \$$

Levelized cost of energy

$0.170 \$ / \mathrm{kWh}$

\section{Net Present Costs}

\begin{tabular}{|c|c|c|c|c|c|c|}
\hline Component & Capital & Replacement & O\&M & Fuel & Salvage & Total \\
\hline SunPower PV & $40,358,332$ & 0 & $1,656,876$ & 0 & 0 & $42,015,208$ \\
\hline Remaining Generic flat plate PV & $4,678,459$ & 0 & 339,013 & 0 & 0 & $5,017,472$ \\
\hline Solar World 320W flat plate PV Copy & $3,083,333$ & 0 & 280,096 & 0 & 0 & $3,363,429$ \\
\hline Grid & 0 & 0 & $154,198,048$ & 0 & 0 & $154,198,048$ \\
\hline Converter & $3,000,000$ & $1,272,821$ & 0 & 0 & $-239,558$ & $4,033,263$ \\
\hline System & $51,120,124$ & $1,272,821$ & $156,474,048$ & 0 & $-239,558$ & $208,627,435$ \\
\hline
\end{tabular}

Annualized Costs

\begin{tabular}{|l|r|r|r|r|r|r|}
\hline Component & Capital & Replacement & O\&M & Fuel & Salvage & Total \\
\hline SunPower PV & $3,121,894$ & 0 & 128,167 & 0 & 0 \\
\hline
\end{tabular}


Bompipinenfeneric flat plate PV

Solar World 320W flat plate PV Copy

Grid

Converter

System
Capisan ,899 Replacement 0 O\&M 26,224 Fuel ${ }^{0}$ Salvage 0 Total 388,123

\begin{tabular}{ll|l|l|l|l|}
2238,509 & 0 & 21,667 & 0 & 0 & 260,176
\end{tabular}

0

\begin{tabular}{l|l|l}
0 & $11,927,894$ & 0
\end{tabular}

\begin{tabular}{l|l}
0 & $11,927,894$
\end{tabular}

98,458

$0 \quad 0$

$-18,531$

311,990

98,458

$12,103,953$

\begin{tabular}{l|l}
0 & $-18,531$
\end{tabular}

$16,138,246$

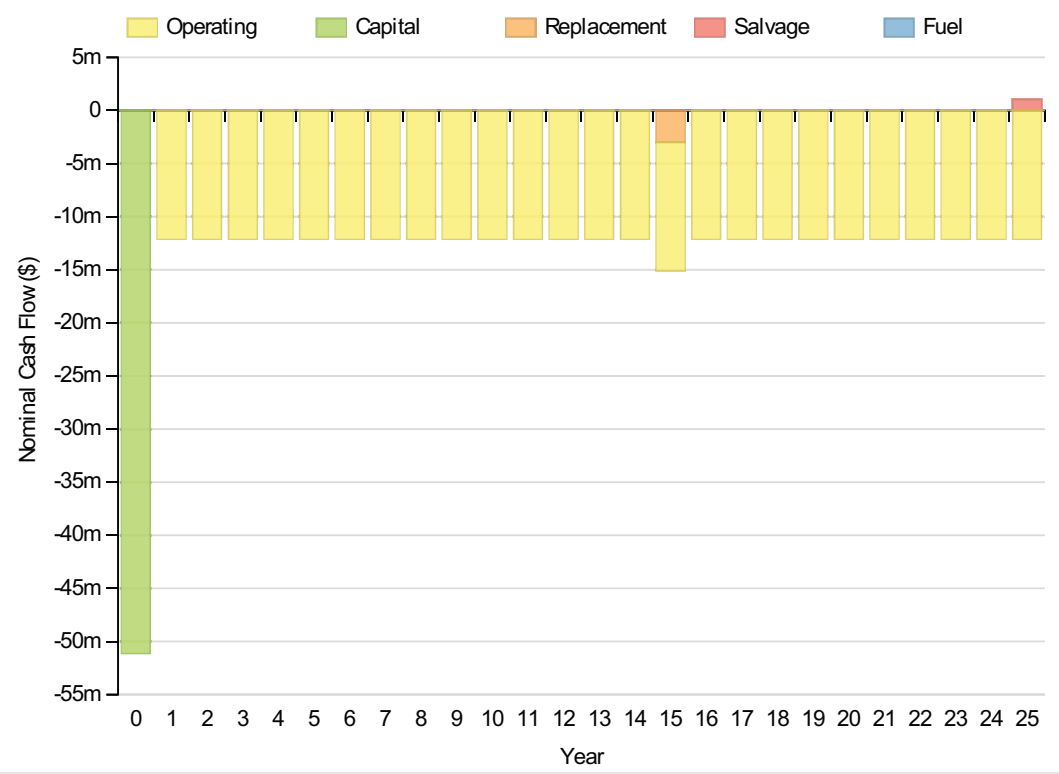

\section{Electrical}

\section{Quantity}

Excess electricity

Unmet load

Capacity shortage

Renewable fraction
Value

Value

\begin{tabular}{|c|c|c|}
\hline Component & Production(kWh/yr) & Fraction (\%) \\
\hline PV & $24,644,568$ & 25 \\
\hline PV & $2,766,525$ & 3 \\
\hline PV & $1,788,429$ & 2 \\
\hline Grid Purchases & $70,901,448$ & 71 \\
\hline Total & $100,100,976$ & 100 \\
\hline Load & Consumption(kWh/yr) & Fraction (\%) \\
\hline AC primary load & $95,052,456$ & 100 \\
\hline DC primary load & & 0 \\
\hline Total & $95,052,456$ & 100 \\
\hline
\end{tabular}




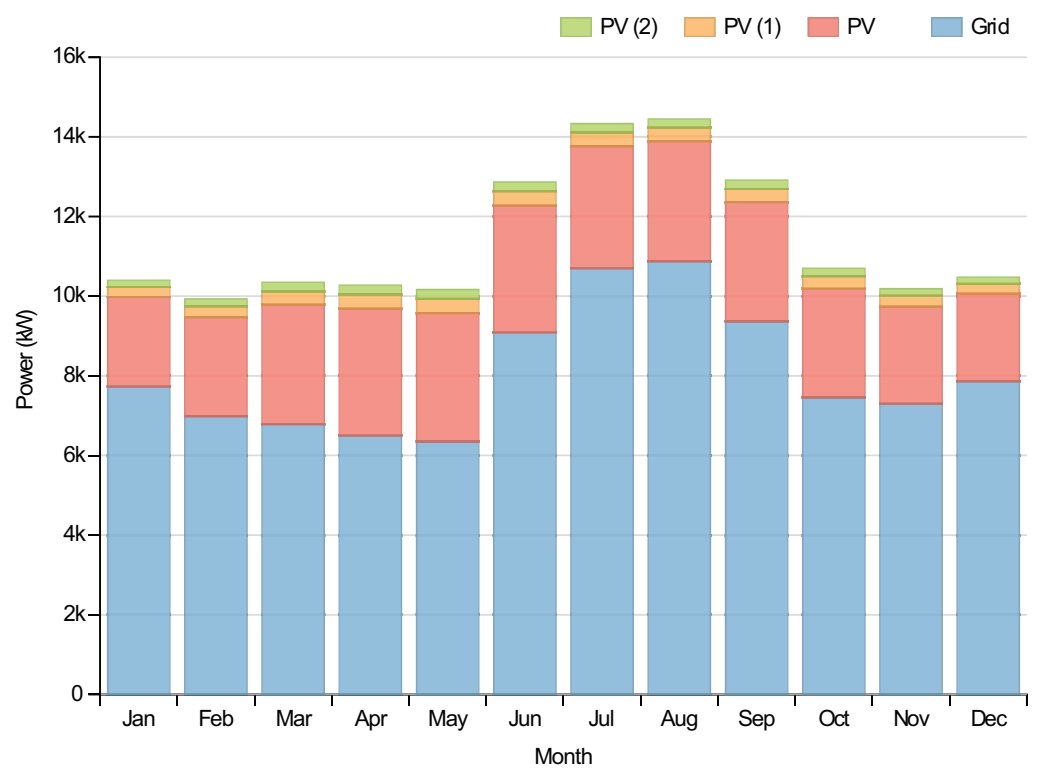

\section{PV:SunPower PV}

\begin{tabular}{|c|c|c|}
\hline Quantity & Value & Units \\
\hline Rated capacity & 13780 & $\mathrm{~kW}$ \\
\hline Mean output & 2813 & kW \\
\hline Mean output & 67519.00 & $\mathrm{kWh} / \mathrm{d}$ \\
\hline Capacity factor & 20.42 & $\%$ \\
\hline Total production & 24644568 & $\mathrm{kWh} / \mathrm{yr}$ \\
\hline Minimum output & 0.00 & kW \\
\hline Maximum output & 13941.00 & kW \\
\hline PV penetration & 25.93 & $\%$ \\
\hline Hours of operation & 4386 & $\mathrm{hrs} / \mathrm{yr}$ \\
\hline Levelized cost & 0.111 & $\$ / \mathrm{kWh}$ \\
\hline
\end{tabular}

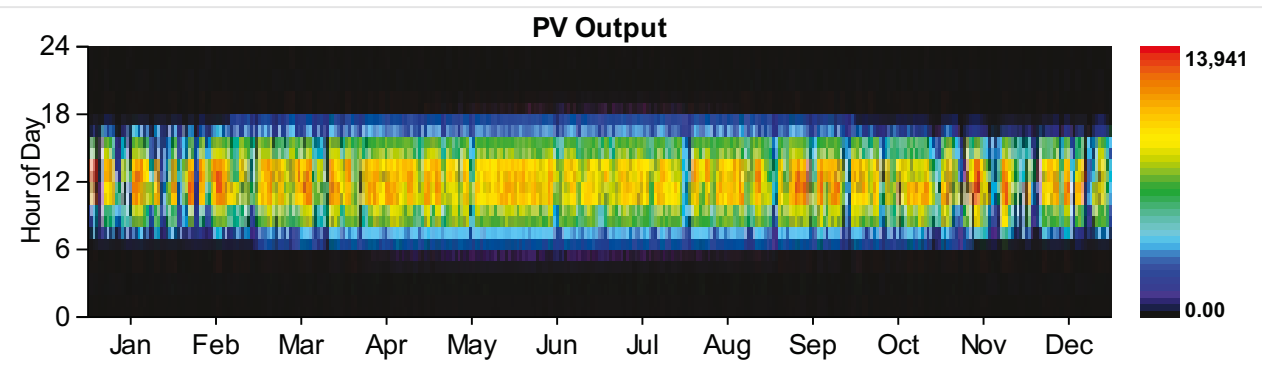

\section{PV:Remaining Generic flat plate PV}

\begin{tabular}{|l|c|c|}
\hline Quantity & Value & Units \\
\hline Rated capacity & $1547 \mathrm{~kW}$ \\
\hline Mean output & $316 \mathrm{~kW}$ \\
\hline Mean output & $7579.50 \mathrm{kWh} / \mathrm{d}$ \\
\hline Capacity factor & $20.42 \%$ \\
\hline
\end{tabular}


§etahtripduction

Minimum output

Maximum output

PV penetration

Hours of operation

Levelized cost

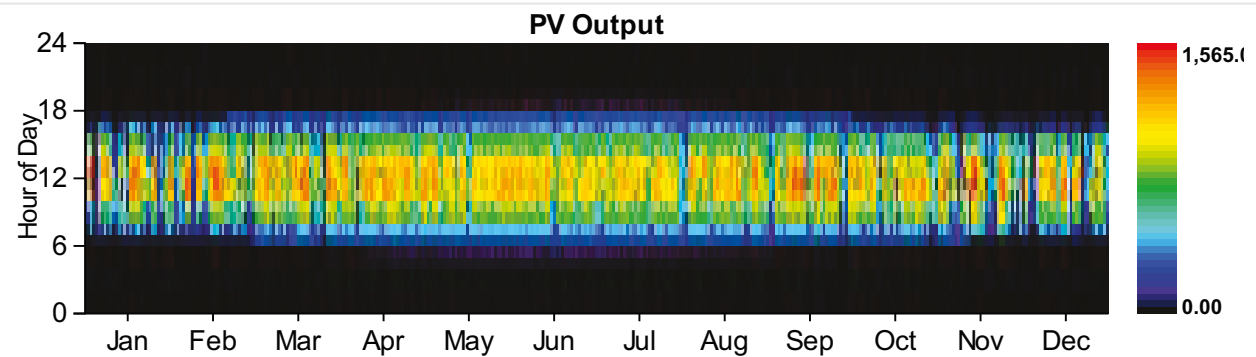

\section{PV:Solar World 320W flat plate PV Copy}

\section{Quantity}

Rated capacity

Mean output

Mean output

Capacity factor

Total production

Minimum output

Maximum output

PV penetration

Hours of operation

Levelized cost

\section{5 vNAtesr}

$0.00 \mathrm{~kW}$

$1565.00 \mathrm{~kW}$

$2.91 \%$

$4386 \mathrm{hrs} / \mathrm{yr}$

$0.013 \$ / \mathrm{kWh}$

\begin{tabular}{|l|l|l|}
\hline Value & Units \\
\hline 1000 & $\mathrm{~kW}$ \\
204 & $\mathrm{~kW}$ \\
\hline 4899.80 & $\mathrm{kWh} / \mathrm{d}$ \\
\hline 20.42 & $\%$ \\
\hline 1788429 & $\mathrm{kWh} / \mathrm{yr}$ \\
\hline 0.00 & $\mathrm{~kW}$ \\
\hline 1011.70 & $\mathrm{~kW}$ \\
\hline 1.88 & $\%$ \\
\hline 4386 & $\mathrm{hrs} / \mathrm{yr}$ \\
\hline 0.009 & $\$ / \mathrm{kWh}$ \\
\hline
\end{tabular}

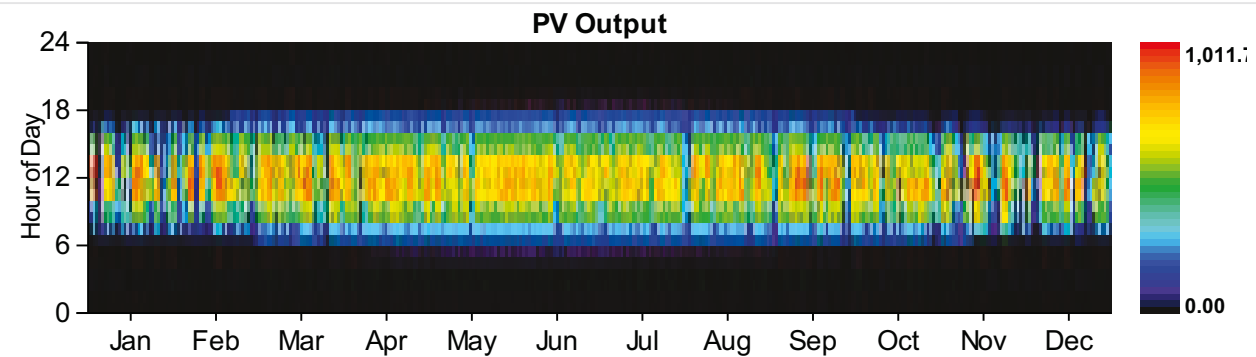

\section{Converter}

\begin{tabular}{|l|r|r|r|}
\hline Quantity & Inverter & Rectifier & Units \\
\hline Capacity & 10,000 & 9,000 & $\mathrm{~kW}$ \\
\hline Mean output & 2,757 & $0 \mathrm{~kW}$ \\
\hline
\end{tabular}




\begin{tabular}{|l|r|r|r|}
\hline Qinimitity output & Inverter & 0 & Rectifier \\
\hline Maximum output & 10,000 & 0 \\
\hline Capacity factor & 28 & 0 \\
\hline Hours of operation & 4,386 & 0 \\
\hline Energy in & $26,834,786$ & 0 \\
\hline Energy out & $24,151,150$ & 0 \\
\hline Losses & $2,683,636$ & $\mathrm{kWh} / \mathrm{yr}$ \\
\hline
\end{tabular}
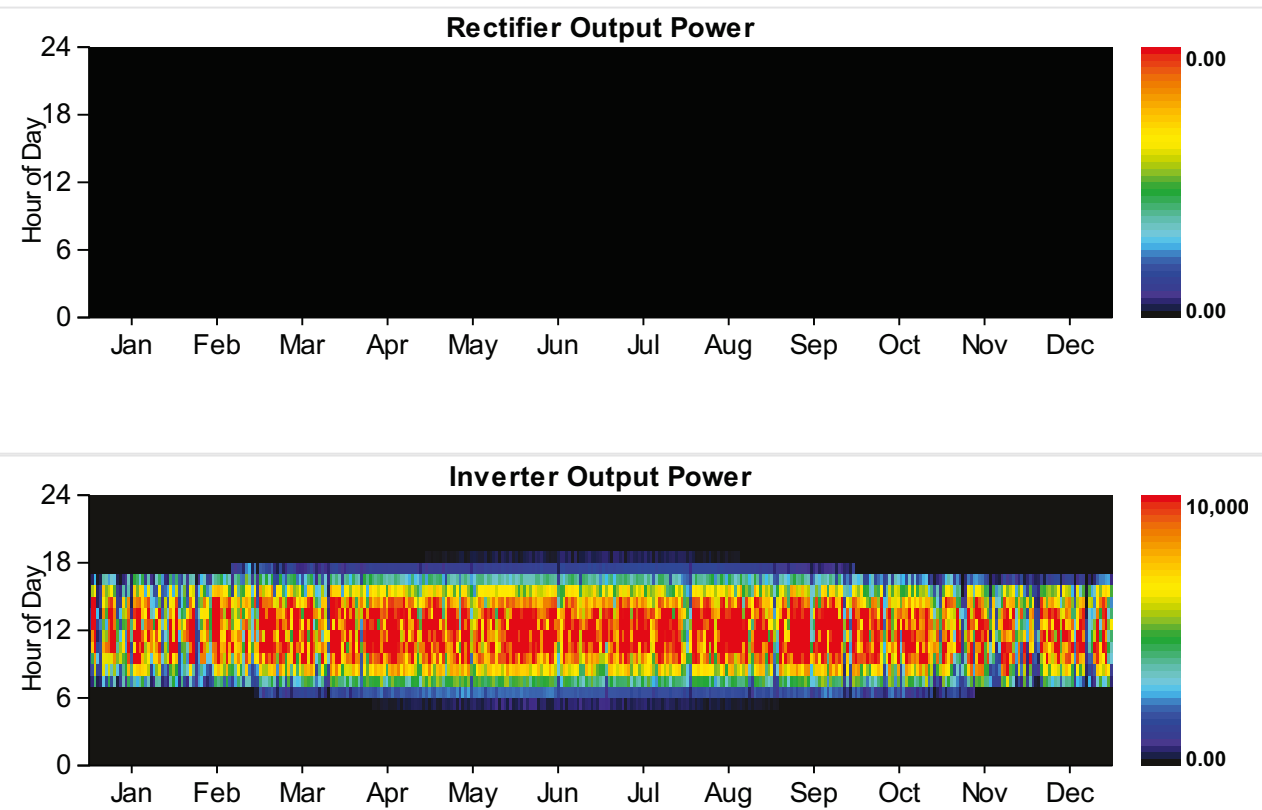

Grid

Rate: Rate 1 Demand

\begin{tabular}{|c|c|c|c|c|c|c|}
\hline Resources.ReportingService_GeneratelnputsReport_Month & $\begin{array}{l}\text { Energy } \\
\text { Purchased } \\
\text { (kWh) }\end{array}$ & $\begin{array}{l}\text { Energy } \\
\text { Sold } \\
\text { (kWh) }\end{array}$ & $\begin{array}{l}\text { Net } \\
\text { Purchases } \\
\text { (kWh) }\end{array}$ & $\begin{array}{l}\text { Peak } \\
\text { Demand } \\
(\mathbf{k W})\end{array}$ & $\begin{array}{l}\text { Energy } \\
\text { Charge } \\
\text { (\$) }\end{array}$ & $\begin{array}{l}\text { Demand } \\
\text { Charge } \\
(\$)\end{array}$ \\
\hline January & 0 & 0 & 0 & 17,079 & 0 & 273,262 \\
\hline February & 0 & 0 & 0 & 15,668 & 0 & 250,685 \\
\hline March & 0 & 0 & 0 & 17,075 & 0 & 273,199 \\
\hline April & 0 & 0 & 0 & 14,394 & 0 & 230,299 \\
\hline May & 0 & 0 & 0 & 14,850 & 0 & 237,602 \\
\hline June & 0 & 0 & 0 & 20,019 & 0 & 320,305 \\
\hline July & 0 & 0 & 0 & 24,158 & 0 & 386,525 \\
\hline August & 0 & 0 & 0 & 22,709 & 0 & 363,351 \\
\hline September & 0 & 0 & 0 & 18,833 & 0 & 301,321 \\
\hline October & 0 & 0 & 0 & 16,050 & 0 & 256,794 \\
\hline November & 0 & 0 & 0 & 15,751 & 0 & 252,018 \\
\hline
\end{tabular}


December

Annual

Resources.ReportingService_GeneratelnputsReport_Month

Rate: Rate 1

\begin{tabular}{|c|c|c|c|c|c|c|}
\hline Resources.ReportingService_GeneratelnputsReport_Month & $\begin{array}{l}\text { Energy } \\
\text { Purchased } \\
\text { (kWh) }\end{array}$ & $\begin{array}{l}\text { Energy } \\
\text { Sold } \\
\text { (kWh) }\end{array}$ & $\begin{array}{l}\text { Net } \\
\text { Purchases } \\
\text { (kWh) }\end{array}$ & $\begin{array}{l}\text { Peak } \\
\text { Demand } \\
\text { (kW) }\end{array}$ & $\begin{array}{l}\text { Energy } \\
\text { Charge } \\
\text { (\$) }\end{array}$ & $\begin{array}{l}\text { Demand } \\
\text { Charge } \\
\text { (\$) }\end{array}$ \\
\hline January & $5,751,815$ & 0 & $5,751,815$ & 0 & 690,218 & 0 \\
\hline February & $4,692,684$ & 0 & $4,692,684$ & 0 & 563,122 & 0 \\
\hline March & $5,049,504$ & 0 & $5,049,504$ & 0 & 605,940 & 0 \\
\hline April & $4,681,443$ & 0 & $4,681,443$ & 0 & 561,773 & 0 \\
\hline May & $4,726,141$ & 0 & $4,726,141$ & 0 & 567,137 & 0 \\
\hline June & $6,541,892$ & 0 & $6,541,892$ & 0 & 785,027 & 0 \\
\hline July & $7,964,076$ & 0 & $7,964,076$ & 0 & 955,689 & 0 \\
\hline August & $8,092,122$ & 0 & $8,092,122$ & 0 & 971,055 & 0 \\
\hline September & $6,744,112$ & 0 & $6,744,112$ & 0 & 809,293 & 0 \\
\hline October & $5,548,274$ & 0 & $5,548,274$ & 0 & 665,793 & 0 \\
\hline November & $5,255,797$ & 0 & $5,255,797$ & 0 & 630,696 & 0 \\
\hline December & $5,853,593$ & 0 & $5,853,593$ & 0 & 702,431 & 0 \\
\hline Annual & $70,901,448$ & 0 & $70,901,448$ & 0 & $8,508,174$ & 0 \\
\hline
\end{tabular}

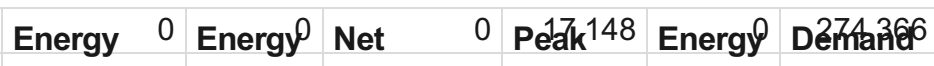

Purchased Sold 0 Purchaseo Denąag Charge \&,4aggre6

(kWh)

(kWh)

(kWh)

(kW)

(\$)

(\$)

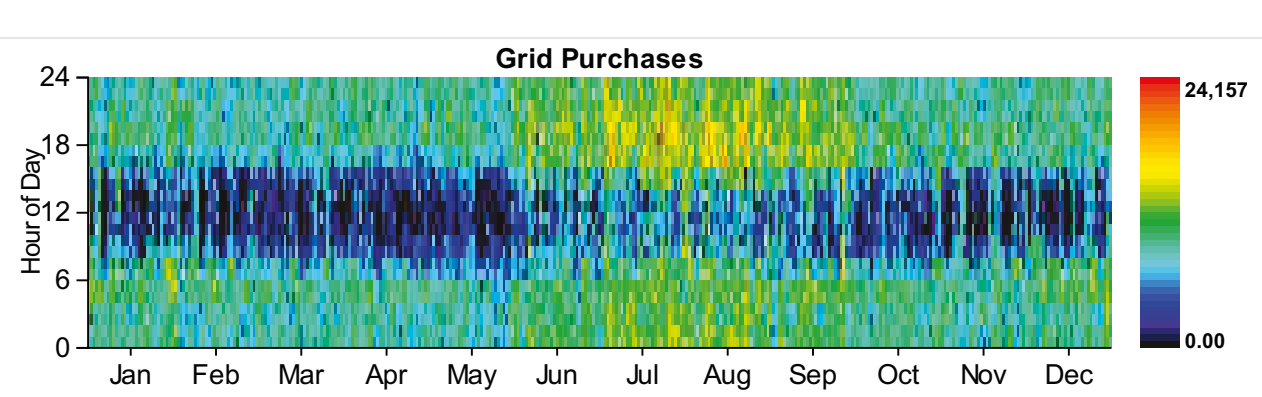

\section{Emissions}

\begin{tabular}{|l|r|l|}
\hline Pollutant & Emissions & Units \\
\hline Carbon dioxide & $44809716 \mathrm{~kg} / \mathrm{yr}$ \\
\hline Carbon monoxide & $0 \mathrm{~kg} / \mathrm{yr}$ \\
\hline Unburned hydrocarbons & $0 \mathrm{~kg} / \mathrm{yr}$ \\
\hline Particulate matter & $0 \mathrm{~kg} / \mathrm{yr}$ \\
\hline Sulfur dioxide & $194270 \mathrm{~kg} / \mathrm{yr}$ \\
\hline Nitrogen oxides & $95008 \mathrm{~kg} / \mathrm{yr}$ \\
\hline
\end{tabular}


System Report

System architecture

\begin{tabular}{|c|c|c|c|}
\hline PV & SunPower PV & 13,780 & $\mathrm{~kW}$ \\
\hline PV \#2 & Remaining Generic flat plate PV & 1,547 & $\mathrm{~kW}$ \\
\hline PV \#3 & Solar World 320W flat plate PV Copy & 1,000 & $\mathrm{~kW}$ \\
\hline Battery & GS200 flow & 1 & strings \\
\hline Converter & System Converter & 10,000 & $\mathrm{~kW}$ \\
\hline Grid & Grid & 27,200 & $\mathrm{~kW}$ \\
\hline Dispatch Strategy & Cycle Charging & & \\
\hline
\end{tabular}

\section{Cost summary}

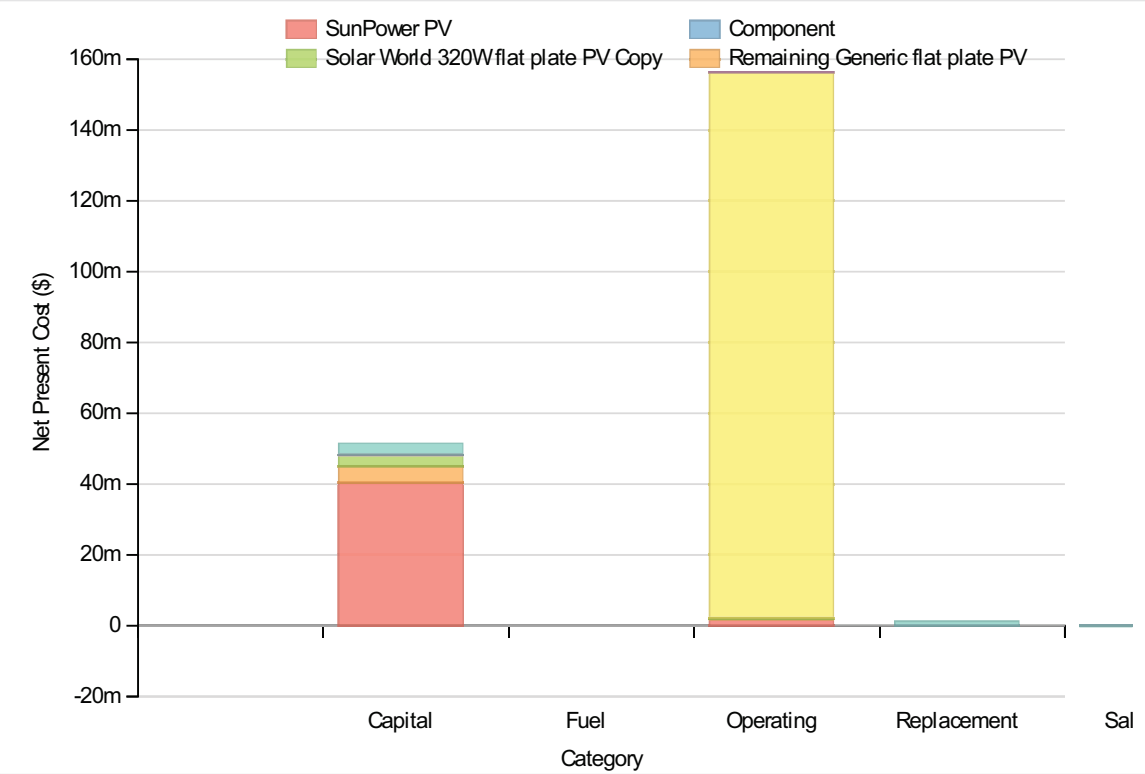

Cost Summary

Total net present cost

$208789744 \$$

Levelized cost of energy

$0.170 \$ / k W h$

Net Present Costs

\begin{tabular}{|c|c|c|c|c|c|c|}
\hline Component & Capital & Replacement & O\&M & Fuel & Salvage & Total \\
\hline SunPower PV & $40,358,332$ & 0 & $1,656,876$ & 0 & 0 & $42,015,208$ \\
\hline Remaining Generic flat plate PV & $4,678,459$ & 0 & 339,013 & 0 & 0 & $5,017,472$ \\
\hline Solar World 320W flat plate PV Copy & $3,083,333$ & 0 & 280,096 & 0 & 0 & $3,363,429$ \\
\hline Grid & 0 & 0 & $153,998,464$ & 0 & 0 & $153,998,464$ \\
\hline GS200 flow & 324,589 & 7,176 & 31,026 & 0 & -973 & 361,818 \\
\hline Converter & $3,000,000$ & $1,272,821$ & 0 & 0 & $-239,558$ & $4,033,263$ \\
\hline System & $51,444,712$ & $1,279,996$ & $156,305,472$ & 0 & $-240,530$ & $208,789,650$ \\
\hline
\end{tabular}




\begin{tabular}{|c|c|c|c|c|c|c|}
\hline Component & Capital & Replacement & O\&M & Fuel & Salvage & Total \\
\hline SunPower PV & $3,121,894$ & 0 & 128,167 & 0 & 0 & $3,250,061$ \\
\hline Remaining Generic flat plate PV & 361,899 & 0 & 26,224 & 0 & 0 & 388,123 \\
\hline Solar World 320W flat plate PV Copy & 238,509 & 0 & 21,667 & 0 & 0 & 260,176 \\
\hline Grid & 0 & 0 & $11,912,456$ & 0 & 0 & $11,912,456$ \\
\hline GS200 flow & 25,108 & 555 & 2,400 & 0 & -75 & 27,988 \\
\hline Converter & 232,063 & 98,458 & 0 & 0 & $-18,531$ & 311,990 \\
\hline System & $3,979,474$ & 99,013 & $12,090,913$ & 0 & $-18,606$ & $16,150,794$ \\
\hline
\end{tabular}

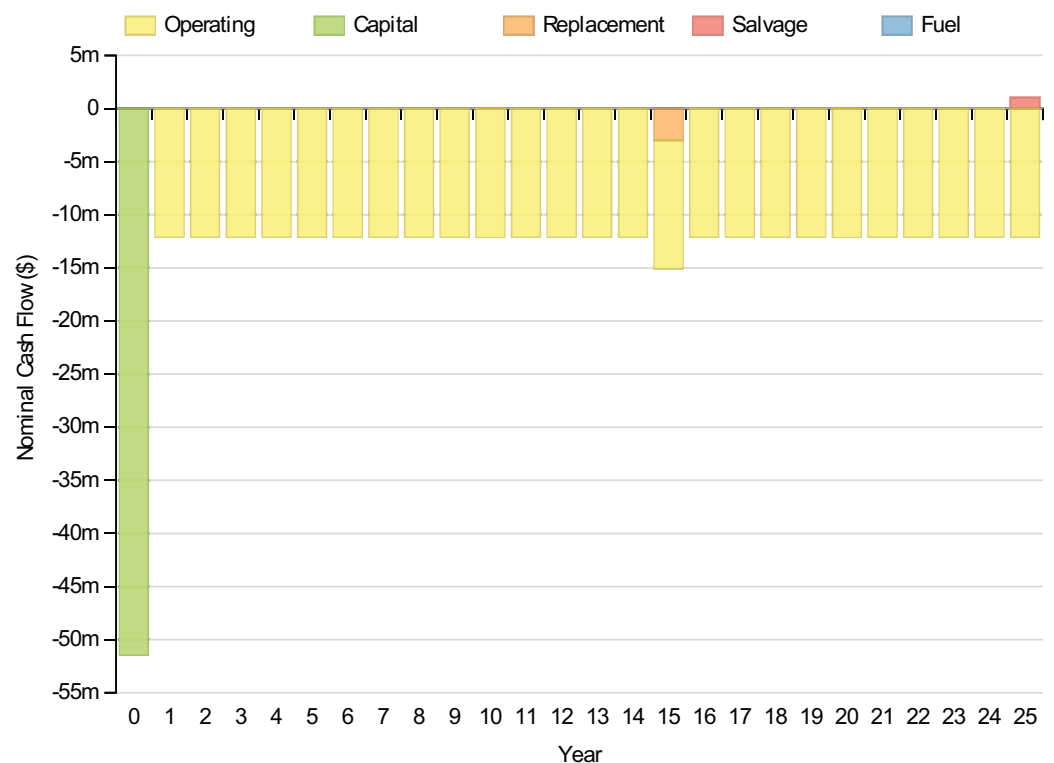

\section{Electrical}

\section{Quantity}

Excess electricity

Unmet load

Capacity shortage

Renewable fraction

\section{Component}

PV

PV

PV

Grid Purchases

Total

Load

Consumption(kWh/yr)

Production(kWh/yr)

\section{Value}

Fraction (\%)

$24,644,568$

25

$2,766,525$

$1,788,429$

$70,794,880$

$99,994,408$ 


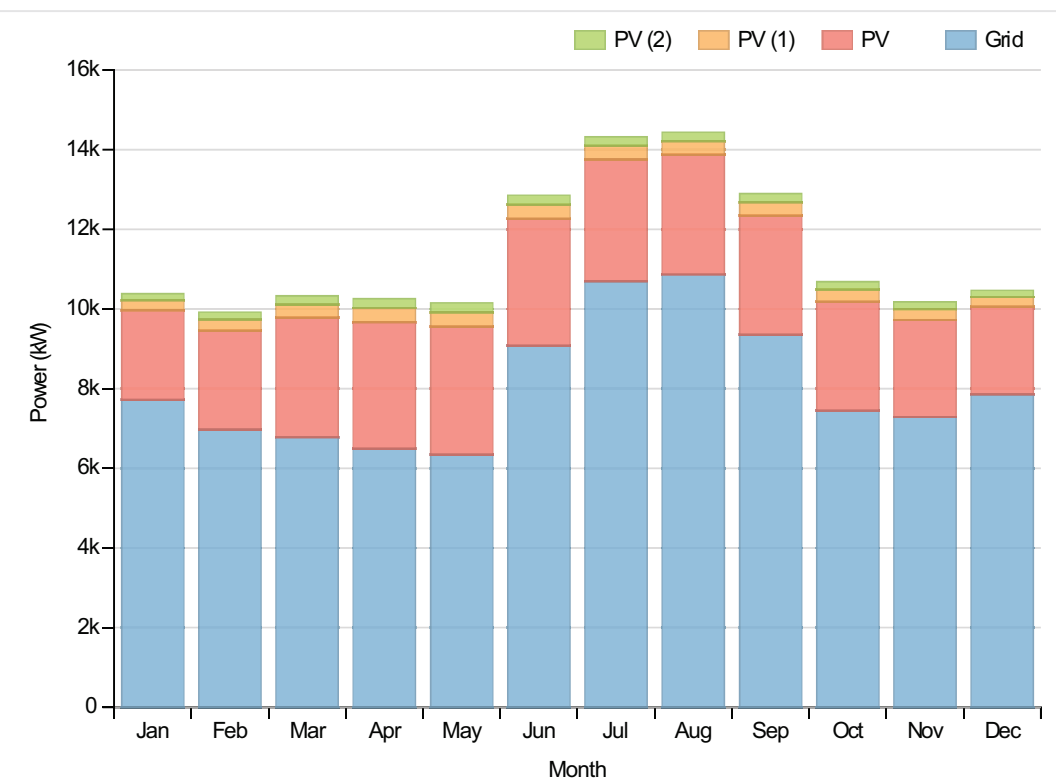

PV:SunPower PV

\begin{tabular}{|c|c|c|}
\hline Quantity & Value & Units \\
\hline Rated capacity & 13780 & $\mathrm{~kW}$ \\
\hline Mean output & 2813 & $\mathrm{~kW}$ \\
\hline Mean output & 67519.00 & $\mathrm{kWh} / \mathrm{d}$ \\
\hline Capacity factor & 20.42 & $\%$ \\
\hline Total production & 24644568 & $\mathrm{kWh} / \mathrm{yr}$ \\
\hline Minimum output & 0.00 & $\mathrm{~kW}$ \\
\hline Maximum output & 13941.00 & $\mathrm{~kW}$ \\
\hline PV penetration & 25.93 & $\%$ \\
\hline Hours of operation & 4386 & hrs/yr \\
\hline Levelized cost & 0.111 & $\$ / \mathrm{kWh}$ \\
\hline
\end{tabular}

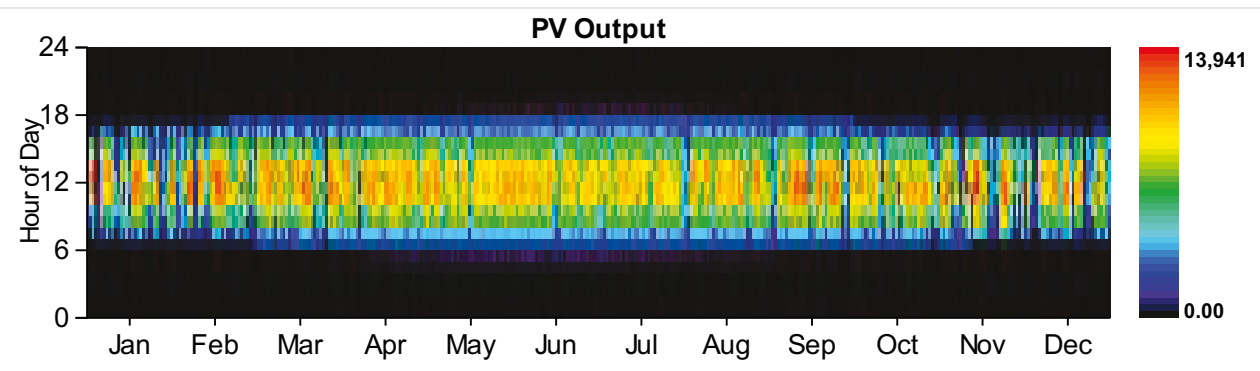

\section{$\mathrm{PV}$ :Remaining Generic flat plate PV}

\section{Quantity}

Rated capacity

Mean output

Mean output

\section{Value}

Units

1547 kW

$316 \mathrm{~kW}$ 


\begin{tabular}{|c|c|c|c|}
\hline QuRaratity factor & Value & 20.42 & Units \\
\hline Total production & & 2766525 & $\mathrm{kWh} / \mathrm{yr}$ \\
\hline Minimum output & & 0.00 & $\mathrm{~kW}$ \\
\hline Maximum output & & 1565.00 & $\mathrm{~kW}$ \\
\hline PV penetration & & 2.91 & $\%$ \\
\hline Hours of operation & & 4386 & $\mathrm{hrs} / \mathrm{yr}$ \\
\hline Levelized cost & & 0.013 & $\$ / k W h$ \\
\hline
\end{tabular}

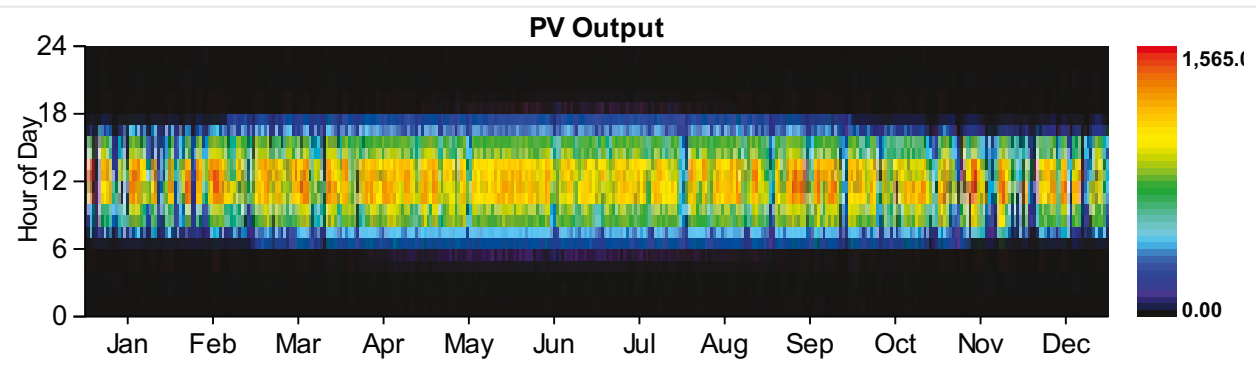

\section{PV:Solar World 320W flat plate PV Copy}

\section{Quantity}

Rated capacity

Mean output

Mean output

Capacity factor

Total production

Minimum output

Maximum output

PV penetration

Hours of operation

Levelized cost

\section{Value}

ran

$(20)$




\section{Quantity}

Value

Units

Nominal capacity

Usable nominal capacity

Autonomy

$0 \mathrm{hr}$

Lifetime throughput

Battery wear cost

$0.000 \$ / \mathrm{kWh}$

Average energy cost

$0.000 \$ / k W h$

Energy in

$168438 \mathrm{kWh} / \mathrm{yr}$

Energy out

$118409 \mathrm{kWh} / \mathrm{yr}$

Storage depletion

$600 \mathrm{kWh} / \mathrm{yr}$

Losses

$49429 \mathrm{kWh} / \mathrm{yr}$

Annual throughput

$141526 \mathrm{kWh} / \mathrm{yr}$

Expected life

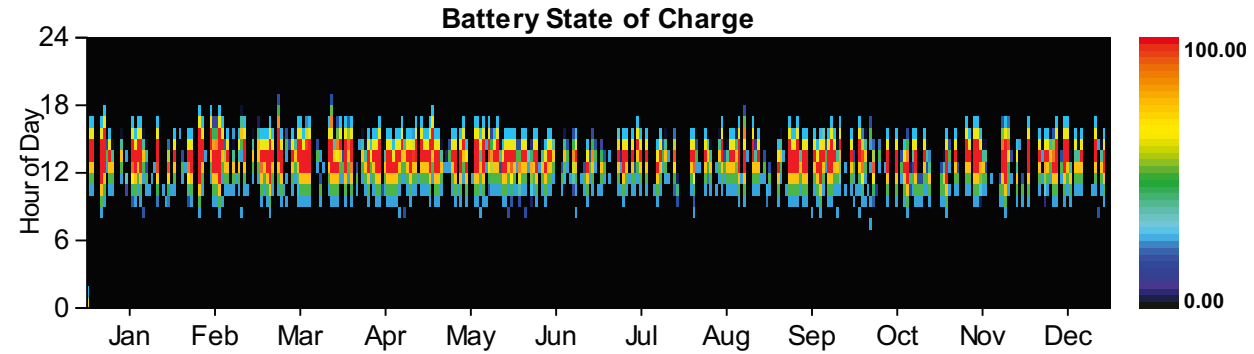

\section{Converter}

\begin{tabular}{|l|r|r|r|}
\hline Quantity & Inverter & Rectifier & Units \\
\hline Capacity & 10,000 & 9,000 & $\mathrm{~kW}$ \\
\hline Mean output & 2,769 & 0 \\
\hline Minimum output & 0 & $\mathrm{~kW}$ \\
\hline Maximum output & 10,000 & 0 \\
\hline Capacity factor & $\mathrm{kW}$ & 0 \\
\hline Hours of operation & 28 & 0 \\
\hline Energy in & 4,479 & $\mathrm{hrs} / \mathrm{yr}$ \\
\hline Energy out & $26,953,204$ & 0 \\
\hline Losses & $\mathrm{kWh} / \mathrm{yr}$ \\
\hline
\end{tabular}



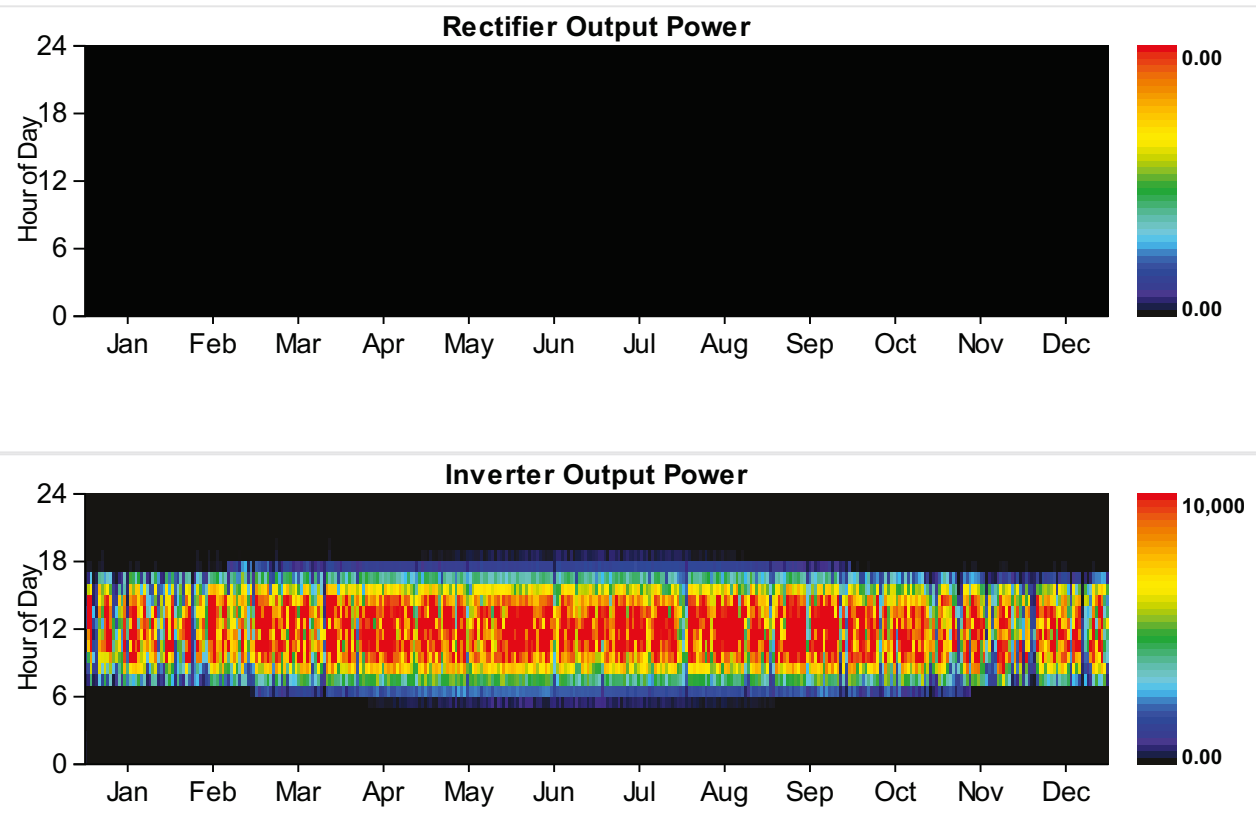

Grid

Rate: Rate 1 Demand

\begin{tabular}{|c|c|c|c|c|c|c|}
\hline Resources.ReportingService_GeneratelnputsReport_Month & $\begin{array}{l}\text { Energy } \\
\text { Purchased } \\
\text { (kWh) }\end{array}$ & $\begin{array}{l}\text { Energy } \\
\text { Sold } \\
\text { (kWh) }\end{array}$ & $\begin{array}{l}\text { Net } \\
\text { Purchases } \\
\text { (kWh) }\end{array}$ & $\begin{array}{l}\text { Peak } \\
\text { Demand } \\
(\mathbf{k W})\end{array}$ & $\begin{array}{l}\text { Energy } \\
\text { Charge } \\
(\$)\end{array}$ & $\begin{array}{l}\text { Demand } \\
\text { Charge } \\
(\$)\end{array}$ \\
\hline January & 0 & 0 & 0 & 17,079 & 0 & 273,262 \\
\hline February & 0 & 0 & 0 & 15,668 & 0 & 250,685 \\
\hline March & 0 & 0 & 0 & 17,075 & 0 & 273,199 \\
\hline April & 0 & 0 & 0 & 14,394 & 0 & 230,299 \\
\hline May & 0 & 0 & 0 & 14,850 & 0 & 237,602 \\
\hline June & 0 & 0 & 0 & 20,019 & 0 & 320,305 \\
\hline July & 0 & 0 & 0 & 24,158 & 0 & 386,525 \\
\hline August & 0 & 0 & 0 & 22,544 & 0 & 360,700 \\
\hline September & 0 & 0 & 0 & 18,833 & 0 & 301,321 \\
\hline October & 0 & 0 & 0 & 16,050 & 0 & 256,794 \\
\hline November & 0 & 0 & 0 & 15,751 & 0 & 252,018 \\
\hline December & 0 & 0 & 0 & 17,148 & 0 & 274,366 \\
\hline Annual & 0 & 0 & 0 & 24,158 & 0 & $3,417,076$ \\
\hline
\end{tabular}

Rate: Rate 1

\begin{tabular}{|c|c|c|c|c|c|c|}
\hline Resources.ReportingService_GeneratelnputsReport_Month & $\begin{array}{l}\text { Energy } \\
\text { Purchased } \\
\text { (kWh) }\end{array}$ & $\begin{array}{l}\text { Energy } \\
\text { Sold } \\
\text { (kWh) }\end{array}$ & $\begin{array}{l}\text { Net } \\
\text { Purchases } \\
\text { (kWh) }\end{array}$ & $\begin{array}{l}\text { Peak } \\
\text { Demand } \\
(\mathbf{k W})\end{array}$ & $\begin{array}{l}\text { Energy } \\
\text { Charge } \\
(\$)\end{array}$ & $\begin{array}{l}\text { Demand } \\
\text { Charge } \\
(\$)\end{array}$ \\
\hline January & $5,743,823$ & 0 & $5,743,823$ & 0 & 689,259 & 0 \\
\hline February & $4,684,560$ & 0 & $4,684,560$ & 0 & 562,147 & 0 \\
\hline March & $5,039,282$ & 0 & $5,039,282$ & 0 & 604,714 & 0 \\
\hline
\end{tabular}




\begin{tabular}{|c|c|c|c|c|c|c|}
\hline & $\begin{array}{l}\text { Energy } \\
\text { Purchased }\end{array}$ & $\begin{array}{l}\text { Energy } \\
\text { Sold }\end{array}$ & $\begin{array}{l}\text { Net } \\
\text { Purchaseses }\end{array}$ & $\begin{array}{l}\text { Peak } 0 \\
\text { Demand }\end{array}$ & $\begin{array}{l}\text { Energy } \\
\text { Charge } \\
\text { Charge }\end{array}$ & $\begin{array}{l}\text { Demang } \\
\text { Charge }\end{array}$ \\
\hline Reasources.ReportingService_GeneratelnputsReport_Month & $\left(\mathbf{k} 4,7 \mathrm{p}_{4,417}\right.$ & $(\mathbf{k W h})_{0}$ & $(\mathbf{k}) /, 4)_{4,417}$ & (kW) 0 & $(\$ 565,730$ & (\$) 0 \\
\hline June & $6,532,493$ & 0 & $6,532,493$ & 0 & 783,899 & 0 \\
\hline July & $7,956,805$ & 0 & $7,956,805$ & 0 & 954,817 & 0 \\
\hline August & $8,083,955$ & 0 & $8,083,955$ & 0 & 970,075 & 0 \\
\hline September & $6,734,741$ & 0 & $6,734,741$ & 0 & 808,169 & 0 \\
\hline October & $5,540,203$ & 0 & $5,540,203$ & 0 & 664,824 & 0 \\
\hline November & $5,248,187$ & 0 & $5,248,187$ & 0 & 629,782 & 0 \\
\hline December & $5,846,067$ & 0 & $5,846,067$ & 0 & 701,528 & 0 \\
\hline Annual & $70,794,880$ & 0 & $70,794,880$ & 0 & $8,495,386$ & 0 \\
\hline
\end{tabular}

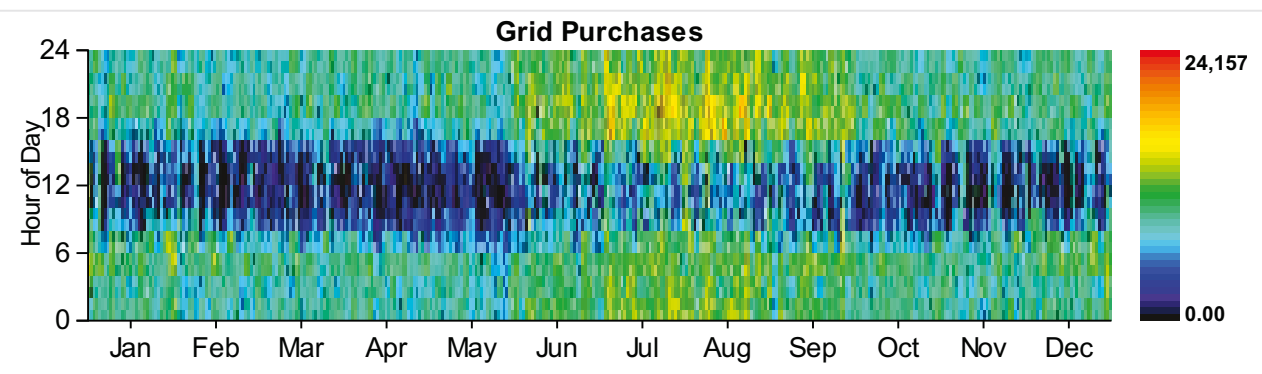

\section{Emissions}

\section{Pollutant}

Carbon dioxide

Carbon monoxide

Unburned hydrocarbons

Particulate matter

Sulfur dioxide

Nitrogen oxides

HOMER Energy, LLC @ 2016

\section{Emissions}

\section{Units}

44742364 kg/yr

$0 \mathrm{~kg} / \mathrm{yr}$

$0 \mathrm{~kg} / \mathrm{yr}$

$0 \mathrm{~kg} / \mathrm{yr}$

193978 kg/yr

$94865 \mathrm{~kg} / \mathrm{yr}$ 
System Report

System architecture

\begin{tabular}{|l|l|l|l|}
\hline PV & SunPower PV & 13,780 & $\mathrm{~kW}$ \\
\hline PV \#2 & Remaining Generic flat plate PV & 1,547 & $\mathrm{~kW}$ \\
\hline PV \#3 & Solar World 320W flat plate PV Copy & 1,000 & $\mathrm{~kW}$ \\
\hline Generator & Kohler 3250 Prime Power & 2,800 \\
\hline Converter & System Converter & 10,000 \\
\hline Grid & GWW & $\mathrm{kW}$ \\
\hline Dispatch Strategy & Cycle Charging & 20,000 \\
\hline
\end{tabular}

\section{Cost summary}

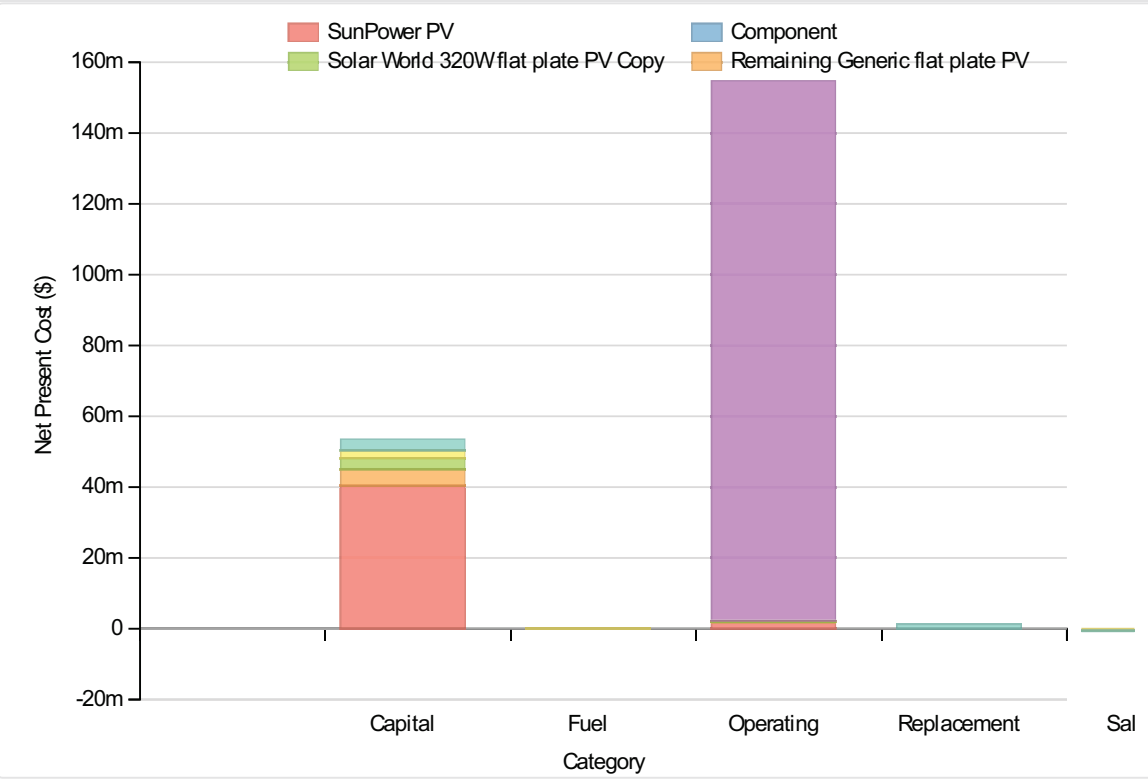

Cost Summary

Total net present cost

$208874000 \$$

Levelized cost of energy

$0.170 \$ / k W h$

Net Present Costs

\begin{tabular}{|c|c|c|c|c|c|c|}
\hline Component & Capital & Replacement & O\&M & Fuel & Salvage & Total \\
\hline SunPower PV & $40,358,332$ & 0 & $1,656,876$ & 0 & 0 & $42,015,208$ \\
\hline Remaining Generic flat plate PV & $4,678,459$ & 0 & 339,013 & 0 & 0 & $5,017,472$ \\
\hline Solar World 320W flat plate PV Copy & $3,083,333$ & 0 & 280,096 & 0 & 0 & $3,363,429$ \\
\hline Kohler 3250 Prime Power & $2,350,000$ & 0 & 38,457 & 168,760 & $-493,528$ & $2,063,689$ \\
\hline Grid & 0 & 0 & $152,380,880$ & 0 & 0 & $152,380,880$ \\
\hline Converter & $3,000,000$ & $1,272,821$ & 0 & 0 & $-239,558$ & $4,033,263$ \\
\hline System & $53,470,124$ & $1,272,821$ & $154,695,296$ & 168,760 & $-733,086$ & $208,873,915$ \\
\hline
\end{tabular}




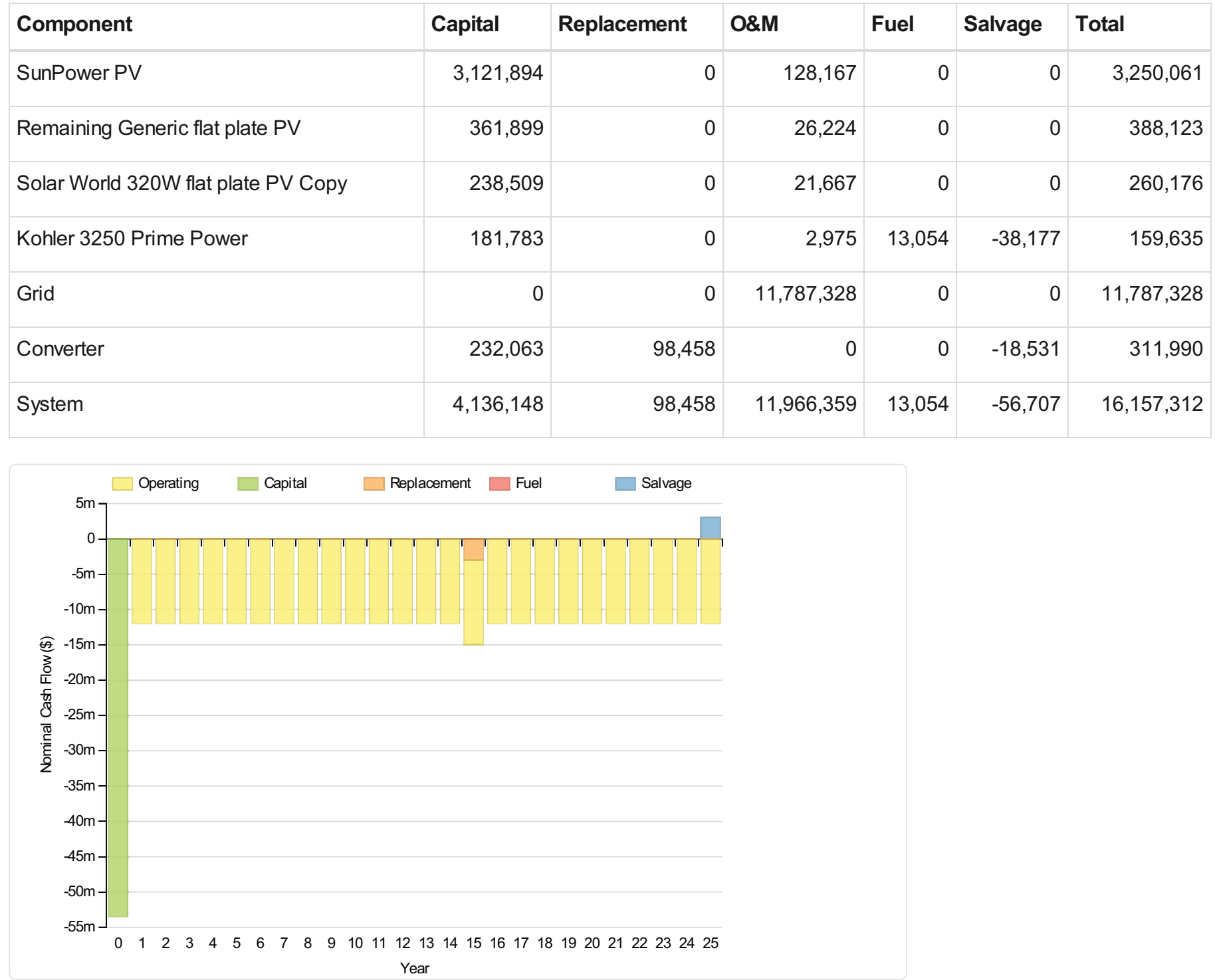

\section{Electrical}

\section{Quantity}

Excess electricity

Unmet load

Capacity shortage

Renewable fraction

\section{Component}

PV

PV

PV

Generator

Grid Purchases

Total

\section{Production(kWh/yr)}

Value

Units

$2364865 \mathrm{kWh} / \mathrm{yr}$

2334 kWh/yr

$33532 \mathrm{kWh} / \mathrm{yr}$

0

\section{Fraction (\%)}

$24,644,568$

$2,766,525$

$1,788,429$

66,764

\section{Load}

Consumption(kWh/yr)

Fraction (\%) 


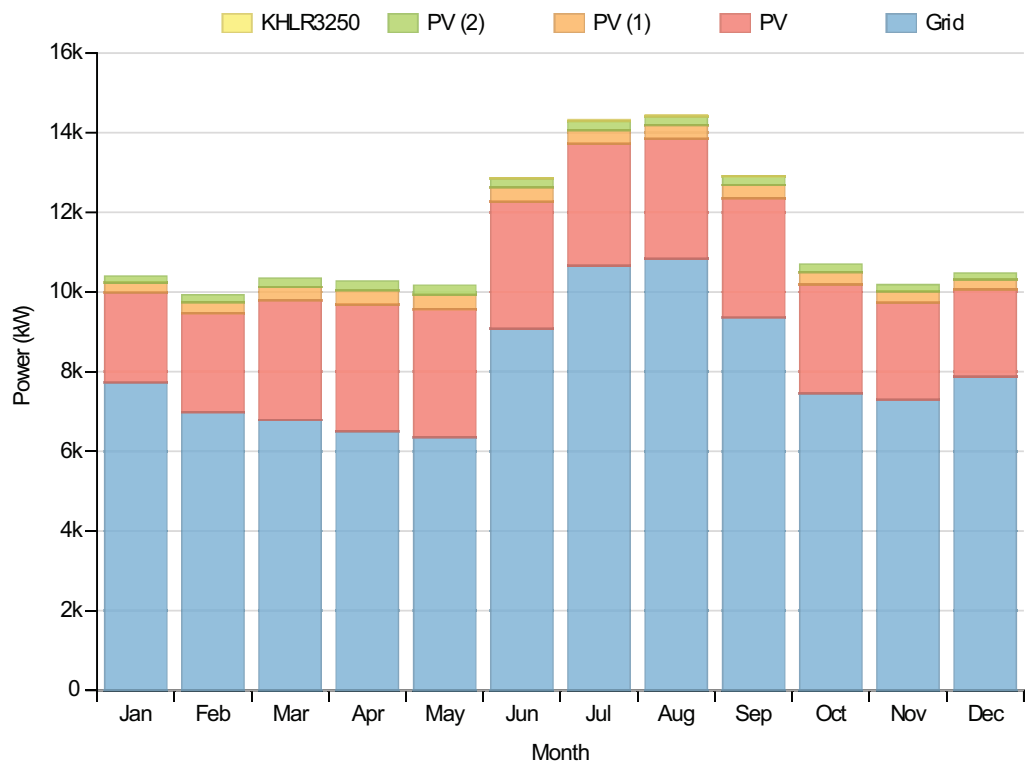

\section{PV:SunPower PV}

\begin{tabular}{|l|l|l|}
\hline Quantity & Value & Units \\
\hline Rated capacity & $13780 \mathrm{~kW}$ \\
\hline Mean output & $2813 \mathrm{~kW}$ \\
\hline Mean output & $67519.00 \mathrm{kWh} / \mathrm{d}$ \\
\hline Capacity factor & $20.42 \%$ & $\%$ \\
\hline Total production & $24644568 \mathrm{kWh} / \mathrm{yr}$ \\
\hline Minimum output & $0.00 \mathrm{~kW}$ \\
\hline Maximum output & $13941.00 \mathrm{~kW}$ \\
\hline PV penetration & $25.93 \%$ & $\%$ \\
\hline Hours of operation & 4386 & $\mathrm{hrs} / \mathrm{yr}$ \\
\hline Levelized cost & $\$ 1 / \mathrm{kWh}$ \\
\hline
\end{tabular}

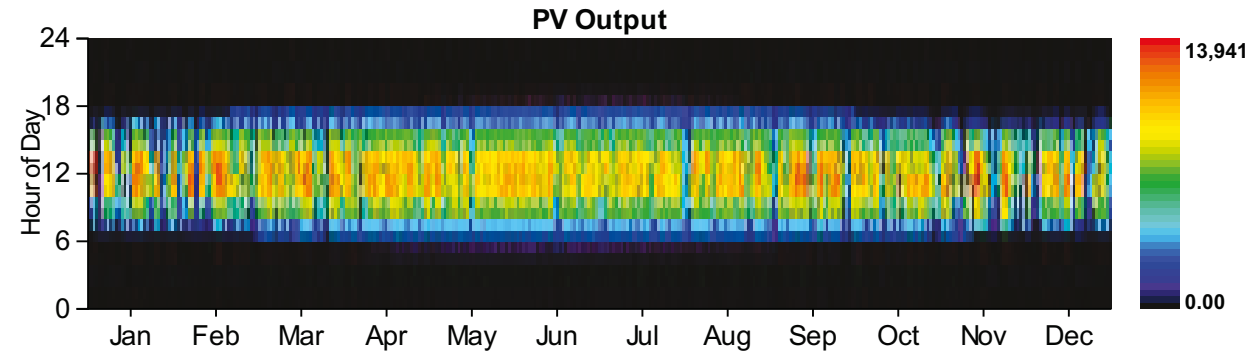

PV:Remaining Generic flat plate PV

\begin{tabular}{|l|l|l|}
\hline Quantity & Value & Units \\
\hline Rated capacity & $1547 \mathrm{~kW}$ \\
\hline Mean output & $316 \mathrm{~kW}$ \\
\hline
\end{tabular}


Qeannfityput

Capacity factor

Total production

Minimum output

Maximum output

PV penetration

Hours of operation

Levelized cost
7579.50 kflifts

$20.42 \%$

$2766525 \mathrm{kWh} / \mathrm{yr}$

$0.00 \mathrm{~kW}$

1565.00 kW

$2.91 \%$

$4386 \mathrm{hrs} / \mathrm{yr}$

$0.013 \$ / \mathrm{kWh}$

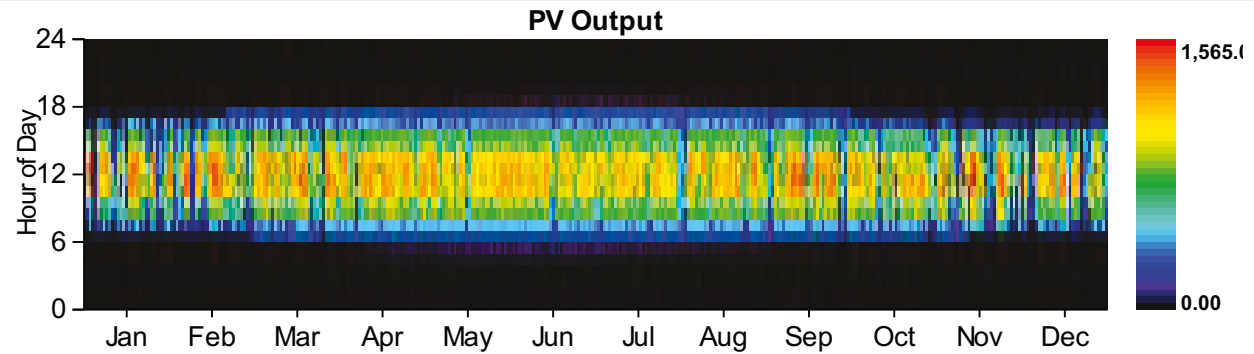

PV:Solar World 320W flat plate PV Copy

\begin{tabular}{|l|c|c|}
\hline Quantity & Value & Units \\
\hline Rated capacity & $1000 \mathrm{~kW}$ \\
\hline Mean output & $204 \mathrm{~kW}$ \\
\hline Mean output & $4899.80 \mathrm{kWh} / \mathrm{d}$ \\
\hline Capacity factor & $20.42 \%$ \\
\hline Total production & $1788429 \mathrm{kWh} / \mathrm{yr}$ \\
\hline Minimum output & $0.00 \mathrm{~kW}$ \\
\hline Maximum output & $1011.70 \mathrm{~kW}$ \\
\hline PV penetration & $1.88 \%$ & $\%$ \\
\hline Hours of operation & $4386 \mathrm{hrs} / \mathrm{yr}$ \\
\hline Levelized cost & $\$ / \mathrm{kWh}$ \\
\hline
\end{tabular}

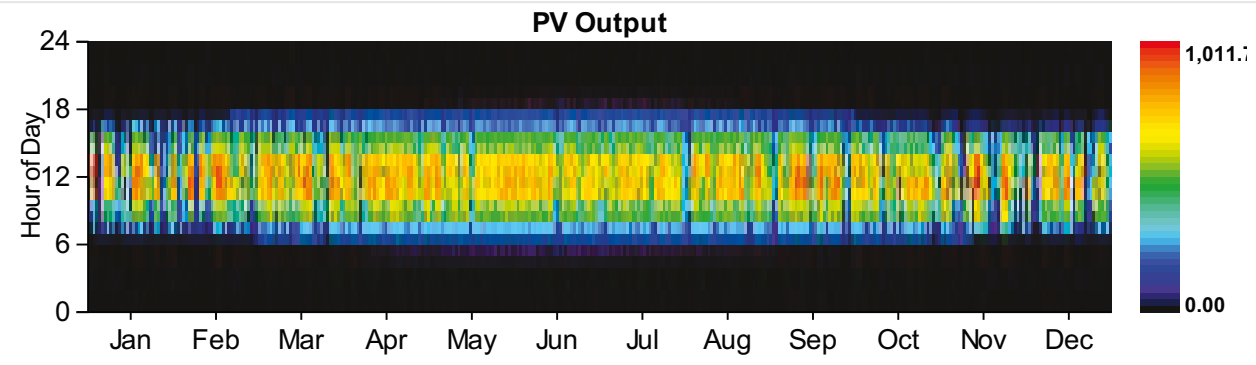

Generator:Kohler 3250 Prime Power 
dedtfiftity

Number of starts

Operational life

Fixed generation cost

Marginal generation cost

Electrical production

Mean electrical output

Min. electrical output

Max. electrical output

Fuel consumption

Specific fuel consumption

Fuel energy input

Mean electrical efficiency
Value

74 brsturs

66 starts/yr

$203 \mathrm{yr}$

$207.82 \$ / h r$

$0.18 \$ / k W h$

66764 kWh/yr

902 kW

$700 \mathrm{~kW}$

$2800 \mathrm{~kW}$

16524 L/yr

$0.25 \mathrm{~L} / \mathrm{kWh}$

$162601 \mathrm{kWh} / \mathrm{yr}$

$41 \%$

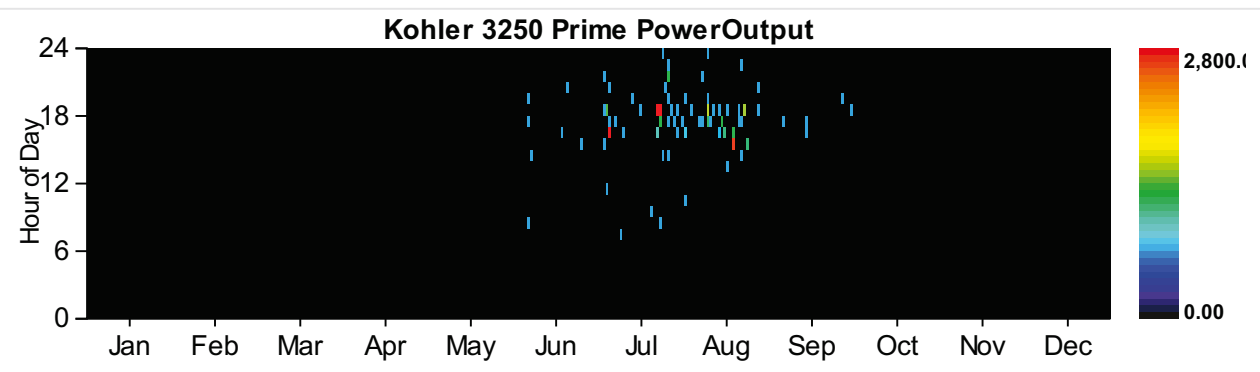

\section{Converter}

\begin{tabular}{|c|c|c|c|}
\hline Quantity & Inverter & Rectifier & Units \\
\hline Capacity & 10,000 & 9,000 & $\mathrm{~kW}$ \\
\hline Mean output & 2,757 & 0 & $\mathrm{~kW}$ \\
\hline Minimum output & 0 & 0 & $\mathrm{~kW}$ \\
\hline Maximum output & 10,000 & 0 & $\mathrm{~kW}$ \\
\hline Capacity factor & 28 & 0 & $\%$ \\
\hline Hours of operation & 4,386 & 0 & $\mathrm{hrs} / \mathrm{yr}$ \\
\hline Energy in & $26,834,786$ & 0 & $\mathrm{kWh} / \mathrm{yr}$ \\
\hline Energy out & $24,151,150$ & 0 & $\mathrm{kWh} / \mathrm{yr}$ \\
\hline Losses & $2,683,636$ & 0 & $\mathrm{kWh} / \mathrm{yr}$ \\
\hline
\end{tabular}



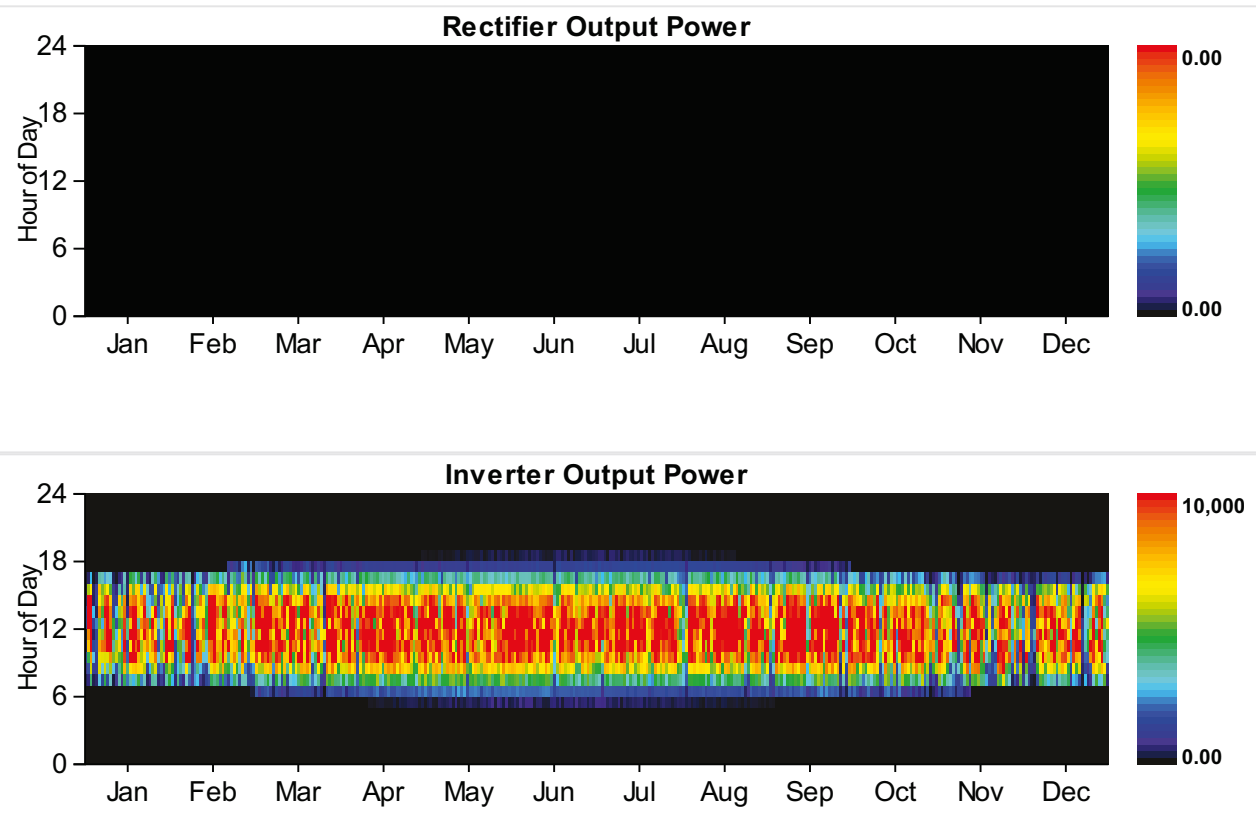

Grid

Rate: Rate 1 Demand

\begin{tabular}{|c|c|c|c|c|c|c|}
\hline Resources.ReportingService_GeneratelnputsReport_Month & $\begin{array}{l}\text { Energy } \\
\text { Purchased } \\
\text { (kWh) }\end{array}$ & $\begin{array}{l}\text { Energy } \\
\text { Sold } \\
\text { (kWh) }\end{array}$ & $\begin{array}{l}\text { Net } \\
\text { Purchases } \\
\text { (kWh) }\end{array}$ & $\begin{array}{l}\text { Peak } \\
\text { Demand } \\
(\mathbf{k W})\end{array}$ & $\begin{array}{l}\text { Energy } \\
\text { Charge } \\
(\$)\end{array}$ & $\begin{array}{l}\text { Demand } \\
\text { Charge } \\
\text { (\$) }\end{array}$ \\
\hline January & 0 & 0 & 0 & 17,079 & 0 & 273,262 \\
\hline February & 0 & 0 & 0 & 15,668 & 0 & 250,685 \\
\hline March & 0 & 0 & 0 & 17,075 & 0 & 273,199 \\
\hline April & 0 & 0 & 0 & 14,394 & 0 & 230,299 \\
\hline May & 0 & 0 & 0 & 14,850 & 0 & 237,602 \\
\hline June & 0 & 0 & 0 & 19,319 & 0 & 309,105 \\
\hline July & 0 & 0 & 0 & 20,000 & 0 & 320,000 \\
\hline August & 0 & 0 & 0 & 20,000 & 0 & 320,000 \\
\hline September & 0 & 0 & 0 & 18,133 & 0 & 290,121 \\
\hline October & 0 & 0 & 0 & 16,050 & 0 & 256,794 \\
\hline November & 0 & 0 & 0 & 15,751 & 0 & 252,018 \\
\hline December & 0 & 0 & 0 & 17,148 & 0 & 274,366 \\
\hline Annual & 0 & 0 & 0 & 20,000 & 0 & $3,287,451$ \\
\hline
\end{tabular}

Rate: Rate 1

\begin{tabular}{|c|c|c|c|c|c|c|}
\hline Resources.ReportingService_GeneratelnputsReport_Month & $\begin{array}{l}\text { Energy } \\
\text { Purchased } \\
\text { (kWh) }\end{array}$ & $\begin{array}{l}\text { Energy } \\
\text { Sold } \\
\text { (kWh) }\end{array}$ & $\begin{array}{l}\text { Net } \\
\text { Purchases } \\
\text { (kWh) }\end{array}$ & $\begin{array}{l}\text { Peak } \\
\text { Demand } \\
(\mathbf{k W})\end{array}$ & $\begin{array}{l}\text { Energy } \\
\text { Charge } \\
(\$)\end{array}$ & $\begin{array}{l}\text { Demand } \\
\text { Charge } \\
(\$)\end{array}$ \\
\hline January & $5,751,815$ & 0 & $5,751,815$ & 0 & 690,218 & 0 \\
\hline February & $4,692,684$ & 0 & $4,692,684$ & 0 & 563,122 & 0 \\
\hline March & $5,049,504$ & 0 & $5,049,504$ & 0 & 605,940 & 0 \\
\hline
\end{tabular}




\begin{tabular}{|c|c|c|c|c|c|c|}
\hline & $\begin{array}{l}\text { Energy } \\
\text { Purch } 443 \\
\text { Pused }\end{array}$ & $\begin{array}{l}\text { Energy } \\
\text { Sold }\end{array}$ & $\begin{array}{l}\text { Net } \\
\text { Purchi,443 } \\
\text { Purch }\end{array}$ & $\begin{array}{l}\text { Peak } 0 \\
\text { Demand }\end{array}$ & $\begin{array}{l}\text { Energy } \\
\text { Chi, } 73 \\
\text { charge }\end{array}$ & $\begin{array}{l}\text { Demang } \\
\text { Charge }\end{array}$ \\
\hline Reasources.ReportingService_GeneratelnputsReport_Month & $(\mathbf{k} 4,426,141$ & $(\mathbf{k W h})_{0}$ & $(\mathbf{k} \mathbf{W h}, 726,141$ & $(\mathbf{k W}) \quad 0$ & $(\$)_{567,137}$ & $(\$)$ \\
\hline June & $6,536,992$ & 0 & $6,536,992$ & 0 & 784,439 & 0 \\
\hline July & $7,931,617$ & 0 & $7,931,617$ & 0 & 951,794 & 0 \\
\hline August & $8,063,882$ & 0 & $8,063,882$ & 0 & 967,666 & 0 \\
\hline September & $6,740,612$ & 0 & $6,740,612$ & 0 & 808,873 & 0 \\
\hline October & $5,548,274$ & 0 & $5,548,274$ & 0 & 665,793 & 0 \\
\hline November & $5,255,797$ & 0 & $5,255,797$ & 0 & 630,696 & 0 \\
\hline December & $5,853,593$ & 0 & $5,853,593$ & 0 & 702,431 & 0 \\
\hline Annual & $70,832,352$ & 0 & $70,832,352$ & 0 & $8,499,882$ & 0 \\
\hline
\end{tabular}

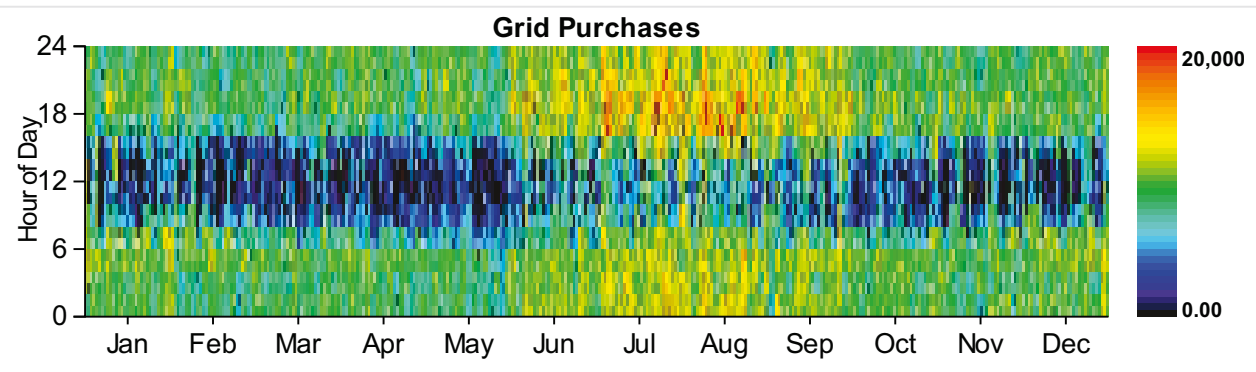

\section{Emissions}

\section{Pollutant}

Carbon dioxide

Carbon monoxide

Unburned hydrocarbons

Particulate matter

Sulfur dioxide

Nitrogen oxides

HOMER Energy, LLC @ 2016
Emissions

Units

44809416 kg/yr

$182 \mathrm{~kg} / \mathrm{yr}$

$21 \mathrm{~kg} / \mathrm{yr}$

$5 \mathrm{~kg} / \mathrm{yr}$

194170 kg/yr

95097 kg/yr 
System Report

System architecture

\begin{tabular}{|l|l|r|}
\hline PV & SunPower PV & 13,780 \\
\hline PV \#2 & Remaining Generic flat plate PV & 1,547 \\
\hline PV \#3 & Solar World 320W flat plate PV Copy & 1,000 \\
\hline Wind Turbine & Siemens $2.3 \mathrm{MW}-108$ & 2 \\
\hline Battery & GS200 flow & 3 \\
\hline Converter & System Converter & 10,000 \\
\hline Grid & kW & 20,000 \\
\hline Dispatch Strategy & Grid & $\mathrm{kW}$ \\
\hline
\end{tabular}

\section{Cost summary}

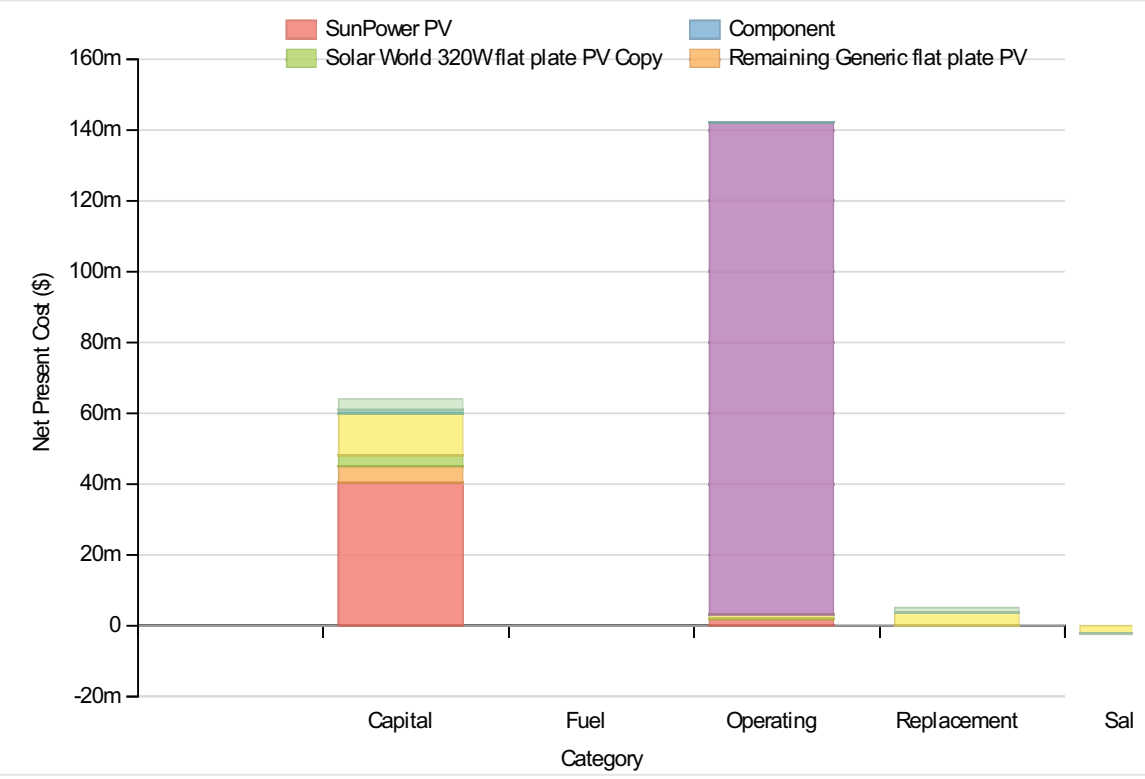

Cost Summary

Total net present cost

208878272 \$

Levelized cost of energy

$0.170 \$ / k W h$

\section{Net Present Costs}

\begin{tabular}{|c|c|c|c|c|c|c|}
\hline Component & Capital & Replacement & O\&M & Fuel & Salvage & Total \\
\hline SunPower PV & $40,358,332$ & 0 & $1,656,876$ & 0 & 0 & $42,015,208$ \\
\hline Remaining Generic flat plate PV & $4,678,459$ & 0 & 339,013 & 0 & 0 & $5,017,472$ \\
\hline Solar World 320W flat plate PV Copy & $3,083,333$ & 0 & 280,096 & 0 & 0 & $3,363,429$ \\
\hline Siemens 2.3 MW - 108 & $11,925,000$ & $3,801,774$ & $1,034,201$ & 0 & $-2,142,543$ & $14,618,432$ \\
\hline Grid & 0 & 0 & $138,744,976$ & 0 & 0 & $138,744,976$ \\
\hline GS200 flow & 973,767 & 21,510 & 93,078 & 0 & $-2,917$ & $1,085,439$ \\
\hline Converter & $3,000,000$ & $1,272,821$ & 0 & 0 & $-239,558$ & $4,033,263$ \\
\hline
\end{tabular}




\section{Annualized Costs}

\begin{tabular}{|c|c|c|c|c|c|c|}
\hline Component & Capital & Replacement & O\&M & Fuel & Salvage & Total \\
\hline SunPower PV & $3,121,894$ & 0 & 128,167 & 0 & 0 & $3,250,061$ \\
\hline Remaining Generic flat plate PV & 361,899 & 0 & 26,224 & 0 & 0 & 388,123 \\
\hline Solar World 320W flat plate PV Copy & 238,509 & 0 & 21,667 & 0 & 0 & 260,176 \\
\hline Siemens 2.3 MW - 108 & 922,451 & 294,084 & 80,000 & 0 & $-165,735$ & $1,130,800$ \\
\hline Grid & 0 & 0 & $10,732,531$ & 0 & 0 & $10,732,531$ \\
\hline GS200 flow & 75,325 & 1,664 & 7,200 & 0 & -226 & 83,963 \\
\hline Converter & 232,063 & 98,458 & 0 & 0 & $-18,531$ & 311,990 \\
\hline System & $4,952,142$ & 394,206 & $10,995,789$ & 0 & $-184,491$ & $16,157,646$ \\
\hline
\end{tabular}

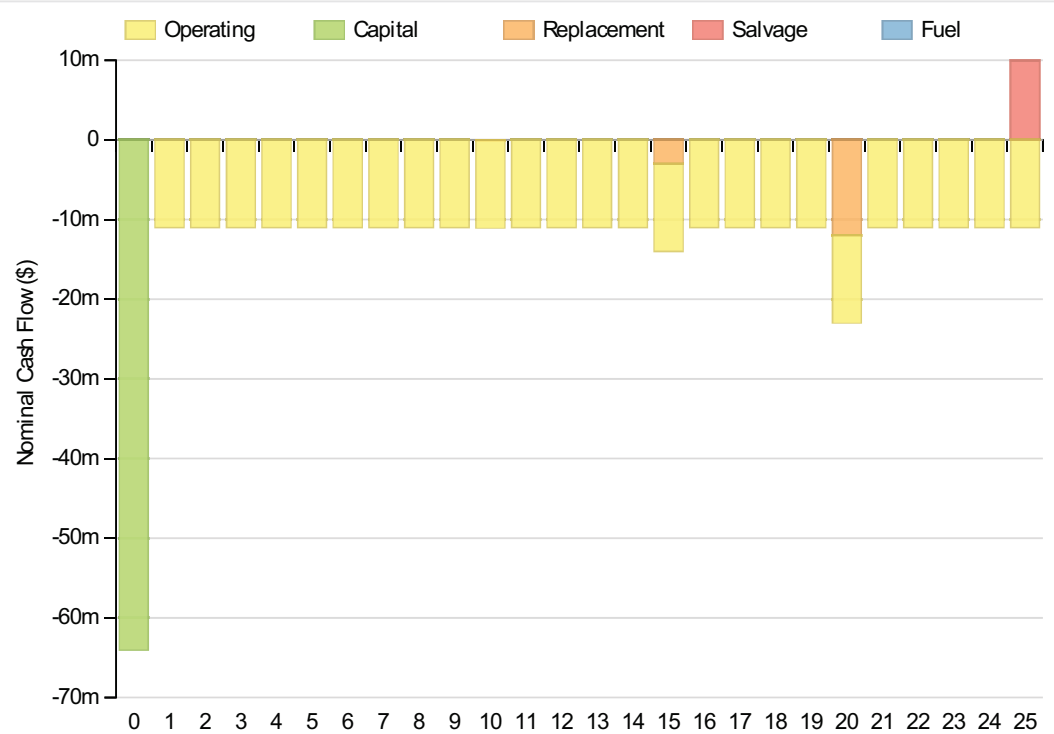

Year

Electrical

\section{Quantity}

Excess electricity

Unmet load

Capacity shortage

Renewable fraction

\begin{tabular}{|r|l|}
\hline Value & Units \\
\hline 3171029 & $\mathrm{kWh} / \mathrm{yr}$ \\
\hline 7341 & $\mathrm{kWh} / \mathrm{yr}$ \\
\hline 94840 & $\mathrm{kWh} / \mathrm{yr}$ \\
\hline 0 & \\
\hline
\end{tabular}

Fraction (\%)

\begin{tabular}{|l|r|r|}
\hline Component & Production(kWh/yr) & \multicolumn{1}{|l|}{ Fraction (\%) } \\
\hline PV & $24,644,568$ & 24 \\
\hline PV & $2,766,525$ & 3 \\
\hline PV & $1,788,429$ & 2 \\
\hline Wind Turbine & $9,348,703$ & 9 \\
\hline Grid Purchases & $62,415,532$ & 62 \\
\hline
\end{tabular}




\section{Load}

AC primary load

DC primary load

Total

\section{Consumption(kWh/yr)}

Fraction (\%)

$95,045,120$

100

0

0

$95,045,120$

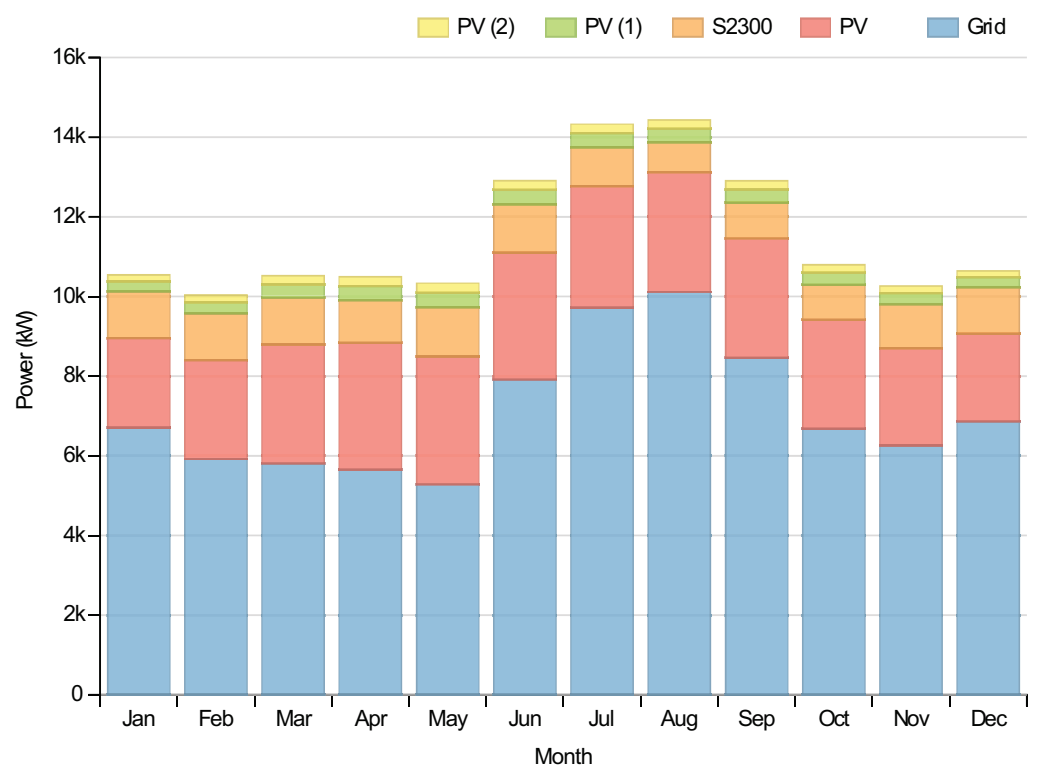

\section{PV:SunPower PV}

\section{Quantity}

Rated capacity

Mean output

Mean output

Capacity factor

Total production

Minimum output

Maximum output

PV penetration

Hours of operation

Levelized cost

\section{Value}

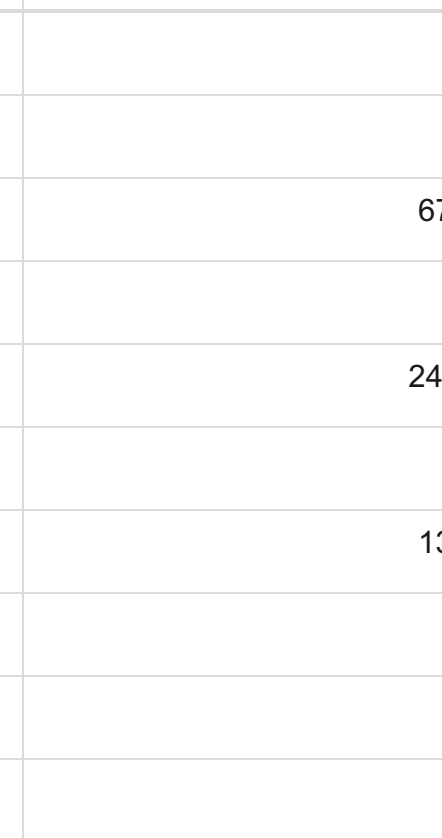

\begin{tabular}{|r|l|}
\hline 13780 & $\mathrm{~kW}$ \\
\hline 2813 & $\mathrm{~kW}$ \\
\hline 67519.00 & $\mathrm{kWh} / \mathrm{d}$ \\
\hline 20.42 & $\%$ \\
\hline 24644568 & $\mathrm{kWh} / \mathrm{yr}$ \\
\hline 0.00 & $\mathrm{~kW}$ \\
\hline 13941.00 & $\mathrm{~kW}$ \\
\hline 25.93 & $\%$ \\
\hline 4386 & $\mathrm{hrs} / \mathrm{yr}$ \\
\hline 0.111 & $\$ / \mathrm{kWh}$ \\
\hline
\end{tabular}

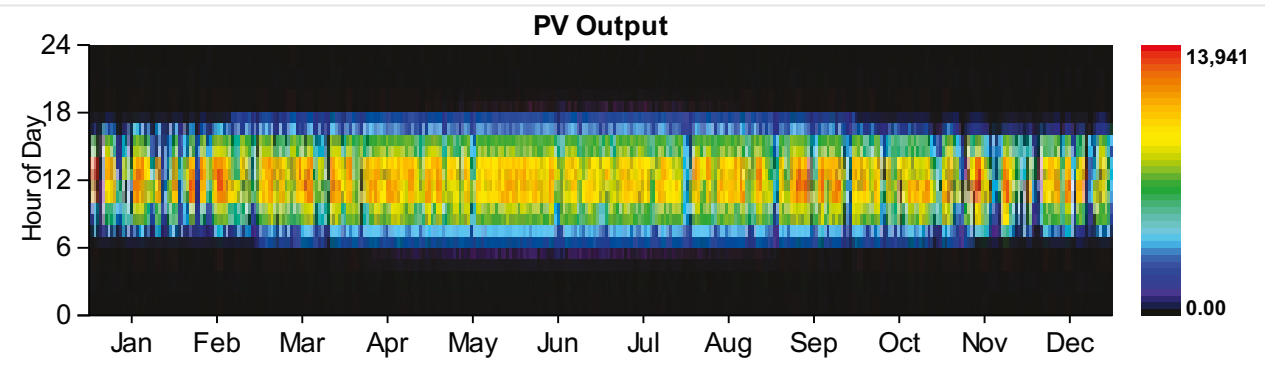




\section{Quantity}

Rated capacity

Mean output

Mean output

Capacity factor

Total production

Minimum output

Maximum output

PV penetration

Hours of operation

Levelized cost
Value

Units

1547 kW

316 kW

$7579.50 \mathrm{kWh} / \mathrm{d}$

$20.42 \%$

$2766525 \mathrm{kWh} / \mathrm{yr}$

$0.00 \mathrm{~kW}$

$1565.00 \mathrm{~kW}$

$2.91 \%$

$4386 \mathrm{hrs} / \mathrm{yr}$

$0.013 \$ / k W h$

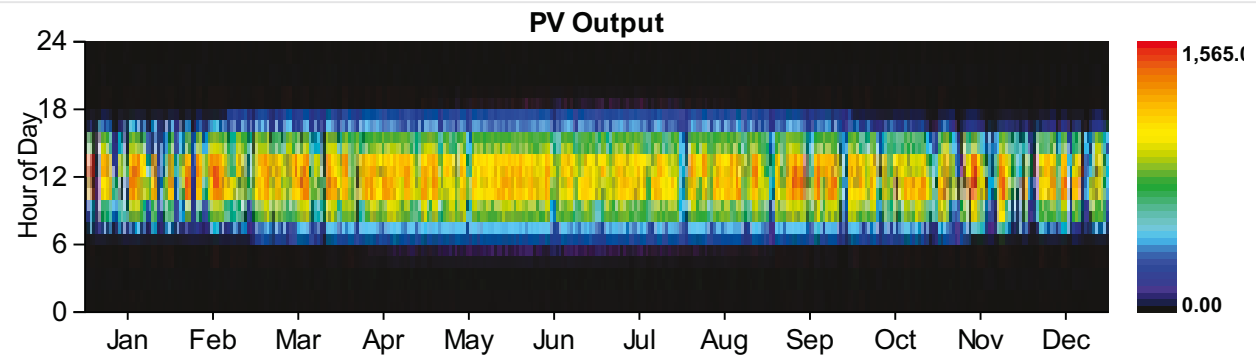

\section{PV:Solar World 320W flat plate PV Copy}

\begin{tabular}{|c|c|c|}
\hline Quantity & Value & Units \\
\hline Rated capacity & 1000 & kW \\
\hline Mean output & 204 & kW \\
\hline Mean output & 4899.80 & $\mathrm{kWh} / \mathrm{d}$ \\
\hline Capacity factor & 20.42 & $\%$ \\
\hline Total production & 1788429 & $\mathrm{kWh} / \mathrm{yr}$ \\
\hline Minimum output & 0.00 & $\mathrm{~kW}$ \\
\hline Maximum output & 1011.70 & $\mathrm{~kW}$ \\
\hline PV penetration & 1.88 & $\%$ \\
\hline Hours of operation & 4386 & $\mathrm{hrs} / \mathrm{yr}$ \\
\hline Levelized cost & 0.009 & $\$ / k W h$ \\
\hline
\end{tabular}




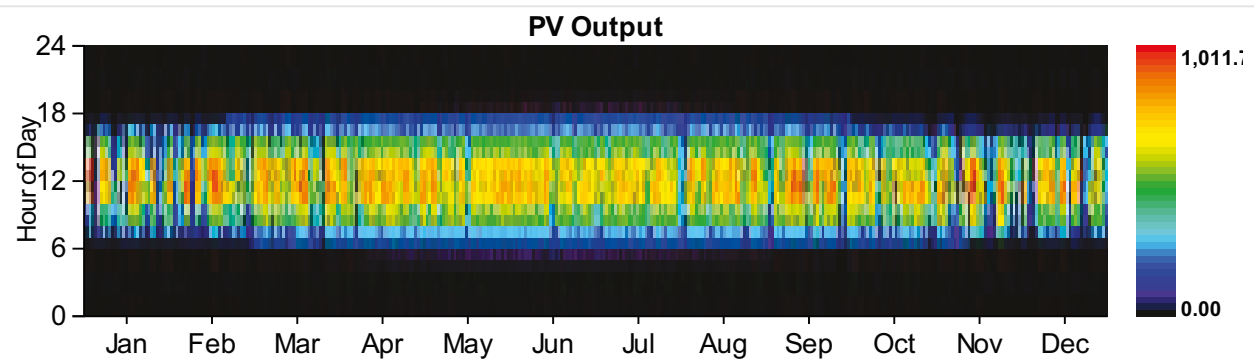

Wind Turbine:Siemens 2.3 MW - 108

\begin{tabular}{|l|r|l|}
\hline Quantity & Value & Units \\
\hline Total rated capacity & $4600 \mathrm{~kW}$ \\
\hline Mean output & $1067 \mathrm{~kW}$ \\
\hline Capacity factor & $23.20 \%$ \\
\hline Total production & $9348703 \mathrm{kWh} / \mathrm{yr}$ \\
\hline Minimum output & $0.99 \mathrm{~kW}$ \\
\hline Maximum output & $4625.40 \mathrm{~kW}$ \\
\hline Wind penetration & $9.84 \%$ \\
\hline Hours of operation & 8760 & $\mathrm{hrs} / \mathrm{yr}$ \\
\hline Levelized cost & $\$ 121 / \mathrm{kWh}$ \\
\hline
\end{tabular}

\section{Battery:GS200 flow}

Quantity $\quad$ Value

String size

Strings in parallel

Batteries

Bus voltage

\begin{tabular}{|c|c|c|}
\hline Quantity & Value & Units \\
\hline Nominal capacity & 1800 & kWh \\
\hline Usable nominal capacity & 1800 & kWh \\
\hline Autonomy & 0 & $\mathrm{hr}$ \\
\hline Lifetime throughput & 0 & \\
\hline Battery wear cost & 0.000 & $\$ / k W h$ \\
\hline Average energy cost & 0.000 & $\$ / k W h$ \\
\hline Energy in & 539759 & $\mathrm{kWh} / \mathrm{yr}$ \\
\hline Energy out & 379338 & $\mathrm{kWh} / \mathrm{yr}$ \\
\hline Storage depletion & 1800 & $\mathrm{kWh} / \mathrm{yr}$ \\
\hline Losses & 158620 & $\mathrm{kWh} / \mathrm{yr}$ \\
\hline
\end{tabular}


Quantity

Annual throughput

Value

Expected life
Units

$453396 \mathrm{kWh} / \mathrm{yr}$

$25 \mathrm{yr}$

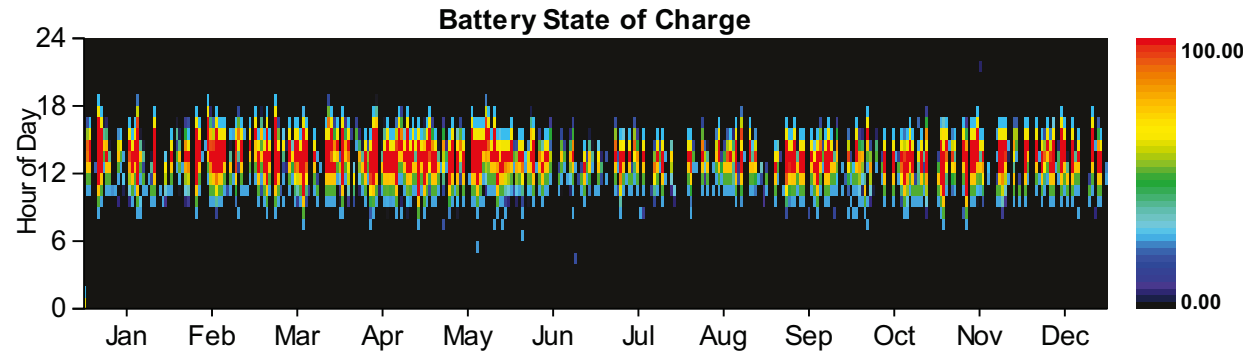

\section{Converter}

\begin{tabular}{|l|r|r|l|}
\hline Quantity & Inverter & Rectifier & Units \\
\hline Capacity & 10,000 & 9,000 & $\mathrm{~kW}$ \\
\hline Mean output & 2,658 & 0 & $\mathrm{~kW}$ \\
\hline Minimum output & 0 & $\mathrm{~kW}$ \\
\hline Maximum output & 10,000 & $366 \mathrm{~kW}$ \\
\hline Capacity factor & 27 & 0 \\
\hline Hours of operation & 4,618 & 83 & $\mathrm{hrs} / \mathrm{yr}$ \\
\hline Energy in & $25,868,908$ & $873 \mathrm{kWh} / \mathrm{yr}$ \\
\hline Energy out & $23,281,934$ & $742 \mathrm{kWh} / \mathrm{yr}$ \\
\hline Losses & $2,586,974$ & 131 & $\mathrm{kWh} / \mathrm{yr}$ \\
\hline
\end{tabular}
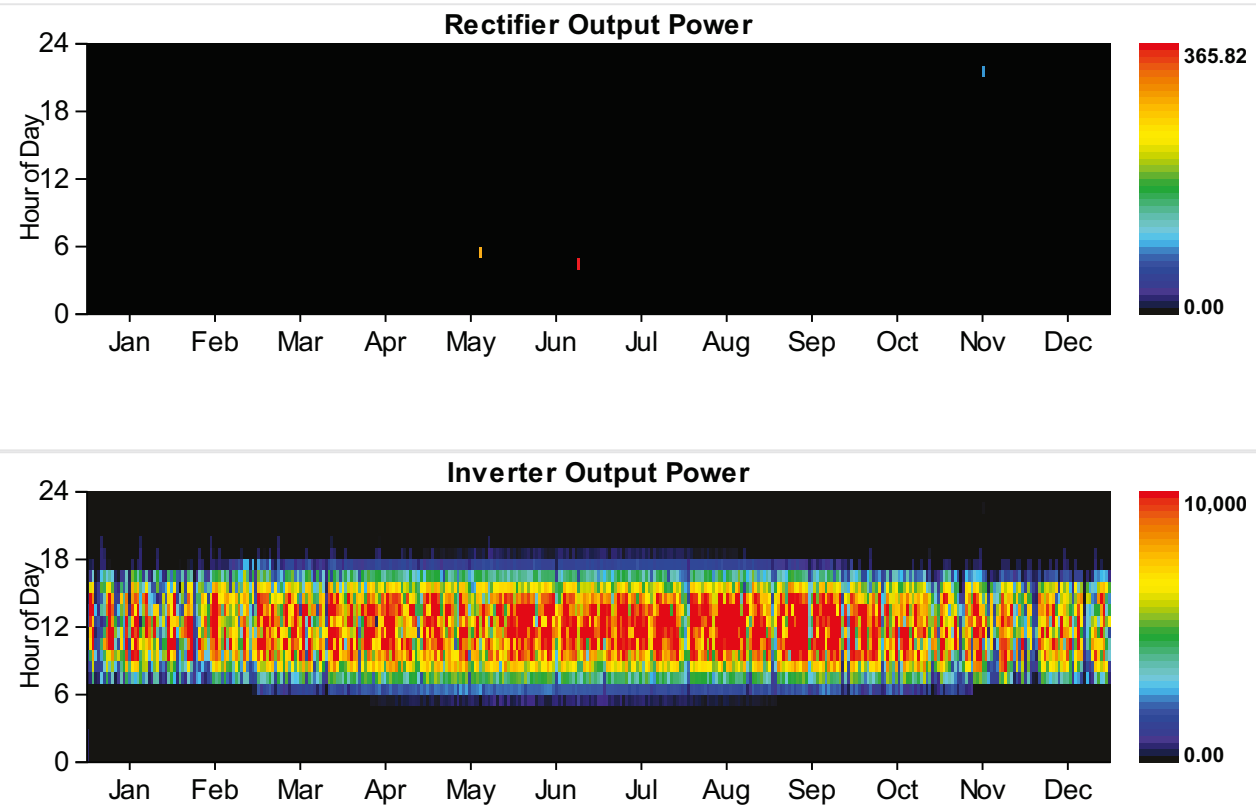

Grid

Rate: Rate 1 Demand 


\begin{tabular}{|c|c|c|c|c|c|c|}
\hline Resources.ReportingService_GeneratelnputsReport_Month & $\begin{array}{l}\text { Energy } \\
\text { Purchased } \\
\text { (kWh) }\end{array}$ & $\begin{array}{l}\text { Energy } \\
\text { Sold } \\
\text { (kWh) }\end{array}$ & $\begin{array}{l}\text { Net } \\
\text { Purchases } \\
\text { (kWh) }\end{array}$ & $\begin{array}{l}\text { Peak } \\
\text { Demand } \\
\text { (kW) }\end{array}$ & $\begin{array}{l}\text { Energy } \\
\text { Charge } \\
\text { (\$) }\end{array}$ & $\begin{array}{l}\text { Demand } \\
\text { Charge } \\
\text { (\$) }\end{array}$ \\
\hline January & 0 & 0 & 0 & 15,872 & 0 & 253,957 \\
\hline February & 0 & 0 & 0 & 15,640 & 0 & 250,233 \\
\hline March & 0 & 0 & 0 & 17,011 & 0 & 272,176 \\
\hline April & 0 & 0 & 0 & 14,338 & 0 & 229,412 \\
\hline May & 0 & 0 & 0 & 13,482 & 0 & 215,718 \\
\hline June & 0 & 0 & 0 & 19,988 & 0 & 319,816 \\
\hline July & 0 & 0 & 0 & 20,000 & 0 & 320,000 \\
\hline August & 0 & 0 & 0 & 20,000 & 0 & 320,000 \\
\hline September & 0 & 0 & 0 & 18,527 & 0 & 296,432 \\
\hline October & 0 & 0 & 0 & 15,997 & 0 & 255,953 \\
\hline November & 0 & 0 & 0 & 15,220 & 0 & 243,516 \\
\hline December & 0 & 0 & 0 & 16,591 & 0 & 265,460 \\
\hline Annual & 0 & 0 & 0 & 20,000 & 0 & $3,242,673$ \\
\hline
\end{tabular}

Rate: Rate 1

\begin{tabular}{|c|c|c|c|c|c|c|}
\hline Resources.ReportingService_GeneratelnputsReport_Month & $\begin{array}{l}\text { Energy } \\
\text { Purchased } \\
\text { (kWh) }\end{array}$ & $\begin{array}{l}\text { Energy } \\
\text { Sold } \\
\text { (kWh) }\end{array}$ & $\begin{array}{l}\text { Net } \\
\text { Purchases } \\
\text { (kWh) }\end{array}$ & $\begin{array}{l}\text { Peak } \\
\text { Demand } \\
\text { (kW) }\end{array}$ & $\begin{array}{l}\text { Energy } \\
\text { Charge } \\
\text { (\$) }\end{array}$ & $\begin{array}{l}\text { Demand } \\
\text { Charge } \\
\text { (\$) }\end{array}$ \\
\hline January & $4,990,218$ & 0 & $4,990,218$ & 0 & 598,826 & 0 \\
\hline February & $3,978,615$ & 0 & $3,978,615$ & 0 & 477,434 & 0 \\
\hline March & $4,319,255$ & 0 & $4,319,255$ & 0 & 518,311 & 0 \\
\hline April & $4,071,989$ & 0 & $4,071,989$ & 0 & 488,639 & 0 \\
\hline May & $3,927,020$ & 0 & $3,927,020$ & 0 & 471,242 & 0 \\
\hline June & $5,697,224$ & 0 & $5,697,224$ & 0 & 683,667 & 0 \\
\hline July & $7,227,189$ & 0 & $7,227,189$ & 0 & 867,263 & 0 \\
\hline August & $7,524,698$ & 0 & $7,524,698$ & 0 & 902,964 & 0 \\
\hline September & $6,095,000$ & 0 & $6,095,000$ & 0 & 731,400 & 0 \\
\hline October & $4,969,575$ & 0 & $4,969,575$ & 0 & 596,349 & 0 \\
\hline November & $4,508,634$ & 0 & $4,508,634$ & 0 & 541,036 & 0 \\
\hline December & $5,106,119$ & 0 & $5,106,119$ & 0 & 612,734 & 0 \\
\hline Annual & $62,415,532$ & 0 & $62,415,532$ & 0 & $7,489,864$ & 0 \\
\hline
\end{tabular}




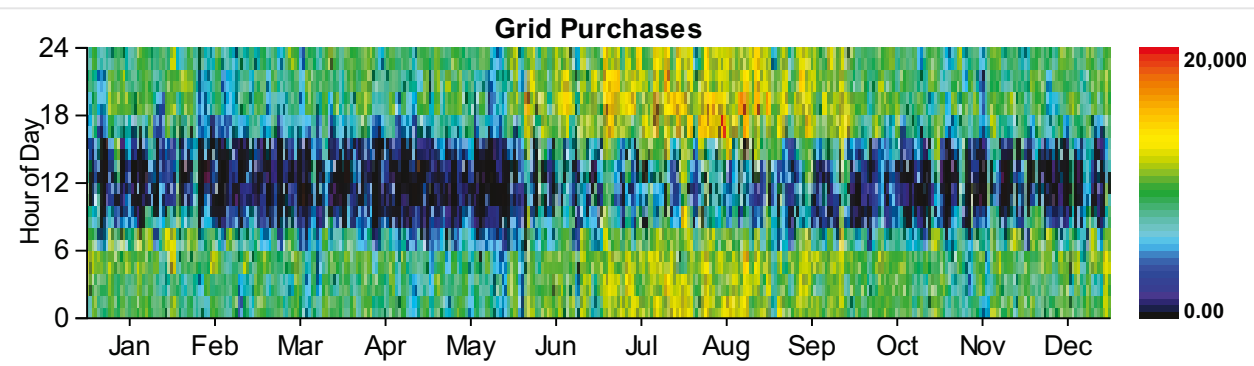

\section{Emissions}

\begin{tabular}{|l|r|l|}
\hline Pollutant & Emissions & Units \\
\hline Carbon dioxide & 39446616 & $\mathrm{~kg} / \mathrm{yr}$ \\
\hline Carbon monoxide & $0 \mathrm{~kg} / \mathrm{yr}$ \\
\hline Unburned hydrocarbons & 0 & $\mathrm{~kg} / \mathrm{yr}$ \\
\hline Particulate matter & $0 \mathrm{~kg} / \mathrm{yr}$ \\
\hline Sulfur dioxide & $171019 \mathrm{~kg} / \mathrm{yr}$ \\
\hline Nitrogen oxides & $83637 \mathrm{~kg} / \mathrm{yr}$ \\
\hline
\end{tabular}

HOMER Energy, LLC @ 2016 
System Report

System architecture

\begin{tabular}{|l|l|r|}
\hline PV & SunPower PV & 13,780 \\
\hline PV \#2 & Remaining Generic flat plate PV & 1,547 \\
\hline PV \#3 & Solar World 320W flat plate PV Copy & 1,000 \\
\hline Generator & Kohler 3250 Prime Power & 5,600 \\
\hline Battery & GS200 flow & $\mathrm{kW}$ \\
\hline Converter & System Converter & 1 \\
\hline Grid & Grid & 10,000 \\
\hline Dispatch Strategy & Cycle Charging & 15,000 \\
\hline
\end{tabular}

\section{Cost summary}

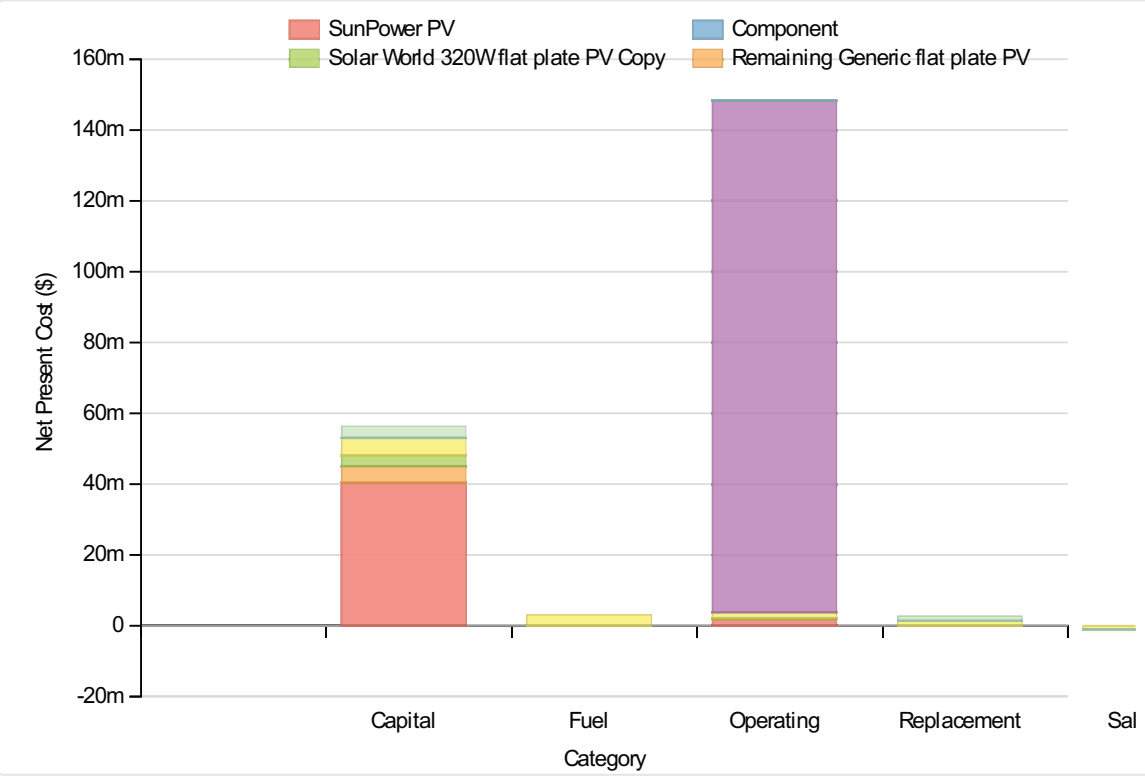

Cost Summary

Total net present cost

$209014160 \$$

Levelized cost of energy

$0.170 \$ / k W h$

\section{Net Present Costs}

\begin{tabular}{|c|c|c|c|c|c|c|}
\hline Component & Capital & Replacement & O\&M & Fuel & Salvage & Total \\
\hline SunPower PV & $40,358,332$ & 0 & $1,656,876$ & 0 & 0 & $42,015,208$ \\
\hline Remaining Generic flat plate PV & $4,678,459$ & 0 & 339,013 & 0 & 0 & $5,017,472$ \\
\hline Solar World 320W flat plate PV Copy & $3,083,333$ & 0 & 280,096 & 0 & 0 & $3,363,429$ \\
\hline Kohler 3250 Prime Power & $4,821,429$ & $1,386,639$ & $1,495,738$ & $3,040,480$ & $-985,608$ & $9,758,678$ \\
\hline Grid & 0 & 0 & $144,464,208$ & 0 & 0 & $144,464,208$ \\
\hline GS200 flow & 324,589 & 7,176 & 31,026 & 0 & -973 & 361,818 \\
\hline Converter & $3,000,000$ & $1,272,821$ & 0 & 0 & $-239,558$ & $4,033,263$ \\
\hline
\end{tabular}




\section{Annualized Costs}

\begin{tabular}{|c|c|c|c|c|c|c|}
\hline Component & Capital & Replacement & O\&M & Fuel & Salvage & Total \\
\hline SunPower PV & $3,121,894$ & 0 & 128,167 & 0 & 0 & $3,250,061$ \\
\hline Remaining Generic flat plate PV & 361,899 & 0 & 26,224 & 0 & 0 & 388,123 \\
\hline Solar World 320W flat plate PV Copy & 238,509 & 0 & 21,667 & 0 & 0 & 260,176 \\
\hline Kohler 3250 Prime Power & 372,959 & 107,263 & 115,702 & 235,194 & $-76,241$ & 754,877 \\
\hline Grid & 0 & 0 & $11,174,939$ & 0 & 0 & $11,174,939$ \\
\hline GS200 flow & 25,108 & 555 & 2,400 & 0 & -75 & 27,988 \\
\hline Converter & 232,063 & 98,458 & 0 & 0 & $-18,531$ & 311,990 \\
\hline System & $4,352,433$ & 206,276 & $11,469,097$ & 235,194 & $-94,847$ & $16,168,153$ \\
\hline
\end{tabular}

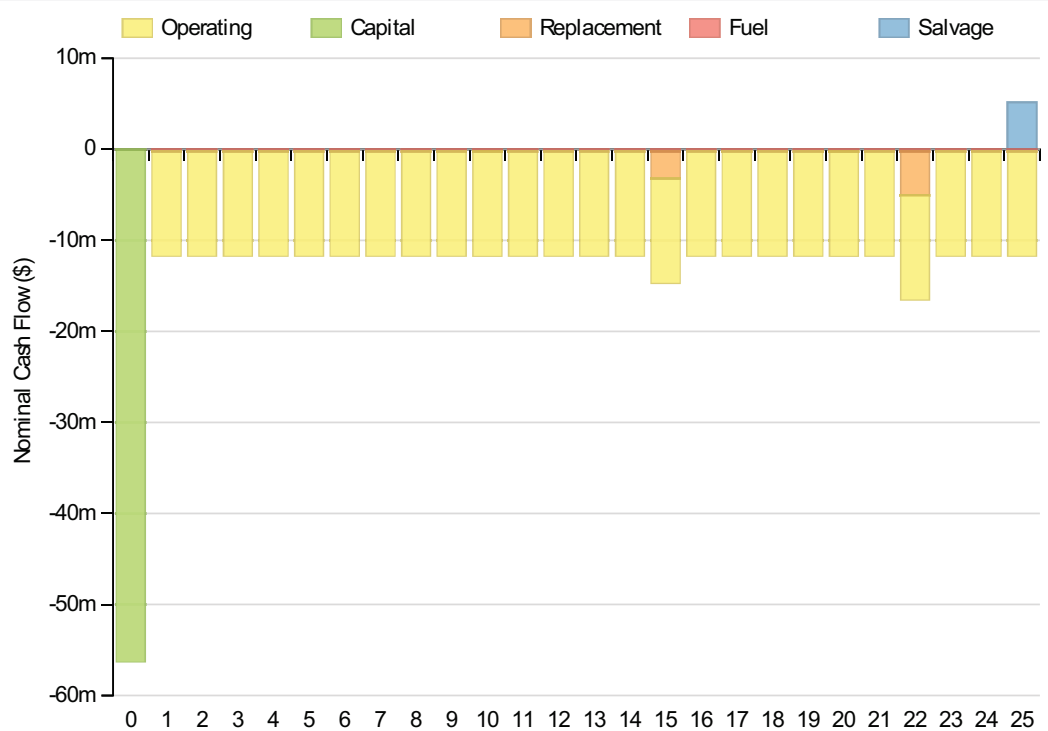
Year

Electrical

\section{Quantity}

Excess electricity

Unmet load

Capacity shortage

Renewable fraction

\begin{tabular}{|r|l|}
\hline Value & Units \\
\hline 2364608 & $\mathrm{kWh} / \mathrm{yr}$ \\
\hline 16214 & $\mathrm{kWh} / \mathrm{yr}$ \\
\hline 91448 & $\mathrm{kWh} / \mathrm{yr}$ \\
\hline 0 & \\
\hline
\end{tabular}

Fraction (\%)

\section{Component}

PV

PV

PV

Generator
Production(kWh/yr)

$24,644,568$ 


\section{Load}

AC primary load

DC primary load

Total

\section{Consumption(kWh/yr)}

Fraction (\%)

$95,036,248$

0

$95,036,248$
100

0

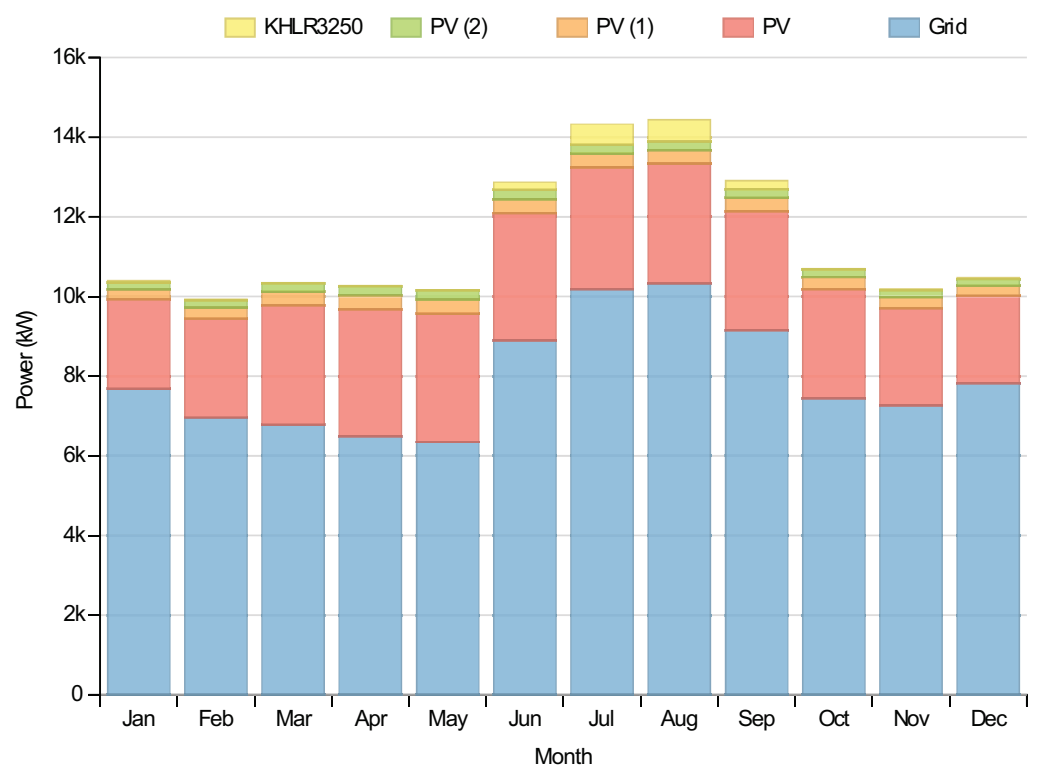

\section{PV:SunPower PV}

\section{Quantity}

Rated capacity

Mean output

Mean output

Capacity factor

Total production

Minimum output

Maximum output

PV penetration

Hours of operation

Levelized cost

\section{Value}

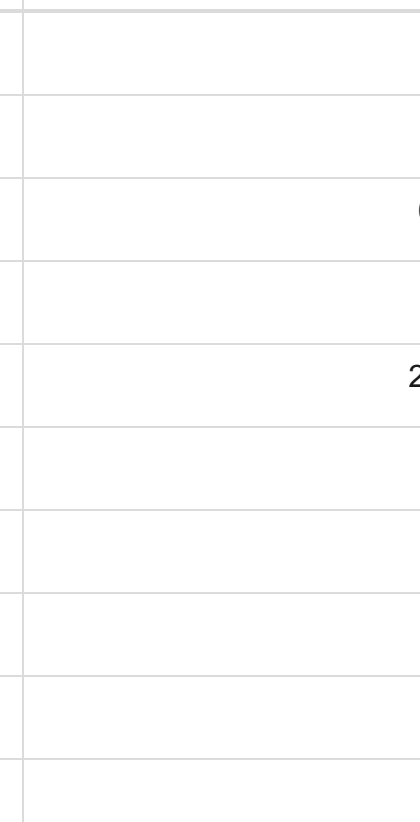

\begin{tabular}{|r|l|}
\hline 13780 & $\mathrm{~kW}$ \\
2813 & $\mathrm{~kW}$ \\
\hline 67519.00 & $\mathrm{kWh} / \mathrm{d}$ \\
\hline 20.42 & $\%$ \\
\hline 24644568 & $\mathrm{kWh} / \mathrm{yr}$ \\
\hline 0.00 & $\mathrm{~kW}$ \\
\hline 13941.00 & $\mathrm{~kW}$ \\
\hline 25.93 & $\%$ \\
\hline 4386 & $\mathrm{hrs} / \mathrm{yr}$ \\
\hline 0.111 & $\$ / \mathrm{kWh}$ \\
\hline
\end{tabular}

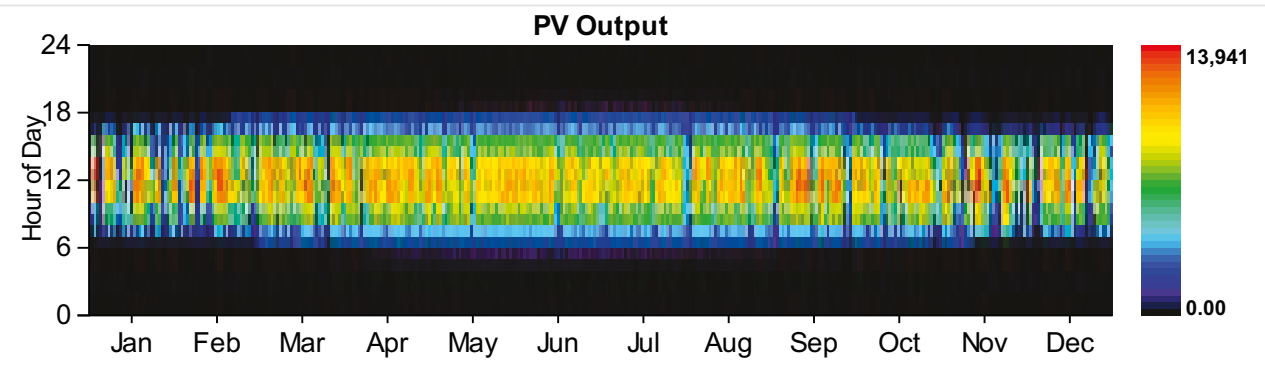




\section{Quantity}

Rated capacity

Mean output

Mean output

Capacity factor

Total production

Minimum output

Maximum output

PV penetration

Hours of operation

Levelized cost
Value

Units

1547 kW

316 kW

$7579.50 \mathrm{kWh} / \mathrm{d}$

$20.42 \%$

$2766525 \mathrm{kWh} / \mathrm{yr}$

$0.00 \mathrm{~kW}$

$1565.00 \mathrm{~kW}$

$2.91 \%$

$4386 \mathrm{hrs} / \mathrm{yr}$

$0.013 \$ / k W h$

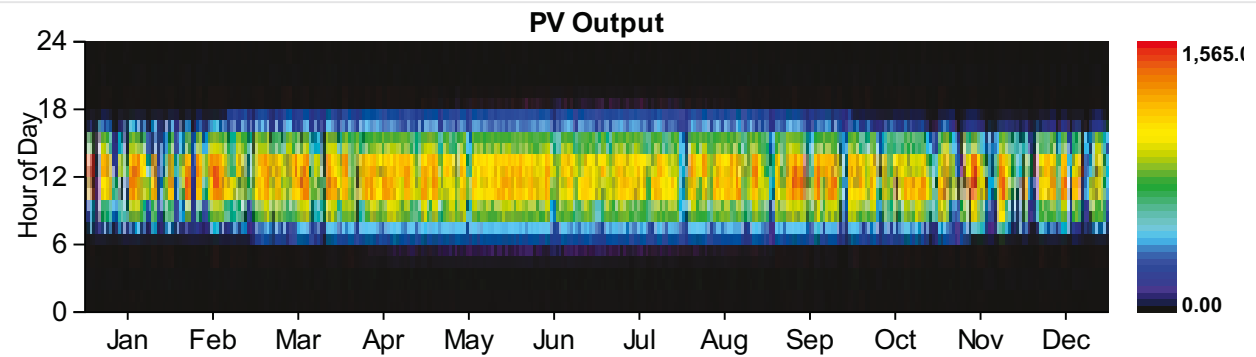

\section{PV:Solar World 320W flat plate PV Copy}

\begin{tabular}{|c|c|c|}
\hline Quantity & Value & Units \\
\hline Rated capacity & 1000 & kW \\
\hline Mean output & 204 & kW \\
\hline Mean output & 4899.80 & $\mathrm{kWh} / \mathrm{d}$ \\
\hline Capacity factor & 20.42 & $\%$ \\
\hline Total production & 1788429 & $\mathrm{kWh} / \mathrm{yr}$ \\
\hline Minimum output & 0.00 & $\mathrm{~kW}$ \\
\hline Maximum output & 1011.70 & $\mathrm{~kW}$ \\
\hline PV penetration & 1.88 & $\%$ \\
\hline Hours of operation & 4386 & $\mathrm{hrs} / \mathrm{yr}$ \\
\hline Levelized cost & 0.009 & $\$ / k W h$ \\
\hline
\end{tabular}




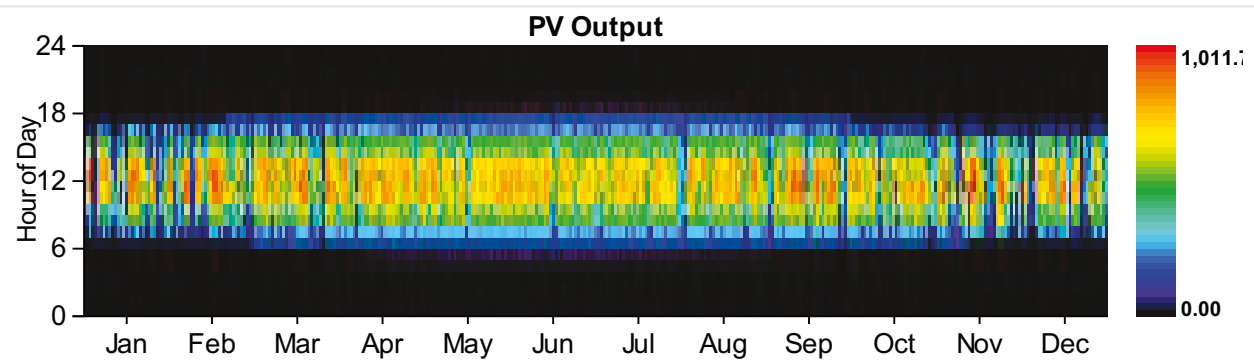

Generator:Kohler 3250 Prime Power

\begin{tabular}{|c|c|c|}
\hline Quantity & Value & Units \\
\hline Hours of operation & 688 & $\mathrm{hrs} / \mathrm{yr}$ \\
\hline Number of starts & 438 & starts/yr \\
\hline Operational life & 22 & $\mathrm{yr}$ \\
\hline Fixed generation cost & 511.50 & $\$ / \mathrm{hr}$ \\
\hline Marginal generation cost & 0.18 & $\$ / k W h$ \\
\hline Electrical production & 1200312 & $\mathrm{kWh} / \mathrm{yr}$ \\
\hline Mean electrical output & 1745 & kW \\
\hline Min. electrical output & 1400 & kW \\
\hline Max. electrical output & 5600 & kW \\
\hline Fuel consumption & 297715 & L/yr \\
\hline Specific fuel consumption & 0.25 & $\mathrm{~L} / \mathrm{kWh}$ \\
\hline Fuel energy input & 2929512 & $\mathrm{kWh} / \mathrm{yr}$ \\
\hline Mean electrical efficiency & 41 & $\%$ \\
\hline
\end{tabular}

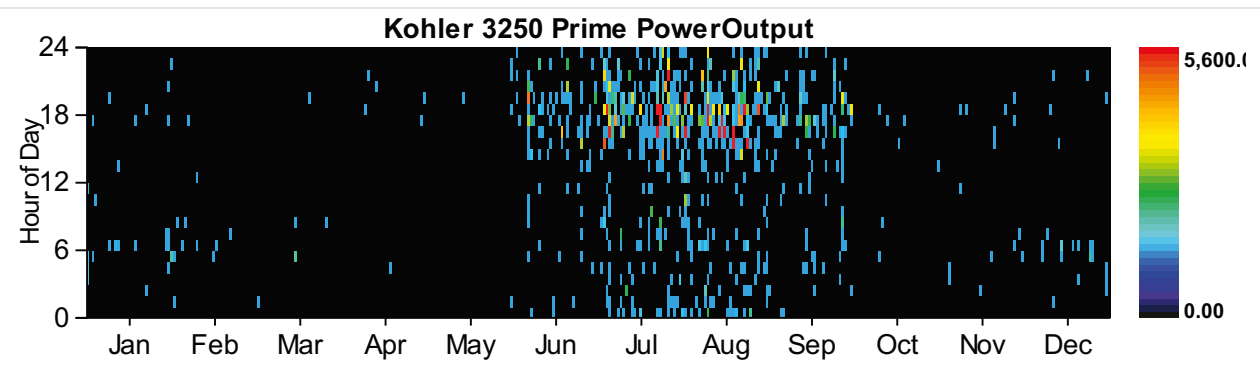

\section{Battery:GS200 flow}

\section{Quantity}

Value

String size

Strings in parallel

Batteries 
Qemarititycapacity

Usable nominal capacity

Autonomy

Lifetime throughput

Battery wear cost

Average energy cost

Energy in

Energy out

Storage depletion

Losses

Annual throughput

Expected life

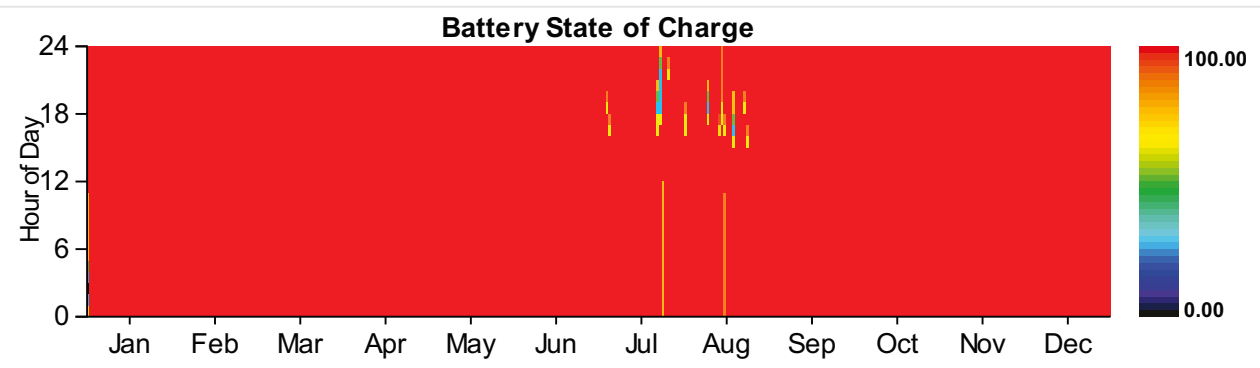

600 vifits

$600 \mathrm{kWh}$

$0 \mathrm{hr}$

0

$0.000 \$ / k W h$

$0.281 \$ / k W h$

$5137 \mathrm{kWh} / \mathrm{yr}$

$3596 \mathrm{kWh} / \mathrm{yr}$

$0 \mathrm{kWh} / \mathrm{yr}$

$1541 \mathrm{kWh} / \mathrm{yr}$

$4298 \mathrm{kWh} / \mathrm{yr}$

$25 \mathrm{yr}$

\section{Converter}

\begin{tabular}{|c|c|c|c|}
\hline Quantity & Inverter & Rectifier & Units \\
\hline Capacity & 10,000 & 9,000 & $\mathrm{~kW}$ \\
\hline Mean output & 2,757 & 0 & $\mathrm{~kW}$ \\
\hline Minimum output & 0 & 0 & $\mathrm{~kW}$ \\
\hline Maximum output & 10,000 & 179 & $\mathrm{~kW}$ \\
\hline Capacity factor & 28 & 0 & $\%$ \\
\hline Hours of operation & 4,389 & 25 & hrs/yr \\
\hline Energy in & $26,836,728$ & 3,795 & $\mathrm{kWh} / \mathrm{yr}$ \\
\hline Energy out & $24,152,896$ & 3,225 & $\mathrm{kWh} / \mathrm{yr}$ \\
\hline Losses & $2,683,832$ & 569 & $\mathrm{kWh} / \mathrm{yr}$ \\
\hline
\end{tabular}



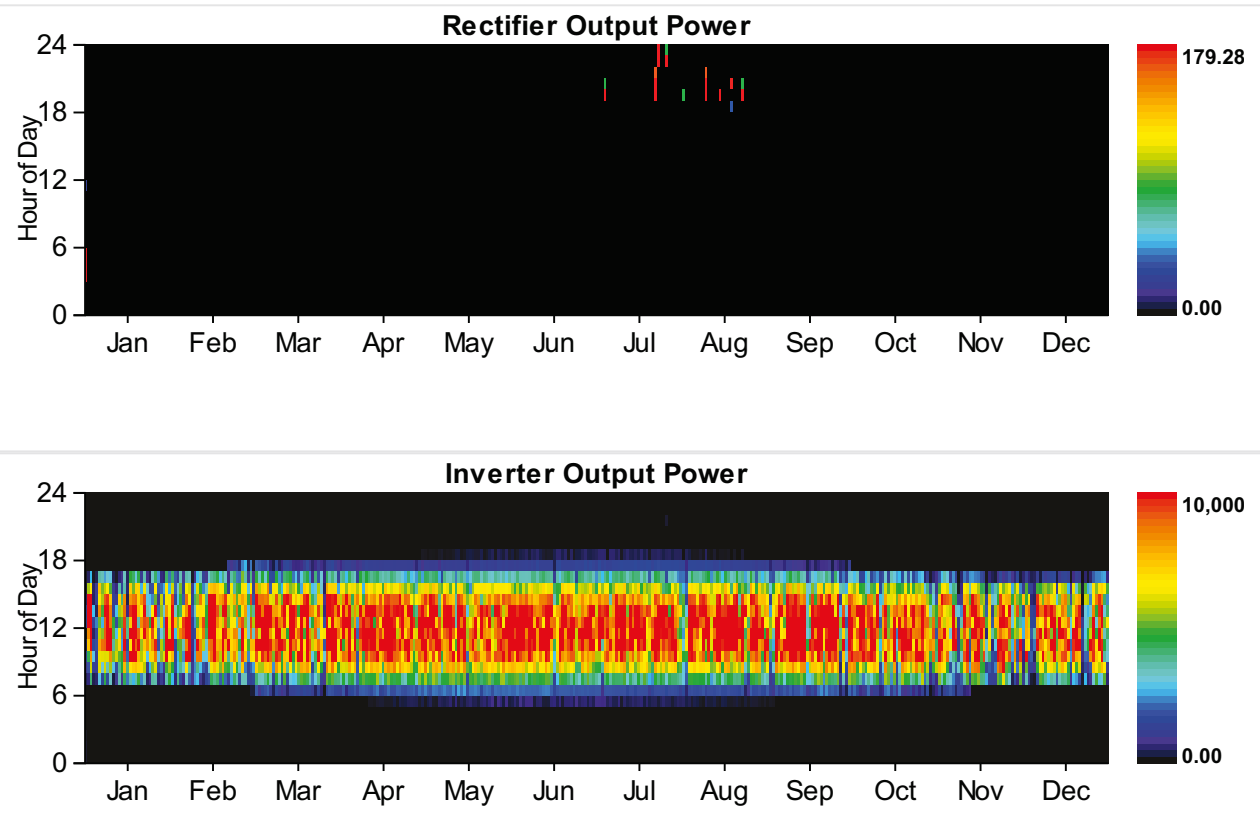

Grid

Rate: Rate 1 Demand

\begin{tabular}{|c|c|c|c|c|c|c|}
\hline Resources.ReportingService_GeneratelnputsReport_Month & $\begin{array}{l}\text { Energy } \\
\text { Purchased } \\
\text { (kWh) }\end{array}$ & $\begin{array}{l}\text { Energy } \\
\text { Sold } \\
\text { (kWh) }\end{array}$ & $\begin{array}{l}\text { Net } \\
\text { Purchases } \\
\text { (kWh) }\end{array}$ & $\begin{array}{l}\text { Peak } \\
\text { Demand } \\
(\mathbf{k W})\end{array}$ & $\begin{array}{l}\text { Energy } \\
\text { Charge } \\
\text { (\$) }\end{array}$ & $\begin{array}{l}\text { Demand } \\
\text { Charge } \\
(\$)\end{array}$ \\
\hline January & 0 & 0 & 0 & 15,000 & 0 & 240,000 \\
\hline February & 0 & 0 & 0 & 14,268 & 0 & 228,285 \\
\hline March & 0 & 0 & 0 & 15,000 & 0 & 240,000 \\
\hline April & 0 & 0 & 0 & 13,781 & 0 & 220,501 \\
\hline May & 0 & 0 & 0 & 13,732 & 0 & 219,711 \\
\hline June & 0 & 0 & 0 & 15,000 & 0 & 240,000 \\
\hline July & 0 & 0 & 0 & 15,000 & 0 & 240,000 \\
\hline August & 0 & 0 & 0 & 15,000 & 0 & 240,000 \\
\hline September & 0 & 0 & 0 & 15,000 & 0 & 240,000 \\
\hline October & 0 & 0 & 0 & 14,650 & 0 & 234,394 \\
\hline November & 0 & 0 & 0 & 14,351 & 0 & 229,618 \\
\hline December & 0 & 0 & 0 & 15,000 & 0 & 240,000 \\
\hline Annual & 0 & 0 & 0 & 15,000 & 0 & $2,812,509$ \\
\hline
\end{tabular}

Rate: Rate 1

\begin{tabular}{|c|c|c|c|c|c|c|}
\hline Resources.ReportingService_GeneratelnputsReport_Month & $\begin{array}{l}\text { Energy } \\
\text { Purchased } \\
\text { (kWh) }\end{array}$ & $\begin{array}{l}\text { Energy } \\
\text { Sold } \\
\text { (kWh) }\end{array}$ & $\begin{array}{l}\text { Net } \\
\text { Purchases } \\
\text { (kWh) }\end{array}$ & $\begin{array}{l}\text { Peak } \\
\text { Demand } \\
(\mathbf{k W})\end{array}$ & $\begin{array}{l}\text { Energy } \\
\text { Charge } \\
(\$)\end{array}$ & $\begin{array}{l}\text { Demand } \\
\text { Charge } \\
(\$)\end{array}$ \\
\hline January & $5,715,638$ & 0 & $5,715,638$ & 0 & 685,877 & 0 \\
\hline February & $4,677,284$ & 0 & $4,677,284$ & 0 & 561,274 & 0 \\
\hline March & $5,041,829$ & 0 & $5,041,829$ & 0 & 605,019 & 0 \\
\hline
\end{tabular}




\begin{tabular}{|c|c|c|c|c|c|c|}
\hline $\begin{array}{l}\text { April } \\
\text { Rẹşayrources.ReportingService_GeneratelnputsReport_Month }\end{array}$ & $\begin{array}{l}\text { Energy } \\
\text { Purchased } \\
\text { (ky/2) } \\
\text { (k,3,341 }\end{array}$ & $\begin{array}{l}\text { Energy } \\
\text { Sold } \\
(\mathbf{k W h})_{0}\end{array}$ & $\begin{array}{l}\text { Net, } 4,674,443 \\
\text { Purchases } \\
\text { (kw/h) } \\
4,72,341\end{array}$ & $\begin{array}{l}\text { Peak } 0 \\
\text { Demand } \\
\text { (kW) } 0\end{array}$ & $\begin{array}{l}\text { Energy }_{33} \\
\text { Charge } \\
(\$)_{566,801}\end{array}$ & $\begin{array}{l}\text { Demang } \\
\text { Charge } \\
\text { (\$) } \quad 0\end{array}$ \\
\hline June & $6,407,944$ & 0 & $6,407,944$ & 0 & 768,953 & 0 \\
\hline July & $7,575,564$ & 0 & $7,575,564$ & 0 & 909,068 & 0 \\
\hline August & $7,684,806$ & 0 & $7,684,806$ & 0 & 922,177 & 0 \\
\hline September & $6,589,743$ & 0 & $6,589,743$ & 0 & 790,769 & 0 \\
\hline October & $5,538,475$ & 0 & $5,538,475$ & 0 & 664,617 & 0 \\
\hline November & $5,234,798$ & 0 & $5,234,798$ & 0 & 628,176 & 0 \\
\hline December & $5,823,112$ & 0 & $5,823,112$ & 0 & 698,773 & 0 \\
\hline Annual & $69,686,976$ & 0 & $69,686,976$ & 0 & $8,362,437$ & 0 \\
\hline
\end{tabular}

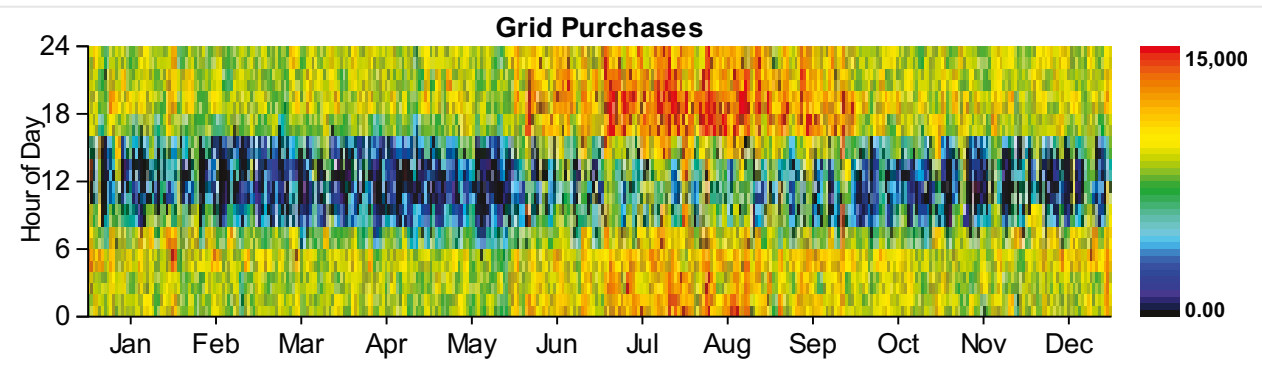

\section{Emissions}

\section{Pollutant}

Carbon dioxide

Carbon monoxide

Unburned hydrocarbons

Particulate matter

Sulfur dioxide

Nitrogen oxides

HOMER Energy, LLC @ 2016
Emissions

Units

44823524 kg/yr

$3275 \mathrm{~kg} / \mathrm{yr}$

$375 \mathrm{~kg} / \mathrm{yr}$

$94 \mathrm{~kg} / \mathrm{yr}$

$192551 \mathrm{~kg} / \mathrm{yr}$

$96655 \mathrm{~kg} / \mathrm{yr}$ 
System Report

System architecture

\begin{tabular}{|c|c|c|c|}
\hline PV & SunPower PV & 13,780 & $\mathrm{~kW}$ \\
\hline PV \#2 & Remaining Generic flat plate PV & 1,547 & $\mathrm{~kW}$ \\
\hline PV \#3 & Solar World 320W flat plate PV Copy & 1,000 & $\mathrm{~kW}$ \\
\hline Wind Turbine & Siemens 2.3 MW - 108 & 1 & \\
\hline Converter & System Converter & 10,000 & $\mathrm{~kW}$ \\
\hline Grid & Grid & 27,200 & $\mathrm{~kW}$ \\
\hline Dispatch Strategy & Cycle Charging & & \\
\hline
\end{tabular}

\section{Cost summary}

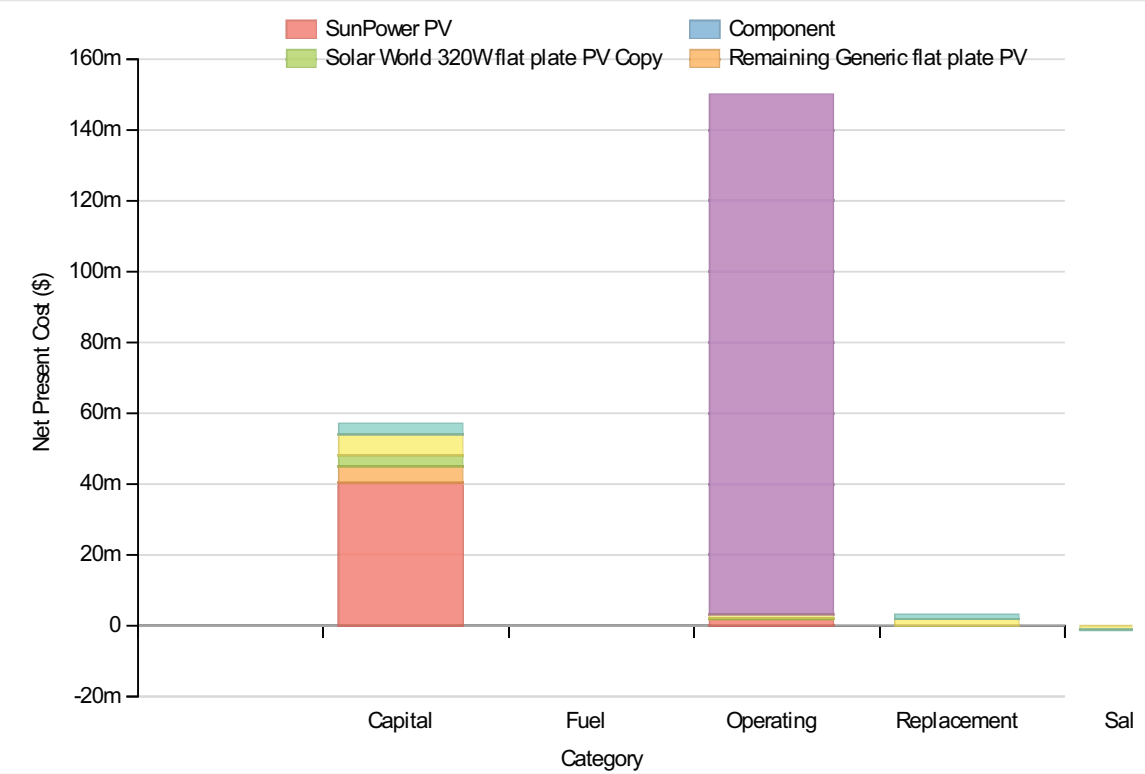

Cost Summary

Total net present cost

$209074208 \$$

Levelized cost of energy

$0.170 \$ / k W h$

Net Present Costs

\begin{tabular}{|c|c|c|c|c|c|c|}
\hline Component & Capital & Replacement & O\&M & Fuel & Salvage & Total \\
\hline SunPower PV & $40,358,332$ & 0 & $1,656,876$ & 0 & 0 & $42,015,208$ \\
\hline Remaining Generic flat plate PV & $4,678,459$ & 0 & 339,013 & 0 & 0 & $5,017,472$ \\
\hline Solar World 320W flat plate PV Copy & $3,083,333$ & 0 & 280,096 & 0 & 0 & $3,363,429$ \\
\hline Siemens $2.3 \mathrm{MW}$ - 108 & $6,000,000$ & $1,912,842$ & 969,563 & 0 & $-1,078,009$ & $7,804,396$ \\
\hline Grid & 0 & 0 & $146,840,352$ & 0 & 0 & $146,840,352$ \\
\hline Converter & $3,000,000$ & $1,272,821$ & 0 & 0 & $-239,558$ & $4,033,263$ \\
\hline System & $57,120,124$ & $3,185,663$ & $150,085,920$ & 0 & $-1,317,567$ & $209,074,140$ \\
\hline
\end{tabular}




\begin{tabular}{|c|c|c|c|c|c|c|}
\hline Component & Capital & Replacement & O\&M & Fuel & Salvage & Total \\
\hline SunPower PV & $3,121,894$ & 0 & 128,167 & 0 & 0 & $3,250,061$ \\
\hline Remaining Generic flat plate PV & 361,899 & 0 & 26,224 & 0 & 0 & 388,123 \\
\hline Solar World 320W flat plate PV Copy & 238,509 & 0 & 21,667 & 0 & 0 & 260,176 \\
\hline Siemens 2.3 MW - 108 & 464,126 & 147,967 & 75,000 & 0 & $-83,389$ & 603,704 \\
\hline Grid & 0 & 0 & $11,358,744$ & 0 & 0 & $11,358,744$ \\
\hline Converter & 232,063 & 98,458 & 0 & 0 & $-18,531$ & 311,990 \\
\hline System & $4,418,492$ & 246,425 & $11,609,803$ & 0 & $-101,920$ & $16,172,800$ \\
\hline
\end{tabular}

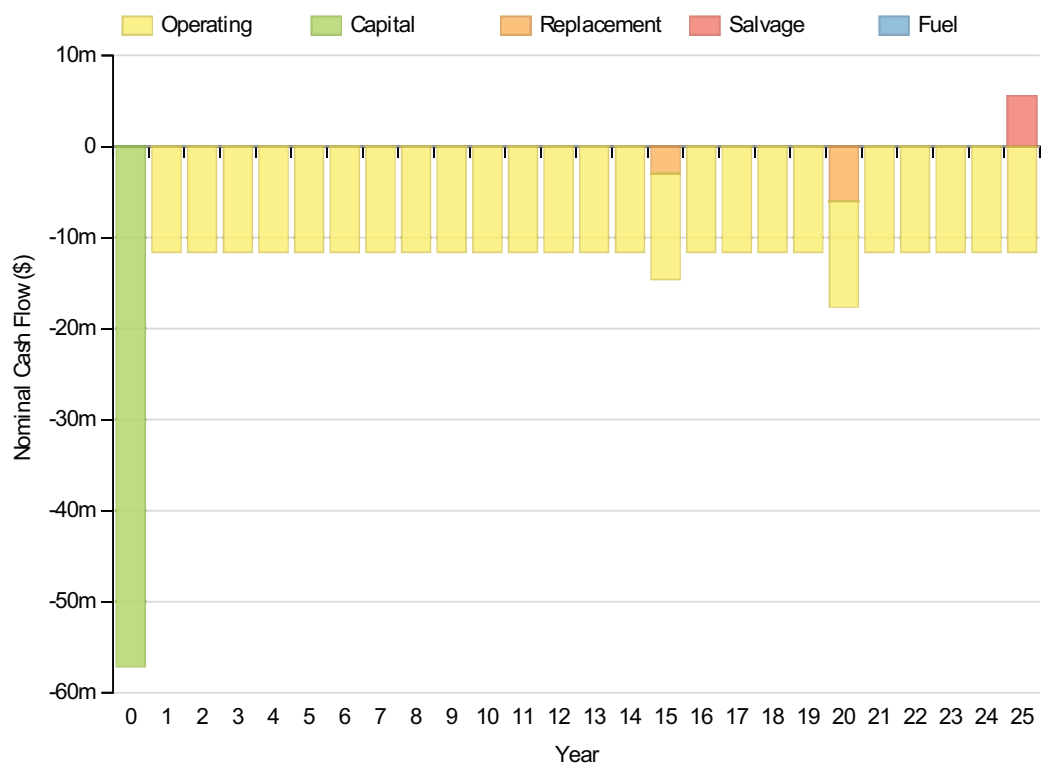

\section{Electrical}

\section{Quantity}

Excess electricity

Unmet load

Capacity shortage

Renewable fraction

\section{Component}

PV

PV

PV

Wind Turbine

Grid Purchases

Total

\section{Value}

$2886962 \mathrm{kWh} / \mathrm{yr}$

$0 \mathrm{kWh} / \mathrm{yr}$

$0 \mathrm{kWh} / \mathrm{yr}$

Production(kWh/yr)

Fraction (\%)

$24,644,568$

25

$2,766,525$

3

$1,788,429$

2

$4,674,352$

5

$66,696,980$

$100,570,864$ 


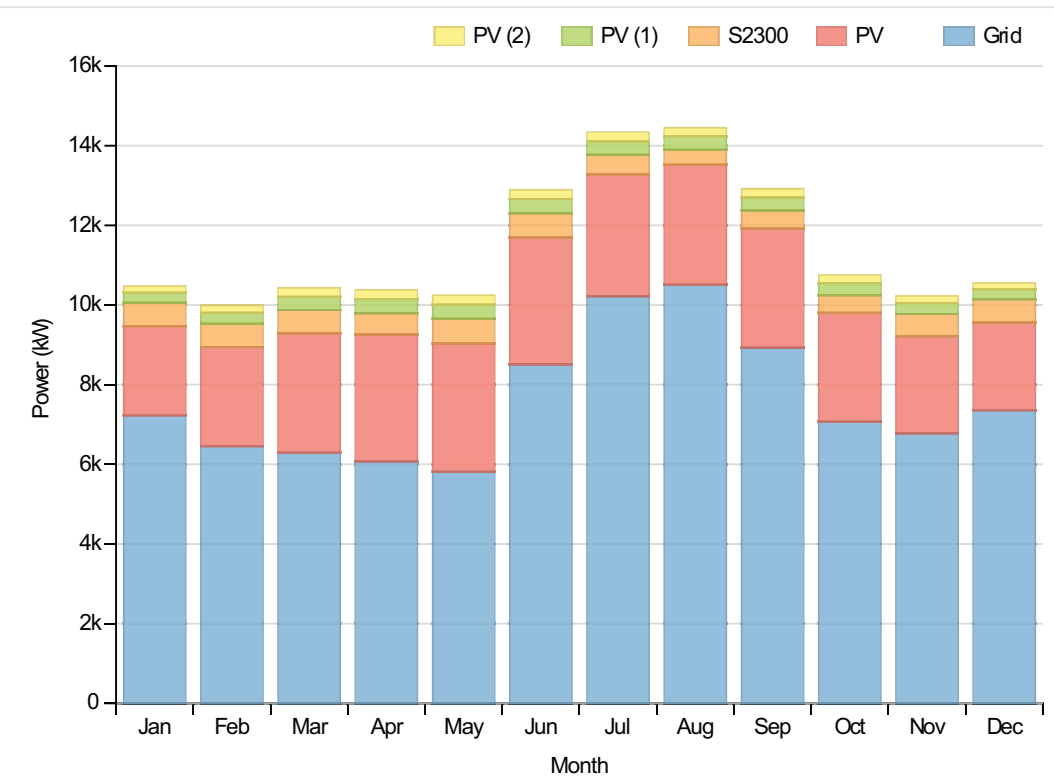

\section{PV:SunPower PV}

\begin{tabular}{|c|c|c|}
\hline Quantity & Value & Units \\
\hline Rated capacity & 13780 & $\mathrm{~kW}$ \\
\hline Mean output & 2813 & $\mathrm{~kW}$ \\
\hline Mean output & 67519.00 & $\mathrm{kWh} / \mathrm{d}$ \\
\hline Capacity factor & 20.42 & $\%$ \\
\hline Total production & 24644568 & $\mathrm{kWh} / \mathrm{yr}$ \\
\hline Minimum output & 0.00 & $\mathrm{~kW}$ \\
\hline Maximum output & 13941.00 & $\mathrm{~kW}$ \\
\hline PV penetration & 25.93 & $\%$ \\
\hline Hours of operation & 4386 & hrs/yr \\
\hline Levelized cost & 0.111 & $\$ / \mathrm{kWh}$ \\
\hline
\end{tabular}

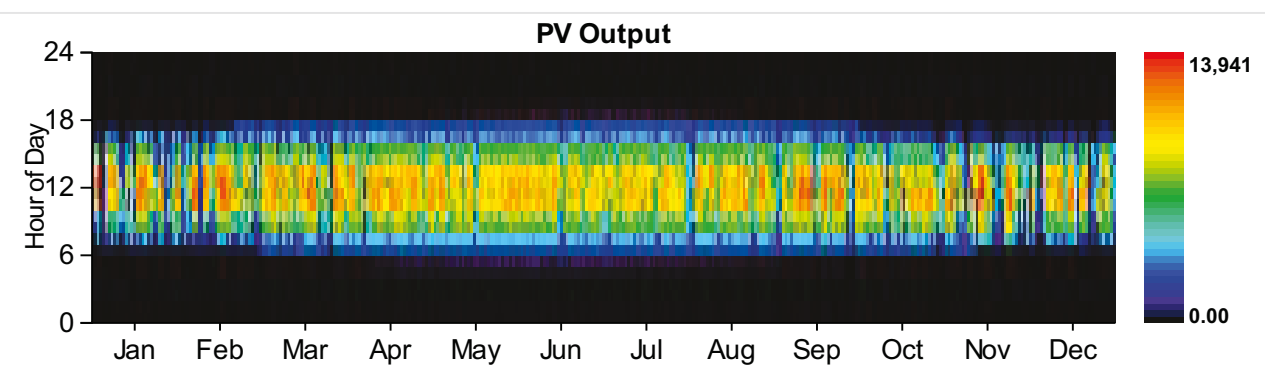

PV:Remaining Generic flat plate PV

\begin{tabular}{|l|c|c|}
\hline Quantity & Value & Units \\
\hline Rated capacity & $1547 \mathrm{~kW}$ \\
\hline Mean output & $316 \mathrm{~kW}$ \\
\hline
\end{tabular}


Qeannfityput

Capacity factor

Total production

Minimum output

Maximum output

PV penetration

Hours of operation

Levelized cost
7579.50 kflifts

$20.42 \%$

$2766525 \mathrm{kWh} / \mathrm{yr}$

$0.00 \mathrm{~kW}$

1565.00 kW

$2.91 \%$

$4386 \mathrm{hrs} / \mathrm{yr}$

$0.013 \$ / \mathrm{kWh}$

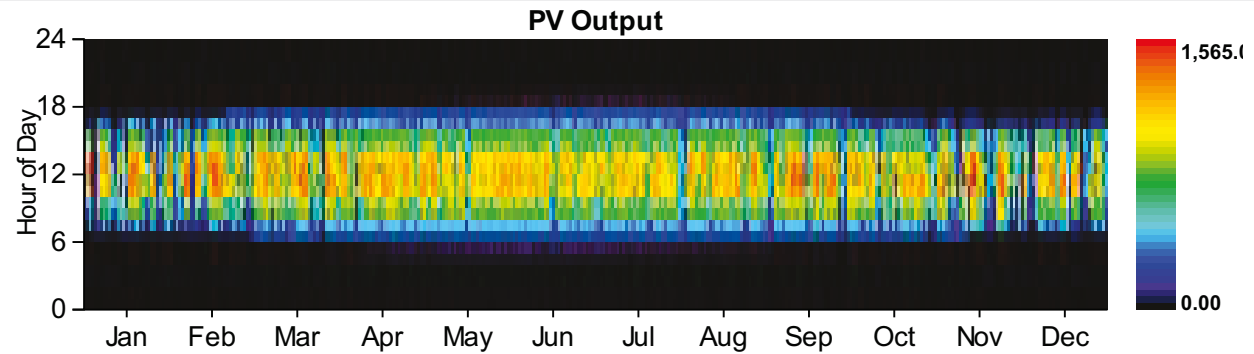

PV:Solar World 320W flat plate PV Copy

\begin{tabular}{|l|c|c|}
\hline Quantity & Value & Units \\
\hline Rated capacity & $1000 \mathrm{~kW}$ \\
\hline Mean output & $204 \mathrm{~kW}$ \\
\hline Mean output & $4899.80 \mathrm{kWh} / \mathrm{d}$ \\
\hline Capacity factor & $20.42 \%$ \\
\hline Total production & $1788429 \mathrm{kWh} / \mathrm{yr}$ \\
\hline Minimum output & $0.00 \mathrm{~kW}$ \\
\hline Maximum output & $1011.70 \mathrm{~kW}$ \\
\hline PV penetration & $1.88 \%$ & $\%$ \\
\hline Hours of operation & $4386 \mathrm{hrs} / \mathrm{yr}$ \\
\hline Levelized cost & $\$ / \mathrm{kWh}$ \\
\hline
\end{tabular}

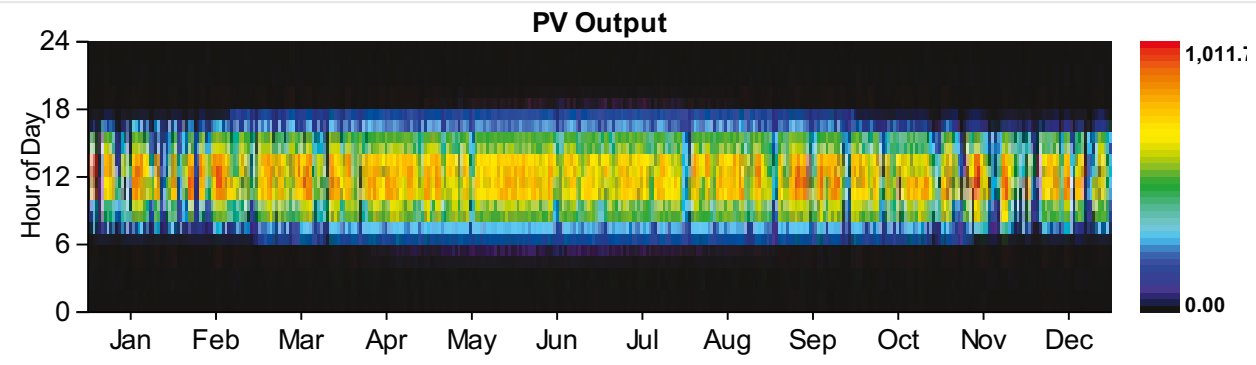

Wind Turbine:Siemens 2.3 MW - 108 
Qetahrifited capacity

Mean output

Capacity factor

Total production

Minimum output

Maximum output

Wind penetration

Hours of operation

Levelized cost
2300 kJits

534 kW

$23.20 \%$

$4674352 \mathrm{kWh} / \mathrm{yr}$

$0.49 \mathrm{~kW}$

$2312.70 \mathrm{~kW}$

$4.92 \%$

8760 hrs/yr

$0.129 \$ / k W h$

\section{Converter}

\section{Quantity}

Capacity

Mean output

Minimum output

Maximum output

Capacity factor

Hours of operation

Energy in

Energy out

Losses
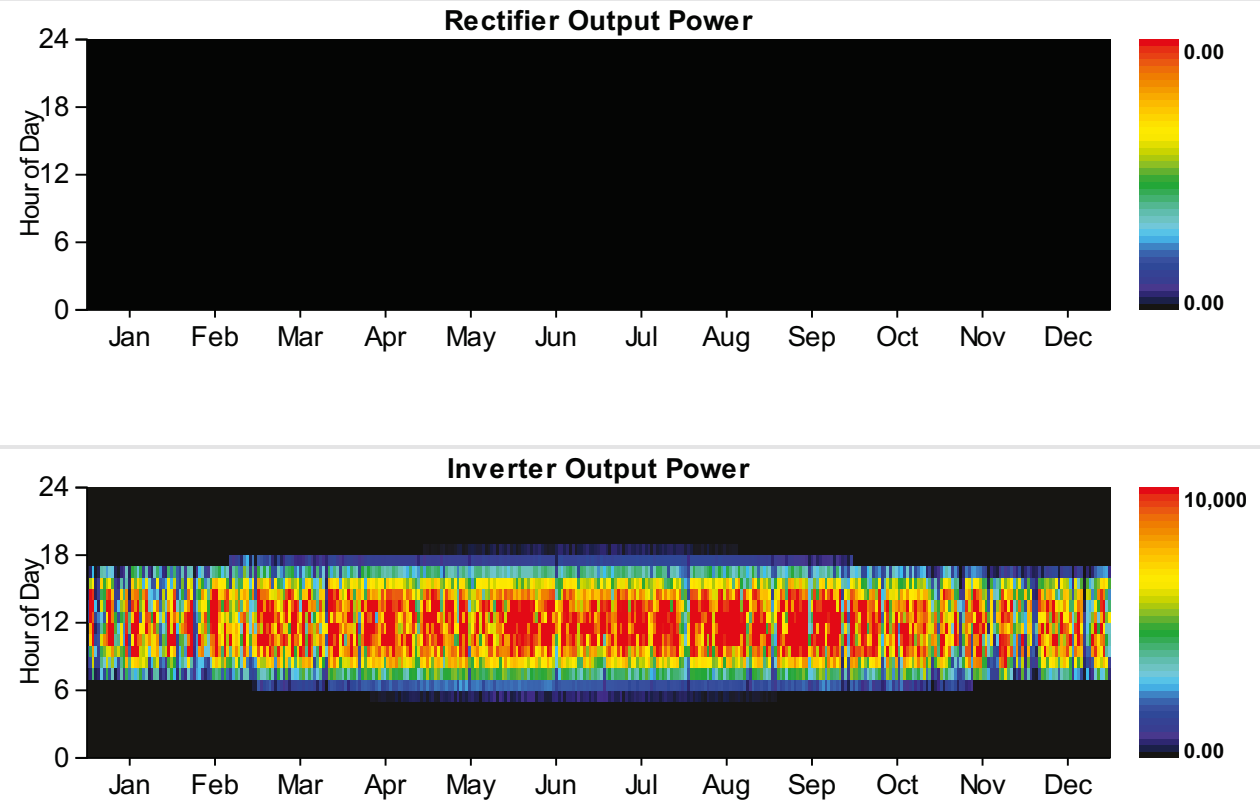

Grid

Rate: Rate 1 Demand

\begin{tabular}{|r|r|l|l|}
\hline Inverter & Rectifier & Units \\
\hline 10,000 & 9,000 & $\mathrm{~kW}$ \\
\hline 2,703 & 0 & $\mathrm{~kW}$ \\
\hline 0 & 0 & $\mathrm{~kW}$ \\
\hline 10,000 & 0 & $\mathrm{~kW}$ \\
\hline 27 & 0 & $\%$ \\
\hline 4,386 & 0 & $\mathrm{hrs} / \mathrm{yr}$ \\
\hline $26,312,666$ & 0 & $\mathrm{kWh} / \mathrm{yr}$ \\
\hline $23,681,260$ & 0 & $\mathrm{kWh} / \mathrm{yr}$ \\
\hline $2,631,406$ & 0 & $\mathrm{kWh} / \mathrm{yr}$ \\
\hline
\end{tabular}




\begin{tabular}{|c|c|c|c|c|c|c|}
\hline Resources.ReportingService_GeneratelnputsReport_Month & $\begin{array}{l}\text { Energy } \\
\text { Purchased } \\
\text { (kWh) }\end{array}$ & $\begin{array}{l}\text { Energy } \\
\text { Sold } \\
\text { (kWh) }\end{array}$ & $\begin{array}{l}\text { Net } \\
\text { Purchases } \\
\text { (kWh) }\end{array}$ & $\begin{array}{l}\text { Peak } \\
\text { Demand } \\
\text { (kW) }\end{array}$ & $\begin{array}{l}\text { Energy } \\
\text { Charge } \\
\text { (\$) }\end{array}$ & $\begin{array}{l}\text { Demand } \\
\text { Charge } \\
\text { (\$) }\end{array}$ \\
\hline January & 0 & 0 & 0 & 16,288 & 0 & 260,605 \\
\hline February & 0 & 0 & 0 & 15,654 & 0 & 250,459 \\
\hline March & 0 & 0 & 0 & 17,043 & 0 & 272,688 \\
\hline April & 0 & 0 & 0 & 14,366 & 0 & 229,856 \\
\hline May & 0 & 0 & 0 & 13,700 & 0 & 219,202 \\
\hline June & 0 & 0 & 0 & 20,004 & 0 & 320,060 \\
\hline July & 0 & 0 & 0 & 23,778 & 0 & 380,443 \\
\hline August & 0 & 0 & 0 & 22,183 & 0 & 354,921 \\
\hline September & 0 & 0 & 0 & 18,548 & 0 & 296,765 \\
\hline October & 0 & 0 & 0 & 16,023 & 0 & 256,374 \\
\hline November & 0 & 0 & 0 & 15,239 & 0 & 243,828 \\
\hline December & 0 & 0 & 0 & 16,870 & 0 & 269,913 \\
\hline Annual & 0 & 0 & 0 & 23,778 & 0 & $3,355,113$ \\
\hline
\end{tabular}

Rate: Rate 1

\begin{tabular}{|c|c|c|c|c|c|c|}
\hline Resources.ReportingService_GeneratelnputsReport_Month & $\begin{array}{l}\text { Energy } \\
\text { Purchased } \\
\text { (kWh) }\end{array}$ & $\begin{array}{l}\text { Energy } \\
\text { Sold } \\
\text { (kWh) }\end{array}$ & $\begin{array}{l}\text { Net } \\
\text { Purchases } \\
\text { (kWh) }\end{array}$ & $\begin{array}{l}\text { Peak } \\
\text { Demand } \\
\text { (kW) }\end{array}$ & $\begin{array}{l}\text { Energy } \\
\text { Charge } \\
\text { (\$) }\end{array}$ & $\begin{array}{l}\text { Demand } \\
\text { Charge } \\
\text { (\$) }\end{array}$ \\
\hline January & $5,374,291$ & 0 & $5,374,291$ & 0 & 644,915 & 0 \\
\hline February & $4,338,702$ & 0 & $4,338,702$ & 0 & 520,644 & 0 \\
\hline March & $4,682,773$ & 0 & $4,682,773$ & 0 & 561,933 & 0 \\
\hline April & $4,374,337$ & 0 & $4,374,337$ & 0 & 524,920 & 0 \\
\hline May & $4,326,311$ & 0 & $4,326,311$ & 0 & 519,157 & 0 \\
\hline June & $6,124,817$ & 0 & $6,124,817$ & 0 & 734,978 & 0 \\
\hline July & $7,604,342$ & 0 & $7,604,342$ & 0 & 912,521 & 0 \\
\hline August & $7,819,220$ & 0 & $7,819,220$ & 0 & 938,306 & 0 \\
\hline September & $6,429,440$ & 0 & $6,429,440$ & 0 & 771,533 & 0 \\
\hline October & $5,262,241$ & 0 & $5,262,241$ & 0 & 631,469 & 0 \\
\hline November & $4,884,078$ & 0 & $4,884,078$ & 0 & 586,089 & 0 \\
\hline December & $5,476,427$ & 0 & $5,476,427$ & 0 & 657,171 & 0 \\
\hline Annual & $66,696,980$ & 0 & $66,696,980$ & 0 & $8,003,638$ & 0 \\
\hline
\end{tabular}




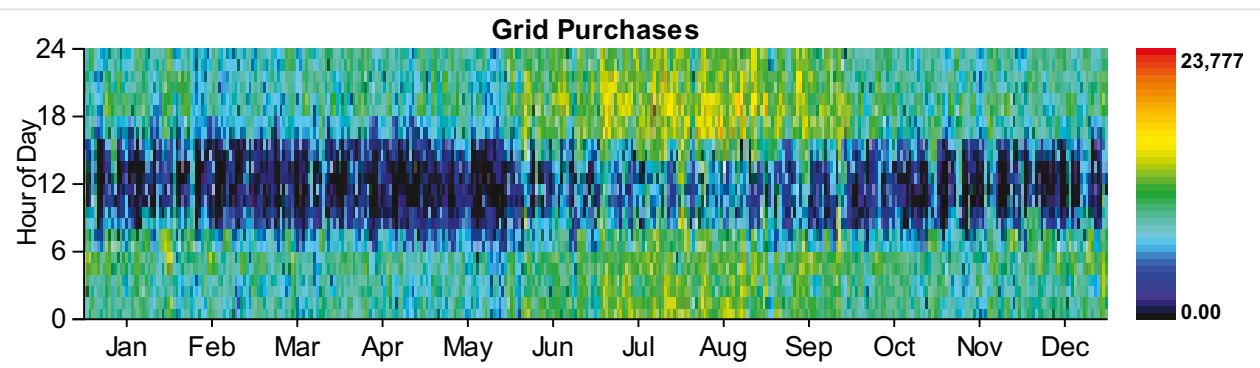

\section{Emissions}

\begin{tabular}{|l|r|l|}
\hline Pollutant & Emissions & Units \\
\hline Carbon dioxide & 42152492 & $\mathrm{~kg} / \mathrm{yr}$ \\
\hline Carbon monoxide & $0 \mathrm{~kg} / \mathrm{yr}$ \\
\hline Unburned hydrocarbons & 0 & $\mathrm{~kg} / \mathrm{yr}$ \\
\hline Particulate matter & $0 \mathrm{~kg} / \mathrm{yr}$ \\
\hline Sulfur dioxide & $182750 \mathrm{~kg} / \mathrm{yr}$ \\
\hline Nitrogen oxides & $89374 \mathrm{~kg} / \mathrm{yr}$ \\
\hline
\end{tabular}

HOMER Energy, LLC @ 2016 
System Report

System architecture

\begin{tabular}{|l|l|r|}
\hline PV & SunPower PV & 13,780 \\
\hline PV \#2 & Remaining Generic flat plate PV & 1,547 \\
\hline PV \#3 & Solar World 320W flat plate PV Copy & 1,000 \\
\hline Wind Turbine & Siemens $2.3 \mathrm{~kW}-108$ & 1 \\
\hline Generator & Kohler 3250 Prime Power & 5,600 \\
\hline Converter & System Converter & 10,000 \\
\hline Grid & Grid & $\mathrm{kW}$ \\
\hline Dispatch Strategy & Cycle Charging & 15,000 \\
\hline
\end{tabular}

\section{Cost summary}

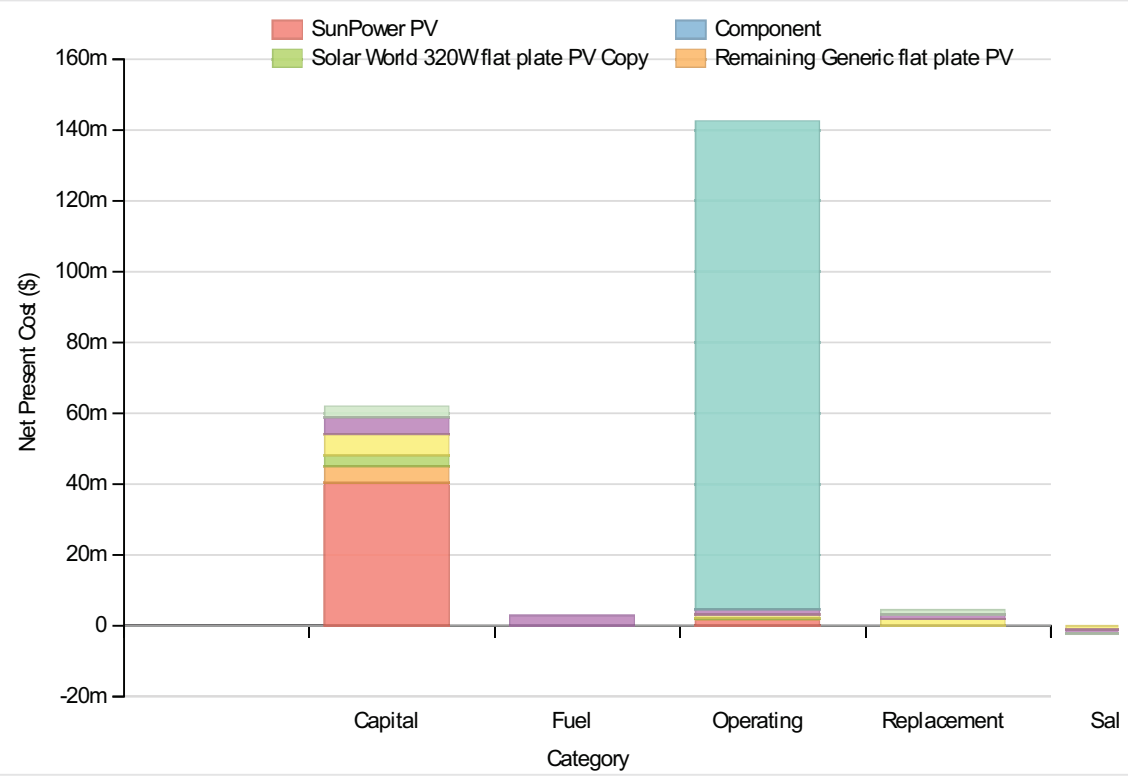

Cost Summary

Total net present cost

209445488 \$

Levelized cost of energy

$0.170 \$ / k W h$

\section{Net Present Costs}

\begin{tabular}{|c|c|c|c|c|c|c|}
\hline Component & Capital & Replacement & O\&M & Fuel & Salvage & Total \\
\hline SunPower PV & $40,358,332$ & 0 & $1,656,876$ & 0 & 0 & $42,015,208$ \\
\hline Remaining Generic flat plate PV & $4,678,459$ & 0 & 339,013 & 0 & 0 & $5,017,472$ \\
\hline Solar World 320W flat plate PV Copy & $3,083,333$ & 0 & 280,096 & 0 & 0 & $3,363,429$ \\
\hline Siemens 2.3 MW - 108 & $6,000,000$ & $1,912,842$ & 969,563 & 0 & $-1,078,009$ & $7,804,396$ \\
\hline Kohler 3250 Prime Power & $4,821,429$ & $1,335,855$ & $1,452,257$ & $2,825,962$ & $-1,024,108$ & $9,411,395$ \\
\hline Grid & 0 & 0 & $137,800,256$ & 0 & 0 & $137,800,256$ \\
\hline Converter & $3,000,000$ & $1,272,821$ & 0 & 0 & $-239,558$ & $4,033,263$ \\
\hline
\end{tabular}




\section{Annualized Costs}

\begin{tabular}{|c|c|c|c|c|c|c|}
\hline Component & Capital & Replacement & O\&M & Fuel & Salvage & Total \\
\hline SunPower PV & $3,121,894$ & 0 & 128,167 & 0 & 0 & $3,250,061$ \\
\hline Remaining Generic flat plate PV & 361,899 & 0 & 26,224 & 0 & 0 & 388,123 \\
\hline Solar World 320W flat plate PV Copy & 238,509 & 0 & 21,667 & 0 & 0 & 260,176 \\
\hline Siemens 2.3 MW - 108 & 464,126 & 147,967 & 75,000 & 0 & $-83,389$ & 603,704 \\
\hline Kohler 3250 Prime Power & 372,959 & 103,334 & 112,338 & 218,601 & $-79,219$ & 728,013 \\
\hline Grid & 0 & 0 & $10,659,453$ & 0 & 0 & $10,659,453$ \\
\hline Converter & 232,063 & 98,458 & 0 & 0 & $-18,531$ & 311,990 \\
\hline System & $4,791,451$ & 349,759 & $11,022,847$ & 218,601 & $-181,139$ & $16,201,519$ \\
\hline
\end{tabular}

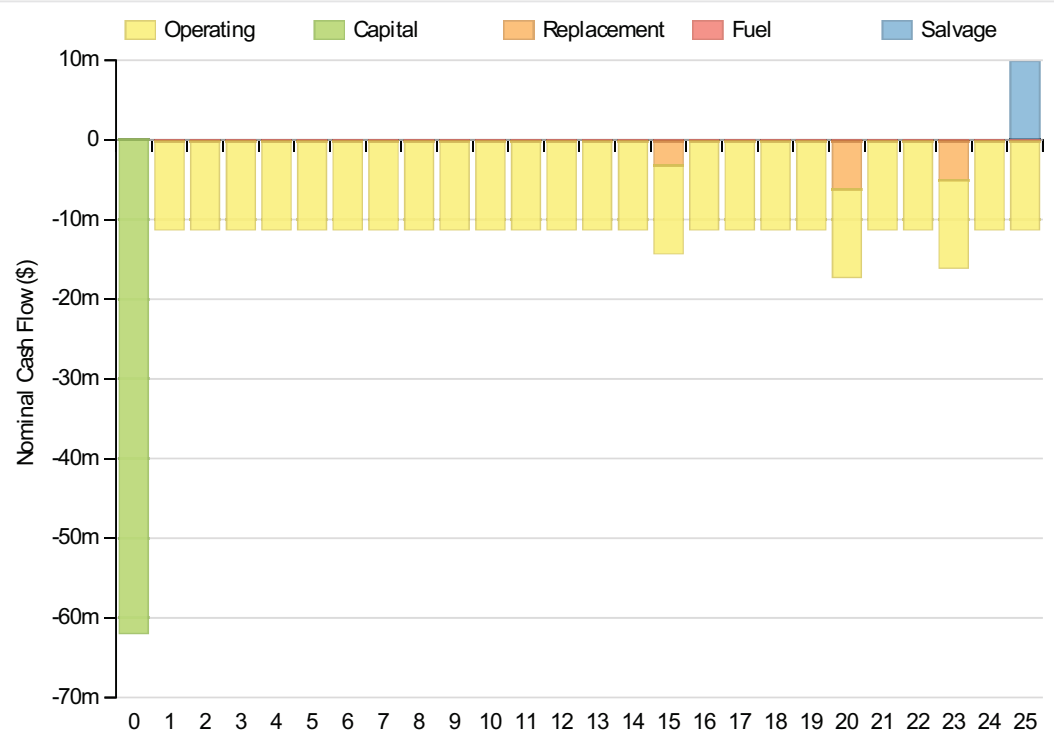
Year

Electrical

\begin{tabular}{|c|c|c|c|c|}
\hline Quantity & & Value & & Units \\
\hline Excess electricity & & & 886962 & $\mathrm{kWh} / \mathrm{yr}$ \\
\hline Unmet load & & & 7555 & $\mathrm{kWh} / \mathrm{yr}$ \\
\hline Capacity shortage & & & 83283 & $\mathrm{kWh} / \mathrm{yr}$ \\
\hline Renewable fraction & & & 0 & \\
\hline Component & Production(kWh/yr) & & Fractio & n (\%) \\
\hline PV & & $24,644,568$ & & 25 \\
\hline PV & & $2,766,525$ & & 3 \\
\hline PV & & $1,788,429$ & & 2 \\
\hline Generator & & $1,112,217$ & & 1 \\
\hline Wind Turbine & & $4,674,352$ & & 5 \\
\hline
\end{tabular}


Load

Consumption(kWh/yr)

Fraction (\%)

AC primary load

$95,044,912$

DC primary load

Total

0

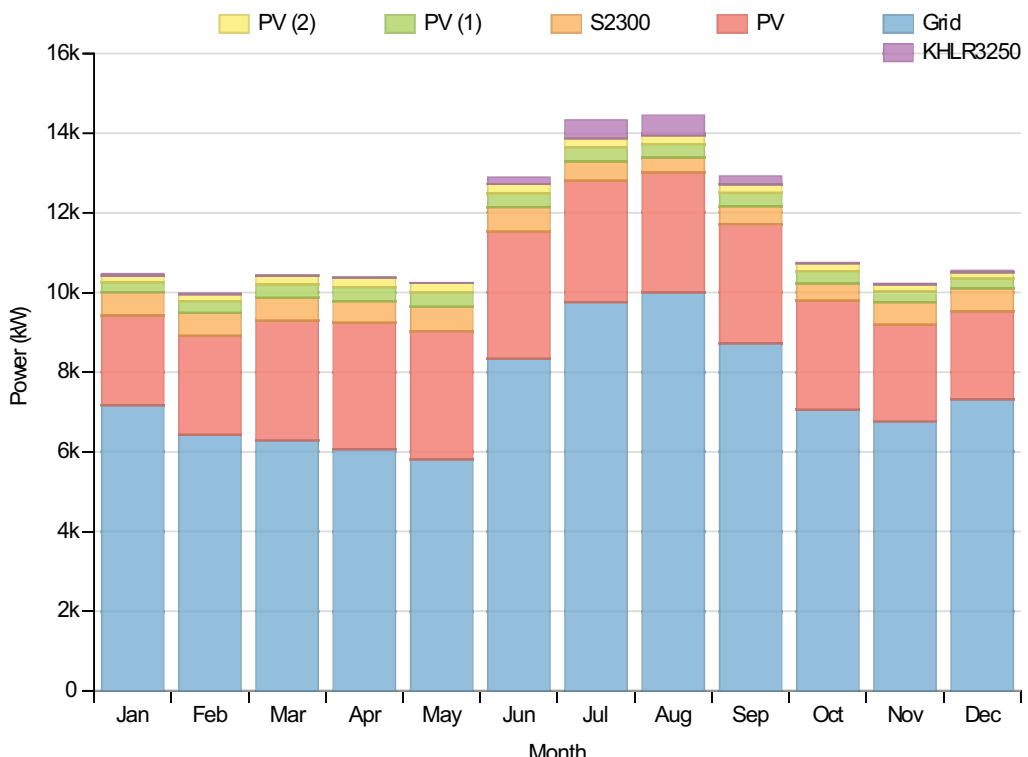

\section{PV:SunPower PV}

\begin{tabular}{|c|c|c|}
\hline Quantity & Value & Units \\
\hline Rated capacity & 13780 & kW \\
\hline Mean output & 2813 & kW \\
\hline Mean output & 67519.00 & $\mathrm{kWh} / \mathrm{d}$ \\
\hline Capacity factor & 20.42 & $\%$ \\
\hline Total production & 24644568 & $\mathrm{kWh} / \mathrm{yr}$ \\
\hline Minimum output & 0.00 & $\mathrm{~kW}$ \\
\hline Maximum output & 13941.00 & $\mathrm{~kW}$ \\
\hline PV penetration & 25.93 & $\%$ \\
\hline Hours of operation & 4386 & $\mathrm{hrs} / \mathrm{yr}$ \\
\hline Levelized cost & 0.111 & $\$ / k W h$ \\
\hline
\end{tabular}




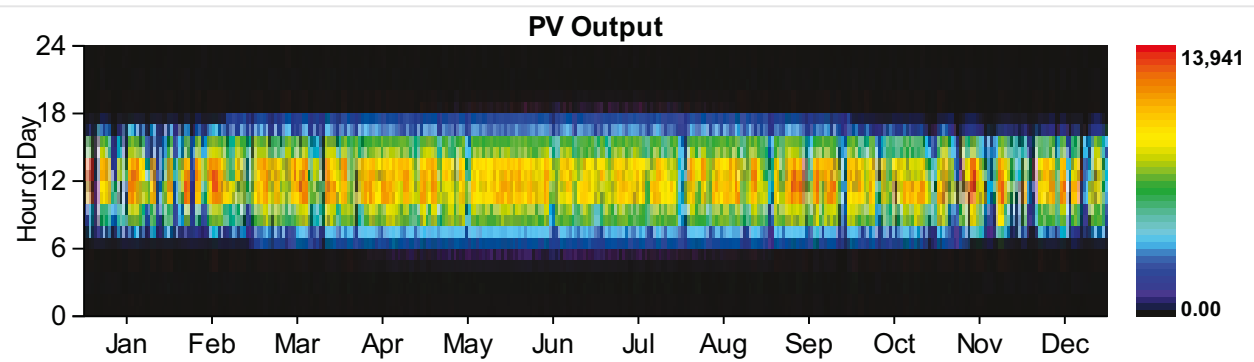

PV:Remaining Generic flat plate PV

\begin{tabular}{|c|c|c|}
\hline Quantity & Value & Units \\
\hline Rated capacity & 1547 & $\mathrm{~kW}$ \\
\hline Mean output & 316 & $\mathrm{~kW}$ \\
\hline Mean output & 7579.50 & $\mathrm{kWh} / \mathrm{d}$ \\
\hline Capacity factor & 20.42 & $\%$ \\
\hline Total production & 2766525 & $\mathrm{kWh} / \mathrm{yr}$ \\
\hline Minimum output & 0.00 & $\mathrm{~kW}$ \\
\hline Maximum output & 1565.00 & $\mathrm{~kW}$ \\
\hline PV penetration & 2.91 & $\%$ \\
\hline Hours of operation & 4386 & $\mathrm{hrs} / \mathrm{yr}$ \\
\hline Levelized cost & 0.013 & $\$ / k W h$ \\
\hline
\end{tabular}

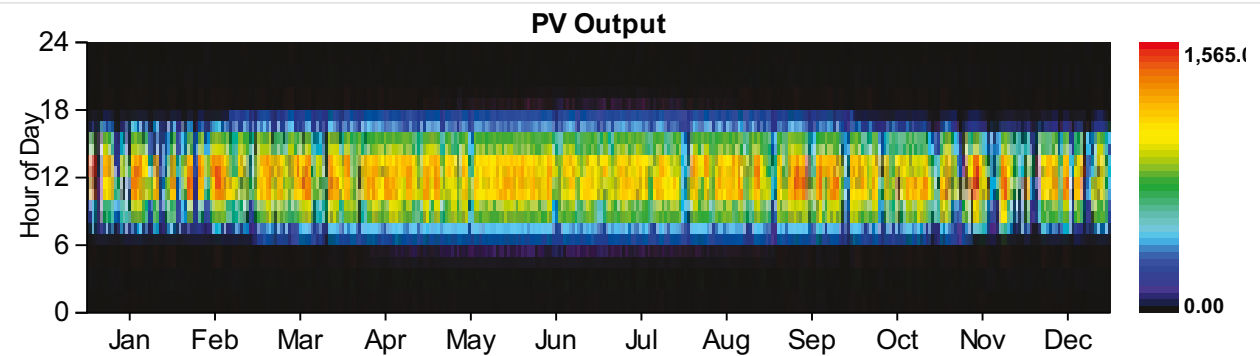

PV:Solar World 320W flat plate PV Copy

\begin{tabular}{|l|r|l|}
\hline Quantity & Value & Units \\
\hline Rated capacity & $1000 \mathrm{~kW}$ \\
\hline Mean output & $204 \mathrm{~kW}$ \\
\hline Mean output & $4899.80 \mathrm{kWh} / \mathrm{d}$ \\
\hline Capacity factor & $20.42 \%$ \\
\hline Total production & $1788429 \mathrm{kWh} / \mathrm{yr}$ \\
\hline Minimum output & $0.00 \mathrm{~kW}$ \\
\hline Maximum output & $1011.70 \mathrm{~kW}$ \\
\hline PV penetration & $1.88 \%$ & $\%$ \\
\hline
\end{tabular}




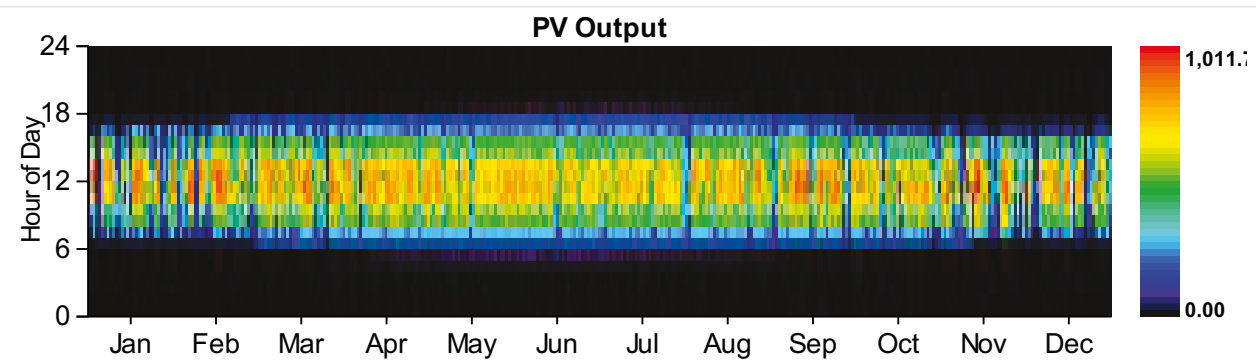

Wind Turbine:Siemens 2.3 MW - 108

\section{Quantity}

Total rated capacity

Mean output

Capacity factor

Total production

Minimum output

Maximum output

Wind penetration

Hours of operation

Levelized cost
Value

Units

$2300 \mathrm{~kW}$

534 kW

$23.20 \%$

$4674352 \mathrm{kWh} / \mathrm{yr}$

$0.49 \mathrm{~kW}$

$2312.70 \mathrm{~kW}$

$4.92 \%$

$8760 \mathrm{hrs} / \mathrm{yr}$

$0.129 \$ / k W h$

\section{Generator:Kohler 3250 Prime Power}

\begin{tabular}{|c|c|c|}
\hline Quantity & Value & Units \\
\hline Hours of operation & 668 & hrs/yr \\
\hline Number of starts & 424 & starts/yr \\
\hline Operational life & 22 & $\mathrm{yr}$ \\
\hline Fixed generation cost & 511.50 & $\$ / h r$ \\
\hline Marginal generation cost & 0.18 & $\$ / k W h$ \\
\hline Electrical production & 1112217 & $\mathrm{kWh} / \mathrm{yr}$ \\
\hline Mean electrical output & 1665 & kW \\
\hline Min. electrical output & 1400 & kW \\
\hline Max. electrical output & 5600 & $\mathrm{~kW}$ \\
\hline Fuel consumption & 276710 & L/yr \\
\hline Specific fuel consumption & 0.25 & $\mathrm{~L} / \mathrm{kWh}$ \\
\hline Fuel energy input & 2722823 & $\mathrm{kWh} / \mathrm{yr}$ \\
\hline Mean electrical efficiency & 41 & $\%$ \\
\hline
\end{tabular}




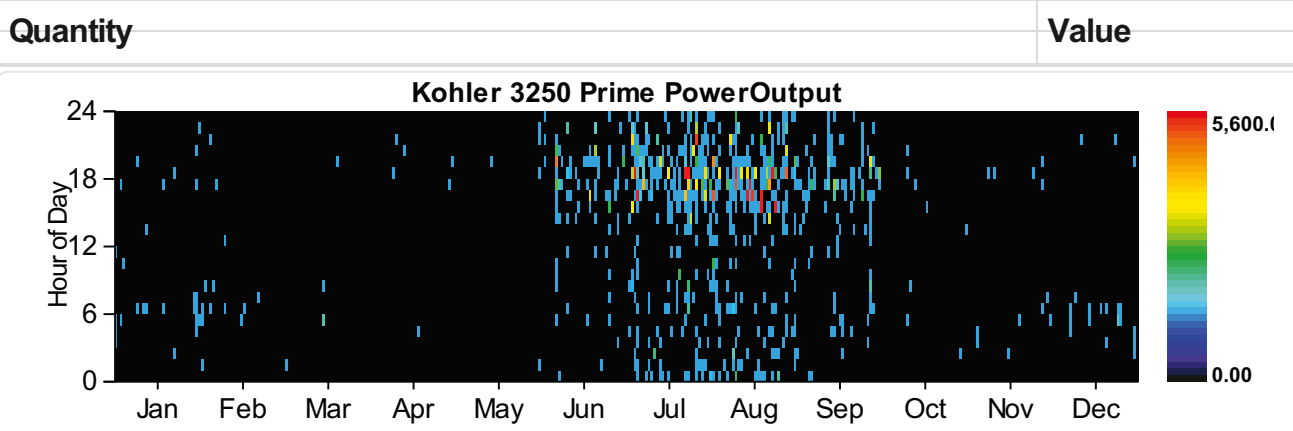

\section{Converter}

\begin{tabular}{|c|c|c|c|}
\hline Quantity & Inverter & Rectifier & Units \\
\hline Capacity & 10,000 & 9,000 & $\mathrm{~kW}$ \\
\hline Mean output & 2,703 & 0 & $\mathrm{~kW}$ \\
\hline Minimum output & 0 & 0 & $\mathrm{~kW}$ \\
\hline Maximum output & 10,000 & 0 & $\mathrm{~kW}$ \\
\hline Capacity factor & 27 & 0 & $\%$ \\
\hline Hours of operation & 4,386 & 0 & hrs/yr \\
\hline Energy in & $26,312,666$ & 0 & $\mathrm{kWh} / \mathrm{yr}$ \\
\hline Energy out & $23,681,260$ & 0 & $\mathrm{kWh} / \mathrm{yr}$ \\
\hline Losses & $2,631,406$ & 0 & $\mathrm{kWh} / \mathrm{yr}$ \\
\hline
\end{tabular}
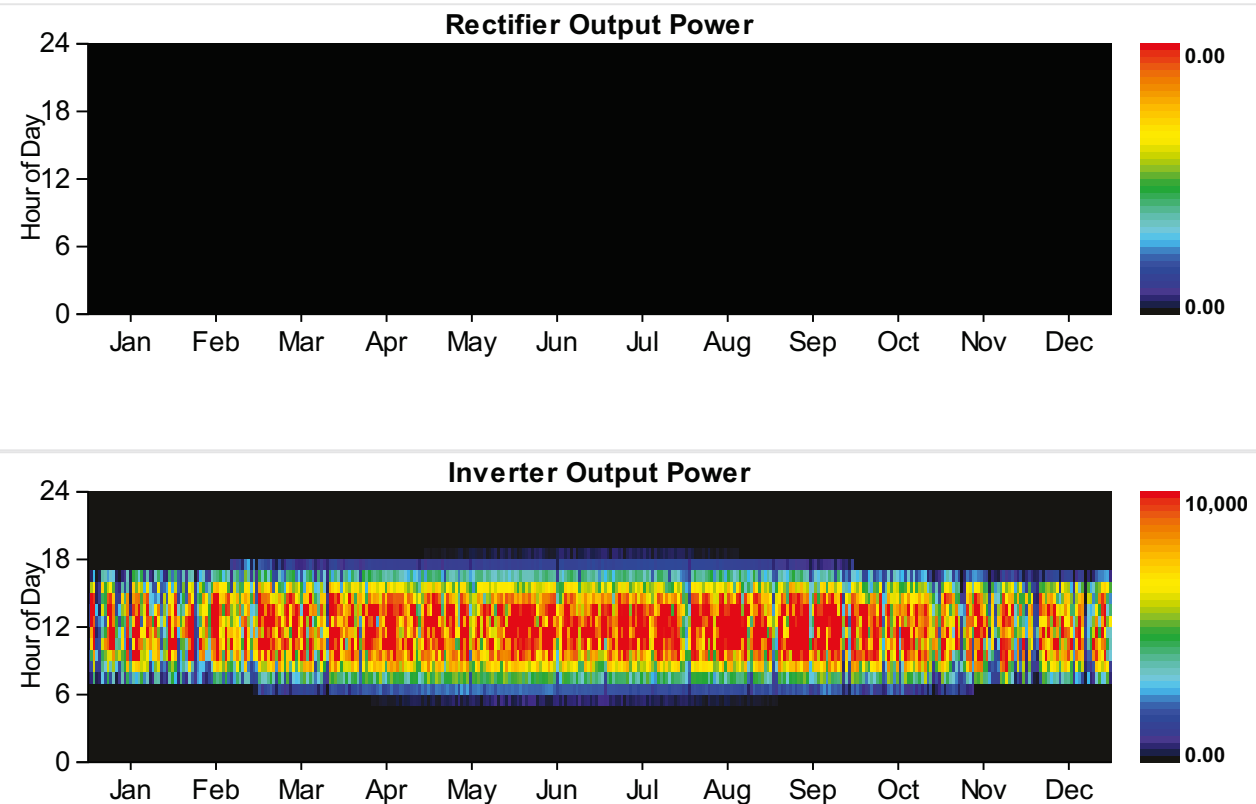

Grid

Rate: Rate 1 Demand

\begin{tabular}{|c|c|c|c|c|c|c|}
\hline Resources.ReportingService_GeneratelnputsReport_Month & $\begin{array}{l}\text { Energy } \\
\text { Purchased } \\
\text { (kWh) }\end{array}$ & $\begin{array}{l}\text { Energy } \\
\text { Sold } \\
\text { (kWh) }\end{array}$ & $\begin{array}{l}\text { Net } \\
\text { Purchases } \\
\text { (kWh) }\end{array}$ & $\begin{array}{l}\text { Peak } \\
\text { Demand } \\
(\mathbf{k W})\end{array}$ & $\begin{array}{l}\text { Energy } \\
\text { Charge } \\
\text { (\$) }\end{array}$ & $\begin{array}{l}\text { Demand } \\
\text { Charge } \\
\text { (\$) }\end{array}$ \\
\hline
\end{tabular}
January

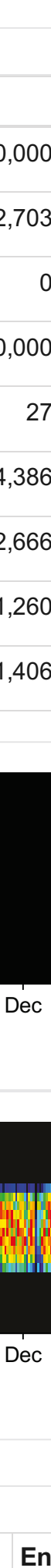

\begin{tabular}{|l|l|}
\hline 0 & 0 \\
\hline
\end{tabular}

$0 \quad 238,205$ 


\begin{tabular}{|c|c|c|c|c|c|c|}
\hline February & Energy 0 & Energy & Net 0 & Peâk254 & Energy & 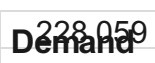 \\
\hline $\begin{array}{l}\text { March } \\
\text { Resources.ReportingService_GeneratelnputsReport_Month }\end{array}$ & $\begin{array}{l}\text { Purchased } \\
\text { (kWh) }\end{array}$ & $\begin{array}{l}\text { Sold } 0 \\
\text { (kWh) }\end{array}$ & $\begin{array}{l}\text { Purchase } \\
\text { (kWh) }\end{array}$ & 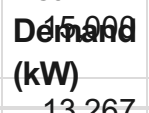 & $\begin{array}{l}\text { Charge } \\
\text { (\$) }\end{array}$ & CRAagge0 \\
\hline May & 0 & 0 & 0 & 13,516 & 0 & 216,263 \\
\hline June & 0 & 0 & 0 & 15,000 & 0 & 240,000 \\
\hline July & 0 & 0 & 0 & 15,000 & 0 & 240,000 \\
\hline August & 0 & 0 & 0 & 15,000 & 0 & 240,000 \\
\hline September & 0 & 0 & 0 & 15,000 & 0 & 240,000 \\
\hline October & 0 & 0 & 0 & 14,623 & 0 & 233,974 \\
\hline November & 0 & 0 & 0 & 13,839 & 0 & 221,428 \\
\hline December & 0 & 0 & 0 & 15,000 & 0 & 240,000 \\
\hline Annual & 0 & 0 & 0 & 15,000 & 0 & $2,790,194$ \\
\hline
\end{tabular}

Rate: Rate 1

\begin{tabular}{|c|c|c|c|c|c|c|}
\hline Resources.ReportingService_GeneratelnputsReport_Month & $\begin{array}{l}\text { Energy } \\
\text { Purchased } \\
\text { (kWh) }\end{array}$ & $\begin{array}{l}\text { Energy } \\
\text { Sold } \\
\text { (kWh) }\end{array}$ & $\begin{array}{l}\text { Net } \\
\text { Purchases } \\
\text { (kWh) }\end{array}$ & $\begin{array}{l}\text { Peak } \\
\text { Demand } \\
(\mathbf{k W})\end{array}$ & $\begin{array}{l}\text { Energy } \\
\text { Charge } \\
(\$)\end{array}$ & $\begin{array}{l}\text { Demand } \\
\text { Charge } \\
\text { (\$) }\end{array}$ \\
\hline January & $5,337,892$ & 0 & $5,337,892$ & 0 & 640,547 & 0 \\
\hline February & $4,320,502$ & 0 & $4,320,502$ & 0 & 518,460 & 0 \\
\hline March & $4,676,530$ & 0 & $4,676,530$ & 0 & 561,184 & 0 \\
\hline April & $4,367,337$ & 0 & $4,367,337$ & 0 & 524,080 & 0 \\
\hline May & $4,323,511$ & 0 & $4,323,511$ & 0 & 518,821 & 0 \\
\hline June & $6,005,034$ & 0 & $6,005,034$ & 0 & 720,604 & 0 \\
\hline July & $7,253,835$ & 0 & $7,253,835$ & 0 & 870,460 & 0 \\
\hline August & $7,440,888$ & 0 & $7,440,888$ & 0 & 892,907 & 0 \\
\hline September & $6,284,002$ & 0 & $6,284,002$ & 0 & 754,080 & 0 \\
\hline October & $5,251,041$ & 0 & $5,251,041$ & 0 & 630,125 & 0 \\
\hline November & $4,867,278$ & 0 & $4,867,278$ & 0 & 584,073 & 0 \\
\hline December & $5,449,358$ & 0 & $5,449,358$ & 0 & 653,923 & 0 \\
\hline Annual & $65,577,200$ & 0 & $65,577,200$ & 0 & $7,869,265$ & 0 \\
\hline
\end{tabular}




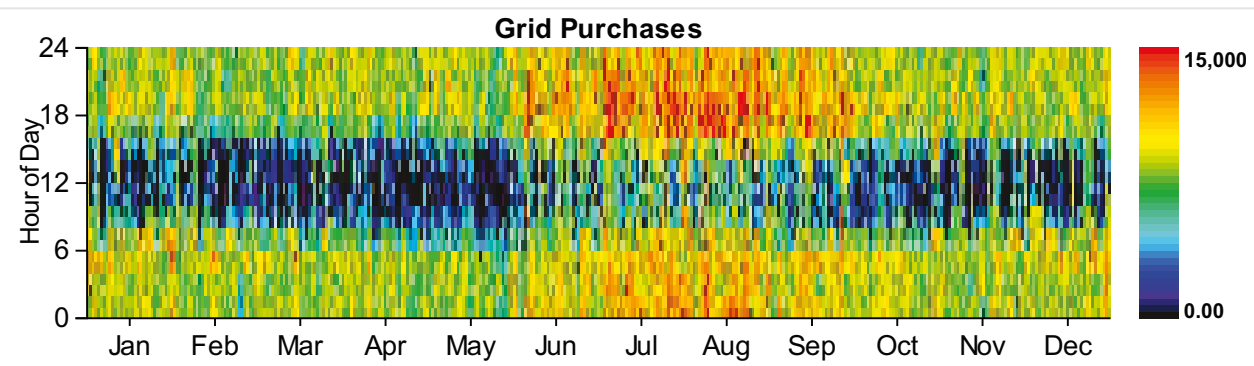

\section{Emissions}

\begin{tabular}{|l|r|l|}
\hline Pollutant & Emissions & Units \\
\hline Carbon dioxide & $42171020 \mathrm{~kg} / \mathrm{yr}$ \\
\hline Carbon monoxide & $3044 \mathrm{~kg} / \mathrm{yr}$ \\
\hline Unburned hydrocarbons & $349 \mathrm{~kg} / \mathrm{yr}$ \\
\hline Particulate matter & $87 \mathrm{~kg} / \mathrm{yr}$ \\
\hline Sulfur dioxide & $181177 \mathrm{~kg} / \mathrm{yr}$ \\
\hline Nitrogen oxides & $90917 \mathrm{~kg} / \mathrm{yr}$ \\
\hline
\end{tabular}

HOMER Energy, LLC @ 2016 
System Report

System architecture

\begin{tabular}{|c|c|c|c|}
\hline PV & SunPower PV & 13,780 & $\mathrm{~kW}$ \\
\hline PV \#2 & Remaining Generic flat plate PV & 1,547 & kW \\
\hline PV \#3 & Solar World 320W flat plate PV Copy & 1,000 & $\mathrm{~kW}$ \\
\hline Wind Turbine & Siemens $2.3 \mathrm{MW}-108$ & 1 & \\
\hline Generator & Kohler 3250 Prime Power & 5,600 & $\mathrm{~kW}$ \\
\hline Battery & GS200 flow & 1 & strings \\
\hline Converter & System Converter & 10,000 & kW \\
\hline Grid & Grid & 15,000 & kW \\
\hline Dispatch Strategy & Cycle Charging & & \\
\hline
\end{tabular}

\section{Cost summary}

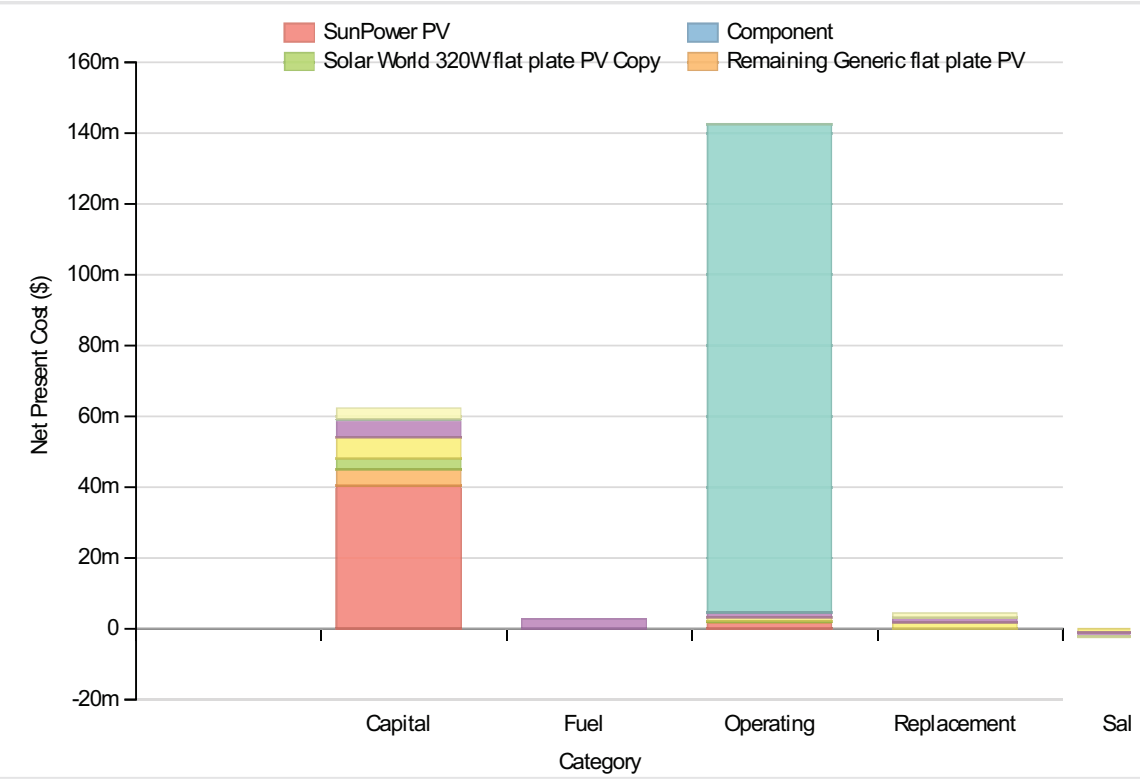

Cost Summary

Total net present cost

$209549552 \$$

Levelized cost of energy

$0.171 \$ / k W h$

\section{Net Present Costs}

\begin{tabular}{|c|c|c|c|c|c|c|}
\hline Component & Capital & Replacement & O\&M & Fuel & Salvage & Total \\
\hline SunPower PV & $40,358,332$ & 0 & $1,656,876$ & 0 & 0 & $42,015,208$ \\
\hline Remaining Generic flat plate PV & $4,678,459$ & 0 & 339,013 & 0 & 0 & $5,017,472$ \\
\hline Solar World 320W flat plate PV Copy & $3,083,333$ & 0 & 280,096 & 0 & 0 & $3,363,429$ \\
\hline Siemens 2.3 MW - 108 & $6,000,000$ & $1,912,842$ & 969,563 & 0 & $-1,078,009$ & $7,804,396$ \\
\hline Kohler 3250 Prime Power & $4,821,429$ & $1,225,634$ & $1,360,948$ & $2,681,734$ & $-1,104,959$ & $8,984,786$ \\
\hline Grid & 0 & 0 & $137,969,088$ & 0 & 0 & $137,969,088$ \\
\hline
\end{tabular}




\begin{tabular}{|c|c|c|c|c|c|c|}
\hline 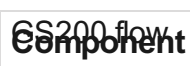 & Capizát4,589 & Replacement 6 & O\&M 31,026 & Fuel & Salvage 973 & Total 361,818 \\
\hline Converter & $3,000,000$ & $1,272,821$ & 0 & 0 & $-239,558$ & $4,033,263$ \\
\hline System & $62,266,140$ & $4,418,472$ & $142,606,624$ & $2,681,734$ & $-2,423,499$ & $209,549,471$ \\
\hline
\end{tabular}

\section{Annualized Costs}

\begin{tabular}{|c|c|c|c|c|c|c|}
\hline Component & Capital & Replacement & O\&M & Fuel & Salvage & Total \\
\hline SunPower PV & $3,121,894$ & 0 & 128,167 & 0 & 0 & $3,250,061$ \\
\hline Remaining Generic flat plate PV & 361,899 & 0 & 26,224 & 0 & 0 & 388,123 \\
\hline Solar World 320W flat plate PV Copy & 238,509 & 0 & 21,667 & 0 & 0 & 260,176 \\
\hline Siemens 2.3 MW - 108 & 464,126 & 147,967 & 75,000 & 0 & $-83,389$ & 603,704 \\
\hline Kohler 3250 Prime Power & 372,959 & 94,808 & 105,275 & 207,444 & $-85,473$ & 695,013 \\
\hline Grid & 0 & 0 & $10,672,513$ & 0 & 0 & $10,672,513$ \\
\hline GS200 flow & 25,108 & 555 & 2,400 & 0 & -75 & 27,988 \\
\hline Converter & 232,063 & 98,458 & 0 & 0 & $-18,531$ & 311,990 \\
\hline System & $4,816,559$ & 341,788 & $11,031,247$ & 207,444 & $-187,468$ & $16,209,570$ \\
\hline
\end{tabular}

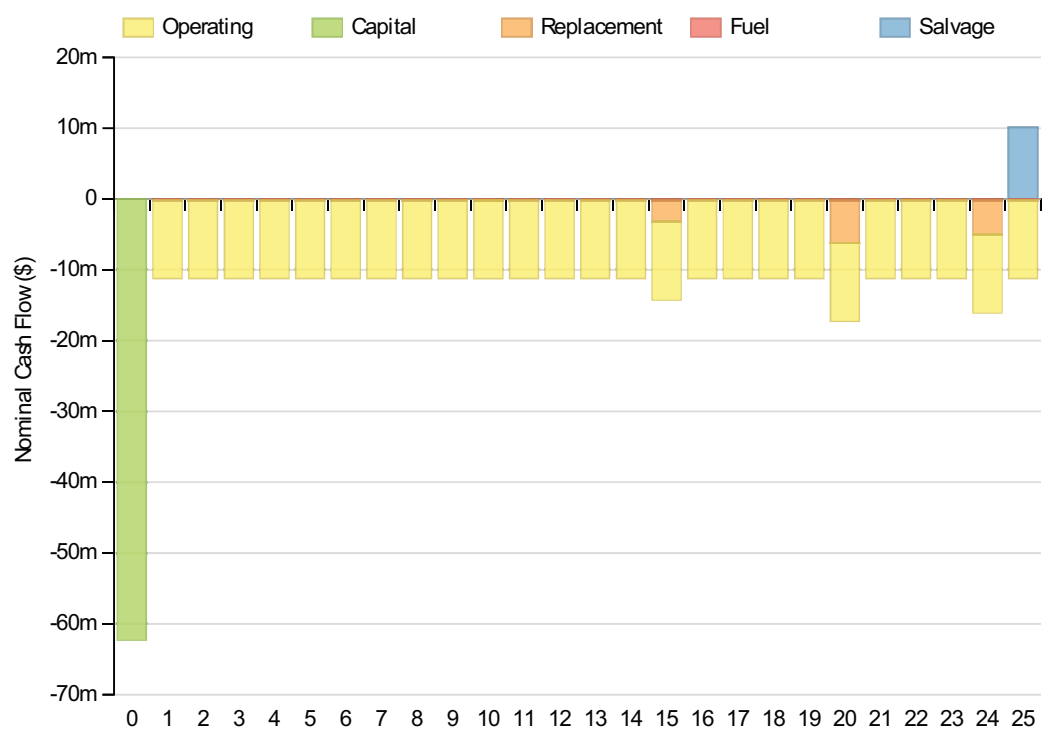

Year

Electrical

\section{Quantity}

Excess electricity

Unmet load

Capacity shortage

Renewable fraction
Value

Units

$2886873 \mathrm{kWh} / \mathrm{yr}$

$6236 \mathrm{kWh} / \mathrm{yr}$

$76596 \mathrm{kWh} / \mathrm{yr}$

0

\section{Component}

PV

PV
Production(kWh/yr)

Fraction (\%) 
Eל̌mponent

\section{Generator}

Wind Turbine

Grid Purchases

Total

\section{Load}

Consumption(kWh/yr)

\section{Fraction (\%)}

AC primary load

DC primary load

0

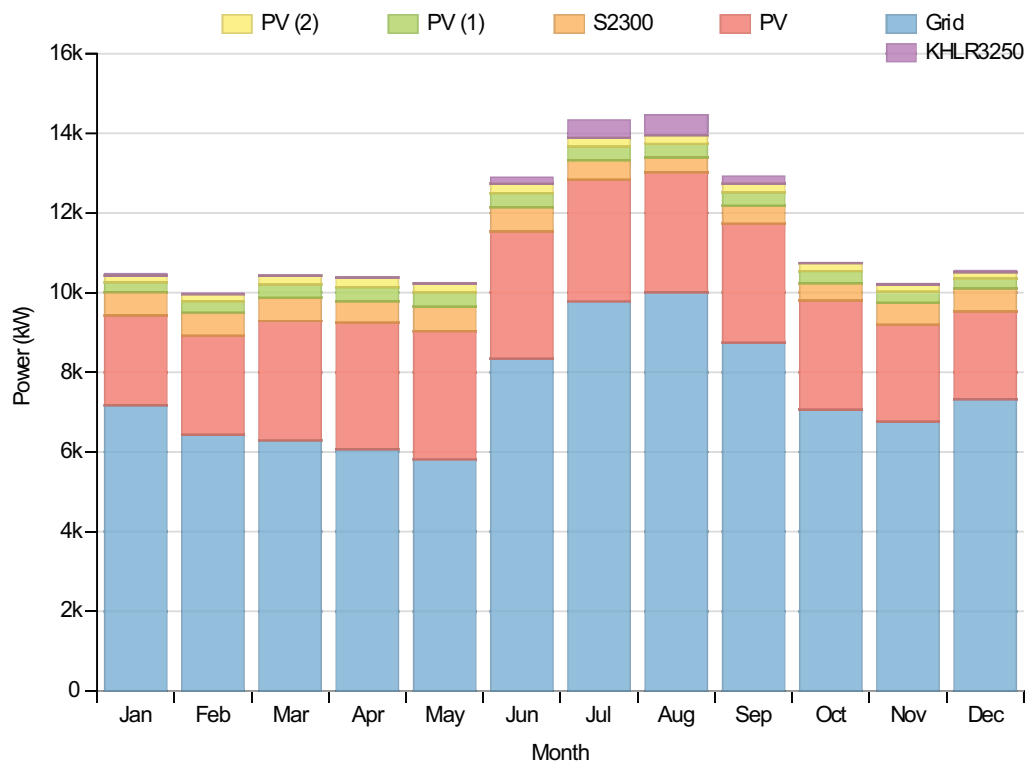

\section{PV:SunPower PV}

\begin{tabular}{|c|c|c|}
\hline Quantity & Value & Units \\
\hline Rated capacity & 13780 & $\mathrm{~kW}$ \\
\hline Mean output & 2813 & $\mathrm{~kW}$ \\
\hline Mean output & 67519.00 & $\mathrm{kWh} / \mathrm{d}$ \\
\hline Capacity factor & 20.42 & $\%$ \\
\hline Total production & 24644568 & $\mathrm{kWh} / \mathrm{yr}$ \\
\hline Minimum output & 0.00 & $\mathrm{~kW}$ \\
\hline Maximum output & 13941.00 & $\mathrm{~kW}$ \\
\hline PV penetration & 25.93 & $\%$ \\
\hline Hours of operation & 4386 & hrs/yr \\
\hline Levelized cost & 0.111 & $\$ / \mathrm{kWh}$ \\
\hline
\end{tabular}




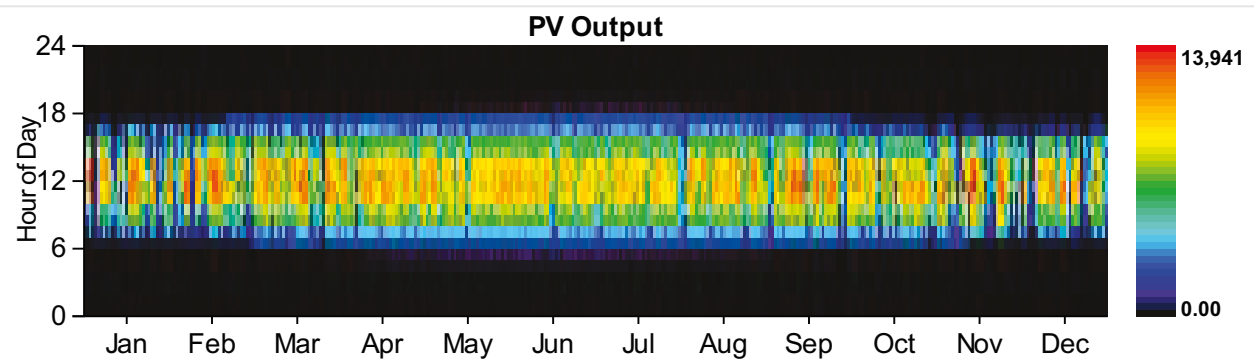

PV:Remaining Generic flat plate PV

\begin{tabular}{|c|c|c|}
\hline Quantity & Value & Units \\
\hline Rated capacity & 1547 & $\mathrm{~kW}$ \\
\hline Mean output & 316 & $\mathrm{~kW}$ \\
\hline Mean output & 7579.50 & $\mathrm{kWh} / \mathrm{d}$ \\
\hline Capacity factor & 20.42 & $\%$ \\
\hline Total production & 2766525 & $\mathrm{kWh} / \mathrm{yr}$ \\
\hline Minimum output & 0.00 & $\mathrm{~kW}$ \\
\hline Maximum output & 1565.00 & $\mathrm{~kW}$ \\
\hline PV penetration & 2.91 & $\%$ \\
\hline Hours of operation & 4386 & $\mathrm{hrs} / \mathrm{yr}$ \\
\hline Levelized cost & 0.013 & $\$ / k W h$ \\
\hline
\end{tabular}

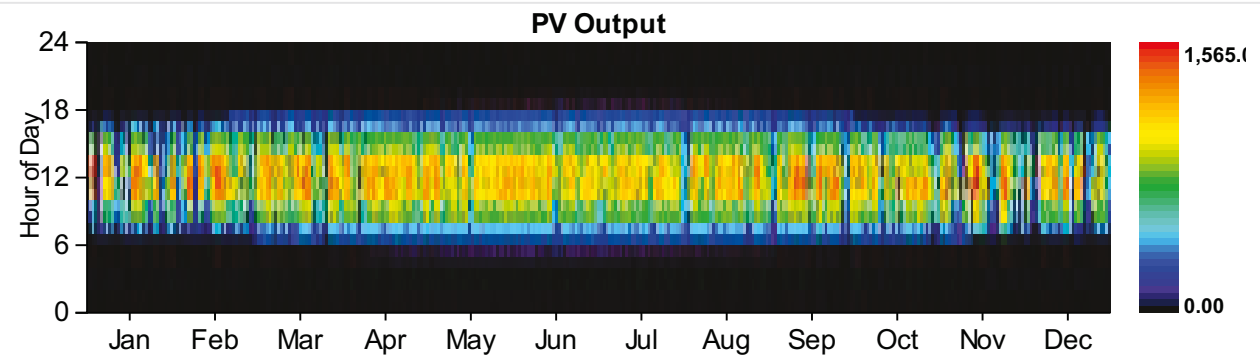

PV:Solar World 320W flat plate PV Copy

\begin{tabular}{|l|r|l|}
\hline Quantity & Value & Units \\
\hline Rated capacity & $1000 \mathrm{~kW}$ \\
\hline Mean output & $204 \mathrm{~kW}$ \\
\hline Mean output & $4899.80 \mathrm{kWh} / \mathrm{d}$ \\
\hline Capacity factor & $20.42 \%$ \\
\hline Total production & $1788429 \mathrm{kWh} / \mathrm{yr}$ \\
\hline Minimum output & $0.00 \mathrm{~kW}$ \\
\hline Maximum output & $1011.70 \mathrm{~kW}$ \\
\hline PV penetration & $1.88 \%$ & $\%$ \\
\hline
\end{tabular}




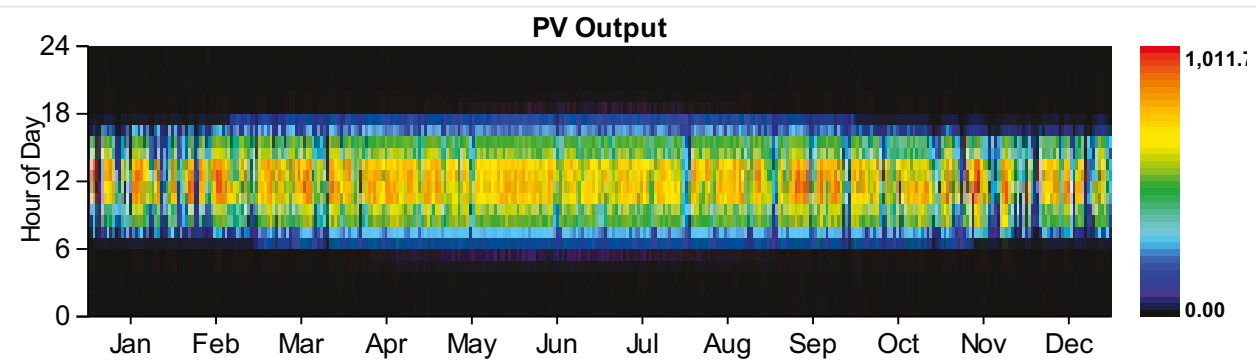

Wind Turbine:Siemens 2.3 MW - 108

\section{Quantity}

Total rated capacity

Mean output

Capacity factor

Total production

Minimum output

Maximum output

Wind penetration

Hours of operation

Levelized cost
Value

Units

$2300 \mathrm{~kW}$

534 kW

$23.20 \%$

$4674352 \mathrm{kWh} / \mathrm{yr}$

$0.49 \mathrm{~kW}$

$2312.70 \mathrm{~kW}$

$4.92 \%$

$8760 \mathrm{hrs} / \mathrm{yr}$

$0.129 \$ / k W h$

\section{Generator:Kohler 3250 Prime Power}

\begin{tabular}{|c|c|c|}
\hline Quantity & Value & Units \\
\hline Hours of operation & 626 & $\mathrm{hrs} / \mathrm{yr}$ \\
\hline Number of starts & 410 & starts/yr \\
\hline Operational life & 24 & $\mathrm{yr}$ \\
\hline Fixed generation cost & 511.50 & $\$ / \mathrm{hr}$ \\
\hline Marginal generation cost & 0.18 & $\$ / k W h$ \\
\hline Electrical production & 1056398 & $\mathrm{kWh} / \mathrm{yr}$ \\
\hline Mean electrical output & 1688 & $\mathrm{~kW}$ \\
\hline Min. electrical output & 1400 & $\mathrm{~kW}$ \\
\hline Max. electrical output & 5600 & $\mathrm{~kW}$ \\
\hline Fuel consumption & 262587 & L/yr \\
\hline Specific fuel consumption & 0.25 & $\mathrm{~L} / \mathrm{kWh}$ \\
\hline Fuel energy input & 2583860 & $\mathrm{kWh} / \mathrm{yr}$ \\
\hline Mean electrical efficiency & 41 & $\%$ \\
\hline
\end{tabular}




\section{Quantity}

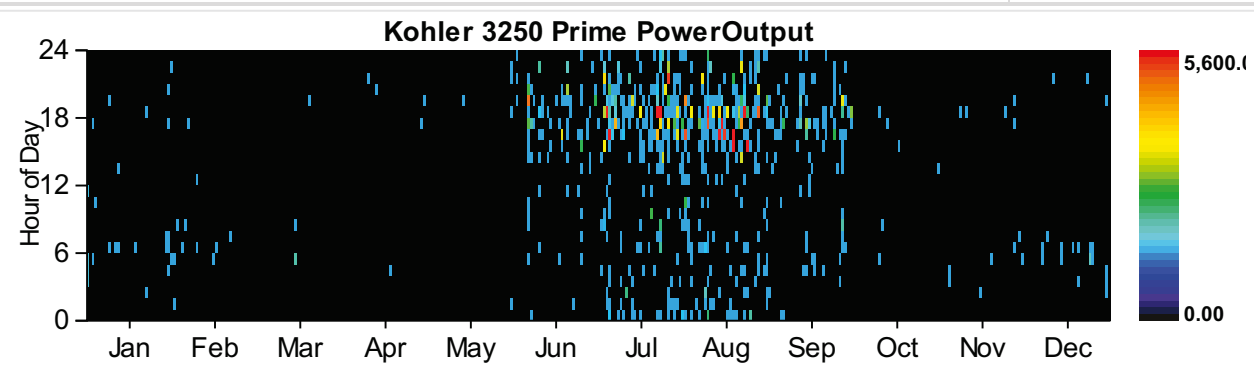

\section{Battery:GS200 flow}

Quantity Value

String size

Strings in parallel

Batteries

Bus voltage

\section{Quantity}

Nominal capacity

Usable nominal capacity

Autonomy

Lifetime throughput

Battery wear cost

Average energy cost

Energy in

Energy out

Storage depletion

Losses

Annual throughput

Expected life

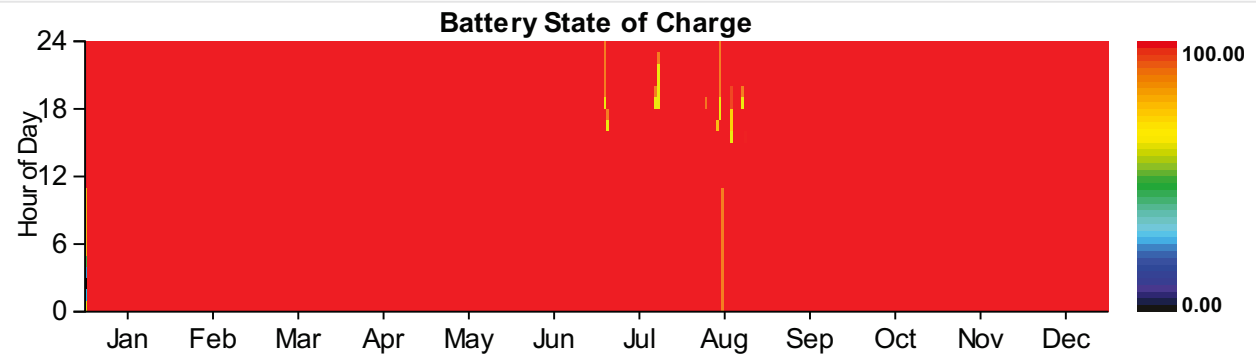

Value

Units

$600 \mathrm{kWh}$

$600 \mathrm{kWh}$

$0 \mathrm{hr}$

0

$0.000 \$ / \mathrm{kWh}$

$0.285 \$ / k W h$

$2810 \mathrm{kWh} / \mathrm{yr}$

1967 kWh/yr

$0 \mathrm{kWh} / \mathrm{yr}$

$843 \mathrm{kWh} / \mathrm{yr}$

$2351 \mathrm{kWh} / \mathrm{yr}$

$25 \mathrm{yr}$

\section{Converter}




\begin{tabular}{|c|c|c|c|}
\hline Qenarafity & Inverter & Rectifier & WNits \\
\hline Mean output & 2,704 & 0 & $\mathrm{~kW}$ \\
\hline Minimum output & 0 & 0 & $\mathrm{~kW}$ \\
\hline Maximum output & 10,000 & 179 & $\mathrm{~kW}$ \\
\hline Capacity factor & 27 & 0 & $\%$ \\
\hline Hours of operation & 4,388 & 18 & hrs/yr \\
\hline Energy in & $26,313,838$ & 2,263 & $\mathrm{kWh} / \mathrm{yr}$ \\
\hline Energy out & $23,682,316$ & 1,924 & kWh/yr \\
\hline Losses & $2,631,522$ & 339 & $\mathrm{kWh} / \mathrm{yr}$ \\
\hline
\end{tabular}
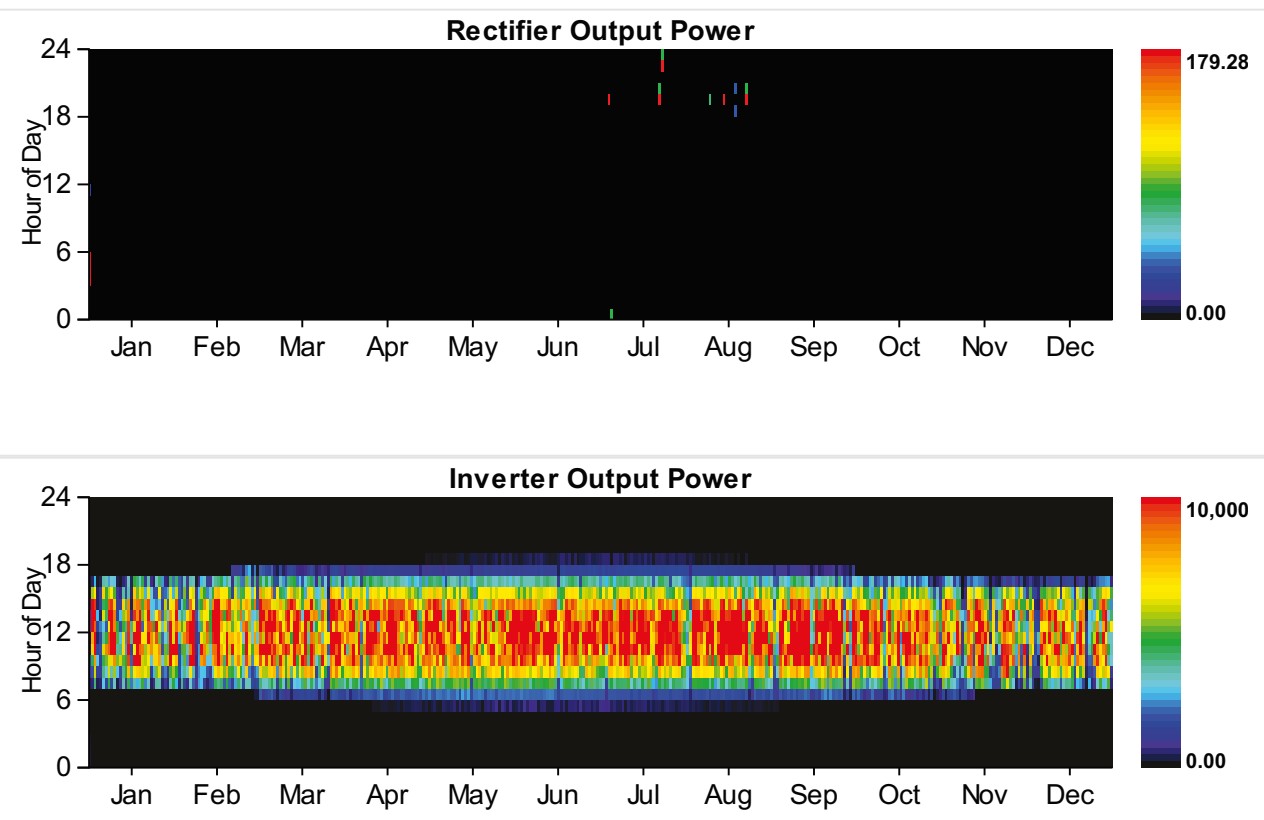

\section{Grid}

Rate: Rate 1 Demand

\begin{tabular}{|c|c|c|c|c|c|c|}
\hline Resources.ReportingService_GeneratelnputsReport_Month & $\begin{array}{l}\text { Energy } \\
\text { Purchased } \\
\text { (kWh) }\end{array}$ & $\begin{array}{l}\text { Energy } \\
\text { Sold } \\
\text { (kWh) }\end{array}$ & $\begin{array}{l}\text { Net } \\
\text { Purchases } \\
\text { (kWh) }\end{array}$ & $\begin{array}{l}\text { Peak } \\
\text { Demand } \\
(\mathbf{k W})\end{array}$ & $\begin{array}{l}\text { Energy } \\
\text { Charge } \\
\text { (\$) }\end{array}$ & $\begin{array}{l}\text { Demand } \\
\text { Charge } \\
(\$)\end{array}$ \\
\hline January & 0 & 0 & 0 & 14,888 & 0 & 238,205 \\
\hline February & 0 & 0 & 0 & 14,254 & 0 & 228,059 \\
\hline March & 0 & 0 & 0 & 15,000 & 0 & 240,000 \\
\hline April & 0 & 0 & 0 & 13,645 & 0 & 218,325 \\
\hline May & 0 & 0 & 0 & 13,516 & 0 & 216,263 \\
\hline June & 0 & 0 & 0 & 15,000 & 0 & 240,000 \\
\hline July & 0 & 0 & 0 & 15,000 & 0 & 240,000 \\
\hline August & 0 & 0 & 0 & 15,000 & 0 & 240,000 \\
\hline September & 0 & 0 & 0 & 15,000 & 0 & 240,000 \\
\hline
\end{tabular}




\begin{tabular}{|c|c|c|c|c|c|c|}
\hline October & Energy 0 & Energy & Net & Peakk623 & Energy & Dénâất \\
\hline $\begin{array}{l}\text { November } \\
\text { Resources.ReportingService_GeneratelnputsReport_Month }\end{array}$ & $\begin{array}{l}\text { Purchased } \\
\text { (kWh) }\end{array}$ & $\begin{array}{l}\text { Sold } 0 \\
\text { (kWh) }\end{array}$ & $\begin{array}{l}\text { Purchases } \\
\text { (kWh) }\end{array}$ & $\begin{array}{l}\text { Dem,abd } \\
(\mathrm{kW})\end{array}$ & $\begin{array}{l}\text { Charge } \\
(\$)\end{array}$ & $\begin{array}{l}\text { Change28 } \\
\text { (\$) }\end{array}$ \\
\hline December & 0 & 0 & 0 & 15,000 & 0 & 240,000 \\
\hline Annual & 0 & 0 & 0 & 15,000 & 0 & $2,796,253$ \\
\hline
\end{tabular}

Rate: Rate 1

\begin{tabular}{|c|c|c|c|c|c|c|}
\hline Resources.ReportingService_GeneratelnputsReport_Month & $\begin{array}{l}\text { Energy } \\
\text { Purchased } \\
\text { (kWh) }\end{array}$ & $\begin{array}{l}\text { Energy } \\
\text { Sold } \\
\text { (kWh) }\end{array}$ & $\begin{array}{l}\text { Net } \\
\text { Purchases } \\
\text { (kWh) }\end{array}$ & $\begin{array}{l}\text { Peak } \\
\text { Demand } \\
(\mathbf{k W})\end{array}$ & $\begin{array}{l}\text { Energy } \\
\text { Charge } \\
(\$)\end{array}$ & $\begin{array}{l}\text { Demand } \\
\text { Charge } \\
(\$)\end{array}$ \\
\hline January & $5,340,240$ & 0 & $5,340,240$ & 0 & 640,829 & 0 \\
\hline February & $4,323,302$ & 0 & $4,323,302$ & 0 & 518,796 & 0 \\
\hline March & $4,677,930$ & 0 & $4,677,930$ & 0 & 561,352 & 0 \\
\hline April & $4,368,737$ & 0 & $4,368,737$ & 0 & 524,248 & 0 \\
\hline May & $4,323,511$ & 0 & $4,323,511$ & 0 & 518,821 & 0 \\
\hline June & $6,009,234$ & 0 & $6,009,234$ & 0 & 721,108 & 0 \\
\hline July & $7,273,435$ & 0 & $7,273,435$ & 0 & 872,812 & 0 \\
\hline August & $7,452,088$ & 0 & $7,452,088$ & 0 & 894,251 & 0 \\
\hline September & $6,295,202$ & 0 & $6,295,202$ & 0 & 755,424 & 0 \\
\hline October & $5,253,841$ & 0 & $5,253,841$ & 0 & 630,461 & 0 \\
\hline November & $4,867,278$ & 0 & $4,867,278$ & 0 & 584,073 & 0 \\
\hline December & $5,450,758$ & 0 & $5,450,758$ & 0 & 654,091 & 0 \\
\hline Annual & $65,635,548$ & 0 & $65,635,548$ & 0 & $7,876,267$ & 0 \\
\hline
\end{tabular}

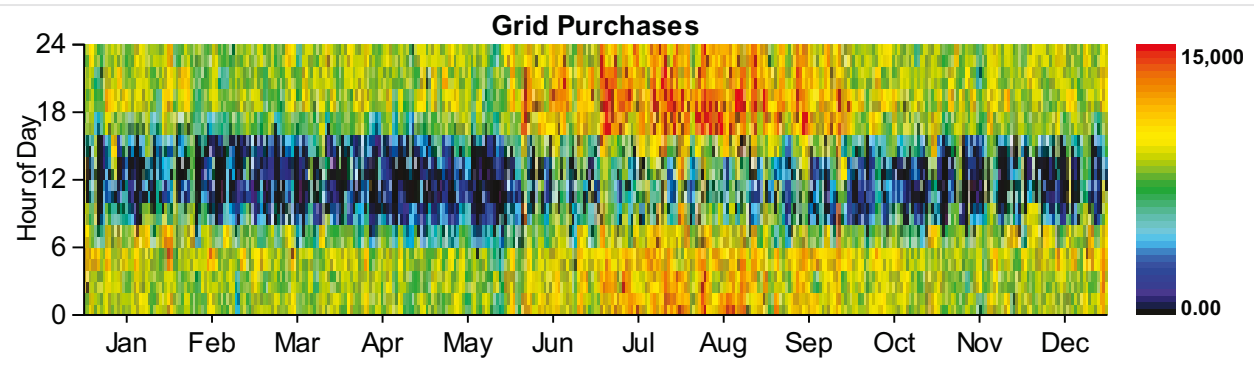

\section{Emissions}

\section{Pollutant}

Carbon dioxide

Carbon monoxide

Unburned hydrocarbons

Particulate matter

Sulfur dioxide

Nitrogen oxides
Emissions

Units

$42170832 \mathrm{~kg} / \mathrm{yr}$

$2889 \mathrm{~kg} / \mathrm{yr}$

$331 \mathrm{~kg} / \mathrm{yr}$

$83 \mathrm{~kg} / \mathrm{yr}$

$181260 \mathrm{~kg} / \mathrm{yr}$

90840 kg/yr 
HOMER Energy, LLC @ 2016 
System Report

System architecture

\begin{tabular}{|c|c|c|c|}
\hline PV & SunPower PV & 13,780 & $\mathrm{~kW}$ \\
\hline PV \#2 & Remaining Generic flat plate PV & 1,547 & $\mathrm{~kW}$ \\
\hline PV \#3 & Solar World 320W flat plate PV Copy & 6,000 & $\mathrm{~kW}$ \\
\hline Wind Turbine & Siemens 2.3 MW - 108 & 6 & \\
\hline Generator & Kohler 3250 Prime Power & 16,800 & $\mathrm{~kW}$ \\
\hline Battery & GS200 flow & 70 & strings \\
\hline Converter & System Converter & 20,000 & $\mathrm{~kW}$ \\
\hline Dispatch Strategy & Cycle Charging & & \\
\hline
\end{tabular}

\section{Cost summary}

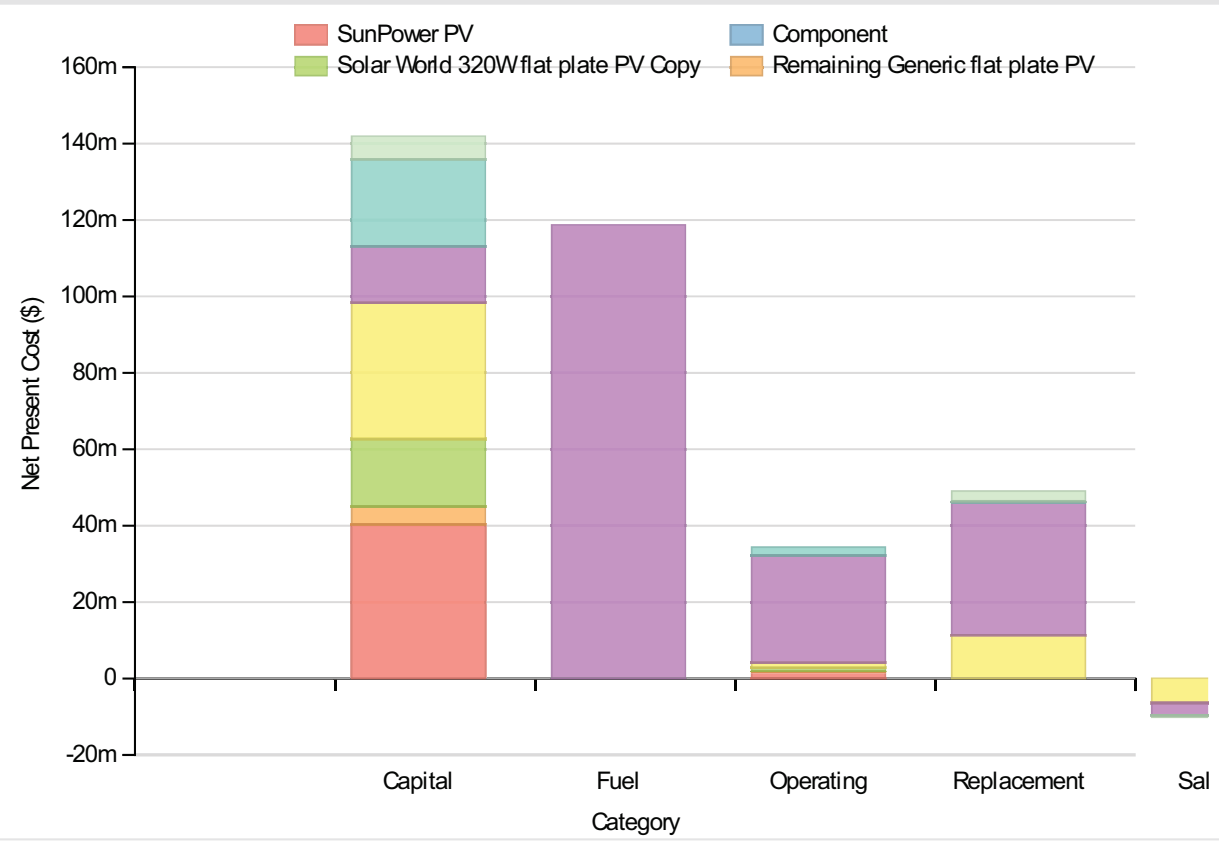

Cost Summary

Total net present cost

$333824096 \$$

Levelized cost of energy

$0.272 \$ / k W h$

\section{Net Present Costs}

\begin{tabular}{|c|c|c|c|c|c|c|}
\hline Component & Capital & Replacement & O\&M & Fuel & Salvage & Total \\
\hline SunPower PV & $40,358,332$ & 0 & $1,656,876$ & 0 & 0 & $42,015,208$ \\
\hline Remaining Generic flat plate PV & $4,678,459$ & 0 & 339,013 & 0 & 0 & $5,017,472$ \\
\hline $\begin{array}{l}\text { Solar World } 320 \mathrm{~W} \text { flat plate PV } \\
\text { Copy }\end{array}$ & $17,666,668$ & 0 & 818,742 & 0 & 0 & $18,485,410$ \\
\hline Siemens 2.3 MW - 108 & $35,625,000$ & $11,357,500$ & $1,292,751$ & 0 & $-6,400,678$ & $41,874,573$ \\
\hline Kohler 3250 Prime Power & $14,821,428$ & $34,618,928$ & $28,073,770$ & $118,660,264$ & $-3,136,350$ & $193,038,040$ \\
\hline
\end{tabular}




\begin{tabular}{|l|r|r|r|r|r|r|}
\hline $\begin{array}{l}\text { Component } \\
\text { GS200 flow }\end{array}$ & $\begin{array}{c}\text { Capital } \\
22,721,244\end{array}$ & Replacement & O\&M & Fuel & Salvage & \multicolumn{1}{c|}{ Total } \\
\hline Converter & $6,000,000$ & $2,545,641$ & 0 & 0 & $-479,115$ & $8,066,526$ \\
\hline System & $141,871,136$ & $49,023,804$ & $34,352,980$ & $118,660,264$ & $-10,084,170$ & $333,824,014$ \\
\hline
\end{tabular}

\section{Annualized Costs}

\begin{tabular}{|l|r|r|r|r|r|r|}
\hline Component & Capital & Replacement & O\&M & Fuel & Salvage & Total \\
\hline SunPower PV & $3,121,894$ & 0 & 128,167 & 0 & 0 & $3,250,061$ \\
\hline Remaining Generic flat plate PV & 361,899 & 0 & 26,224 & 0 & 0 & 388,123 \\
\hline Solar World 320W flat plate PV Copy & $1,366,594$ & 0 & 63,333 & 0 & 0 & $1,429,927$ \\
\hline Siemens 2.3 MW - 108 & $2,755,750$ & 878,552 & 100,000 & 0 & $-495,120$ & $3,239,182$ \\
\hline Kohler 3250 Prime Power & $1,146,502$ & $2,677,926$ & $2,171,629$ & $9,178,891$ & $-242,610$ & $14,932,338$ \\
\hline GS200 flow & $1,757,588$ & 38,811 & 168,000 & 0 & $-5,262$ & $1,959,137$ \\
\hline Converter & 464,126 & 196,916 & 0 & 0 & $-37,062$ & 623,980 \\
\hline System & $10,974,354$ & $3,792,206$ & $2,657,354$ & $9,178,891$ & $-780,055$ & $25,822,750$ \\
\hline
\end{tabular}

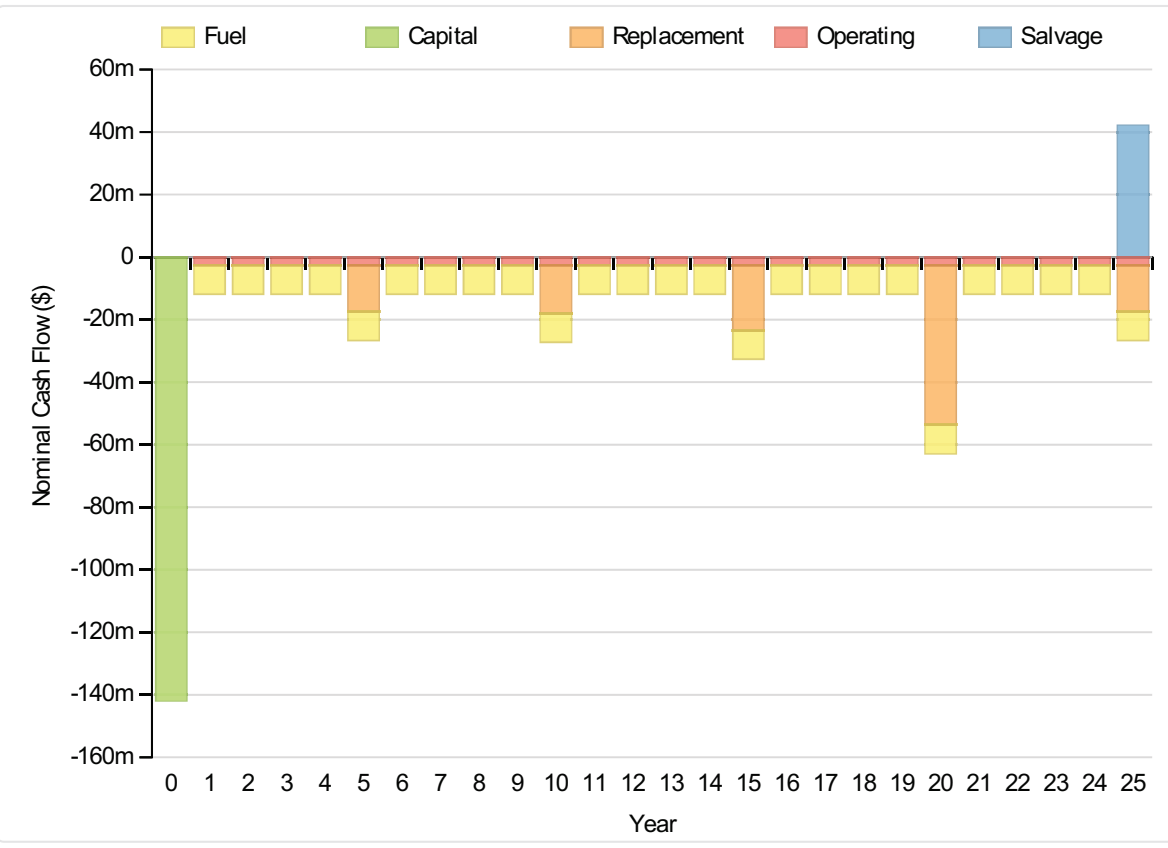

\section{Electrical}

\begin{tabular}{|l|r|c|}
\hline Quantity & Value & Units \\
\hline Excess electricity & $8502696 \mathrm{kWh} / \mathrm{yr}$ \\
\hline Unmet load & $0 \mathrm{kWh} / \mathrm{yr}$ \\
\hline Capacity shortage & $0 \mathrm{kWh} / \mathrm{yr}$ \\
\hline Renewable fraction & 0 \\
\hline
\end{tabular}




\section{Eלmponent}

PV

PV

Generator

Wind Turbine

Total
26,935,180 Fraction (\%)

$2,766,526$

$11,727,956$

$48,951,668$

$28,046,112$

$118,427,440$

Load

Consumption(kWh/yr)

AC primary load

DC primary load

Total

$95,052,456$

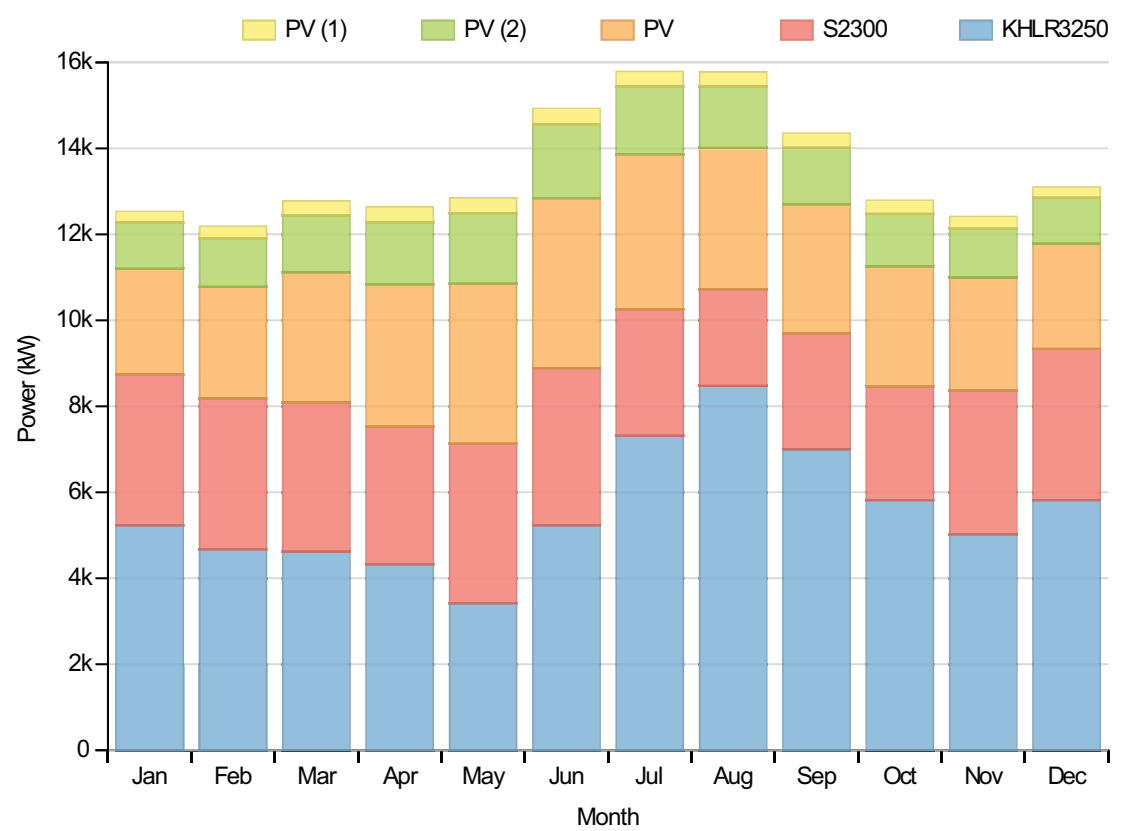

\section{PV:SunPower PV}

\section{Quantity}

Rated capacity

Mean output

Mean output

Capacity factor

Total production

Minimum output

Maximum output

PV penetration

Hours of operation
Fraction (\%)

0 


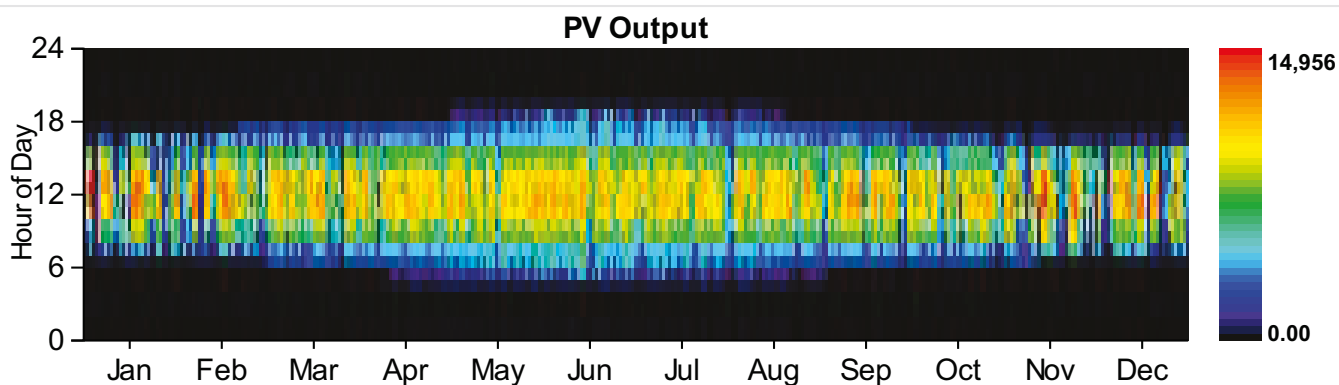

PV:Remaining Generic flat plate PV

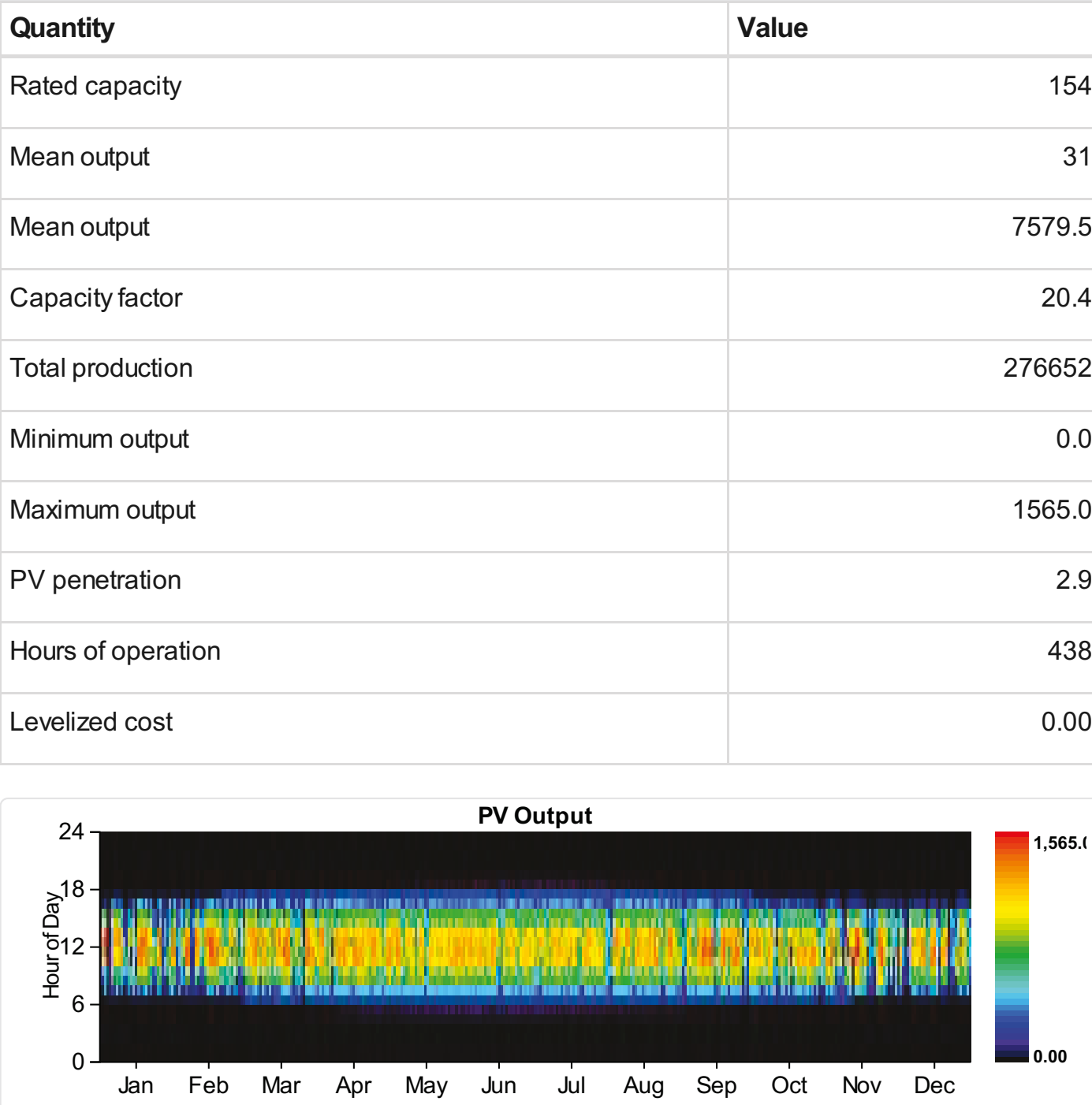

PV:Solar World 320W flat plate PV Copy

\begin{tabular}{|l|r|l|}
\hline Quantity & Value & Units \\
\hline Rated capacity & $6000 \mathrm{~kW}$ \\
\hline Mean output & $1339 \mathrm{~kW}$ \\
\hline Mean output & $32131.00 \mathrm{kWh} / \mathrm{d}$ \\
\hline Capacity factor & $22.31 \%$ \\
\hline
\end{tabular}


Qetahprefduction

Minimum output

Maximum output

PV penetration

Hours of operation

Levelized cost
Value

11727956 kNAtsyr

$0.00 \mathrm{~kW}$

$6512.50 \mathrm{~kW}$

$12.34 \%$

$4386 \mathrm{hrs} / \mathrm{yr}$

$0.035 \$ / k W h$

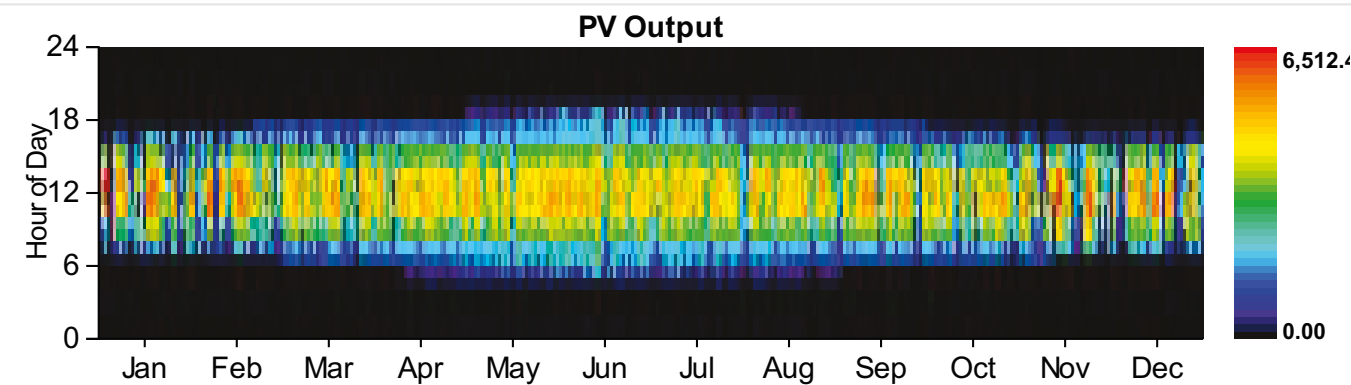

Wind Turbine:Siemens 2.3 MW - 108

\section{Quantity}

Total rated capacity

Mean output

Capacity factor

Total production

Minimum output

Maximum output

Wind penetration

Hours of operation

Levelized cost

\section{Value}

3202 kW

$23.20 \%$

28046112 kWh/yr

$2.96 \mathrm{~kW}$

$13876.00 \mathrm{~kW}$

$29.51 \%$

$8760 \mathrm{hrs} / \mathrm{yr}$

$0.115 \$ / k W h$

Generator:Kohler 3250 Prime Power

\section{Quantity}

Hours of operation

Number of starts

Operational life

Fixed generation cost

Marginal generation cost

Electrical production
Value

Units

$3070 \mathrm{hrs} / \mathrm{yr}$

1604 starts/yr

$5 \mathrm{yr}$

$1761.20 \$ / \mathrm{hr}$

$0.18 \$ / k W h$ 


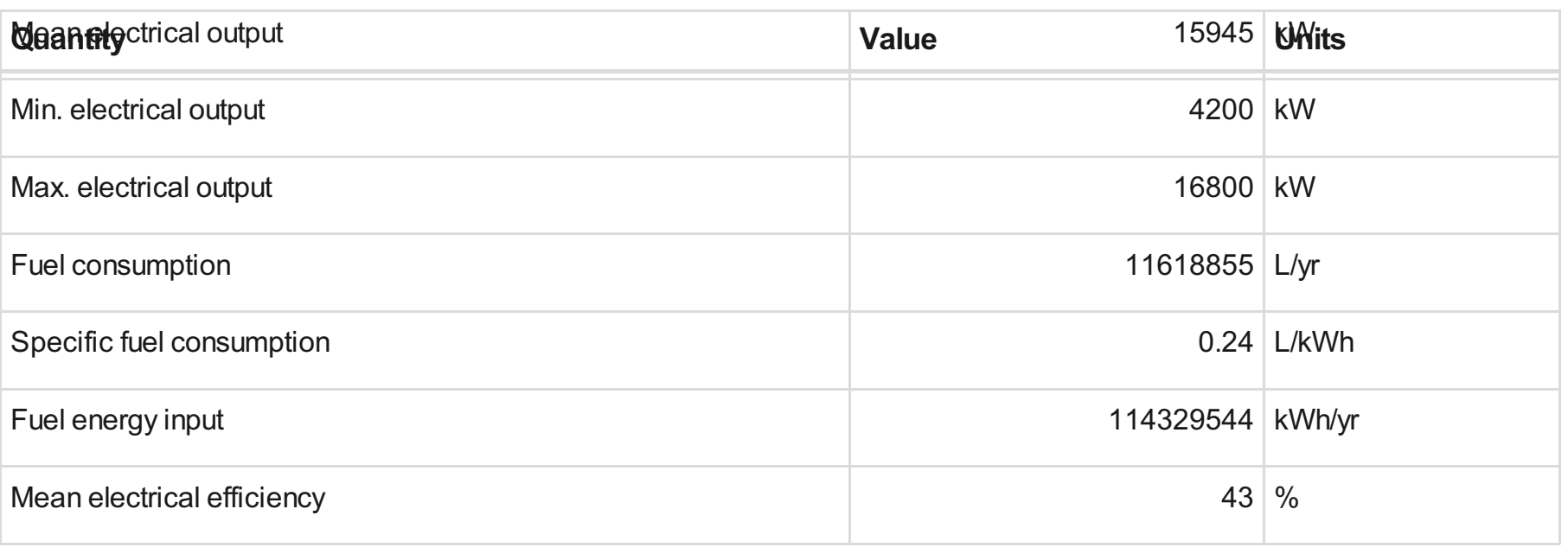

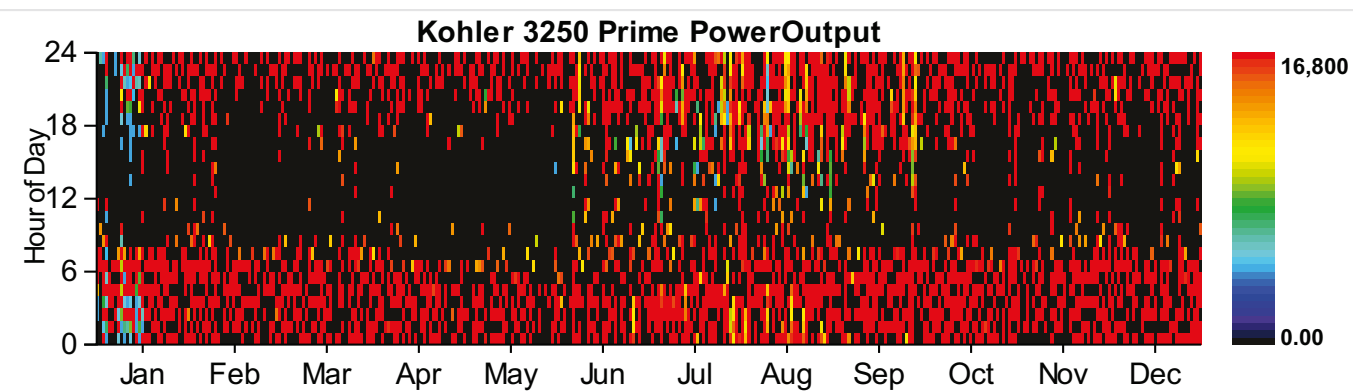

\section{Battery:GS200 flow}

\section{Quantity}

Value

String size

Strings in parallel

Batteries

Bus voltage

\section{Quantity}

Nominal capacity

Usable nominal capacity

Autonomy

Lifetime throughput

Battery wear cost

Average energy cost

Energy in

Energy out

Storage depletion

Losses

Annual throughput
Value

Units

42000 kWh

42000 kWh

$4 \mathrm{hr}$

0

$0.000 \$ / k W h$

$0.184 \$ / k W h$

26938554 kWh/yr

$18877262 \mathrm{kWh} / \mathrm{yr}$

$24254 \mathrm{kWh} / \mathrm{yr}$

8037038 kWh/yr

$22562632 \mathrm{kWh} / \mathrm{yr}$ 


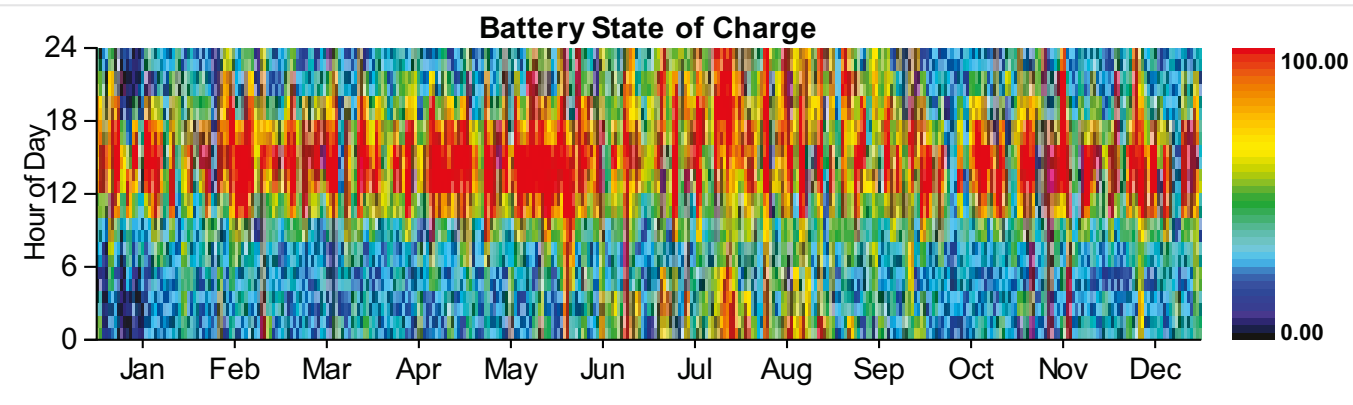

Converter

\begin{tabular}{|l|r|r|l|}
\hline Quantity & Rectifier & Units \\
\hline Capacity & 20,000 & $18,000 \mathrm{~kW}$ \\
\hline Mean output & 4,263 & $1,722 \mathrm{~kW}$ \\
\hline Minimum output & 0 & $0 \mathrm{~kW}$ \\
\hline Maximum output & 18,041 & $12,550 \mathrm{~kW}$ \\
\hline Capacity factor & 21 & $9 \%$ \\
\hline Hours of operation & 5,216 & $2,988 \mathrm{hrs} / \mathrm{yr}$ \\
\hline Energy in & $41,488,572$ & $17,746,810 \mathrm{kWh} / \mathrm{yr}$ \\
\hline Energy out & $37,339,740$ & $15,084,814 \mathrm{kWh} / \mathrm{yr}$ \\
\hline Losses & $4,148,832$ & $2,661,996 \mathrm{kWh} / \mathrm{yr}$ \\
\hline
\end{tabular}
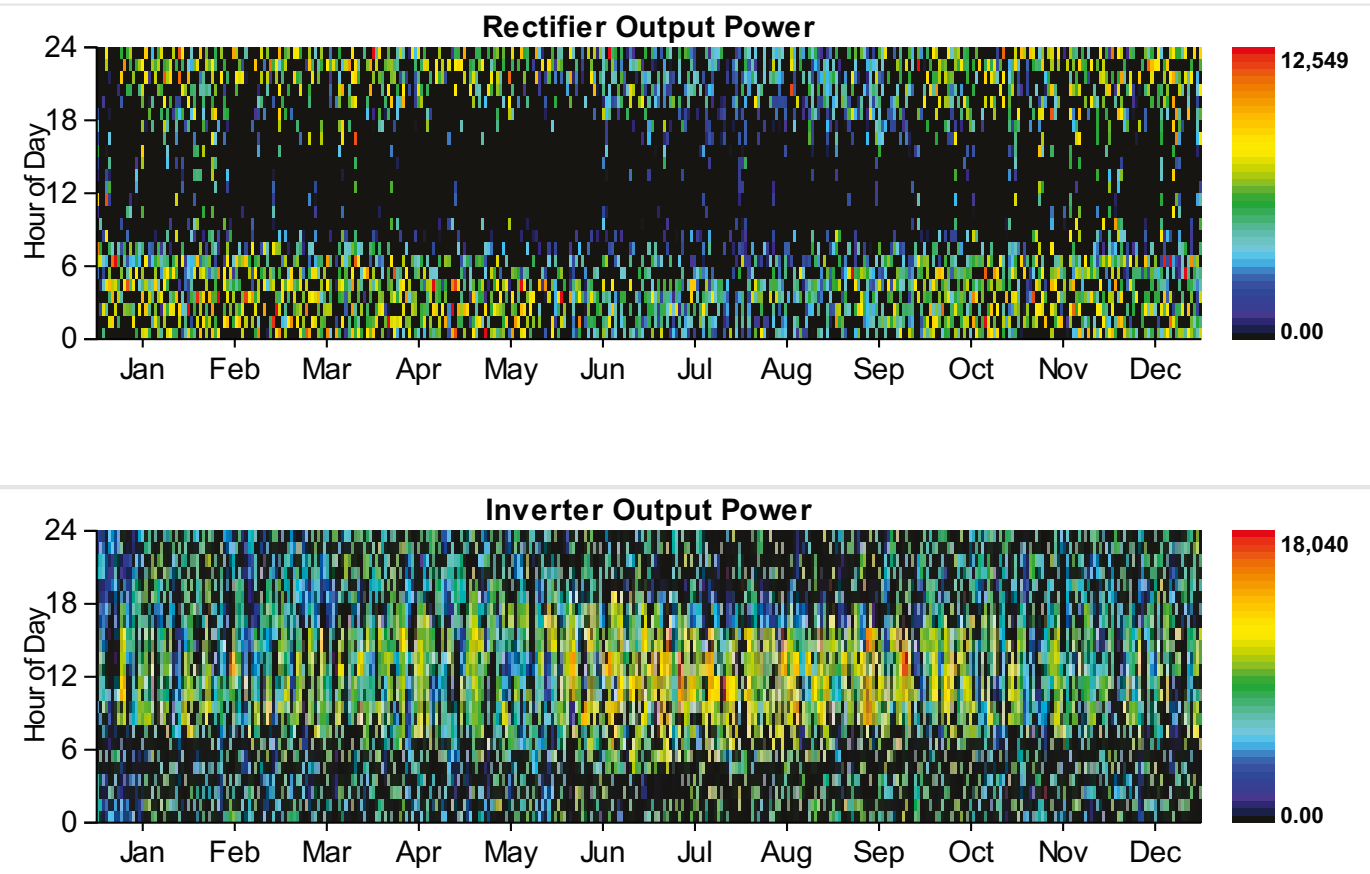

\section{Emissions}


Carbon monoxide $127807 \mathrm{~kg} / \mathrm{yr}$

Unburned hydrocarbons $14640 \mathrm{~kg} / \mathrm{yr}$

Particulate matter $3660 \mathrm{~kg} / \mathrm{yr}$

Sulfur dioxide $62785 \mathrm{~kg} / \mathrm{yr}$

Nitrogen oxides $127807 \mathrm{~kg} / \mathrm{yr}$

HOMER Energy, LLC @ 2016 
System Report

System architecture

\begin{tabular}{|l|l|r|}
\hline PV & SunPower PV & 13,780 \\
\hline PV \#2 & Remaining Generic flat plate PV & 1,547 \\
\hline Wind Turbine & Siemens 2.3 MW - 108 & 8 \\
\hline Generator & Kohler 3250 Prime Power & 16,800 \\
\hline Battery & GS200 flow & 70 \\
\hline Converter & System Converter & 20,000 \\
\hline Dispatch Strategy & Cycle Charging & $\mathrm{kW}$ \\
\hline
\end{tabular}

\section{Cost summary}

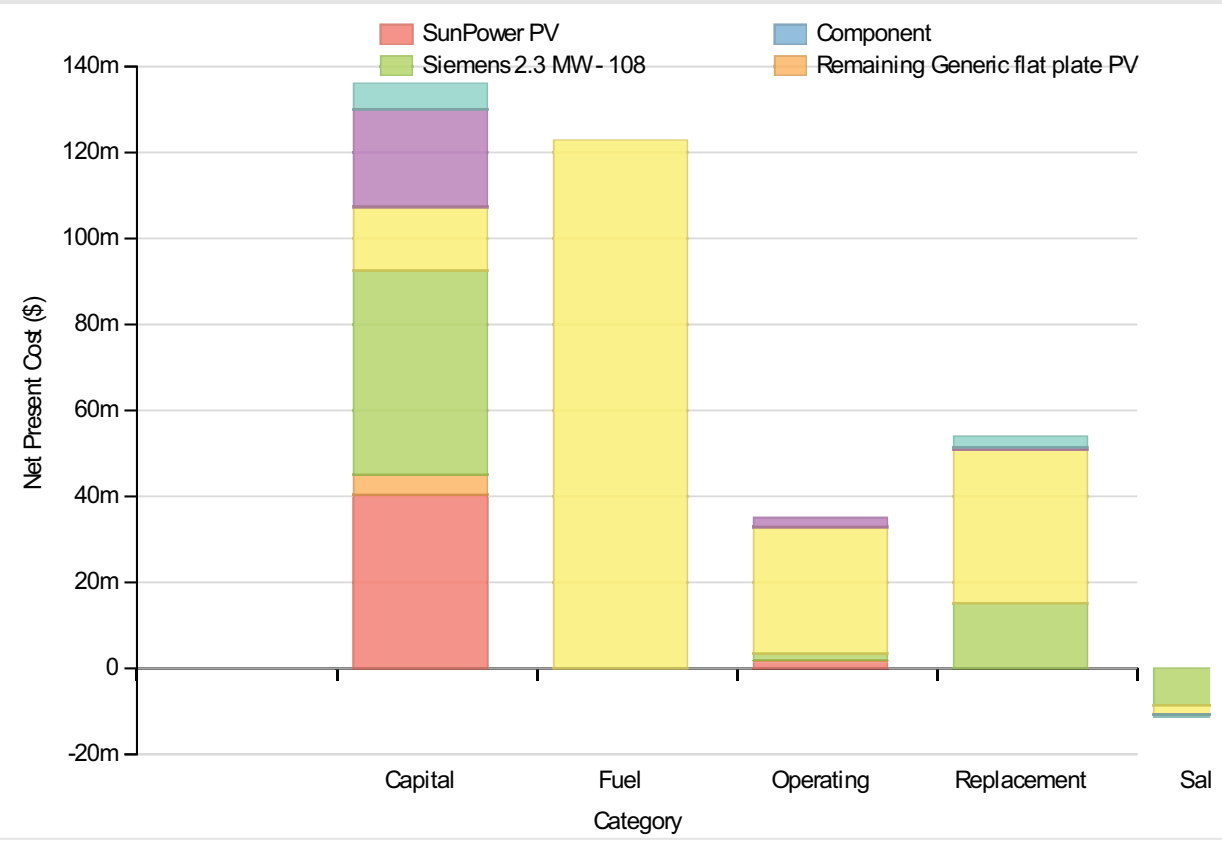

Cost Summary

Total net present cost

$336949504 \$$

Levelized cost of energy

$0.274 \$ / k W h$

\section{Net Present Costs}

\begin{tabular}{|l|r|r|r|r|r|r|}
\hline Component & Capital & Replacement & O\&M & Fuel & Salvage & Total \\
\hline SunPower PV & $40,358,332$ & 0 & $1,656,876$ & 0 & $42,015,208$ \\
\hline Remaining Generic flat plate PV & $4,678,459$ & 0 & 339,013 & 0 & $5,017,472$ \\
\hline Siemens 2.3 MW - 108 & $47,475,000$ & $15,135,363$ & $1,422,026$ & 0 & $-8,529,745$ & $55,502,644$ \\
\hline Kohler 3250 Prime Power & $14,821,428$ & $35,779,392$ & $29,482,028$ & $122,823,920$ & $-2,225,033$ \\
\hline GS200 flow & $22,721,244$ & 501,733 & $2,171,822$ & 0 & $-68,026$ \\
\hline Converter & $6,000,000$ & $2,545,641$ & $25,326,773$ \\
\hline
\end{tabular}




\section{Annualized Costs}

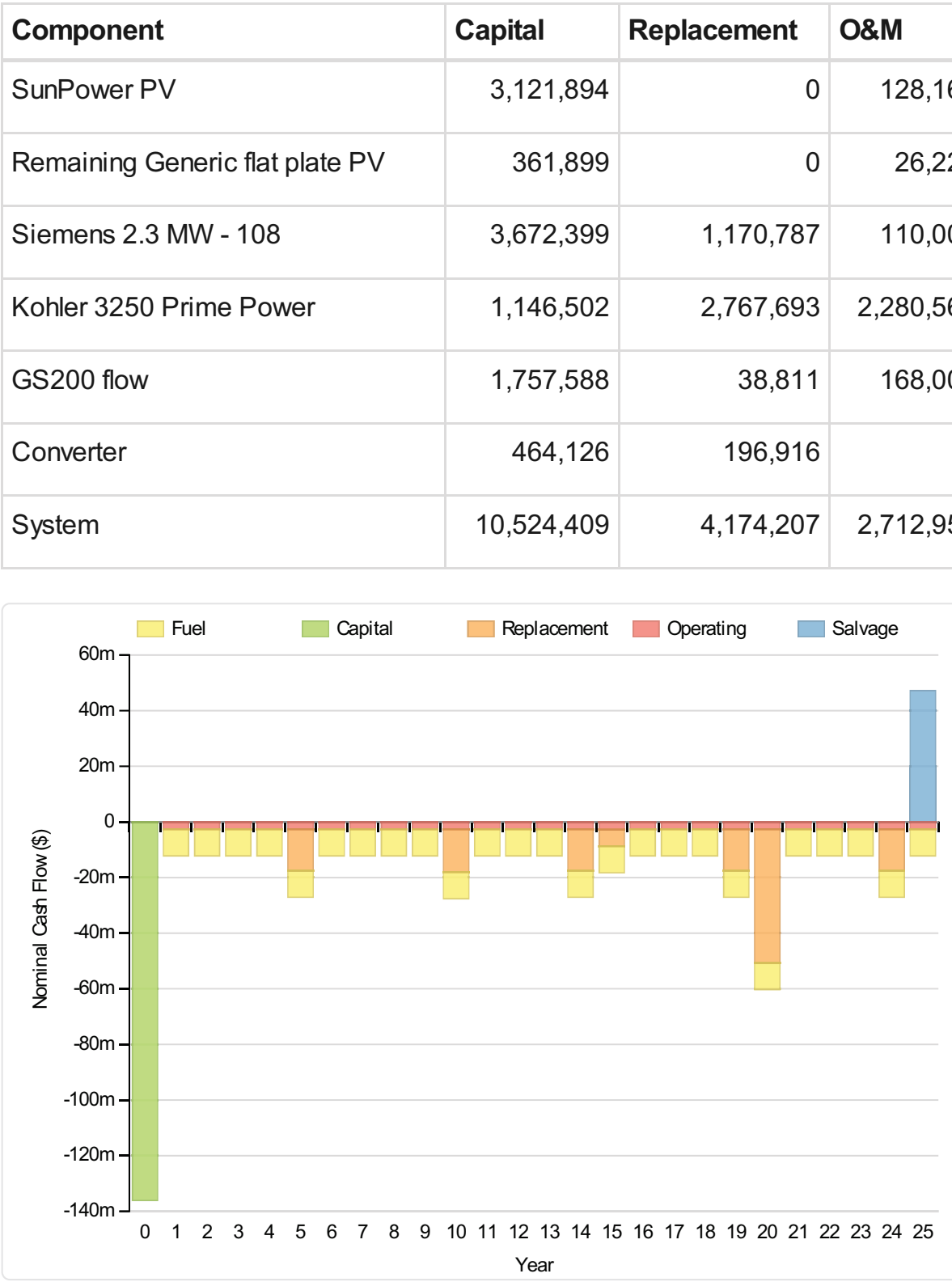

\section{Electrical}

\begin{tabular}{|l|r|r|}
\hline Quantity & Value & \multicolumn{1}{l|}{ Units } \\
\hline Excess electricity & 8300497 kWh/yr \\
\hline Unmet load & 0 kWh/yr \\
\hline Capacity shortage & & $625 \mathrm{kWh} / \mathrm{yr}$ \\
\hline Renewable fraction & Production(kWh/yr) & 0 \\
\hline Component & & Fraction (\%) \\
\hline PV & & \\
\hline PV & $26,935,180$ & 23 \\
\hline Generator & $2,766,526$ & \\
\hline Wind Turbine & $50,652,748$ & \\
\hline
\end{tabular}


Load

Consumption(kWh/yr)

Fraction (\%)

AC primary load

$95,052,456$

DC primary load

0

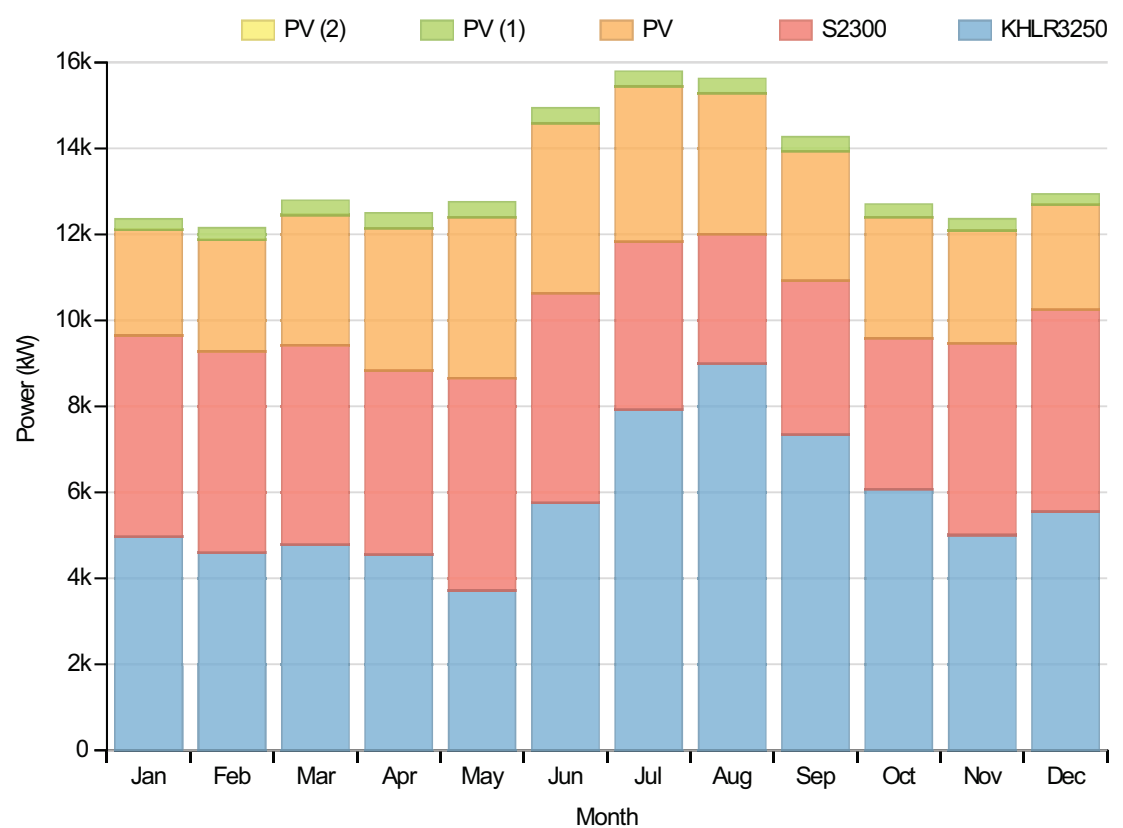

\section{PV:SunPower PV}

\section{Quantity}

Rated capacity

Mean output

Mean output

Capacity factor

Total production

Minimum output

Maximum output

PV penetration

Hours of operation

Levelized cost

\section{Value}

Units

13780 kW

3075 kW

$73795.00 \mathrm{kWh} / \mathrm{d}$

$22.31 \%$

$26935180 \mathrm{kWh} / \mathrm{yr}$

$0.00 \mathrm{~kW}$

$14957.00 \mathrm{~kW}$

$28.34 \%$

$4386 \mathrm{hrs} / \mathrm{yr}$

$0.109 \$ / k W h$ 


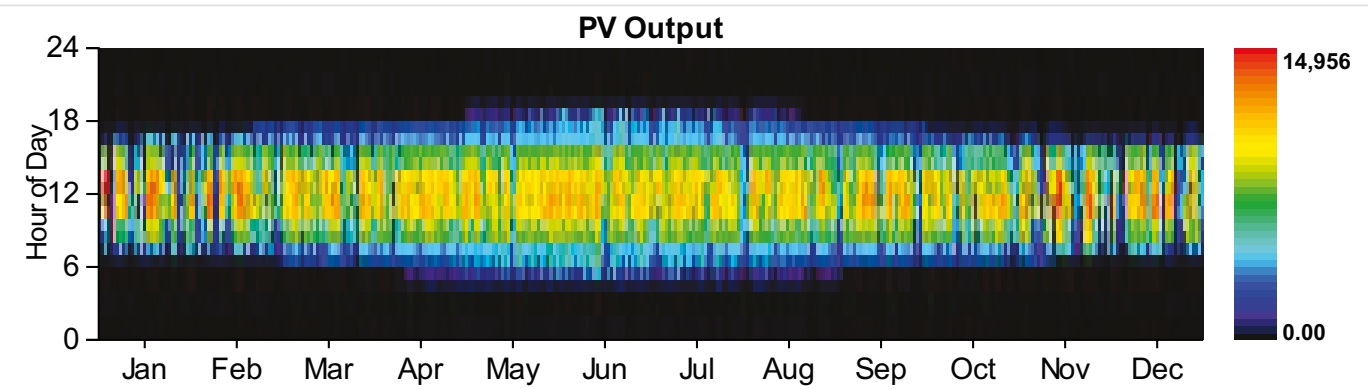

\section{PV:Remaining Generic flat plate PV}

\begin{tabular}{|l|r|l|}
\hline Quantity & Value & Units \\
\hline Rated capacity & $1547 \mathrm{~kW}$ \\
\hline Mean output & $316 \mathrm{~kW}$ \\
\hline Mean output & $7579.50 \mathrm{kWh} / \mathrm{d}$ \\
\hline Capacity factor & $20.42 \%$ \\
\hline Total production & $2766526 \mathrm{kWh} / \mathrm{yr}$ \\
\hline Minimum output & $0.00 \mathrm{~kW}$ \\
\hline Maximum output & $1565.00 \mathrm{~kW}$ \\
\hline PV penetration & $2.91 \%$ \\
\hline Hours of operation & $4386 \mathrm{hrs} / \mathrm{yr}$ \\
\hline Levelized cost & 0.013 \$/kWh \\
\hline
\end{tabular}

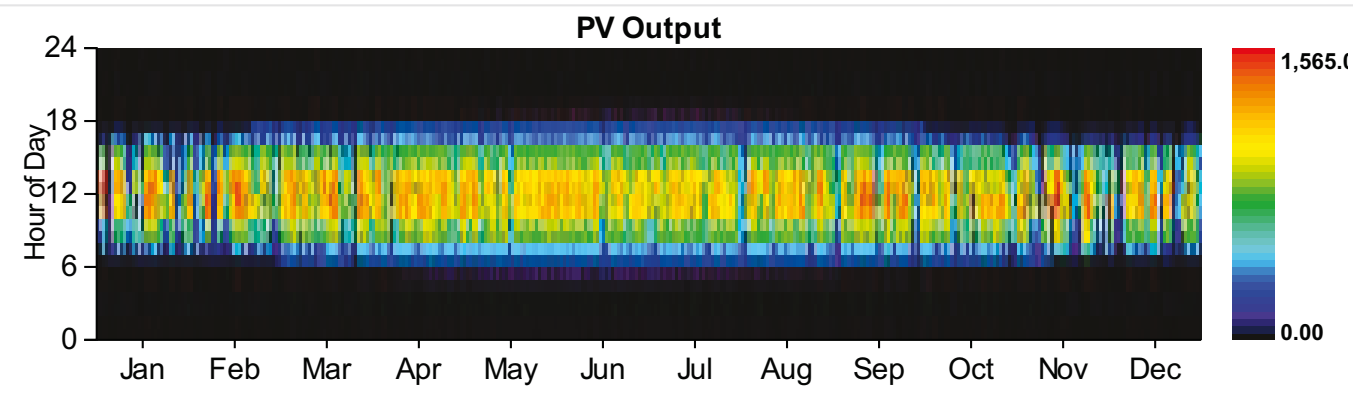

Wind Turbine:Siemens 2.3 MW - 108

\section{Quantity}

Total rated capacity

Mean output

Capacity factor

Total production

Minimum output

Maximum output
Value

Units

18400 kW

4269 kW

$23.20 \%$

37394812 kWh/yr

$3.95 \mathrm{~kW}$

18502.00 kW 
Quizhinetration

Hours of operation

Levelized cost
39.34 Units

$8760 \mathrm{hrs} / \mathrm{yr}$

$0.115 \$ / k W h$

\section{Generator:Kohler 3250 Prime Power}

\section{Quantity}

Hours of operation

Number of starts

Operational life

Fixed generation cost

Marginal generation cost

Electrical production

Mean electrical output

Min. electrical output

Max. electrical output

Fuel consumption

Specific fuel consumption

Fuel energy input

Mean electrical efficiency

\begin{tabular}{|r|l|l|}
\hline Value & Units \\
\hline 3224 & hrs/yr \\
1621 & starts/yr \\
5 & $\mathrm{yr}$ \\
\hline 1761.20 & $\$ / \mathrm{hr}$ \\
\hline 0.18 & $\$ / \mathrm{kWh}$ \\
\hline 50652748 & $\mathrm{kWh} / \mathrm{yr}$ \\
\hline 15711 & $\mathrm{~kW}$ \\
\hline 4200 & $\mathrm{~kW}$ \\
\hline 16800 & $\mathrm{~kW}$ \\
\hline 12026548 & $\mathrm{~L} / \mathrm{yr}$ \\
\hline 0.24 & $\mathrm{~L} / \mathrm{kWh}$ \\
\hline 118341248 & $\mathrm{kWh} / \mathrm{yr}$ \\
\hline 43 & $\%$ \\
\hline
\end{tabular}

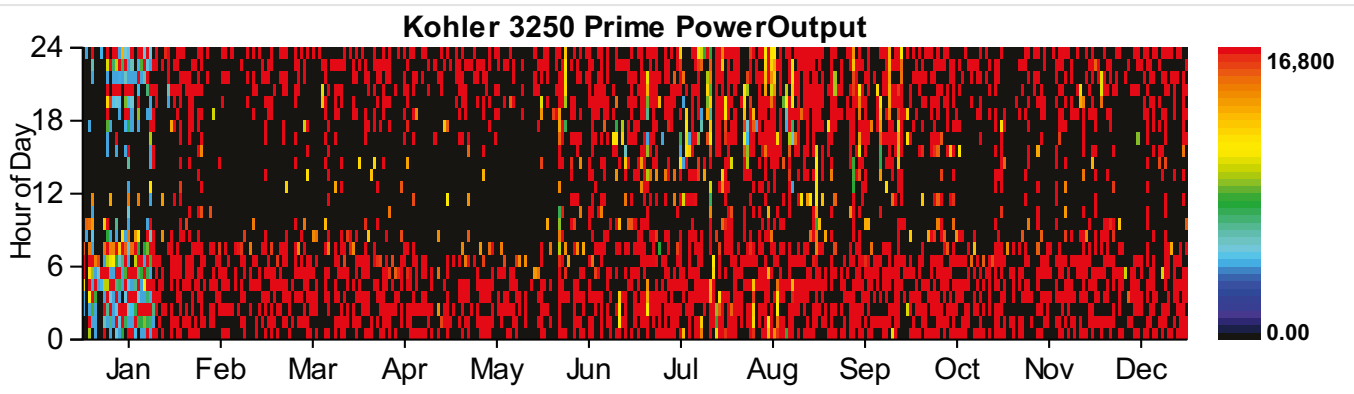

\section{Battery:GS200 flow}

\section{Quantity}

Value

String size

Strings in parallel

Batteries

Bus voltage 


\section{Quantity}

Nominal capacity

Usable nominal capacity

Autonomy

Lifetime throughput

Battery wear cost

Average energy cost

Energy in

Energy out

Storage depletion

Losses

Annual throughput

Expected life
Value

Units

42000 kWh

42000 kWh

$4 \mathrm{hr}$

0

$0.000 \$ / \mathrm{kWh}$

$0.189 \$ / k W h$

$26827478 \mathrm{kWh} / \mathrm{yr}$

$18808224 \mathrm{kWh} / \mathrm{yr}$

$34694 \mathrm{kWh} / \mathrm{yr}$

$7984560 \mathrm{kWh} / \mathrm{yr}$

$22480140 \mathrm{kWh} / \mathrm{yr}$

$25 \mathrm{yr}$

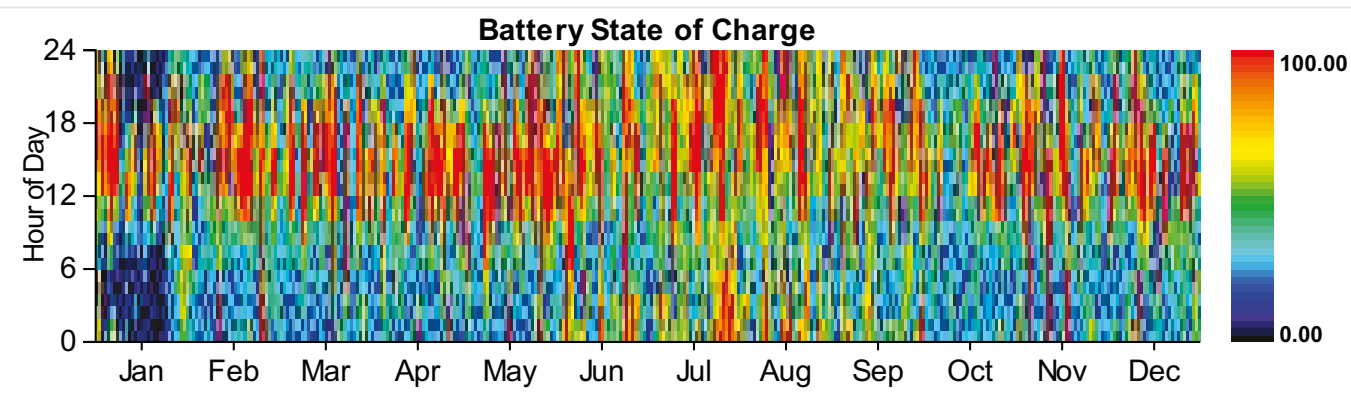

\section{Converter}

\begin{tabular}{|l|r|r|l|}
\hline Quantity & Inverter & Rectifier & Units \\
\hline Capacity & 20,000 & $18,000 \mathrm{~kW}$ \\
\hline Mean output & 3,518 & $1,910 \mathrm{~kW}$ \\
\hline Minimum output & 0 & $0 \mathrm{~kW}$ \\
\hline Maximum output & 17,970 & $12,550 \mathrm{~kW}$ \\
\hline Capacity factor & 18 & $10 \%$ \\
\hline Hours of operation & 4,697 & $3,337 \mathrm{hrs} / \mathrm{yr}$ \\
\hline Energy in & $34,241,788$ & $19,685,400 \mathrm{kWh} / \mathrm{yr}$ \\
\hline Energy out & $30,817,560$ & $16,732,604 \mathrm{kWh} / \mathrm{yr}$ \\
\hline Losses & $3,424,228$ & $2,952,796 \mathrm{kWh} / \mathrm{yr}$ \\
\hline
\end{tabular}



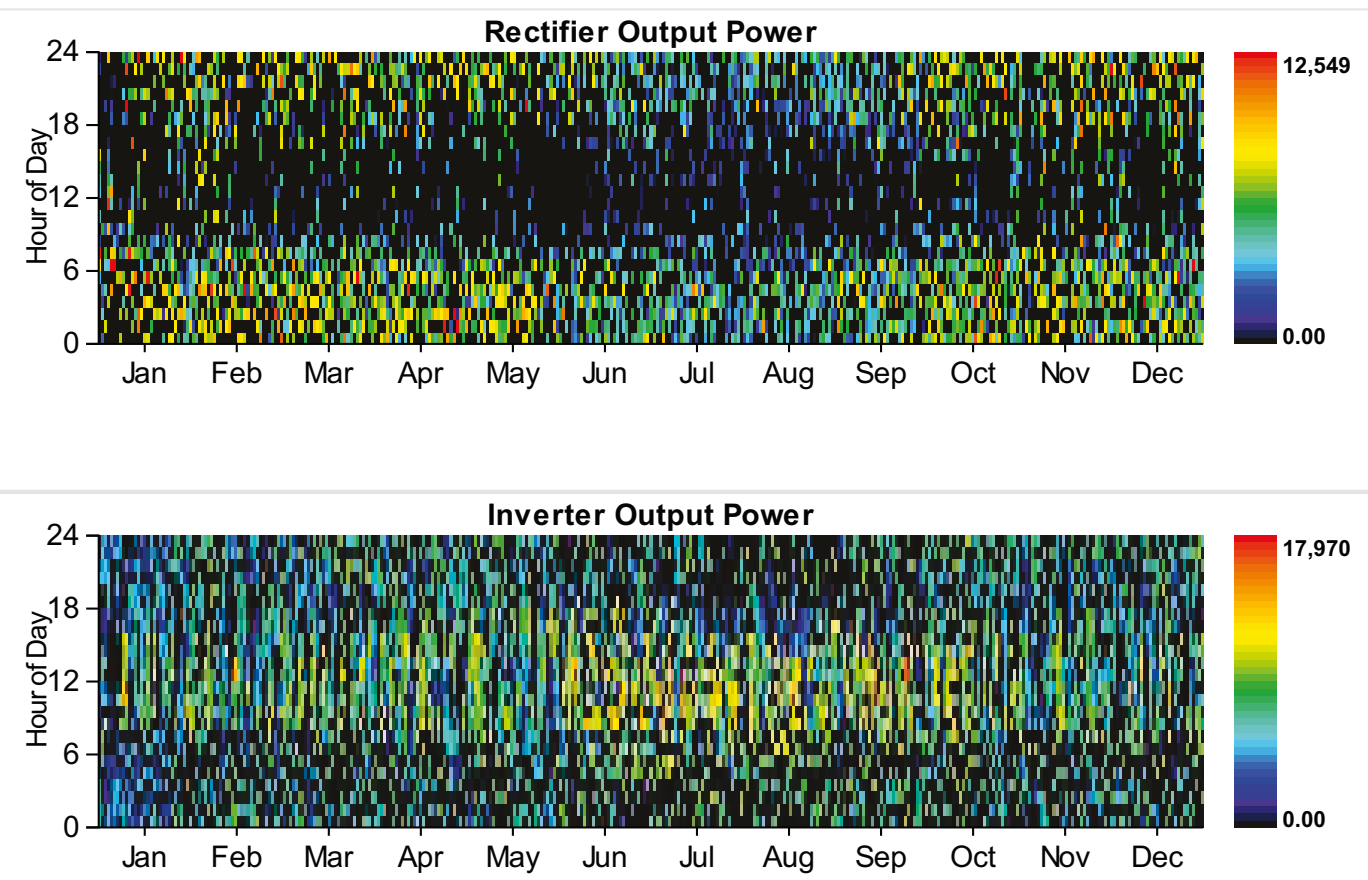

\section{Emissions}

\begin{tabular}{|l|r|}
\hline Pollutant & Emissions \\
\hline Carbon dioxide & $31563860 \mathrm{~kg} / \mathrm{yr}$ \\
\hline Carbon monoxide & $132292 \mathrm{~kg} / \mathrm{yr}$ \\
\hline Unburned hydrocarbons & $15153 \mathrm{~kg} / \mathrm{yr}$ \\
\hline Particulate matter & $3788 \mathrm{~kg} / \mathrm{yr}$ \\
\hline Sulfur dioxide & $64988 \mathrm{~kg} / \mathrm{yr}$ \\
\hline Nitrogen oxides & $132292 \mathrm{~kg} / \mathrm{yr}$ \\
\hline
\end{tabular}

HOMER Energy, LLC @ 2016 


\section{Appendix C - New London Scenarios}

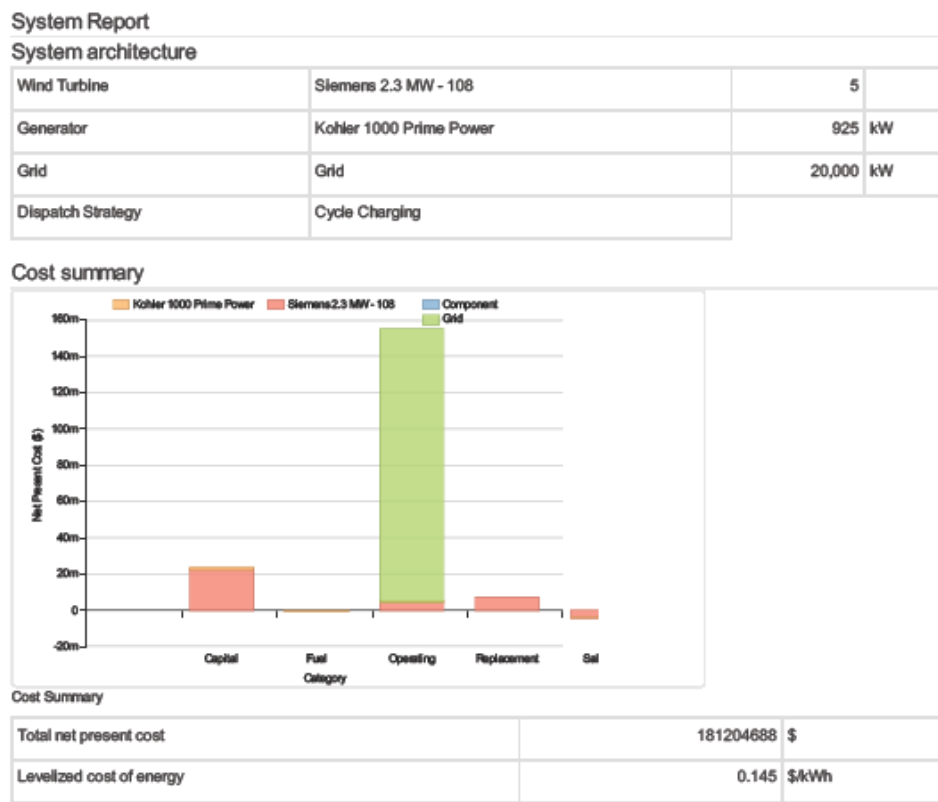

\begin{tabular}{|c|c|c|c|c|c|c|}
\hline Component & Capital & Replacement & Osm & Fuel & Salvage & Total \\
\hline Slemens $2.3 \mathrm{MW}$ - 108 & $22,500,000$ & $7,173,158$ & $4,847,816$ & 0 & $-4,042,534$ & $30,478,440$ \\
\hline Kohler 1000 Prime Power & 925,000 & 0 & 20,807 & 99,446 & $-178,750$ & 866,503 \\
\hline Grid & 0 & 0 & $149,859,664$ & 0 & $\mathbf{0}$ & $149,859,664$ \\
\hline System & $23,425,000$ & $7,173,158$ & $154,728,304$ & 99,446 & $-4,221,283$ & $181,204,625$ \\
\hline \multicolumn{7}{|l|}{ Annualized Costs } \\
\hline Component & Capital & Replacement & $08 \mathrm{~m}$ & Fuel & Salvage & Total \\
\hline Slemens $2.3 \mathrm{MW}$ - 108 & $1,740,474$ & 554,875 & 375,000 & 0 & $-312,708$ & $2,357,641$ \\
\hline Kohter 1000 Prime Power & 71,553 & & 1,610 & 7,693 & $-13,827$ & 67,028 \\
\hline Gind & 0 & 0 & $11,592,301$ & 0 & 0 & $11,592,301$ \\
\hline System & $1,812,026$ & 554,875 & $11,968,912$ & 7,693 & $-326,535$ & $14,016,971$ \\
\hline
\end{tabular}


System Report

System architecture

\begin{tabular}{|l|l|r|}
\hline Wind Turbine & Siemens 2.3 MW - 108 & 5 \\
\hline Generator & Kohler 1000 Prime Power & 925 \\
\hline Grid & Grid & 20,000 \\
\hline Dispatch Strategy & Cycle Charging & $\mathrm{kW}$ \\
\hline
\end{tabular}

\section{Cost summary}

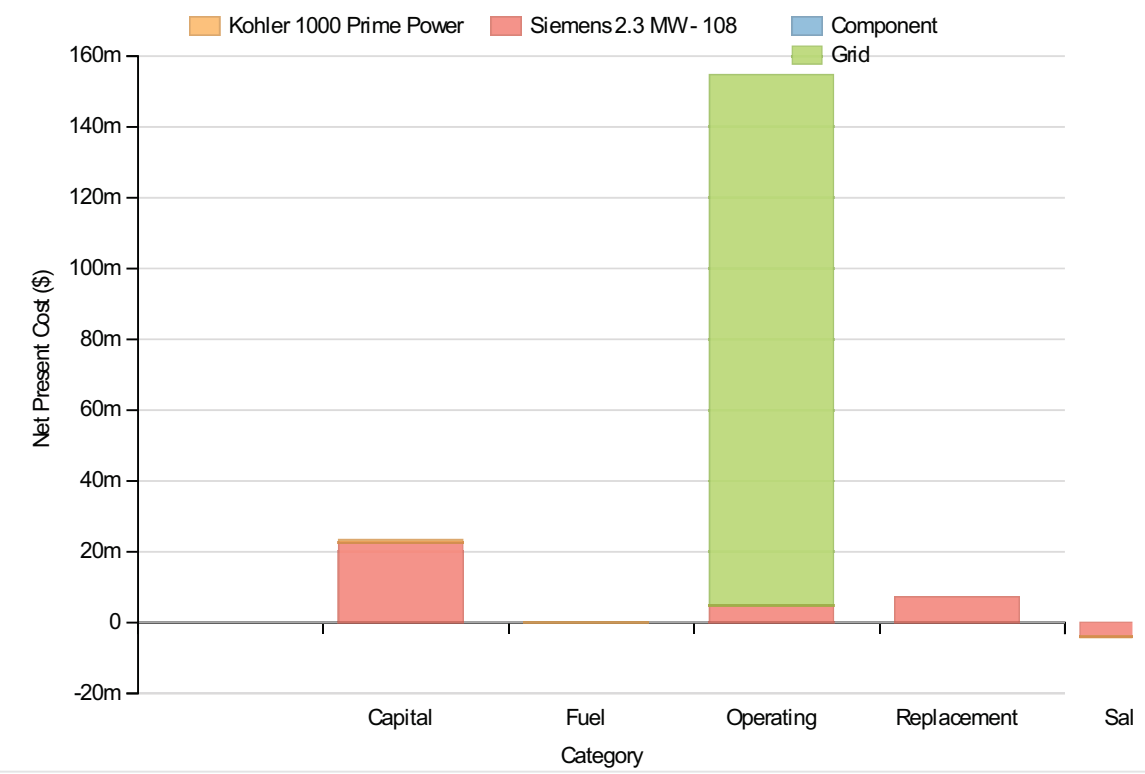

Cost Summary

Total net present cost

$181204688 \$$

Levelized cost of energy

$0.145 \$ / k W h$

\section{Net Present Costs}

\begin{tabular}{|l|r|r|r|r|r|r|}
\hline Component & Capital & Replacement & O\&M & Fuel & \multicolumn{1}{l|}{ Salvage } & \multicolumn{1}{l|}{ Total } \\
\hline Siemens 2.3 MW - 108 & $22,500,000$ & $7,173,158$ & $4,847,816$ & 0 & $-4,042,534$ & $30,478,440$ \\
\hline Kohler 1000 Prime Power & 925,000 & 0 & 20,807 & 99,446 & $-178,750$ & 866,503 \\
\hline Grid & 0 & 0 & $149,859,664$ & 0 & 0 \\
\hline System & $23,425,000$ & $7,173,158$ & $154,728,304$ & 99,446 & $-4,221,283$ & $181,204,625$ \\
\hline
\end{tabular}

Annualized Costs

\begin{tabular}{|l|r|r|r|r|r|r|}
\hline Component & Capital & Replacement & O\&M & Fuel & Salvage & Total \\
\hline Siemens 2.3 MW - 108 & $1,740,474$ & 554,875 & 375,000 & 0 & $-312,708$ & $2,357,641$ \\
\hline Kohler 1000 Prime Power & 71,553 & 0 & 1,610 & 7,693 & $-13,827$ & 67,028 \\
\hline Grid & 0 & 0 & $11,592,301$ & 0 & 0 \\
\hline System & $1,812,026$ & 554,875 & $11,968,912$ & 7,693 & $-326,535$ & $14,016,971$ \\
\hline
\end{tabular}




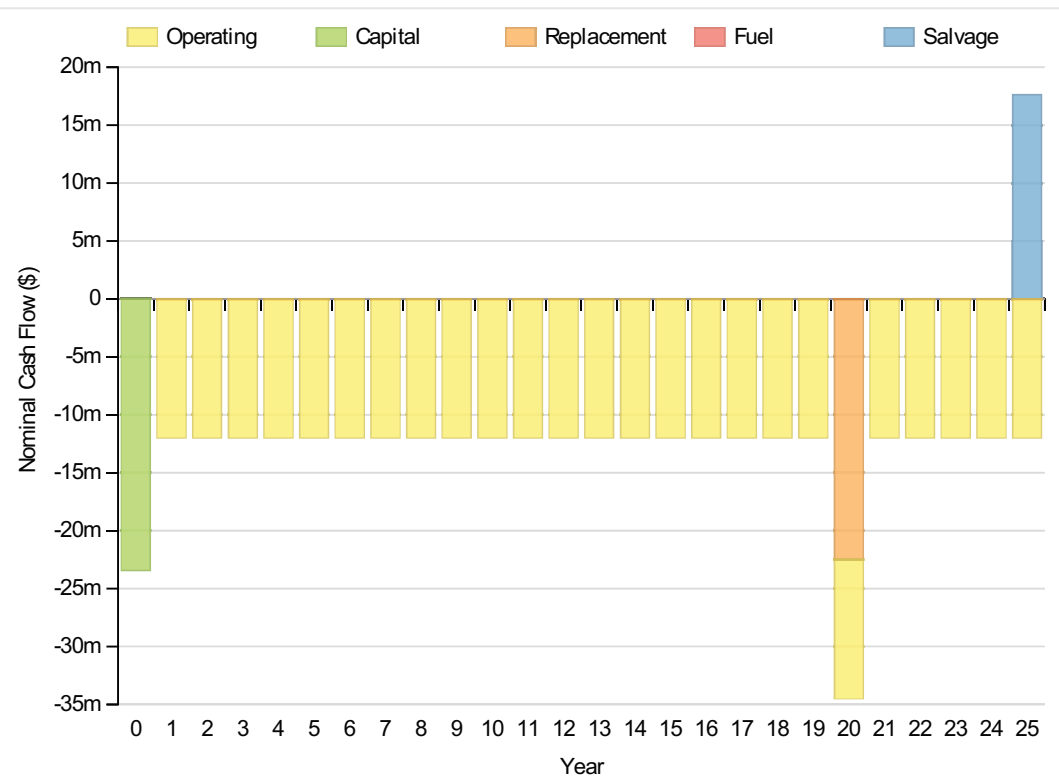

Electrical

\begin{tabular}{|c|c|c|c|c|}
\hline \multicolumn{2}{|l|}{ Quantity } & \multicolumn{2}{|l|}{ Value } & Units \\
\hline Excess electricity & & & 742167 & $\mathrm{kWh} / \mathrm{yr}$ \\
\hline Unmet load & & & 8504 & $\mathrm{kWh} / \mathrm{yr}$ \\
\hline Capacity shortage & & & 80731 & $\mathrm{kWh} / \mathrm{yr}$ \\
\hline Renewable fraction & & & 0 & \\
\hline Component & Production(kWh/yr) & & Fractio & $n(\%)$ \\
\hline Generator & & 33,608 & & 0 \\
\hline Wind Turbine & & $30,268,832$ & & 31 \\
\hline Grid Purchases & & $68,295,392$ & & 69 \\
\hline Total & & $98,597,832$ & & 100 \\
\hline Load & Consumption(kWh/yr) & & Fract & tion (\%) \\
\hline AC primary load & & $96,855,648$ & & 100 \\
\hline DC primary load & & 0 & 0 & 0 \\
\hline Total & & $96,855,648$ & & 100 \\
\hline
\end{tabular}




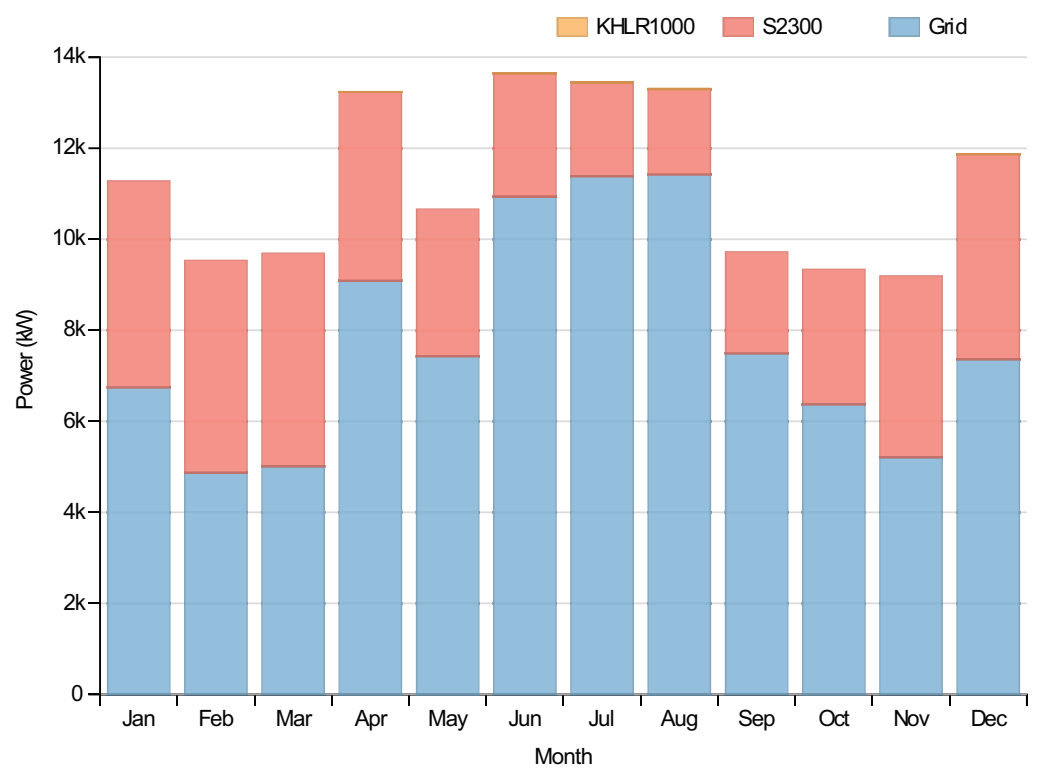

Wind Turbine:Siemens 2.3 MW - 108

\begin{tabular}{|c|c|c|}
\hline Quantity & Value & Units \\
\hline Total rated capacity & 11500 & $\mathrm{~kW}$ \\
\hline Mean output & 3455 & $\mathrm{~kW}$ \\
\hline Capacity factor & 30.05 & $\%$ \\
\hline Total production & 30268832 & $\mathrm{kWh} / \mathrm{yr}$ \\
\hline Minimum output & 7.19 & $\mathrm{~kW}$ \\
\hline Maximum output & 11574.00 & $\mathrm{~kW}$ \\
\hline Wind penetration & 31.25 & $\%$ \\
\hline Hours of operation & 8760 & $\mathrm{hrs} / \mathrm{yr}$ \\
\hline Levelized cost & 0.078 & $\$ / k W h$ \\
\hline
\end{tabular}

\section{Generator:Kohler 1000 Prime Power}

\begin{tabular}{|l|r|l}
\hline Quantity & Value & Units \\
\hline Hours of operation & 116 hrs/yr \\
\hline Number of starts & 108 starts/yr \\
\hline Operational life & $129 \mathrm{yr}$ \\
\hline Fixed generation cost & 76.24 & $\$ / \mathrm{hr}$ \\
\hline Marginal generation cost & 0.23 & $\$ / \mathrm{kWh}$ \\
\hline Electrical production & 33608 & $\mathrm{kWh} / \mathrm{yr}$ \\
\hline Mean electrical output & $290 \mathrm{~kW}$ \\
\hline Min. electrical output & 231 & $\mathrm{~kW}$ \\
\hline Max. electrical output & $925 \mathrm{~kW}$ \\
\hline Fuel consumption & $9738 \mathrm{~L} / \mathrm{yr}$ \\
\hline Specific fuel consumption & $0.29 \mathrm{~L} / \mathrm{kWh}$
\end{tabular}


Quantity

Fuel energy input

Mean electrical efficiency
95817 kWh/yr

$35 \%$

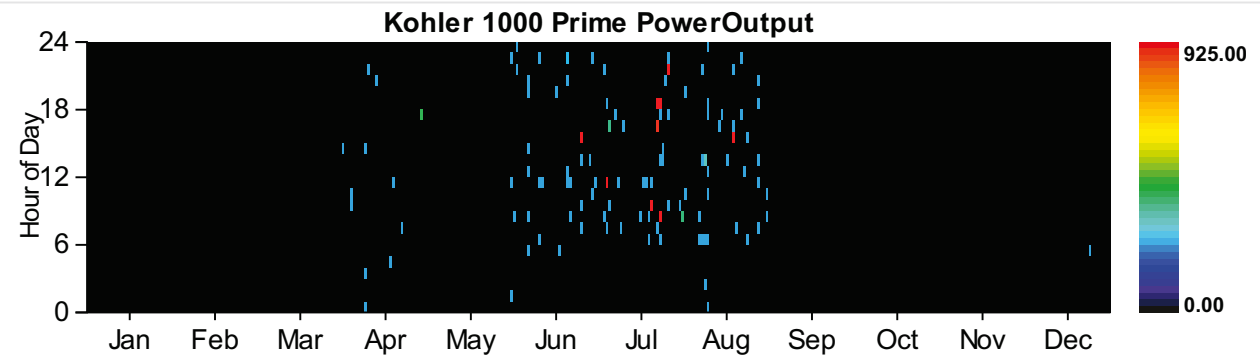

Grid

Rate: Demand 1

\begin{tabular}{|c|c|c|c|c|c|c|}
\hline Resources.ReportingService_GeneratelnputsReport_Month & $\begin{array}{l}\text { Energy } \\
\text { Purchased } \\
\text { (kWh) }\end{array}$ & $\begin{array}{l}\text { Energy } \\
\text { Sold } \\
\text { (kWh) }\end{array}$ & $\begin{array}{l}\text { Net } \\
\text { Purchases } \\
\text { (kWh) }\end{array}$ & $\begin{array}{l}\text { Peak } \\
\text { Demand } \\
(\mathbf{k W})\end{array}$ & $\begin{array}{l}\text { Energy } \\
\text { Charge } \\
\text { (\$) }\end{array}$ & $\begin{array}{l}\text { Demand } \\
\text { Charge } \\
\text { (\$) }\end{array}$ \\
\hline January & 0 & 0 & 0 & 18,095 & 0 & 289,519 \\
\hline February & 0 & 0 & 0 & 15,686 & 0 & 250,977 \\
\hline March & 0 & 0 & 0 & 16,699 & 0 & 267,178 \\
\hline April & 0 & 0 & 0 & 20,000 & 0 & 320,000 \\
\hline May & 0 & 0 & 0 & 17,784 & 0 & 284,539 \\
\hline June & 0 & 0 & 0 & 20,000 & 0 & 320,000 \\
\hline July & 0 & 0 & 0 & 20,000 & 0 & 320,000 \\
\hline August & 0 & 0 & 0 & 20,000 & 0 & 320,000 \\
\hline September & 0 & 0 & 0 & 15,890 & 0 & 254,236 \\
\hline October & 0 & 0 & 0 & 13,843 & 0 & 221,494 \\
\hline November & 0 & 0 & 0 & 15,876 & 0 & 254,021 \\
\hline December & 0 & 0 & 0 & 18,431 & 0 & 294,896 \\
\hline Annual & 0 & 0 & 0 & 20,000 & 0 & $3,396,860$ \\
\hline
\end{tabular}

Rate: Rate 1

\begin{tabular}{|c|c|c|c|c|c|c|}
\hline Resources.ReportingService_GeneratelnputsReport_Month & $\begin{array}{l}\text { Energy } \\
\text { Purchased } \\
\text { (kWh) }\end{array}$ & $\begin{array}{l}\text { Energy } \\
\text { Sold } \\
\text { (kWh) }\end{array}$ & $\begin{array}{l}\text { Net } \\
\text { Purchases } \\
\text { (kWh) }\end{array}$ & $\begin{array}{l}\text { Peak } \\
\text { Demand } \\
(\mathbf{k W})\end{array}$ & $\begin{array}{l}\text { Energy } \\
\text { Charge } \\
(\$)\end{array}$ & $\begin{array}{l}\text { Demand } \\
\text { Charge } \\
(\$)\end{array}$ \\
\hline January & $5,015,457$ & 0 & $5,015,457$ & 0 & 601,855 & 0 \\
\hline February & $3,272,783$ & 0 & $3,272,783$ & 0 & 392,734 & 0 \\
\hline March & $3,728,512$ & 0 & $3,728,512$ & 0 & 447,421 & 0 \\
\hline April & $6,542,809$ & 0 & $6,542,809$ & 0 & 785,137 & 0 \\
\hline May & $5,524,917$ & 0 & $5,524,917$ & 0 & 662,990 & 0 \\
\hline
\end{tabular}




\begin{tabular}{|c|c|c|c|c|c|c|c|}
\hline-9 & $\begin{array}{l}\text { En:®gy,156 } \\
\text { Purchased }\end{array}$ & $\begin{array}{l}\text { Energy } \\
\text { Sold }\end{array}$ & $\begin{array}{l}\text { Net,873,156 } \\
\text { Purchases }\end{array}$ & $\begin{array}{l}\text { Peak } \\
\text { Dema }\end{array}$ & & $\begin{array}{l}\text { Engragy/9 } \\
\text { Charge }\end{array}$ & $\begin{array}{l}\text { Demand } \\
\text { Charge }\end{array}$ \\
\hline Rassources.ReportingService_GeneratelnputsReport_Month & (k\$d,4) & $(\mathbf{k W h}) 0$ & $(\mathbf{k}, 4 \mathbf{4}) 9,009$ & $(\mathrm{~kW})$ & 0 & $\mathbf{k} \downarrow 16,281$ & (\$) 0 \\
\hline August & $8,498,816$ & 0 & $8,498,816$ & & 0 & $1,019,858$ & 0 \\
\hline September & $5,395,241$ & 0 & $5,395,241$ & & 0 & 647,429 & 0 \\
\hline October & $4,744,924$ & 0 & $4,744,924$ & & 0 & 569,391 & 0 \\
\hline November & $3,750,403$ & 0 & $3,750,403$ & & 0 & 450,048 & 0 \\
\hline December & $5,479,366$ & 0 & $5,479,366$ & & 0 & 657,524 & 0 \\
\hline Annual & $68,295,392$ & 0 & $68,295,392$ & & 0 & $8,195,448$ & 0 \\
\hline
\end{tabular}

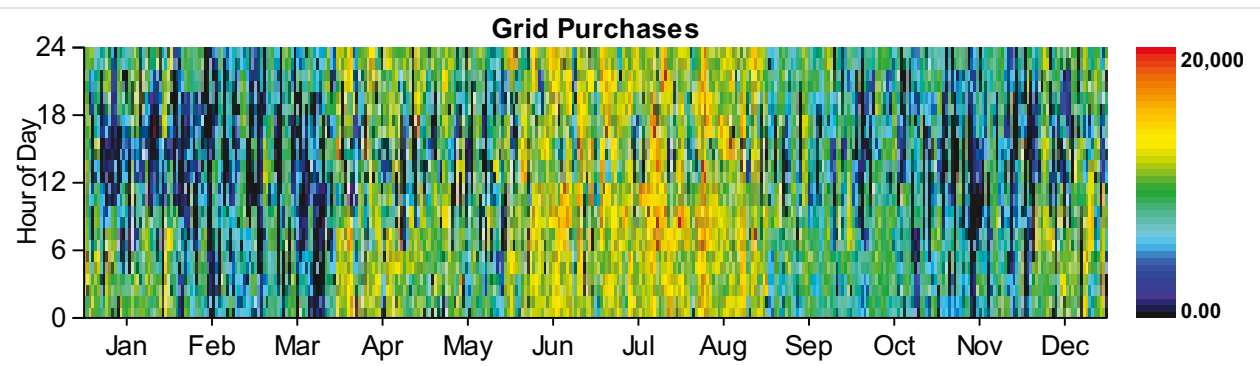

\section{Emissions}

\begin{tabular}{|l|r|l|}
\hline Pollutant & Emissions & Units \\
\hline Carbon dioxide & $43188244 \mathrm{~kg} / \mathrm{yr}$ \\
\hline Carbon monoxide & $107 \mathrm{~kg} / \mathrm{yr}$ \\
\hline Unburned hydrocarbons & $12 \mathrm{~kg} / \mathrm{yr}$ \\
\hline Particulate matter & $3 \mathrm{~kg} / \mathrm{yr}$ \\
\hline Sulfur dioxide & $187182 \mathrm{~kg} / \mathrm{yr}$ \\
\hline Nitrogen oxides & $91623 \mathrm{~kg} / \mathrm{yr}$ \\
\hline
\end{tabular}

HOMER Energy, LLC $\odot 2016$ 
System Report

System architecture

\begin{tabular}{|l|l|r|}
\hline Wind Turbine & Siemens 2.3 MW - 108 & 5 \\
\hline Generator \#2 & Kohler 750 Prime Power & $690 \mathrm{~kW}$ \\
\hline Battery & GS200 flow & $1 \mathrm{strings}$ \\
\hline Converter & System Converter & $250 \mathrm{~kW}$ \\
\hline Grid & Grid & $20,000 \mathrm{~kW}$ \\
\hline Dispatch Strategy & Cycle Charging & \\
\hline
\end{tabular}

Cost summary

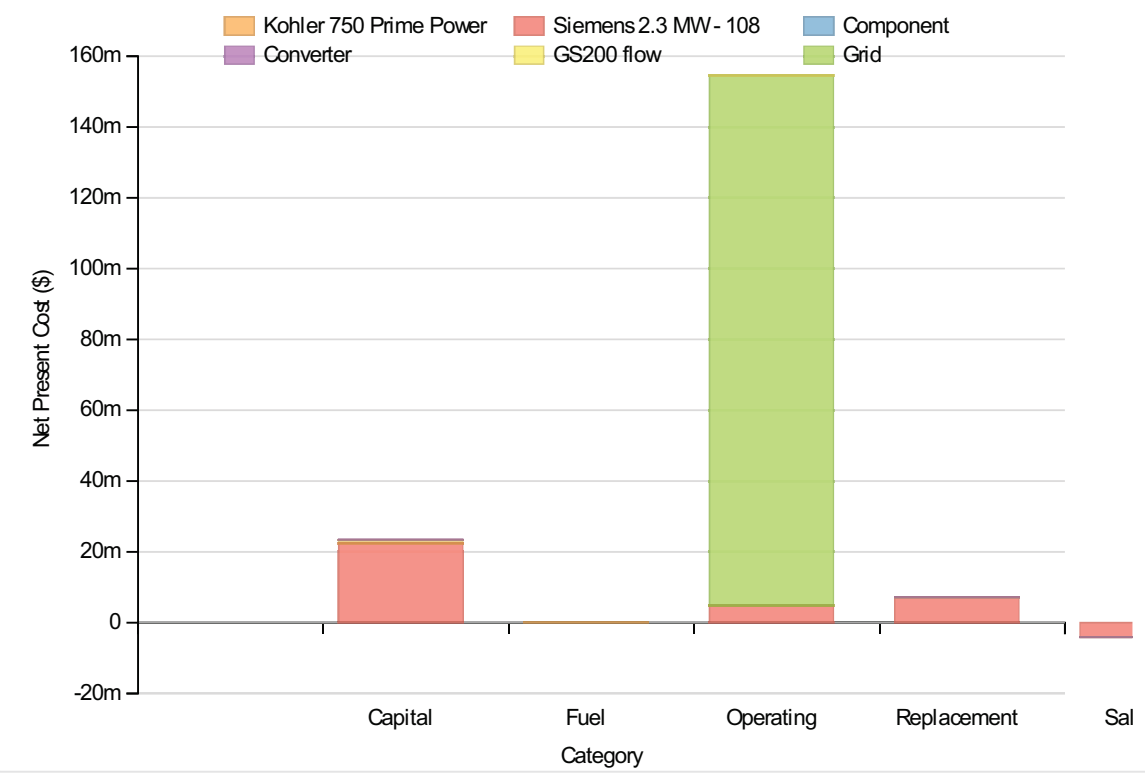

Cost Summary

Total net present cost

$181403424 \$$

Levelized cost of energy

$0.145 \$ / k W h$

\section{Net Present Costs}

\begin{tabular}{|l|r|r|r|r|r|r|}
\hline Component & Capital & Replacement & O\&M & Fuel & Salvage & \multicolumn{1}{l|}{ Total } \\
\hline Siemens 2.3 MW - 108 & $22,500,000$ & $7,173,158$ & $4,847,816$ & 0 & $-4,042,534$ & $30,478,440$ \\
\hline Kohler 750 Prime Power & 690,000 & 0 & 15,387 & 139,257 & $-133,613$ & 711,031 \\
\hline Grid & 0 & 0 & $149,751,216$ & 0 & $149,751,216$ \\
\hline GS200 flow & 324,589 & 7,176 & 31,026 & 0 & -973 \\
\hline Converter & 75,000 & 31,821 & 0 & 0 & $-5,989$ \\
\hline System & $23,589,588$ & $7,212,154$ & $154,645,456$ & 139,257 & $-4,183,109$ \\
\hline
\end{tabular}

Annualized Costs

\begin{tabular}{|l|r|r|r|r|r|r|}
\hline Component & Capital & Replacement & O\&M & Fuel & Salvage & Total \\
\hline Siemens 2.3 MW - 108 & $1,740,474$ & 554,875 & 375,000 & 0 & $-312,708$ & $2,357,641$ \\
\hline
\end{tabular}




\begin{tabular}{|c|c|c|c|c|c|c|}
\hline 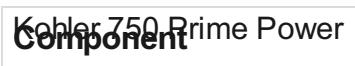 & Capita $\lceil 3,375$ & Replacement 0 & O\&M & Fuê $\rho, 772$ & Salvabe336 & Total \\
\hline Grid & 0 & 0 & $11,583,912$ & 0 & 0 & $11,583,912$ \\
\hline GS200 flow & 25,108 & 555 & 2,400 & 0 & -75 & 27,988 \\
\hline Converter & 5,802 & 2,462 & 0 & 0 & -463 & 7,800 \\
\hline System & $1,824,758$ & 557,892 & $11,962,503$ & 10,772 & $-323,582$ & $14,032,343$ \\
\hline
\end{tabular}

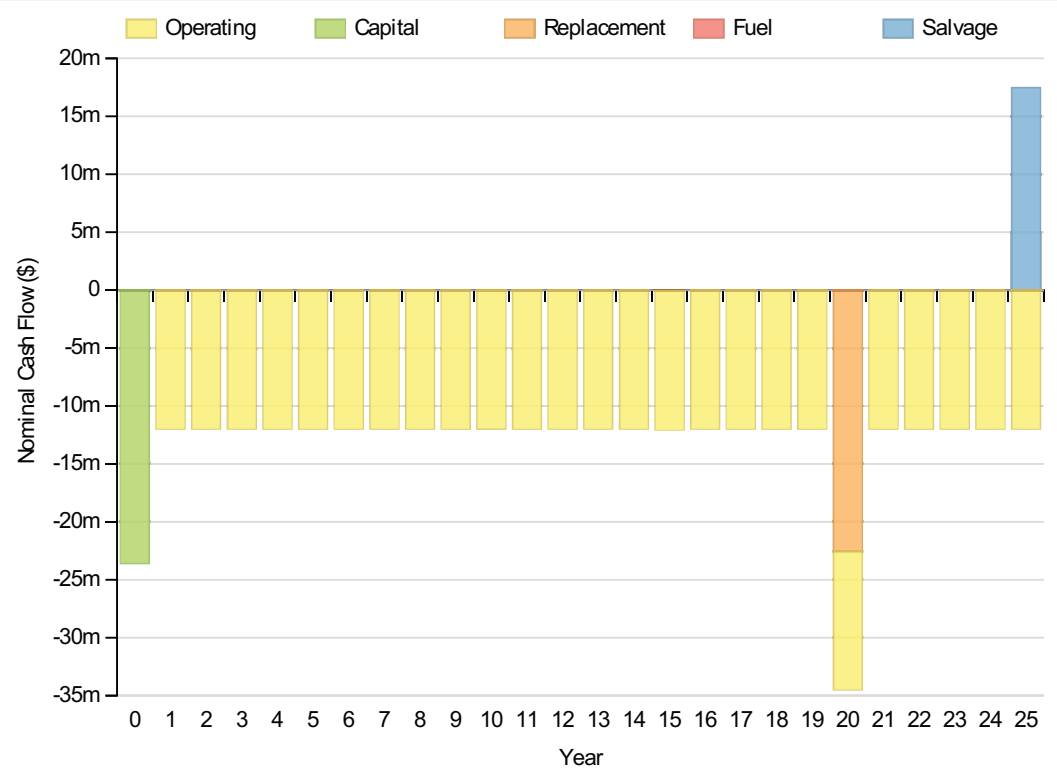

\section{Electrical}

\section{Quantity}

Excess electricity

Unmet load

Capacity shortage

Renewable fraction

\section{Component}

Generator

Wind Turbine

Grid Purchases

Total
Value

Production(kWh/yr)

Consumption(kWh/yr)
Units

$1607580 \mathrm{kWh} / \mathrm{yr}$

$10486 \mathrm{kWh} / \mathrm{yr}$

96779 kWh/yr

Load

$96,853,672$

100

AC primary load

$30,268,832$

$68,217,656$

DC primary load

0 


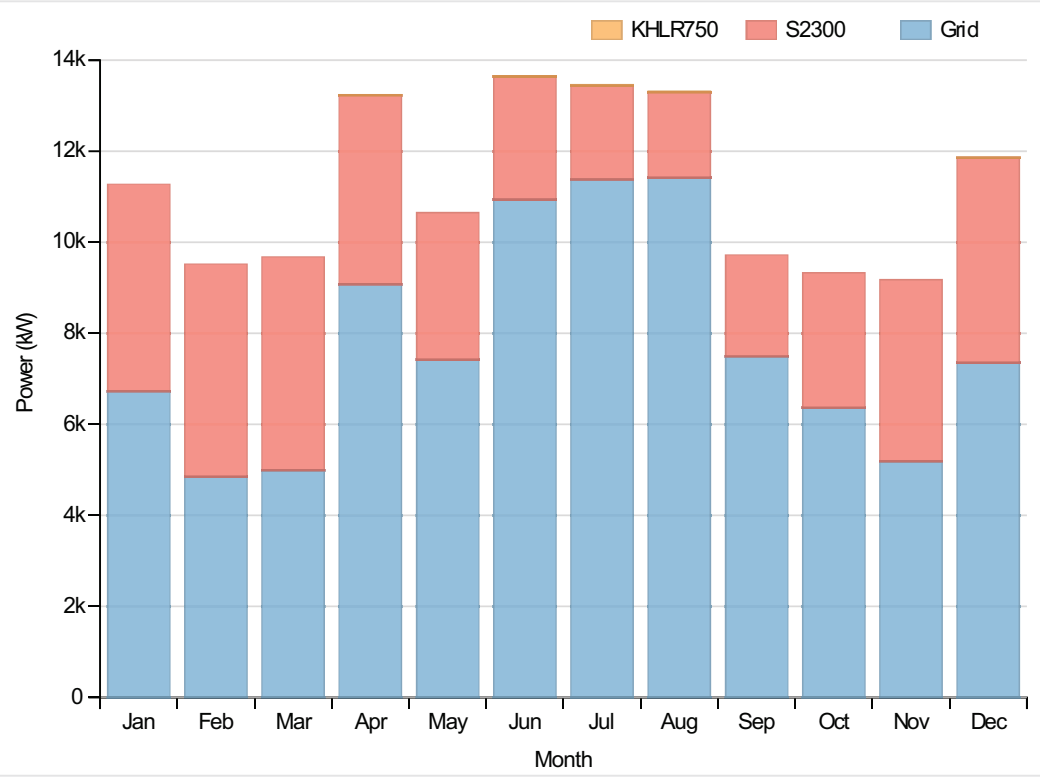

Wind Turbine:Siemens 2.3 MW - 108

\begin{tabular}{|c|c|c|}
\hline Quantity & Value & Units \\
\hline Total rated capacity & 11500 & $\mathrm{~kW}$ \\
\hline Mean output & 3455 & $\mathrm{~kW}$ \\
\hline Capacity factor & 30.05 & $\%$ \\
\hline Total production & 30268832 & $\mathrm{kWh} / \mathrm{yr}$ \\
\hline Minimum output & 7.19 & $\mathrm{~kW}$ \\
\hline Maximum output & 11574.00 & $\mathrm{~kW}$ \\
\hline Wind penetration & 31.25 & $\%$ \\
\hline Hours of operation & 8760 & $\mathrm{hrs} / \mathrm{yr}$ \\
\hline Levelized cost & 0.078 & $\$ / k W h$ \\
\hline
\end{tabular}

\section{Generator:Kohler 750 Prime Power}

\begin{tabular}{|l|r|l|}
\hline Quantity & Value & Units \\
\hline Hours of operation & 115 hrs/yr \\
\hline Number of starts & 108 starts/yr \\
\hline Operational life & $130 \mathrm{yr}$ \\
\hline Fixed generation cost & 56.87 & $\$ / \mathrm{hr}$ \\
\hline Marginal generation cost & 0.23 & $\$ / \mathrm{kWh}$ \\
\hline Electrical production & $46728 \mathrm{kWh} / \mathrm{yr}$ \\
\hline Mean electrical output & $406 \mathrm{~kW}$ \\
\hline Min. electrical output & $173 \mathrm{~kW}$ \\
\hline Max. electrical output & $690 \mathrm{~kW}$ \\
\hline Fuel consumption & 13636 & $\mathrm{~L} / \mathrm{yr}$ \\
\hline Specific fuel consumption & $0.29 \mathrm{~L} / \mathrm{kWh}$ \\
\hline
\end{tabular}




\begin{tabular}{|l|r|l|}
\hline Putantityrgy input & Value & 134175 \\
\hline Mean electrical efficiency & $35 \%$ \\
\hline
\end{tabular}

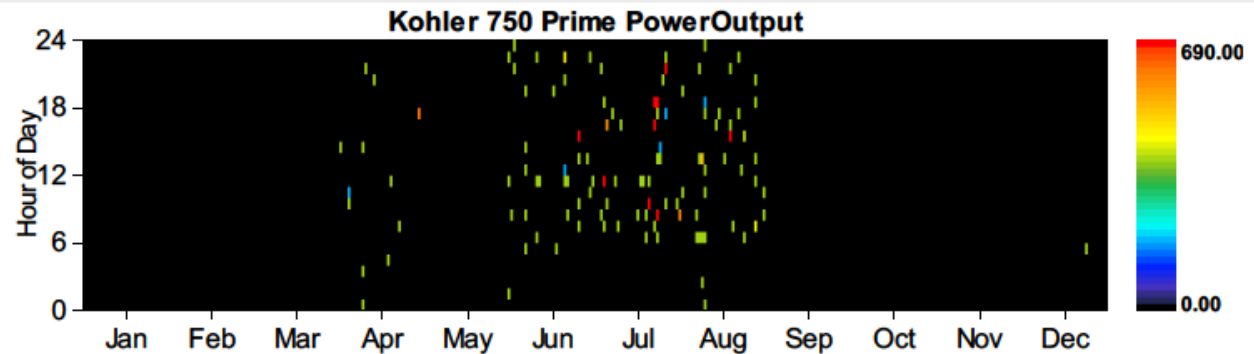

\section{Battery:GS200 flow}

\begin{tabular}{|l|r|}
\hline Quantity & Value \\
\hline String size & 1 \\
\hline Strings in parallel & 1 \\
\hline Batteries & 1 \\
\hline Bus voltage & 100 \\
\hline
\end{tabular}

\begin{tabular}{|c|c|c|}
\hline Quantity & Value & Units \\
\hline Nominal capacity & 600 & kWh \\
\hline Usable nominal capacity & 600 & kWh \\
\hline Autonomy & 0 & $\mathrm{hr}$ \\
\hline Lifetime throughput & 0 & \\
\hline Battery wear cost & 0.000 & $\$ / \mathrm{kWh}$ \\
\hline Average energy cost & 0.035 & $\$ / k W h$ \\
\hline Energy in & 132507 & kWh/yr \\
\hline Energy out & 93257 & $\mathrm{kWh} / \mathrm{yr}$ \\
\hline Storage depletion & 600 & $\mathrm{kWh} / \mathrm{yr}$ \\
\hline Losses & 38649 & kWh/yr \\
\hline Annual throughput & 111464 & $\mathrm{kWh} / \mathrm{yr}$ \\
\hline Expected life & 25 & $\mathrm{yr}$ \\
\hline
\end{tabular}

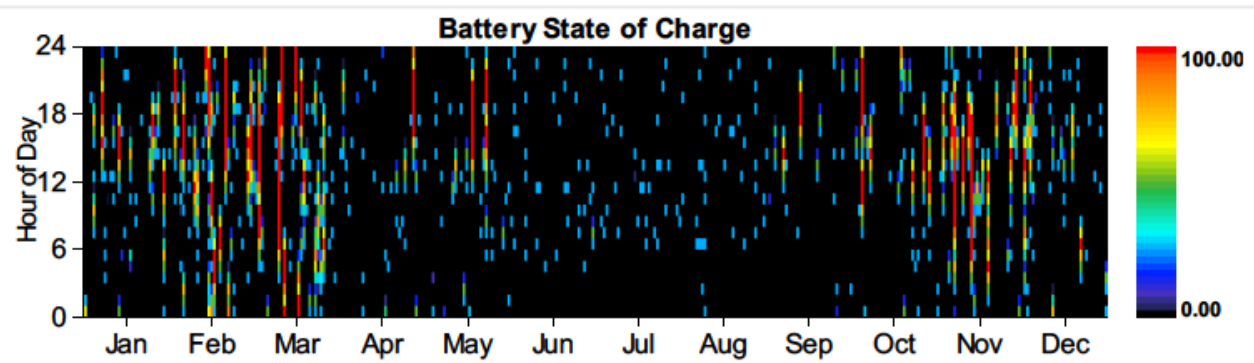


Converter

\begin{tabular}{|c|c|c|c|}
\hline Quantity & Inverter & Rectifier & Units \\
\hline Capacity & 250 & 225 & kW \\
\hline Mean output & 10 & 15 & $\mathrm{~kW}$ \\
\hline Minimum output & 0 & 0 & $\mathrm{~kW}$ \\
\hline Maximum output & 166 & 179 & $\mathrm{~kW}$ \\
\hline Capacity factor & 4 & 6 & $\%$ \\
\hline Hours of operation & 872 & 1,014 & $\mathrm{hrs} / \mathrm{yr}$ \\
\hline Energy in & 93,257 & 155,891 & $\mathrm{kWh} / \mathrm{yr}$ \\
\hline Energy out & 83,931 & 132,507 & $\mathrm{kWh} / \mathrm{yr}$ \\
\hline Losses & 9,326 & 23,384 & $\mathrm{kWh} / \mathrm{yr}$ \\
\hline
\end{tabular}
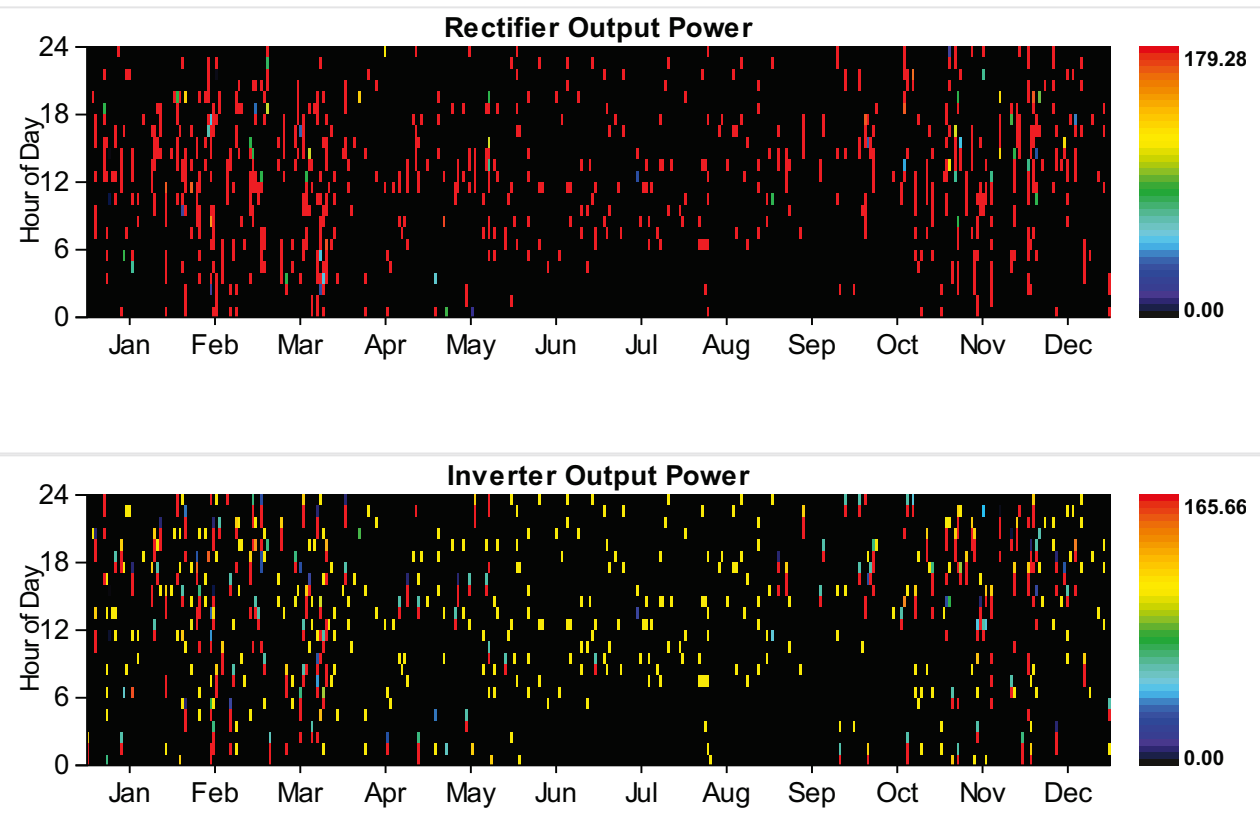

\section{Grid}

Rate: Demand 1

\begin{tabular}{|c|c|c|c|c|c|c|}
\hline Resources.ReportingService_GeneratelnputsReport_Month & $\begin{array}{l}\text { Energy } \\
\text { Purchased } \\
\text { (kWh) }\end{array}$ & $\begin{array}{l}\text { Energy } \\
\text { Sold } \\
\text { (kWh) }\end{array}$ & $\begin{array}{l}\text { Net } \\
\text { Purchases } \\
\text { (kWh) }\end{array}$ & $\begin{array}{l}\text { Peak } \\
\text { Demand } \\
(\mathbf{k W})\end{array}$ & $\begin{array}{l}\text { Energy } \\
\text { Charge } \\
\text { (\$) }\end{array}$ & $\begin{array}{l}\text { Demand } \\
\text { Charge } \\
(\$)\end{array}$ \\
\hline January & 0 & 0 & 0 & 18,095 & 0 & 289,519 \\
\hline February & 0 & 0 & 0 & 15,686 & 0 & 250,977 \\
\hline March & 0 & 0 & 0 & 16,699 & 0 & 267,178 \\
\hline April & 0 & 0 & 0 & 20,000 & 0 & 320,000 \\
\hline May & 0 & 0 & 0 & 17,784 & 0 & 284,539 \\
\hline June & 0 & 0 & 0 & 20,000 & 0 & 320,000 \\
\hline July & 0 & 0 & 0 & 20,000 & 0 & 320,000 \\
\hline August & 0 & 0 & 0 & 20,000 & 0 & 320,000 \\
\hline
\end{tabular}




\begin{tabular}{|c|c|c|c|c|c|c|}
\hline Retswarces.ReportingService_GeneratelnputsReport_Month & $\begin{array}{l}\text { Energy } \\
\text { Purchased } \\
\text { (kWh) }\end{array}$ & $\begin{array}{l}\text { Energy } \\
\text { Sold } 0 \\
(\mathbf{k W h})_{0}\end{array}$ & $\begin{array}{l}\text { Net } \\
\text { Purchases } \\
\text { (kWh) }\end{array}$ & $\begin{array}{l}\text { Peak } \\
15,899 \\
\text { Demañ } \\
\text { (kW) } \\
13,843\end{array}$ & $\begin{array}{l}\text { Energy } \\
\text { Charge } \\
(\$) \quad 0\end{array}$ & $\begin{array}{l}\text { Demand } \\
\text { Ch54,236 } \\
\text { Charge } \\
\text { (\$) } \\
{ }_{221,494}\end{array}$ \\
\hline November & 0 & 0 & 0 & 15,876 & 0 & 254,021 \\
\hline December & 0 & 0 & 0 & 18,490 & 0 & 295,836 \\
\hline Annual & 0 & 0 & 0 & 20,000 & 0 & $3,397,800$ \\
\hline
\end{tabular}

Rate: Rate 1

\begin{tabular}{|c|c|c|c|c|c|c|}
\hline Resources.ReportingService_GeneratelnputsReport_Month & $\begin{array}{l}\text { Energy } \\
\text { Purchased } \\
\text { (kWh) }\end{array}$ & $\begin{array}{l}\text { Energy } \\
\text { Sold } \\
(\mathbf{k W h})\end{array}$ & $\begin{array}{l}\text { Net } \\
\text { Purchases } \\
\text { (kWh) }\end{array}$ & $\begin{array}{l}\text { Peak } \\
\text { Demand } \\
(\mathbf{k W})\end{array}$ & $\begin{array}{l}\text { Energy } \\
\text { Charge } \\
(\$)\end{array}$ & $\begin{array}{l}\text { Demand } \\
\text { Charge } \\
(\$)\end{array}$ \\
\hline January & $5,007,178$ & 0 & $5,007,178$ & 0 & 600,861 & 0 \\
\hline February & $3,260,560$ & 0 & $3,260,560$ & 0 & 391,267 & 0 \\
\hline March & $3,715,573$ & 0 & $3,715,573$ & 0 & 445,869 & 0 \\
\hline April & $6,538,779$ & 0 & $6,538,779$ & 0 & 784,653 & 0 \\
\hline May & $5,519,575$ & 0 & $5,519,575$ & 0 & 662,349 & 0 \\
\hline June & $7,870,852$ & 0 & $7,870,852$ & 0 & 944,502 & 0 \\
\hline July & $8,467,073$ & 0 & $8,467,073$ & 0 & $1,016,049$ & 0 \\
\hline August & $8,496,675$ & 0 & $8,496,675$ & 0 & $1,019,601$ & 0 \\
\hline September & $5,392,027$ & 0 & $5,392,027$ & 0 & 647,043 & 0 \\
\hline October & $4,738,349$ & 0 & $4,738,349$ & 0 & 568,602 & 0 \\
\hline November & $3,739,770$ & 0 & $3,739,770$ & 0 & 448,772 & 0 \\
\hline December & $5,471,249$ & 0 & $5,471,249$ & 0 & 656,550 & 0 \\
\hline Annual & $68,217,656$ & 0 & $68,217,656$ & 0 & $8,186,120$ & 0 \\
\hline
\end{tabular}

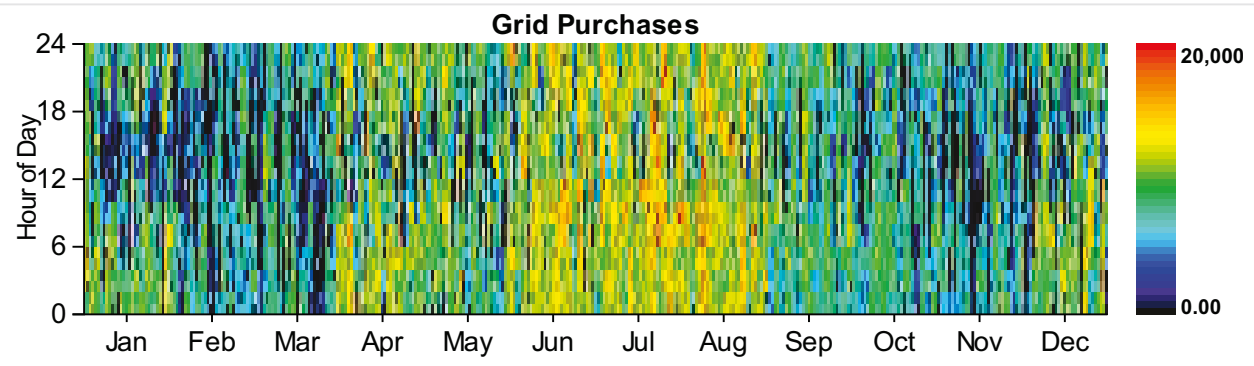

\section{Emissions}

\section{Pollutant}

Carbon dioxide

Carbon monoxide

Unburned hydrocarbons

Particulate matter

\section{Emissions}

Units

$43149348 \mathrm{~kg} / \mathrm{yr}$

$150 \mathrm{~kg} / \mathrm{yr}$

$17 \mathrm{~kg} / \mathrm{yr}$

$4 \mathrm{~kg} / \mathrm{yr}$ 
HOMER Energy, LLC @ 2016 
System Report

System architecture

\begin{tabular}{|l|l|r|}
\hline Wind Turbine & Siemens 2.3 MW - 108 & 5 \\
\hline Generator \#2 & Kohler 750 Prime Power & 1,380 \\
\hline Grid & GW \\
\hline Dispatch Strategy & Cycle Charging & 20,000 \\
\hline
\end{tabular}

\section{Cost summary}

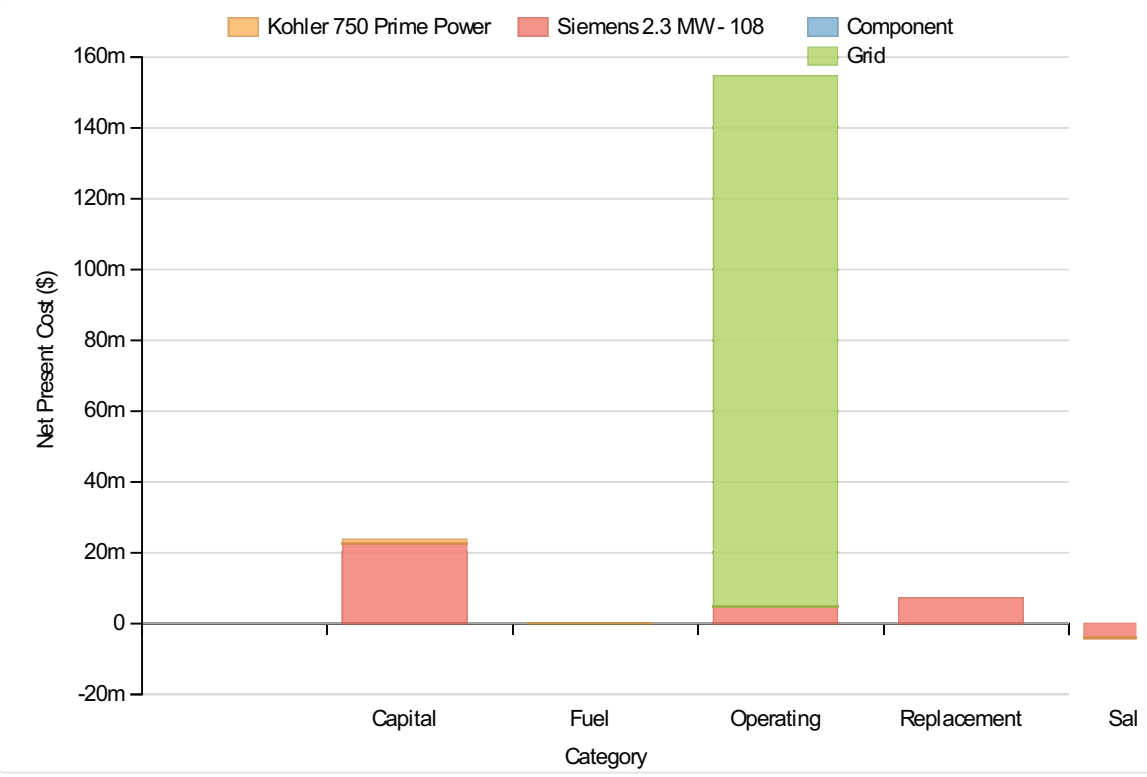

Cost Summary

Total net present cost

$181509216 \$$

Levelized cost of energy

$0.145 \$ / k W h$

\section{Net Present Costs}

\begin{tabular}{|l|r|r|r|r|r|r|}
\hline Component & Capital & Replacement & O\&M & Fuel & Salvage & \multicolumn{1}{l|}{ Total } \\
\hline Siemens 2.3 MW - 108 & $22,500,000$ & $7,173,158$ & $4,847,816$ & 0 & $-4,042,534$ & $30,478,440$ \\
\hline Kohler 750 Prime Power & $1,285,000$ & 0 & 30,472 & 145,623 & $-248,317$ & $1,212,778$ \\
\hline Grid & 0 & 0 & $149,817,920$ & 0 & 0 \\
\hline System & $23,785,000$ & $7,173,158$ & $154,696,192$ & 145,623 & $-4,290,851$ & $181,509,122$ \\
\hline
\end{tabular}

Annualized Costs

\begin{tabular}{|l|r|r|r|r|r|r|}
\hline Component & Capital & Replacement & O\&M & Fuel & Salvage & Total \\
\hline Siemens 2.3 MW - 108 & $1,740,474$ & 554,875 & 375,000 & 0 & $-312,708$ & $2,357,641$ \\
\hline Kohler 750 Prime Power & 99,400 & 0 & 2,357 & 11,265 & $-19,208$ & 93,814 \\
\hline Grid & 0 & 0 & $11,589,072$ & 0 & 0 \\
\hline System & $1,839,874$ & 554,875 & $11,966,428$ & 11,265 & $-331,916$ & $14,040,526$ \\
\hline
\end{tabular}




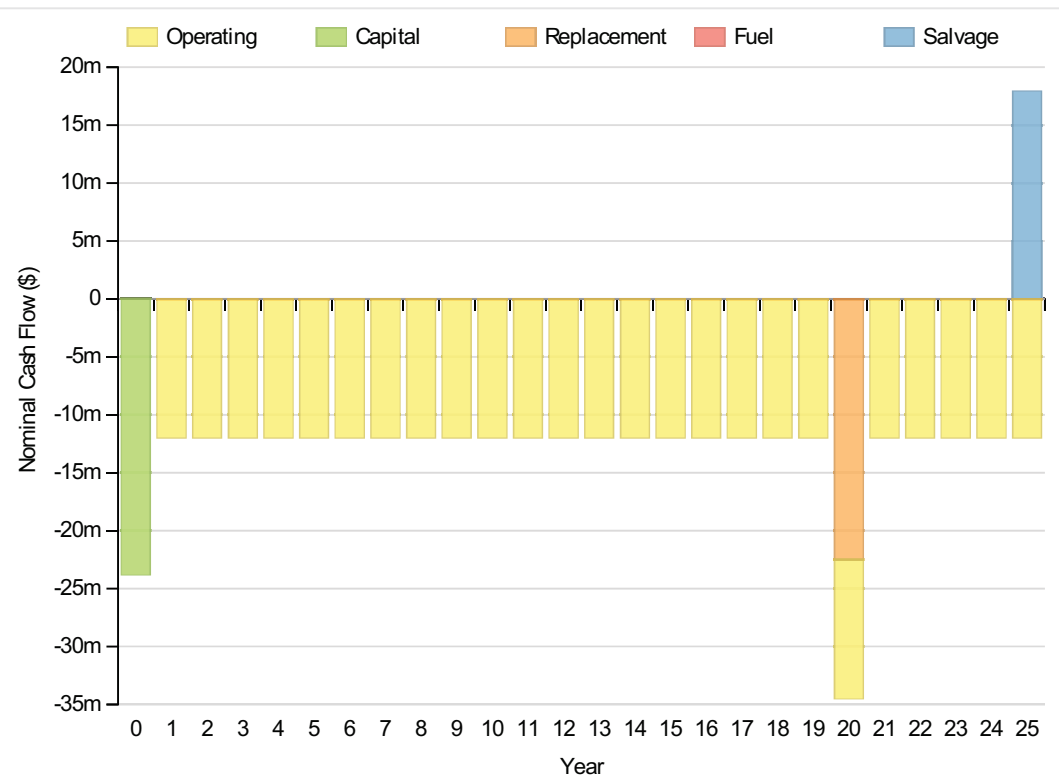

Electrical

\begin{tabular}{|c|c|c|c|c|}
\hline \multicolumn{2}{|l|}{ Quantity } & \multicolumn{2}{|l|}{ Value } & Units \\
\hline Excess electricity & & & 742167 & $\mathrm{kWh} / \mathrm{yr}$ \\
\hline Unmet load & & & 5237 & $\mathrm{kWh} / \mathrm{yr}$ \\
\hline Capacity shortage & & & 55203 & $\mathrm{kWh} / \mathrm{yr}$ \\
\hline Renewable fraction & & & 0 & \\
\hline Component & Production(kWh/yr) & & Fractio & $n(\%)$ \\
\hline Generator & & 48,613 & & 0 \\
\hline Wind Turbine & & $30,268,832$ & & 31 \\
\hline Grid Purchases & & $68,283,648$ & & 69 \\
\hline Total & & $98,601,096$ & & 100 \\
\hline Load & Consumption(kWh/yr) & & Fract & tion (\%) \\
\hline AC primary load & & $96,858,912$ & & 100 \\
\hline DC primary load & & & 0 & 0 \\
\hline Total & & $96,858,912$ & & 100 \\
\hline
\end{tabular}




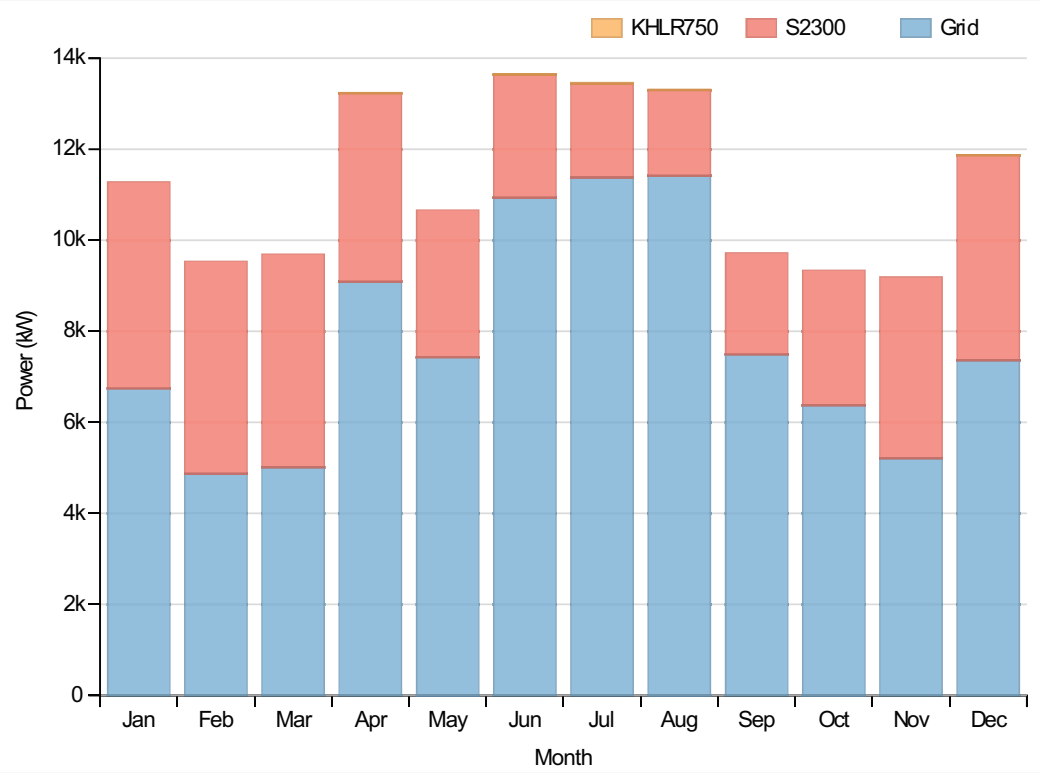

Wind Turbine:Siemens 2.3 MW - 108

\begin{tabular}{|c|c|c|}
\hline Quantity & Value & Units \\
\hline Total rated capacity & 11500 & $\mathrm{~kW}$ \\
\hline Mean output & 3455 & $\mathrm{~kW}$ \\
\hline Capacity factor & 30.05 & $\%$ \\
\hline Total production & 30268832 & $\mathrm{kWh} / \mathrm{yr}$ \\
\hline Minimum output & 7.19 & $\mathrm{~kW}$ \\
\hline Maximum output & 11574.00 & $\mathrm{~kW}$ \\
\hline Wind penetration & 31.25 & $\%$ \\
\hline Hours of operation & 8760 & $\mathrm{hrs} / \mathrm{yr}$ \\
\hline Levelized cost & 0.078 & $\$ / k W h$ \\
\hline
\end{tabular}

\section{Generator:Kohler 750 Prime Power}

\begin{tabular}{|l|r|l|}
\hline Quantity & Value & Units \\
\hline Hours of operation & 116 hrs/yr \\
\hline Number of starts & 108 starts/yr \\
\hline Operational life & 129 yr \\
\hline Fixed generation cost & 107.02 & $\$ / \mathrm{hr}$ \\
\hline Marginal generation cost & 0.23 & $\$ / \mathrm{kWh}$ \\
\hline Electrical production & $48613 \mathrm{kWh} / \mathrm{yr}$ \\
\hline Mean electrical output & $419 \mathrm{~kW}$ \\
\hline Min. electrical output & 345 & $\mathrm{~kW}$ \\
\hline Max. electrical output & 1380 & $\mathrm{~kW}$ \\
\hline Fuel consumption & $14259 \mathrm{~L} / \mathrm{yr}$ \\
\hline Specific fuel consumption & $0.29 \mathrm{~L} / \mathrm{kWh}$ \\
\hline
\end{tabular}


Quantity

Fuel energy input

Mean electrical efficiency
Units

140309 kWh/yr

$35 \%$

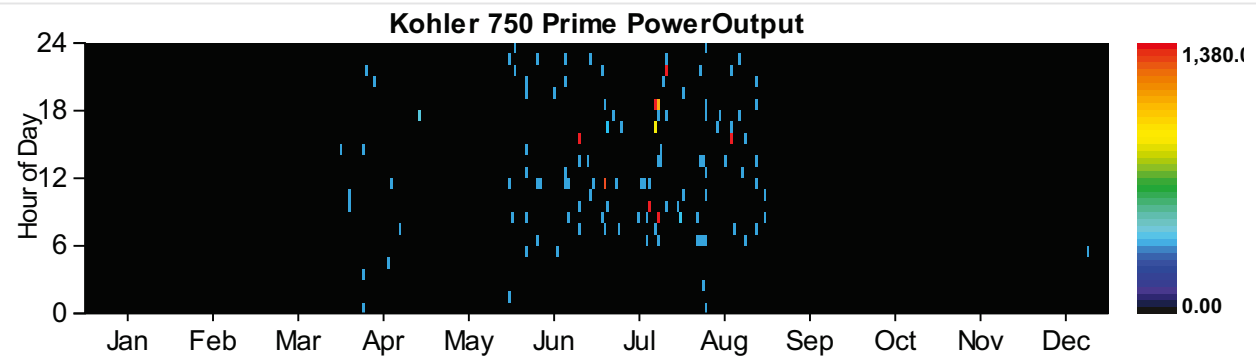

Grid

Rate: Demand 1

\begin{tabular}{|c|c|c|c|c|c|c|}
\hline Resources.ReportingService_GeneratelnputsReport_Month & $\begin{array}{l}\text { Energy } \\
\text { Purchased } \\
\text { (kWh) }\end{array}$ & $\begin{array}{l}\text { Energy } \\
\text { Sold } \\
\text { (kWh) }\end{array}$ & $\begin{array}{l}\text { Net } \\
\text { Purchases } \\
\text { (kWh) }\end{array}$ & $\begin{array}{l}\text { Peak } \\
\text { Demand } \\
(\mathbf{k W})\end{array}$ & $\begin{array}{l}\text { Energy } \\
\text { Charge } \\
\text { (\$) }\end{array}$ & $\begin{array}{l}\text { Demand } \\
\text { Charge } \\
(\$)\end{array}$ \\
\hline January & 0 & 0 & 0 & 18,095 & 0 & 289,519 \\
\hline February & 0 & 0 & 0 & 15,686 & 0 & 250,977 \\
\hline March & 0 & 0 & 0 & 16,699 & 0 & 267,178 \\
\hline April & 0 & 0 & 0 & 20,000 & 0 & 320,000 \\
\hline May & 0 & 0 & 0 & 17,784 & 0 & 284,539 \\
\hline June & 0 & 0 & 0 & 20,000 & 0 & 320,000 \\
\hline July & 0 & 0 & 0 & 20,000 & 0 & 320,000 \\
\hline August & 0 & 0 & 0 & 20,000 & 0 & 320,000 \\
\hline September & 0 & 0 & 0 & 15,890 & 0 & 254,236 \\
\hline October & 0 & 0 & 0 & 13,843 & 0 & 221,494 \\
\hline November & 0 & 0 & 0 & 15,876 & 0 & 254,021 \\
\hline December & 0 & 0 & 0 & 18,317 & 0 & 293,076 \\
\hline Annual & 0 & 0 & 0 & 20,000 & 0 & $3,395,040$ \\
\hline
\end{tabular}

Rate: Rate 1

\begin{tabular}{|c|c|c|c|c|c|c|}
\hline Resources.ReportingService_GeneratelnputsReport_Month & $\begin{array}{l}\text { Energy } \\
\text { Purchased } \\
\text { (kWh) }\end{array}$ & $\begin{array}{l}\text { Energy } \\
\text { Sold } \\
\text { (kWh) }\end{array}$ & $\begin{array}{l}\text { Net } \\
\text { Purchases } \\
\text { (kWh) }\end{array}$ & $\begin{array}{l}\text { Peak } \\
\text { Demand } \\
(\mathbf{k W})\end{array}$ & $\begin{array}{l}\text { Energy } \\
\text { Charge } \\
(\$)\end{array}$ & $\begin{array}{l}\text { Demand } \\
\text { Charge } \\
(\$)\end{array}$ \\
\hline January & $5,015,457$ & 0 & $5,015,457$ & 0 & 601,855 & 0 \\
\hline February & $3,272,783$ & 0 & $3,272,783$ & 0 & 392,734 & 0 \\
\hline March & $3,728,512$ & 0 & $3,728,512$ & 0 & 447,421 & 0 \\
\hline April & $6,541,557$ & 0 & $6,541,557$ & 0 & 784,987 & 0 \\
\hline May & $5,524,917$ & 0 & $5,524,917$ & 0 & 662,990 & 0 \\
\hline
\end{tabular}




\begin{tabular}{|c|c|c|c|c|c|c|c|}
\hline June & $\begin{array}{l}\text { En@,@g,754 } \\
\text { Purchased }\end{array}$ & $\begin{array}{l}\text { Energy } \\
\text { Sold }\end{array}$ & $\begin{array}{l}\text { Nett869,754 } \\
\text { Purchases }\end{array}$ & $\begin{array}{l}\text { Peak } \\
\text { Dema }\end{array}$ & & $\begin{array}{l}\text { Engengy/70 } \\
\text { Charge }\end{array}$ & $\begin{array}{l}\text { Demand } \\
\text { Charge }\end{array}$ \\
\hline Rassources.ReportingService_GeneratelnputsReport_Month & $(\mathbf{k}, 4,4) 5,938$ & $(\mathbf{k W h}) 0$ & $(\mathbf{k}+4 \mathbf{4}) 5,938$ & $(\mathbf{k W})$ & 0 & $\mathbf{k} \downarrow 15,913$ & (\$) $\quad 0$ \\
\hline August & $8,494,914$ & 0 & $8,494,914$ & & 0 & $1,019,390$ & 0 \\
\hline September & $5,395,241$ & 0 & $5,395,241$ & & 0 & 647,429 & 0 \\
\hline October & $4,744,924$ & 0 & $4,744,924$ & & 0 & 569,391 & 0 \\
\hline November & $3,750,403$ & 0 & $3,750,403$ & & 0 & 450,048 & 0 \\
\hline December & $5,479,252$ & 0 & $5,479,252$ & & 0 & 657,510 & 0 \\
\hline Annual & $68,283,648$ & 0 & $68,283,648$ & & 0 & $8,194,038$ & 0 \\
\hline
\end{tabular}

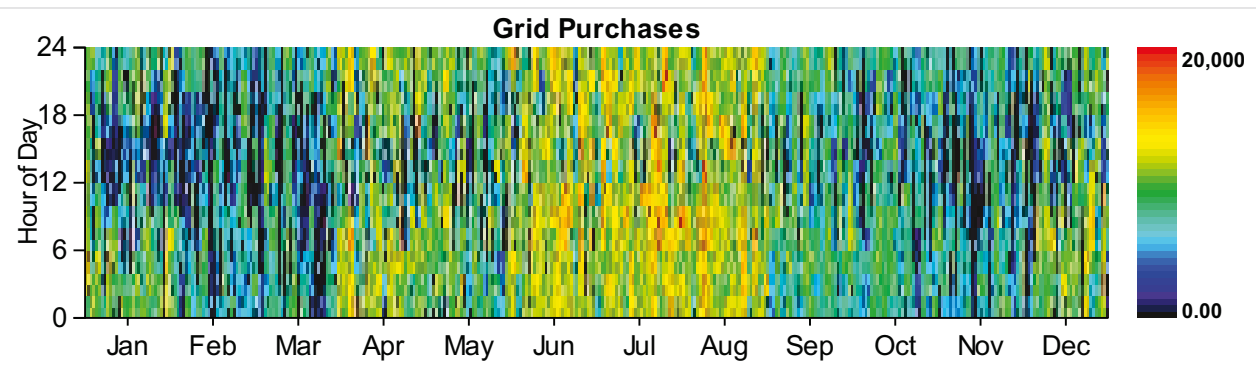

\section{Emissions}

\begin{tabular}{|l|r|l|}
\hline Pollutant & Emissions & Units \\
\hline Carbon dioxide & $43192688 \mathrm{~kg} / \mathrm{yr}$ \\
\hline Carbon monoxide & $157 \mathrm{~kg} / \mathrm{yr}$ \\
\hline Unburned hydrocarbons & $18 \mathrm{~kg} / \mathrm{yr}$ \\
\hline Particulate matter & $4 \mathrm{~kg} / \mathrm{yr}$ \\
\hline Sulfur dioxide & $187174 \mathrm{~kg} / \mathrm{yr}$ \\
\hline Nitrogen oxides & $91657 \mathrm{~kg} / \mathrm{yr}$ \\
\hline
\end{tabular}

HOMER Energy, LLC $\odot 2016$ 
System Report

System architecture

\begin{tabular}{|l|l|r|}
\hline Wind Turbine & Siemens 2.3 MW - 108 & 5 \\
\hline Generator & Kohler 1000 Prime Power & 925 \\
\hline Battery & GS200 flow & $\mathrm{kW}$ \\
\hline Converter & System Converter & 1 \\
\hline Grid & Grid & 250 \\
\hline Dispatch Strategy & $\mathrm{kW}$ \\
\hline
\end{tabular}

Cost summary

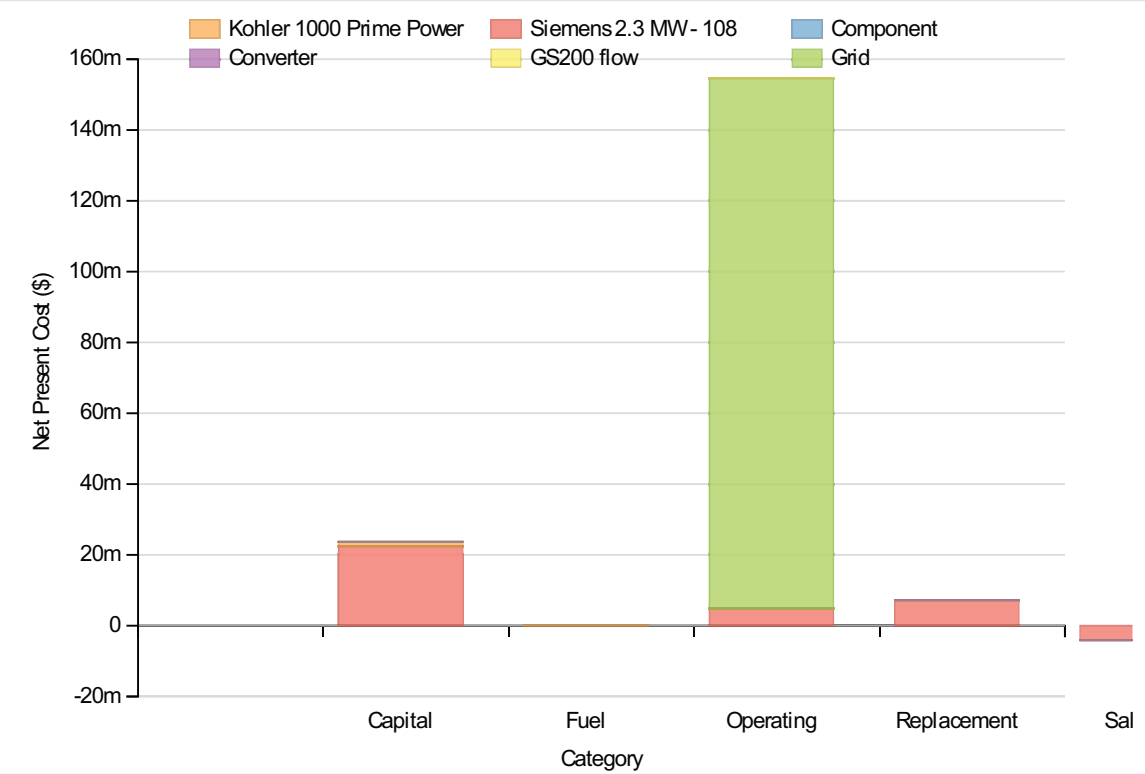

Cost Summary

Total net present cost

$181599280 \$$

Levelized cost of energy

$0.145 \$ / k W h$

\section{Net Present Costs}

\begin{tabular}{|c|c|c|c|c|c|c|}
\hline Component & Capital & Replacement & O\&M & Fuel & Salvage & Total \\
\hline Siemens 2.3 MW - 108 & $22,500,000$ & $7,173,158$ & $4,847,816$ & 0 & $-4,042,534$ & $30,478,440$ \\
\hline Kohler 1000 Prime Power & 925,000 & 0 & 20,627 & 161,812 & $-179,119$ & 928,320 \\
\hline Grid & 0 & 0 & $149,729,776$ & 0 & 0 & $149,729,776$ \\
\hline GS200 flow & 324,589 & 7,176 & 31,026 & 0 & -973 & 361,818 \\
\hline Converter & 75,000 & 31,821 & 0 & 0 & $-5,989$ & 100,832 \\
\hline System & $23,824,588$ & $7,212,154$ & $154,629,264$ & 161,812 & $-4,228,615$ & $181,599,203$ \\
\hline
\end{tabular}

\section{Annualized Costs}

\begin{tabular}{|l|r|r|r|r|r|r|}
\hline Component & Capital & Replacement & O\&M & Fuel & Salvage & Total \\
\hline Siemens 2.3 MW - 108 & $1,740,474$ & 554,875 & 375,000 & 0 & $-312,708$ & $2,357,641$ \\
\hline
\end{tabular}




\begin{tabular}{|c|c|c|c|c|c|c|}
\hline 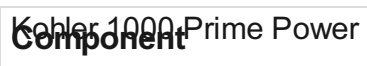 & Capita 71,553 & Replacement 0 & O\&M & Fuê?,517 & Salvał'e 856 & Total \\
\hline Grid & 0 & 0 & $11,582,254$ & 0 & 0 & $11,582,254$ \\
\hline GS200 flow & 25,108 & 555 & 2,400 & 0 & -75 & 27,988 \\
\hline Converter & 5,802 & 2,462 & 0 & 0 & -463 & 7,800 \\
\hline System & $1,842,936$ & 557,892 & $11,961,251$ & 12,517 & $-327,102$ & $14,047,494$ \\
\hline
\end{tabular}

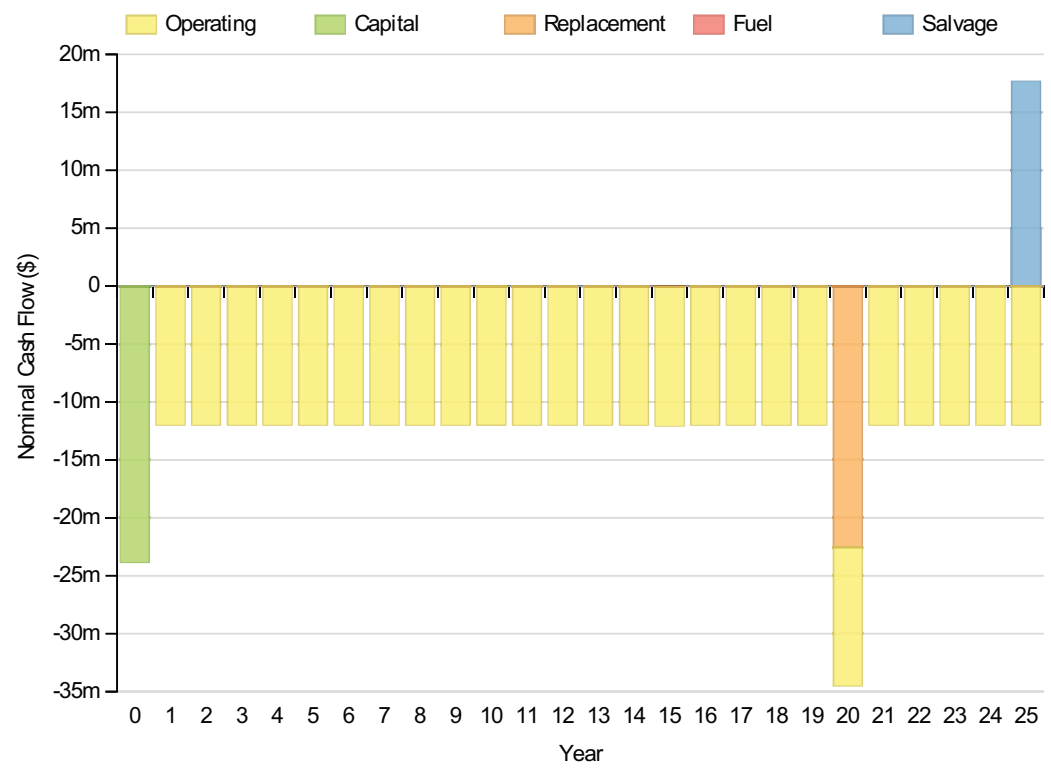

\section{Electrical}

\section{Quantity}

Excess electricity

Unmet load

Capacity shortage

Renewable fraction

\section{Component}

Generator

Wind Turbine

Grid Purchases

Total
Value

Production(kWh/yr)

Consumption(kWh/yr)
Units

$1607580 \mathrm{kWh} / \mathrm{yr}$

$8391 \mathrm{kWh} / \mathrm{yr}$

80318 kWh/yr

\begin{tabular}{|r|r|} 
& Fraction (\%) \\
\hline 54,910 & 0 \\
\hline $30,268,832$ & 31 \\
\hline $68,211,680$ & 69 \\
\hline $98,535,424$ & 100 \\
\hline
\end{tabular}

\section{Load}

AC primary load

$96,855,760$

DC primary load

0 


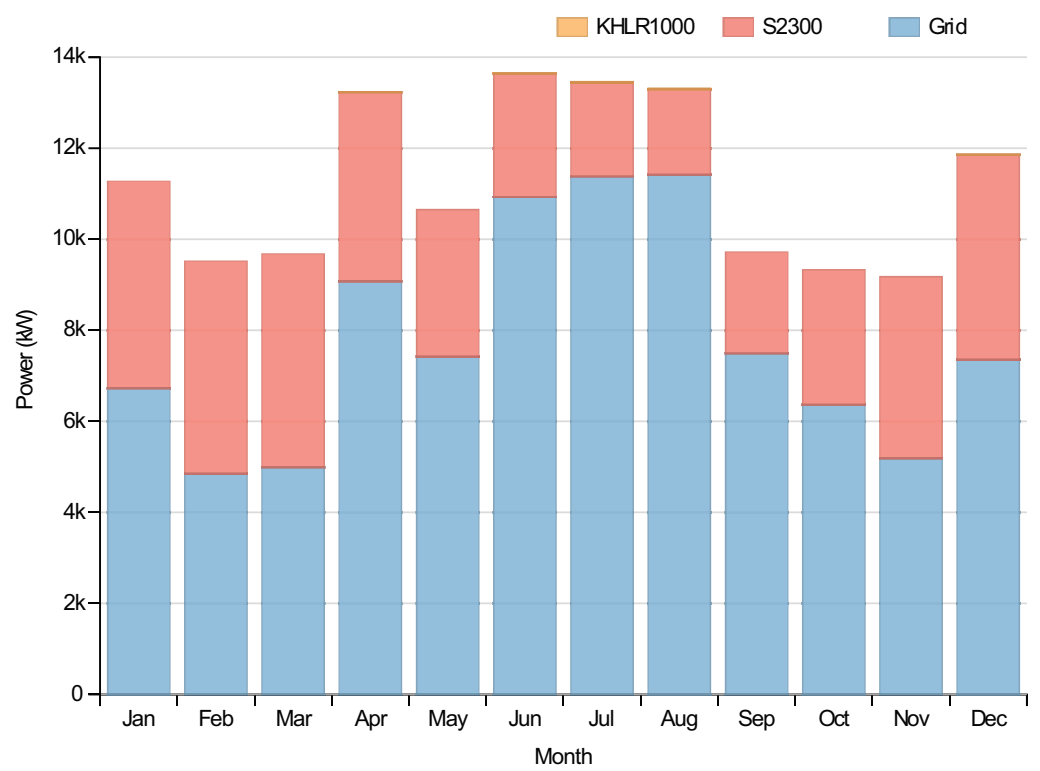

Wind Turbine:Siemens 2.3 MW - 108

\begin{tabular}{|c|c|c|}
\hline Quantity & Value & Units \\
\hline Total rated capacity & 11500 & $\mathrm{~kW}$ \\
\hline Mean output & 3455 & $\mathrm{~kW}$ \\
\hline Capacity factor & 30.05 & $\%$ \\
\hline Total production & 30268832 & $\mathrm{kWh} / \mathrm{yr}$ \\
\hline Minimum output & 7.19 & $\mathrm{~kW}$ \\
\hline Maximum output & 11574.00 & $\mathrm{~kW}$ \\
\hline Wind penetration & 31.25 & $\%$ \\
\hline Hours of operation & 8760 & $\mathrm{hrs} / \mathrm{yr}$ \\
\hline Levelized cost & 0.078 & $\$ / k W h$ \\
\hline
\end{tabular}

\section{Generator:Kohler 1000 Prime Power}

\begin{tabular}{|l|r|l|}
\hline Quantity & Value & Units \\
\hline Hours of operation & 115 hrs/yr \\
\hline Number of starts & 108 starts/yr \\
\hline Operational life & $130 \mathrm{yr}$ \\
\hline Fixed generation cost & 76.24 \$/hr \\
\hline Marginal generation cost & 0.23 & $\$ / \mathrm{kWh}$ \\
\hline Electrical production & $54910 \mathrm{kWh} / \mathrm{yr}$ \\
\hline Mean electrical output & $477 \mathrm{~kW}$ \\
\hline Min. electrical output & 231 & $\mathrm{~kW}$ \\
\hline Max. electrical output & $\mathrm{kW}$ \\
\hline Fuel consumption & 925 & $\mathrm{~kW}$ \\
\hline Specific fuel consumption & $15844 \mathrm{~L} / \mathrm{yr}$ \\
\hline
\end{tabular}




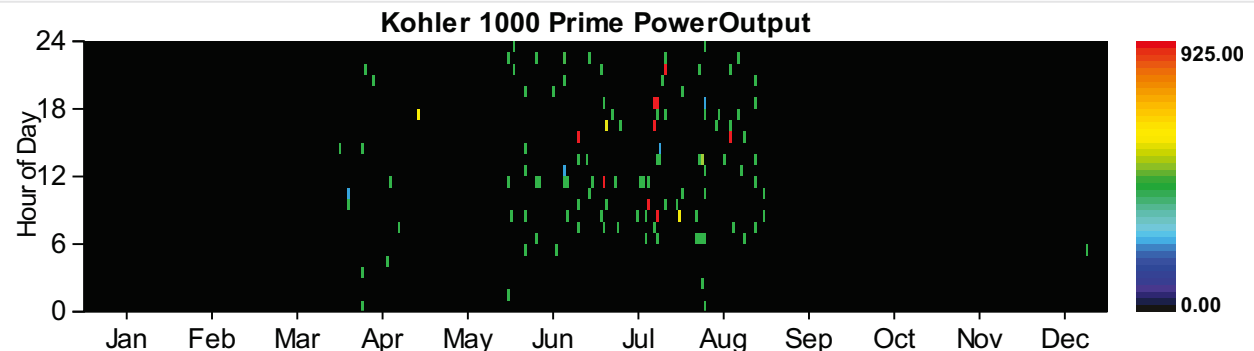

\section{Battery:GS200 flow}

Quantity

Value

String size

Strings in parallel

Batteries

Bus voltage

\section{Quantity}

Nominal capacity

Usable nominal capacity

Autonomy

Lifetime throughput

Battery wear cost

Average energy cost

Energy in

Energy out

Storage depletion

Losses

Annual throughput

Expected life
Value

\section{Units}

600 kWh

$600 \mathrm{kWh}$

$0 \mathrm{hr}$

0

$0.000 \$ / k W h$

$0.035 \$ / \mathrm{kWh}$

$132703 \mathrm{kWh} / \mathrm{yr}$

$93395 \mathrm{kWh} / \mathrm{yr}$

$600 \mathrm{kWh} / \mathrm{yr}$

$38708 \mathrm{kWh} / \mathrm{yr}$

$111628 \mathrm{kWh} / \mathrm{yr}$

$25 \mathrm{yr}$

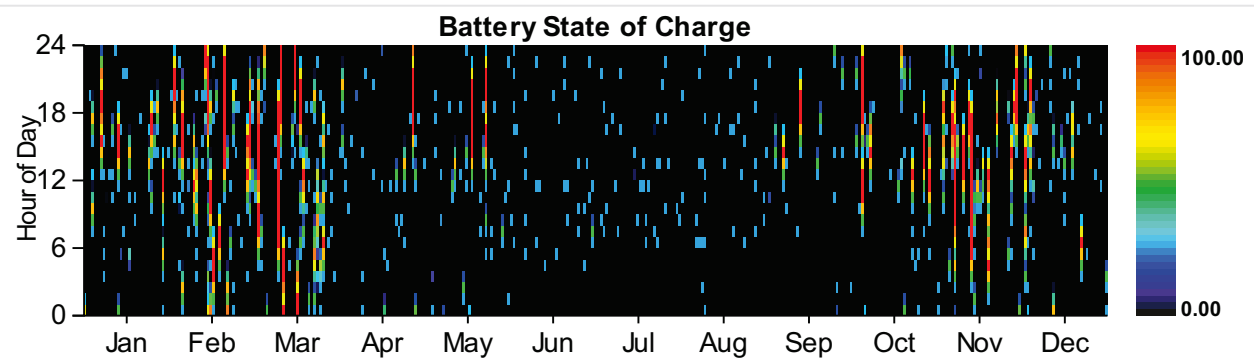


Converter

\begin{tabular}{|c|c|c|c|}
\hline Quantity & Inverter & Rectifier & Units \\
\hline Capacity & 250 & 225 & kW \\
\hline Mean output & 10 & 15 & $\mathrm{~kW}$ \\
\hline Minimum output & 0 & 0 & $\mathrm{~kW}$ \\
\hline Maximum output & 166 & 179 & $\mathrm{~kW}$ \\
\hline Capacity factor & 4 & 6 & $\%$ \\
\hline Hours of operation & 873 & 1,017 & $\mathrm{hrs} / \mathrm{yr}$ \\
\hline Energy in & 93,394 & 156,121 & $\mathrm{kWh} / \mathrm{yr}$ \\
\hline Energy out & 84,055 & 132,703 & $\mathrm{kWh} / \mathrm{yr}$ \\
\hline Losses & 9,340 & 23,419 & $\mathrm{kWh} / \mathrm{yr}$ \\
\hline
\end{tabular}
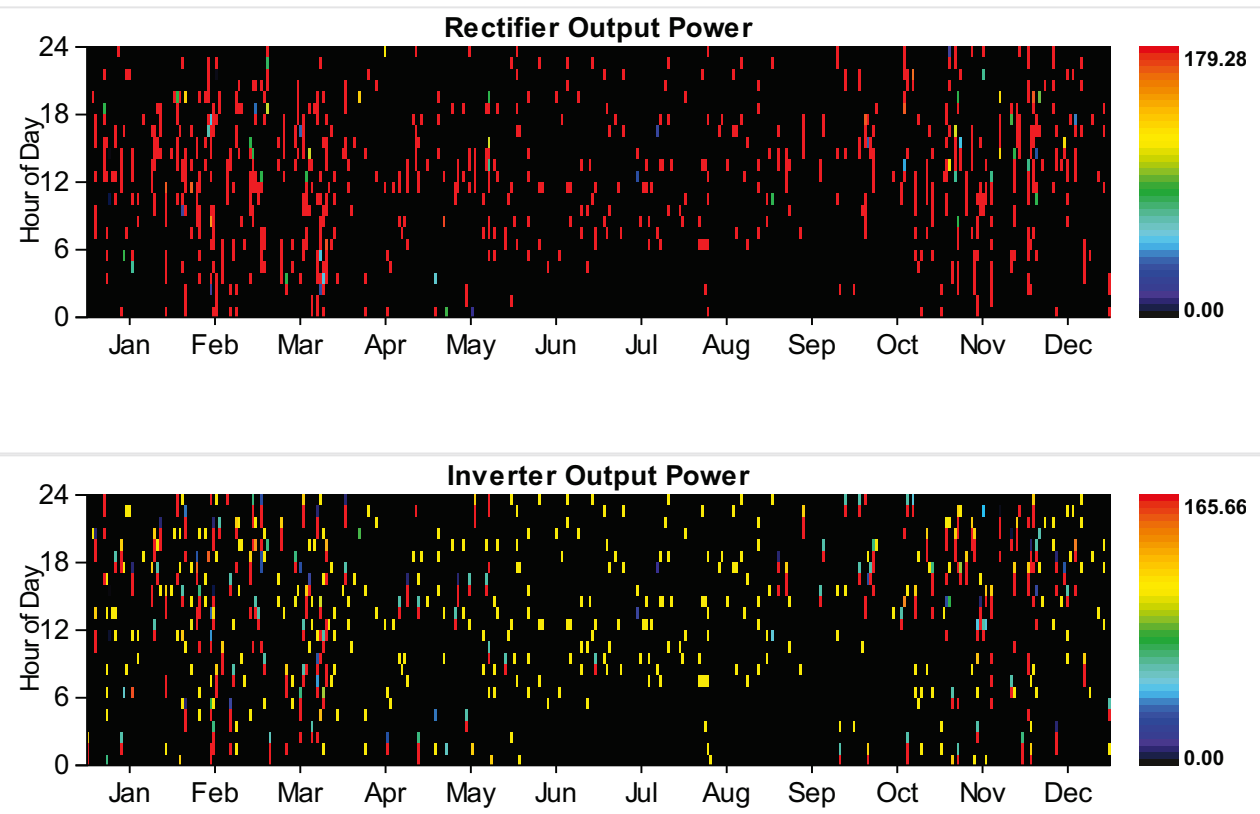

\section{Grid}

Rate: Demand 1

\begin{tabular}{|c|c|c|c|c|c|c|}
\hline Resources.ReportingService_GeneratelnputsReport_Month & $\begin{array}{l}\text { Energy } \\
\text { Purchased } \\
\text { (kWh) }\end{array}$ & $\begin{array}{l}\text { Energy } \\
\text { Sold } \\
\text { (kWh) }\end{array}$ & $\begin{array}{l}\text { Net } \\
\text { Purchases } \\
\text { (kWh) }\end{array}$ & $\begin{array}{l}\text { Peak } \\
\text { Demand } \\
(\mathbf{k W})\end{array}$ & $\begin{array}{l}\text { Energy } \\
\text { Charge } \\
\text { (\$) }\end{array}$ & $\begin{array}{l}\text { Demand } \\
\text { Charge } \\
(\$)\end{array}$ \\
\hline January & 0 & 0 & 0 & 18,095 & 0 & 289,519 \\
\hline February & 0 & 0 & 0 & 15,686 & 0 & 250,977 \\
\hline March & 0 & 0 & 0 & 16,699 & 0 & 267,178 \\
\hline April & 0 & 0 & 0 & 20,000 & 0 & 320,000 \\
\hline May & 0 & 0 & 0 & 17,784 & 0 & 284,539 \\
\hline June & 0 & 0 & 0 & 20,000 & 0 & 320,000 \\
\hline July & 0 & 0 & 0 & 20,000 & 0 & 320,000 \\
\hline August & 0 & 0 & 0 & 20,000 & 0 & 320,000 \\
\hline
\end{tabular}




\begin{tabular}{|c|c|c|c|c|c|c|}
\hline Retswarces.ReportingService_GeneratelnputsReport_Month & $\begin{array}{l}\text { Energy } \\
\text { Purchased } \\
\text { (kWh) }\end{array}$ & $\begin{array}{l}\text { Energy } \\
\text { Sold } 0 \\
(\mathbf{k W h})_{0}\end{array}$ & $\begin{array}{l}\text { Net } \\
\text { Purchases } \\
\text { (kWh) }\end{array}$ & $\begin{array}{l}\text { Peak } \\
15,899 \\
\text { Demañ } \\
\text { (kW) } \\
13,843\end{array}$ & $\begin{array}{l}\text { Energy } \\
\text { Charge } \\
\text { (\$) } \quad 0\end{array}$ & $\begin{array}{l}\text { Demand } \\
\text { Ch54,236 } \\
\text { Charge } \\
\text { (\$) } \\
{ }_{221,494}\end{array}$ \\
\hline November & 0 & 0 & 0 & 15,876 & 0 & 254,021 \\
\hline December & 0 & 0 & 0 & 18,431 & 0 & 294,896 \\
\hline Annual & 0 & 0 & 0 & 20,000 & 0 & $3,396,860$ \\
\hline
\end{tabular}

Rate: Rate 1

\begin{tabular}{|c|c|c|c|c|c|c|}
\hline Resources.ReportingService_GeneratelnputsReport_Month & $\begin{array}{l}\text { Energy } \\
\text { Purchased } \\
\text { (kWh) }\end{array}$ & $\begin{array}{l}\text { Energy } \\
\text { Sold } \\
\text { (kWh) }\end{array}$ & $\begin{array}{l}\text { Net } \\
\text { Purchases } \\
\text { (kWh) }\end{array}$ & $\begin{array}{l}\text { Peak } \\
\text { Demand } \\
(\mathbf{k W})\end{array}$ & $\begin{array}{l}\text { Energy } \\
\text { Charge } \\
\text { (\$) }\end{array}$ & $\begin{array}{l}\text { Demand } \\
\text { Charge } \\
(\$)\end{array}$ \\
\hline January & $5,007,178$ & 0 & $5,007,178$ & 0 & 600,861 & 0 \\
\hline February & $3,260,560$ & 0 & $3,260,560$ & 0 & 391,267 & 0 \\
\hline March & $3,715,573$ & 0 & $3,715,573$ & 0 & 445,869 & 0 \\
\hline April & $6,538,133$ & 0 & $6,538,133$ & 0 & 784,576 & 0 \\
\hline May & $5,519,575$ & 0 & $5,519,575$ & 0 & 662,349 & 0 \\
\hline June & $7,869,208$ & 0 & $7,869,208$ & 0 & 944,305 & 0 \\
\hline July & $8,465,368$ & 0 & $8,465,368$ & 0 & $1,015,844$ & 0 \\
\hline August & $8,494,749$ & 0 & $8,494,749$ & 0 & $1,019,370$ & 0 \\
\hline September & $5,392,027$ & 0 & $5,392,027$ & 0 & 647,043 & 0 \\
\hline October & $4,738,349$ & 0 & $4,738,349$ & 0 & 568,602 & 0 \\
\hline November & $3,739,770$ & 0 & $3,739,770$ & 0 & 448,772 & 0 \\
\hline December & $5,471,190$ & 0 & $5,471,190$ & 0 & 656,543 & 0 \\
\hline Annual & $68,211,680$ & 0 & $68,211,680$ & 0 & $8,185,402$ & 0 \\
\hline
\end{tabular}

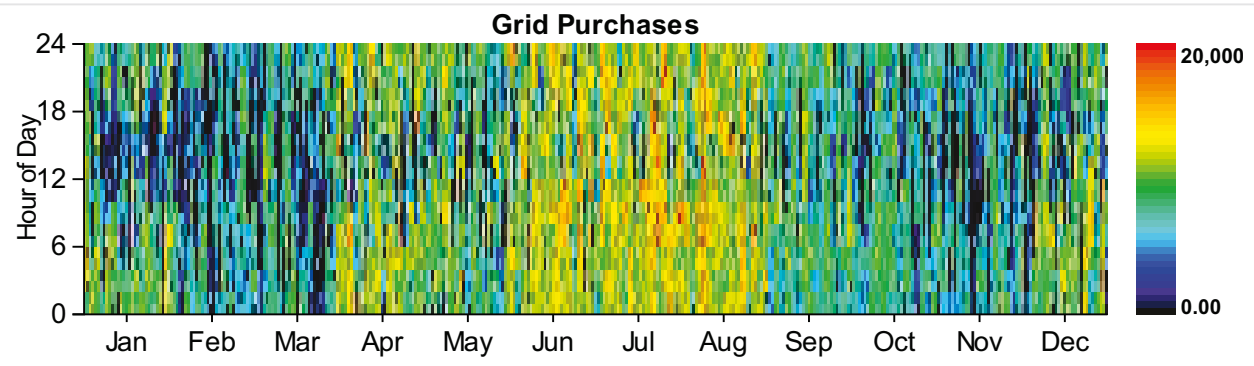

\section{Emissions}

\section{Pollutant}

Carbon dioxide

Carbon monoxide

Unburned hydrocarbons

Particulate matter

\section{Emissions}

Units

$43151364 \mathrm{~kg} / \mathrm{yr}$

174 kg/yr

$20 \mathrm{~kg} / \mathrm{yr}$

$5 \mathrm{~kg} / \mathrm{yr}$ 
HOMER Energy, LLC @ 2016 
System Report

System architecture

\begin{tabular}{|l|l|r|}
\hline Wind Turbine & Siemens 2.3 MW - 108 & 5 \\
\hline Generator & Kohler 1000 Prime Power & 925 \\
\hline Generator \#2 & Kohler 750 Prime Power & 690 \\
\hline Grid & Grid & 20,000 \\
\hline Dispatch Strategy & Cycle Charging & $\mathrm{kW}$ \\
\hline
\end{tabular}

\section{Cost summary}

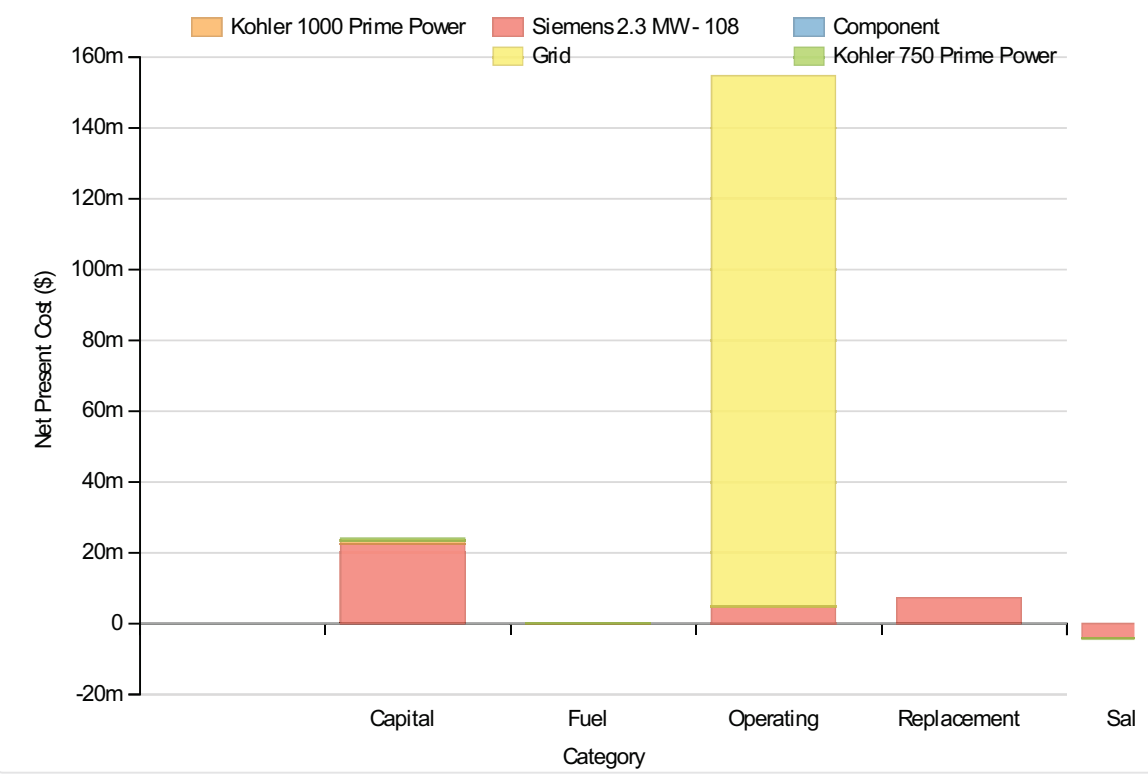

Cost Summary

Total net present cost

181785888 \$

Levelized cost of energy

$0.145 \$ / k W h$

Net Present Costs

\begin{tabular}{|l|r|r|r|r|r|r|}
\hline Component & Capital & Replacement & O\&M & Fuel & Salvage & \multicolumn{1}{l|}{ Total } \\
\hline Siemens 2.3 MW - 108 & $22,500,000$ & $7,173,158$ & $4,847,816$ & 0 & $-4,042,534$ \\
\hline Kohler 1000 Prime Power & 925,000 & 0 & 13,991 & 71,206 & $-192,784$ \\
\hline Kohler 750 Prime Power & 690,000 & 0 & 13,648 & 62,102 & $-137,195$ \\
\hline Grid & 0 & 0 & $149,861,392$ & 0 & 0 \\
\hline System & $24,115,000$ & $7,173,158$ & $154,736,864$ & 133,307 & $-4,372,512$ \\
\hline
\end{tabular}

\section{Annualized Costs}

\begin{tabular}{|l|r|r|r|r|r|r|}
\hline Component & Capital & Replacement & O\&M & Fuel & Salvage & Total \\
\hline Siemens 2.3 MW - 108 & $1,740,474$ & 554,875 & 375,000 & 0 & $-312,708$ & $2,357,641$ \\
\hline Kohler 1000 Prime Power & 71,553 & 0 & 1,082 & 5,508 & $-14,913$ & 63,230 \\
\hline Kohler 750 Prime Power & 53,375 & 0 & 1,056 & 4,804 & $-10,613$ \\
\hline
\end{tabular}




\begin{tabular}{l|llllllllllll} 
Ebimponent & Capital & 0 & Replacement & 0 & $\mathbf{O} \& \mathbf{M} 1,592,435$ & Fuel & 0 & Salvage & 0 & Total $1,592,435$
\end{tabular}

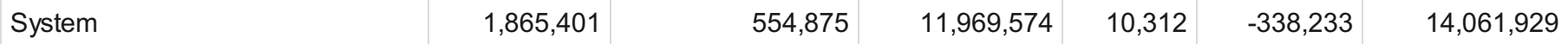

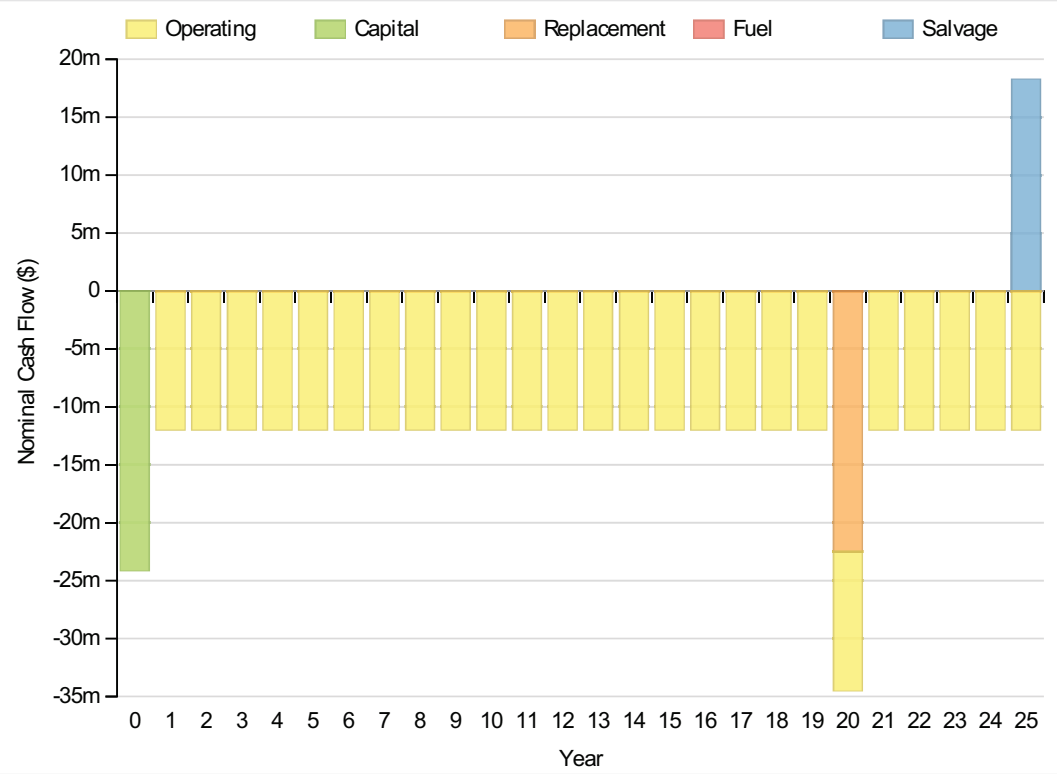

\section{Electrical}

\begin{tabular}{|c|c|c|c|c|}
\hline \multicolumn{2}{|l|}{ Quantity } & \multicolumn{2}{|l|}{ Value } & Units \\
\hline & & & 742167 & $\mathrm{kWh} / \mathrm{yr}$ \\
\hline Unmet load & & & 4013 & $\mathrm{kWh} / \mathrm{yr}$ \\
\hline Capacity shortage & & & 44902 & $\mathrm{kWh} / \mathrm{yr}$ \\
\hline Renewable fraction & & & 0 & \\
\hline Component & Production(kWh/yr) & & Fractio & $n(\%)$ \\
\hline Generator & & 24,079 & & 0 \\
\hline Generator & & 20,724 & & 0 \\
\hline Wind Turbine & & $30,268,832$ & & 31 \\
\hline Grid Purchases & & $68,288,688$ & & 69 \\
\hline Total & & $98,602,328$ & & 100 \\
\hline Load & Consumption(kWh/yr) & & Fract & tion (\%) \\
\hline AC primary load & & $96,860,144$ & & 100 \\
\hline DC primary load & & & 0 & 0 \\
\hline Total & & $96,860,144$ & & 100 \\
\hline
\end{tabular}




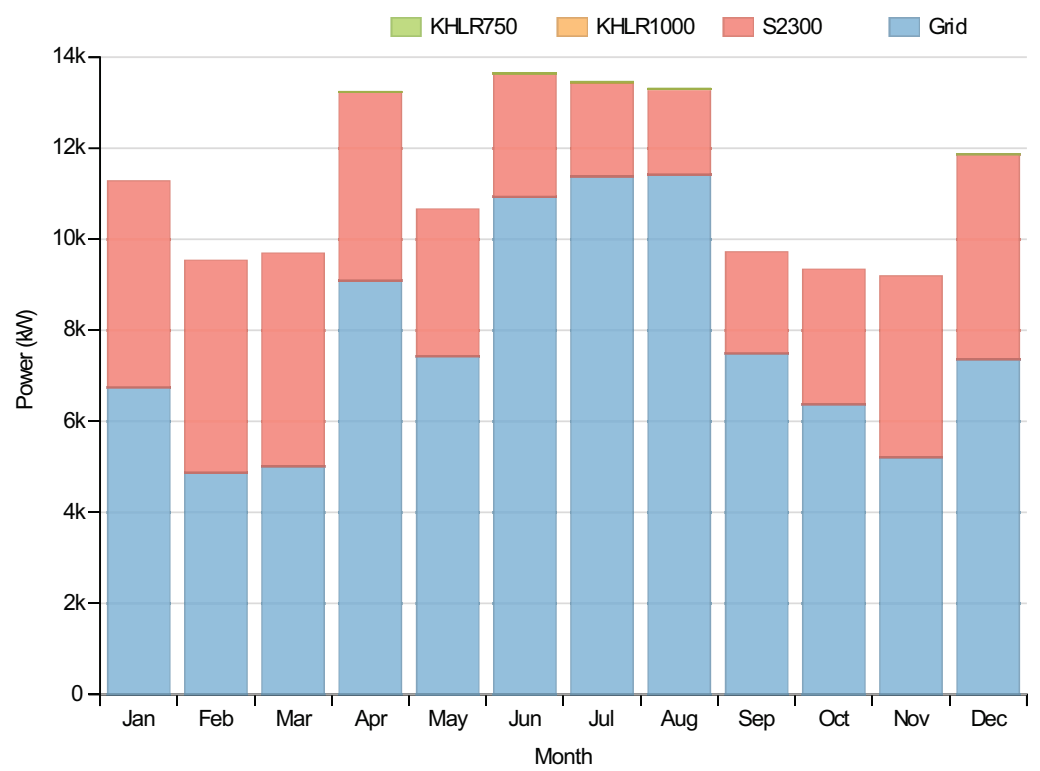

Wind Turbine:Siemens 2.3 MW - 108

\begin{tabular}{|c|c|c|}
\hline Quantity & Value & Units \\
\hline Total rated capacity & 11500 & $\mathrm{~kW}$ \\
\hline Mean output & 3455 & $\mathrm{~kW}$ \\
\hline Capacity factor & 30.05 & $\%$ \\
\hline Total production & 30268832 & $\mathrm{kWh} / \mathrm{yr}$ \\
\hline Minimum output & 7.19 & $\mathrm{~kW}$ \\
\hline Maximum output & 11574.00 & $\mathrm{~kW}$ \\
\hline Wind penetration & 31.25 & $\%$ \\
\hline Hours of operation & 8760 & $\mathrm{hrs} / \mathrm{yr}$ \\
\hline Levelized cost & 0.078 & $\$ / k W h$ \\
\hline
\end{tabular}

\section{Generator:Kohler 1000 Prime Power}

\begin{tabular}{|l|r|l}
\hline Quantity & Value & Units \\
\hline Hours of operation & 78 hrs/yr \\
\hline Number of starts & 73 starts/yr \\
\hline Operational life & 192 & $\mathrm{yr}$ \\
\hline Fixed generation cost & 76.24 & $\$ / \mathrm{hr}$ \\
\hline Marginal generation cost & 0.23 & $\$ / \mathrm{kWh}$ \\
\hline Electrical production & $24079 \mathrm{kWh} / \mathrm{yr}$ \\
\hline Mean electrical output & $309 \mathrm{~kW}$ \\
\hline Min. electrical output & 231 & $\mathrm{~kW}$ \\
\hline Max. electrical output & 925 & $\mathrm{~kW}$ \\
\hline Fuel consumption & 6972 & $\mathrm{~L} / \mathrm{yr}$ \\
\hline Specific fuel consumption & $0.29 \mathrm{~L} / \mathrm{kWh}$
\end{tabular}


Quantity

Fuel energy input

Mean electrical efficiency
Value

Units

$68607 \mathrm{kWh} / \mathrm{yr}$

$35 \%$

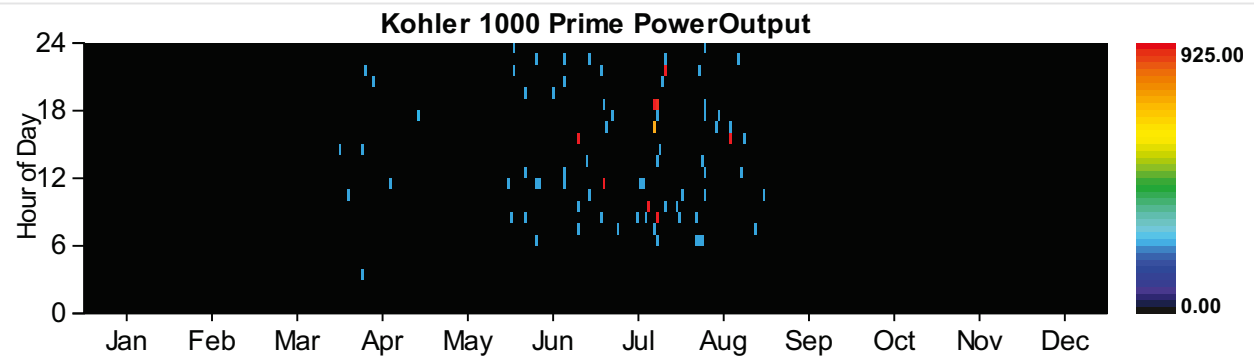

Generator:Kohler 750 Prime Power

\section{Quantity}

Hours of operation

Number of starts

Operational life

Fixed generation cost

Marginal generation cost

Electrical production

Mean electrical output

Min. electrical output

Max. electrical output

Fuel consumption

Specific fuel consumption

Fuel energy input

Mean electrical efficiency

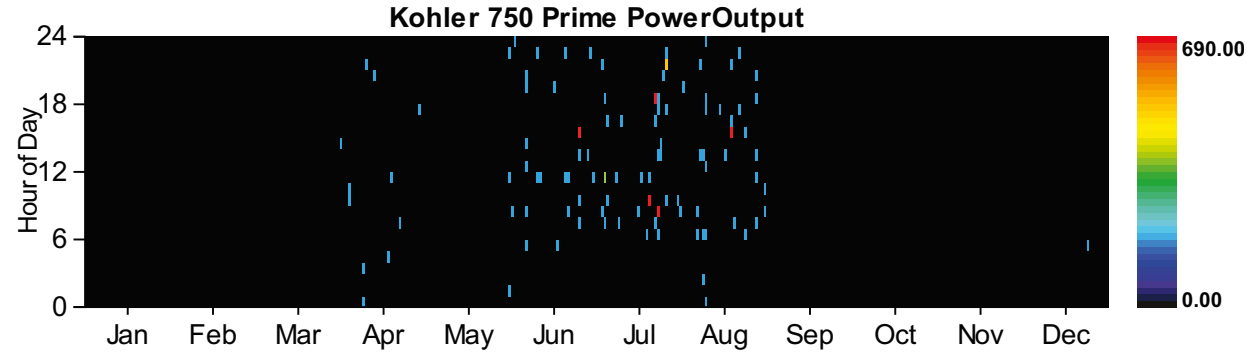

Value

Units

102 hrs/yr

95 starts/yr

$147 \mathrm{yr}$

$56.87 \$ / h r$

$0.23 \$ / k W h$

$20724 \mathrm{kWh} / \mathrm{yr}$

203 kW

$173 \mathrm{~kW}$

690 kW

6081 L/yr

$0.29 \mathrm{~L} / \mathrm{kWh}$

$59835 \mathrm{kWh} / \mathrm{yr}$

$35 \%$

\section{Grid}

Rate: Demand 1

\begin{tabular}{|l|l|l|l|l|l|}
\begin{tabular}{|l|l|l|l|} 
Energy \\
Purchased \\
$\mathbf{( k W h )}$
\end{tabular} & $\begin{array}{l}\text { Energy } \\
\text { Sold } \\
\mathbf{( k W h )}\end{array}$ & $\begin{array}{l}\text { Net } \\
\text { Purchases } \\
\mathbf{( k W h )}\end{array}$ & $\begin{array}{l}\text { Peak } \\
\text { Demand } \\
\mathbf{( k W )}\end{array}$ & $\begin{array}{l}\text { Energy } \\
\text { Charge } \\
\mathbf{( \$ )}\end{array}$ & $\begin{array}{l}\text { Demand } \\
\text { Charge } \\
\mathbf{( \$ )}\end{array}$ \\
\hline 0 & 0 & 0 & 18,095 & 0 & 289,519 \\
\hline
\end{tabular}




\begin{tabular}{|c|c|c|c|c|c|c|c|}
\hline February & $\begin{array}{l}\text { Energy } 0 \\
\text { Purchased }\end{array}$ & $\begin{array}{l}\text { Energy } \\
\text { Sold }\end{array}$ & $\begin{array}{l}\text { Net } \\
\text { Purcha }\end{array}$ & & $\begin{array}{l}\text { Peak, } 686 \\
\text { Demand }\end{array}$ & $\begin{array}{l}\text { Energy } \\
\text { Charge }\end{array}$ & $\begin{array}{l}\text { Deparagd } \\
\text { Charge }\end{array}$ \\
\hline Reasqurces.ReportingService_GeneratelnputsReport_Month & $(\mathbf{k W h}) \quad 0$ & $(\mathbf{k W h})_{0}$ & (kWh) & 0 & $(\mathbf{k W}, 699$ & (\$) 0 & $(\$ 267,178$ \\
\hline April & 0 & 0 & & 0 & 20,000 & 0 & 320,000 \\
\hline May & 0 & 0 & & 0 & 17,784 & 0 & 284,539 \\
\hline June & 0 & 0 & & 0 & 20,000 & 0 & 320,000 \\
\hline July & 0 & 0 & & 0 & 20,000 & 0 & 320,000 \\
\hline August & 0 & 0 & & 0 & 20,000 & 0 & 320,000 \\
\hline September & 0 & 0 & & 0 & 15,890 & 0 & 254,236 \\
\hline October & 0 & 0 & & 0 & 13,843 & 0 & 221,494 \\
\hline November & 0 & 0 & & 0 & 15,876 & 0 & 254,021 \\
\hline December & 0 & 0 & & 0 & 18,490 & 0 & 295,836 \\
\hline Annual & 0 & 0 & & 0 & 20,000 & 0 & $3,397,800$ \\
\hline
\end{tabular}

Rate: Rate 1

\begin{tabular}{|c|c|c|c|c|c|c|}
\hline Resources.ReportingService_GeneratelnputsReport_Month & $\begin{array}{l}\text { Energy } \\
\text { Purchased } \\
\text { (kWh) }\end{array}$ & $\begin{array}{l}\text { Energy } \\
\text { Sold } \\
\text { (kWh) }\end{array}$ & $\begin{array}{l}\text { Net } \\
\text { Purchases } \\
\text { (kWh) }\end{array}$ & $\begin{array}{l}\text { Peak } \\
\text { Demand } \\
\text { (kW) }\end{array}$ & $\begin{array}{l}\text { Energy } \\
\text { Charge } \\
(\$)\end{array}$ & $\begin{array}{l}\text { Demand } \\
\text { Charge } \\
\text { (\$) }\end{array}$ \\
\hline January & $5,015,457$ & 0 & $5,015,457$ & 0 & 601,855 & 0 \\
\hline February & $3,272,783$ & 0 & $3,272,783$ & 0 & 392,734 & 0 \\
\hline March & $3,728,512$ & 0 & $3,728,512$ & 0 & 447,421 & 0 \\
\hline April & $6,542,009$ & 0 & $6,542,009$ & 0 & 785,041 & 0 \\
\hline May & $5,524,917$ & 0 & $5,524,917$ & 0 & 662,990 & 0 \\
\hline June & $7,870,936$ & 0 & $7,870,936$ & 0 & 944,512 & 0 \\
\hline July & $8,466,915$ & 0 & $8,466,915$ & 0 & $1,016,030$ & 0 \\
\hline August & $8,497,167$ & 0 & $8,497,167$ & 0 & $1,019,660$ & 0 \\
\hline September & $5,395,241$ & 0 & $5,395,241$ & 0 & 647,429 & 0 \\
\hline October & $4,744,924$ & 0 & $4,744,924$ & 0 & 569,391 & 0 \\
\hline November & $3,750,403$ & 0 & $3,750,403$ & 0 & 450,048 & 0 \\
\hline December & $5,479,425$ & 0 & $5,479,425$ & 0 & 657,531 & 0 \\
\hline Annual & $68,288,688$ & 0 & $68,288,688$ & 0 & $8,194,643$ & 0 \\
\hline
\end{tabular}




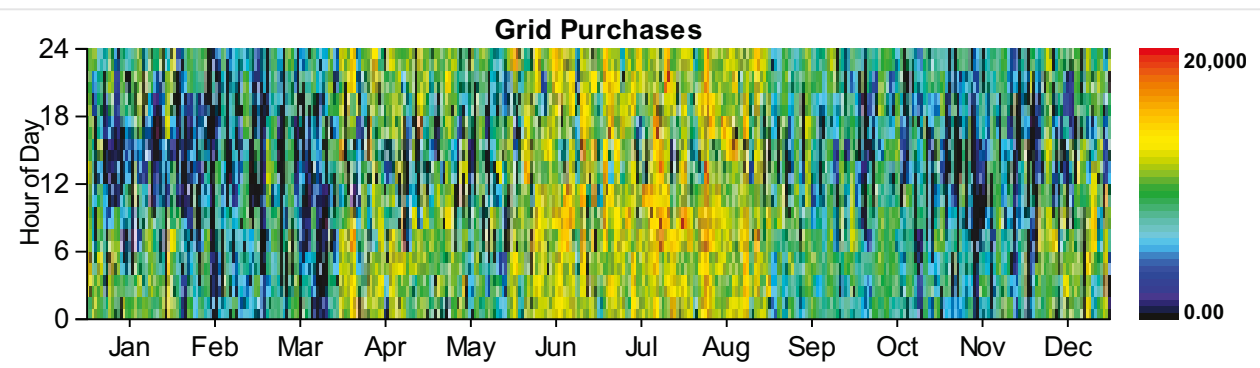

\section{Emissions}

\begin{tabular}{|l|r|l|}
\hline Pollutant & Emissions & Units \\
\hline Carbon dioxide & 43192712 & $\mathrm{~kg} / \mathrm{yr}$ \\
\hline Carbon monoxide & $144 \mathrm{~kg} / \mathrm{yr}$ \\
\hline Unburned hydrocarbons & $16 \mathrm{~kg} / \mathrm{yr}$ \\
\hline Particulate matter & $4 \mathrm{~kg} / \mathrm{yr}$ \\
\hline Sulfur dioxide & $187182 \mathrm{~kg} / \mathrm{yr}$ \\
\hline Nitrogen oxides & $91650 \mathrm{~kg} / \mathrm{yr}$ \\
\hline
\end{tabular}

HOMER Energy, LLC @ 2016 
System Report

System architecture

\begin{tabular}{|l|l|r|}
\hline Wind Turbine & Siemens 2.3 MW - 108 & 5 \\
\hline Grid & Grid & 25,000 \\
\hline Dispatch Strategy & Cycle Charging & \\
\hline
\end{tabular}

Cost summary

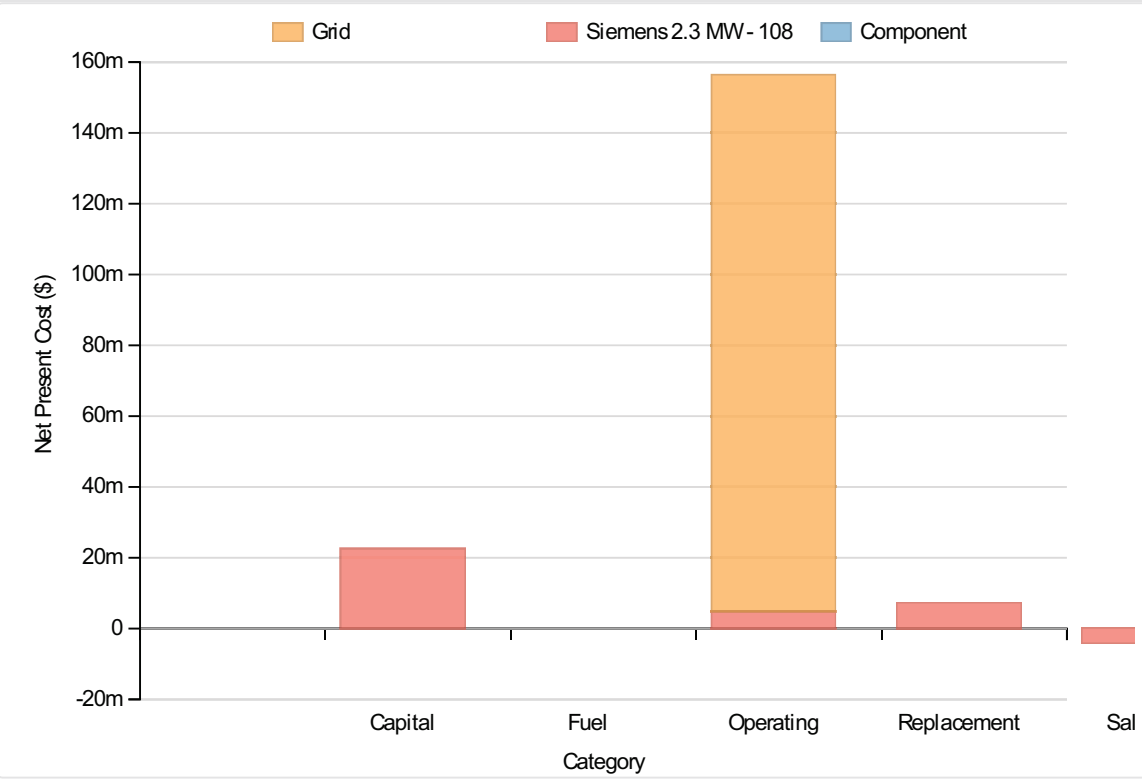

Cost Summary

Total net present cost

$182065680 \$$

Levelized cost of energy

$0.145 \$ / k W h$

Net Present Costs

\begin{tabular}{|l|r|r|r|r|r|r|}
\hline Component & Capital & Replacement & O\&M & Fuel & \multicolumn{2}{l|}{ Salvage } \\
\hline Siemens 2.3 MW-108 & $22,500,000$ & $7,173,158$ & $4,847,816$ & 0 & $-4,042,534$ & $30,478,440$ \\
\hline Grid & 0 & 0 & $151,587,152$ & 0 & 0 & $151,587,152$ \\
\hline System & $22,500,000$ & $7,173,158$ & $156,434,976$ & 0 & $-4,042,534$ & $182,065,600$ \\
\hline
\end{tabular}

Annualized Costs

\begin{tabular}{|l|r|r|r|r|r|r|}
\hline Component & Capital & Replacement & O\&M & Fuel & Salvage & Total \\
\hline Siemens 2.3 MW - 108 & $1,740,474$ & 554,875 & 375,000 & 0 & $-312,708$ & $2,357,641$ \\
\hline Grid & 0 & 0 & $11,725,930$ & 0 & 0 & $11,725,930$ \\
\hline System & $1,740,474$ & 554,875 & $12,100,930$ & 0 & $-312,708$ & $14,083,571$ \\
\hline
\end{tabular}




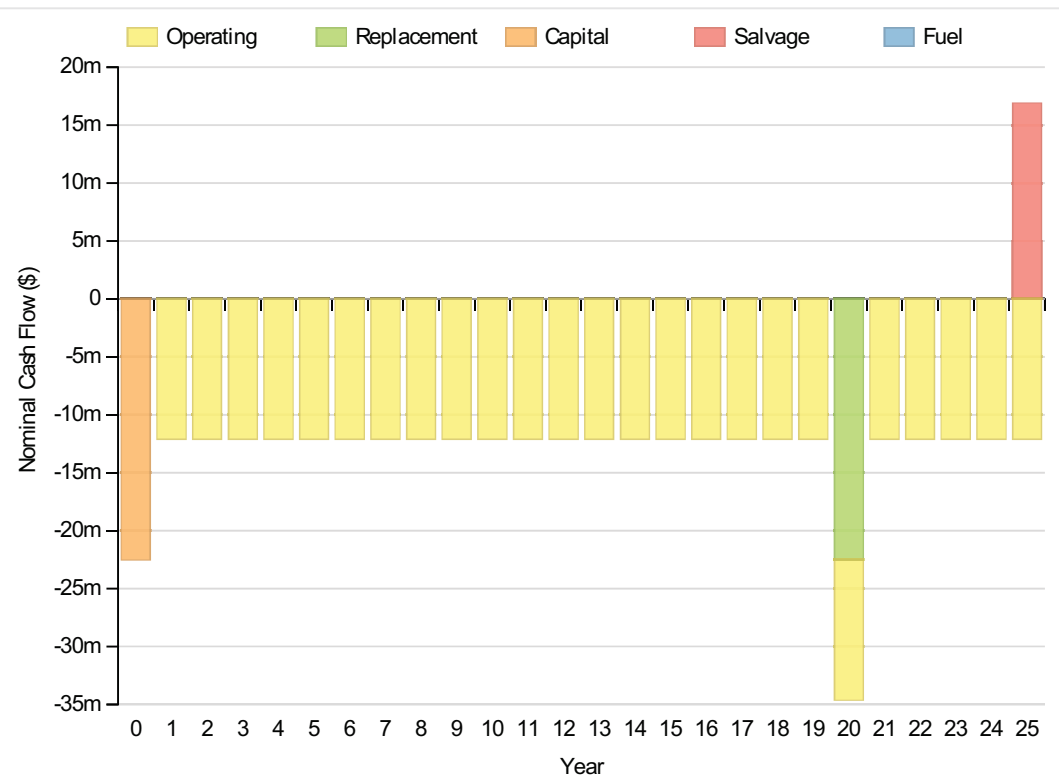

Electrical

\begin{tabular}{|c|c|c|c|c|}
\hline \multirow{2}{*}{\multicolumn{2}{|c|}{$\begin{array}{l}\text { Quantity } \\
\text { Excess electricity }\end{array}$}} & \multicolumn{2}{|l|}{ Value } & Units \\
\hline & & 174 & 42167 & $\mathrm{kWh} / \mathrm{yr}$ \\
\hline Unmet load & & & 0 & $\mathrm{kWh} / \mathrm{yr}$ \\
\hline Capacity shortage & & & 1840 & $\mathrm{kWh} / \mathrm{yr}$ \\
\hline Renewable fraction & & & 0 & \\
\hline Component & Production(kWh/yr) & & =ractio & n (\%) \\
\hline Wind Turbine & & $30,268,832$ & & 31 \\
\hline Grid Purchases & & $68,337,504$ & & 69 \\
\hline Total & & $98,606,336$ & & 100 \\
\hline Load & Consumption(kWh/yr) & & Fract & tion (\%) \\
\hline AC primary load & & $96,864,160$ & & 100 \\
\hline DC primary load & & 0 & & 0 \\
\hline Total & & $96,864,160$ & & 100 \\
\hline
\end{tabular}




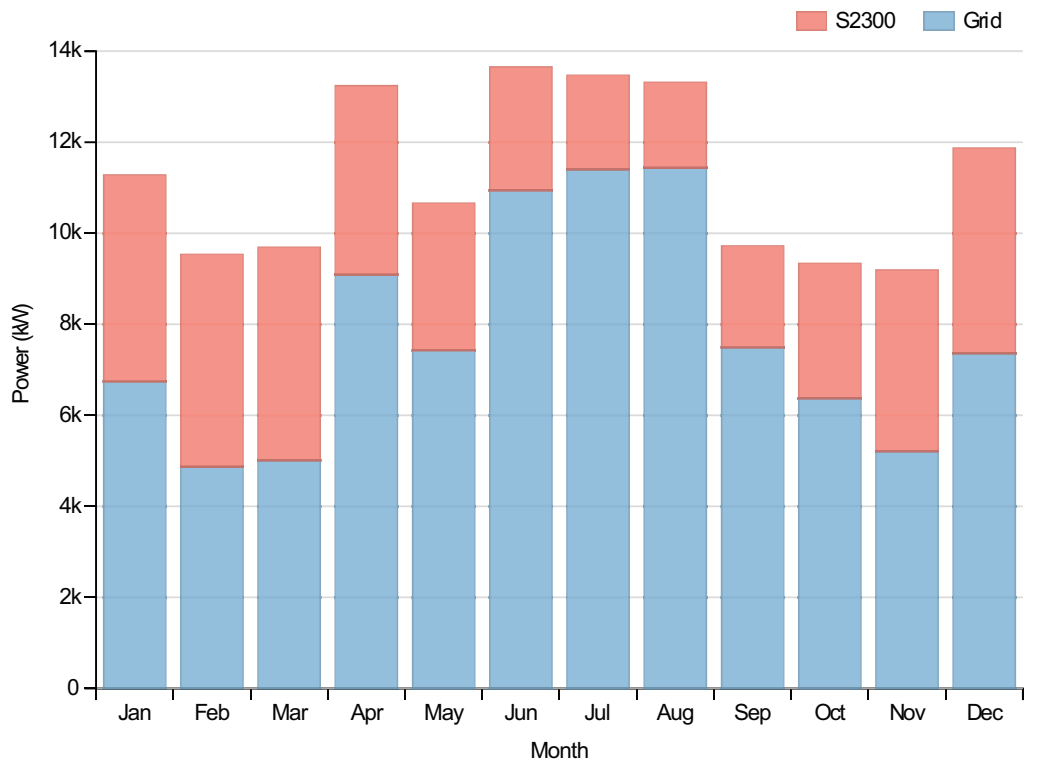

Wind Turbine:Siemens 2.3 MW - 108

\begin{tabular}{|c|c|c|}
\hline Quantity & Value & Units \\
\hline Total rated capacity & 11500 & kW \\
\hline Mean output & 3455 & kW \\
\hline Capacity factor & 30.05 & $\%$ \\
\hline Total production & 30268832 & $\mathrm{kWh} / \mathrm{yr}$ \\
\hline Minimum output & 7.19 & $\mathrm{~kW}$ \\
\hline Maximum output & 11574.00 & kW \\
\hline Wind penetration & 31.25 & $\%$ \\
\hline Hours of operation & 8760 & $\mathrm{hrs} / \mathrm{yr}$ \\
\hline Levelized cost & 0.078 & $\$ / k W h$ \\
\hline
\end{tabular}

\section{Grid}

Rate: Demand 1

\begin{tabular}{|c|c|c|c|c|c|c|}
\hline Resources.ReportingService_GeneratelnputsReport_Month & $\begin{array}{l}\text { Energy } \\
\text { Purchased } \\
\text { (kWh) }\end{array}$ & $\begin{array}{l}\text { Energy } \\
\text { Sold } \\
\text { (kWh) }\end{array}$ & $\begin{array}{l}\text { Net } \\
\text { Purchases } \\
\text { (kWh) }\end{array}$ & $\begin{array}{l}\text { Peak } \\
\text { Demand } \\
(\mathbf{k W})\end{array}$ & $\begin{array}{l}\text { Energy } \\
\text { Charge } \\
(\$)\end{array}$ & $\begin{array}{l}\text { Demand } \\
\text { Charge } \\
\text { (\$) }\end{array}$ \\
\hline January & 0 & 0 & 0 & 18,095 & 0 & 289,519 \\
\hline February & 0 & 0 & 0 & 15,686 & 0 & 250,977 \\
\hline March & 0 & 0 & 0 & 16,699 & 0 & 267,178 \\
\hline April & 0 & 0 & 0 & 20,413 & 0 & 326,604 \\
\hline May & 0 & 0 & 0 & 17,784 & 0 & 284,539 \\
\hline June & 0 & 0 & 0 & 21,607 & 0 & 345,713 \\
\hline July & 0 & 0 & 0 & 23,804 & 0 & 380,861 \\
\hline August & 0 & 0 & 0 & 21,981 & 0 & 351,699 \\
\hline September & 0 & 0 & 0 & 15,890 & 0 & 254,236 \\
\hline
\end{tabular}




\begin{tabular}{|c|c|c|c|c|c|c|}
\hline Rovember_ReportingService_GeneratelnputsReport_Month & $\begin{array}{l}\text { Energy } 0 \\
\text { Purchased } \\
\text { (kWh) } 0\end{array}$ & $\begin{array}{l}\text { Energy } \\
\text { Sold } \\
\text { (kWh) } 0\end{array}$ & $\begin{array}{l}\text { Net } \\
\text { Purchases } \\
\text { (kWh) }\end{array}$ & $\begin{array}{l}\text { Peak } 843 \\
\text { Demand } \\
\text { (kWh), } 876\end{array}$ & $\begin{array}{l}\text { Energy } \\
\text { Charge } \\
(\$)\end{array}$ & $\begin{array}{l}\text { Dérianient } \\
\text { Charge } \\
\text { (\$5 }^{54,021}\end{array}$ \\
\hline December & 0 & 0 & 0 & 18,662 & 0 & 298,596 \\
\hline Annual & 0 & 0 & 0 & 23,804 & 0 & $3,525,437$ \\
\hline
\end{tabular}

Rate: Rate 1

\begin{tabular}{|c|c|c|c|c|c|c|}
\hline Resources.ReportingService_GeneratelnputsReport_Month & $\begin{array}{l}\text { Energy } \\
\text { Purchased } \\
\text { (kWh) }\end{array}$ & $\begin{array}{l}\text { Energy } \\
\text { Sold } \\
\text { (kWh) }\end{array}$ & $\begin{array}{l}\text { Net } \\
\text { Purchases } \\
\text { (kWh) }\end{array}$ & $\begin{array}{l}\text { Peak } \\
\text { Demand } \\
(\mathbf{k W})\end{array}$ & $\begin{array}{l}\text { Energy } \\
\text { Charge } \\
\text { (\$) }\end{array}$ & $\begin{array}{l}\text { Demand } \\
\text { Charge } \\
(\$)\end{array}$ \\
\hline January & $5,015,457$ & 0 & $5,015,457$ & 0 & 601,855 & 0 \\
\hline February & $3,272,783$ & 0 & $3,272,783$ & 0 & 392,734 & 0 \\
\hline March & $3,728,512$ & 0 & $3,728,512$ & 0 & 447,421 & 0 \\
\hline April & $6,545,765$ & 0 & $6,545,765$ & 0 & 785,492 & 0 \\
\hline May & $5,524,917$ & 0 & $5,524,917$ & 0 & 662,990 & 0 \\
\hline June & $7,881,712$ & 0 & $7,881,712$ & 0 & 945,805 & 0 \\
\hline July & $8,488,840$ & 0 & $8,488,840$ & 0 & $1,018,661$ & 0 \\
\hline August & $8,509,350$ & 0 & $8,509,350$ & 0 & $1,021,122$ & 0 \\
\hline September & $5,395,241$ & 0 & $5,395,241$ & 0 & 647,429 & 0 \\
\hline October & $4,744,924$ & 0 & $4,744,924$ & 0 & 569,391 & 0 \\
\hline November & $3,750,403$ & 0 & $3,750,403$ & 0 & 450,048 & 0 \\
\hline December & $5,479,597$ & 0 & $5,479,597$ & 0 & 657,552 & 0 \\
\hline Annual & $68,337,504$ & 0 & $68,337,504$ & 0 & $8,200,500$ & 0 \\
\hline
\end{tabular}

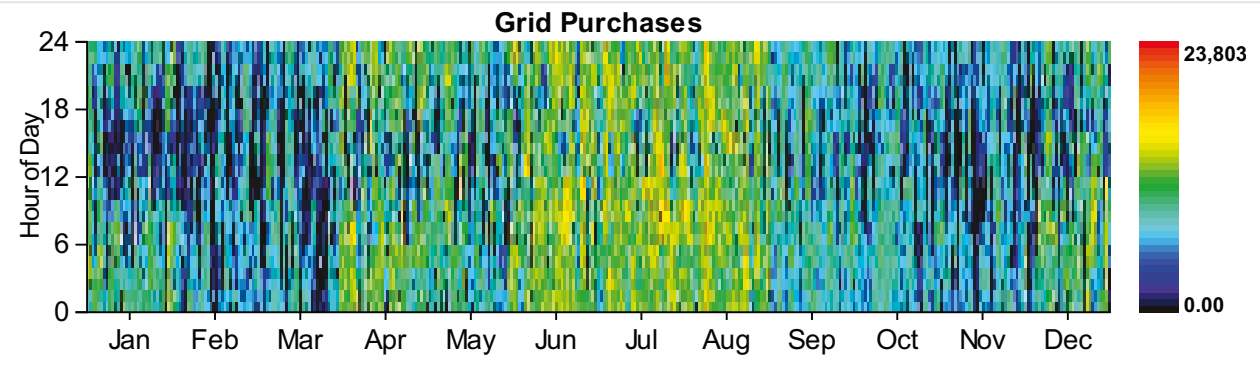

\section{Emissions}

\section{Pollutant}

Emissions

Units

Carbon dioxide

$43189304 \mathrm{~kg} / \mathrm{yr}$

Carbon monoxide

$0 \mathrm{~kg} / \mathrm{yr}$

Unburned hydrocarbons

$0 \mathrm{~kg} / \mathrm{yr}$

Particulate matter

$0 \mathrm{~kg} / \mathrm{yr}$

Sulfur dioxide

$187245 \mathrm{~kg} / \mathrm{yr}$

Nitrogen oxides 
HOMER Energy, LLC @ 2016 
System Report

System architecture

\begin{tabular}{|l|l|r|}
\hline Wind Turbine & Siemens 2.3 MW - 108 & 5 \\
\hline Generator & Kohler 1000 Prime Power & 925 \\
\hline Generator \#2 & Kohler 750 Prime Power & 690 \\
\hline Battery & GS200 flow & 1 \\
\hline Converter & System Converter & strings \\
\hline Grid & Grid & 250 \\
\hline Dispatch Strategy & Cycle Charging & 20,000 \\
\hline
\end{tabular}

\section{Cost summary}

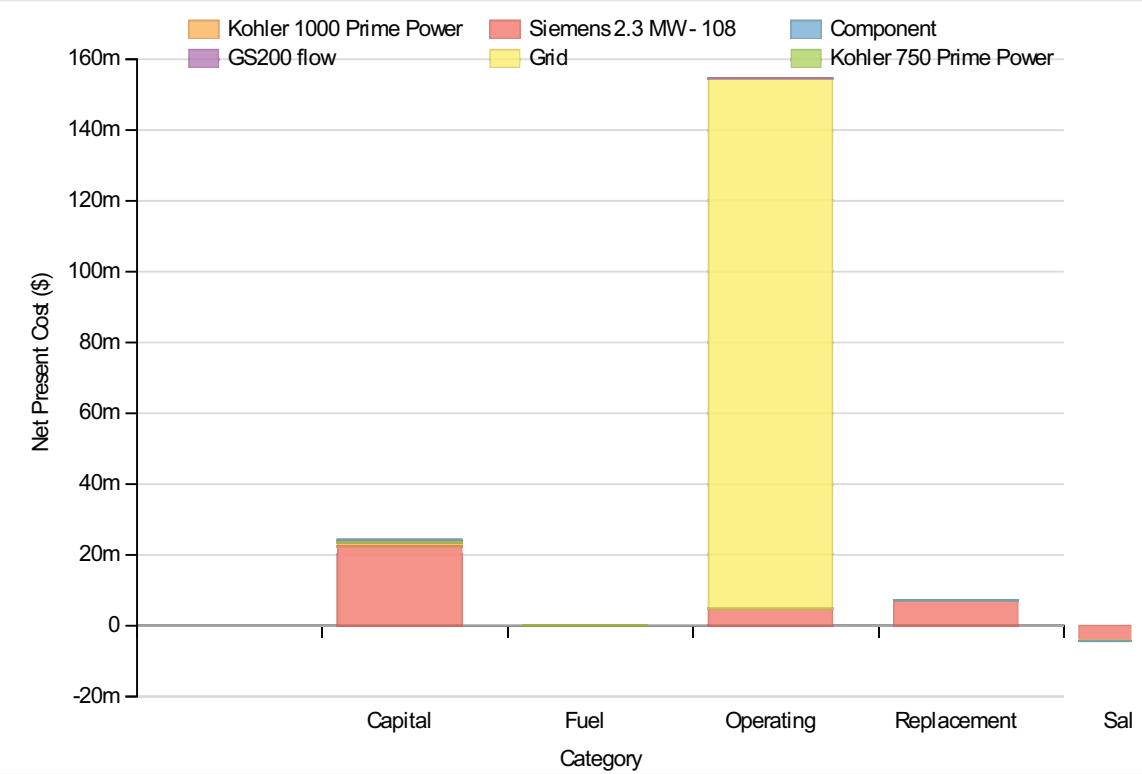

Cost Summary

Total net present cost

$182180144 \$$

Levelized cost of energy

$0.145 \$ / k W h$

Net Present Costs

\begin{tabular}{|c|c|c|c|c|c|c|}
\hline Component & Capital & Replacement & O\&M & Fuel & Salvage & Total \\
\hline Siemens 2.3 MW - 108 & $22,500,000$ & $7,173,158$ & $4,847,816$ & 0 & $-4,042,534$ & $30,478,440$ \\
\hline Kohler 1000 Prime Power & 925,000 & 0 & 13,811 & 110,277 & $-193,153$ & 855,935 \\
\hline Kohler 750 Prime Power & 690,000 & 0 & 13,514 & 85,319 & $-137,470$ & 651,363 \\
\hline Grid & 0 & 0 & $149,731,712$ & 0 & 0 & $149,731,712$ \\
\hline GS200 flow & 324,589 & 7,176 & 31,026 & 0 & -973 & 361,818 \\
\hline Converter & 75,000 & 31,821 & 0 & 0 & $-5,989$ & 100,832 \\
\hline System & $24,514,588$ & $7,212,154$ & $154,637,856$ & 195,597 & $-4,380,119$ & $182,180,076$ \\
\hline
\end{tabular}




\begin{tabular}{|c|c|c|c|c|c|c|}
\hline Component & Capital & Replacement & O\&M & Fuel & Salvage & Total \\
\hline Siemens 2.3 MW - 108 & $1,740,474$ & 554,875 & 375,000 & 0 & $-312,708$ & $2,357,641$ \\
\hline Kohler 1000 Prime Power & 71,553 & 0 & 1,068 & 8,530 & $-14,941$ & 66,211 \\
\hline Kohler 750 Prime Power & 53,375 & 0 & 1,045 & 6,600 & $-10,634$ & 50,386 \\
\hline Grid & 0 & 0 & $11,582,404$ & 0 & 0 & $11,582,404$ \\
\hline GS200 flow & 25,108 & 555 & 2,400 & 0 & -75 & 27,988 \\
\hline Converter & 5,802 & 2,462 & 0 & 0 & -463 & 7,800 \\
\hline System & $1,896,311$ & 557,892 & $11,961,915$ & 15,130 & $-338,821$ & $14,092,427$ \\
\hline
\end{tabular}

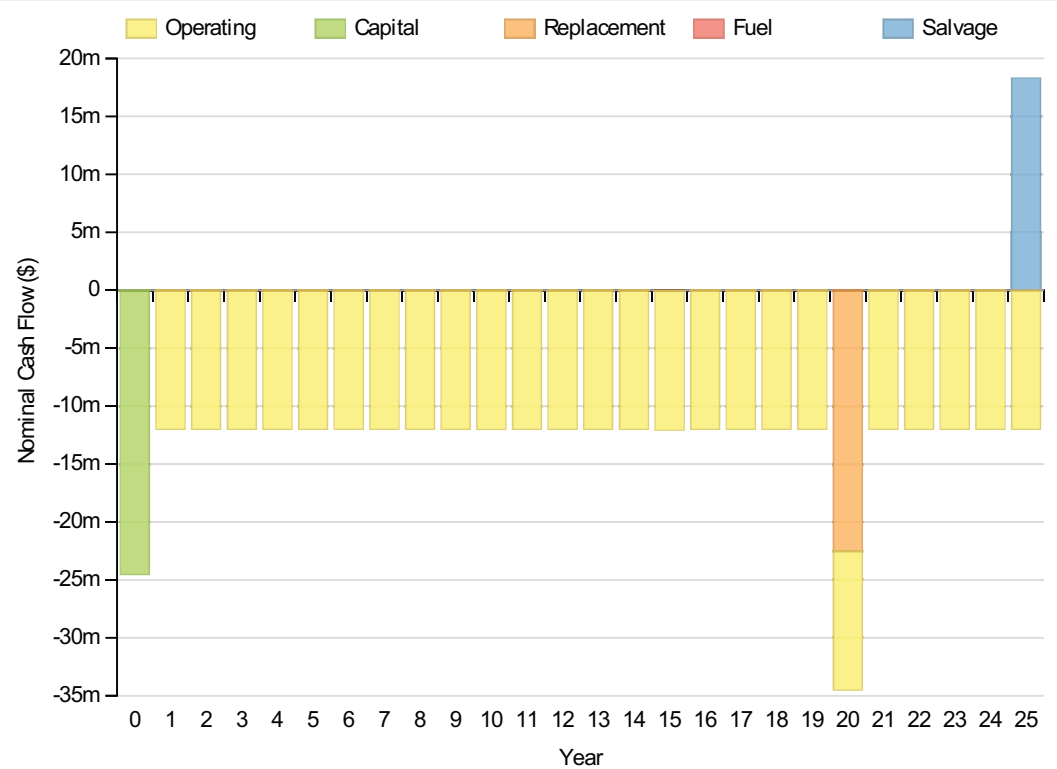

\section{Electrical}

\section{Quantity}

Excess electricity

Unmet load

Capacity shortage

Renewable fraction

\section{Component}

Generator

Generator

Wind Turbine

Grid Purchases

Total

Load

Consumption(kWh/yr)

\section{Value}

Production(kWh/yr)

37,426

28,561

$30,268,832$

$68,205,056$

$98,539,872$

\section{Fraction (\%)}

$1607580 \mathrm{kWh} / \mathrm{yr}$

$4013 \mathrm{kWh} / \mathrm{yr}$

$44676 \mathrm{kWh} / \mathrm{yr}$

0 


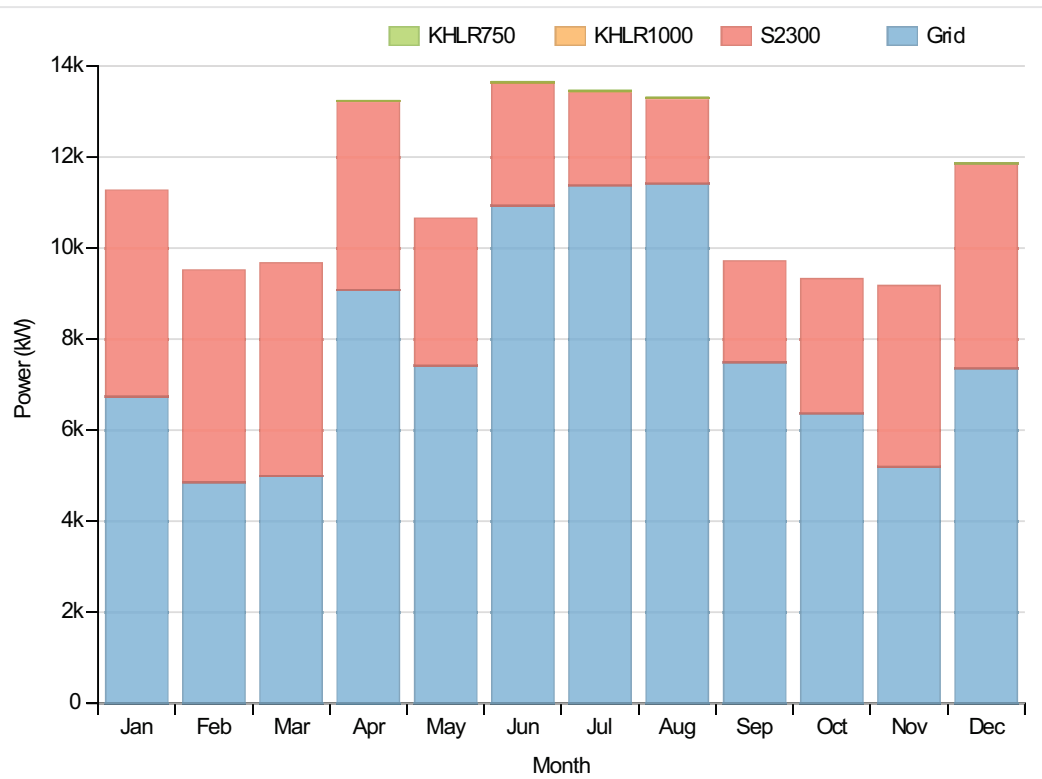

Wind Turbine:Siemens 2.3 MW - 108

\begin{tabular}{|c|c|c|}
\hline Quantity & Value & Units \\
\hline Total rated capacity & 11500 & $\mathrm{~kW}$ \\
\hline Mean output & 3455 & $\mathrm{~kW}$ \\
\hline Capacity factor & 30.05 & $\%$ \\
\hline Total production & 30268832 & $\mathrm{kWh} / \mathrm{yr}$ \\
\hline Minimum output & 7.19 & $\mathrm{~kW}$ \\
\hline Maximum output & 11574.00 & $\mathrm{~kW}$ \\
\hline Wind penetration & 31.25 & $\%$ \\
\hline Hours of operation & 8760 & $\mathrm{hrs} / \mathrm{yr}$ \\
\hline Levelized cost & 0.078 & $\$ / k W h$ \\
\hline
\end{tabular}

\section{Generator:Kohler 1000 Prime Power}

\section{Quantity}

Hours of operation

Number of starts

Operational life

Fixed generation cost

Marginal generation cost

Electrical production

Mean electrical output

Min. electrical output

Max. electrical output

\begin{tabular}{|r|l|l|}
\hline Value & Units \\
\hline 77 & $\mathrm{hrs} / \mathrm{yr}$ \\
\hline 73 & starts/yr \\
\hline 195 & $\mathrm{yr}$ \\
\hline 76.24 & $\$ / \mathrm{hr}$ \\
\hline 0.23 & $\$ / \mathrm{kWh}$ \\
\hline 37426 & $\mathrm{kWh} / \mathrm{yr}$ \\
\hline 486 & $\mathrm{~kW}$ \\
\hline 231 & $\mathrm{~kW}$ \\
\hline 925 & $\mathrm{~kW}$ \\
\hline
\end{tabular}




\begin{tabular}{|c|c|c|c|}
\hline Qutififfifsumption & Value & 10798 & UMits \\
\hline Specific fuel consumption & & 0.29 & $\mathrm{~L} / \mathrm{kWh}$ \\
\hline Fuel energy input & & 106252 & $\mathrm{kWh} / \mathrm{yr}$ \\
\hline Mean electrical efficiency & & 35 & $\%$ \\
\hline
\end{tabular}

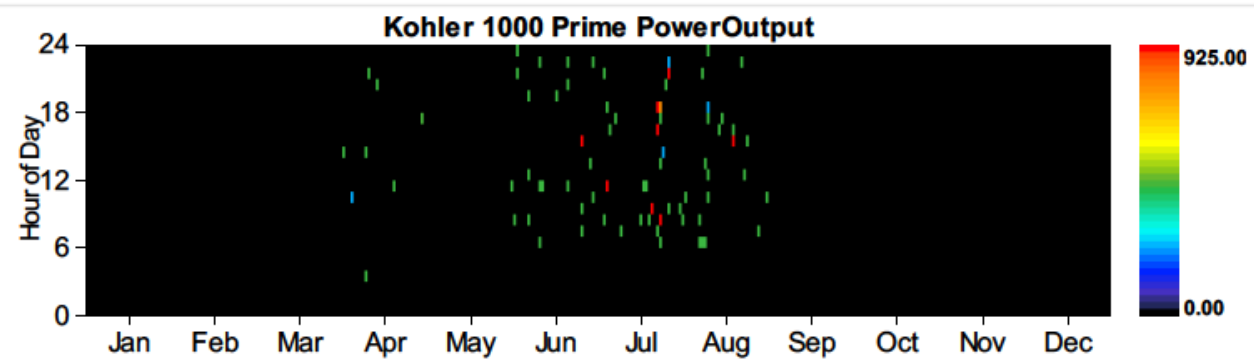

\section{Generator:Kohler 750 Prime Power}

\begin{tabular}{|c|c|c|}
\hline Quantity & Value & Units \\
\hline Hours of operation & 101 & hrs/yr \\
\hline Number of starts & 95 & starts/yr \\
\hline Operational life & 149 & yr \\
\hline Fixed generation cost & 56.87 & $\$ / \mathrm{hr}$ \\
\hline Marginal generation cost & 0.23 & $\$ / \mathrm{kWh}$ \\
\hline Electrical production & 28561 & $\mathrm{kWh} / \mathrm{yr}$ \\
\hline Mean electrical output & 283 & $\mathrm{~kW}$ \\
\hline Min. electrical output & 173 & $\mathrm{~kW}$ \\
\hline Max. electrical output & 690 & $\mathrm{~kW}$ \\
\hline Fuel consumption & 8354 & L/yr \\
\hline Specific fuel consumption & 0.29 & $\mathrm{~L} / \mathrm{kWh}$ \\
\hline Fuel energy input & 82206 & $\mathrm{kWh} / \mathrm{yr}$ \\
\hline Mean electrical efficiency & 35 & $\%$ \\
\hline
\end{tabular}

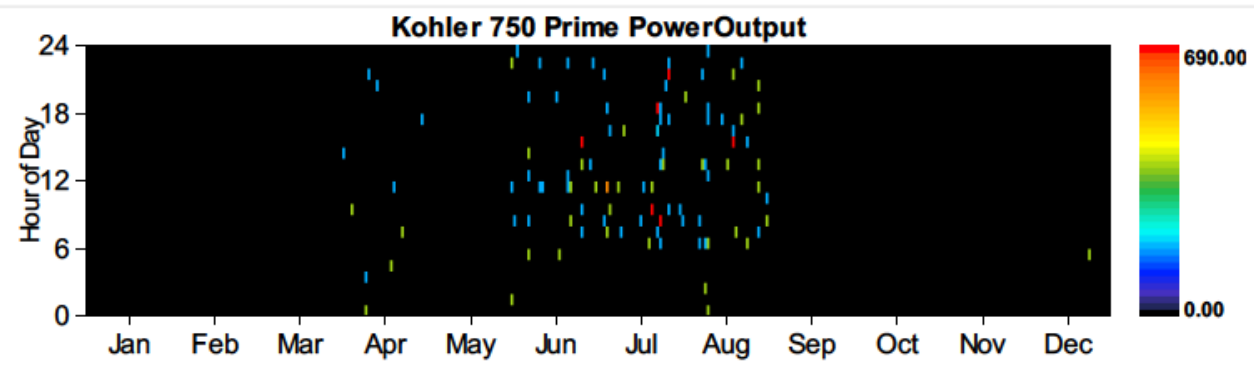

\section{Battery:GS200 flow}

\section{Quantity}

Value

String size 


\section{Quantity}

Value

Units

Nominal capacity

$600 \mathrm{kWh}$

Usable nominal capacity

600 kWh

Autonomy

$0 \mathrm{hr}$

Lifetime throughput

Battery wear cost

$0.000 \$ / \mathrm{kWh}$

Average energy cost

$0.036 \$ / k W h$

Energy in

$132844 \mathrm{kWh} / \mathrm{yr}$

Energy out

$93493 \mathrm{kWh} / \mathrm{yr}$

Storage depletion

$600 \mathrm{kWh} / \mathrm{yr}$

Losses

$38751 \mathrm{kWh} / \mathrm{yr}$

Annual throughput

111746 kWh/yr

Expected life

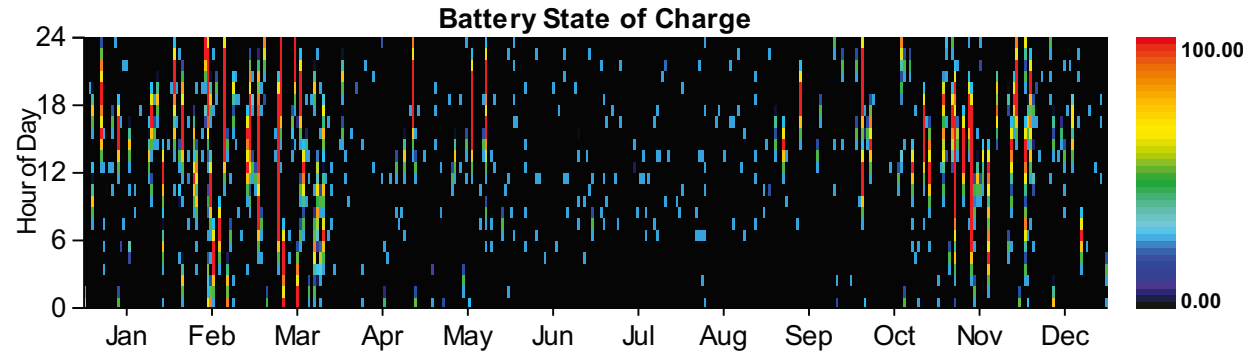

\section{Converter}

\section{Quantity}

Capacity

Mean output

Minimum output

Maximum output

Capacity factor

Hours of operation

Energy in

Energy out

Losses

\section{Inverter}

Rectifier

250

10

0

166

4

874

93,493

84,144

9,350
Units

225 kW

$15 \mathrm{~kW}$

$0 \mathrm{~kW}$

179 kW

$6 \%$

$1,016 \mathrm{hrs} / \mathrm{yr}$

$156,288 \mathrm{kWh} / \mathrm{yr}$

$132,844 \mathrm{kWh} / \mathrm{yr}$

23,444 kWh/yr 

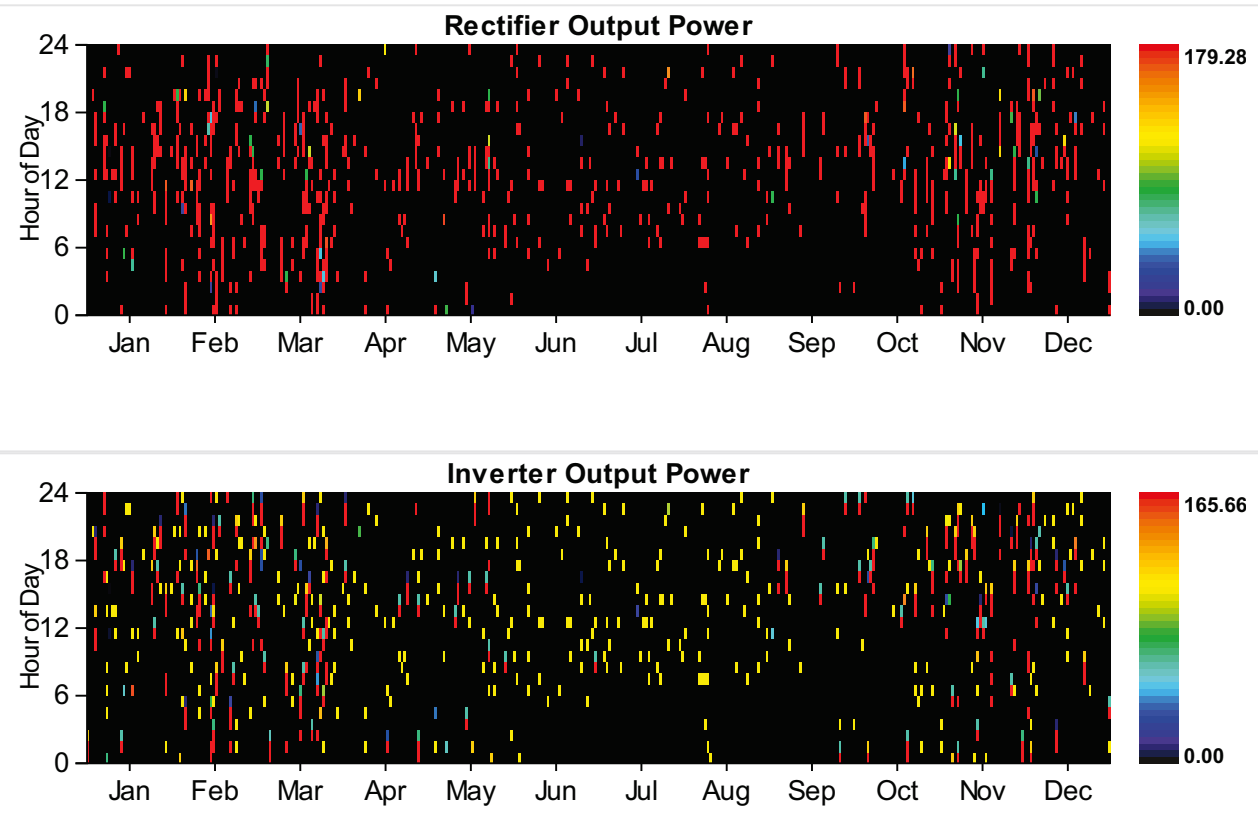

\section{Grid}

Rate: Demand 1

\begin{tabular}{|c|c|c|c|c|c|c|}
\hline Resources.ReportingService_GeneratelnputsReport_Month & $\begin{array}{l}\text { Energy } \\
\text { Purchased } \\
\text { (kWh) }\end{array}$ & $\begin{array}{l}\text { Energy } \\
\text { Sold } \\
\text { (kWh) }\end{array}$ & $\begin{array}{l}\text { Net } \\
\text { Purchases } \\
\text { (kWh) }\end{array}$ & $\begin{array}{l}\text { Peak } \\
\text { Demand } \\
(\mathbf{k W})\end{array}$ & $\begin{array}{l}\text { Energy } \\
\text { Charge } \\
\text { (\$) }\end{array}$ & $\begin{array}{l}\text { Demand } \\
\text { Charge } \\
\text { (\$) }\end{array}$ \\
\hline January & 0 & 0 & 0 & 18,095 & 0 & 289,519 \\
\hline February & 0 & 0 & 0 & 15,686 & 0 & 250,977 \\
\hline March & 0 & 0 & 0 & 16,699 & 0 & 267,178 \\
\hline April & 0 & 0 & 0 & 20,000 & 0 & 320,000 \\
\hline May & 0 & 0 & 0 & 17,784 & 0 & 284,539 \\
\hline June & 0 & 0 & 0 & 20,000 & 0 & 320,000 \\
\hline July & 0 & 0 & 0 & 20,000 & 0 & 320,000 \\
\hline August & 0 & 0 & 0 & 20,000 & 0 & 320,000 \\
\hline September & 0 & 0 & 0 & 15,890 & 0 & 254,236 \\
\hline October & 0 & 0 & 0 & 13,843 & 0 & 221,494 \\
\hline November & 0 & 0 & 0 & 15,876 & 0 & 254,021 \\
\hline December & 0 & 0 & 0 & 18,490 & 0 & 295,836 \\
\hline Annual & 0 & 0 & 0 & 20,000 & 0 & $3,397,800$ \\
\hline
\end{tabular}

Rate: Rate 1

\begin{tabular}{|c|c|c|c|c|c|c|}
\hline Resources.ReportingService_GeneratelnputsReport_Month & $\begin{array}{l}\text { Energy } \\
\text { Purchased } \\
\text { (kWh) }\end{array}$ & $\begin{array}{l}\text { Energy } \\
\text { Sold } \\
\text { (kWh) }\end{array}$ & $\begin{array}{l}\text { Net } \\
\text { Purchases } \\
\text { (kWh) }\end{array}$ & $\begin{array}{l}\text { Peak } \\
\text { Demand } \\
(\mathbf{k W})\end{array}$ & $\begin{array}{l}\text { Energy } \\
\text { Charge } \\
(\$)\end{array}$ & $\begin{array}{l}\text { Demand } \\
\text { Charge } \\
(\$)\end{array}$ \\
\hline January & $5,007,178$ & 0 & $5,007,178$ & 0 & 600,861 & 0 \\
\hline February & $3,260,560$ & 0 & $3,260,560$ & 0 & 391,267 & 0 \\
\hline March & $3,715,573$ & 0 & $3,715,573$ & 0 & 445,869 & 0 \\
\hline
\end{tabular}




\begin{tabular}{|c|c|c|c|c|c|c|}
\hline $\begin{array}{l}\text { April } \\
\text { Reaşources.ReportingService_GeneratelnputsReport_Month }\end{array}$ & $\begin{array}{l}\text { Energy }_{\text {b.j. }}, 505 \\
\text { Purchased } \\
\text { (kWh) } \\
5,5,575\end{array}$ & $\begin{array}{l}\text { Energy } \\
\text { Sold } \\
(\mathbf{k W h})_{0}\end{array}$ & $\begin{array}{l}\text { Net } \\
\text { Purchases } \\
\text { (kwh) } \\
5,5,575\end{array}$ & $\begin{array}{l}\text { Peak } 0 \\
\text { Demand } \\
(\mathbf{k W}) \quad 0\end{array}$ & $\begin{array}{l}\text { Energy } \\
\text { Charge } \\
\text { (\$\$62,349 }\end{array}$ & $\begin{array}{l}\text { Demand } \\
\text { Charge } \\
\text { (\$) } \quad 0\end{array}$ \\
\hline June & $7,866,983$ & 0 & $7,866,983$ & 0 & 944,038 & 0 \\
\hline July & $8,463,189$ & 0 & $8,463,189$ & 0 & $1,015,583$ & 0 \\
\hline August & $8,493,100$ & 0 & $8,493,100$ & 0 & $1,019,172$ & 0 \\
\hline September & $5,392,027$ & 0 & $5,392,027$ & 0 & 647,043 & 0 \\
\hline October & $4,738,349$ & 0 & $4,738,349$ & 0 & 568,602 & 0 \\
\hline November & $3,739,770$ & 0 & $3,739,770$ & 0 & 448,772 & 0 \\
\hline December & $5,471,249$ & 0 & $5,471,249$ & 0 & 656,550 & 0 \\
\hline Annual & $68,205,056$ & 0 & $68,205,056$ & 0 & $8,184,608$ & 0 \\
\hline
\end{tabular}

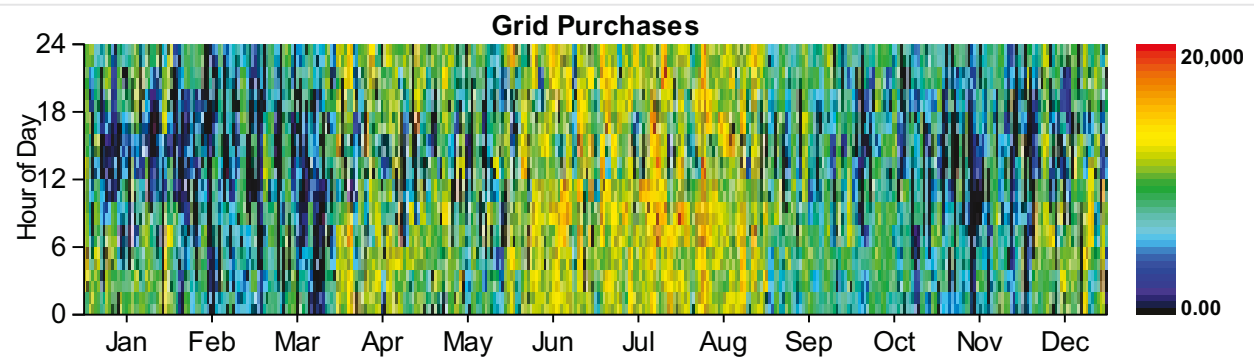

\section{Emissions}

\section{Pollutant}

Carbon dioxide

Carbon monoxide

Unburned hydrocarbons

Particulate matter

Sulfur dioxide

Nitrogen oxides

HOMER Energy, LLC @ 2016
Emissions

Units

$43155860 \mathrm{~kg} / \mathrm{yr}$

$211 \mathrm{~kg} / \mathrm{yr}$

$24 \mathrm{~kg} / \mathrm{yr}$

$6 \mathrm{~kg} / \mathrm{yr}$

186985 kg/yr

91605 kg/yr 
System Report

System architecture

\begin{tabular}{|l|l|r|}
\hline Wind Turbine & Siemens 2.3 MW - 108 & 5 \\
\hline Battery & GS200 flow & 1 \\
\hline Converter & System Converter & 250 \\
\hline Grid & Grid & $\mathrm{kW}$ \\
\hline Dispatch Strategy & Cycle Charging & 25,000 \\
\hline
\end{tabular}

\section{Cost summary}

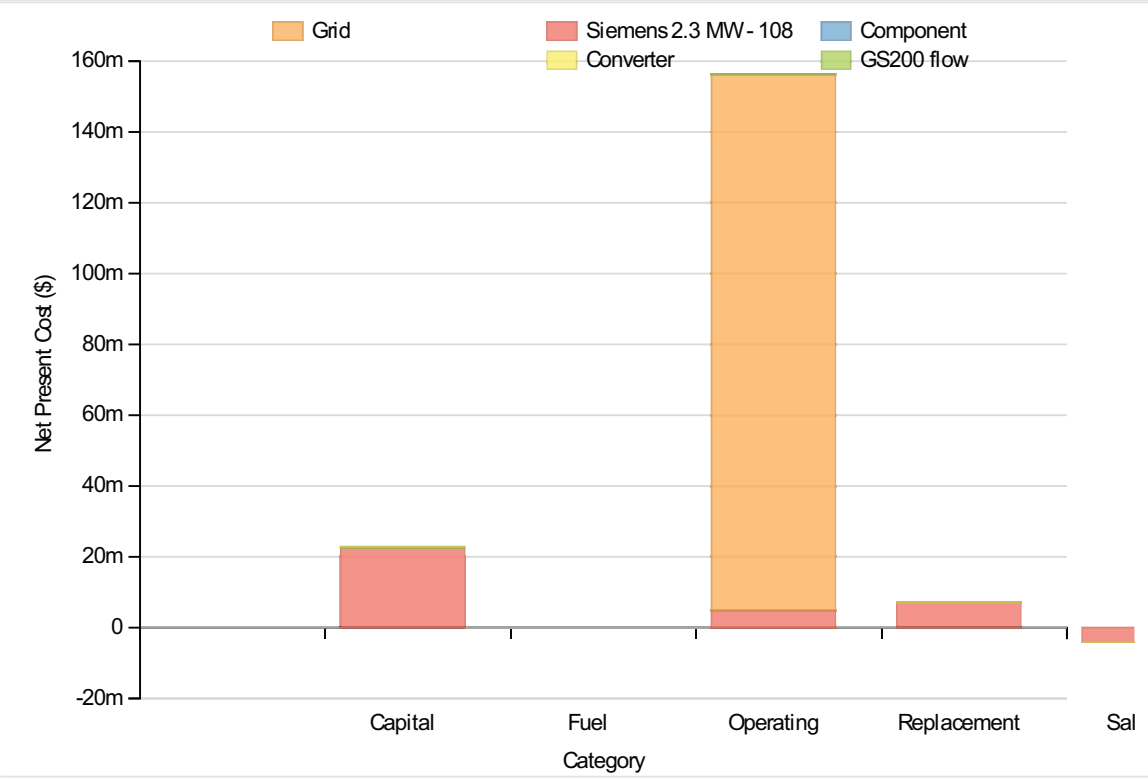

Cost Summary

Total net present cost

$182415824 \$$

Levelized cost of energy

$0.146 \$ / k W h$

Net Present Costs

\begin{tabular}{|l|r|r|r|r|r|r|}
\hline Component & Capital & Replacement & O\&M & Fuel & \multicolumn{1}{l|}{ Salvage } & \multicolumn{1}{l|}{ Total } \\
\hline Siemens 2.3 MW - 108 & $22,500,000$ & $7,173,158$ & $4,847,816$ & 0 & $-4,042,534$ \\
\hline Grid & 0 & 0 & $151,474,640$ & 0 & $151,474,640$ \\
\hline GS200 flow & 324,589 & 7,176 & 31,026 & 0 & -973 \\
\hline Converter & 75,000 & 31,821 & 0 & 0 & $-5,989$ \\
\hline System & $22,899,588$ & $7,212,154$ & $156,353,488$ & 0 & $-4,049,496$ \\
\hline
\end{tabular}

\section{Annualized Costs}

\begin{tabular}{|l|r|r|r|r|r|r|}
\hline Component & Capital & Replacement & O\&M & Fuel & Salvage & Total \\
\hline Siemens 2.3 MW - 108 & $1,740,474$ & 554,875 & 375,000 & 0 & $-312,708$ & $2,357,641$ \\
\hline Grid & 0 & 0 & $11,717,227$ & 0 & 0 & $11,717,227$ \\
\hline GS200 flow & 25,108 & 555 & 2,400 & 0 & -75 & 27,988 \\
\hline
\end{tabular}




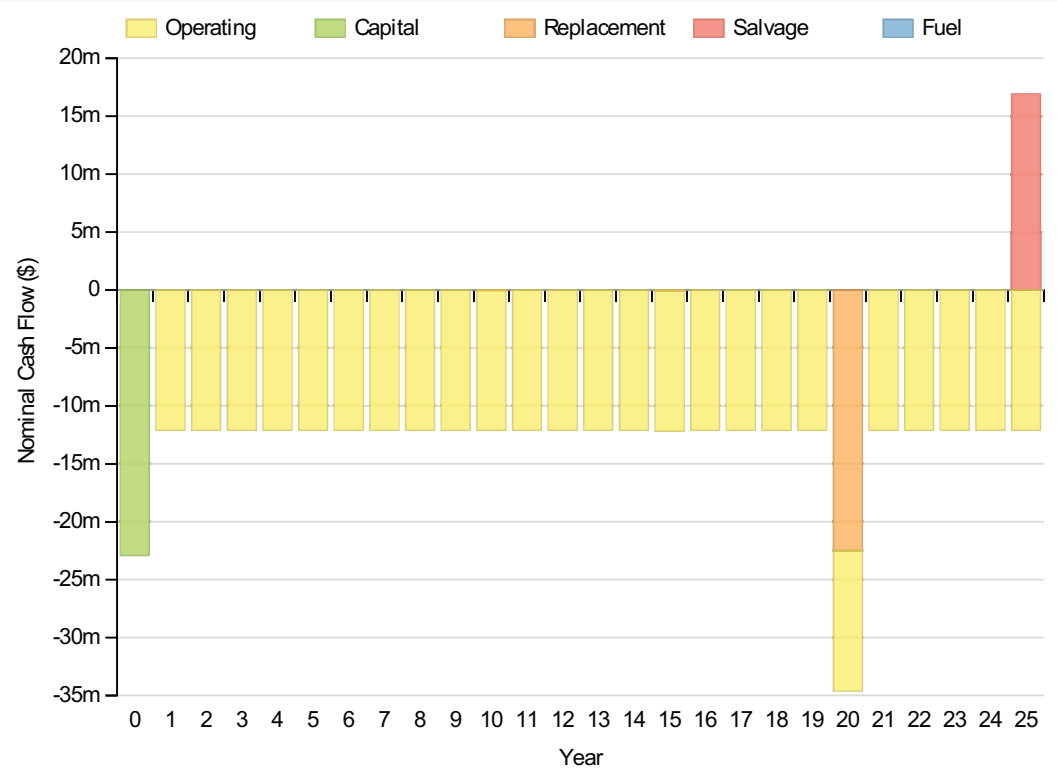

\section{Electrical}

\begin{tabular}{|c|c|c|c|c|}
\hline \multicolumn{2}{|l|}{ Quantity } & \multicolumn{2}{|l|}{ Value } & Units \\
\hline Excess electricity & & 160 & 07580 & $\mathrm{kWh} / \mathrm{yr}$ \\
\hline Unmet load & & & 0 & $\mathrm{kWh} / \mathrm{yr}$ \\
\hline Capacity shortage & & & 1840 & $\mathrm{kWh} / \mathrm{yr}$ \\
\hline Renewable fraction & & & 0 & \\
\hline Component & Production(kWh/yr) & & Eractio & $n(\%)$ \\
\hline Wind Turbine & & $30,268,832$ & & 31 \\
\hline Grid Purchases & & $68,264,976$ & & 69 \\
\hline Total & & $98,533,808$ & & 100 \\
\hline Load & Consumption(kWh/yr) & & Fract & tion $(\%)$ \\
\hline AC primary load & & $96,864,160$ & & 100 \\
\hline DC primary load & & 0 & & 0 \\
\hline Total & & $96,864,160$ & & 100 \\
\hline
\end{tabular}




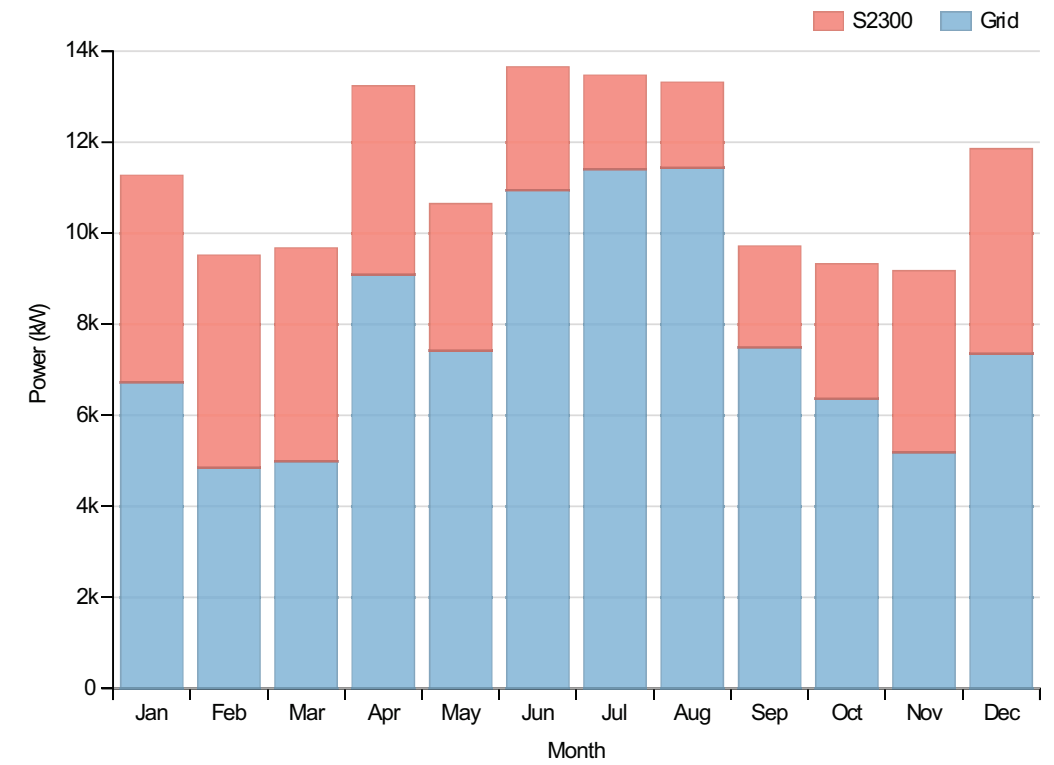

Wind Turbine:Siemens 2.3 MW - 108

\begin{tabular}{|c|c|c|}
\hline Quantity & Value & Units \\
\hline Total rated capacity & 11500 & $\mathrm{~kW}$ \\
\hline Mean output & 3455 & $\mathrm{~kW}$ \\
\hline Capacity factor & 30.05 & $\%$ \\
\hline Total production & 30268832 & $\mathrm{kWh} / \mathrm{yr}$ \\
\hline Minimum output & 7.19 & $\mathrm{~kW}$ \\
\hline Maximum output & 11574.00 & $\mathrm{~kW}$ \\
\hline Wind penetration & 31.25 & $\%$ \\
\hline Hours of operation & 8760 & $\mathrm{hrs} / \mathrm{yr}$ \\
\hline Levelized cost & 0.078 & $\$ / k W h$ \\
\hline
\end{tabular}

\section{Battery:GS200 flow}

\begin{tabular}{|l|l|l}
\hline Quantity & Value
\end{tabular}

String size

Strings in parallel

\section{Quantity}

Nominal capacity

Usable nominal capacity

\section{Autonomy}

Lifetime throughput

Battery wear cost

Average energy cost

\section{Value}

\section{Units}

$600 \mathrm{kWh}$

$600 \mathrm{kWh}$

$0 \mathrm{hr}$

0

$0.000 \$ / k W h$ 


\section{Quantity}

Energy in

Energy out

Storage depletion

Losses

Annual throughput

Expected life

\section{Value}

Units

114399 kWh/yr

80582 kWh/yr

$600 \mathrm{kWh} / \mathrm{yr}$

$33217 \mathrm{kWh} / \mathrm{yr}$

96314 kWh/yr

$25 \mathrm{yr}$

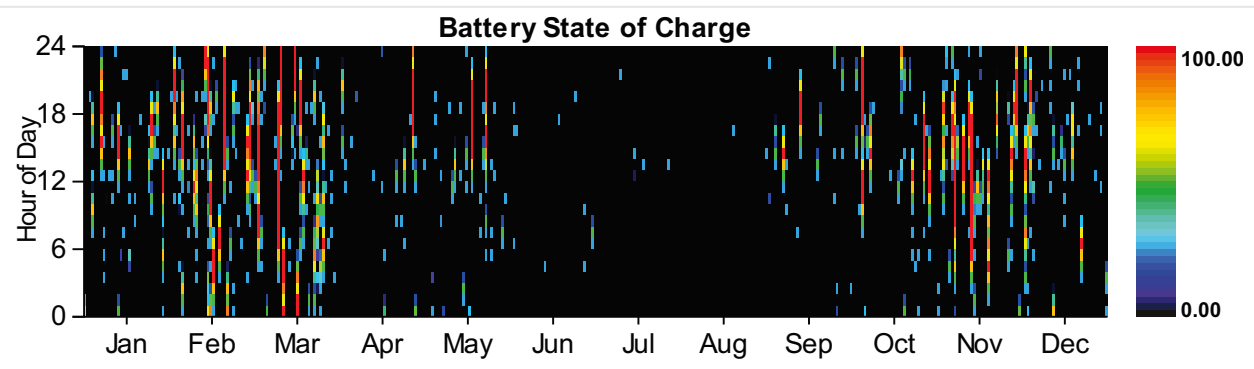

Converter

\begin{tabular}{|c|c|c|c|}
\hline Quantity & Inverter & Rectifier & Units \\
\hline Capacity & 250 & 225 & $\mathrm{~kW}$ \\
\hline Mean output & 8 & 13 & $\mathrm{~kW}$ \\
\hline Minimum output & 0 & 0 & $\mathrm{~kW}$ \\
\hline Maximum output & 166 & 179 & $\mathrm{~kW}$ \\
\hline Capacity factor & 3 & 5 & $\%$ \\
\hline Hours of operation & 777 & 917 & hrs/yr \\
\hline Energy in & 80,582 & 134,588 & $\mathrm{kWh} / \mathrm{yr}$ \\
\hline Energy out & 72,523 & 114,399 & $\mathrm{kWh} / \mathrm{yr}$ \\
\hline Losses & 8,058 & 20,188 & $\mathrm{kWh} / \mathrm{yr}$ \\
\hline
\end{tabular}

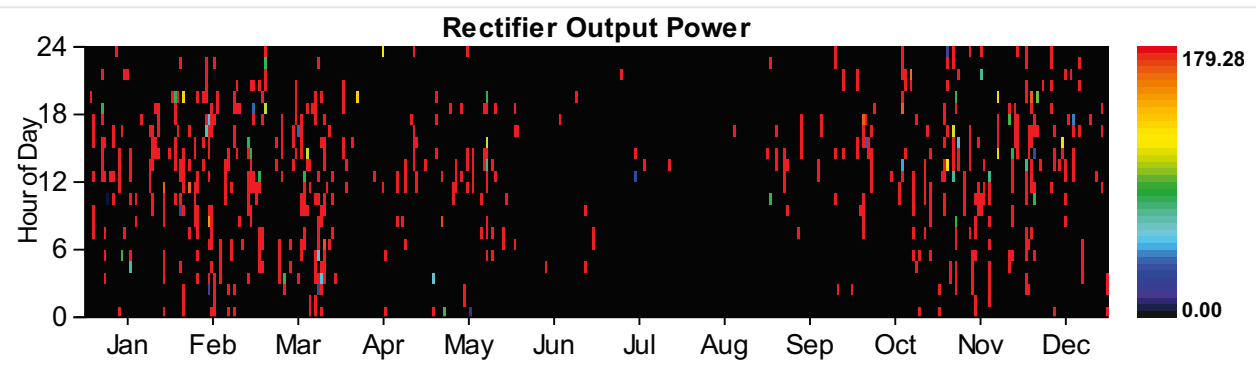




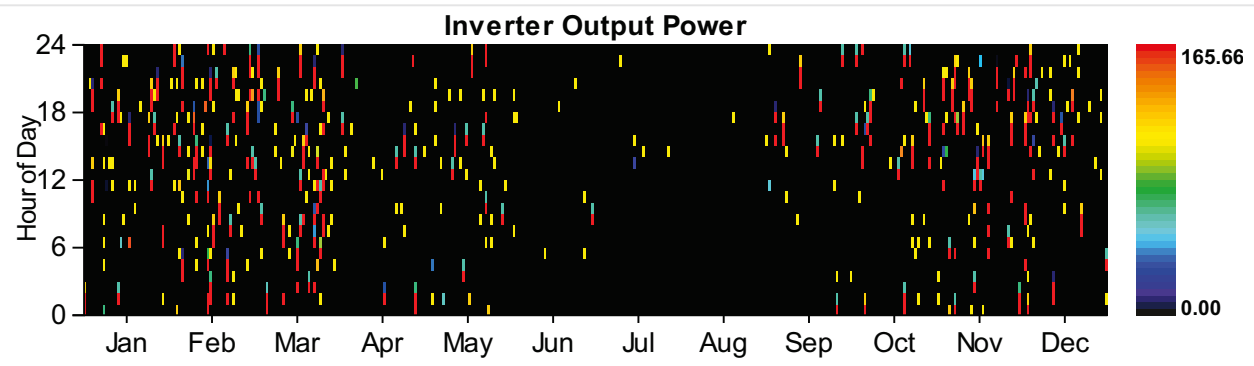

Grid

Rate: Demand 1

\begin{tabular}{|c|c|c|c|c|c|c|}
\hline Resources.ReportingService_GeneratelnputsReport_Month & $\begin{array}{l}\text { Energy } \\
\text { Purchased } \\
\text { (kWh) }\end{array}$ & $\begin{array}{l}\text { Energy } \\
\text { Sold } \\
\text { (kWh) }\end{array}$ & $\begin{array}{l}\text { Net } \\
\text { Purchases } \\
\text { (kWh) }\end{array}$ & $\begin{array}{l}\text { Peak } \\
\text { Demand } \\
(\mathbf{k W})\end{array}$ & $\begin{array}{l}\text { Energy } \\
\text { Charge } \\
\text { (\$) }\end{array}$ & $\begin{array}{l}\text { Demand } \\
\text { Charge } \\
(\$)\end{array}$ \\
\hline January & 0 & 0 & 0 & 18,095 & 0 & 289,519 \\
\hline February & 0 & 0 & 0 & 15,686 & 0 & 250,977 \\
\hline March & 0 & 0 & 0 & 16,699 & 0 & 267,178 \\
\hline April & 0 & 0 & 0 & 20,413 & 0 & 326,604 \\
\hline May & 0 & 0 & 0 & 17,784 & 0 & 284,539 \\
\hline June & 0 & 0 & 0 & 21,607 & 0 & 345,713 \\
\hline July & 0 & 0 & 0 & 23,804 & 0 & 380,861 \\
\hline August & 0 & 0 & 0 & 21,981 & 0 & 351,699 \\
\hline September & 0 & 0 & 0 & 15,890 & 0 & 254,236 \\
\hline October & 0 & 0 & 0 & 13,843 & 0 & 221,494 \\
\hline November & 0 & 0 & 0 & 15,876 & 0 & 254,021 \\
\hline December & 0 & 0 & 0 & 18,662 & 0 & 298,596 \\
\hline Annual & 0 & 0 & 0 & 23,804 & 0 & $3,525,437$ \\
\hline
\end{tabular}

Rate: Rate 1

\begin{tabular}{|c|c|c|c|c|c|c|}
\hline Resources.ReportingService_GeneratelnputsReport_Month & $\begin{array}{l}\text { Energy } \\
\text { Purchased } \\
\text { (kWh) }\end{array}$ & $\begin{array}{l}\text { Energy } \\
\text { Sold } \\
\text { (kWh) }\end{array}$ & $\begin{array}{l}\text { Net } \\
\text { Purchases } \\
\text { (kWh) }\end{array}$ & $\begin{array}{l}\text { Peak } \\
\text { Demand } \\
(\mathbf{k W})\end{array}$ & $\begin{array}{l}\text { Energy } \\
\text { Charge } \\
(\$)\end{array}$ & $\begin{array}{l}\text { Demand } \\
\text { Charge } \\
\text { (\$) }\end{array}$ \\
\hline January & $5,007,178$ & 0 & $5,007,178$ & 0 & 600,861 & 0 \\
\hline February & $3,260,560$ & 0 & $3,260,560$ & 0 & 391,267 & 0 \\
\hline March & $3,715,573$ & 0 & $3,715,573$ & 0 & 445,869 & 0 \\
\hline April & $6,542,332$ & 0 & $6,542,332$ & 0 & 785,080 & 0 \\
\hline May & $5,519,575$ & 0 & $5,519,575$ & 0 & 662,349 & 0 \\
\hline June & $7,880,695$ & 0 & $7,880,695$ & 0 & 945,683 & 0 \\
\hline July & $8,488,147$ & 0 & $8,488,147$ & 0 & $1,018,578$ & 0 \\
\hline August & $8,509,237$ & 0 & $8,509,237$ & 0 & $1,021,108$ & 0 \\
\hline
\end{tabular}




\begin{tabular}{|c|c|c|c|c|c|c|c|}
\hline September & $\begin{array}{l}\text { Ë,Ð9y,027 } \\
\text { Purchased }\end{array}$ & $\begin{array}{l}\text { Energy } \\
\text { Sold }\end{array}$ & $\begin{array}{l}\text { Net392,027 } \\
\text { Purchases }\end{array}$ & $\begin{array}{l}\text { Peak } \\
\text { Dema }\end{array}$ & & $\begin{array}{l}\text { Ebargo } 43 \\
\text { Charge }\end{array}$ & $\begin{array}{l}\text { Demand } \\
\text { Charge }\end{array}$ \\
\hline Besarces.ReportingService_GeneratelnputsReport_Month & $(\mathbf{k}, \mathbf{n} / \mathrm{h}) 8,349$ & $(\mathbf{k W h}) 0$ & $(\mathbf{k}, \mathbf{n}, \mathbf{1}) 8,349$ & $(\mathbf{k W})$ & 0 & $(\$ \$ 68,602$ & (\$) 0 \\
\hline November & $3,739,770$ & 0 & $3,739,770$ & & 0 & 448,772 & 0 \\
\hline December & $5,471,535$ & 0 & $5,471,535$ & & 0 & 656,584 & 0 \\
\hline Annual & $68,264,976$ & 0 & $68,264,976$ & & 0 & $8,191,798$ & 0 \\
\hline
\end{tabular}

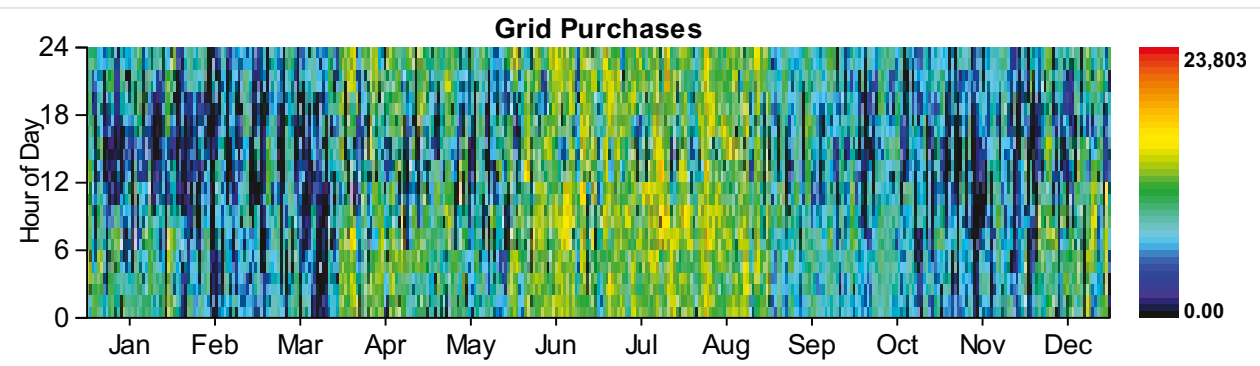

\section{Emissions}

\begin{tabular}{|l|r|l|}
\hline Pollutant & Emissions & Units \\
\hline Carbon dioxide & $43143464 \mathrm{~kg} / \mathrm{yr}$ \\
\hline Carbon monoxide & 0 & $\mathrm{~kg} / \mathrm{yr}$ \\
\hline Unburned hydrocarbons & 0 & $\mathrm{~kg} / \mathrm{yr}$ \\
\hline Particulate matter & 0 & $\mathrm{~kg} / \mathrm{yr}$ \\
\hline Sulfur dioxide & $187046 \mathrm{~kg} / \mathrm{yr}$ \\
\hline Nitrogen oxides & $91475 \mathrm{~kg} / \mathrm{yr}$ \\
\hline
\end{tabular}

HOMER Energy, LLC @ 2016 
System Report

System architecture

\begin{tabular}{|l|l|l|}
\hline PV & Solar World 320W flat plate PV Copy & 1,000 \\
\hline Wind Turbine & Siemens 2.3 MW - 108 & 5 \\
\hline Generator \#2 & Kohler 750 Prime Power & 690 \\
\hline Converter & System Converter & kW \\
\hline Grid & Grid & 750 \\
\hline Dispatch Strategy & Cycle Charging & 20,000 \\
\hline
\end{tabular}

Cost summary

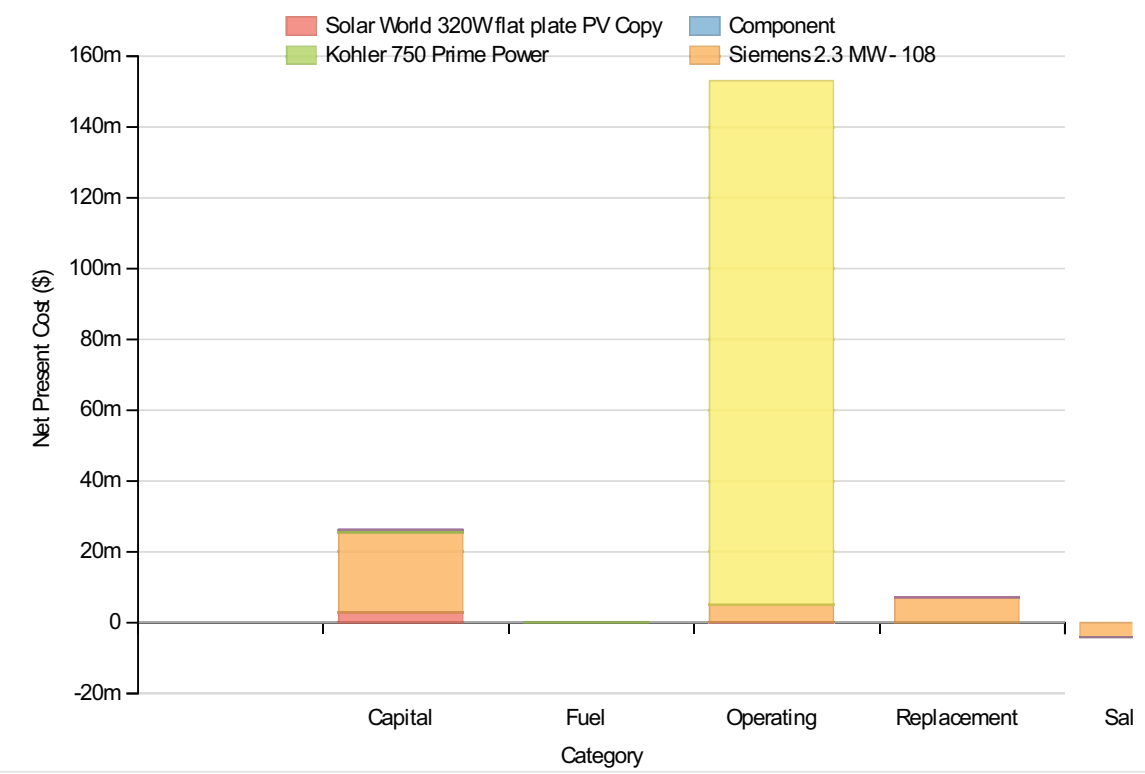

Cost Summary

Total net present cost

$182618288 \$$

Levelized cost of energy

$0.146 \$ / k W h$

\section{Net Present Costs}

\begin{tabular}{|c|c|c|c|c|c|c|}
\hline Component & Capital & Replacement & O\&M & Fuel & Salvage & Total \\
\hline Solar World 320W flat plate PV Copy & $3,000,000$ & 0 & 193,913 & 0 & 0 & $3,193,913$ \\
\hline Siemens 2.3 MW - 108 & $22,500,000$ & $7,173,158$ & $4,847,816$ & 0 & $-4,042,534$ & $30,478,440$ \\
\hline Kohler 750 Prime Power & 690,000 & 0 & 13,648 & 67,521 & $-137,195$ & 633,974 \\
\hline Grid & 0 & 0 & $148,009,376$ & 0 & 0 & $148,009,376$ \\
\hline Converter & 225,000 & 95,462 & 0 & 0 & $-17,967$ & 302,495 \\
\hline System & $26,415,000$ & $7,268,619$ & $153,064,752$ & 67,521 & $-4,197,695$ & $182,618,197$ \\
\hline
\end{tabular}

\section{Annualized Costs}

\begin{tabular}{|l|r|r|r|r|r|r|}
\hline Component & Capital & Replacement & O\&M & Fuel & Salvage & Total \\
\hline Solar World 320W flat plate PV Copy & 232,063 & 0 & 15,000 & 0 & 0 \\
\hline
\end{tabular}




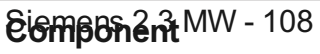

Kohler 750 Prime Power

Grid

Converter

System

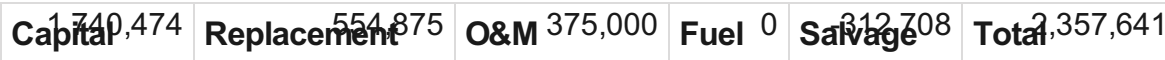

53,375

$0 \quad 1,056$

$5,223-10,613$

49,041

0

\begin{tabular}{l}
$0 \quad 11,449,173$ \\
\hline
\end{tabular}

0

0

$11,449,173$

17,405

7,384

0

$0 \quad-1,390$

23,400

$2,043,316$

562,260

$11,840,229$

5,223

$-324,710$

$14,126,318$

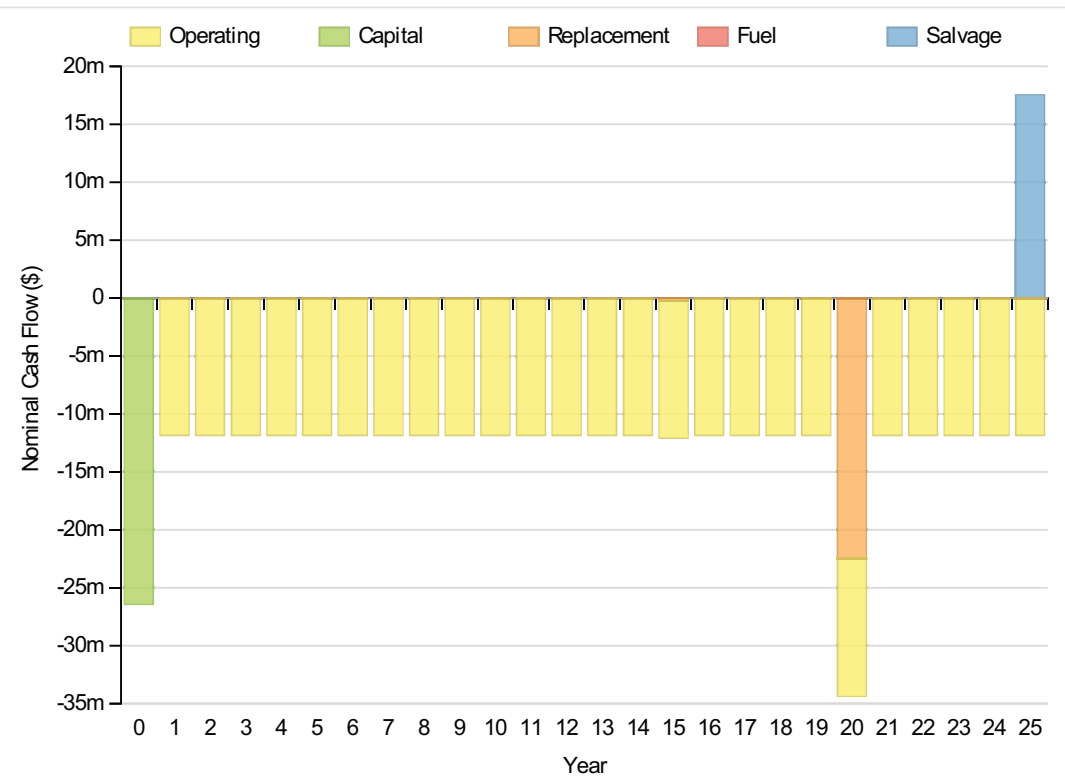

\section{Electrical}

\section{Quantity}

Excess electricity

Unmet load

Capacity shortage

Renewable fraction
Value

\begin{tabular}{|r|r|}
\hline 1907658 & $\mathrm{kWh} / \mathrm{yr}$ \\
\hline 9237 & $\mathrm{kWh} / \mathrm{yr}$ \\
\hline 87708 & $\mathrm{kWh} / \mathrm{yr}$ \\
\hline
\end{tabular}

\section{Component}

PV

Generator

Wind Turbine

Grid Purchases

Total

Load

AC primary load

DC primary load

Total

\section{Production(kWh/yr)}

Fraction (\%)

\begin{tabular}{|c|c|}
\hline & raction (\%) \\
\hline $1,288,675$ & 1 \\
\hline 22,553 & 0 \\
\hline $30,268,832$ & 31 \\
\hline $67,294,856$ & 68 \\
\hline \multirow[t]{2}{*}{$98,874,912$} & 100 \\
\hline & Fraction (\%) \\
\hline $96,854,920$ & 100 \\
\hline 0 & 0 \\
\hline $96,854,920$ & 100 \\
\hline
\end{tabular}




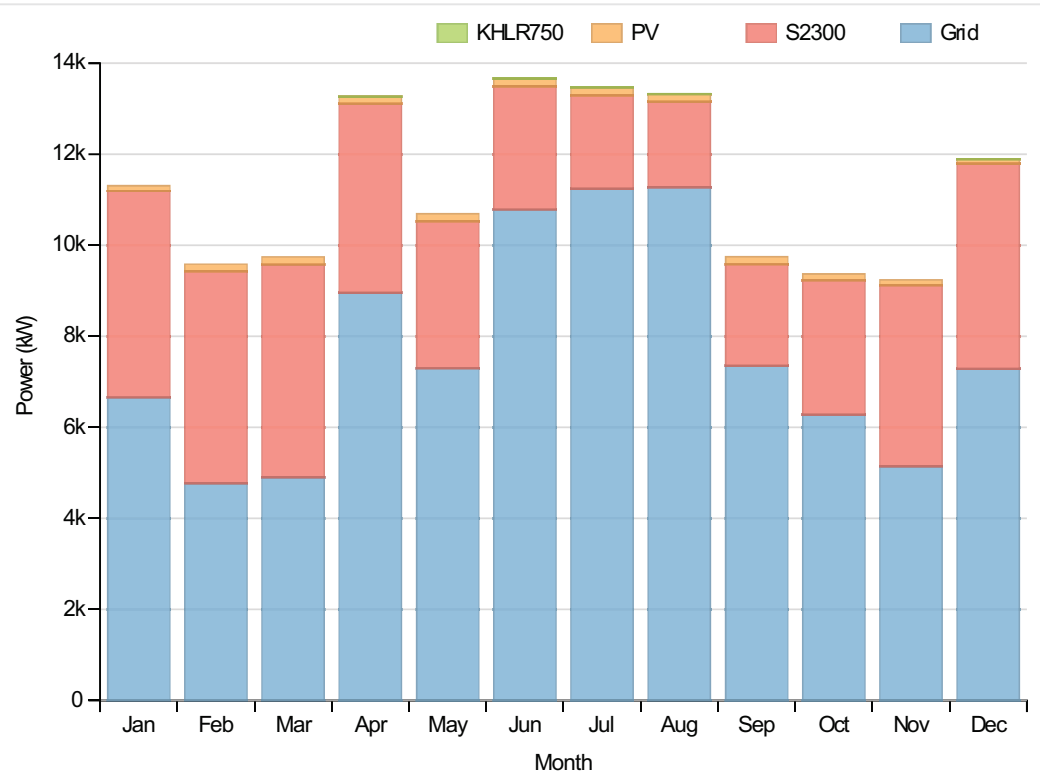

PV:Solar World 320W flat plate PV Copy

\begin{tabular}{|c|c|c|}
\hline Quantity & Value & Units \\
\hline Rated capacity & 1000 & $\mathrm{~kW}$ \\
\hline Mean output & 147 & $\mathrm{~kW}$ \\
\hline Mean output & 3530.60 & $\mathrm{kWh} / \mathrm{d}$ \\
\hline Capacity factor & 14.71 & $\%$ \\
\hline Total production & 1288675 & $\mathrm{kWh} / \mathrm{yr}$ \\
\hline Minimum output & 0.00 & $\mathrm{~kW}$ \\
\hline Maximum output & 1022.50 & $\mathrm{~kW}$ \\
\hline PV penetration & 1.33 & $\%$ \\
\hline Hours of operation & 4377 & $\mathrm{hrs} / \mathrm{yr}$ \\
\hline Levelized cost & 0.192 & $\$ / k W h$ \\
\hline
\end{tabular}

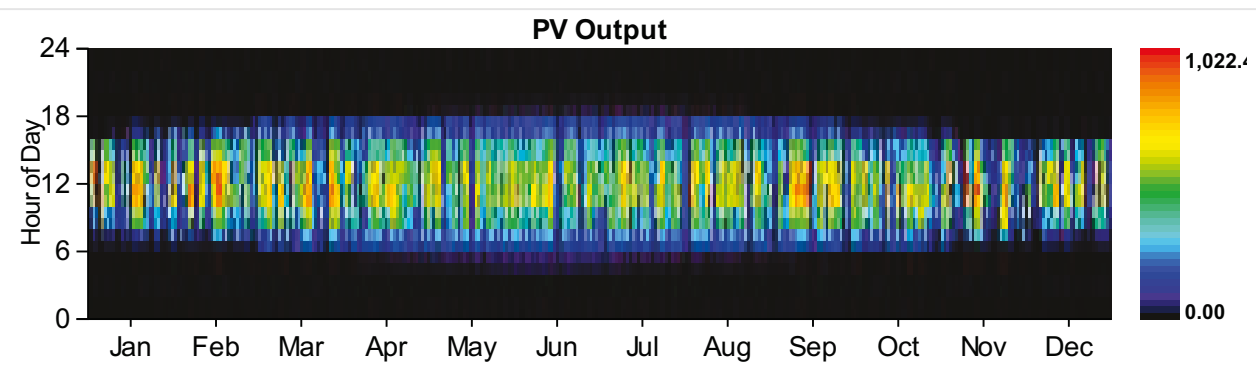

Wind Turbine:Siemens 2.3 MW - 108

\begin{tabular}{|l|r|l|}
\hline Quantity & Value & Units \\
\hline Total rated capacity & 11500 & $\mathrm{~kW}$ \\
\hline Mean output & 3455 & $\mathrm{~kW}$ \\
\hline Capacity factor & $30.05 \%$ \\
\hline Total production & $30268832 \mathrm{kWh} / \mathrm{yr}$ \\
\hline
\end{tabular}




\begin{tabular}{|c|c|c|c|}
\hline Qinimutity output & Value & 7.19 & Elyits \\
\hline Maximum output & & 11574.00 & $\mathrm{~kW}$ \\
\hline Wind penetration & & 31.25 & $\%$ \\
\hline Hours of operation & & 8760 & $\mathrm{hrs} / \mathrm{yr}$ \\
\hline Levelized cost & & 0.078 & $\$ / \mathrm{kWh}$ \\
\hline
\end{tabular}

\section{Generator:Kohler 750 Prime Power}

\begin{tabular}{|c|c|c|}
\hline Quantity & Value & Units \\
\hline Hours of operation & 102 & $\mathrm{hrs} / \mathrm{yr}$ \\
\hline Number of starts & 96 & starts/yr \\
\hline Operational life & 147 & yr \\
\hline Fixed generation cost & 56.87 & $\$ / \mathrm{hr}$ \\
\hline Marginal generation cost & 0.23 & $\$ / k W h$ \\
\hline Electrical production & 22553 & kWh/yr \\
\hline Mean electrical output & 221 & $\mathrm{~kW}$ \\
\hline Min. electrical output & 173 & $\mathrm{~kW}$ \\
\hline Max. electrical output & 690 & $\mathrm{~kW}$ \\
\hline Fuel consumption & 6611 & L/yr \\
\hline Specific fuel consumption & 0.29 & $\mathrm{~L} / \mathrm{kWh}$ \\
\hline Fuel energy input & 65056 & $\mathrm{kWh} / \mathrm{yr}$ \\
\hline Mean electrical efficiency & 35 & $\%$ \\
\hline
\end{tabular}

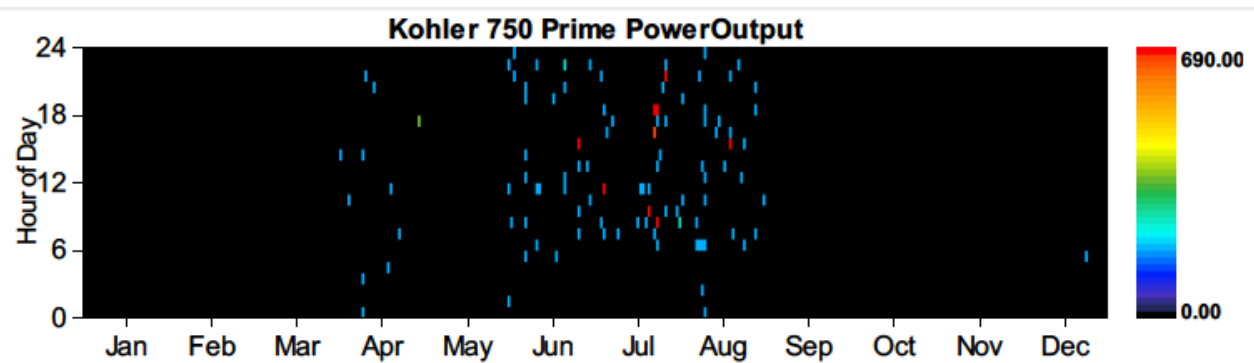

\section{Converter}

\begin{tabular}{|l|r|r|l|}
\hline Quantity & Inverter & Rectifier & Units \\
\hline Capacity & 750 & 675 & $\mathrm{~kW}$ \\
\hline Mean output & 115 & $0 \mathrm{~kW}$ \\
\hline Minimum output & 0 & $0 \mathrm{~kW}$ \\
\hline Maximum output & 750 & $0 \mathrm{~kW}$ \\
\hline Capacity factor & 15 & 0 & $\%$ \\
\hline
\end{tabular}




\begin{tabular}{|c|c|c|c|c|c|}
\hline deptafítifyoperation & Inverter & 3,902 & Rectifier & 0 & brshirs \\
\hline Energy in & & $1,123,182$ & & 0 & kWh/yr \\
\hline Energy out & & $1,010,865$ & & 0 & kWh/yr \\
\hline Losses & & 112,317 & & 0 & $\mathrm{kWh} / \mathrm{yr}$ \\
\hline
\end{tabular}
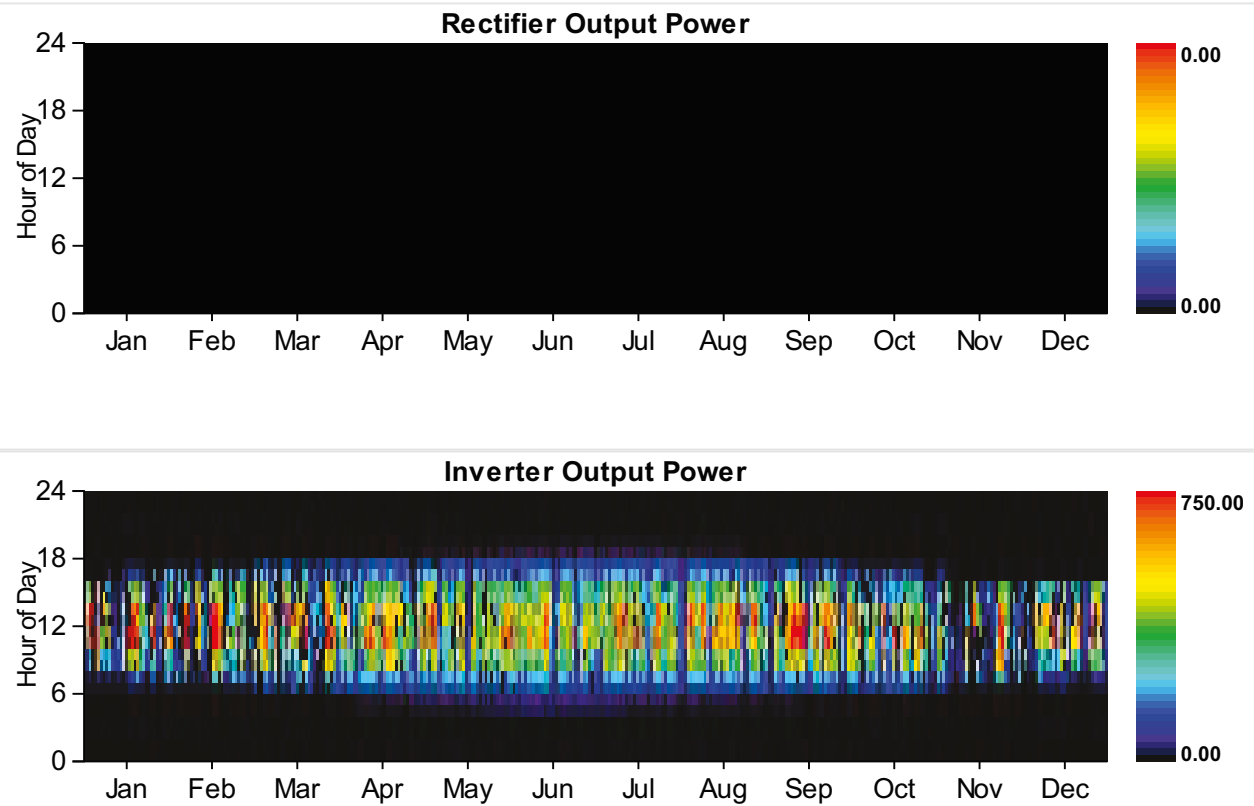

Grid

Rate: Demand 1

\begin{tabular}{|c|c|c|c|c|c|c|}
\hline Resources.ReportingService_GeneratelnputsReport_Month & $\begin{array}{l}\text { Energy } \\
\text { Purchased } \\
\text { (kWh) }\end{array}$ & $\begin{array}{l}\text { Energy } \\
\text { Sold } \\
\text { (kWh) }\end{array}$ & $\begin{array}{l}\text { Net } \\
\text { Purchases } \\
\text { (kWh) }\end{array}$ & $\begin{array}{l}\text { Peak } \\
\text { Demand } \\
(\mathbf{k W})\end{array}$ & $\begin{array}{l}\text { Energy } \\
\text { Charge } \\
(\$)\end{array}$ & $\begin{array}{l}\text { Demand } \\
\text { Charge } \\
(\$)\end{array}$ \\
\hline January & 0 & 0 & 0 & 18,095 & 0 & 289,519 \\
\hline February & 0 & 0 & 0 & 14,936 & 0 & 238,977 \\
\hline March & 0 & 0 & 0 & 16,699 & 0 & 267,178 \\
\hline April & 0 & 0 & 0 & 20,000 & 0 & 320,000 \\
\hline May & 0 & 0 & 0 & 17,572 & 0 & 281,151 \\
\hline June & 0 & 0 & 0 & 20,000 & 0 & 320,000 \\
\hline July & 0 & 0 & 0 & 20,000 & 0 & 320,000 \\
\hline August & 0 & 0 & 0 & 20,000 & 0 & 320,000 \\
\hline September & 0 & 0 & 0 & 15,468 & 0 & 247,493 \\
\hline October & 0 & 0 & 0 & 13,726 & 0 & 219,623 \\
\hline November & 0 & 0 & 0 & 15,876 & 0 & 254,021 \\
\hline December & 0 & 0 & 0 & 18,490 & 0 & 295,836 \\
\hline Annual & 0 & 0 & 0 & 20,000 & 0 & $3,373,798$ \\
\hline
\end{tabular}




\begin{tabular}{|c|c|c|c|c|c|c|}
\hline Resources.ReportingService_GeneratelnputsReport_Month & $\begin{array}{l}\text { Energy } \\
\text { Purchased } \\
\text { (kWh) }\end{array}$ & $\begin{array}{l}\text { Energy } \\
\text { Sold } \\
\text { (kWh) }\end{array}$ & $\begin{array}{l}\text { Net } \\
\text { Purchases } \\
\text { (kWh) }\end{array}$ & $\begin{array}{l}\text { Peak } \\
\text { Demand } \\
\text { (kW) }\end{array}$ & $\begin{array}{l}\text { Energy } \\
\text { Charge } \\
\text { (\$) }\end{array}$ & $\begin{array}{l}\text { Demand } \\
\text { Charge } \\
\text { (\$) }\end{array}$ \\
\hline January & $4,952,182$ & 0 & $4,952,182$ & 0 & 594,262 & 0 \\
\hline February & $3,207,647$ & 0 & $3,207,647$ & 0 & 384,918 & 0 \\
\hline March & $3,646,840$ & 0 & $3,646,840$ & 0 & 437,621 & 0 \\
\hline April & $6,450,498$ & 0 & $6,450,498$ & 0 & 774,060 & 0 \\
\hline May & $5,429,449$ & 0 & $5,429,449$ & 0 & 651,534 & 0 \\
\hline June & $7,766,616$ & 0 & $7,766,616$ & 0 & 931,994 & 0 \\
\hline July & $8,362,597$ & 0 & $8,362,597$ & 0 & $1,003,512$ & 0 \\
\hline August & $8,391,706$ & 0 & $8,391,706$ & 0 & $1,007,005$ & 0 \\
\hline September & $5,297,231$ & 0 & $5,297,231$ & 0 & 635,668 & 0 \\
\hline October & $4,667,008$ & 0 & $4,667,008$ & 0 & 560,041 & 0 \\
\hline November & $3,703,306$ & 0 & $3,703,306$ & 0 & 444,397 & 0 \\
\hline December & $5,419,775$ & 0 & $5,419,775$ & 0 & 650,373 & 0 \\
\hline Annual & $67,294,856$ & 0 & $67,294,856$ & 0 & $8,075,382$ & 0 \\
\hline
\end{tabular}

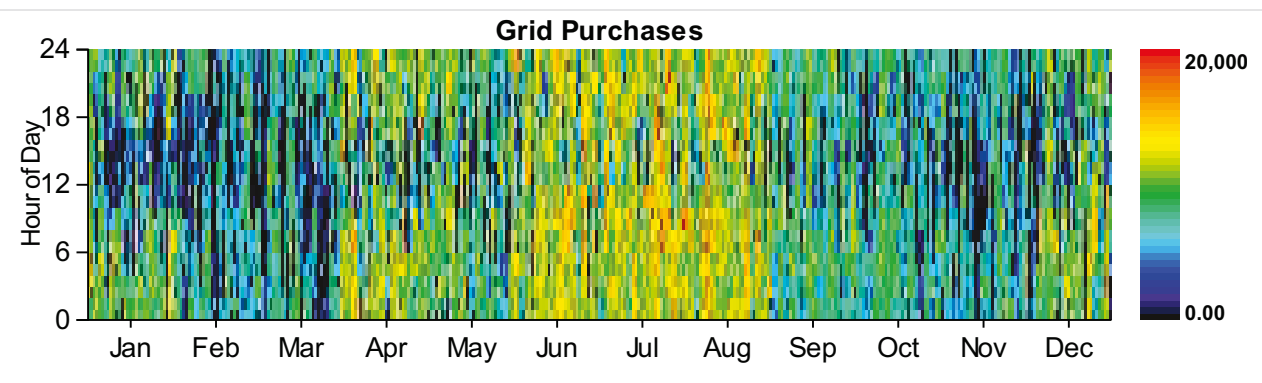

\section{Emissions}

\begin{tabular}{|c|c|c|}
\hline Pollutant & Emissions & Units \\
\hline Carbon dioxide & 42547700 & $\mathrm{~kg} / \mathrm{yr}$ \\
\hline Carbon monoxide & 73 & $\mathrm{~kg} / \mathrm{yr}$ \\
\hline Unburned hydrocarbons & 8 & $\mathrm{~kg} / \mathrm{yr}$ \\
\hline Particulate matter & 2 & $\mathrm{~kg} / \mathrm{yr}$ \\
\hline Sulfur dioxide & 184424 & $\mathrm{~kg} / \mathrm{yr}$ \\
\hline Nitrogen oxides & 90248 & $\mathrm{~kg} / \mathrm{yr}$ \\
\hline
\end{tabular}


System Report

System architecture

\begin{tabular}{|l|l|l|}
\hline PV & Solar World 320W flat plate PV Copy & 1,000 \\
\hline Wind Turbine & Siemens 2.3 MW - 108 & 5 \\
\hline Generator & Kohler 1000 Prime Power & 925 \\
\hline Converter & System Converter & kW \\
\hline Grid & Grid & 750 \\
\hline Dispatch Strategy & Cycle Charging & 20,000 \\
\hline
\end{tabular}

Cost summary

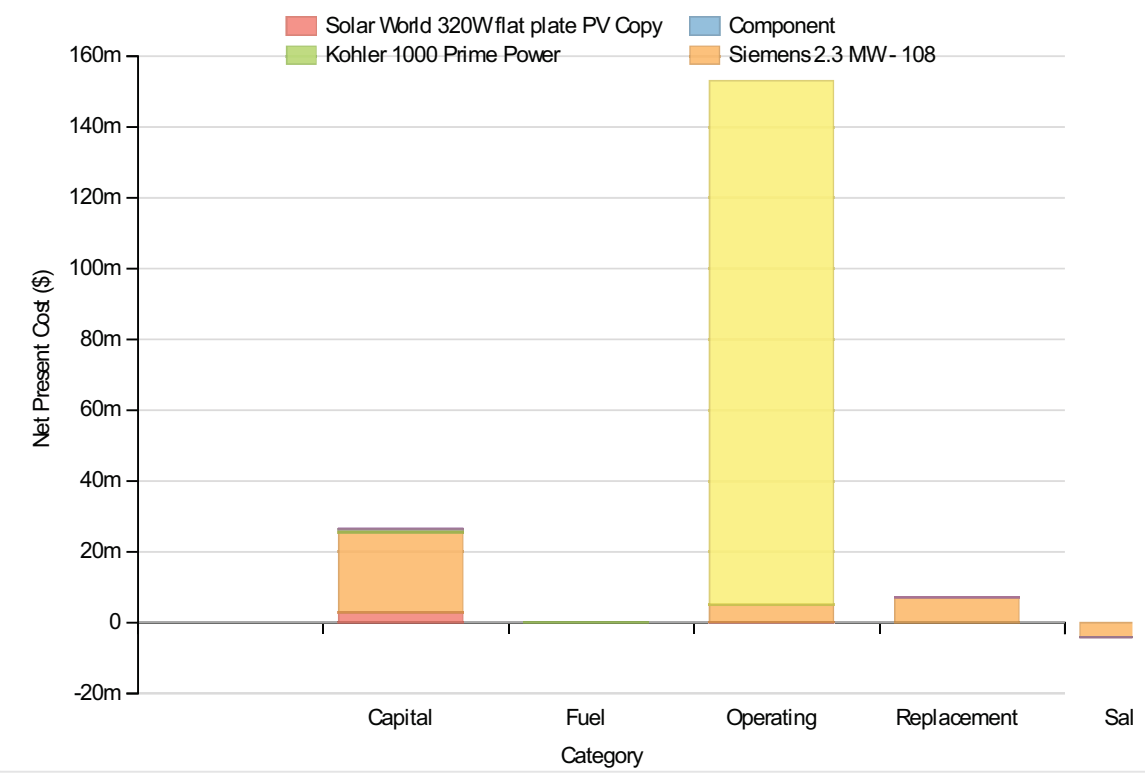

Cost Summary

Total net present cost

$182811264 \$$

Levelized cost of energy

$0.146 \$ / \mathrm{kWh}$

\section{Net Present Costs}

\begin{tabular}{|l|r|r|r|r|r|r|}
\hline Component & Capital & Replacement & O\&M & Fuel & Salvage & Total \\
\hline Solar World 320W flat plate PV Copy & $3,000,000$ & 0 & 193,913 & 0 & 0 \\
\hline Siemens 2.3 MW - 108 & $22,500,000$ & $7,173,158$ & $4,847,816$ & 0 & $-4,042,534$ \\
\hline Kohler 1000 Prime Power & 925,000 & 0 & 18,296 & 87,939 & $-183,920$ \\
\hline Grid & 0 & 0 & $147,989,040$ & 0 & 0 \\
\hline Converter & 225,000 & 95,462 & $147,989,040$ \\
\hline System & $26,650,000$ & $7,268,619$ & $153,049,056$ & 87,939 & $-4,244,421$ & $182,811,193$ \\
\hline
\end{tabular}

\section{Annualized Costs}

\begin{tabular}{|l|r|r|r|r|r|r|}
\hline Component & Capital & Replacement & O\&M & Fuel & Salvage & Total \\
\hline Solar World 320W flat plate PV Copy & 232,063 & 0 & 15,000 & 0 & 0 \\
\hline & & 247,063 \\
\hline
\end{tabular}


Ėempresenent MW - 108

Kohler 1000 Prime Power

Grid

Converter

System

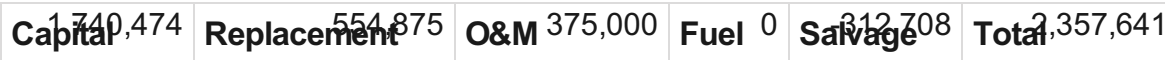

71,553

0

1,415

6,802

$-14,227$

65,544

0

0
$11,447,600 \quad 0$

$0 \quad 11,447,600$

17,405

7,384

0

0

$-1,390$

23,400

$2,061,494$

562,260

$11,839,015$

6,802

$-328,325$

$14,141,246$

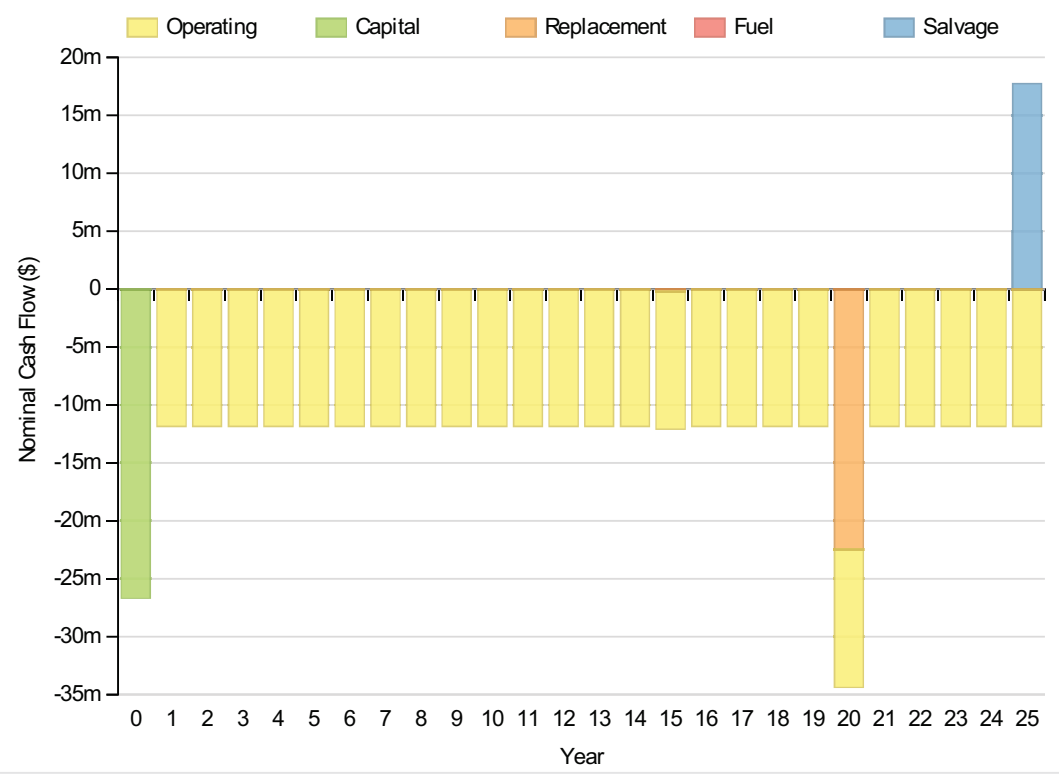

\section{Electrical}

\section{Quantity}

Excess electricity

Unmet load

Capacity shortage

Renewable fraction
Value

\begin{tabular}{|r|r|}
\hline 1907658 & $\mathrm{kWh} / \mathrm{yr}$ \\
\hline 7357 & $\mathrm{kWh} / \mathrm{yr}$ \\
\hline 73139 & $\mathrm{kWh} / \mathrm{yr}$ \\
\hline
\end{tabular}

\section{Component}

PV

Generator

Wind Turbine

Grid Purchases

Total

Load

AC primary load

DC primary load

Total

\section{Production(kWh/yr)}

Fraction (\%)

\begin{tabular}{|r|r|}
\hline $1,288,675$ & 1 \\
\hline 29,720 & 0 \\
\hline $30,268,832$ & 31 \\
\hline $67,289,568$ & 68 \\
\hline $98,876,792$ & 100 \\
\hline
\end{tabular}

\section{Consumption(kWh/yr)}

Fraction (\%) 


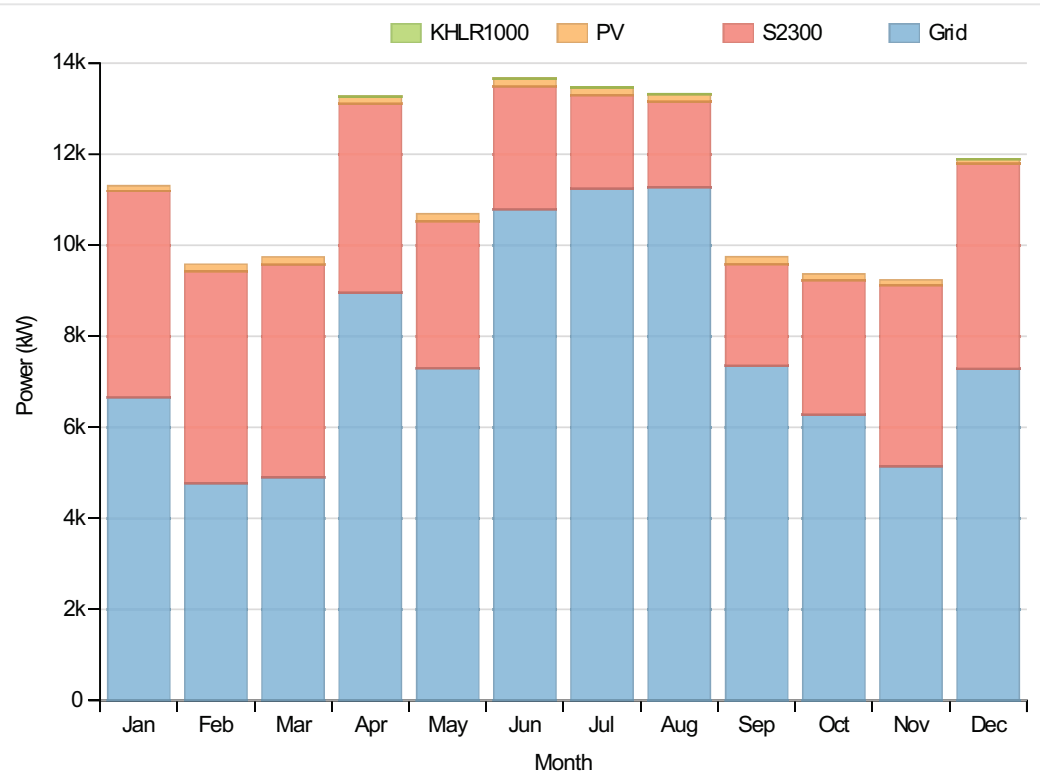

PV:Solar World 320W flat plate PV Copy

\begin{tabular}{|c|c|c|}
\hline Quantity & Value & Units \\
\hline Rated capacity & 1000 & $\mathrm{~kW}$ \\
\hline Mean output & 147 & $\mathrm{~kW}$ \\
\hline Mean output & 3530.60 & $\mathrm{kWh} / \mathrm{d}$ \\
\hline Capacity factor & 14.71 & $\%$ \\
\hline Total production & 1288675 & $\mathrm{kWh} / \mathrm{yr}$ \\
\hline Minimum output & 0.00 & $\mathrm{~kW}$ \\
\hline Maximum output & 1022.50 & $\mathrm{~kW}$ \\
\hline PV penetration & 1.33 & $\%$ \\
\hline Hours of operation & 4377 & $\mathrm{hrs} / \mathrm{yr}$ \\
\hline Levelized cost & 0.192 & $\$ / k W h$ \\
\hline
\end{tabular}

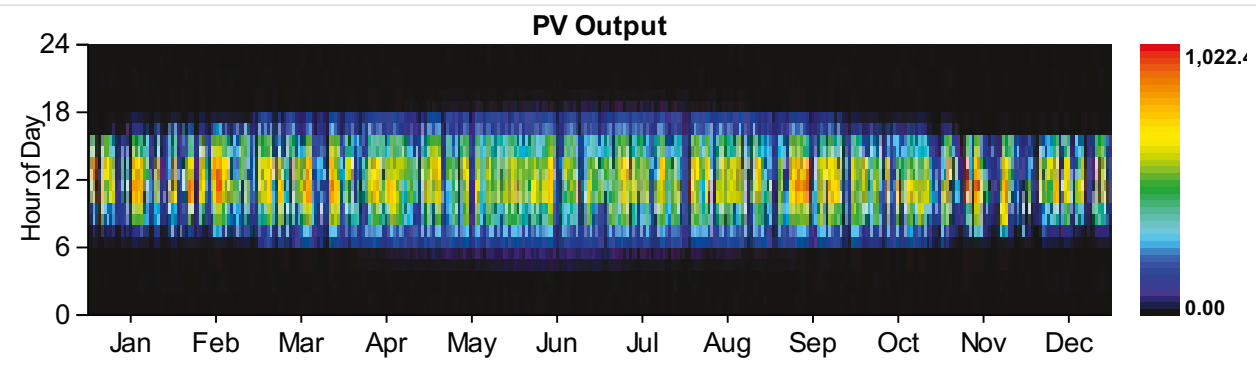

Wind Turbine:Siemens 2.3 MW - 108

\begin{tabular}{|l|r|l|}
\hline Quantity & Value & Units \\
\hline Total rated capacity & 11500 & $\mathrm{~kW}$ \\
\hline Mean output & 3455 & $\mathrm{~kW}$ \\
\hline Capacity factor & $30.05 \%$ \\
\hline Total production & $30268832 \mathrm{kWh} / \mathrm{yr}$ \\
\hline
\end{tabular}


Qiirimitity output

Maximum output

Wind penetration

Hours of operation

Levelized cost
Value

7.19 UNits

11574.00 kW

$31.25 \%$

8760 hrs/yr

$0.078 \$ / k W h$

\section{Generator:Kohler 1000 Prime Power}

\section{Quantity}

Hours of operation

Number of starts

Operational life

Fixed generation cost

Marginal generation cost

Electrical production

Mean electrical output

Min. electrical output

Max. electrical output

Fuel consumption

Specific fuel consumption

Fuel energy input

Mean electrical efficiency

\begin{tabular}{|r|l|l|}
\hline Value & Units \\
\hline 102 & $\mathrm{hrs} / \mathrm{yr}$ \\
\hline 96 & starts/yr \\
\hline 147 & $\mathrm{yr}$ \\
\hline 76.24 & $\$ / \mathrm{hr}$ \\
\hline 0.23 & $\$ / \mathrm{kWh}$ \\
\hline 29720 & $\mathrm{kWh} / \mathrm{yr}$ \\
\hline 291 & $\mathrm{~kW}$ \\
\hline 231 & $\mathrm{~kW}$ \\
\hline 925 & $\mathrm{~kW}$ \\
\hline 8611 & $\mathrm{~L} / \mathrm{yr}$ \\
\hline 0.29 & $\mathrm{~L} / \mathrm{kWh}$ \\
\hline 84729 & $\mathrm{kWh} / \mathrm{yr}$ \\
\hline 35 & $\%$ \\
\hline
\end{tabular}

Units

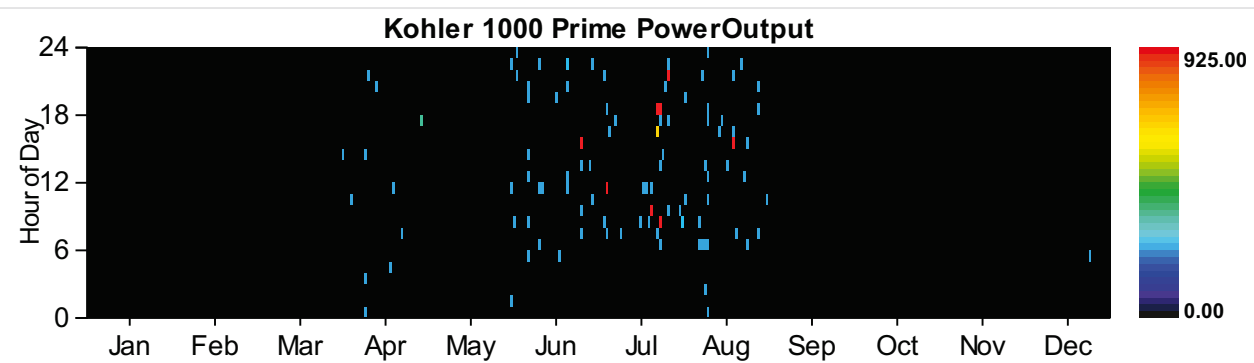

\section{Converter}

\begin{tabular}{|l|r|r|l|}
\hline Quantity & Inverter & Rectifier & Units \\
\hline Capacity & 750 & 675 & $\mathrm{~kW}$ \\
\hline Mean output & 115 & $\mathrm{~kW}$ & $0 \mathrm{~kW}$ \\
\hline Minimum output & 0 & $0 \mathrm{~kW}$ & 0 \\
\hline Maximum output & 750 & 15 & $\%$ \\
\hline Capacity factor & 15 & \\
\hline
\end{tabular}




\begin{tabular}{|c|c|c|c|c|c|}
\hline deptafítifyoperation & Inverter & 3,902 & Rectifier & 0 & brshirs \\
\hline Energy in & & $1,123,182$ & & 0 & kWh/yr \\
\hline Energy out & & $1,010,865$ & & 0 & kWh/yr \\
\hline Losses & & 112,317 & & 0 & $\mathrm{kWh} / \mathrm{yr}$ \\
\hline
\end{tabular}
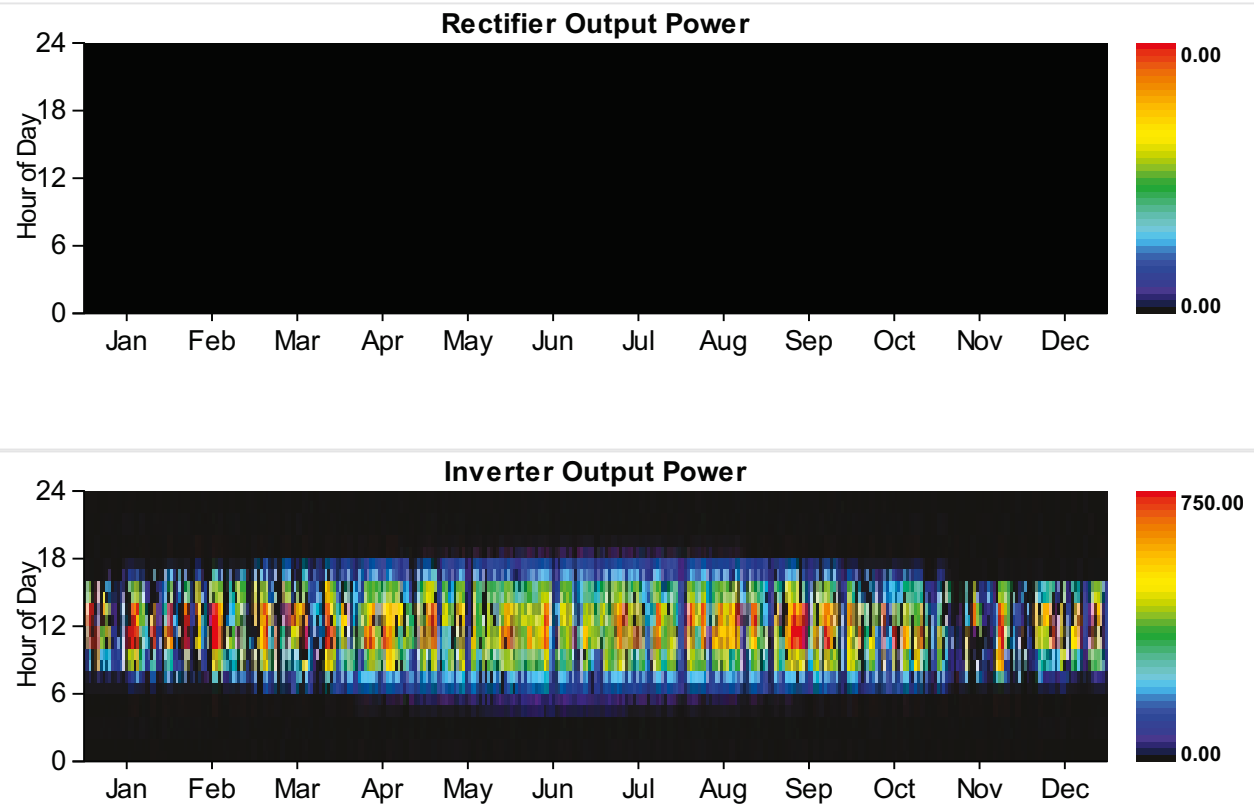

Grid

Rate: Demand 1

\begin{tabular}{|c|c|c|c|c|c|c|}
\hline Resources.ReportingService_GeneratelnputsReport_Month & $\begin{array}{l}\text { Energy } \\
\text { Purchased } \\
\text { (kWh) }\end{array}$ & $\begin{array}{l}\text { Energy } \\
\text { Sold } \\
\text { (kWh) }\end{array}$ & $\begin{array}{l}\text { Net } \\
\text { Purchases } \\
\text { (kWh) }\end{array}$ & $\begin{array}{l}\text { Peak } \\
\text { Demand } \\
(\mathbf{k W})\end{array}$ & $\begin{array}{l}\text { Energy } \\
\text { Charge } \\
(\$)\end{array}$ & $\begin{array}{l}\text { Demand } \\
\text { Charge } \\
(\$)\end{array}$ \\
\hline January & 0 & 0 & 0 & 18,095 & 0 & 289,519 \\
\hline February & 0 & 0 & 0 & 14,936 & 0 & 238,977 \\
\hline March & 0 & 0 & 0 & 16,699 & 0 & 267,178 \\
\hline April & 0 & 0 & 0 & 20,000 & 0 & 320,000 \\
\hline May & 0 & 0 & 0 & 17,572 & 0 & 281,151 \\
\hline June & 0 & 0 & 0 & 20,000 & 0 & 320,000 \\
\hline July & 0 & 0 & 0 & 20,000 & 0 & 320,000 \\
\hline August & 0 & 0 & 0 & 20,000 & 0 & 320,000 \\
\hline September & 0 & 0 & 0 & 15,468 & 0 & 247,493 \\
\hline October & 0 & 0 & 0 & 13,726 & 0 & 219,623 \\
\hline November & 0 & 0 & 0 & 15,876 & 0 & 254,021 \\
\hline December & 0 & 0 & 0 & 18,431 & 0 & 294,896 \\
\hline Annual & 0 & 0 & 0 & 20,000 & 0 & $3,372,858$ \\
\hline
\end{tabular}




\begin{tabular}{|c|c|c|c|c|c|c|}
\hline Resources.ReportingService_GeneratelnputsReport_Month & $\begin{array}{l}\text { Energy } \\
\text { Purchased } \\
\text { (kWh) }\end{array}$ & $\begin{array}{l}\text { Energy } \\
\text { Sold } \\
\text { (kWh) }\end{array}$ & $\begin{array}{l}\text { Net } \\
\text { Purchases } \\
\text { (kWh) }\end{array}$ & $\begin{array}{l}\text { Peak } \\
\text { Demand } \\
\text { (kW) }\end{array}$ & $\begin{array}{l}\text { Energy } \\
\text { Charge } \\
\text { (\$) }\end{array}$ & $\begin{array}{l}\text { Demand } \\
\text { Charge } \\
\text { (\$) }\end{array}$ \\
\hline January & $4,952,182$ & 0 & $4,952,182$ & 0 & 594,262 & 0 \\
\hline February & $3,207,647$ & 0 & $3,207,647$ & 0 & 384,918 & 0 \\
\hline March & $3,646,840$ & 0 & $3,646,840$ & 0 & 437,621 & 0 \\
\hline April & $6,449,910$ & 0 & $6,449,910$ & 0 & 773,989 & 0 \\
\hline May & $5,429,449$ & 0 & $5,429,449$ & 0 & 651,534 & 0 \\
\hline June & $7,765,030$ & 0 & $7,765,030$ & 0 & 931,804 & 0 \\
\hline July & $8,361,306$ & 0 & $8,361,306$ & 0 & $1,003,357$ & 0 \\
\hline August & $8,389,944$ & 0 & $8,389,944$ & 0 & $1,006,793$ & 0 \\
\hline September & $5,297,231$ & 0 & $5,297,231$ & 0 & 635,668 & 0 \\
\hline October & $4,667,008$ & 0 & $4,667,008$ & 0 & 560,041 & 0 \\
\hline November & $3,703,306$ & 0 & $3,703,306$ & 0 & 444,397 & 0 \\
\hline December & $5,419,716$ & 0 & $5,419,716$ & 0 & 650,366 & 0 \\
\hline Annual & $67,289,568$ & 0 & $67,289,568$ & 0 & $8,074,748$ & 0 \\
\hline
\end{tabular}

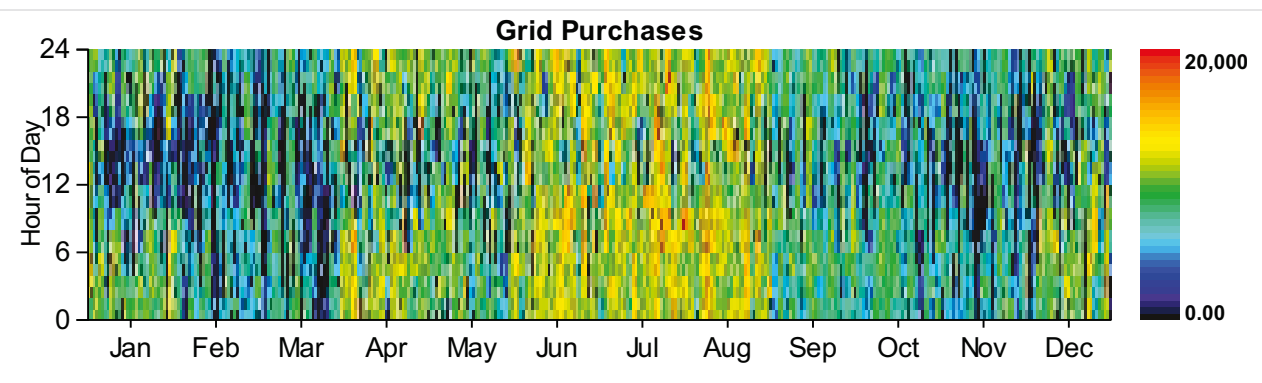

\section{Emissions}

\begin{tabular}{|c|c|c|}
\hline Pollutant & Emissions & Units \\
\hline Carbon dioxide & 42549608 & $\mathrm{~kg} / \mathrm{yr}$ \\
\hline Carbon monoxide & 95 & $\mathrm{~kg} / \mathrm{yr}$ \\
\hline Unburned hydrocarbons & 11 & $\mathrm{~kg} / \mathrm{yr}$ \\
\hline Particulate matter & 3 & $\mathrm{~kg} / \mathrm{yr}$ \\
\hline Sulfur dioxide & 184420 & $\mathrm{~kg} / \mathrm{yr}$ \\
\hline Nitrogen oxides & 90263 & $\mathrm{~kg} / \mathrm{yr}$ \\
\hline
\end{tabular}


System Report

System architecture

\begin{tabular}{|c|c|c|c|}
\hline PV & Solar World 320W flat plate PV Copy & 1,000 & kW \\
\hline Wind Turbine & Siemens 2.3 MW - 108 & 5 & \\
\hline Generator \#2 & Kohler 750 Prime Power & 690 & kW \\
\hline Battery & GS200 flow & 1 & strings \\
\hline Converter & System Converter & 750 & kW \\
\hline Grid & Grid & 20,000 & kW \\
\hline Dispatch Strategy & Cycle Charging & & \\
\hline
\end{tabular}

\section{Cost summary}

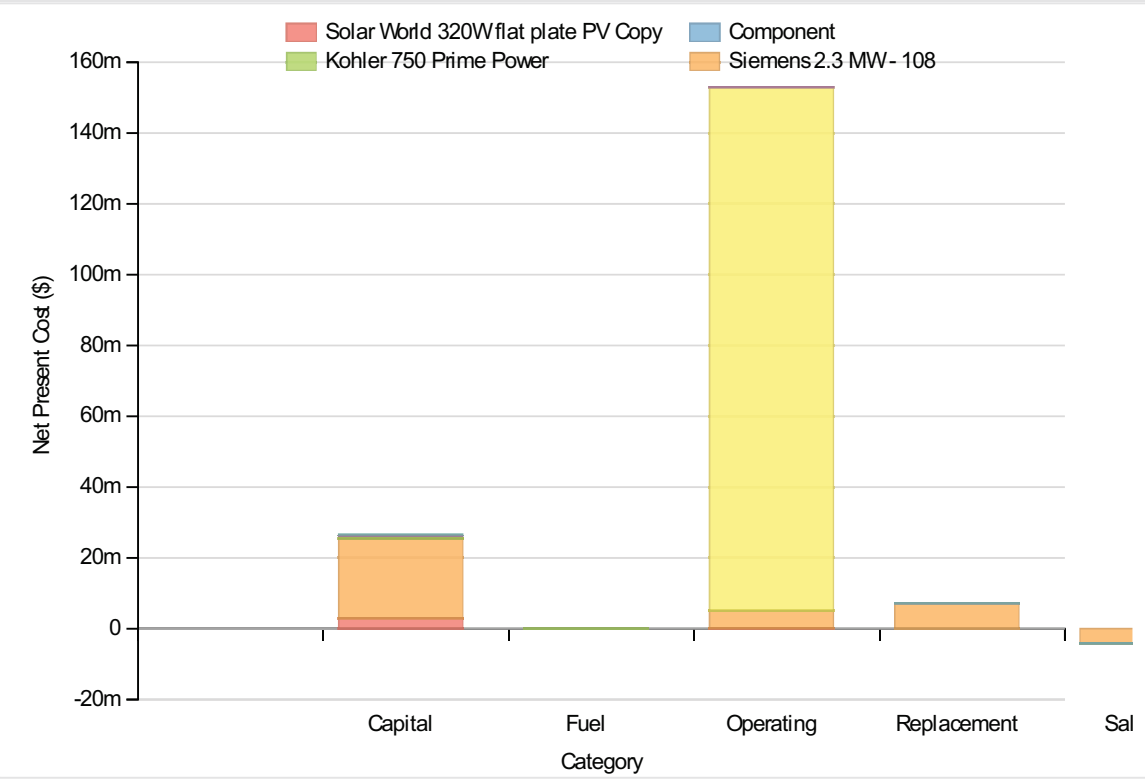

Cost Summary

Total net present cost

$182886224 \$$

Levelized cost of energy

$0.146 \$ / k W h$

Net Present Costs

\begin{tabular}{|c|c|c|c|c|c|c|}
\hline Component & Capital & Replacement & O\&M & Fuel & Salvage & Total \\
\hline Solar World 320W flat plate PV Copy & $3,000,000$ & 0 & 193,913 & 0 & 0 & $3,193,913$ \\
\hline Siemens 2.3 MW - 108 & $22,500,000$ & $7,173,158$ & $4,847,816$ & 0 & $-4,042,534$ & $30,478,440$ \\
\hline Kohler 750 Prime Power & 690,000 & 0 & 13,514 & 108,816 & $-137,470$ & 674,860 \\
\hline Grid & 0 & 0 & $147,874,608$ & 0 & 0 & $147,874,608$ \\
\hline GS200 flow & 324,589 & 7,176 & 31,026 & 0 & -973 & 361,818 \\
\hline Converter & 225,000 & 95,462 & 0 & 0 & $-17,967$ & 302,495 \\
\hline System & $26,739,588$ & $7,275,795$ & $152,960,896$ & 108,816 & $-4,198,944$ & $182,886,151$ \\
\hline
\end{tabular}




\begin{tabular}{|c|c|c|c|c|c|c|}
\hline Component & Capital & Replacement & O\&M & Fuel & Salvage & Total \\
\hline Solar World 320W flat plate PV Copy & 232,063 & 0 & 15,000 & 0 & 0 & 247,063 \\
\hline Siemens 2.3 MW - 108 & $1,740,474$ & 554,875 & 375,000 & 0 & $-312,708$ & $2,357,641$ \\
\hline Kohler 750 Prime Power & 53,375 & 0 & 1,045 & 8,417 & $-10,634$ & 52,204 \\
\hline Grid & 0 & 0 & $11,438,748$ & 0 & 0 & $11,438,748$ \\
\hline GS200 flow & 25,108 & 555 & 2,400 & 0 & -75 & 27,988 \\
\hline Converter & 17,405 & 7,384 & 0 & 0 & $-1,390$ & 23,400 \\
\hline System & $2,068,424$ & 562,815 & $11,832,195$ & 8,417 & $-324,807$ & $14,147,044$ \\
\hline
\end{tabular}

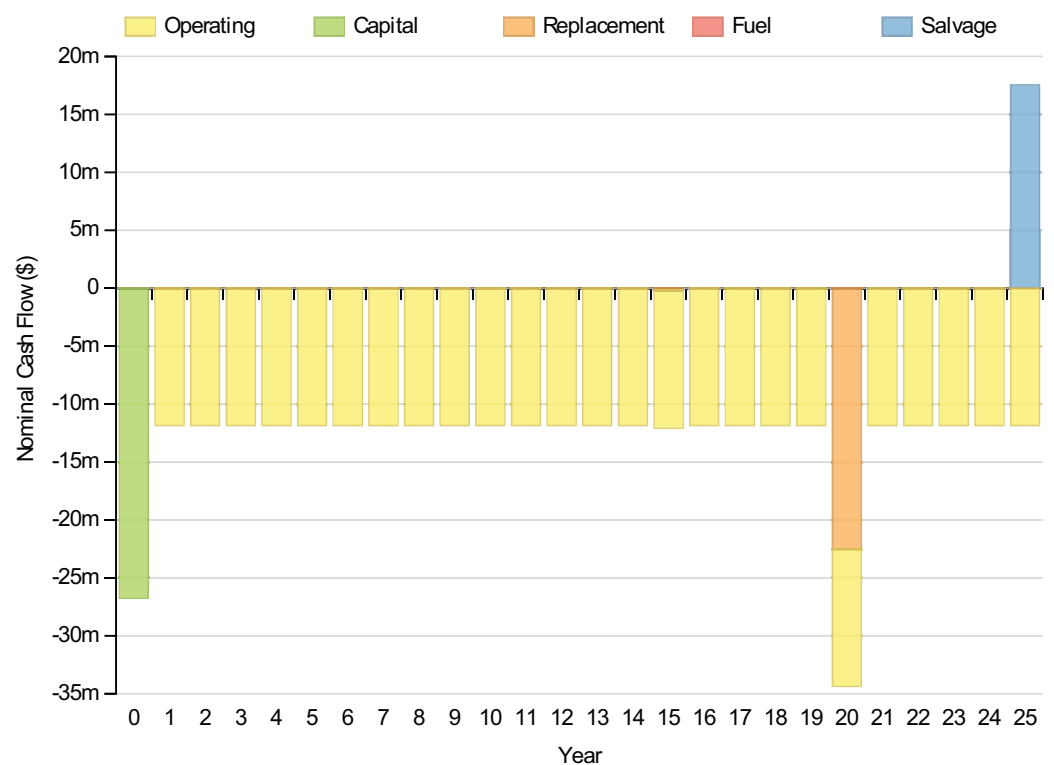

\section{Electrical}

\section{Quantity}

Excess electricity

Unmet load

Capacity shortage

Renewable fraction

\section{Component}

PV

Generator

Wind Turbine

Grid Purchases

Total

Load

Consumption(kWh/yr)

Production(kWh/yr)

\section{Value}

Fraction (\%)

\begin{tabular}{|r|r|}
\hline $1,288,675$ & 1 \\
\hline 36,489 & 0 \\
\hline $67,268,832$ & 31 \\
\hline $98,801,984$ & 68 \\
\hline
\end{tabular}

Fraction (\%) 


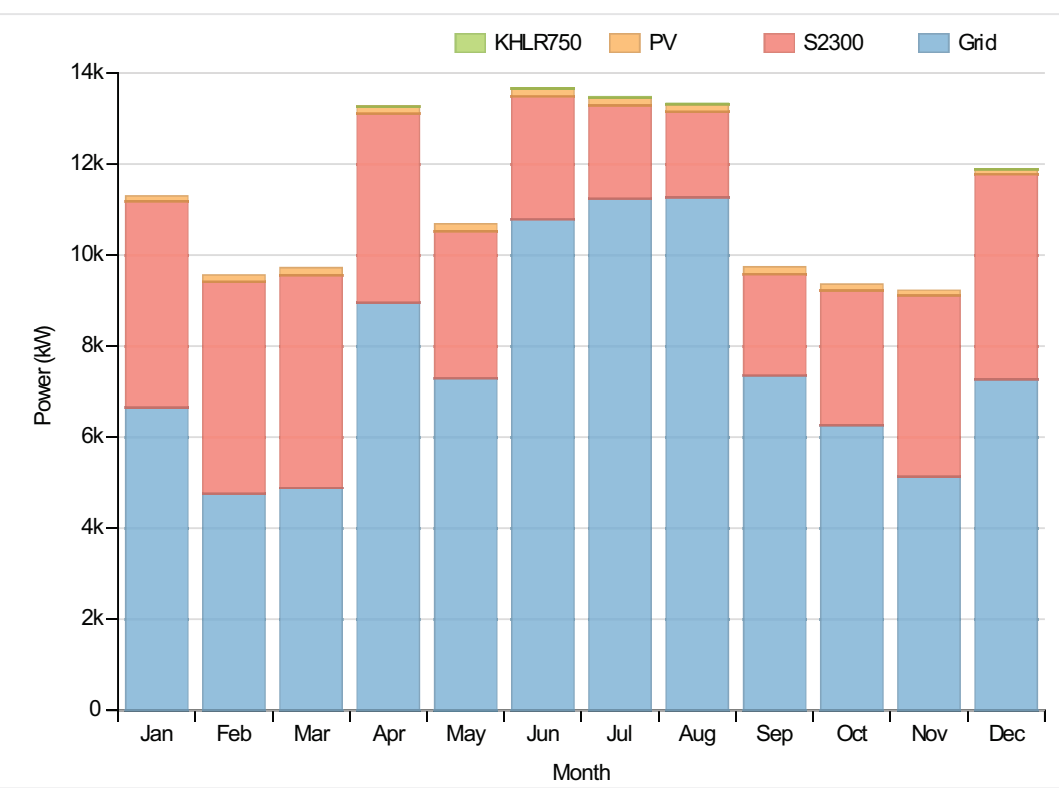

PV:Solar World 320W flat plate PV Copy

\begin{tabular}{|l|c|c|}
\hline Quantity & Value & Units \\
\hline Rated capacity & $1000 \mathrm{~kW}$ \\
\hline Mean output & $147 \mathrm{~kW}$ \\
\hline Mean output & $3530.60 \mathrm{kWh} / \mathrm{d}$ \\
\hline Capacity factor & $14.71 \%$ \\
\hline Total production & $1288675 \mathrm{kWh} / \mathrm{yr}$ \\
\hline Minimum output & $0.00 \mathrm{~kW}$ \\
\hline Maximum output & $1022.50 \mathrm{~kW}$ \\
\hline PV penetration & $1.33 \%$ \\
\hline Hours of operation & $\%$ hrs/yr \\
\hline Levelized cost & 4377 \\
\hline
\end{tabular}

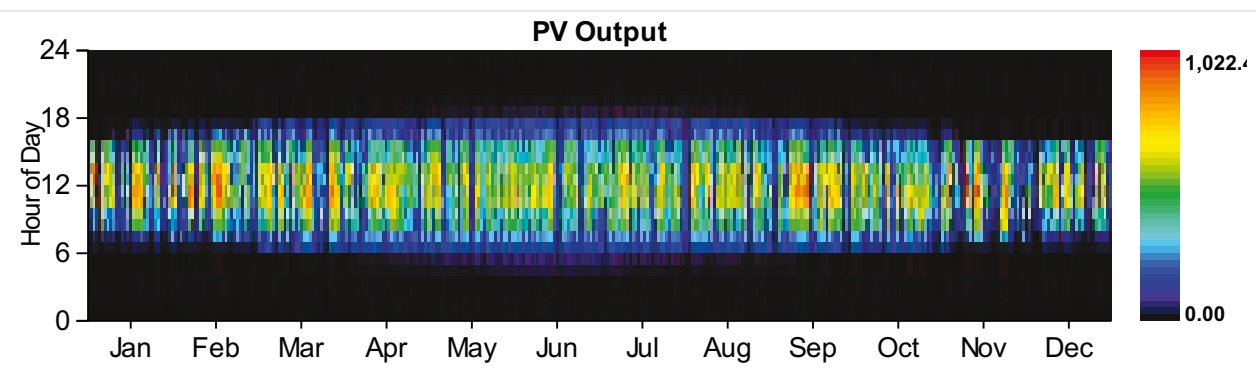

Wind Turbine:Siemens 2.3 MW - 108

\section{Quantity}

Total rated capacity

Mean output

Capacity factor

\begin{tabular}{|l|l|l|}
\hline Value & Units \\
\hline 11500 & $\mathrm{~kW}$ \\
\hline 3455 & $\mathrm{~kW}$ \\
\hline 30.05 & $\%$ \\
\hline
\end{tabular}


¿talheftyduction

Minimum output

Maximum output

Wind penetration

Hours of operation

Levelized cost
Value

30268832 units

$7.19 \mathrm{~kW}$

11574.00 kW

$31.25 \%$

$8760 \mathrm{hrs} / \mathrm{yr}$

$0.078 \$ / k W h$

\section{Generator:Kohler 750 Prime Power}

\section{Quantity}

Hours of operation

Number of starts

Operational life

Fixed generation cost

Marginal generation cost

Electrical production

Mean electrical output

Min. electrical output

Max. electrical output

Fuel consumption

Specific fuel consumption

Fuel energy input

Mean electrical efficiency

\begin{tabular}{|r|l|l|}
\hline Value & Units \\
\hline 101 & $\mathrm{hrs} / \mathrm{yr}$ \\
\hline 149 & $\mathrm{starts} / \mathrm{yr}$ \\
\hline 56.87 & $\mathrm{yr} / \mathrm{hr}$ \\
\hline 0.23 & $\$ / \mathrm{kWh}$ \\
\hline 36489 & $\mathrm{kWh} / \mathrm{yr}$ \\
\hline 361 & $\mathrm{~kW}$ \\
\hline 173 & $\mathrm{~kW}$ \\
\hline 690 & $\mathrm{~kW}$ \\
\hline 10655 & $\mathrm{~L} / \mathrm{yr}$ \\
\hline 0.29 & $\mathrm{~L} / \mathrm{kWh}$ \\
\hline 104845 & $\mathrm{kWh} / \mathrm{yr}$ \\
\hline 35 & $\%$ \\
\hline
\end{tabular}

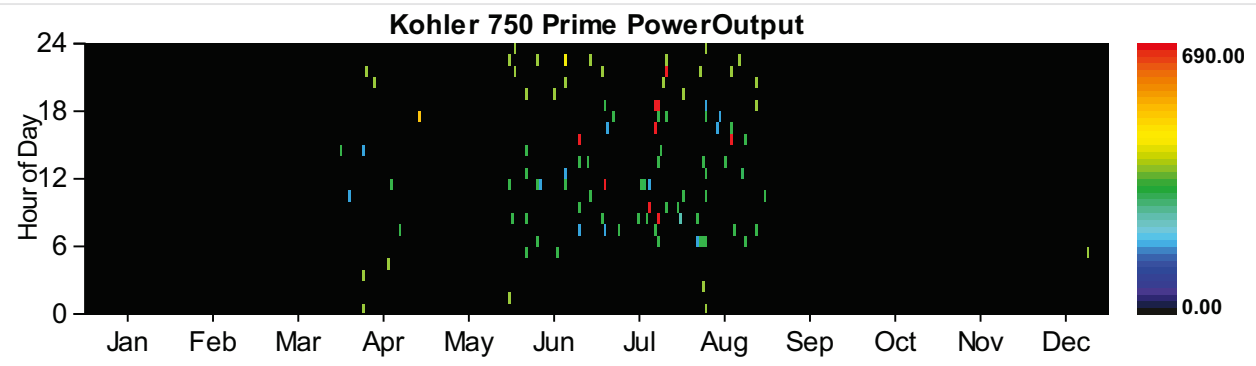

\section{Battery:GS200 flow}

\section{Quantity}

Value

String size

Strings in parallel

\section{Batteries}

Bus voltage 


\section{Quantity}

Nominal capacity

Usable nominal capacity

Autonomy

Lifetime throughput

Battery wear cost

Average energy cost

Energy in

Energy out

Storage depletion

Losses

Annual throughput

Expected life
Value

Units

$00 \mathrm{kWh}$

$600 \mathrm{kWh}$

$0 \mathrm{hr}$

0

$0.000 \$ / k W h$

$0.023 \$ / k W h$

$137578 \mathrm{kWh} / \mathrm{yr}$

96807 kWh/yr

$600 \mathrm{kWh} / \mathrm{yr}$

40171 kWh/yr

$115706 \mathrm{kWh} / \mathrm{yr}$

$25 \mathrm{yr}$

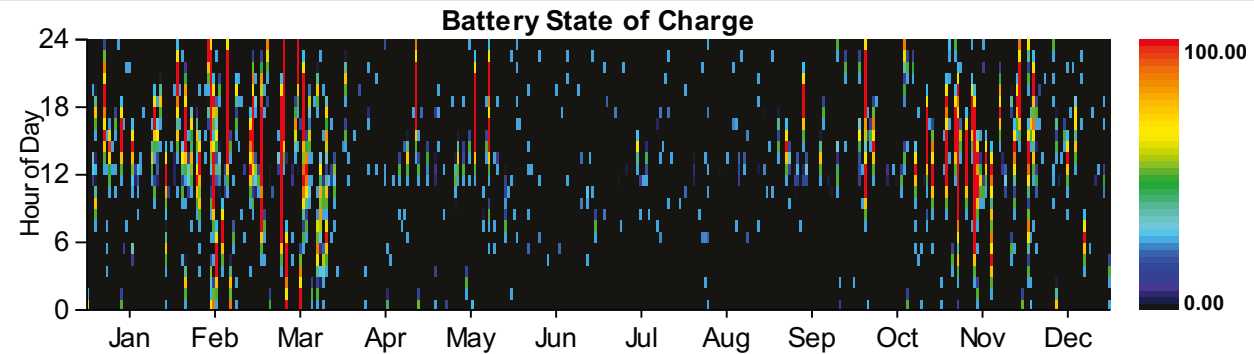

\section{Converter}

\begin{tabular}{|l|r|r|l|}
\hline Quantity & Inverter & Rectifier & Units \\
\hline Capacity & 750 & 675 & $\mathrm{~kW}$ \\
\hline Mean output & 125 & $\mathrm{~kW}$ \\
\hline Minimum output & 0 & $\mathrm{~kW}$ \\
\hline Maximum output & 750 & $179 \mathrm{~kW}$ \\
\hline Capacity factor & 17 & 1 \\
\hline Hours of operation & 4,379 & 585 \\
\hline Energy in & $1,212,861$ & $\mathrm{hrs} / \mathrm{yr}$ \\
\hline Energy out & $1,091,576$ & 79,536 & $\mathrm{kWh} / \mathrm{yr}$ \\
\hline Losses & 121,286 & 67,606 & $\mathrm{kWh} / \mathrm{yr}$ \\
\hline
\end{tabular}



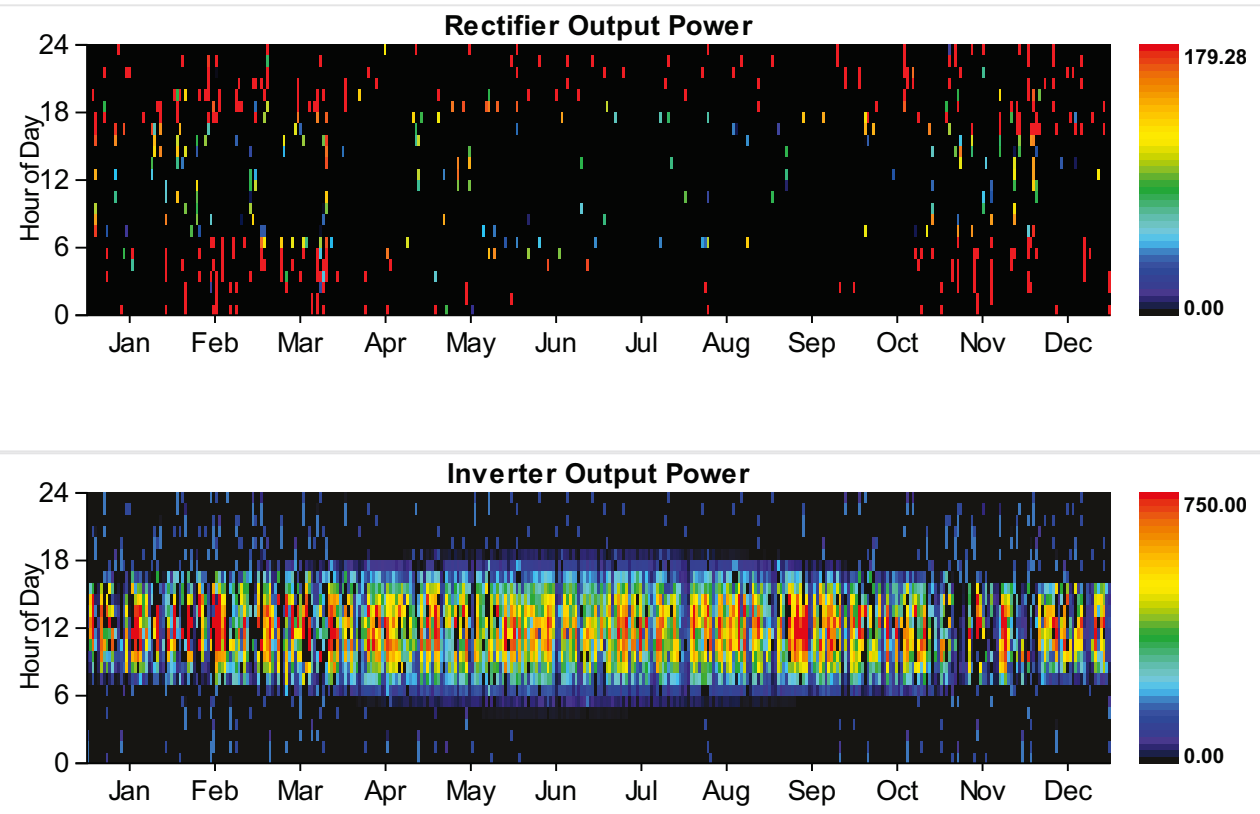

\section{Grid}

Rate: Demand 1

\begin{tabular}{|c|c|c|c|c|c|c|}
\hline Resources.ReportingService_GeneratelnputsReport_Month & $\begin{array}{l}\text { Energy } \\
\text { Purchased } \\
\text { (kWh) }\end{array}$ & $\begin{array}{l}\text { Energy } \\
\text { Sold } \\
\text { (kWh) }\end{array}$ & $\begin{array}{l}\text { Net } \\
\text { Purchases } \\
\text { (kWh) }\end{array}$ & $\begin{array}{l}\text { Peak } \\
\text { Demand } \\
(\mathbf{k W})\end{array}$ & $\begin{array}{l}\text { Energy } \\
\text { Charge } \\
\text { (\$) }\end{array}$ & $\begin{array}{l}\text { Demand } \\
\text { Charge } \\
\text { (\$) }\end{array}$ \\
\hline January & 0 & 0 & 0 & 18,095 & 0 & 289,519 \\
\hline February & 0 & 0 & 0 & 14,936 & 0 & 238,977 \\
\hline March & 0 & 0 & 0 & 16,699 & 0 & 267,178 \\
\hline April & 0 & 0 & 0 & 20,000 & 0 & 320,000 \\
\hline May & 0 & 0 & 0 & 17,572 & 0 & 281,151 \\
\hline June & 0 & 0 & 0 & 20,000 & 0 & 320,000 \\
\hline July & 0 & 0 & 0 & 20,000 & 0 & 320,000 \\
\hline August & 0 & 0 & 0 & 20,000 & 0 & 320,000 \\
\hline September & 0 & 0 & 0 & 15,468 & 0 & 247,493 \\
\hline October & 0 & 0 & 0 & 13,726 & 0 & 219,623 \\
\hline November & 0 & 0 & 0 & 15,876 & 0 & 254,021 \\
\hline December & 0 & 0 & 0 & 18,490 & 0 & 295,836 \\
\hline Annual & 0 & 0 & 0 & 20,000 & 0 & $3,373,798$ \\
\hline
\end{tabular}

Rate: Rate 1

\begin{tabular}{|c|c|c|c|c|c|c|}
\hline Resources.ReportingService_GeneratelnputsReport_Month & $\begin{array}{l}\text { Energy } \\
\text { Purchased } \\
\text { (kWh) }\end{array}$ & $\begin{array}{l}\text { Energy } \\
\text { Sold } \\
\text { (kWh) }\end{array}$ & $\begin{array}{l}\text { Net } \\
\text { Purchases } \\
\text { (kWh) }\end{array}$ & $\begin{array}{l}\text { Peak } \\
\text { Demand } \\
(\mathbf{k W})\end{array}$ & $\begin{array}{l}\text { Energy } \\
\text { Charge } \\
(\$)\end{array}$ & $\begin{array}{l}\text { Demand } \\
\text { Charge } \\
\text { (\$) }\end{array}$ \\
\hline January & $4,942,838$ & 0 & $4,942,838$ & 0 & 593,141 & 0 \\
\hline February & $3,194,187$ & 0 & $3,194,187$ & 0 & 383,302 & 0 \\
\hline March & $3,633,528$ & 0 & $3,633,528$ & 0 & 436,023 & 0 \\
\hline
\end{tabular}




\begin{tabular}{|c|c|c|c|c|c|c|}
\hline $\begin{array}{l}\text { April } \\
\text { Reasources.ReportingService_GeneratelnputsReport_Month }\end{array}$ & $\begin{array}{l}\text { Energy } \\
\text { Purchased } \\
\text { (kwh) } \\
\text { (k,423,759 }\end{array}$ & $\begin{array}{l}\text { Energy } \\
\text { Sold } \\
(\mathbf{k W h})_{0}\end{array}$ & $\begin{array}{l}\text { Nett } \\
\text { Purchases } \\
\text { (kWh) } \\
\text { (k,423,759 }\end{array}$ & $\begin{array}{l}\text { Peak } 0 \\
\text { Demand } \\
\text { (kW) } 0\end{array}$ & $\begin{array}{l}\text { Energy } \\
\text { Charge } \\
(\$ \$ \$ 50,851\end{array}$ & $\begin{array}{l}\text { Demang } \\
\text { Charge } \\
\text { (\$) } \quad 0\end{array}$ \\
\hline June & $7,763,009$ & 0 & $7,763,009$ & 0 & 931,561 & 0 \\
\hline July & $8,359,714$ & 0 & $8,359,714$ & 0 & $1,003,166$ & 0 \\
\hline August & $8,388,528$ & 0 & $8,388,528$ & 0 & $1,006,623$ & 0 \\
\hline September & $5,293,275$ & 0 & $5,293,275$ & 0 & 635,193 & 0 \\
\hline October & $4,660,071$ & 0 & $4,660,071$ & 0 & 559,209 & 0 \\
\hline November & $3,692,188$ & 0 & $3,692,188$ & 0 & 443,063 & 0 \\
\hline December & $5,411,146$ & 0 & $5,411,146$ & 0 & 649,338 & 0 \\
\hline Annual & $67,207,992$ & 0 & $67,207,992$ & 0 & $8,064,959$ & 0 \\
\hline
\end{tabular}

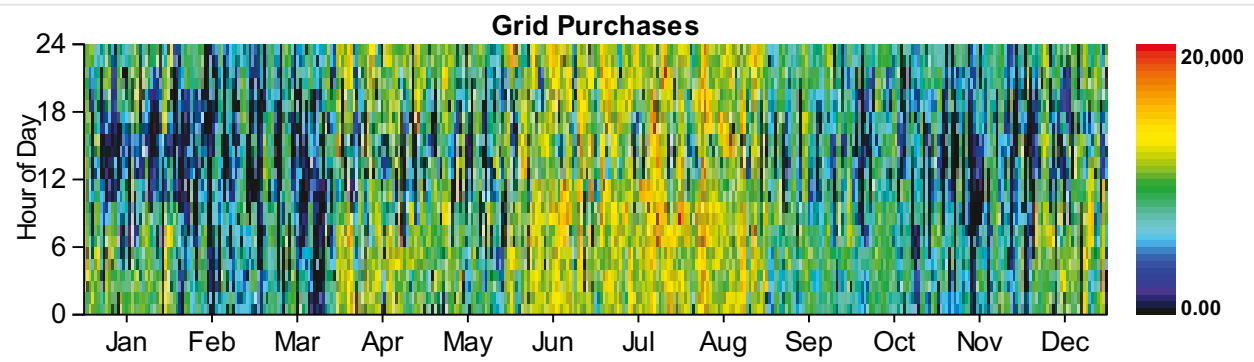

\section{Emissions}

\section{Pollutant}

Carbon dioxide

Carbon monoxide

Unburned hydrocarbons

Particulate matter

Sulfur dioxide

Nitrogen oxides

HOMER Energy, LLC @ 2016
Emissions

Units

42503416 kg/yr

$117 \mathrm{~kg} / \mathrm{yr}$

$13 \mathrm{~kg} / \mathrm{yr}$

$3 \mathrm{~kg} / \mathrm{yr}$

184207 kg/yr

90176 kg/yr 
System Report

System architecture

\begin{tabular}{|c|c|c|c|}
\hline PV & Solar World 320W flat plate PV Copy & 1,000 & kW \\
\hline Wind Turbine & Siemens 2.3 MW - 108 & 5 & \\
\hline Generator & Kohler 1000 Prime Power & 925 & kW \\
\hline Battery & GS200 flow & 1 & strings \\
\hline Converter & System Converter & 750 & kW \\
\hline Grid & Grid & 20,000 & kW \\
\hline Dispatch Strategy & Cycle Charging & & \\
\hline
\end{tabular}

\section{Cost summary}

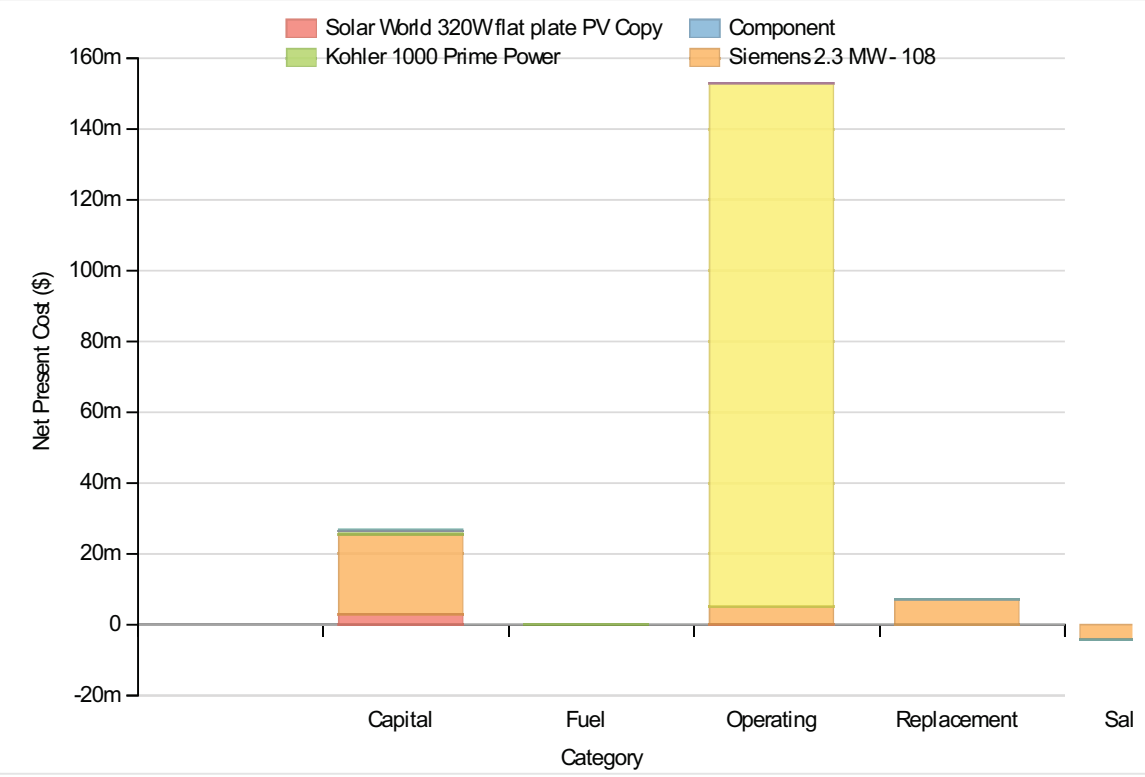

Cost Summary

Total net present cost

$183079984 \$$

Levelized cost of energy

$0.146 \$ / k W h$

Net Present Costs

\begin{tabular}{|c|c|c|c|c|c|c|}
\hline Component & Capital & Replacement & O\&M & Fuel & Salvage & Total \\
\hline Solar World 320W flat plate PV Copy & $3,000,000$ & 0 & 193,913 & 0 & 0 & $3,193,913$ \\
\hline Siemens 2.3 MW - 108 & $22,500,000$ & $7,173,158$ & $4,847,816$ & 0 & $-4,042,534$ & $30,478,440$ \\
\hline Kohler 1000 Prime Power & 925,000 & 0 & 18,116 & 130,850 & $-184,290$ & 889,676 \\
\hline Grid & 0 & 0 & $147,853,584$ & 0 & 0 & $147,853,584$ \\
\hline GS200 flow & 324,589 & 7,176 & 31,026 & 0 & -973 & 361,818 \\
\hline Converter & 225,000 & 95,462 & 0 & 0 & $-17,967$ & 302,495 \\
\hline System & $26,974,588$ & $7,275,795$ & $152,944,432$ & 130,850 & $-4,245,763$ & $183,079,902$ \\
\hline
\end{tabular}




\begin{tabular}{|c|c|c|c|c|c|c|}
\hline Component & Capital & Replacement & O\&M & Fuel & Salvage & Total \\
\hline Solar World 320W flat plate PV Copy & 232,063 & 0 & 15,000 & 0 & 0 & 247,063 \\
\hline Siemens 2.3 MW - 108 & $1,740,474$ & 554,875 & 375,000 & 0 & $-312,708$ & $2,357,641$ \\
\hline Kohler 1000 Prime Power & 71,553 & 0 & 1,401 & 10,122 & $-14,256$ & 68,820 \\
\hline Grid & 0 & 0 & $11,437,122$ & 0 & 0 & $11,437,122$ \\
\hline GS200 flow & 25,108 & 555 & 2,400 & 0 & -75 & 27,988 \\
\hline Converter & 17,405 & 7,384 & 0 & 0 & $-1,390$ & 23,400 \\
\hline System & $2,086,603$ & 562,815 & $11,830,922$ & 10,122 & $-328,428$ & $14,162,034$ \\
\hline
\end{tabular}

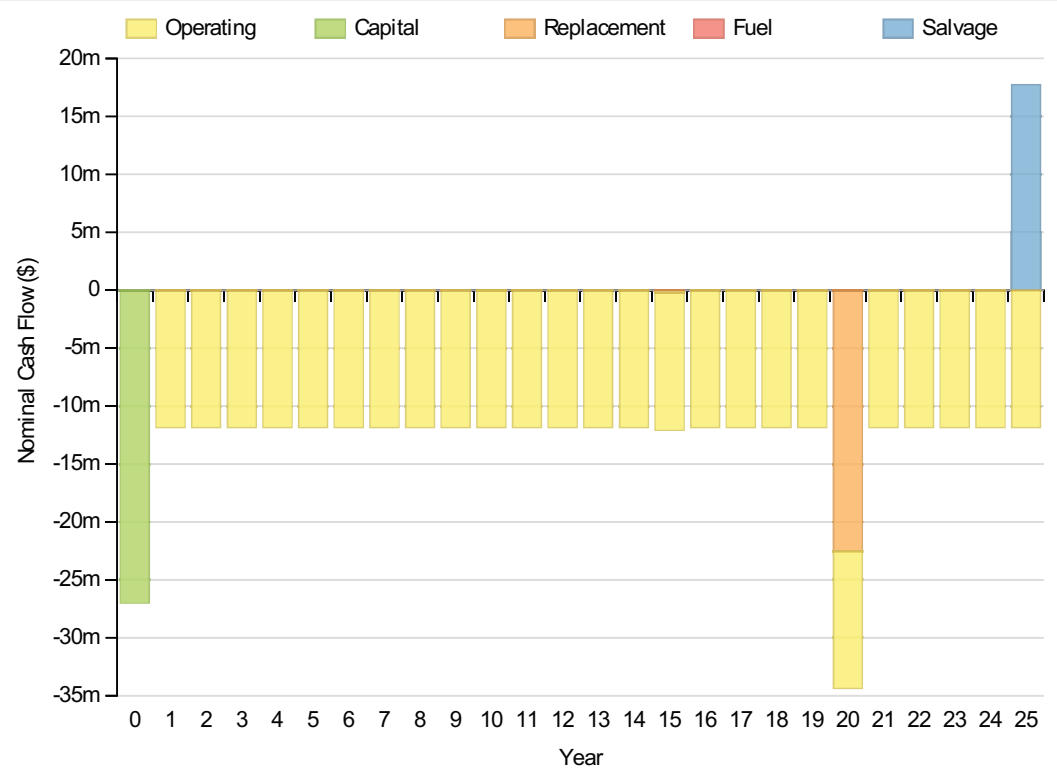

\section{Electrical}

\section{Quantity}

Excess electricity

Unmet load

Capacity shortage

Renewable fraction

\section{Component}

PV

Generator

Wind Turbine

Grid Purchases

Total

Load

Consumption(kWh/yr)

Production(kWh/yr)
Value

Fraction (\%)

$1,288,675$

44,379

$30,268,832$

31

$67,202,248$

$98,804,128$

100 


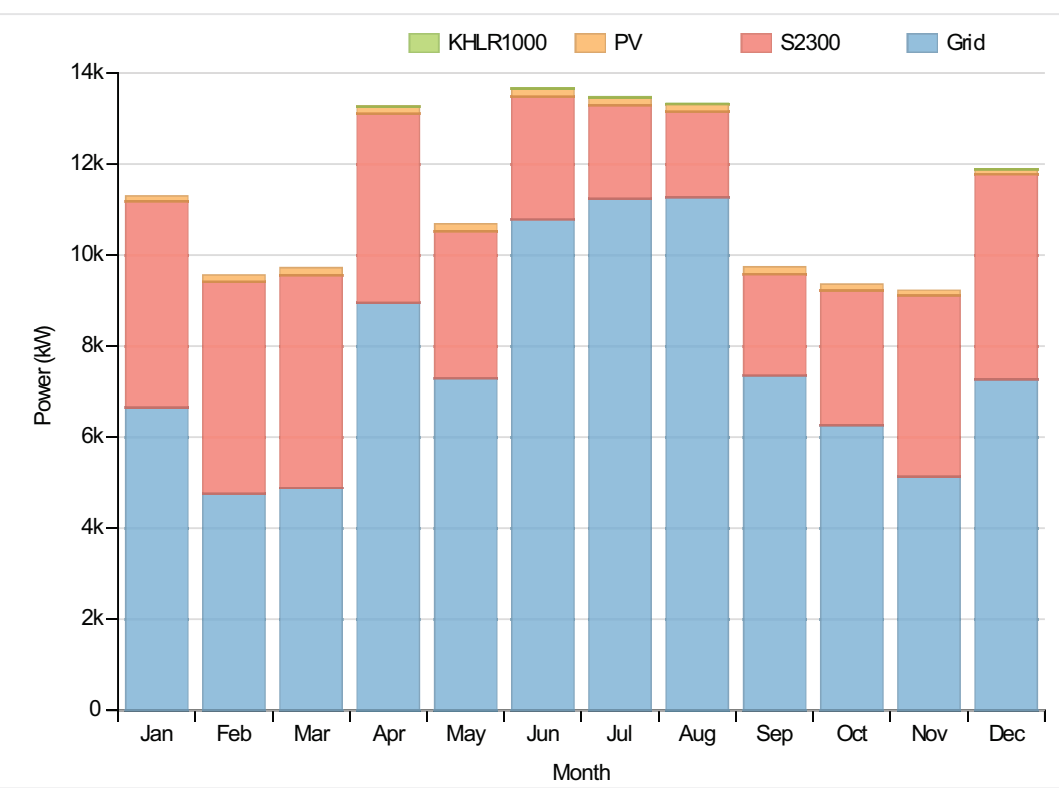

PV:Solar World 320W flat plate PV Copy

\begin{tabular}{|l|c|c|}
\hline Quantity & Value & Units \\
\hline Rated capacity & $1000 \mathrm{~kW}$ \\
\hline Mean output & $147 \mathrm{~kW}$ \\
\hline Mean output & $3530.60 \mathrm{kWh} / \mathrm{d}$ \\
\hline Capacity factor & $14.71 \%$ \\
\hline Total production & $1288675 \mathrm{kWh} / \mathrm{yr}$ \\
\hline Minimum output & $0.00 \mathrm{~kW}$ \\
\hline Maximum output & $1022.50 \mathrm{~kW}$ \\
\hline PV penetration & $1.33 \%$ \\
\hline Hours of operation & $\%$ hrs/yr \\
\hline Levelized cost & 4377 \\
\hline
\end{tabular}

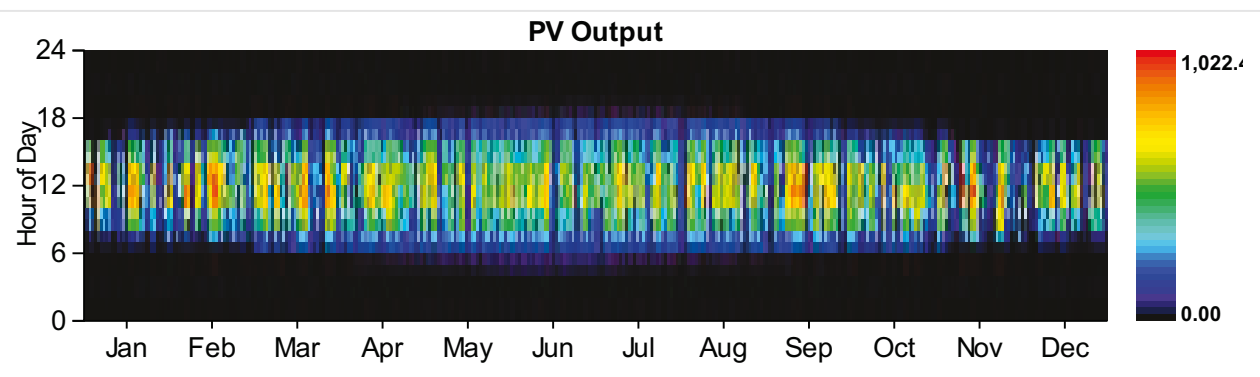

Wind Turbine:Siemens 2.3 MW - 108

\section{Quantity}

Total rated capacity

Mean output

Capacity factor

\begin{tabular}{|l|l|l|}
\hline Value & Units \\
\hline 11500 & $\mathrm{~kW}$ \\
\hline 3455 & $\mathrm{~kW}$ \\
\hline 30.05 & $\%$ \\
\hline
\end{tabular}


¿talheftyduction

Minimum output

Maximum output

Wind penetration

Hours of operation

Levelized cost
Value

30268832 units

$7.19 \mathrm{~kW}$

11574.00 kW

$31.25 \%$

$8760 \mathrm{hrs} / \mathrm{yr}$

$0.078 \$ / k W h$

\section{Generator:Kohler 1000 Prime Power}

\section{Quantity}

Hours of operation

Number of starts

Operational life

Fixed generation cost

Marginal generation cost

Electrical production

Mean electrical output

Min. electrical output

Max. electrical output

Fuel consumption

Specific fuel consumption

Fuel energy input

Mean electrical efficiency

\begin{tabular}{|r|l|l|}
\hline Value & Units \\
\hline 101 & $\mathrm{hrs} / \mathrm{yr}$ \\
\hline 96 & starts/yr \\
\hline 149 & $\mathrm{yr}$ \\
\hline 76.24 & $\$ / \mathrm{hr}$ \\
\hline 0.23 & $\$ / \mathrm{kWh}$ \\
\hline 44379 & $\mathrm{kWh} / \mathrm{yr}$ \\
\hline 439 & $\mathrm{~kW}$ \\
\hline 231 & $\mathrm{~kW}$ \\
\hline 925 & $\mathrm{~kW}$ \\
\hline 12812 & $\mathrm{~L} / \mathrm{yr}$ \\
\hline 0.29 & $\mathrm{~L} / \mathrm{kWh}$ \\
\hline 126075 & $\mathrm{kWh} / \mathrm{yr}$ \\
\hline 35 & $\%$ \\
\hline
\end{tabular}

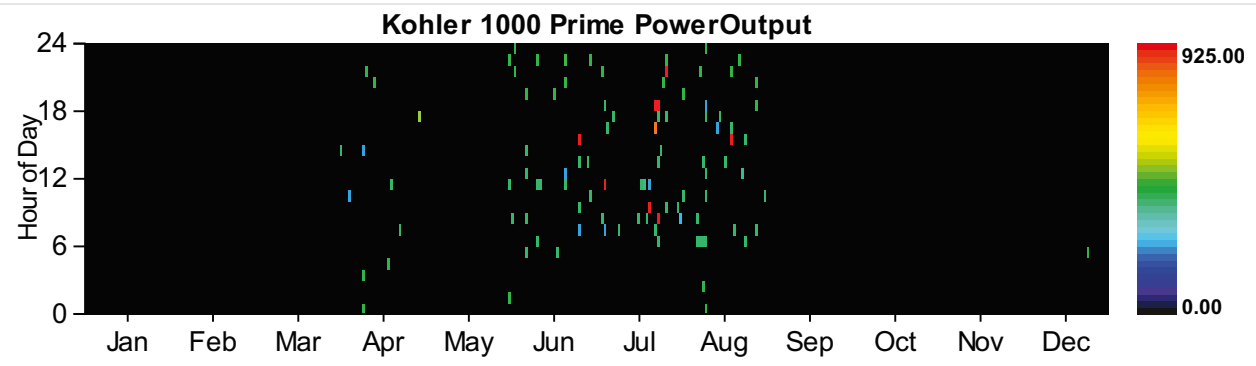

\section{Battery:GS200 flow}

\section{Quantity}

Value

String size

Strings in parallel

\section{Batteries}

Bus voltage 


\section{Quantity}

Nominal capacity

Usable nominal capacity

Autonomy

Lifetime throughput

Battery wear cost

Average energy cost

Energy in

Energy out

Storage depletion

Losses

Annual throughput

Expected life
Value

Units

000 kWh

$600 \mathrm{kWh}$

$0 \mathrm{hr}$

0

$0.000 \$ / k W h$

$0.024 \$ / k W h$

$138406 \mathrm{kWh} / \mathrm{yr}$

97387 kWh/yr

$600 \mathrm{kWh} / \mathrm{yr}$

40419 kWh/yr

$116400 \mathrm{kWh} / \mathrm{yr}$

$25 \mathrm{yr}$

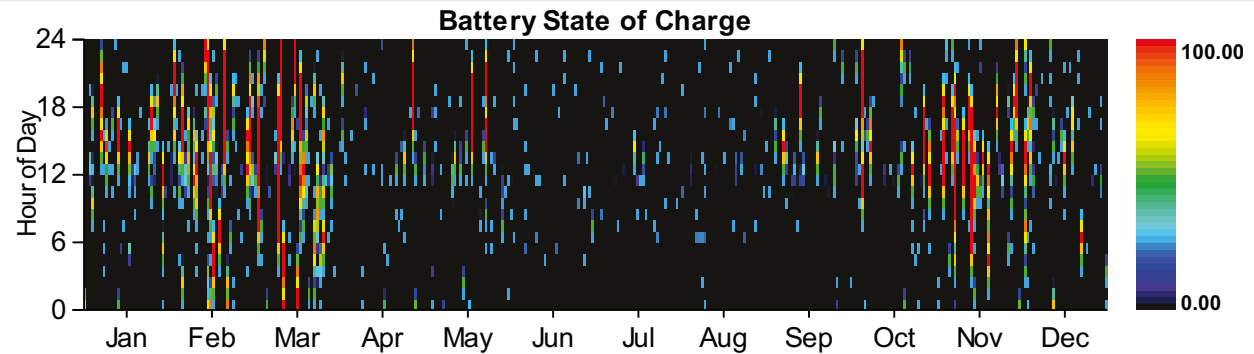

\section{Converter}

\begin{tabular}{|l|r|r|l|}
\hline Quantity & Inverter & Rectifier & Units \\
\hline Capacity & 750 & 675 & $\mathrm{~kW}$ \\
\hline Mean output & 125 & $\mathrm{~kW}$ \\
\hline Minimum output & 0 & $\mathrm{~kW}$ \\
\hline Maximum output & 750 & $179 \mathrm{~kW}$ \\
\hline Capacity factor & 17 & 1 \\
\hline Hours of operation & $\%, 377$ & 587 \\
\hline Energy in & $1,212,743$ & $\mathrm{hrs} / \mathrm{yr}$ \\
\hline Energy out & $1,091,470$ & 79,690 & $\mathrm{kWh} / \mathrm{yr}$ \\
\hline Losses & 121,274 & 67,737 & $\mathrm{kWh} / \mathrm{yr}$ \\
\hline
\end{tabular}



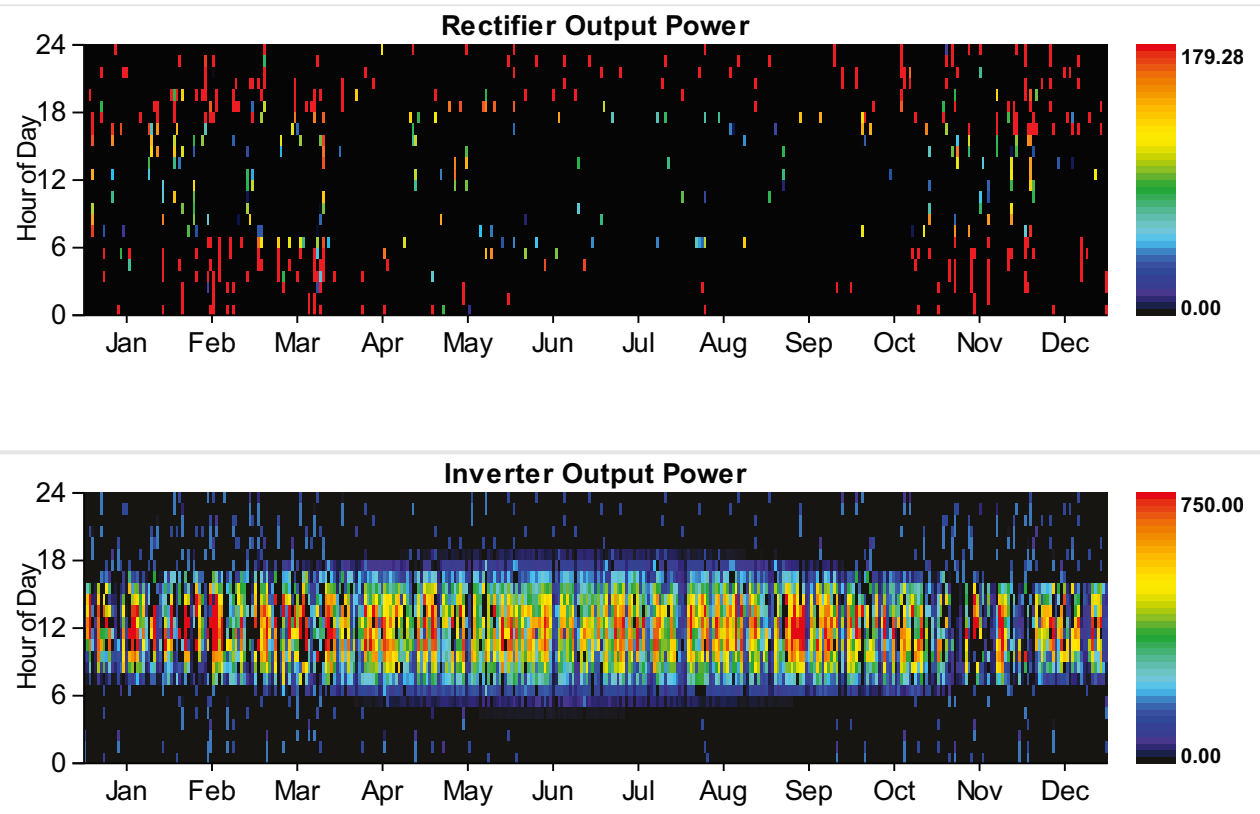

\section{Grid}

Rate: Demand 1

\begin{tabular}{|c|c|c|c|c|c|c|}
\hline Resources.ReportingService_GeneratelnputsReport_Month & $\begin{array}{l}\text { Energy } \\
\text { Purchased } \\
\text { (kWh) }\end{array}$ & $\begin{array}{l}\text { Energy } \\
\text { Sold } \\
\text { (kWh) }\end{array}$ & $\begin{array}{l}\text { Net } \\
\text { Purchases } \\
\text { (kWh) }\end{array}$ & $\begin{array}{l}\text { Peak } \\
\text { Demand } \\
(\mathbf{k W})\end{array}$ & $\begin{array}{l}\text { Energy } \\
\text { Charge } \\
\text { (\$) }\end{array}$ & $\begin{array}{l}\text { Demand } \\
\text { Charge } \\
\text { (\$) }\end{array}$ \\
\hline January & 0 & 0 & 0 & 18,095 & 0 & 289,519 \\
\hline February & 0 & 0 & 0 & 14,936 & 0 & 238,977 \\
\hline March & 0 & 0 & 0 & 16,699 & 0 & 267,178 \\
\hline April & 0 & 0 & 0 & 20,000 & 0 & 320,000 \\
\hline May & 0 & 0 & 0 & 17,572 & 0 & 281,151 \\
\hline June & 0 & 0 & 0 & 20,000 & 0 & 320,000 \\
\hline July & 0 & 0 & 0 & 20,000 & 0 & 320,000 \\
\hline August & 0 & 0 & 0 & 20,000 & 0 & 320,000 \\
\hline September & 0 & 0 & 0 & 15,468 & 0 & 247,493 \\
\hline October & 0 & 0 & 0 & 13,726 & 0 & 219,623 \\
\hline November & 0 & 0 & 0 & 15,876 & 0 & 254,021 \\
\hline December & 0 & 0 & 0 & 18,431 & 0 & 294,896 \\
\hline Annual & 0 & 0 & 0 & 20,000 & 0 & $3,372,858$ \\
\hline
\end{tabular}

Rate: Rate 1

\begin{tabular}{|c|c|c|c|c|c|c|}
\hline Resources.ReportingService_GeneratelnputsReport_Month & $\begin{array}{l}\text { Energy } \\
\text { Purchased } \\
\text { (kWh) }\end{array}$ & $\begin{array}{l}\text { Energy } \\
\text { Sold } \\
\text { (kWh) }\end{array}$ & $\begin{array}{l}\text { Net } \\
\text { Purchases } \\
\text { (kWh) }\end{array}$ & $\begin{array}{l}\text { Peak } \\
\text { Demand } \\
(\mathbf{k W})\end{array}$ & $\begin{array}{l}\text { Energy } \\
\text { Charge } \\
(\$)\end{array}$ & $\begin{array}{l}\text { Demand } \\
\text { Charge } \\
\text { (\$) }\end{array}$ \\
\hline January & $4,942,838$ & 0 & $4,942,838$ & 0 & 593,141 & 0 \\
\hline February & $3,194,187$ & 0 & $3,194,187$ & 0 & 383,302 & 0 \\
\hline March & $3,633,528$ & 0 & $3,633,528$ & 0 & 436,023 & 0 \\
\hline
\end{tabular}




\begin{tabular}{|c|c|c|c|c|c|c|}
\hline - & $\begin{array}{l}\text { Energy } \\
\text { Purchased }\end{array}$ & $\begin{array}{l}\text { Energy } \\
\text { Sold }\end{array}$ & $\begin{array}{l}\text { Net }_{445,165} \\
\text { Purchases }\end{array}$ & $\begin{array}{l}\text { Peak } 0 \\
\text { Demand }\end{array}$ & $\begin{array}{l}\text { Energy } \\
\text { Charge }\end{array}$ & $\begin{array}{l}\text { Demang } \\
\text { Charge }\end{array}$ \\
\hline Reasources.ReportingService_GeneratelnputsReport_Month & $\left(\mathbf{k}_{5,42}\right)_{3,759}$ & $(\mathbf{k W h})_{0}$ & $\left(\mathbf{k}_{5,42} \mathbf{2}\right), 759$ & $(\mathbf{k W}) \quad 0$ & $(\$ \$ 50,851$ & (\$) $\quad 0$ \\
\hline June & $7,761,369$ & 0 & $7,761,369$ & 0 & 931,364 & 0 \\
\hline July & $8,358,215$ & 0 & $8,358,215$ & 0 & $1,002,986$ & 0 \\
\hline August & $8,386,565$ & 0 & $8,386,565$ & 0 & $1,006,388$ & 0 \\
\hline September & $5,293,275$ & 0 & $5,293,275$ & 0 & 635,193 & 0 \\
\hline October & $4,660,071$ & 0 & $4,660,071$ & 0 & 559,209 & 0 \\
\hline November & $3,692,188$ & 0 & $3,692,188$ & 0 & 443,063 & 0 \\
\hline December & $5,411,088$ & 0 & $5,411,088$ & 0 & 649,331 & 0 \\
\hline Annual & $67,202,248$ & 0 & $67,202,248$ & 0 & $8,064,269$ & 0 \\
\hline
\end{tabular}

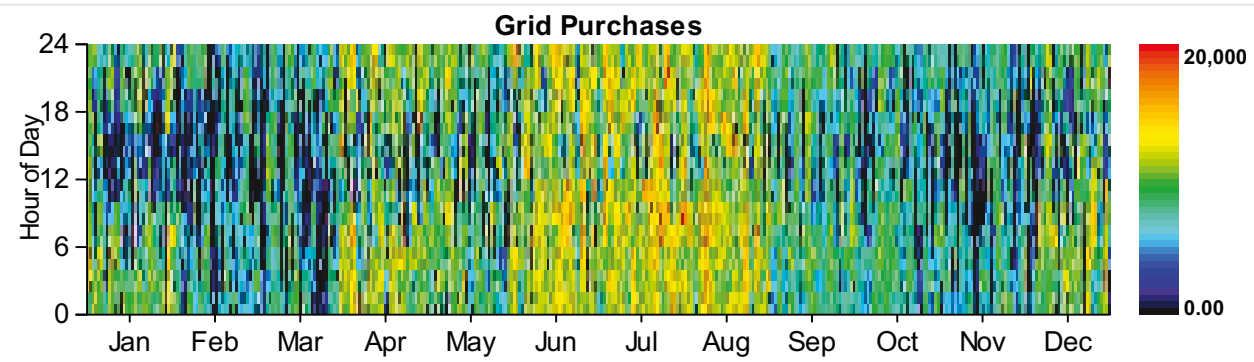

\section{Emissions}

\section{Pollutant}

Carbon dioxide

Carbon monoxide

Unburned hydrocarbons

Particulate matter

Sulfur dioxide

Nitrogen oxides

HOMER Energy, LLC @ 2016
Emissions

Units

42505448 kg/yr

$141 \mathrm{~kg} / \mathrm{yr}$

$16 \mathrm{~kg} / \mathrm{yr}$

$4 \mathrm{~kg} / \mathrm{yr}$

$184203 \mathrm{~kg} / \mathrm{yr}$

90192 kg/yr 
System Report

System architecture

\begin{tabular}{|l|l|l|}
\hline PV & Solar World 320W flat plate PV Copy & 1,000 \\
\hline Wind Turbine & Siemens 2.3 MW - 108 & 5 \\
\hline Generator & Kohler 1000 Prime Power & 925 \\
\hline Generator \#2 & Kohler 750 Prime Power & 690 \\
\hline Converter & System Converter & kW \\
\hline Grid & Grid & 750 \\
\hline Dispatch Strategy & Cycle Charging & 20,000 \\
\hline
\end{tabular}

\section{Cost summary}

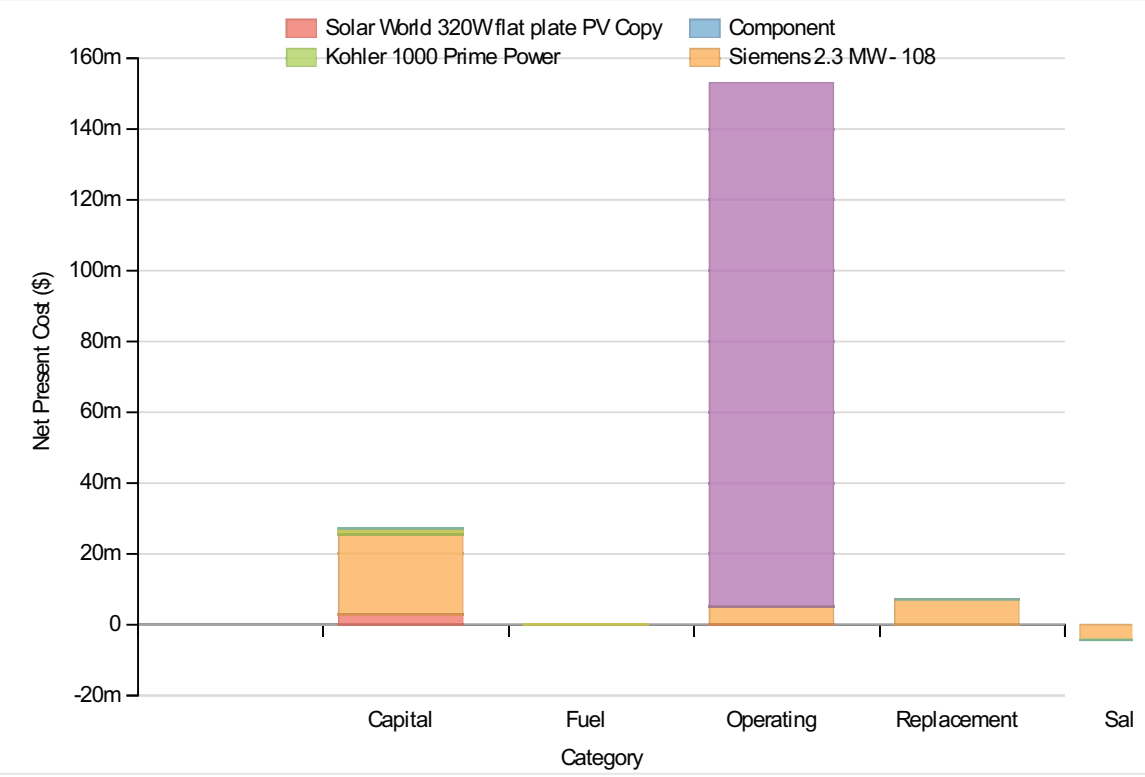

Cost Summary

Total net present cost

183377696 \$

Levelized cost of energy

$0.146 \$ / k W h$

Net Present Costs

\begin{tabular}{|c|c|c|c|c|c|c|}
\hline Component & Capital & Replacement & O\&M & Fuel & Salvage & Total \\
\hline Solar World 320W flat plate PV Copy & $3,000,000$ & 0 & 193,913 & 0 & 0 & $3,193,913$ \\
\hline Siemens 2.3 MW - 108 & $22,500,000$ & $7,173,158$ & $4,847,816$ & 0 & $-4,042,534$ & $30,478,440$ \\
\hline Kohler 1000 Prime Power & 925,000 & 0 & 11,838 & 61,768 & $-197,216$ & 801,390 \\
\hline Kohler 750 Prime Power & 690,000 & 0 & 12,577 & 56,875 & $-139,399$ & 620,053 \\
\hline Grid & 0 & 0 & $147,981,344$ & 0 & 0 & $147,981,344$ \\
\hline Converter & 225,000 & 95,462 & 0 & 0 & $-17,967$ & 302,495 \\
\hline System & $27,340,000$ & $7,268,619$ & $153,047,488$ & 118,642 & $-4,397,115$ & $183,377,634$ \\
\hline
\end{tabular}




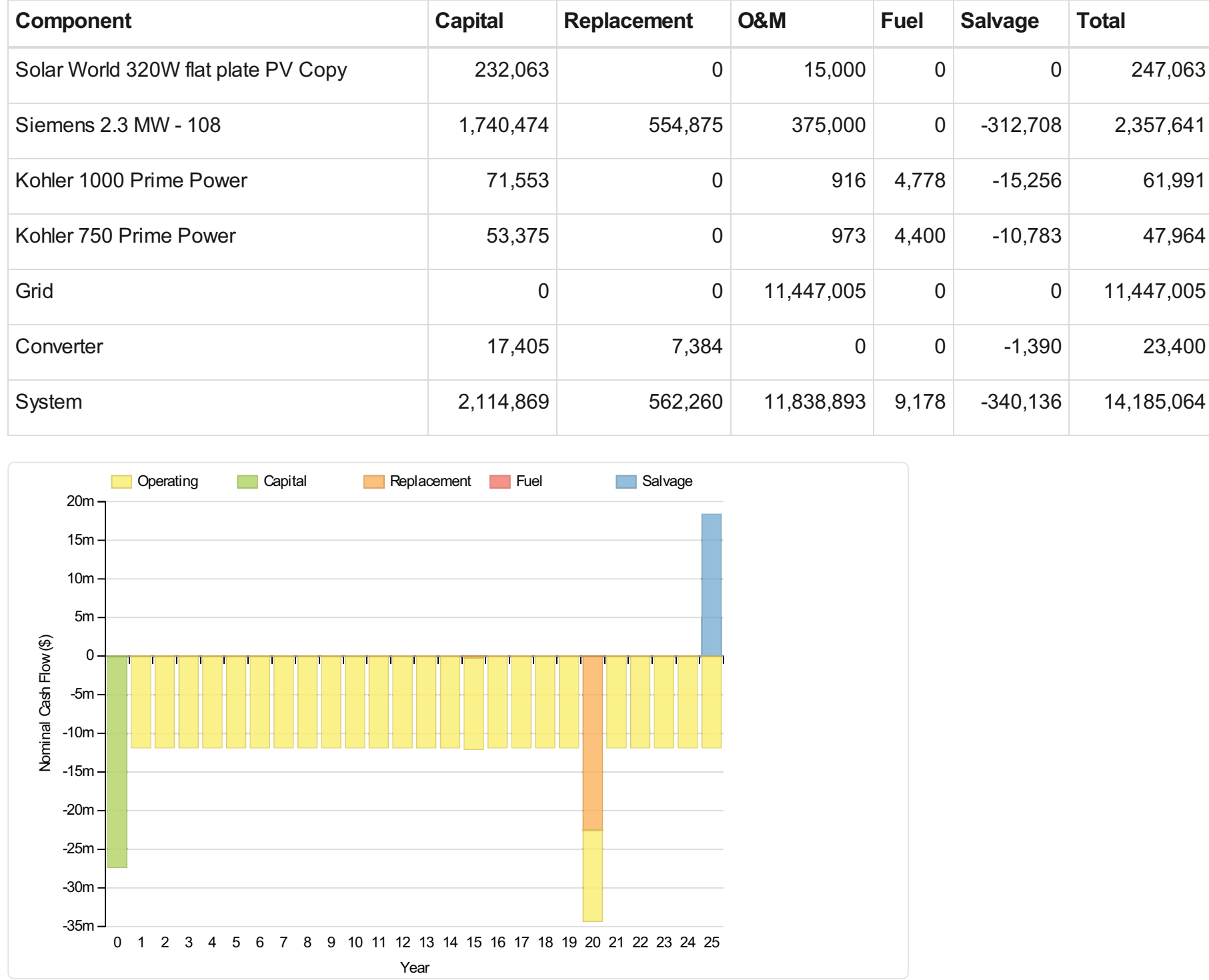

\section{Electrical}

\section{Quantity}

Excess electricity

Unmet load

Capacity shortage

Renewable fraction

\section{Component}

PV

Generator

Generator

Wind Turbine

Grid Purchases

Total

\section{Value}

Production(kWh/yr)

Fraction (\%)

\section{$3395 \mathrm{kWh} / \mathrm{yr}$ \\ 39956 kWh/yr}

0

\begin{tabular}{|r|r|} 
& Fraction (\%) \\
\hline $1,288,675$ & 1 \\
\hline 20,893 & 0 \\
\hline 18,979 & 0 \\
\hline $30,268,832$ & 31 \\
\hline $67,283,376$ & 68 \\
\hline $98,880,752$ & 100 \\
\hline
\end{tabular}

\section{Load}

Consumption(kWh/yr)

Fraction (\%) 


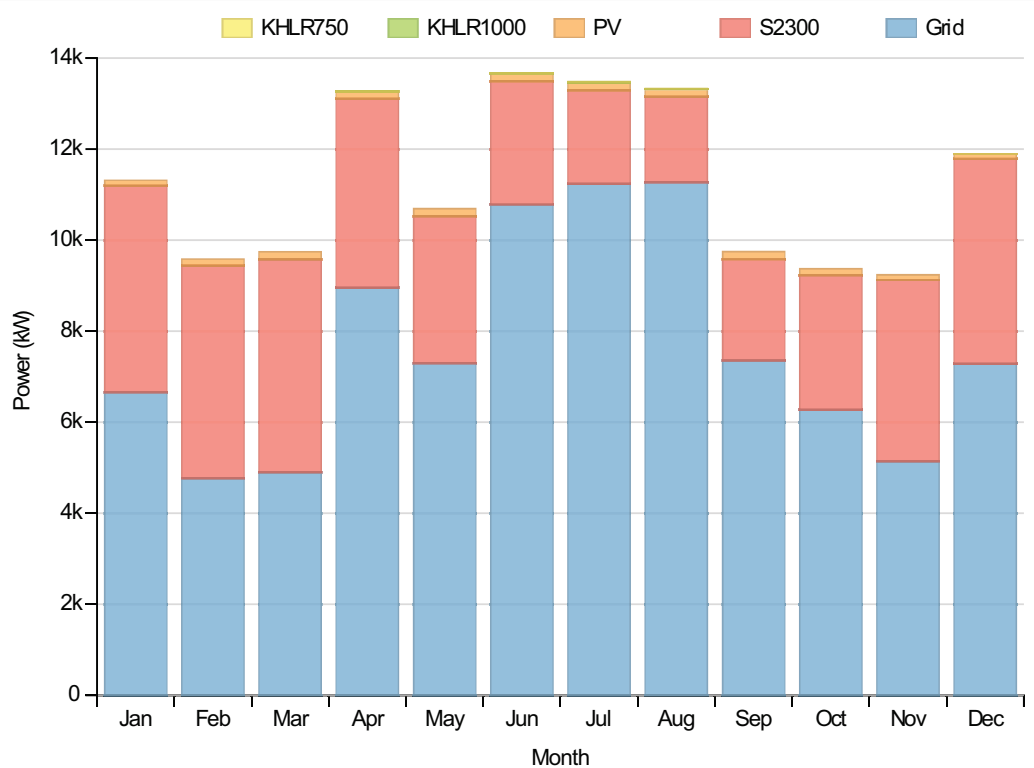

PV:Solar World 320W flat plate PV Copy

\begin{tabular}{|c|c|c|}
\hline Quantity & Value & Units \\
\hline Rated capacity & 1000 & $\mathrm{~kW}$ \\
\hline Mean output & 147 & $\mathrm{~kW}$ \\
\hline Mean output & 3530.60 & $\mathrm{kWh} / \mathrm{d}$ \\
\hline Capacity factor & 14.71 & $\%$ \\
\hline Total production & 1288675 & $\mathrm{kWh} / \mathrm{yr}$ \\
\hline Minimum output & 0.00 & $\mathrm{~kW}$ \\
\hline Maximum output & 1022.50 & $\mathrm{~kW}$ \\
\hline PV penetration & 1.33 & $\%$ \\
\hline Hours of operation & 4377 & $\mathrm{hrs} / \mathrm{yr}$ \\
\hline Levelized cost & 0.192 & $\$ / \mathrm{kWh}$ \\
\hline
\end{tabular}

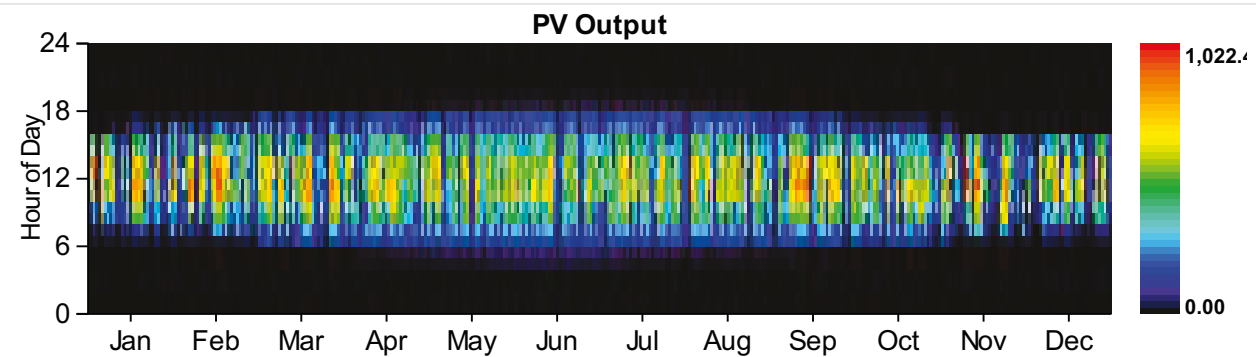

Wind Turbine:Siemens 2.3 MW - 108

\begin{tabular}{|l|r|l|}
\hline Quantity & Value & Units \\
\hline Total rated capacity & $11500 \mathrm{~kW}$ \\
\hline Mean output & $3455 \mathrm{~kW}$ \\
\hline
\end{tabular}




\begin{tabular}{|c|c|c|c|}
\hline Quparntity factor & Value & 30.05 & Upnits \\
\hline Total production & & 30268832 & $\mathrm{kWh} / \mathrm{yr}$ \\
\hline Minimum output & & 7.19 & $\mathrm{~kW}$ \\
\hline Maximum output & & 11574.00 & $\mathrm{~kW}$ \\
\hline Wind penetration & & 31.25 & $\%$ \\
\hline Hours of operation & & 8760 & hrs/yr \\
\hline Levelized cost & & 0.078 & $\$ / k W h$ \\
\hline
\end{tabular}

\section{Generator:Kohler 1000 Prime Power}

\begin{tabular}{|c|c|c|}
\hline Quantity & Value & Units \\
\hline Hours of operation & 66 & $\mathrm{hrs} / \mathrm{yr}$ \\
\hline Number of starts & 62 & starts/yr \\
\hline Operational life & 227 & $\mathrm{yr}$ \\
\hline Fixed generation cost & 76.24 & $\$ / h r$ \\
\hline Marginal generation cost & 0.23 & $\$ / k W h$ \\
\hline Electrical production & 20893 & $\mathrm{kWh} / \mathrm{yr}$ \\
\hline Mean electrical output & 317 & $\mathrm{~kW}$ \\
\hline Min. electrical output & 231 & $\mathrm{~kW}$ \\
\hline Max. electrical output & 925 & $\mathrm{~kW}$ \\
\hline Fuel consumption & 6048 & L/yr \\
\hline Specific fuel consumption & 0.29 & $\mathrm{~L} / \mathrm{kWh}$ \\
\hline Fuel energy input & 59513 & $\mathrm{kWh} / \mathrm{yr}$ \\
\hline Mean electrical efficiency & 35 & $\%$ \\
\hline
\end{tabular}

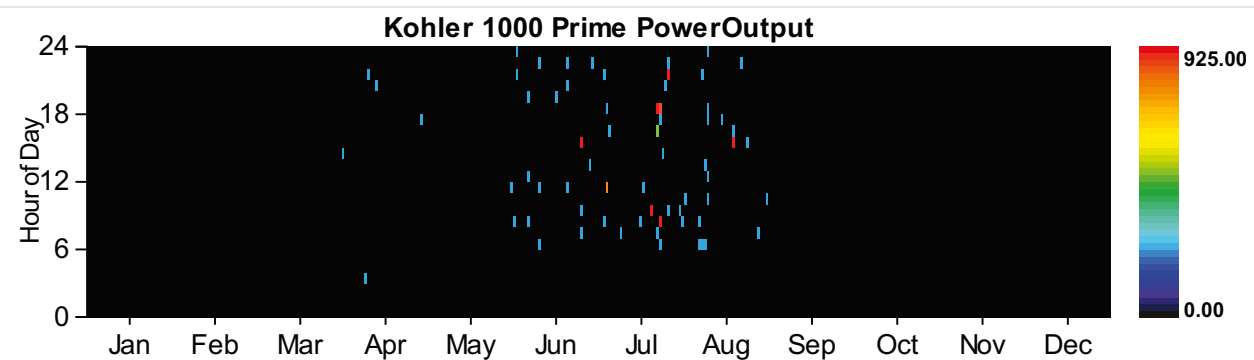

\section{Generator:Kohler 750 Prime Power}

\begin{tabular}{|l|r|r|}
\hline Quantity & Value & Units \\
\hline Hours of operation & 94 hrs/yr \\
\hline Number of starts & 88 starts/yr \\
\hline Operational life & $160 \mathrm{yr}$ \\
\hline
\end{tabular}


Qixâิ

Marginal generation cost

Electrical production

Mean electrical output

Min. electrical output

Max. electrical output

Fuel consumption

Specific fuel consumption

Fuel energy input

Mean electrical efficiency
Value

56.87 Bhlits

$0.23 \$ / k W h$

18979 kWh/yr

202 kW

173 kW

690 kW

5569 L/yr

$0.29 \mathrm{~L} / \mathrm{kWh}$

54799 kWh/yr

$35 \%$

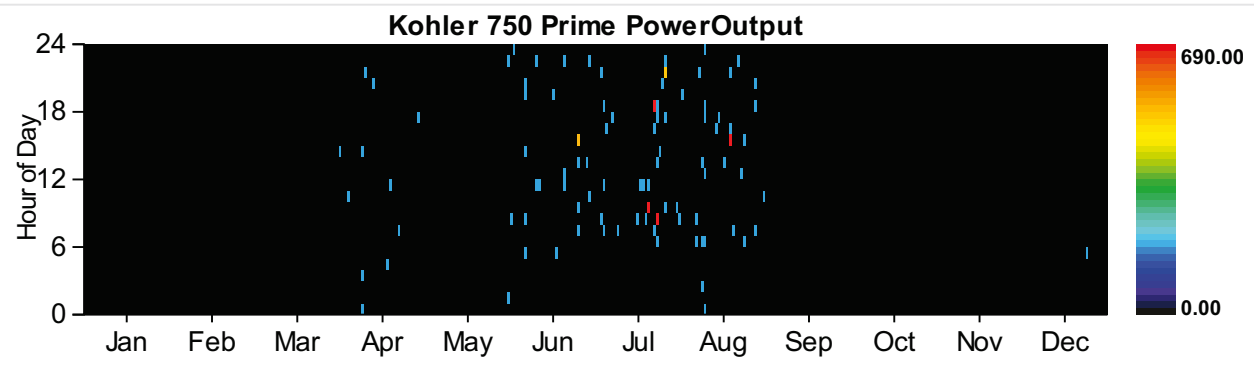

Converter

\begin{tabular}{|c|c|c|c|}
\hline Quantity & Inverter & Rectifier & Units \\
\hline Capacity & 750 & 675 & $\mathrm{~kW}$ \\
\hline Mean output & 115 & 0 & $\mathrm{~kW}$ \\
\hline Minimum output & 0 & 0 & $\mathrm{~kW}$ \\
\hline Maximum output & 750 & 0 & $\mathrm{~kW}$ \\
\hline Capacity factor & 15 & 0 & $\%$ \\
\hline Hours of operation & 3,902 & 0 & $\mathrm{hrs} / \mathrm{yr}$ \\
\hline Energy in & $1,123,182$ & 0 & $\mathrm{kWh} / \mathrm{yr}$ \\
\hline Energy out & $1,010,865$ & 0 & $\mathrm{kWh} / \mathrm{yr}$ \\
\hline Losses & 112,317 & 0 & $\mathrm{kWh} / \mathrm{yr}$ \\
\hline
\end{tabular}

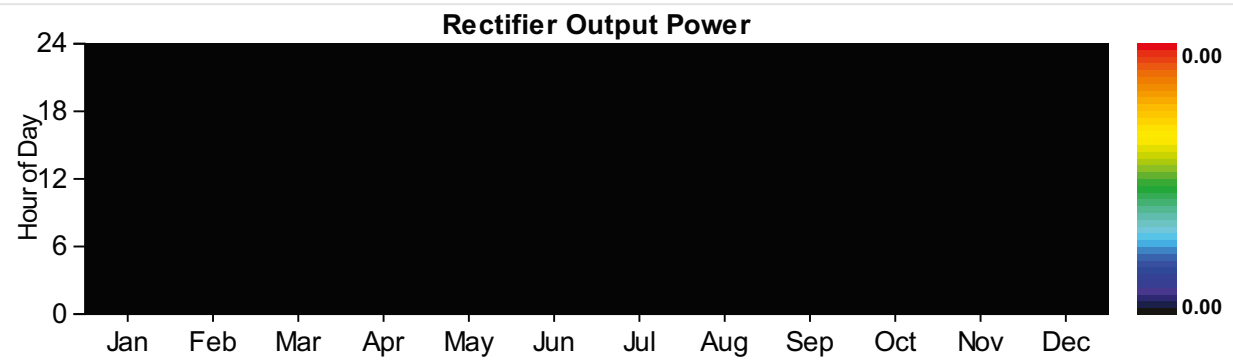




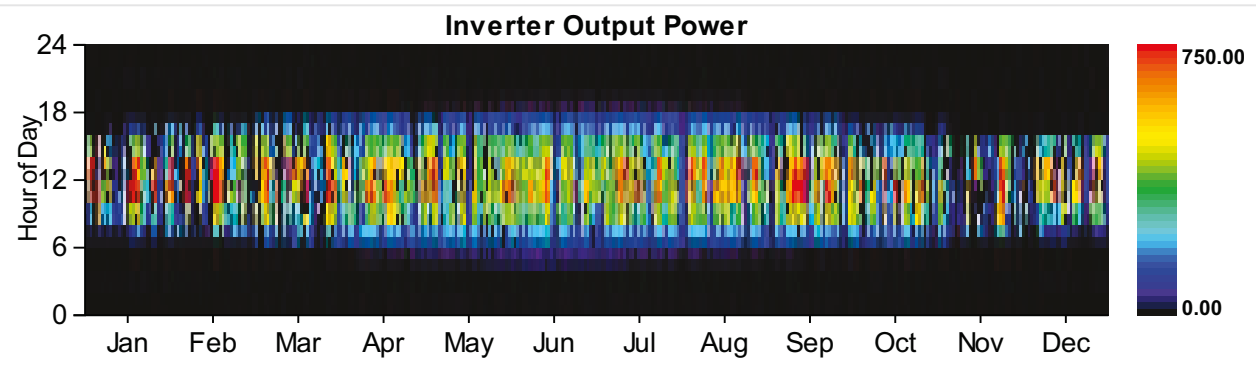

Grid

Rate: Demand 1

\begin{tabular}{|c|c|c|c|c|c|c|}
\hline Resources.ReportingService_GeneratelnputsReport_Month & $\begin{array}{l}\text { Energy } \\
\text { Purchased } \\
\text { (kWh) }\end{array}$ & $\begin{array}{l}\text { Energy } \\
\text { Sold } \\
\text { (kWh) }\end{array}$ & $\begin{array}{l}\text { Net } \\
\text { Purchases } \\
\text { (kWh) }\end{array}$ & $\begin{array}{l}\text { Peak } \\
\text { Demand } \\
(\mathbf{k W})\end{array}$ & $\begin{array}{l}\text { Energy } \\
\text { Charge } \\
\text { (\$) }\end{array}$ & $\begin{array}{l}\text { Demand } \\
\text { Charge } \\
(\$)\end{array}$ \\
\hline January & 0 & 0 & 0 & 18,095 & 0 & 289,519 \\
\hline February & 0 & 0 & 0 & 14,936 & 0 & 238,977 \\
\hline March & 0 & 0 & 0 & 16,699 & 0 & 267,178 \\
\hline April & 0 & 0 & 0 & 19,950 & 0 & 319,208 \\
\hline May & 0 & 0 & 0 & 17,572 & 0 & 281,151 \\
\hline June & 0 & 0 & 0 & 20,000 & 0 & 320,000 \\
\hline July & 0 & 0 & 0 & 20,000 & 0 & 320,000 \\
\hline August & 0 & 0 & 0 & 20,000 & 0 & 320,000 \\
\hline September & 0 & 0 & 0 & 15,468 & 0 & 247,493 \\
\hline October & 0 & 0 & 0 & 13,726 & 0 & 219,623 \\
\hline November & 0 & 0 & 0 & 15,876 & 0 & 254,021 \\
\hline December & 0 & 0 & 0 & 18,490 & 0 & 295,836 \\
\hline Annual & 0 & 0 & 0 & 20,000 & 0 & $3,373,005$ \\
\hline
\end{tabular}

Rate: Rate 1

\begin{tabular}{|c|c|c|c|c|c|c|}
\hline Resources.ReportingService_GeneratelnputsReport_Month & $\begin{array}{l}\text { Energy } \\
\text { Purchased } \\
\text { (kWh) }\end{array}$ & $\begin{array}{l}\text { Energy } \\
\text { Sold } \\
\text { (kWh) }\end{array}$ & $\begin{array}{l}\text { Net } \\
\text { Purchases } \\
\text { (kWh) }\end{array}$ & $\begin{array}{l}\text { Peak } \\
\text { Demand } \\
\text { (kW) }\end{array}$ & $\begin{array}{l}\text { Energy } \\
\text { Charge } \\
(\$)\end{array}$ & $\begin{array}{l}\text { Demand } \\
\text { Charge } \\
(\$)\end{array}$ \\
\hline January & $4,952,182$ & 0 & $4,952,182$ & 0 & 594,262 & 0 \\
\hline February & $3,207,647$ & 0 & $3,207,647$ & 0 & 384,918 & 0 \\
\hline March & $3,646,840$ & 0 & $3,646,840$ & 0 & 437,621 & 0 \\
\hline April & $6,449,524$ & 0 & $6,449,524$ & 0 & 773,943 & 0 \\
\hline May & $5,429,449$ & 0 & $5,429,449$ & 0 & 651,534 & 0 \\
\hline June & $7,763,387$ & 0 & $7,763,387$ & 0 & 931,606 & 0 \\
\hline July & $8,359,129$ & 0 & $8,359,129$ & 0 & $1,003,095$ & 0 \\
\hline August & $8,387,901$ & 0 & $8,387,901$ & 0 & $1,006,548$ & 0 \\
\hline
\end{tabular}


Retobources.ReportingService_GeneratelnputsReport_Month

\section{November}

$3,703,306$

$0 \quad 3,703,306$

\begin{tabular}{l|l}
$0 \quad 444,397$
\end{tabular}

(\$)

\begin{tabular}{|l|r|r|r|r|r|r|r|r|r|r|r|r|r|r|r|r|}
\hline December & $5,419,775$ & 0 & $5,419,775$ & 0 \\
\hline Annual & $67,283,376$ & 0 & $67,283,376$ & 0 & $8,074,005$ & 0 \\
\hline
\end{tabular}

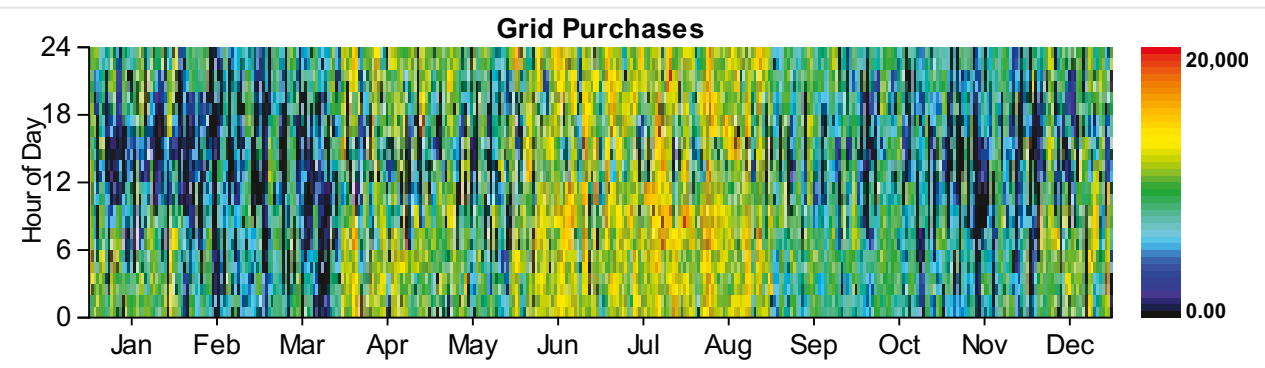

\section{Emissions}

\begin{tabular}{|l|r|l|}
\hline Pollutant & Emissions & Units \\
\hline Carbon dioxide & 42553580 & $\mathrm{~kg} / \mathrm{yr}$ \\
\hline Carbon monoxide & $128 \mathrm{~kg} / \mathrm{yr}$ \\
\hline Unburned hydrocarbons & $15 \mathrm{~kg} / \mathrm{yr}$ \\
\hline Particulate matter & $4 \mathrm{~kg} / \mathrm{yr}$ \\
\hline Sulfur dioxide & $184419 \mathrm{~kg} / \mathrm{yr}$ \\
\hline Nitrogen oxides & $90288 \mathrm{~kg} / \mathrm{yr}$ \\
\hline
\end{tabular}


System Report

System architecture

\begin{tabular}{|l|l|l|l|}
\hline PV & Solar World 320W flat plate PV Copy & 1,000 & $\mathrm{~kW}$ \\
\hline Wind Turbine & Siemens 2.3 MW - 108 & 5 & 750 \\
\hline Converter & System Converter & $\mathrm{kW}$ \\
\hline Grid & Grid & 25,000 \\
\hline Dispatch Strategy & Cycle Charging & $\mathrm{kW}$ \\
\hline
\end{tabular}

\section{Cost summary}

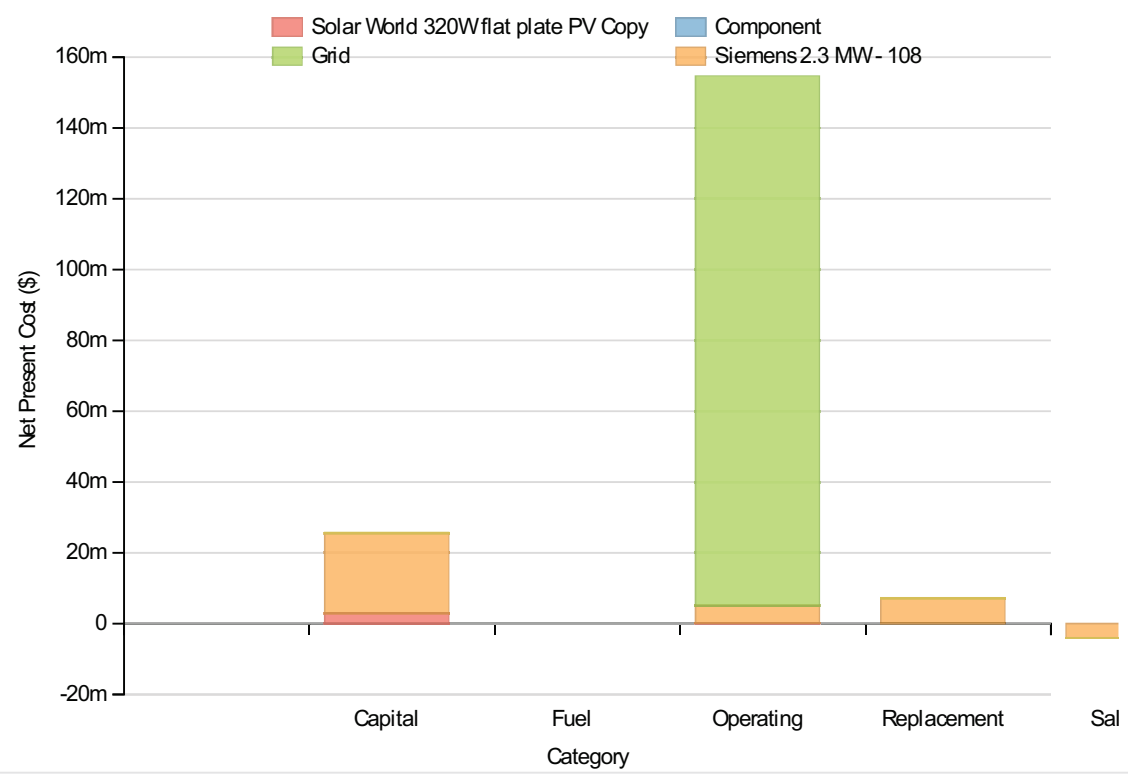

Cost Summary

Total net present cost

$183534320 \$$

Levelized cost of energy

$0.147 \$ / k W h$

Net Present Costs

\begin{tabular}{|c|c|c|c|c|c|c|}
\hline Component & Capital & Replacement & O\&M & Fuel & Salvage & Total \\
\hline Solar World 320W flat plate PV Copy & $3,000,000$ & 0 & 193,913 & 0 & 0 & $3,193,913$ \\
\hline Siemens 2.3 MW - 108 & $22,500,000$ & $7,173,158$ & $4,847,816$ & 0 & $-4,042,534$ & $30,478,440$ \\
\hline Grid & 0 & 0 & $149,559,376$ & 0 & 0 & $149,559,376$ \\
\hline Converter & 225,000 & 95,462 & 0 & 0 & $-17,967$ & 302,495 \\
\hline System & $25,725,000$ & $7,268,619$ & $154,601,104$ & 0 & $-4,060,500$ & $183,534,223$ \\
\hline
\end{tabular}

\section{Annualized Costs}

\begin{tabular}{|l|r|r|r|r|r|r|}
\hline Component & Capital & Replacement & O\&M & Fuel & Salvage & Total \\
\hline Solar World 320W flat plate PV Copy & 232,063 & 0 & 15,000 & 0 & 0 \\
\hline Siemens 2.3 MW - 108 & $1,740,474$ & 554,875 & 375,000 & 0 & $-312,708$ \\
\hline Grid & 0 & 0 & $11,569,073$ & 0 & 0 \\
\hline
\end{tabular}




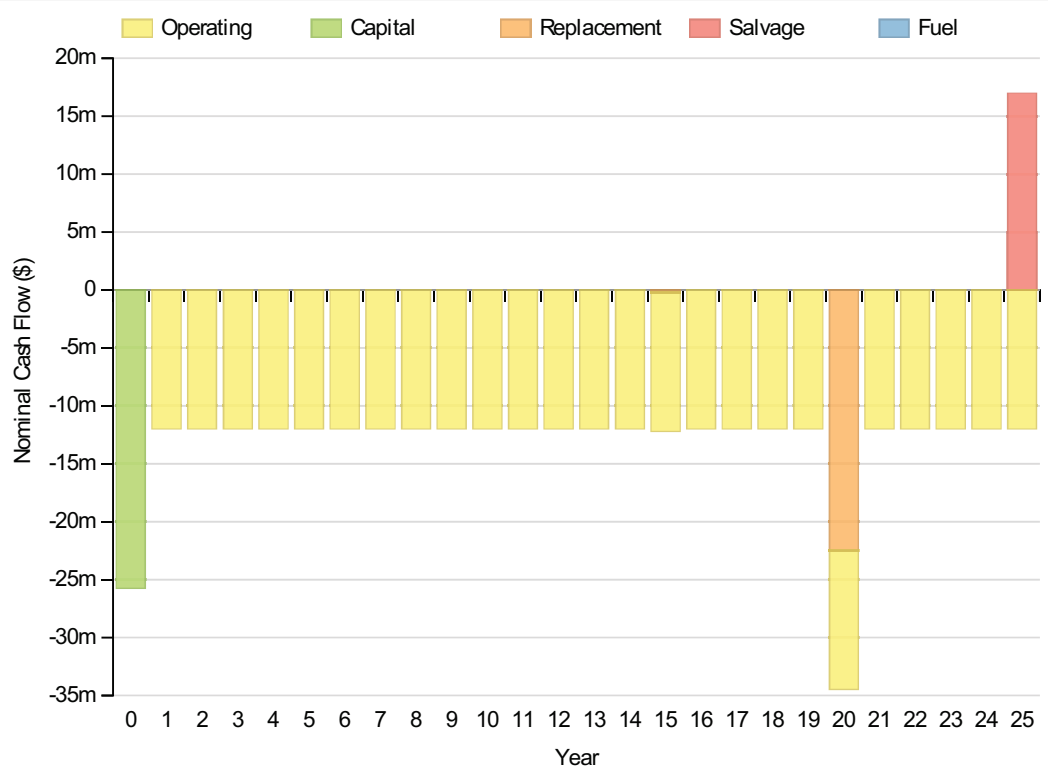

\section{Electrical}

\section{Quantity}

Excess electricity

Unmet load

Capacity shortage

Renewable fraction

\section{Value}

Production(kWh/yr)

PV

Wind Turbine

Grid Purchases

Total

Load

Consumption(kWh/yr)
Component

\section{Fraction (\%)}

\begin{tabular}{r|r}
\hline $1,288,675$ & 1 \\
\hline $30,268,832$ & 31 \\
\hline $67,326,648$ & 68 \\
\hline $98,884,152$ & 100 \\
\hline
\end{tabular}

\section{Fraction (\%)}

AC primary load

$96,864,160$

DC primary load

0

0

Total

$96,864,160$ 


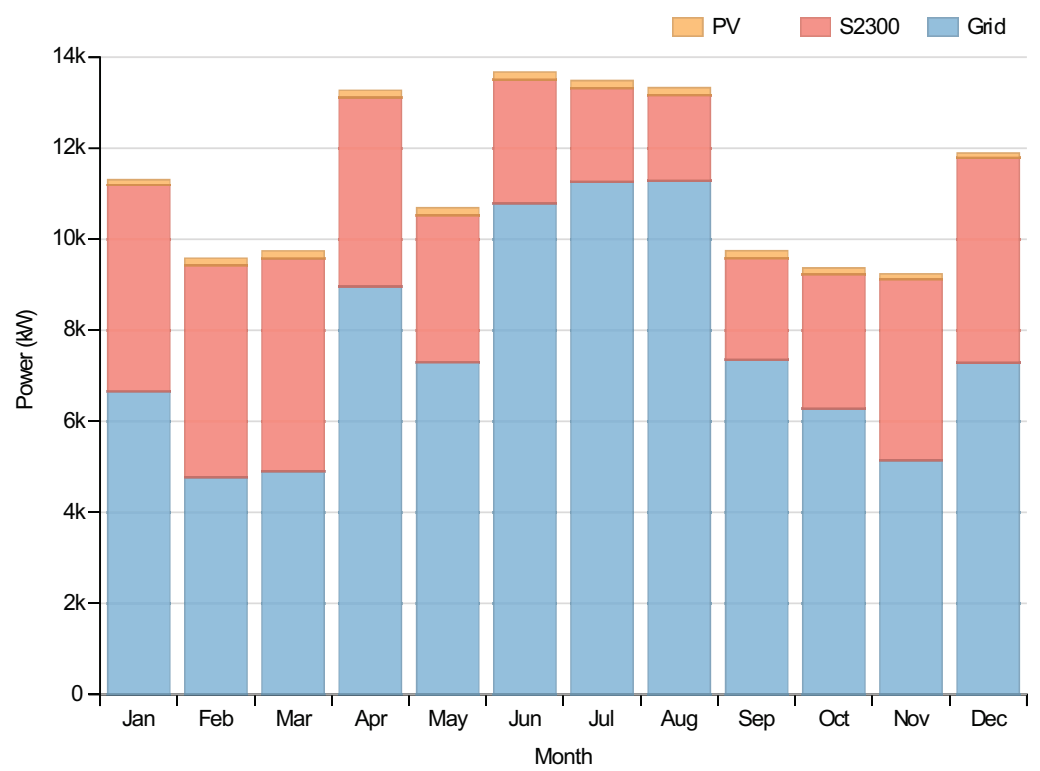

PV:Solar World 320W flat plate PV Copy

\begin{tabular}{|c|c|c|}
\hline Quantity & Value & Units \\
\hline Rated capacity & 1000 & $\mathrm{~kW}$ \\
\hline Mean output & 147 & $\mathrm{~kW}$ \\
\hline Mean output & 3530.60 & $\mathrm{kWh} / \mathrm{d}$ \\
\hline Capacity factor & 14.71 & $\%$ \\
\hline Total production & 1288675 & $\mathrm{kWh} / \mathrm{yr}$ \\
\hline Minimum output & 0.00 & $\mathrm{~kW}$ \\
\hline Maximum output & 1022.50 & $\mathrm{~kW}$ \\
\hline PV penetration & 1.33 & $\%$ \\
\hline Hours of operation & 4377 & $\mathrm{hrs} / \mathrm{yr}$ \\
\hline Levelized cost & 0.192 & $\$ / k W h$ \\
\hline
\end{tabular}

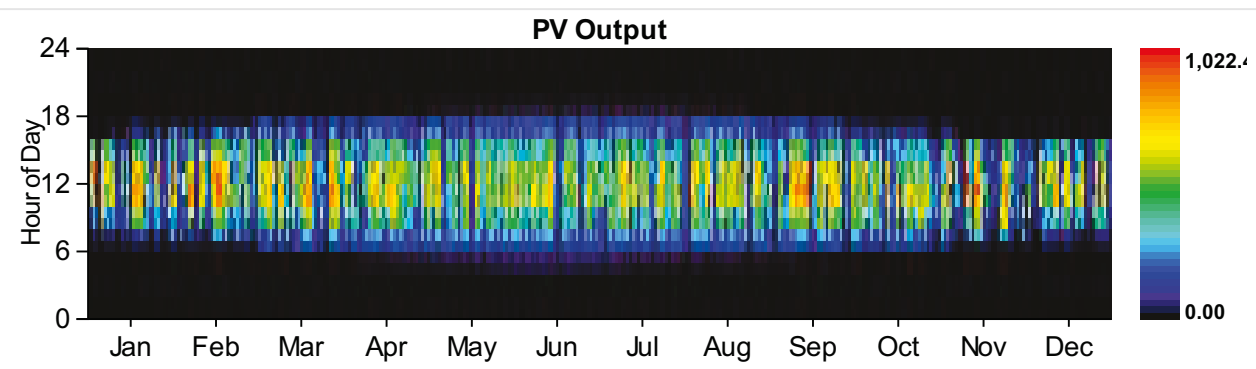

Wind Turbine:Siemens 2.3 MW - 108

\begin{tabular}{|l|r|l|}
\hline Quantity & Value & Units \\
\hline Total rated capacity & 11500 & $\mathrm{~kW}$ \\
\hline Mean output & 3455 & $\mathrm{~kW}$ \\
\hline Capacity factor & $30.05 \%$ \\
\hline Total production & $30268832 \mathrm{kWh} / \mathrm{yr}$ \\
\hline
\end{tabular}


Qinamitity output

Maximum output

Wind penetration

Hours of operation

Levelized cost

\subsection{UUNits}

11574.00 kW

$31.25 \%$

8760 hrs/yr

$0.078 \$ / k W h$

\section{Converter}

\begin{tabular}{|l|r|r|l|}
\hline Quantity & Inverter & Rectifier & Units \\
\hline Capacity & 750 & 675 & $\mathrm{~kW}$ \\
\hline Mean output & 115 & $\mathrm{~kW}$ & $\mathrm{~kW}$ \\
\hline Minimum output & 0 & 0 & $\mathrm{~kW}$ \\
\hline Maximum output & 750 & 0 & $\%$ \\
\hline Capacity factor & 15 & 0 & $\mathrm{krs} / \mathrm{yr}$ \\
\hline Hours of operation & 3,902 & 0 \\
\hline Energy in & $1,123,182$ & $\mathrm{kWh} / \mathrm{yr}$ \\
\hline Energy out & $1,010,865$ & $\mathrm{kWh} / \mathrm{yr}$ \\
\hline Losses & 112,317 & $\mathrm{kWh} / \mathrm{yr}$ \\
\hline
\end{tabular}
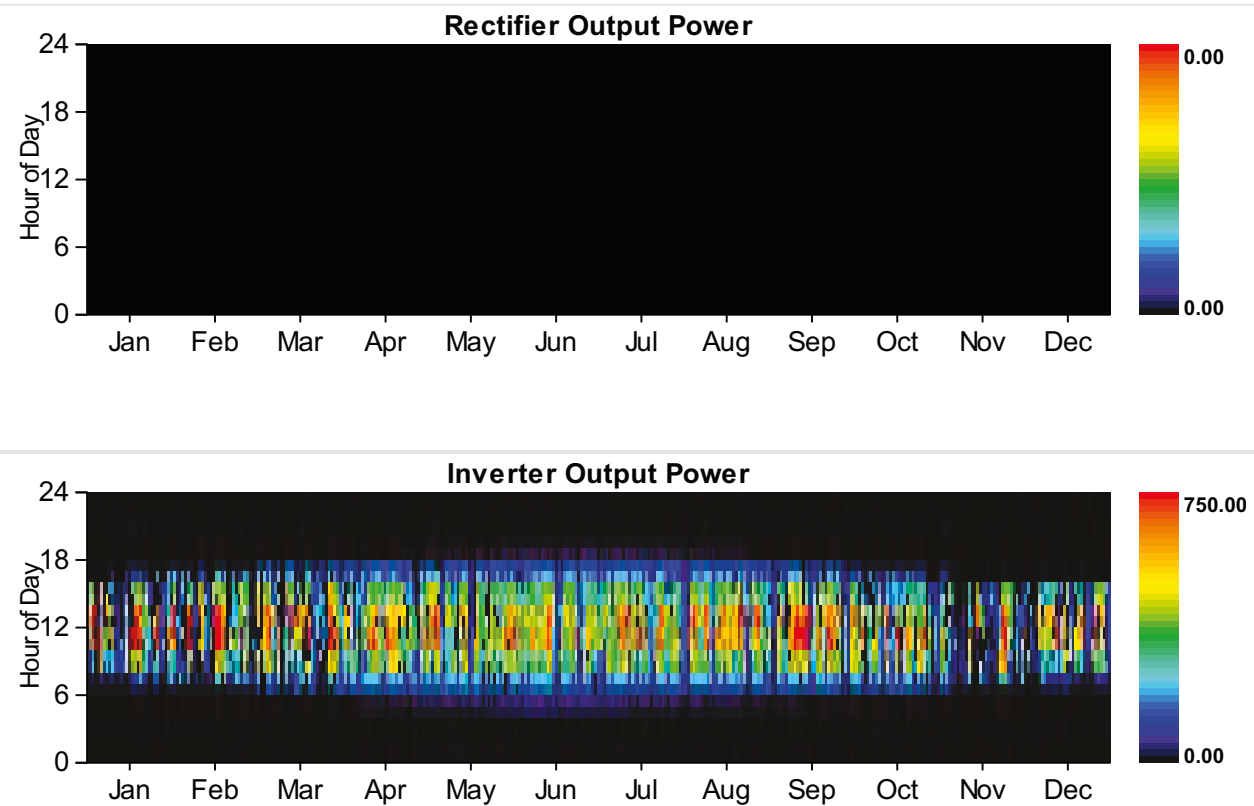

Grid

Rate: Demand 1

\begin{tabular}{|c|c|c|c|c|c|c|}
\hline Resources.ReportingService_GeneratelnputsReport_Month & $\begin{array}{l}\text { Energy } \\
\text { Purchased } \\
\text { (kWh) }\end{array}$ & $\begin{array}{l}\text { Energy } \\
\text { Sold } \\
\text { (kWh) }\end{array}$ & $\begin{array}{l}\text { Net } \\
\text { Purchases } \\
\text { (kWh) }\end{array}$ & $\begin{array}{l}\text { Peak } \\
\text { Demand } \\
(\mathbf{k W})\end{array}$ & $\begin{array}{l}\text { Energy } \\
\text { Charge } \\
\text { (\$) }\end{array}$ & $\begin{array}{l}\text { Demand } \\
\text { Charge } \\
(\$)\end{array}$ \\
\hline January & 0 & 0 & 0 & 18,095 & 0 & 289,519 \\
\hline February & 0 & 0 & 0 & 14,936 & 0 & 238,977 \\
\hline
\end{tabular}




\begin{tabular}{|c|c|c|c|c|c|c|}
\hline March & Energy 0 & Energy 9 & Net $\quad 0$ & Pedak699 & Energy & Déñẳ̆ \\
\hline $\begin{array}{l}\text { April } \\
\text { Resources.ReportingService_GeneratelnputsReport_Month }\end{array}$ & $\begin{array}{l}\text { Purchased } \\
\text { (kWh) }\end{array}$ & $\begin{array}{l}\text { Sold } 0 \\
\text { (kWh) }\end{array}$ & $\begin{array}{l}\text { Purchases } \\
\text { (kWh) }\end{array}$ & $\begin{array}{l}\text { Derm,ang } \\
\text { (kW) }\end{array}$ & $\begin{array}{l}\text { Charge } \\
\text { (\$) }\end{array}$ & $\begin{array}{l}\text { Cbagso68 } \\
\text { (\$) }\end{array}$ \\
\hline May & 0 & 0 & 0 & 17,572 & 0 & 281,151 \\
\hline June & 0 & 0 & 0 & 21,452 & 0 & 343,228 \\
\hline July & 0 & 0 & 0 & 23,398 & 0 & 374,367 \\
\hline August & 0 & 0 & 0 & 21,879 & 0 & 350,064 \\
\hline September & 0 & 0 & 0 & 15,468 & 0 & 247,493 \\
\hline October & 0 & 0 & 0 & 13,726 & 0 & 219,623 \\
\hline November & 0 & 0 & 0 & 15,876 & 0 & 254,021 \\
\hline December & 0 & 0 & 0 & 18,662 & 0 & 298,596 \\
\hline Annual & 0 & 0 & 0 & 23,398 & 0 & $3,489,884$ \\
\hline
\end{tabular}

Rate: Rate 1

\begin{tabular}{|c|c|c|c|c|c|c|}
\hline Resources.ReportingService_GeneratelnputsReport_Month & $\begin{array}{l}\text { Energy } \\
\text { Purchased } \\
\text { (kWh) }\end{array}$ & $\begin{array}{l}\text { Energy } \\
\text { Sold } \\
\text { (kWh) }\end{array}$ & $\begin{array}{l}\text { Net } \\
\text { Purchases } \\
\text { (kWh) }\end{array}$ & $\begin{array}{l}\text { Peak } \\
\text { Demand } \\
\text { (kW) }\end{array}$ & $\begin{array}{l}\text { Energy } \\
\text { Charge } \\
\text { (\$) }\end{array}$ & $\begin{array}{l}\text { Demand } \\
\text { Charge } \\
\text { (\$) }\end{array}$ \\
\hline January & $4,952,182$ & 0 & $4,952,182$ & 0 & 594,262 & 0 \\
\hline February & $3,207,647$ & 0 & $3,207,647$ & 0 & 384,918 & 0 \\
\hline March & $3,646,840$ & 0 & $3,646,840$ & 0 & 437,621 & 0 \\
\hline April & $6,452,577$ & 0 & $6,452,577$ & 0 & 774,309 & 0 \\
\hline May & $5,429,449$ & 0 & $5,429,449$ & 0 & 651,534 & 0 \\
\hline June & $7,772,968$ & 0 & $7,772,968$ & 0 & 932,756 & 0 \\
\hline July & $8,378,485$ & 0 & $8,378,485$ & 0 & $1,005,418$ & 0 \\
\hline August & $8,399,006$ & 0 & $8,399,006$ & 0 & $1,007,881$ & 0 \\
\hline September & $5,297,231$ & 0 & $5,297,231$ & 0 & 635,668 & 0 \\
\hline October & $4,667,008$ & 0 & $4,667,008$ & 0 & 560,041 & 0 \\
\hline November & $3,703,306$ & 0 & $3,703,306$ & 0 & 444,397 & 0 \\
\hline December & $5,419,948$ & 0 & $5,419,948$ & 0 & 650,394 & 0 \\
\hline Annual & $67,326,648$ & 0 & $67,326,648$ & 0 & $8,079,197$ & 0 \\
\hline
\end{tabular}

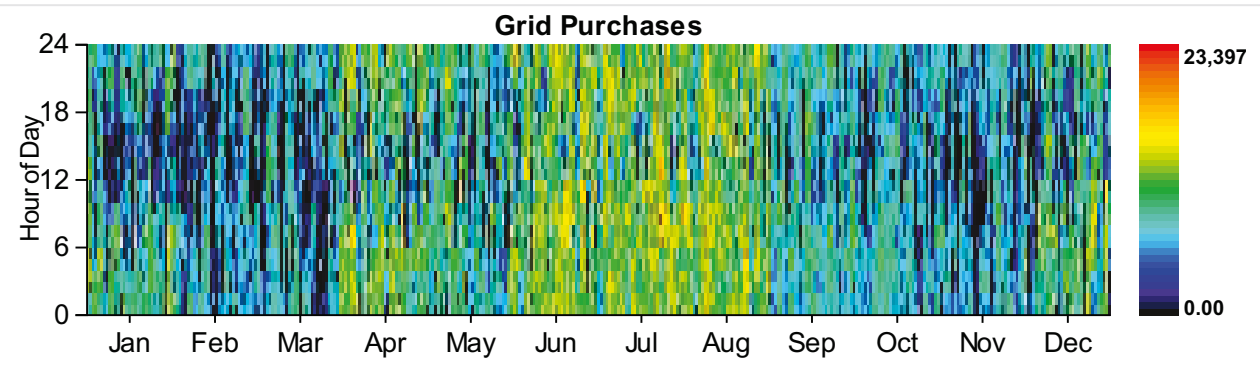




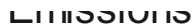

\section{Pollutant}

Emissions

Units

Carbon dioxide

$42550440 \mathrm{~kg} / \mathrm{yr}$

Carbon monoxide

$0 \mathrm{~kg} / \mathrm{yr}$

Unburned hydrocarbons

$0 \mathrm{~kg} / \mathrm{yr}$

Particulate matter

$0 \mathrm{~kg} / \mathrm{yr}$

Sulfur dioxide

$184475 \mathrm{~kg} / \mathrm{yr}$

Nitrogen oxides

$90218 \mathrm{~kg} / \mathrm{yr}$

HOMER Energy, LLC @ 2016 
System Report

System architecture

\begin{tabular}{|l|l|r|}
\hline PV & Solar World 320W flat plate PV Copy & 1,000 \\
\hline Wind Turbine & Siemens 2.3 MW - 108 & 5 \\
\hline Generator & Kohler 1000 Prime Power & 925 \\
\hline Generator \#2 & Kohler 750 Prime Power & 690 \\
\hline Battery & GS200 flow & $\mathrm{kW}$ \\
\hline Converter & System Converter & 1 \\
\hline Grid & Grid & 750 \\
\hline Dispatch Strategy & Cycle Charging & 20,000 \\
\hline
\end{tabular}

\section{Cost summary}

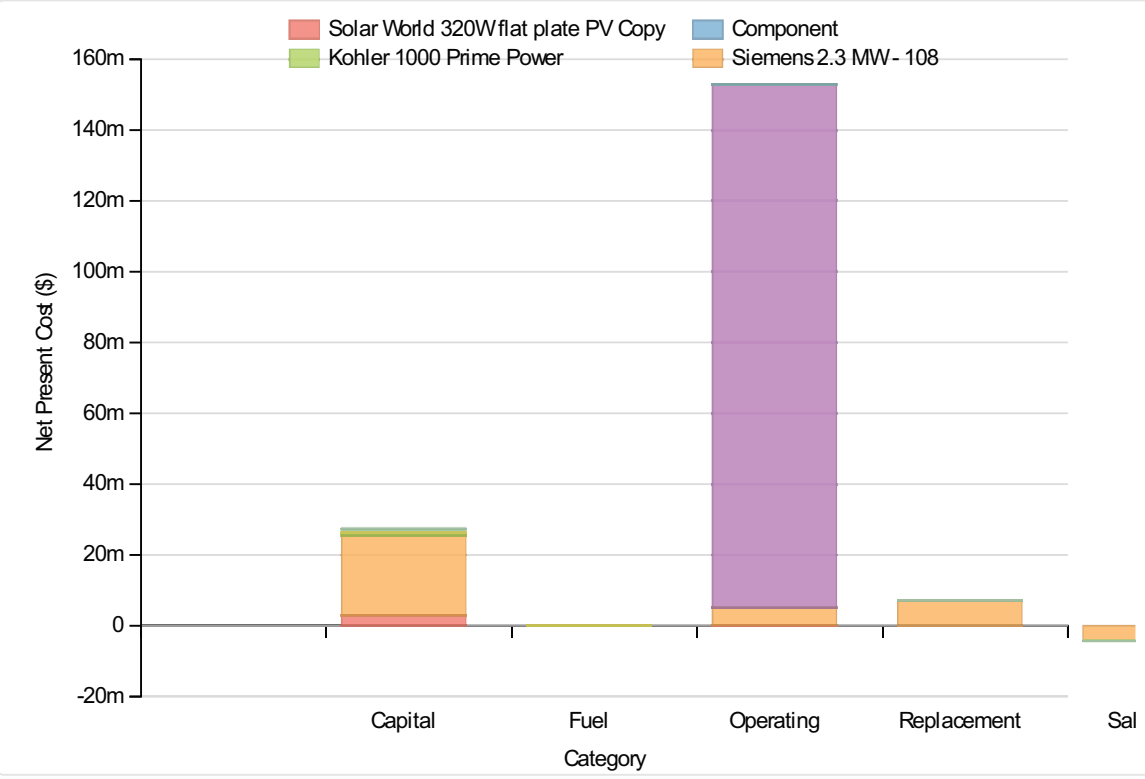

Cost Summary

Total net present cost

$183645824 \$$

Levelized cost of energy

$0.147 \$ / k W h$

Net Present Costs

\begin{tabular}{|c|c|c|c|c|c|c|}
\hline Component & Capital & Replacement & O\&M & Fuel & Salvage & Total \\
\hline Solar World 320W flat plate PV Copy & $3,000,000$ & 0 & 193,913 & 0 & 0 & $3,193,913$ \\
\hline Siemens 2.3 MW - 108 & $22,500,000$ & $7,173,158$ & $4,847,816$ & 0 & $-4,042,534$ & $30,478,440$ \\
\hline Kohler 1000 Prime Power & 925,000 & 0 & 11,838 & 88,287 & $-197,216$ & 827,909 \\
\hline Kohler 750 Prime Power & 690,000 & 0 & 12,443 & 72,292 & $-139,674$ & 635,061 \\
\hline Grid & 0 & 0 & $147,846,112$ & 0 & 0 & $147,846,112$ \\
\hline GS200 flow & 324,589 & 7,176 & 31,026 & 0 & -973 & 361,818 \\
\hline Converter & 225,000 & 95,462 & 0 & 0 & $-17,967$ & 302,495 \\
\hline
\end{tabular}




\section{Annualized Costs}

\begin{tabular}{|c|c|c|c|c|c|c|}
\hline Component & Capital & Replacement & O\&M & Fuel & Salvage & Total \\
\hline Solar World 320W flat plate PV Copy & 232,063 & 0 & 15,000 & 0 & 0 & 247,063 \\
\hline Siemens 2.3 MW - 108 & $1,740,474$ & 554,875 & 375,000 & 0 & $-312,708$ & $2,357,641$ \\
\hline Kohler 1000 Prime Power & 71,553 & 0 & 916 & 6,829 & $-15,256$ & 64,042 \\
\hline Kohler 750 Prime Power & 53,375 & 0 & 963 & 5,592 & $-10,804$ & 49,126 \\
\hline Grid & 0 & 0 & $11,436,544$ & 0 & 0 & $11,436,544$ \\
\hline GS200 flow & 25,108 & 555 & 2,400 & 0 & -75 & 27,988 \\
\hline Converter & 17,405 & 7,384 & 0 & 0 & $-1,390$ & 23,400 \\
\hline System & $2,139,977$ & 562,815 & $11,830,823$ & 12,421 & $-340,233$ & $14,205,803$ \\
\hline
\end{tabular}

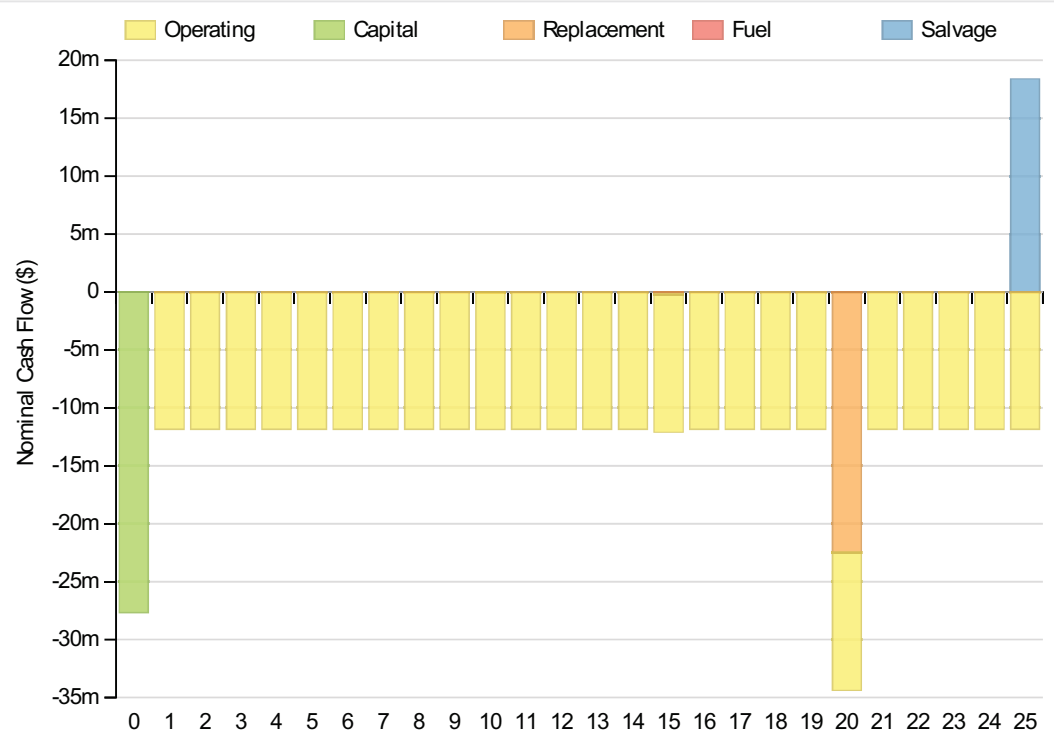

Year

Electrical

\section{Quantity}

Excess electricity

Unmet load

Capacity shortage

Renewable fraction

Value

Value

Production(kWh/yr)

Fraction (\%)

\section{Component}

PV

Generator

Generator

Wind Turbine

Grid Purchases
$1,288,675$ 


\section{Load}

Consumption(kWh/yr)

AC primary load

DC primary load

Total
Fraction (\%)

$96,860,760$

0

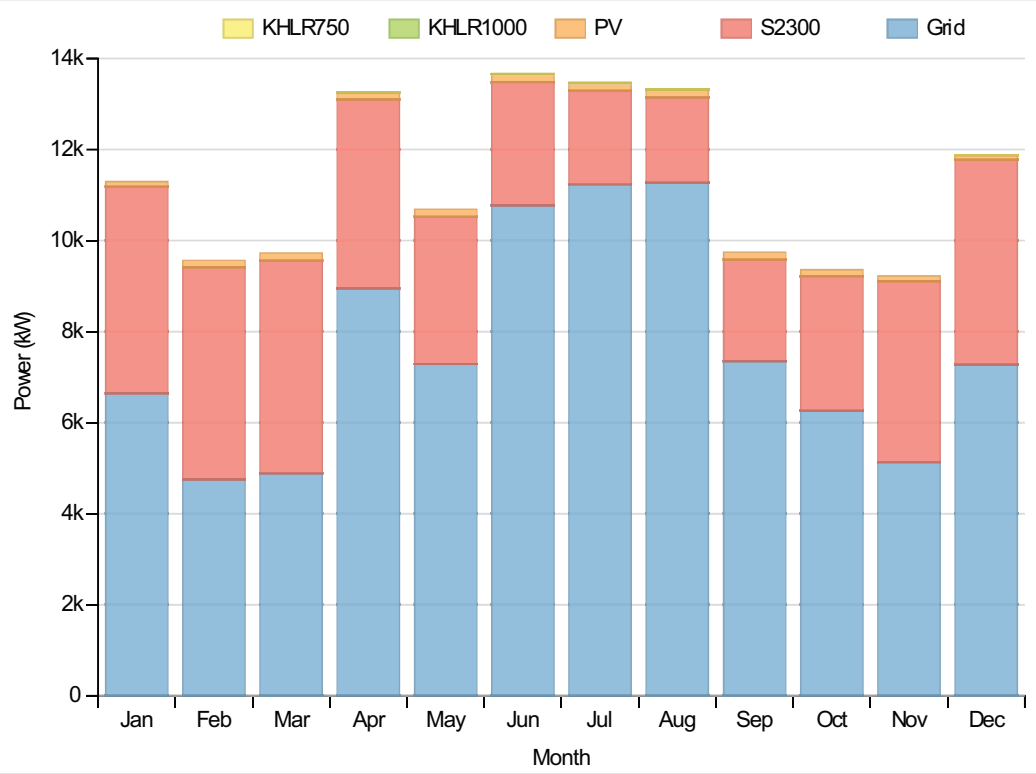

PV:Solar World 320W flat plate PV Copy

\begin{tabular}{|c|c|c|}
\hline Quantity & Value & Units \\
\hline Rated capacity & 1000 & $\mathrm{~kW}$ \\
\hline Mean output & 147 & $\mathrm{~kW}$ \\
\hline Mean output & 3530.60 & $\mathrm{kWh} / \mathrm{d}$ \\
\hline Capacity factor & 14.71 & $\%$ \\
\hline Total production & 1288675 & $\mathrm{kWh} / \mathrm{yr}$ \\
\hline Minimum output & 0.00 & kW \\
\hline Maximum output & 1022.50 & $\mathrm{~kW}$ \\
\hline PV penetration & 1.33 & $\%$ \\
\hline Hours of operation & 4377 & hrs/yr \\
\hline Levelized cost & 0.192 & $\$ / k W h$ \\
\hline
\end{tabular}

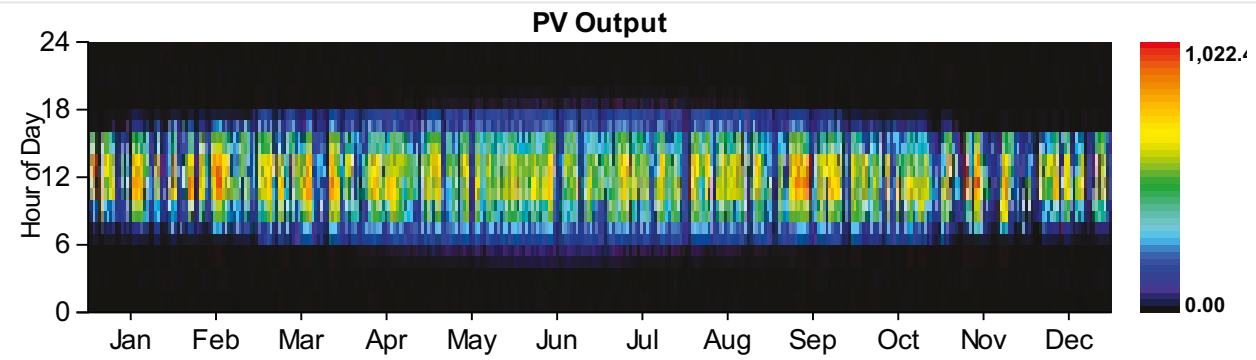




\section{Quantity}

Total rated capacity

Mean output

Capacity factor

Total production

Minimum output

Maximum output

Wind penetration

Hours of operation

Levelized cost

\section{Value}

Units

11500 kW

$3455 \mathrm{~kW}$

$30.05 \%$

$30268832 \mathrm{kWh} / \mathrm{yr}$

$7.19 \mathrm{~kW}$

$11574.00 \mathrm{~kW}$

$31.25 \%$

$8760 \mathrm{hrs} / \mathrm{yr}$

$0.078 \$ / k W h$

\section{Generator:Kohler 1000 Prime Power}

\section{Quantity}

Hours of operation

Number of starts

Operational life

Fixed generation cost

Marginal generation cost

Electrical production

Mean electrical output

Min. electrical output

Max. electrical output

Fuel consumption

Specific fuel consumption

Fuel energy input

Mean electrical efficiency
Value

Units

$66 \mathrm{hrs} / \mathrm{yr}$

62 starts/yr

227 yr

$76.24 \$ / h r$

$0.23 \$ / \mathrm{kWh}$

$29950 \mathrm{kWh} / \mathrm{yr}$

454 kW

231 kW

$925 \mathrm{~kW}$

8645 L/yr

$0.29 \mathrm{~L} / \mathrm{kWh}$

85064 kWh/yr

$35 \%$

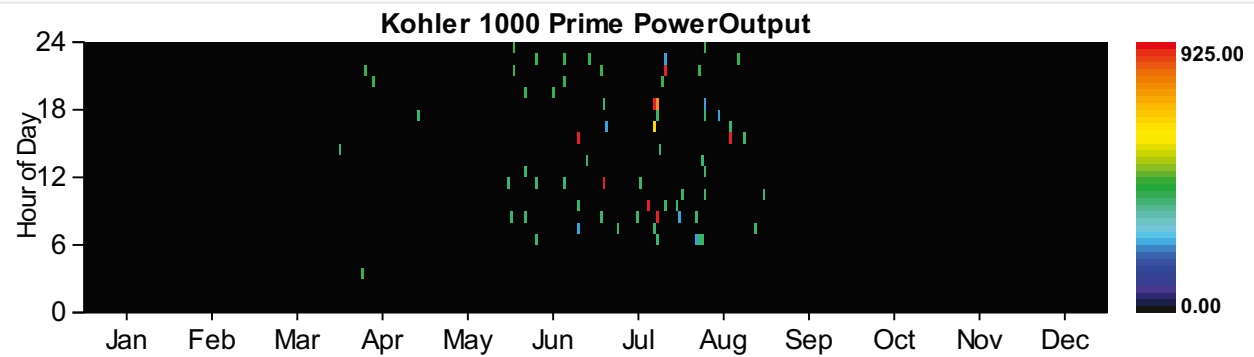

Generator:Kohler 750 Prime Power 
Qdedfiftity

Number of starts

Operational life

Fixed generation cost

Marginal generation cost

Electrical production

Mean electrical output

Min. electrical output

Max. electrical output

Fuel consumption

Specific fuel consumption

Fuel energy input

Mean electrical efficiency

\section{Value}

93 brâts

88 starts/yr

$161 \mathrm{yr}$

$56.87 \$ / h r$

$0.23 \$ / k W h$

$24183 \mathrm{kWh} / \mathrm{yr}$

$260 \mathrm{~kW}$

$173 \mathrm{~kW}$

$690 \mathrm{~kW}$

7079 L/yr

$0.29 \mathrm{~L} / \mathrm{kWh}$

$69653 \mathrm{kWh} / \mathrm{yr}$

$35 \%$

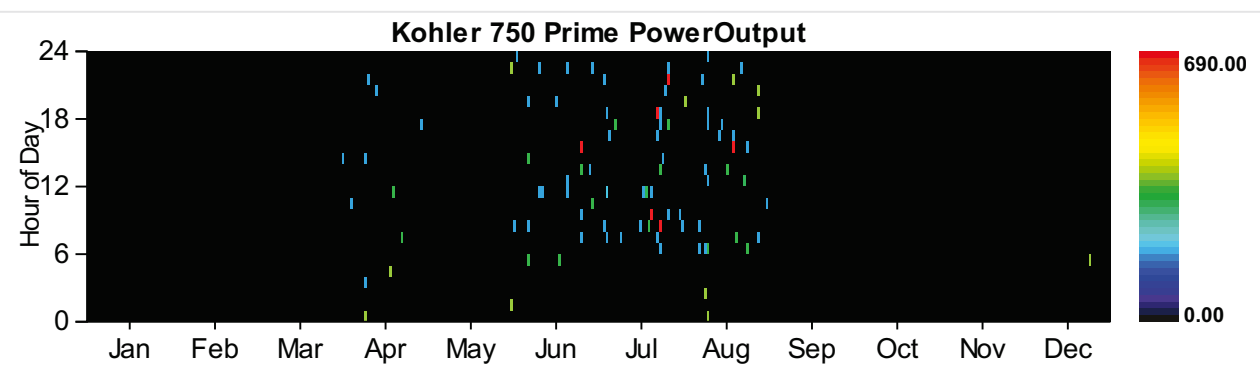

\section{Battery:GS200 flow}

Quantity $\quad$ Value

String size

Strings in parallel

Batteries

Bus voltage

\section{Quantity}

Value

\section{Units}

Nominal capacity

Usable nominal capacity

$600 \mathrm{kWh}$

600 kWh

Autonomy

Lifetime throughput

$0 \mathrm{hr}$

Battery wear cost

0

Average energy cost

$0.000 \$ / k W h$

Energy in 
Losses

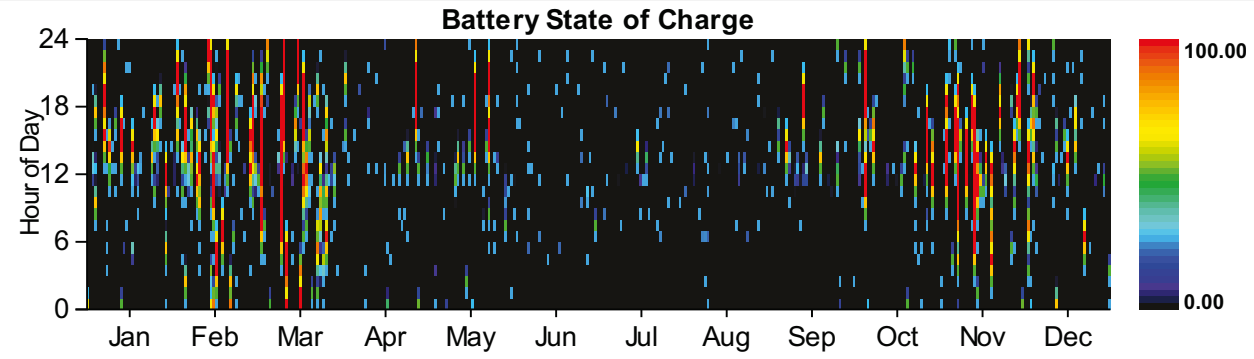

\section{Converter}

\begin{tabular}{|l|r|r|l|}
\hline Quantity & Inverter & Rectifier & Units \\
\hline Capacity & 750 & $675 \mathrm{~kW}$ \\
\hline Mean output & 125 & $8 \mathrm{~kW}$ \\
\hline Minimum output & 0 & $0 \mathrm{~kW}$ \\
\hline Maximum output & 750 & $179 \mathrm{~kW}$ \\
\hline Capacity factor & 17 & $1 \%$ \\
\hline Hours of operation & 4,378 & $586 \mathrm{hrs} / \mathrm{yr}$ \\
\hline Energy in & $1,212,694$ & $79,509 \mathrm{kWh} / \mathrm{yr}$ \\
\hline Energy out & $1,091,425$ & $67,583 \mathrm{kWh} / \mathrm{yr}$ \\
\hline Losses & 121,269 & $11,926 \mathrm{kWh} / \mathrm{yr}$ \\
\hline
\end{tabular}
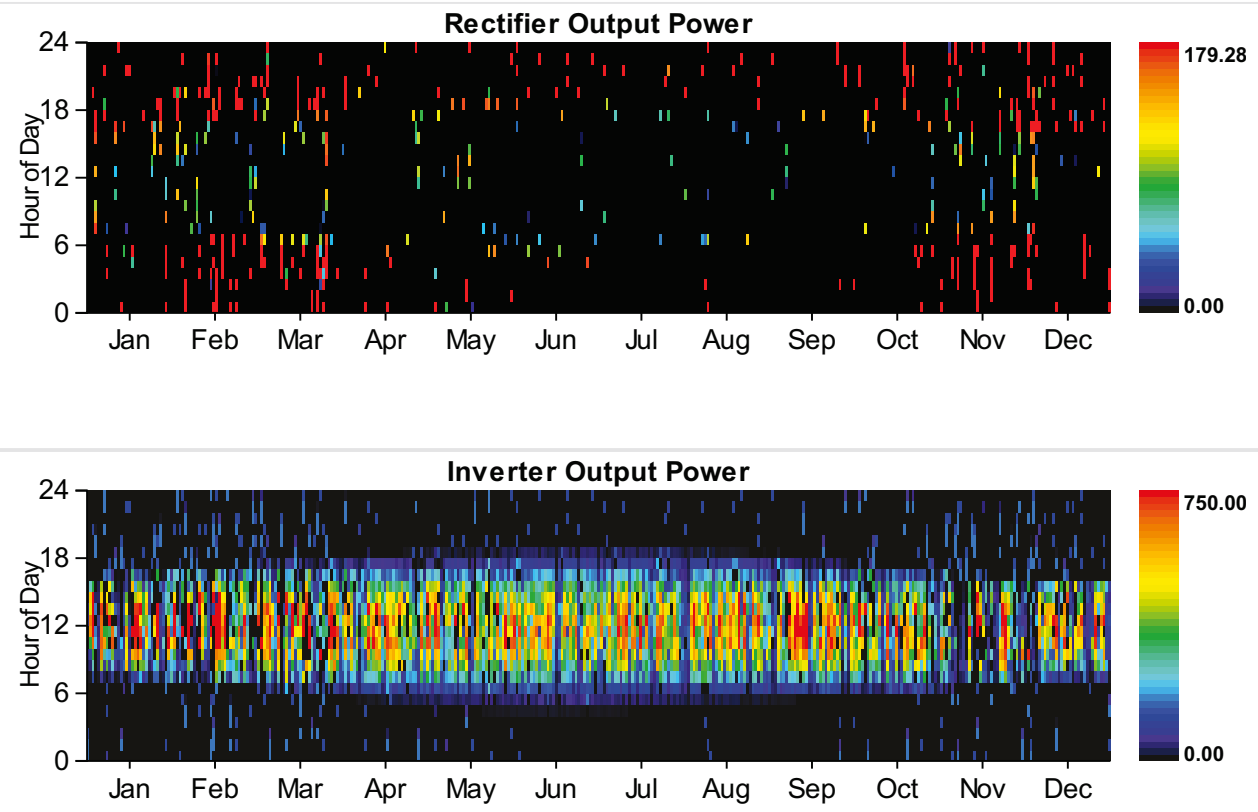
जाu

Rate: Demand 1

\begin{tabular}{|c|c|c|c|c|c|c|}
\hline Resources.ReportingService_GeneratelnputsReport_Month & $\begin{array}{l}\text { Energy } \\
\text { Purchased } \\
\text { (kWh) }\end{array}$ & $\begin{array}{l}\text { Energy } \\
\text { Sold } \\
\text { (kWh) }\end{array}$ & $\begin{array}{l}\text { Net } \\
\text { Purchases } \\
\text { (kWh) }\end{array}$ & $\begin{array}{l}\text { Peak } \\
\text { Demand } \\
(\mathbf{k W})\end{array}$ & $\begin{array}{l}\text { Energy } \\
\text { Charge } \\
\text { (\$) }\end{array}$ & $\begin{array}{l}\text { Demand } \\
\text { Charge } \\
(\$)\end{array}$ \\
\hline January & 0 & 0 & 0 & 18,095 & 0 & 289,519 \\
\hline February & 0 & 0 & 0 & 14,936 & 0 & 238,977 \\
\hline March & 0 & 0 & 0 & 16,699 & 0 & 267,178 \\
\hline April & 0 & 0 & 0 & 19,950 & 0 & 319,208 \\
\hline May & 0 & 0 & 0 & 17,572 & 0 & 281,151 \\
\hline June & 0 & 0 & 0 & 20,000 & 0 & 320,000 \\
\hline July & 0 & 0 & 0 & 20,000 & 0 & 320,000 \\
\hline August & 0 & 0 & 0 & 20,000 & 0 & 320,000 \\
\hline September & 0 & 0 & 0 & 15,468 & 0 & 247,493 \\
\hline October & 0 & 0 & 0 & 13,726 & 0 & 219,623 \\
\hline November & 0 & 0 & 0 & 15,876 & 0 & 254,021 \\
\hline December & 0 & 0 & 0 & 18,490 & 0 & 295,836 \\
\hline Annual & 0 & 0 & 0 & 20,000 & 0 & $3,373,005$ \\
\hline
\end{tabular}

Rate: Rate 1

\begin{tabular}{|c|c|c|c|c|c|c|}
\hline Resources.ReportingService_GeneratelnputsReport_Month & $\begin{array}{l}\text { Energy } \\
\text { Purchased } \\
\text { (kWh) }\end{array}$ & $\begin{array}{l}\text { Energy } \\
\text { Sold } \\
\text { (kWh) }\end{array}$ & $\begin{array}{l}\text { Net } \\
\text { Purchases } \\
\text { (kWh) }\end{array}$ & $\begin{array}{l}\text { Peak } \\
\text { Demand } \\
(\mathbf{k W})\end{array}$ & $\begin{array}{l}\text { Energy } \\
\text { Charge } \\
\text { (\$) }\end{array}$ & $\begin{array}{l}\text { Demand } \\
\text { Charge } \\
(\$)\end{array}$ \\
\hline January & $4,942,838$ & 0 & $4,942,838$ & 0 & 593,141 & 0 \\
\hline February & $3,194,187$ & 0 & $3,194,187$ & 0 & 383,302 & 0 \\
\hline March & $3,633,528$ & 0 & $3,633,528$ & 0 & 436,023 & 0 \\
\hline April & $6,444,779$ & 0 & $6,444,779$ & 0 & 773,373 & 0 \\
\hline May & $5,423,759$ & 0 & $5,423,759$ & 0 & 650,851 & 0 \\
\hline June & $7,759,668$ & 0 & $7,759,668$ & 0 & 931,160 & 0 \\
\hline July & $8,356,055$ & 0 & $8,356,055$ & 0 & $1,002,727$ & 0 \\
\hline August & $8,384,725$ & 0 & $8,384,725$ & 0 & $1,006,167$ & 0 \\
\hline September & $5,293,275$ & 0 & $5,293,275$ & 0 & 635,193 & 0 \\
\hline October & $4,660,071$ & 0 & $4,660,071$ & 0 & 559,209 & 0 \\
\hline November & $3,692,188$ & 0 & $3,692,188$ & 0 & 443,063 & 0 \\
\hline December & $5,411,146$ & 0 & $5,411,146$ & 0 & 649,338 & 0 \\
\hline Annual & $67,196,216$ & 0 & $67,196,216$ & 0 & $8,063,546$ & 0 \\
\hline
\end{tabular}




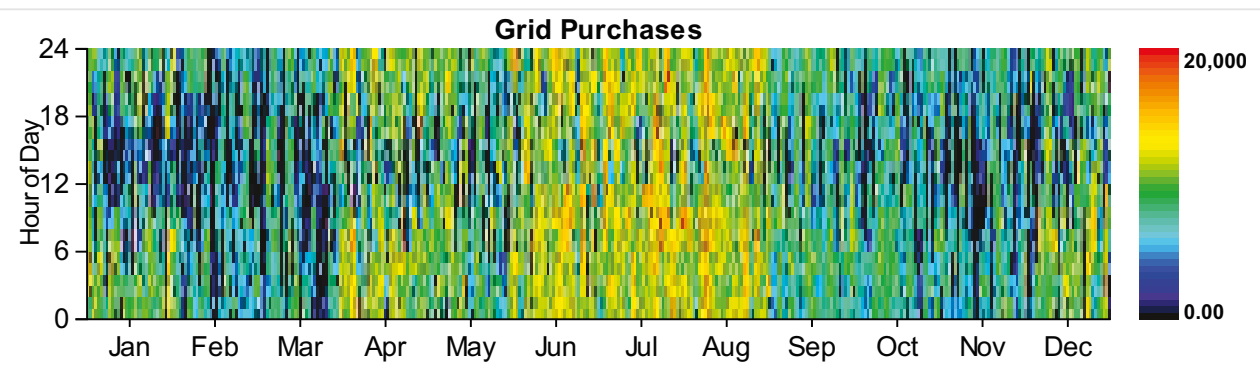

\section{Emissions}

\begin{tabular}{|l|r|l|}
\hline Pollutant & Emissions & Units \\
\hline Carbon dioxide & $42509272 \mathrm{~kg} / \mathrm{yr}$ \\
\hline Carbon monoxide & $173 \mathrm{~kg} / \mathrm{yr}$ \\
\hline Unburned hydrocarbons & $20 \mathrm{~kg} / \mathrm{yr}$ \\
\hline Particulate matter & $5 \mathrm{~kg} / \mathrm{yr}$ \\
\hline Sulfur dioxide & $184203 \mathrm{~kg} / \mathrm{yr}$ \\
\hline Nitrogen oxides & $90216 \mathrm{~kg} / \mathrm{yr}$ \\
\hline
\end{tabular}

HOMER Energy, LLC @ 2016 
System Report

System architecture

\begin{tabular}{|l|l|r|}
\hline PV & Solar World 320W flat plate PV Copy & 1,000 \\
\hline Wind Turbine & Siemens $2.3 \mathrm{~kW}-108$ & 5 \\
\hline Battery & GS200 flow & 1 strings \\
\hline Converter & System Converter & $750 \mathrm{~kW}$ \\
\hline Grid & Grid & 25,000 \\
\hline Dispatch Strategy & Cycle Charging & $\mathrm{kW}$ \\
\hline
\end{tabular}

\section{Cost summary}

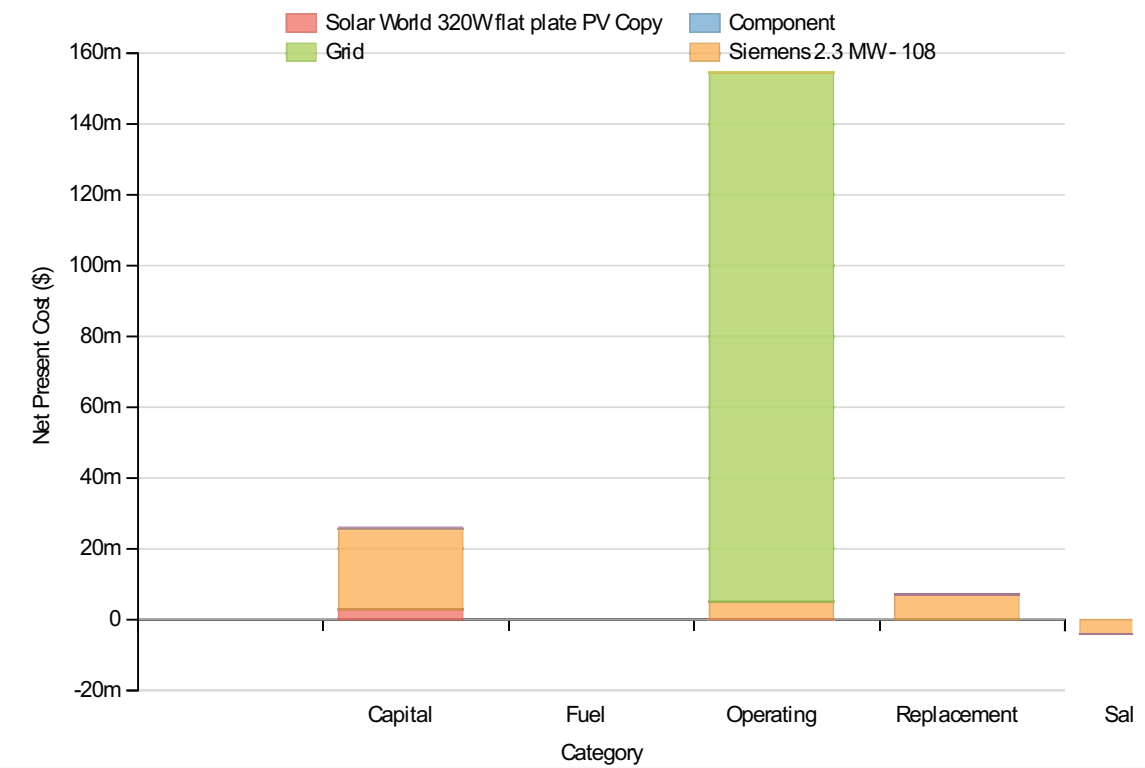

Cost Summary

Total net present cost

$183774336 \$$

Levelized cost of energy

$0.147 \$ / k W h$

\section{Net Present Costs}

\begin{tabular}{|c|c|c|c|c|c|c|}
\hline Component & Capital & Replacement & O\&M & Fuel & Salvage & Total \\
\hline Solar World 320W flat plate PV Copy & $3,000,000$ & 0 & 193,913 & 0 & 0 & $3,193,913$ \\
\hline Siemens 2.3 MW - 108 & $22,500,000$ & $7,173,158$ & $4,847,816$ & 0 & $-4,042,534$ & $30,478,440$ \\
\hline Grid & 0 & 0 & $149,437,584$ & 0 & 0 & $149,437,584$ \\
\hline GS200 flow & 324,589 & 7,176 & 31,026 & 0 & -973 & 361,818 \\
\hline Converter & 225,000 & 95,462 & 0 & 0 & $-17,967$ & 302,495 \\
\hline System & $26,049,588$ & $7,275,795$ & $154,510,368$ & 0 & $-4,061,473$ & $183,774,278$ \\
\hline
\end{tabular}

Annualized Costs

\begin{tabular}{|l|r|r|r|r|r|r|}
\hline Component & Capital & Replacement & O\&M & Fuel & Salvage & Total \\
\hline Solar World 320W flat plate PV Copy & 232,063 & 0 & 15,000 & 0 & 0 \\
\hline
\end{tabular}




\begin{tabular}{|l|r|r|r|r|r|r|r|r|r|r|r|r|}
\hline Grid & 0 & 0 & $11,559,652$ & 0 & 0 \\
\hline GS200 flow & 25,108 & 555 & 2,400 & 0 & -75 \\
\hline Converter & 17,405 & 7,384 & 0 & 0 & $-1,390$ \\
\hline System & $2,015,050$ & 562,815 & $11,952,054$ & 0 & $-314,173$ & $14,215,746$ \\
\hline
\end{tabular}

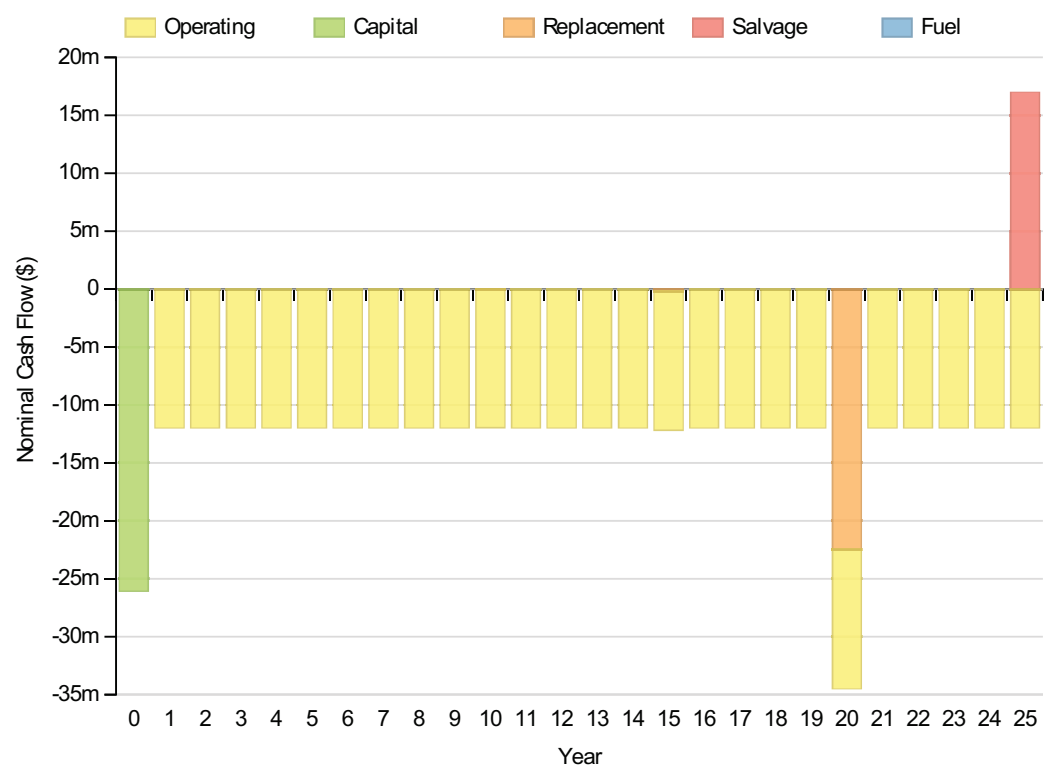

\section{Electrical}

\section{Quantity}

Excess electricity

Unmet load

Capacity shortage

Renewable fraction

\section{Component}

PV

Wind Turbine

Grid Purchases

Total

Load
AC primary load

DC primary load

Total
Value

Production(kWh/yr)

Fraction (\%)

$1,288,675$

$30,268,832$

$67,248,128$

$98,805,632$

Consumption(kWh/yr)

Fraction (\%)
$1772971 \mathrm{kWh} / \mathrm{yr}$

$1 \mathrm{kWh} / \mathrm{yr}$

$1536 \mathrm{kWh} / \mathrm{yr}$ 


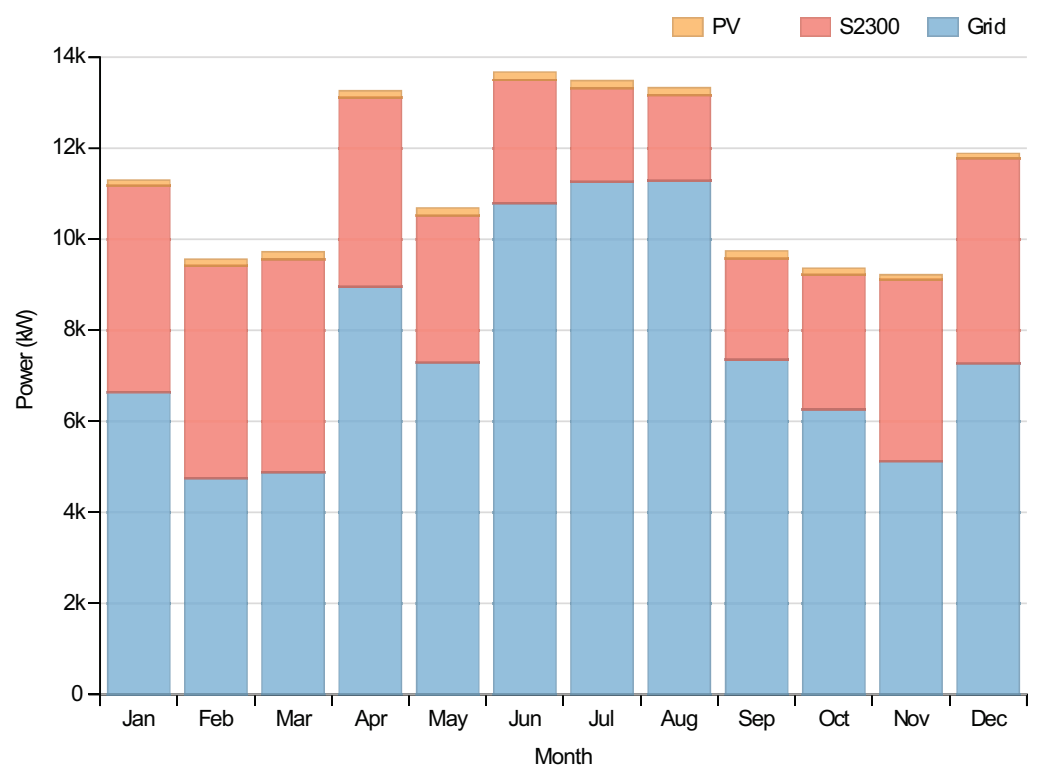

PV:Solar World 320W flat plate PV Copy

\begin{tabular}{|c|c|c|}
\hline Quantity & Value & Units \\
\hline Rated capacity & 1000 & $\mathrm{~kW}$ \\
\hline Mean output & 147 & $\mathrm{~kW}$ \\
\hline Mean output & 3530.60 & $\mathrm{kWh} / \mathrm{d}$ \\
\hline Capacity factor & 14.71 & $\%$ \\
\hline Total production & 1288675 & $\mathrm{kWh} / \mathrm{yr}$ \\
\hline Minimum output & 0.00 & $\mathrm{~kW}$ \\
\hline Maximum output & 1022.50 & $\mathrm{~kW}$ \\
\hline PV penetration & 1.33 & $\%$ \\
\hline Hours of operation & 4377 & $\mathrm{hrs} / \mathrm{yr}$ \\
\hline Levelized cost & 0.192 & $\$ / k W h$ \\
\hline
\end{tabular}

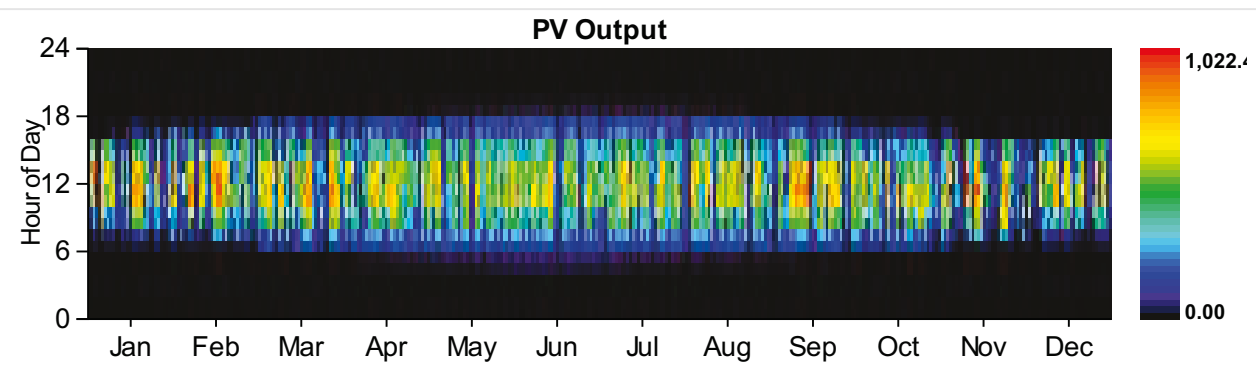

Wind Turbine:Siemens 2.3 MW - 108

\begin{tabular}{|l|r|l|}
\hline Quantity & Value & Units \\
\hline Total rated capacity & 11500 & $\mathrm{~kW}$ \\
\hline Mean output & 3455 & $\mathrm{~kW}$ \\
\hline Capacity factor & $30.05 \%$ \\
\hline Total production & $30268832 \mathrm{kWh} / \mathrm{yr}$ \\
\hline
\end{tabular}


Qinimitity output

Maximum output

Wind penetration

Hours of operation

Levelized cost
7.19 UWits

11574.00 kW

$31.25 \%$

$8760 \mathrm{hrs} / \mathrm{yr}$

$0.078 \$ / k W h$

\section{Battery:GS200 flow}

\begin{tabular}{l|l} 
Quantity & Value
\end{tabular}

String size

Strings in parallel

Batteries

Batteries

Bus voltage

\section{Quantity}

Nominal capacity

Usable nominal capacity

Autonomy

Lifetime throughput

Battery wear cost

Average energy cost

Energy in

Energy out

Storage depletion

Losses

Annual throughput

Expected life
Value

Units

600 kWh

600 kWh

$0 \mathrm{hr}$

0

$0.000 \$ / k W h$

$0.000 \$ / k W h$

$123910 \mathrm{kWh} / \mathrm{yr}$

87240 kWh/yr

$600 \mathrm{kWh} / \mathrm{yr}$

36070 kWh/yr

$104271 \mathrm{kWh} / \mathrm{yr}$

$25 \mathrm{yr}$

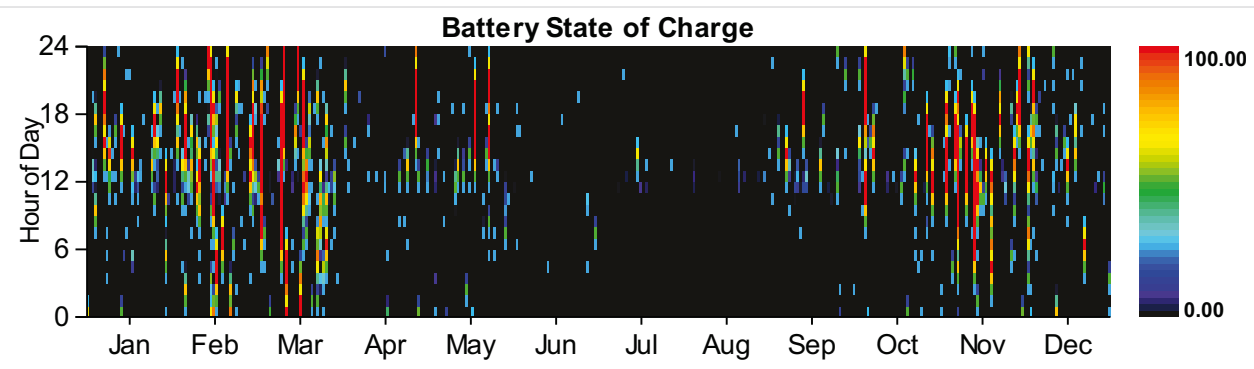

\section{Converter}

\section{Quantity}

Capacity
Inverter

Rectifier
Units

675 kW 


\begin{tabular}{|c|c|c|c|}
\hline Qląnetytput & Inverter & Rectifier & UNits \\
\hline Minimum output & 0 & 0 & $\mathrm{~kW}$ \\
\hline Maximum output & 750 & 179 & $\mathrm{~kW}$ \\
\hline Capacity factor & 17 & 1 & $\%$ \\
\hline Hours of operation & 4,378 & 536 & hrs/yr \\
\hline Energy in & $1,210,425$ & 71,846 & $\mathrm{kWh} / \mathrm{yr}$ \\
\hline Energy out & $1,089,383$ & 61,070 & $\mathrm{kWh} / \mathrm{yr}$ \\
\hline Losses & 121,042 & 10,777 & $\mathrm{kWh} / \mathrm{yr}$ \\
\hline
\end{tabular}
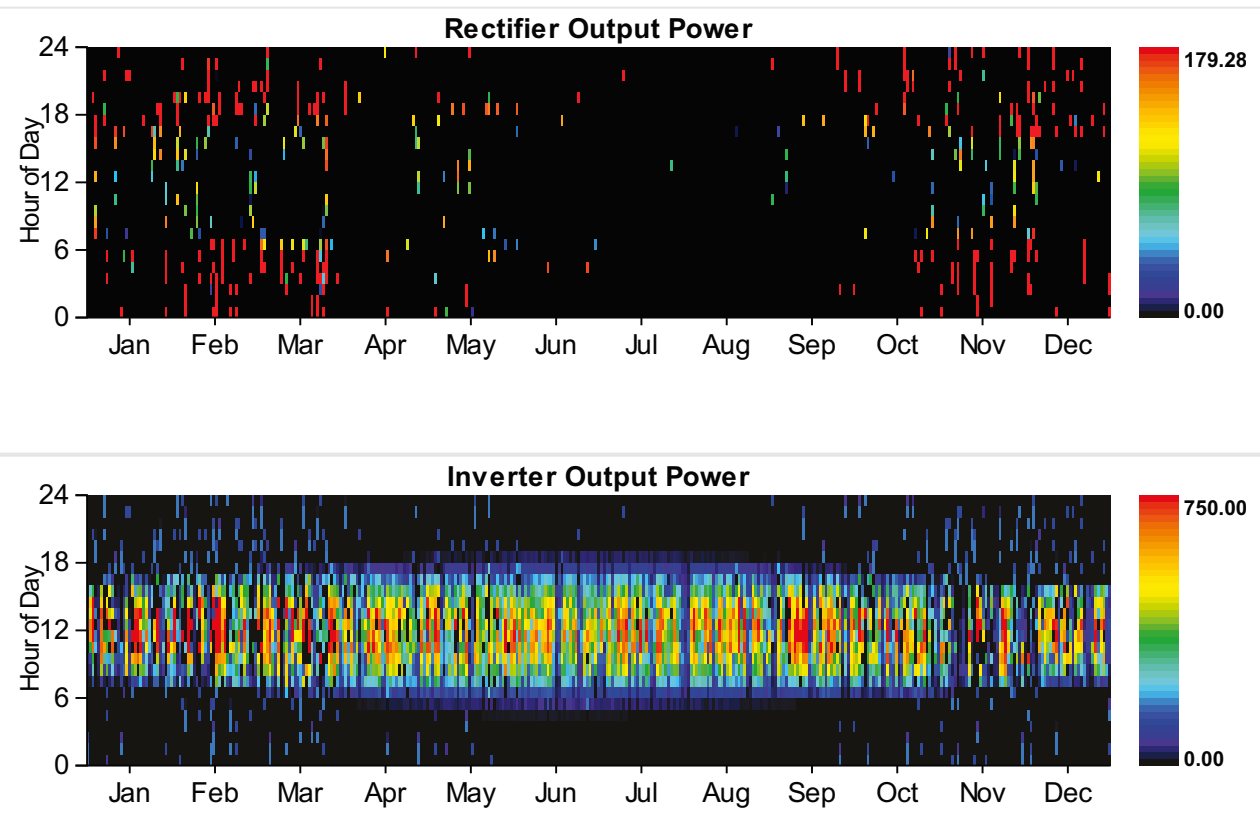

Grid

Rate: Demand 1

\begin{tabular}{|c|c|c|c|c|c|c|}
\hline Resources.ReportingService_GeneratelnputsReport_Month & $\begin{array}{l}\text { Energy } \\
\text { Purchased } \\
\text { (kWh) }\end{array}$ & $\begin{array}{l}\text { Energy } \\
\text { Sold } \\
\text { (kWh) }\end{array}$ & $\begin{array}{l}\text { Net } \\
\text { Purchases } \\
\text { (kWh) }\end{array}$ & $\begin{array}{l}\text { Peak } \\
\text { Demand } \\
(\mathbf{k W})\end{array}$ & $\begin{array}{l}\text { Energy } \\
\text { Charge } \\
\text { (\$) }\end{array}$ & $\begin{array}{l}\text { Demand } \\
\text { Charge } \\
(\$)\end{array}$ \\
\hline January & 0 & 0 & 0 & 18,095 & 0 & 289,519 \\
\hline February & 0 & 0 & 0 & 14,936 & 0 & 238,977 \\
\hline March & 0 & 0 & 0 & 16,699 & 0 & 267,178 \\
\hline April & 0 & 0 & 0 & 20,354 & 0 & 325,668 \\
\hline May & 0 & 0 & 0 & 17,572 & 0 & 281,151 \\
\hline June & 0 & 0 & 0 & 21,452 & 0 & 343,228 \\
\hline July & 0 & 0 & 0 & 23,398 & 0 & 374,367 \\
\hline August & 0 & 0 & 0 & 21,879 & 0 & 350,064 \\
\hline September & 0 & 0 & 0 & 15,468 & 0 & 247,493 \\
\hline October & 0 & 0 & 0 & 13,726 & 0 & 219,623 \\
\hline
\end{tabular}




\begin{tabular}{|c|c|c|c|c|c|c|}
\hline November & Energy 0 & Energy & Net 0 & Peak'876 & Energy & DéF̂ââ1 \\
\hline $\begin{array}{l}\text { December } \\
\text { Resources.ReportingService_GeneratelnputsReport_Month }\end{array}$ & $\begin{array}{l}\text { Purchased } \\
\text { (kWh) }\end{array}$ & $\begin{array}{l}\text { Sold } 0 \\
\text { (kWh) }\end{array}$ & $\begin{array}{l}\text { Purchases } \\
\text { (kWh) }\end{array}$ & $\begin{array}{l}\text { Dєाஜा, } \\
\text { (kW) }\end{array}$ & $\begin{array}{l}\text { Charge } \\
\text { (\$) }\end{array}$ & $\begin{array}{l}\text { Chargeg6 } \\
\text { (\$) }\end{array}$ \\
\hline Annual & 0 & 0 & 0 & 23,398 & 0 & $3,489,884$ \\
\hline
\end{tabular}

Rate: Rate 1

\begin{tabular}{|c|c|c|c|c|c|c|}
\hline Resources.ReportingService_GeneratelnputsReport_Month & $\begin{array}{l}\text { Energy } \\
\text { Purchased } \\
\text { (kWh) }\end{array}$ & $\begin{array}{l}\text { Energy } \\
\text { Sold } \\
\text { (kWh) }\end{array}$ & $\begin{array}{l}\text { Net } \\
\text { Purchases } \\
\text { (kWh) }\end{array}$ & $\begin{array}{l}\text { Peak } \\
\text { Demand } \\
(\mathbf{k W})\end{array}$ & $\begin{array}{l}\text { Energy } \\
\text { Charge } \\
\text { (\$) }\end{array}$ & $\begin{array}{l}\text { Demand } \\
\text { Charge } \\
\text { (\$) }\end{array}$ \\
\hline January & $4,942,838$ & 0 & $4,942,838$ & 0 & 593,141 & 0 \\
\hline February & $3,194,187$ & 0 & $3,194,187$ & 0 & 383,302 & 0 \\
\hline March & $3,633,528$ & 0 & $3,633,528$ & 0 & 436,023 & 0 \\
\hline April & $6,448,824$ & 0 & $6,448,824$ & 0 & 773,859 & 0 \\
\hline May & $5,423,759$ & 0 & $5,423,759$ & 0 & 650,851 & 0 \\
\hline June & $7,771,824$ & 0 & $7,771,824$ & 0 & 932,619 & 0 \\
\hline July & $8,377,567$ & 0 & $8,377,567$ & 0 & $1,005,308$ & 0 \\
\hline August & $8,398,634$ & 0 & $8,398,634$ & 0 & $1,007,836$ & 0 \\
\hline September & $5,293,275$ & 0 & $5,293,275$ & 0 & 635,193 & 0 \\
\hline October & $4,660,071$ & 0 & $4,660,071$ & 0 & 559,209 & 0 \\
\hline November & $3,692,188$ & 0 & $3,692,188$ & 0 & 443,063 & 0 \\
\hline December & $5,411,432$ & 0 & $5,411,432$ & 0 & 649,372 & 0 \\
\hline Annual & $67,248,128$ & 0 & $67,248,128$ & 0 & $8,069,775$ & 0 \\
\hline
\end{tabular}

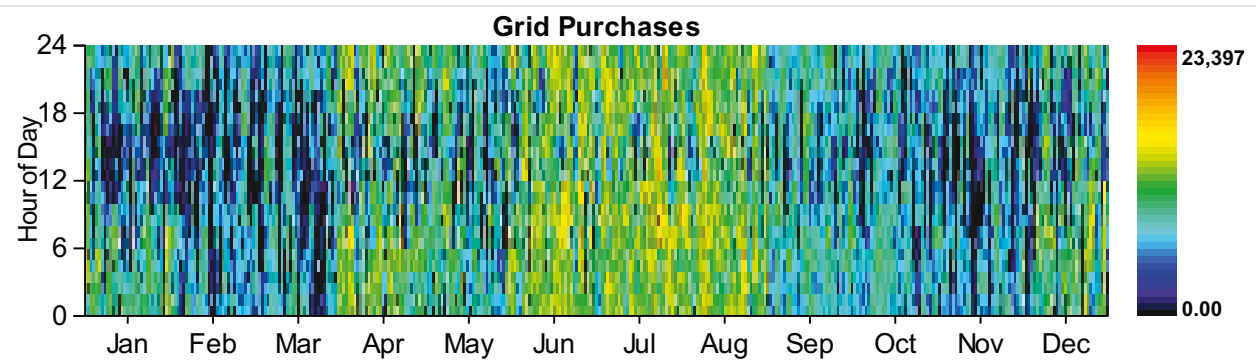

\section{Emissions}

\section{Pollutant}

Carbon dioxide

Carbon monoxide

Unburned hydrocarbons

Particulate matter

Sulfur dioxide

Nitrogen oxides
Emissions

Units

42500816 kg/yr

$0 \mathrm{~kg} / \mathrm{yr}$

$0 \mathrm{~kg} / \mathrm{yr}$

$0 \mathrm{~kg} / \mathrm{yr}$

$184260 \mathrm{~kg} / \mathrm{yr}$

90112 kg/yr 
HOMER Energy, LLC ๑ 2016 
System Report

System architecture

\begin{tabular}{|l|l|r|r|}
\hline Generator & Kohler 1000 Prime Power & 1,850 & $\mathrm{~kW}$ \\
\hline Grid & Grid & 20,000 & $\mathrm{~kW}$ \\
\hline Dispatch Strategy & Cycle Charging & \\
\hline
\end{tabular}

Cost summary

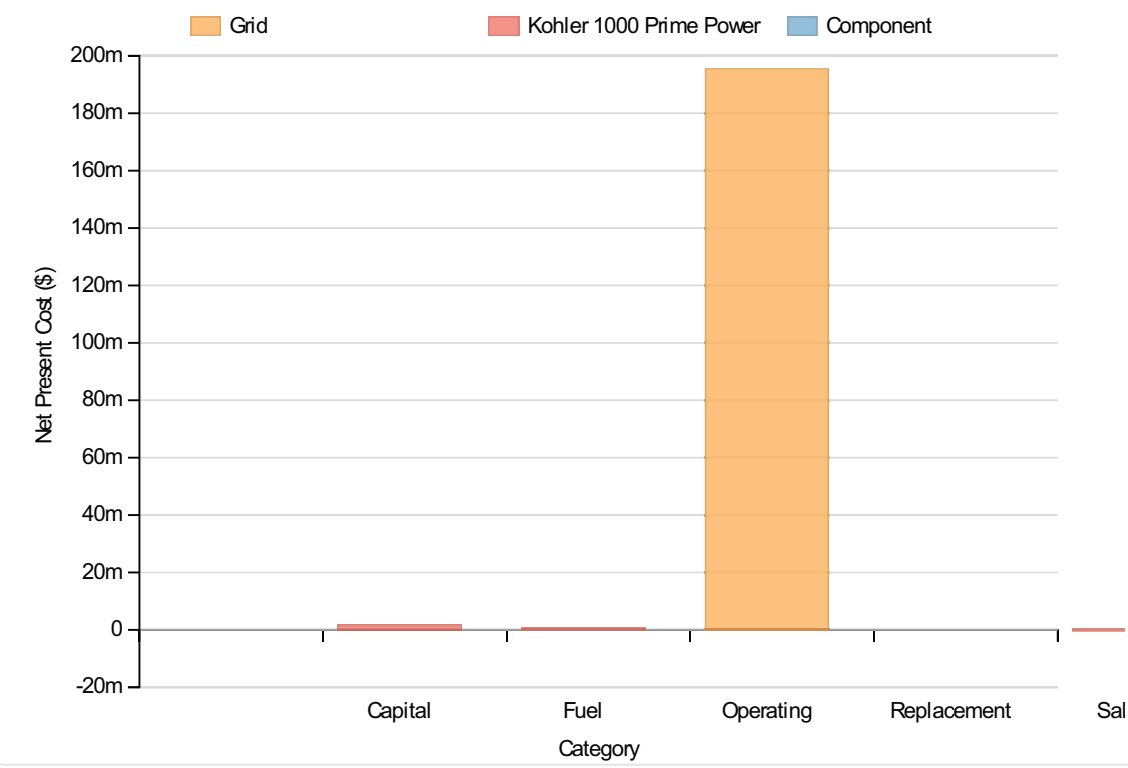

Cost Summary

Total net present cost

$197132240 \$$

Levelized cost of energy

0.157 \$/kWh

Net Present Costs

\begin{tabular}{|l|r|r|r|r|r|r|}
\hline Component & Capital & Replacement & O\&M & Fuel & Salvage & Total \\
\hline Kohler 1000 Prime Power & $1,637,500$ & 0 & 66,073 & 360,152 & $-268,055$ & $1,795,670$ \\
\hline Grid & 0 & 0 & $195,336,464$ & 0 & 0 & $195,336,464$ \\
\hline System & $1,637,500$ & 0 & $195,402,544$ & 360,152 & $-268,055$ & $197,132,141$ \\
\hline
\end{tabular}

Annualized Costs

\begin{tabular}{|l|r|r|r|r|r|r|}
\hline Component & Capital & Replacement & O\&M & Fuel & Salvage & Total \\
\hline Kohler 1000 Prime Power & 126,668 & 0 & 5,111 & 27,859 & $-20,735$ & 138,903 \\
\hline Grid & 0 & 0 & $15,110,131$ & 0 & 0 \\
\hline System & 126,668 & 0 & $15,115,242$ & 27,859 & $-20,735$ & $15,249,034$ \\
\hline
\end{tabular}




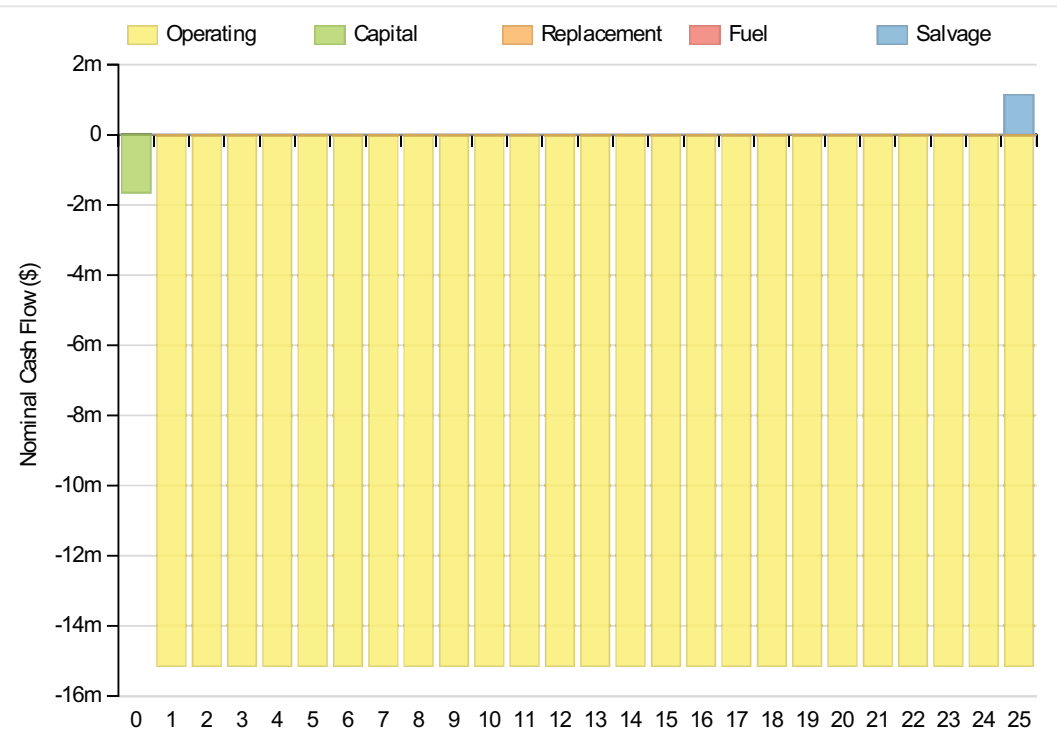

Year

\section{Electrical}

\begin{tabular}{|c|c|c|c|c|}
\hline Quantity & & Value & & Units \\
\hline Excess electricity & & & 0 & kWh/yr \\
\hline Unmet load & & $10 c$ & 32 & kWh/yr \\
\hline Capacity shortage & & 827 & 92 & $\mathrm{kWh} / \mathrm{yr}$ \\
\hline Renewable fraction & & & 0 & \\
\hline Component & Production(kWh/yr) & & racti & ion $(\%)$ \\
\hline Generator & & 121,836 & & 0 \\
\hline Grid Purchases & & $96,732,328$ & & 100 \\
\hline Total & & $96,854,160$ & & 100 \\
\hline Load & Consumption(kWh/yr) & & Frac & action (\%) \\
\hline AC primary load & & $96,854,128$ & & 100 \\
\hline DC primary load & & 0 & & 0 \\
\hline Total & & $96,854,128$ & & 100 \\
\hline
\end{tabular}




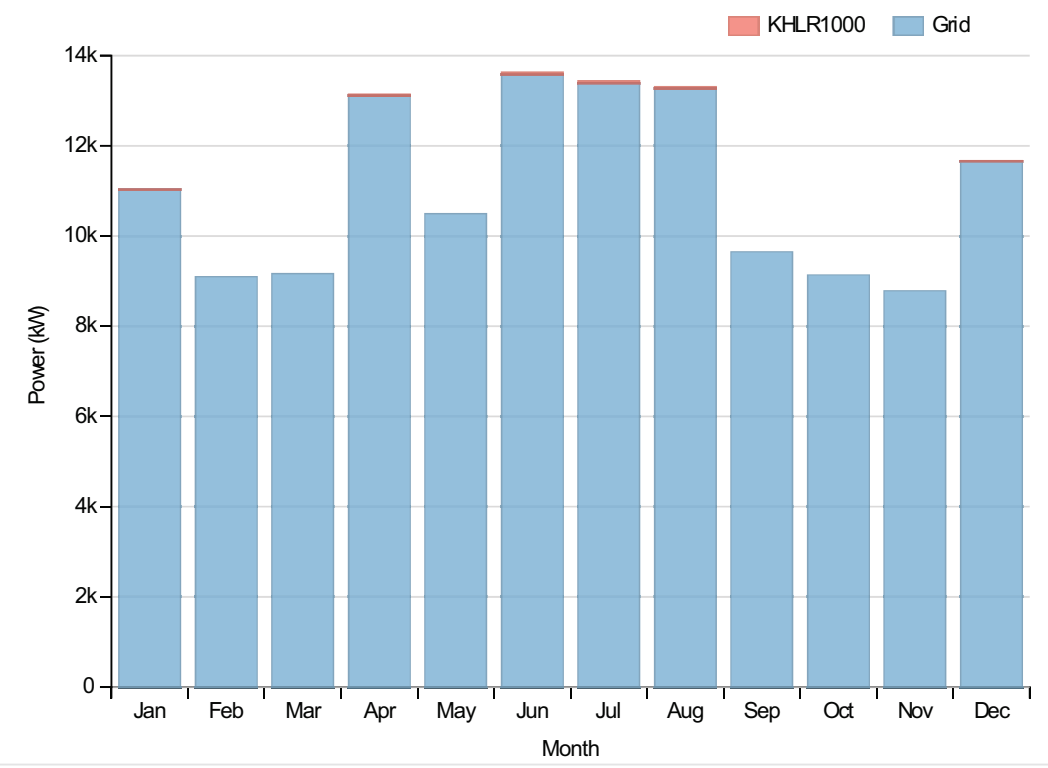

Generator:Kohler 1000 Prime Power

\begin{tabular}{|c|c|c|}
\hline Quantity & Value & Units \\
\hline Hours of operation & 190 & $\mathrm{hrs} / \mathrm{yr}$ \\
\hline Number of starts & 170 & starts/yr \\
\hline Operational life & 79 & $\mathrm{yr}$ \\
\hline Fixed generation cost & 137.46 & $\$ / \mathrm{hr}$ \\
\hline Marginal generation cost & 0.23 & $\$ / \mathrm{kWh}$ \\
\hline Electrical production & 121836 & $\mathrm{kWh} / \mathrm{yr}$ \\
\hline Mean electrical output & 641 & kW \\
\hline Min. electrical output & 463 & $\mathrm{~kW}$ \\
\hline Max. electrical output & 1850 & $\mathrm{~kW}$ \\
\hline Fuel consumption & 35265 & L/yr \\
\hline Specific fuel consumption & 0.29 & $\mathrm{~L} / \mathrm{kWh}$ \\
\hline Fuel energy input & 347008 & $\mathrm{kWh} / \mathrm{yr}$ \\
\hline Mean electrical efficiency & 35 & $\%$ \\
\hline
\end{tabular}

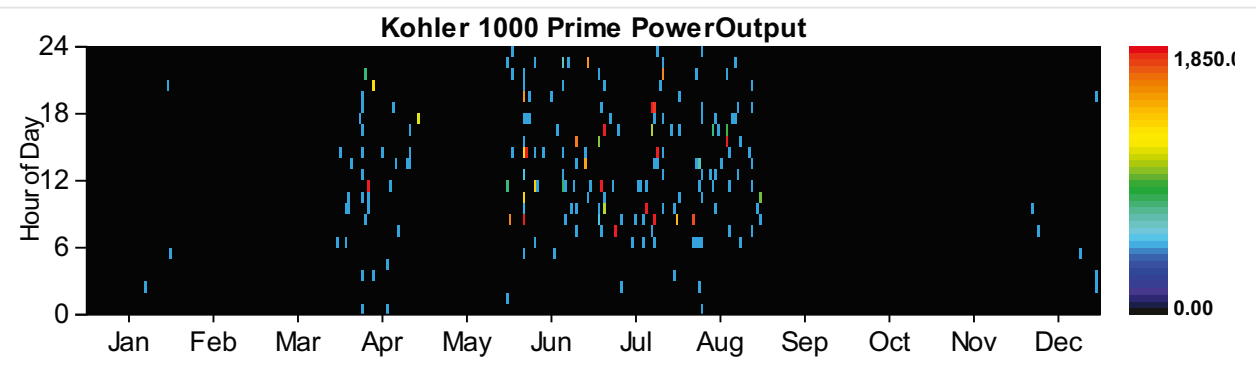

Grid

Rate: Demand 1

\begin{tabular}{|c|c|c|c|c|c|c|}
\hline Resources.ReportingService_GeneratelnputsReport_Month & $\begin{array}{l}\text { Energy } \\
\text { Purchased } \\
\text { (kWh) }\end{array}$ & $\begin{array}{l}\text { Energy } \\
\text { Sold } \\
\text { (kWh) }\end{array}$ & $\begin{array}{l}\text { Net } \\
\text { Purchases } \\
\text { (kWh) }\end{array}$ & $\begin{array}{l}\text { Peak } \\
\text { Demand } \\
(\mathrm{kW})\end{array}$ & $\begin{array}{l}\text { Energy } \\
\text { Charge } \\
(\$)\end{array}$ & $\begin{array}{l}\text { Demand } \\
\text { Charge } \\
\text { (\$) }\end{array}$ \\
\hline
\end{tabular}




\begin{tabular}{|c|c|c|c|c|c|c|c|}
\hline Rébruartes.ReportingService_GeneratelnputsReport_Month & $\begin{array}{l}\text { Energy } 0 \\
\text { Purchased } \\
\text { (kWh) }\end{array}$ & $\begin{array}{l}\text { Energy } 0 \\
\text { Sold } \\
\text { (kWh) } 0\end{array}$ & $\begin{array}{l}\text { Net } \\
\text { Purchase } \\
\text { (kWh) }\end{array}$ & & $\begin{array}{l}\text { Peâk } \\
\text { Demand } \\
\text { (kWh),561 }\end{array}$ & $\begin{array}{l}\text { Energy } \\
\text { Charge } \\
\text { (\$) } 0\end{array}$ & 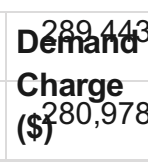 \\
\hline March & 0 & 0 & & 0 & 17,425 & 0 & 278,807 \\
\hline April & 0 & 0 & & 0 & 20,000 & 0 & 320,000 \\
\hline May & 0 & 0 & & 0 & 17,869 & 0 & 285,902 \\
\hline June & 0 & 0 & & 0 & 20,000 & 0 & 320,000 \\
\hline July & 0 & 0 & & 0 & 20,000 & 0 & 320,000 \\
\hline August & 0 & 0 & & 0 & 20,000 & 0 & 320,000 \\
\hline September & 0 & 0 & & 0 & 16,926 & 0 & 270,820 \\
\hline October & 0 & 0 & & 0 & 14,895 & 0 & 238,325 \\
\hline November & 0 & 0 & & 0 & 16,518 & 0 & 264,286 \\
\hline December & 0 & 0 & & 0 & 19,606 & 0 & 313,698 \\
\hline Annual & 0 & 0 & & 0 & 20,000 & 0 & $3,502,259$ \\
\hline
\end{tabular}

Rate: Rate 1

\begin{tabular}{|c|c|c|c|c|c|c|}
\hline Resources.ReportingService_GeneratelnputsReport_Month & $\begin{array}{l}\text { Energy } \\
\text { Purchased } \\
\text { (kWh) }\end{array}$ & $\begin{array}{l}\text { Energy } \\
\text { Sold } \\
\text { (kWh) }\end{array}$ & $\begin{array}{l}\text { Net } \\
\text { Purchases } \\
\text { (kWh) }\end{array}$ & $\begin{array}{l}\text { Peak } \\
\text { Demand } \\
\text { (kW) }\end{array}$ & $\begin{array}{l}\text { Energy } \\
\text { Charge (\$) }\end{array}$ & $\begin{array}{l}\text { Demand } \\
\text { Charge } \\
\text { (\$) }\end{array}$ \\
\hline January & $8,208,348$ & 0 & $8,208,348$ & 0 & 985,002 & 0 \\
\hline February & $6,110,456$ & 0 & $6,110,456$ & 0 & 733,255 & 0 \\
\hline March & $6,816,916$ & 0 & $6,816,916$ & 0 & 818,030 & 0 \\
\hline April & $9,441,886$ & 0 & $9,441,886$ & 0 & $1,133,026$ & 0 \\
\hline May & $7,805,883$ & 0 & $7,805,883$ & 0 & 936,706 & 0 \\
\hline June & $9,779,835$ & 0 & $9,779,835$ & 0 & $1,173,580$ & 0 \\
\hline July & $9,962,285$ & 0 & $9,962,285$ & 0 & $1,195,474$ & 0 \\
\hline August & $9,872,852$ & 0 & $9,872,852$ & 0 & $1,184,742$ & 0 \\
\hline September & $6,943,139$ & 0 & $6,943,139$ & 0 & 833,177 & 0 \\
\hline October & $6,792,313$ & 0 & $6,792,313$ & 0 & 815,078 & 0 \\
\hline November & $6,321,105$ & 0 & $6,321,105$ & 0 & 758,533 & 0 \\
\hline December & $8,677,315$ & 0 & $8,677,315$ & 0 & $1,041,278$ & 0 \\
\hline Annual & $96,732,328$ & 0 & $96,732,328$ & 0 & $11,607,880$ & 0 \\
\hline
\end{tabular}




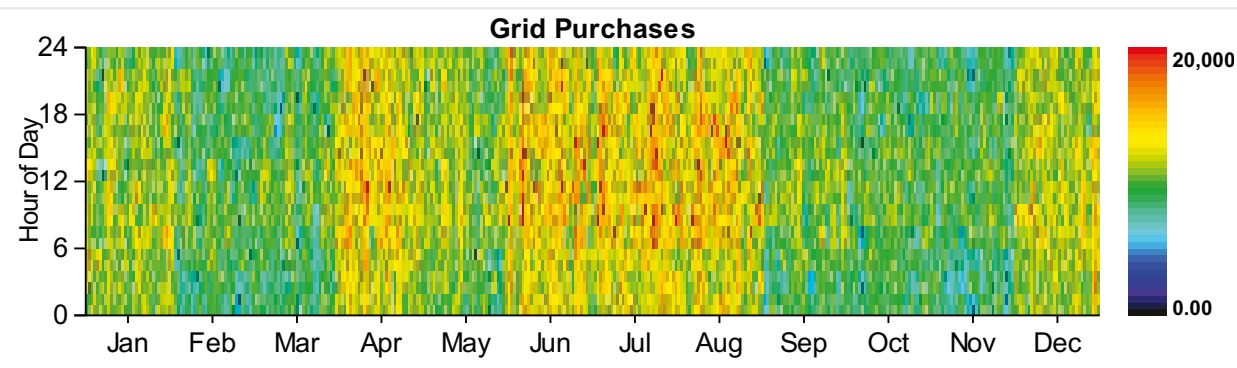

\section{Emissions}

\begin{tabular}{|l|r|l|}
\hline Pollutant & Emissions & Units \\
\hline Carbon dioxide & $61227384 \mathrm{~kg} / \mathrm{yr}$ \\
\hline Carbon monoxide & $388 \mathrm{~kg} / \mathrm{yr}$ \\
\hline Unburned hydrocarbons & $44 \mathrm{~kg} / \mathrm{yr}$ \\
\hline Particulate matter & $11 \mathrm{~kg} / \mathrm{yr}$ \\
\hline Sulfur dioxide & $265237 \mathrm{~kg} / \mathrm{yr}$ \\
\hline Nitrogen oxides & $130009 \mathrm{~kg} / \mathrm{yr}$ \\
\hline
\end{tabular}

HOMER Energy, LLC @ 2016 
System Report

System architecture

\begin{tabular}{|l|l|r|}
\hline Generator \#2 & Kohler 750 Prime Power & 2,070 \\
\hline Grid & Grid & 20,000 \\
\hline Dispatch Strategy & Cycle Charging & $\mathrm{kW}$ \\
\hline
\end{tabular}

Cost summary

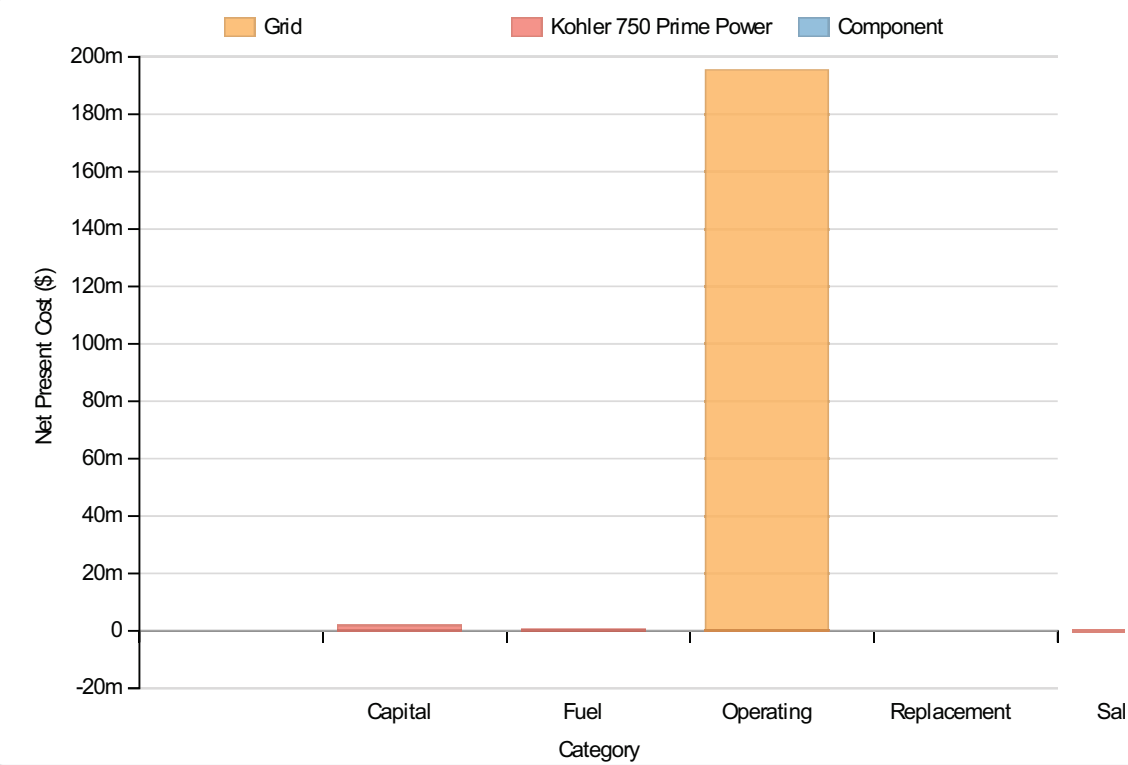

Cost Summary

Total net present cost

$197277584 \$$

Levelized cost of energy

$0.158 \$ / k W h$

Net Present Costs

\begin{tabular}{|l|r|r|r|r|r|r|}
\hline Component & Capital & Replacement & O\&M & Fuel & Salvage & Total \\
\hline Kohler 750 Prime Power & $1,802,500$ & 0 & 73,638 & 395,627 & $-295,065$ & $1,976,700$ \\
\hline Grid & 0 & 0 & $195,300,800$ & 0 & 0 \\
\hline System & $1,802,500$ & 0 & $195,374,432$ & 395,627 & $-295,065$ & $197,277,494$ \\
\hline
\end{tabular}

Annualized Costs

\begin{tabular}{|l|r|r|r|r|r|r|}
\hline Component & Capital & Replacement & O\&M & Fuel & Salvage & Total \\
\hline Kohler 750 Prime Power & 139,431 & 0 & 5,696 & 30,604 & $-22,825$ & 152,906 \\
\hline Grid & 0 & 0 & $15,107,372$ & 0 & 0 \\
\hline System & 139,431 & 0 & $15,113,068$ & 30,604 & $-22,825$ & $15,260,278$ \\
\hline
\end{tabular}




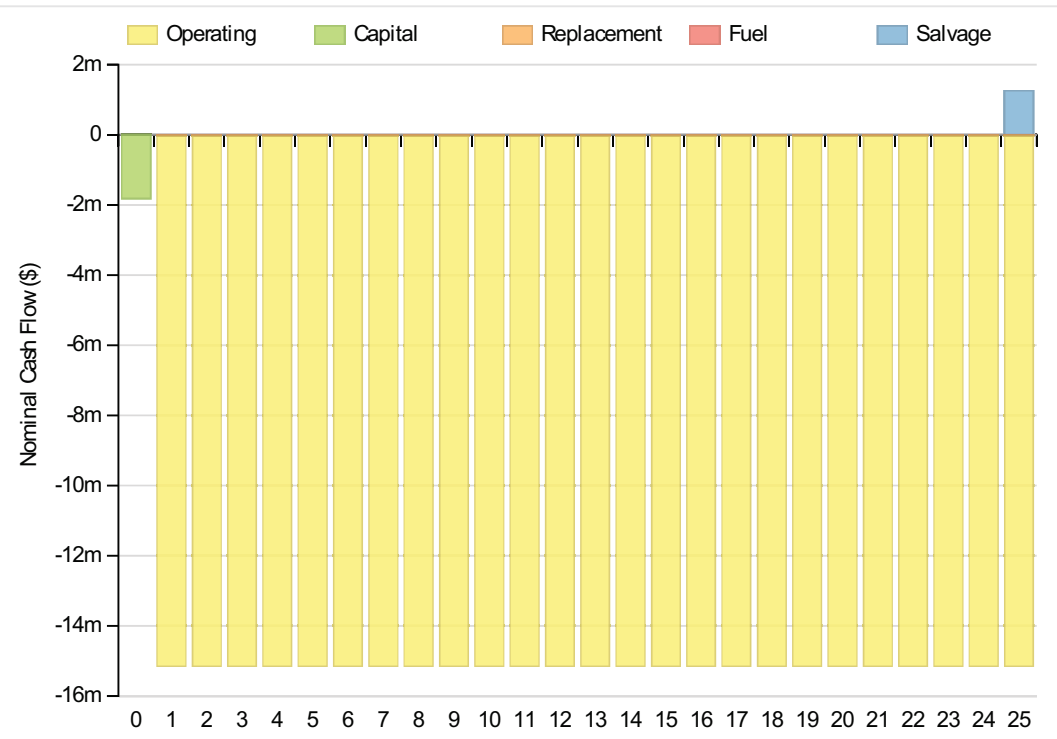

Year

\section{Electrical}

\begin{tabular}{|c|c|c|c|c|}
\hline Quantity & & Value & & Units \\
\hline Excess electricity & & & 0 & kWh/yr \\
\hline Unmet load & & & 997 & kWh/yr \\
\hline Capacity shortage & & 688 & 306 & $\mathrm{kWh} / \mathrm{yr}$ \\
\hline Renewable fraction & & & 0 & \\
\hline Component & Production(kWh/yr) & & racti & ion (\%) \\
\hline Generator & & 132,207 & & 0 \\
\hline Grid Purchases & & $96,723,992$ & & 100 \\
\hline Total & & $96,856,200$ & & 100 \\
\hline Load & Consumption(kWh/yr) & & Fra & ction (\%) \\
\hline AC primary load & & $96,856,160$ & & 100 \\
\hline DC primary load & & 0 & & 0 \\
\hline Total & & $96,856,160$ & & 100 \\
\hline
\end{tabular}




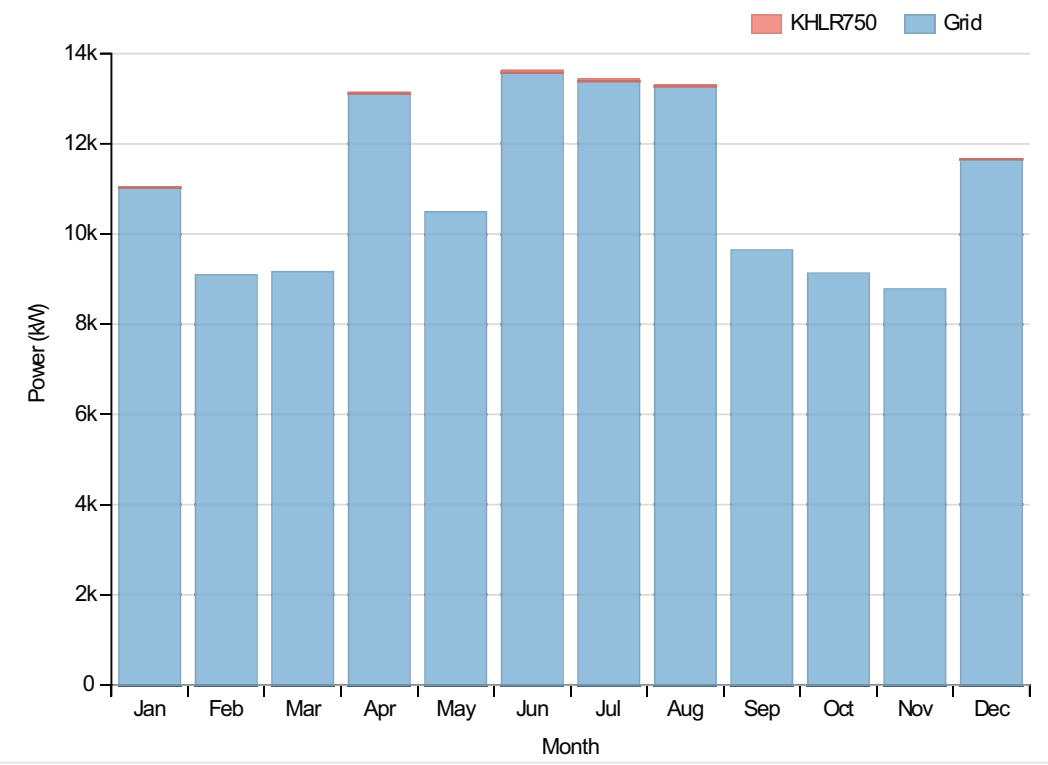

Generator:Kohler 750 Prime Power

\begin{tabular}{|c|c|c|}
\hline Quantity & Value & Units \\
\hline Hours of operation & 190 & $\mathrm{hrs} / \mathrm{yr}$ \\
\hline Number of starts & 170 & starts/yr \\
\hline Operational life & 79 & $\mathrm{yr}$ \\
\hline Fixed generation cost & 151.69 & $\$ / \mathrm{hr}$ \\
\hline Marginal generation cost & 0.23 & $\$ / k W h$ \\
\hline Electrical production & 132207 & $\mathrm{kWh} / \mathrm{yr}$ \\
\hline Mean electrical output & 696 & kW \\
\hline Min. electrical output & 518 & $\mathrm{~kW}$ \\
\hline Max. electrical output & 2070 & $\mathrm{~kW}$ \\
\hline Fuel consumption & 38739 & L/yr \\
\hline Specific fuel consumption & 0.29 & $\mathrm{~L} / \mathrm{kWh}$ \\
\hline Fuel energy input & 381188 & $\mathrm{kWh} / \mathrm{yr}$ \\
\hline Mean electrical efficiency & 35 & $\%$ \\
\hline
\end{tabular}

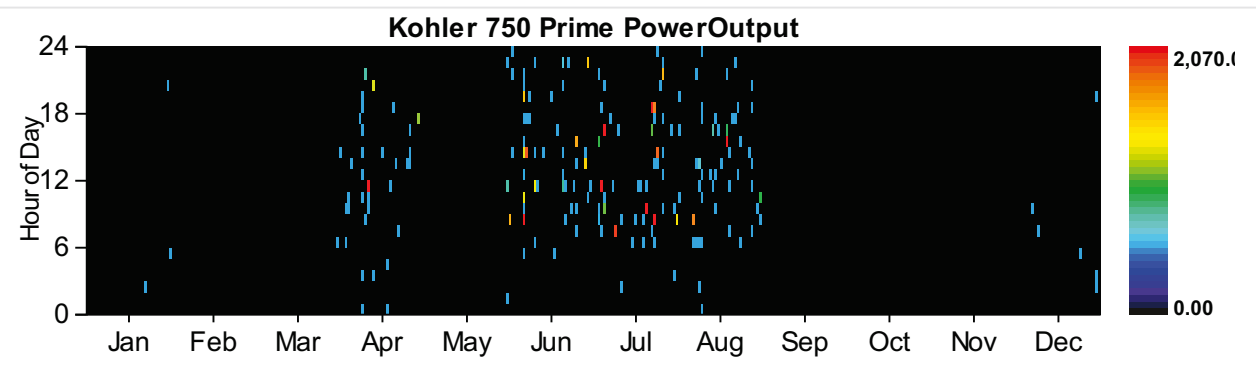

Grid

Rate: Demand 1

\begin{tabular}{|c|c|c|c|c|c|c|}
\hline Resources.ReportingService_GeneratelnputsReport_Month & $\begin{array}{l}\text { Energy } \\
\text { Purchased } \\
\text { (kWh) }\end{array}$ & $\begin{array}{l}\text { Energy } \\
\text { Sold } \\
\text { (kWh) }\end{array}$ & $\begin{array}{l}\text { Net } \\
\text { Purchases } \\
\text { (kWh) }\end{array}$ & $\begin{array}{l}\text { Peak } \\
\text { Demand } \\
(\mathrm{kW})\end{array}$ & $\begin{array}{l}\text { Energy } \\
\text { Charge } \\
(\$)\end{array}$ & $\begin{array}{l}\text { Demand } \\
\text { Charge } \\
\text { (\$) }\end{array}$ \\
\hline
\end{tabular}




\begin{tabular}{|c|c|c|c|c|c|c|c|}
\hline Rébruartes.ReportingService_GeneratelnputsReport_Month & $\begin{array}{l}\text { Energy } 0 \\
\text { Purchased } \\
\text { (kWh) }\end{array}$ & $\begin{array}{l}\text { Energy } 0 \\
\text { Sold } \\
\text { (kWh) } 0\end{array}$ & $\begin{array}{l}\text { Net } \\
\text { Purchase } \\
\text { (kWh) }\end{array}$ & & $\begin{array}{l}\text { Peâk } \\
\text { Demand } \\
\text { (kWh),561 }\end{array}$ & $\begin{array}{l}\text { Energy } \\
\text { Charge } \\
\text { (\$) } 0\end{array}$ & $\begin{array}{l}\text { Denhangas } \\
\text { Charge } \\
\text { (\$2 } \$)^{80,978}\end{array}$ \\
\hline March & 0 & 0 & & 0 & 17,425 & 0 & 278,807 \\
\hline April & 0 & 0 & & 0 & 20,000 & 0 & 320,000 \\
\hline May & 0 & 0 & & 0 & 17,869 & 0 & 285,902 \\
\hline June & 0 & 0 & & 0 & 20,000 & 0 & 320,000 \\
\hline July & 0 & 0 & & 0 & 20,000 & 0 & 320,000 \\
\hline August & 0 & 0 & & 0 & 20,000 & 0 & 320,000 \\
\hline September & 0 & 0 & & 0 & 16,926 & 0 & 270,820 \\
\hline October & 0 & 0 & & 0 & 14,895 & 0 & 238,325 \\
\hline November & 0 & 0 & & 0 & 16,518 & 0 & 264,286 \\
\hline December & 0 & 0 & & 0 & 19,551 & 0 & 312,818 \\
\hline Annual & 0 & 0 & & 0 & 20,000 & 0 & $3,500,499$ \\
\hline
\end{tabular}

Rate: Rate 1

\begin{tabular}{|c|c|c|c|c|c|c|}
\hline Resources.ReportingService_GeneratelnputsReport_Month & $\begin{array}{l}\text { Energy } \\
\text { Purchased } \\
\text { (kWh) }\end{array}$ & $\begin{array}{l}\text { Energy } \\
\text { Sold } \\
\text { (kWh) }\end{array}$ & $\begin{array}{l}\text { Net } \\
\text { Purchases } \\
\text { (kWh) }\end{array}$ & $\begin{array}{l}\text { Peak } \\
\text { Demand } \\
\text { (kW) }\end{array}$ & $\begin{array}{l}\text { Energy } \\
\text { Charge (\$) }\end{array}$ & $\begin{array}{l}\text { Demand } \\
\text { Charge } \\
\text { (\$) }\end{array}$ \\
\hline January & $8,208,183$ & 0 & $8,208,183$ & 0 & 984,982 & 0 \\
\hline February & $6,110,456$ & 0 & $6,110,456$ & 0 & 733,255 & 0 \\
\hline March & $6,816,916$ & 0 & $6,816,916$ & 0 & 818,030 & 0 \\
\hline April & $9,440,184$ & 0 & $9,440,184$ & 0 & $1,132,822$ & 0 \\
\hline May & $7,805,883$ & 0 & $7,805,883$ & 0 & 936,706 & 0 \\
\hline June & $9,777,930$ & 0 & $9,777,930$ & 0 & $1,173,352$ & 0 \\
\hline July & $9,960,415$ & 0 & $9,960,415$ & 0 & $1,195,250$ & 0 \\
\hline August & $9,870,487$ & 0 & $9,870,487$ & 0 & $1,184,458$ & 0 \\
\hline September & $6,943,139$ & 0 & $6,943,139$ & 0 & 833,177 & 0 \\
\hline October & $6,792,313$ & 0 & $6,792,313$ & 0 & 815,078 & 0 \\
\hline November & $6,321,105$ & 0 & $6,321,105$ & 0 & 758,533 & 0 \\
\hline December & $8,676,985$ & 0 & $8,676,985$ & 0 & $1,041,238$ & 0 \\
\hline Annual & $96,723,992$ & 0 & $96,723,992$ & 0 & $11,606,880$ & 0 \\
\hline
\end{tabular}




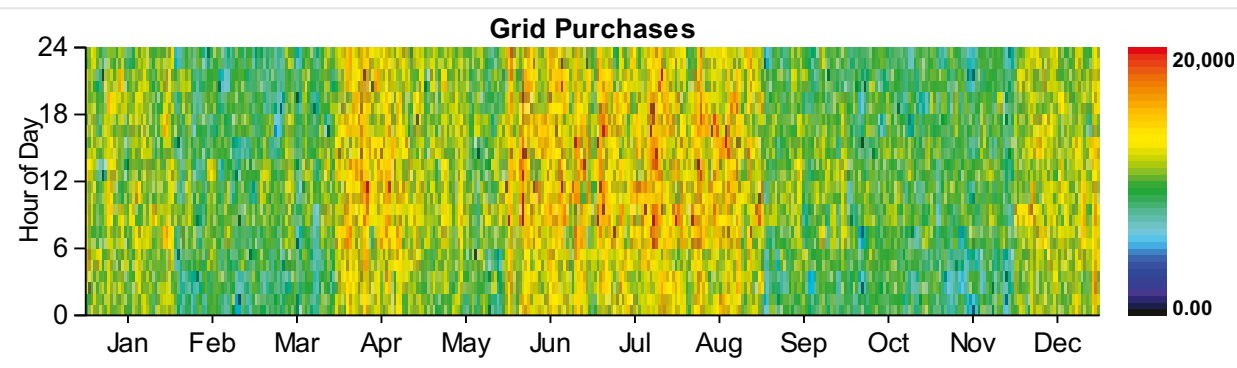

\section{Emissions}

\begin{tabular}{|l|r|l|}
\hline Pollutant & Emissions & Units \\
\hline Carbon dioxide & $61231236 \mathrm{~kg} / \mathrm{yr}$ \\
\hline Carbon monoxide & $426 \mathrm{~kg} / \mathrm{yr}$ \\
\hline Unburned hydrocarbons & $49 \mathrm{~kg} / \mathrm{yr}$ \\
\hline Particulate matter & $12 \mathrm{~kg} / \mathrm{yr}$ \\
\hline Sulfur dioxide & $265233 \mathrm{~kg} / \mathrm{yr}$ \\
\hline Nitrogen oxides & $130036 \mathrm{~kg} / \mathrm{yr}$ \\
\hline
\end{tabular}

HOMER Energy, LLC @ 2016 
System Report

System architecture

\begin{tabular}{|l|l|r|}
\hline Generator & Kohler 1000 Prime Power & $925 \mathrm{~kW}$ \\
\hline Generator \#2 & Kohler 750 Prime Power & $690 \mathrm{~kW}$ \\
\hline Battery & GS200 flow & 1 strings \\
\hline Converter & System Converter & $250 \mathrm{~kW}$ \\
\hline Grid & Grid & $20,000 \mathrm{~kW}$ \\
\hline Dispatch Strategy & Cycle Charging & \\
\hline
\end{tabular}

Cost summary

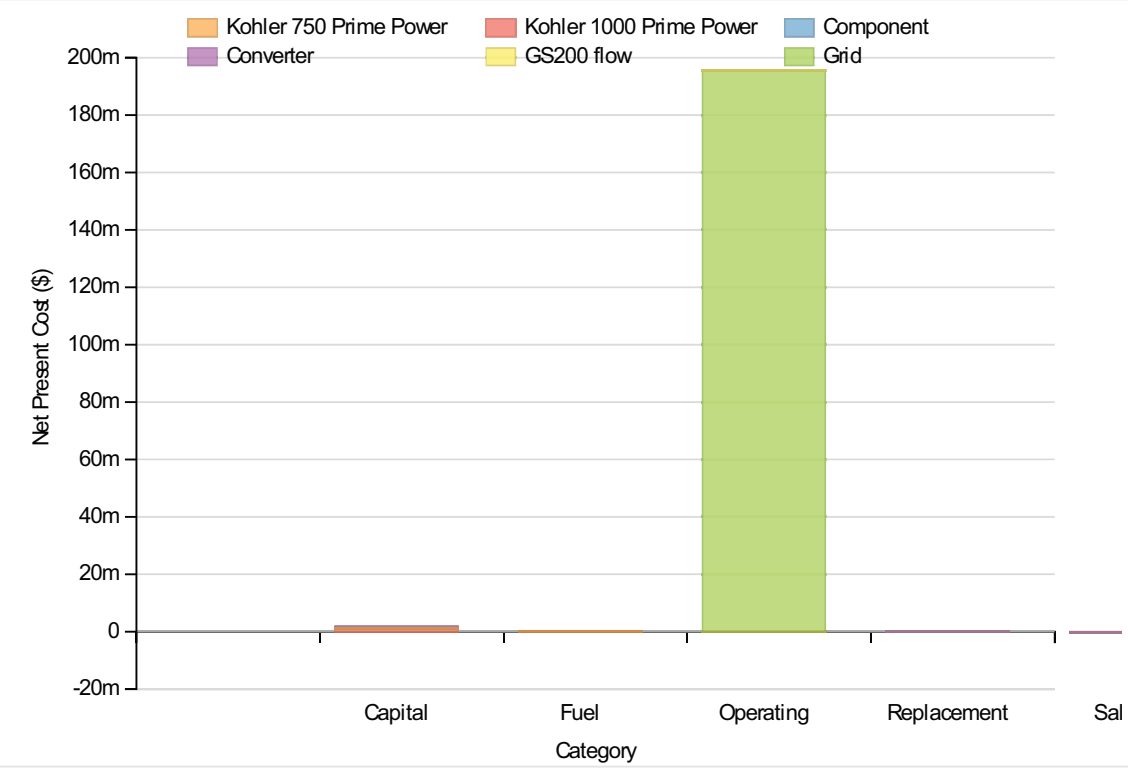

Cost Summary

Total net present cost

197556960 \$

Levelized cost of energy

$0.158 \$ / k W h$

Net Present Costs

\begin{tabular}{|l|r|r|r|r|r|r|}
\hline Component & Capital & Replacement & O\&M & Fuel & Salvage & Total \\
\hline Kohler 1000 Prime Power & 925,000 & 0 & 22,421 & 159,833 & $-175,426$ & 931,828 \\
\hline Kohler 750 Prime Power & 690,000 & 0 & 21,007 & 119,719 & $-122,043$ & 708,683 \\
\hline Grid & 0 & 0 & $195,453,680$ & 0 & 0 \\
\hline GS200 flow & 324,589 & 7,176 & 31,026 & 0 & -973 \\
\hline Converter & 75,000 & 31,821 & 0 & 0 & $-5,953,989$ \\
\hline System & $2,014,589$ & 38,996 & $195,528,160$ & 279,551 & $-304,430$ \\
\hline
\end{tabular}

Annualized Costs

\begin{tabular}{|l|r|r|r|r|r|r|}
\hline Component & Capital & Replacement & O\&M & Fuel & Salvage & Total \\
\hline Kohler 1000 Prime Power & 71,553 & 0 & 1,734 & 12,364 & $-13,570$ & 72,081 \\
\hline Kohler 750 Prime Power & 53,375 & 0 & 1,625 & 9,261 & $-9,441$ & 54,820 \\
\hline
\end{tabular}




\begin{tabular}{|c|c|c|c|c|c|c|}
\hline $\begin{array}{l}\text { Component } \\
\text { Grid }\end{array}$ & $\begin{array}{ll}\text { Capital } & 0\end{array}$ & Replacement & $\mathbf{O} \& \mathbf{M}_{15,119,198}$ & Fuel & Salvage & Total $_{15,119,198}$ \\
\hline GS200 flow & 25,108 & 555 & 2,400 & 0 & -75 & 27,988 \\
\hline Converter & 5,802 & 2,462 & 0 & 0 & -463 & 7,800 \\
\hline System & 155,837 & 3,017 & $15,124,959$ & 21,625 & $-23,549$ & $15,281,889$ \\
\hline
\end{tabular}

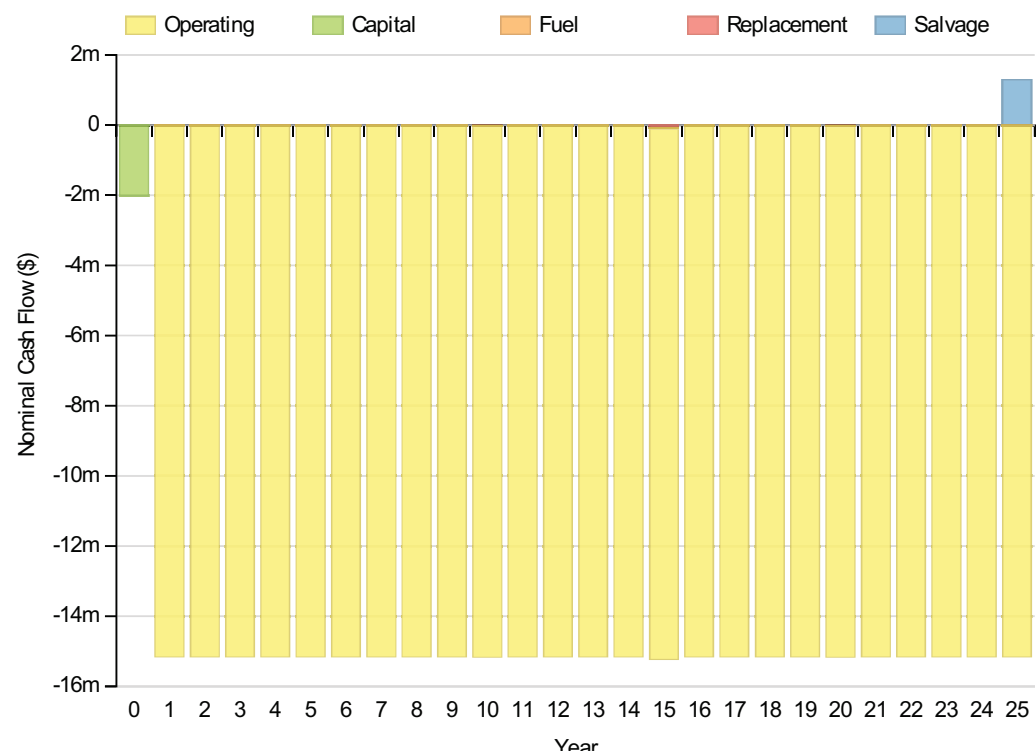

\section{Electrical}

\section{Quantity}

Excess electricity

Unmet load

Capacity shortage

Renewable fraction

\section{Value}

$0816 \mathrm{kWh} / \mathrm{yr}$

$87614 \mathrm{kWh} / \mathrm{yr}$

0

\begin{tabular}{|c|c|c|}
\hline Component & Production(kWh/yr) & Fraction (\%) \\
\hline Generator & 54,204 & 0 \\
\hline Generator & 40,041 & 0 \\
\hline Grid Purchases & $96,761,408$ & 100 \\
\hline Total & $96,855,648$ & 100 \\
\hline Load & Consumption(kWh/yr) & Fraction $(\%)$ \\
\hline AC primary load & $96,853,336$ & 100 \\
\hline DC primary load & & 0 \\
\hline Total & $96,853,336$ & 100 \\
\hline
\end{tabular}




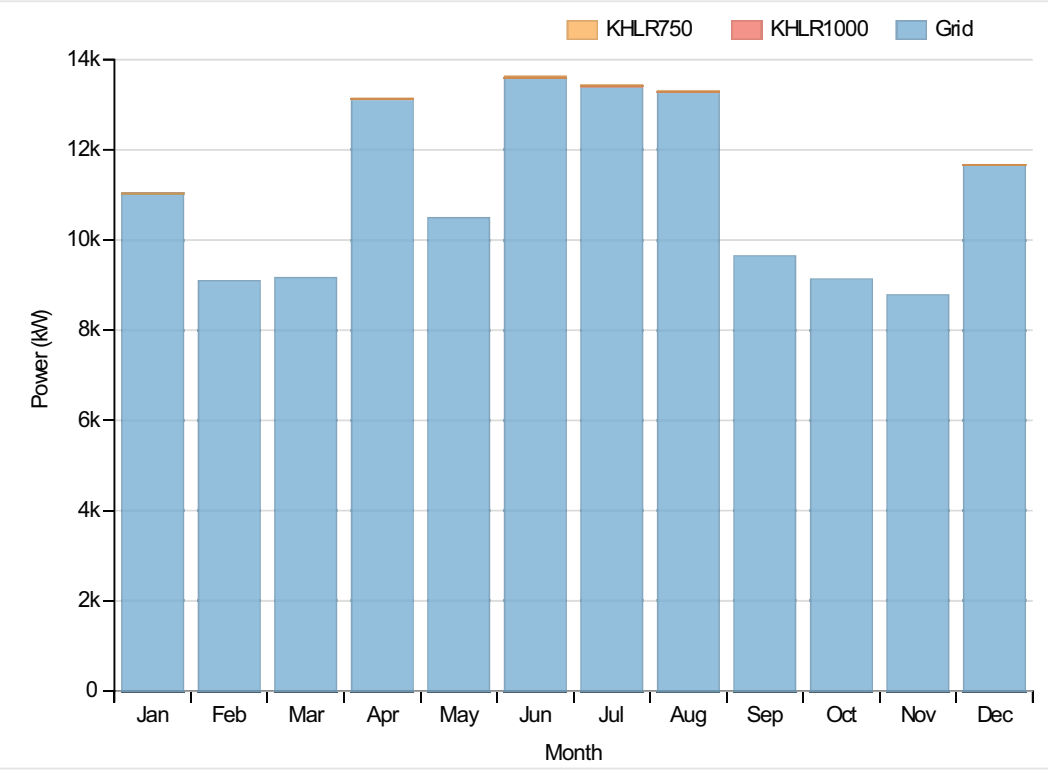

Generator:Kohler 1000 Prime Power

\begin{tabular}{|c|c|c|}
\hline Quantity & Value & Units \\
\hline Hours of operation & 125 & $\mathrm{hrs} / \mathrm{yr}$ \\
\hline Number of starts & 113 & starts/yr \\
\hline Operational life & 120 & $\mathrm{yr}$ \\
\hline Fixed generation cost & 76.24 & $\$ / h r$ \\
\hline Marginal generation cost & 0.23 & $\$ / k W h$ \\
\hline Electrical production & 54204 & $\mathrm{kWh} / \mathrm{yr}$ \\
\hline Mean electrical output & 434 & kW \\
\hline Min. electrical output & 231 & kW \\
\hline Max. electrical output & 925 & $\mathrm{~kW}$ \\
\hline Fuel consumption & 15650 & $\mathrm{~L} / \mathrm{yr}$ \\
\hline Specific fuel consumption & 0.29 & $\mathrm{~L} / \mathrm{kWh}$ \\
\hline Fuel energy input & 153999 & $\mathrm{kWh} / \mathrm{yr}$ \\
\hline Mean electrical efficiency & 35 & $\%$ \\
\hline
\end{tabular}

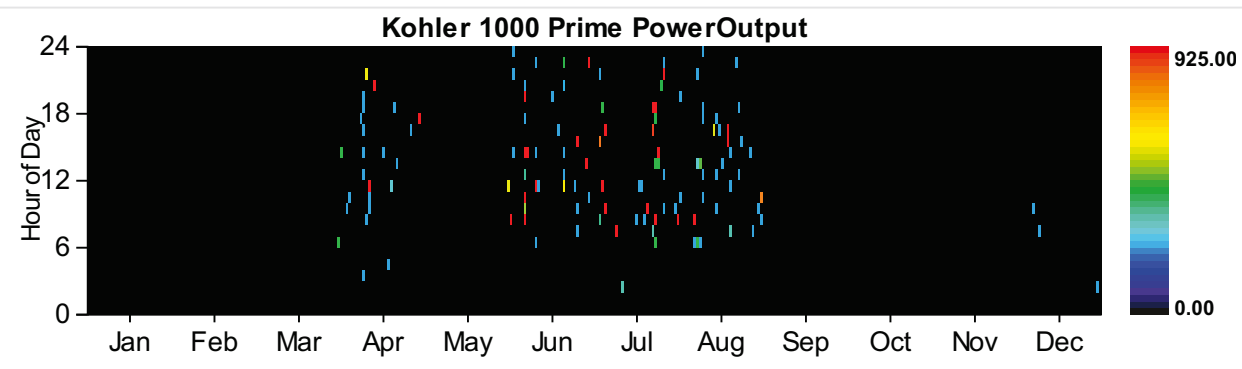

Generator:Kohler 750 Prime Power

\begin{tabular}{|l|r|l|}
\hline Quantity & Value & Units \\
\hline Hours of operation & 157 hrs/yr \\
\hline Number of starts & 143 starts/yr
\end{tabular}


Fixed generation cost

Marginal generation cost

Electrical production

Mean electrical output

Min. electrical output

Max. electrical output

Fuel consumption

Specific fuel consumption

Fuel energy input

Mean electrical efficiency
$56.87 \$ / \mathrm{hr}$

$0.23 \$ / \mathrm{kWh}$

$40041 \mathrm{kWh} / \mathrm{yr}$

255 kW

173 kW

690 kW

11723 L/yr

$0.29 \mathrm{~L} / \mathrm{kWh}$

$115350 \mathrm{kWh} / \mathrm{yr}$

$35 \%$

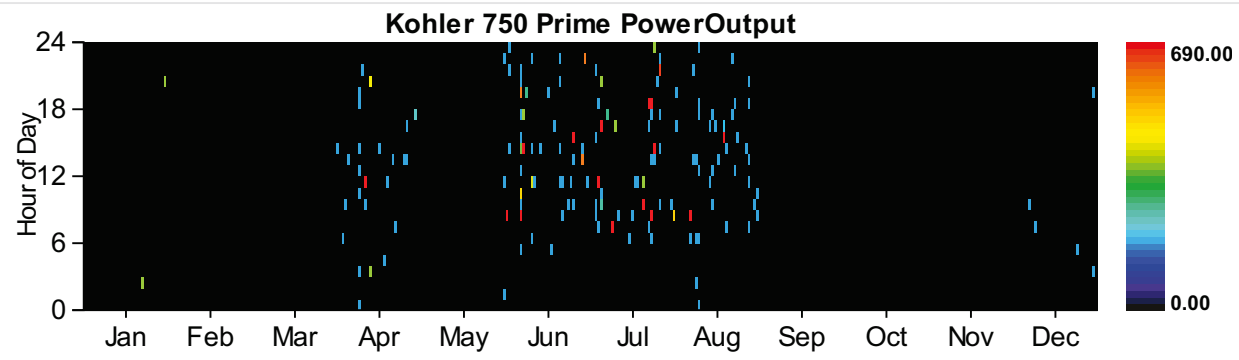

\section{Battery:GS200 flow}

\section{Quantity}

Value

String size

Strings in parallel

Batteries

Bus voltage

\section{Quantity}

Value

Units

Nominal capacity

$600 \mathrm{kWh}$

Usable nominal capacity

$600 \mathrm{kWh}$

Autonomy

$0 \mathrm{hr}$

Lifetime throughput

0

Battery wear cost

$0.000 \$ / k W h$

Average energy cost

$0.361 \$ / k W h$

Energy in

$4149 \mathrm{kWh} / \mathrm{yr}$

Energy out

2905 kWh/yr

Storage depletion

$0 \mathrm{kWh} / \mathrm{yr}$ 


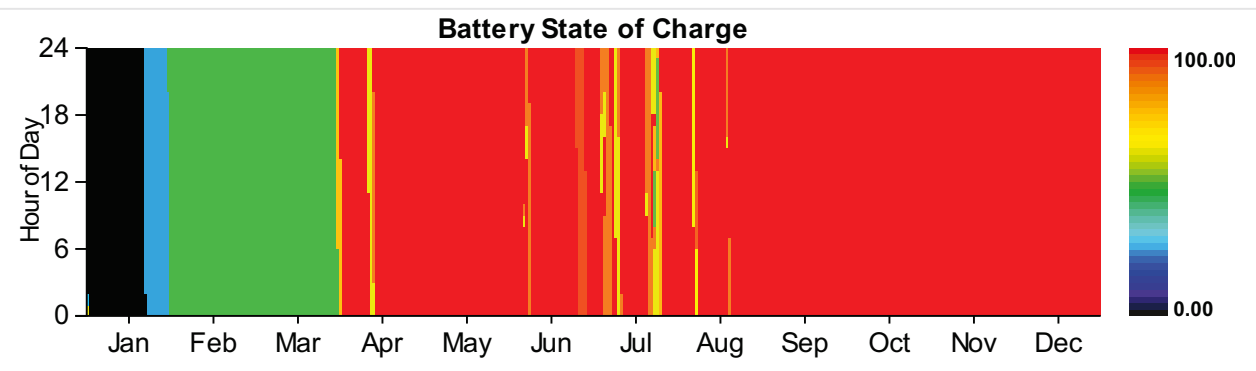

\section{Converter}

\begin{tabular}{|l|r|r|r|}
\hline Quantity & Inverter & Rectifier & Units \\
\hline Capacity & 250 & 225 & $\mathrm{~kW}$ \\
\hline Mean output & 0 & $\mathrm{~kW}$ & $\mathrm{~kW}$ \\
\hline Minimum output & 0 & $179 \mathrm{~kW}$ \\
\hline Maximum output & 166 & 0 & $\%$ \\
\hline Capacity factor & 0 & 30 & $\mathrm{hrs} / \mathrm{yr}$ \\
\hline Hours of operation & 17 & 4,881 kWh/yr \\
\hline Energy in & 2,905 & $4,149 \mathrm{kWh} / \mathrm{yr}$ \\
\hline Energy out & 2,614 & $732 \mathrm{kWh} / \mathrm{yr}$ \\
\hline Losses & 290 & \\
\hline
\end{tabular}
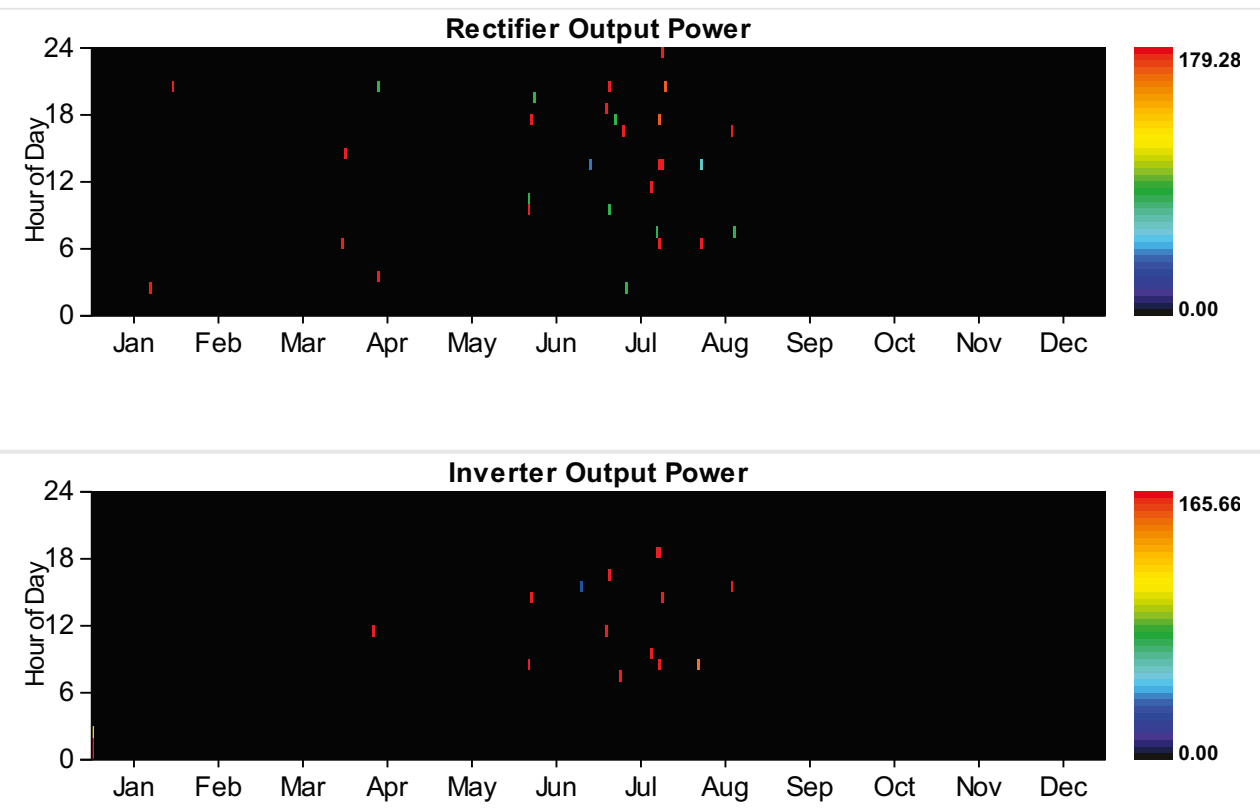

Grid

Rate: Demand 1 


\begin{tabular}{|c|c|c|c|c|c|c|}
\hline Resources.ReportingService_GeneratelnputsReport_Month & $\begin{array}{l}\text { Energy } \\
\text { Purchased } \\
\text { (kWh) }\end{array}$ & $\begin{array}{l}\text { Energy } \\
\text { Sold } \\
\text { (kWh) }\end{array}$ & $\begin{array}{l}\text { Net } \\
\text { Purchases } \\
\text { (kWh) }\end{array}$ & $\begin{array}{l}\text { Peak } \\
\text { Demand } \\
(\mathbf{k W})\end{array}$ & $\begin{array}{l}\text { Energy } \\
\text { Charge } \\
(\$)\end{array}$ & $\begin{array}{l}\text { Demand } \\
\text { Charge } \\
(\$)\end{array}$ \\
\hline January & 0 & 0 & 0 & 18,380 & 0 & 294,083 \\
\hline February & 0 & 0 & 0 & 17,561 & 0 & 280,978 \\
\hline March & 0 & 0 & 0 & 17,425 & 0 & 278,807 \\
\hline April & 0 & 0 & 0 & 20,000 & 0 & 320,000 \\
\hline May & 0 & 0 & 0 & 17,869 & 0 & 285,902 \\
\hline June & 0 & 0 & 0 & 20,000 & 0 & 320,000 \\
\hline July & 0 & 0 & 0 & 20,000 & 0 & 320,000 \\
\hline August & 0 & 0 & 0 & 20,000 & 0 & 320,000 \\
\hline September & 0 & 0 & 0 & 16,926 & 0 & 270,820 \\
\hline October & 0 & 0 & 0 & 14,895 & 0 & 238,325 \\
\hline November & 0 & 0 & 0 & 16,518 & 0 & 264,286 \\
\hline December & 0 & 0 & 0 & 19,665 & 0 & 314,638 \\
\hline Annual & 0 & 0 & 0 & 20,000 & 0 & $3,507,839$ \\
\hline
\end{tabular}

Rate: Rate 1

\begin{tabular}{|c|c|c|c|c|c|c|}
\hline Resources.ReportingService_GeneratelnputsReport_Month & $\begin{array}{l}\text { Energy } \\
\text { Purchased } \\
\text { (kWh) }\end{array}$ & $\begin{array}{l}\text { Energy } \\
\text { Sold } \\
(\mathbf{k W h})\end{array}$ & $\begin{array}{l}\text { Net } \\
\text { Purchases } \\
\text { (kWh) }\end{array}$ & $\begin{array}{l}\text { Peak } \\
\text { Demand } \\
(\mathbf{k W})\end{array}$ & $\begin{array}{l}\text { Energy } \\
\text { Charge (\$) }\end{array}$ & $\begin{array}{l}\text { Demand } \\
\text { Charge } \\
(\$)\end{array}$ \\
\hline January & $8,208,939$ & 0 & $8,208,939$ & 0 & 985,073 & 0 \\
\hline February & $6,110,456$ & 0 & $6,110,456$ & 0 & 733,255 & 0 \\
\hline March & $6,816,916$ & 0 & 6,816,916 & 0 & 818,030 & 0 \\
\hline April & $9,447,916$ & 0 & $9,447,916$ & 0 & $1,133,750$ & 0 \\
\hline May & $7,805,883$ & 0 & $7,805,883$ & 0 & 936,706 & 0 \\
\hline June & $9,786,386$ & 0 & $9,786,386$ & 0 & $1,174,366$ & 0 \\
\hline July & $9,969,422$ & 0 & $9,969,422$ & 0 & $1,196,331$ & 0 \\
\hline August & $9,880,399$ & 0 & $9,880,399$ & 0 & $1,185,648$ & 0 \\
\hline September & $6,943,139$ & 0 & $6,943,139$ & 0 & 833,177 & 0 \\
\hline October & $6,792,313$ & 0 & $6,792,313$ & 0 & 815,078 & 0 \\
\hline November & $6,321,105$ & 0 & $6,321,105$ & 0 & 758,533 & 0 \\
\hline December & $8,678,534$ & 0 & $8,678,534$ & 0 & $1,041,424$ & 0 \\
\hline Annual & $96,761,408$ & 0 & $96,761,408$ & 0 & $11,611,368$ & 0 \\
\hline
\end{tabular}




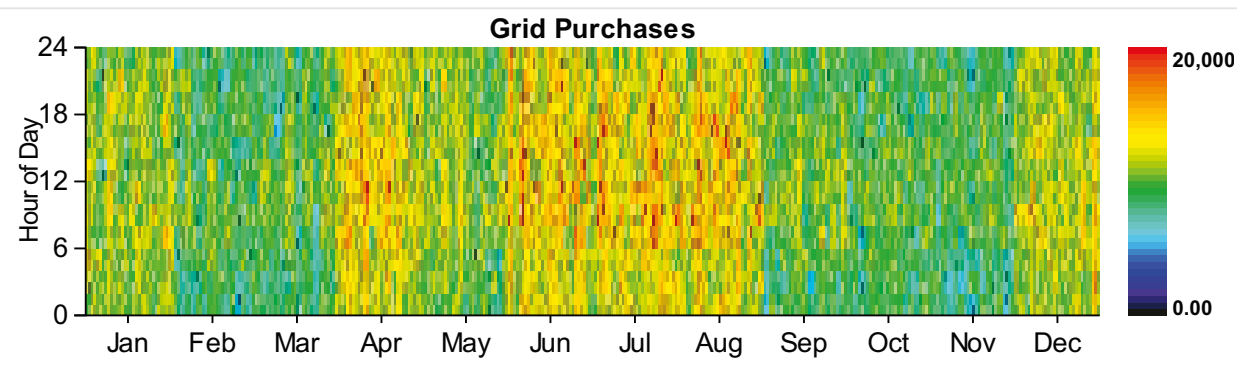

\section{Emissions}

\begin{tabular}{|l|r|l|}
\hline Pollutant & Emissions & Units \\
\hline Carbon dioxide & 61225048 & $\mathrm{~kg} / \mathrm{yr}$ \\
\hline Carbon monoxide & $301 \mathrm{~kg} / \mathrm{yr}$ \\
\hline Unburned hydrocarbons & $34 \mathrm{~kg} / \mathrm{yr}$ \\
\hline Particulate matter & $9 \mathrm{~kg} / \mathrm{yr}$ \\
\hline Sulfur dioxide & $265274 \mathrm{~kg} / \mathrm{yr}$ \\
\hline Nitrogen oxides & $129961 \mathrm{~kg} / \mathrm{yr}$ \\
\hline
\end{tabular}

HOMER Energy, LLC @ 2016 
System Report

System architecture

\begin{tabular}{|l|l|r|}
\hline Generator & Kohler 1000 Prime Power & 925 \\
\hline Generator \#2 & Kohler 750 Prime Power & 1,380 \\
\hline Grid & GW & 20,000 \\
\hline Dispatch Strategy & Cycle Charging & $\mathrm{kW}$ \\
\hline
\end{tabular}

\section{Cost summary}

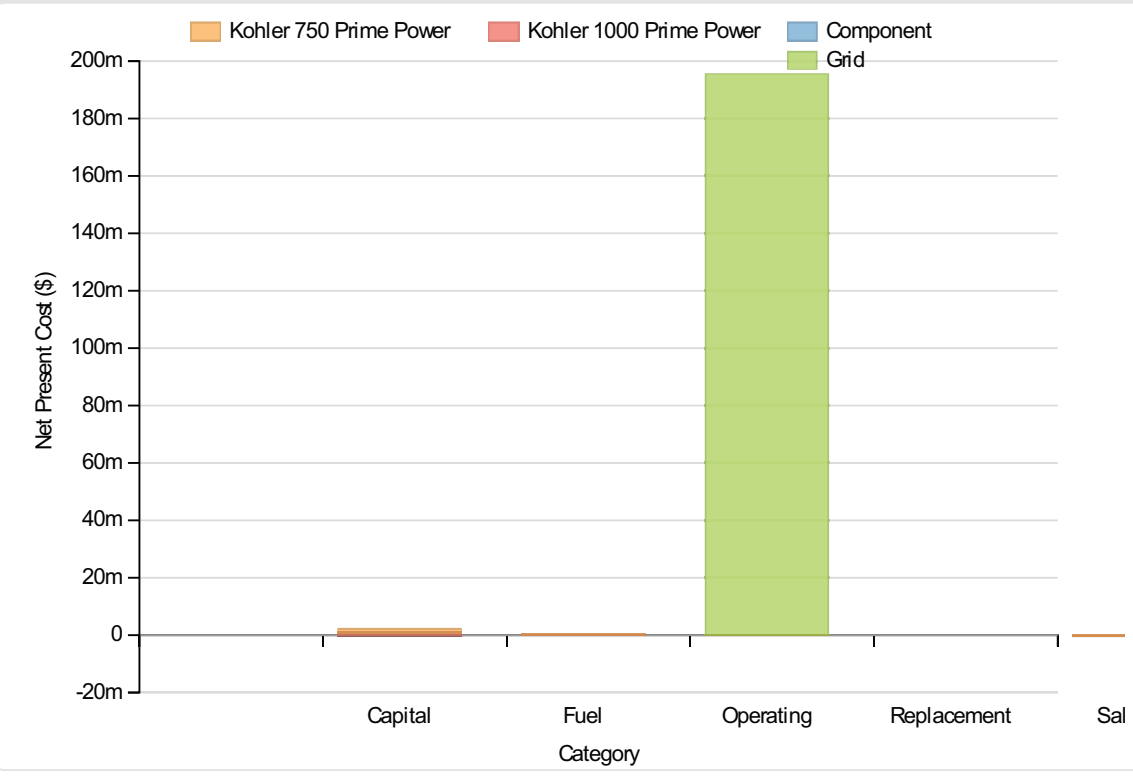

Cost Summary

Total net present cost

Levelized cost of energy
$197574432 \$$

$0.158 \$ / k W h$

Net Present Costs

\begin{tabular}{|l|r|r|r|r|r|r|}
\hline Component & Capital & Replacement & O\&M & Fuel & Salvage & Total \\
\hline Kohler 1000 Prime Power & 925,000 & 0 & 28,520 & 168,299 & $-162,869$ & 958,950 \\
\hline Kohler 750 Prime Power & $1,285,000$ & 0 & 31,260 & 162,939 & $-246,778$ & $1,232,421$ \\
\hline Grid & 0 & 0 & $195,382,944$ & 0 & 0 \\
\hline System & $2,210,000$ & 0 & $195,442,736$ & 331,238 & $-409,647$ & $197,382,944$ \\
\hline
\end{tabular}

Annualized Costs

\begin{tabular}{|l|r|r|r|r|r|r|}
\hline Component & Capital & Replacement & O\&M & Fuel & Salvage & Total \\
\hline Kohler 1000 Prime Power & 71,553 & 0 & 2,206 & 13,019 & $-12,599$ & 74,179 \\
\hline Kohler 750 Prime Power & 99,400 & 0 & 2,418 & 12,604 & $-19,089$ & 95,333 \\
\hline Grid & 0 & 0 & $15,113,726$ & 0 & 0 \\
\hline System & 170,953 & 0 & $15,118,351$ & 25,623 & $-31,688$ & $15,283,239$ \\
\hline
\end{tabular}




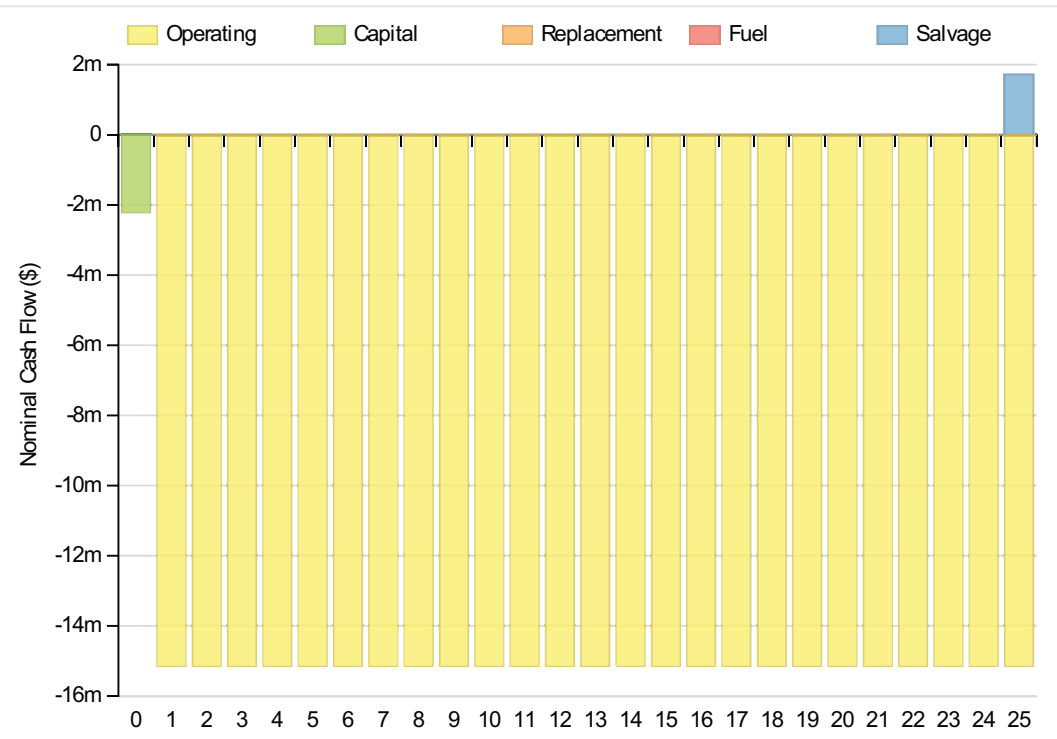

Year

\section{Electrical}

\begin{tabular}{|c|c|c|c|c|}
\hline \multicolumn{2}{|l|}{ Quantity } & \multicolumn{2}{|l|}{ Value } & Units \\
\hline Excess electricity & & & 0 & $\mathrm{kWh} / \mathrm{yr}$ \\
\hline Unmet load & & & 6127 & kWh/yr \\
\hline Capacity shortage & & & 7094 & kWh/yr \\
\hline Renewable fraction & & & 0 & \\
\hline Component & Production(kWh/yr) & & Fracti & ion $(\%)$ \\
\hline Generator & & 56,991 & & 0 \\
\hline Generator & & 54,442 & & 0 \\
\hline Grid Purchases & & $96,746,632$ & & 100 \\
\hline Total & & $96,858,064$ & & 100 \\
\hline Load & Consumption(kWh/yr) & & Fra & Iction (\%) \\
\hline AC primary load & & $96,858,024$ & & 100 \\
\hline DC primary load & & & 0 & 0 \\
\hline Total & & $96,858,024$ & & 100 \\
\hline
\end{tabular}




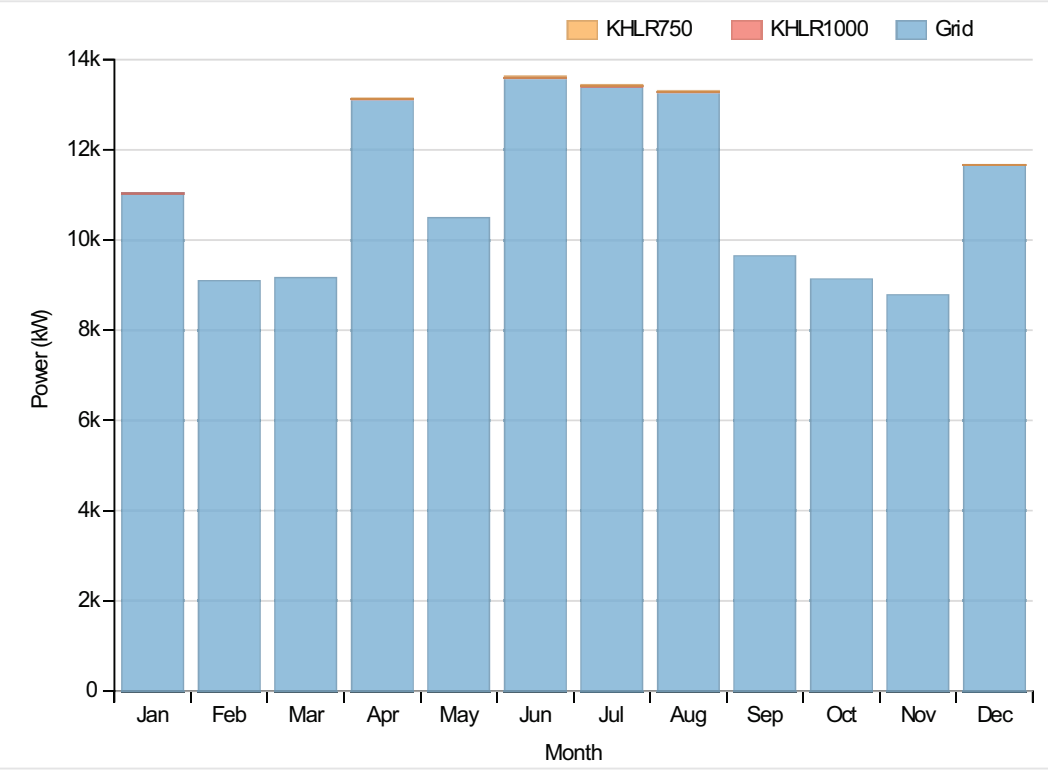

Generator:Kohler 1000 Prime Power

\begin{tabular}{|c|c|c|}
\hline Quantity & Value & Units \\
\hline Hours of operation & 159 & $\mathrm{hrs} / \mathrm{yr}$ \\
\hline Number of starts & 144 & starts/yr \\
\hline Operational life & 94 & $\mathrm{yr}$ \\
\hline Fixed generation cost & 76.24 & $\$ / \mathrm{hr}$ \\
\hline Marginal generation cost & 0.23 & $\$ / k W h$ \\
\hline Electrical production & 56991 & $\mathrm{kWh} / \mathrm{yr}$ \\
\hline Mean electrical output & 358 & kW \\
\hline Min. electrical output & 231 & kW \\
\hline Max. electrical output & 925 & $\mathrm{~kW}$ \\
\hline Fuel consumption & 16479 & $\mathrm{~L} / \mathrm{yr}$ \\
\hline Specific fuel consumption & 0.29 & $\mathrm{~L} / \mathrm{kWh}$ \\
\hline Fuel energy input & 162156 & $\mathrm{kWh} / \mathrm{yr}$ \\
\hline Mean electrical efficiency & 35 & $\%$ \\
\hline
\end{tabular}

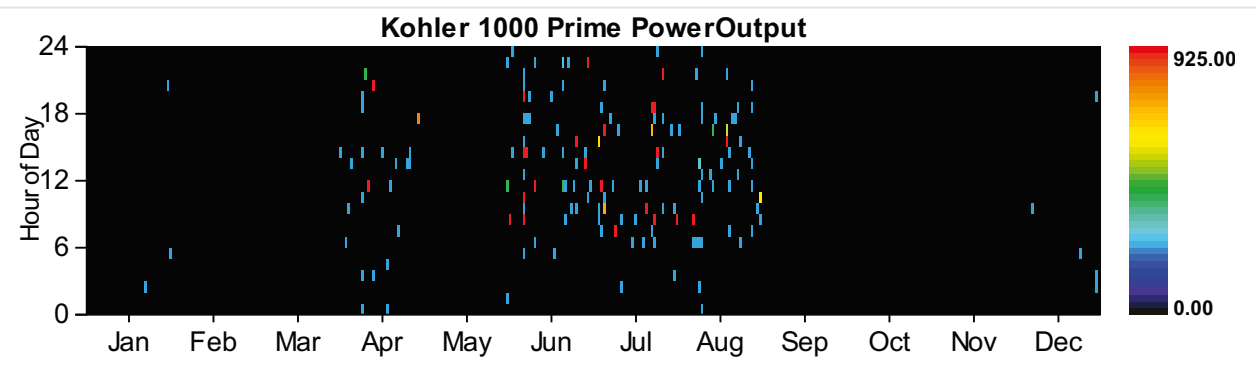

Generator:Kohler 750 Prime Power

\begin{tabular}{|l|r|l|}
\hline Quantity & Value & Units \\
\hline Hours of operation & 119 hrs/yr \\
\hline Number of starts & 107 & starts/yr \\
\hline
\end{tabular}


Fixed generation cost

Marginal generation cost

Electrical production

Mean electrical output

Min. electrical output

Max. electrical output

Fuel consumption

Specific fuel consumption

Fuel energy input

Mean electrical efficiency
$107.02 \$ / h r$

\begin{tabular}{|r|l|l|}
\hline 0.23 & $\$ / \mathrm{kWh}$ \\
\hline 54442 & $\mathrm{kWh} / \mathrm{yr}$ \\
\hline 458 & $\mathrm{~kW}$ \\
\hline 345 & $\mathrm{~kW}$ \\
\hline 1380 & $\mathrm{~kW}$ \\
\hline 15955 & $\mathrm{~L} / \mathrm{yr}$ \\
\hline 0.29 & $\mathrm{~L} / \mathrm{kWh}$ \\
\hline 56993 & $\mathrm{kWh} / \mathrm{yr}$ \\
\hline 35 & $\%$ \\
\hline
\end{tabular}

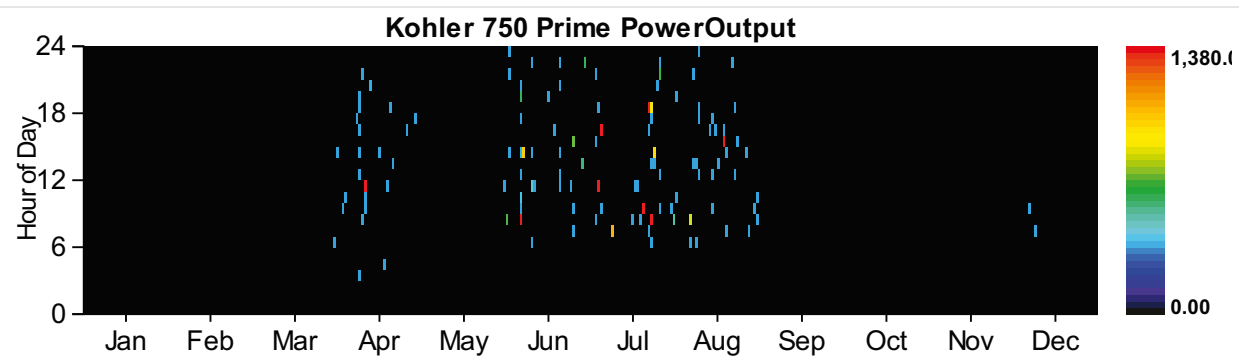

Grid

Rate: Demand 1

\begin{tabular}{|c|c|c|c|c|c|c|}
\hline Resources.ReportingService_GeneratelnputsReport_Month & $\begin{array}{l}\text { Energy } \\
\text { Purchased } \\
\text { (kWh) }\end{array}$ & $\begin{array}{l}\text { Energy } \\
\text { Sold } \\
\text { (kWh) }\end{array}$ & $\begin{array}{l}\text { Net } \\
\text { Purchases } \\
\text { (kWh) }\end{array}$ & $\begin{array}{l}\text { Peak } \\
\text { Demand } \\
(\mathbf{k W})\end{array}$ & $\begin{array}{l}\text { Energy } \\
\text { Charge } \\
(\$)\end{array}$ & $\begin{array}{l}\text { Demand } \\
\text { Charge } \\
(\$)\end{array}$ \\
\hline January & 0 & 0 & 0 & 18,321 & 0 & 293,143 \\
\hline February & 0 & 0 & 0 & 17,561 & 0 & 280,978 \\
\hline March & 0 & 0 & 0 & 17,425 & 0 & 278,807 \\
\hline April & 0 & 0 & 0 & 20,000 & 0 & 320,000 \\
\hline May & 0 & 0 & 0 & 17,869 & 0 & 285,902 \\
\hline June & 0 & 0 & 0 & 20,000 & 0 & 320,000 \\
\hline July & 0 & 0 & 0 & 20,000 & 0 & 320,000 \\
\hline August & 0 & 0 & 0 & 20,000 & 0 & 320,000 \\
\hline September & 0 & 0 & 0 & 16,926 & 0 & 270,820 \\
\hline October & 0 & 0 & 0 & 14,895 & 0 & 238,325 \\
\hline November & 0 & 0 & 0 & 16,518 & 0 & 264,286 \\
\hline December & 0 & 0 & 0 & 19,492 & 0 & 311,878 \\
\hline
\end{tabular}




\begin{tabular}{|c|c|c|c|c|c|c|}
\hline $\begin{array}{l}\text { Annual } \\
\text { Rate: Rate } 1 \text { Resources.ReportingService_GeneratelnputsReport_Month }\end{array}$ & $\begin{array}{l}\text { Energy } 0 \\
\text { Purchased } \\
\text { (kWh) }\end{array}$ & $\begin{array}{l}\text { Energy } \\
\text { Sold } \\
(\mathbf{k W h})\end{array}$ & $\begin{array}{l}\text { Net } 0 \\
\text { Purchases } \\
\text { (kWh) }\end{array}$ & $\begin{array}{l}\text { Peak000 } \\
\text { Demand } \\
(\mathrm{kW})\end{array}$ & $\begin{array}{l}\text { Energy } \\
\text { Charge } \\
(\$)\end{array}$ & 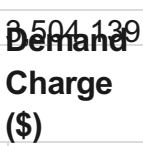 \\
\hline Resources.ReportingService_GeneratelnputsReport_Month & $\begin{array}{l}\text { Energy } \\
\text { Purchased } \\
\text { (kWh) }\end{array}$ & \begin{tabular}{l|} 
Energy \\
Sold \\
(kWh)
\end{tabular} & $\begin{array}{l}\text { Net } \\
\text { Purchases } \\
\text { (kWh) }\end{array}$ & $\begin{array}{l}\text { Peak } \\
\text { Demand } \\
\text { (kW) }\end{array}$ & $\begin{array}{l}\text { Energy } \\
\text { Charge (\$) }\end{array}$ & $\begin{array}{l}\text { Demand } \\
\text { Charge } \\
(\$)\end{array}$ \\
\hline January & $8,209,042$ & 0 & $8,209,042$ & 0 & 985,085 & 0 \\
\hline February & $6,110,456$ & 0 & $6,110,456$ & 0 & 733,255 & 0 \\
\hline March & $6,816,916$ & 0 & $6,816,916$ & 0 & 818,030 & 0 \\
\hline April & $9,444,701$ & 0 & $9,444,701$ & 0 & $1,133,364$ & 0 \\
\hline May & $7,805,883$ & 0 & $7,805,883$ & 0 & 936,706 & 0 \\
\hline June & $9,782,846$ & 0 & $9,782,846$ & 0 & $1,173,942$ & 0 \\
\hline July & $9,966,205$ & 0 & $9,966,205$ & 0 & $1,195,945$ & 0 \\
\hline August & $9,875,789$ & 0 & $9,875,789$ & 0 & $1,185,095$ & 0 \\
\hline September & $6,943,139$ & 0 & $6,943,139$ & 0 & 833,177 & 0 \\
\hline October & $6,792,313$ & 0 & $6,792,313$ & 0 & 815,078 & 0 \\
\hline November & $6,321,105$ & 0 & $6,321,105$ & 0 & 758,533 & 0 \\
\hline December & $8,678,243$ & 0 & $8,678,243$ & 0 & $1,041,389$ & 0 \\
\hline Annual & $96,746,632$ & 0 & $96,746,632$ & 0 & $11,609,597$ & 0 \\
\hline
\end{tabular}

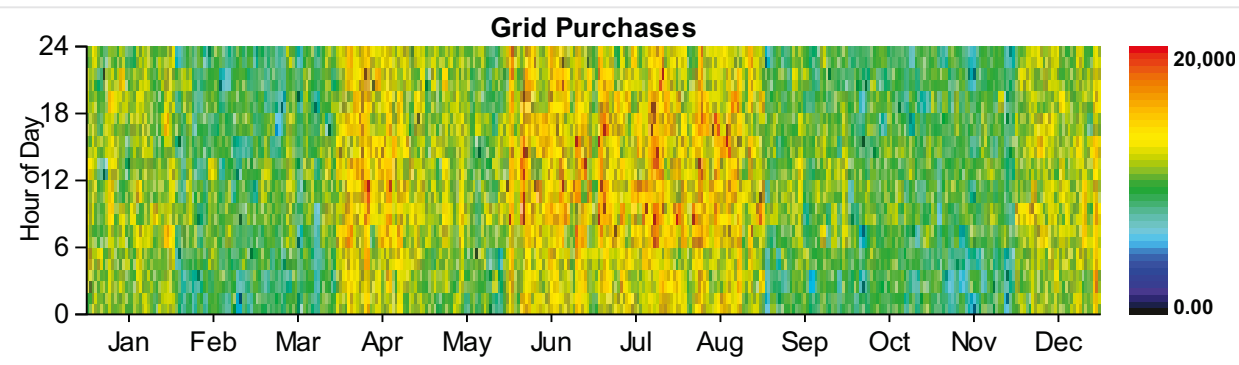

\section{Emissions}

\begin{tabular}{|l|r|l|}
\hline Pollutant & Emissions & Units \\
\hline Carbon dioxide & $61228996 \mathrm{~kg} / \mathrm{yr}$ \\
\hline Carbon monoxide & $357 \mathrm{~kg} / \mathrm{yr}$ \\
\hline Unburned hydrocarbons & $41 \mathrm{~kg} / \mathrm{yr}$ \\
\hline Particulate matter & $10 \mathrm{~kg} / \mathrm{yr}$ \\
\hline Sulfur dioxide & $265261 \mathrm{~kg} / \mathrm{yr}$ \\
\hline Nitrogen oxides & $129997 \mathrm{~kg} / \mathrm{yr}$ \\
\hline
\end{tabular}


System Report

System architecture

\begin{tabular}{|l|l|r|}
\hline Generator & Kohler 1000 Prime Power & $1,850 \mathrm{~kW}$ \\
\hline Battery & GS200 flow & 1 strings \\
\hline Converter & System Converter & $250 \mathrm{~kW}$ \\
\hline Grid & Grid & $20,000 \mathrm{~kW}$ \\
\hline Dispatch Strategy & Cycle Charging & \\
\hline
\end{tabular}

Cost summary

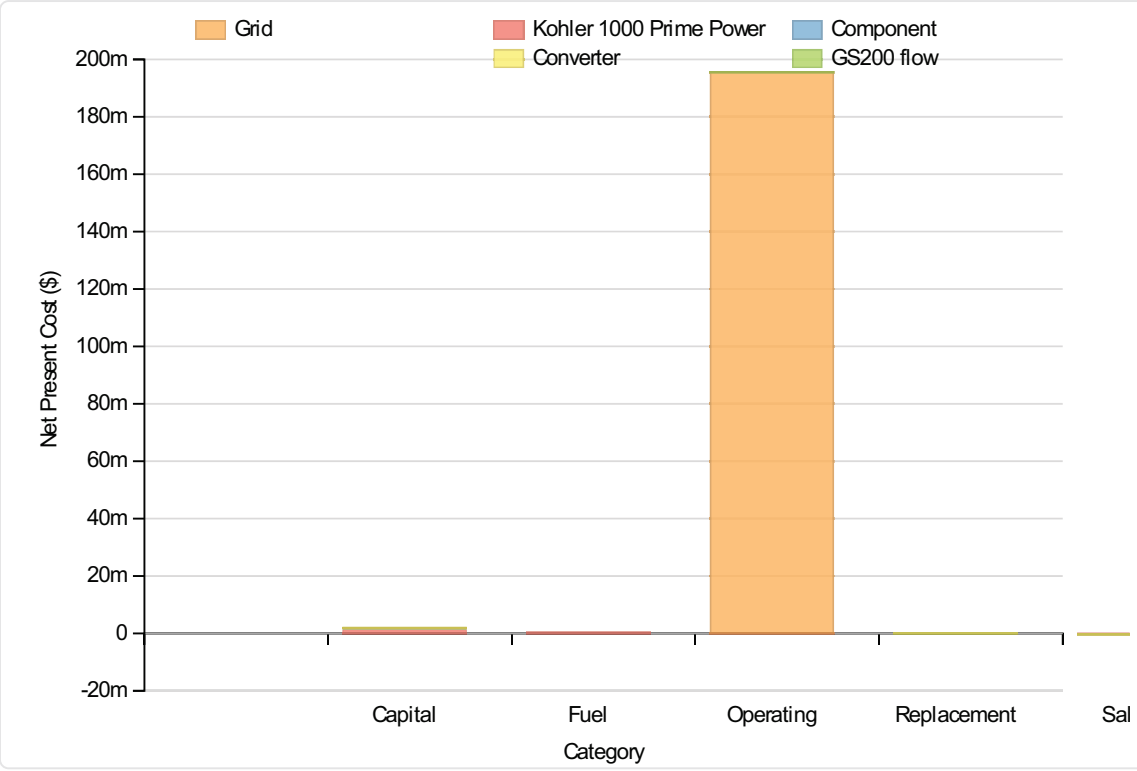

Cost Summary

Total net present cost

$197614656 \$$

Levelized cost of energy

$0.158 \$ / k W h$

Net Present Costs

\begin{tabular}{|l|r|r|r|r|r|r|}
\hline Component & Capital & Replacement & O\&M & Fuel & Salvage & Total \\
\hline Kohler 1000 Prime Power & $1,637,500$ & 0 & 61,204 & 352,161 & $-277,208$ & $1,773,657$ \\
\hline Grid & 0 & 0 & $195,378,288$ & 0 & 0 \\
\hline GS200 flow & 324,589 & 7,176 & 31,026 & 0 & -973 \\
\hline Converter & 75,000 & 31,821 & 0 & 0 & $-5,989$ \\
\hline System & $2,037,089$ & 38,996 & $195,470,464$ & 352,161 & $-284,170$ \\
\hline
\end{tabular}

\section{Annualized Costs}

\begin{tabular}{|l|r|r|r|r|r|r|r|}
\hline Component & Capital & Replacement & O\&M & Fuel & Salvage & Total \\
\hline Kohler 1000 Prime Power & 126,668 & 0 & 4,734 & 27,241 & $-21,443$ & 137,200 \\
\hline Grid & 0 & 0 & $15,113,366$ & 0 & 0 \\
\hline GS200 flow & 25,108 & 555 & 2,400 & 0 & -75 \\
\hline Converter & 5,802 & 2,462 & 0 & 0 & -463 \\
\hline
\end{tabular}




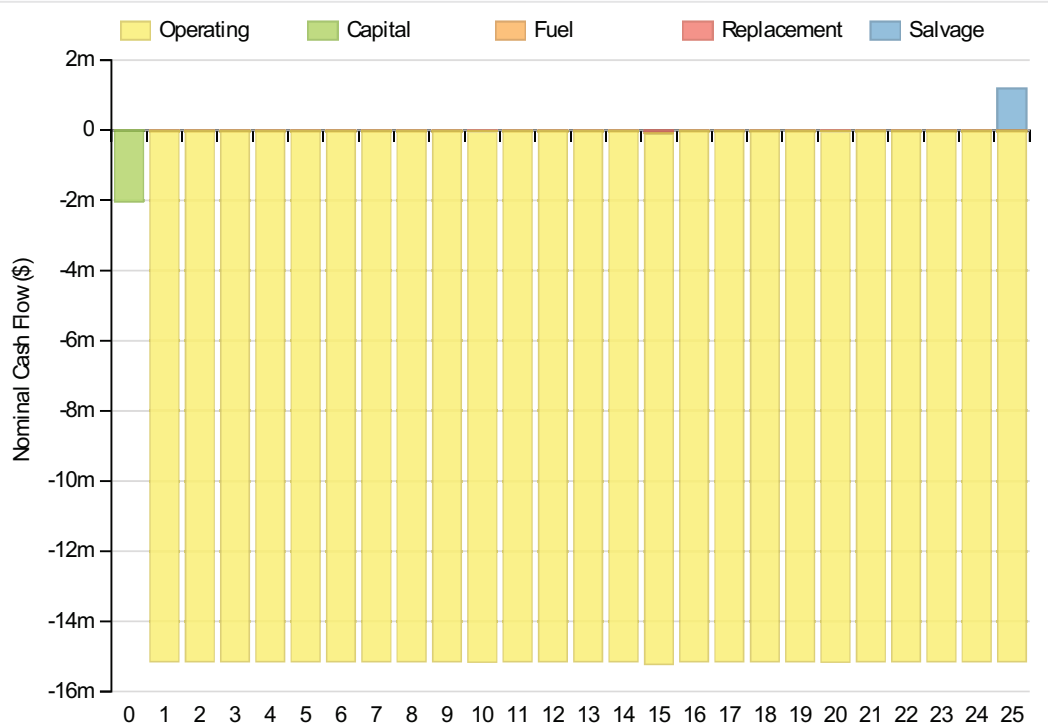

Year

\section{Electrical}

\begin{tabular}{|c|c|c|c|c|}
\hline \multicolumn{2}{|l|}{ Quantity } & \multicolumn{2}{|l|}{ Value } & Units \\
\hline Excess electricity & & & 0 & $\mathrm{kWh} / \mathrm{yr}$ \\
\hline Unmet load & & & 8432 & kWh/yr \\
\hline Capacity shortage & & & 2100 & kWh/yr \\
\hline Renewable fraction & & & 0 & \\
\hline Component & Production(kWh/yr) & & Fract & ion $(\%)$ \\
\hline Generator & & 119,192 & & 0 \\
\hline Grid Purchases & & $96,738,344$ & & 100 \\
\hline Total & & $96,857,536$ & & 100 \\
\hline Load & Consumption(kWh/yr) & & Fra & iction (\%) \\
\hline AC primary load & & $96,855,720$ & & 100 \\
\hline DC primary load & & & 0 & 0 \\
\hline Total & & $96,855,720$ & & 100 \\
\hline
\end{tabular}




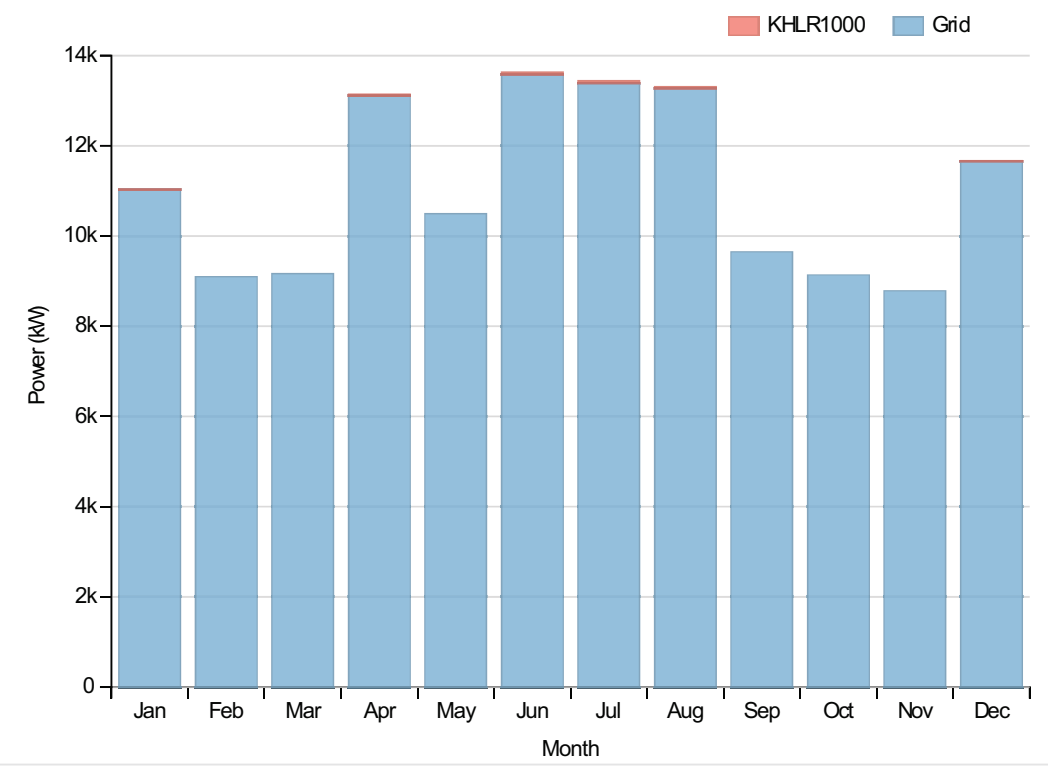

Generator:Kohler 1000 Prime Power

\begin{tabular}{|c|c|c|}
\hline Quantity & Value & Units \\
\hline Hours of operation & 176 & $\mathrm{hrs} / \mathrm{yr}$ \\
\hline Number of starts & 157 & starts/yr \\
\hline Operational life & 85 & $\mathrm{yr}$ \\
\hline Fixed generation cost & 137.46 & $\$ / \mathrm{hr}$ \\
\hline Marginal generation cost & 0.23 & $\$ / k W h$ \\
\hline Electrical production & 119192 & $\mathrm{kWh} / \mathrm{yr}$ \\
\hline Mean electrical output & 677 & $\mathrm{~kW}$ \\
\hline Min. electrical output & 463 & $\mathrm{~kW}$ \\
\hline Max. electrical output & 1850 & $\mathrm{~kW}$ \\
\hline Fuel consumption & 34483 & L/yr \\
\hline Specific fuel consumption & 0.29 & $\mathrm{~L} / \mathrm{kWh}$ \\
\hline Fuel energy input & 339308 & $\mathrm{kWh} / \mathrm{yr}$ \\
\hline Mean electrical efficiency & 35 & $\%$ \\
\hline
\end{tabular}

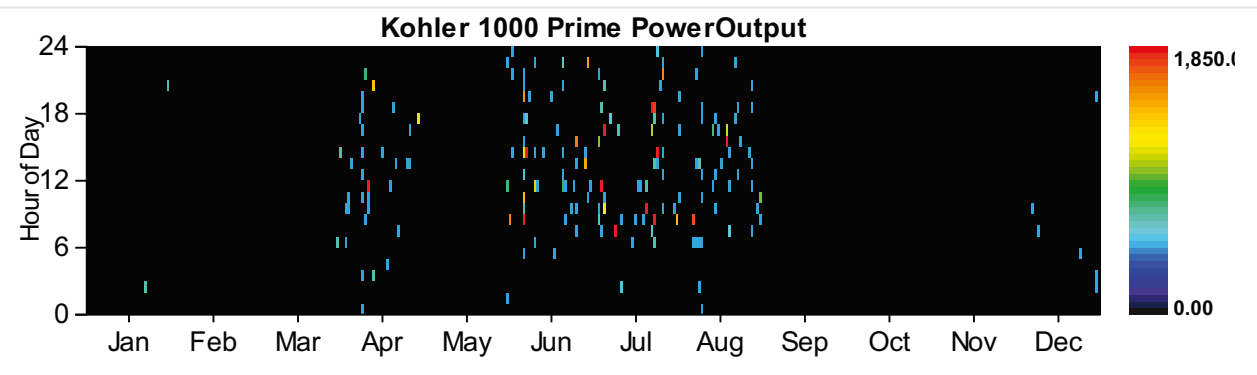

\section{Battery:GS200 flow}

\section{Quantity}

Value

String size 


\begin{tabular}{|c|c|c|}
\hline Quantity & Value & Units \\
\hline Nominal capacity & 600 & kWh \\
\hline Usable nominal capacity & 600 & kWh \\
\hline Autonomy & 0 & $\mathrm{hr}$ \\
\hline Lifetime throughput & 0 & \\
\hline Battery wear cost & 0.000 & $\$ / k W h$ \\
\hline Average energy cost & 0.359 & $\$ / k W h$ \\
\hline Energy in & 3257 & $\mathrm{kWh} / \mathrm{yr}$ \\
\hline Energy out & 2280 & $\mathrm{kWh} / \mathrm{yr}$ \\
\hline Storage depletion & 0 & $\mathrm{kWh} / \mathrm{yr}$ \\
\hline Losses & 977 & $\mathrm{kWh} / \mathrm{yr}$ \\
\hline Annual throughput & 2725 & $\mathrm{kWh} / \mathrm{yr}$ \\
\hline Expected life & 25 & $\mathrm{yr}$ \\
\hline
\end{tabular}

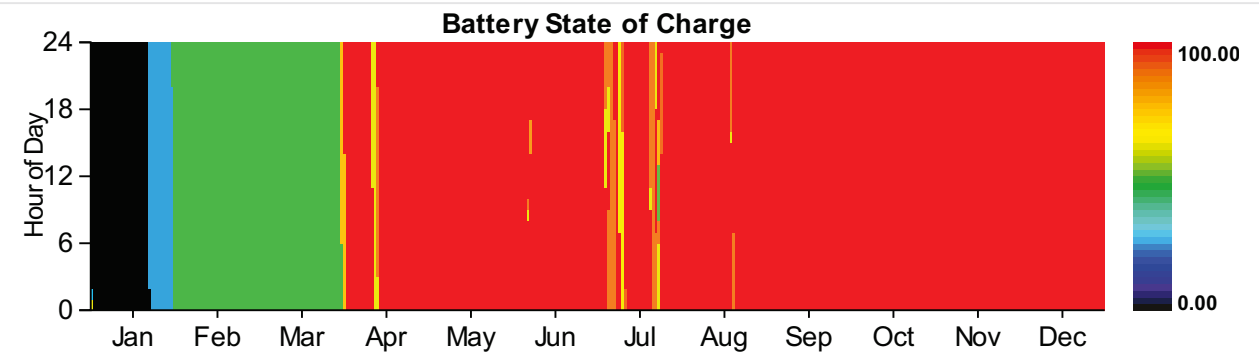

\section{Converter}

\begin{tabular}{|c|c|c|c|}
\hline Quantity & Inverter & Rectifier & Units \\
\hline Capacity & 250 & 225 & $\mathrm{~kW}$ \\
\hline Mean output & 0 & 0 & $\mathrm{~kW}$ \\
\hline Minimum output & 0 & 0 & $\mathrm{~kW}$ \\
\hline Maximum output & 166 & 179 & kW \\
\hline Capacity factor & 0 & 0 & $\%$ \\
\hline Hours of operation & 14 & 24 & $\mathrm{hrs} / \mathrm{yr}$ \\
\hline Energy in & 2,280 & 3,832 & $\mathrm{kWh} / \mathrm{yr}$ \\
\hline Energy out & 2,052 & 3,257 & $\mathrm{kWh} / \mathrm{yr}$ \\
\hline Losses & 228 & 575 & $\mathrm{kWh} / \mathrm{yr}$ \\
\hline
\end{tabular}



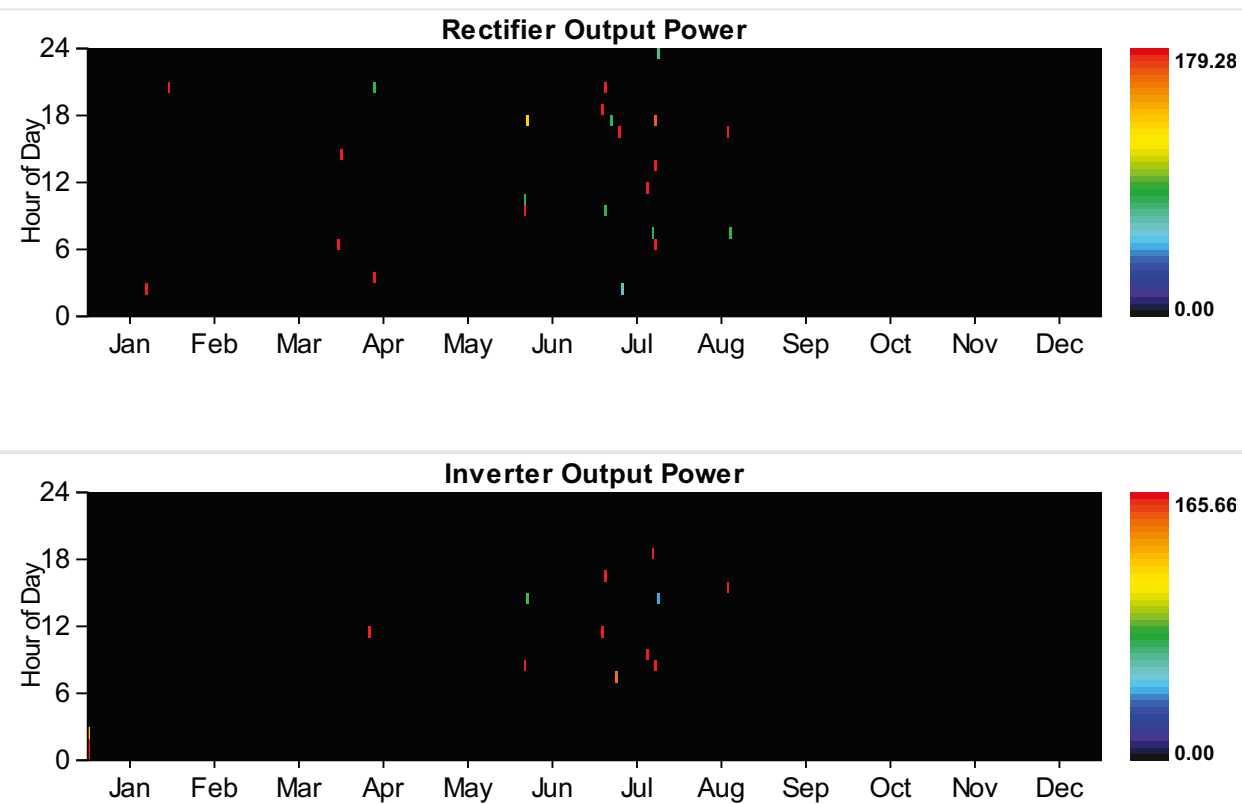

Grid

Rate: Demand 1

\begin{tabular}{|c|c|c|c|c|c|c|}
\hline Resources.ReportingService_GeneratelnputsReport_Month & $\begin{array}{l}\text { Energy } \\
\text { Purchased } \\
\text { (kWh) }\end{array}$ & $\begin{array}{l}\text { Energy } \\
\text { Sold } \\
\text { (kWh) }\end{array}$ & $\begin{array}{l}\text { Net } \\
\text { Purchases } \\
\text { (kWh) }\end{array}$ & $\begin{array}{l}\text { Peak } \\
\text { Demand } \\
(\mathbf{k W})\end{array}$ & $\begin{array}{l}\text { Energy } \\
\text { Charge } \\
\text { (\$) }\end{array}$ & $\begin{array}{l}\text { Demand } \\
\text { Charge } \\
(\$)\end{array}$ \\
\hline January & 0 & 0 & 0 & 18,247 & 0 & 291,953 \\
\hline February & 0 & 0 & 0 & 17,561 & 0 & 280,978 \\
\hline March & 0 & 0 & 0 & 17,425 & 0 & 278,807 \\
\hline April & 0 & 0 & 0 & 20,000 & 0 & 320,000 \\
\hline May & 0 & 0 & 0 & 17,869 & 0 & 285,902 \\
\hline June & 0 & 0 & 0 & 20,000 & 0 & 320,000 \\
\hline July & 0 & 0 & 0 & 20,000 & 0 & 320,000 \\
\hline August & 0 & 0 & 0 & 20,000 & 0 & 320,000 \\
\hline September & 0 & 0 & 0 & 16,926 & 0 & 270,820 \\
\hline October & 0 & 0 & 0 & 14,895 & 0 & 238,325 \\
\hline November & 0 & 0 & 0 & 16,518 & 0 & 264,286 \\
\hline December & 0 & 0 & 0 & 19,606 & 0 & 313,698 \\
\hline Annual & 0 & 0 & 0 & 20,000 & 0 & $3,504,769$ \\
\hline
\end{tabular}

Rate: Rate 1

\begin{tabular}{|c|c|c|c|c|c|c|}
\hline Resources.ReportingService_GeneratelnputsReport_Month & $\begin{array}{l}\text { Energy } \\
\text { Purchased } \\
\text { (kWh) }\end{array}$ & $\begin{array}{l}\text { Energy } \\
\text { Sold } \\
\text { (kWh) }\end{array}$ & $\begin{array}{l}\text { Net } \\
\text { Purchases } \\
\text { (kWh) }\end{array}$ & $\begin{array}{l}\text { Peak } \\
\text { Demand } \\
(\mathbf{k W})\end{array}$ & $\begin{array}{l}\text { Energy } \\
\text { Charge (\$) }\end{array}$ & $\begin{array}{l}\text { Demand } \\
\text { Charge } \\
\text { (\$) }\end{array}$ \\
\hline January & $8,208,359$ & 0 & $8,208,359$ & 0 & 985,003 & 0 \\
\hline February & $6,110,456$ & 0 & $6,110,456$ & 0 & 733,255 & 0 \\
\hline March & $6,816,916$ & 0 & $6,816,916$ & 0 & 818,030 & 0 \\
\hline
\end{tabular}




\begin{tabular}{|c|c|c|c|c|c|c|}
\hline Rełources.ReportingService_GeneratelnputsReport_Month & 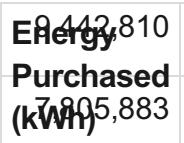 & $\begin{array}{l}\text { Energ9 } \\
\text { Sold } \\
(\mathbf{k W h})^{0}\end{array}$ & $\begin{array}{l}\text { Net442,810 } \\
\text { Purchases } \\
\text { (kª̂95,883 }\end{array}$ & $\begin{array}{l}\text { Peak } 0 \\
\text { Demand } \\
(\mathbf{k W}) \quad 0\end{array}$ & $\begin{array}{l}\text { 1,133,137 } \\
\text { Energy } \\
\text { ChâfGeł\$ }\end{array}$ & $\begin{array}{l}\text { Demand } \\
\text { Charge } \\
\text { (\$) } 0\end{array}$ \\
\hline June & $9,780,760$ & 0 & $9,780,760$ & 0 & $1,173,691$ & 0 \\
\hline July & $9,964,135$ & 0 & $9,964,135$ & 0 & $1,195,696$ & 0 \\
\hline August & $9,875,164$ & 0 & $9,875,164$ & 0 & $1,185,020$ & 0 \\
\hline September & $6,943,139$ & 0 & $6,943,139$ & 0 & 833,177 & 0 \\
\hline October & $6,792,313$ & 0 & $6,792,313$ & 0 & 815,078 & 0 \\
\hline November & $6,321,105$ & 0 & $6,321,105$ & 0 & 758,533 & 0 \\
\hline December & $8,677,315$ & 0 & $8,677,315$ & 0 & $1,041,278$ & 0 \\
\hline Annual & $96,738,344$ & 0 & $96,738,344$ & 0 & $11,608,602$ & 0 \\
\hline
\end{tabular}

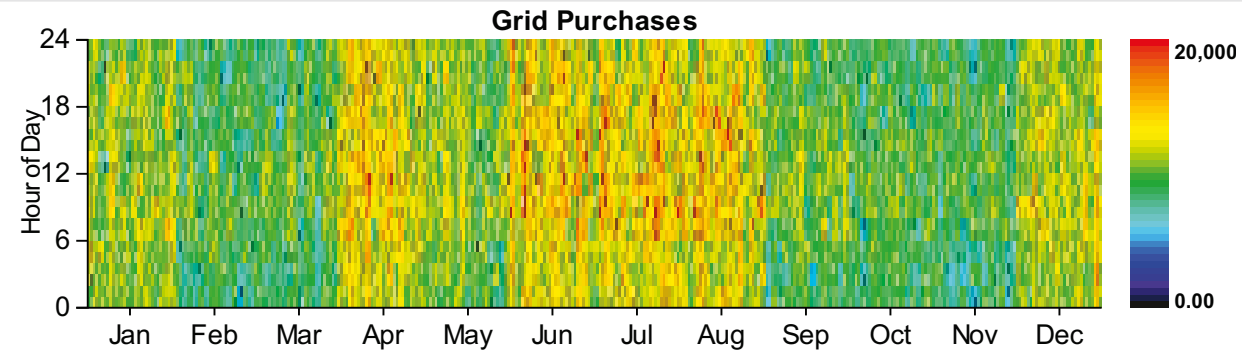

\section{Emissions}

\begin{tabular}{|l|r|l|}
\hline Pollutant & Emissions & Units \\
\hline Carbon dioxide & 61229132 & $\mathrm{~kg} / \mathrm{yr}$ \\
\hline Carbon monoxide & $379 \mathrm{~kg} / \mathrm{yr}$ \\
\hline Unburned hydrocarbons & $43 \mathrm{~kg} / \mathrm{yr}$ \\
\hline Particulate matter & $11 \mathrm{~kg} / \mathrm{yr}$ \\
\hline Sulfur dioxide & $265249 \mathrm{~kg} / \mathrm{yr}$ \\
\hline Nitrogen oxides & $130009 \mathrm{~kg} / \mathrm{yr}$ \\
\hline
\end{tabular}


System Report

System architecture

\begin{tabular}{|l|r|r|}
\hline Generator \#2 & Kohler 750 Prime Power & 1,380 \\
\hline Battery & GS200 flow & 2 strings \\
\hline Converter & System Converter & $500 \mathrm{~kW}$ \\
\hline Grid & Grid & $20,000 \mathrm{~kW}$ \\
\hline Dispatch Strategy & Cycle Charging & \\
\hline
\end{tabular}

Cost summary

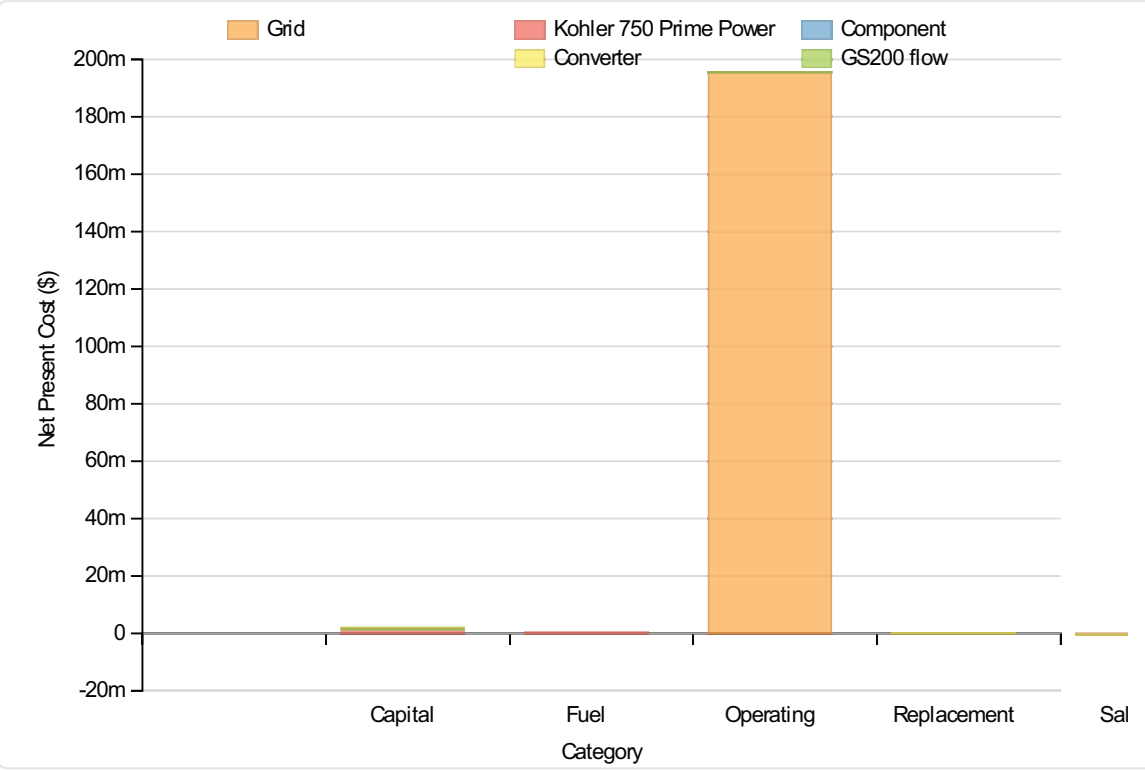

Cost Summary

Total net present cost

$197753920 \$$

Levelized cost of energy

$0.158 \$ / k W h$

Net Present Costs

\begin{tabular}{|l|r|r|r|r|r|r|}
\hline Component & Capital & Replacement & O\&M & Fuel & Salvage & Total \\
\hline Kohler 750 Prime Power & $1,285,000$ & 0 & 41,505 & 292,709 & $-226,769$ & $1,392,445$ \\
\hline Grid & 0 & 0 & $195,436,096$ & 0 & 0 \\
\hline GS200 flow & 649,178 & 14,343 & 62,052 & 0 & $-1,945$ \\
\hline Converter & 150,000 & 63,641 & 0 & 0 & $-11,978$ \\
\hline System & $2,084,178$ & 77,984 & $195,539,664$ & 292,709 & $-240,692$ \\
\hline
\end{tabular}

\section{Annualized Costs}

\begin{tabular}{|l|r|r|r|r|r|r|}
\hline Component & Capital & Replacement & O\&M & Fuel & Salvage & Total \\
\hline Kohler 750 Prime Power & 99,400 & 0 & 3,211 & 22,642 & $-17,542$ & 107,711 \\
\hline Grid & 0 & 0 & $15,117,838$ & 0 & $15,117,838$ \\
\hline GS200 flow & 50,217 & 1,110 & 4,800 & 0 & -150 \\
\hline Converter & 11,603 & 4,923 & 0 & 0 & -927 \\
\hline
\end{tabular}




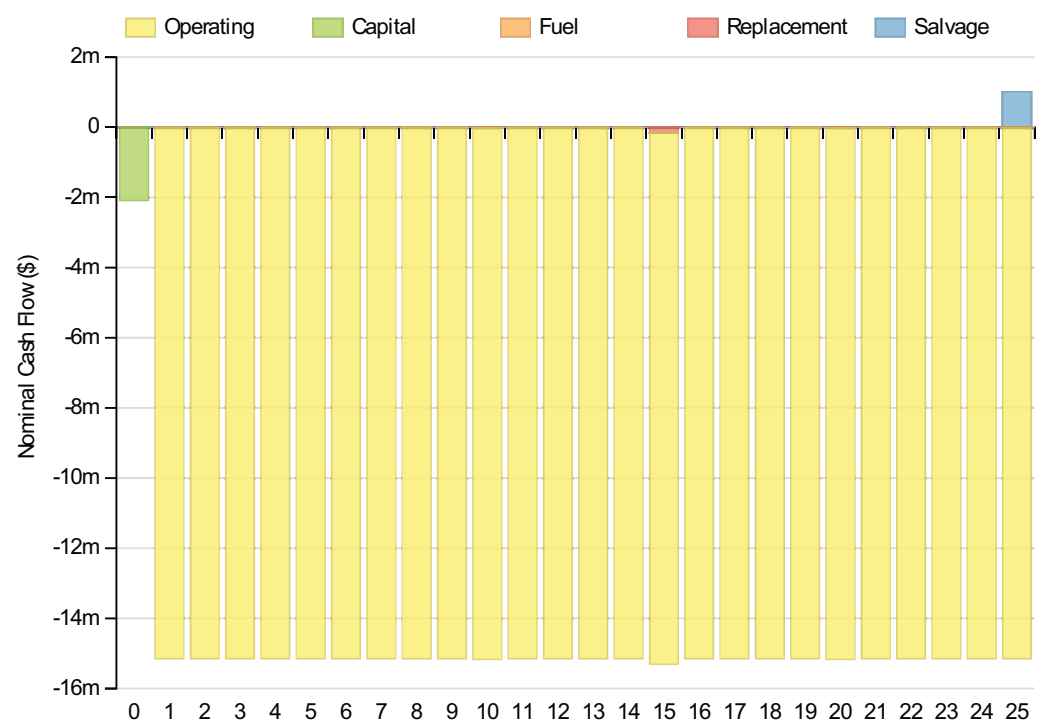

Year

\section{Electrical}

\begin{tabular}{|c|c|c|c|c|}
\hline \multicolumn{2}{|l|}{ Quantity } & \multicolumn{2}{|l|}{ Value } & Units \\
\hline Excess electricity & & & 0 & kWh/yr \\
\hline Unmet load & & & 701 & kWh/yr \\
\hline Capacity shortage & & & 542 & $\mathrm{kWh} / \mathrm{yr}$ \\
\hline Renewable fraction & & & 0 & \\
\hline Component & Production(kWh/yr) & & racti & ion (\%) \\
\hline Generator & & 98,052 & & 0 \\
\hline Grid Purchases & & $96,759,968$ & & 100 \\
\hline Total & & $96,858,024$ & & 100 \\
\hline Load & Consumption(kWh/yr) & & Fra & action (\%) \\
\hline AC primary load & & $96,852,464$ & & 100 \\
\hline DC primary load & & 0 & & 0 \\
\hline Total & & $96,852,464$ & & 100 \\
\hline
\end{tabular}




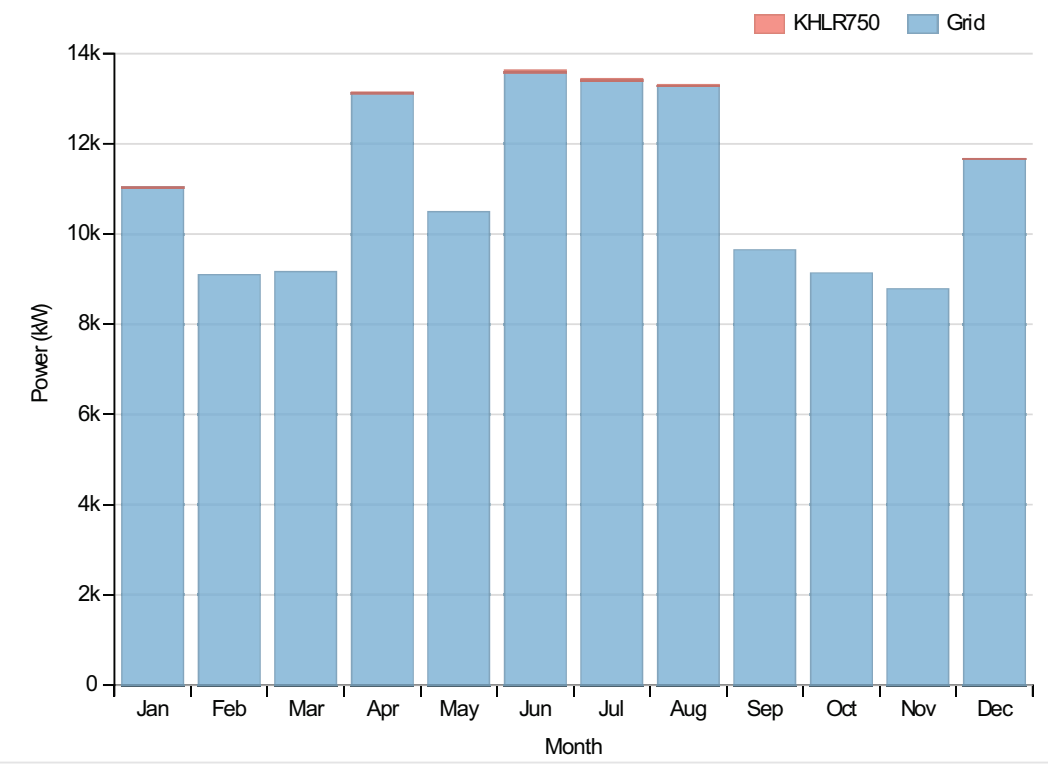

Generator:Kohler 750 Prime Power

\begin{tabular}{|c|c|c|}
\hline Quantity & Value & Units \\
\hline Hours of operation & 158 & hrs/yr \\
\hline Number of starts & 143 & starts/yr \\
\hline Operational life & 95 & $\mathrm{yr}$ \\
\hline Fixed generation cost & 107.02 & $\$ / \mathrm{hr}$ \\
\hline Marginal generation cost & 0.23 & $\$ / k W h$ \\
\hline Electrical production & 98052 & $\mathrm{kWh} / \mathrm{yr}$ \\
\hline Mean electrical output & 621 & $\mathrm{~kW}$ \\
\hline Min. electrical output & 345 & $\mathrm{~kW}$ \\
\hline Max. electrical output & 1380 & $\mathrm{~kW}$ \\
\hline Fuel consumption & 28661 & L/yr \\
\hline Specific fuel consumption & 0.29 & $\mathrm{~L} / \mathrm{kWh}$ \\
\hline Fuel energy input & 282026 & $\mathrm{kWh} / \mathrm{yr}$ \\
\hline Mean electrical efficiency & 35 & $\%$ \\
\hline
\end{tabular}

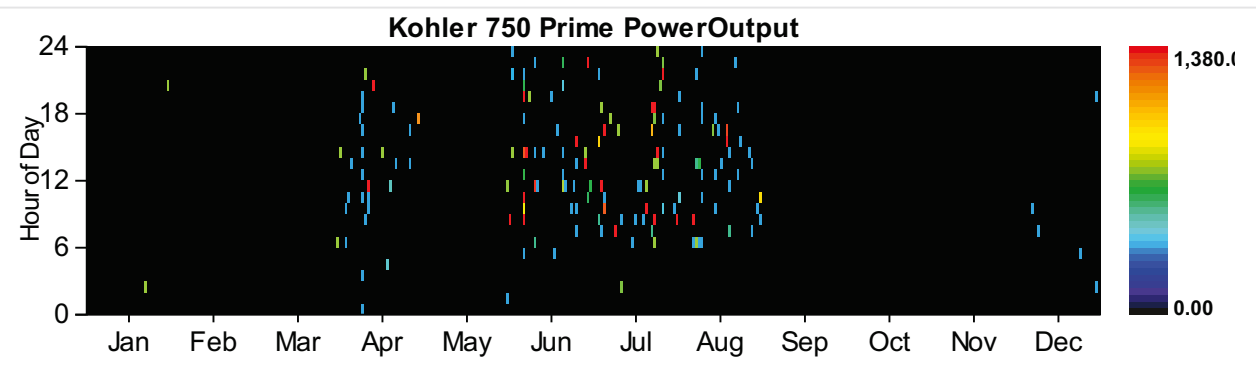

\section{Battery:GS200 flow}

\section{Quantity}

Value

String size 


\begin{tabular}{|c|c|c|}
\hline Quantity & Value & Units \\
\hline Nominal capacity & 1200 & kWh \\
\hline Usable nominal capacity & 1200 & kWh \\
\hline Autonomy & 0 & $\mathrm{hr}$ \\
\hline Lifetime throughput & 0 & \\
\hline Battery wear cost & 0.000 & $\$ / k W h$ \\
\hline Average energy cost & 0.363 & $\$ / k W h$ \\
\hline Energy in & 10100 & $\mathrm{kWh} / \mathrm{yr}$ \\
\hline Energy out & 7070 & $\mathrm{kWh} / \mathrm{yr}$ \\
\hline Storage depletion & 0 & $\mathrm{kWh} / \mathrm{yr}$ \\
\hline Losses & 3030 & $\mathrm{kWh} / \mathrm{yr}$ \\
\hline Annual throughput & 8451 & $\mathrm{kWh} / \mathrm{yr}$ \\
\hline Expected life & 25 & $\mathrm{yr}$ \\
\hline
\end{tabular}

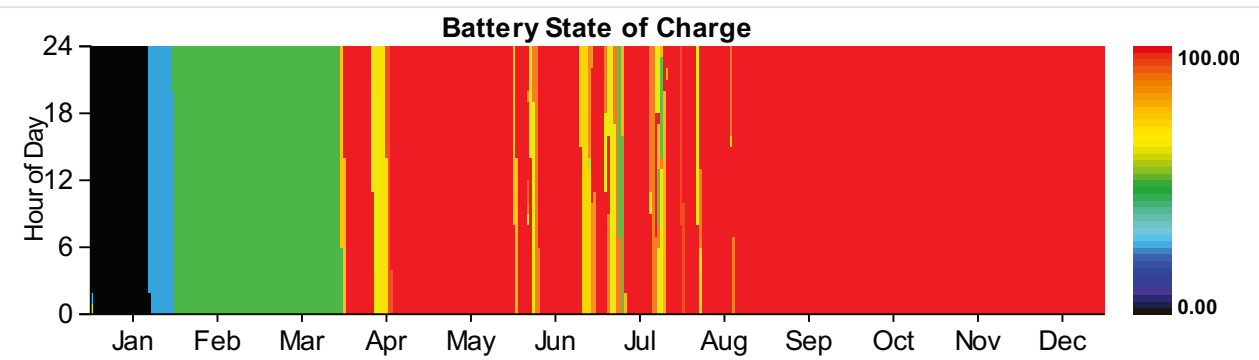

\section{Converter}

\begin{tabular}{|c|c|c|c|}
\hline Quantity & Inverter & Rectifier & Units \\
\hline Capacity & 500 & 450 & kW \\
\hline Mean output & 1 & 1 & kW \\
\hline Minimum output & 0 & 0 & kW \\
\hline Maximum output & 331 & 359 & $\mathrm{~kW}$ \\
\hline Capacity factor & 0 & 0 & $\%$ \\
\hline Hours of operation & 23 & 38 & $\mathrm{hrs} / \mathrm{yr}$ \\
\hline Energy in & 7,070 & 11,883 & $\mathrm{kWh} / \mathrm{yr}$ \\
\hline Energy out & 6,363 & 10,100 & $\mathrm{kWh} / \mathrm{yr}$ \\
\hline Losses & 707 & 1,782 & $\mathrm{kWh} / \mathrm{yr}$ \\
\hline
\end{tabular}



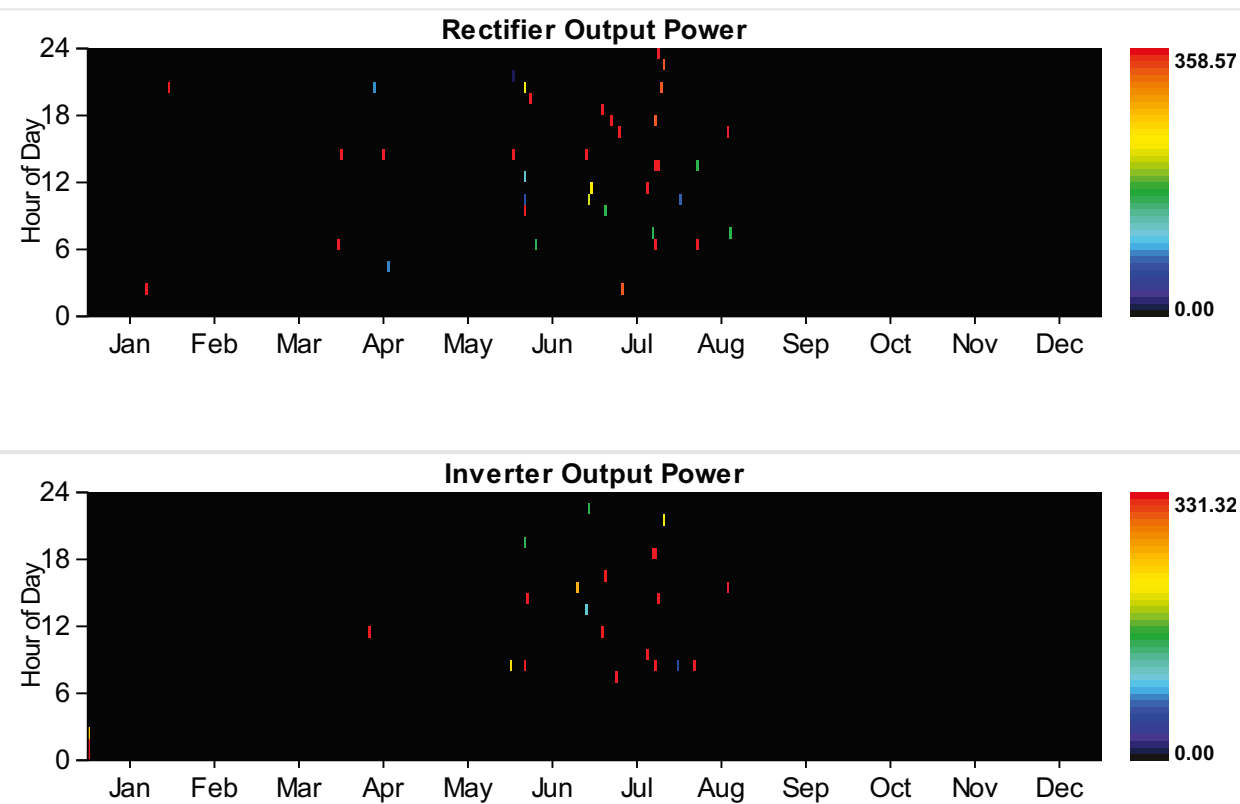

Grid

Rate: Demand 1

\begin{tabular}{|c|c|c|c|c|c|c|}
\hline Resources.ReportingService_GeneratelnputsReport_Month & $\begin{array}{l}\text { Energy } \\
\text { Purchased } \\
\text { (kWh) }\end{array}$ & $\begin{array}{l}\text { Energy } \\
\text { Sold } \\
\text { (kWh) }\end{array}$ & $\begin{array}{l}\text { Net } \\
\text { Purchases } \\
\text { (kWh) }\end{array}$ & $\begin{array}{l}\text { Peak } \\
\text { Demand } \\
(\mathbf{k W})\end{array}$ & $\begin{array}{l}\text { Energy } \\
\text { Charge } \\
\text { (\$) }\end{array}$ & $\begin{array}{l}\text { Demand } \\
\text { Charge } \\
\text { (\$) }\end{array}$ \\
\hline January & 0 & 0 & 0 & 18,247 & 0 & 291,953 \\
\hline February & 0 & 0 & 0 & 17,561 & 0 & 280,978 \\
\hline March & 0 & 0 & 0 & 17,425 & 0 & 278,807 \\
\hline April & 0 & 0 & 0 & 20,000 & 0 & 320,000 \\
\hline May & 0 & 0 & 0 & 17,869 & 0 & 285,902 \\
\hline June & 0 & 0 & 0 & 20,000 & 0 & 320,000 \\
\hline July & 0 & 0 & 0 & 20,000 & 0 & 320,000 \\
\hline August & 0 & 0 & 0 & 20,000 & 0 & 320,000 \\
\hline September & 0 & 0 & 0 & 16,926 & 0 & 270,820 \\
\hline October & 0 & 0 & 0 & 14,895 & 0 & 238,325 \\
\hline November & 0 & 0 & 0 & 16,518 & 0 & 264,286 \\
\hline December & 0 & 0 & 0 & 19,724 & 0 & 315,578 \\
\hline Annual & 0 & 0 & 0 & 20,000 & 0 & $3,506,649$ \\
\hline
\end{tabular}

Rate: Rate 1

\begin{tabular}{|c|c|c|c|c|c|c|}
\hline Resources.ReportingService_GeneratelnputsReport_Month & $\begin{array}{l}\text { Energy } \\
\text { Purchased } \\
\text { (kWh) }\end{array}$ & $\begin{array}{l}\text { Energy } \\
\text { Sold } \\
\text { (kWh) }\end{array}$ & $\begin{array}{l}\text { Net } \\
\text { Purchases } \\
\text { (kWh) }\end{array}$ & $\begin{array}{l}\text { Peak } \\
\text { Demand } \\
(\mathbf{k W})\end{array}$ & $\begin{array}{l}\text { Energy } \\
\text { Charge (\$) }\end{array}$ & $\begin{array}{l}\text { Demand } \\
\text { Charge } \\
\text { (\$) }\end{array}$ \\
\hline January & $8,208,142$ & 0 & $8,208,142$ & 0 & 984,977 & 0 \\
\hline February & $6,110,456$ & 0 & $6,110,456$ & 0 & 733,255 & 0 \\
\hline March & $6,816,916$ & 0 & $6,816,916$ & 0 & 818,030 & 0 \\
\hline
\end{tabular}




\begin{tabular}{|c|c|c|c|c|c|c|}
\hline Rałources.ReportingService_GeneratelnputsReport_Month & $\begin{array}{l}\text { ERefty } 481 \\
\text { Purchased } \\
\text { (kWh95,883 }\end{array}$ & $\begin{array}{l}\text { Energy } \\
\text { Sold } \\
\text { (kWh) } 0\end{array}$ & $\begin{array}{l}\text { Net447,481 } \\
\text { Purchases } \\
\text { (kª̂95,883 }\end{array}$ & $\begin{array}{l}\text { Peak } 0 \\
\text { Demand } \\
(\mathbf{k W}) \quad 0\end{array}$ & $\begin{array}{l}\text { 1,133,698 } \\
\text { Energy } \\
\text { Chåłgeł\$9 }\end{array}$ & $\begin{array}{l}\text { Demand } \\
\text { Charge } \\
\text { (\$) } 0\end{array}$ \\
\hline June & $9,785,834$ & 0 & $9,785,834$ & 0 & $1,174,300$ & 0 \\
\hline July & $9,968,291$ & 0 & $9,968,291$ & 0 & $1,196,195$ & 0 \\
\hline August & $9,882,045$ & 0 & $9,882,045$ & 0 & $1,185,845$ & 0 \\
\hline September & $6,943,139$ & 0 & $6,943,139$ & 0 & 833,177 & 0 \\
\hline October & $6,792,313$ & 0 & $6,792,313$ & 0 & 815,078 & 0 \\
\hline November & $6,321,105$ & 0 & $6,321,105$ & 0 & 758,533 & 0 \\
\hline December & $8,678,365$ & 0 & $8,678,365$ & 0 & $1,041,404$ & 0 \\
\hline Annual & $96,759,968$ & 0 & $96,759,968$ & 0 & $11,611,196$ & 0 \\
\hline
\end{tabular}

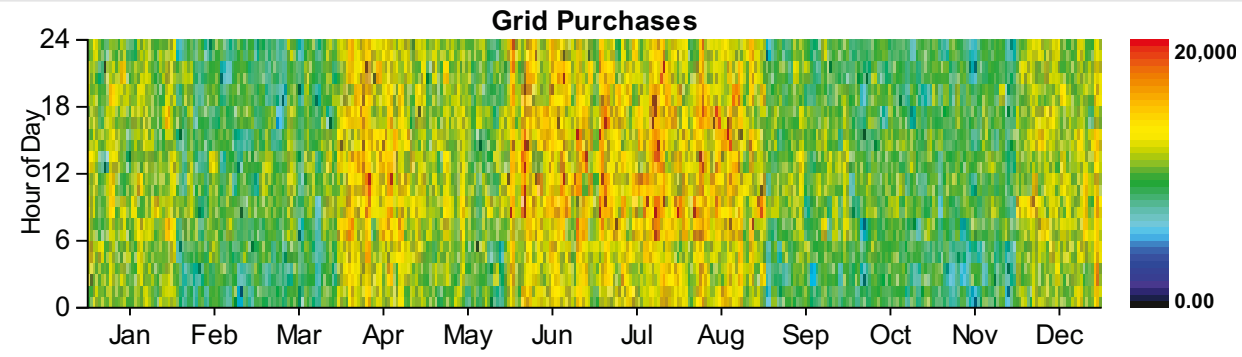

\section{Emissions}

\begin{tabular}{|l|r|l|}
\hline Pollutant & Emissions & Units \\
\hline Carbon dioxide & 61227520 & $\mathrm{~kg} / \mathrm{yr}$ \\
\hline Carbon monoxide & $315 \mathrm{~kg} / \mathrm{yr}$ \\
\hline Unburned hydrocarbons & $36 \mathrm{~kg} / \mathrm{yr}$ \\
\hline Particulate matter & $9 \mathrm{~kg} / \mathrm{yr}$ \\
\hline Sulfur dioxide & $265277 \mathrm{~kg} / \mathrm{yr}$ \\
\hline Nitrogen oxides & 129974 \\
\hline
\end{tabular}


System Report

System architecture

\begin{tabular}{|l|l|l|}
\hline PV & Solar World 320W flat plate PV Copy \\
\hline Generator & Kohler 1000 Prime Power \\
\hline Generator \#2 & Kohler 750 Prime Power \\
\hline Converter & System Converter \\
\hline Grid & Grid \\
\hline Dispatch Strategy & Cycle Charging \\
\hline
\end{tabular}

\section{Cost summary}

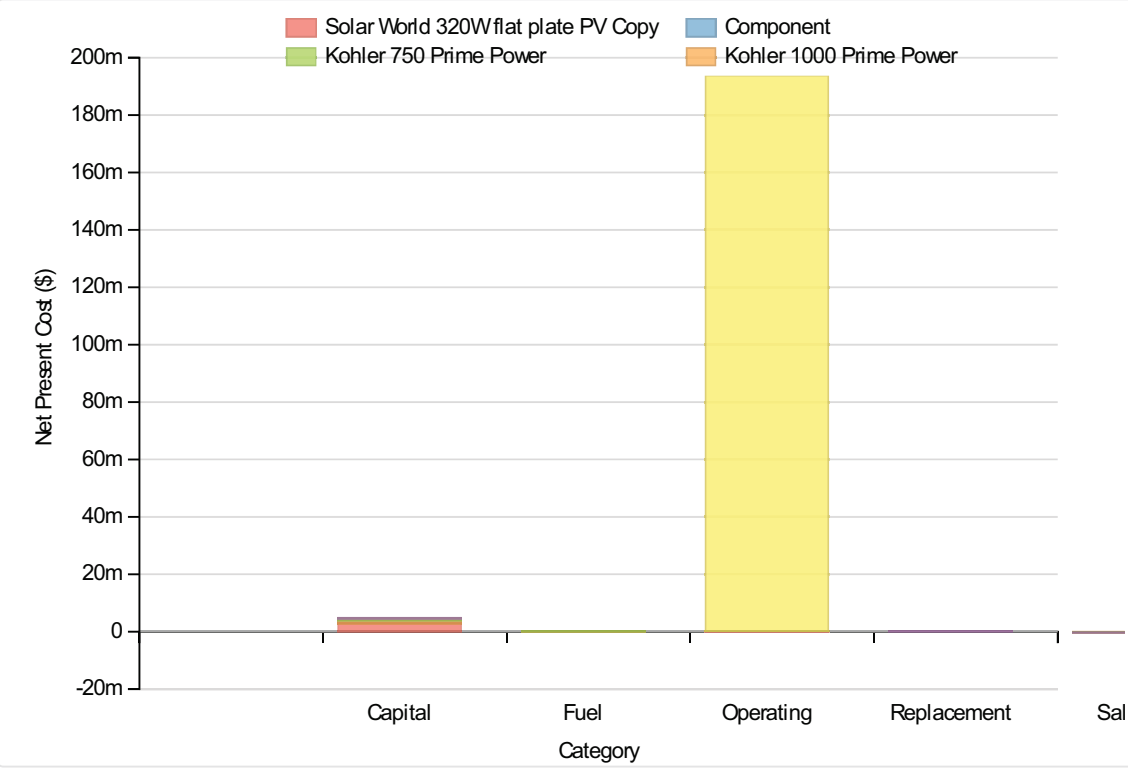

Cost Summary

Total net present cost

$198261296 \$$

Levelized cost of energy

$0.158 \$ / k W h$

\section{Net Present Costs}

\begin{tabular}{|c|c|c|c|c|c|c|}
\hline Component & Capital & Replacement & O\&M & Fuel & Salvage & Total \\
\hline Solar World 320W flat plate PV Copy & $3,000,000$ & 0 & 193,913 & 0 & 0 & $3,193,913$ \\
\hline Kohler 1000 Prime Power & 925,000 & 0 & 22,242 & 141,871 & $-175,795$ & 913,318 \\
\hline Kohler 750 Prime Power & 690,000 & 0 & 21,408 & 108,781 & $-121,216$ & 698,973 \\
\hline Grid & 0 & 0 & $193,152,480$ & 0 & 0 & $193,152,480$ \\
\hline Converter & 225,000 & 95,462 & 0 & 0 & $-17,967$ & 302,495 \\
\hline System & $4,840,000$ & 95,462 & $193,390,064$ & 250,652 & $-314,978$ & $198,261,200$ \\
\hline
\end{tabular}

\section{Annualized Costs}

\begin{tabular}{|l|r|r|r|r|r|r|}
\hline Component & Capital & Replacement & O\&M & Fuel & Salvage & Total \\
\hline Solar World 320W flat plate PV Copy & 232,063 & 0 & 15,000 & 0 & 0 \\
\hline Kohler 1000 Prime Power & 71,553 & 0 & 1,721 & 10,974 & $-13,599$ \\
\hline
\end{tabular}




\begin{tabular}{|c|c|c|c|c|c|c|}
\hline $\begin{array}{l}\text { Component } \\
\text { Kohler } 750 \text { Prime Power }\end{array}$ & $\begin{array}{l}\text { Capital } \\
53,375\end{array}$ & Replacement 0 & O\&M & $\begin{array}{l}\text { Fuel } \\
8,415\end{array}$ & $\begin{array}{r}\text { Salvage } \\
-9,377\end{array}$ & $\begin{array}{l}\text { Total } \quad 54,069\end{array}$ \\
\hline Grid & 0 & 0 & $14,941,190$ & 0 & 0 & $14,941,190$ \\
\hline Converter & 17,405 & 7,384 & 0 & 0 & $-1,390$ & 23,400 \\
\hline System & 374,395 & 7,384 & $14,959,568$ & 19,389 & $-24,365$ & $15,336,371$ \\
\hline
\end{tabular}

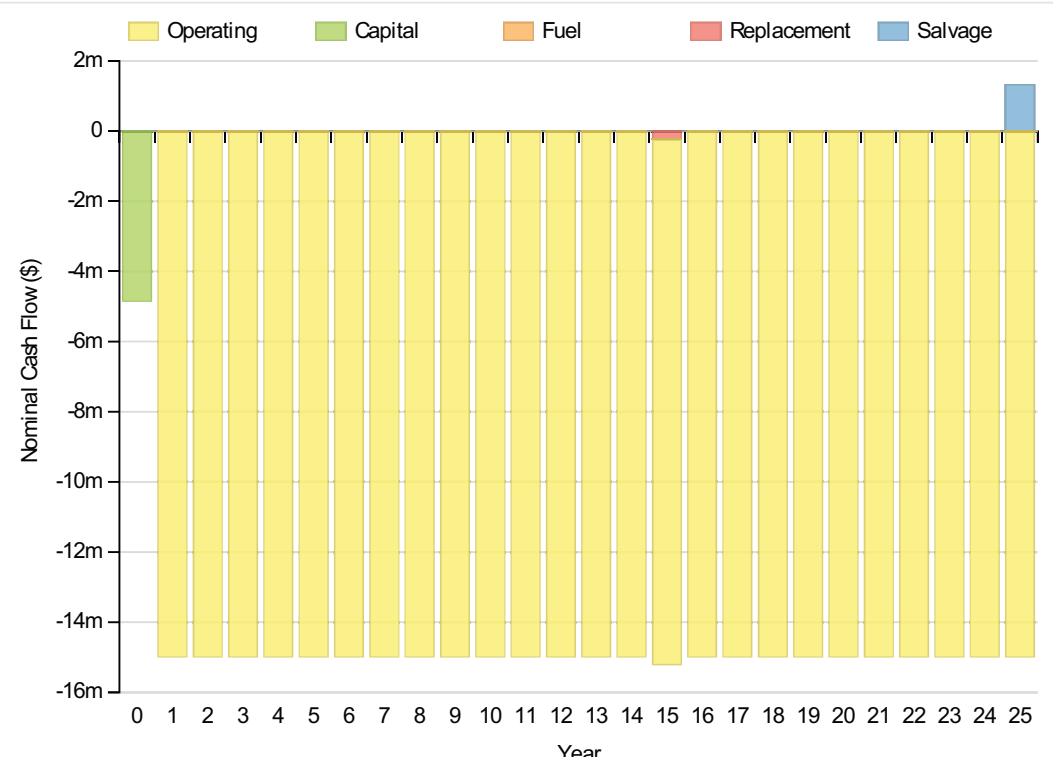

\section{Electrical}

\section{Quantity}

Excess electricity

Unmet load

Capacity shortage

Renewable fraction
Value

Production(kWh/yr)

Component

PV

Generator

Generator

Grid Purchases

Total

Load

Consumption(kWh/yr)

AC primary load

DC primary load

Total
Fraction (\%)

$5225 \mathrm{kWh} / \mathrm{yr}$

10040 kWh/yr

88587 kWh/yr

0

\begin{tabular}{|c|c|}
\hline$F$ & raction (\%) \\
\hline $1,288,675$ & 1 \\
\hline 48,072 & c \\
\hline 36,344 & c \\
\hline $95,614,640$ & 99 \\
\hline \multirow[t]{2}{*}{$96,987,728$} & 100 \\
\hline & Fraction (\%) \\
\hline $96,854,112$ & 100 \\
\hline 0 & c \\
\hline $96,854,112$ & 100 \\
\hline
\end{tabular}




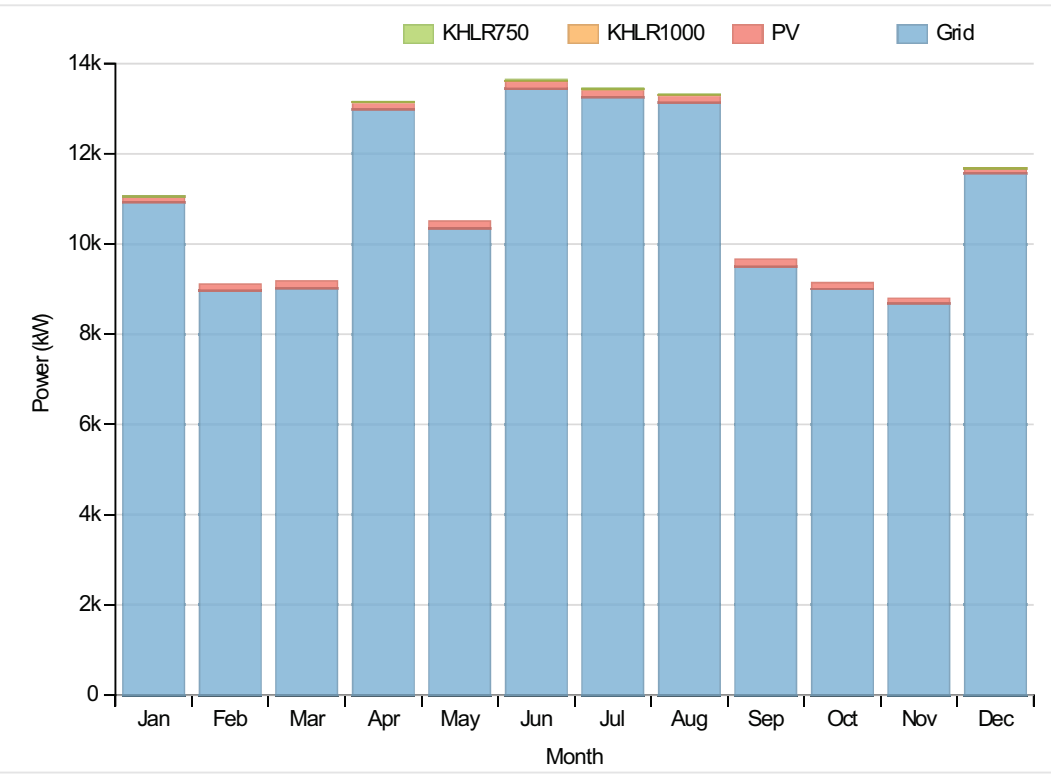

PV:Solar World 320W flat plate PV Copy

\begin{tabular}{|l|c|c|}
\hline Quantity & Value & Units \\
\hline Rated capacity & $1000 \mathrm{~kW}$ \\
\hline Mean output & $147 \mathrm{~kW}$ \\
\hline Mean output & $3530.60 \mathrm{kWh} / \mathrm{d}$ \\
\hline Capacity factor & $14.71 \%$ \\
\hline Total production & $1288675 \mathrm{kWh} / \mathrm{yr}$ \\
\hline Minimum output & $0.00 \mathrm{~kW}$ \\
\hline Maximum output & $1022.50 \mathrm{~kW}$ \\
\hline PV penetration & $1.33 \%$ \\
\hline Hours of operation & $4377 \mathrm{hrs} / \mathrm{yr}$ \\
\hline Levelized cost & $\$ 1 / \mathrm{kWh}$ \\
\hline
\end{tabular}

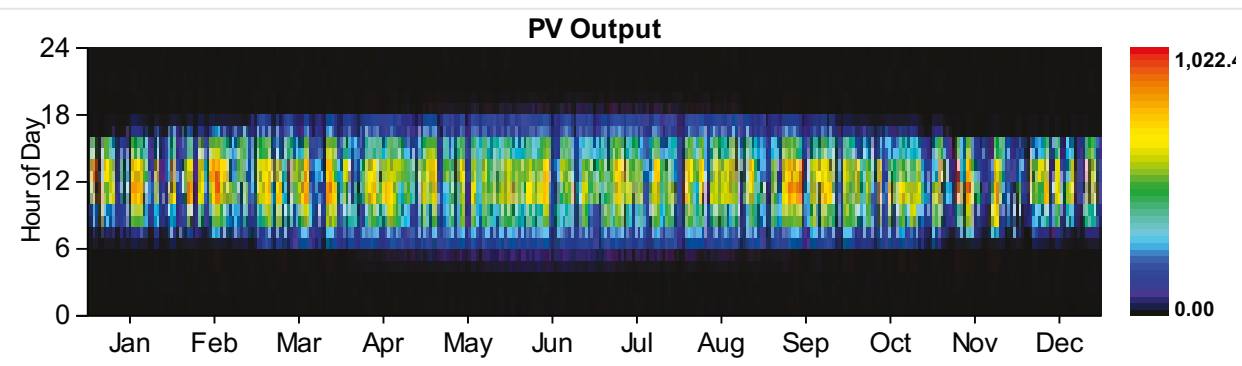

Generator:Kohler 1000 Prime Power

\section{Quantity}

Hours of operation

Number of starts

Operational life

Fixed generation cost

Marginal generation cost

\section{Value}

\section{Units}

$124 \mathrm{hrs} / \mathrm{yr}$

114 starts/yr

$121 \mathrm{yr}$

$76.24 \$ / h r$

$0.23 \$ / k W h$ 


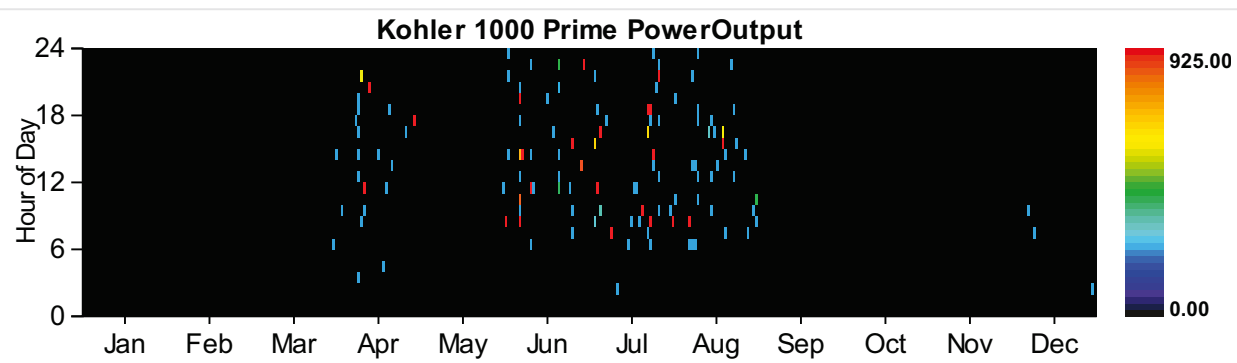

\section{Generator:Kohler 750 Prime Power}

\section{Quantity}

Hours of operation

Number of starts

Operational life

Fixed generation cost

Marginal generation cost

Electrical production

Mean electrical output

Min. electrical output

Max. electrical output

Fuel consumption

Specific fuel consumption

Fuel energy input

Mean electrical efficiency

\section{Value}

Units

$160 \mathrm{hrs} / \mathrm{yr}$

144 starts/yr

$94 \mathrm{yr}$

$56.87 \$ / h r$

$0.23 \$ / k W h$

$36344 \mathrm{kWh} / \mathrm{yr}$

227 kW

173 kW

690 kW

10652 L/yr

$0.29 \mathrm{~L} / \mathrm{kWh}$

$104811 \mathrm{kWh} / \mathrm{yr}$

$35 \%$ 


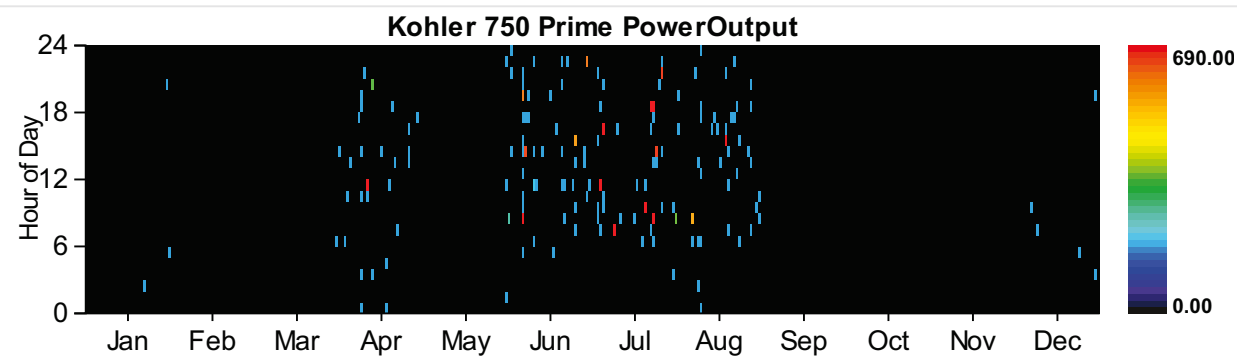

\section{Converter}

\begin{tabular}{|c|c|c|c|}
\hline Quantity & Inverter & Rectifier & Units \\
\hline Capacity & 750 & 675 & kW \\
\hline Mean output & 132 & 0 & kW \\
\hline Minimum output & 0 & 0 & kW \\
\hline Maximum output & 750 & 0 & kW \\
\hline Capacity factor & 18 & 0 & $\%$ \\
\hline Hours of operation & 4,377 & 0 & $\mathrm{hrs} / \mathrm{yr}$ \\
\hline Energy in & $1,283,450$ & 0 & $\mathrm{kWh} / \mathrm{yr}$ \\
\hline Energy out & $1,155,104$ & 0 & kWh/yr \\
\hline Losses & 128,346 & 0 & $\mathrm{kWh} / \mathrm{yr}$ \\
\hline
\end{tabular}
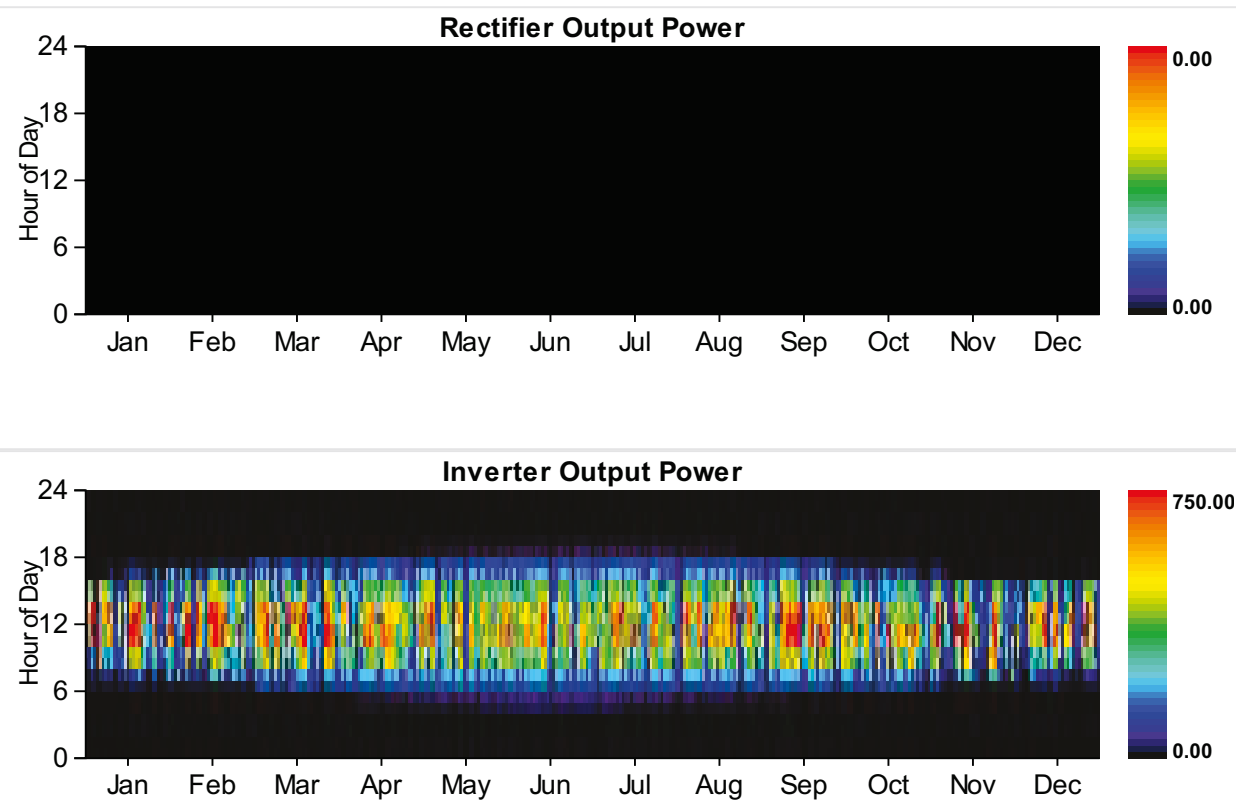

Grid

Rate: Demand 1

\begin{tabular}{|c|c|c|c|c|c|c|}
\hline Resources.ReportingService_GeneratelnputsReport_Month & $\begin{array}{l}\text { Energy } \\
\text { Purchased } \\
\text { (kWh) }\end{array}$ & $\begin{array}{l}\text { Energy } \\
\text { Sold } \\
\text { (kWh) }\end{array}$ & $\begin{array}{l}\text { Net } \\
\text { Purchases } \\
\text { (kWh) }\end{array}$ & $\begin{array}{l}\text { Peak } \\
\text { Demand } \\
(\mathbf{k W})\end{array}$ & $\begin{array}{l}\text { Energy } \\
\text { Charge } \\
\text { (\$) }\end{array}$ & $\begin{array}{l}\text { Demand } \\
\text { Charge } \\
(\$)\end{array}$ \\
\hline January & 0 & 0 & 0 & 18,380 & 0 & 294,083 \\
\hline February & 0 & 0 & 0 & 16,811 & 0 & 268,978 \\
\hline March & 0 & 0 & 0 & 16,941 & 0 & 271,062 \\
\hline
\end{tabular}




\begin{tabular}{|c|c|c|c|c|c|c|}
\hline $\begin{array}{l}\text { April } \\
\text { Resources.ReportingService_GeneratelnputsReport_Month } \\
\text { May }\end{array}$ & $\begin{array}{l}\text { Energy } \\
\text { Purchased } \\
\text { (kWh) } \quad 0\end{array}$ & $\begin{array}{l}\text { Energy } \\
\text { Sold } 0 \\
(\mathbf{k W h})_{0}\end{array}$ & $\begin{array}{l}\text { Net } \\
\text { Purchases } \\
\text { (kWh) } \quad 0\end{array}$ & $\begin{array}{l}\text { Peak } \\
\text { Demano } \\
\text { (kW) } \\
17,657\end{array}$ & $\begin{array}{l}\text { Energy } \\
\text { Charge } \\
\text { (\$) } \quad 0\end{array}$ & $\begin{array}{l}\text { Demand } \\
\text { Ch20000 } \\
\text { (\$) } 282,514\end{array}$ \\
\hline June & 0 & 0 & 0 & 20,000 & 0 & 320,000 \\
\hline July & 0 & 0 & 0 & 20,000 & 0 & 320,000 \\
\hline August & 0 & 0 & 0 & 20,000 & 0 & 320,000 \\
\hline September & 0 & 0 & 0 & 16,176 & 0 & 258,820 \\
\hline October & 0 & 0 & 0 & 14,744 & 0 & 235,900 \\
\hline November & 0 & 0 & 0 & 16,518 & 0 & 264,286 \\
\hline December & 0 & 0 & 0 & 19,488 & 0 & 311,800 \\
\hline Annual & 0 & 0 & 0 & 20,000 & 0 & $3,467,443$ \\
\hline
\end{tabular}

Rate: Rate 1

\begin{tabular}{|c|c|c|c|c|c|c|}
\hline Resources.ReportingService_GeneratelnputsReport_Month & $\begin{array}{l}\text { Energy } \\
\text { Purchased } \\
\text { (kWh) }\end{array}$ & $\begin{array}{l}\text { Energy } \\
\text { Sold } \\
\text { (kWh) }\end{array}$ & $\begin{array}{l}\text { Net } \\
\text { Purchases } \\
\text { (kWh) }\end{array}$ & $\begin{array}{l}\text { Peak } \\
\text { Demand } \\
(\mathbf{k W})\end{array}$ & $\begin{array}{l}\text { Energy } \\
\text { Charge (\$) }\end{array}$ & $\begin{array}{l}\text { Demand } \\
\text { Charge } \\
\text { (\$) }\end{array}$ \\
\hline January & $8,131,761$ & 0 & $8,131,761$ & 0 & 975,811 & 0 \\
\hline February & $6,023,890$ & 0 & $6,023,890$ & 0 & 722,867 & 0 \\
\hline March & $6,708,956$ & 0 & $6,708,956$ & 0 & 805,075 & 0 \\
\hline April & $9,347,045$ & 0 & $9,347,045$ & 0 & $1,121,645$ & 0 \\
\hline May & $7,697,129$ & 0 & $7,697,129$ & 0 & 923,655 & 0 \\
\hline June & $9,679,243$ & 0 & $9,679,243$ & 0 & $1,161,509$ & 0 \\
\hline July & $9,859,910$ & 0 & $9,859,910$ & 0 & $1,183,189$ & 0 \\
\hline August & $9,771,679$ & 0 & $9,771,679$ & 0 & $1,172,602$ & 0 \\
\hline September & $6,837,491$ & 0 & $6,837,491$ & 0 & 820,499 & 0 \\
\hline October & $6,699,125$ & 0 & $6,699,125$ & 0 & 803,895 & 0 \\
\hline November & $6,249,541$ & 0 & $6,249,541$ & 0 & 749,945 & 0 \\
\hline December & $8,608,863$ & 0 & $8,608,863$ & 0 & $1,033,064$ & 0 \\
\hline Annual & $95,614,640$ & 0 & $95,614,640$ & 0 & $11,473,756$ & 0 \\
\hline
\end{tabular}

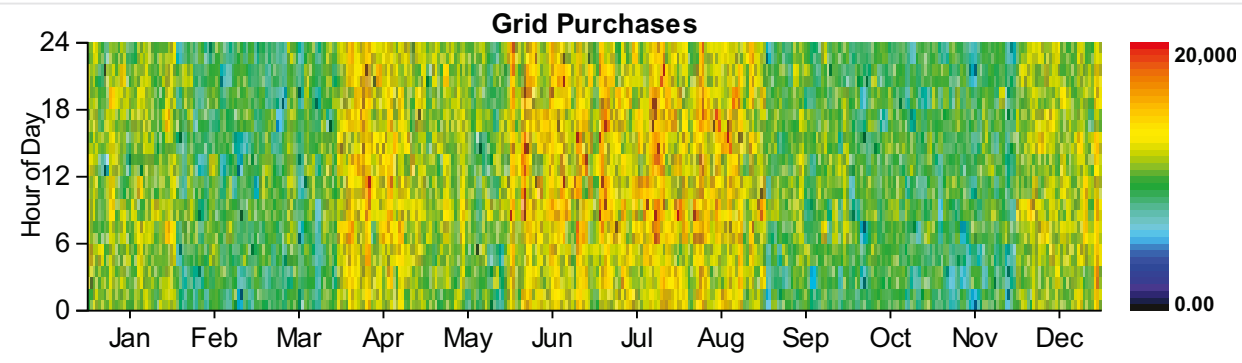




\section{Emissions}

Pollutant

Carbon dioxide

Carbon monoxide

Unburned hydrocarbons

Particulate matter

Sulfur dioxide

Nitrogen oxides
Emissions

Units

$60492868 \mathrm{~kg} / \mathrm{yr}$

$270 \mathrm{~kg} / \mathrm{yr}$

$31 \mathrm{~kg} / \mathrm{yr}$

$8 \mathrm{~kg} / \mathrm{yr}$

$262117 \mathrm{~kg} / \mathrm{yr}$

$128394 \mathrm{~kg} / \mathrm{yr}$

HOMER Energy, LLC @ 2016 
System Report

System architecture

\begin{tabular}{|l|l|l|}
\hline PV & Solar World 320W flat plate PV Copy & 1,000 \\
\hline Generator & Kohler 1000 Prime Power & 1,850 \\
\hline Converter & System Converter & 750 \\
\hline Grid & Grid & $\mathrm{kW}$ \\
\hline Dispatch Strategy & Cycle Charging & 20,000 \\
\hline
\end{tabular}

Cost summary

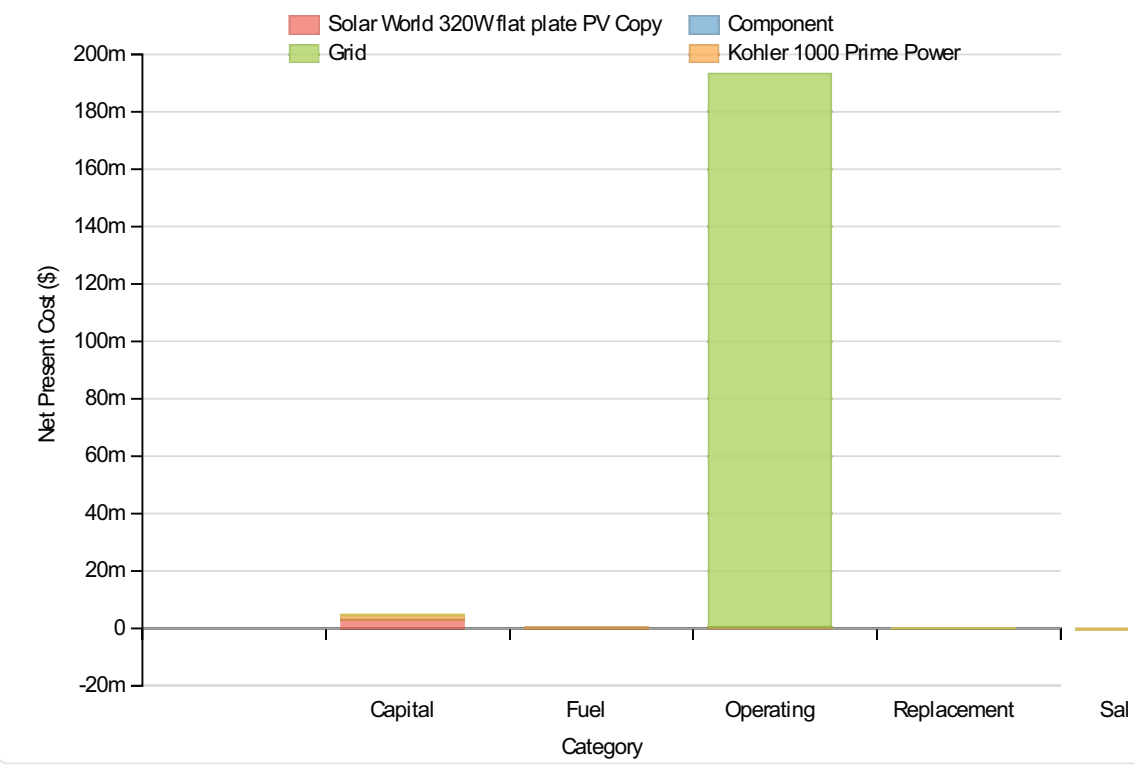

Cost Summary

Total net present cost

$198295184 \$$

Levelized cost of energy

$0.158 \$ / k W h$

Net Present Costs

\begin{tabular}{|c|c|c|c|c|c|c|}
\hline Component & Capital & Replacement & O\&M & Fuel & Salvage & Total \\
\hline Solar World 320W flat plate PV Copy & $3,000,000$ & 0 & 193,913 & 0 & 0 & $3,193,913$ \\
\hline Kohler 1000 Prime Power & $1,637,500$ & 0 & 62,943 & 331,191 & $-273,939$ & $1,757,695$ \\
\hline Grid & 0 & 0 & $193,040,976$ & 0 & 0 & $193,040,976$ \\
\hline Converter & 225,000 & 95,462 & 0 & 0 & $-17,967$ & 302,495 \\
\hline System & $4,862,500$ & 95,462 & $193,297,856$ & 331,191 & $-291,906$ & $198,295,103$ \\
\hline
\end{tabular}

\section{Annualized Costs}

\section{Component}

Solar World 320W flat plate PV Copy

Kohler 1000 Prime Power

Grid

Converter

\begin{tabular}{|r|r|r|r|r|r|r|}
\hline Capital & Replacement & O\&M & Fuel & Salvage & \multicolumn{1}{l|}{ Total } \\
\hline 232,063 & 0 & 15,000 & 0 & 0 & 247,063 \\
\hline 126,668 & 0 & 4,869 & 25,619 & $-21,190$ & 135,966 \\
\hline 0 & 0 & $14,932,565$ & 0 & 0 & $14,932,565$ \\
\hline 17,405 & 7,384 & 0 & 0 & $-1,390$ & 23,400 \\
\hline
\end{tabular}




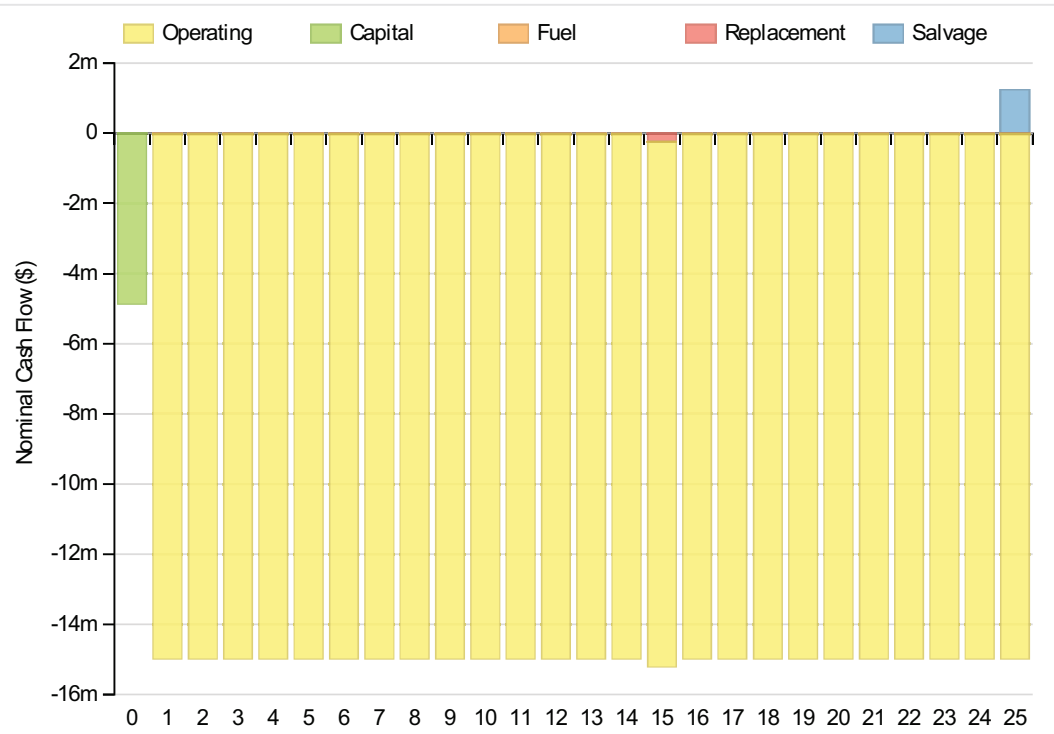

Year

\section{Electrical}

\begin{tabular}{|c|c|c|c|c|}
\hline \multicolumn{2}{|l|}{ Quantity } & \multicolumn{2}{|l|}{ Value } & Units \\
\hline Excess electricity & & & 5225 & $\mathrm{kWh} / \mathrm{yr}$ \\
\hline Unmet load & & & 7831 & $\mathrm{kWh} / \mathrm{yr}$ \\
\hline Capacity shortage & & & 73016 & $\mathrm{kWh} / \mathrm{yr}$ \\
\hline Renewable fraction & & & 0 & \\
\hline Component & Production(kWh/yr) & & Fracti & ion $(\%)$ \\
\hline PV & & $1,288,675$ & & 1 \\
\hline Generator & & 112,000 & & 0 \\
\hline Grid Purchases & & $95,589,264$ & & 99 \\
\hline Total & & $96,989,936$ & & 100 \\
\hline Load & Consumption(kWh/yr) & & Fra & Iction (\%) \\
\hline AC primary load & & $96,856,328$ & & 100 \\
\hline DC primary load & & & 0 & 0 \\
\hline Total & & $96,856,328$ & & 100 \\
\hline
\end{tabular}




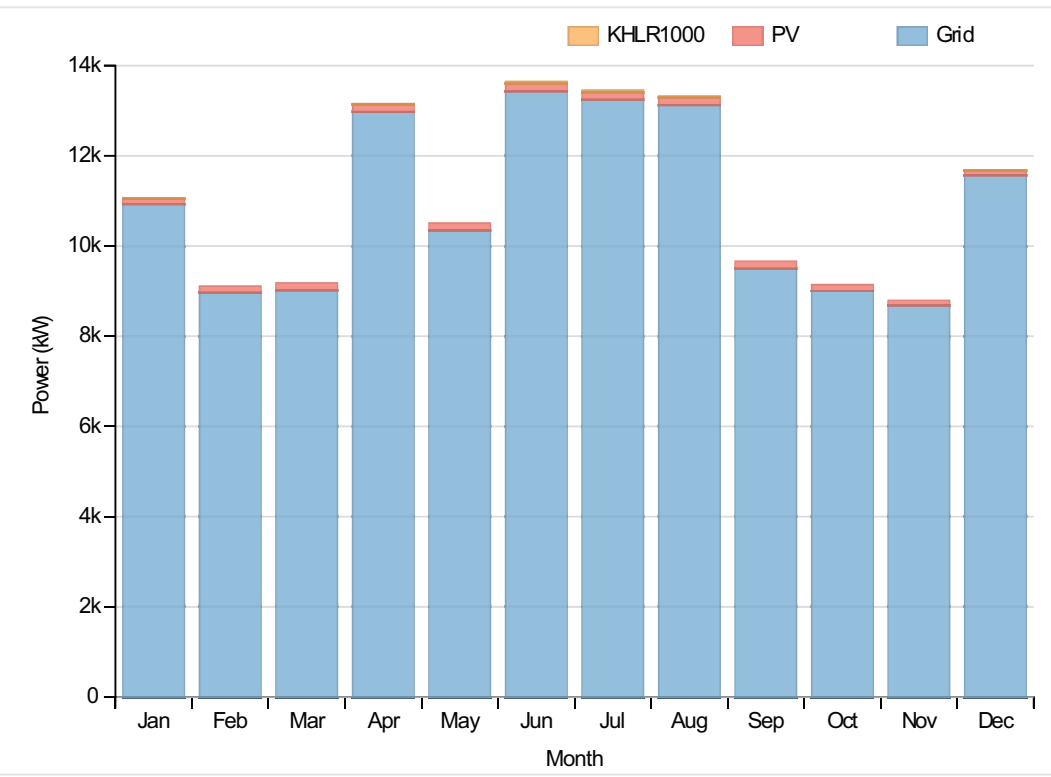

PV:Solar World 320W flat plate PV Copy

\begin{tabular}{|l|c|c|}
\hline Quantity & Value & Units \\
\hline Rated capacity & $1000 \mathrm{~kW}$ \\
\hline Mean output & $147 \mathrm{~kW}$ \\
\hline Mean output & $3530.60 \mathrm{kWh} / \mathrm{d}$ \\
\hline Capacity factor & $14.71 \%$ \\
\hline Total production & $1288675 \mathrm{kWh} / \mathrm{yr}$ \\
\hline Minimum output & $0.00 \mathrm{~kW}$ \\
\hline Maximum output & $1022.50 \mathrm{~kW}$ \\
\hline PV penetration & $1.33 \%$ \\
\hline Hours of operation & $4377 \mathrm{hrs} / \mathrm{yr}$ \\
\hline Levelized cost & $\$ 1 / \mathrm{kWh}$ \\
\hline
\end{tabular}

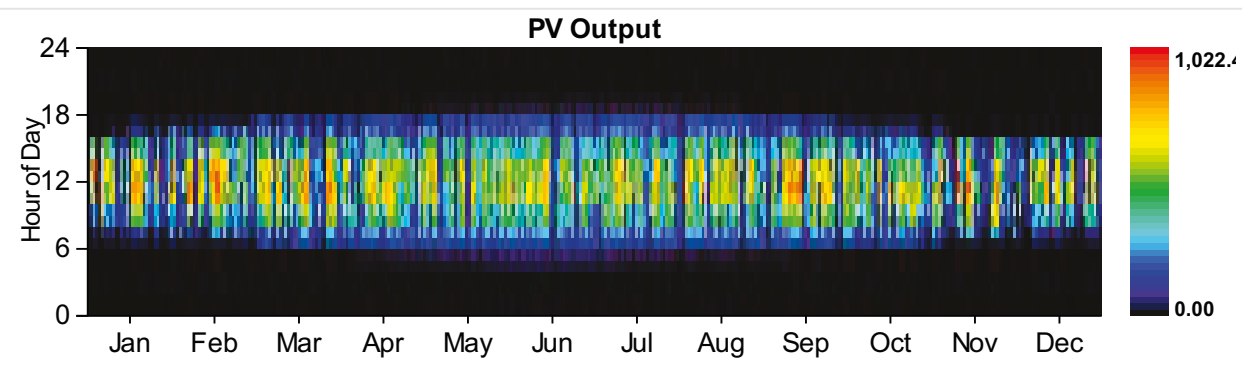

Generator:Kohler 1000 Prime Power

\section{Quantity}

Hours of operation

Number of starts

Operational life

Fixed generation cost

Marginal generation cost

\section{Value}

\section{Units}

$181 \mathrm{hrs} / \mathrm{yr}$

162 starts/yr

$83 \mathrm{yr}$

$137.46 \$ / \mathrm{hr}$

$0.23 \$ / k W h$ 


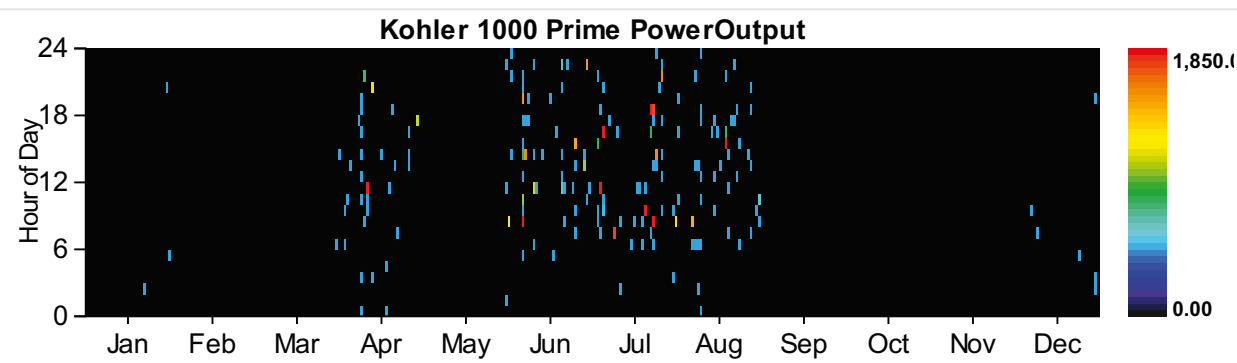

\section{Converter}

\section{Quantity}

Capacity

Mean output

Minimum output

Maximum output

Capacity factor

Hours of operation

Energy in

Energy out

Losses

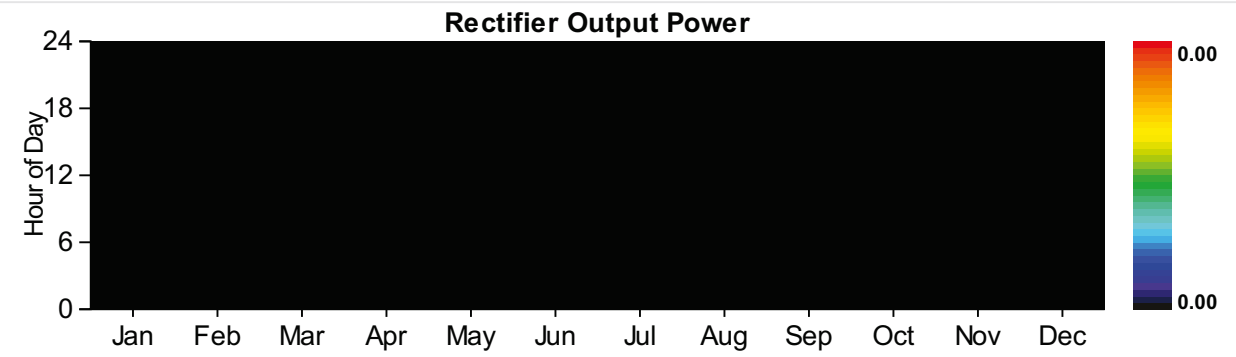

\section{Inverter}

750

132

0

750

18

4,377

$1,283,450$

$1,155,104$

128,346

Rectifier

Units

675 kW

$0 \mathrm{~kW}$

$0 \mathrm{~kW}$

$0 \mathrm{~kW}$

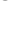

$0 \%$

hrs/yr

0 kWh/yr

$0 \mathrm{kWh} / \mathrm{yr}$

$0 \mathrm{kWh} / \mathrm{yr}$ 


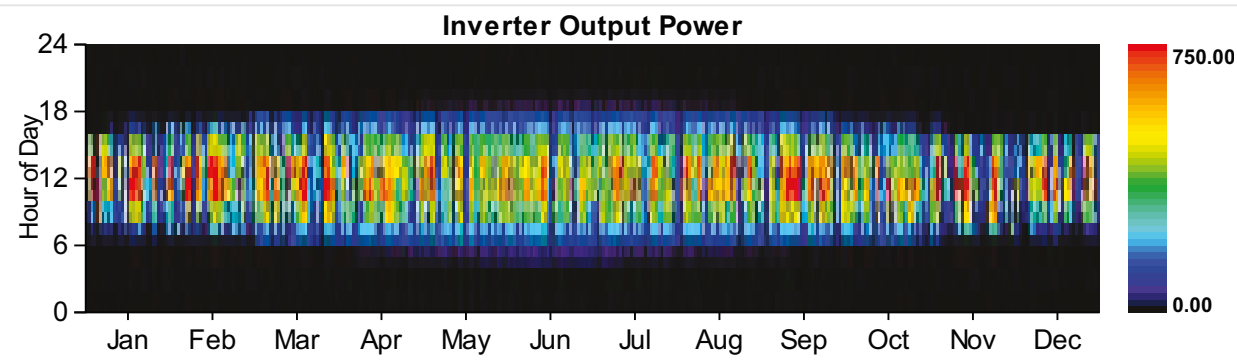

Grid

Rate: Demand 1

\begin{tabular}{|c|c|c|c|c|c|c|}
\hline Resources.ReportingService_GeneratelnputsReport_Month & $\begin{array}{l}\text { Energy } \\
\text { Purchased } \\
\text { (kWh) }\end{array}$ & $\begin{array}{l}\text { Energy } \\
\text { Sold } \\
\text { (kWh) }\end{array}$ & $\begin{array}{l}\text { Net } \\
\text { Purchases } \\
\text { (kWh) }\end{array}$ & $\begin{array}{l}\text { Peak } \\
\text { Demand } \\
(\mathbf{k W})\end{array}$ & $\begin{array}{l}\text { Energy } \\
\text { Charge } \\
\text { (\$) }\end{array}$ & $\begin{array}{l}\text { Demand } \\
\text { Charge } \\
(\$)\end{array}$ \\
\hline January & 0 & 0 & 0 & 18,090 & 0 & 289,443 \\
\hline February & 0 & 0 & 0 & 16,811 & 0 & 268,978 \\
\hline March & 0 & 0 & 0 & 16,941 & 0 & 271,062 \\
\hline April & 0 & 0 & 0 & 20,000 & 0 & 320,000 \\
\hline May & 0 & 0 & 0 & 17,657 & 0 & 282,514 \\
\hline June & 0 & 0 & 0 & 20,000 & 0 & 320,000 \\
\hline July & 0 & 0 & 0 & 20,000 & 0 & 320,000 \\
\hline August & 0 & 0 & 0 & 20,000 & 0 & 320,000 \\
\hline September & 0 & 0 & 0 & 16,176 & 0 & 258,820 \\
\hline October & 0 & 0 & 0 & 14,744 & 0 & 235,900 \\
\hline November & 0 & 0 & 0 & 16,518 & 0 & 264,286 \\
\hline December & 0 & 0 & 0 & 19,429 & 0 & 310,860 \\
\hline Annual & 0 & 0 & 0 & 20,000 & 0 & $3,461,863$ \\
\hline
\end{tabular}

Rate: Rate 1

\begin{tabular}{|c|c|c|c|c|c|c|}
\hline Resources.ReportingService_GeneratelnputsReport_Month & $\begin{array}{l}\text { Energy } \\
\text { Purchased } \\
\text { (kWh) }\end{array}$ & $\begin{array}{l}\text { Energy } \\
\text { Sold } \\
\text { (kWh) }\end{array}$ & $\begin{array}{l}\text { Net } \\
\text { Purchases } \\
\text { (kWh) }\end{array}$ & $\begin{array}{l}\text { Peak } \\
\text { Demand } \\
(\mathbf{k W})\end{array}$ & $\begin{array}{l}\text { Energy } \\
\text { Charge (\$) }\end{array}$ & $\begin{array}{l}\text { Demand } \\
\text { Charge } \\
(\$)\end{array}$ \\
\hline January & $8,130,891$ & 0 & $8,130,891$ & 0 & 975,707 & 0 \\
\hline February & $6,023,890$ & 0 & $6,023,890$ & 0 & 722,867 & 0 \\
\hline March & $6,708,956$ & 0 & $6,708,956$ & 0 & 805,075 & 0 \\
\hline April & $9,341,935$ & 0 & $9,341,935$ & 0 & $1,121,032$ & 0 \\
\hline May & $7,697,129$ & 0 & $7,697,129$ & 0 & 923,655 & 0 \\
\hline June & $9,673,265$ & 0 & $9,673,265$ & 0 & $1,160,792$ & 0 \\
\hline July & $9,853,874$ & 0 & $9,853,874$ & 0 & $1,182,465$ & 0 \\
\hline August & $9,765,519$ & 0 & $9,765,519$ & 0 & $1,171,862$ & 0 \\
\hline
\end{tabular}




\begin{tabular}{|c|c|c|c|c|c|c|}
\hline September & ERergaty 491 & Energy & Nêt837,491 & Peak 0 & & Demand \\
\hline $\begin{array}{l}\text { Rctober } \\
\text { Resources.ReportingService_GeneratelnputsReport_Month }\end{array}$ & $\begin{array}{l}\text { Purchased } \\
\text { (kWh9,125 }\end{array}$ & $\begin{array}{l}\text { Sold } \\
(\mathbf{k W h})\end{array}$ & $\begin{array}{l}\text { Purchases } \\
\text { 6W99,125 } \\
\text { (kWh) }\end{array}$ & $\begin{array}{l}\text { Demand } \\
(\mathrm{kW})\end{array}$ & $\begin{array}{l}\text { Energy } \\
\text { Charges.895 (\$) }\end{array}$ & $\begin{array}{l}\text { Charge }_{0} \\
(\$)\end{array}$ \\
\hline November & $6,249,541$ & 0 & $6,249,541$ & 0 & 749,945 & 0 \\
\hline December & $8,607,644$ & 0 & $8,607,644$ & 0 & $1,032,917$ & 0 \\
\hline Annual & $95,589,264$ & 0 & $95,589,264$ & 0 & $11,470,711$ & 0 \\
\hline
\end{tabular}

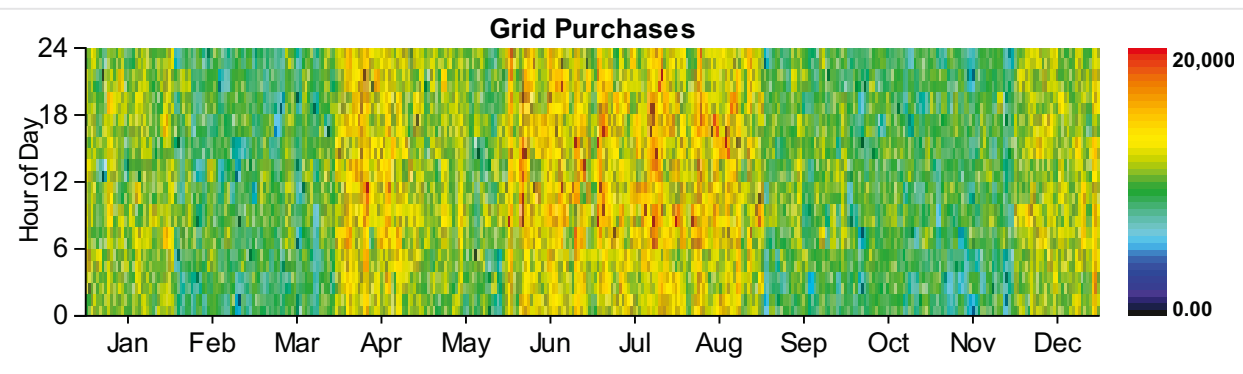

\section{Emissions}

\begin{tabular}{|l|r|l|}
\hline Pollutant & Emissions & Units \\
\hline Carbon dioxide & 60497528 & $\mathrm{~kg} / \mathrm{yr}$ \\
\hline Carbon monoxide & $357 \mathrm{~kg} / \mathrm{yr}$ \\
\hline Unburned hydrocarbons & $41 \mathrm{~kg} / \mathrm{yr}$ \\
\hline Particulate matter & $10 \mathrm{~kg} / \mathrm{yr}$ \\
\hline Sulfur dioxide & $262090 \mathrm{~kg} / \mathrm{yr}$ \\
\hline Nitrogen oxides & $128446 \mathrm{~kg} / \mathrm{yr}$ \\
\hline
\end{tabular}


System Report

System architecture

\begin{tabular}{|c|c|c|c|}
\hline PV & Solar World 320W flat plate PV Copy & 1,000 & $\mathrm{~kW}$ \\
\hline Generator \#2 & Kohler 750 Prime Power & 1,380 & $\mathrm{~kW}$ \\
\hline Battery & GS200 flow & 1 & strings \\
\hline Converter & System Converter & 750 & $\mathrm{~kW}$ \\
\hline Grid & Grid & 20,000 & $\mathrm{~kW}$ \\
\hline Dispatch Strategy & Cycle Charging & & \\
\hline
\end{tabular}

\section{Cost summary}

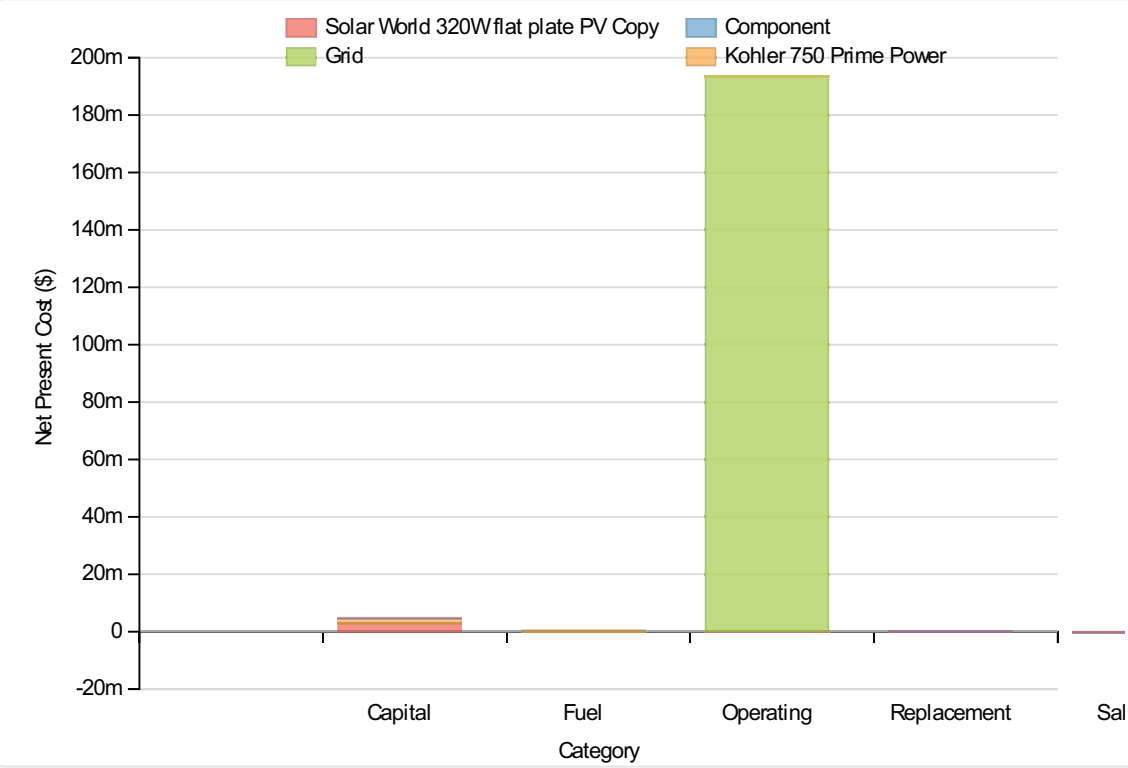

Cost Summary

Total net present cost

$198358448 \$$

Levelized cost of energy

$0.158 \$ / k W h$

Net Present Costs

\begin{tabular}{|l|r|r|r|r|r|r|}
\hline Component & Capital & Replacement & O\&M & Fuel & Salvage & Total \\
\hline Solar World 320W flat plate PV Copy & $3,000,000$ & 0 & 193,913 & 0 & 0 \\
\hline Kohler 750 Prime Power & $1,285,000$ & 0 & 42,555 & 266,901 & $-224,717$ \\
\hline Grid & 0 & 0 & $193,130,400$ & 0 & 0 \\
\hline GS200 flow & 324,589 & 7,176 & 31,026 & 0 & -973 \\
\hline Converter & 225,000 & $95,130,400$ \\
\hline System & $4,834,589$ & 102,637 & $193,397,920$ & 266,901 & $-243,657$ \\
\hline
\end{tabular}

Annualized Costs

\begin{tabular}{|l|r|r|r|r|r|r|}
\hline Component & Capital & Replacement & O\&M & Fuel & Salvage & Total \\
\hline Solar World 320W flat plate PV Copy & 232,063 & 0 & 15,000 & 0 & 0 \\
\hline Kohler 750 Prime Power & 99,400 & 0 & 3,292 & 20,646 & $-17,383$ \\
\hline
\end{tabular}




\begin{tabular}{|c|c|c|c|c|c|c|}
\hline Component & 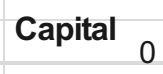 & Replacement 0 & O\&M $14,939,482$ & Fuel 0 & Salvage $_{0}$ & $\begin{array}{l}\text { Total } \\
14,939,482\end{array}$ \\
\hline GS200 flow & 25,108 & 555 & 2,400 & 0 & -75 & 27,988 \\
\hline Converter & 17,405 & 7,384 & 0 & 0 & $-1,390$ & 23,400 \\
\hline System & 373,977 & 7,939 & $14,960,176$ & 20,646 & $-18,848$ & $15,343,890$ \\
\hline
\end{tabular}

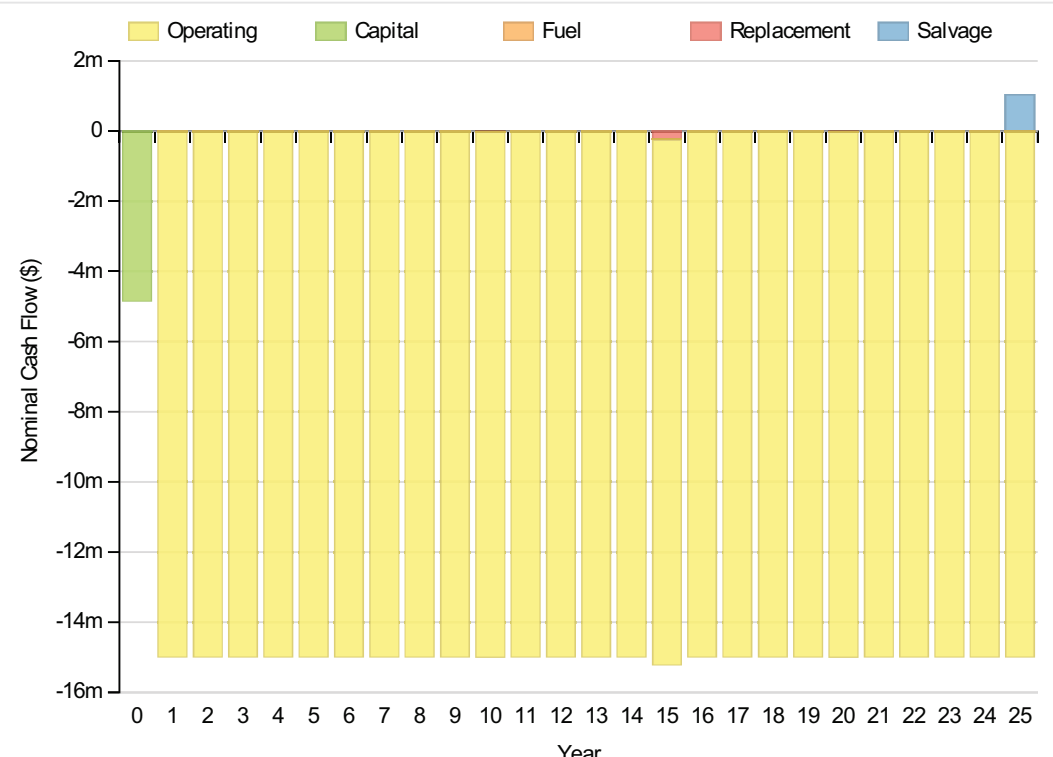

\section{Electrical}

\section{Quantity}

Excess electricity

Unmet load

Capacity shortage

Renewable fraction
Value

Units

$2745 \mathrm{kWh} / \mathrm{yr}$

10858 kWh/yr

93644 kWh/yr

0

\begin{tabular}{|c|c|c|}
\hline Component & Production(kWh/yr) & Fraction $(\%)$ \\
\hline PV & $1,288,675$ & 1 \\
\hline Generator & 89,327 & 0 \\
\hline Grid Purchases & $95,610,304$ & 99 \\
\hline Total & $96,988,304$ & 100 \\
\hline Load & Consumption(kWh/yr) & Fraction $(\%)$ \\
\hline AC primary load & $96,853,304$ & 100 \\
\hline DC primary load & & 0 \\
\hline Total & $96,853,304$ & 100 \\
\hline
\end{tabular}




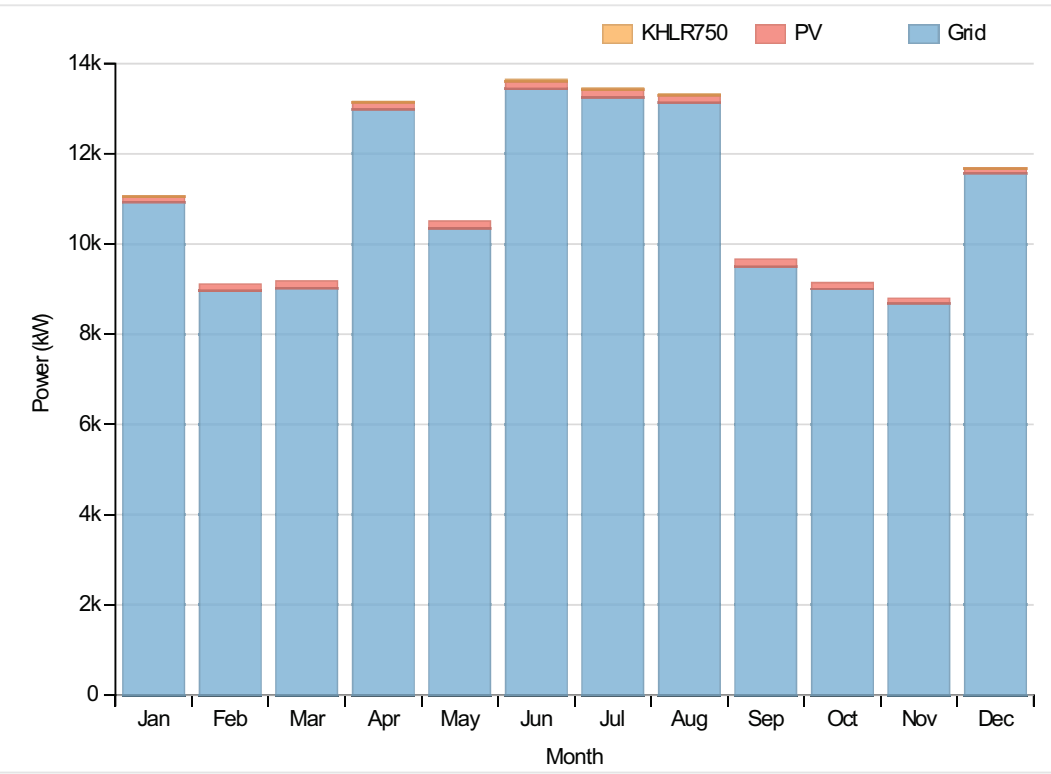

PV:Solar World 320W flat plate PV Copy

\begin{tabular}{|l|c|c|}
\hline Quantity & Value & Units \\
\hline Rated capacity & $1000 \mathrm{~kW}$ \\
\hline Mean output & $147 \mathrm{~kW}$ \\
\hline Mean output & $3530.60 \mathrm{kWh} / \mathrm{d}$ \\
\hline Capacity factor & $14.71 \%$ \\
\hline Total production & $1288675 \mathrm{kWh} / \mathrm{yr}$ \\
\hline Minimum output & $0.00 \mathrm{~kW}$ \\
\hline Maximum output & $1022.50 \mathrm{~kW}$ \\
\hline PV penetration & $1.33 \%$ \\
\hline Hours of operation & $4377 \mathrm{hrs} / \mathrm{yr}$ \\
\hline Levelized cost & $\$ 1 / \mathrm{kWh}$ \\
\hline
\end{tabular}

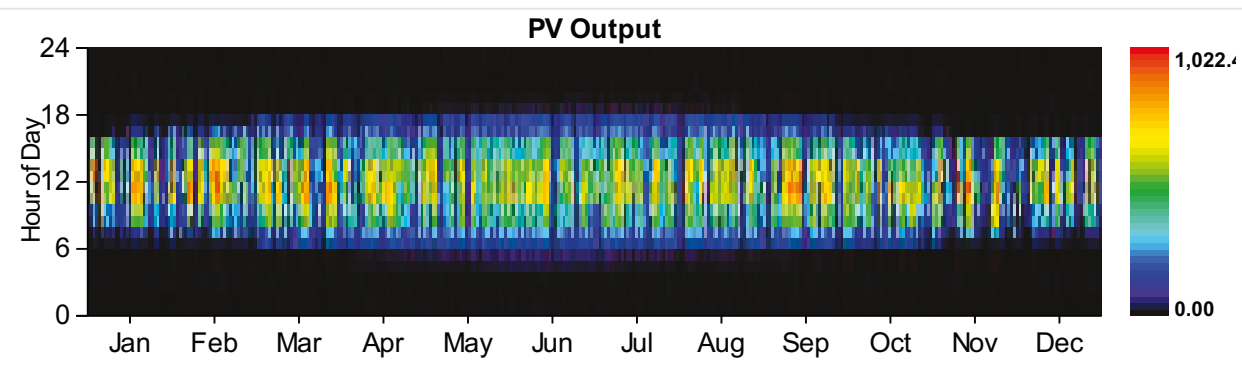

Generator:Kohler 750 Prime Power

\section{Quantity}

Hours of operation

Number of starts

Operational life

Fixed generation cost

Marginal generation cost

\section{Value}

\section{Units}

$162 \mathrm{hrs} / \mathrm{yr}$

146 starts/yr

$93 \mathrm{yr}$

$107.02 \$ / h r$

$0.23 \$ / k W h$ 


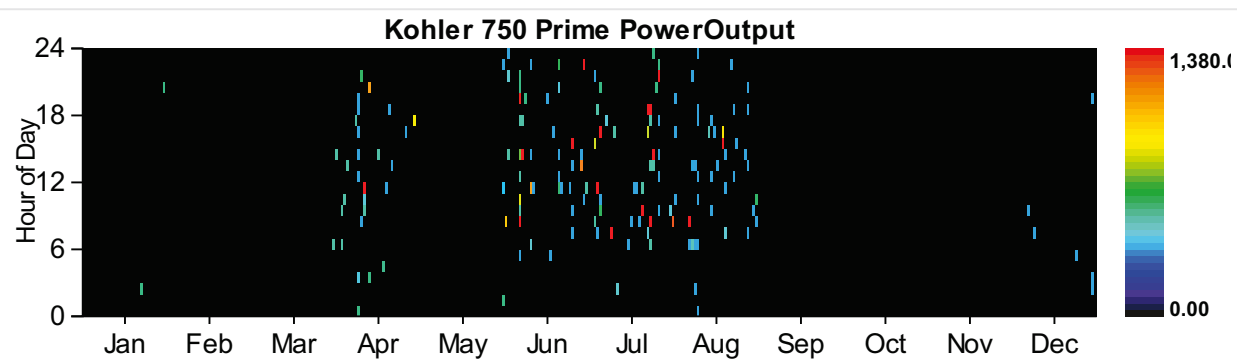

\section{Battery:GS200 flow}

\section{Quantity}

Value

String size

Strings in parallel

\section{Batteries}

Bus voltage

\section{Quantity}

Value

Units

Nominal capacity

Usable nominal capacity

$600 \mathrm{kWh}$

Autonomy

Lifetime throughput

Battery wear cost

$600 \mathrm{kWh}$

Average energy cost

Energy in

Energy out

Storage depletion

Losses

$0 \mathrm{hr}$

Annual throughput

Expected life

0

$0.000 \$ / k W h$

\section{$0.184 \$ / k W h$}

9945 kWh/yr

$6961 \mathrm{kWh} / \mathrm{yr}$

$0 \mathrm{kWh} / \mathrm{yr}$

$2983 \mathrm{kWh} / \mathrm{yr}$

8320 kWh/yr

$25 \mathrm{yr}$ 


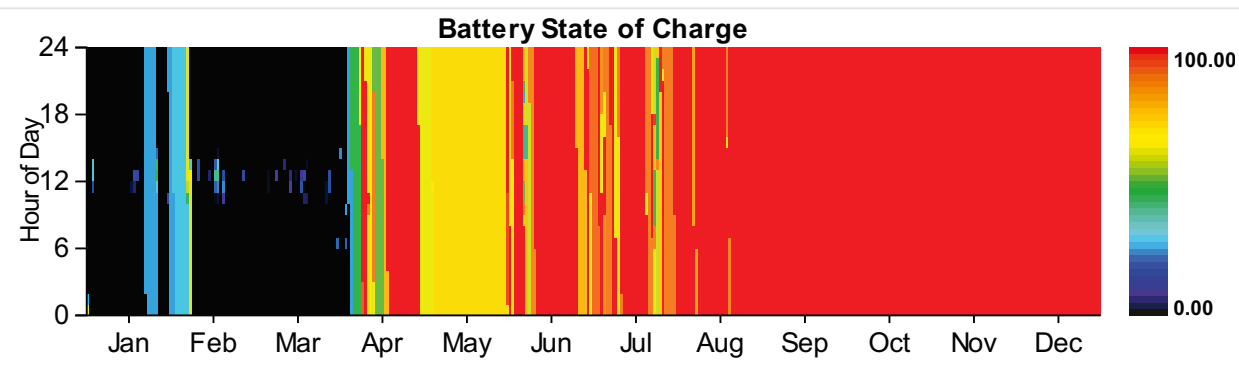

Converter

\begin{tabular}{|c|c|c|c|}
\hline Quantity & Inverter & Rectifier & Units \\
\hline Capacity & 750 & 675 & kW \\
\hline Mean output & 132 & 0 & kW \\
\hline Minimum output & 0 & 0 & $\mathrm{~kW}$ \\
\hline Maximum output & 750 & 179 & $\mathrm{~kW}$ \\
\hline Capacity factor & 18 & 0 & $\%$ \\
\hline Hours of operation & 4,390 & 29 & $\mathrm{hrs} / \mathrm{yr}$ \\
\hline Energy in & $1,286,347$ & 4,001 & kWh/yr \\
\hline Energy out & $1,157,711$ & 3,401 & $\mathrm{kWh} / \mathrm{yr}$ \\
\hline Losses & 128,636 & 600 & kWh/yr \\
\hline
\end{tabular}
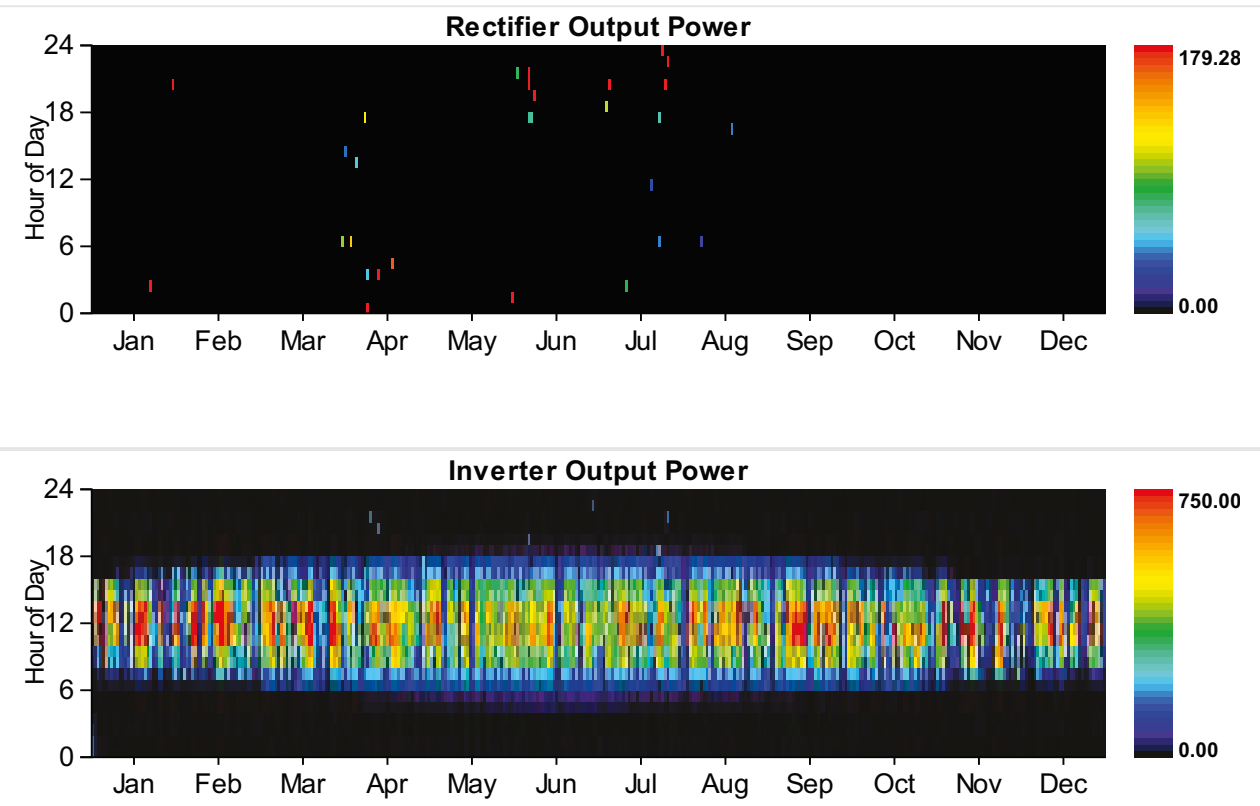

Grid

Rate: Demand 1

\begin{tabular}{|c|c|c|c|c|c|c|}
\hline Resources.ReportingService_GeneratelnputsReport_Month & $\begin{array}{l}\text { Energy } \\
\text { Purchased } \\
\text { (kWh) }\end{array}$ & $\begin{array}{l}\text { Energy } \\
\text { Sold } \\
\text { (kWh) }\end{array}$ & $\begin{array}{l}\text { Net } \\
\text { Purchases } \\
\text { (kWh) }\end{array}$ & $\begin{array}{l}\text { Peak } \\
\text { Demand } \\
(\mathbf{k W})\end{array}$ & $\begin{array}{l}\text { Energy } \\
\text { Charge } \\
(\$)\end{array}$ & $\begin{array}{l}\text { Demand } \\
\text { Charge } \\
\text { (\$) }\end{array}$ \\
\hline January & 0 & 0 & 0 & 18,247 & 0 & 291,953 \\
\hline February & 0 & 0 & 0 & 16,811 & 0 & 268,978 \\
\hline March & 0 & 0 & 0 & 16,941 & 0 & 271,062 \\
\hline
\end{tabular}




\begin{tabular}{|c|c|c|c|c|c|c|}
\hline $\begin{array}{l}\text { April } \\
\text { Resources.ReportingService_GeneratelnputsReport_Month } \\
\text { May }\end{array}$ & $\begin{array}{l}\text { Energy } \\
\text { Purchased } \\
\text { (kWh) } \quad 0\end{array}$ & $\begin{array}{l}\text { Energy }_{0} \\
\text { Sold } \\
\text { (kWh) }\end{array}$ & $\begin{array}{l}\text { Net } \\
\text { Purchases } \\
(\mathrm{kWh}) \quad 0\end{array}$ & $\begin{array}{l}\text { Peak } \\
\text { Demiona } \\
\text { (kW) } \\
17,657\end{array}$ & $\begin{array}{l}\text { Energy } \\
\text { Charge } \\
\text { (\$) } \quad 0\end{array}$ & $\begin{array}{l}\text { Demand } \\
\text { charge } \\
(\$ 282,514\end{array}$ \\
\hline June & 0 & 0 & 0 & 20,000 & 0 & 320,000 \\
\hline July & 0 & 0 & 0 & 20,000 & 0 & 320,000 \\
\hline August & 0 & 0 & 0 & 20,000 & 0 & 320,000 \\
\hline September & 0 & 0 & 0 & 16,176 & 0 & 258,820 \\
\hline October & 0 & 0 & 0 & 14,744 & 0 & 235,900 \\
\hline November & 0 & 0 & 0 & 16,518 & 0 & 264,286 \\
\hline December & 0 & 0 & 0 & 19,546 & 0 & 312,740 \\
\hline Annual & 0 & 0 & 0 & 20,000 & 0 & $3,466,253$ \\
\hline
\end{tabular}

Rate: Rate 1

\begin{tabular}{|c|c|c|c|c|c|c|}
\hline Resources.ReportingService_GeneratelnputsReport_Month & $\begin{array}{l}\text { Energy } \\
\text { Purchased } \\
\text { (kWh) }\end{array}$ & $\begin{array}{l}\text { Energy } \\
\text { Sold } \\
\text { (kWh) }\end{array}$ & $\begin{array}{l}\text { Net } \\
\text { Purchases } \\
\text { (kWh) }\end{array}$ & $\begin{array}{l}\text { Peak } \\
\text { Demand } \\
(\mathbf{k W})\end{array}$ & $\begin{array}{l}\text { Energy } \\
\text { Charge (\$) }\end{array}$ & $\begin{array}{l}\text { Demand } \\
\text { Charge } \\
\text { (\$) }\end{array}$ \\
\hline January & $8,130,624$ & 0 & $8,130,624$ & 0 & 975,675 & 0 \\
\hline February & $6,023,001$ & 0 & $6,023,001$ & 0 & 722,760 & 0 \\
\hline March & $6,708,603$ & 0 & $6,708,603$ & 0 & 805,032 & 0 \\
\hline April & $9,346,668$ & 0 & $9,346,668$ & 0 & $1,121,600$ & 0 \\
\hline May & $7,697,129$ & 0 & $7,697,129$ & 0 & 923,655 & 0 \\
\hline June & $9,679,151$ & 0 & $9,679,151$ & 0 & $1,161,498$ & 0 \\
\hline July & $9,859,018$ & 0 & $9,859,018$ & 0 & $1,183,082$ & 0 \\
\hline August & $9,771,599$ & 0 & $9,771,599$ & 0 & $1,172,592$ & 0 \\
\hline September & $6,837,491$ & 0 & $6,837,491$ & 0 & 820,499 & 0 \\
\hline October & $6,699,125$ & 0 & $6,699,125$ & 0 & 803,895 & 0 \\
\hline November & $6,249,541$ & 0 & $6,249,541$ & 0 & 749,945 & 0 \\
\hline December & $8,608,349$ & 0 & $8,608,349$ & 0 & $1,033,002$ & 0 \\
\hline Annual & $95,610,304$ & 0 & $95,610,304$ & 0 & $11,473,236$ & 0 \\
\hline
\end{tabular}

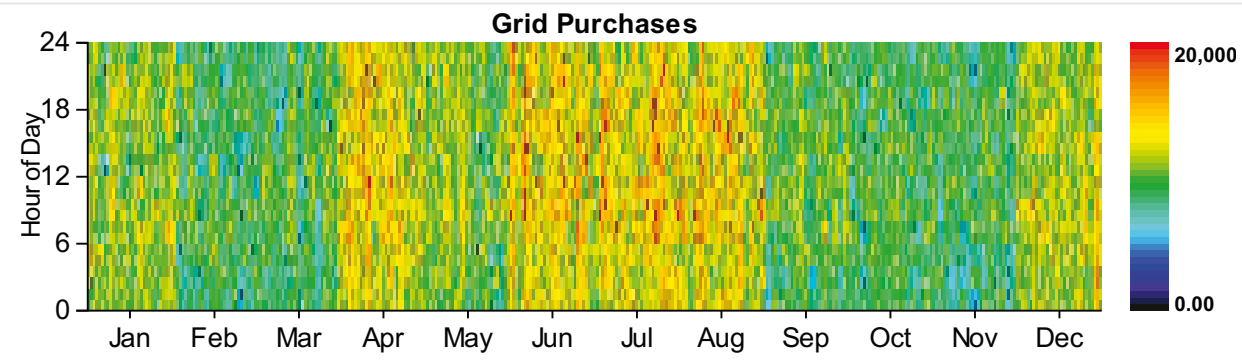




\section{Emissions}

Pollutant

Carbon dioxide

Carbon monoxide

Unburned hydrocarbons

Particulate matter

Sulfur dioxide

Nitrogen oxides
Emissions

Units

$60494300 \mathrm{~kg} / \mathrm{yr}$

287 kg/yr

$33 \mathrm{~kg} / \mathrm{yr}$

$8 \mathrm{~kg} / \mathrm{yr}$

$262113 \mathrm{~kg} / \mathrm{yr}$

$128405 \mathrm{~kg} / \mathrm{yr}$

HOMER Energy, LLC $\odot 2016$ 
System Report

System architecture

\begin{tabular}{|l|l|l|}
\hline PV & Solar World 320W flat plate PV Copy & 1,000 \\
\hline Generator \#2 & Kohler 750 Prime Power & 2,070 \\
\hline Converter & System Converter & 750 \\
\hline Grid & Grid & $\mathrm{kW}$ \\
\hline Dispatch Strategy & Cycle Charging & 20,000 \\
\hline
\end{tabular}

Cost summary

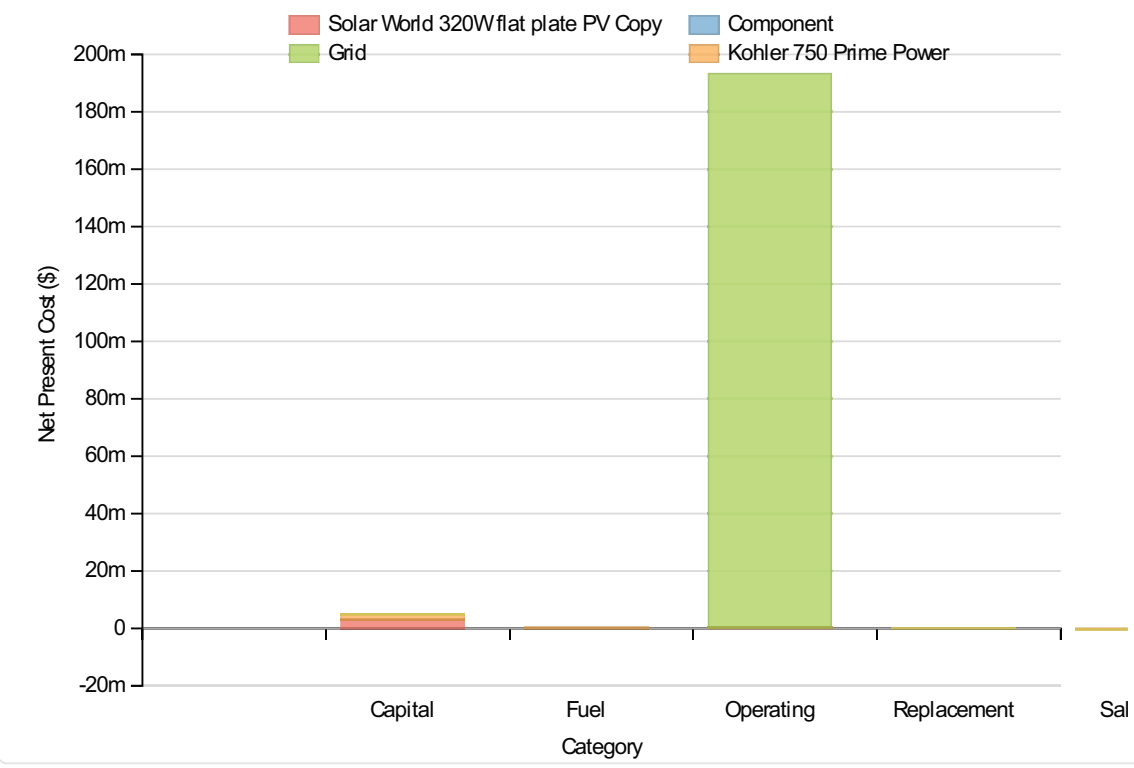

Cost Summary

Total net present cost

$198437776 \$$

Levelized cost of energy

$0.158 \$ / k W h$

Net Present Costs

\begin{tabular}{|l|r|r|r|r|r|r|}
\hline Component & Capital & Replacement & O\&M & Fuel & Salvage & Total \\
\hline Solar World 320W flat plate PV Copy & $3,000,000$ & 0 & 193,913 & 0 & 0 \\
\hline Kohler 750 Prime Power & $1,802,500$ & 0 & 70,150 & 364,555 & $-301,542$ \\
\hline Grid & 0 & 0 & $193,905,632$ & 0 & 0 \\
\hline Converter & 225,000 & 95,462 & 0 & $-17,963,005,632$ \\
\hline System & $5,027,500$ & 95,462 & $193,269,680$ & 364,555 & $-319,509$ \\
\hline
\end{tabular}

\section{Annualized Costs}

\section{Component}

Solar World 320W flat plate PV Copy

Kohler 750 Prime Power

Grid

Converter

\begin{tabular}{|r|r|r|r|r|r|r|}
\hline Capital & Replacement & O\&M & Fuel & Salvage & Total \\
\hline 232,063 & 0 & 15,000 & 0 & 0 & 247,063 \\
\hline 139,431 & 0 & 5,426 & 28,200 & $-23,326$ & 149,731 \\
\hline 0 & 0 & $14,929,831$ & 0 & 0 & $14,929,831$ \\
\hline 17,405 & 7,384 & 0 & 0 & $-1,390$ & 23,400 \\
\hline
\end{tabular}




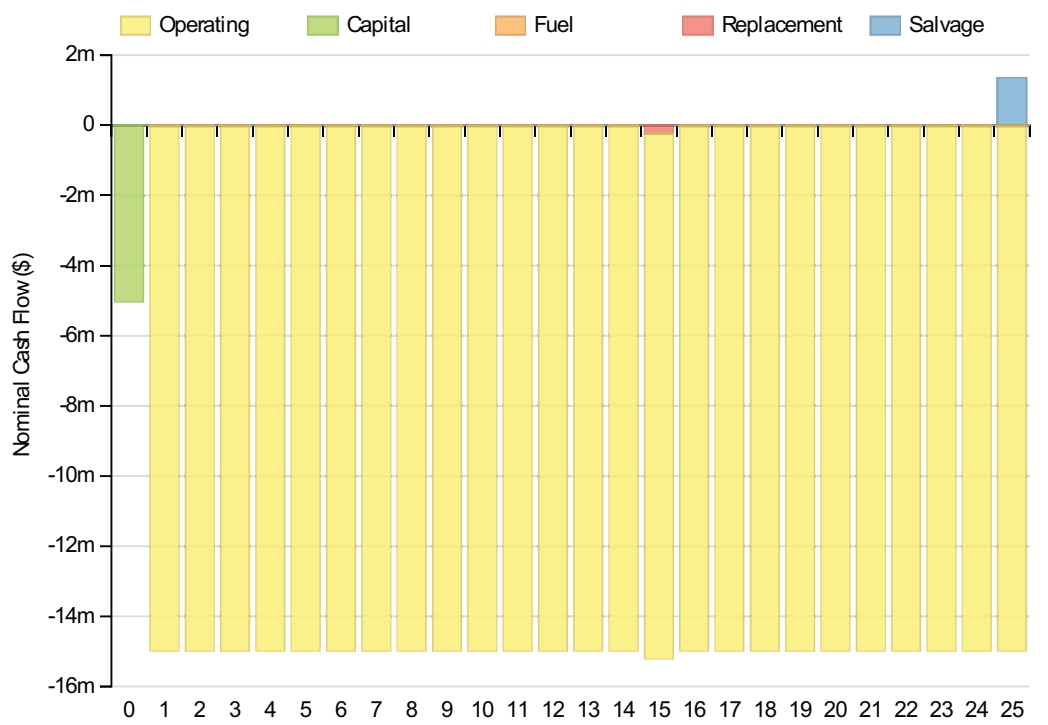

Year

\section{Electrical}

\begin{tabular}{|c|c|c|c|c|}
\hline \multicolumn{2}{|l|}{ Quantity } & \multicolumn{2}{|l|}{ Value } & Units \\
\hline Excess electricity & & & 5225 & $\mathrm{kWh} / \mathrm{yr}$ \\
\hline Unmet load & & & 6192 & $\mathrm{kWh} / \mathrm{yr}$ \\
\hline Capacity shortage & & & 0344 & kWh/yr \\
\hline Renewable fraction & & & 0 & \\
\hline Component & Production(kWh/yr) & & Fract & ion (\%) \\
\hline PV & & $1,288,675$ & & 1 \\
\hline Generator & & 121,784 & & 0 \\
\hline Grid Purchases & & $95,581,120$ & & 99 \\
\hline Total & & $96,991,576$ & & 100 \\
\hline Load & Consumption(kWh/yr) & & Fra & Iction (\%) \\
\hline AC primary load & & $96,857,960$ & & 100 \\
\hline DC primary load & & & 0 & 0 \\
\hline Total & & $96,857,960$ & & 100 \\
\hline
\end{tabular}




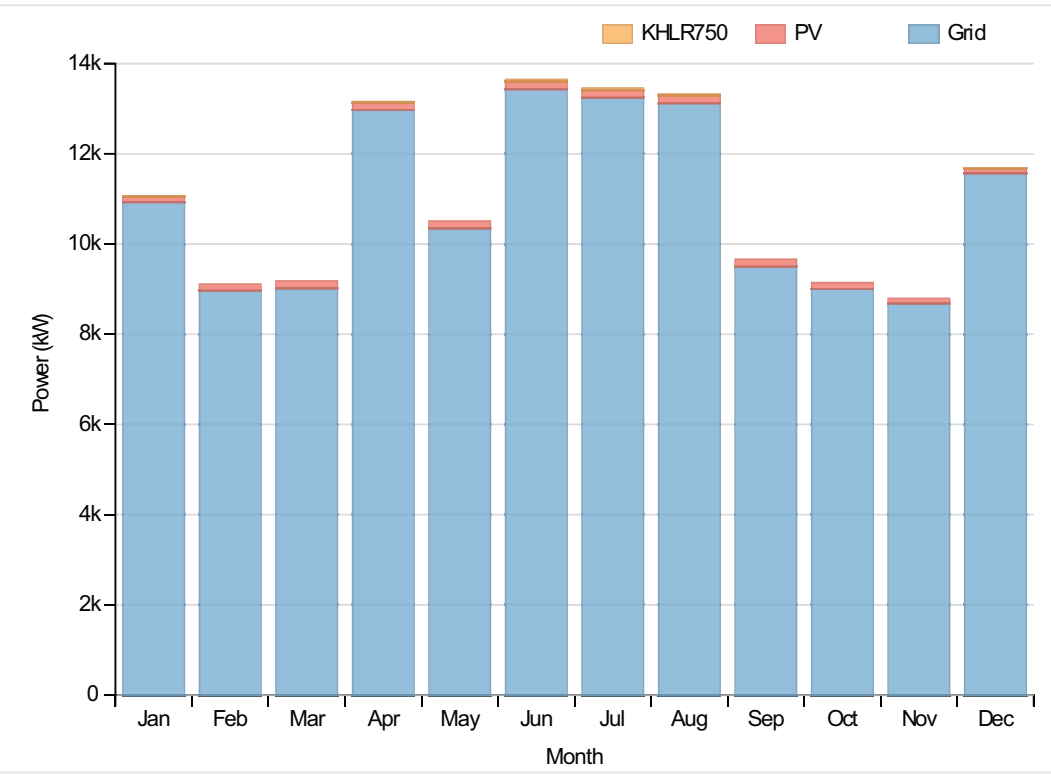

PV:Solar World 320W flat plate PV Copy

\begin{tabular}{|l|c|c|}
\hline Quantity & Value & Units \\
\hline Rated capacity & $1000 \mathrm{~kW}$ \\
\hline Mean output & $147 \mathrm{~kW}$ \\
\hline Mean output & $3530.60 \mathrm{kWh} / \mathrm{d}$ \\
\hline Capacity factor & $14.71 \%$ \\
\hline Total production & $1288675 \mathrm{kWh} / \mathrm{yr}$ \\
\hline Minimum output & $0.00 \mathrm{~kW}$ \\
\hline Maximum output & $1022.50 \mathrm{~kW}$ \\
\hline PV penetration & $1.33 \%$ \\
\hline Hours of operation & $4377 \mathrm{hrs} / \mathrm{yr}$ \\
\hline Levelized cost & $\$ 1 / \mathrm{kWh}$ \\
\hline
\end{tabular}

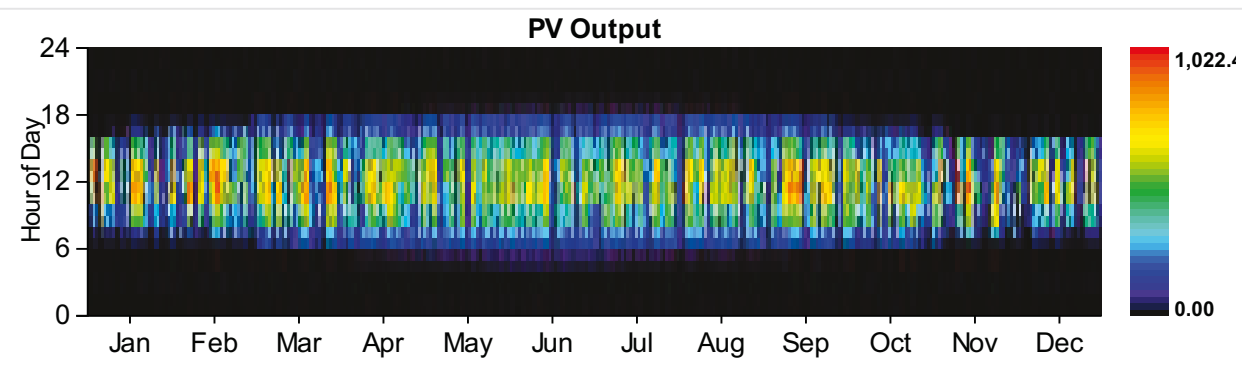

Generator:Kohler 750 Prime Power

\section{Quantity}

Hours of operation

Number of starts

Operational life

Fixed generation cost

Marginal generation cost

\section{Value}

\section{Units}

$181 \mathrm{hrs} / \mathrm{yr}$

162 starts/yr

$83 \mathrm{yr}$

$151.69 \$ / \mathrm{hr}$ 
Mean electrical output

Min. electrical output

Max. electrical output

Fuel consumption

Specific fuel consumption

Fuel energy input

Mean electrical efficiency

\begin{tabular}{|r|l|l|}
\hline 673 & $\mathrm{~kW}$ \\
\hline 518 & $\mathrm{~kW}$ \\
\hline 2070 & $\mathrm{~kW}$ \\
\hline 35696 & $\mathrm{~L} / \mathrm{yr}$ \\
\hline 0.29 & $\mathrm{~L} / \mathrm{kWh}$ \\
\hline 351250 & $\mathrm{kWh} / \mathrm{yr}$ \\
\hline 35 & $\%$ \\
\hline
\end{tabular}

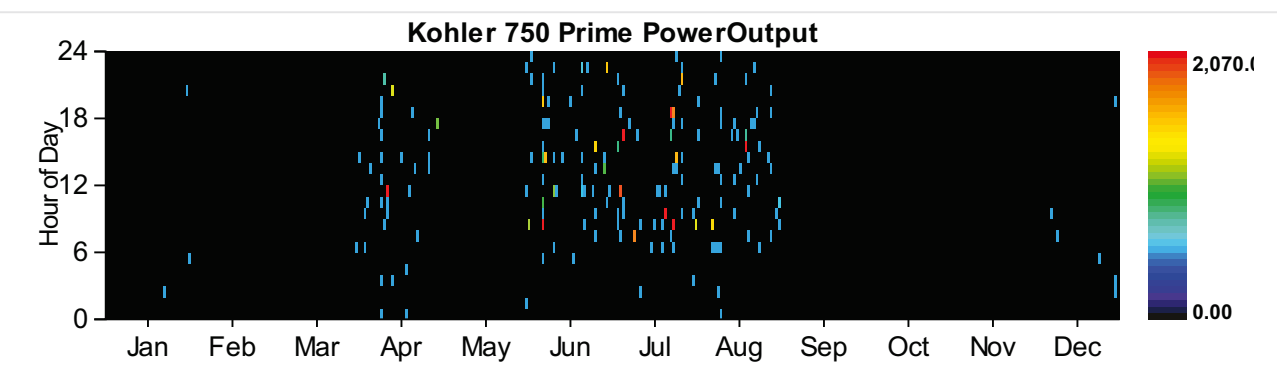

\section{Converter}

\section{Quantity}

Capacity

Mean output

Minimum output

Maximum output

Capacity factor

Hours of operation

Energy in

Energy out

Losses

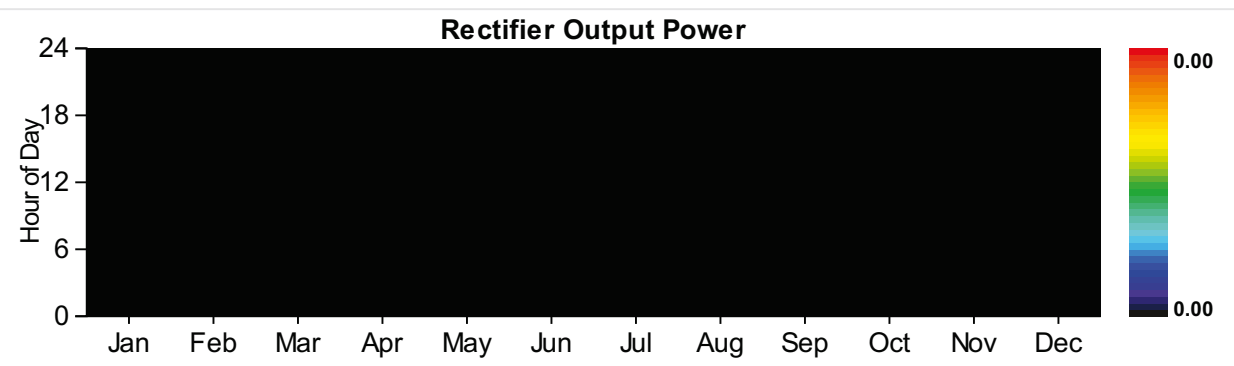

\begin{tabular}{|r|r|l|l|}
\hline Inverter & Rectifier & Units \\
\hline 750 & 675 & $\mathrm{~kW}$ \\
\hline 132 & 0 & $\mathrm{~kW}$ \\
\hline 750 & 0 & $\mathrm{~kW}$ \\
\hline 18 & 0 & $\mathrm{~kW}$ \\
\hline 4,377 & 0 & $\%$ \\
\hline $1,283,450$ & 0 & $\mathrm{hrs} / \mathrm{yr}$ \\
\hline $1,155,104$ & 0 & $\mathrm{kWh} / \mathrm{yr}$ \\
\hline 128,346 & 0 & $\mathrm{kWh} / \mathrm{yr}$ \\
\hline & 0 & $\mathrm{kWh} / \mathrm{yr}$ \\
\hline
\end{tabular}




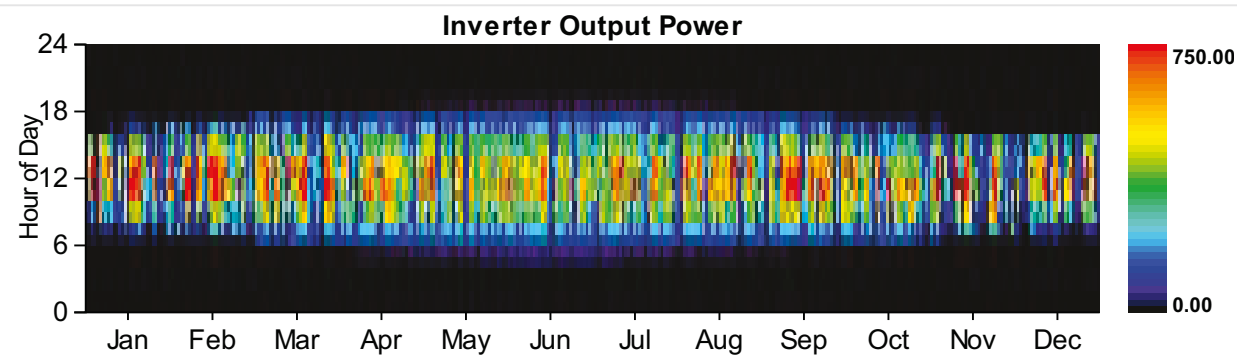

Grid

Rate: Demand 1

\begin{tabular}{|c|c|c|c|c|c|c|}
\hline Resources.ReportingService_GeneratelnputsReport_Month & $\begin{array}{l}\text { Energy } \\
\text { Purchased } \\
\text { (kWh) }\end{array}$ & $\begin{array}{l}\text { Energy } \\
\text { Sold } \\
\text { (kWh) }\end{array}$ & $\begin{array}{l}\text { Net } \\
\text { Purchases } \\
\text { (kWh) }\end{array}$ & $\begin{array}{l}\text { Peak } \\
\text { Demand } \\
(\mathbf{k W})\end{array}$ & $\begin{array}{l}\text { Energy } \\
\text { Charge } \\
\text { (\$) }\end{array}$ & $\begin{array}{l}\text { Demand } \\
\text { Charge } \\
(\$)\end{array}$ \\
\hline January & 0 & 0 & 0 & 18,035 & 0 & 288,563 \\
\hline February & 0 & 0 & 0 & 16,811 & 0 & 268,978 \\
\hline March & 0 & 0 & 0 & 16,941 & 0 & 271,062 \\
\hline April & 0 & 0 & 0 & 20,000 & 0 & 320,000 \\
\hline May & 0 & 0 & 0 & 17,657 & 0 & 282,514 \\
\hline June & 0 & 0 & 0 & 20,000 & 0 & 320,000 \\
\hline July & 0 & 0 & 0 & 20,000 & 0 & 320,000 \\
\hline August & 0 & 0 & 0 & 20,000 & 0 & 320,000 \\
\hline September & 0 & 0 & 0 & 16,176 & 0 & 258,820 \\
\hline October & 0 & 0 & 0 & 14,744 & 0 & 235,900 \\
\hline November & 0 & 0 & 0 & 16,518 & 0 & 264,286 \\
\hline December & 0 & 0 & 0 & 19,374 & 0 & 309,980 \\
\hline Annual & 0 & 0 & 0 & 20,000 & 0 & $3,460,103$ \\
\hline
\end{tabular}

Rate: Rate 1

\begin{tabular}{|c|c|c|c|c|c|c|}
\hline Resources.ReportingService_GeneratelnputsReport_Month & $\begin{array}{l}\text { Energy } \\
\text { Purchased } \\
\text { (kWh) }\end{array}$ & $\begin{array}{l}\text { Energy } \\
\text { Sold } \\
\text { (kWh) }\end{array}$ & $\begin{array}{l}\text { Net } \\
\text { Purchases } \\
\text { (kWh) }\end{array}$ & $\begin{array}{l}\text { Peak } \\
\text { Demand } \\
(\mathbf{k W})\end{array}$ & $\begin{array}{l}\text { Energy } \\
\text { Charge (\$) }\end{array}$ & $\begin{array}{l}\text { Demand } \\
\text { Charge } \\
(\$)\end{array}$ \\
\hline January & $8,130,726$ & 0 & $8,130,726$ & 0 & 975,687 & 0 \\
\hline February & $6,023,890$ & 0 & $6,023,890$ & 0 & 722,867 & 0 \\
\hline March & $6,708,956$ & 0 & $6,708,956$ & 0 & 805,075 & 0 \\
\hline April & $9,340,340$ & 0 & $9,340,340$ & 0 & $1,120,841$ & 0 \\
\hline May & $7,697,129$ & 0 & $7,697,129$ & 0 & 923,655 & 0 \\
\hline June & $9,671,285$ & 0 & $9,671,285$ & 0 & $1,160,554$ & 0 \\
\hline July & $9,852,042$ & 0 & $9,852,042$ & 0 & $1,182,245$ & 0 \\
\hline August & $9,763,277$ & 0 & $9,763,277$ & 0 & $1,171,593$ & 0 \\
\hline
\end{tabular}




\begin{tabular}{|c|c|c|c|c|c|c|}
\hline $\begin{array}{l}\text { Qctober } \\
\text { Resources.ReportingService_GeneratelnputsReport_Month }\end{array}$ & $\begin{array}{l}\text { EÂerğy } 491 \\
\text { Purchased } \\
\text { (kWh) }\end{array}$ & $\begin{array}{l}\text { Energy } \\
\text { Sold } 0 \\
(\mathbf{k W h})\end{array}$ & $\begin{array}{l}\text { Nêt837,491 } \\
\text { Purchases } \\
\text { (kWh) }\end{array}$ & $\begin{array}{l}\text { Peak } 0 \\
\text { Demand } \\
(\mathbf{k W})\end{array}$ & $\begin{array}{l}\text { 820,499 } \\
\text { Energy } \\
\text { Charges, } \\
\text { Charg) }\end{array}$ & $\begin{array}{l}\text { Demand } \\
\text { Charge } \\
(\$)\end{array}$ \\
\hline November & $6,249,541$ & 0 & $6,249,541$ & 0 & 749,945 & 0 \\
\hline December & $8,607,314$ & 0 & $8,607,314$ & 0 & $1,032,878$ & 0 \\
\hline Annual & $95,581,120$ & 0 & $95,581,120$ & 0 & $11,469,734$ & 0 \\
\hline
\end{tabular}

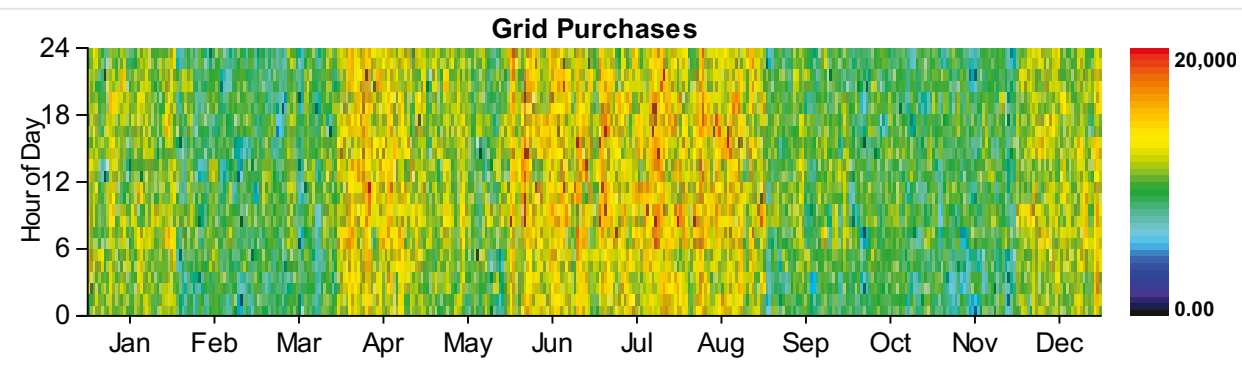

\section{Emissions}

\begin{tabular}{|l|r|l|}
\hline Pollutant & Emissions & Units \\
\hline Carbon dioxide & $60500952 \mathrm{~kg} / \mathrm{yr}$ \\
\hline Carbon monoxide & $393 \mathrm{~kg} / \mathrm{yr}$ \\
\hline Unburned hydrocarbons & $45 \mathrm{~kg} / \mathrm{yr}$ \\
\hline Particulate matter & $11 \mathrm{~kg} / \mathrm{yr}$ \\
\hline Sulfur dioxide & $262085 \mathrm{~kg} / \mathrm{yr}$ \\
\hline Nitrogen oxides & $128471 \mathrm{~kg} / \mathrm{yr}$ \\
\hline
\end{tabular}


System Report

System architecture

\begin{tabular}{|l|l|r|}
\hline PV & Solar World 320W flat plate PV Copy & 1,000 \\
\hline Generator & Kohler 1000 Prime Power & kW \\
\hline Generator \#2 & Kohler 750 Prime Power & kW \\
\hline Battery & GS200 flow & 690 \\
\hline Converter & System Converter & 1 \\
\hline Grid & Grid & strings \\
\hline Dispatch Strategy & Cycle Charging & 20,000 \\
\hline
\end{tabular}

\section{Cost summary}

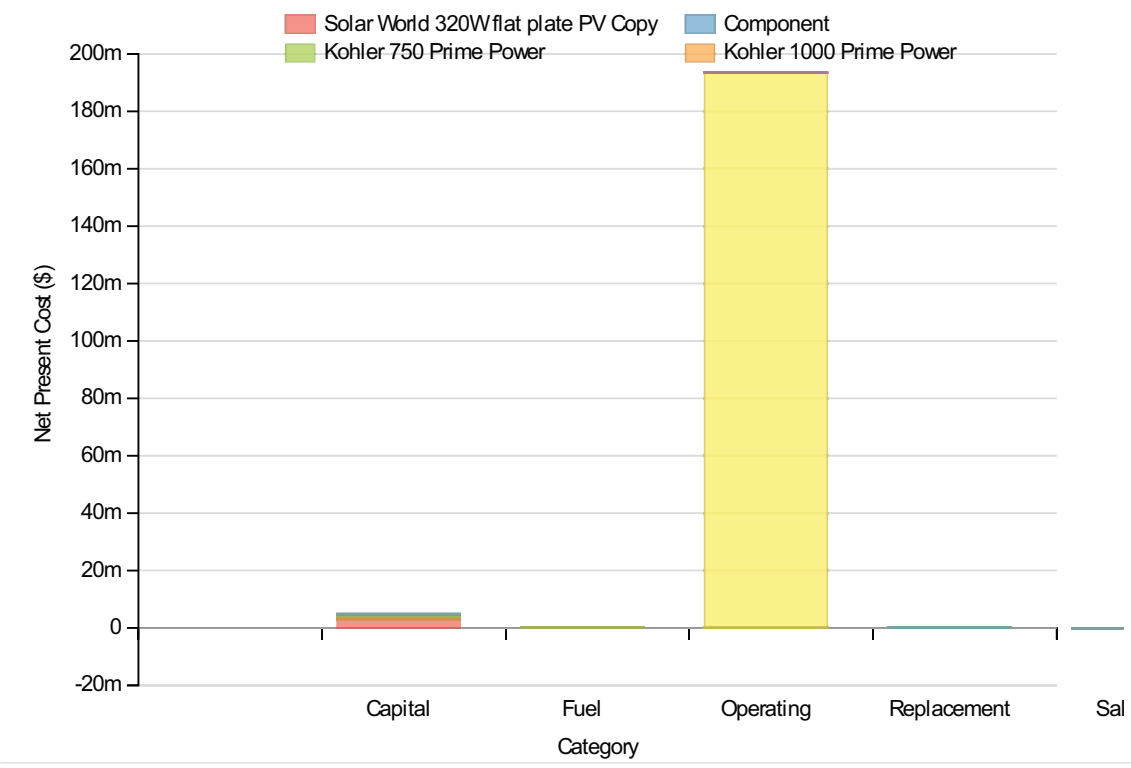

Cost Summary

Total net present cost

198614768 \$

Levelized cost of energy

$0.159 \$ / k W h$

\section{Net Present Costs}

\begin{tabular}{|c|c|c|c|c|c|c|}
\hline Component & Capital & Replacement & O\&M & Fuel & Salvage & Total \\
\hline Solar World 320W flat plate PV Copy & $3,000,000$ & 0 & 193,913 & 0 & 0 & $3,193,913$ \\
\hline Kohler 1000 Prime Power & 925,000 & 0 & 19,910 & 140,672 & $-180,596$ & 904,986 \\
\hline Kohler 750 Prime Power & 690,000 & 0 & 19,802 & 109,855 & $-124,522$ & 695,135 \\
\hline Grid & 0 & 0 & $193,156,336$ & 0 & 0 & $193,156,336$ \\
\hline GS200 flow & 324,589 & 7,176 & 31,026 & 0 & -973 & 361,818 \\
\hline Converter & 225,000 & 95,462 & 0 & 0 & $-17,967$ & 302,495 \\
\hline System & $5,164,589$ & 102,637 & $193,420,960$ & 250,527 & $-324,058$ & $198,614,655$ \\
\hline
\end{tabular}




\begin{tabular}{|c|c|c|c|c|c|c|}
\hline Component & Capital & Replacement & O\&M & Fuel & Salvage & Total \\
\hline Solar World 320W flat plate PV Copy & 232,063 & 0 & 15,000 & 0 & 0 & 247,063 \\
\hline Kohler 1000 Prime Power & 71,553 & 0 & 1,540 & 10,882 & $-13,970$ & 70,005 \\
\hline Kohler 750 Prime Power & 53,375 & 0 & 1,532 & 8,498 & $-9,632$ & 53,772 \\
\hline Grid & 0 & 0 & $14,941,488$ & 0 & 0 & $14,941,488$ \\
\hline GS200 flow & 25,108 & 555 & 2,400 & 0 & -75 & 27,988 \\
\hline Converter & 17,405 & 7,384 & 0 & 0 & $-1,390$ & 23,400 \\
\hline System & 399,504 & 7,939 & $14,961,958$ & 19,379 & $-25,067$ & $15,363,713$ \\
\hline
\end{tabular}

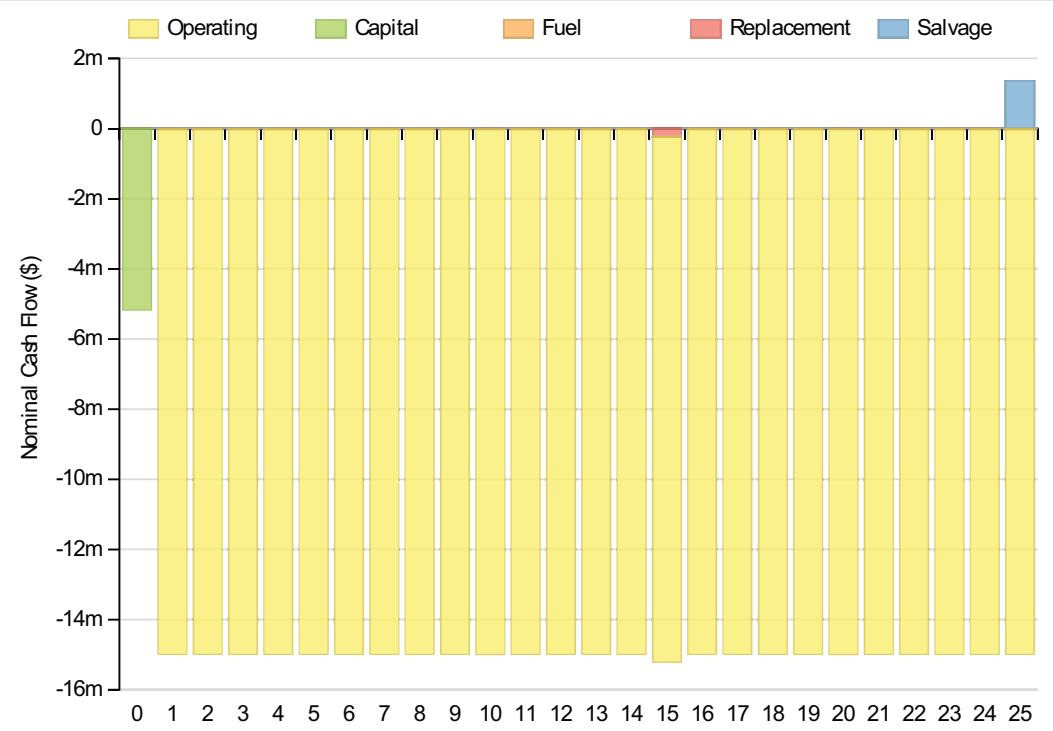

Year

\section{Electrical}

\begin{tabular}{|c|c|c|c|c|}
\hline \multirow{2}{*}{\multicolumn{2}{|c|}{$\begin{array}{l}\text { Quantity } \\
\text { Excess electricity }\end{array}$}} & \multicolumn{2}{|l|}{ Value } & Units \\
\hline & & & 745 & $\mathrm{kWh} / \mathrm{yr}$ \\
\hline Unmet load & & & 389 & $\mathrm{kWh} / \mathrm{yr}$ \\
\hline Capacity shortage & & & 683 & $\mathrm{kWh} / \mathrm{yr}$ \\
\hline Renewable fraction & & & 0 & \\
\hline Component & Production(kWh/yr) & & iracti & tion (\%) \\
\hline PV & & $1,288,675$ & & 1 \\
\hline Generator & & 47,703 & & 0 \\
\hline Generator & & 36,734 & & 0 \\
\hline Grid Purchases & & $95,617,096$ & & 99 \\
\hline Total & & $96,990,208$ & & 100 \\
\hline Load & Consumption(kWh/yr) & & Fra & action (\%) \\
\hline AC primary load & & $96,855,776$ & & 100 \\
\hline DC primary load & & 0 & & 0 \\
\hline
\end{tabular}




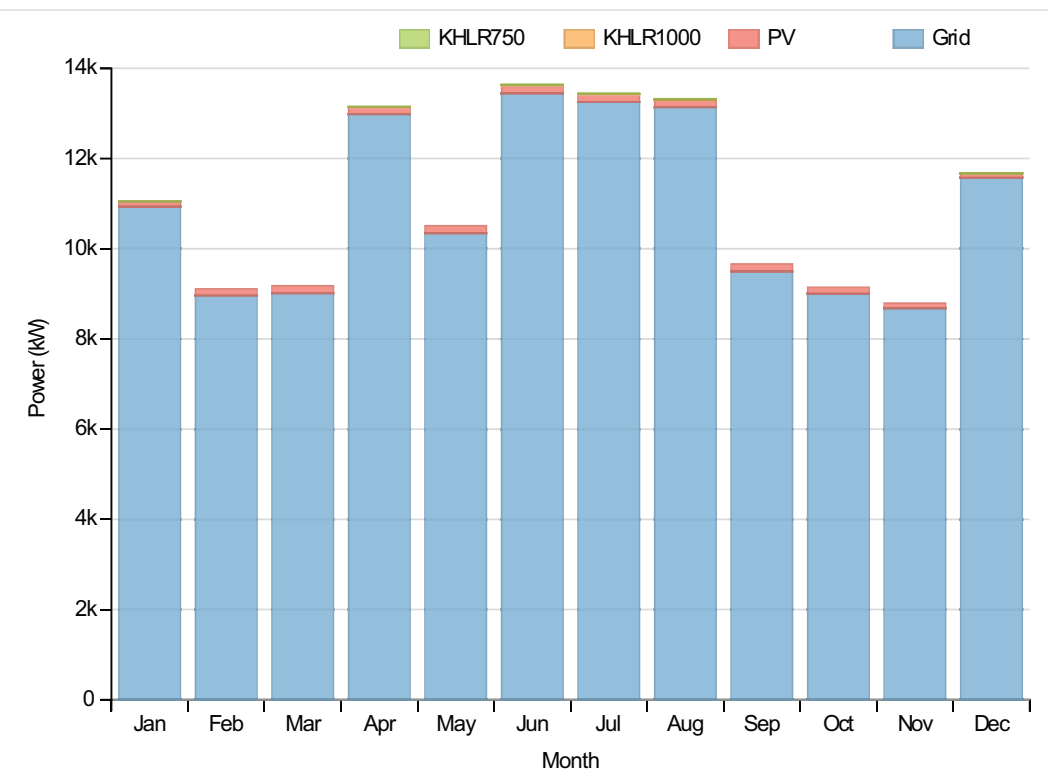

PV:Solar World 320W flat plate PV Copy

\begin{tabular}{|c|c|c|}
\hline Quantity & Value & Units \\
\hline Rated capacity & 1000 & $\mathrm{~kW}$ \\
\hline Mean output & 147 & $\mathrm{~kW}$ \\
\hline Mean output & 3530.60 & $\mathrm{kWh} / \mathrm{d}$ \\
\hline Capacity factor & 14.71 & $\%$ \\
\hline Total production & 1288675 & $\mathrm{kWh} / \mathrm{yr}$ \\
\hline Minimum output & 0.00 & $\mathrm{~kW}$ \\
\hline Maximum output & 1022.50 & $\mathrm{~kW}$ \\
\hline PV penetration & 1.33 & $\%$ \\
\hline Hours of operation & 4377 & $\mathrm{hrs} / \mathrm{yr}$ \\
\hline Levelized cost & 0.192 & $\$ / k W h$ \\
\hline
\end{tabular}

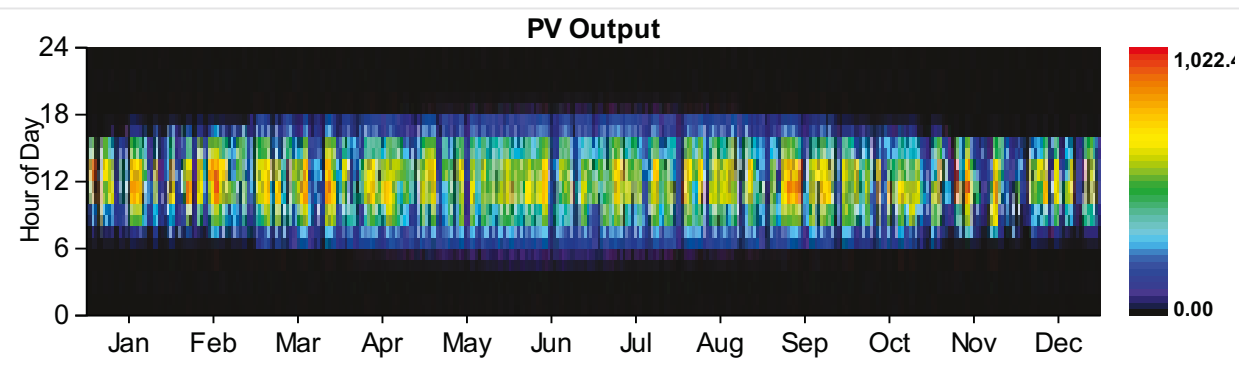

Generator:Kohler 1000 Prime Power

\section{Quantity}

Hours of operation

Number of starts

Operational life

\section{Value}

Units

$111 \mathrm{hrs} / \mathrm{yr}$

102 starts/yr

$135 \mathrm{yr}$ 
Quxąhtieyeryeration cost

Marginal generation cost

Electrical production

Mean electrical output

Min. electrical output

Max. electrical output

Fuel consumption

Specific fuel consumption

Fuel energy input

Mean electrical efficiency

\subsection{Bhlits}

$0.23 \$ / k W h$

$47703 \mathrm{kWh} / \mathrm{yr}$

$430 \mathrm{~kW}$

$231 \mathrm{~kW}$

$925 \mathrm{~kW}$

13774 L/yr

$0.29 \mathrm{~L} / \mathrm{kWh}$

$135538 \mathrm{kWh} / \mathrm{yr}$

$35 \%$

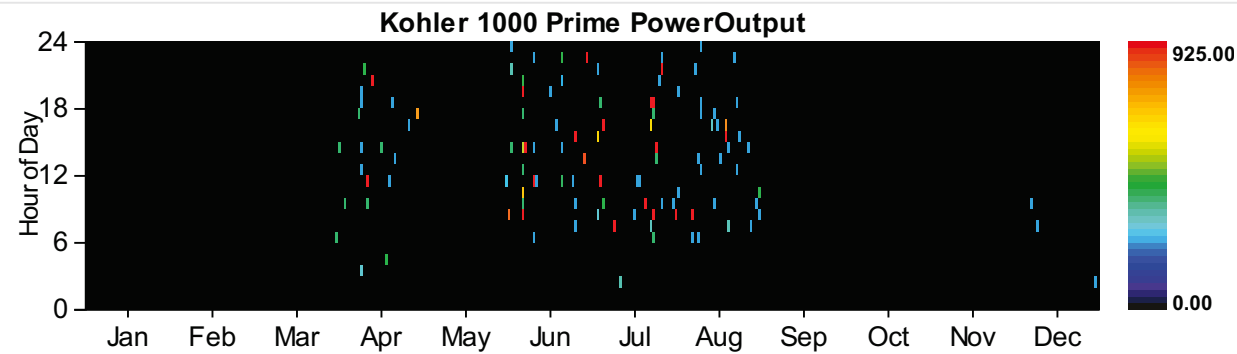

\section{Generator:Kohler 750 Prime Power}

\section{Quantity}

Hours of operation

Number of starts

Operational life

Fixed generation cost

Marginal generation cost

Electrical production

Mean electrical output

Min. electrical output

Max. electrical output

Fuel consumption

Specific fuel consumption

Fuel energy input

Mean electrical efficiency

\begin{tabular}{|r|l|l} 
Value & Units \\
\hline 148 & $\mathrm{hrs} / \mathrm{yr}$ \\
\hline 134 & $\mathrm{starts} / \mathrm{yr}$ \\
\hline 101 & $\mathrm{yr}$ \\
\hline 56.87 & $\$ / \mathrm{hr}$ \\
\hline 0.23 & $\$ / \mathrm{kWh}$ \\
\hline 36734 & $\mathrm{kWh} / \mathrm{yr}$ \\
\hline 248 & $\mathrm{~kW}$ \\
\hline 173 & $\mathrm{~kW}$ \\
\hline 690 & $\mathrm{~kW}$ \\
\hline 10757 & $\mathrm{~L} / \mathrm{yr}$ \\
\hline 0.29 & $\mathrm{~L} / \mathrm{kWh}$ \\
\hline 105846 & $\mathrm{kWh} / \mathrm{yr}$ \\
\hline 35 & $\%$ \\
\hline
\end{tabular}




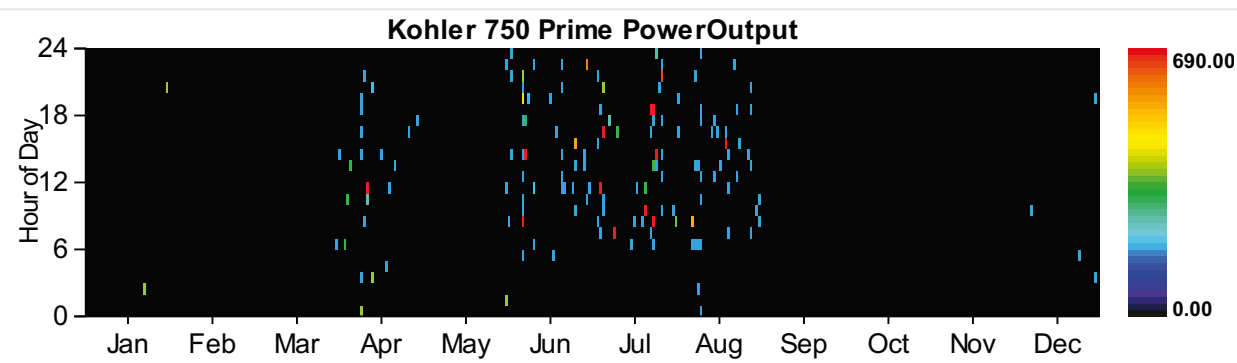

\section{Battery:GS200 flow}

\section{Quantity}

\section{Value}

String size

Strings in parallel

\section{Batteries}

Bus voltage

\section{Quantity}

Value

Units

Nominal capacity

$600 \mathrm{kWh}$

Usable nominal capacity

600 kWh

\section{Autonomy}

Lifetime throughput

$\mathrm{hr}$

Battery wear cost

0

Average energy cost

$0.000 \$ / \mathrm{kWh}$

Energy in

$0.176 \$ / k W h$

Energy out

Storage depletion

Losses

$8596 \mathrm{kWh} / \mathrm{yr}$

6017 kWh/yr

$0 \mathrm{kWh} / \mathrm{yr}$

$2579 \mathrm{kWh} / \mathrm{yr}$

Annual throughput

$7192 \mathrm{kWh} / \mathrm{yr}$

Expected life

$25 \mathrm{yr}$

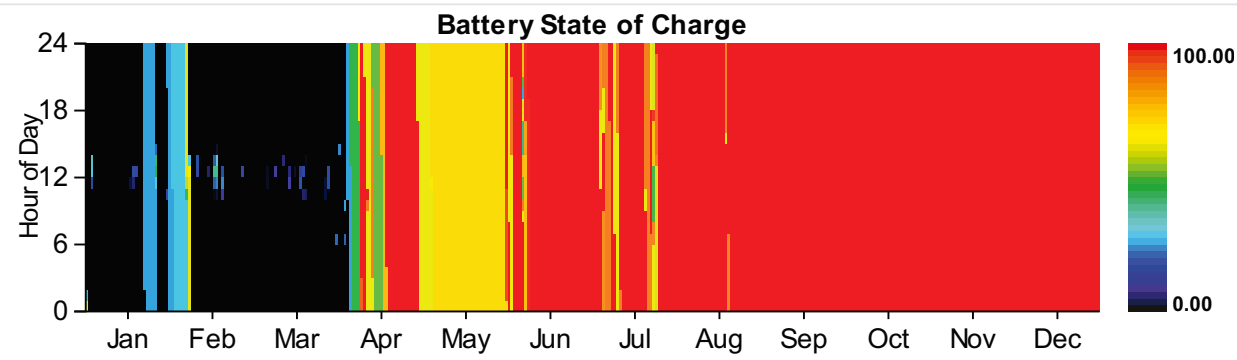

\section{Converter}

\section{Quantity}

Capacity

\section{Inverter}

\section{Units}

$675 \mathrm{~kW}$ 


\begin{tabular}{|c|c|c|c|}
\hline Qeanetityput & Inverter & Rectifier & WNits \\
\hline Minimum output & 0 & 0 & $\mathrm{~kW}$ \\
\hline Maximum output & 750 & 179 & $\mathrm{~kW}$ \\
\hline Capacity factor & 18 & 0 & $\%$ \\
\hline Hours of operation & 4,389 & 26 & $\mathrm{hrs} / \mathrm{yr}$ \\
\hline Energy in & $1,286,067$ & 3,196 & $\mathrm{kWh} / \mathrm{yr}$ \\
\hline Energy out & $1,157,460$ & 2,716 & $\mathrm{kWh} / \mathrm{yr}$ \\
\hline Losses & 128,608 & 479 & $\mathrm{kWh} / \mathrm{yr}$ \\
\hline
\end{tabular}
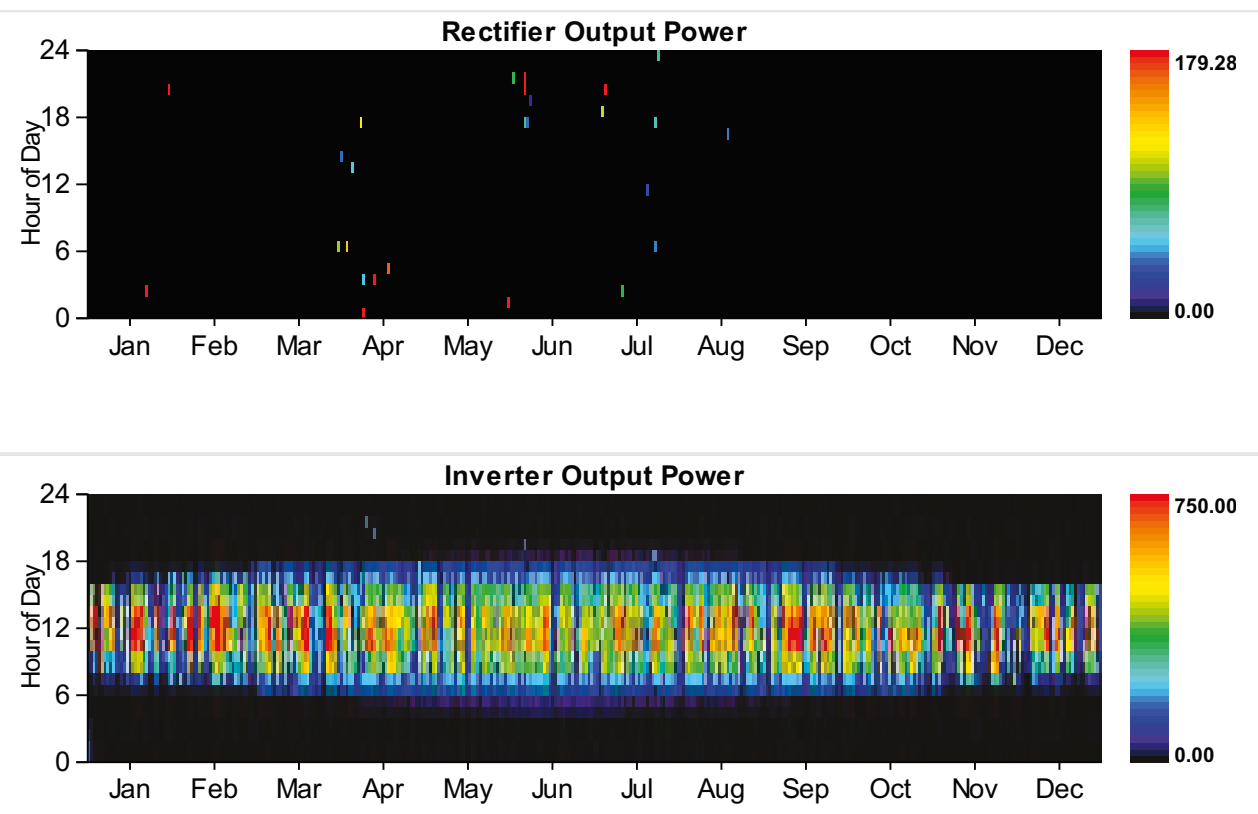

Grid

Rate: Demand 1

\begin{tabular}{|c|c|c|c|c|c|c|}
\hline Resources.ReportingService_GeneratelnputsReport_Month & $\begin{array}{l}\text { Energy } \\
\text { Purchased } \\
\text { (kWh) }\end{array}$ & $\begin{array}{l}\text { Energy } \\
\text { Sold } \\
\text { (kWh) }\end{array}$ & $\begin{array}{l}\text { Net } \\
\text { Purchases } \\
\text { (kWh) }\end{array}$ & $\begin{array}{l}\text { Peak } \\
\text { Demand } \\
(\mathbf{k W})\end{array}$ & $\begin{array}{l}\text { Energy } \\
\text { Charge } \\
(\$)\end{array}$ & $\begin{array}{l}\text { Demand } \\
\text { Charge } \\
(\$)\end{array}$ \\
\hline January & 0 & 0 & 0 & 18,380 & 0 & 294,083 \\
\hline February & 0 & 0 & 0 & 16,811 & 0 & 268,978 \\
\hline March & 0 & 0 & 0 & 16,941 & 0 & 271,062 \\
\hline April & 0 & 0 & 0 & 20,000 & 0 & 320,000 \\
\hline May & 0 & 0 & 0 & 17,657 & 0 & 282,514 \\
\hline June & 0 & 0 & 0 & 20,000 & 0 & 320,000 \\
\hline July & 0 & 0 & 0 & 20,000 & 0 & 320,000 \\
\hline August & 0 & 0 & 0 & 20,000 & 0 & 320,000 \\
\hline September & 0 & 0 & 0 & 16,176 & 0 & 258,820 \\
\hline October & 0 & 0 & 0 & 14,744 & 0 & 235,900 \\
\hline November & 0 & 0 & 0 & 16,518 & 0 & 264,286 \\
\hline
\end{tabular}




\begin{tabular}{|c|c|c|c|c|c|c|}
\hline $\begin{array}{l}\text { December } \\
\text { Resources.ReportingService_GeneratelnputsReport_Month } \\
\text { Annual }\end{array}$ & $\begin{array}{l}\text { Energy } \\
\text { Purchased } \\
\text { (kWh) } \quad 0\end{array}$ & $\begin{array}{l}\text { Energy }_{0} \\
\text { Sold } \\
(\mathbf{k W h})_{0}\end{array}$ & $\begin{array}{l}\text { Net } \\
\text { Purchases } \\
\text { (kWh) } \quad 0\end{array}$ & $\begin{array}{l}\text { Peak } \\
19,488 \\
\text { Demand } \\
\text { (ky, }\end{array}$ & $\begin{array}{l}\text { Energy } \\
\text { Charge } \\
\text { (\$) } \quad 0\end{array}$ & $\begin{array}{l}\text { Demand } \\
\text { charge } \\
\text { (\$), } \$ 67,443\end{array}$ \\
\hline
\end{tabular}

Rate: Rate 1

\begin{tabular}{|c|c|c|c|c|c|c|}
\hline Resources.ReportingService_GeneratelnputsReport_Month & $\begin{array}{l}\text { Energy } \\
\text { Purchased } \\
\text { (kWh) }\end{array}$ & $\begin{array}{l}\text { Energy } \\
\text { Sold } \\
\text { (kWh) }\end{array}$ & $\begin{array}{l}\text { Net } \\
\text { Purchases } \\
\text { (kWh) }\end{array}$ & $\begin{array}{l}\text { Peak } \\
\text { Demand } \\
(\mathbf{k W})\end{array}$ & $\begin{array}{l}\text { Energy } \\
\text { Charge (\$) }\end{array}$ & $\begin{array}{l}\text { Demand } \\
\text { Charge } \\
\text { (\$) }\end{array}$ \\
\hline January & $8,130,969$ & 0 & $8,130,969$ & 0 & 975,716 & 0 \\
\hline February & $6,023,001$ & 0 & $6,023,001$ & 0 & 722,760 & 0 \\
\hline March & $6,708,603$ & 0 & $6,708,603$ & 0 & 805,032 & 0 \\
\hline April & $9,347,970$ & 0 & $9,347,970$ & 0 & $1,121,756$ & 0 \\
\hline May & $7,697,129$ & 0 & $7,697,129$ & 0 & 923,655 & 0 \\
\hline June & $9,680,682$ & 0 & $9,680,682$ & 0 & $1,161,682$ & 0 \\
\hline July & $9,860,954$ & 0 & $9,860,954$ & 0 & $1,183,315$ & 0 \\
\hline August & $9,772,604$ & 0 & $9,772,604$ & 0 & $1,172,713$ & 0 \\
\hline September & $6,837,491$ & 0 & $6,837,491$ & 0 & 820,499 & 0 \\
\hline October & $6,699,125$ & 0 & $6,699,125$ & 0 & 803,895 & 0 \\
\hline November & $6,249,541$ & 0 & $6,249,541$ & 0 & 749,945 & 0 \\
\hline December & $8,609,035$ & 0 & $8,609,035$ & 0 & $1,033,084$ & 0 \\
\hline Annual & $95,617,096$ & 0 & $95,617,096$ & 0 & $11,474,052$ & 0 \\
\hline
\end{tabular}

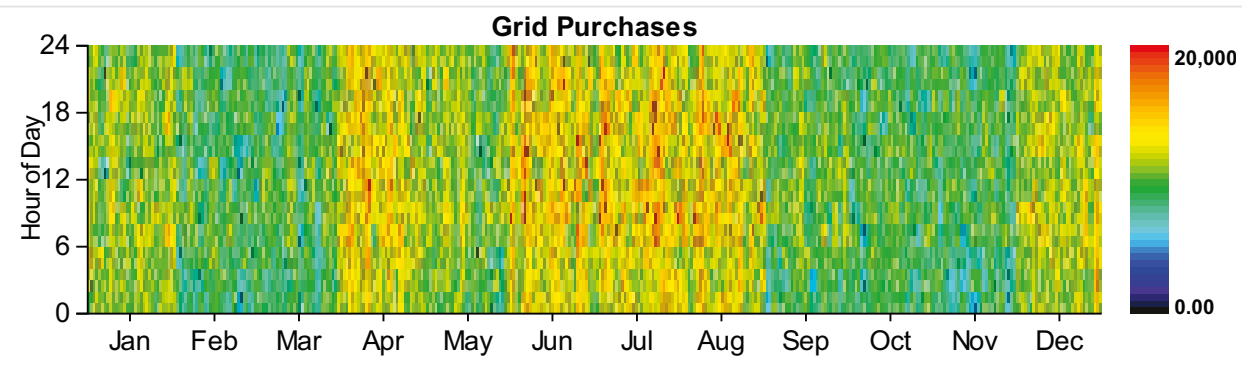

\section{Emissions}

\begin{tabular}{|l|r|l|}
\hline Pollutant & Emissions & Units \\
\hline Carbon dioxide & $60494388 \mathrm{~kg} / \mathrm{yr}$ \\
\hline Carbon monoxide & $270 \mathrm{~kg} / \mathrm{yr}$ \\
\hline Unburned hydrocarbons & $31 \mathrm{~kg} / \mathrm{yr}$ \\
\hline Particulate matter & $8 \mathrm{~kg} / \mathrm{yr}$ \\
\hline Sulfur dioxide & $262123 \mathrm{~kg} / \mathrm{yr}$ \\
\hline Nitrogen oxides & $128397 \mathrm{~kg} / \mathrm{yr}$ \\
\hline
\end{tabular}


System Report

System architecture

\begin{tabular}{|c|c|c|c|}
\hline PV & Solar World 320W flat plate PV Copy & 1,000 & kW \\
\hline Generator & Kohler 1000 Prime Power & 1,850 & kW \\
\hline Battery & GS200 flow & 1 & strings \\
\hline Converter & System Converter & 750 & kW \\
\hline Grid & Grid & 20,000 & kW \\
\hline Dispatch Strategy & Cycle Charging & & \\
\hline
\end{tabular}

\section{Cost summary}

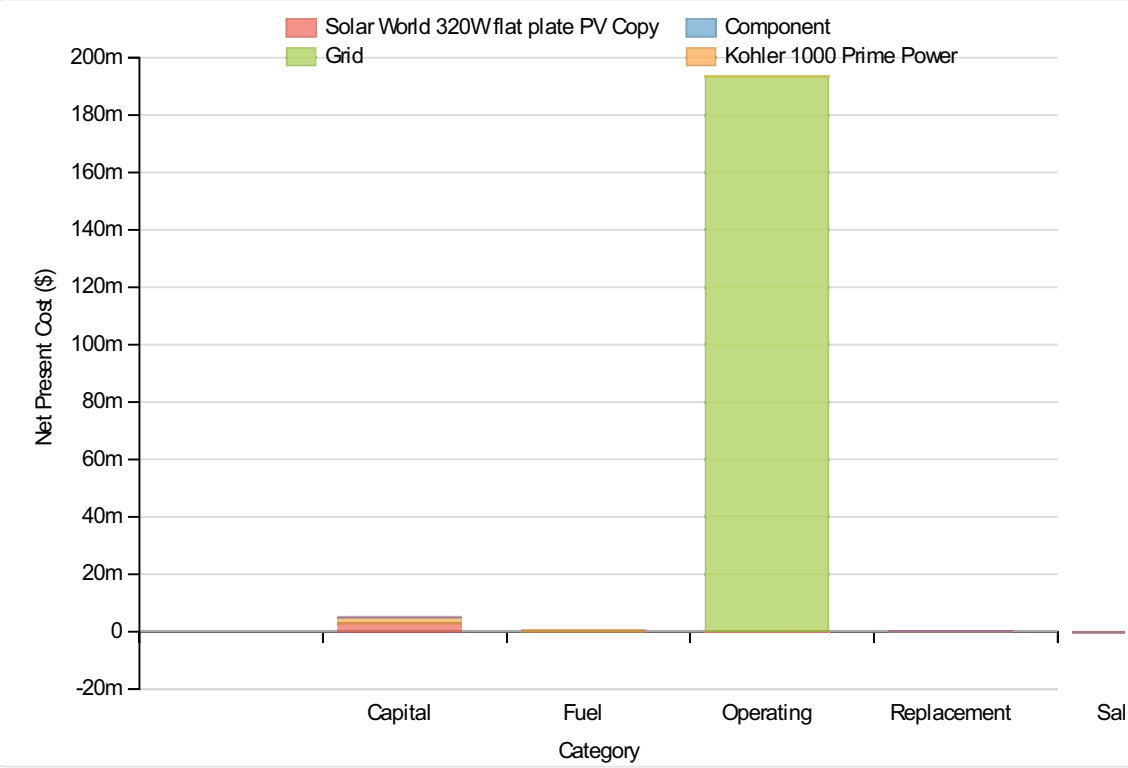

Cost Summary

Total net present cost

198667040 \$

Levelized cost of energy

$0.159 \$ / k W h$

\section{Net Present Costs}

\begin{tabular}{|l|r|r|r|r|r|r|}
\hline Component & Capital & Replacement & O\&M & Fuel & Salvage & Total \\
\hline Solar World 320W flat plate PV Copy & $3,000,000$ & 0 & 193,913 & 0 & 0 \\
\hline Kohler 1000 Prime Power & $1,637,500$ & 0 & 56,336 & 318,225 & $-286,361$ \\
\hline Grid & 0 & 0 & $193,083,040$ & 0 & 0 \\
\hline GS200 flow & 324,589 & $7,176,725,700$ \\
\hline Converter & 225,000 & 31,026 & 0 & -973 \\
\hline System & $5,187,089$ & $102,637,040$ & 361,818 \\
\hline
\end{tabular}

Annualized Costs

\begin{tabular}{|l|r|r|r|r|r|r|}
\hline Component & Capital & Replacement & O\&M & Fuel & Salvage & Total \\
\hline Solar World 320W flat plate PV Copy & 232,063 & 0 & 15,000 & 0 & 0 \\
\hline Kohler 1000 Prime Power & 126,668 & 0 & 4,358 & 24,616 & $-22,151$ & 133,491 \\
\hline
\end{tabular}




\begin{tabular}{|c|c|c|c|c|c|c|}
\hline $\begin{array}{l}\text { Component } \\
\text { Grid }\end{array}$ & 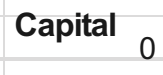 & Replacement 0 & O\&M & Fuel & ${ }_{\text {Salvage }} 0$ & $\begin{array}{l}\text { Total } \\
14,935,819\end{array}$ \\
\hline GS200 flow & 25,108 & 555 & 2,400 & 0 & -75 & 27,988 \\
\hline Converter & 17,405 & 7,384 & 0 & 0 & $-1,390$ & 23,400 \\
\hline System & 401,244 & 7,939 & $14,957,576$ & 24,616 & $-23,616$ & $15,367,759$ \\
\hline
\end{tabular}

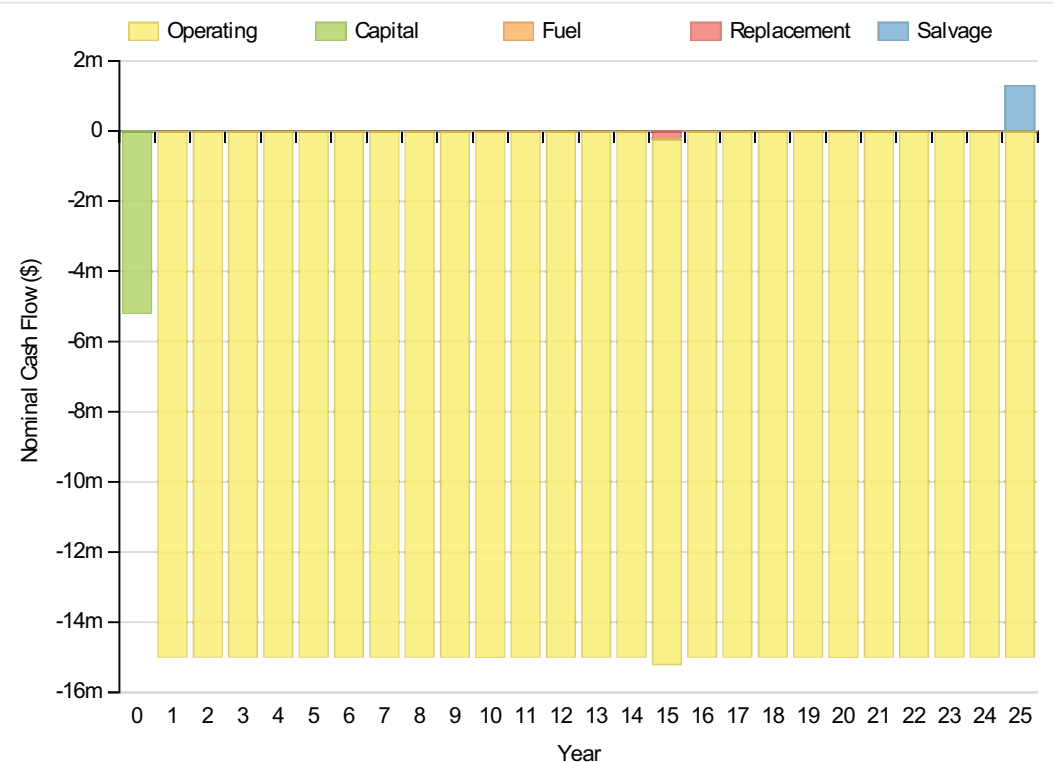

\section{Electrical}

\section{Quantity}

Excess electricity

Unmet load

Capacity shortage

Renewable fraction
Value

Production(kWh/yr)

Component

PV

Generator

Grid Purchases

Total

Load

Consumption(kWh/yr)

AC primary load

DC primary load

Total

Consumption(kWh/yr)

$1,288,675$
107,688
$95,595,440$
$96,991,800$

\section{Fraction (\%)}

\begin{abstract}
$2745 \mathrm{kWh} / \mathrm{yr}$
$6573 \mathrm{kWh} / \mathrm{yr}$

$63347 \mathrm{kWh} / \mathrm{yr}$
\end{abstract}

0

$96,857,576$

100

0

0

$96,857,576$ 


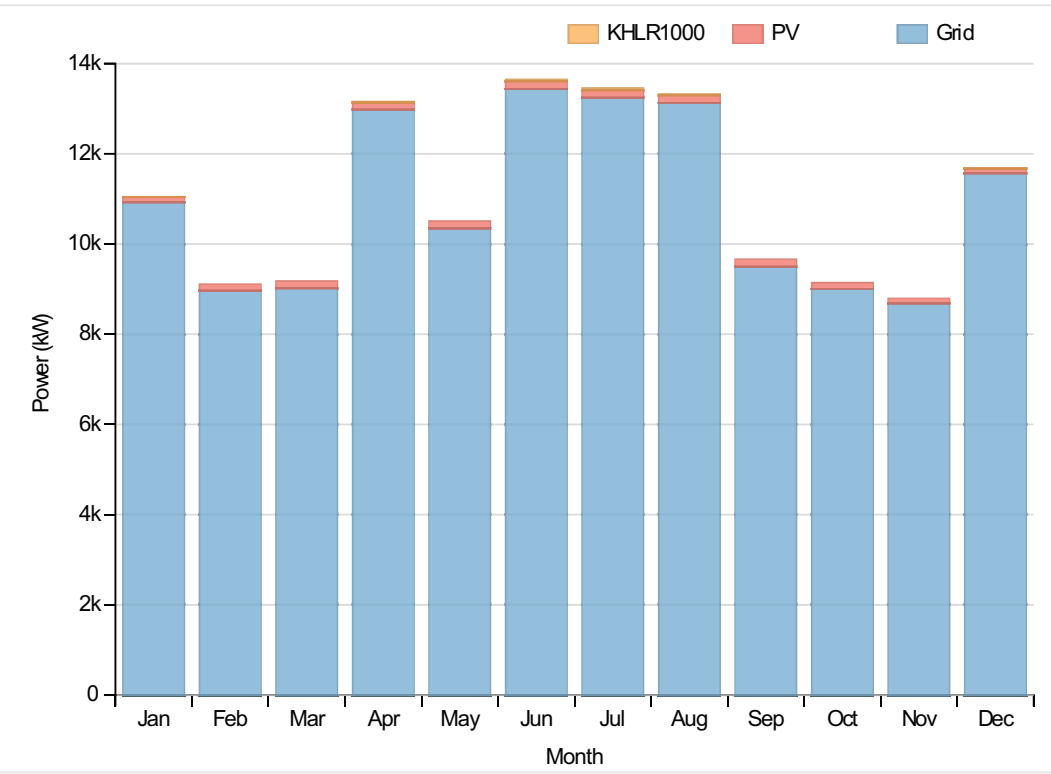

PV:Solar World 320W flat plate PV Copy

\begin{tabular}{|l|c|c|}
\hline Quantity & Value & Units \\
\hline Rated capacity & $1000 \mathrm{~kW}$ \\
\hline Mean output & $147 \mathrm{~kW}$ \\
\hline Mean output & $3530.60 \mathrm{kWh} / \mathrm{d}$ \\
\hline Capacity factor & $14.71 \%$ \\
\hline Total production & $1288675 \mathrm{kWh} / \mathrm{yr}$ \\
\hline Minimum output & $0.00 \mathrm{~kW}$ \\
\hline Maximum output & $1022.50 \mathrm{~kW}$ \\
\hline PV penetration & $1.33 \%$ \\
\hline Hours of operation & $4377 \mathrm{hrs} / \mathrm{yr}$ \\
\hline Levelized cost & $\$ 1 / \mathrm{kWh}$ \\
\hline
\end{tabular}

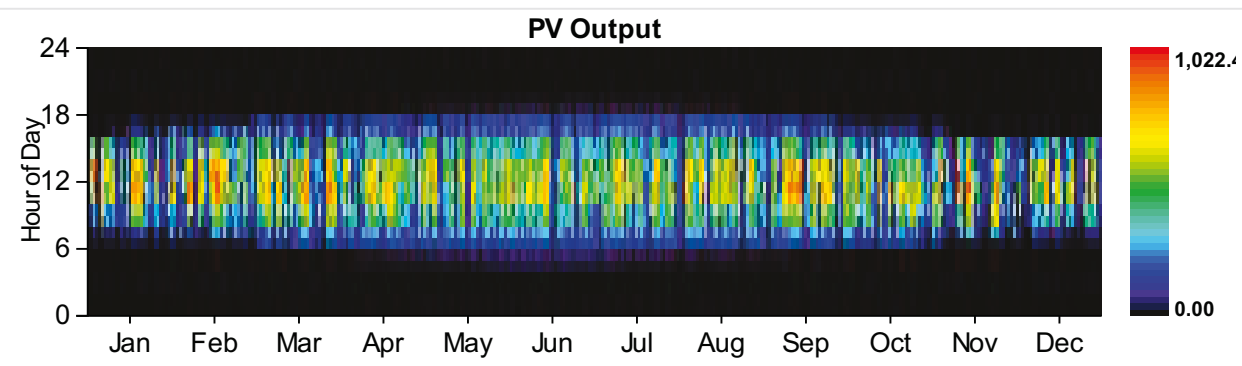

Generator:Kohler 1000 Prime Power

\section{Quantity}

Hours of operation

Number of starts

Operational life

Fixed generation cost

Marginal generation cost

\section{Value}

\section{Units}

$162 \mathrm{hrs} / \mathrm{yr}$

146 starts/yr

$93 \mathrm{yr}$

$137.46 \$ / \mathrm{hr}$

$0.23 \$ / \mathrm{kWh}$ 
Mean electrical output

Min. electrical output

Max. electrical output

Fuel consumption

Specific fuel consumption

Fuel energy input

Mean electrical efficiency

\begin{tabular}{|r|l|}
\hline 665 & $\mathrm{~kW}$ \\
\hline 463 & $\mathrm{~kW}$ \\
\hline 1850 & $\mathrm{~kW}$ \\
\hline 31160 & $\mathrm{~L} / \mathrm{yr}$ \\
\hline 0.29 & $\mathrm{~L} / \mathrm{kWh}$ \\
\hline 06611 & $\mathrm{kWh} / \mathrm{yr}$ \\
\hline 35 & $\%$ \\
\hline
\end{tabular}

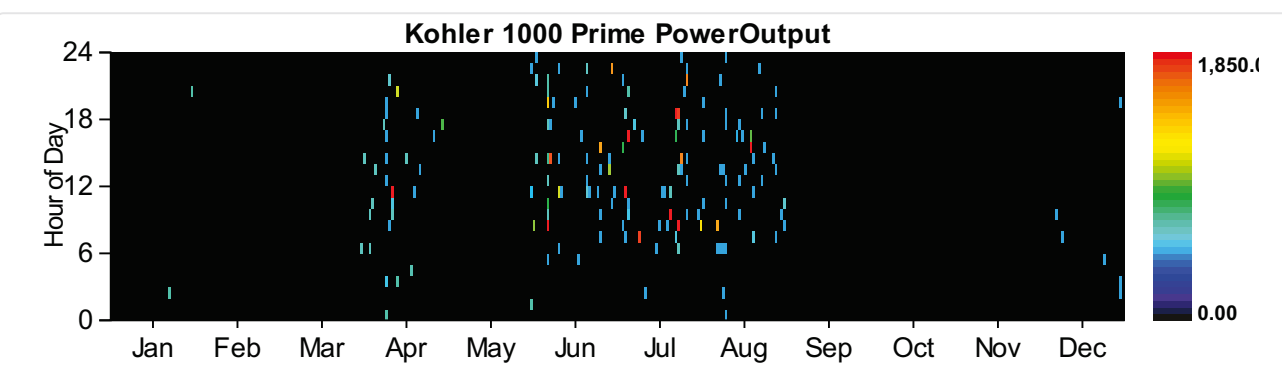

\section{Battery:GS200 flow}

\section{Quantity}

Value

String size

Strings in parallel

Batteries

Bus voltage

\section{Quantity}

Value

Units

Nominal capacity

Usable nominal capacity

$600 \mathrm{kWh}$

Autonomy

Lifetime throughput

Battery wear cost

Average energy cost

Energy in

Energy out

Storage depletion

Losses

Annual throughput

Expected life

600 kWh

$0 \mathrm{hr}$

0

$0.000 \$ / k W h$

\section{$0.172 \$ / k W h$}

$7974 \mathrm{kWh} / \mathrm{yr}$

$5582 \mathrm{kWh} / \mathrm{yr}$

$0 \mathrm{kWh} / \mathrm{yr}$

$2392 \mathrm{kWh} / \mathrm{yr}$

$6671 \mathrm{kWh} / \mathrm{yr}$

$25 \mathrm{yr}$ 


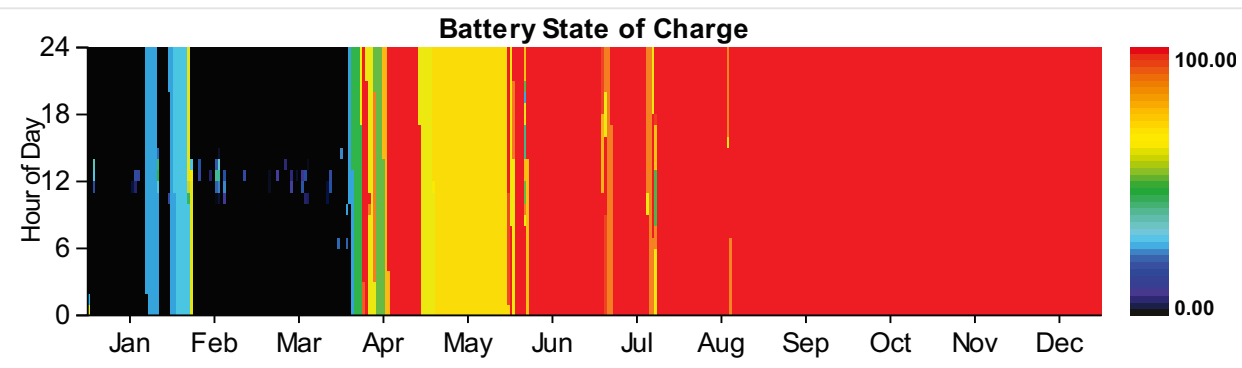

Converter

\begin{tabular}{|l|r|r|l|}
\hline Quantity & Inverter & Rectifier & Units \\
\hline Capacity & 750 & 675 & $\mathrm{~kW}$ \\
\hline Mean output & 132 & $\mathrm{~kW}$ \\
\hline Minimum output & 0 & $\mathrm{~kW}$ \\
\hline Maximum output & 750 & $179 \mathrm{~kW}$ \\
\hline Capacity factor & 18 & 0 \\
\hline Hours of operation & 4,390 & 22 \\
\hline Energy in & $1,286,038$ & $\mathrm{hrs} / \mathrm{yr}$ \\
\hline Energy out & $1,157,434$ & 2,942 & $\mathrm{kWh} / \mathrm{yr}$ \\
\hline Losses & 128,605 & 2,501 & $\mathrm{kWh} / \mathrm{yr}$ \\
\hline
\end{tabular}
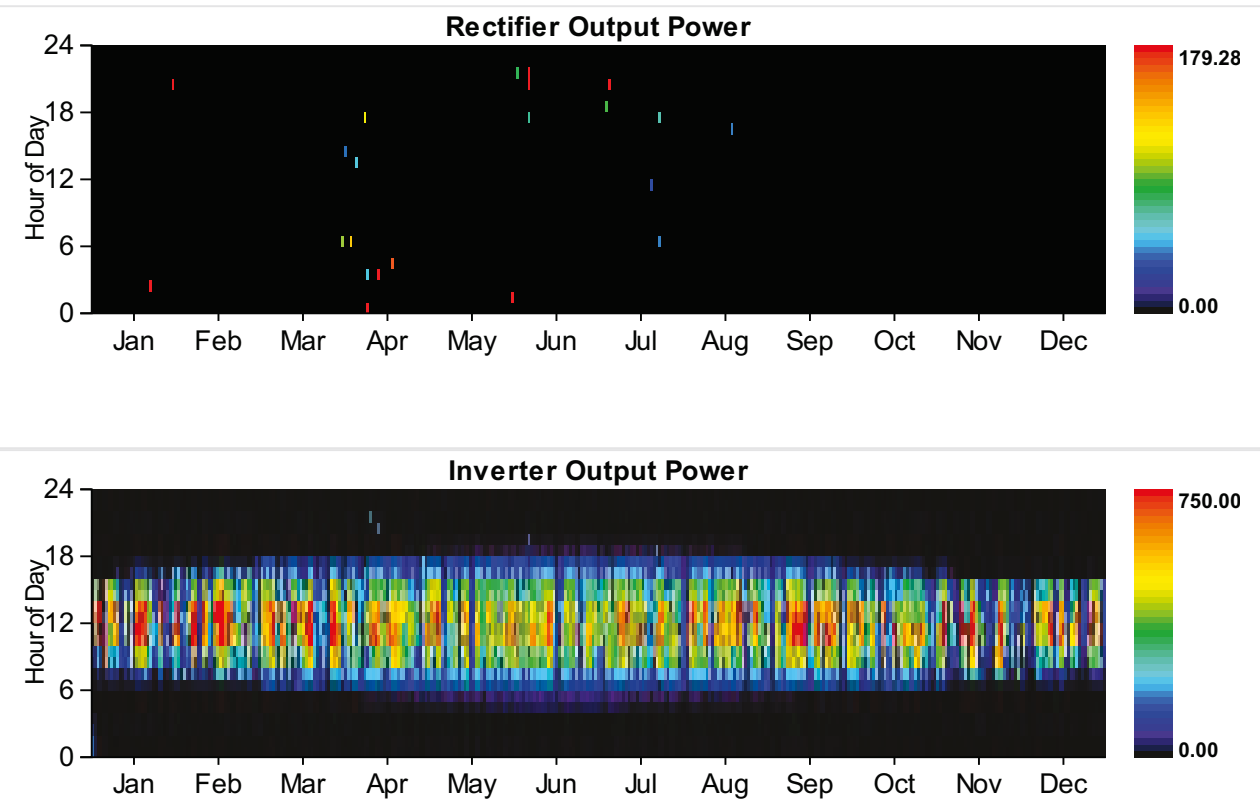

Grid

Rate: Demand 1

\begin{tabular}{|c|c|c|c|c|c|c|}
\hline Resources.ReportingService_GeneratelnputsReport_Month & $\begin{array}{l}\text { Energy } \\
\text { Purchased } \\
\text { (kWh) }\end{array}$ & $\begin{array}{l}\text { Energy } \\
\text { Sold } \\
\text { (kWh) }\end{array}$ & $\begin{array}{l}\text { Net } \\
\text { Purchases } \\
\text { (kWh) }\end{array}$ & $\begin{array}{l}\text { Peak } \\
\text { Demand } \\
(\mathbf{k W})\end{array}$ & $\begin{array}{l}\text { Energy } \\
\text { Charge } \\
(\$)\end{array}$ & $\begin{array}{l}\text { Demand } \\
\text { Charge } \\
\text { (\$) }\end{array}$ \\
\hline January & 0 & 0 & 0 & 18,247 & 0 & 291,953 \\
\hline February & 0 & 0 & 0 & 16,811 & 0 & 268,978 \\
\hline March & 0 & 0 & 0 & 16,941 & 0 & 271,062 \\
\hline
\end{tabular}




\begin{tabular}{|c|c|c|c|c|c|c|}
\hline $\begin{array}{l}\text { April } \\
\text { Resources.ReportingService_GeneratelnputsReport_Month } \\
\text { May }\end{array}$ & $\begin{array}{l}\text { Energy } \\
\text { Purchased } \\
\text { (kWh) } \quad 0\end{array}$ & $\begin{array}{l}\text { Energy }_{0} \\
\text { Sold } \\
\text { (kWh) }\end{array}$ & $\begin{array}{l}\text { Net } \\
\text { Purchases } \\
\text { (kWh) } \quad 0\end{array}$ & $\begin{array}{l}\text { Peak } \\
\text { Demionad } \\
\text { (kW) } \\
17,657\end{array}$ & $\begin{array}{l}\text { Energy } \\
\text { Charge } \\
\text { (\$) } \quad 0\end{array}$ & $\begin{array}{l}\text { Demand } \\
\text { charge } \\
(\$ 282,514\end{array}$ \\
\hline June & 0 & 0 & 0 & 20,000 & 0 & 320,000 \\
\hline July & 0 & 0 & 0 & 20,000 & 0 & 320,000 \\
\hline August & 0 & 0 & 0 & 20,000 & 0 & 320,000 \\
\hline September & 0 & 0 & 0 & 16,176 & 0 & 258,820 \\
\hline October & 0 & 0 & 0 & 14,744 & 0 & 235,900 \\
\hline November & 0 & 0 & 0 & 16,518 & 0 & 264,286 \\
\hline December & 0 & 0 & 0 & 19,429 & 0 & 310,860 \\
\hline Annual & 0 & 0 & 0 & 20,000 & 0 & $3,464,373$ \\
\hline
\end{tabular}

Rate: Rate 1

\begin{tabular}{|c|c|c|c|c|c|c|}
\hline Resources.ReportingService_GeneratelnputsReport_Month & $\begin{array}{l}\text { Energy } \\
\text { Purchased } \\
\text { (kWh) }\end{array}$ & $\begin{array}{l}\text { Energy } \\
\text { Sold } \\
\text { (kWh) }\end{array}$ & $\begin{array}{l}\text { Net } \\
\text { Purchases } \\
\text { (kWh) }\end{array}$ & $\begin{array}{l}\text { Peak } \\
\text { Demand } \\
(\mathbf{k W})\end{array}$ & $\begin{array}{l}\text { Energy } \\
\text { Charge (\$) }\end{array}$ & $\begin{array}{l}\text { Demand } \\
\text { Charge } \\
\text { (\$) }\end{array}$ \\
\hline January & $8,130,389$ & 0 & $8,130,389$ & 0 & 975,647 & 0 \\
\hline February & $6,023,001$ & 0 & $6,023,001$ & 0 & 722,760 & 0 \\
\hline March & $6,708,603$ & 0 & $6,708,603$ & 0 & 805,032 & 0 \\
\hline April & $9,343,847$ & 0 & $9,343,847$ & 0 & $1,121,262$ & 0 \\
\hline May & $7,697,129$ & 0 & $7,697,129$ & 0 & 923,655 & 0 \\
\hline June & $9,675,577$ & 0 & $9,675,577$ & 0 & $1,161,069$ & 0 \\
\hline July & $9,855,724$ & 0 & $9,855,724$ & 0 & $1,182,687$ & 0 \\
\hline August & $9,767,369$ & 0 & $9,767,369$ & 0 & $1,172,084$ & 0 \\
\hline September & $6,837,491$ & 0 & $6,837,491$ & 0 & 820,499 & 0 \\
\hline October & $6,699,125$ & 0 & $6,699,125$ & 0 & 803,895 & 0 \\
\hline November & $6,249,541$ & 0 & $6,249,541$ & 0 & 749,945 & 0 \\
\hline December & $8,607,644$ & 0 & $8,607,644$ & 0 & $1,032,917$ & 0 \\
\hline Annual & $95,595,440$ & 0 & $95,595,440$ & 0 & $11,471,452$ & 0 \\
\hline
\end{tabular}

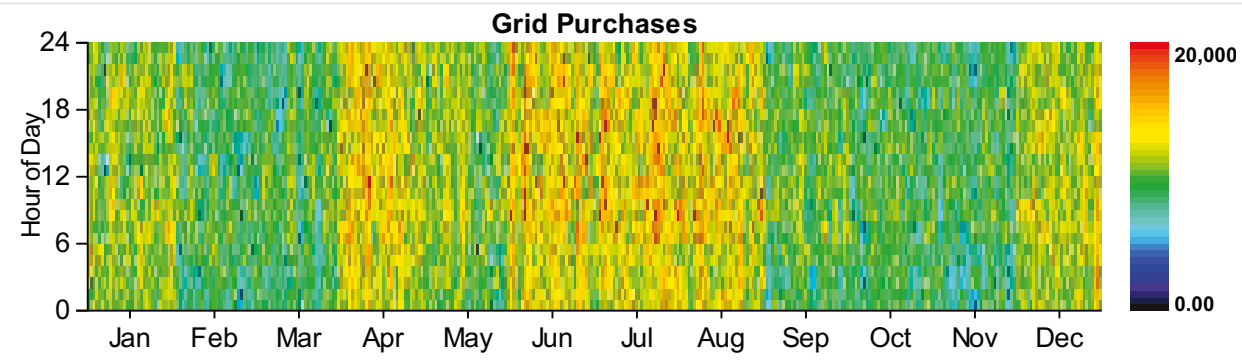




\section{Emissions}

Pollutant

Carbon dioxide

Carbon monoxide

Unburned hydrocarbons

Particulate matter

Sulfur dioxide

Nitrogen oxides
Emissions

Units

$60498100 \mathrm{~kg} / \mathrm{yr}$

$343 \mathrm{~kg} / \mathrm{yr}$

$39 \mathrm{~kg} / \mathrm{yr}$

$10 \mathrm{~kg} / \mathrm{yr}$

262100 kg/yr

$128441 \mathrm{~kg} / \mathrm{yr}$

HOMER Energy, LLC @ 2016 
System Report

System architecture

\begin{tabular}{|l|l|l|}
\hline Grid & Grid & $\mathbf{2 5 , 0 0 0}$ \\
\hline Dispatch Strategy & Cycle Charging \\
\hline
\end{tabular}

\section{Cost summary}

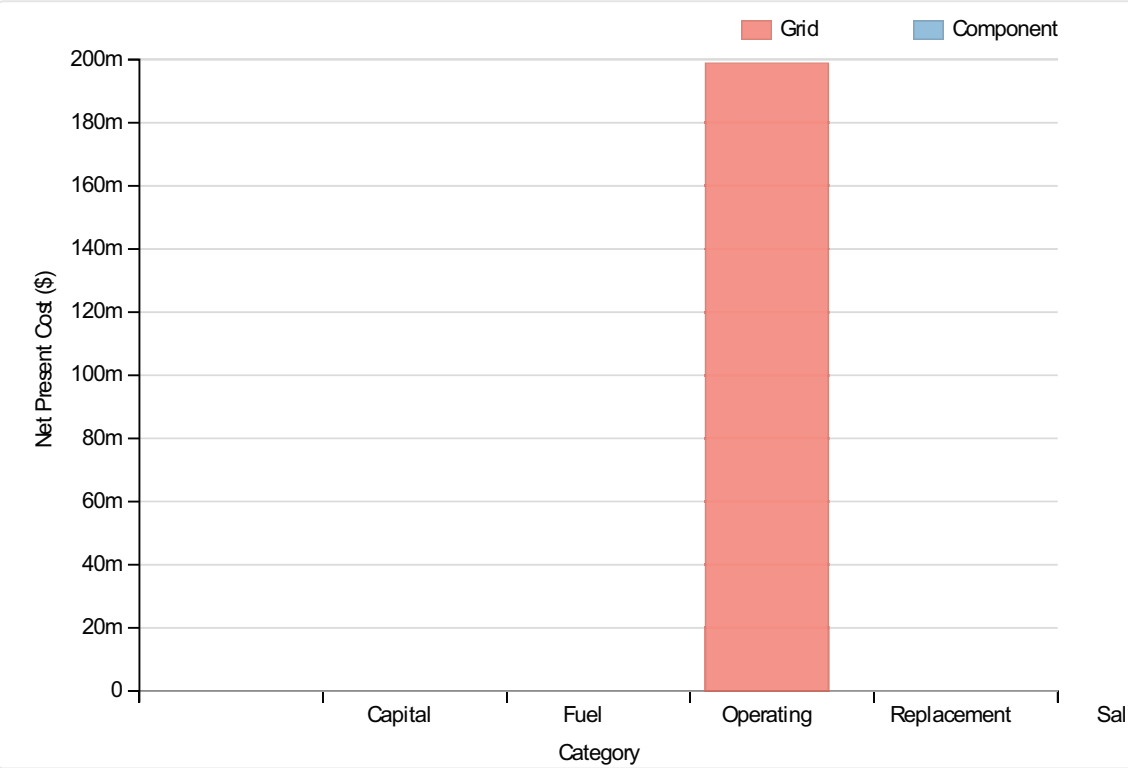

Cost Summary

Total net present cost

$198776640 \$$

Levelized cost of energy

$0.159 \$ / \mathrm{kWh}$

\section{Net Present Costs}

\begin{tabular}{|l|r|r|r|r|r|r|}
\hline Component & Capital & Replacement & O\&M & Fuel & Salvage & Total \\
\hline Grid & 0 & 0 & $198,776,528$ & 0 & 0 \\
\hline System & 0 & 0 & $198,776,528$ & 0 & 0 \\
\hline
\end{tabular}

\section{Annualized Costs}

\begin{tabular}{|l|r|r|r|r|r|r|}
\hline Component & Capital & Replacement & O\&M & Fuel & Salvage & Total \\
\hline Grid & 0 & 0 & $15,376,235$ & 0 & $15,376,235$ \\
\hline System & 0 & 0 & $15,376,235$ & 0 & 0 & $15,376,235$ \\
\hline
\end{tabular}




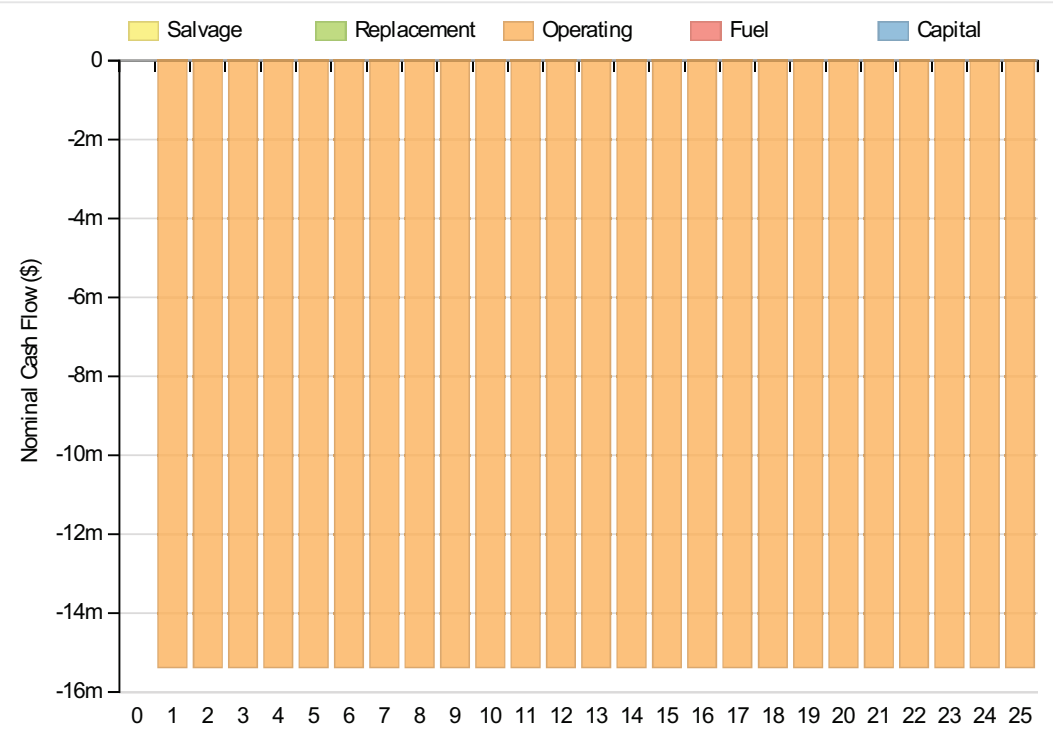

Year

\section{Electrical}

\begin{tabular}{|c|c|c|c|c|}
\hline Quantity & & Value & & Units \\
\hline Excess electricity & & & 0 & kWh/yr \\
\hline Unmet load & & & 0 & kWh/yr \\
\hline Capacity shortage & & & 252 & $\mathrm{kWh} / \mathrm{yr}$ \\
\hline Renewable fraction & & & 0 & \\
\hline Component & Production(kWh/yr) & & Fracti & tion $(\%)$ \\
\hline Grid Purchases & & $96,864,192$ & & 100 \\
\hline Total & & $96,864,192$ & & 100 \\
\hline Load & Consumption(kWh/yr) & & Fra & action $(\%)$ \\
\hline AC primary load & & $96,864,160$ & & 100 \\
\hline DC primary load & & 0 & & 0 \\
\hline Total & & $96,864,160$ & & 100 \\
\hline
\end{tabular}

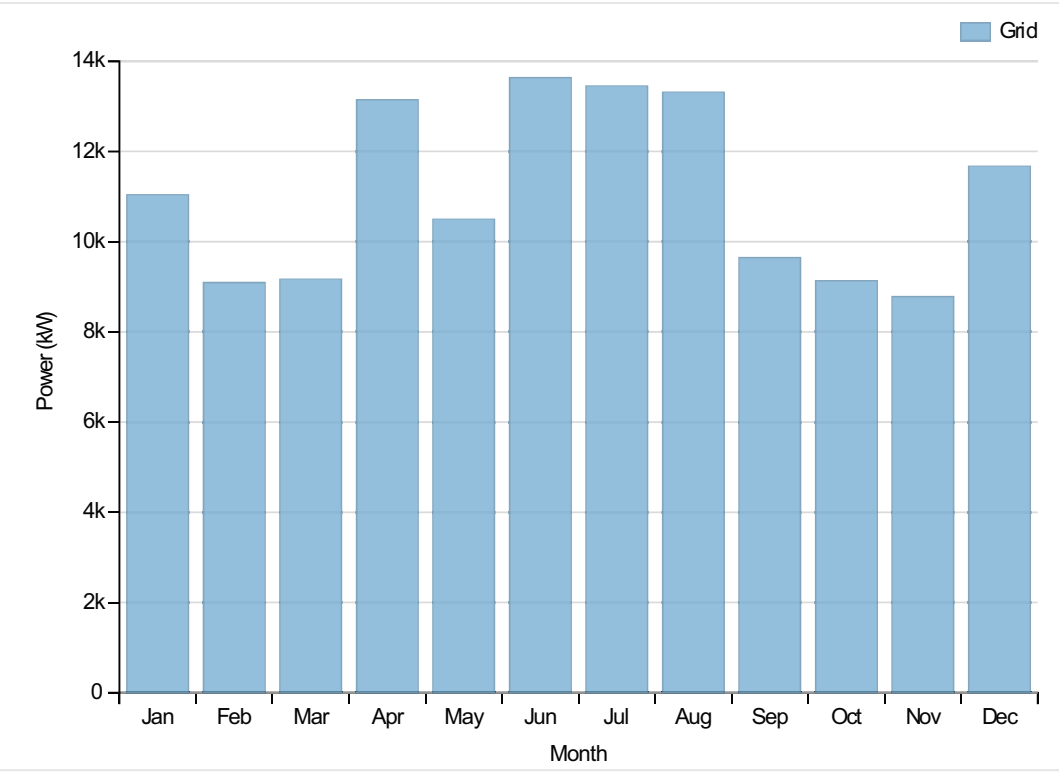


Grid

Rate: Demand 1

\begin{tabular}{|c|c|c|c|c|c|c|}
\hline Resources.ReportingService_GeneratelnputsReport_Month & $\begin{array}{l}\text { Energy } \\
\text { Purchased } \\
\text { (kWh) }\end{array}$ & $\begin{array}{l}\text { Energy } \\
\text { Sold } \\
\text { (kWh) }\end{array}$ & $\begin{array}{l}\text { Net } \\
\text { Purchases } \\
\text { (kWh) }\end{array}$ & $\begin{array}{l}\text { Peak } \\
\text { Demand } \\
(\mathbf{k W})\end{array}$ & $\begin{array}{l}\text { Energy } \\
\text { Charge } \\
\text { (\$) }\end{array}$ & $\begin{array}{l}\text { Demand } \\
\text { Charge } \\
(\$)\end{array}$ \\
\hline January & 0 & 0 & 0 & 18,553 & 0 & 296,843 \\
\hline February & 0 & 0 & 0 & 17,561 & 0 & 280,978 \\
\hline March & 0 & 0 & 0 & 17,425 & 0 & 278,807 \\
\hline April & 0 & 0 & 0 & 23,104 & 0 & 369,666 \\
\hline May & 0 & 0 & 0 & 17,869 & 0 & 285,902 \\
\hline June & 0 & 0 & 0 & 24,046 & 0 & 384,739 \\
\hline July & 0 & 0 & 0 & 24,897 & 0 & 398,345 \\
\hline August & 0 & 0 & 0 & 22,671 & 0 & 362,729 \\
\hline September & 0 & 0 & 0 & 16,926 & 0 & 270,820 \\
\hline October & 0 & 0 & 0 & 14,895 & 0 & 238,325 \\
\hline November & 0 & 0 & 0 & 16,518 & 0 & 264,286 \\
\hline December & 0 & 0 & 0 & 20,069 & 0 & 321,098 \\
\hline Annual & 0 & 0 & 0 & 24,897 & 0 & $3,752,539$ \\
\hline
\end{tabular}

Rate: Rate 1

\begin{tabular}{|c|c|c|c|c|c|c|}
\hline Resources.ReportingService_GeneratelnputsReport_Month & $\begin{array}{l}\text { Energy } \\
\text { Purchased } \\
\text { (kWh) }\end{array}$ & $\begin{array}{l}\text { Energy } \\
\text { Sold } \\
\text { (kWh) }\end{array}$ & $\begin{array}{l}\text { Net } \\
\text { Purchases } \\
\text { (kWh) }\end{array}$ & $\begin{array}{l}\text { Peak } \\
\text { Demand } \\
(\mathbf{k W})\end{array}$ & $\begin{array}{l}\text { Energy } \\
\text { Charge (\$) }\end{array}$ & $\begin{array}{l}\text { Demand } \\
\text { Charge } \\
\text { (\$) }\end{array}$ \\
\hline January & $8,209,736$ & 0 & $8,209,736$ & 0 & 985,168 & 0 \\
\hline February & $6,110,456$ & 0 & $6,110,456$ & 0 & 733,255 & 0 \\
\hline March & $6,816,916$ & 0 & $6,816,916$ & 0 & 818,030 & 0 \\
\hline April & $9,462,522$ & 0 & $9,462,522$ & 0 & $1,135,503$ & 0 \\
\hline May & $7,805,883$ & 0 & $7,805,883$ & 0 & 936,706 & 0 \\
\hline June & $9,816,466$ & 0 & $9,816,466$ & 0 & $1,177,976$ & 0 \\
\hline July & $10,003,713$ & 0 & $10,003,713$ & 0 & $1,200,446$ & 0 \\
\hline August & $9,901,865$ & 0 & $9,901,865$ & 0 & $1,188,224$ & 0 \\
\hline September & $6,943,139$ & 0 & $6,943,139$ & 0 & 833,177 & 0 \\
\hline October & $6,792,313$ & 0 & $6,792,313$ & 0 & 815,078 & 0 \\
\hline November & $6,321,105$ & 0 & $6,321,105$ & 0 & 758,533 & 0 \\
\hline December & $8,680,090$ & 0 & $8,680,090$ & 0 & $1,041,611$ & 0 \\
\hline Annual & $96,864,192$ & 0 & $96,864,192$ & 0 & $11,623,705$ & 0 \\
\hline
\end{tabular}




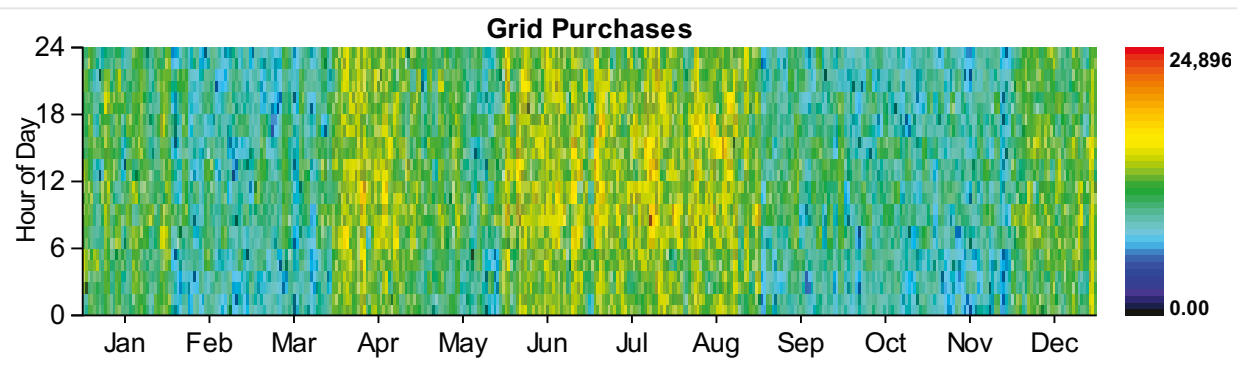

\section{Emissions}

\begin{tabular}{|l|r|l|}
\hline Pollutant & Emissions & Units \\
\hline Carbon dioxide & $61218168 \mathrm{~kg} / \mathrm{yr}$ \\
\hline Carbon monoxide & 0 & $\mathrm{~kg} / \mathrm{yr}$ \\
\hline Unburned hydrocarbons & $0 \mathrm{~kg} / \mathrm{yr}$ \\
\hline Particulate matter & $0 \mathrm{~kg} / \mathrm{yr}$ \\
\hline Sulfur dioxide & $265408 \mathrm{~kg} / \mathrm{yr}$ \\
\hline Nitrogen oxides & $129798 \mathrm{~kg} / \mathrm{yr}$ \\
\hline
\end{tabular}

HOMER Energy, LLC @ 2016 
System Report

System architecture

\begin{tabular}{|l|l|r|}
\hline Battery & GS200 flow & 1 strings \\
\hline Converter & System Converter & 250 \\
\hline Grid & Grid & 25,000 \\
\hline Dispatch Strategy & Cycle Charging & $\mathrm{kW}$ \\
\hline
\end{tabular}

\section{Cost summary}

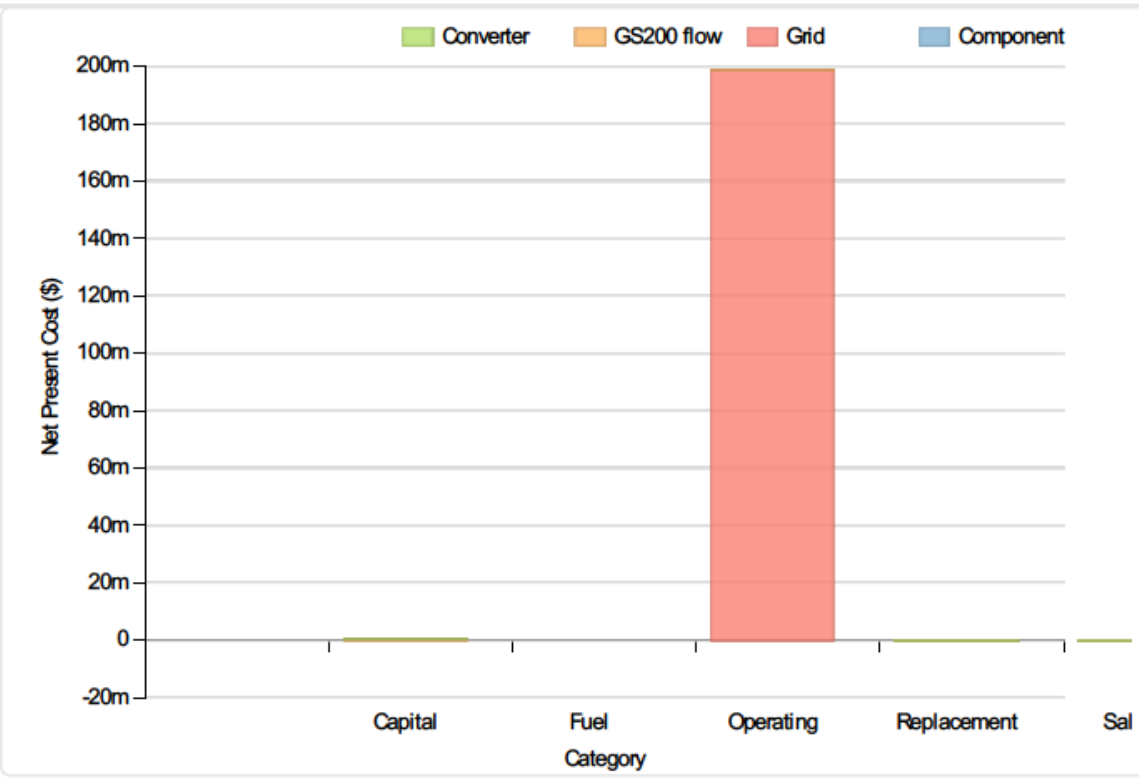

Cost Summary

Total net present cost

Levelized cost of energy
$199238592 \$$

$0.159 \$ / k W h$

Net Present Costs

\begin{tabular}{|l|r|r|r|r|r|r|}
\hline Component & Capital & Replacement & O\&M & Fuel & Salvage & \multicolumn{1}{l|}{ Total } \\
\hline Grid & 0 & 0 & $198,775,856$ & 0 & 0 & $198,775,856$ \\
\hline GS200 flow & 324,589 & 7,176 & 31,026 & 0 & -973 & 361,818 \\
\hline Converter & 75,000 & 31,821 & 0 & 0 & $-5,989$ & 100,832 \\
\hline System & 399,589 & 38,996 & $198,806,896$ & 0 & $-6,962$ & $199,238,519$ \\
\hline
\end{tabular}

Annualized Costs

\begin{tabular}{|l|r|r|r|r|r|r|}
\hline Component & Capital & Replacement & O\&M & Fuel & Salvage & \multicolumn{1}{l|}{ Total } \\
\hline Grid & 0 & 0 & $15,376,183$ & 0 & 0 \\
\hline GS200 flow & 25,108 & 555 & 2,400 & 0 & -75 & $15,376,183$ \\
\hline Converter & 5,802 & 2,462 & 0 & 0 & -463 & 7,800 \\
\hline System & 30,910 & 3,017 & $15,378,584$ & 0 & -539 & $15,411,972$ \\
\hline
\end{tabular}




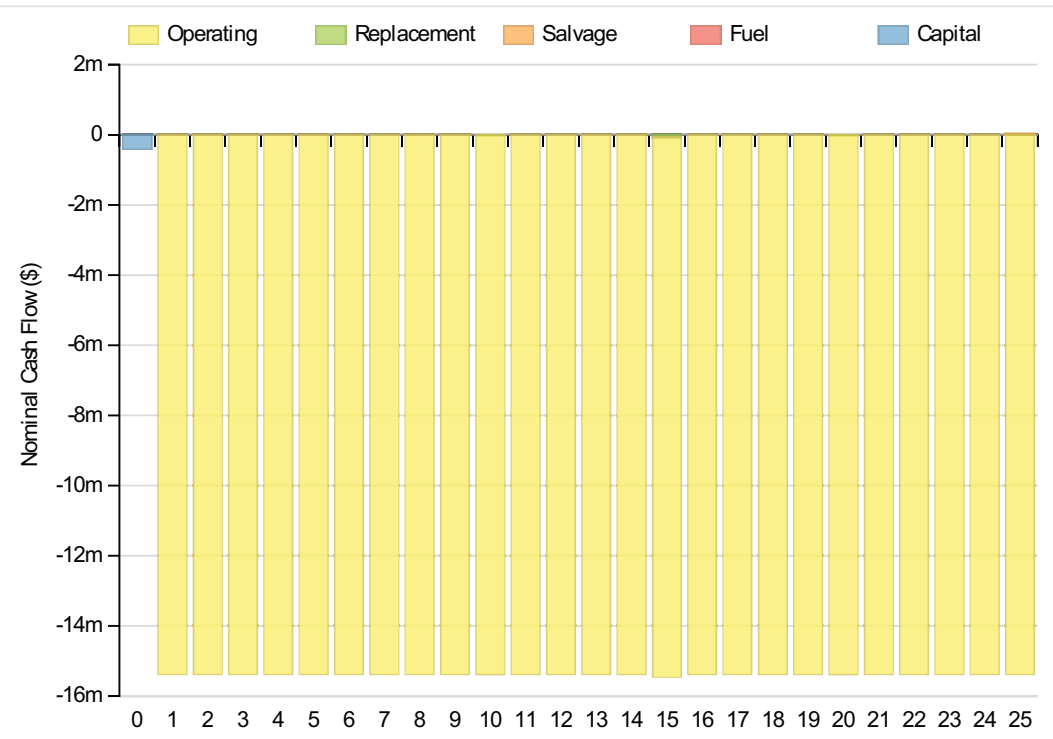

Year

\section{Electrical}

\begin{tabular}{|c|c|c|c|c|}
\hline Quantity & & Value & & Units \\
\hline Excess electricity & & & 0 & kWh/yr \\
\hline Unmet load & & & 0 & kWh/yr \\
\hline Capacity shortage & & & 252 & $\mathrm{kWh} / \mathrm{yr}$ \\
\hline Renewable fraction & & & 0 & \\
\hline Component & Production(kWh/yr) & & Fracti & tion $(\%)$ \\
\hline Grid Purchases & & $96,863,744$ & & 100 \\
\hline Total & & $96,863,744$ & & 100 \\
\hline Load & Consumption(kWh/yr) & & Fra & action $(\%)$ \\
\hline AC primary load & & $96,864,160$ & & 100 \\
\hline DC primary load & & 0 & & 0 \\
\hline Total & & $96,864,160$ & & 100 \\
\hline
\end{tabular}

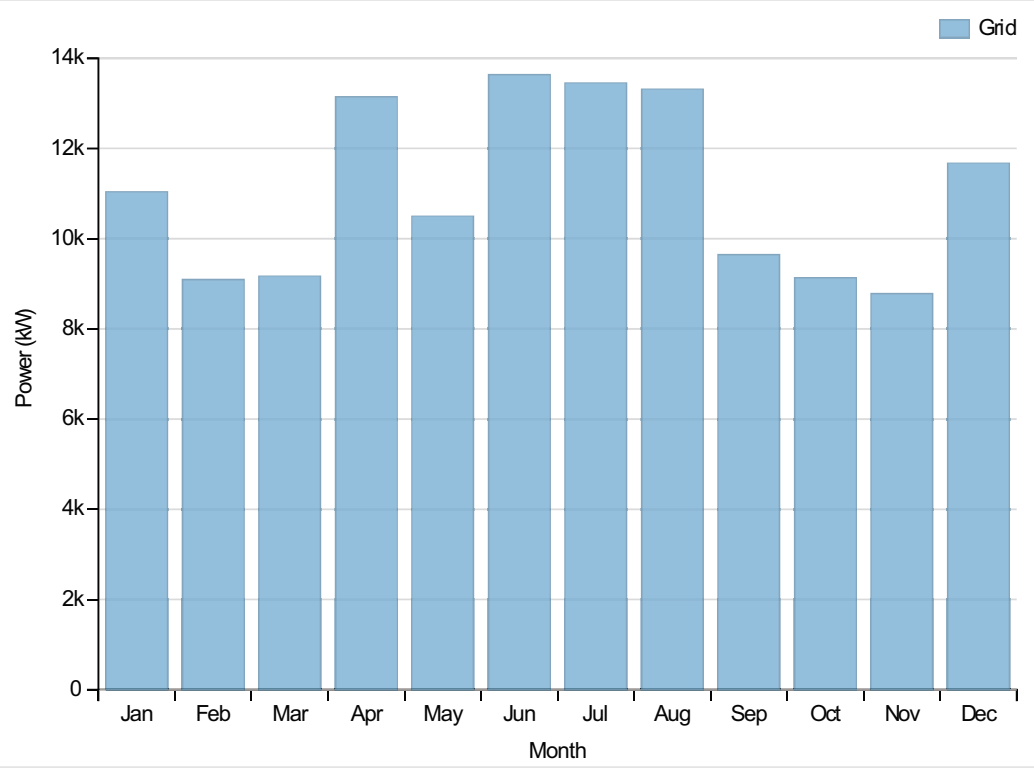


Battery:GS200 flow

\begin{tabular}{|l|r|}
\hline Quantity & Value \\
\hline String size & 1 \\
\hline Strings in parallel & 1 \\
\hline Batteries & 1 \\
\hline Bus voltage & 100 \\
\hline
\end{tabular}

\begin{tabular}{|c|c|c|}
\hline Quantity & Value & Units \\
\hline Nominal capacity & 600 & kWh \\
\hline Usable nominal capacity & 600 & kWh \\
\hline Autonomy & 0 & $\mathrm{hr}$ \\
\hline Lifetime throughput & 0 & \\
\hline Battery wear cost & 0.000 & $\$ / \mathrm{kWh}$ \\
\hline Average energy cost & 0.000 & $\$ / \mathrm{kWh}$ \\
\hline Energy in & 0 & $\mathrm{kWh} / \mathrm{yr}$ \\
\hline Energy out & 502 & $\mathrm{kWh} / \mathrm{yr}$ \\
\hline Storage depletion & 600 & $\mathrm{kWh} / \mathrm{yr}$ \\
\hline Losses & -1102 & $\mathrm{kWh} / \mathrm{yr}$ \\
\hline Annual throughput & 600 & $\mathrm{kWh} / \mathrm{yr}$ \\
\hline Expected life & 25 & yr \\
\hline
\end{tabular}

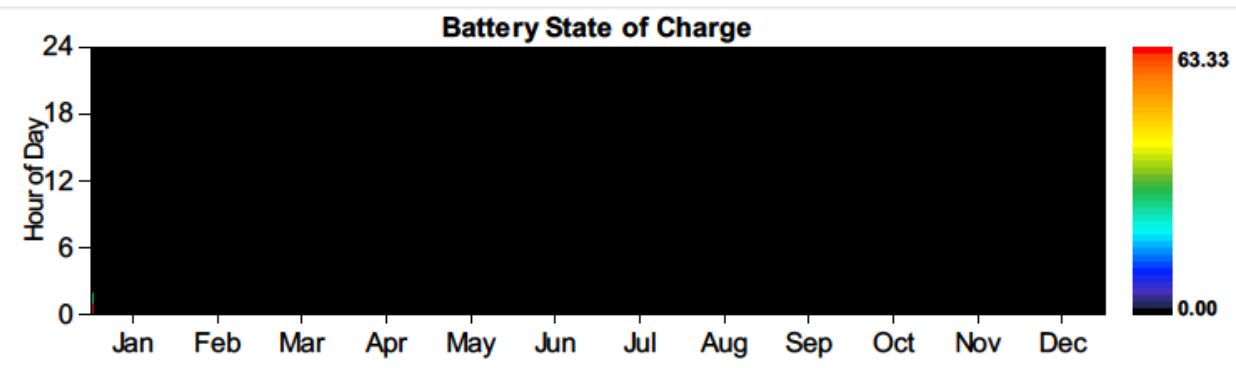

\section{Converter}

\begin{tabular}{|l|r|r|l|}
\hline Quantity & Inverter & Rectifier & Units \\
\hline Capacity & 250 & 225 & $\mathrm{~kW}$ \\
\hline Mean output & 0 & $\mathrm{~kW}$ \\
\hline Minimum output & 0 & $\mathrm{~kW}$ \\
\hline Maximum output & 166 & 0 & $\mathrm{~kW}$ \\
\hline Capacity factor & 0 & 0 & $\%$ \\
\hline Hours of operation & 3 & 0 & $\mathrm{hrs} / \mathrm{yr}$ \\
\hline Energy in & 502 & 0 & $\mathrm{kWh} / \mathrm{yr}$ \\
\hline
\end{tabular}



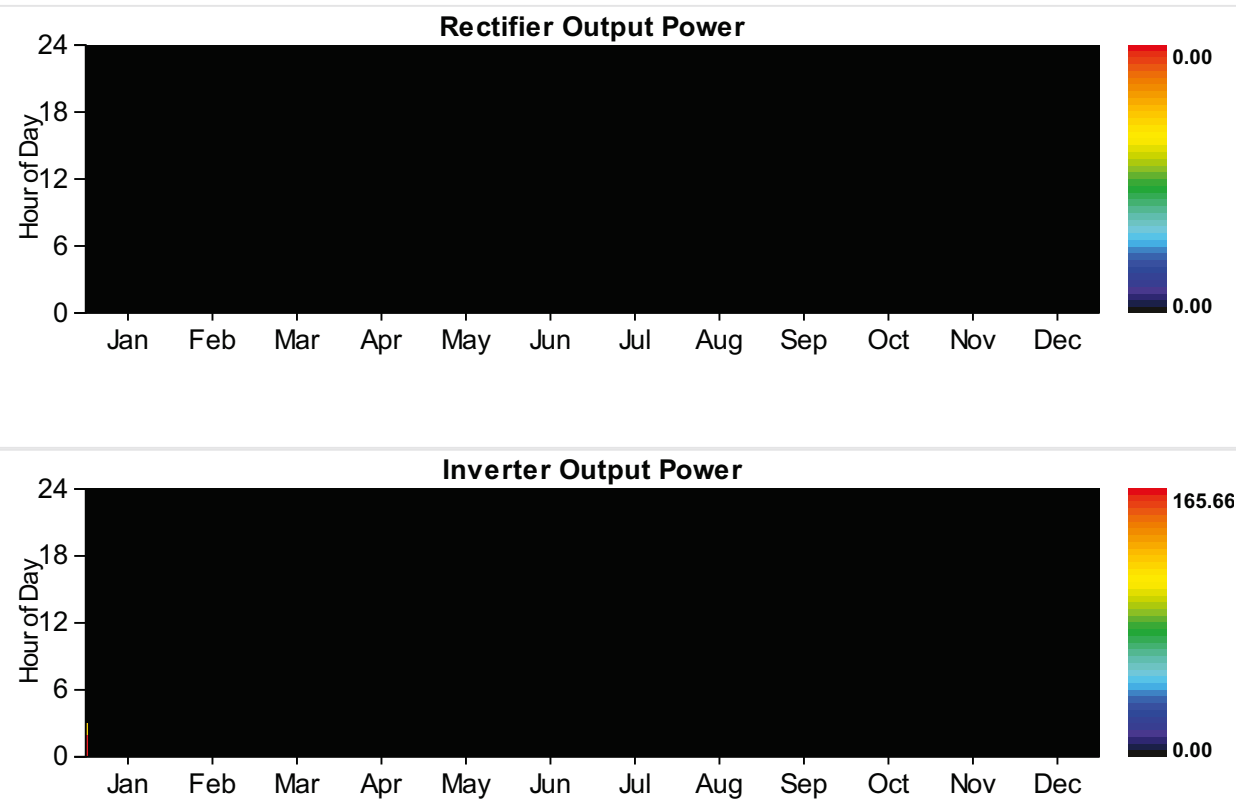

Grid

Rate: Demand 1

\begin{tabular}{|c|c|c|c|c|c|c|}
\hline Resources.ReportingService_GeneratelnputsReport_Month & $\begin{array}{l}\text { Energy } \\
\text { Purchased } \\
\text { (kWh) }\end{array}$ & $\begin{array}{l}\text { Energy } \\
\text { Sold } \\
\text { (kWh) }\end{array}$ & $\begin{array}{l}\text { Net } \\
\text { Purchases } \\
\text { (kWh) }\end{array}$ & $\begin{array}{l}\text { Peak } \\
\text { Demand } \\
(\mathbf{k W})\end{array}$ & $\begin{array}{l}\text { Energy } \\
\text { Charge } \\
(\$)\end{array}$ & $\begin{array}{l}\text { Demand } \\
\text { Charge } \\
(\$)\end{array}$ \\
\hline January & 0 & 0 & 0 & 18,553 & 0 & 296,843 \\
\hline February & 0 & 0 & 0 & 17,561 & 0 & 280,978 \\
\hline March & 0 & 0 & 0 & 17,425 & 0 & 278,807 \\
\hline April & 0 & 0 & 0 & 23,104 & 0 & 369,666 \\
\hline May & 0 & 0 & 0 & 17,869 & 0 & 285,902 \\
\hline June & 0 & 0 & 0 & 24,046 & 0 & 384,739 \\
\hline July & 0 & 0 & 0 & 24,897 & 0 & 398,345 \\
\hline August & 0 & 0 & 0 & 22,671 & 0 & 362,729 \\
\hline September & 0 & 0 & 0 & 16,926 & 0 & 270,820 \\
\hline October & 0 & 0 & 0 & 14,895 & 0 & 238,325 \\
\hline November & 0 & 0 & 0 & 16,518 & 0 & 264,286 \\
\hline December & 0 & 0 & 0 & 20,069 & 0 & 321,098 \\
\hline Annual & 0 & 0 & 0 & 24,897 & 0 & $3,752,539$ \\
\hline
\end{tabular}

Rate: Rate 1

\begin{tabular}{|c|c|c|c|c|c|c|}
\hline Resources.ReportingService_GeneratelnputsReport_Month & $\begin{array}{l}\text { Energy } \\
\text { Purchased } \\
\text { (kWh) }\end{array}$ & $\begin{array}{l}\text { Energy } \\
\text { Sold } \\
\text { (kWh) }\end{array}$ & $\begin{array}{l}\text { Net } \\
\text { Purchases } \\
\text { (kWh) }\end{array}$ & $\begin{array}{l}\text { Peak } \\
\text { Demand } \\
(\mathrm{kW})\end{array}$ & $\begin{array}{l}\text { Energy } \\
\text { Charge (\$) }\end{array}$ & $\begin{array}{l}\text { Demand } \\
\text { Charge } \\
\text { (\$) }\end{array}$ \\
\hline
\end{tabular}




\begin{tabular}{|c|c|c|c|c|c|c|}
\hline January & 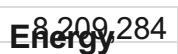 & Energy & Nêt209,284 & Peak 0 & 985,114 & Demand \\
\hline February & PGrchasted & Sold 0 & PGruthas & Deman日 & Enezposy255 & Charge 0 \\
\hline $\begin{array}{l}\text { Resources.ReportingService_GeneratelnputsReport_Month } \\
\text { March }\end{array}$ & $\begin{array}{l}\text { (kWh) } \\
6,816,916\end{array}$ & ${ }_{0}^{(k W h)}$ & $\begin{array}{l}\text { (kWh) } \\
6,816,916\end{array}$ & $(\mathbf{k W}) \quad 0$ & $\begin{array}{r}\text { Charge (\$) } \\
818,030\end{array}$ & (\$) $\quad 0$ \\
\hline April & $9,462,522$ & 0 & $9,462,522$ & 0 & $1,135,503$ & 0 \\
\hline May & $7,805,883$ & 0 & $7,805,883$ & 0 & 936,706 & 0 \\
\hline June & $9,816,466$ & 0 & $9,816,466$ & 0 & $1,177,976$ & 0 \\
\hline July & $10,003,713$ & 0 & $10,003,713$ & 0 & $1,200,446$ & 0 \\
\hline August & $9,901,865$ & 0 & $9,901,865$ & 0 & $1,188,224$ & 0 \\
\hline September & $6,943,139$ & 0 & $6,943,139$ & 0 & 833,177 & 0 \\
\hline October & $6,792,313$ & 0 & $6,792,313$ & 0 & 815,078 & 0 \\
\hline November & $6,321,105$ & 0 & $6,321,105$ & 0 & 758,533 & 0 \\
\hline December & $8,680,090$ & 0 & $8,680,090$ & 0 & $1,041,611$ & 0 \\
\hline Annual & $96,863,744$ & 0 & $96,863,744$ & 0 & $11,623,651$ & 0 \\
\hline
\end{tabular}

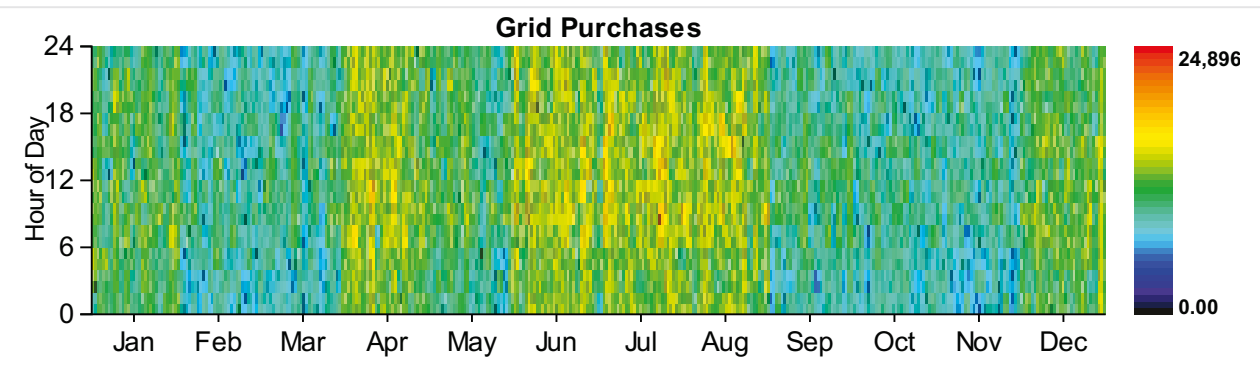

\section{Emissions}

\begin{tabular}{|l|r|l|}
\hline Pollutant & Emissions & Units \\
\hline Carbon dioxide & $61217888 \mathrm{~kg} / \mathrm{yr}$ \\
\hline Carbon monoxide & 0 & $\mathrm{~kg} / \mathrm{yr}$ \\
\hline Unburned hydrocarbons & 0 & $\mathrm{~kg} / \mathrm{yr}$ \\
\hline Particulate matter & 0 & $\mathrm{~kg} / \mathrm{yr}$ \\
\hline Sulfur dioxide & $265407 \mathrm{~kg} / \mathrm{yr}$ \\
\hline Nitrogen oxides & $129797 \mathrm{~kg} / \mathrm{yr}$ \\
\hline
\end{tabular}


System Report

System architecture

\begin{tabular}{|l|l|l|}
\hline PV & Solar World 320W flat plate PV Copy & 1,000 \\
\hline Converter & System Converter & 750 \\
\hline Grid & Grid & 25,000 \\
\hline Dispatch Strategy & Cycle Charging & $\mathrm{kW}$ \\
\hline
\end{tabular}

\section{Cost summary}

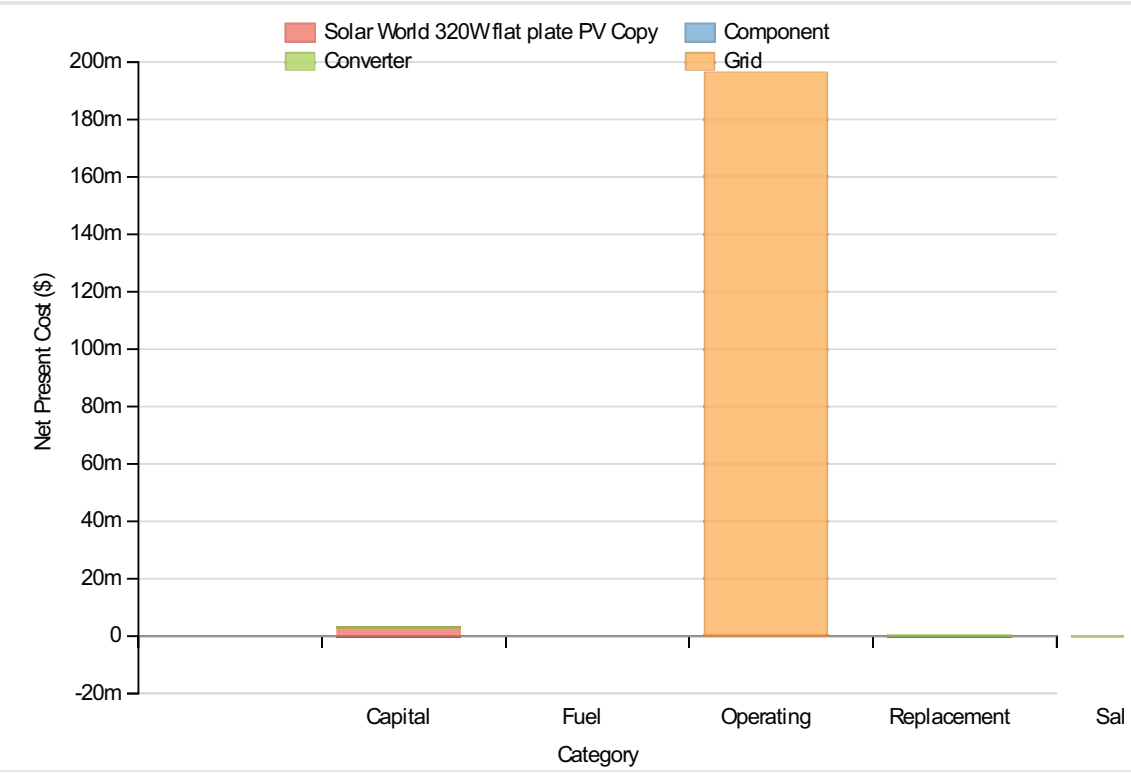

Cost Summary

Total net present cost

$199704592 \$$

Levelized cost of energy

$0.159 \$ / k W h$

Net Present Costs

\begin{tabular}{|l|r|r|r|r|r|r|}
\hline Component & Capital & Replacement & O\&M & Fuel & Salvage & Total \\
\hline Solar World 320W flat plate PV Copy & $3,000,000$ & 0 & 193,913 & 0 & 0 & $3,193,913$ \\
\hline Grid & 0 & 0 & $196,208,096$ & 0 & 0 & $196,208,096$ \\
\hline Converter & 225,000 & 95,462 & 0 & 0 & $-17,967$ \\
\hline System & $3,225,000$ & 95,462 & $196,401,968$ & 0 & $-17,967$ & $199,704,463$ \\
\hline
\end{tabular}

Annualized Costs

\begin{tabular}{|l|r|r|r|r|r|r|}
\hline Component & Capital & Replacement & O\&M & Fuel & Salvage & Total \\
\hline Solar World 320W flat plate PV Copy & 232,063 & 0 & 15,000 & 0 & 0 \\
\hline Grid & 0 & 0 & $15,177,555$ & 0 & 0 \\
\hline Converter & 17,405 & 7,384 & 0 & 0 & $-1,390$ \\
\hline System & 249,468 & 7,384 & $15,192,552$ & 0 & $-1,390$ \\
\hline
\end{tabular}




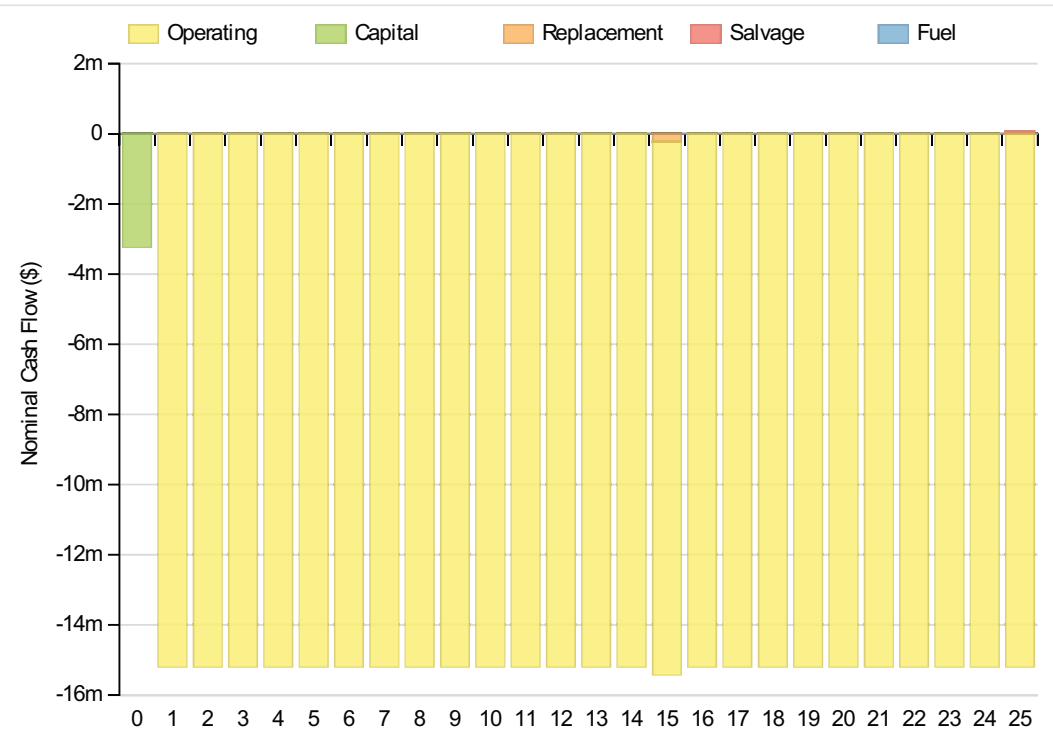

Year

\section{Electrical}

\begin{tabular}{|c|c|c|c|c|}
\hline Quantity & & Value & & Units \\
\hline Excess electricity & & & 5225 & kWh/yr \\
\hline Unmet load & & & 0 & $\mathrm{kWh} / \mathrm{yr}$ \\
\hline Capacity shortage & & & 3418 & $\mathrm{kWh} / \mathrm{yr}$ \\
\hline Renewable fraction & & & 0 & \\
\hline Component & Production(kWh/yr) & & Fracti & tion (\%) \\
\hline PV & & $1,288,675$ & & 1 \\
\hline Grid Purchases & & $95,709,088$ & & 99 \\
\hline Total & & $96,997,760$ & & 100 \\
\hline Load & Consumption(kWh/yr) & & Fra & action (\%) \\
\hline AC primary load & & $96,864,160$ & & 100 \\
\hline DC primary load & & 0 & 0 & 0 \\
\hline Total & & $96,864,160$ & & 100 \\
\hline
\end{tabular}




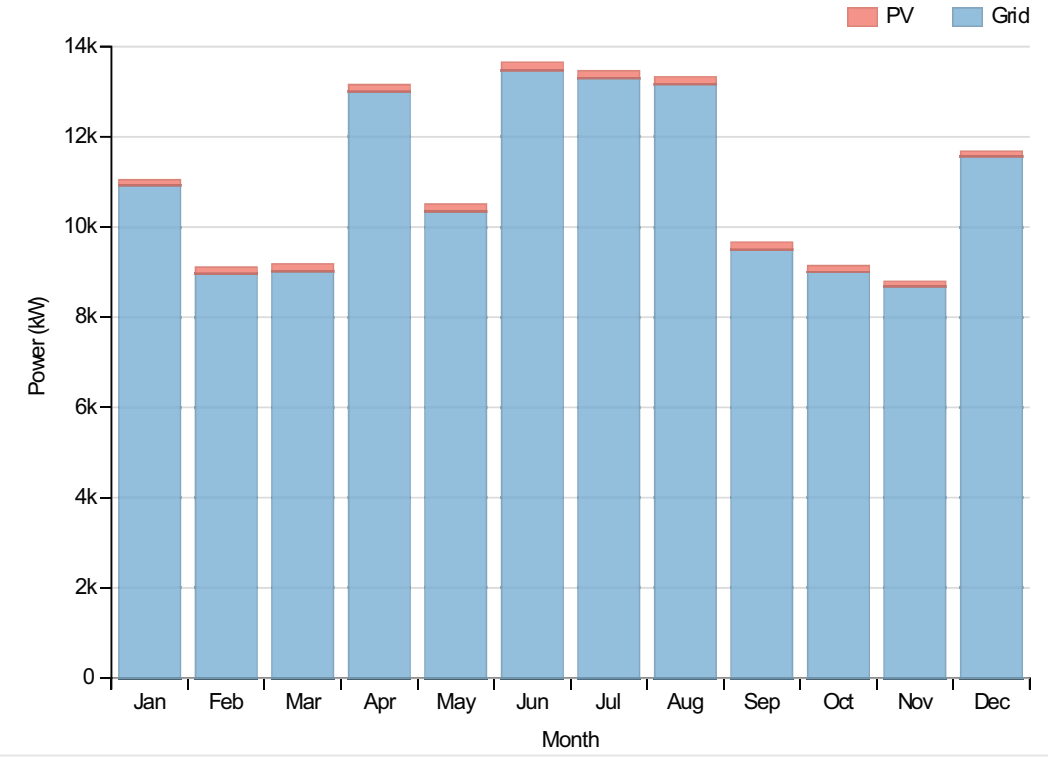

PV:Solar World 320W flat plate PV Copy

\begin{tabular}{|l|c|c|}
\hline Quantity & Value & Units \\
\hline Rated capacity & $1000 \mathrm{~kW}$ \\
\hline Mean output & $147 \mathrm{~kW}$ \\
\hline Mean output & $3530.60 \mathrm{kWh} / \mathrm{d}$ \\
\hline Capacity factor & $14.71 \%$ \\
\hline Total production & $1288675 \mathrm{kWh} / \mathrm{yr}$ \\
\hline Minimum output & $0.00 \mathrm{~kW}$ \\
\hline Maximum output & $1022.50 \mathrm{~kW}$ \\
\hline PV penetration & $1.33 \%$ \\
\hline Hours of operation & $4377 \mathrm{hrs} / \mathrm{yr}$ \\
\hline Levelized cost & $\$ 1 / \mathrm{kWh}$ \\
\hline
\end{tabular}

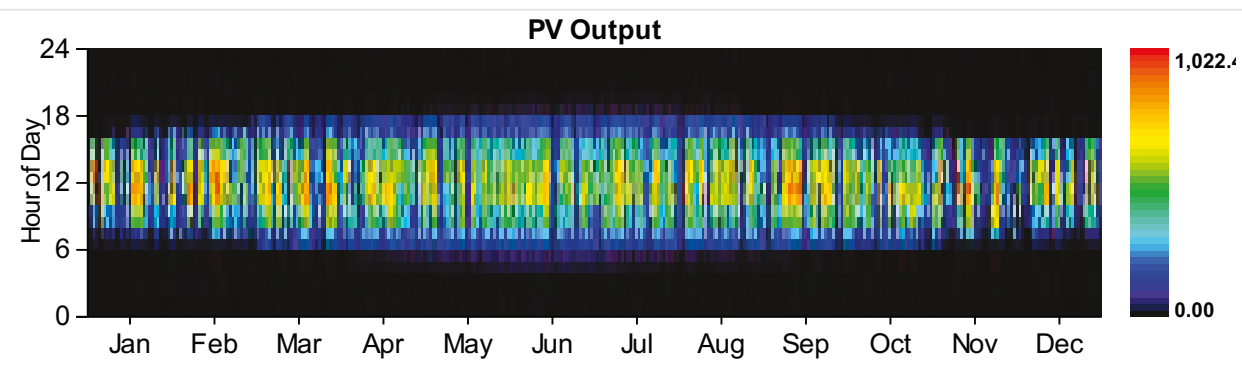

\section{Converter}

\section{Quantity}

Capacity

Mean output

Minimum output

Maximum output

Capacity factor

\section{Inverter}

Rectifier

750

132

0

750

18 


\begin{tabular}{|c|c|c|c|}
\hline Ruarntifyoperation & Inverter & Rectifier 0 & Hejits \\
\hline Energy in & $1,283,450$ & 0 & $\mathrm{kWh} / \mathrm{yr}$ \\
\hline Energy out & $1,155,104$ & 0 & $\mathrm{kWh} / \mathrm{yr}$ \\
\hline Losses & 128,346 & 0 & $\mathrm{kWh} / \mathrm{yr}$ \\
\hline
\end{tabular}
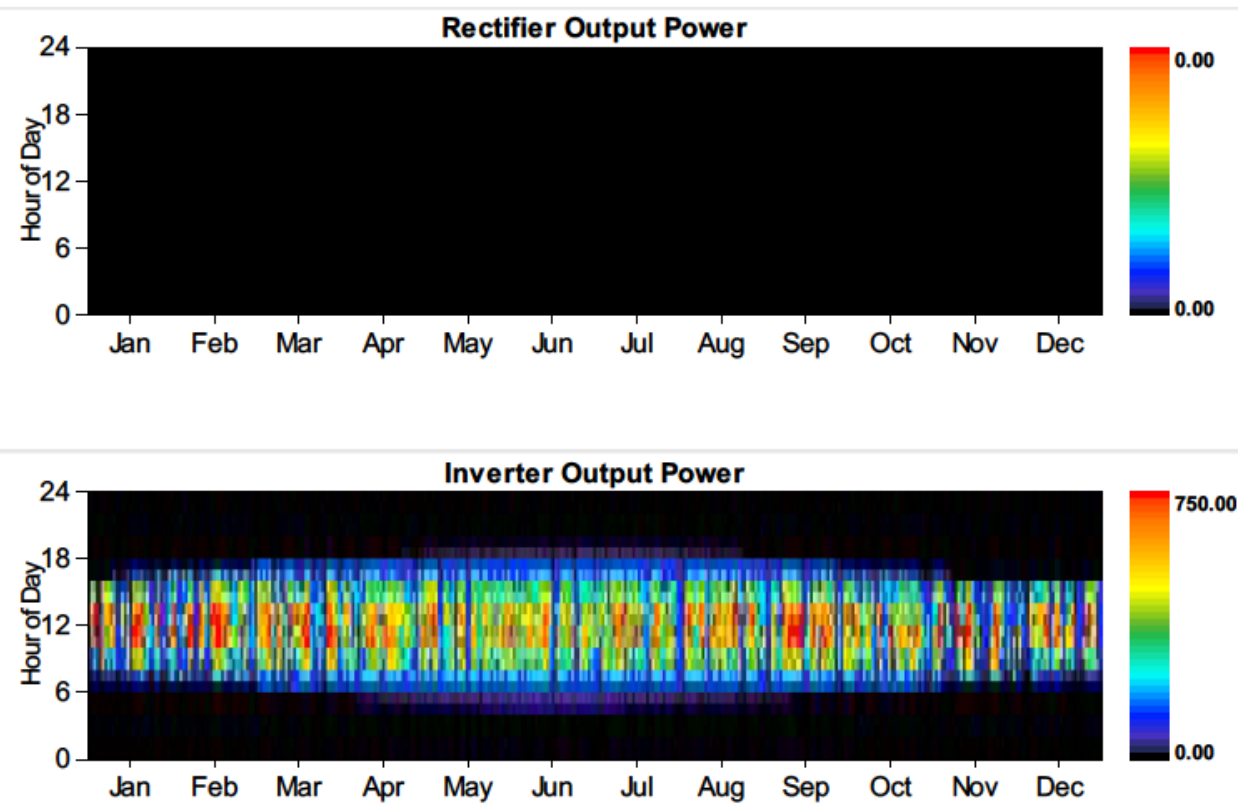

Grid

Rate: Demand 1

\begin{tabular}{|c|c|c|c|c|c|c|}
\hline Resources.ReportingService_GeneratelnputsReport_Month & $\begin{array}{l}\text { Energy } \\
\text { Purchased } \\
\text { (kWh) }\end{array}$ & $\begin{array}{l}\text { Energy } \\
\text { Sold } \\
\text { (kWh) }\end{array}$ & $\begin{array}{l}\text { Net } \\
\text { Purchases } \\
\text { (kWh) }\end{array}$ & $\begin{array}{l}\text { Peak } \\
\text { Demand } \\
(\mathbf{k W})\end{array}$ & $\begin{array}{l}\text { Energy } \\
\text { Charge } \\
\text { (\$) }\end{array}$ & $\begin{array}{l}\text { Demand } \\
\text { Charge } \\
(\$)\end{array}$ \\
\hline January & 0 & 0 & 0 & 18,553 & 0 & 296,843 \\
\hline February & 0 & 0 & 0 & 16,811 & 0 & 268,978 \\
\hline March & 0 & 0 & 0 & 16,941 & 0 & 271,062 \\
\hline April & 0 & 0 & 0 & 22,535 & 0 & 360,567 \\
\hline May & 0 & 0 & 0 & 17,657 & 0 & 282,514 \\
\hline June & 0 & 0 & 0 & 23,894 & 0 & 382,297 \\
\hline July & 0 & 0 & 0 & 24,491 & 0 & 391,852 \\
\hline August & 0 & 0 & 0 & 22,568 & 0 & 361,094 \\
\hline September & 0 & 0 & 0 & 16,176 & 0 & 258,820 \\
\hline October & 0 & 0 & 0 & 14,744 & 0 & 235,900 \\
\hline November & 0 & 0 & 0 & 16,518 & 0 & 264,286 \\
\hline December & 0 & 0 & 0 & 19,891 & 0 & 318,260 \\
\hline Annual & 0 & 0 & 0 & 24,491 & 0 & $3,692,473$ \\
\hline
\end{tabular}

Rate: Rate 1 


\begin{tabular}{|c|c|c|c|c|c|c|}
\hline Resources.ReportingService_GeneratelnputsReport_Month & $\begin{array}{l}\text { Energy } \\
\text { Purchased } \\
\text { (kWh) }\end{array}$ & $\begin{array}{l}\text { Energy } \\
\text { Sold } \\
(\mathbf{k W h})\end{array}$ & $\begin{array}{l}\text { Net } \\
\text { Purchases } \\
\text { (kWh) }\end{array}$ & $\begin{array}{l}\text { Peak } \\
\text { Demand } \\
(\mathbf{k W})\end{array}$ & $\begin{array}{l}\text { Energy } \\
\text { Charge (\$) }\end{array}$ & $\begin{array}{l}\text { Demand } \\
\text { Charge } \\
(\$)\end{array}$ \\
\hline January & $8,132,278$ & 0 & $8,132,278$ & 0 & 975,873 & 0 \\
\hline February & $6,023,890$ & 0 & $6,023,890$ & 0 & 722,867 & 0 \\
\hline March & $6,708,956$ & 0 & $6,708,956$ & 0 & 805,075 & 0 \\
\hline April & $9,361,015$ & 0 & $9,361,015$ & 0 & $1,123,322$ & 0 \\
\hline May & $7,697,129$ & 0 & $7,697,129$ & 0 & 923,655 & 0 \\
\hline June & $9,706,376$ & 0 & $9,706,376$ & 0 & $1,164,765$ & 0 \\
\hline July & $9,891,672$ & 0 & $9,891,672$ & 0 & $1,187,001$ & 0 \\
\hline August & $9,791,200$ & 0 & $9,791,200$ & 0 & $1,174,944$ & 0 \\
\hline September & $6,837,491$ & 0 & $6,837,491$ & 0 & 820,499 & 0 \\
\hline October & $6,699,125$ & 0 & $6,699,125$ & 0 & 803,895 & 0 \\
\hline November & $6,249,541$ & 0 & $6,249,541$ & 0 & 749,945 & 0 \\
\hline December & $8,610,419$ & 0 & $8,610,419$ & 0 & $1,033,250$ & 0 \\
\hline Annual & $95,709,088$ & 0 & $95,709,088$ & 0 & $11,485,090$ & 0 \\
\hline
\end{tabular}

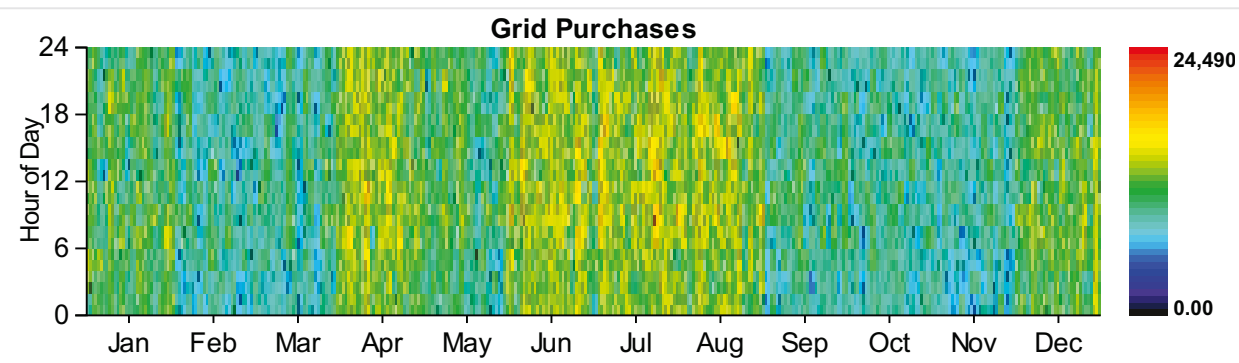

\section{Emissions}

\begin{tabular}{|l|r|l|}
\hline Pollutant & Emissions & Units \\
\hline Carbon dioxide & 60488144 & $\mathrm{~kg} / \mathrm{yr}$ \\
\hline Carbon monoxide & 0 & $\mathrm{~kg} / \mathrm{yr}$ \\
\hline Unburned hydrocarbons & $0 \mathrm{~kg} / \mathrm{yr}$ \\
\hline Particulate matter & $0 \mathrm{~kg} / \mathrm{yr}$ \\
\hline Sulfur dioxide & $262243 \mathrm{~kg} / \mathrm{yr}$ \\
\hline Nitrogen oxides & $128250 \mathrm{~kg} / \mathrm{yr}$ \\
\hline
\end{tabular}


System Report

System architecture

\begin{tabular}{|l|l|r|r|}
\hline PV & Solar World 320W flat plate PV Copy & 1,000 & $\mathrm{~kW}$ \\
\hline Battery & GS200 flow & 1 strings \\
\hline Converter & System Converter & $750 \mathrm{~kW}$ \\
\hline Grid & Grid & $25,000 \mathrm{~kW}$ \\
\hline Dispatch Strategy & Cycle Charging & \\
\hline
\end{tabular}

Cost summary

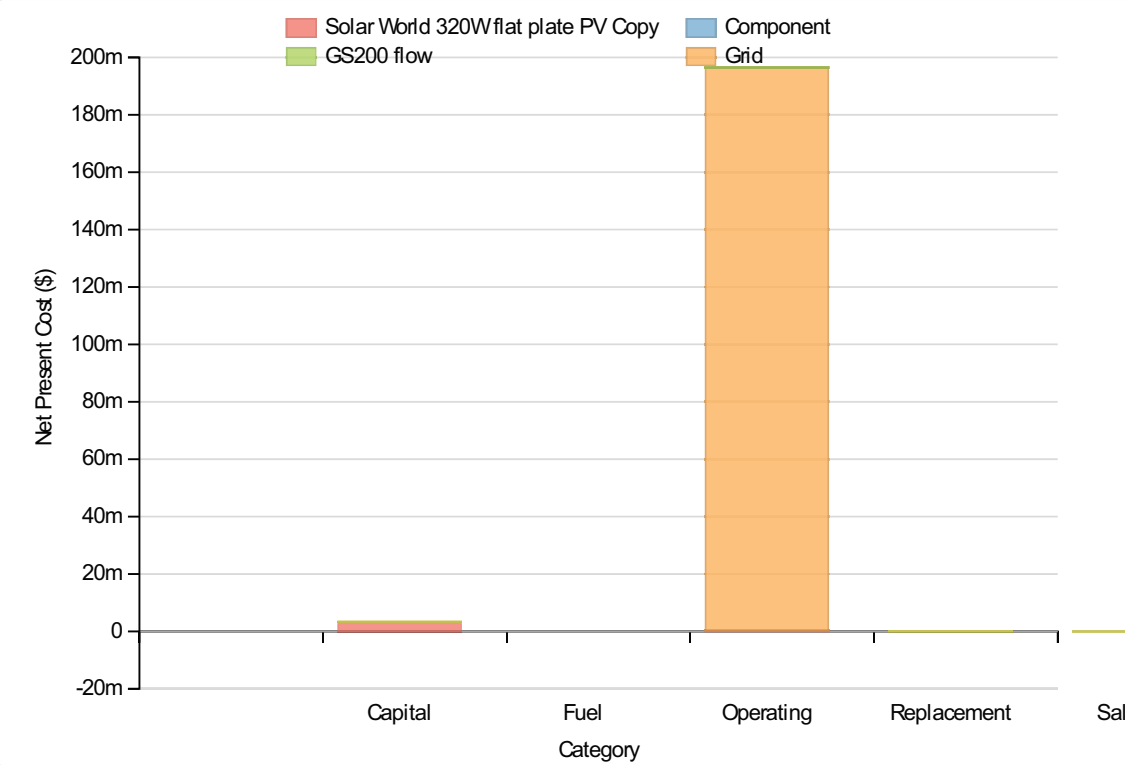

Cost Summary

Total net present cost

$200060624 \$$

Levelized cost of energy

$0.160 \$ / \mathrm{kWh}$

Net Present Costs

\begin{tabular}{|l|r|r|r|r|r|r|}
\hline Component & Capital & Replacement & O\&M & Fuel & Salvage & Total \\
\hline Solar World 320W flat plate PV Copy & $3,000,000$ & 0 & 193,913 & 0 & 0 \\
\hline Grid & 0 & 0 & $196,202,320$ & 0 & 0 \\
\hline GS200 flow & 324,589 & 7,176 & 31,026 & 0 & -973 \\
\hline Converter & 225,000 & 95,462 & 0 & 0 & $-17,967$ \\
\hline System & $3,549,589$ & 102,637 & $196,427,320$ \\
\hline
\end{tabular}

\section{Annualized Costs}

\section{Component}

Solar World 320W flat plate PV Copy

\begin{tabular}{|l|r|r|r|r|r|r|}
\hline Component & Capital & Replacement & O\&M & Fuel & Salvage & Total \\
\hline Solar World 320W flat plate PV Copy & 232,063 & 0 & 15,000 & 0 & 0 \\
\hline Grid & 0 & 0 & $15,177,109$ & 0 & 0 \\
\hline GS200 flow & 25,108 & 555 & 2,400 & 0 & -75 \\
\hline Converter & 17,405 & 27,063 & 27,109 \\
\hline
\end{tabular}




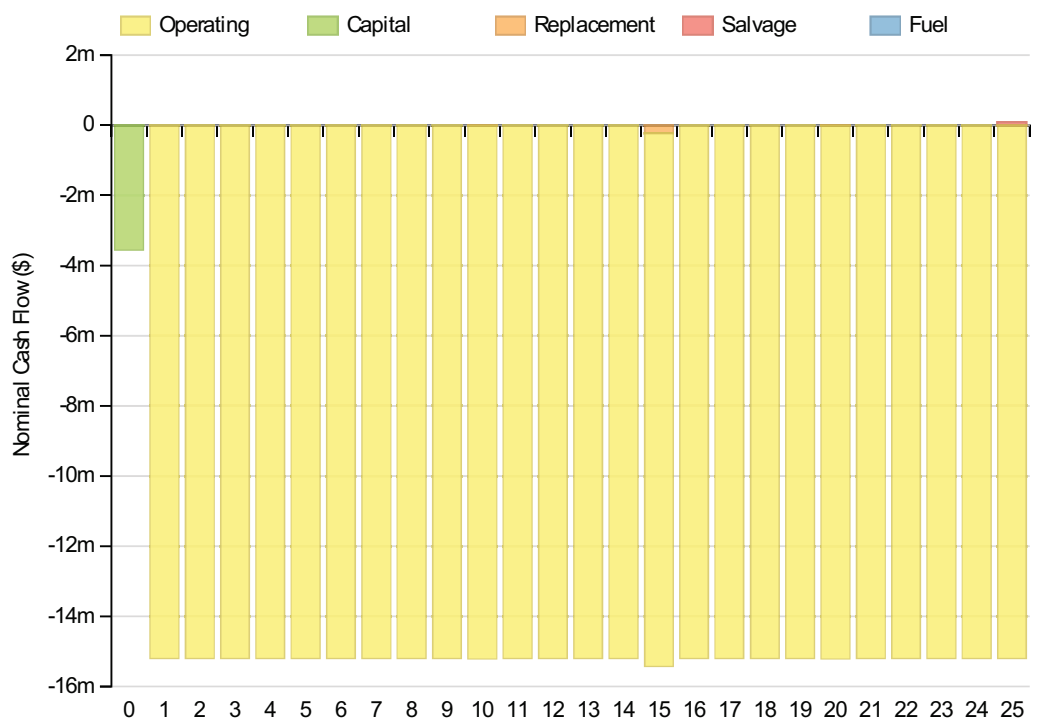

Year

\section{Electrical}

\begin{tabular}{|c|c|c|c|c|}
\hline Quantity & & Value & & Units \\
\hline Excess electricity & & & 10 & $\mathrm{kWh} / \mathrm{yr}$ \\
\hline Unmet load & & & 0 & $\mathrm{kWh} / \mathrm{yr}$ \\
\hline Capacity shortage & & & 3418 & $\mathrm{kWh} / \mathrm{yr}$ \\
\hline Renewable fraction & & & 0 & \\
\hline Component & Production(kWh/yr) & & Fract & tion (\%) \\
\hline PV & & $1,288,675$ & & 1 \\
\hline Grid Purchases & & $95,705,352$ & & 99 \\
\hline Total & & $96,994,024$ & & 100 \\
\hline Load & Consumption(kWh/yr) & & Fra & action (\%) \\
\hline AC primary load & & $96,864,160$ & & 100 \\
\hline DC primary load & & & 0 & 0 \\
\hline Total & & $96,864,160$ & & 100 \\
\hline
\end{tabular}




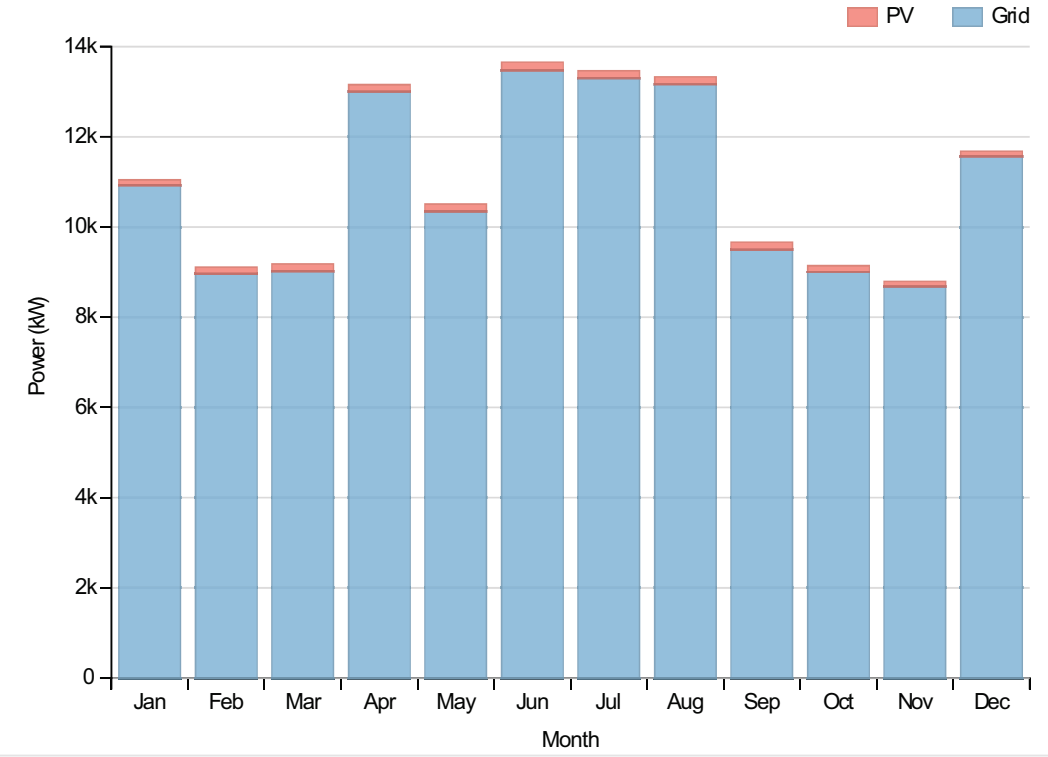

PV:Solar World 320W flat plate PV Copy

\begin{tabular}{|l|c|c|}
\hline Quantity & Value & Units \\
\hline Rated capacity & $1000 \mathrm{~kW}$ \\
\hline Mean output & $147 \mathrm{~kW}$ \\
\hline Mean output & $3530.60 \mathrm{kWh} / \mathrm{d}$ \\
\hline Capacity factor & $14.71 \%$ \\
\hline Total production & $1288675 \mathrm{kWh} / \mathrm{yr}$ \\
\hline Minimum output & $0.00 \mathrm{~kW}$ \\
\hline Maximum output & $1022.50 \mathrm{~kW}$ \\
\hline PV penetration & $1.33 \%$ \\
\hline Hours of operation & $4377 \mathrm{hrs} / \mathrm{yr}$ \\
\hline Levelized cost & $\$ 1 / \mathrm{kWh}$ \\
\hline
\end{tabular}

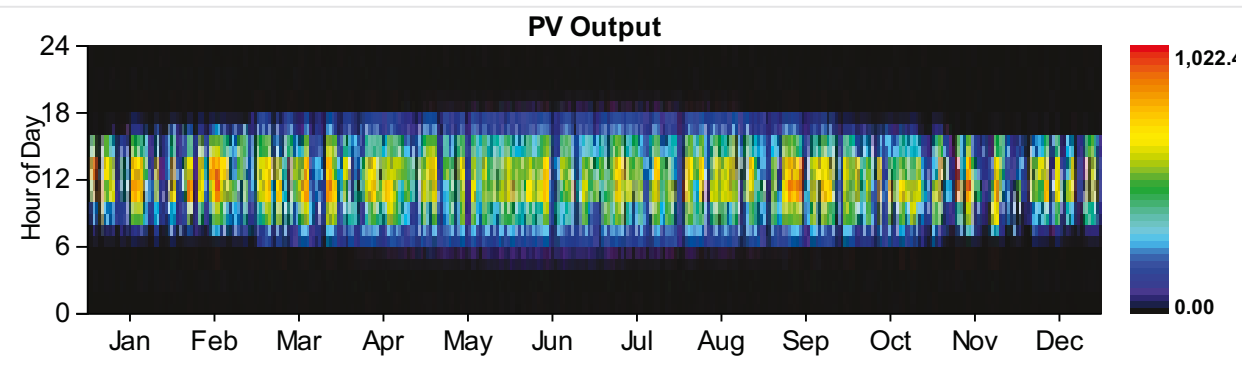

\section{Battery:GS200 flow}

\section{Quantity}

Value

String size

Strings in parallel 


\section{Quantity}

Value

Units

Nominal capacity

600 kWh

Usable nominal capacity

$600 \mathrm{kWh}$

Autonomy

$0 \mathrm{hr}$

Lifetime throughput

0

Battery wear cost

$0.000 \$ / k W h$

Average energy cost

$0.000 \$ / k W h$

Energy in

$5215 \mathrm{kWh} / \mathrm{yr}$

Energy out

$4152 \mathrm{kWh} / \mathrm{yr}$

Storage depletion

$600 \mathrm{kWh} / \mathrm{yr}$

Losses

462 kWh/yr

Annual throughput

$4963 \mathrm{kWh} / \mathrm{yr}$

Expected life

$25 \mathrm{yr}$

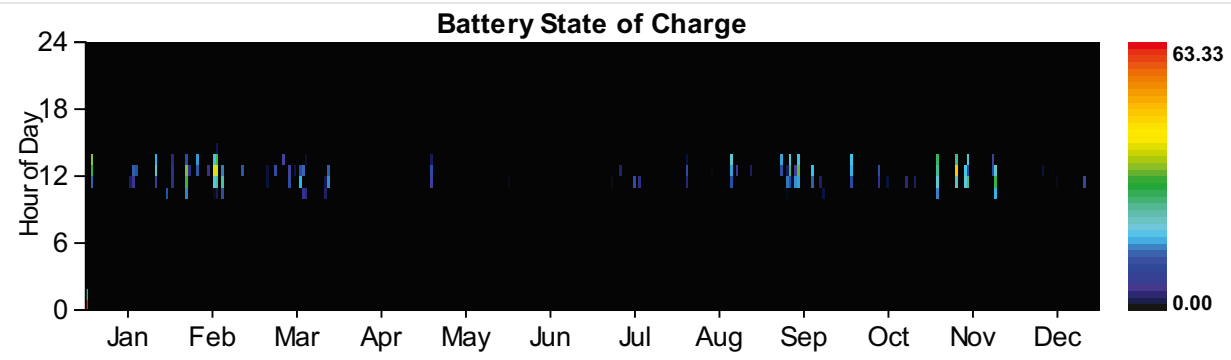

\section{Converter}

\begin{tabular}{|c|c|c|c|}
\hline Quantity & Inverter & Rectifier & Units \\
\hline Capacity & 750 & 675 & $\mathrm{~kW}$ \\
\hline Mean output & 132 & 0 & kW \\
\hline Minimum output & 0 & 0 & $\mathrm{~kW}$ \\
\hline Maximum output & 750 & 0 & $\mathrm{~kW}$ \\
\hline Capacity factor & 18 & 0 & $\%$ \\
\hline Hours of operation & 4,463 & 0 & $\mathrm{hrs} / \mathrm{yr}$ \\
\hline Energy in & $1,287,602$ & 0 & $\mathrm{kWh} / \mathrm{yr}$ \\
\hline Energy out & $1,158,841$ & 0 & $\mathrm{kWh} / \mathrm{yr}$ \\
\hline Losses & 128,761 & 0 & $\mathrm{kWh} / \mathrm{yr}$ \\
\hline
\end{tabular}



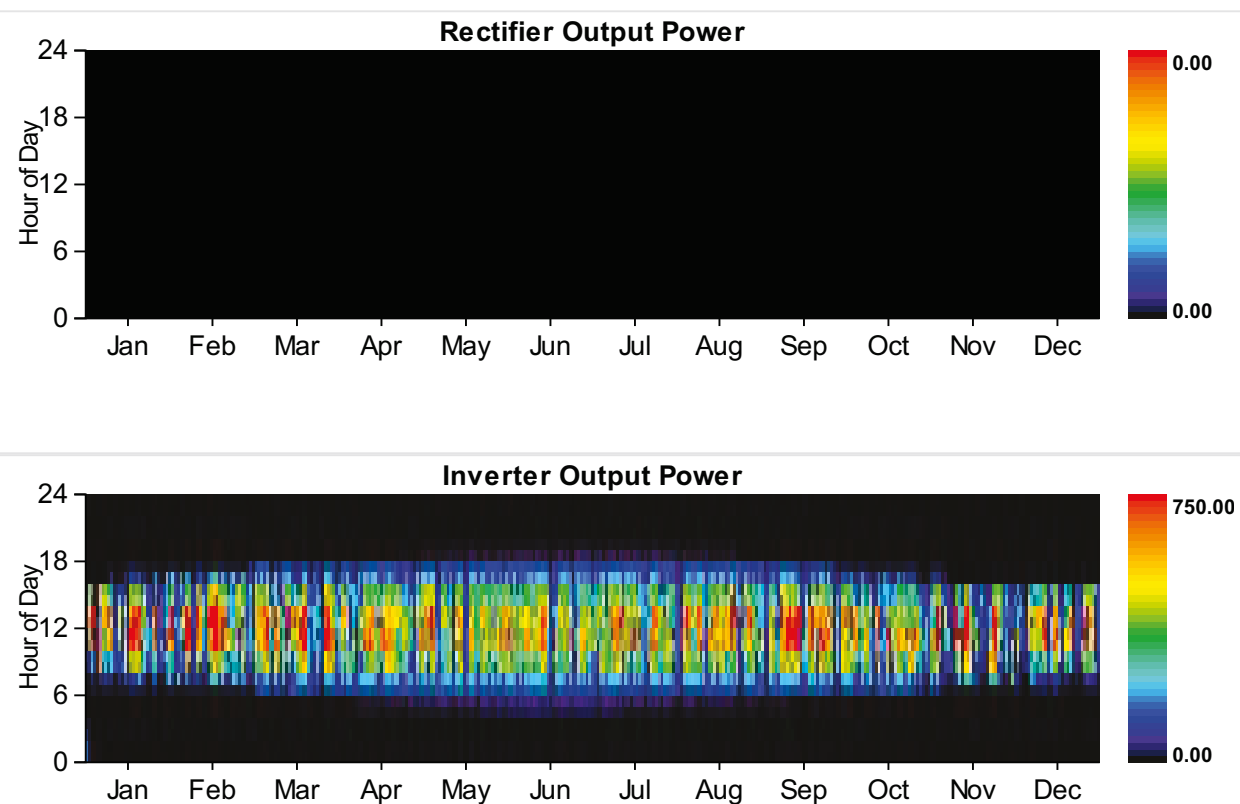

Grid

Rate: Demand 1

\begin{tabular}{|c|c|c|c|c|c|c|}
\hline Resources.ReportingService_GeneratelnputsReport_Month & $\begin{array}{l}\text { Energy } \\
\text { Purchased } \\
\text { (kWh) }\end{array}$ & $\begin{array}{l}\text { Energy } \\
\text { Sold } \\
\text { (kWh) }\end{array}$ & $\begin{array}{l}\text { Net } \\
\text { Purchases } \\
\text { (kWh) }\end{array}$ & $\begin{array}{l}\text { Peak } \\
\text { Demand } \\
(\mathbf{k W})\end{array}$ & $\begin{array}{l}\text { Energy } \\
\text { Charge } \\
(\$)\end{array}$ & $\begin{array}{l}\text { Demand } \\
\text { Charge } \\
(\$)\end{array}$ \\
\hline January & 0 & 0 & 0 & 18,553 & 0 & 296,843 \\
\hline February & 0 & 0 & 0 & 16,811 & 0 & 268,978 \\
\hline March & 0 & 0 & 0 & 16,941 & 0 & 271,062 \\
\hline April & 0 & 0 & 0 & 22,535 & 0 & 360,567 \\
\hline May & 0 & 0 & 0 & 17,657 & 0 & 282,514 \\
\hline June & 0 & 0 & 0 & 23,894 & 0 & 382,297 \\
\hline July & 0 & 0 & 0 & 24,491 & 0 & 391,852 \\
\hline August & 0 & 0 & 0 & 22,568 & 0 & 361,094 \\
\hline September & 0 & 0 & 0 & 16,176 & 0 & 258,820 \\
\hline October & 0 & 0 & 0 & 14,744 & 0 & 235,900 \\
\hline November & 0 & 0 & 0 & 16,518 & 0 & 264,286 \\
\hline December & 0 & 0 & 0 & 19,891 & 0 & 318,260 \\
\hline Annual & 0 & 0 & 0 & 24,491 & 0 & $3,692,473$ \\
\hline
\end{tabular}

Rate: Rate 1

\begin{tabular}{|c|c|c|c|c|c|c|}
\hline Resources.ReportingService_GeneratelnputsReport_Month & $\begin{array}{l}\text { Energy } \\
\text { Purchased } \\
\text { (kWh) }\end{array}$ & $\begin{array}{l}\text { Energy } \\
\text { Sold } \\
(\mathbf{k W h})\end{array}$ & $\begin{array}{l}\text { Net } \\
\text { Purchases } \\
\text { (kWh) }\end{array}$ & $\begin{array}{l}\text { Peak } \\
\text { Demand } \\
(\mathbf{k W})\end{array}$ & $\begin{array}{l}\text { Energy } \\
\text { Charge (\$) }\end{array}$ & $\begin{array}{l}\text { Demand } \\
\text { Charge } \\
(\$)\end{array}$ \\
\hline January & $8,131,427$ & 0 & $8,131,427$ & 0 & 975,771 & 0 \\
\hline February & $6,023,114$ & 0 & $6,023,114$ & 0 & 722,774 & 0 \\
\hline March & $6,708,603$ & 0 & $6,708,603$ & 0 & 805,032 & 0 \\
\hline
\end{tabular}




\begin{tabular}{|c|c|c|c|c|c|c|}
\hline Rałources.ReportingService_GeneratelnputsReport_Month & 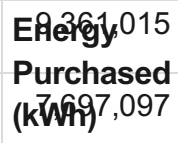 & $\begin{array}{l}\text { Energy } \\
\text { Sold } \\
\text { (kWh) } 0\end{array}$ & 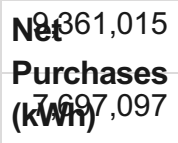 & $\begin{array}{l}\text { Peak } 0 \\
\text { Demand } \\
(\mathbf{k W}) \quad 0\end{array}$ & $\begin{array}{l}\text { 1,123,322 } \\
\text { Energy } \\
\text { Chafge } 6 \text { \$sq }\end{array}$ & $\begin{array}{l}\text { Demand } \\
\text { Charge } \\
\text { (\$) } 0\end{array}$ \\
\hline June & $9,706,373$ & 0 & $9,706,373$ & 0 & $1,164,765$ & 0 \\
\hline July & $9,891,607$ & 0 & $9,891,607$ & 0 & $1,186,993$ & 0 \\
\hline August & $9,791,049$ & 0 & $9,791,049$ & 0 & $1,174,926$ & 0 \\
\hline September & $6,836,924$ & 0 & $6,836,924$ & 0 & 820,431 & 0 \\
\hline October & $6,698,983$ & 0 & $6,698,983$ & 0 & 803,878 & 0 \\
\hline November & $6,248,778$ & 0 & $6,248,778$ & 0 & 749,853 & 0 \\
\hline December & $8,610,384$ & 0 & $8,610,384$ & 0 & $1,033,246$ & 0 \\
\hline Annual & $95,705,352$ & 0 & $95,705,352$ & 0 & $11,484,642$ & 0 \\
\hline
\end{tabular}

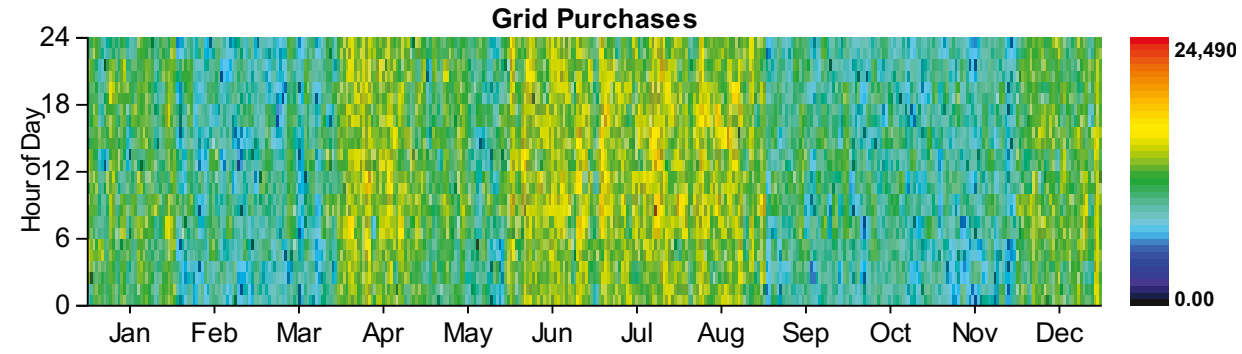

\section{Emissions}

\begin{tabular}{|l|r|l|}
\hline Pollutant & Emissions & Units \\
\hline Carbon dioxide & 60485784 & $\mathrm{~kg} / \mathrm{yr}$ \\
\hline Carbon monoxide & 0 & $\mathrm{~kg} / \mathrm{yr}$ \\
\hline Unburned hydrocarbons & $\mathrm{kg} / \mathrm{yr}$ \\
\hline Particulate matter & 0 & $\mathrm{~kg} / \mathrm{yr}$ \\
\hline Sulfur dioxide & $262233 \mathrm{~kg} / \mathrm{yr}$ \\
\hline Nitrogen oxides & $128245 \mathrm{~kg} / \mathrm{yr}$ \\
\hline
\end{tabular}


System Report

System architecture

\begin{tabular}{|l|l|r|}
\hline Wind Turbine & Siemens 2.3 MW - 108 & 15 \\
\hline Generator & Kohler 3250 Prime Power & 16,800 \\
\hline Battery & GS200 flow & 60 \\
\hline Converter & System Converter & strings \\
\hline Dispatch Strategy & Cycle Charging & 24,000 \\
\hline
\end{tabular}

\section{Cost summary}

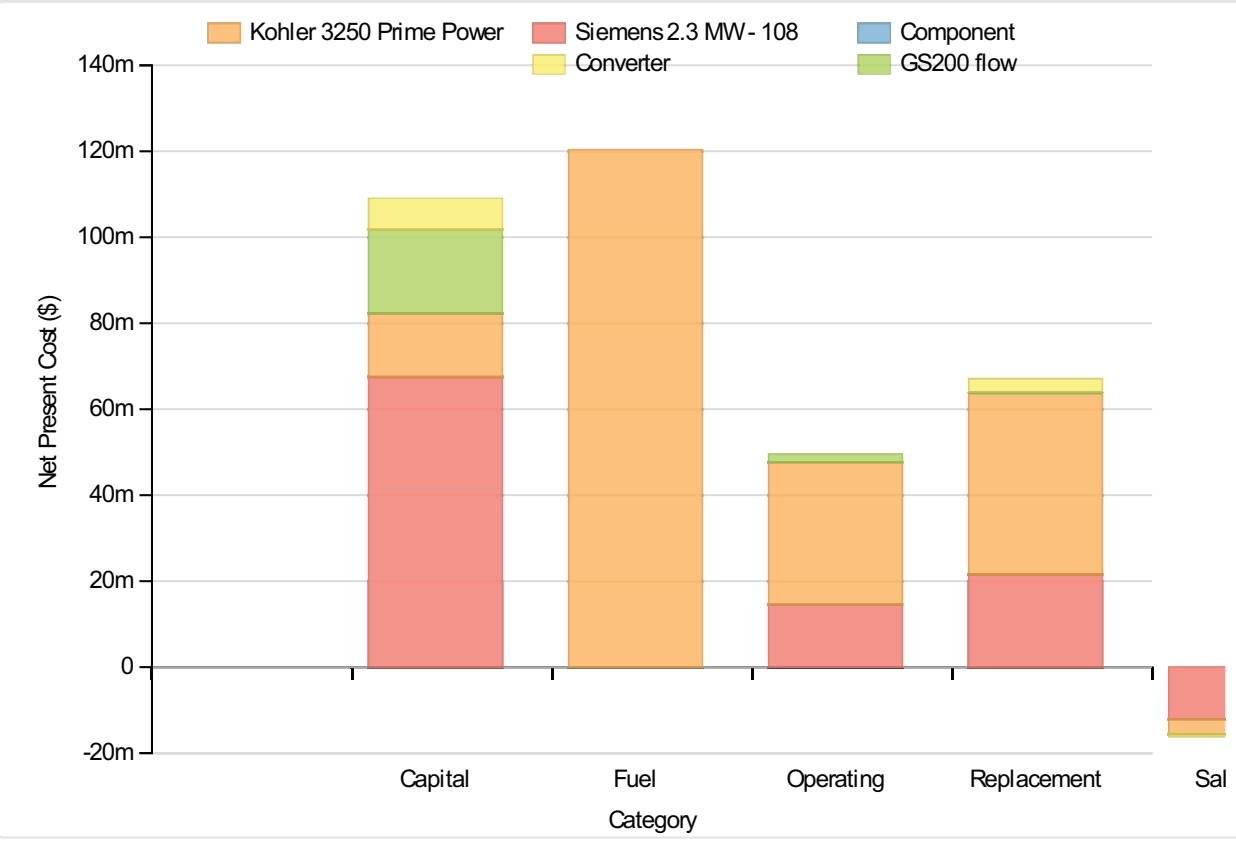

Cost Summary

Total net present cost

$329803680 \$$

Levelized cost of energy

$0.263 \$ / k W h$

Net Present Costs

\begin{tabular}{|l|r|r|r|r|r|r|}
\hline Component & Capital & Replacement & O\&M & Fuel & Salvage & Total \\
\hline Siemens 2.3 MW - 108 & $67,500,000$ & $21,519,472$ & $14,543,448$ & 0 & $-12,127,600$ & $91,435,320$ \\
\hline Kohler 3250 Prime Power & $14,821,428$ & $42,092,420$ & $33,130,706$ & $120,349,664$ & $-3,414,479$ & $206,979,739$ \\
\hline GS200 flow & $19,475,352$ & 430,058 & $1,861,562$ & 0 & $-58,308$ & $21,708,664$ \\
\hline Converter & $7,200,000$ & $3,054,769$ & 0 & $-574,938$ & $9,679,831$ \\
\hline System & $108,996,776$ & $67,096,720$ & $49,535,724$ & $120,349,664$ & $-16,175,325$ & $329,803,559$ \\
\hline
\end{tabular}

Annualized Costs

\begin{tabular}{|l|r|r|r|r|r|r|}
\hline Component & Capital & Replacement & O\&M & Fuel & Salvage & Total \\
\hline Siemens 2.3 MW - 108 & $5,221,421$ & $1,664,625$ & $1,124,999$ & 0 & $-938,123$ & $7,072,922$ \\
\hline
\end{tabular}




\begin{tabular}{|l|r|r|r|r|r|r|}
\hline GS200 flow & $1,506,504$ & 33,267 & 144,000 & 0 & $-4,510$ & $1,679,261$ \\
\hline Converter & 556,952 & 236,300 & 0 & 0 & $-44,474$ \\
\hline System & $8,431,378$ & $5,190,225$ & $3,831,805$ & $9,309,574$ & $-1,251,232$ & $25,511,750$ \\
\hline
\end{tabular}

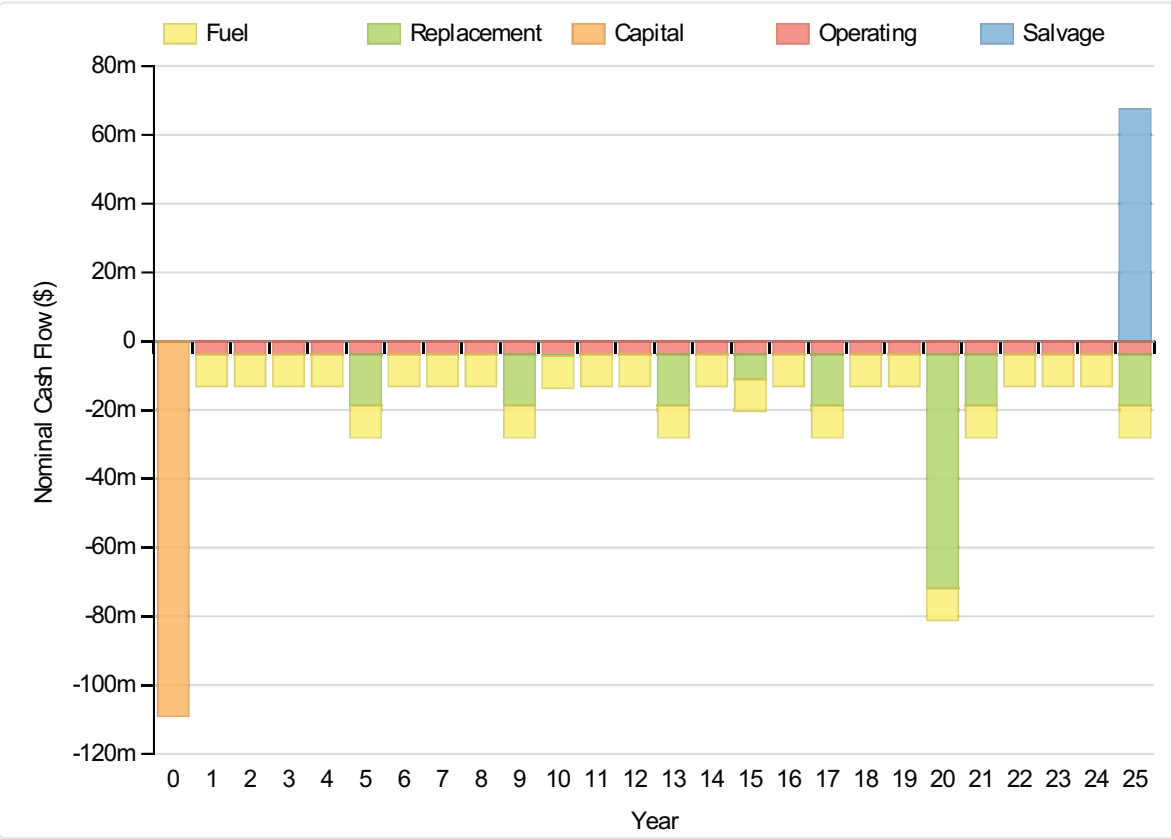

\section{Electrical}

\section{Quantity}

Excess electricity

Unmet load

Capacity shortage

Renewable fraction

\section{Value}

Units

$35579732 \mathrm{kWh} / \mathrm{yr}$

$0 \mathrm{kWh} / \mathrm{yr}$

$2329 \mathrm{kWh} / \mathrm{yr}$

0

\section{Component}

Generator

Wind Turbine

Total

Load

Consumption(kWh/yr)

\section{Fraction (\%)}

$50,113,132$

$90,806,648$

64

$140,919,776$

100

AC primary load

$96,864,160$

100

DC primary load

0

0

Total

$96,864,160$ 


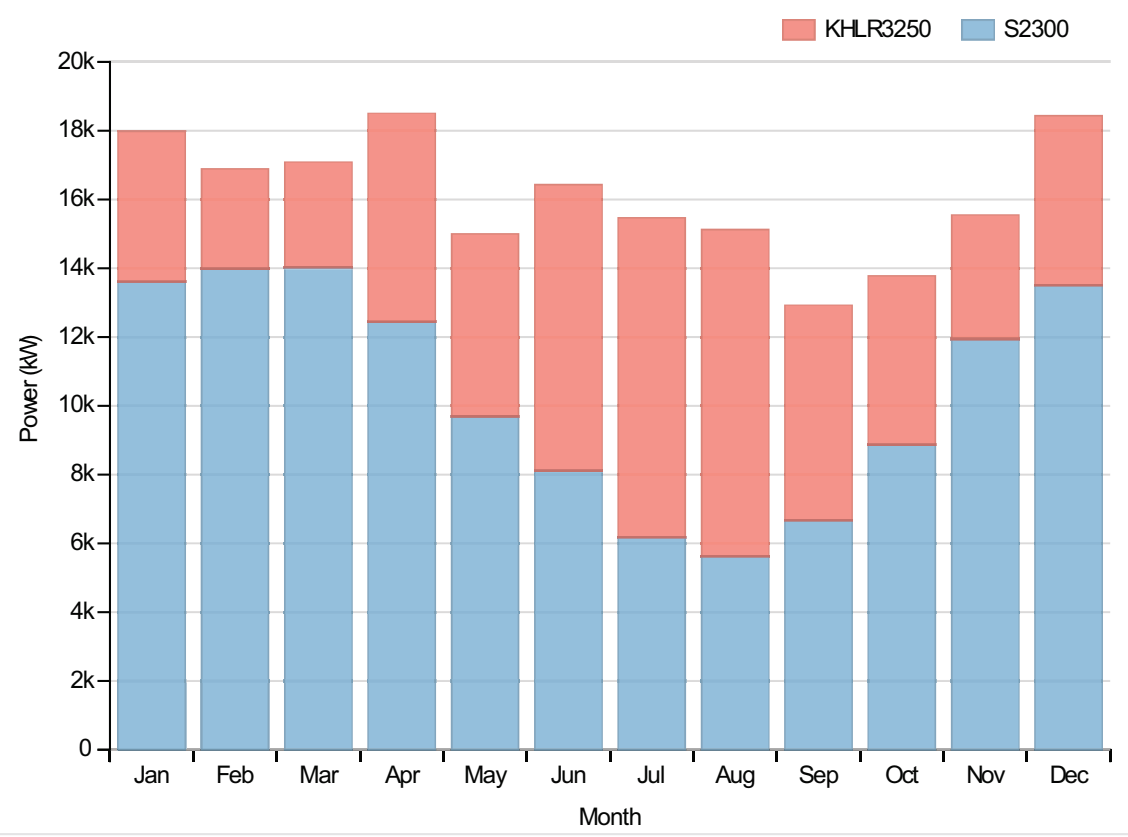

Wind Turbine:Siemens 2.3 MW - 108

\begin{tabular}{|l|r|l|}
\hline Quantity & Value & Units \\
\hline Total rated capacity & $34500 \mathrm{~kW}$ \\
\hline Mean output & $10366 \mathrm{~kW}$ \\
\hline Capacity factor & $30.05 \%$ \\
\hline Total production & $90806648 \mathrm{kWh} / \mathrm{yr}$ \\
\hline Minimum output & $21.57 \mathrm{~kW}$ \\
\hline Maximum output & $34723.00 \mathrm{~kW}$ \\
\hline Wind penetration & $93.75 \%$ \\
\hline Hours of operation & $8760 \mathrm{hrs} / \mathrm{yr}$ \\
\hline Levelized cost & 0.078 & $\$ / \mathrm{kWh}$ \\
\hline
\end{tabular}

\section{Generator:Kohler 3250 Prime Power}

\section{Quantity}

Hours of operation

Number of starts

Operational life

Fixed generation cost

Marginal generation cost

Electrical production

Mean electrical output

Min. electrical output

\section{Value}

Units

$3623 \mathrm{hrs} / \mathrm{yr}$

1209 starts/yr

$4 \mathrm{yr}$

$1728.30 \$ / h r$

$0.18 \$ / k W h$

$50113132 \mathrm{kWh} / \mathrm{yr}$

$13832 \mathrm{~kW}$

4200 kW 
Qaănitegtrical output

Fuel consumption

Specific fuel consumption

Fuel energy input

Mean electrical efficiency
Value

16800 kinits

11784276 L/yr

$0.24 \mathrm{~L} / \mathrm{kWh}$

$115957288 \mathrm{kWh} / \mathrm{yr}$

$43 \%$

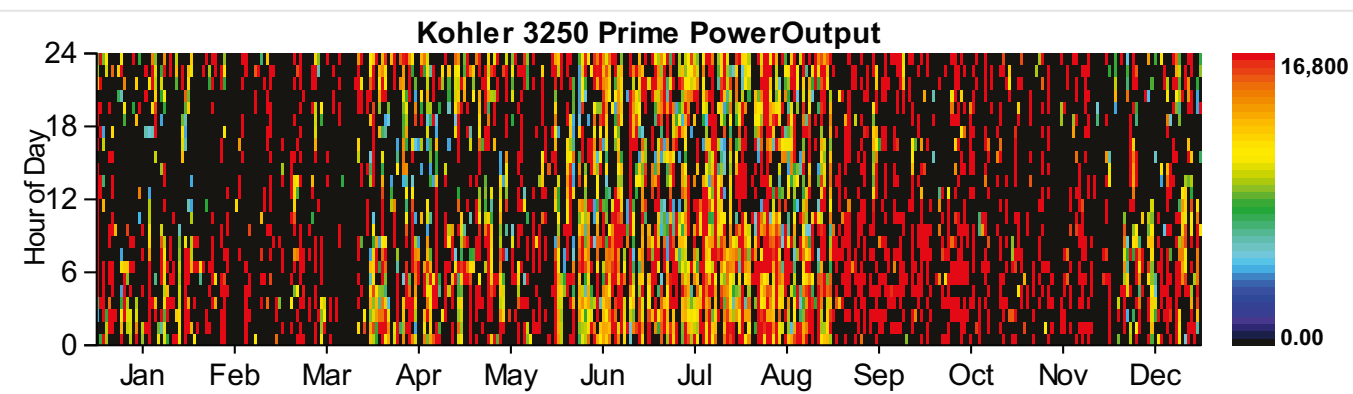

\section{Battery:GS200 flow}

\section{Quantity}

Value

String size

Strings in parallel

Batteries

Bus voltage

\section{Quantity}

Nominal capacity

Usable nominal capacity

Autonomy

Lifetime throughput

Battery wear cost

Average energy cost

Energy in

Energy out

Storage depletion

Losses

Annual throughput

Expected life

\section{Value}

Units

36000 kWh

36000 kWh

$3 \mathrm{hr}$

0

$0.000 \$ / k W h$

$0.202 \$ / k W h$

$15509890 \mathrm{kWh} / \mathrm{yr}$

$10856936 \mathrm{kWh} / \mathrm{yr}$

$0 \mathrm{kWh} / \mathrm{yr}$

4652954 kWh/yr

$12976515 \mathrm{kWh} / \mathrm{yr}$

$25 \mathrm{yr}$ 


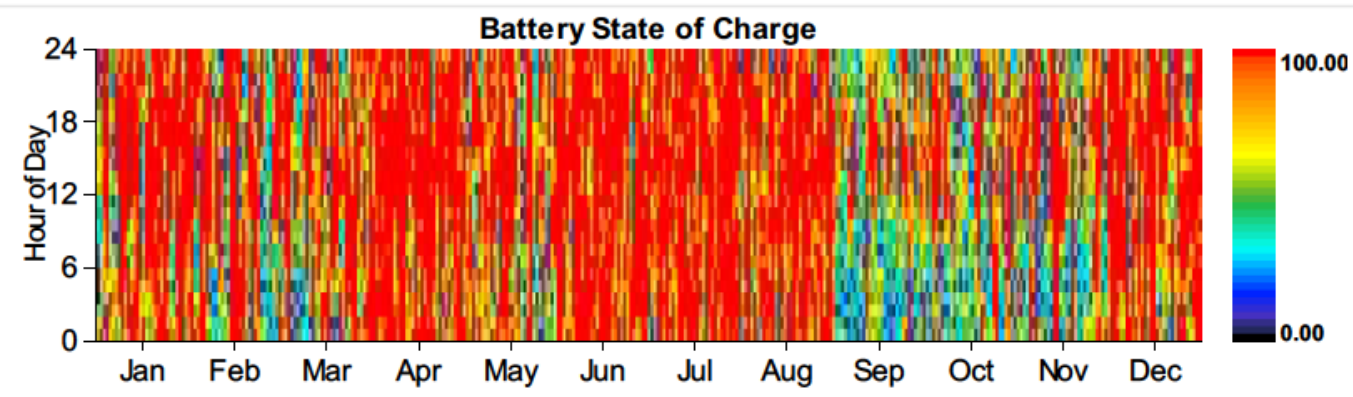

Converter

\begin{tabular}{|l|r|r|l|}
\hline Quantity & Inverter & Rectifier & Units \\
\hline Capacity & 24,000 & 21,600 & $\mathrm{~kW}$ \\
\hline Mean output & 1,115 & $1,771 \mathrm{~kW}$ \\
\hline Minimum output & 0 & $0 \mathrm{~kW}$ \\
\hline Maximum output & 8,983 & 10,757 & $\mathrm{~kW}$ \\
\hline Capacity factor & 5 & $7 \%$ \\
\hline Hours of operation & 2,201 & 2,998 & $\mathrm{hrs} / \mathrm{yr}$ \\
\hline Energy in & $10,856,936$ & $18,246,954$ & $\mathrm{kWh} / \mathrm{yr}$ \\
\hline Energy out & $9,771,234$ & $15,509,890 \mathrm{kWh} / \mathrm{yr}$ \\
\hline Losses & $1,085,702$ & $2,737,064$ & $\mathrm{kWh} / \mathrm{yr}$ \\
\hline
\end{tabular}
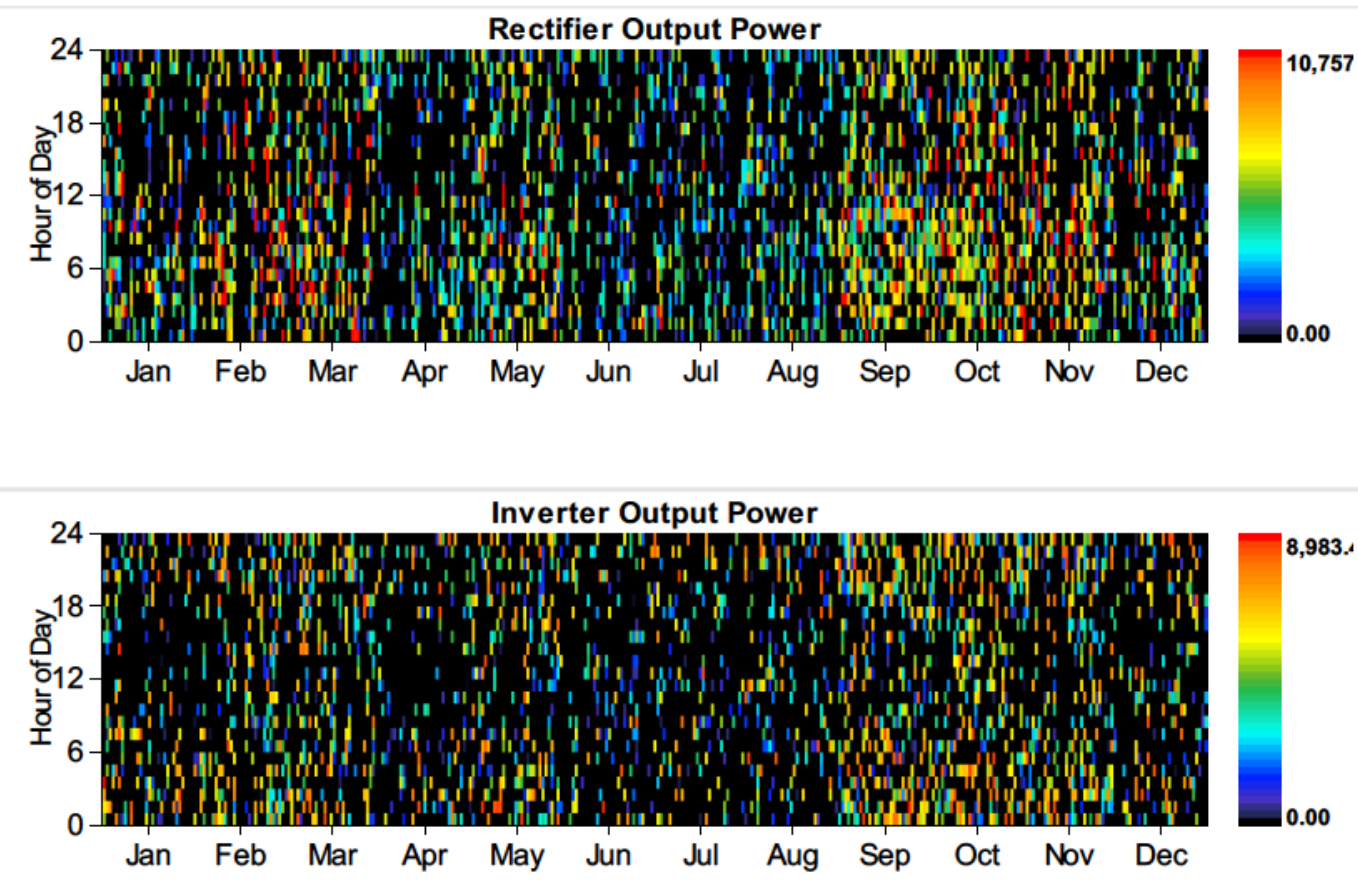

\section{Emissions}

\begin{tabular}{|l|r|l|}
\hline Pollutant & Emissions & Units \\
\hline Carbon dioxide & 30928012 & $\mathrm{~kg} / \mathrm{yr}$ \\
\hline Carbon monoxide & 129627 & $\mathrm{~kg} / \mathrm{yr}$ \\
\hline
\end{tabular}


Pollutant
Unburned hydrocarbons

Emissions

Particulate matter

Sulfur dioxide

Nitrogen oxides

HOMER Energy, LLC @ 2016
Units

$14848 \mathrm{~kg} / \mathrm{yr}$

$3712 \mathrm{~kg} / \mathrm{yr}$

$63679 \mathrm{~kg} / \mathrm{yr}$

$129627 \mathrm{~kg} / \mathrm{yr}$ 
System Report

System architecture

\begin{tabular}{|l|l|r|}
\hline PV & Solar World 320W flat plate PV Copy & 5,000 \\
\hline Wind Turbine & Siemens 2.3 MW - 108 & 15 \\
\hline Generator & Kohler 3250 Prime Power & $16,800 \mathrm{~kW}$ \\
\hline Battery & GS200 flow & 60 strings \\
\hline Converter & System Converter & 24,000 \\
\hline Dispatch Strategy & Cycle Charging & $\mathrm{kW}$ \\
\hline
\end{tabular}

\section{Cost summary}

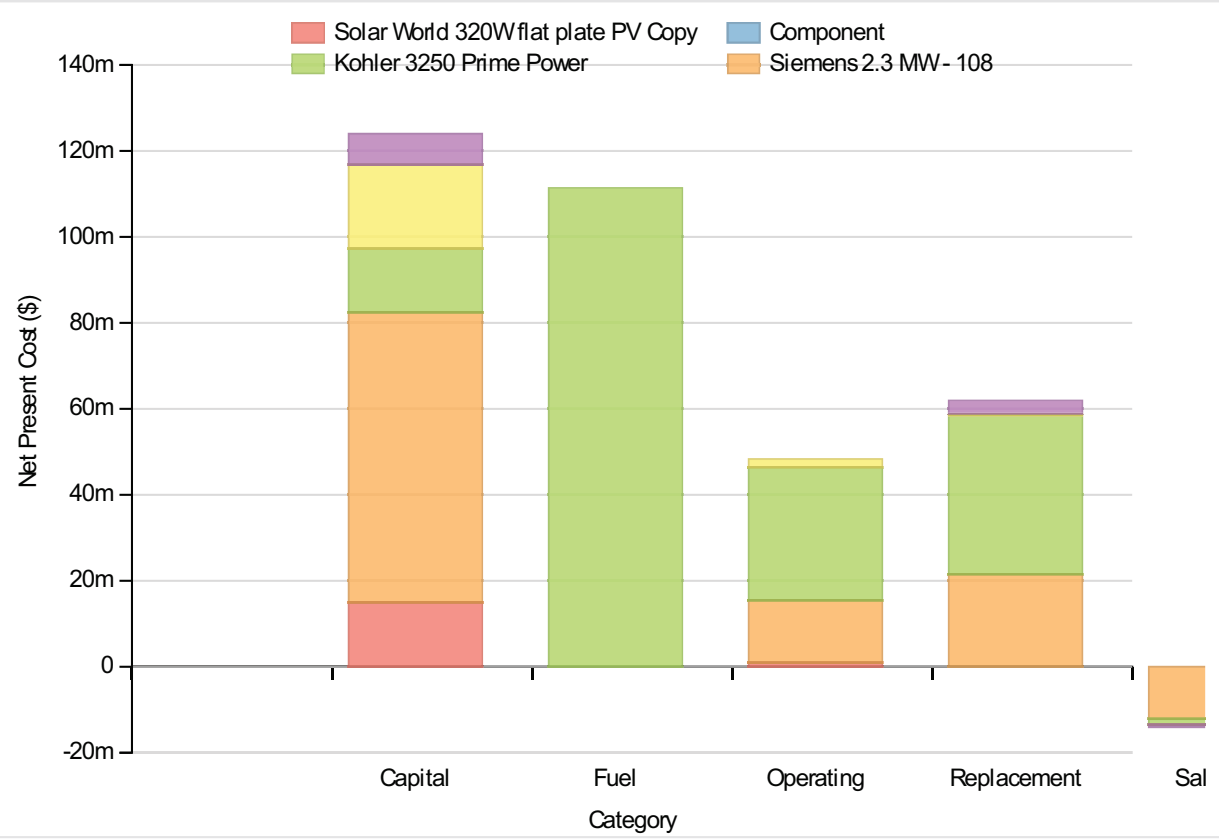

Cost Summary

Total net present cost

$331481728 \$$

Levelized cost of energy

$0.265 \$ / k W h$

\section{Net Present Costs}

\begin{tabular}{|c|c|c|c|c|c|c|}
\hline Component & Capital & Replacement & O\&M & Fuel & Salvage & Total \\
\hline $\begin{array}{l}\text { Solar World } 320 \mathrm{~W} \text { flat plate PV } \\
\text { Copy }\end{array}$ & $15,000,000$ & 0 & 969,563 & 0 & 0 & $15,969,563$ \\
\hline Siemens 2.3 MW - 108 & $67,500,000$ & $21,519,472$ & $14,543,448$ & 0 & $-12,127,600$ & $91,435,320$ \\
\hline Kohler 3250 Prime Power & $14,821,428$ & $36,898,980$ & $30,917,726$ & $111,346,072$ & $-1,295,964$ & $192,688,242$ \\
\hline GS200 flow & $19,475,352$ & 430,058 & $1,861,562$ & 0 & $-58,308$ & $21,708,664$ \\
\hline Converter & $7,200,000$ & $3,054,769$ & 0 & 0 & $-574,938$ & $9,679,831$ \\
\hline System & $123,996,776$ & $61,903,280$ & $48,292,304$ & $111,346,072$ & $-14,056,811$ & $331,481,621$ \\
\hline
\end{tabular}




\section{Annualized Costs}

\begin{tabular}{|l|r|r|r|r|r|r|}
\hline Component & Capital & Replacement & O\&M & Fuel & Salvage & \multicolumn{1}{l|}{ Total } \\
\hline Solar World 320W flat plate PV Copy & $1,160,316$ & 0 & 75,000 & 0 & 0 & $1,235,316$ \\
\hline Siemens 2.3 MW - 108 & $5,221,421$ & $1,664,625$ & $1,124,999$ & 0 & $-938,123$ & $7,072,922$ \\
\hline Kohler 3250 Prime Power & $1,146,502$ & $2,854,298$ & $2,391,622$ & $8,613,106$ & $-100,249$ & $14,905,279$ \\
\hline GS200 flow & $1,506,504$ & 33,267 & 144,000 & 0 & $-4,510$ & $1,679,261$ \\
\hline Converter & 556,952 & 236,300 & 0 & 0 & $-44,474$ & 748,778 \\
\hline System & $9,591,694$ & $4,788,490$ & $3,735,621$ & $8,613,106$ & $-1,087,356$ & $25,641,555$ \\
\hline
\end{tabular}

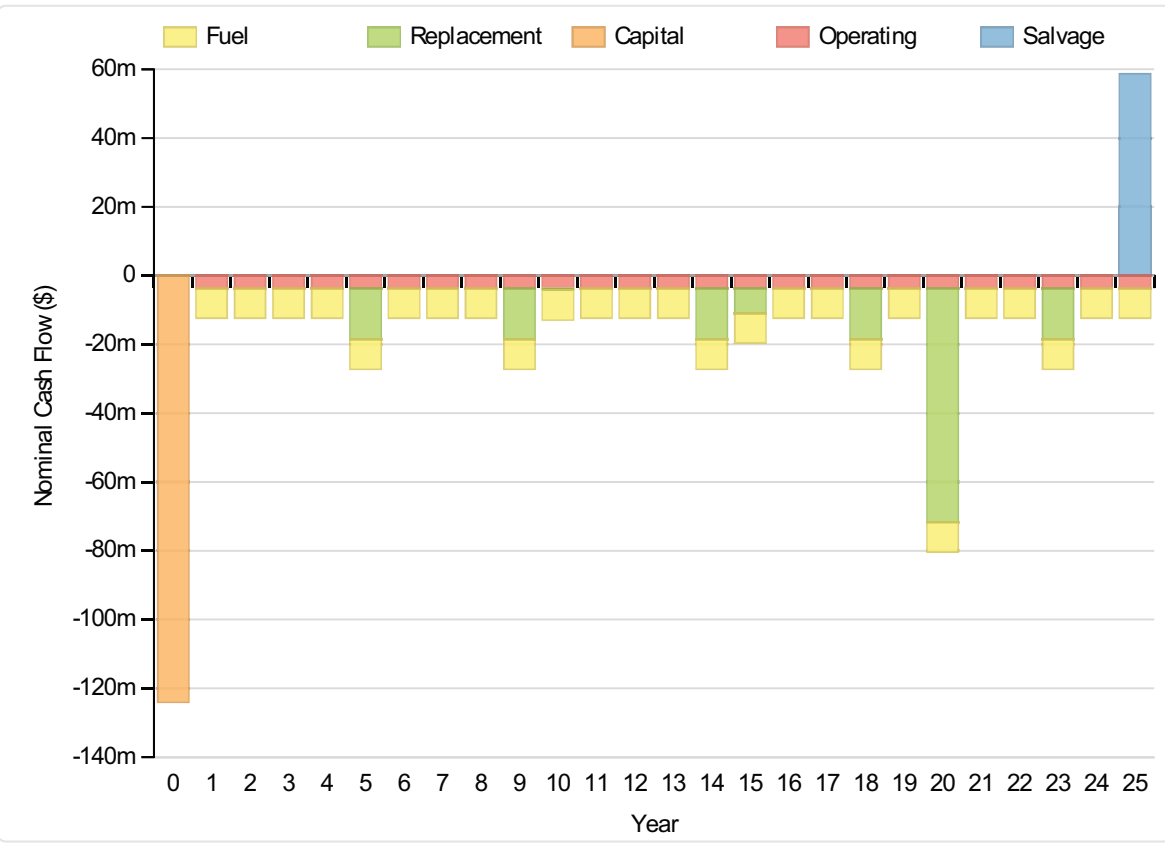

\section{Electrical}

\begin{tabular}{|c|c|c|c|c|}
\hline \multirow{2}{*}{\multicolumn{2}{|c|}{$\begin{array}{l}\text { Quantity } \\
\text { Excess electricity }\end{array}$}} & \multicolumn{2}{|l|}{ Value } & Units \\
\hline & & \multicolumn{2}{|r|}{38246392} & $\mathrm{kWh} / \mathrm{yr}$ \\
\hline \multicolumn{2}{|l|}{ Unmet load } & \multicolumn{2}{|r|}{0} & $\mathrm{kWh} / \mathrm{yr}$ \\
\hline \multicolumn{2}{|l|}{ Capacity shortage } & \multicolumn{2}{|r|}{2329} & $\mathrm{kWh} / \mathrm{yr}$ \\
\hline \multicolumn{2}{|l|}{ Renewable fraction } & \multicolumn{2}{|r|}{1} & \\
\hline Component & \multicolumn{2}{|l|}{ Production(kWh/yr) } & \multicolumn{2}{|c|}{ Fraction (\%) } \\
\hline PV & & $6,443,367$ & & 4 \\
\hline Generator & & $46,359,196$ & & 32 \\
\hline Wind Turbine & & $90,806,648$ & & 63 \\
\hline Total & & $143,609,216$ & & 100 \\
\hline Load & Consumption(kWh/yr) & & Fract & tion (\%) \\
\hline AC primary load & & $96,864,1$ & 160 & 100 \\
\hline
\end{tabular}




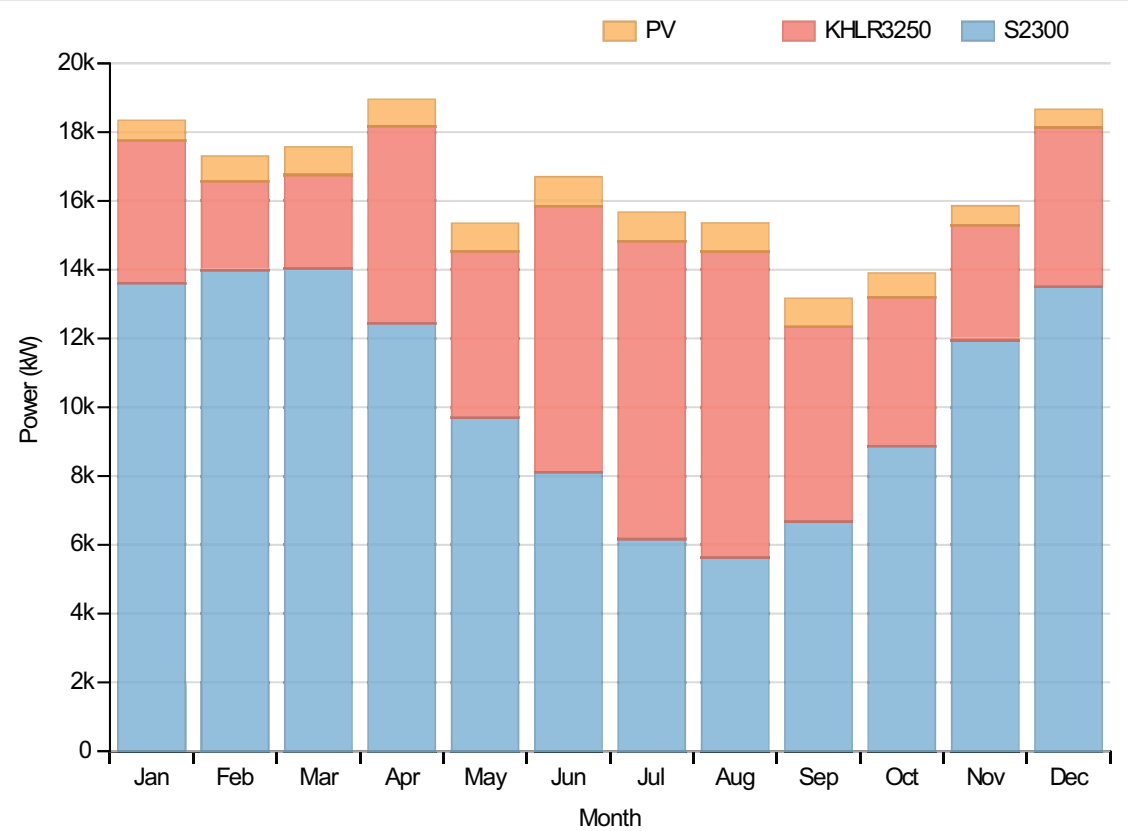

PV:Solar World 320W flat plate PV Copy

\begin{tabular}{|l|r|l|}
\hline Quantity & Value & Units \\
\hline Rated capacity & $5000 \mathrm{~kW}$ \\
\hline Mean output & $736 \mathrm{~kW}$ \\
\hline Mean output & $17653.00 \mathrm{kWh} / \mathrm{d}$ \\
\hline Capacity factor & $14.71 \%$ \\
\hline Total production & $6443367 \mathrm{kWh} / \mathrm{yr}$ \\
\hline Minimum output & $0.00 \mathrm{~kW}$ \\
\hline Maximum output & $5112.50 \mathrm{~kW}$ \\
\hline PV penetration & $6.65 \%$ \\
\hline Hours of operation & $4377 \mathrm{hrs} / \mathrm{yr}$ \\
\hline Levelized cost & $0.192 \mathrm{~S} / \mathrm{kWh}$ \\
\hline
\end{tabular}

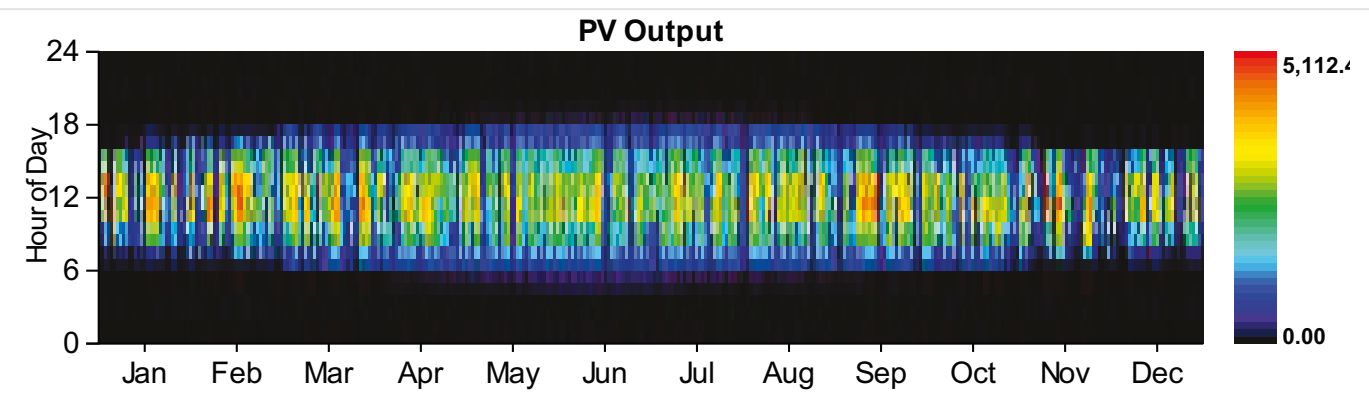


Wind Turbine:Siemens 2.3 MW - 108

\begin{tabular}{|l|l|l|}
\hline Quantity & Value & Units \\
\hline Total rated capacity & 34500 & $\mathrm{~kW}$ \\
\hline Mean output & 10366 & $\mathrm{~kW}$ \\
\hline Capacity factor & 30.05 & $\%$ \\
\hline Total production & 90806648 & $\mathrm{kWh} / \mathrm{yr}$ \\
\hline Minimum output & 21.57 & $\mathrm{~kW}$ \\
\hline Maximum output & 34723.00 & $\mathrm{~kW}$ \\
\hline Wind penetration & 93.75 & $\%$ \\
\hline Hours of operation & 8760 & $\mathrm{hrs} / \mathrm{yr}$ \\
\hline Levelized cost & & $\$ \mathrm{kWh}$ \\
\hline
\end{tabular}

\section{Generator:Kohler 3250 Prime Power}

\begin{tabular}{|c|c|c|}
\hline Quantity & Value & Units \\
\hline Hours of operation & 3381 & $\mathrm{hrs} / \mathrm{yr}$ \\
\hline Number of starts & 1209 & starts/yr \\
\hline Operational life & 4 & yr \\
\hline Fixed generation cost & 1728.30 & $\$ / \mathrm{hr}$ \\
\hline Marginal generation cost & 0.18 & $\$ / k W h$ \\
\hline Electrical production & 46359196 & $\mathrm{kWh} / \mathrm{yr}$ \\
\hline Mean electrical output & 13712 & $\mathrm{~kW}$ \\
\hline Min. electrical output & 4200 & $\mathrm{~kW}$ \\
\hline Max. electrical output & 16800 & $\mathrm{~kW}$ \\
\hline Fuel consumption & 10902671 & L/yr \\
\hline Specific fuel consumption & 0.24 & L/kWh \\
\hline Fuel energy input & 107282296 & $\mathrm{kWh} / \mathrm{yr}$ \\
\hline Mean electrical efficiency & 43 & $\%$ \\
\hline
\end{tabular}




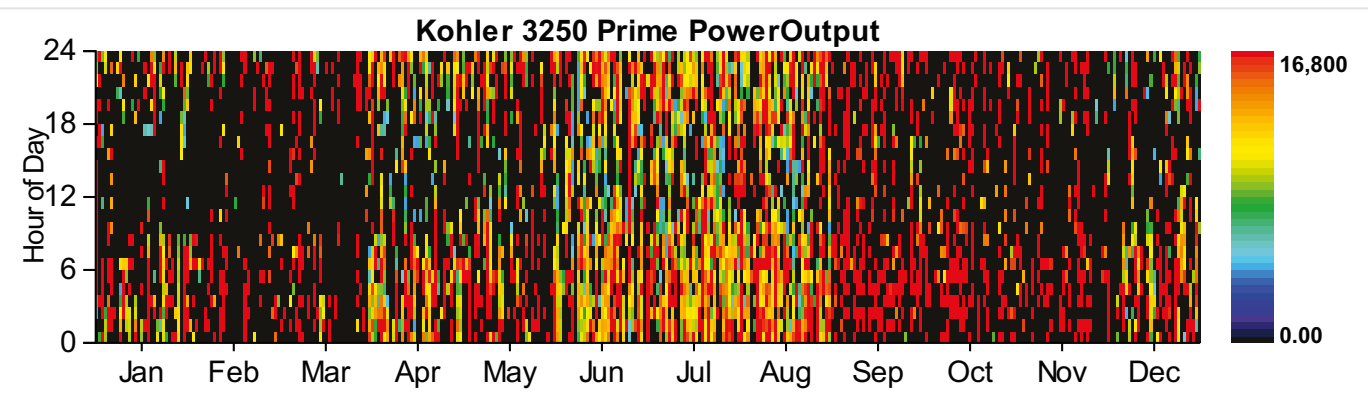

Battery:GS200 flow

Quantity Value

String size

Strings in parallel

Batteries

Bus voltage

\section{Quantity}

Nominal capacity

Value

Units

Usable nominal capacity

$36000 \mathrm{kWh}$

Autonomy

36000 kWh

Lifetime throughput

$3 \mathrm{hr}$

Battery wear cost

0

Average energy cost

$0.000 \$ / k W h$

Energy in

$0.193 \$ / k W h$

Energyin

Energy out

15720101 kWh/yr

Storage depletion

11004080 kWh/yr

Losses

$0 \mathrm{kWh} / \mathrm{yr}$

Annual throughput

$4716021 \mathrm{kWh} / \mathrm{yr}$

Expected life

13152383 kWh/yr

$25 \mathrm{yr}$

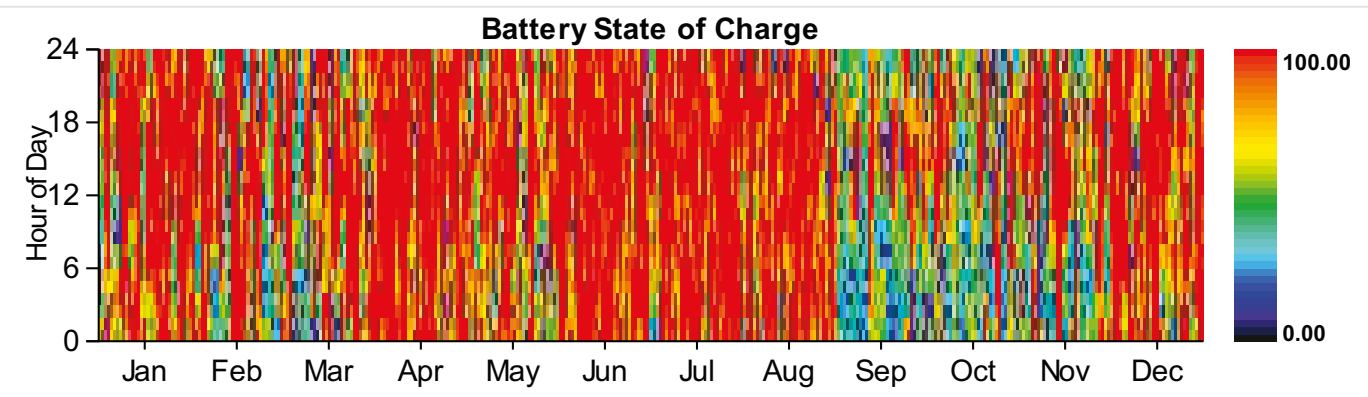


UUiveı เেı

\begin{tabular}{|c|c|c|c|}
\hline Quantity & Inverter & Rectifier & Units \\
\hline Capacity & 24,000 & 21,600 & $\mathrm{~kW}$ \\
\hline Mean output & 1,381 & 1,577 & $\mathrm{~kW}$ \\
\hline Minimum output & 0 & 0 & $\mathrm{~kW}$ \\
\hline Maximum output & 11,091 & 10,757 & $\mathrm{~kW}$ \\
\hline Capacity factor & 6 & 7 & $\%$ \\
\hline Hours of operation & 3,031 & 2,856 & $\mathrm{hrs} / \mathrm{yr}$ \\
\hline Energy in & $13,443,823$ & $16,254,018$ & $\mathrm{kWh} / \mathrm{yr}$ \\
\hline Energy out & $12,099,451$ & $13,815,923$ & $\mathrm{kWh} / \mathrm{yr}$ \\
\hline Losses & $1,344,372$ & 2,438,095 & $\mathrm{kWh} / \mathrm{yr}$ \\
\hline
\end{tabular}
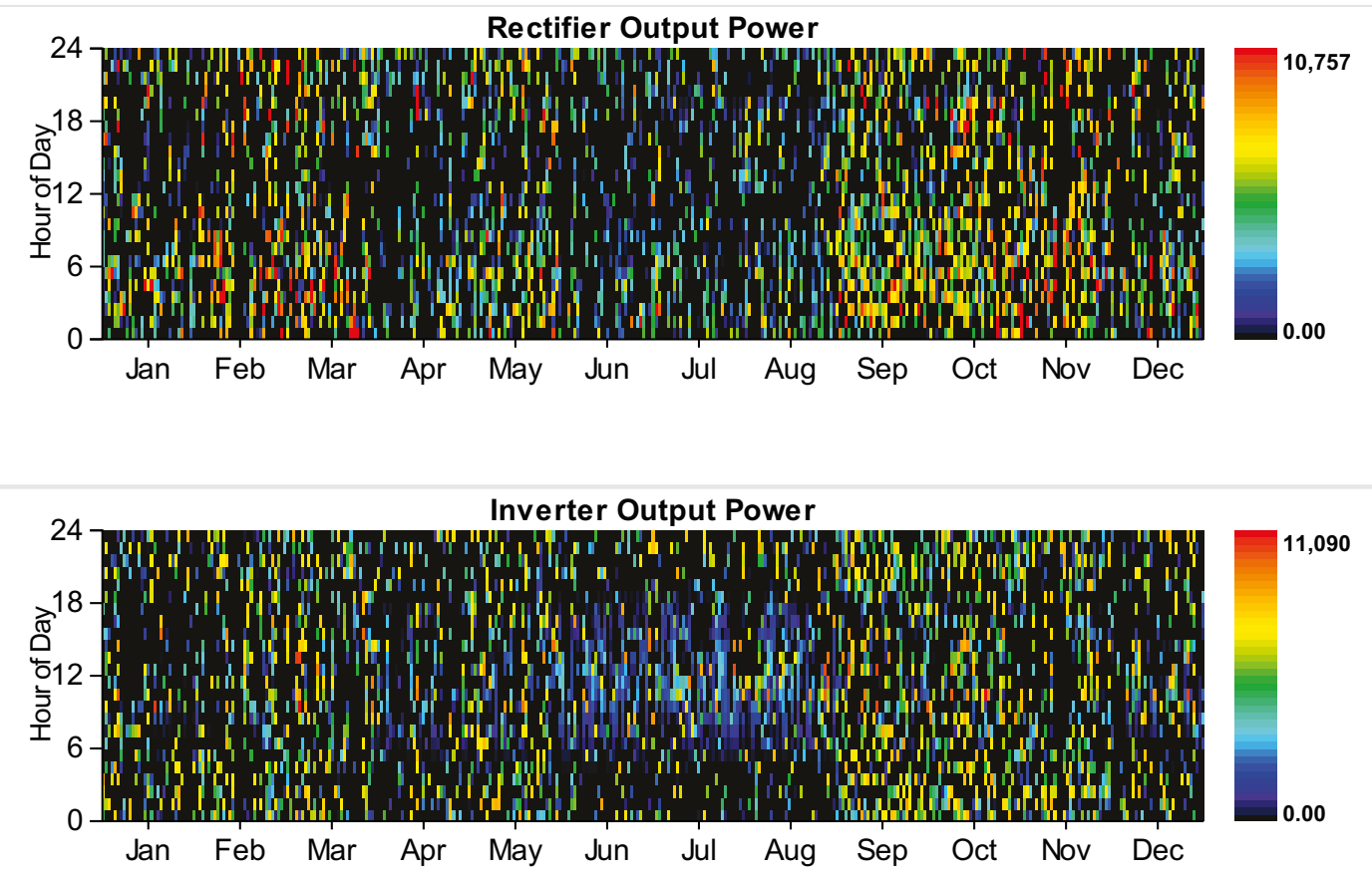

\section{Emissions}

\section{Pollutant}

Carbon dioxide

Carbon monoxide

Unburned hydrocarbons

Particulate matter

Sulfur dioxide

Nitrogen oxides

\section{Emissions}

Units

$28614226 \mathrm{~kg} / \mathrm{yr}$

$119929 \mathrm{~kg} / \mathrm{yr}$

$13737 \mathrm{~kg} / \mathrm{yr}$

$3434 \mathrm{~kg} / \mathrm{yr}$

58915 kg/yr

$119929 \mathrm{~kg} / \mathrm{yr}$ 
System Report

System architecture

\begin{tabular}{|l|l|r|}
\hline PV & Solar World 320W flat plate PV Copy & 30,000 \\
\hline Generator & Kohler 3250 Prime Power & 16,800 \\
\hline Battery & GS200 flow & 80 \\
\hline Converter & System Converter & 24,000 \\
\hline Dispatch Strategy & Cycle Charging & $\mathrm{kW}$ \\
\hline
\end{tabular}

\section{Cost summary}

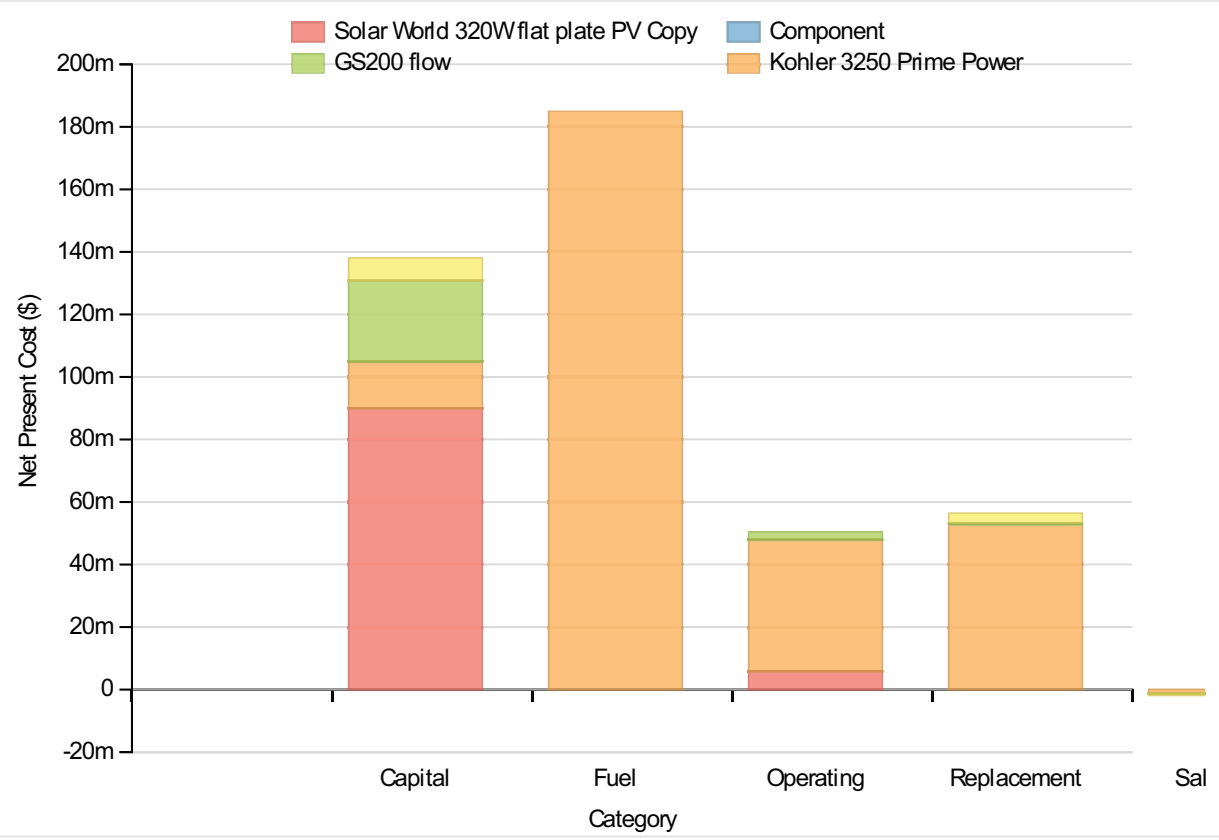

Cost Summary

Total net present cost

$427814592 \$$

Levelized cost of energy

$0.342 \$ / k W h$

Net Present Costs

\begin{tabular}{|c|c|c|c|c|c|c|}
\hline Component & Capital & Replacement & O\&M & Fuel & Salvage & Total \\
\hline Solar World 320W flat plate PV Copy & $90,000,000$ & 0 & $5,817,379$ & 0 & 0 & $95,817,379$ \\
\hline Kohler 3250 Prime Power & $14,821,428$ & $52,738,052$ & $42,092,368$ & $184,886,256$ & $-1,165,772$ & $293,372,332$ \\
\hline GS200 flow & $25,967,136$ & 573,408 & $2,482,082$ & 0 & $-77,744$ & $28,944,882$ \\
\hline Converter & $7,200,000$ & $3,054,769$ & 0 & 0 & $-574,938$ & $9,679,831$ \\
\hline System & $137,988,560$ & $56,366,228$ & $50,391,828$ & $184,886,256$ & $-1,818,454$ & $427,814,418$ \\
\hline
\end{tabular}

\section{Annualized Costs}

\begin{tabular}{|l|l|r|r|r|r|r|}
\hline Component & Capital & Replacement & O\&M & Fuel & Salvage & Total \\
\hline Solar World 320W flat plate PV Copy & $6,961,894$ & 0 & 450,000 & 0 & 0 & $7,411,894$ \\
\hline
\end{tabular}




\begin{tabular}{|l|r|r|r|r|r|r|}
\hline GS200 flow & $2,008,672$ & 44,356 & 192,000 & 0 & $-6,014$ & $2,239,014$ \\
\hline Converter & 556,952 & 236,300 & 0 & 0 & $-44,474$ \\
\hline System & $10,674,019$ & $4,360,175$ & $3,898,029$ & $14,301,762$ & $-140,665$ & $33,093,320$ \\
\hline
\end{tabular}

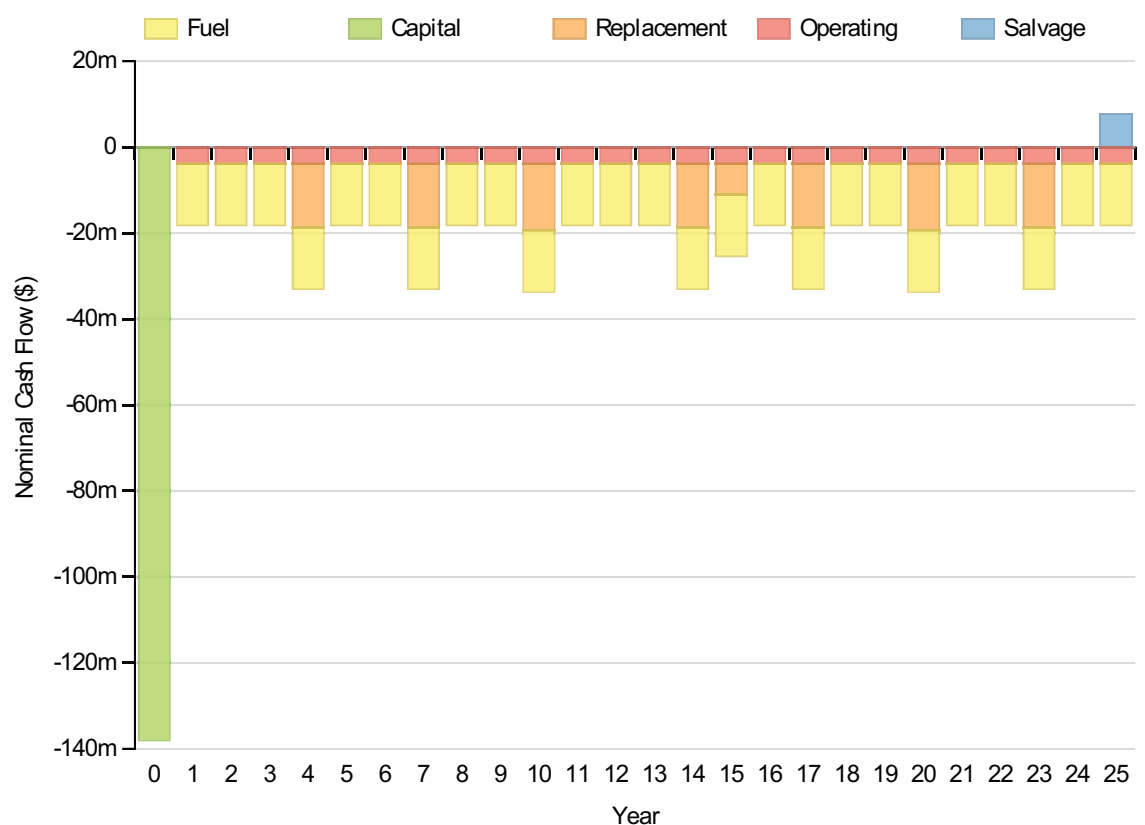

\section{Electrical}

\begin{tabular}{|c|c|c|c|c|}
\hline \multicolumn{2}{|l|}{ Quantity } & \multicolumn{2}{|l|}{ Value } & Units \\
\hline \multicolumn{2}{|l|}{ Excess electricity } & \multicolumn{2}{|r|}{1550152} & $\mathrm{kWh} / \mathrm{yr}$ \\
\hline \multicolumn{2}{|l|}{ Unmet load } & \multicolumn{2}{|r|}{4993} & kWh/yr \\
\hline \multicolumn{2}{|l|}{ Capacity shortage } & \multicolumn{2}{|r|}{27529} & $\mathrm{kWh} / \mathrm{yr}$ \\
\hline \multicolumn{2}{|l|}{ Renewable fraction } & \multicolumn{3}{|c|}{0} \\
\hline Component & \multicolumn{2}{|l|}{ Production(kWh/yr) } & \multicolumn{2}{|c|}{ Fraction (\%) } \\
\hline PV & \multicolumn{2}{|r|}{$38,660,180$} & \multicolumn{2}{|r|}{33} \\
\hline Generator & \multicolumn{2}{|r|}{$77,155,992$} & \multicolumn{2}{|r|}{67} \\
\hline Total & \multicolumn{2}{|r|}{$115,816,176$} & \multicolumn{2}{|r|}{100} \\
\hline Load & \multicolumn{2}{|l|}{ Consumption(kWh/yr) } & \multicolumn{2}{|c|}{ Fraction (\%) } \\
\hline AC primary load & \multicolumn{2}{|c|}{$96,859,168$} & \multicolumn{2}{|r|}{100} \\
\hline DC primary load & \multicolumn{2}{|c|}{0} & & 0 \\
\hline Total & \multicolumn{2}{|c|}{$96,859,168$} & \multicolumn{2}{|r|}{100} \\
\hline
\end{tabular}




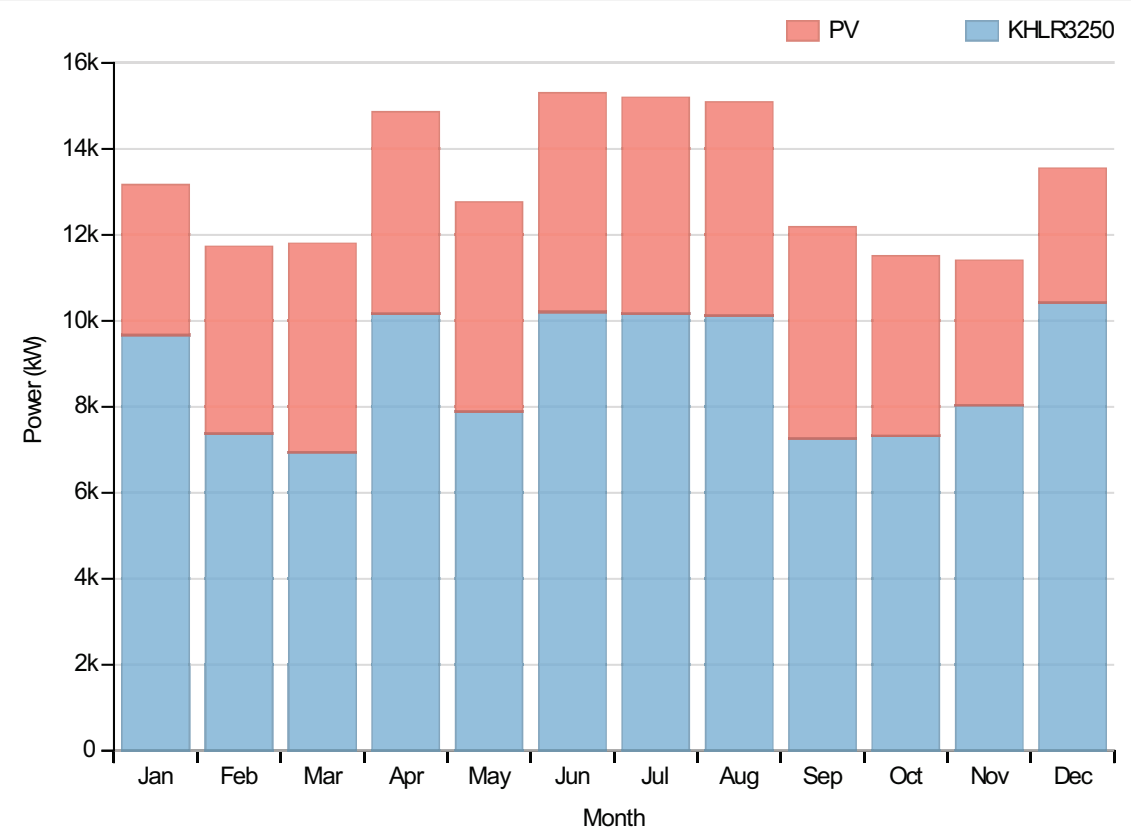

PV:Solar World 320W flat plate PV Copy

\begin{tabular}{|l|r|l|}
\hline Quantity & Value & Units \\
\hline Rated capacity & $30000 \mathrm{~kW}$ \\
\hline Mean output & $4413 \mathrm{~kW}$ \\
\hline Mean output & $105918.00 \mathrm{kWh} / \mathrm{d}$ \\
\hline Capacity factor & $14.71 \%$ \\
\hline Total production & $38660180 \mathrm{kWh} / \mathrm{yr}$ \\
\hline Minimum output & $0.00 \mathrm{~kW}$ \\
\hline Maximum output & $30675.00 \mathrm{~kW}$ \\
\hline PV penetration & $39.91 \%$ \\
\hline Hours of operation & $4377 \mathrm{hrs} / \mathrm{yr}$ \\
\hline Levelized cost & $0.192 \mathrm{~S} / \mathrm{kWh}$ \\
\hline
\end{tabular}

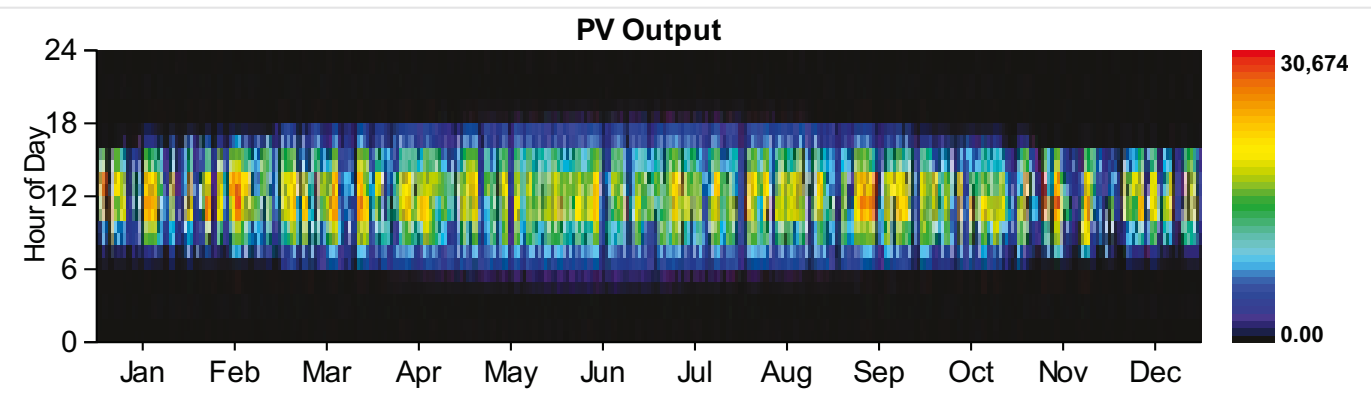

Generator:Kohler 3250 Prime Power

\section{Quantity}

Hours of operation

Number of starts

\section{Value}

Units

$4603 \mathrm{hrs} / \mathrm{yr}$

1922 starts/yr 


\begin{tabular}{|l|r|l|}
\hline Qe日arititienal life & Value & Units \\
\hline Fixed generation cost & 1728.30 & $\$ / \mathrm{hr}$ \\
\hline Marginal generation cost & 0.18 \$/kWh \\
\hline Electrical production & $77155992 \mathrm{kWh} / \mathrm{yr}$ \\
\hline Mean electrical output & $16762 \mathrm{~kW}$ \\
\hline Min. electrical output & $4200 \mathrm{~kW}$ \\
\hline Max. electrical output & $16800 \mathrm{~kW}$ \\
\hline Fuel consumption & $18103504 \mathrm{~L} / \mathrm{yr}$ \\
\hline Specific fuel consumption & $0.23 \mathrm{~L} / \mathrm{kWh}$ \\
\hline Fuel energy input & $178138496 \mathrm{kWh} / \mathrm{yr}$ \\
\hline Mean electrical efficiency & $43 \%$ \\
\hline
\end{tabular}

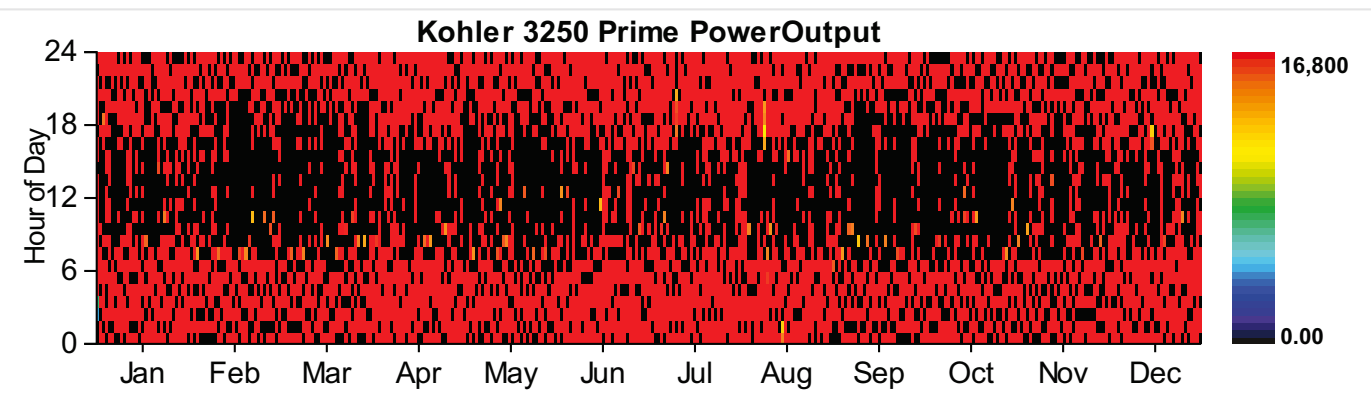

\section{Battery:GS200 flow}

\section{Quantity}

Value

String size

Strings in parallel

Batteries

Bus voltage

\section{Quantity}

Value

Units

Nominal capacity

48000 kWh

Usable nominal capacity

$48000 \mathrm{kWh}$

Autonomy

$4 \mathrm{hr}$

Lifetime throughput

0

Battery wear cost

$0.000 \$ / k W h$

Average energy cost

Energy in 


\section{Q14argyjtyut}

Storage depletion

Losses

Annual throughput

Expected life

\section{Value}

21708802 KWitsyr

$43801 \mathrm{kWh} / \mathrm{yr}$

$9207541 \mathrm{kWh} / \mathrm{yr}$

$25946942 \mathrm{kWh} / \mathrm{yr}$

$25 \mathrm{yr}$

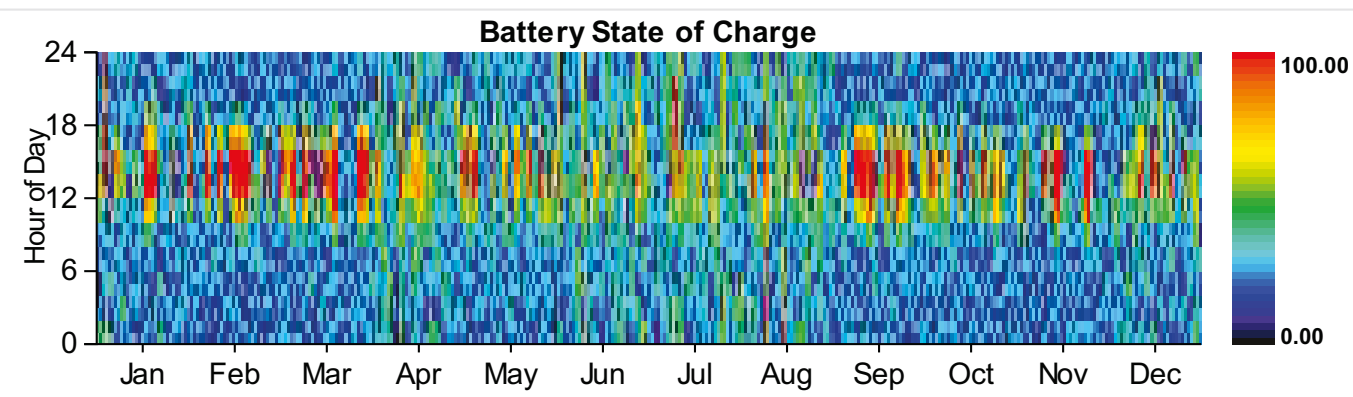

\section{Converter}

\begin{tabular}{|l|r|r|r|}
\hline Quantity & Inverter & Rectifier & Units \\
\hline Capacity & 24,000 & 21,600 & $\mathrm{~kW}$ \\
\hline Mean output & 4,858 & 2,217 & $\mathrm{~kW}$ \\
\hline Minimum output & 0 & $\mathrm{~kW}$ \\
\hline Maximum output & 20,756 & 11,474 & $\mathrm{~kW}$ \\
\hline Capacity factor & 20 & $9 \%$ \\
\hline Hours of operation & 4,498 & 4,255 & $\mathrm{hrs} / \mathrm{yr}$ \\
\hline Energy in & $47,280,436$ & $22,849,226$ & $\mathrm{kWh} / \mathrm{yr}$ \\
\hline Energy out & $42,552,432$ & $19,421,814$ & $\mathrm{kWh} / \mathrm{yr}$ \\
\hline Losses & $4,728,004$ & $3,427,412$ & $\mathrm{kWh} / \mathrm{yr}$ \\
\hline
\end{tabular}

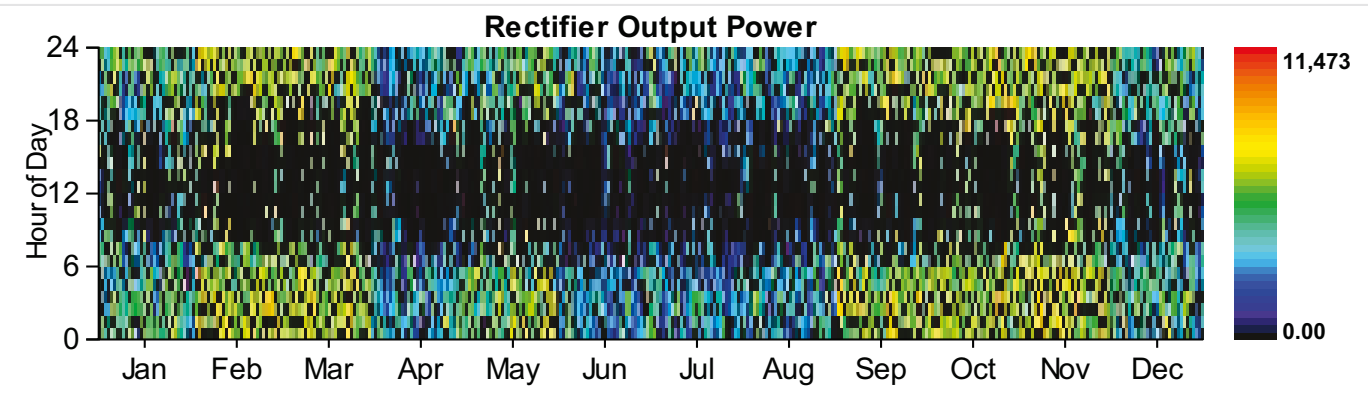




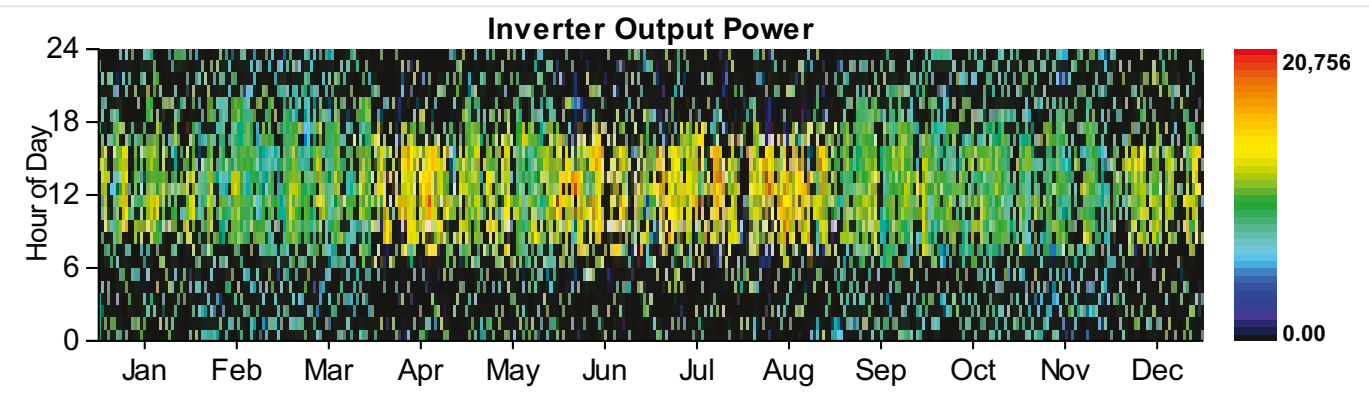

\section{Emissions}

\begin{tabular}{|l|r|l|}
\hline Pollutant & Emissions & Units \\
\hline Carbon dioxide & 47512924 & $\mathrm{~kg} / \mathrm{yr}$ \\
\hline Carbon monoxide & 199139 & $\mathrm{~kg} / \mathrm{yr}$ \\
\hline Unburned hydrocarbons & 22810 & $\mathrm{~kg} / \mathrm{yr}$ \\
\hline Particulate matter & 5703 & $\mathrm{~kg} / \mathrm{yr}$ \\
\hline Sulfur dioxide & 97826 & $\mathrm{~kg} / \mathrm{yr}$ \\
\hline Nitrogen oxides & $199139 \mathrm{~kg} / \mathrm{yr}$ \\
\hline
\end{tabular}

HOMER Energy, LLC @ 2016 
System Report

System architecture

\begin{tabular}{|l|l|l|}
\hline PV & Solar World 320W flat plate PV Copy & 5,000 \\
\hline Wind Turbine & Siemens $2.3 \mathrm{MW}-108$ & 15 \\
\hline Generator & Kohler 3250 Prime Power & 19,600 \\
\hline Converter & System Converter & 24,000 \\
\hline Dispatch Strategy & Cycle Charging & $\mathrm{kW}$ \\
\hline
\end{tabular}

\section{Cost summary}

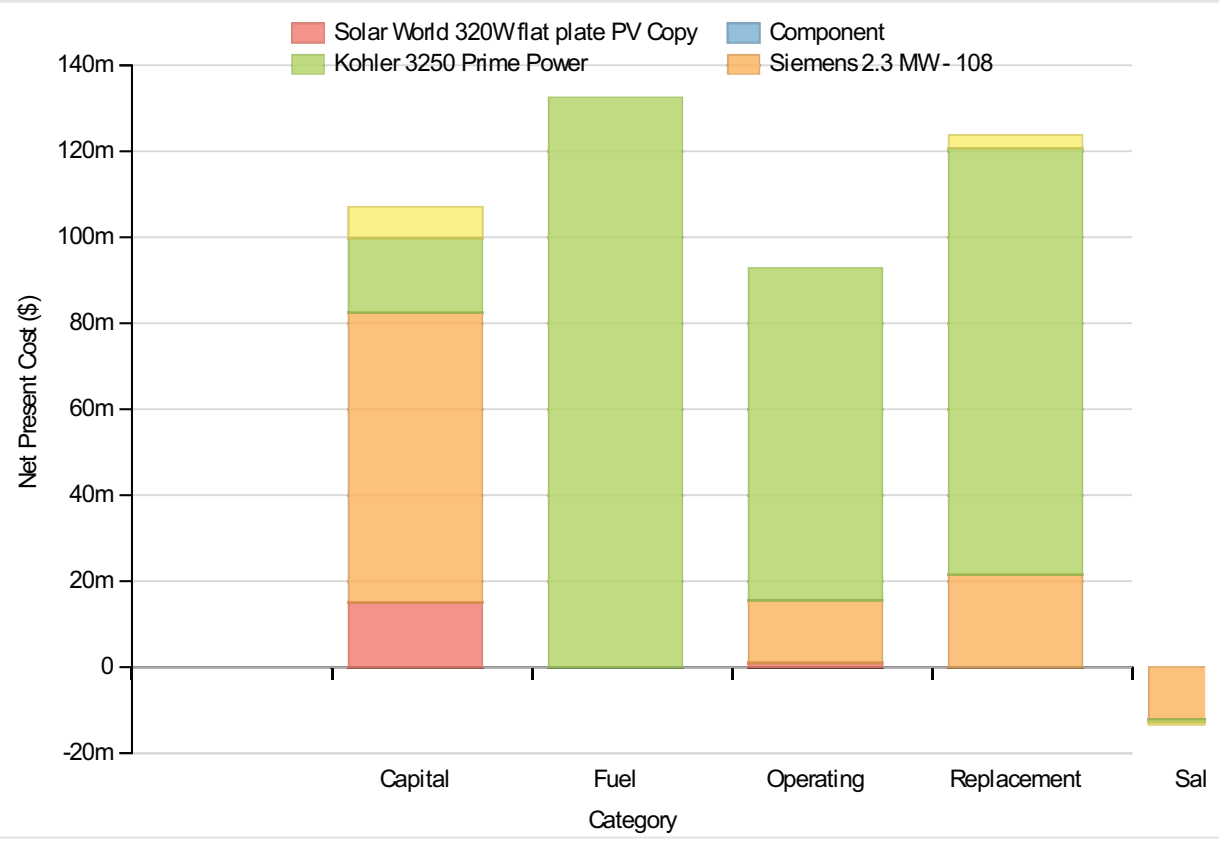

Cost Summary

Total net present cost

$442710560 \$$

Levelized cost of energy

$0.354 \$ / k W h$

Net Present Costs

\begin{tabular}{|c|c|c|c|c|c|c|}
\hline Component & Capital & Replacement & O\&M & Fuel & Salvage & Total \\
\hline $\begin{array}{l}\text { Solar World 320W flat plate PV } \\
\text { Copy }\end{array}$ & $15,000,000$ & 0 & 969,563 & 0 & 0 & $15,969,563$ \\
\hline Siemens 2.3 MW - 108 & $67,500,000$ & $21,519,472$ & $14,543,448$ & 0 & $-12,127,600$ & $91,435,320$ \\
\hline Kohler 3250 Prime Power & $17,321,428$ & $99,201,744$ & $77,298,968$ & $132,495,112$ & $-691,588$ & $325,625,664$ \\
\hline Converter & $7,200,000$ & $3,054,769$ & 0 & 0 & $-574,938$ & $9,679,831$ \\
\hline System & $107,021,424$ & $123,775,984$ & $92,811,984$ & $132,495,112$ & $-13,394,126$ & $442,710,378$ \\
\hline
\end{tabular}

Annualized Costs 


\begin{tabular}{|c|c|c|c|c|c|c|}
\hline Élaipldatghe $320 \mathrm{~W}$ flat plate PV Copy & dapftap16 & Replacement 0 & $0 \& \mathbb{T} 5,000$ & Fuel & Salvage 0 & Total 35,316 \\
\hline Siemens $2.3 \mathrm{MW}-108$ & $5,221,421$ & $1,664,625$ & $1,124,999$ & 0 & $-938,123$ & $7,072,922$ \\
\hline Kohler 3250 Prime Power & $1,339,888$ & $7,673,690$ & $5,979,414$ & $10,249,077$ & $-53,497$ & $25,188,572$ \\
\hline Converter & 556,952 & 236,300 & 0 & 0 & $-44,474$ & 748,778 \\
\hline System & $8,278,576$ & $9,574,614$ & $7,179,414$ & $10,249,077$ & $-1,036,094$ & $34,245,587$ \\
\hline
\end{tabular}

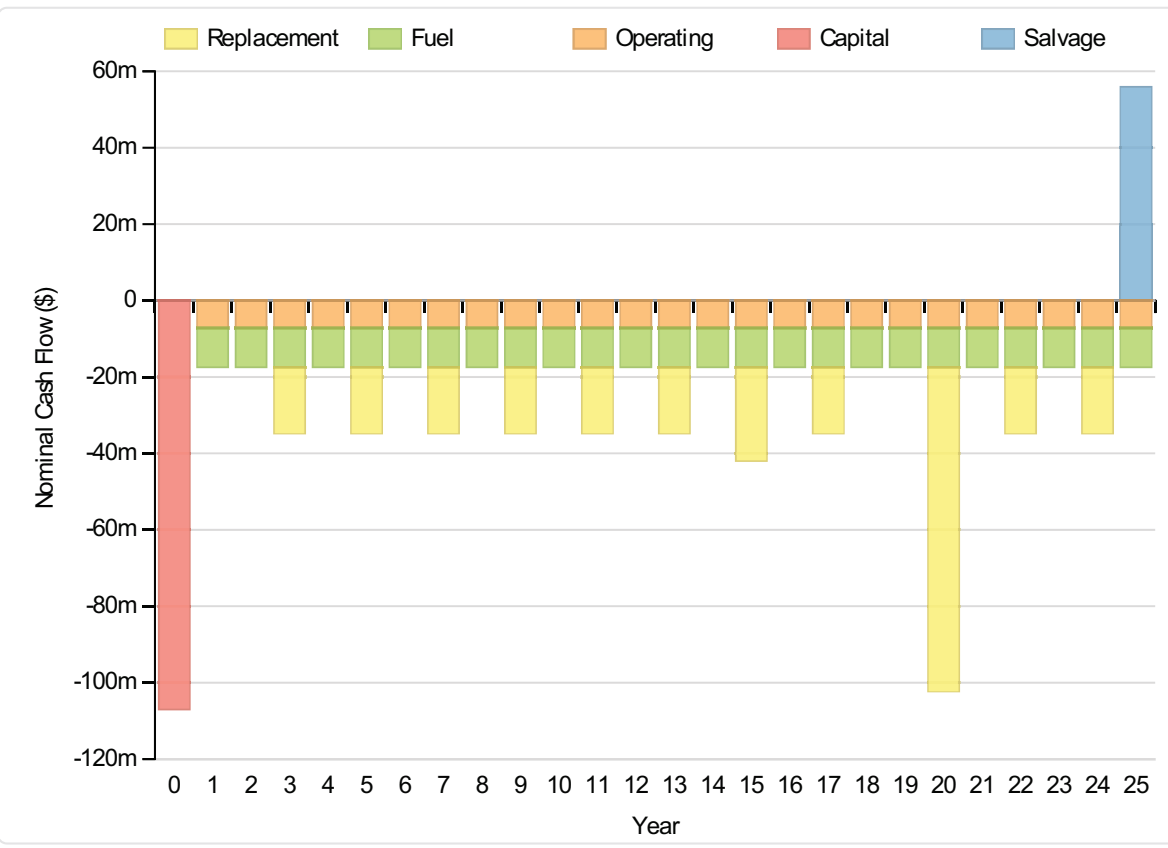

\section{Electrical}

\begin{tabular}{|c|c|c|c|c|}
\hline \multicolumn{2}{|l|}{ Quantity } & \multicolumn{2}{|l|}{ Value } & Units \\
\hline & & \multicolumn{2}{|c|}{54552108} & $\mathrm{kWh} / \mathrm{yr}$ \\
\hline \multicolumn{2}{|l|}{ Unmet load } & \multicolumn{2}{|r|}{7857} & $\mathrm{kWh} / \mathrm{yr}$ \\
\hline \multicolumn{2}{|l|}{ Capacity shortage } & \multicolumn{2}{|c|}{95673} & $\mathrm{kWh} / \mathrm{yr}$ \\
\hline \multicolumn{2}{|l|}{ Renewable fraction } & \multicolumn{3}{|c|}{0} \\
\hline Component & \multicolumn{2}{|l|}{ Production(kWh/yr) } & \multicolumn{2}{|c|}{ Fraction (\%) } \\
\hline PV & & $6,443,367$ & & 4 \\
\hline Generator & & $54,402,400$ & & 36 \\
\hline Wind Turbine & & $90,806,648$ & & 60 \\
\hline Total & & $151,652,416$ & & 100 \\
\hline Load & Consumption(kWh/yr) & & Fract & tion (\%) \\
\hline AC primary load & & $96,856,296$ & & 100 \\
\hline DC primary load & & c & & 0 \\
\hline Total & & $96,856,296$ & & 100 \\
\hline
\end{tabular}




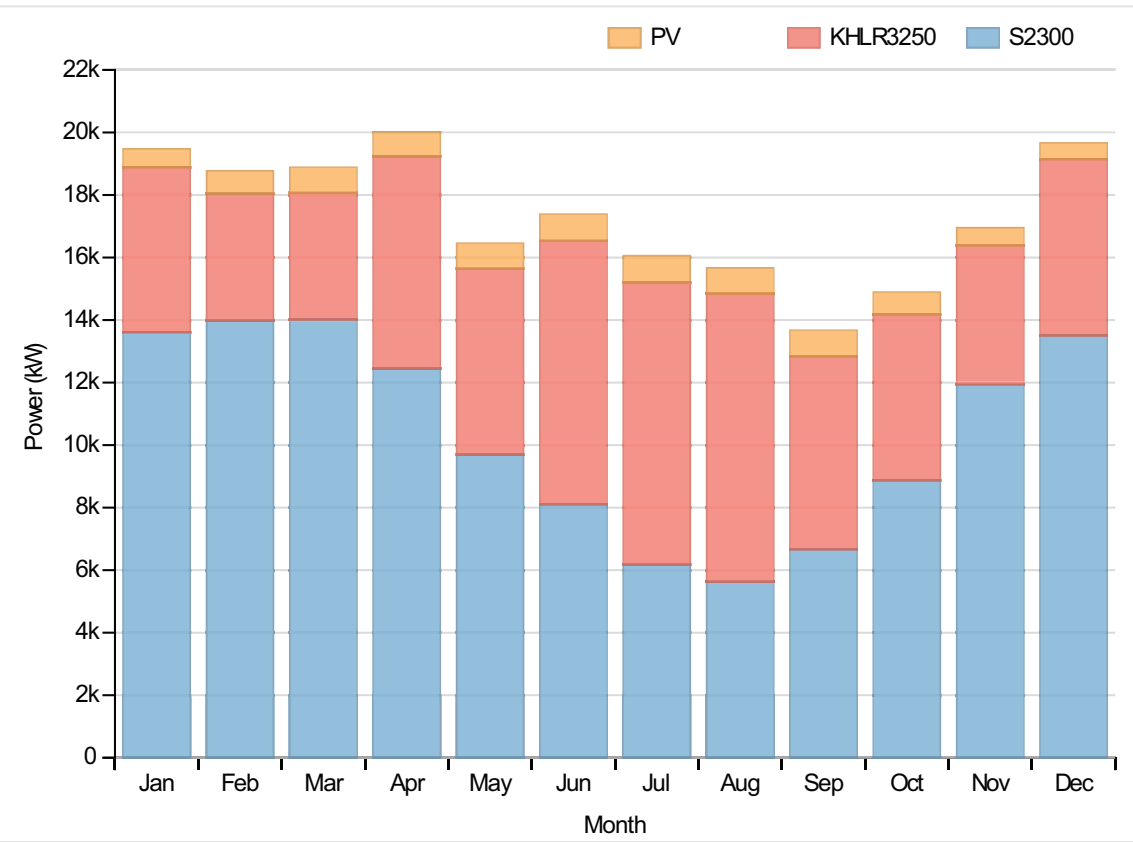

PV:Solar World 320W flat plate PV Copy

\begin{tabular}{|l|r|l|}
\hline Quantity & Value & Units \\
\hline Rated capacity & $5000 \mathrm{~kW}$ \\
\hline Mean output & $736 \mathrm{~kW}$ \\
\hline Mean output & $17653.00 \mathrm{kWh} / \mathrm{d}$ \\
\hline Capacity factor & $14.71 \%$ \\
\hline Total production & $6443367 \mathrm{kWh} / \mathrm{yr}$ \\
\hline Minimum output & $0.00 \mathrm{~kW}$ \\
\hline Maximum output & $5112.50 \mathrm{~kW}$ \\
\hline PV penetration & $6.65 \%$ & $\%$ \\
\hline Hours of operation & $4377 \mathrm{hrs} / \mathrm{yr}$ \\
\hline Levelized cost & 0.192 & $\$ / \mathrm{kWh}$ \\
\hline
\end{tabular}

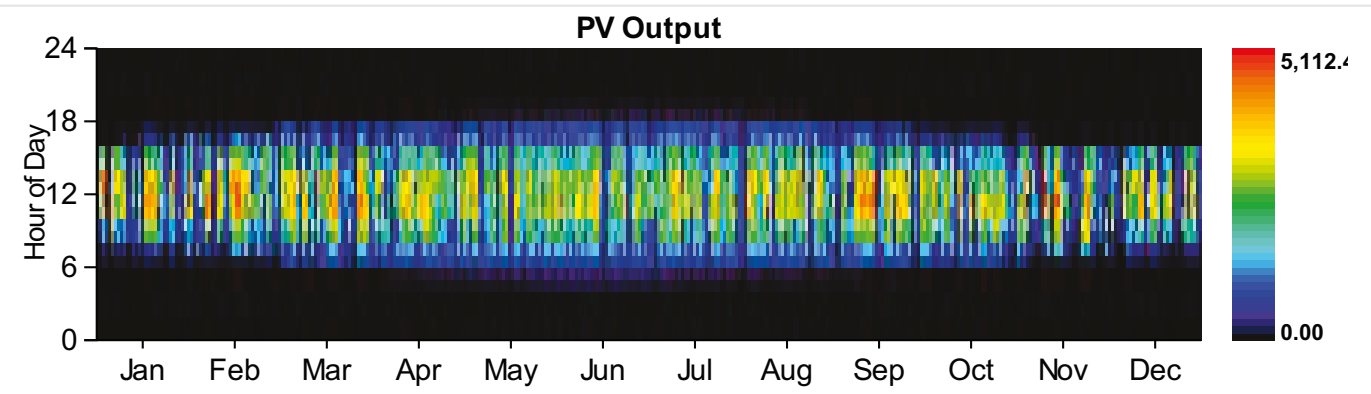

Wind Turbine:Siemens 2.3 MW - 108

\section{Quantity}

Total rated capacity

Mean output

\section{Value}

Units

$34500 \mathrm{~kW}$

10366 kW 


\begin{tabular}{|c|c|c|c|}
\hline 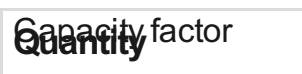 & Value & 30.05 & Units \\
\hline Total production & & 90806648 & $\mathrm{kWh} / \mathrm{yr}$ \\
\hline Minimum output & & 21.57 & kW \\
\hline Maximum output & & 34723.00 & kW \\
\hline Wind penetration & & 93.75 & $\%$ \\
\hline Hours of operation & & 8760 & $\mathrm{hrs} / \mathrm{yr}$ \\
\hline Levelized cost & & 0.078 & $\$ / k W h$ \\
\hline
\end{tabular}

\section{Generator:Kohler 3250 Prime Power}

\begin{tabular}{|l|r|l|}
\hline Quantity & Value & Units \\
\hline Hours of operation & 7100 hrs/yr \\
\hline Number of starts & 512 starts/yr \\
\hline Operational life & 2 yr \\
\hline Fixed generation cost & 2035.30 \$/hr \\
\hline Marginal generation cost & 0.18 \$/kWh \\
\hline Electrical production & $54402400 \mathrm{kWh} / \mathrm{yr}$ \\
\hline Mean electrical output & $7662 \mathrm{~kW}$ \\
\hline Min. electrical output & $4900 \mathrm{~kW}$ \\
\hline Max. electrical output & $19600 \mathrm{~kW}$ \\
\hline Fuel consumption & $12973523 \mathrm{~L} / \mathrm{yr}$ \\
\hline Specific fuel consumption & $0.24 \mathrm{~L} / \mathrm{kWh}$ \\
\hline Fuel energy input & $127659480 \mathrm{kWh} / \mathrm{yr}$ \\
\hline Mean electrical efficiency & $43 \%$ \\
\hline
\end{tabular}

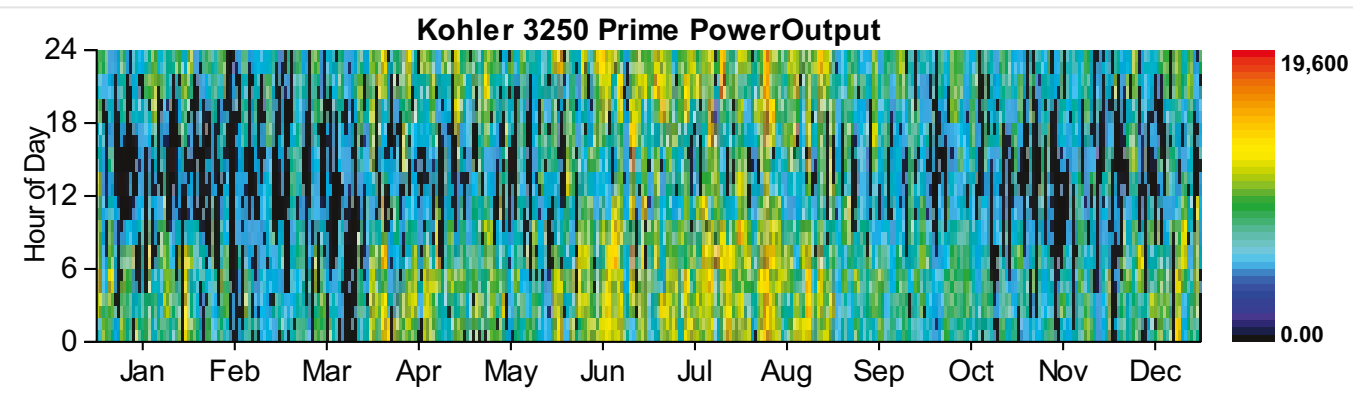

\section{Converter}

\section{Quantity}

Capacity
Inverter

Rectifier

24,000
Units

$21,600 \mathrm{~kW}$ 


\begin{tabular}{|c|c|c|c|}
\hline Qearanfityput & Inverter & Rectifier & 0 kinits \\
\hline Minimum output & 0 & 0 & $0 \mathrm{~kW}$ \\
\hline Maximum output & 4,348 & 0 & $0 \mathrm{~kW}$ \\
\hline Capacity factor & 1 & 0 & $0 \%$ \\
\hline Hours of operation & 1,972 & 0 & 0 hrs/yr \\
\hline Energy in & $2,439,241$ & 0 & $0 \mathrm{kWh} / \mathrm{yr}$ \\
\hline Energy out & $2,195,318$ & 0 & $0 \mathrm{kWh} / \mathrm{yr}$ \\
\hline Losses & 243,924 & 0 & $0 \mathrm{kWh} / \mathrm{yr}$ \\
\hline
\end{tabular}
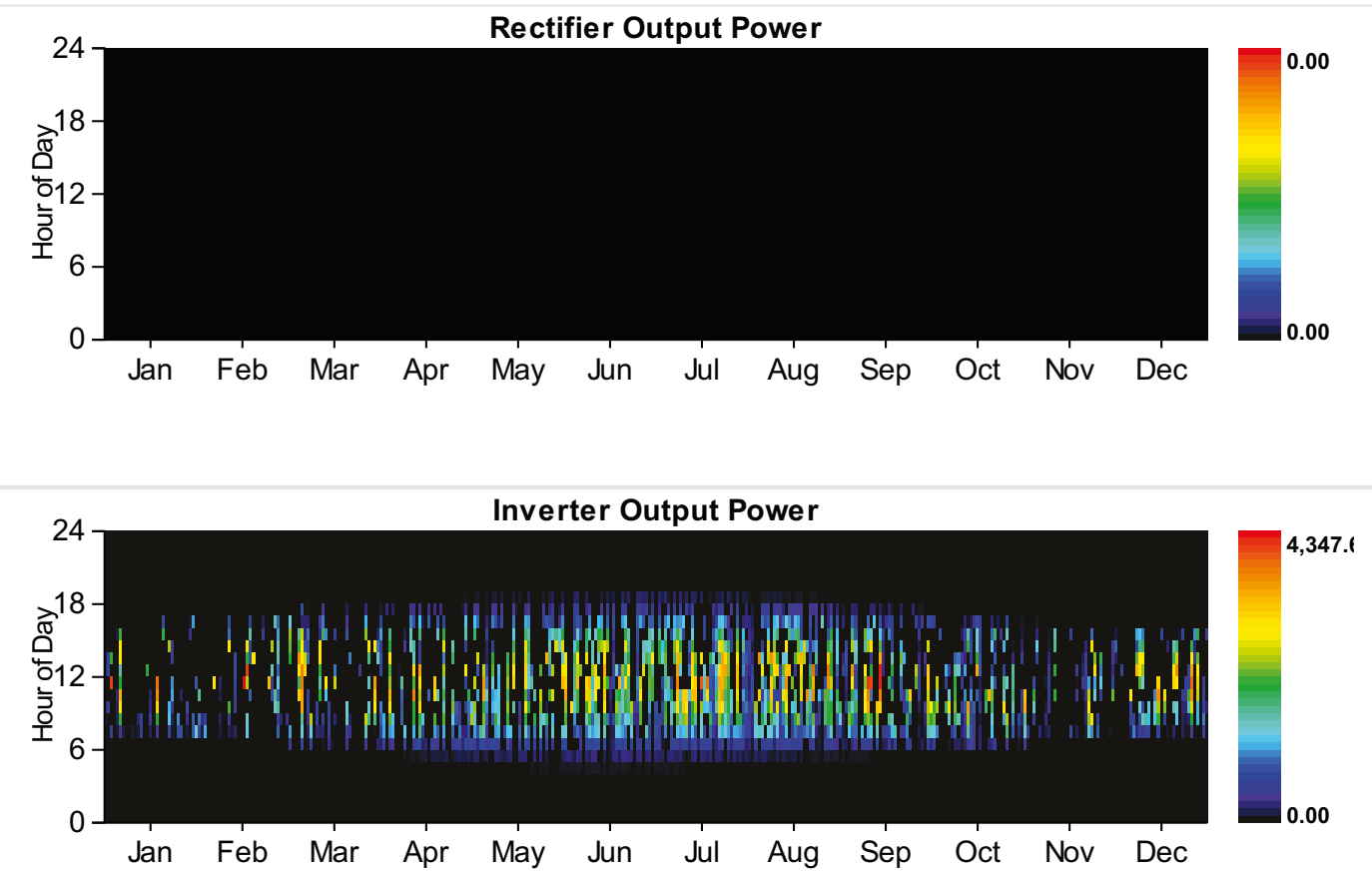

\section{Emissions}

\section{Pollutant}

Emissions

Units

Carbon dioxide

$34049208 \mathrm{~kg} / \mathrm{yr}$

Carbon monoxide

$142709 \mathrm{~kg} / \mathrm{yr}$

Unburned hydrocarbons

16347 kg/yr

Particulate matter

$4087 \mathrm{~kg} / \mathrm{yr}$

Sulfur dioxide

70105 kg/yr

Nitrogen oxides

$142709 \mathrm{~kg} / \mathrm{yr}$

HOMER Energy, LLC @ 2016 
System Report

System architecture

\begin{tabular}{|l|l|r|}
\hline Wind Turbine & Siemens $2.3 \mathrm{MW}-108$ & 30 \\
\hline Generator & Kohler 3250 Prime Power & $19,600 \mathrm{~kW}$ \\
\hline Dispatch Strategy & Cycle Charging & \\
\hline
\end{tabular}

Cost summary

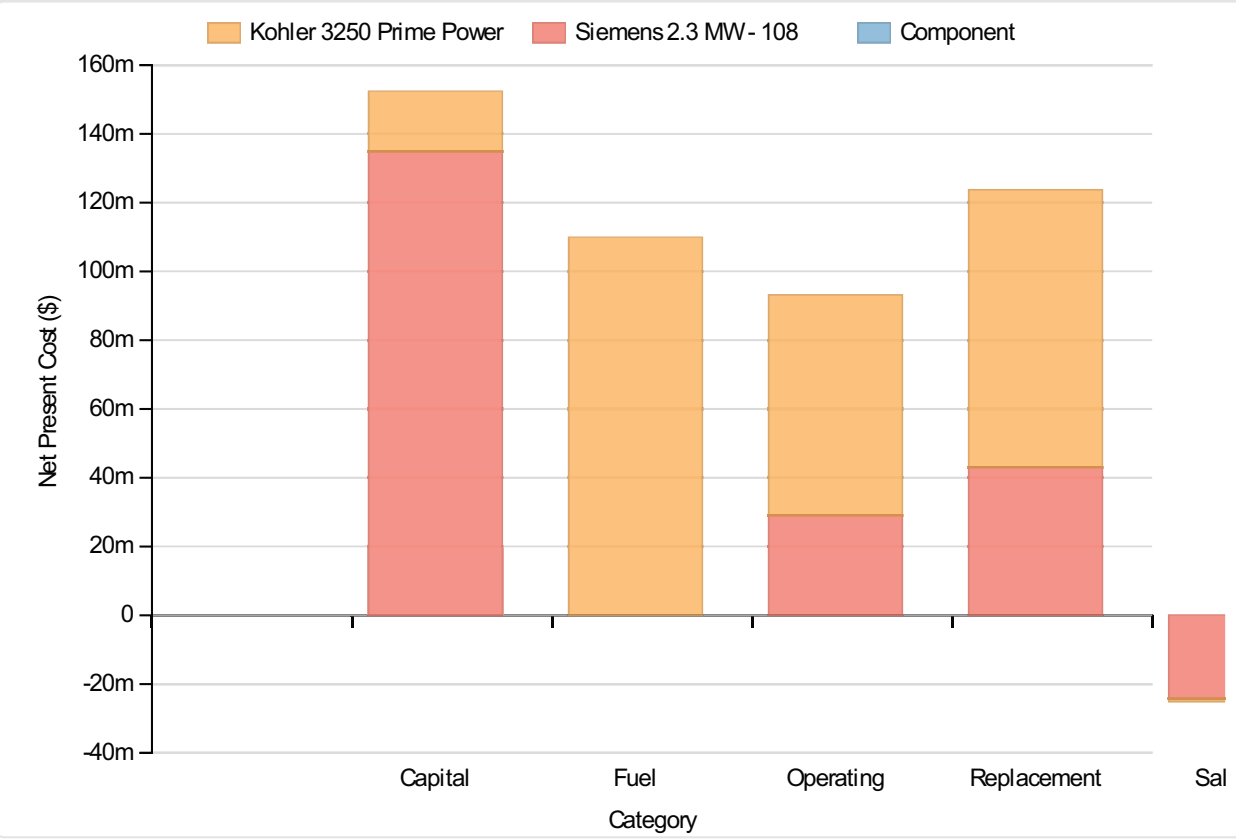

Cost Summary

Total net present cost

$453894880 \$$

Levelized cost of energy

$0.363 \$ / k W h$

\section{Net Present Costs}

\begin{tabular}{|l|r|r|r|r|r|r|}
\hline Component & Capital & Replacement & O\&M & Fuel & Salvage & Total \\
\hline Siemens 2.3 MW - 108 & $135,000,000$ & $43,038,944$ & $29,086,896$ & 0 & $-24,255,200$ & $182,870,640$ \\
\hline Kohler 3250 Prime Power & $17,321,428$ & $80,646,536$ & $64,027,496$ & $109,851,592$ & $-822,981$ & $271,024,071$ \\
\hline System & $152,321,424$ & $123,685,480$ & $93,114,384$ & $109,851,592$ & $-25,078,182$ & $453,894,698$ \\
\hline
\end{tabular}

\section{Annualized Costs}

\begin{tabular}{|l|r|r|r|r|r|r|}
\hline Component & Capital & Replacement & O\&M & Fuel & Salvage & Total \\
\hline Siemens 2.3 MW - 108 & $10,442,841$ & $3,329,251$ & $2,249,999$ & 0 & $-1,876,246$ & $14,145,845$ \\
\hline Kohler 3250 Prime Power & $1,339,888$ & $6,238,363$ & $4,952,807$ & $8,497,502$ & $-63,661$ & $20,964,899$ \\
\hline System & $11,782,729$ & $9,567,613$ & $7,202,806$ & $8,497,502$ & $-1,939,907$ & $35,110,743$ \\
\hline
\end{tabular}




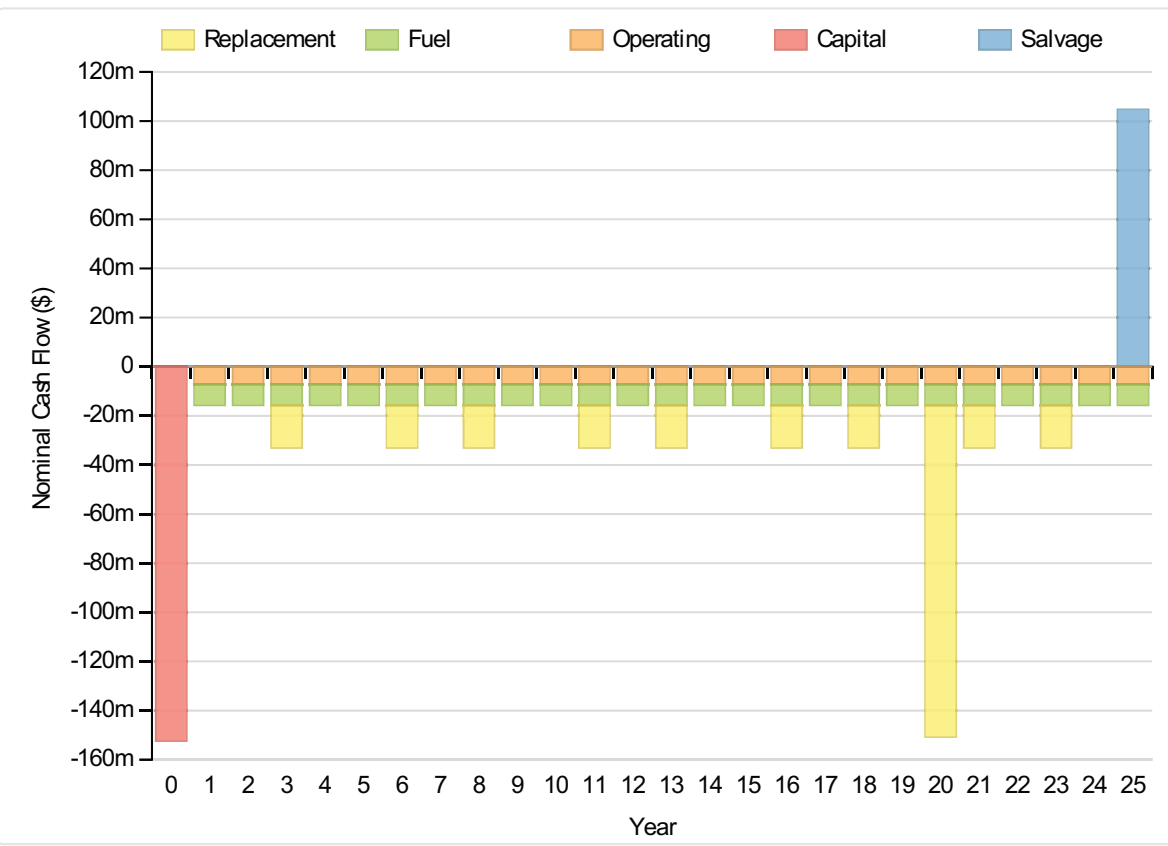

\section{Electrical}

\begin{tabular}{|c|c|c|c|c|}
\hline \multirow{2}{*}{\multicolumn{2}{|c|}{$\begin{array}{l}\text { Quantity } \\
\text { Excess electricity }\end{array}$}} & \multicolumn{2}{|l|}{ Value } & Units \\
\hline & & \multicolumn{2}{|c|}{129863544} & $\mathrm{kWh} / \mathrm{yr}$ \\
\hline \multicolumn{2}{|l|}{ Unmet load } & \multicolumn{2}{|r|}{8264} & kWh/yr \\
\hline \multicolumn{2}{|l|}{ Capacity shortage } & \multicolumn{2}{|r|}{94180} & $\mathrm{kWh} / \mathrm{yr}$ \\
\hline \multicolumn{2}{|l|}{ Renewable fraction } & \multicolumn{2}{|r|}{1} & \\
\hline Component & \multicolumn{2}{|l|}{ Production(kWh/yr) } & \multicolumn{2}{|c|}{ Fraction $(\%)$} \\
\hline Generator & \multicolumn{2}{|r|}{$45,106,168$} & \multicolumn{2}{|r|}{20} \\
\hline Wind Turbine & \multicolumn{2}{|r|}{$181,613,296$} & \multicolumn{2}{|r|}{80} \\
\hline Total & \multicolumn{2}{|r|}{$226,719,456$} & \multicolumn{2}{|r|}{100} \\
\hline Load & \multicolumn{2}{|c|}{ Consumption(kWh/yr) } & \multicolumn{2}{|c|}{ Fraction (\%) } \\
\hline AC primary load & \multicolumn{2}{|c|}{$96,855,888$} & \multicolumn{2}{|r|}{100} \\
\hline DC primary load & \multicolumn{2}{|c|}{0} & \multicolumn{2}{|c|}{0} \\
\hline Total & \multicolumn{2}{|c|}{$96,855,888$} & \multicolumn{2}{|r|}{100} \\
\hline
\end{tabular}




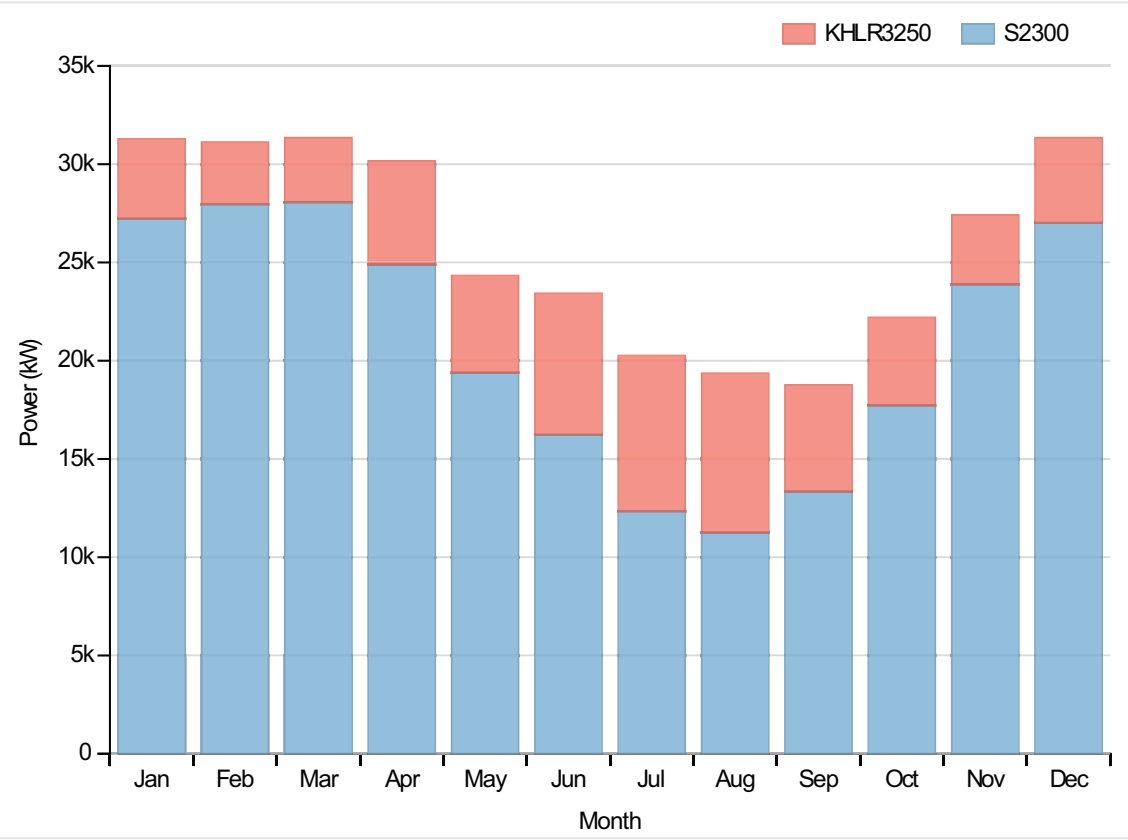

Wind Turbine:Siemens 2.3 MW - 108

\begin{tabular}{|c|c|c|}
\hline Quantity & Value & Units \\
\hline Total rated capacity & 69000 & kW \\
\hline Mean output & 20732 & $\mathrm{~kW}$ \\
\hline Capacity factor & 30.05 & $\%$ \\
\hline Total production & 181613296 & $\mathrm{kWh} / \mathrm{yr}$ \\
\hline Minimum output & 43.13 & $\mathrm{~kW}$ \\
\hline Maximum output & 69447.00 & $\mathrm{~kW}$ \\
\hline Wind penetration & 187.49 & $\%$ \\
\hline Hours of operation & 8760 & hrs/yr \\
\hline Levelized cost & 0.078 & $\$ / k W h$ \\
\hline
\end{tabular}

\section{Generator:Kohler 3250 Prime Power}

\section{Quantity}

Hours of operation

Number of starts

Operational life

Fixed generation cost

Marginal generation cost

Electrical production

Mean electrical output

Min. electrical output

\section{Value}

Units

$5881 \mathrm{hrs} / \mathrm{yr}$

643 starts/yr

$3 \mathrm{yr}$

$2035.30 \$ / h r$

$0.18 \$ / k W h$

45106168 kWh/yr

7670 kW

4900 kW 


\begin{tabular}{|c|c|c|c|}
\hline Qaxrititeytrical output & Value & 19600 & UAits \\
\hline Fuel consumption & & 10756336 & L/yr \\
\hline Specific fuel consumption & & 0.24 & L/kWh \\
\hline Fuel energy input & & 105842360 & $\mathrm{kWh} / \mathrm{yr}$ \\
\hline Mean electrical efficiency & & 43 & $\%$ \\
\hline
\end{tabular}

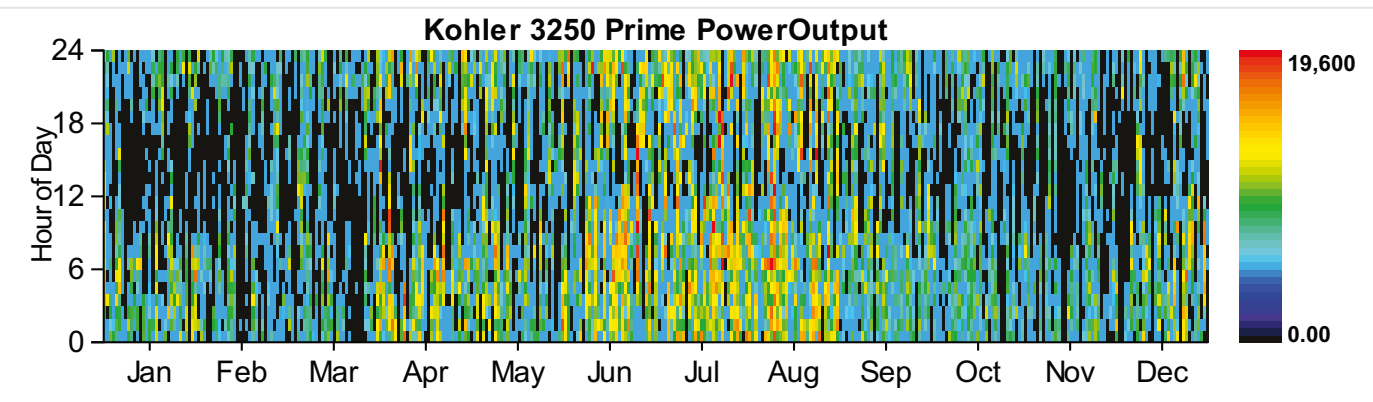

\section{Emissions}

\begin{tabular}{|l|r|l|}
\hline Pollutant & Emissions & Units \\
\hline Carbon dioxide & 28230168 & $\mathrm{~kg} / \mathrm{yr}$ \\
\hline Carbon monoxide & 118320 & $\mathrm{~kg} / \mathrm{yr}$ \\
\hline Unburned hydrocarbons & 13553 & $\mathrm{~kg} / \mathrm{yr}$ \\
\hline Particulate matter & 3388 & $\mathrm{~kg} / \mathrm{yr}$ \\
\hline Sulfur dioxide & 58124 & $\mathrm{~kg} / \mathrm{yr}$ \\
\hline Nitrogen oxides & 118320 & $\mathrm{~kg} / \mathrm{yr}$ \\
\hline
\end{tabular}

HOMER Energy, LLC @ 2016 
System Report

System architecture

\begin{tabular}{|l|l|r|}
\hline Generator & Kohler 3250 Prime Power & 16,800 \\
\hline Battery & GS200 flow & 80 \\
\hline Converter & System Converter & strings \\
\hline Dispatch Strategy & Cycle Charging & 24,000 \\
\hline
\end{tabular}

Cost summary

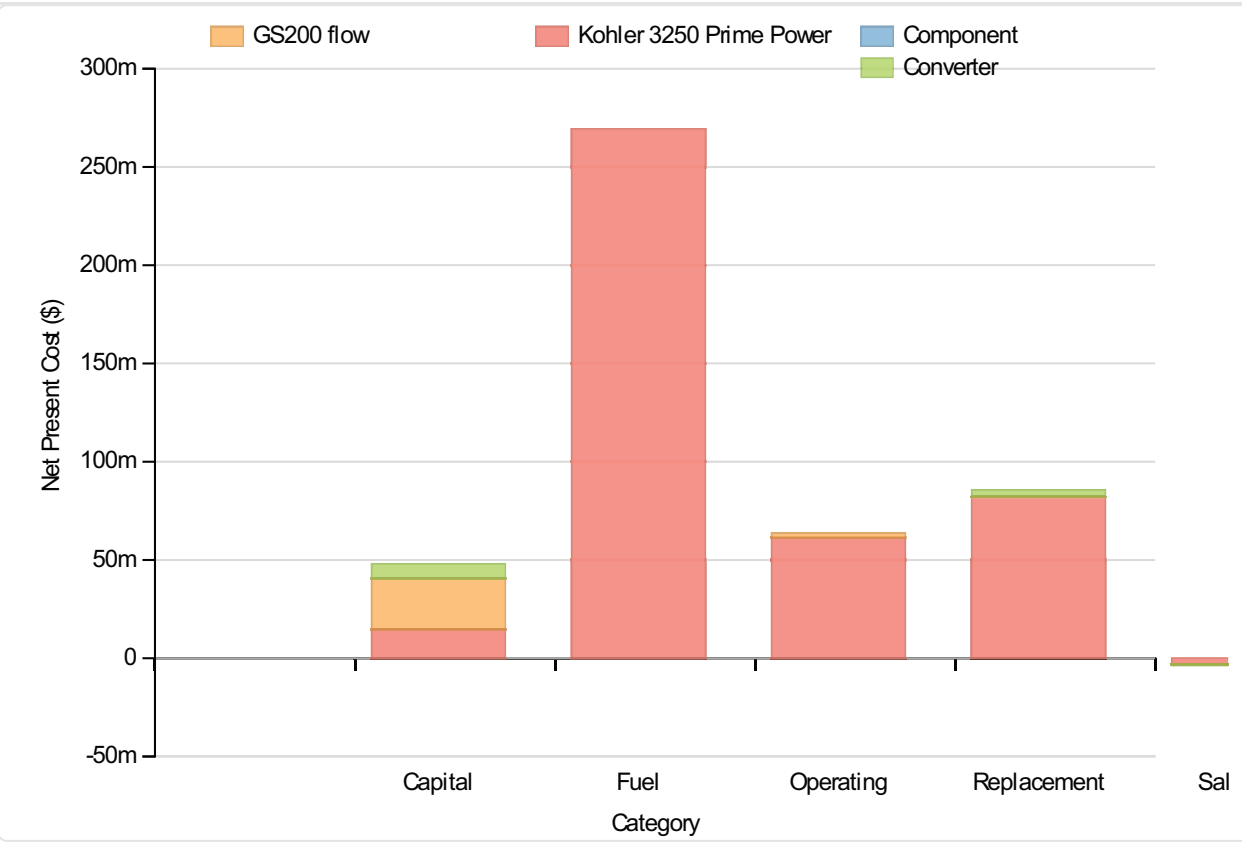

Cost Summary

Total net present cost

$462819808 \$$

Levelized cost of energy

$0.370 \$ / k W h$

Net Present Costs

\begin{tabular}{|l|r|r|r|r|r|r|}
\hline Component & Capital & Replacement & O\&M & Fuel & Salvage & Total \\
\hline Kohler 3250 Prime Power & $14,821,428$ & $81,992,984$ & $61,259,348$ & $269,085,888$ & $-2,964,737$ & $424,194,911$ \\
\hline GS200 flow & $25,967,136$ & 573,408 & $2,482,082$ & 0 & $-77,744$ & $28,944,882$ \\
\hline Converter & $7,200,000$ & $3,054,769$ & 0 & $-574,938$ & $9,679,831$ \\
\hline System & $47,988,564$ & $85,621,160$ & $63,741,424$ & $269,085,888$ & $-3,617,419$ & $462,819,617$ \\
\hline
\end{tabular}

\section{Annualized Costs}

\begin{tabular}{|l|r|r|r|r|r|r|}
\hline Component & Capital & Replacement & O\&M & Fuel & Salvage & Total \\
\hline Kohler 3250 Prime Power & $1,146,502$ & $6,342,517$ & $4,738,679$ & $20,814,972$ & $-229,335$ & $32,813,335$ \\
\hline GS200 flow & $2,008,672$ & 44,356 & 192,000 & 0 & $-6,014$ & $2,239,014$ \\
\hline Converter & 556,952 & 236,300 & 0 & 0 & $-44,474$ \\
\hline
\end{tabular}




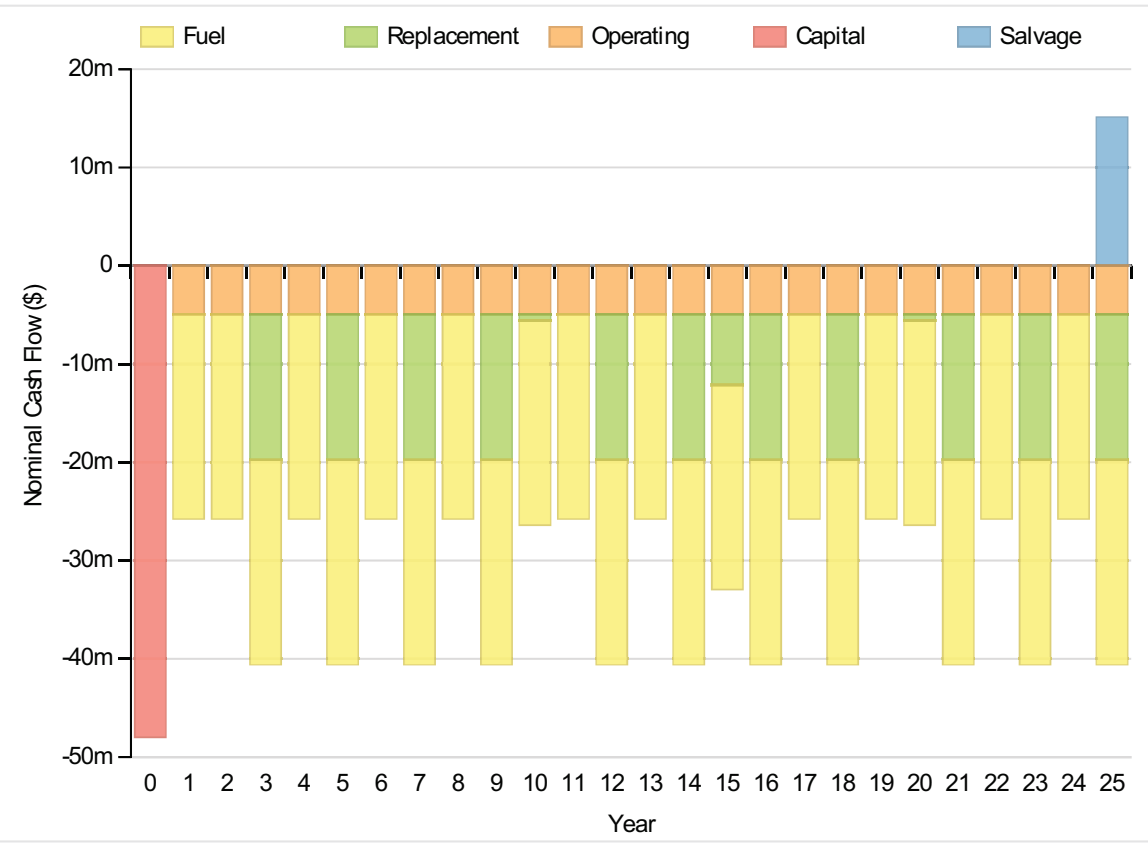

\section{Electrical}

\begin{tabular}{|c|c|c|c|c|}
\hline \multicolumn{2}{|l|}{ Quantity } & \multicolumn{2}{|l|}{ Value } & Units \\
\hline \multicolumn{2}{|l|}{ Excess electricity } & & 0 & $\mathrm{kWh} / \mathrm{yr}$ \\
\hline \multicolumn{2}{|l|}{ Unmet load } & \multicolumn{2}{|r|}{42667} & $\mathrm{kWh} / \mathrm{yr}$ \\
\hline \multicolumn{2}{|l|}{ Capacity shortage } & \multicolumn{2}{|r|}{95013} & $\mathrm{kWh} / \mathrm{yr}$ \\
\hline \multicolumn{2}{|l|}{ Renewable fraction } & \multicolumn{2}{|c|}{0} & \\
\hline Component & \multicolumn{2}{|l|}{ Production(kWh/yr) } & \multicolumn{2}{|c|}{ Fraction (\%) } \\
\hline Generator & \multicolumn{3}{|c|}{$112,291,864$} & 100 \\
\hline Total & \multicolumn{3}{|c|}{$112,291,864$} & 100 \\
\hline Load & Consumption(kWh/yr) & \multicolumn{3}{|c|}{ Fraction (\%) } \\
\hline AC primary load & & \multicolumn{2}{|c|}{$96,821,488$} & 100 \\
\hline DC primary load & & \multicolumn{2}{|r|}{0} & 0 \\
\hline Total & & \multicolumn{2}{|c|}{$96,821,488$} & 100 \\
\hline
\end{tabular}




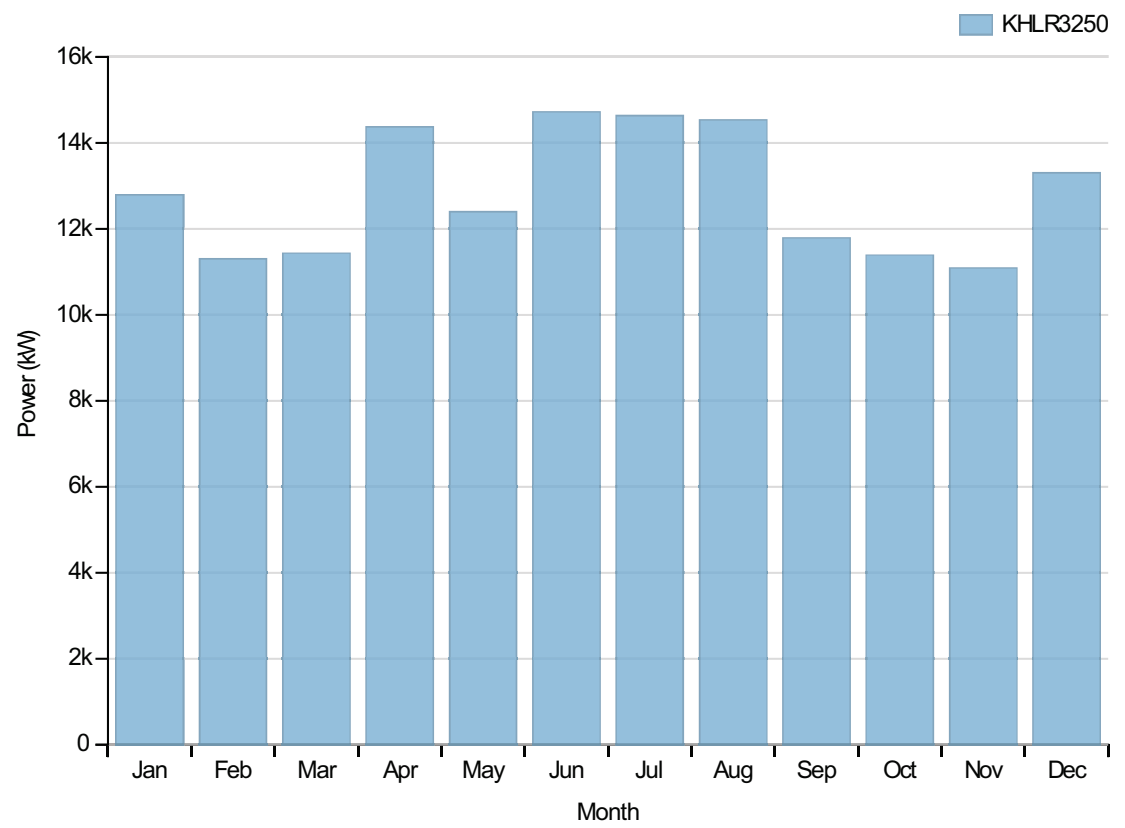

Generator:Kohler 3250 Prime Power

\begin{tabular}{|l|r|l|}
\hline Quantity & Value & Units \\
\hline Hours of operation & 6699 hrs/yr \\
\hline Number of starts & 1948 starts/yr \\
\hline Operational life & 2 yr \\
\hline Fixed generation cost & 1728.30 & $\$ / \mathrm{hr}$ \\
\hline Marginal generation cost & 0.18 \$/kWh \\
\hline Electrical production & $112291864 \mathrm{kWh} / \mathrm{yr}$ \\
\hline Mean electrical output & $16762 \mathrm{~kW}$ \\
\hline Min. electrical output & $4200 \mathrm{~kW}$ \\
\hline Max. electrical output & $16800 \mathrm{~kW}$ \\
\hline Fuel consumption & $26348078 \mathrm{~L} / \mathrm{yr}$ \\
\hline Specific fuel consumption & $0.23 \mathrm{~L} / \mathrm{kWh}$ \\
\hline Fuel energy input & $259265120 \mathrm{kWh} / \mathrm{yr}$ \\
\hline Mean electrical efficiency & $43 \%$ \\
\hline
\end{tabular}

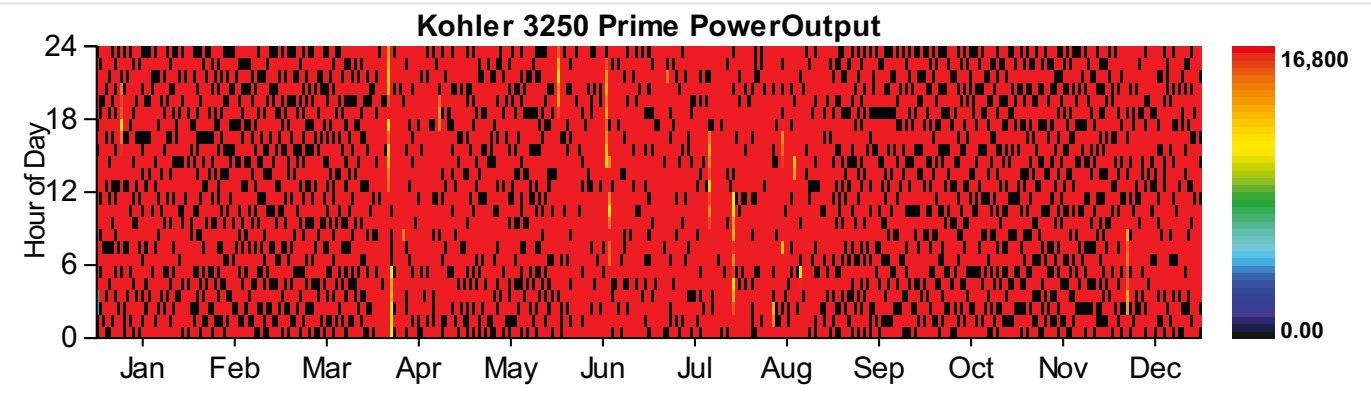




\section{Quantity}

Value

String size

Strings in parallel

Batteries

Bus voltage

\section{Quantity}

Value

Units

Nominal capacity

Usable nominal capacity

Autonomy

Lifetime throughput

Battery wear cost

$0.000 \$ / k W h$

Average energy cost

$0.308 \$ / k W h$

Energy in

$28359858 \mathrm{kWh} / \mathrm{yr}$

Energy out

$19882438 \mathrm{kWh} / \mathrm{yr}$

Storage depletion

$36487 \mathrm{kWh} / \mathrm{yr}$

Losses

$8440933 \mathrm{kWh} / \mathrm{yr}$

Annual throughput

$23764098 \mathrm{kWh} / \mathrm{yr}$

Expected life

$25 \mathrm{yr}$

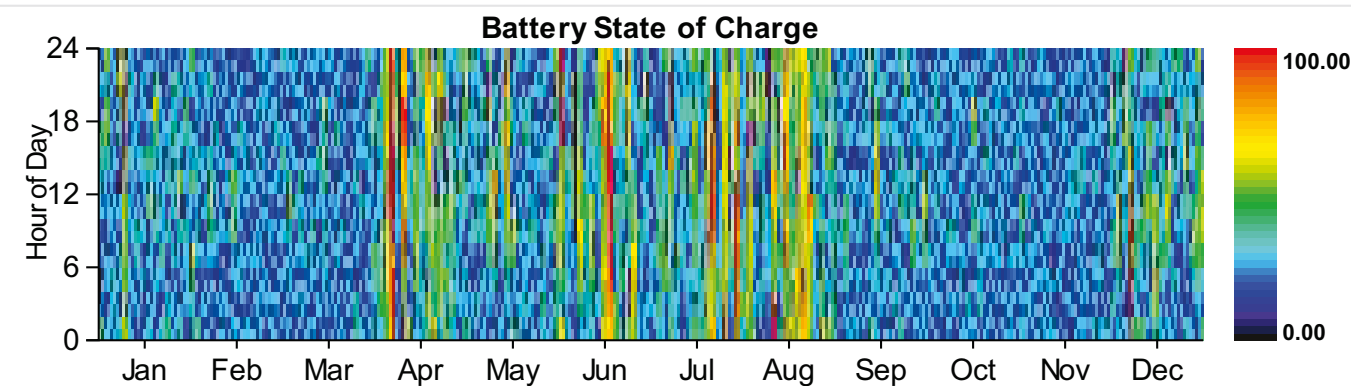

\section{Converter}

\section{Quantity} Inverter

Rectifier

Units

Capacity

24,000

2,043

Mean output

Minimum output

Maximum output

Capacity factor

\begin{tabular}{|r|r|r|}
24,000 & 21,600 & $\mathrm{~kW}$ \\
\hline 2,043 & 3,237 & $\mathrm{~kW}$ \\
\hline 0 & $0 \mathrm{~kW}$ \\
\hline 10,874 & $12,988 \mathrm{~kW}$ \\
\hline 9 & $13 \%$ \\
\hline
\end{tabular}




\begin{tabular}{|c|c|c|c|c|}
\hline Retanstifyoperation & Inverter & Rectifier & 6,211 & Heitss \\
\hline Energy in & $19,882,438$ & & $33,364,564$ & $\mathrm{kWh} / \mathrm{yr}$ \\
\hline Energy out & $17,894,204$ & & $28,359,858$ & $\mathrm{kWh} / \mathrm{yr}$ \\
\hline Losses & $1,988,234$ & & $5,004,706$ & $\mathrm{kWh} / \mathrm{yr}$ \\
\hline
\end{tabular}
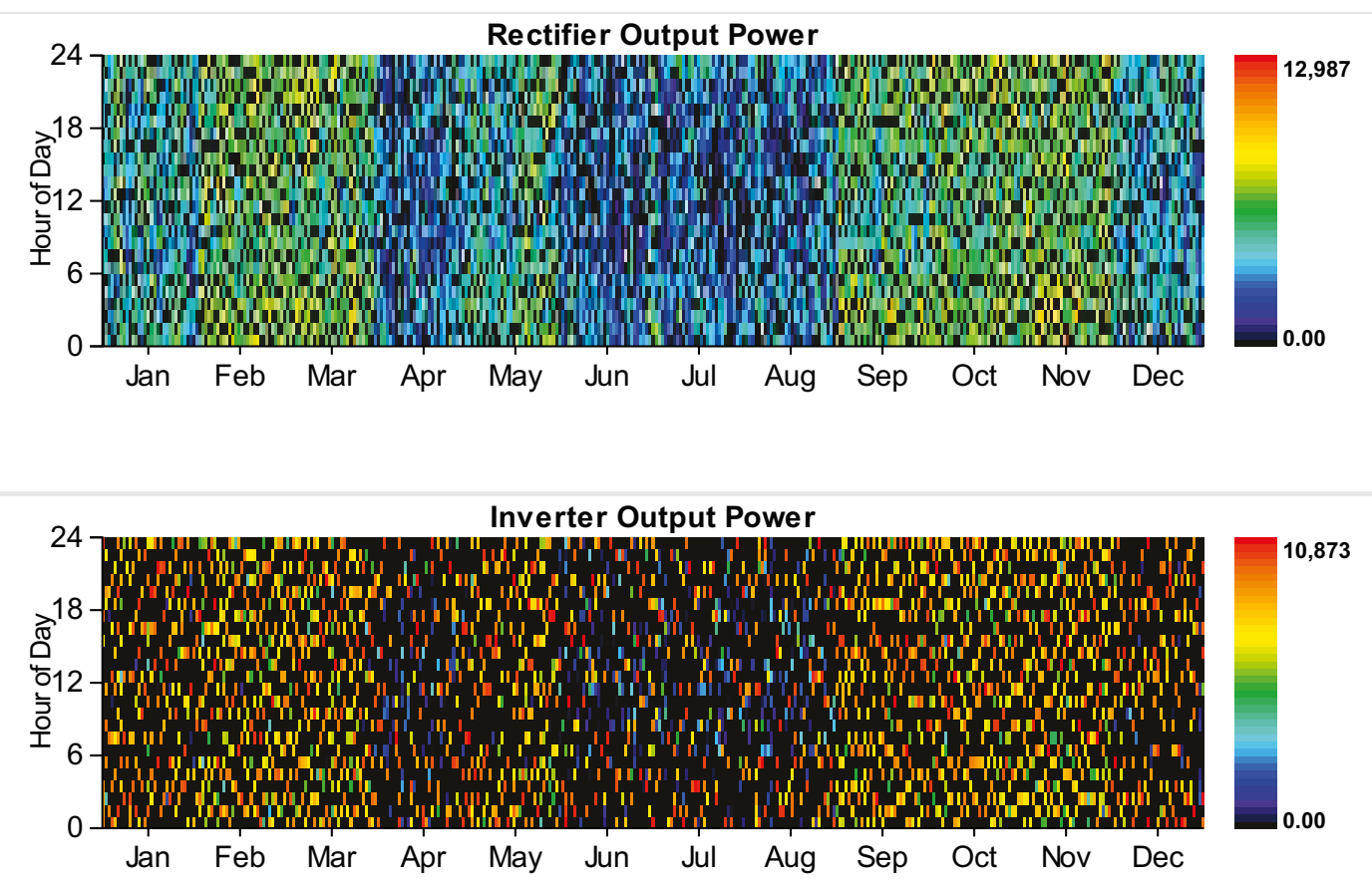

\section{Emissions}

\begin{tabular}{|l|r|}
\hline Pollutant & Emissions \\
\hline Carbon dioxide & $69150936 \mathrm{~kg} / \mathrm{yr}$ \\
\hline Carbon monoxide & $289829 \mathrm{~kg} / \mathrm{yr}$ \\
\hline Unburned hydrocarbons & $33199 \mathrm{~kg} / \mathrm{yr}$ \\
\hline Particulate matter & $8300 \mathrm{~kg} / \mathrm{yr}$ \\
\hline Sulfur dioxide & $142377 \mathrm{~kg} / \mathrm{yr}$ \\
\hline Nitrogen oxides & $289829 \mathrm{~kg} / \mathrm{yr}$ \\
\hline
\end{tabular}

HOMER Energy, LLC @ 2016 
System Report

System architecture

\begin{tabular}{|l|l|l|}
\hline Generator & Kohler 3250 Prime Power & 22,400 \\
\hline Dispatch Strategy & Cycle Charging & \\
\hline
\end{tabular}

\section{Cost summary}

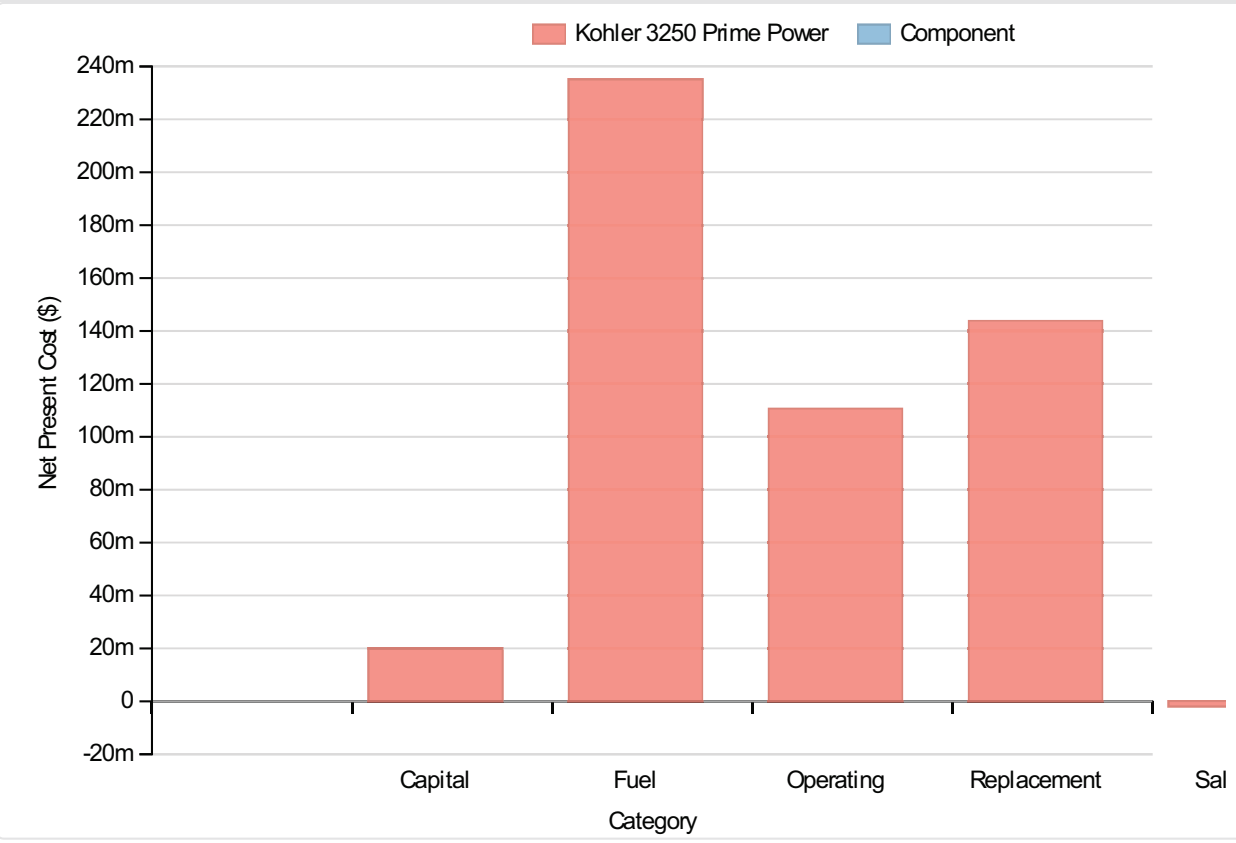

Cost Summary

Total net present cost

$507364928 \$$

Levelized cost of energy

$0.405 \$ / k W h$

\section{Net Present Costs}

\begin{tabular}{|l|l|l|l|l|l|l|}
\hline Component & Capital & Replacement & O\&M & Fuel & Salvage & Total \\
\hline Kohler 3250 Prime Power & $19,821,428$ & $143,797,872$ & $110,637,144$ & $235,007,568$ & $-1,899,339$ & $507,364,673$ \\
\hline System & $19,821,428$ & $143,797,872$ & $110,637,144$ & $235,007,568$ & $-1,899,339$ & $507,364,673$ \\
\hline
\end{tabular}

Annualized Costs

\begin{tabular}{|l|r|r|r|r|r|r|}
\hline Component & Capital & Replacement & O\&M & Fuel & Salvage & Total \\
\hline Kohler 3250 Prime Power & $1,533,274$ & $11,123,395$ & $8,558,268$ & $18,178,864$ & $-146,922$ & $39,246,879$ \\
\hline System & $1,533,274$ & $11,123,395$ & $8,558,268$ & $18,178,864$ & $-146,922$ & $39,246,879$ \\
\hline
\end{tabular}




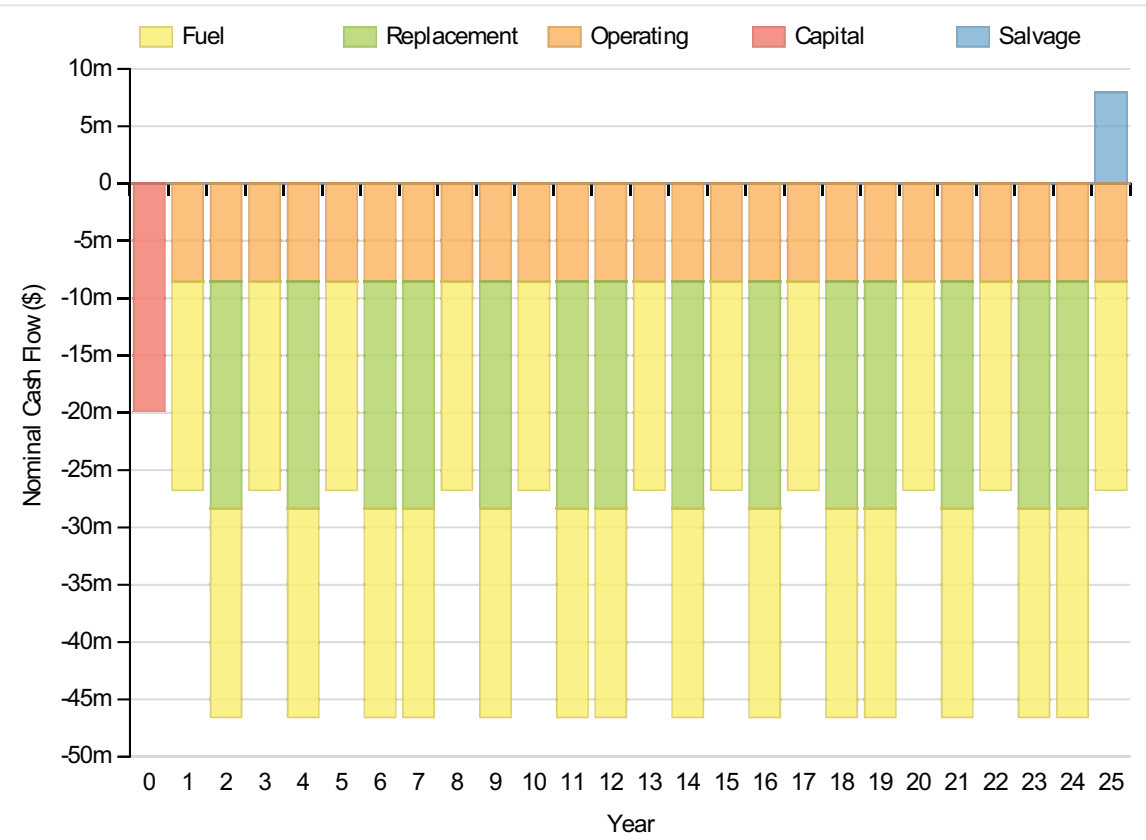

\section{Electrical}

\begin{tabular}{|c|c|c|c|c|}
\hline \multirow{2}{*}{\multicolumn{2}{|c|}{$\begin{array}{l}\text { Quantity } \\
\text { Excess electricity }\end{array}$}} & \multicolumn{2}{|l|}{ Value } & Units \\
\hline & & & 174601 & $\mathrm{kWh} / \mathrm{yr}$ \\
\hline Unmet load & & & 5507 & $\mathrm{kWh} / \mathrm{yr}$ \\
\hline Capacity shortage & & & 52841 & $\mathrm{kWh} / \mathrm{yr}$ \\
\hline Renewable fraction & & & 0 & \\
\hline Component & Production(kWh/yr) & & Fractio & n (\%) \\
\hline Generator & & $97,033,256$ & & 100 \\
\hline Total & & $97,033,256$ & & 100 \\
\hline Load & Consumption(kWh/yr) & & Fra & ction (\%) \\
\hline AC primary load & & $96,858,6$ & 648 & 100 \\
\hline DC primary load & & & 0 & 0 \\
\hline Total & & $96,858,6$ & 648 & 100 \\
\hline
\end{tabular}




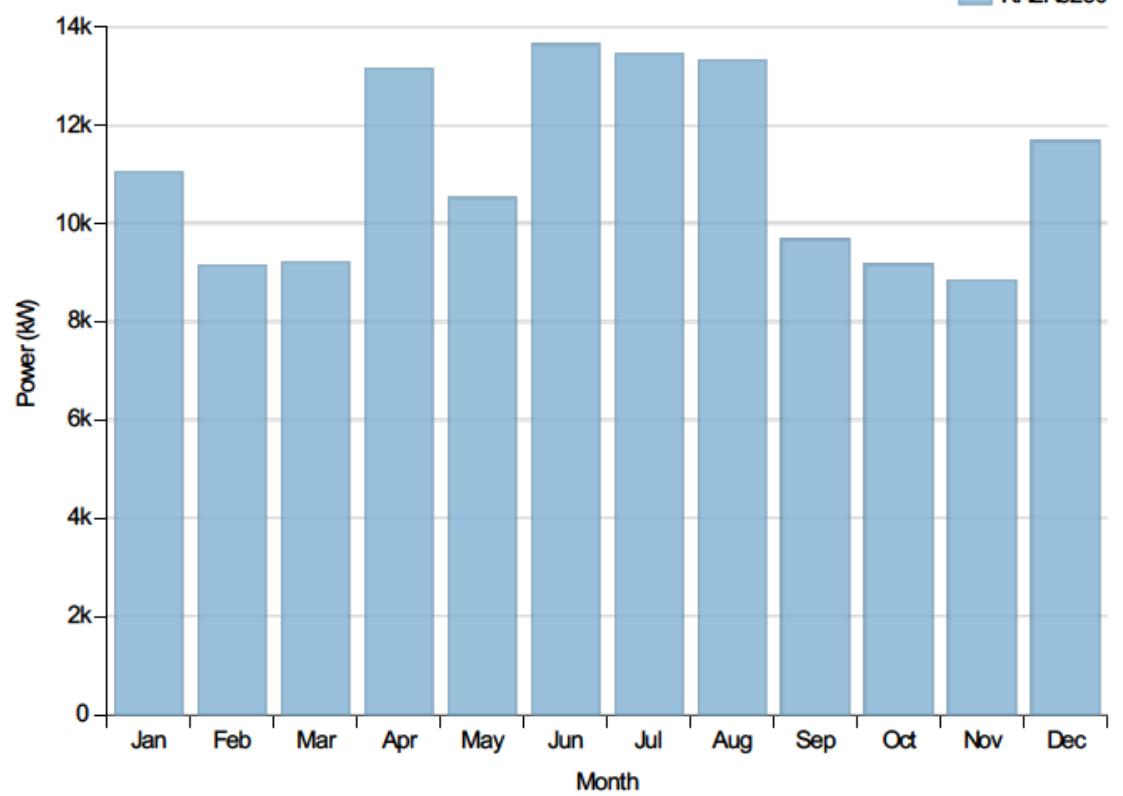

Generator:Kohler 3250 Prime Power

\begin{tabular}{|l|r|l|}
\hline Quantity & Value & Units \\
\hline Hours of operation & 8760 & hrs/yr \\
\hline Number of starts & 1 & starts/yr \\
\hline Operational life & 2 & yr \\
\hline Fixed generation cost & 2342.20 & $\$ / \mathrm{hr}$ \\
\hline Marginal generation cost & 0.18 & $\$ / \mathrm{kWh}$ \\
\hline Electrical production & 97033256 & $\mathrm{kWh} / \mathrm{yr}$ \\
\hline Mean electrical output & 11077 & $\mathrm{~kW}$ \\
\hline Min. electrical output & 5600 & $\mathrm{~kW}$ \\
\hline Max. electrical output & 22400 & $\mathrm{~kW}$ \\
\hline Fuel consumption & 23011234 & $\mathrm{~L} / \mathrm{yr}$ \\
\hline Specific fuel consumption & 0.24 & $\mathrm{~L} / \mathrm{kWh}$ \\
\hline Fuel energy input & 226430576 & $\mathrm{kWh} / \mathrm{yr}$ \\
\hline Mean electrical efficiency & $43 \%$ & $\%$ \\
\hline
\end{tabular}

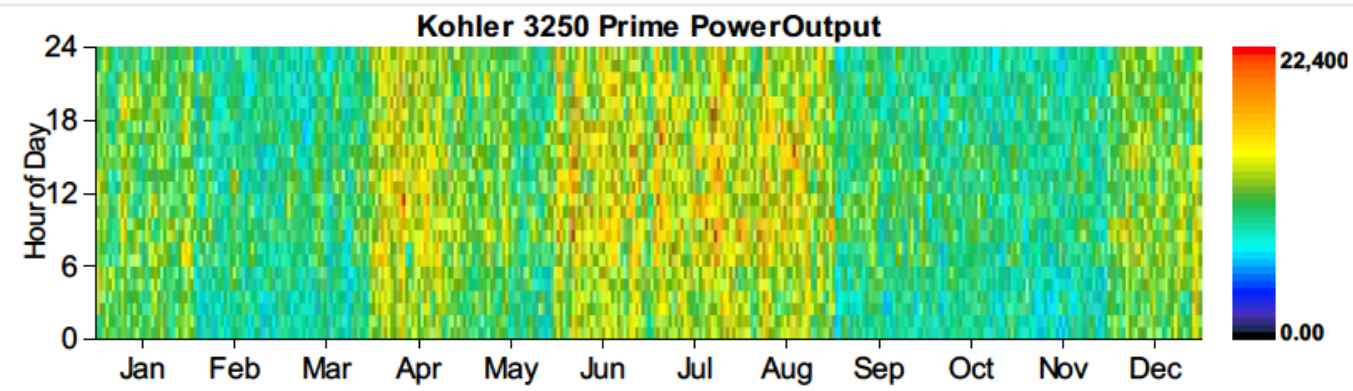

\section{Emissions}




\begin{tabular}{|l|r|l|}
\hline Pollutant & Emissions & Units \\
\hline Carbon dioxide & 60393336 & $\mathrm{~kg} / \mathrm{yr}$ \\
\hline Carbon monoxide & $253124 \mathrm{~kg} / \mathrm{yr}$ \\
\hline Unburned hydrocarbons & 28994 \\
\hline Particulate matter & $\mathrm{kg} / \mathrm{yr}$ \\
\hline Sulfur dioxide & 7249 & $\mathrm{~kg} / \mathrm{yr}$ \\
\hline Nitrogen oxides & 124345 & $\mathrm{~kg} / \mathrm{yr}$ \\
\hline
\end{tabular}

HOMER Energy, LLC @ 2016 
System Report

System architecture

\begin{tabular}{|l|l|r|}
\hline PV & Solar World 320W flat plate PV Copy & 5,000 \\
\hline Generator & Kohler 3250 Prime Power & 22,400 \\
\hline Converter & System Converter & 24,000 \\
\hline Dispatch Strategy & Cycle Charging & $\mathrm{kW}$ \\
\hline
\end{tabular}

\section{Cost summary}

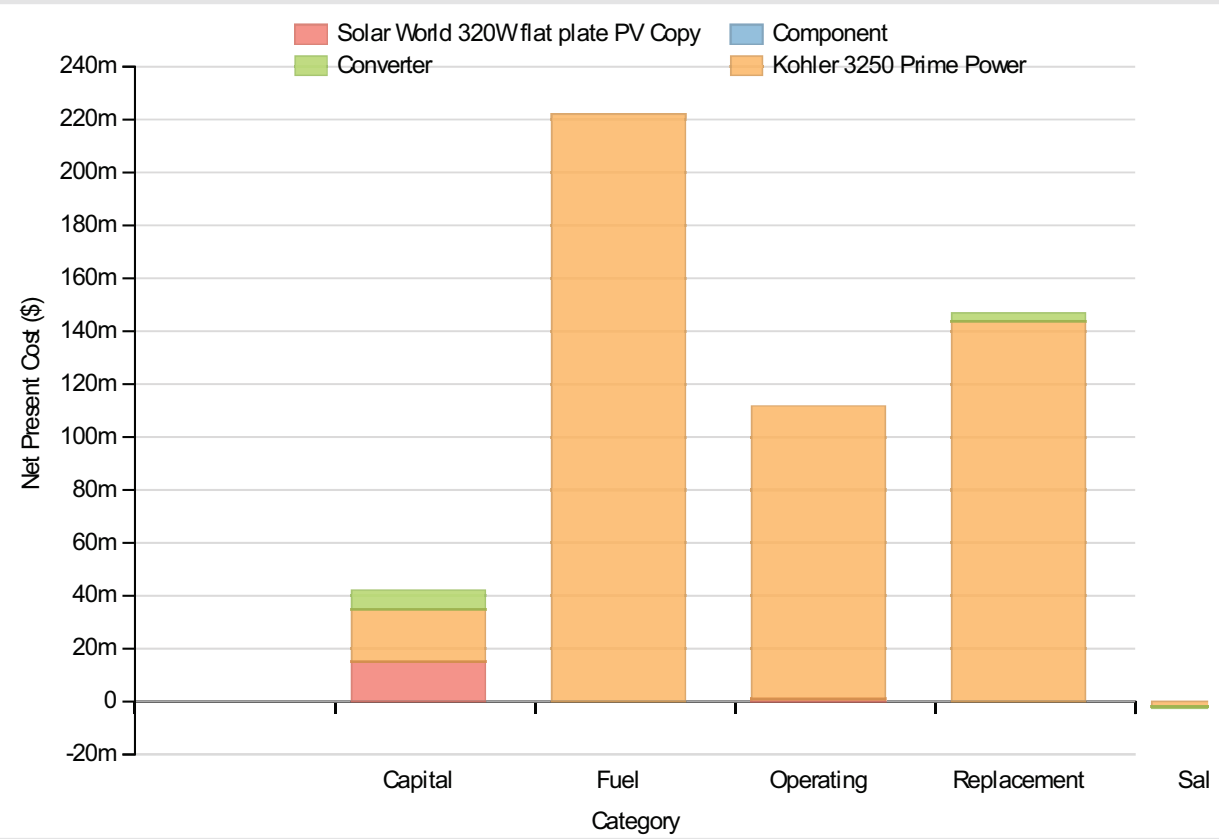

Cost Summary

Total net present cost

$520071488 \$$

Levelized cost of energy

$0.415 \$ / k W h$

\section{Net Present Costs}

\begin{tabular}{|c|c|c|c|c|c|c|}
\hline Component & Capital & Replacement & O\&M & Fuel & Salvage & Total \\
\hline Solar World 320W flat plate PV Copy & $15,000,000$ & 0 & 969,563 & 0 & 0 & $15,969,563$ \\
\hline Kohler 3250 Prime Power & $19,821,428$ & $143,797,872$ & $110,637,144$ & $222,064,784$ & $-1,899,339$ & $494,421,889$ \\
\hline Converter & $7,200,000$ & $3,054,769$ & 0 & 0 & $-574,938$ & $9,679,831$ \\
\hline System & $42,021,428$ & $146,852,640$ & $111,606,664$ & $222,064,784$ & $-2,474,277$ & $520,071,239$ \\
\hline
\end{tabular}

\section{Annualized Costs}

\begin{tabular}{|l|r|r|r|r|r|r|}
\hline Component & Capital & Replacement & O\&M & Fuel & Salvage & Total \\
\hline Solar World 320W flat plate PV Copy & $1,160,316$ & 0 & 75,000 & 0 & 0 \\
\hline Kohler 3250 Prime Power & $1,533,274$ & $11,123,395$ & $8,558,268$ & $17,177,684$ & $-146,922$ & $38,245,699$ \\
\hline Converter & 556,952 & 236,300 & 0 & 0 & $-44,474$ \\
\hline
\end{tabular}




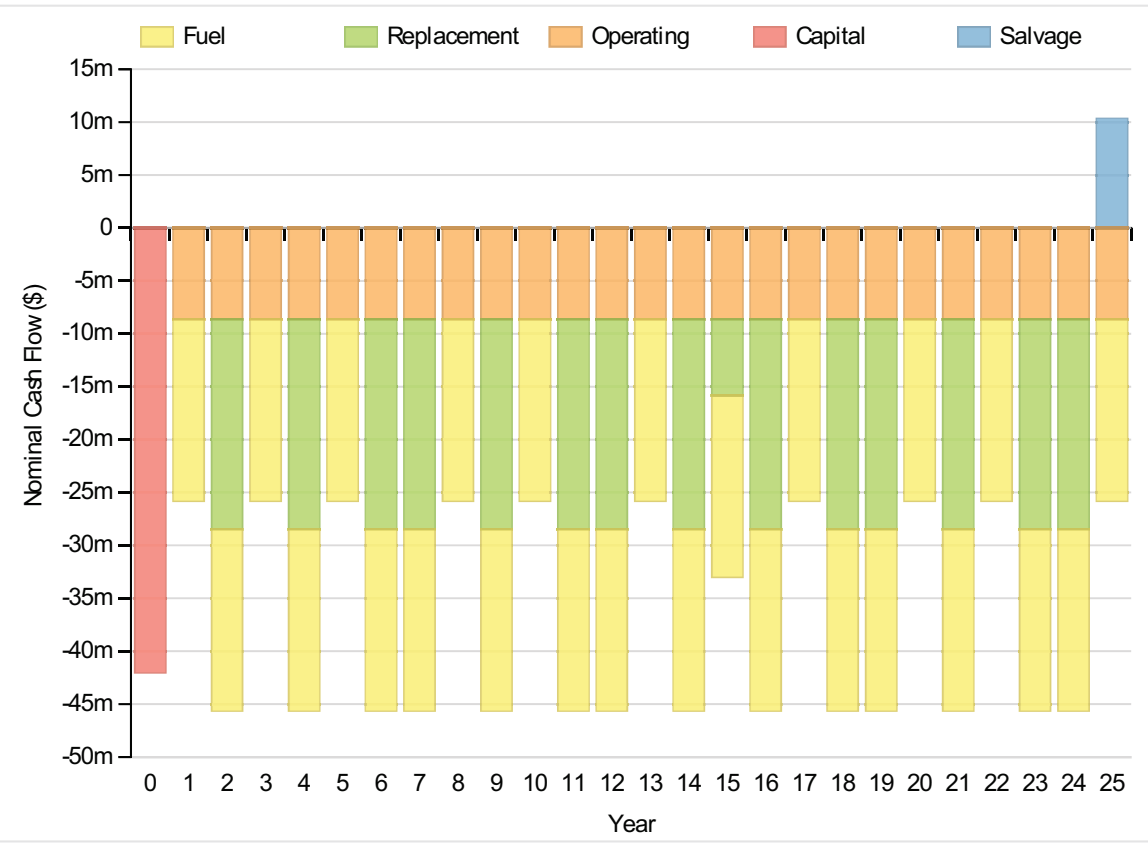

\section{Electrical}

\begin{tabular}{|c|c|c|c|c|}
\hline \multirow{2}{*}{\multicolumn{2}{|c|}{ Quantity }} & \multicolumn{2}{|l|}{ Value } & Units \\
\hline & & & 547357 & $\mathrm{kWh} / \mathrm{yr}$ \\
\hline Unmet load & & & 1351 & $\mathrm{kWh} / \mathrm{yr}$ \\
\hline Capacity shortage & & & 28736 & $\mathrm{kWh} / \mathrm{yr}$ \\
\hline Renewable fraction & & & 0 & \\
\hline Component & Production(kWh/yr) & & Fractio & on (\%) \\
\hline PV & & $6,443,367$ & & 7 \\
\hline Generator & & $91,574,000$ & & 93 \\
\hline Total & & $98,017,368$ & & 100 \\
\hline Load & Consumption(kWh/yr) & & Fra & ction $(\%)$ \\
\hline AC primary load & & $96,862,8$ & & 100 \\
\hline DC primary load & & & 0 & 0 \\
\hline Total & & $96,862,8$ & 308 & 100 \\
\hline
\end{tabular}




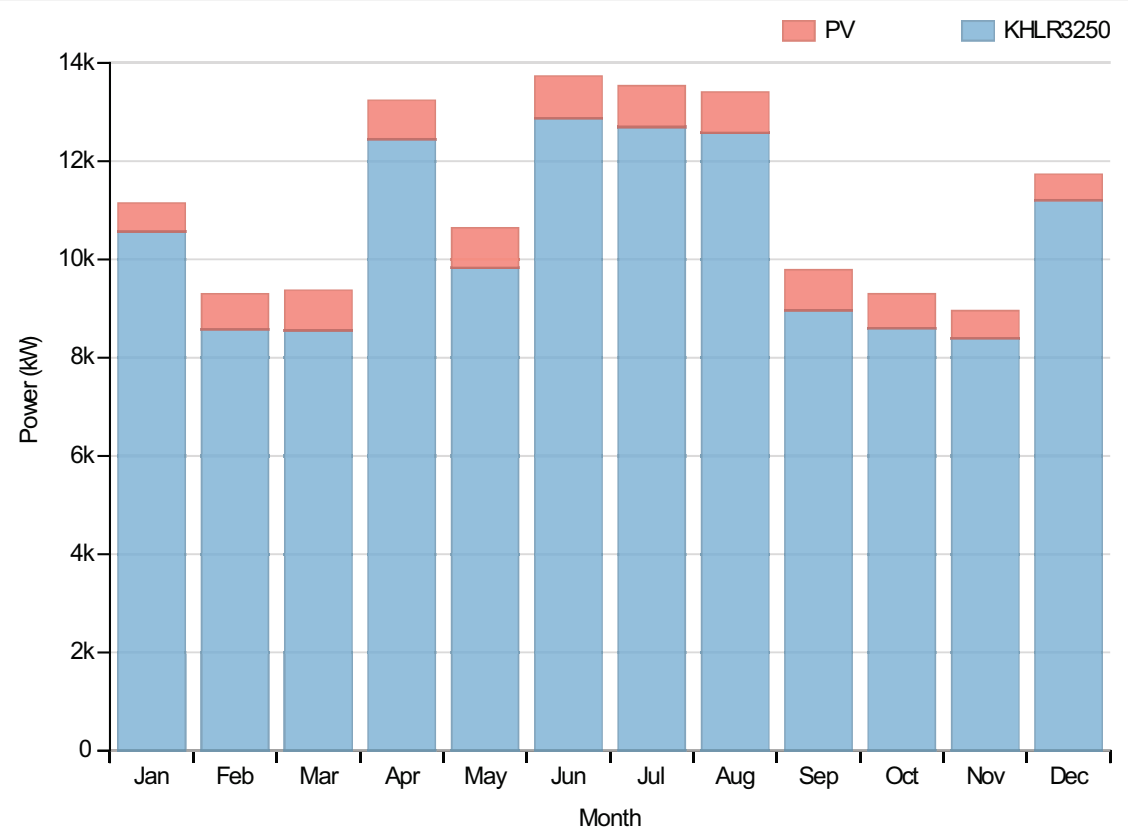

PV:Solar World 320W flat plate PV Copy

\begin{tabular}{|l|r|l|}
\hline Quantity & Value & Units \\
\hline Rated capacity & $5000 \mathrm{~kW}$ \\
\hline Mean output & $736 \mathrm{~kW}$ \\
\hline Mean output & $17653.00 \mathrm{kWh} / \mathrm{d}$ \\
\hline Capacity factor & $14.71 \%$ \\
\hline Total production & $6443367 \mathrm{kWh} / \mathrm{yr}$ \\
\hline Minimum output & $0.00 \mathrm{~kW}$ \\
\hline Maximum output & $5112.50 \mathrm{~kW}$ \\
\hline PV penetration & $6.65 \%$ & $\%$ \\
\hline Hours of operation & $4377 \mathrm{hrs} / \mathrm{yr}$ \\
\hline Levelized cost & 0.192 & $\$ / \mathrm{kWh}$ \\
\hline
\end{tabular}

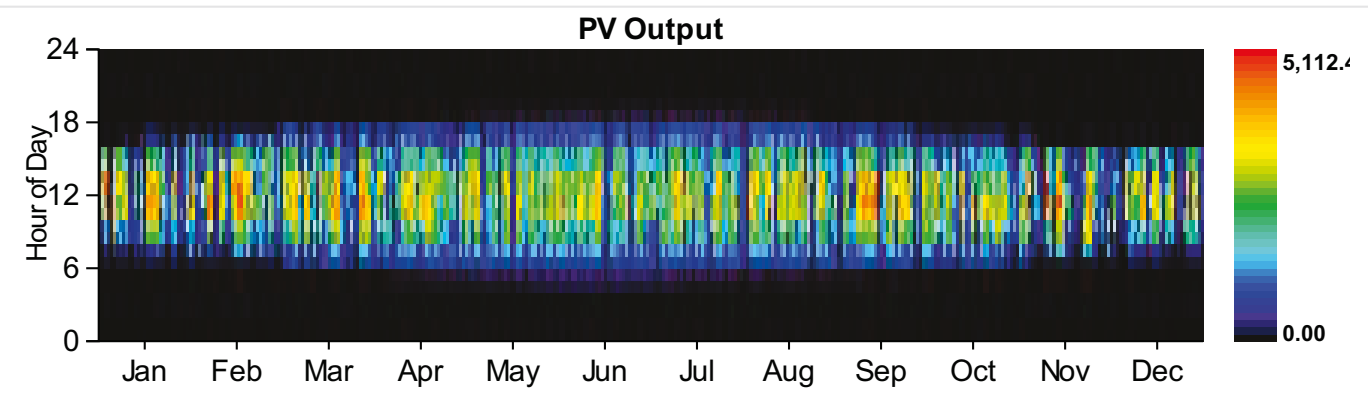

Generator:Kohler 3250 Prime Power

\section{Quantity}

Hours of operation

Number of starts

\section{Value}

Units

$8760 \mathrm{hrs} / \mathrm{yr}$

1 starts/yr 


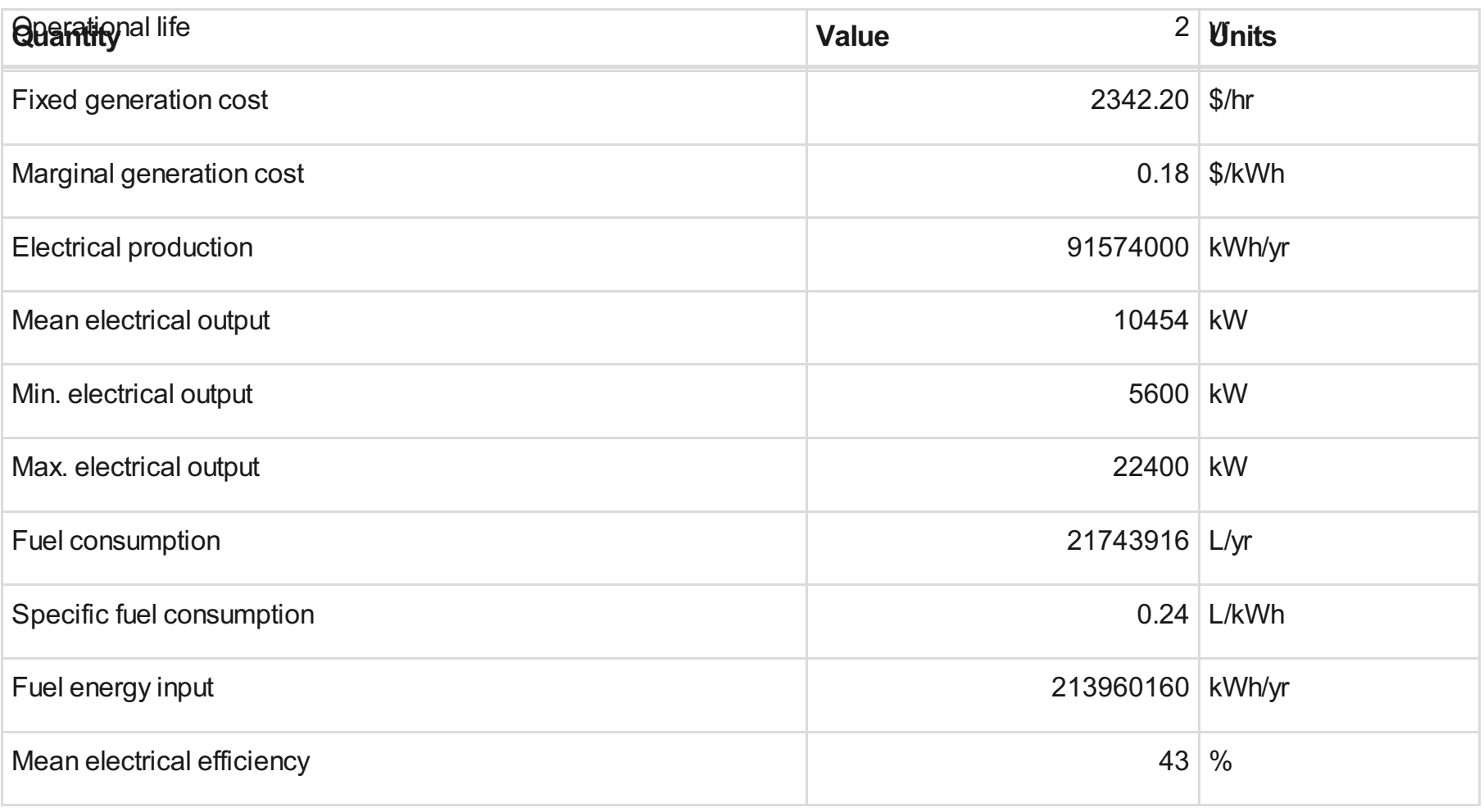

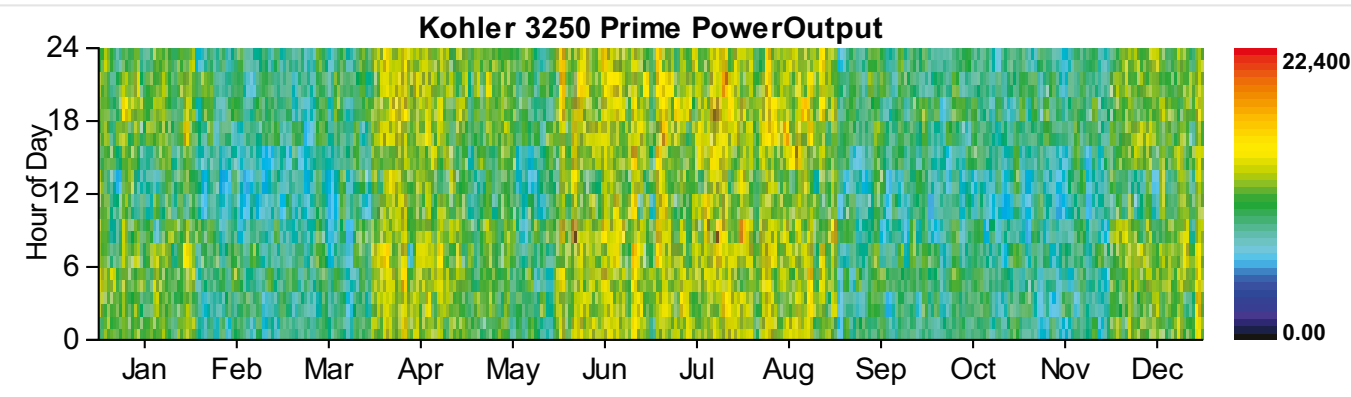

\section{Converter}

\begin{tabular}{|l|r|r|l|}
\hline Quantity & Inverter & Rectifier & Units \\
\hline Capacity & 24,000 & 21,600 & $\mathrm{~kW}$ \\
\hline Mean output & 624 & $\mathrm{~kW}$ \\
\hline Minimum output & 0 & $\mathrm{~kW}$ \\
\hline Maximum output & 4,601 & $\mathrm{~kW}$ \\
\hline Capacity factor & 3 & 0 & $\%$ \\
\hline Hours of operation & 4,311 & 0 \\
\hline Energy in & $6,070,614$ & $\mathrm{hrs} / \mathrm{yr}$ \\
\hline Energy out & $5,463,549$ & $\mathrm{kWh} / \mathrm{yr}$ \\
\hline Losses & 607,065 & $\mathrm{kWh} / \mathrm{yr}$ \\
\hline
\end{tabular}



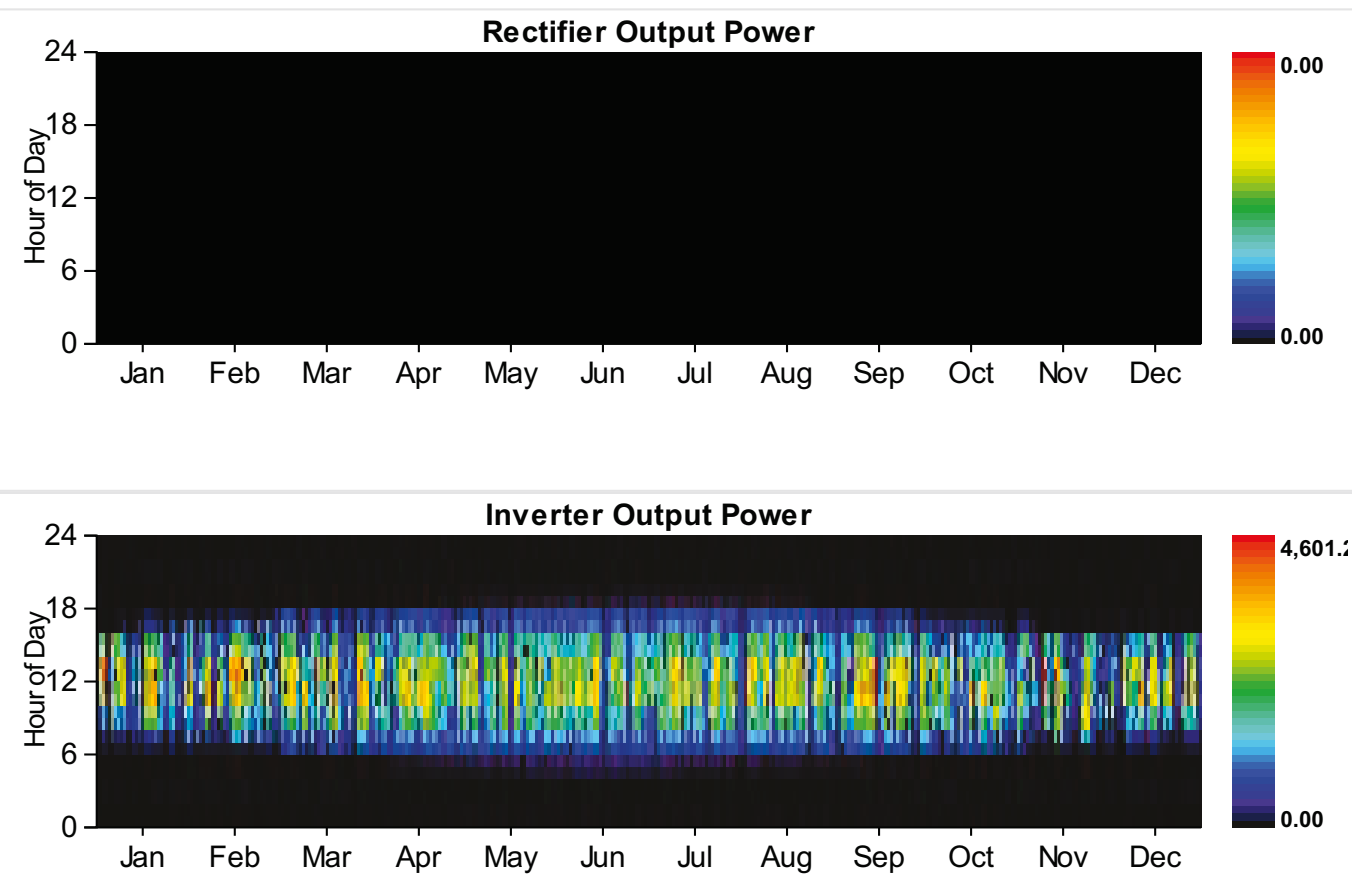

\section{Emissions}

\begin{tabular}{|l|r|l|}
\hline Pollutant & Emissions & Units \\
\hline Carbon dioxide & 57067240 & $\mathrm{~kg} / \mathrm{yr}$ \\
\hline Carbon monoxide & $239183 \mathrm{~kg} / \mathrm{yr}$ \\
\hline Unburned hydrocarbons & 27397 & $\mathrm{~kg} / \mathrm{yr}$ \\
\hline Particulate matter & $6849 \mathrm{~kg} / \mathrm{yr}$ \\
\hline Sulfur dioxide & $117497 \mathrm{~kg} / \mathrm{yr}$ \\
\hline Nitrogen oxides & $239183 \mathrm{~kg} / \mathrm{yr}$ \\
\hline
\end{tabular}

HOMER Energy, LLC @ 2016 


\section{Appendix D - AUTEC Scenarios}

\begin{tabular}{l|l|r|}
$\begin{array}{l}\text { System Report } \\
\text { System architecture }\end{array}$ & 2 \\
\hline Wind Turbine & Siemens 2.3 MW - 108 & $3,250 \mathrm{~kW}$ \\
\hline Generator & Kohler 3250 Prime Power & 12 stings \\
\hline Battery & GS200 flow & $2,000 \mathrm{~kW}$ \\
\hline Converter & System Converter & \\
\hline Dispatch Strategy & Cycle Charging & \\
\hline
\end{tabular}

\section{Cost summary}

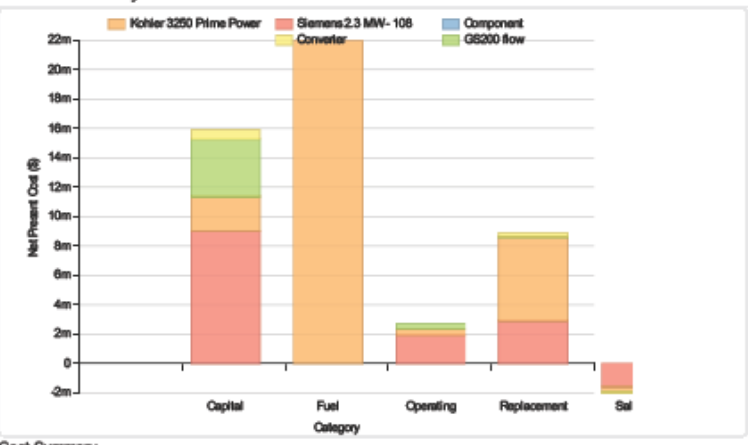

Cost Summary

\begin{tabular}{|c|c|c|}
\hline Total net present cost & 47442712 & s \\
\hline Levelized cost of energy & 0.261 & $\$ / k W h$ \\
\hline
\end{tabular}

Net Present Costs

\begin{tabular}{|l|r|r|r|r|r|r|}
\hline Component & Capital & Replacement & O\&M & Fuel & \multicolumn{1}{l}{ Salvage } & \multicolumn{1}{l}{ Total } \\
\hline Siemens 2.3 MW - 108 & $9,000,000$ & $2,869,263$ & $1,939,126$ & 0 & $-1,617,013$ & $12,191,376$ \\
\hline Koller 3250 Prime Power & $2,346,591$ & $5,707,372$ & 426,261 & $21,940,282$ & $-317,611$ & $30,102,895$ \\
\hline GS200 flow & $3,895,070$ & 86,018 & 372,312 & 0 & $-11,663$ & $4,341,737$ \\
\hline Converter & 600,000 & 254,564 & 0 & 0 & $-47,912$ & 806,652 \\
\hline System & $15,841,661$ & $8,917,217$ & $2,737,699$ & $21,940,282$ & $-1,994,198$ & $47,442,661$ \\
\hline
\end{tabular}

Annualized Costs

\begin{tabular}{|l|r|r|r|r|r|r|}
\hline Component & Capital & Replacement & O\&M & Fuel & \multicolumn{1}{l}{ Salvage } & \multicolumn{1}{l}{ Total } \\
\hline Siemens 2.3 MW - 108 & 696,189 & 221,950 & 150,000 & 0 & $-125,083$ & 943,056 \\
\hline
\end{tabular}


System Report

System architecture

\begin{tabular}{|l|l|r|}
\hline Wind Turbine & Siemens 2.3 MW - 108 & 2 \\
\hline Generator & Kohler 3250 Prime Power & 3,250 \\
\hline Battery & GS200 flow & 12 \\
\hline Converter & System Converter & 2,000 \\
\hline Dispatch Strategy & Cycle Charging & $\mathrm{kW}$ \\
\hline
\end{tabular}

\section{Cost summary}

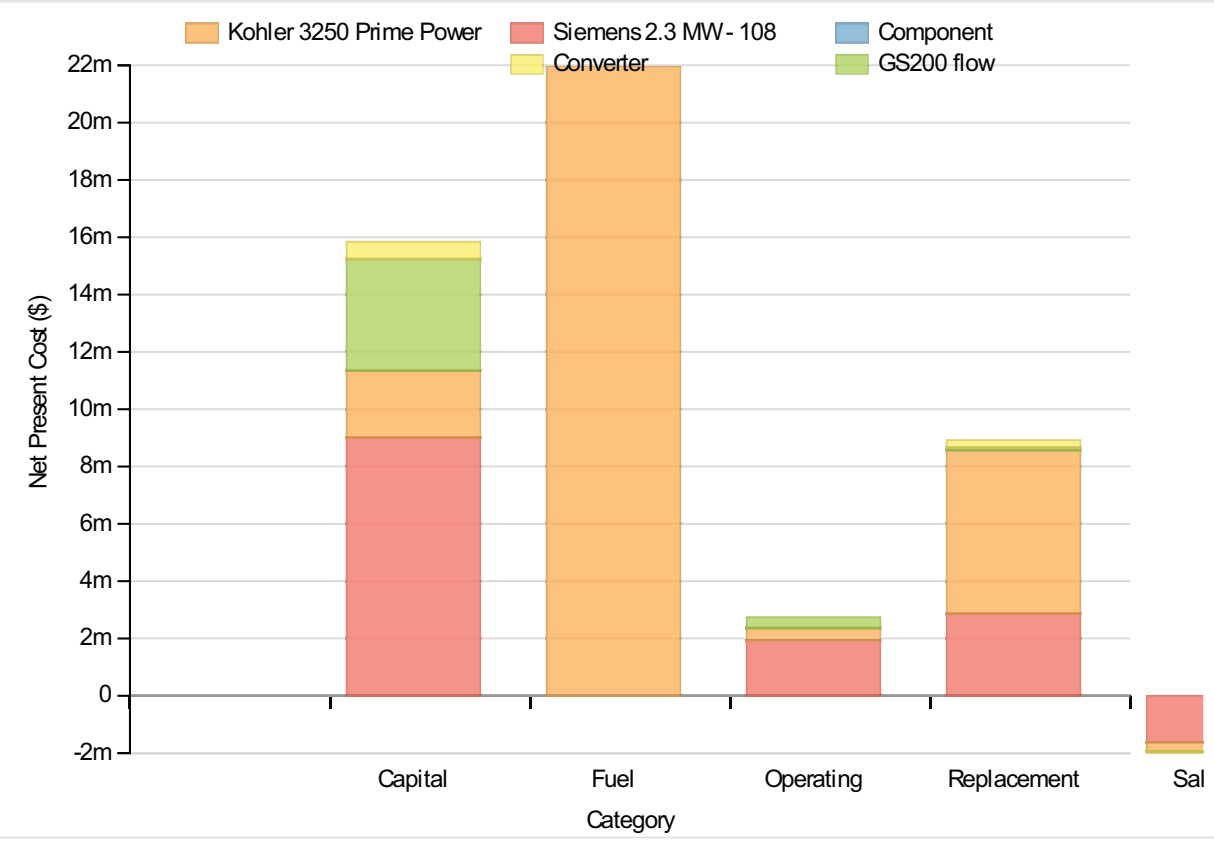

Cost Summary

Total net present cost

$47442712 \$$

Levelized cost of energy

$0.261 \$ / k W h$

Net Present Costs

\begin{tabular}{|l|r|r|r|r|r|r|}
\hline Component & Capital & Replacement & O\&M & Fuel & Salvage & Total \\
\hline Siemens 2.3 MW - 108 & $9,000,000$ & $2,869,263$ & $1,939,126$ & 0 & $-1,617,013$ & $12,191,376$ \\
\hline Kohler 3250 Prime Power & $2,346,591$ & $5,707,372$ & 426,261 & $21,940,282$ & $-317,611$ & $30,102,895$ \\
\hline GS200 flow & $3,895,070$ & 86,018 & 372,312 & 0 & $-11,663$ & $4,341,737$ \\
\hline Converter & 600,000 & 254,564 & 0 & $-47,912$ & 806,652 \\
\hline System & $15,841,661$ & $8,917,217$ & $2,737,699$ & $21,940,282$ & $-1,994,198$ & $47,442,661$ \\
\hline
\end{tabular}

\section{Annualized Costs}

\begin{tabular}{|l|r|r|r|r|r|r|}
\hline Component & Capital & Replacement & O\&M & Fuel & Salvage & \multicolumn{1}{l|}{ Total } \\
\hline Siemens 2.3 MW - 108 & 696,189 & 221,950 & 150,000 & 0 & $-125,083$ & 943,056 \\
\hline
\end{tabular}




\begin{tabular}{|l|r|r|r|r|r|r|}
\hline GS200 flow & 301,301 & 6,654 & 28,800 & 0 & -902 & 335,853 \\
\hline Converter & 46,413 & 19,692 & 0 & $-3,706$ & 62,399 \\
\hline System & $1,225,422$ & 689,786 & 211,773 & $1,697,177$ & $-154,260$ & $3,669,898$ \\
\hline
\end{tabular}

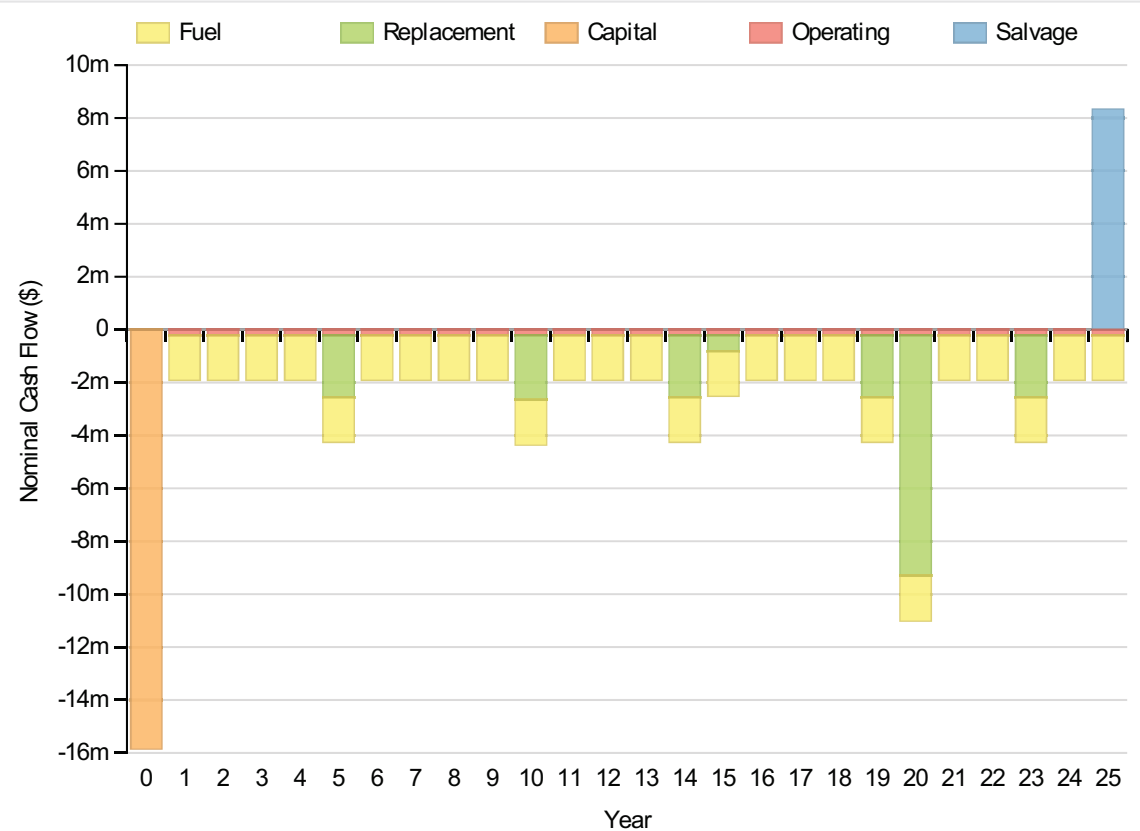

\section{Electrical}

\begin{tabular}{|c|c|c|c|c|}
\hline \multicolumn{2}{|l|}{ Quantity } & \multicolumn{2}{|l|}{ Value } & Units \\
\hline \multicolumn{2}{|l|}{ Excess electricity } & \multicolumn{2}{|c|}{4217891} & $\mathrm{kWh} / \mathrm{yr}$ \\
\hline \multicolumn{2}{|l|}{ Unmet load } & \multicolumn{2}{|r|}{488} & $\mathrm{kWh} / \mathrm{yr}$ \\
\hline \multicolumn{2}{|l|}{ Capacity shortage } & \multicolumn{2}{|r|}{3675} & $\mathrm{kWh} / \mathrm{yr}$ \\
\hline \multicolumn{2}{|l|}{ Renewable fraction } & \multicolumn{2}{|r|}{0} & \\
\hline Component & \multicolumn{2}{|l|}{ Production(kWh/yr) } & \multicolumn{2}{|c|}{ Fraction $(\%)$} \\
\hline Generator & \multicolumn{2}{|r|}{$7,356,061$} & \multicolumn{2}{|r|}{36} \\
\hline Wind Turbine & \multicolumn{2}{|r|}{$13,081,490$} & \multicolumn{2}{|r|}{64} \\
\hline Total & \multicolumn{2}{|r|}{$20,437,550$} & \multicolumn{2}{|r|}{100} \\
\hline Load & \multicolumn{2}{|l|}{ Consumption(kWh/yr) } & \multicolumn{2}{|c|}{ Fraction (\%) } \\
\hline AC primary load & \multicolumn{2}{|r|}{$14,060,971$} & \multicolumn{2}{|c|}{100} \\
\hline DC primary load & \multicolumn{2}{|r|}{0} & & 0 \\
\hline Total & \multicolumn{2}{|r|}{$14,060,971$} & \multicolumn{2}{|c|}{100} \\
\hline
\end{tabular}




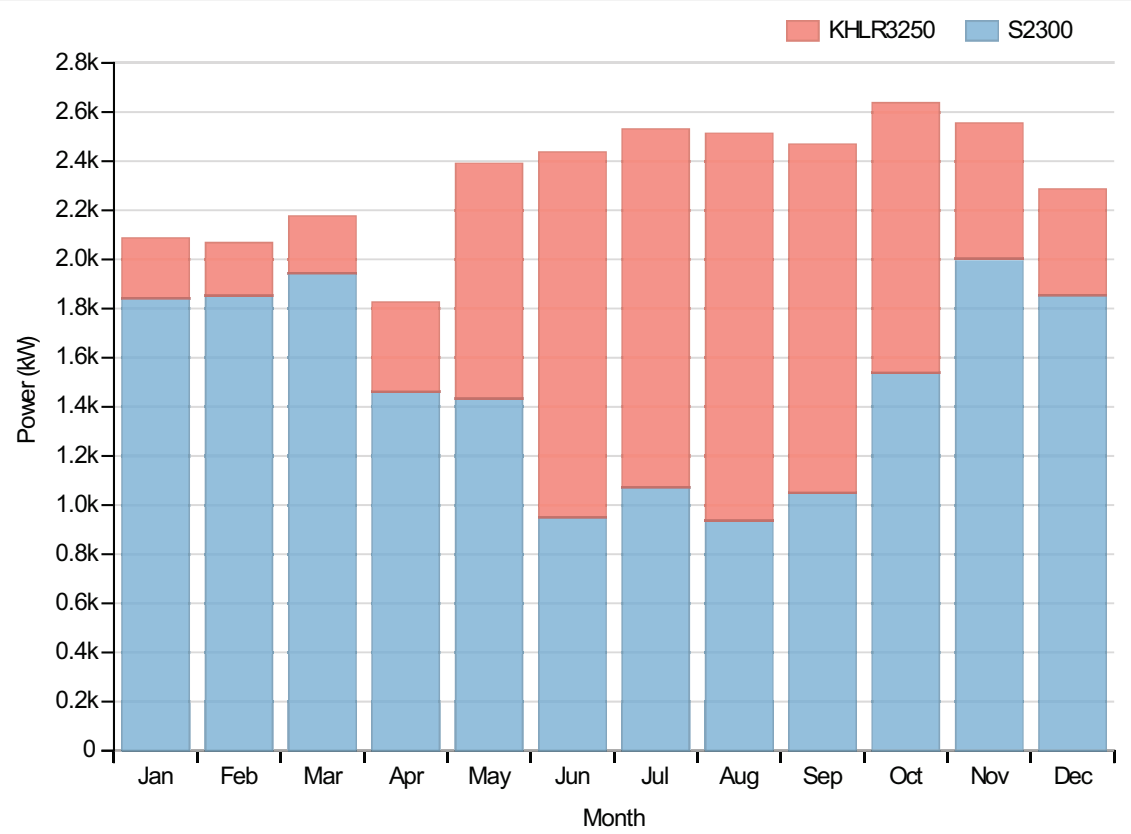

Wind Turbine:Siemens 2.3 MW - 108

\begin{tabular}{|l|l|l|}
\hline Quantity & Value & Units \\
\hline Total rated capacity & 4600 & $\mathrm{~kW}$ \\
\hline Mean output & 1493 & $\mathrm{~kW}$ \\
\hline Capacity factor & 32.46 & $\%$ \\
\hline Total production & 13081490 & $\mathrm{kWh} / \mathrm{yr}$ \\
\hline Minimum output & 3.12 & $\mathrm{~kW}$ \\
\hline Maximum output & 4629.60 & $\mathrm{~kW}$ \\
\hline Wind penetration & 93.03 & $\%$ \\
\hline Hours of operation & 8760 & $\mathrm{hrs} / \mathrm{yr}$ \\
\hline Levelized cost & $\$ \mathrm{kWh}$ \\
\hline
\end{tabular}

\section{Generator:Kohler 3250 Prime Power}

\section{Quantity}

Hours of operation

Number of starts

Operational life

Fixed generation cost

Marginal generation cost

Electrical production

Mean electrical output

Min. electrical output

\section{Value}

Units

$3261 \mathrm{hrs} / \mathrm{yr}$

911 starts/yr

$5 \mathrm{yr}$

$235.85 \$ / h r$

$0.20 \$ / \mathrm{kWh}$

$7356061 \mathrm{kWh} / \mathrm{yr}$

2256 kW 
Qaănitegtrical output

Fuel consumption

Specific fuel consumption

Fuel energy input

Mean electrical efficiency
Value

3250 UNits

1697178 L/yr

$0.23 \mathrm{~L} / \mathrm{kWh}$

$16700232 \mathrm{kWh} / \mathrm{yr}$

$44 \%$

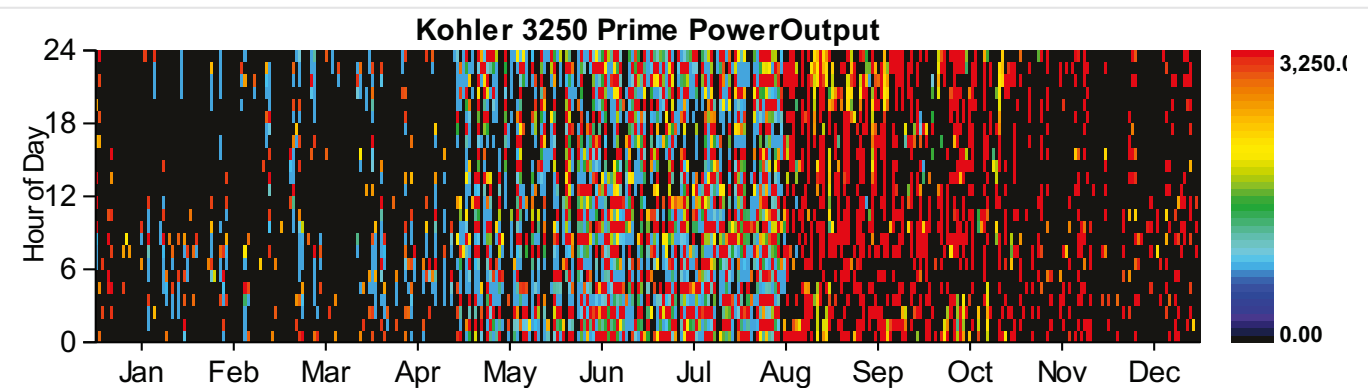

\section{Battery:GS200 flow}

\section{Quantity}

Value

String size

Strings in parallel

Batteries

Bus voltage

\section{Quantity}

Nominal capacity

Usable nominal capacity

Autonomy

Lifetime throughput

Battery wear cost

Average energy cost

Energy in

Energy out

Storage depletion

Losses

Annual throughput

Expected life

\section{Value}

Units

7200 kWh

7200 kWh

$4 \mathrm{hr}$

0

$0.000 \$ / k W h$

$0.159 \$ / k W h$

$3953248 \mathrm{kWh} / \mathrm{yr}$

$2769061 \mathrm{kWh} / \mathrm{yr}$

$2134 \mathrm{kWh} / \mathrm{yr}$

$1182053 \mathrm{kWh} / \mathrm{yr}$

$3309657 \mathrm{kWh} / \mathrm{yr}$

$25 \mathrm{yr}$ 


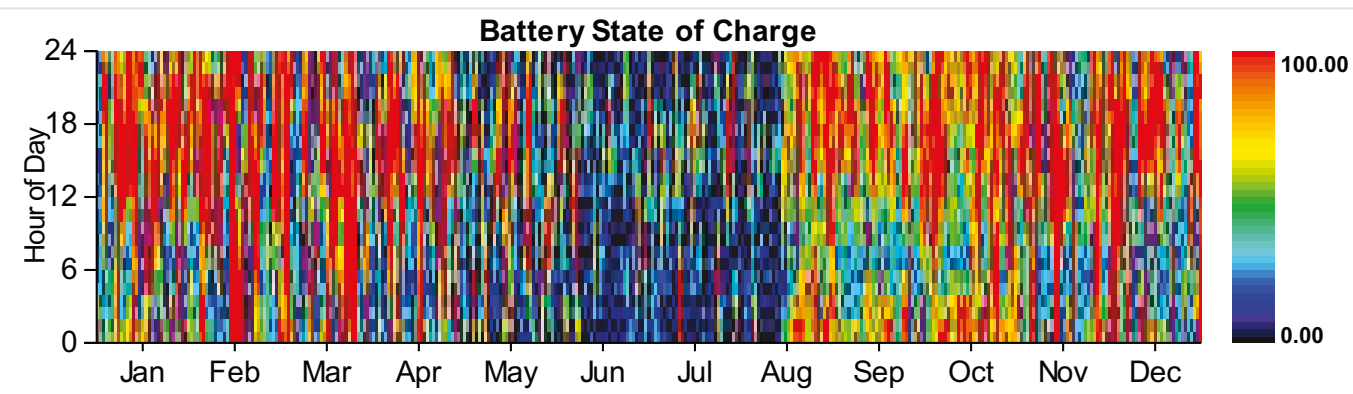

Converter

\begin{tabular}{|l|r|r|l|}
\hline Quantity & Inverter & Rectifier & Units \\
\hline Capacity & 2,000 & $1,800 \mathrm{~kW}$ \\
\hline Mean output & 284 & $451 \mathrm{~kW}$ \\
\hline Minimum output & 0 & $0 \mathrm{~kW}$ \\
\hline Maximum output & 1,988 & $2,000 \mathrm{~kW}$ \\
\hline Capacity factor & 14 & $23 \%$ \\
\hline Hours of operation & 3,651 & $3,335 \mathrm{hrs} / \mathrm{yr}$ \\
\hline Energy in & $2,769,061$ & $4,650,904 \mathrm{kWh} / \mathrm{yr}$ \\
\hline Energy out & $2,492,151$ & $3,953,248 \mathrm{kWh} / \mathrm{yr}$ \\
\hline Losses & 276,910 & 697,656 & $\mathrm{kWh} / \mathrm{yr}$ \\
\hline
\end{tabular}
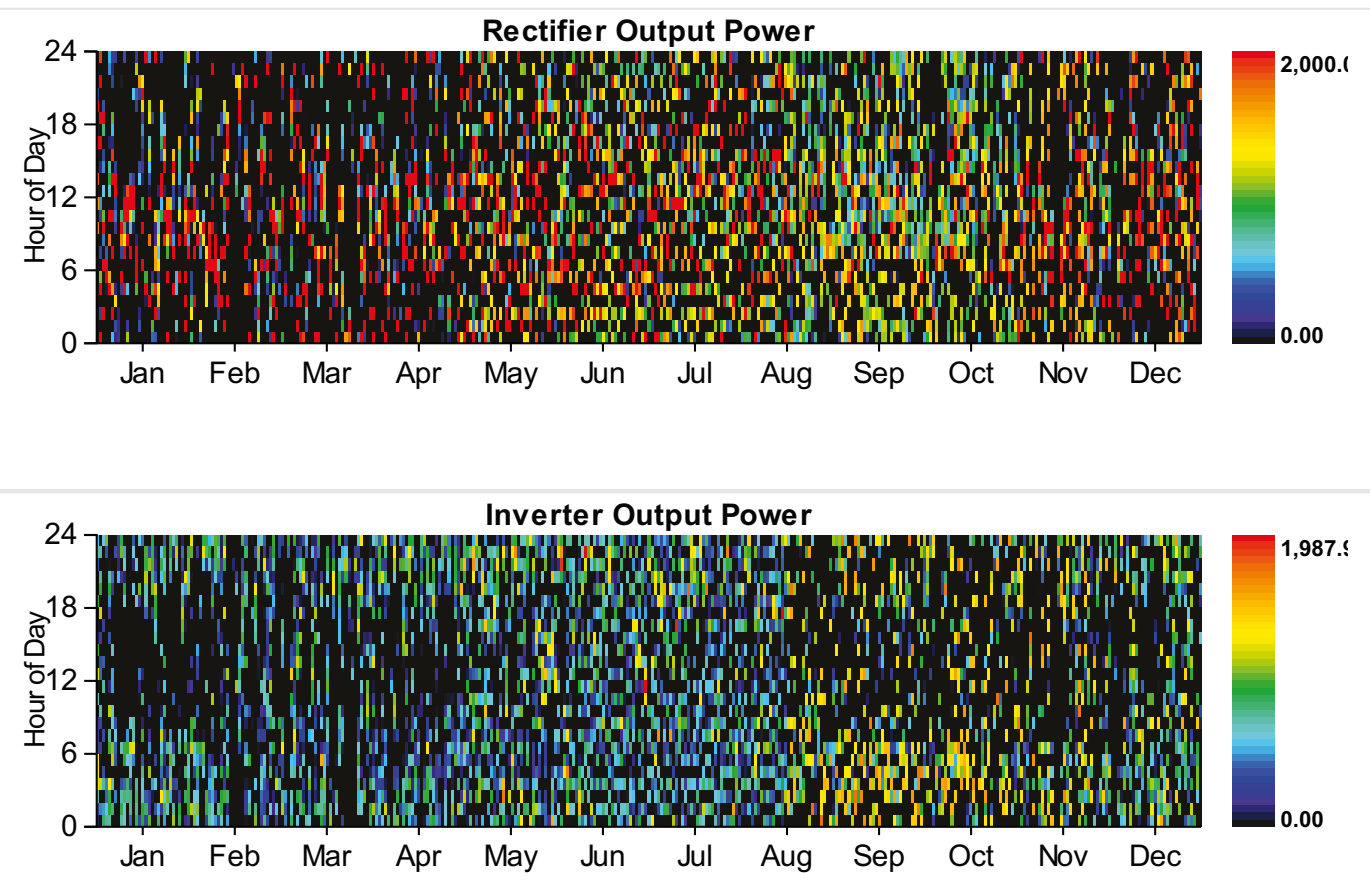

\section{Emissions}

\begin{tabular}{|l|r|l|}
\hline Pollutant & Emissions & Units \\
\hline Carbon dioxide & $4454270 \mathrm{~kg} / \mathrm{yr}$ \\
\hline Carbon monoxide & $18669 \mathrm{~kg} / \mathrm{yr}$ \\
\hline
\end{tabular}


Pollutant

Unburned hydrocarbons

Particulate matter

Sulfur dioxide

Nitrogen oxides

HOMER Energy, LLC @ 2016
2138 Units

$535 \mathrm{~kg} / \mathrm{yr}$

$9171 \mathrm{~kg} / \mathrm{yr}$

$18669 \mathrm{~kg} / \mathrm{yr}$ 
System Report

System architecture

\begin{tabular}{|l|l|r|}
\hline PV & Solar World 320W flat plate PV Copy & 2,000 \\
\hline Wind Turbine & Siemens 2.3 MW - 108 & 2 \\
\hline Generator & Kohler 3250 Prime Power & 3,250 \\
\hline Battery & GS200 flow & 15 \\
\hline Converter & System Converter & strings \\
\hline Dispatch Strategy & Cycle Charging & 3,000 \\
\hline
\end{tabular}

\section{Cost summary}

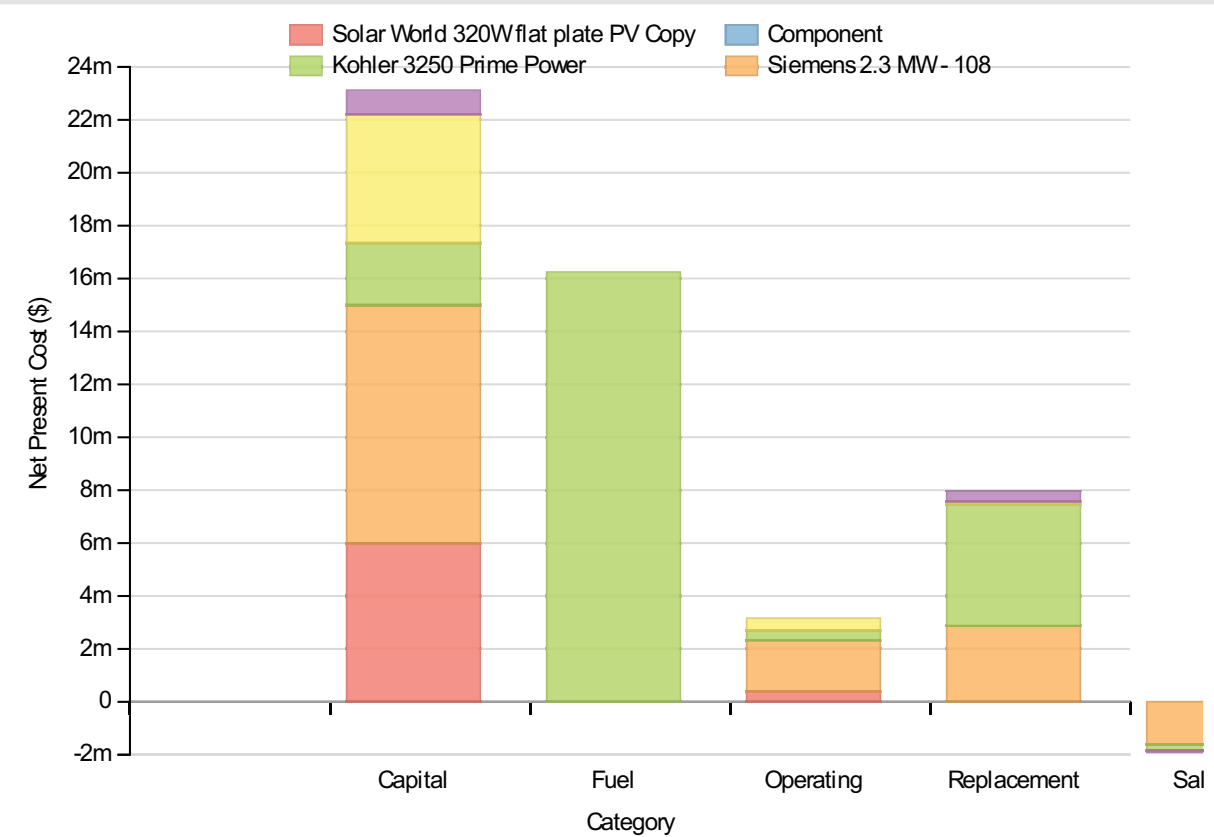

Cost Summary

Total net present cost

$48557108 \$$

Levelized cost of energy

$0.267 \$ / k W h$

\section{Net Present Costs}

\begin{tabular}{|c|c|c|c|c|c|c|}
\hline Component & Capital & Replacement & O\&M & Fuel & Salvage & Total \\
\hline Solar World 320W flat plate PV Copy & $6,000,000$ & 0 & 387,825 & 0 & 0 & $6,387,825$ \\
\hline Siemens 2.3 MW - 108 & $9,000,000$ & $2,869,263$ & $1,939,126$ & 0 & $-1,617,013$ & $12,191,376$ \\
\hline Kohler 3250 Prime Power & $2,346,591$ & $4,600,629$ & 362,995 & $16,239,458$ & $-208,930$ & $23,340,743$ \\
\hline GS200 flow & $4,868,838$ & 107,521 & 465,390 & 0 & $-14,578$ & $5,427,171$ \\
\hline Converter & 900,000 & 381,846 & 0 & 0 & $-71,867$ & $1,209,979$ \\
\hline System & $23,115,428$ & $7,959,259$ & $3,155,337$ & $16,239,458$ & $-1,912,389$ & $48,557,093$ \\
\hline
\end{tabular}




\begin{tabular}{|l|r|r|r|r|r|r|}
\hline Component & Capital & Replacement & O\&M & Fuel & Salvage & \multicolumn{1}{l|}{ Total } \\
\hline Solar World 320W flat plate PV Copy & 464,126 & 0 & 30,000 & 0 & 0 & 494,126 \\
\hline Siemens 2.3 MW - 108 & 696,189 & 221,950 & 150,000 & 0 & $-125,083$ & 943,056 \\
\hline Kohler 3250 Prime Power & 181,519 & 355,879 & 28,079 & $1,256,193$ & $-16,162$ & $1,805,508$ \\
\hline GS200 flow & 376,626 & 8,317 & 36,000 & 0 & $-1,128$ & 419,816 \\
\hline Converter & 69,619 & 29,537 & 0 & 0 & $-5,559$ & 93,597 \\
\hline System & $1,788,080$ & 615,684 & 244,079 & $1,256,193$ & $-147,932$ & $3,756,104$ \\
\hline
\end{tabular}

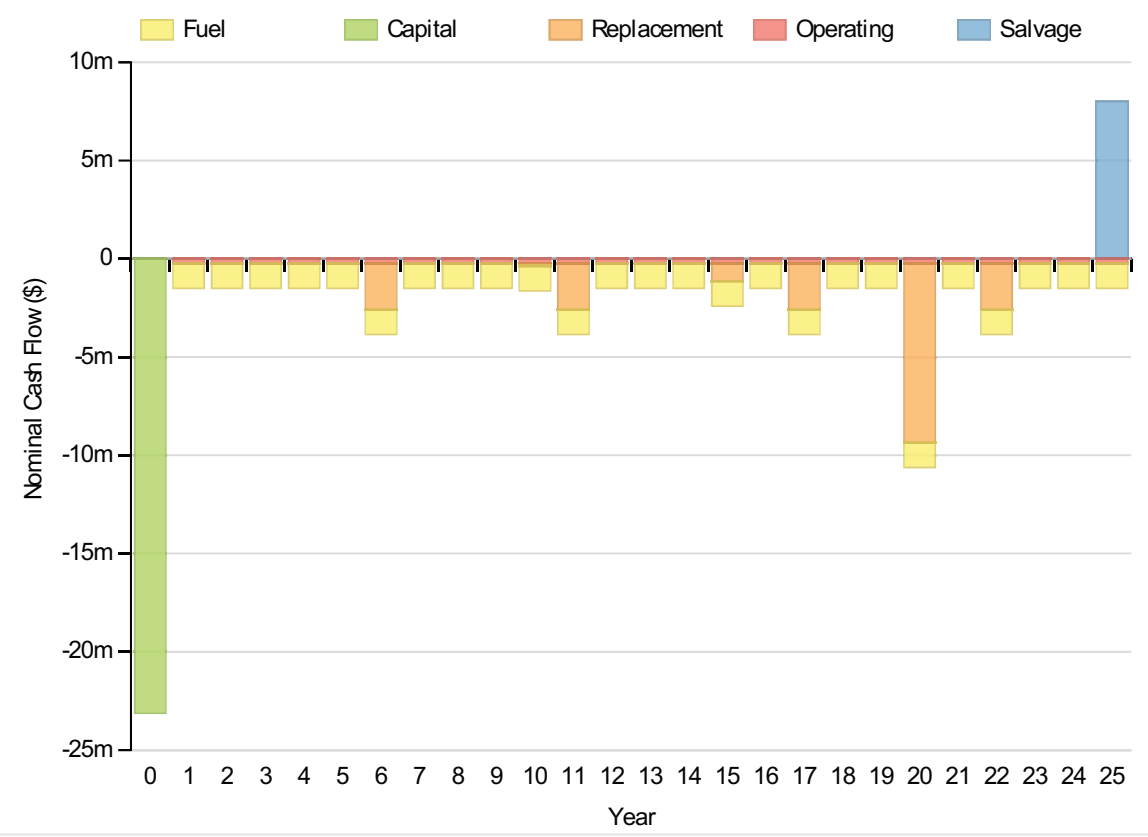

\section{Electrical}

\begin{tabular}{|c|c|c|c|c|}
\hline \multicolumn{2}{|l|}{ Quantity } & \multicolumn{2}{|l|}{ Value } & Units \\
\hline \multicolumn{2}{|l|}{ Excess electricity } & \multicolumn{2}{|c|}{5480098} & $\mathrm{kWh} / \mathrm{yr}$ \\
\hline \multicolumn{2}{|l|}{ Unmet load } & \multicolumn{2}{|r|}{157} & $\mathrm{kWh} / \mathrm{yr}$ \\
\hline \multicolumn{2}{|l|}{ Capacity shortage } & \multicolumn{2}{|r|}{2506} & $\mathrm{kWh} / \mathrm{yr}$ \\
\hline \multicolumn{2}{|l|}{ Renewable fraction } & \multicolumn{2}{|r|}{1} & \\
\hline Component & \multicolumn{2}{|l|}{ Production(kWh/yr) } & \multicolumn{2}{|c|}{ Fraction (\%) } \\
\hline PV & & $3,250,128$ & & 15 \\
\hline Generator & & $5,318,757$ & & 25 \\
\hline Wind Turbine & & $13,081,490$ & & 60 \\
\hline Total & & $21,650,376$ & & 100 \\
\hline Load & Consumption(kWh/yr) & & Frac & tion (\%) \\
\hline AC primary load & & $14,061,302$ & & 100 \\
\hline DC primary load & & 0 & & 0 \\
\hline
\end{tabular}




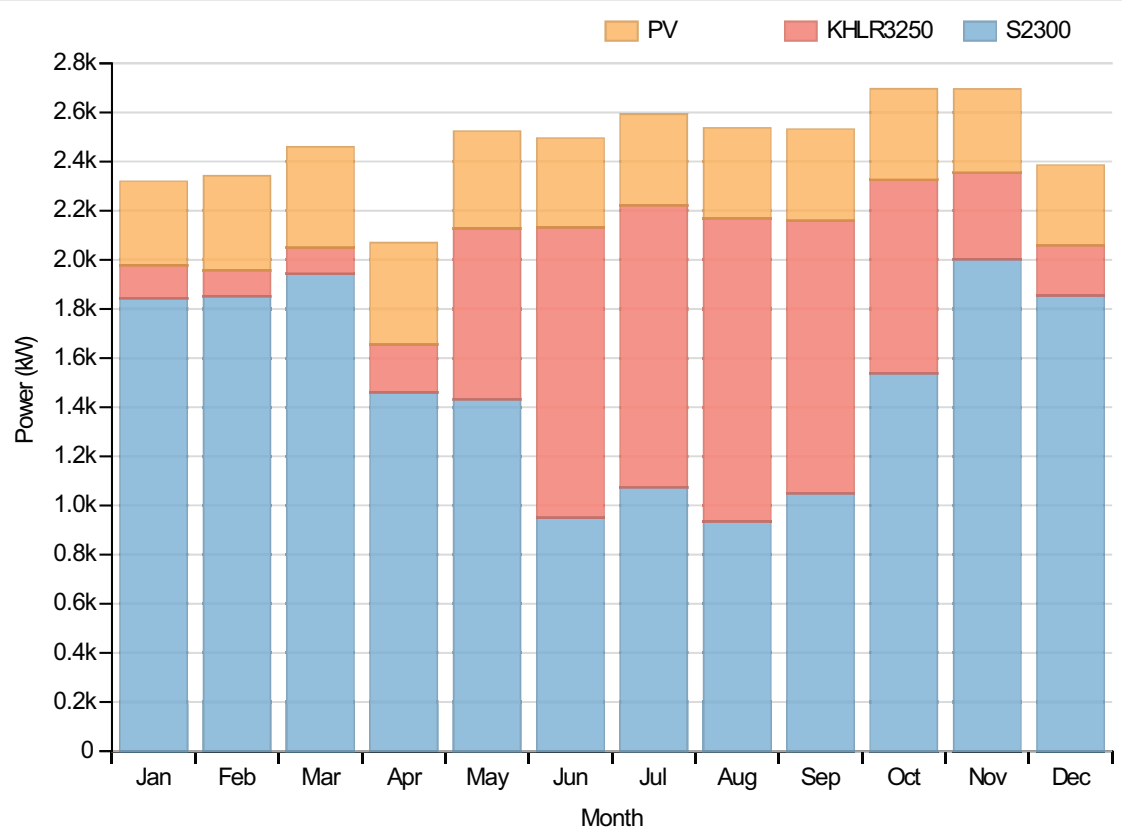

PV:Solar World 320W flat plate PV Copy

\begin{tabular}{|l|r|l|}
\hline Quantity & Value & Units \\
\hline Rated capacity & $2000 \mathrm{~kW}$ \\
\hline Mean output & $371 \mathrm{~kW}$ \\
\hline Mean output & $8904.50 \mathrm{kWh} / \mathrm{d}$ \\
\hline Capacity factor & $18.55 \%$ \\
\hline Total production & $3250128 \mathrm{kWh} / \mathrm{yr}$ \\
\hline Minimum output & $0.00 \mathrm{~kW}$ \\
\hline Maximum output & $1996.60 \mathrm{~kW}$ \\
\hline PV penetration & $23.11 \%$ \\
\hline Hours of operation & 4370 & $\mathrm{hrs} / \mathrm{yr}$ \\
\hline Levelized cost & 0.152 & $\$ / \mathrm{kWh}$ \\
\hline
\end{tabular}

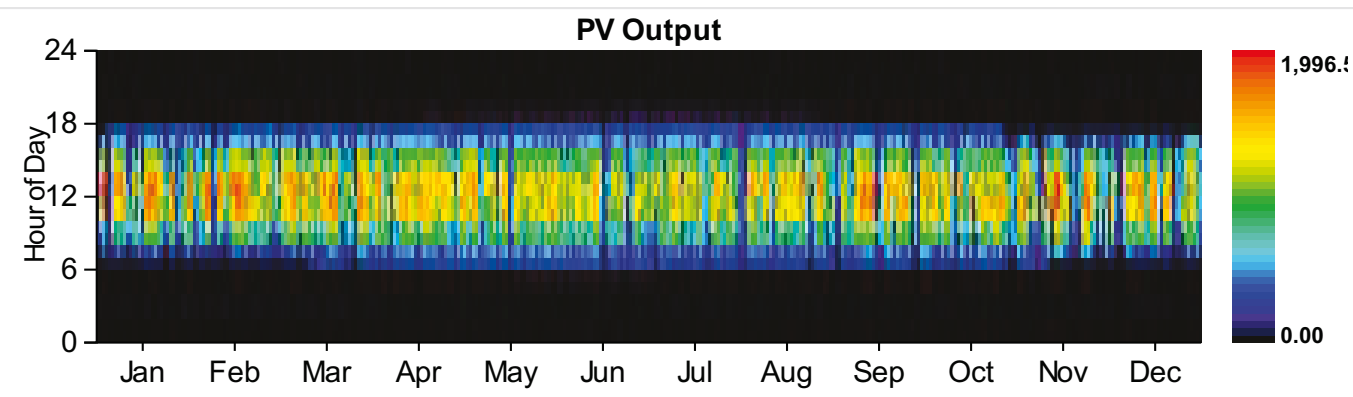

Wind Turbine:Siemens 2.3 MW - 108 


\begin{tabular}{|c|c|c|c|}
\hline Qrtahrefted capacity & Value & 4600 & UNits \\
\hline Mean output & & 1493 & $\mathrm{~kW}$ \\
\hline Capacity factor & & 32.46 & $\%$ \\
\hline Total production & & 13081490 & $\mathrm{kWh} / \mathrm{yr}$ \\
\hline Minimum output & & 3.12 & $\mathrm{~kW}$ \\
\hline Maximum output & & 4629.60 & kW \\
\hline Wind penetration & & 93.03 & $\%$ \\
\hline Hours of operation & & 8760 & $\mathrm{hrs} / \mathrm{yr}$ \\
\hline Levelized cost & & 0.072 & $\$ / k W h$ \\
\hline
\end{tabular}

\section{Generator:Kohler 3250 Prime Power}

\section{Quantity}

Hours of operation

Number of starts

Operational life

Fixed generation cost

Marginal generation cost

Electrical production

Mean electrical output

Min. electrical output

Max. electrical output

Fuel consumption

Specific fuel consumption

Fuel energy input

Mean electrical efficiency

\section{Value}

\section{Units}

$2777 \mathrm{hrs} / \mathrm{yr}$

532 starts/yr

$5 \mathrm{yr}$

$235.85 \$ / h r$

$0.20 \$ / \mathrm{kWh}$

$5318757 \mathrm{kWh} / \mathrm{yr}$

1915 kW

813 kW

$3250 \mathrm{~kW}$

1256194 L/yr

$0.24 \mathrm{~L} / \mathrm{kWh}$

$12360949 \mathrm{kWh} / \mathrm{yr}$

$43 \%$

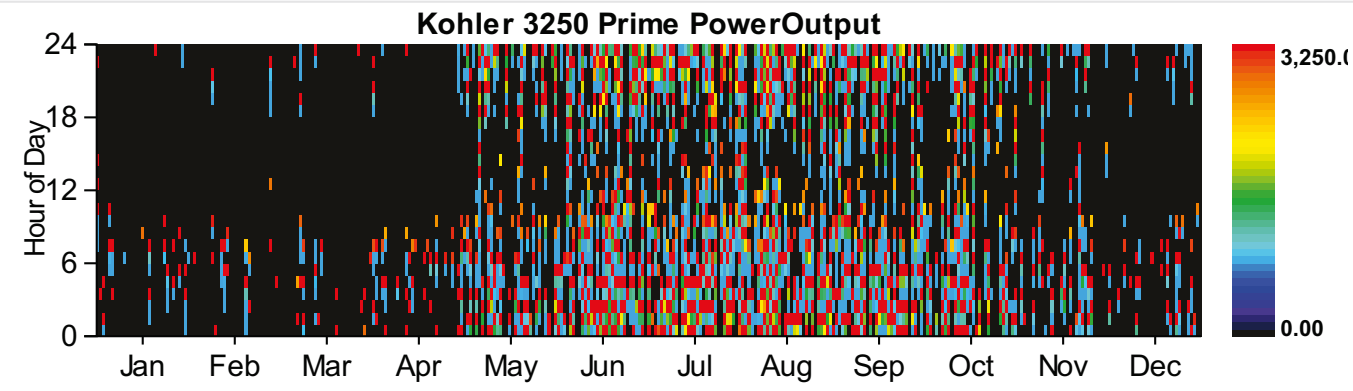


String size

Strings in parallel

Batteries

Bus voltage

\section{Quantity}

Value

Units

Nominal capacity

Usable nominal capacity

Autonomy

Lifetime throughput

Battery wear cost

Average energy cost

Energy in

Energy out

Storage depletion

Losses

Annual throughput

Expected life

$25 \mathrm{yr}$

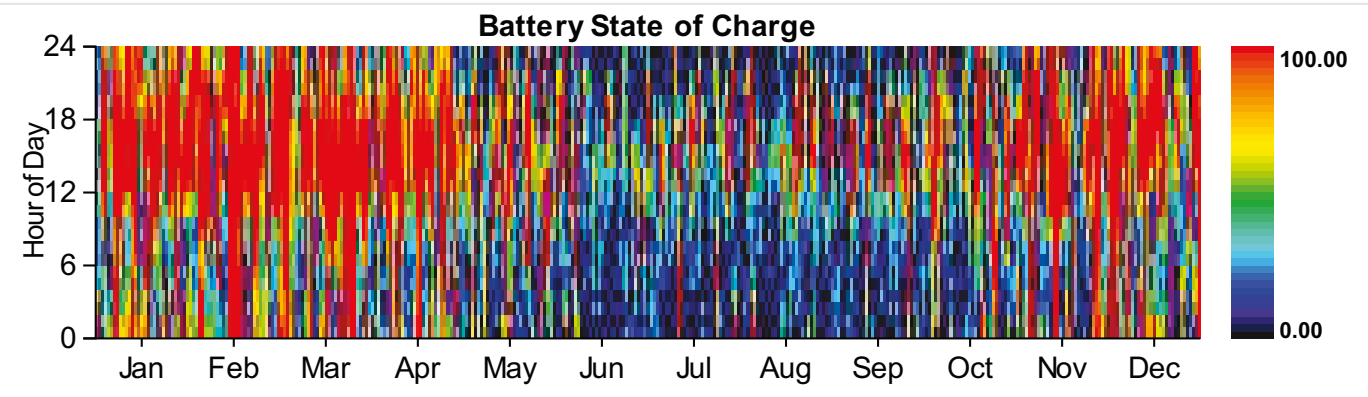

\section{Converter}

\section{Quantity}

Capacity

Mean output

Minimum output

Maximum output

Capacity factor
Inverter

Rectifier

Units

3,000

408

0

2,691

14

$2,700 \mathrm{~kW}$

330 kW

$0 \mathrm{~kW}$

2,689 kW

$11 \%$ 


\begin{tabular}{|c|c|c|c|}
\hline Ruanstifyoperation & Inverter & Rectifier & Hrsitss \\
\hline Energy in & $3,975,608$ & $3,401,441$ & $\mathrm{kWh} / \mathrm{yr}$ \\
\hline Energy out & $3,578,049$ & $2,891,229$ & $\mathrm{kWh} / \mathrm{yr}$ \\
\hline Losses & 397,560 & 510,212 & $\mathrm{kWh} / \mathrm{yr}$ \\
\hline
\end{tabular}
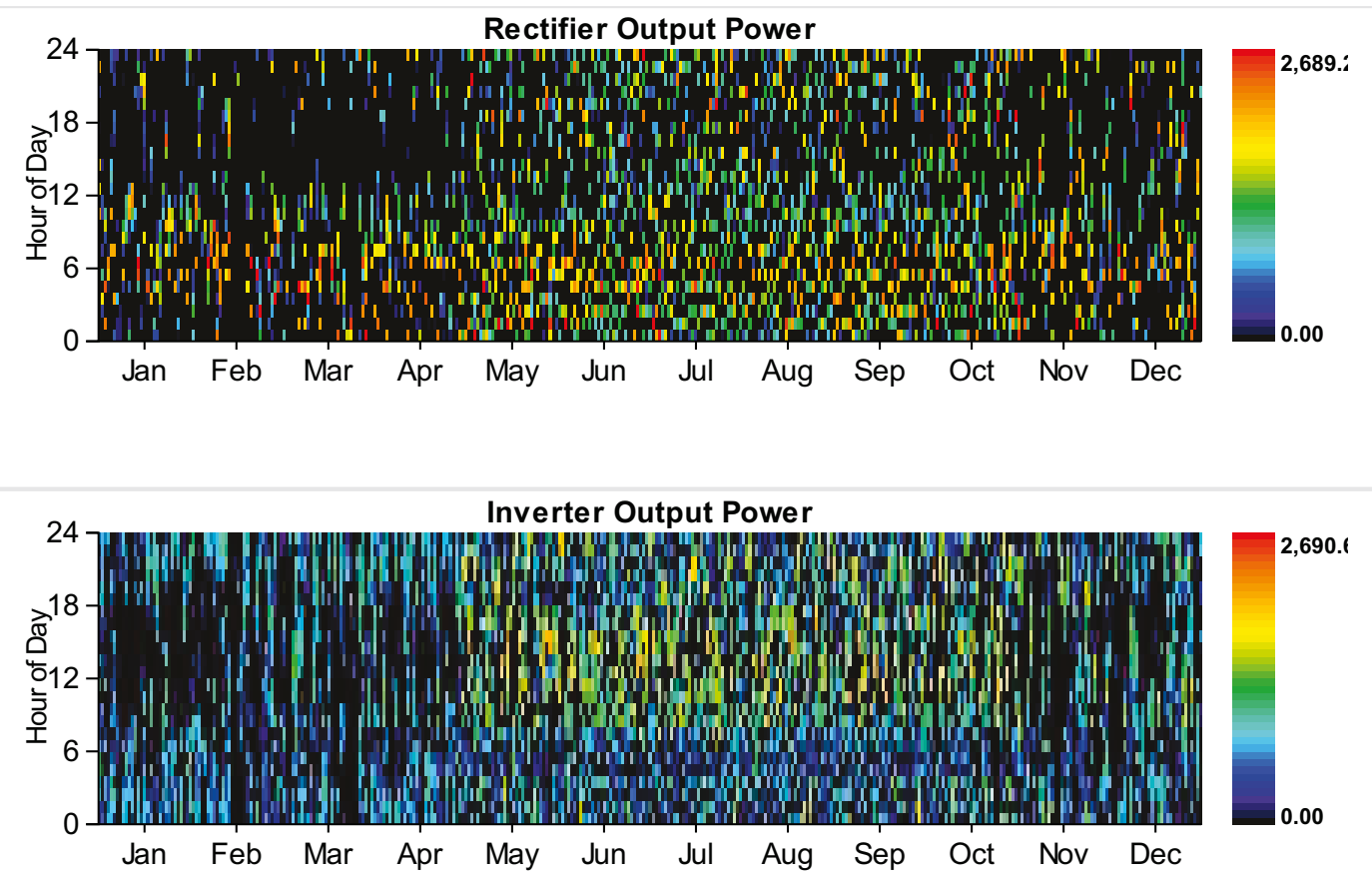

\section{Emissions}

\begin{tabular}{|l|r|l|}
\hline Pollutant & Emissions & Units \\
\hline Carbon dioxide & 3296900 & $\mathrm{~kg} / \mathrm{yr}$ \\
\hline Carbon monoxide & 13818 & $\mathrm{~kg} / \mathrm{yr}$ \\
\hline Unburned hydrocarbons & 1583 & $\mathrm{~kg} / \mathrm{yr}$ \\
\hline Particulate matter & 396 & $\mathrm{~kg} / \mathrm{yr}$ \\
\hline Sulfur dioxide & 6788 & $\mathrm{~kg} / \mathrm{yr}$ \\
\hline Nitrogen oxides & 13818 \\
\hline
\end{tabular}


System Report

System architecture

\begin{tabular}{|l|l|r|}
\hline PV & Solar World 320W flat plate PV Copy & 4,000 \\
\hline Generator & Kohler 3250 Prime Power & 3,250 \\
\hline Battery & GS200 flow & 10 \\
\hline Converter & System Converter & 3,000 \\
\hline Dispatch Strategy & Cycle Charging & $\mathrm{kW}$ \\
\hline
\end{tabular}

\section{Cost summary}

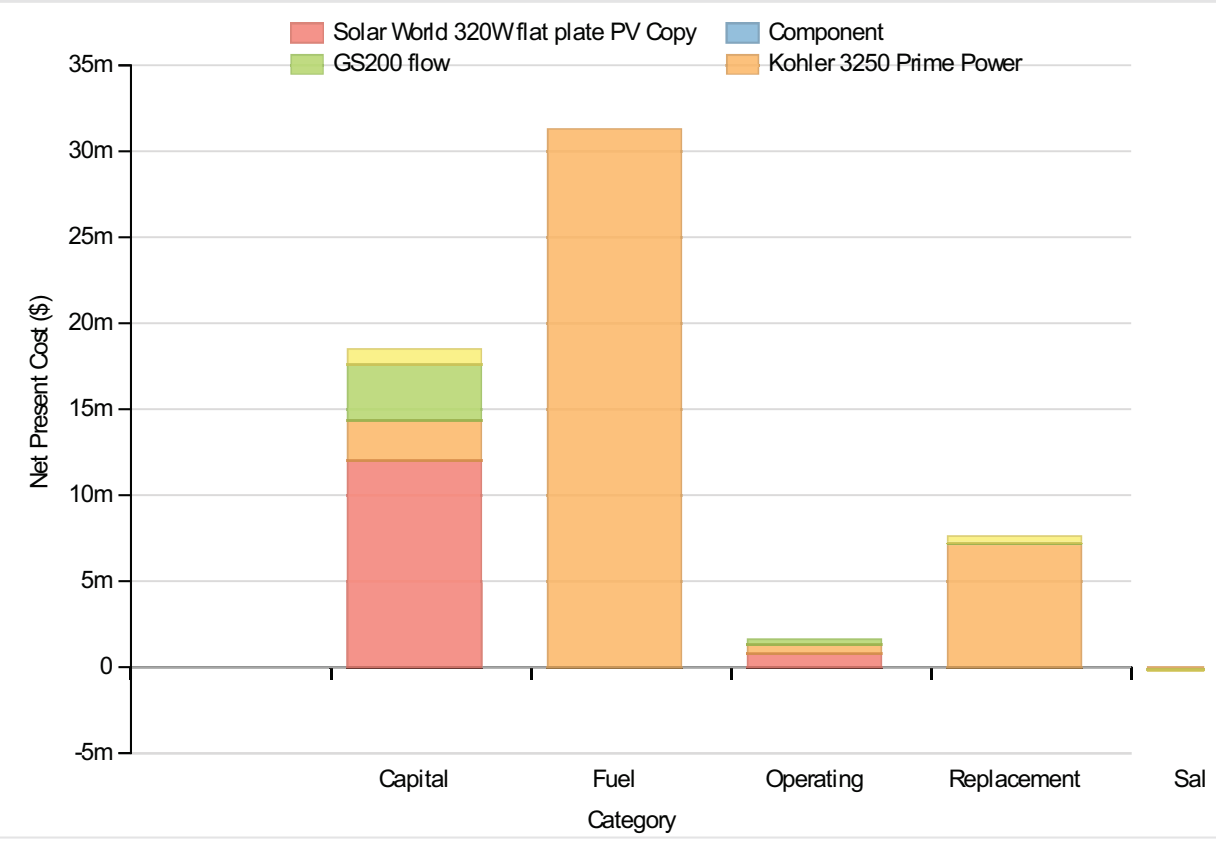

Cost Summary

Total net present cost

$58783660 \$$

Levelized cost of energy

$0.323 \$ / k W h$

Net Present Costs

\begin{tabular}{|l|r|r|r|r|r|r|}
\hline Component & Capital & Replacement & O\&M & Fuel & Salvage & Total \\
\hline Solar World 320W flat plate PV Copy & $12,000,000$ & 0 & 775,651 & 0 & $12,775,651$ \\
\hline Kohler 3250 Prime Power & $2,346,591$ & $7,159,266$ & 529,525 & $31,284,102$ & $-139,599$ & $41,179,885$ \\
\hline GS200 flow & $3,245,892$ & 71,683 & 310,260 & 0 & $-9,719$ & $3,618,116$ \\
\hline Converter & 900,000 & 381,846 & 0 & 0 & $-71,867$ & $1,209,979$ \\
\hline System & $18,492,482$ & $7,612,796$ & $1,615,436$ & $31,284,102$ & $-221,185$ & $58,783,631$ \\
\hline
\end{tabular}

\section{Annualized Costs}

\begin{tabular}{|l|r|r|r|r|r|r|}
\hline Component & Capital & Replacement & O\&M & Fuel & Salvage & Total \\
\hline Solar World 320W flat plate PV Copy & 928,253 & 0 & 60,000 & 0 & 0 & 988,253 \\
\hline
\end{tabular}




\begin{tabular}{|l|r|r|r|r|r|r|}
\hline GS200 flow & 251,084 & 5,545 & 24,000 & 0 & -752 & 279,877 \\
\hline Converter & 69,619 & 29,537 & 0 & 0 & $-5,559$ \\
\hline System & $1,430,475$ & 588,883 & 124,961 & $2,419,962$ & $-17,110$ & $4,547,171$ \\
\hline
\end{tabular}

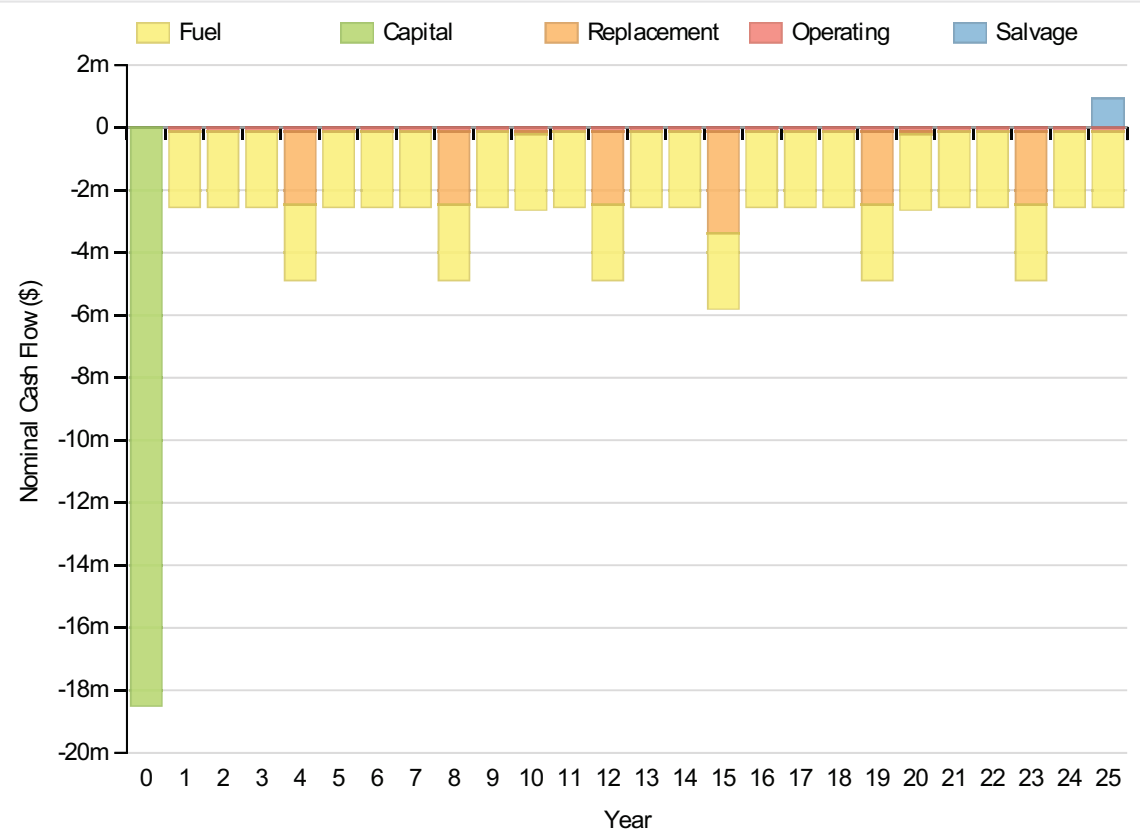

\section{Electrical}

\begin{tabular}{|c|c|c|c|c|c|}
\hline \multicolumn{2}{|l|}{ Quantity } & \multicolumn{3}{|l|}{ Value } & Units \\
\hline \multicolumn{2}{|l|}{ Excess electricity } & \multicolumn{3}{|c|}{604374} & $\mathrm{kWh} / \mathrm{yr}$ \\
\hline \multicolumn{2}{|l|}{ Unmet load } & \multicolumn{4}{|c|}{$0 \mathrm{kWh} / \mathrm{yr}$} \\
\hline \multicolumn{2}{|l|}{ Capacity shortage } & \multicolumn{4}{|c|}{$0 \mathrm{kWh} / \mathrm{yr}$} \\
\hline \multicolumn{2}{|l|}{ Renewable fraction } & \multicolumn{4}{|c|}{0} \\
\hline Component & \multicolumn{2}{|l|}{ Production(kWh/yr) } & \multicolumn{3}{|c|}{ Fraction (\%) } \\
\hline PV & \multicolumn{2}{|r|}{$6,500,257$} & \multicolumn{3}{|r|}{38} \\
\hline Generator & \multicolumn{2}{|r|}{$10,696,306$} & \multicolumn{3}{|r|}{62} \\
\hline Total & \multicolumn{2}{|r|}{$17,196,562$} & \multicolumn{3}{|r|}{100} \\
\hline Load & \multicolumn{2}{|l|}{ Consumption(kWh/yr) } & \multicolumn{3}{|c|}{ Fraction (\%) } \\
\hline AC primary load & \multicolumn{3}{|c|}{$14,061,459$} & \multicolumn{2}{|r|}{100} \\
\hline DC primary load & \multicolumn{3}{|r|}{0} & \multicolumn{2}{|c|}{0} \\
\hline Total & \multicolumn{3}{|c|}{$14,061,459$} & \multicolumn{2}{|r|}{100} \\
\hline
\end{tabular}




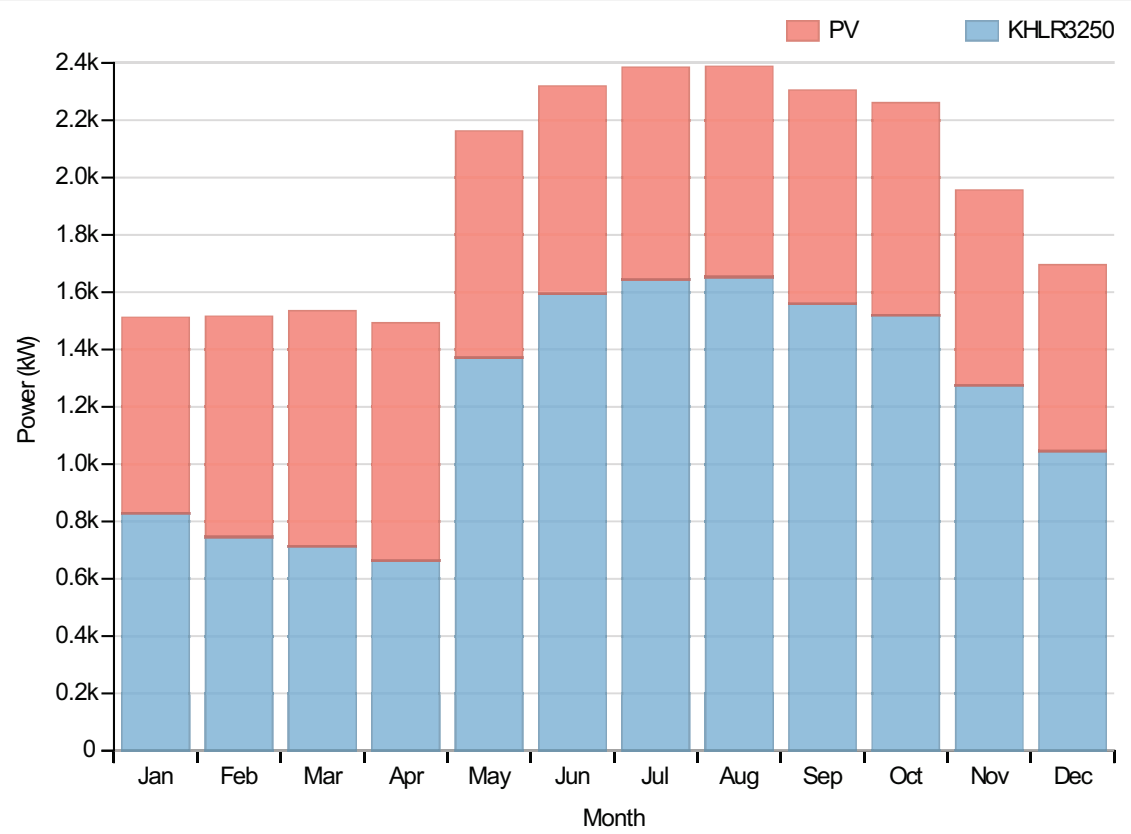

PV:Solar World 320W flat plate PV Copy

\begin{tabular}{|l|r|l|}
\hline Quantity & Value & Units \\
\hline Rated capacity & $4000 \mathrm{~kW}$ \\
\hline Mean output & $742 \mathrm{~kW}$ \\
\hline Mean output & $17809.00 \mathrm{kWh} / \mathrm{d}$ \\
\hline Capacity factor & $18.55 \%$ \\
\hline Total production & $6500257 \mathrm{kWh} / \mathrm{yr}$ \\
\hline Minimum output & $0.00 \mathrm{~kW}$ \\
\hline Maximum output & $3993.10 \mathrm{~kW}$ \\
\hline PV penetration & $46.23 \%$ \\
\hline Hours of operation & $4370 \mathrm{hrs} / \mathrm{yr}$ \\
\hline Levelized cost & $0.152 \mathrm{~S} / \mathrm{kWh}$ \\
\hline
\end{tabular}

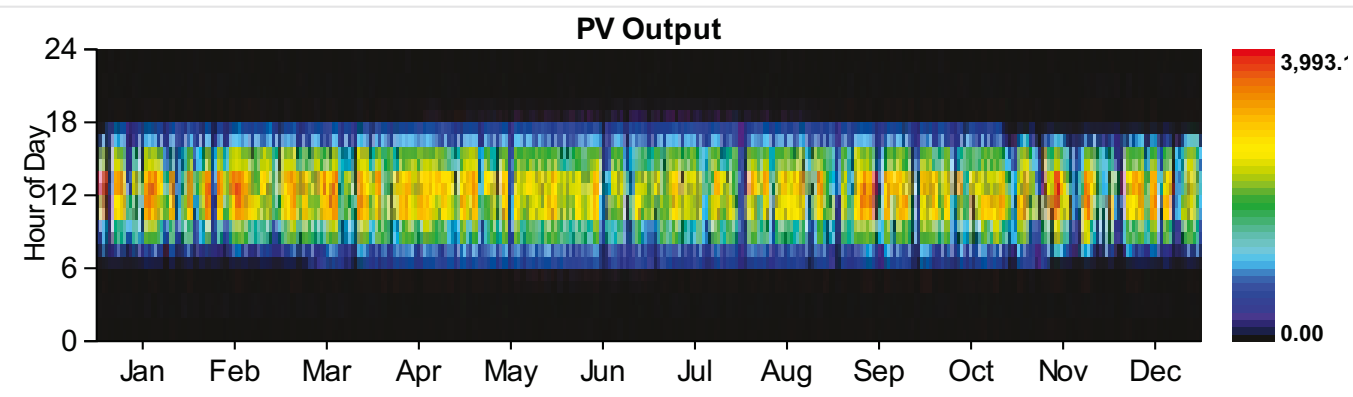

Generator:Kohler 3250 Prime Power

\section{Quantity}

Hours of operation

Number of starts

\section{Value}

Units

$4051 \mathrm{hrs} / \mathrm{yr}$

1758 starts/yr 


\begin{tabular}{|c|c|c|}
\hline Quartatipyal life & Value & Vnits \\
\hline Fixed generation cost & 235.85 & $\$ / h r$ \\
\hline Marginal generation cost & 0.20 & $\$ / \mathrm{kWh}$ \\
\hline Electrical production & 10696306 & $\mathrm{kWh} / \mathrm{yr}$ \\
\hline Mean electrical output & 2640 & kW \\
\hline Min. electrical output & 813 & kW \\
\hline Max. electrical output & 3250 & kW \\
\hline Fuel consumption & 2419964 & L/yr \\
\hline Specific fuel consumption & 0.23 & $\mathrm{~L} / \mathrm{kWh}$ \\
\hline Fuel energy input & 23812448 & $\mathrm{kWh} / \mathrm{yr}$ \\
\hline Mean electrical efficiency & 45 & $\%$ \\
\hline
\end{tabular}

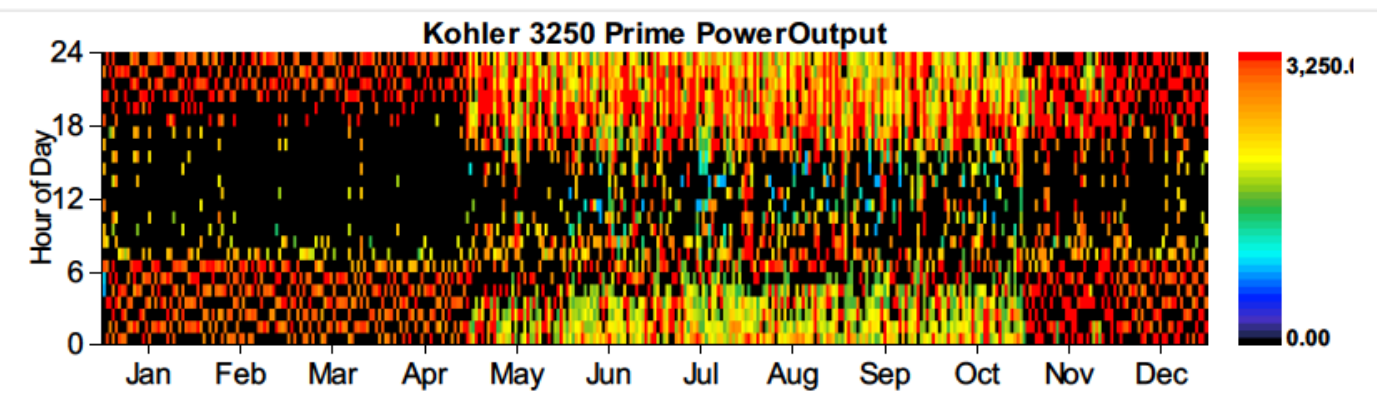

Battery:GS200 flow

\begin{tabular}{|l|r|}
\hline Quantity & Value \\
\hline String size & \\
\hline Strings in parallel & 1 \\
\hline Batteries & 10 \\
\hline Bus voltage & 10 \\
\hline
\end{tabular}

\begin{tabular}{|l|r|l|}
\hline Quantity & Value & Units \\
\hline Nominal capacity & $6000 \mathrm{kWh}$ \\
\hline Usable nominal capacity & $6000 \mathrm{kWh}$ \\
\hline Autonomy & $4 \mathrm{hr}$ \\
\hline Lifetime throughput & 0 & \\
\hline Battery wear cost & 0.000 & $\$ / \mathrm{kWh}$ \\
\hline Average energy cost & 0.241 \$/kWh \\
\hline Energy in & $4366512 \mathrm{kWh} / \mathrm{yr}$ \\
\hline
\end{tabular}




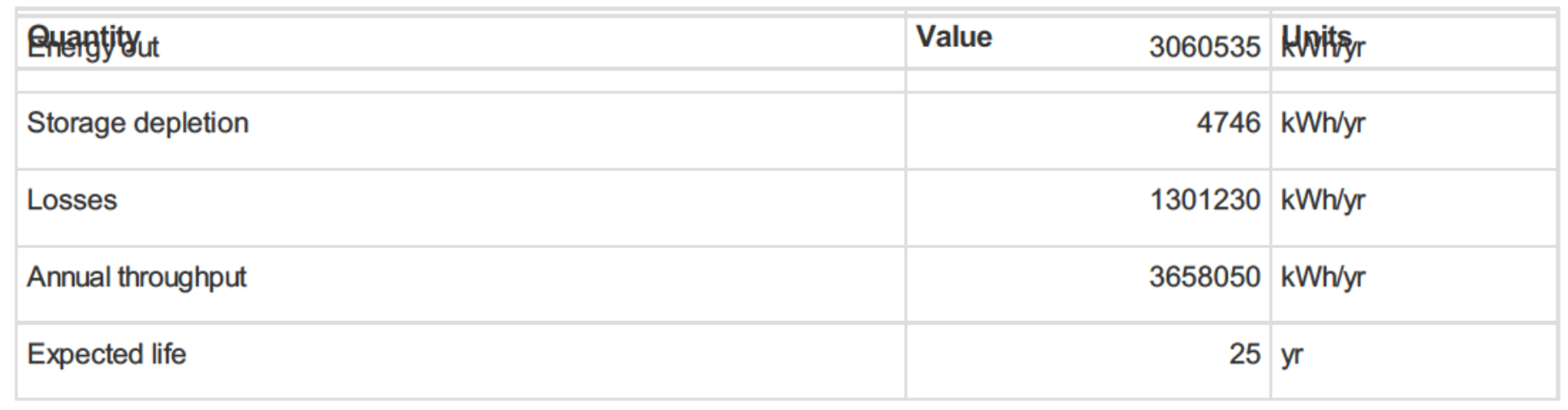

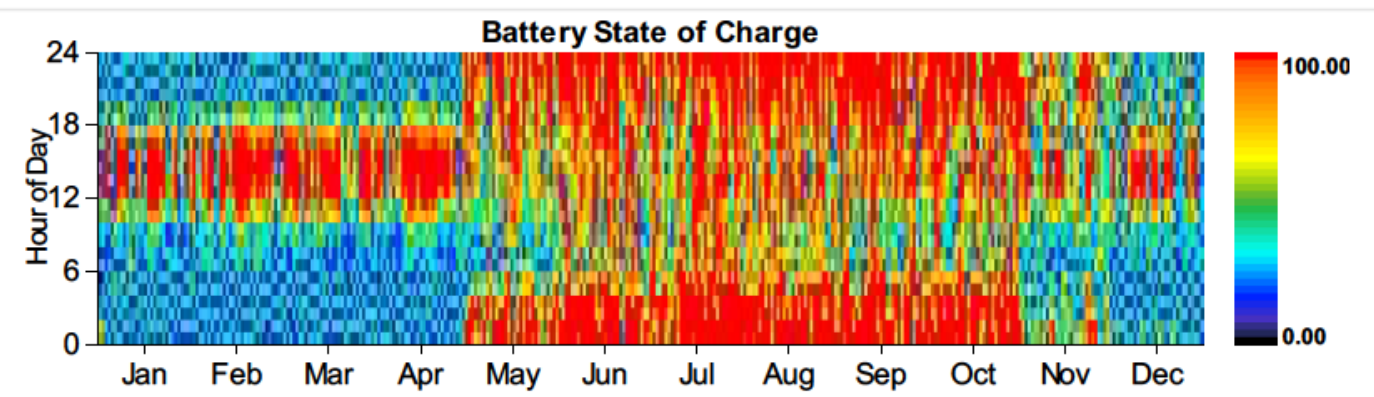

Converter

\begin{tabular}{|l|r|r|l|}
\hline Quantity & Inverter & Rectifier & Units \\
\hline Capacity & 3,000 & 756 & 316 \\
\hline Mean output & 0 & $\mathrm{~kW}$ \\
\hline Minimum output & 2,754 & $0 \mathrm{~kW}$ \\
\hline Maximum output & 25 & 1,793 & $\mathrm{~kW}$ \\
\hline Capacity factor & 5,168 & $11 \%$ \\
\hline Hours of operation & $7,359,752$ & 2,482 & $\mathrm{hrs} / \mathrm{yr}$ \\
\hline Energy in & $6,623,762$ & $3,258,656$ & $\mathrm{kWh} / \mathrm{yr}$ \\
\hline Energy out & 735,990 & $2,769,838$ & $\mathrm{kWh} / \mathrm{yr}$ \\
\hline Losses & & 488,819 & $\mathrm{kWh} / \mathrm{yr}$ \\
\hline
\end{tabular}

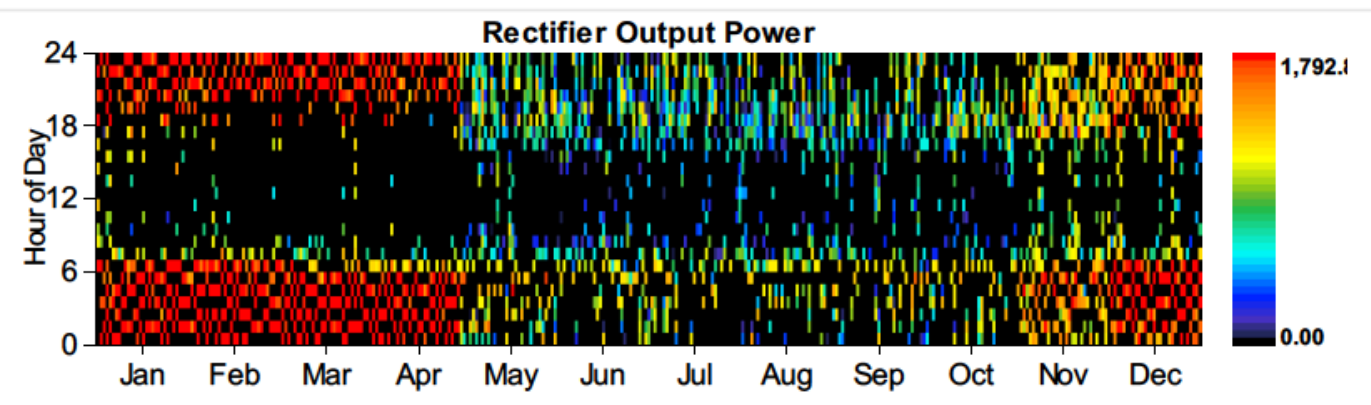




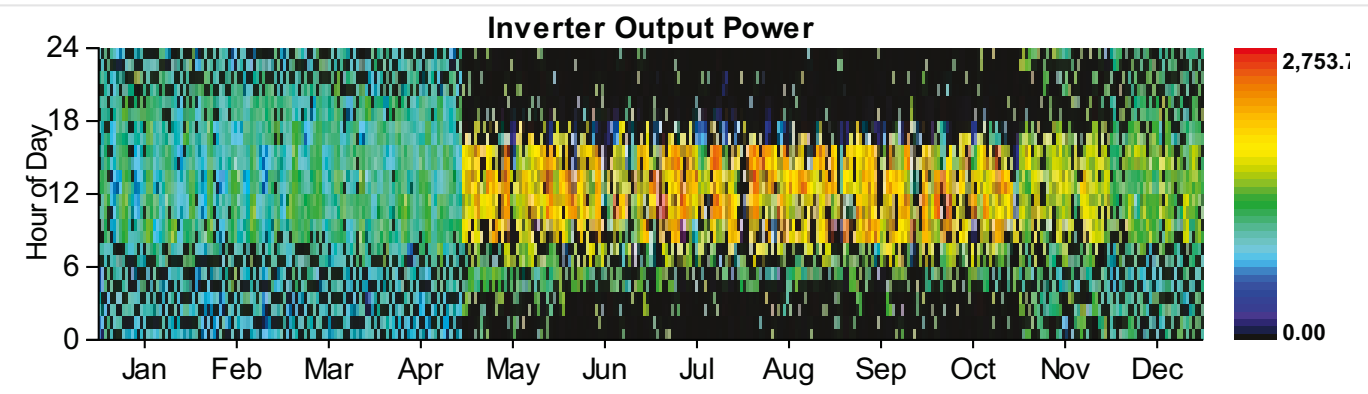

\section{Emissions}

\begin{tabular}{|l|r|l|}
\hline Pollutant & Emissions & Units \\
\hline Carbon dioxide & $6351233 \mathrm{~kg} / \mathrm{yr}$ \\
\hline Carbon monoxide & $26620 \mathrm{~kg} / \mathrm{yr}$ \\
\hline Unburned hydrocarbons & $3049 \mathrm{~kg} / \mathrm{yr}$ \\
\hline Particulate matter & $762 \mathrm{~kg} / \mathrm{yr}$ \\
\hline Sulfur dioxide & $13077 \mathrm{~kg} / \mathrm{yr}$ \\
\hline Nitrogen oxides & $26620 \mathrm{~kg} / \mathrm{yr}$ \\
\hline
\end{tabular}

HOMER Energy, LLC @ 2016 
System Report

System architecture

\begin{tabular}{|l|l|r|}
\hline PV & Solar World 320W flat plate PV Copy & 2,000 \\
\hline Wind Turbine & Siemens 2.3 MW - 108 & 2 \\
\hline Generator & Kohler 3250 Prime Power & 3,250 \\
\hline Converter & System Converter & 1,000 \\
\hline Dispatch Strategy & Cycle Charging & $\mathrm{kW}$ \\
\hline
\end{tabular}

\section{Cost summary}

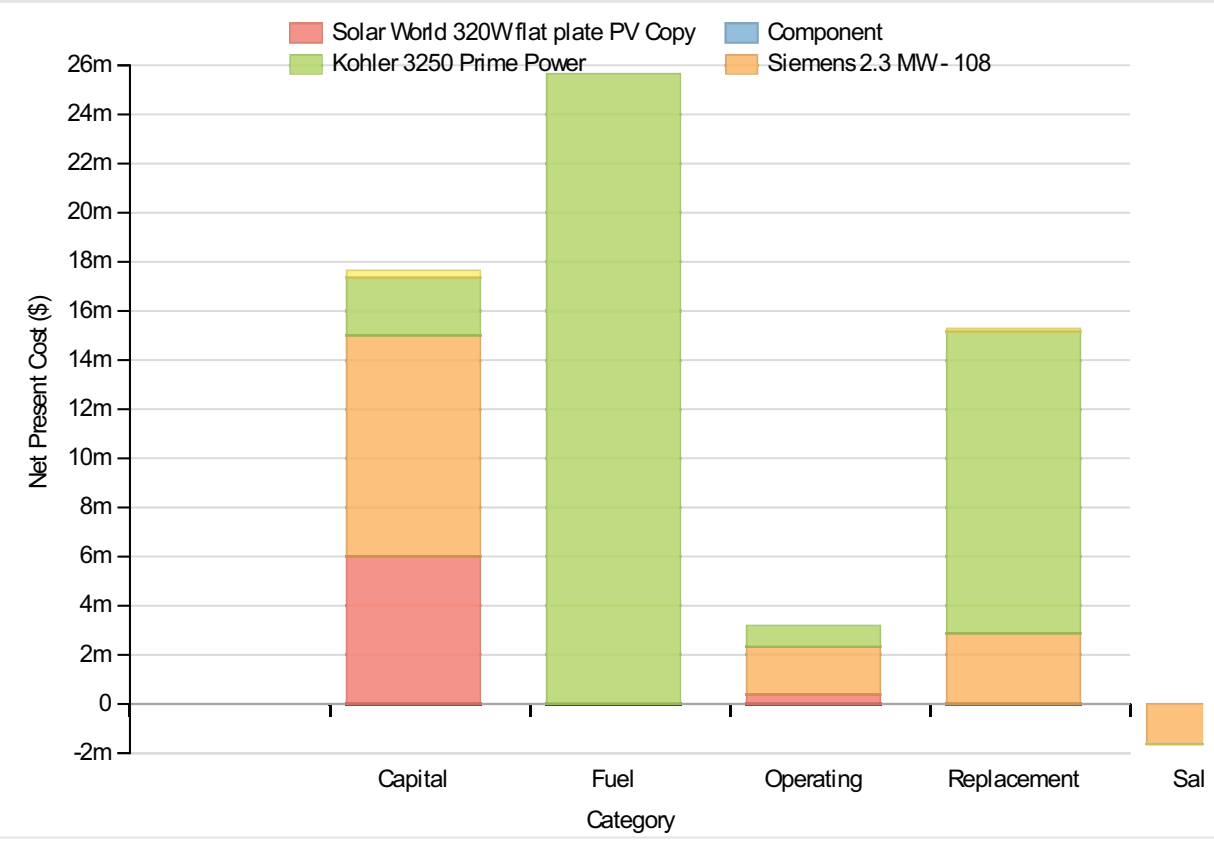

Cost Summary

Total net present cost

$60113544 \$$

Levelized cost of energy

$0.331 \$ / k W h$

Net Present Costs

\begin{tabular}{|c|c|c|c|c|c|c|}
\hline Component & Capital & Replacement & O\&M & Fuel & Salvage & Total \\
\hline Solar World 320W flat plate PV Copy & $6,000,000$ & 0 & 387,825 & 0 & 0 & $6,387,825$ \\
\hline Siemens 2.3 MW - 108 & $9,000,000$ & $2,869,263$ & $1,939,126$ & 0 & $-1,617,013$ & $12,191,376$ \\
\hline Kohler 3250 Prime Power & $2,346,591$ & $12,285,225$ & 860,757 & $25,652,476$ & $-14,054$ & $41,130,995$ \\
\hline Converter & 300,000 & 127,282 & 0 & 0 & $-23,956$ & 403,326 \\
\hline System & $17,646,590$ & $15,281,769$ & $3,187,708$ & $25,652,476$ & $-1,655,023$ & $60,113,520$ \\
\hline
\end{tabular}

\section{Annualized Costs}

\section{Component}

Solar World 320W flat plate PV Copy

\begin{tabular}{|r|r|r|r|r|r|}
\hline Capital & Replacement & O\&M & Fuel & Salvage & Total \\
\hline 464,126 & 0 & 30,000 & 0 & 0 & 494,126 \\
\hline
\end{tabular}


Kohler 3250 Prime Power

Converter

System

\begin{tabular}{|r|r|r|r|r|r|}
\hline 181,519 & 950,316 & 66,583 & $1,984,331$ & $-1,087$ & $3,181,662$ \\
\hline 23,206 & 9,846 & 0 & 0 & $-1,853$ & 31,199 \\
\hline $1,365,041$ & $1,182,112$ & 246,583 & $1,984,331$ & $-128,023$ & $4,650,044$ \\
\hline
\end{tabular}

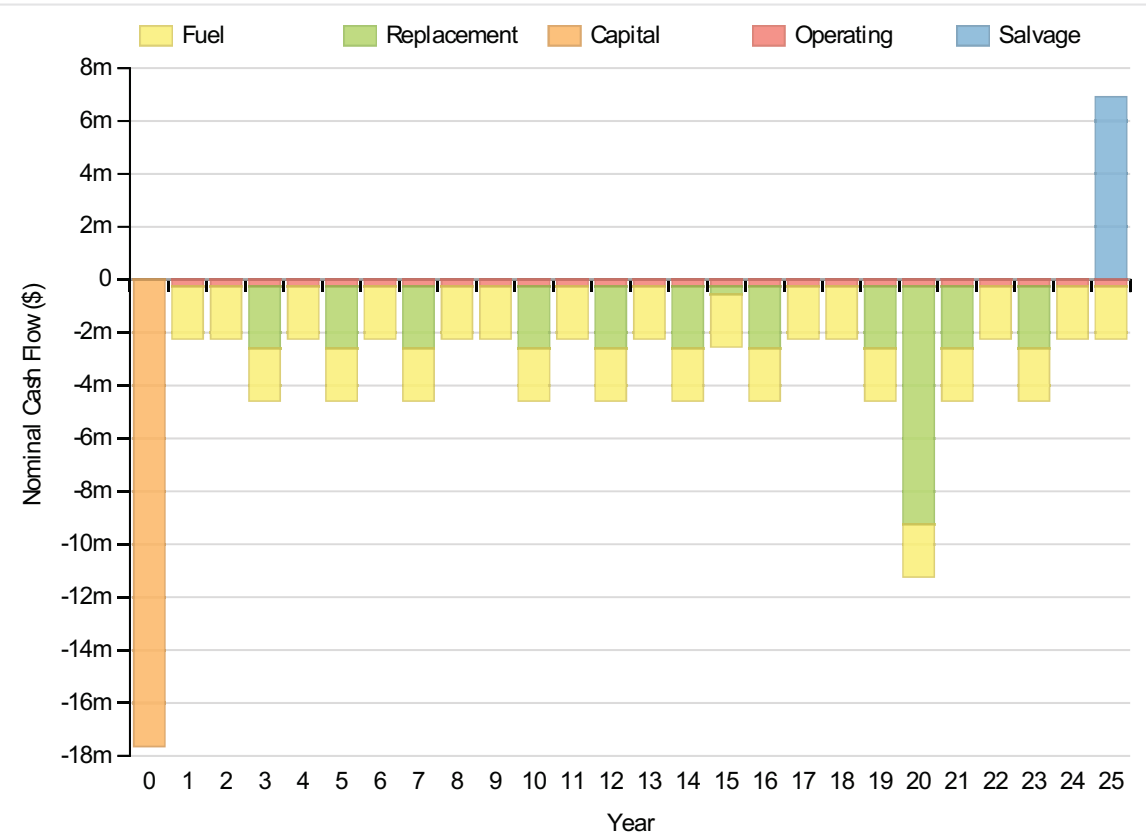

Electrical

\section{Quantity}

Excess electricity

Unmet load

Capacity shortage

Renewable fraction

\section{Value}

Units

$9828963 \mathrm{kWh} / \mathrm{yr}$

$716 \mathrm{kWh} / \mathrm{yr}$

$6247 \mathrm{kWh} / \mathrm{yr}$

0

\section{Component}

PV

\section{Generator}

Wind Turbine

Total

Load

AC primary load

DC primary load

Total
Production(kWh/yr)

$3,250,128$

$7,640,236$

$13,081,490$

$23,971,854$

Consumption(kWh/yr)

$14,060,743$

100

0

0

$14,060,743$ 


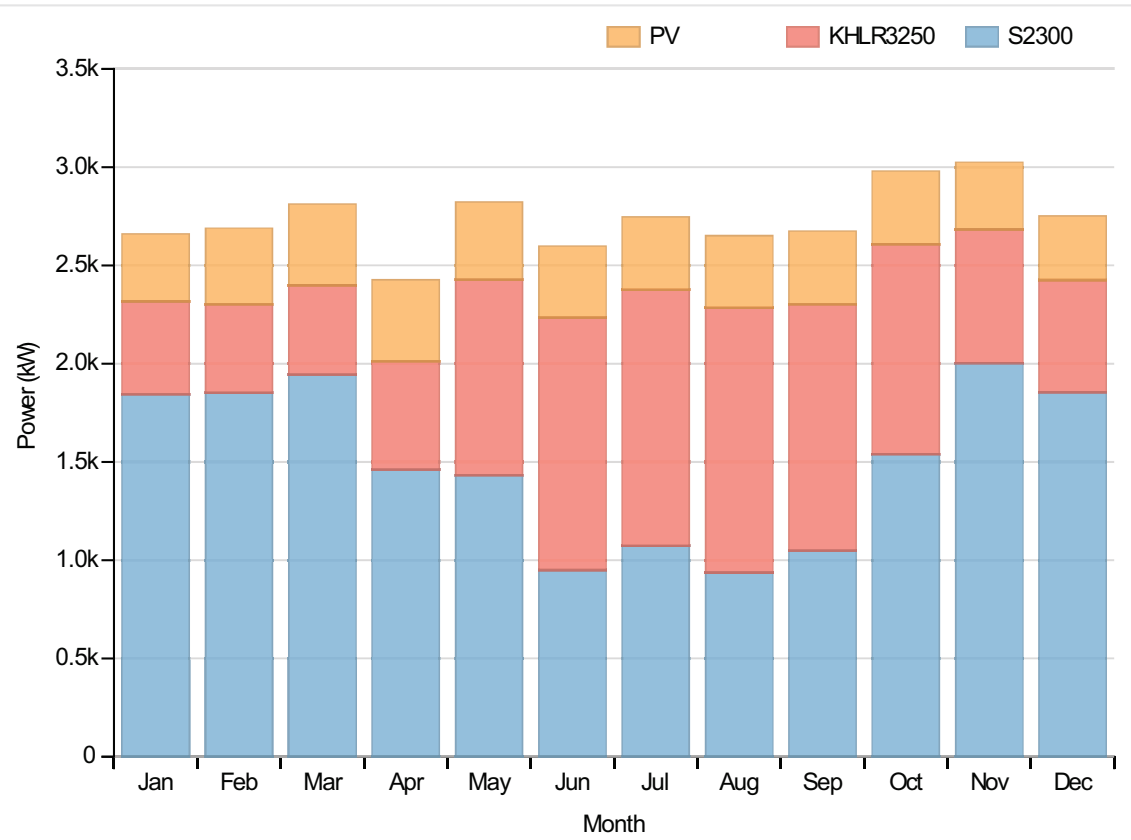

PV:Solar World 320W flat plate PV Copy

\begin{tabular}{|l|r|l|}
\hline Quantity & Value & Units \\
\hline Rated capacity & $2000 \mathrm{~kW}$ \\
\hline Mean output & $371 \mathrm{~kW}$ \\
\hline Mean output & $8904.50 \mathrm{kWh} / \mathrm{d}$ \\
\hline Capacity factor & $18.55 \%$ \\
\hline Total production & $3250128 \mathrm{kWh} / \mathrm{yr}$ \\
\hline Minimum output & $0.00 \mathrm{~kW}$ \\
\hline Maximum output & $1996.60 \mathrm{~kW}$ \\
\hline PV penetration & $23.11 \%$ \\
\hline Hours of operation & $4370 \mathrm{hrs} / \mathrm{yr}$ \\
\hline Levelized cost & $0.152 \mathrm{~S} / \mathrm{kWh}$ \\
\hline
\end{tabular}

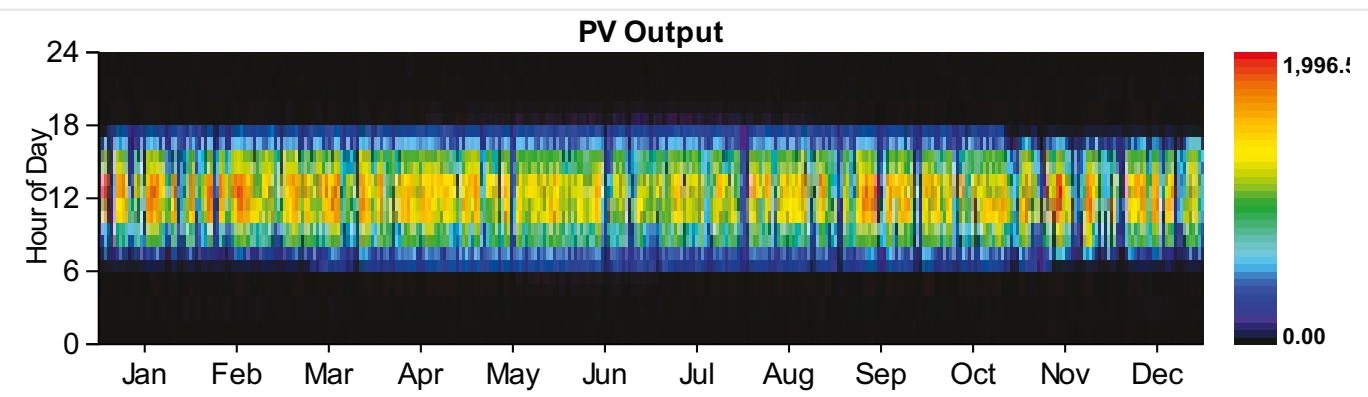

Wind Turbine:Siemens 2.3 MW - 108

\section{Quantity}

Total rated capacity

Mean output

\section{Value}

Units

$4600 \mathrm{~kW}$

1493 kW 


\begin{tabular}{|c|c|c|c|}
\hline Quparatity factor & Value & 32.46 & UPnits \\
\hline Total production & & 13081490 & $\mathrm{kWh} / \mathrm{yr}$ \\
\hline Minimum output & & 3.12 & kW \\
\hline Maximum output & & 4629.60 & kW \\
\hline Wind penetration & & 93.03 & $\%$ \\
\hline Hours of operation & & 8760 & $\mathrm{hrs} / \mathrm{yr}$ \\
\hline Levelized cost & & 0.072 & $\$ / k W h$ \\
\hline
\end{tabular}

\section{Generator:Kohler 3250 Prime Power}

\section{Quantity}

Hours of operation

Number of starts

Operational life

Fixed generation cost

Marginal generation cost

Electrical production

Mean electrical output

Min. electrical output

Max. electrical output

Fuel consumption

Specific fuel consumption

Fuel energy input

Mean electrical efficiency

\section{Value}

Units

$6585 \mathrm{hrs} / \mathrm{yr}$

550 starts/yr

$2 \mathrm{yr}$

$235.85 \$ / h r$

$0.20 \$ / k W h$

$7640236 \mathrm{kWh} / \mathrm{yr}$

1160 kW

813 kW

$3250 \mathrm{~kW}$

1984332 L/yr

$0.26 \mathrm{~L} / \mathrm{kWh}$

$19525830 \mathrm{kWh} / \mathrm{yr}$

$39 \%$

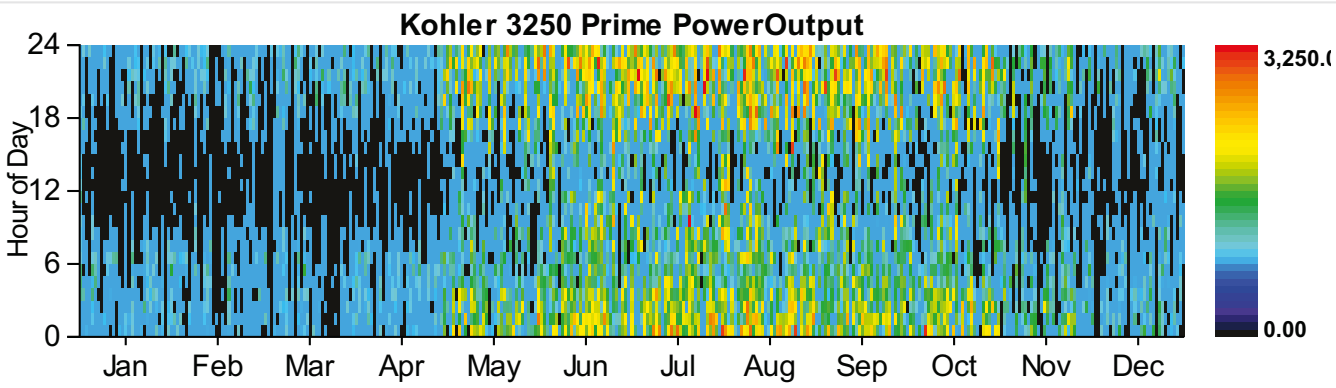

\section{Converter}

\section{Quantity}

Capacity

\section{Inverter}

Rectifier

Units 


\begin{tabular}{|c|c|c|c|}
\hline Qearanfityput & Inverter & Rectifier & UNits \\
\hline Minimum output & 0 & 0 & $\mathrm{~kW}$ \\
\hline Maximum output & 1,000 & 0 & $\mathrm{~kW}$ \\
\hline Capacity factor & 8 & 0 & $\%$ \\
\hline Hours of operation & 1,813 & 0 & $\mathrm{hrs} / \mathrm{yr}$ \\
\hline Energy in & 821,853 & 0 & $\mathrm{kWh} / \mathrm{yr}$ \\
\hline Energy out & 739,666 & 0 & $\mathrm{kWh} / \mathrm{yr}$ \\
\hline Losses & 82,186 & 0 & $\mathrm{kWh} / \mathrm{yl}$ \\
\hline
\end{tabular}
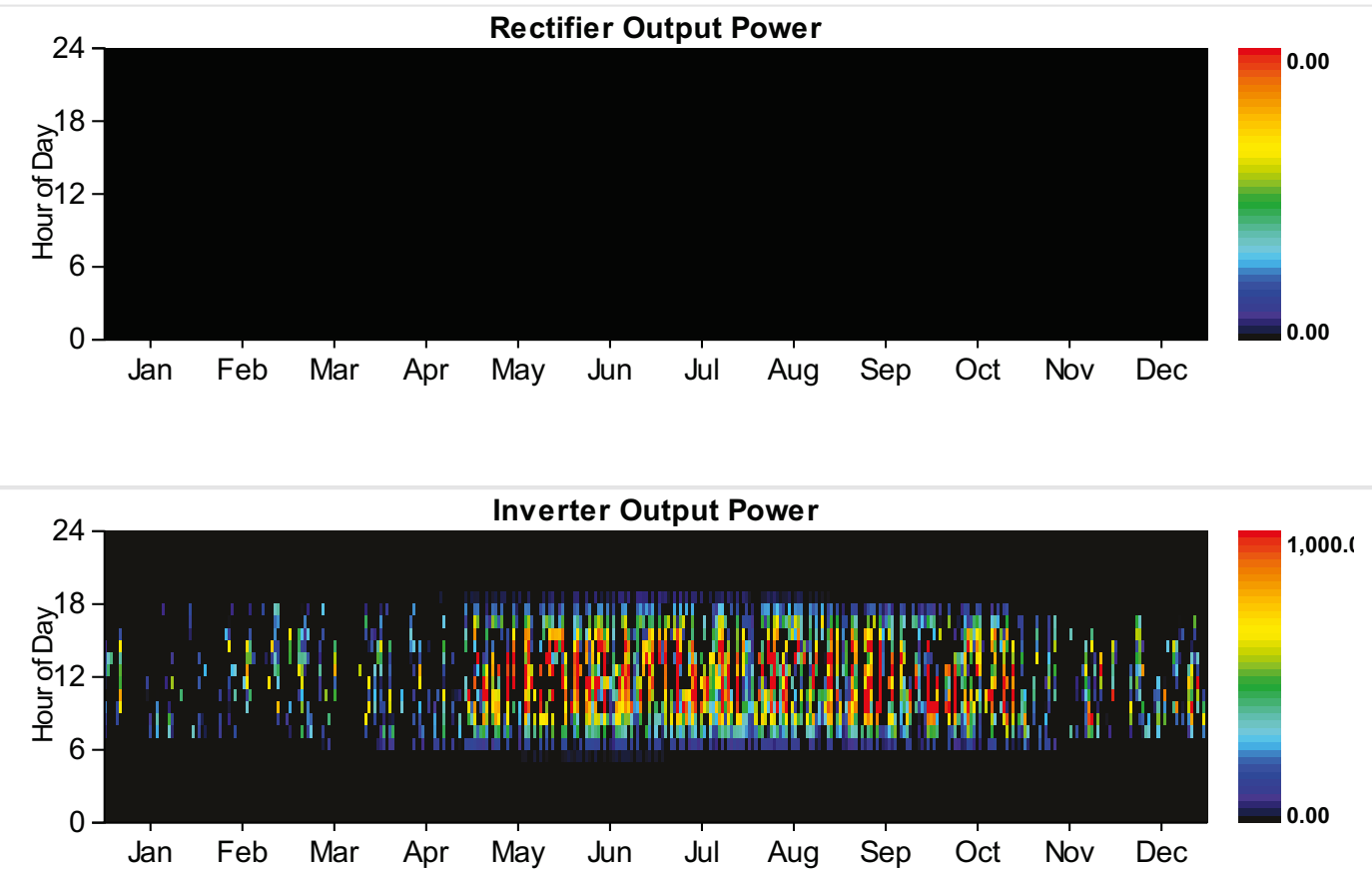

\section{Emissions}

\section{Pollutant}

Emissions

Units

Carbon dioxide

$5207910 \mathrm{~kg} / \mathrm{yr}$

Carbon monoxide

$21828 \mathrm{~kg} / \mathrm{yr}$

Unburned hydrocarbons

$2500 \mathrm{~kg} / \mathrm{yr}$

Particulate matter

$625 \mathrm{~kg} / \mathrm{yr}$

Sulfur dioxide

$10723 \mathrm{~kg} / \mathrm{yr}$

Nitrogen oxides

$21828 \mathrm{~kg} / \mathrm{yr}$

HOMER Energy, LLC @ 2016 
System Report

System architecture

\begin{tabular}{|l|l|r|}
\hline Generator & Kohler 3250 Prime Power & 3,250 \\
\hline Battery & GS200 flow & 6 \\
\hline Converter & System Converter & 1,000 \\
\hline Dispatch Strategy & Cycle Charging & $\mathrm{kW}$ \\
\hline
\end{tabular}

Cost summary

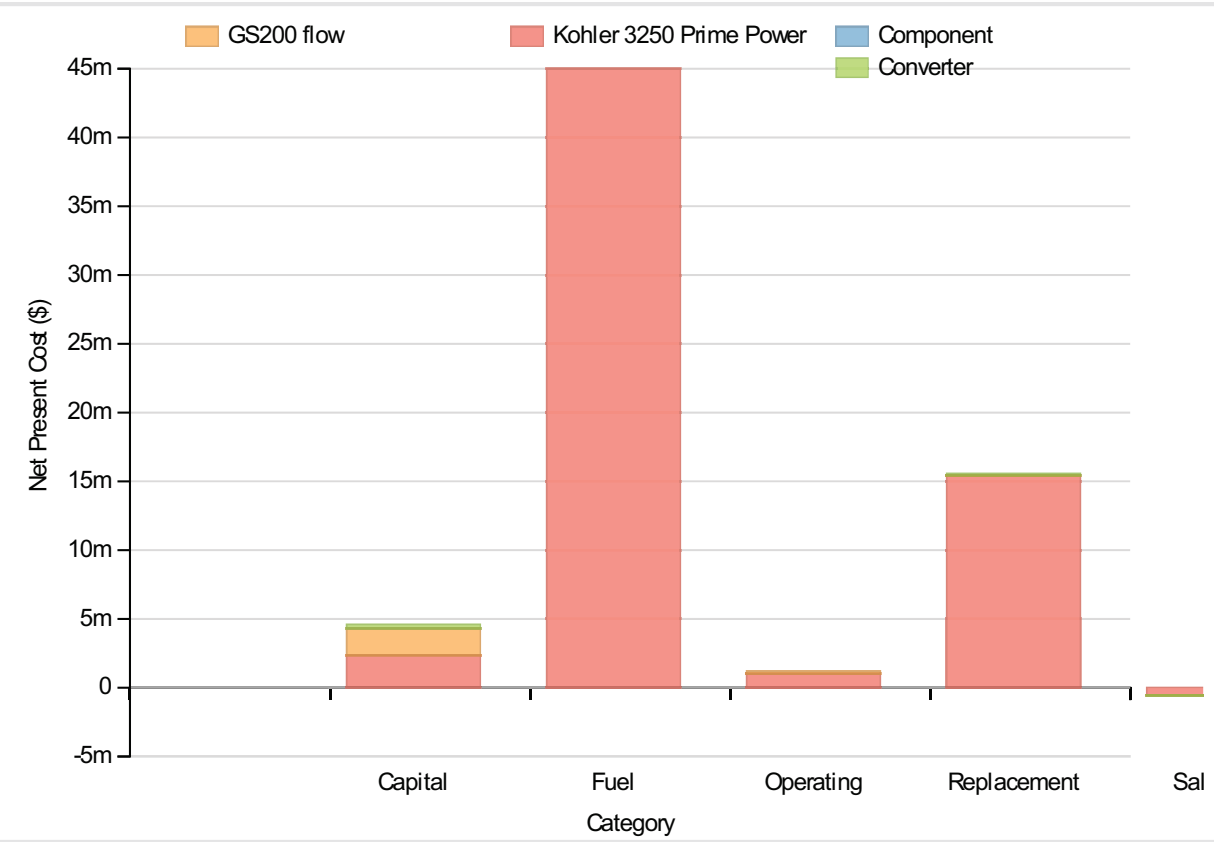

Cost Summary

Total net present cost

$65802344 \$$

Levelized cost of energy

$0.362 \$ / k W h$

\section{Net Present Costs}

\begin{tabular}{|l|r|r|r|r|r|r|}
\hline Component & Capital & Replacement & O\&M & Fuel & Salvage & Total \\
\hline Kohler 3250 Prime Power & $2,346,591$ & $15,398,611$ & $1,024,020$ & $44,989,192$ & $-530,288$ & $63,228,126$ \\
\hline GS200 flow & $1,947,535$ & 43,013 & 186,156 & 0 & $-5,832$ & $2,170,872$ \\
\hline Converter & 300,000 & 127,282 & 0 & 0 & $-23,956$ \\
\hline System & $4,594,126$ & $15,568,906$ & $1,210,175$ & $44,989,192$ & $-560,076$ & $65,802,323$ \\
\hline
\end{tabular}

\section{Annualized Costs}

\begin{tabular}{|l|r|r|r|r|r|r|}
\hline Component & Capital & Replacement & O\&M & Fuel & Salvage & Total \\
\hline Kohler 3250 Prime Power & 181,519 & $1,191,150$ & 79,212 & $3,480,111$ & $-41,020$ & $4,890,972$ \\
\hline GS200 flow & 150,650 & 3,327 & 14,400 & 0 & -451 \\
\hline Converter & 23,206 & 9,846 & 0 & 0 & $-1,853$ \\
\hline
\end{tabular}




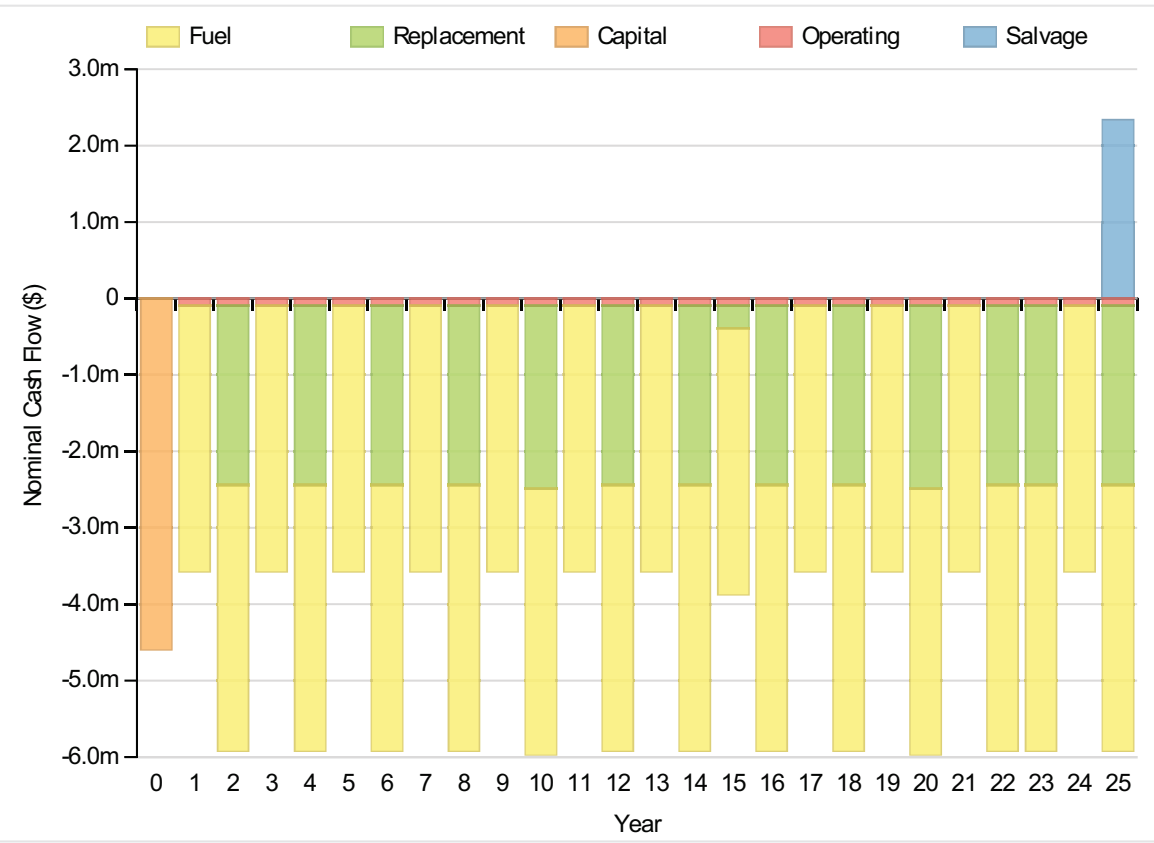

\section{Electrical}

\begin{tabular}{|c|c|c|c|c|c|}
\hline \multicolumn{2}{|l|}{ Quantity } & \multicolumn{2}{|l|}{ Value } & \multicolumn{2}{|c|}{ Units } \\
\hline \multicolumn{2}{|l|}{ Excess electricity } & \multicolumn{3}{|c|}{0} & $\mathrm{kWh} / \mathrm{yr}$ \\
\hline \multicolumn{2}{|l|}{ Unmet load } & \multicolumn{3}{|c|}{0} & $\mathrm{kWh} / \mathrm{yr}$ \\
\hline \multicolumn{2}{|l|}{ Capacity shortage } & \multicolumn{3}{|c|}{0} & $\mathrm{kWh} / \mathrm{yr}$ \\
\hline \multicolumn{2}{|l|}{ Renewable fraction } & \multicolumn{4}{|c|}{0} \\
\hline Component & \multicolumn{2}{|l|}{ Production(kWh/yr) } & \multicolumn{3}{|c|}{ Fraction $(\%)$} \\
\hline Generator & \multicolumn{4}{|c|}{$14,686,122$} & 100 \\
\hline Total & \multicolumn{4}{|c|}{$14,686,122$} & 100 \\
\hline Load & Consumption(kWh/yr) & & & \multicolumn{2}{|c|}{ Fraction (\%) } \\
\hline AC primary load & & \multicolumn{2}{|c|}{$14,061,459$} & & 100 \\
\hline DC primary load & & \multicolumn{3}{|c|}{0} & 0 \\
\hline Total & & \multicolumn{2}{|c|}{$14,061,459$} & & 100 \\
\hline
\end{tabular}




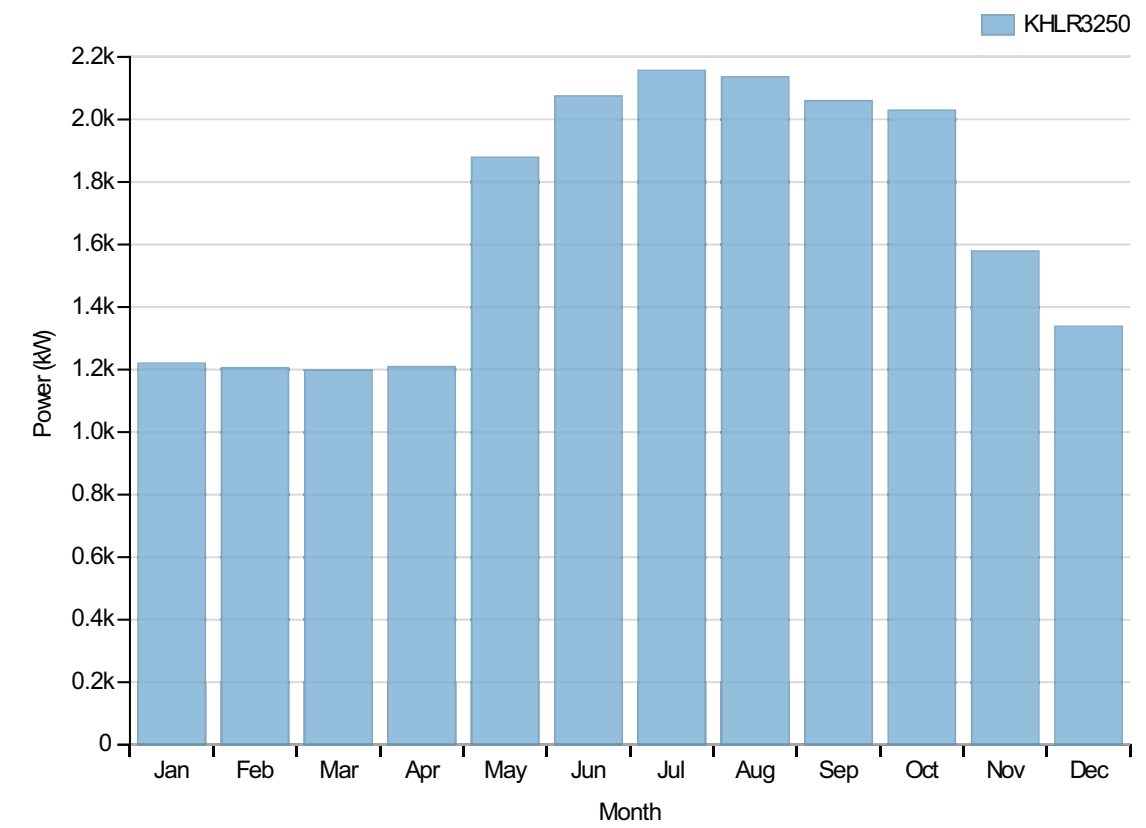

Generator:Kohler 3250 Prime Power

\begin{tabular}{|l|r|l|}
\hline Quantity & Value & Units \\
\hline Hours of operation & 7834 hrs/yr \\
\hline Number of starts & 694 starts/yr \\
\hline Operational life & 2 yr \\
\hline Fixed generation cost & 235.85 & $\$ / \mathrm{hr}$ \\
\hline Marginal generation cost & 0.20 & $\$ / \mathrm{kWh}$ \\
\hline Electrical production & $14686122 \mathrm{kWh} / \mathrm{yr}$ \\
\hline Mean electrical output & $1875 \mathrm{~kW}$ \\
\hline Min. electrical output & $813 \mathrm{~kW}$ \\
\hline Max. electrical output & $3250 \mathrm{~kW}$ \\
\hline Fuel consumption & $3480113 \mathrm{~L} / \mathrm{yr}$ \\
\hline Specific fuel consumption & $0.24 \mathrm{~L} / \mathrm{kWh}$ \\
\hline Fuel energy input & $34244312 \mathrm{kWh} / \mathrm{yr}$ \\
\hline Mean electrical efficiency & $43 \%$ \\
\hline
\end{tabular}

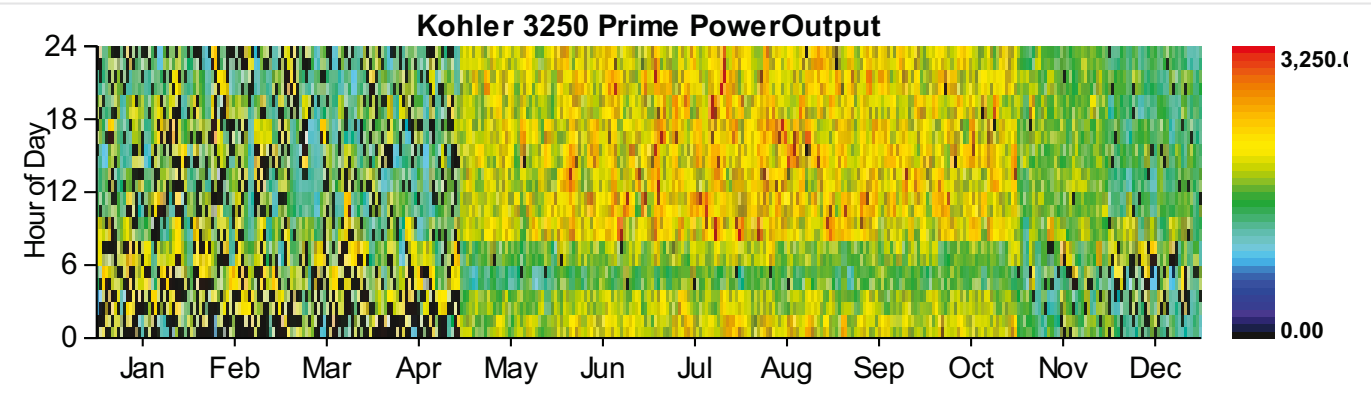




\section{Quantity}

Value

String size

Strings in parallel

Batteries

Bus voltage

\section{Quantity}

Nominal capacity

Usable nominal capacity

Autonomy

Lifetime throughput

Battery wear cost

$0.000 \$ / k W h$

Average energy cost

Energy in

Energy out

Value

Units

Storage depletion

$800171 \mathrm{kWh} / \mathrm{yr}$

Losses

$0 \mathrm{kWh} / \mathrm{yr}$

Annual throughput

$0.334 \$ / k W h$

$1143102 \mathrm{kWh} / \mathrm{yr}$

Expected life

$342931 \mathrm{kWh} / \mathrm{yr}$

956387 kWh/yr

$25 \mathrm{yr}$

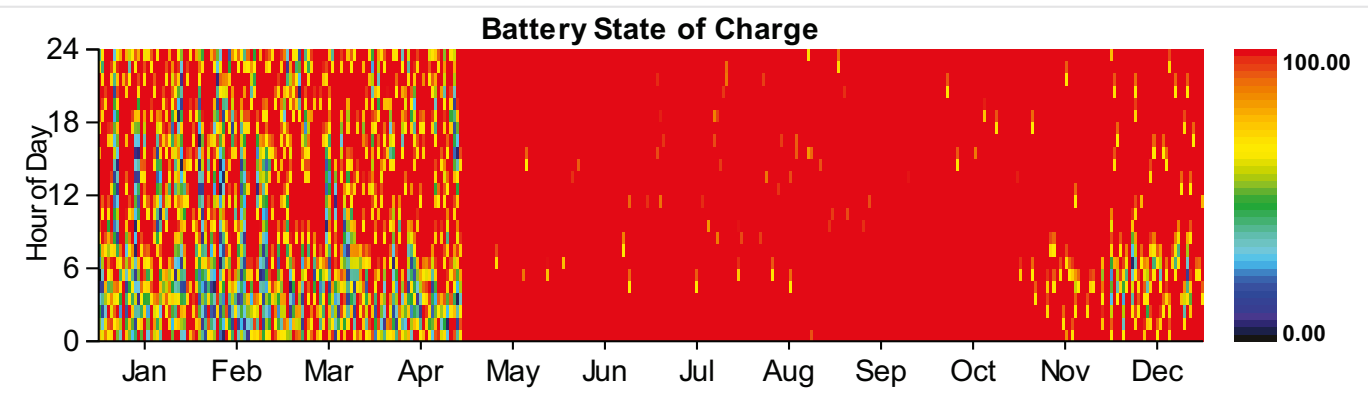

\section{Converter}

\section{Quantity}

Capacity

Mean output

Minimum output

Maximum output

Capacity factor

\begin{tabular}{|r|r|l|}
\hline Inverter & Rectifier & Units \\
\hline 1,000 & 900 & $\mathrm{~kW}$ \\
\hline 82 & 130 & $\mathrm{~kW}$ \\
\hline 0 & 0 & $\mathrm{~kW}$ \\
\hline 904 & 1,000 & $\mathrm{~kW}$ \\
\hline 8 & 13 & $\%$ \\
\hline
\end{tabular}




\begin{tabular}{|c|c|c|c|c|}
\hline Retarstiftyoperation & Inverter & Rectifier & 1,411 & Hritss \\
\hline Energy in & 800,171 & & $1,344,841$ & $\mathrm{kWh} / \mathrm{yr}$ \\
\hline Energy out & 720,153 & & $1,143,102$ & $\mathrm{kWh} / \mathrm{yr}$ \\
\hline Losses & 80,017 & & 201,739 & $\mathrm{kWh} / \mathrm{yr}$ \\
\hline
\end{tabular}
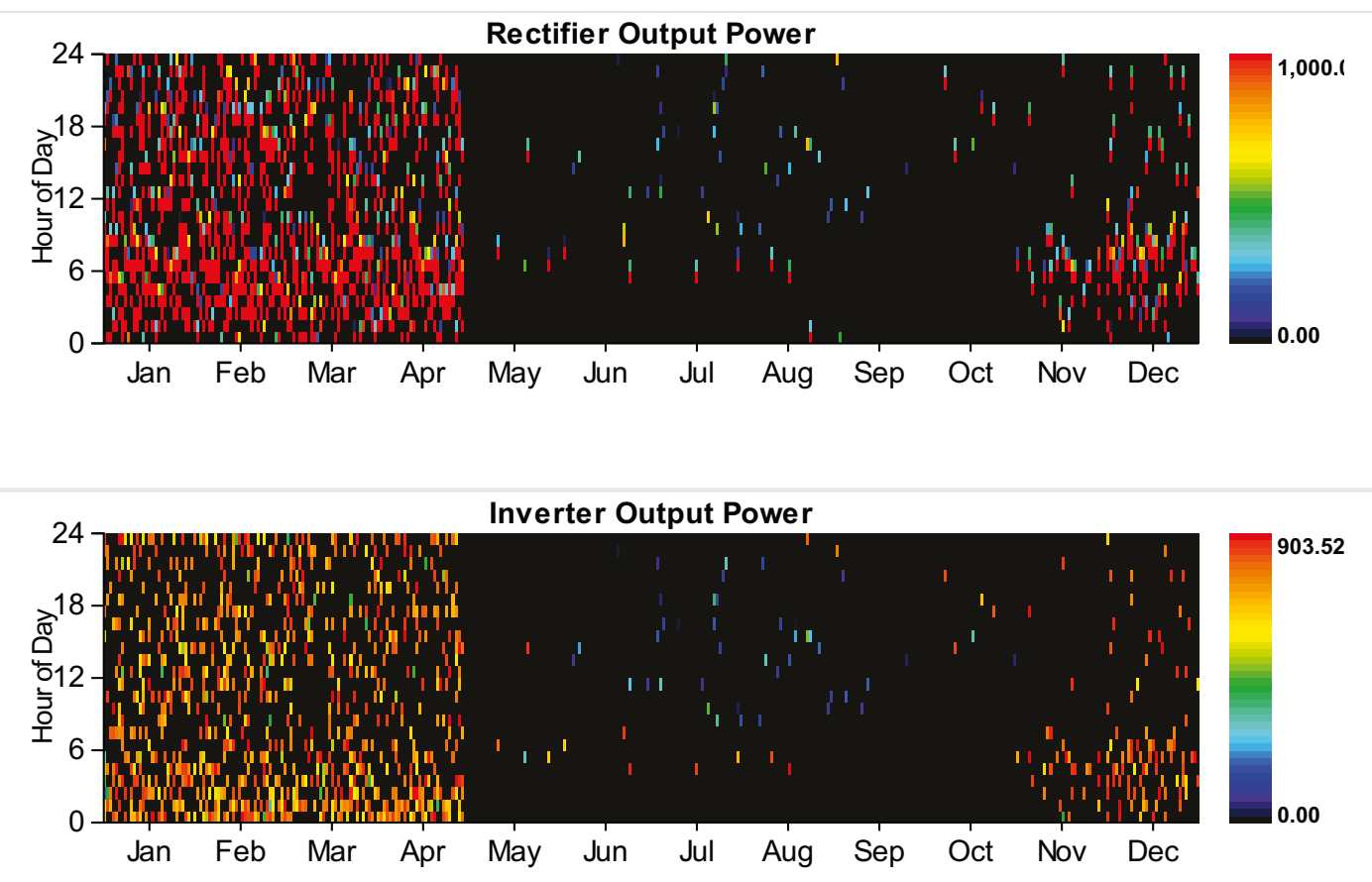

\section{Emissions}

\begin{tabular}{|l|r|l|}
\hline Pollutant & Emissions & Units \\
\hline Carbon dioxide & 9133608 & $\mathrm{~kg} / \mathrm{yr}$ \\
\hline Carbon monoxide & 38281 & $\mathrm{~kg} / \mathrm{yr}$ \\
\hline Unburned hydrocarbons & 4385 & $\mathrm{~kg} / \mathrm{yr}$ \\
\hline Particulate matter & 1096 \\
\hline Sulfur dioxide & $\mathrm{kg} / \mathrm{yr}$ \\
\hline Nitrogen oxides & 18805 & $\mathrm{~kg} / \mathrm{yr}$ \\
\hline
\end{tabular}

HOMER Energy, LLC @ 2016 
System Report

System architecture

\begin{tabular}{|l|l|r|}
\hline PV & Solar World 320W flat plate PV Copy & 3,000 \\
\hline Generator & Kohler 3250 Prime Power & 3,250 \\
\hline Converter & System Converter & 2,000 \\
\hline Dispatch Strategy & Cycle Charging & $\mathrm{kW}$ \\
\hline
\end{tabular}

\section{Cost summary}

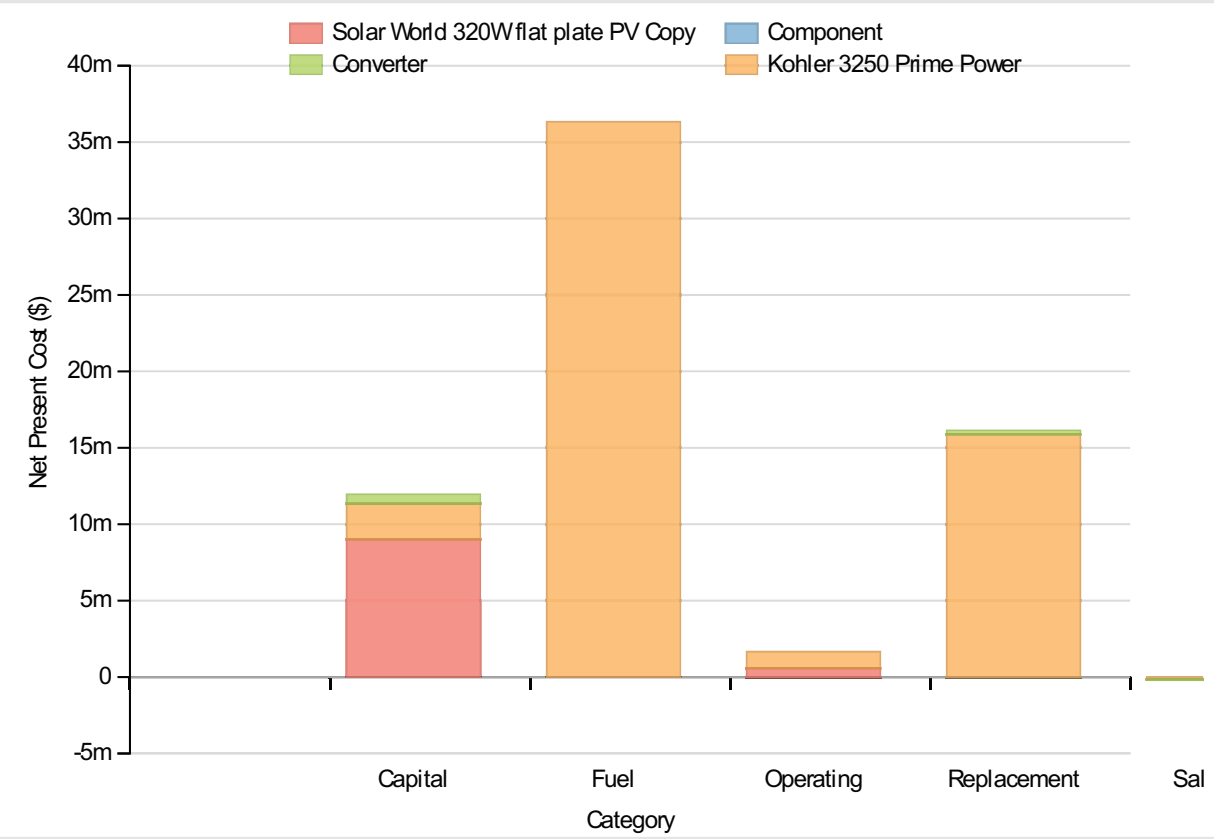

Cost Summary

Total net present cost

$65865580 \$$

Levelized cost of energy

$0.362 \$ / k W h$

\section{Net Present Costs}

\begin{tabular}{|l|r|r|r|r|r|r|}
\hline Component & Capital & Replacement & O\&M & Fuel & Salvage & Total \\
\hline Solar World 320W flat plate PV Copy & $9,000,000$ & 0 & 581,738 & 0 & 0 \\
\hline Kohler 3250 Prime Power & $2,346,591$ & $15,872,167$ & $1,077,743$ & $36,325,876$ & $-145,221$ & $55,477,156$ \\
\hline Converter & 600,000 & 254,564 & 0 & 0 & $-47,912$ \\
\hline System & & & 806,652 \\
\hline & $11,946,591$ & $16,126,731$ & $1,659,481$ & $36,325,876$ & $-193,132$ & $65,865,547$ \\
\hline
\end{tabular}

\section{Annualized Costs}

\begin{tabular}{|l|r|r|r|r|r|r|}
\hline Component & Capital & Replacement & O\&M & Fuel & Salvage & Total \\
\hline Solar World 320W flat plate PV Copy & 696,189 & 0 & 45,000 & 0 & 0 \\
\hline Kohler 3250 Prime Power & 181,519 & $1,227,782$ & 83,368 & $2,809,966$ & $-11,233$ & $4,291,402$ \\
\hline Converter & 46,413 & 19,692 & 0 & 0 & $-3,706$ \\
\hline
\end{tabular}




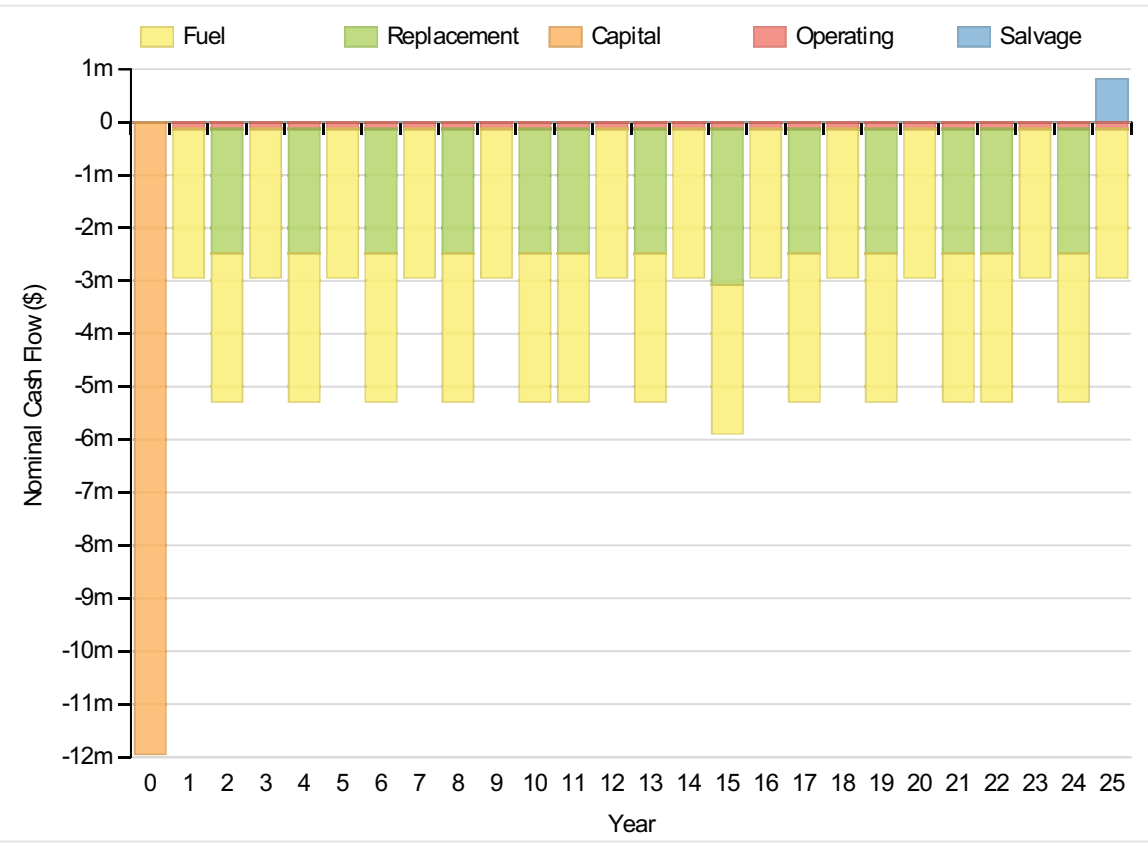

\section{Electrical}

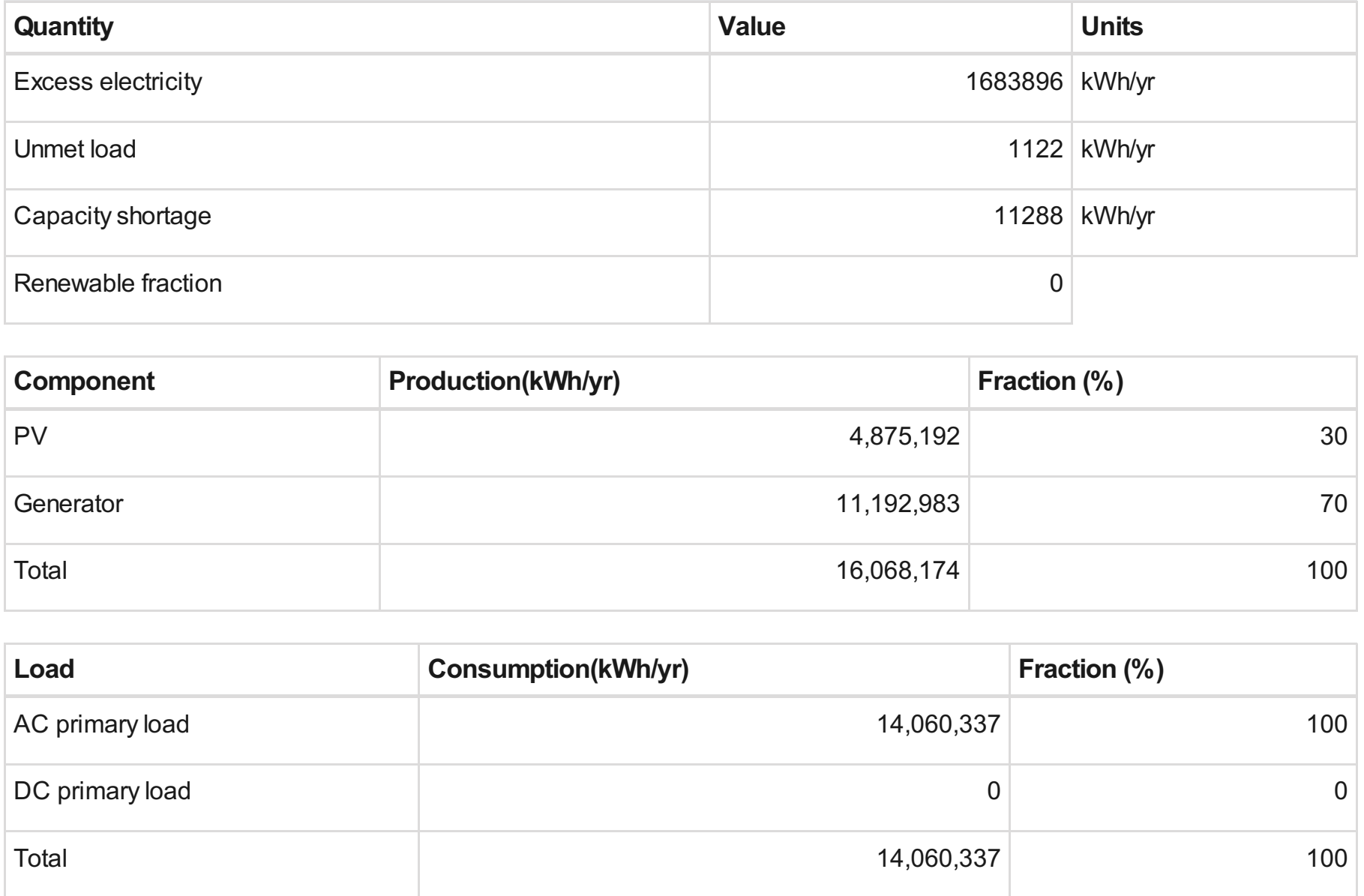




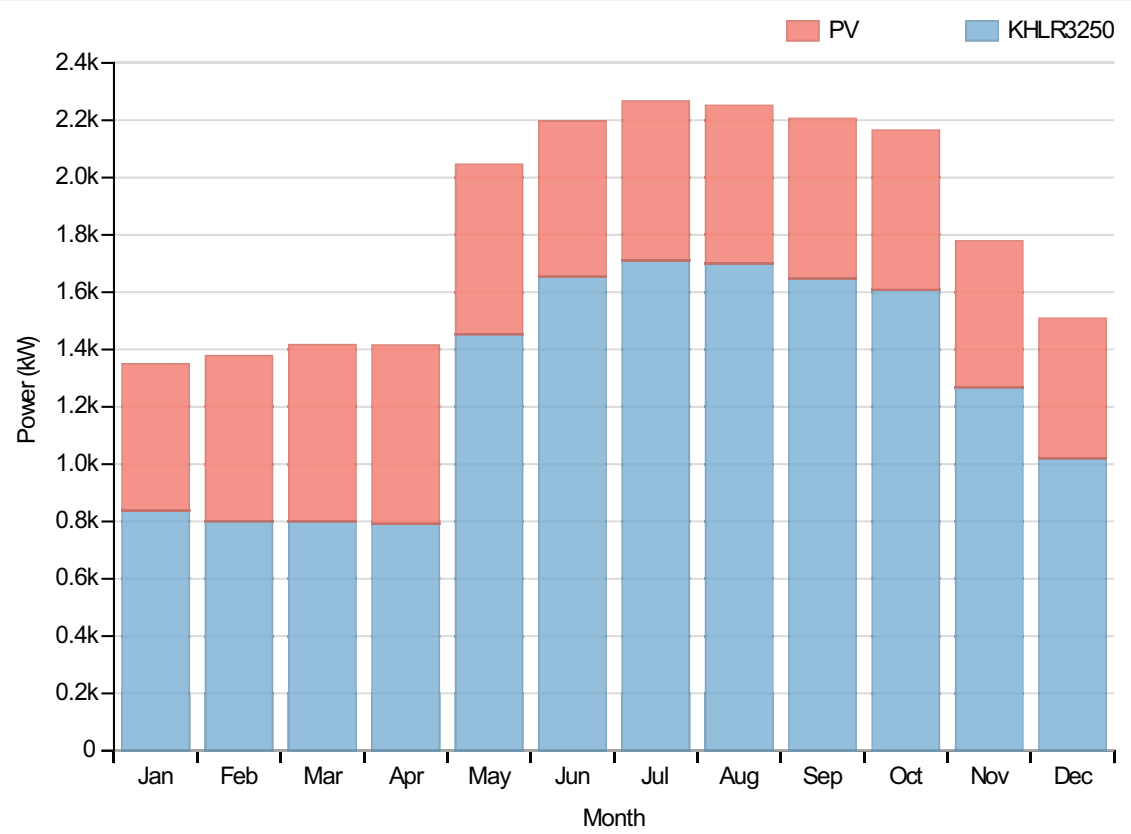

PV:Solar World 320W flat plate PV Copy

\begin{tabular}{|l|r|l|}
\hline Quantity & Value & Units \\
\hline Rated capacity & $3000 \mathrm{~kW}$ \\
\hline Mean output & $557 \mathrm{~kW}$ \\
\hline Mean output & $13357.00 \mathrm{kWh} / \mathrm{d}$ \\
\hline Capacity factor & $18.55 \%$ \\
\hline Total production & $4875192 \mathrm{kWh} / \mathrm{yr}$ \\
\hline Minimum output & $0.00 \mathrm{~kW}$ \\
\hline Maximum output & $2994.80 \mathrm{~kW}$ \\
\hline PV penetration & $34.67 \%$ \\
\hline Hours of operation & $4370 \mathrm{hrs} / \mathrm{yr}$ \\
\hline Levelized cost & $0.152 \mathrm{~S} / \mathrm{kWh}$ \\
\hline
\end{tabular}

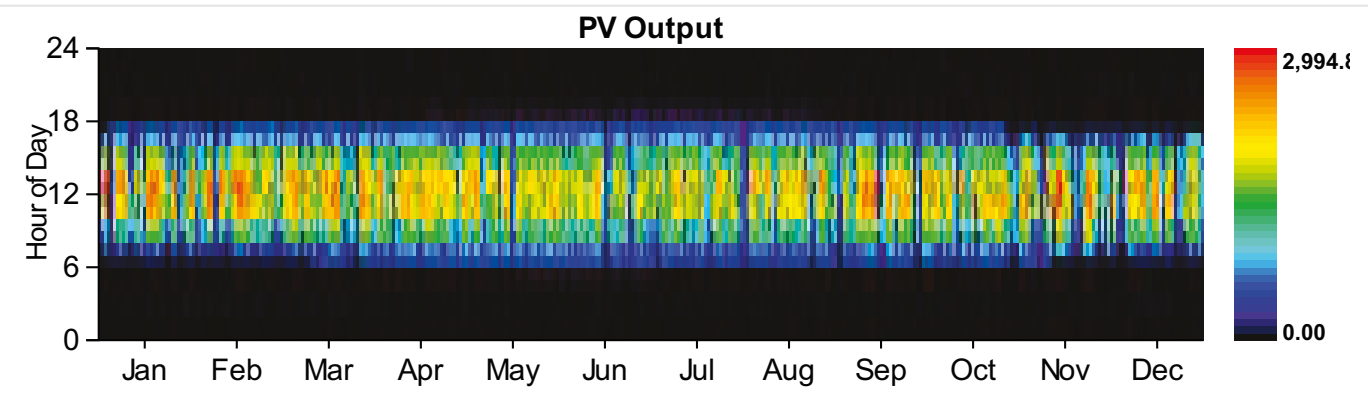

Generator:Kohler 3250 Prime Power

\section{Quantity}

Hours of operation

Number of starts

\section{Value}

\section{Units}

$8245 \mathrm{hrs} / \mathrm{yr}$

191 starts/yr 
Quaratitienal life

Fixed generation cost

Marginal generation cost

Electrical production

Mean electrical output

Min. electrical output

Max. electrical output

Fuel consumption

Specific fuel consumption

Fuel energy input

Mean electrical efficiency
2 Vnits

$235.85 \$ / h r$

$0.20 \$ / k W h$

11192983 kWh/yr

1358 kW

813 kW

$3250 \mathrm{~kW}$

2809968 L/yr

$0.25 \mathrm{~L} / \mathrm{kWh}$

$27650084 \mathrm{kWh} / \mathrm{yr}$

$40 \%$

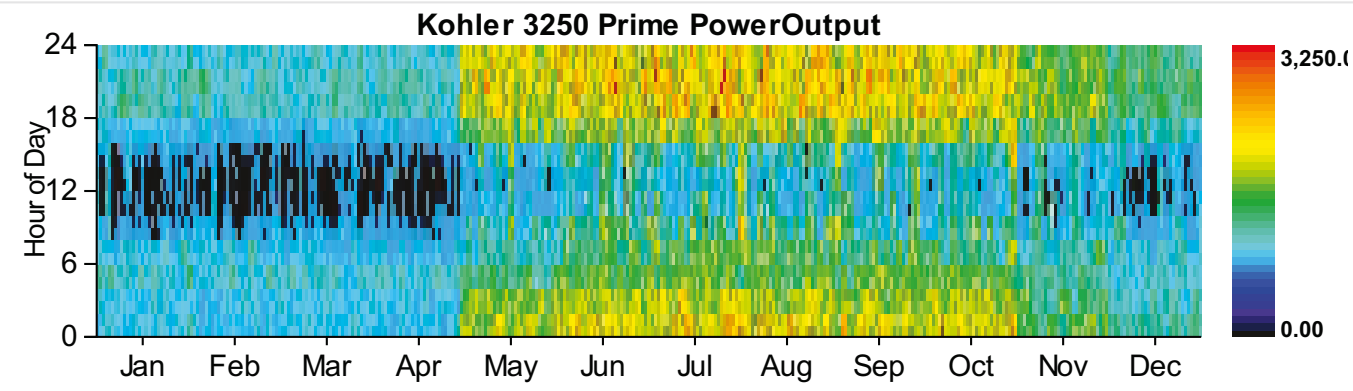

\section{Converter}

\begin{tabular}{|l|r|r|l|}
\hline Quantity & Inverter & Rectifier & Units \\
\hline Capacity & 2,000 & $1,800 \mathrm{~kW}$ \\
\hline Mean output & 333 & $0 \mathrm{~kW}$ \\
\hline Minimum output & 0 & $0 \mathrm{~kW}$ \\
\hline Maximum output & 2,000 & $0 \mathrm{~kW}$ \\
\hline Capacity factor & 17 & $0 \%$ \\
\hline Hours of operation & 4,247 & $0 \mathrm{hrs} / \mathrm{yr}$ \\
\hline Energy in & $3,241,253$ & $0 \mathrm{kWh} / \mathrm{yr}$ \\
\hline Energy out & $2,917,129$ & $0 \mathrm{kWh} / \mathrm{yr}$ \\
\hline Losses & 324,124 & $0 \mathrm{kWh} / \mathrm{yr}$ \\
\hline
\end{tabular}



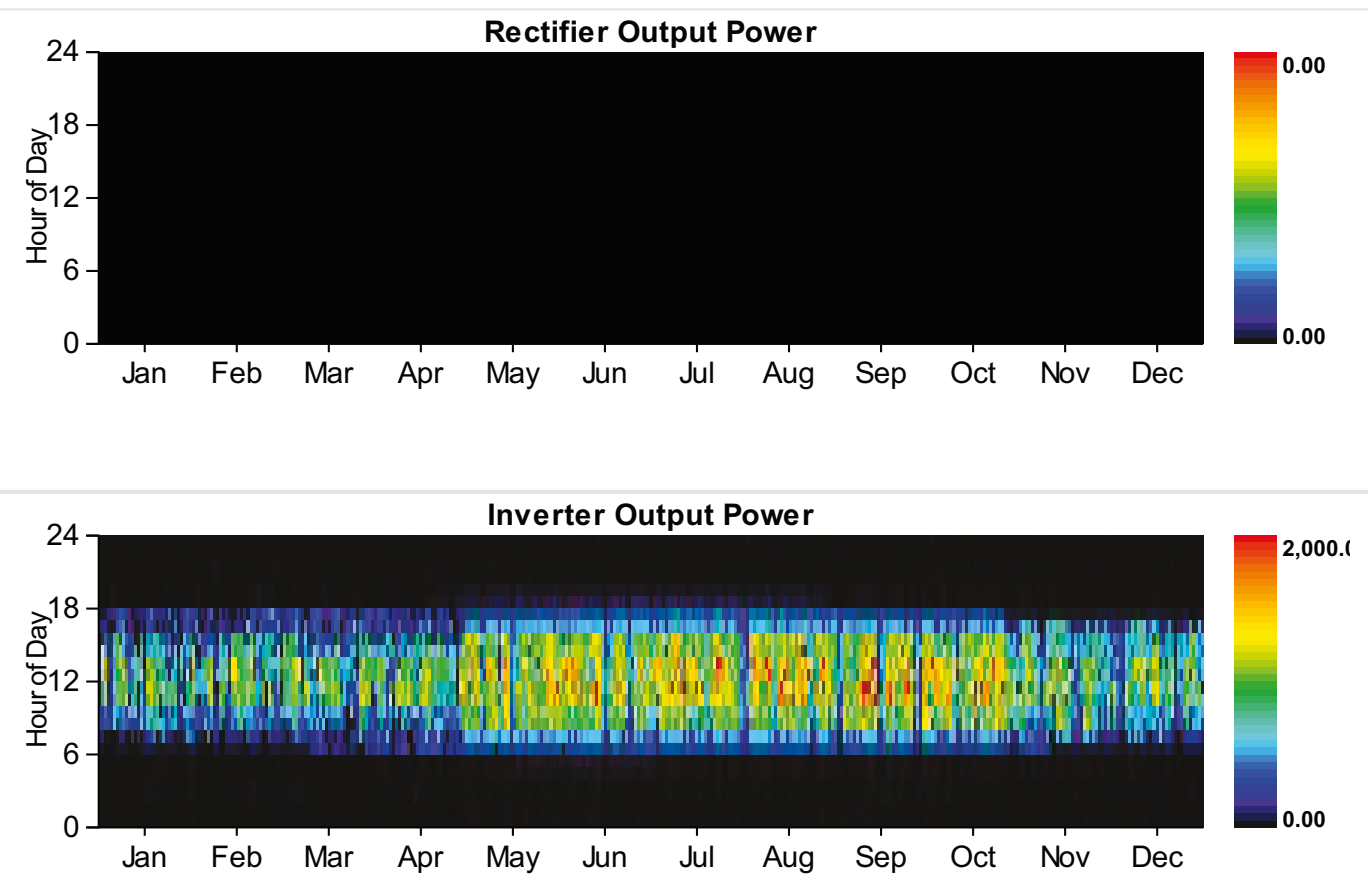

\section{Emissions}

\begin{tabular}{|l|r|l|}
\hline Pollutant & Emissions & Units \\
\hline Carbon dioxide & 7374803 & $\mathrm{~kg} / \mathrm{yr}$ \\
\hline Carbon monoxide & $30910 \mathrm{~kg} / \mathrm{yr}$ \\
\hline Unburned hydrocarbons & $3541 \mathrm{~kg} / \mathrm{yr}$ \\
\hline Particulate matter & $885 \mathrm{~kg} / \mathrm{yr}$ \\
\hline Sulfur dioxide & $15184 \mathrm{~kg} / \mathrm{yr}$ \\
\hline Nitrogen oxides & $30910 \mathrm{~kg} / \mathrm{yr}$ \\
\hline
\end{tabular}

HOMER Energy, LLC @ 2016 